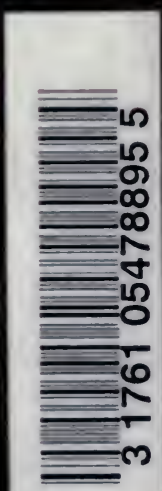

rANDARD FIELD TABLES

GENERAL LAND OFFICE

SEM

A

606 

A.E.NOLISE Tororto A4g.lII 

GENERAL LAND OFFICE

\section{TABLES AND FORMULAS}

FOR THE USE OF

U. S. SURVEYORS AND ENGINEERS ON

\section{PUBLIC LAND SURVEYS, \#}

A SUPPLEMENT TO THE MANUAL OF SURVEYING INSTRUCTIONS

COMPILED UNDER THE DIRECTION OF

FRED DENNETT

COMMISSIONER OF THE GENERAL LAND OFFICE

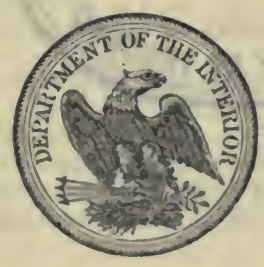

WASHINGTON

2) GOVERNMENT PRINTING OFfice \# 
United States surveyors and engineers, and all others who may find it convenient to use the tables included in this volume, are requested to report to the Commissioner of the General Land Office, Washington, D. C., any errors detected in the tables as printed.

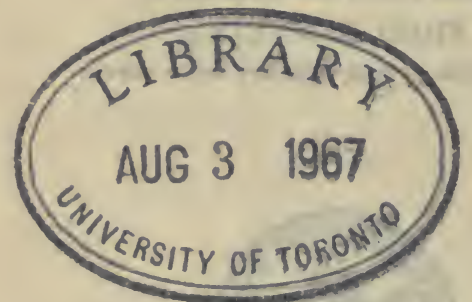




\section{CONTENTS.}

No.

1. Units of linear measure, units of area expansion of steel tapes, and conversion tables chains to feet and feet to chains.

2. Reduction in latitude to south boundary of township, and corrections for

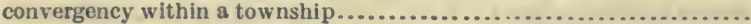

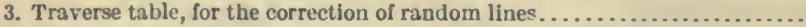

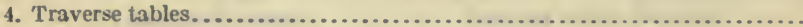

5. Correction of error in stadia wire interval . . . . . . . . . . . . . . . . . . . . . .

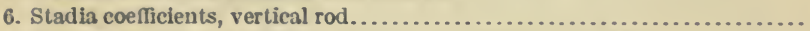

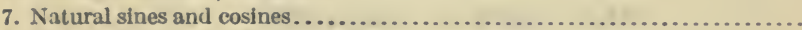

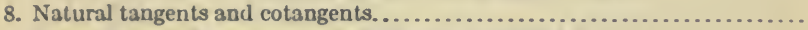

9. Logarithmic sines, cosines, tangents, and cotangents...................

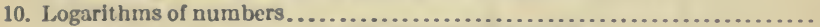

11. Convergency of meridians, and differences of latitude and longitude.......

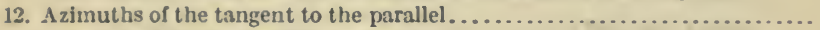

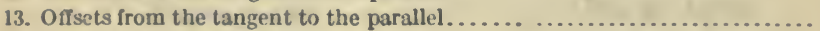

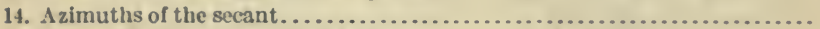

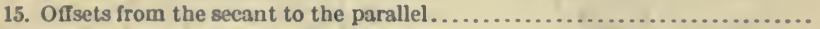

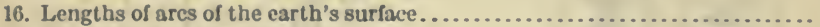

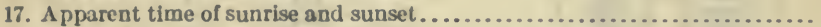

18. Conversion tables, degrees to time, and time to degrees.................

19. Sidereal conversions, and reductions to the local mean time of upper culmination of Polaris.

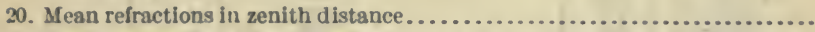

21. Coefficients to apply to mean refractions for variations in barometer and temperature.

22. Coefficients for computing errors in azimuth, due to small errors in declination or latitude.

23. Mean refractions in polar distance.

25. Trigonometric formulas for the solution of stadia measurements, observations for time, latitude, and azimuth, and problems in convergency..... 


\section{To. TYYY}

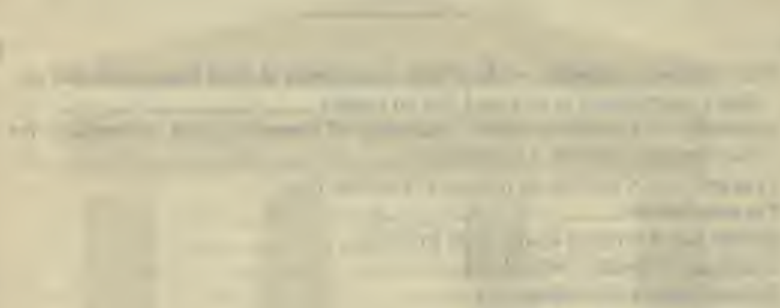

Digitized by the Internet Archive in 2007 with funding from Microsoft Corporation 


\section{Standard Field Tables.}

TABLE 1.-UNITS OF MEASURE, ETC.

Units of Linear Measure.

$$
\begin{aligned}
1 \text { chain } & =100 \text { links. } \\
& =66 \text { feet. } \\
1 \text { mile } & =80 \text { chains. } \\
& =5,250 \text { feet. }
\end{aligned}
$$

Units of AREA.

$$
\begin{aligned}
1 \text { acre } & =10 \text { square chains. } \\
& =43,560 \text { square feet. } \\
1 \text { square mile } & =640 \text { acres. }
\end{aligned}
$$

\section{Expansion of Steel Tapes.}

The coefficient of expansion of steel tapes $=0.0000065$ for $1^{\circ} \mathrm{F}$.

\section{Conversion Tables.}

Chains to feet.

\begin{tabular}{|r|r|}
\hline 1 & 66 \\
2 & 132 \\
3 & 198 \\
4 & 264 \\
5 & 330 \\
6 & 396 \\
7 & 462 \\
8 & 528 \\
9 & 594 \\
10 & 660 \\
\hline
\end{tabular}

Links to feet.

\begin{tabular}{|r|l|}
\hline 1 & 0.66 \\
2 & 1.32 \\
3 & 1.98 \\
4 & 2.64 \\
5 & 3.30 \\
6 & 3.96 \\
7 & 4.62 \\
8 & 5.28 \\
9 & 5.94 \\
10 & 6.60 \\
\hline
\end{tabular}

Feet to chains.

\begin{tabular}{|r|r|}
\hline 100 & 1.51515 \\
200 & 3.03030 \\
300 & 4.54546 \\
400 & 6.06061 \\
500 & 7.57576 \\
600 & 9.09091 \\
700 & 10.60606 \\
800 & 12.12121 \\
900 & 13.63636 \\
1,000 & 15.15152 \\
\hline
\end{tabular}

Feet to links.

\begin{tabular}{|r|r|}
\hline 1 & 1.5 \\
2 & 3.0 \\
3 & 4.5 \\
4 & 6.1 \\
5 & 7.6 \\
6 & 9.1 \\
7 & 10.6 \\
8 & 12.1 \\
9 & 13.6 \\
10 & 15.2 \\
\hline
\end{tabular}


TABI.E 2.-REDUCTION IN LATITLDF TO SOITI BOUNDARY OF TOWNSIIP.

\begin{tabular}{|c|c|c|c|c|c|c|}
\hline \multirow{2}{*}{ Chs. } & \multicolumn{6}{|c|}{ Miles. } \\
\hline & 0 & 1 & 2 & 3 & 4 & 5 \\
\hline & , & , & , & , & , & , \\
\hline 0 & 0.0 & 0.9 & 1.7 & 2.6 & 3.5 & 4.3 \\
\hline 10 & 0.1 & 1.0 & 1.8 & 2.7 & 3.6 & 4. 4 \\
\hline 20 & 0.2 & 1.1 & 2.0 & 2.8 & 3.7 & 4.6 \\
\hline 30 & 0.3 & 1.2 & 2.1 & 2.9 & 3.8 & 4.7 \\
\hline 40 & 0.4 & 1.3 & 2.2 & 3.0 & 3.9 & 4.8 \\
\hline 50 & 0.5 & 1.4 & 2.3 & 3.1 & 4.0 & 4.9 \\
\hline 60 & 0.6 & 1.5 & 2.4 & 3.2 & 4.1 & 5.0 \\
\hline 70 & 0.8 & 1.6 & 2.5 & 3.4 & 4.2 & 5.1 \\
\hline 80 & 0.9 & 1.7 & 2.6 & 3.5 & 4.3 & 5.2 \\
\hline
\end{tabular}

\section{CORRECTIONS FOI CONVERGENCY WITHIN A TOWNSHIP.}

\begin{tabular}{|c|c|c|c|c|c|}
\hline Lat. & $1 \mathrm{mi}$ & $2 \mathrm{mi}$ & $3 \mathrm{mi}$. & $4 \mathrm{mi}$. & $5 \mathrm{mi}$. \\
\hline $\begin{array}{l}- \\
25 \text { to } 30 \\
30 \text { to } 35 \\
35 \text { to } 40 \\
40 \text { to } 45 \\
45 \text { to } 50 \\
50 \text { to } 55 \\
55 \text { to } 60 \\
60 \text { to } 65 \\
65 \text { to } 70\end{array}$ & $\begin{array}{l}1 \\
0 \\
1 \\
1 \\
1 \\
1 \\
1 \\
1 \\
1 \\
2 \\
2\end{array}$ & $\begin{array}{l}1 \\
1 \\
1 \\
1 \\
2 \\
2 \\
2 \\
2 \\
3 \\
3 \\
4\end{array}$ & $\begin{array}{l}1 \\
1 \\
2 \\
2 \\
2 \\
3 \\
3 \\
3 \\
4 \\
5 \\
6\end{array}$ & $\begin{array}{l}i \\
2 \\
2 \\
3 \\
3 \\
4 \\
5 \\
5 \\
5 \\
7 \\
8\end{array}$ & $\begin{array}{r} \\
2 \\
2 \\
3 \\
3 \\
4 \\
4 \\
5 \\
6 \\
7 \\
8 \\
8 \\
10\end{array}$ \\
\hline
\end{tabular}


TABLE 3.-TRIVERE TABLF FOR TIE COREECTION OF RANDOM IINES.

\begin{tabular}{|c|c|c|c|c|c|c|c|c|c|c|c|}
\hline \multirow[b]{3}{*}{$0^{\circ}$, } & \multicolumn{10}{|c|}{ Distance In chains. } & \\
\hline & 10 & 20 & 30 & 10 & 50 & 60 & 70 & $8 !$ & 90 & 100 & \\
\hline & \multicolumn{10}{|c|}{ Departure in links. } & \\
\hline 0 & 0.0 & 0.0 & 0.0 & 0.0 & 0.0 & 0.0 & 0.0 & 0.0 & 0.0 & 0.0 & 60 \\
\hline 1 & 0.3 & 0.6 & 0.9 & 1.2 & 1.5 & 1.7 & 2.0 & 2.3 & 2.6 & 2.9 & 59 \\
\hline 2 & 0.6 & 1.2 & 1.7 & 2.3 & 2.9 & 3.5 & 4. 1 & 4.7 & 5.2 & 5.8 & 58 \\
\hline 3 & 0.9 & 1.7 & 2.6 & 3.5 & 4.4 & 5.2 & 6.1 & 7.0 & 7.9 & 8.7 & 57 \\
\hline 4 & 1.2 & 2.3 & 3.5 & 4.7 & 5.8 & 7.0 & 8.1 & 9.3 & 10.5 & 11.6 & 56 \\
\hline 5 & 1.5 & 2.9 & 4. 4 & 5.8 & 7.3 & 8.7 & 10.2 & 11.6 & 13.1 & 11.5 & 55 \\
\hline 6 & 1.7 & 3.5 & 5.2 & 7.0 & 8.7 & 10.5 & 12.2 & 14.0 & 15.7 & 17.5 & 54 \\
\hline 7 & 2.0 & 4.1 & 6.1 & 8.1 & 10.2 & 12.2 & 14.3 & 16.3 & 18.3 & 20.4 & 53 \\
\hline 8 & 2.3 & 4.7 & 7.0 & 9.3 & 11.6 & 14.0 & 16.3 & 18.6 & 20.9 & 23.3 & 52 \\
\hline 9 & 2.6 & 5.2 & 7.9 & 10.5 & 13.1 & 15.7 & 18.3 & 20.9 & 23.6 & 26.2 & 51 \\
\hline 10 & 2.9 & 5. 8 & 8.7 & 11.6 & 14.5 & 17.5 & 20.4 & 23.3 & 26.2 & 29.1 & 53 \\
\hline 11 & 3.2 & 6.4 & 9.6 & 12.8 & 16.0 & 19.2 & 22.4 & 25.6 & 28.8 & 32.0 & 49 \\
\hline 12 & 3.5 & 7.0 & 10.5 & 14.0 & 17.5 & 20.9 & 24.4 & 27.9 & 31.4 & 34.9 & 4.9 \\
\hline 13 & 3.8 & 7.6 & 11.3 & 15.1 & 18.9 & 22.7 & 26.5 & 30.3 & 34.0 & 37.8 & 47 \\
\hline $1 \pm$ & 4.1 & 8.1 & 12.2 & 16.3 & 20.4 & 24.4 & 28.5 & 32.6 & 36.7 & 40.7 & 46 \\
\hline 15 & 4.4 & 8.7 & 13.1 & 17.5 & 21.8 & 26.2 & 30.5 & 34.9 & 39.3 & 43. 6 & 45 \\
\hline 16 & 4.7 & 9.3 & 14.0 & 18. 6 & 23.3 & 27.9 & 32.6 & 37.2 & 41.9 & 46.5 & 44 \\
\hline 17 & 4.9 & 9.9 & 14.8 & 19.8 & 24.7 & 29.7 & 34.6 & 39.6 & 44.5 & 49.5 & 43 \\
\hline is' & 5.2 & 10.5 & 15.7 & 20.9 & 21. 2 & 31.4 & 36.7 & 41.9 & 47.1 & 52.4 & 42 \\
\hline 19 & $5 . \overline{5}$ & 11.1 & 16.6 & 22.1 & 27. 6 & 33.2 & 38.7 & 44.2 & 49.7 & 55.3 & 41 \\
\hline 20 & 5.8 & 11.6 & 17.5 & 23.3 & 23.1 & 34.9 & 40.7 & 46.5 & 52.4 & 58.2 & 40 \\
\hline 21 & 6.1 & 12. 2 & 18. 3 & 24.4 & 30. & 36.7 & 42.8 & 45.9 & 55.0 & 61.1 & 39 \\
\hline 22 & 6.4 & 12.8 & 19.2 & 25. 6 & 32.0 & 38.4 & 44.8 & 51.2 & 57.6 & 64.0 & 38 \\
\hline 23 & 6.7 & 13.4 & 20.1 & 26.8 & 33.5 & 40. 1 & 46.8 & 53.5 & 60.2 & 66.9 & 37 \\
\hline 24 & 7.0 & 14.0 & 20.9 & 27.0 & 34.9 & 41.9 & 48.9 & 55.9 & 62.8 & 69.8 & 36 \\
\hline 25 & 7.3 & 14.5 & 21.8 & 29.1 & 36.4 & 43. 6 & 50.9 & 58.2 & 65.4 & 72.7 & 35 \\
\hline 26 & 7.6 & 15.1 & 22.7 & 30.3 & 37.8 & 45.4 & 52.9 & 60.5 & 68.1 & 75.6 & 34 \\
\hline $2 \vec{i}$ & 7.9 & 15.7 & 23.6 & 31.4 & 39.3 & 47.1 & 55.0 & 62.8 & 70.7 & 78.5 & 33 \\
\hline 28 & 8.1 & 16.3 & 24.4 & 32.6 & 40.7 & 48.9 & 57.0 & 65.2 & 73.3 & 81.4 & 32 \\
\hline 29 & 8.4 & 16.9 & 25.3 & 33.7 & 42.2 & 50). 6 & 59.0 & 67.5 & 75.9 & 84.4 & 31 \\
\hline 30 & 8.7 & 17.5 & 26.2 & 34.9 & 43. 6 & 52.4 & 61.1 & 69.8 & 78.5 & 87.3 & 30 \\
\hline 31 & 9.0 & 18.0 & 27.1 & 36.1 & 45.1 & 54.1 & 63.1 & 72.1 & 81.2 & 90.2 & 29 \\
\hline 32 & 9.3 & 18.6 & 27.9 & 37.2 & 46.5 & 55.8 & 65.2 & 74.5 & 83.8 & 93.1 & 28 \\
\hline 33 & 9.6 & 19.2 & $2 \mathrm{~S} .8$ & 38.4 & 48.0 & 57.6 & 67.2 & 76.8 & 86.4 & 96.0 & 27 \\
\hline 34 & 9.9 & 19.8 & 29.7 & 39.6 & 49.5 & 59.3 & 69.2 & 79.1 & 89.0 & 98.9 & 26 \\
\hline 35 & 10.2 & 20.4 & 30.5 & 40.7 & 50.0 & 61.1 & 71.3 & 81.4 & 91.6 & 101.8 & 25 \\
\hline 36 & 10.5 & 20.9 & 31.4 & 41.9 & 52.4 & 62.8 & 73.3 & 83.8 & 94.2 & 104.7 & 24 \\
\hline 37 & 10.8 & 21.5 & 32.3 & 43. 1 & 53.8 & 64.6 & 75.3 & 86.1 & 96.9 & 107.6 & 23 \\
\hline 38 & 11.1 & 22.1 & 33.2 & 44. 2 & 55.3 & 66.3 & 77.4 & 8.9 .4 & 99.5 & 110.5 & 22 \\
\hline 39 & 11.3 & 22.7 & 34.0 & 45.4 & 56.7 & 68.1 & 79.4 & 90.8 & 102.1 & 113.4 & 21 \\
\hline 40 & 11.6 & 23.3 & 34.9 & 46.5 & 58.2 & 69.8 & 81.4 & 93.1 & 104.7 & 1115.4 & 20 \\
\hline 41 & 11.9 & 23.9 & 35.8 & 47.7 & 59.6 & 71.6 & \$3. 5 & 95.4 & 107.3 & 119.3 & 19 \\
\hline 42 & 12.2 & 24.4 & 36.7 & 48.9 & (i1.1 & 73. 3 & 8.. 5 & 97.7 & 110.0 & 122.2 & 18 \\
\hline 43 & 12.5 & 25.0 & 37.5 & 50.0 & 62.5 & 75.0 & 87.6 & 100.1 & 112.6 & 125.1 & 17 \\
\hline 41 & 12.8 & 25.6 & $3 S .4$ & 51.2 & 64.0 & 76.8 & 89.6 & 102.4 & 115.2 & 128.0 & 16 \\
\hline 45 & 13.1 & 26.2 & 39.3 & 52.4 & 65.4 & 78.5 & 91.6 & 104.7 & 117.8 & 130.9 & 15 \\
\hline 46 & 13.4 & $2 f .8$ & 40.1 & 53.5 & 66.9 & 80.3 & 93.7 & 107.0 & 120.4 & 133.8 & 14 \\
\hline 47 & 13.7 & 27.3 & 41.0 & 54.7 & 69.4 & 82.0 & 95.7 & 109.4 & 123.0 & 136.7 & 13 \\
\hline 48 & 14.0 & 27.9 & 41.9 & 5.5 .8 & 69.8 & 83.8 & 97.7 & 111.7 & 135.7 & มะว. 6 & 12 \\
\hline 49 & 14.3 & 28.5 & 42.8 & 57.0 & 71.3 & 85.5 & 99.8 & 114.0 & 128.3 & 142.5 & 11 \\
\hline 50 & 14.5 & 29.1 & 43.6 & 58.2 & 72.7 & 87.3 & 101.8 & 116.4 & 130.9 & 145.4 & 10 \\
\hline 51 & 14.8 & 29.7 & 44.5 & 59.3 & 74.2 & 89.0 & 103. S & 118. 7 & 133.5 & 148.3 & 9 \\
\hline 52 & 15.1 & 30. 3 & 45.4 & 60.5 & 75.6 & 90.8 & 105.9 & 121.0 & 136.1 & 101.3 & 8 \\
\hline 53 & 15. 4 & 30.8 & 46.2 & 61.7 & 77.1 & 92.5 & 107.9 & 123.3 & 138.7 & 154.2 & 7 \\
\hline 54 & 15.7 & 31.4 & 47.1 & 62.8 & 78.5 & 94.2 & 110.0 & 125.7 & 141.4 & 157.1 & 6 \\
\hline 55 & 16.0 & 32.0 & 48.0 & 64.0 & 80.0 & 96.0 & 112.0 & 125.0 & 144.0 & 160.0 & 5 \\
\hline 56 & 16.3 & 32.6 & 49. 9 & 65. 2 & 81.4 & 97.7 & 114.0 & 130.3 & 146.6 & 102.9 & 4 \\
\hline 57 & 16.6 & 33.2 & 49.7 & 66.3 & 82.9 & 99.5 & 116.1 & 132.6 & 149.2 & $165 . \mathrm{S}$ & 3 \\
\hline 58 & 16.9 & 33.7 & 50. 6 & 67.5 & 84.4 & 101.2 & 118.1 & 135.0 & 151.8 & $1 \mathrm{RS} .7$ & 2 \\
\hline & 17.2 & 34.3 & 51.3 & 69.6 & 85.8 & 103.0 & 120.1 & 137.3 & 154.5 & 171.6 & 1 \\
\hline \multirow[t]{2}{*}{60} & 17.5 & 34.9 & 52.4 & 69.8 & 87.3 & 104.7 & 122.2 & 139.6 & 157.1 & 174.5 & 0 \\
\hline & \multicolumn{10}{|c|}{ Latitude in links. } & $89^{\circ}$ \\
\hline
\end{tabular}


TABLE 4.-TRAVERSE TABLE.

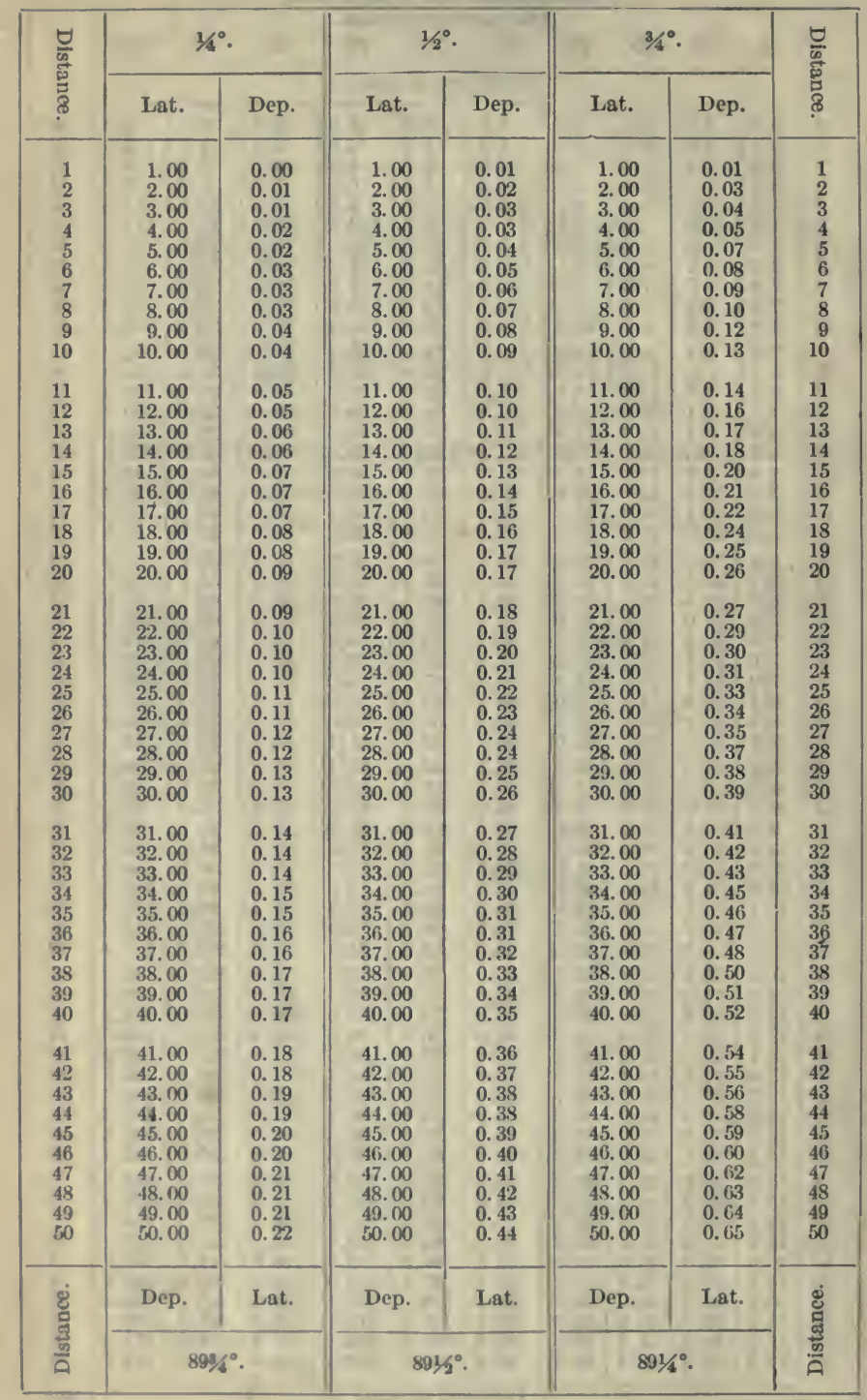


TABLE 4.-TRAVERSE TABLE.

\begin{tabular}{|c|c|c|c|c|c|c|c|}
\hline \multirow{2}{*}{ 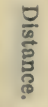 } & \multicolumn{2}{|c|}{$1 / 4^{\circ}}$. & \multicolumn{2}{|c|}{$1 / 2^{\circ}}$. & \multicolumn{2}{|c|}{$34^{\circ}}$. & \multirow{2}{*}{ 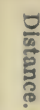 } \\
\hline & Lat. & Dep. & Lat. & Dep. & Lat. & Dep. & \\
\hline $\begin{array}{l}51 \\
52 \\
53 \\
54 \\
55 \\
56 \\
57 \\
58 \\
59 \\
60\end{array}$ & $\begin{array}{l}51.00 \\
52.00 \\
53.00 \\
54.00 \\
55.00 \\
56.00 \\
57.00 \\
58.00 \\
59.00 \\
60.00\end{array}$ & $\begin{array}{l}0.22 \\
0.23 \\
0.23 \\
0.24 \\
0.24 \\
0.24 \\
0.25 \\
0.25 \\
0.26 \\
0.26\end{array}$ & $\begin{array}{l}51.00 \\
52.00 \\
53.00 \\
54.00 \\
55.00 \\
56.00 \\
57.00 \\
58.00 \\
59.00 \\
60.00\end{array}$ & $\begin{array}{l}0.45 \\
0.45 \\
0.46 \\
0.47 \\
0.48 \\
0.49 \\
0.50 \\
0.51 \\
0.51 \\
0.52\end{array}$ & $\begin{array}{l}51.00 \\
52.00 \\
53.00 \\
54.00 \\
55.00 \\
56.00 \\
57.00 \\
57.99 \\
58.99 \\
59.99\end{array}$ & $\begin{array}{l}0.67 \\
0.68 \\
0.69 \\
0.71 \\
0.72 \\
0.73 \\
0.75 \\
0.76 \\
0.77 \\
0.79\end{array}$ & $\begin{array}{l}51 \\
52 \\
53 \\
54 \\
55 \\
56 \\
57 \\
58 \\
59 \\
60\end{array}$ \\
\hline $\begin{array}{l}61 \\
62 \\
63 \\
64 \\
65 \\
66 \\
67 \\
68 \\
69 \\
70\end{array}$ & $\begin{array}{l}61.00 \\
62.00 \\
63.00 \\
64.00 \\
65.00 \\
66.00 \\
67.00 \\
68.00 \\
69.00 \\
70.00\end{array}$ & $\begin{array}{l}0.27 \\
0.27 \\
0.27 \\
0.28 \\
0.28 \\
0.29 \\
0.29 \\
0.30 \\
0.30 \\
0.31\end{array}$ & $\begin{array}{l}61.00 \\
62.00 \\
63.00 \\
64.00 \\
65.00 \\
66.00 \\
67.00 \\
68.00 \\
69.00 \\
70.00\end{array}$ & $\begin{array}{l}0.53 \\
0.54 \\
0.55 \\
0.56 \\
0.57 \\
0.58 \\
0.58 \\
0.59 \\
0.60 \\
0.61\end{array}$ & $\begin{array}{l}60.99 \\
61.99 \\
62.99 \\
63.99 \\
64.99 \\
65.99 \\
66.99 \\
67.99 \\
68.99 \\
69.99\end{array}$ & $\begin{array}{l}0.80 \\
0.81 \\
0.82 \\
0.84 \\
0.85 \\
0.86 \\
0.88 \\
0.89 \\
0.30 \\
0.92\end{array}$ & $\begin{array}{l}61 \\
62 \\
63 \\
64 \\
65 \\
66 \\
67 \\
68 \\
69 \\
70\end{array}$ \\
\hline $\begin{array}{l}71 \\
72 \\
73 \\
74 \\
75 \\
76 \\
77 \\
78 \\
79 \\
80\end{array}$ & $\begin{array}{l}71.00 \\
72.00 \\
73.00 \\
74.00 \\
75.00 \\
76.00 \\
77.00 \\
78.00 \\
79.00 \\
80.00\end{array}$ & $\begin{array}{l}0.31 \\
0.31 \\
0.32 \\
0.32 \\
0.33 \\
0.33 \\
0.34 \\
0.34 \\
0.34 \\
0.35\end{array}$ & $\begin{array}{l}71.00 \\
72.00 \\
73.00 \\
74.00 \\
75.00 \\
76.00 \\
77.00 \\
78.00 \\
79.00 \\
80.00\end{array}$ & $\begin{array}{l}0.62 \\
0.63 \\
0.64 \\
0.65 \\
0.65 \\
0.66 \\
0.67 \\
0.68 \\
0.69 \\
0.70\end{array}$ & $\begin{array}{l}70.99 \\
71.99 \\
72.99 \\
73.99 \\
74.99 \\
75.99 \\
76.99 \\
77.99 \\
78.99 \\
79.99\end{array}$ & $\begin{array}{l}0.93 \\
0.94 \\
0.96 \\
0.97 \\
0.98 \\
0.99 \\
1.01 \\
1.02 \\
1.03 \\
1.05\end{array}$ & $\begin{array}{l}71 \\
72 \\
73 \\
74 \\
75 \\
76 \\
77 \\
78 \\
79 \\
80\end{array}$ \\
\hline $\begin{array}{l}81 \\
82 \\
83 \\
84 \\
85 \\
86 \\
87 \\
88 \\
89 \\
90\end{array}$ & $\begin{array}{l}81.00 \\
82.00 \\
83.00 \\
84.00 \\
85.00 \\
86.00 \\
87.00 \\
85.00 \\
89.00 \\
90.00\end{array}$ & $\begin{array}{l}0.35 \\
0.36 \\
0.36 \\
0.37 \\
0.37 \\
0.38 \\
0.38 \\
0.39 \\
0.39 \\
0.39\end{array}$ & $\begin{array}{l}81.00 \\
82.00 \\
83.00 \\
84.00 \\
85.00 \\
88.00 \\
87.00 \\
88.00 \\
89.00 \\
90.00\end{array}$ & $\begin{array}{l}0.71 \\
0.72 \\
0.72 \\
0.73 \\
0.74 \\
0.75 \\
0.76 \\
0.77 \\
0.78 \\
0.79\end{array}$ & $\begin{array}{l}80.99 \\
81.99 \\
82.99 \\
83.99 \\
81.99 \\
85.99 \\
86.99 \\
87.99 \\
88.99 \\
89.99\end{array}$ & $\begin{array}{l}1.06 \\
1.07 \\
1.09 \\
1.10 \\
1.11 \\
1.13 \\
1.14 \\
1.15 \\
1.16 \\
1.18\end{array}$ & $\begin{array}{l}81 \\
82 \\
83 \\
81 \\
85 \\
86 \\
87 \\
88 \\
89 \\
90\end{array}$ \\
\hline $\begin{array}{r}91 \\
92 \\
93 \\
94 \\
95 \\
96 \\
97 \\
98 \\
99 \\
100\end{array}$ & $\begin{array}{r}91.00 \\
92.00 \\
93.00 \\
94.00 \\
95.00 \\
96.00 \\
97.00 \\
98.00 \\
99.00 \\
100.00\end{array}$ & $\begin{array}{l}0.40 \\
0.40 \\
0.41 \\
0.41 \\
0.41 \\
0.42 \\
0.42 \\
0.43 \\
0.43 \\
0.44\end{array}$ & $\begin{array}{r}91.00 \\
92.00 \\
93.00 \\
94.00 \\
95.00 \\
96.00 \\
97.00 \\
98.00 \\
99.00 \\
100.00\end{array}$ & $\begin{array}{l}0.79 \\
0.80 \\
0.81 \\
0.82 \\
0.83 \\
0.84 \\
0.85 \\
0.86 \\
0.86 \\
0.87\end{array}$ & $\begin{array}{l}90.99 \\
91.99 \\
92.99 \\
93.99 \\
94.99 \\
95.99 \\
91.99 \\
97.99 \\
98.99 \\
99.99\end{array}$ & $\begin{array}{l}1.19 \\
1.20 \\
1.22 \\
1.23 \\
1.24 \\
1.26 \\
1.27 \\
1.28 \\
1.30 \\
1.31\end{array}$ & $\begin{array}{r}91 \\
92 \\
93 \\
94 \\
95 \\
96 \\
87 \\
98 \\
99 \\
100\end{array}$ \\
\hline \multirow{2}{*}{ 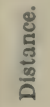 } & Dep. & Lat. & Dep. & Lat. & Dep. & Lat. & $\ddot{\Xi}$ \\
\hline & \multicolumn{2}{|c|}{$8934^{\circ}$. } & \multicolumn{2}{|c|}{$8912^{\circ}$. } & \multicolumn{2}{|c|}{$8914^{\circ}$} & $\doteqdot \frac{n}{0}$ \\
\hline
\end{tabular}


TABLE 1.-TAIVERSE TABLE.

\begin{tabular}{|c|c|c|c|c|c|c|c|c|c|}
\hline \multirow{2}{*}{ 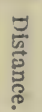 } & \multicolumn{2}{|l|}{1} & \multicolumn{2}{|c|}{$11 / 4^{\circ}$. } & \multicolumn{2}{|c|}{$11 / 2^{\circ}$. } & \multicolumn{2}{|c|}{$134^{\circ}$} & \multirow{2}{*}{ 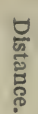 } \\
\hline & Lat. & Dep. & Lat. & Dep. & Lat. & Dep. & Lat. & Dep. & \\
\hline $\begin{array}{r}1 \\
2 \\
3 \\
4 \\
5 \\
6 \\
7 \\
8 \\
9 \\
10\end{array}$ & $\begin{array}{r}1.00 \\
2.00 \\
3.00 \\
4.00 \\
5.00 \\
6.00 \\
7.00 \\
8.00 \\
9.00 \\
10.00\end{array}$ & $\begin{array}{l}0.02 \\
0.03 \\
0.05 \\
0.07 \\
0.09 \\
0.10 \\
0.12 \\
0.14 \\
0.16 \\
0.17\end{array}$ & $\begin{array}{r}1.00 \\
2.00 \\
3.00 \\
4.00 \\
5.00 \\
6.00 \\
7.00 \\
8.00 \\
9.00 \\
10.00\end{array}$ & $\begin{array}{l}0.02 \\
0.04 \\
0.07 \\
0.09 \\
0.11 \\
0.13 \\
0.15 \\
0.17 \\
0.20 \\
0.22\end{array}$ & $\begin{array}{r}1.00 \\
2.00 \\
3.00 \\
4.00 \\
5.00 \\
6.00 \\
7.00 \\
8.00 \\
9.00 \\
10.00\end{array}$ & $\begin{array}{l}0.03 \\
0.05 \\
0.08 \\
0.10 \\
0.13 \\
0.16 \\
0.18 \\
0.21 \\
0.24 \\
0.26\end{array}$ & $\begin{array}{r}1.00 \\
2.00 \\
3.00 \\
4.00 \\
5.00 \\
6.00 \\
7.00 \\
8.00 \\
9.00 \\
10.00\end{array}$ & $\begin{array}{l}0.03 \\
0.06 \\
0.09 \\
0.12 \\
0.15 \\
0.18 \\
0.21 \\
0.24 \\
0.27 \\
0.31\end{array}$ & $\begin{array}{r}1 \\
2 \\
3 \\
4 \\
5 \\
6 \\
7 \\
8 \\
9 \\
10\end{array}$ \\
\hline $\begin{array}{l}11 \\
12 \\
13 \\
14 \\
15 \\
16 \\
17 \\
18 \\
19 \\
20\end{array}$ & $\begin{array}{l}11.00 \\
12.00 \\
13.00 \\
14.00 \\
15.00 \\
16.00 \\
17.00 \\
18.00 \\
19.00 \\
20.00\end{array}$ & $\begin{array}{l}0.19 \\
0.21 \\
0.23 \\
0.24 \\
0.26 \\
0.28 \\
0.30 \\
0.31 \\
0.33 \\
0.35\end{array}$ & $\begin{array}{l}11.00 \\
12.00 \\
13.00 \\
14.00 \\
15.00 \\
16.00 \\
17.00 \\
18.00 \\
19.00 \\
20.00\end{array}$ & $\begin{array}{l}0.24 \\
0.26 \\
0.28 \\
0.31 \\
0.33 \\
0.35 \\
0.37 \\
0.39 \\
0.41 \\
0.44\end{array}$ & $\begin{array}{l}11.00 \\
12.00 \\
13.00 \\
14.09 \\
14.99 \\
15.99 \\
16.99 \\
17.99 \\
18.99 \\
19.99\end{array}$ & $\begin{array}{l}0.29 \\
0.31 \\
0.34 \\
0.37 \\
0.39 \\
0.42 \\
0.45 \\
0.47 \\
0.50 \\
0.52\end{array}$ & $\begin{array}{l}10.99 \\
11.99 \\
12.99 \\
13.99 \\
14.99 \\
15.99 \\
16.99 \\
17.99 \\
18.99 \\
19.99\end{array}$ & $\begin{array}{l}-0.34 \\
0.37 \\
0.40 \\
0.43 \\
0.46 \\
0.49 \\
0.52 \\
0.55 \\
0.58 \\
0.61\end{array}$ & $\begin{array}{l}11 \\
12 \\
13 \\
14 \\
15 \\
16 \\
17 \\
18 \\
19 \\
20\end{array}$ \\
\hline $\begin{array}{l}21 \\
22 \\
23 \\
24 \\
25 \\
26 \\
27 \\
28 \\
29 \\
30\end{array}$ & $\begin{array}{l}21.00 \\
22.00 \\
23.00 \\
24.00 \\
25.00 \\
26.00 \\
27.00 \\
28.00 \\
29.00 \\
30.00\end{array}$ & $\begin{array}{l}0.37 \\
0.38 \\
0.40 \\
0.42 \\
0.44 \\
0.45 \\
0.47 \\
0.49 \\
0.51 \\
0.52\end{array}$ & $\begin{array}{l}21.00 \\
21.99 \\
22.99 \\
23.99 \\
24.99 \\
25.99 \\
26.99 \\
27.99 \\
28.99 \\
29.99\end{array}$ & $\begin{array}{l}0.46 \\
0.48 \\
0.50 \\
0.52 \\
0.55 \\
0.57 \\
0.59 \\
0.61 \\
0.63 \\
0.65\end{array}$ & $\begin{array}{l}20.99 \\
21.99 \\
22.99 \\
23.99 \\
24.99 \\
25.99 \\
26.99 \\
27.99 \\
28.99 \\
29.99\end{array}$ & $\begin{array}{l}0.55 \\
0.58 \\
0.60 \\
0.63 \\
0.65 \\
0.68 \\
0.71 \\
0.73 \\
0.76 \\
0.79\end{array}$ & $\begin{array}{l}20.99 \\
21.99 \\
22.99 \\
23.99 \\
24.99 \\
25.99 \\
26.99 \\
27.99 \\
28.99 \\
29.99\end{array}$ & $\begin{array}{l}0.64 \\
0.67 \\
0.70 \\
0.73 \\
0.76 \\
0.79 \\
0.82 \\
0.86 \\
0.89 \\
0.92\end{array}$ & $\begin{array}{l}21 \\
22 \\
23 \\
24 \\
25 \\
26 \\
27 \\
28 \\
29 \\
30\end{array}$ \\
\hline $\begin{array}{l}31 \\
32 \\
33 \\
34 \\
35 \\
36 \\
37 \\
38 \\
39 \\
40\end{array}$ & $\begin{array}{l}31.00 \\
32.00 \\
32.99 \\
33.99 \\
34.99 \\
35.99 \\
36.99 \\
37.99 \\
38.99 \\
39.99\end{array}$ & $\begin{array}{l}0.54 \\
0.56 \\
0.58 \\
0.59 \\
0.61 \\
0.63 \\
0.65 \\
0.66 \\
0.68 \\
0.70\end{array}$ & $\begin{array}{l}30.99 \\
31.99 \\
32.99 \\
33.99 \\
34.99 \\
35.99 \\
36.99 \\
37.99 \\
38.99 \\
39.99\end{array}$ & $\begin{array}{l}0.68 \\
0.70 \\
0.72 \\
0.74 \\
0.76 \\
0.79 \\
0.81 \\
0.83 \\
0.85 \\
0.87\end{array}$ & $\begin{array}{l}30.99 \\
31.99 \\
32.99 \\
33.99 \\
34.99 \\
35.99 \\
36.99 \\
37.99 \\
38.99 \\
39.99\end{array}$ & $\begin{array}{l}0.81 \\
0.84 \\
0.86 \\
0.89 \\
0.92 \\
0.94 \\
0.97 \\
0.99 \\
1.02 \\
1.05\end{array}$ & $\begin{array}{l}30.99 \\
31.99 \\
32.98 \\
33.98 \\
34.98 \\
35.98 \\
36.98 \\
37.98 \\
38.98 \\
39.98\end{array}$ & $\begin{array}{l}0.95 \\
0.98 \\
1.01 \\
1.04 \\
1.07 \\
1.10 \\
1.13 \\
1.16 \\
1.19 \\
1.22\end{array}$ & $\begin{array}{l}31 \\
32 \\
33 \\
34 \\
35 \\
36 \\
37 \\
38 \\
39 \\
40\end{array}$ \\
\hline $\begin{array}{l}41 \\
42 \\
43 \\
44 \\
45 \\
46 \\
47 \\
48 \\
49 \\
50\end{array}$ & $\begin{array}{l}40.99 \\
41.99 \\
42.99 \\
43.99 \\
44.99 \\
45.99 \\
46.99 \\
47.99 \\
48.99 \\
49.99\end{array}$ & $\begin{array}{l}0.72 \\
0.73 \\
0.75 \\
0.77 \\
0.79 \\
0.80 \\
0.82 \\
0.84 \\
0.86 \\
0.87\end{array}$ & $\begin{array}{l}40.99 \\
41.99 \\
42.99 \\
43.99 \\
44.99 \\
45.99 \\
46.99 \\
47.99 \\
48.99 \\
49.99\end{array}$ & $\begin{array}{l}0.89 \\
0.92 \\
0.94 \\
0.96 \\
0.98 \\
1.00 \\
1.03 \\
1.05 \\
1.07 \\
1.09\end{array}$ & $\begin{array}{l}40.99 \\
41.99 \\
42.99 \\
43.98 \\
44.98 \\
45.98 \\
46.98 \\
47.98 \\
48.98 \\
49.98\end{array}$ & $\begin{array}{l}1.07 \\
1.10 \\
1.13 \\
1.15 \\
1.18 \\
1.20 \\
1.23 \\
1.26 \\
1.28 \\
1.31\end{array}$ & $\begin{array}{l}40.98 \\
41.98 \\
42.98 \\
43.98 \\
44.98 \\
45.98 \\
46.98 \\
47.98 \\
48.98 \\
49.98\end{array}$ & $\begin{array}{l}1.25 \\
1.28 \\
1.31 \\
1.34 \\
1.37 \\
1.40 \\
1.44 \\
1.47 \\
1.50 \\
1.53\end{array}$ & $\begin{array}{l}41 \\
42 \\
43 \\
44 \\
45 \\
46 \\
47 \\
48 \\
49 \\
50\end{array}$ \\
\hline \multirow{2}{*}{ 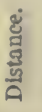 } & Dep. & Lat. & Dep. & Lat. & Dep. & Lat. & Dep. & Lat. & \multirow{2}{*}{ 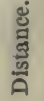 } \\
\hline & & & & & & & & & \\
\hline
\end{tabular}


TABLE A.-TRAVEIRE TABLE.

\begin{tabular}{|c|c|c|c|c|c|c|c|c|c|}
\hline \multirow{2}{*}{ 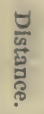 } & \multicolumn{2}{|c|}{$1^{\circ}$} & \multicolumn{2}{|c|}{$13 / 4^{\circ}}$. & \multicolumn{2}{|c|}{$1 ! / 20}$. & \multicolumn{2}{|c|}{$13 / 4^{\circ}$} & \multirow{2}{*}{ 흘 } \\
\hline & Lat. & Dep. & Lat. & Dep. & Lat. & Dep. & Lat. & Dep. & \\
\hline $\begin{array}{l}51 \\
52 \\
53 \\
54 \\
55 \\
56 \\
57 \\
58 \\
59 \\
60\end{array}$ & $\begin{array}{l}50.99 \\
51.99 \\
52.99 \\
53.99 \\
54.99 \\
55.99 \\
56.99 \\
57.99 \\
58.99 \\
59.99\end{array}$ & $\begin{array}{l}0.89 \\
0.91 \\
0.92 \\
0.94 \\
0.96 \\
0.98 \\
0.99 \\
1.01 \\
1.03 \\
1.05\end{array}$ & $\begin{array}{l}50.99 \\
51.99 \\
52.99 \\
53.99 \\
54.99 \\
55.99 \\
56.99 \\
57.99 \\
58.99 \\
59.99\end{array}$ & $\begin{array}{l}1.11 \\
1.13 \\
1.16 \\
1.18 \\
1.20 \\
1.22 \\
1.24 \\
1.27 \\
1.29 \\
1.31\end{array}$ & $\begin{array}{l}50.99 \\
51.98 \\
52.98 \\
53.98 \\
54.98 \\
55.98 \\
56.98 \\
57.98 \\
58.98 \\
59.98\end{array}$ & $\begin{array}{l}1.34 \\
1.36 \\
1.39 \\
1.41 \\
1.44 \\
1.47 \\
1.49 \\
1.52 \\
1.54 \\
1.57\end{array}$ & $\begin{array}{l}50.98 \\
51.99 \\
52.98 \\
53.97 \\
54.97 \\
55.97 \\
56.97 \\
57.97 \\
58.97 \\
59.97\end{array}$ & $\begin{array}{l}1.56 \\
1.59 \\
1.62 \\
1.65 \\
1.68 \\
1.71 \\
1.74 \\
1.77 \\
1.80 \\
1.83\end{array}$ & $\begin{array}{l}51 \\
52 \\
53 \\
54 \\
55 \\
56 \\
57 \\
58 \\
59 \\
60\end{array}$ \\
\hline $\begin{array}{l}61 \\
62 \\
63 \\
64 \\
65 \\
663 \\
67 \\
68 \\
69 \\
70\end{array}$ & $\begin{array}{l}60.99 \\
61.99 \\
62.99 \\
63.99 \\
64.99 \\
65.99 \\
66.99 \\
67.99 \\
68.99 \\
69.99\end{array}$ & $\begin{array}{l}1.06 \\
1.08 \\
1.10 \\
1.12 \\
1.13 \\
1.15 \\
1.17 \\
1.19 \\
1.20 \\
1.22\end{array}$ & $\begin{array}{l}60.99 \\
61.99 \\
62.99 \\
63.98 \\
64.98 \\
65.98 \\
61.98 \\
67.98 \\
62.99 \\
69.98\end{array}$ & $\begin{array}{l}1.33 \\
1.35 \\
1.37 \\
1.40 \\
1.42 \\
1.44 \\
1.46 \\
1.48 \\
1.51 \\
1.53\end{array}$ & $\begin{array}{l}60.98 \\
61.98 \\
62.98 \\
63.98 \\
64.98 \\
65.98 \\
66.98 \\
67.98 \\
68.98 \\
69.98\end{array}$ & $\begin{array}{l}1.60 \\
1.62 \\
1.65 \\
1.68 \\
1.70 \\
1.73 \\
1.75 \\
1.78 \\
1.81 \\
1.83\end{array}$ & $\begin{array}{l}60.97 \\
61.97 \\
62.97 \\
63.97 \\
64.97 \\
6.5 .97 \\
66.97 \\
67.97 \\
68.97 \\
63.97\end{array}$ & $\begin{array}{l}1.86 \\
1.89 \\
1.92 \\
1.95 \\
1.99 \\
2.02 \\
2.05 \\
2.08 \\
2.11 \\
2.14\end{array}$ & $\begin{array}{l}61 \\
62 \\
63 \\
64 \\
65 \\
66 \\
67 \\
65 \\
69 \\
70\end{array}$ \\
\hline $\begin{array}{l}71 \\
72 \\
73 \\
74 \\
75 \\
76 \\
77 \\
78 \\
79 \\
80\end{array}$ & $\begin{array}{l}70.99 \\
71.99 \\
72.99 \\
73.99 \\
74.99 \\
75.99 \\
76.99 \\
77.99 \\
78.99 \\
79.99\end{array}$ & $\begin{array}{l}1.24 \\
1.26 \\
1.27 \\
1.29 \\
1.31 \\
1.33 \\
1.34 \\
1.36 \\
1.39 \\
1.40\end{array}$ & $\begin{array}{l}70.9 .9 \\
71.98 \\
72.98 \\
73.98 \\
74.98 \\
75.98 \\
76.98 \\
77.98 \\
78.98 \\
79.98\end{array}$ & $\begin{array}{l}1.55 \\
1.57 \\
1.59 \\
1.61 \\
1.64 \\
1.66 \\
1.68 \\
1.70 \\
1.72 \\
1.75\end{array}$ & $\begin{array}{l}70.98 \\
71.98 \\
72.97 \\
73.97 \\
74.97 \\
75.97 \\
76.97 \\
77.97 \\
78.97 \\
79.97\end{array}$ & $\begin{array}{l}1.86 \\
1.88 \\
1.91 \\
1.94 \\
1.96 \\
1.99 \\
2.02 \\
2.04 \\
2.07 \\
2.09\end{array}$ & $\begin{array}{l}70.97 \\
71.97 \\
72.97 \\
73.97 \\
74.97 \\
75.96 \\
76.96 \\
77.96 \\
78.96 \\
79.96\end{array}$ & $\begin{array}{l}2.17 \\
2.20 \\
2.23 \\
2.26 \\
2.29 \\
2.32 \\
2.35 \\
2.38 \\
2.41 \\
2.44\end{array}$ & $\begin{array}{l}71 \\
72 \\
73 \\
74 \\
75 \\
76 \\
77 \\
78 \\
79 \\
80\end{array}$ \\
\hline $\begin{array}{l}81 \\
82 \\
83 \\
84 \\
85 \\
86 \\
87 \\
88 \\
89 \\
90\end{array}$ & $\begin{array}{l}\text { \$0. } 99 \\
81.99 \\
82.99 \\
83.99 \\
84.99 \\
85.99 \\
86.99 \\
87.99 \\
85.99 \\
89.99\end{array}$ & $\begin{array}{l}1.41 \\
1.43 \\
1.45 \\
1.47 \\
1.48 \\
1.50 \\
1.52 \\
1.54 \\
1.55 \\
1.57\end{array}$ & $\begin{array}{l}80.98 \\
81.98 \\
82.98 \\
83.98 \\
84.98 \\
8.5 .98 \\
86.98 \\
87.98 \\
85.98 \\
89.98\end{array}$ & $\begin{array}{l}1.77 \\
1.79 \\
1.81 \\
1.83 \\
1.85 \\
1.88 \\
1.90 \\
1.92 \\
1.94 \\
1.96\end{array}$ & $\begin{array}{l}80.97 \\
81.97 \\
82.97 \\
83.97 \\
84.97 \\
85.97 \\
81.97 \\
87.97 \\
88.97 \\
89.97\end{array}$ & $\begin{array}{l}2.12 \\
2.15 \\
2.17 \\
2.20 \\
2.23 \\
2.25 \\
2.28 \\
2.30 \\
2.33 \\
2.36\end{array}$ & $\begin{array}{l}80.96 \\
81.96 \\
82.96 \\
83.96 \\
81.96 \\
85.96 \\
86.96 \\
87.96 \\
88.96 \\
89.96\end{array}$ & $\begin{array}{l}2.47 \\
2.50 \\
2.53 \\
2.57 \\
2.60 \\
2.63 \\
2.66 \\
2.69 \\
2.72 \\
2.75\end{array}$ & $\begin{array}{l}81 \\
82 \\
83 \\
84 \\
85 \\
86 \\
87 \\
89 \\
89 \\
90\end{array}$ \\
\hline $\begin{array}{r}91 \\
92 \\
93 \\
94 \\
95 \\
96 \\
97 \\
98 \\
99 \\
100\end{array}$ & $\begin{array}{l}90.99 \\
91.99 \\
92.99 \\
93.99 \\
94.99 \\
95.99 \\
96.99 \\
97.99 \\
98.99 \\
99.98\end{array}$ & $\begin{array}{l}1.59 \\
1.61 \\
1.62 \\
1.64 \\
1.66 \\
1.68 \\
1.69 \\
1.71 \\
1.73 \\
1.75\end{array}$ & $\begin{array}{l}90.98 \\
91.98 \\
92.98 \\
93.98 \\
94.98 \\
95.98 \\
96.98 \\
97.98 \\
98.98 \\
99.98\end{array}$ & $\begin{array}{l}1.99 \\
2.01 \\
2.03 \\
2.05 \\
2.07 \\
2.09 \\
2.12 \\
2.14 \\
2.16 \\
2.18\end{array}$ & $\begin{array}{l}90.97 \\
91.97 \\
92.97 \\
93.97 \\
94.97 \\
95.97 \\
94.97 \\
97.97 \\
98.97 \\
99.97\end{array}$ & $\begin{array}{l}2.38 \\
2.41 \\
2.43 \\
2.46 \\
2.49 \\
2.51 \\
2.54 \\
2.57 \\
2.59 \\
2.62\end{array}$ & $\begin{array}{l}90.96 \\
91.96 \\
92.96 \\
93.96 \\
94.96 i \\
95.96 \\
96.95 \\
97.95 \\
98.95 \\
99.95\end{array}$ & $\begin{array}{l}2.78 \\
2.81 \\
2.84 \\
2.87 \\
2.90 \\
2.93 \\
2.96 \\
2.99 \\
3.02 \\
3.05\end{array}$ & $\begin{array}{r}91 \\
92 \\
93 \\
94 \\
95 \\
96 \\
97 \\
98 \\
99 \\
100\end{array}$ \\
\hline 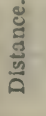 & Dep. & Iat. & \multicolumn{2}{|c|}{$8 S^{3} 4^{\circ}}$. & Dep. & Iat. & Dep. & Lat. & 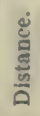 \\
\hline
\end{tabular}


TABLE 4.-TRAVERSE TA BLE.

\begin{tabular}{|c|c|c|c|c|c|c|c|c|c|}
\hline \multirow{2}{*}{ 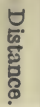 } & \multicolumn{2}{|c|}{$2^{\circ}$. } & \multicolumn{2}{|c|}{$214^{\circ}$. } & \multicolumn{2}{|c|}{$21 / 2^{\circ}$. } & \multicolumn{2}{|c|}{$234^{\circ}$. } & \multirow{2}{*}{ 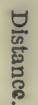 } \\
\hline & Lat. & Dep. & Lat. & Dep. & Lat. & Dep. & Lat. & Dep. & \\
\hline $\begin{array}{r}1 \\
2 \\
3 \\
4 \\
5 \\
6 \\
7 \\
8 \\
9 \\
10\end{array}$ & $\begin{array}{l}1.00 \\
2.00 \\
3.00 \\
4.00 \\
5.00 \\
6.00 \\
7.00 \\
7.99 \\
8.99 \\
9.99\end{array}$ & $\begin{array}{l}0.03 \\
0.07 \\
0.10 \\
0.14 \\
0.17 \\
0.21 \\
0.24 \\
0.28 \\
0.31 \\
0.35\end{array}$ & $\begin{array}{l}1.00 \\
2.00 \\
3.00 \\
4.00 \\
5.00 \\
6.00 \\
6.99 \\
7.99 \\
8.99 \\
9.99\end{array}$ & $\begin{array}{l}0.04 \\
0.08 \\
0.12 \\
0.16 \\
0.20 \\
0.24 \\
0.27 \\
0.31 \\
0.35 \\
0.39\end{array}$ & $\begin{array}{l}1.00 \\
2.00 \\
3.00 \\
4.00 \\
5.00 \\
5.99 \\
6.99 \\
7.99 \\
8.99 \\
9.99\end{array}$ & $\begin{array}{l}0.04 \\
0.09 \\
0.13 \\
0.17 \\
0.22 \\
0.26 \\
0.31 \\
0.35 \\
0.39 \\
0.44\end{array}$ & $\begin{array}{l}1.00 \\
2.00 \\
3.00 \\
4.00 \\
4.99 \\
5.99 \\
6.99 \\
7.99 \\
8.99 \\
9.99\end{array}$ & $\begin{array}{l}0.05 \\
0.10 \\
0.14 \\
0.19 \\
0.24 \\
0.29 \\
0.34 \\
0.38 \\
0.43 \\
0.48\end{array}$ & $\begin{array}{r}1 \\
2 \\
3 \\
4 \\
5 \\
6 \\
7 \\
8 \\
9 \\
10\end{array}$ \\
\hline $\begin{array}{l}11 \\
12 \\
13 \\
14 \\
15 \\
16 \\
17 \\
18 \\
19 \\
20\end{array}$ & $\begin{array}{l}10.99 \\
11.99 \\
12.99 \\
13.99 \\
14.99 \\
15.99 \\
16.99 \\
17.99 \\
18.99 \\
19.99\end{array}$ & $\begin{array}{l}0.38 \\
0.42 \\
0.45 \\
0.49 \\
0.52 \\
0.56 \\
0.59 \\
0.63 \\
0.66 \\
0.70\end{array}$ & $\begin{array}{l}10.99 \\
11.99 \\
12.99 \\
13.99 \\
14.99 \\
15.99 \\
16.99 \\
17.99 \\
18.99 \\
19.98\end{array}$ & $\begin{array}{l}0.43 \\
0.47 \\
0.51 \\
0.55 \\
0.59 \\
0.63 \\
0.67 \\
0.71 \\
0.75 \\
0.79\end{array}$ & $\begin{array}{l}10.99 \\
11.99 \\
12.99 \\
13.99 \\
14.99 \\
15.98 \\
16.98 \\
17.98 \\
18.98 \\
19.98\end{array}$ & $\begin{array}{l}0.48 \\
0.52 \\
0.57 \\
0.61 \\
0.65 \\
0.70 \\
0.74 \\
0.79 \\
0.83 \\
0.87\end{array}$ & $\begin{array}{l}10.99 \\
11.99 \\
12.99 \\
13.98 \\
14.98 \\
15.98 \\
16.98 \\
17.98 \\
18.98 \\
19.98\end{array}$ & $\begin{array}{l}0.53 \\
0.58 \\
0.62 \\
0.67 \\
0.72 \\
0.77 \\
0.82 \\
0.86 \\
0.91 \\
0.96\end{array}$ & $\begin{array}{l}11 \\
12 \\
13 \\
14 \\
15 \\
16 \\
17 \\
18 \\
19 \\
20\end{array}$ \\
\hline $\begin{array}{l}21 \\
22 \\
23 \\
24 \\
25 \\
26 \\
27 \\
28 \\
29 \\
30\end{array}$ & $\begin{array}{l}20.99 \\
21.99 \\
22.99 \\
23.99 \\
24.98 \\
25.98 \\
26.98 \\
27.98 \\
28.98 \\
29.98\end{array}$ & $\begin{array}{l}0.73 \\
0.77 \\
0.80 \\
0.84 \\
0.87 \\
0.91 \\
0.94 \\
0.98 \\
1.01 \\
1.05\end{array}$ & $\begin{array}{l}20.98 \\
21.98 \\
22.98 \\
23.98 \\
24.98 \\
25.98 \\
26.98 \\
27.98 \\
28.98 \\
29.98\end{array}$ & $\begin{array}{l}0.82 \\
0.86 \\
0.90 \\
0.94 \\
0.98 \\
1.02 \\
1.06 \\
1.10 \\
1.14 \\
1.18\end{array}$ & $\begin{array}{l}20.98 \\
21.98 \\
22.98 \\
23.98 \\
24.98 \\
25.98 \\
26.97 \\
27.97 \\
28.97 \\
29.97\end{array}$ & $\begin{array}{l}0.92 \\
0.96 \\
1.00 \\
1.05 \\
1.09 \\
1.13 \\
1.18 \\
1.22 \\
1.26 \\
1.31\end{array}$ & $\begin{array}{l}20.98 \\
21.97 \\
22.97 \\
23.97 \\
24.97 \\
25.97 \\
26.97 \\
27.97 \\
28.97 \\
29.97\end{array}$ & $\begin{array}{l}1.01 \\
1.06 \\
1.10 \\
1.15 \\
1.20 \\
1.25 \\
1.30 \\
1.34 \\
1.39 \\
1.44\end{array}$ & $\begin{array}{l}21 \\
22 \\
23 \\
24 \\
25 \\
26 \\
27 \\
28 \\
29 \\
30\end{array}$ \\
\hline $\begin{array}{l}31 \\
32 \\
33 \\
34 \\
35 \\
36 \\
37 \\
38 \\
39 \\
40\end{array}$ & $\begin{array}{l}30.98 \\
31.98 \\
32.98 \\
33.98 \\
34.98 \\
35.98 \\
36.98 \\
37.98 \\
38.98 \\
39.98\end{array}$ & $\begin{array}{l}1.08 \\
1.12 \\
1.15 \\
1.19 \\
1.22 \\
1.26 \\
1.29 \\
1.33 \\
1.36 \\
1.40\end{array}$ & $\begin{array}{l}30.98 \\
31.98 \\
32.97 \\
33.97 \\
34.97 \\
35.97 \\
36.97 \\
37.97 \\
38.97 \\
39.97\end{array}$ & $\begin{array}{l}1.22 \\
1.26 \\
1.30 \\
1.33 \\
1.37 \\
1.41 \\
1.45 \\
1.49 \\
1.53 \\
1.57\end{array}$ & $\begin{array}{l}30.97 \\
31.97 \\
32.97 \\
33.97 \\
34.97 \\
35.97 \\
36.96 \\
37.96 \\
38.96 \\
39.96\end{array}$ & $\begin{array}{l}1.35 \\
1.40 \\
1.44 \\
1.48 \\
1.53 \\
1.57 \\
1.61 \\
1.66 \\
1.70 \\
1.74\end{array}$ & $\begin{array}{l}30.96 \\
31.96 \\
32.96 \\
33.96 \\
34.96 \\
35.96 \\
36.96 \\
37.96 \\
38.96 \\
39.95\end{array}$ & $\begin{array}{l}1.49 \\
1.54 \\
1.58 \\
1.63 \\
1.68 \\
1.73 \\
1.78 \\
1.82 \\
1.87 \\
1.92\end{array}$ & $\begin{array}{l}31 \\
32 \\
33 \\
34 \\
35 \\
36 \\
37 \\
38 \\
39 \\
40\end{array}$ \\
\hline $\begin{array}{l}41 \\
42 \\
43 \\
44 \\
45 \\
46 \\
47 \\
48 \\
49 \\
50\end{array}$ & $\begin{array}{l}40.98 \\
41.97 \\
42.97 \\
43.97 \\
44.97 \\
45.97 \\
46.97 \\
47.97 \\
48.97 \\
49.97\end{array}$ & $\begin{array}{l}1.43 \\
1.47 \\
1.50 \\
1.54 \\
1.57 \\
1.61 \\
1.64 \\
1.68 \\
1.71 \\
1.74\end{array}$ & $\begin{array}{l}40.97 \\
41.97 \\
42.97 \\
43.97 \\
44.97 \\
45.96 \\
46.96 \\
47.96 \\
48.96 \\
49.96\end{array}$ & $\begin{array}{l}1.61 \\
1.65 \\
1.69 \\
1.73 \\
1.77 \\
1.81 \\
1.85 \\
1.88 \\
1.92 \\
1.96\end{array}$ & $\begin{array}{r}40.96 \\
41.96 \\
42.96 \\
43.96 \\
44.96 \\
45.96 \\
46.96 \\
47.95 \\
48.95 \\
49.95\end{array}$ & $\begin{array}{l}1.79 \\
1.83 \\
1.88 \\
1.92 \\
1.96 \\
2.01 \\
2.05 \\
2.09 \\
2.14 \\
2.18\end{array}$ & $\begin{array}{l}40.95 \\
41.95 \\
42.95 \\
43.95 \\
44.95 \\
45.95 \\
46.95 \\
47.94 \\
48.94 \\
49.94\end{array}$ & $\begin{array}{l}1.97 \\
2.02 \\
2.06 \\
2.11 \\
2.16 \\
2.21 \\
2.25 \\
2.30 \\
2.35 \\
2.40\end{array}$ & $\begin{array}{l}41 \\
42 \\
43 \\
44 \\
45 \\
46 \\
47 \\
48 \\
49 \\
50\end{array}$ \\
\hline ஜ் & Dep. & Lat. & Dep. & Lat. & Dep. & Lat. & Dep. & Lat. & \\
\hline 口 & \multicolumn{2}{|c|}{$88^{\circ}$. } & \multicolumn{2}{|c|}{$87 \% 4^{\circ}$. } & \multicolumn{2}{|c|}{$871 / 2^{\circ}$. } & \multicolumn{2}{|c|}{$871 / 4^{\circ}$. } & A \\
\hline
\end{tabular}


TABLE 4.-TRAVERSE TABIE.

\begin{tabular}{|c|c|c|c|c|c|c|c|c|c|}
\hline \multirow{2}{*}{ 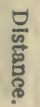 } & \multicolumn{2}{|c|}{$2^{\circ}$} & \multicolumn{2}{|c|}{$21 / 4^{\circ}$. } & \multicolumn{2}{|c|}{$21 / 2^{\circ}$. } & \multicolumn{2}{|c|}{$2 \% 4^{\circ}$. } & \multirow{2}{*}{ 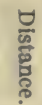 } \\
\hline & Lat. & Dep. & Lat. & Dep. & Lat. & Dep. & Lat. & Dep. & \\
\hline $\begin{array}{l}51 \\
52 \\
53 \\
54 \\
55 \\
56 \\
57 \\
58 \\
59 \\
60\end{array}$ & $\begin{array}{l}50.97 \\
51.97 \\
52.97 \\
53.97 \\
54.97 \\
55.97 \\
56.97 \\
57.96 \\
58.96 \\
59.96\end{array}$ & $\begin{array}{l}1.78 \\
1.81 \\
1.85 \\
1.88 \\
1.92 \\
1.95 \\
1.99 \\
2.02 \\
2.06 \\
2.09\end{array}$ & $\begin{array}{l}50.96 \\
51.96 \\
52.96 \\
53.96 \\
54.96 \\
55.96 \\
56.96 \\
57.96 \\
58.95 \\
59.95\end{array}$ & $\begin{array}{l}2.00 \\
2.04 \\
2.08 \\
2.12 \\
2.16 \\
2.20 \\
2.24 \\
2.28 \\
2.32 \\
2.36\end{array}$ & $\begin{array}{l}50.95 \\
51.95 \\
52.95 \\
53.95 \\
54.95 \\
55.95 \\
56.95 \\
57.94 \\
58.94 \\
59.94\end{array}$ & $\begin{array}{l}2.22 \\
2.27 \\
2.31 \\
2.36 \\
2.40 \\
2.44 \\
2.49 \\
2.53 \\
2.57 \\
2.62\end{array}$ & $\begin{array}{l}50.94 \\
51.94 \\
52.94 \\
53.94 \\
54.94 \\
55.94 \\
56.93 \\
57.93 \\
58.93 \\
59.93\end{array}$ & $\begin{array}{l}2.45 \\
2.49 \\
2.54 \\
2.59 \\
2.64 \\
2.69 \\
2.73 \\
2.78 \\
2.83 \\
2.88\end{array}$ & $\begin{array}{l}51 \\
52 \\
53 \\
54 \\
55 \\
56 \\
57 \\
58 \\
59 \\
60\end{array}$ \\
\hline $\begin{array}{l}61 \\
62 \\
63 \\
64 \\
65 \\
66 \\
67 \\
68 \\
69 \\
70\end{array}$ & $\begin{array}{l}60.96 \\
61.96 \\
62.96 \\
63.96 \\
64.96 \\
65.96 \\
66.96 \\
67.96 \\
68.96 \\
69.96\end{array}$ & $\begin{array}{l}2.13 \\
2.16 \\
2.20 \\
2.23 \\
2.27 \\
2.30 \\
2.34 \\
2.37 \\
2.41 \\
2.44\end{array}$ & $\begin{array}{l}60.95 \\
61.95 \\
62.95 \\
63.95 \\
64.95 \\
65.95 \\
66.95 \\
67.95 \\
68.95 \\
69.95\end{array}$ & $\begin{array}{l}2.39 \\
2.43 \\
2.47 \\
2.51 \\
2.55 \\
2.59 \\
2.63 \\
2.67 \\
2.71 \\
2.75\end{array}$ & $\begin{array}{l}60.94 \\
61.94 \\
62.94 \\
63.94 \\
64.94 \\
65.94 \\
66.94 \\
67.94 \\
68.93 \\
69.93\end{array}$ & $\begin{array}{l}2.66 \\
2.70 \\
2.75 \\
2.79 \\
2.84 \\
2.88 \\
2.92 \\
2.97 \\
3.01 \\
3.05\end{array}$ & $\begin{array}{l}60.93 \\
61.93 \\
62.93 \\
63.93 \\
64.93 \\
65.92 \\
66.92 \\
67.92 \\
68.92 \\
69.92\end{array}$ & $\begin{array}{l}2.93 \\
2.97 \\
3.02 \\
3.07 \\
3.12 \\
3.17 \\
3.21 \\
3.26 \\
3.31 \\
3.36\end{array}$ & $\begin{array}{l}61 \\
62 \\
63 \\
64 \\
65 \\
66 \\
67 \\
68 \\
69 \\
70\end{array}$ \\
\hline $\begin{array}{l}71 \\
72 \\
73 \\
74 \\
75 \\
76 \\
77 \\
78 \\
79 \\
80\end{array}$ & $\begin{array}{l}70.96 \\
71.96 \\
72.96 \\
73.95 \\
74.95 \\
75.95 \\
76.95 \\
77.95 \\
78.95 \\
79.95\end{array}$ & $\begin{array}{l}2.48 \\
2.51 \\
2.55 \\
2.55 \\
2.62 \\
2.65 \\
2.69 \\
2.72 \\
2.76 \\
2.79\end{array}$ & $\begin{array}{l}70.95 \\
71.94 \\
72.94 \\
73.94 \\
74.94 \\
75.94 \\
76.94 \\
77.94 \\
78.94 \\
79.94\end{array}$ & $\begin{array}{l}2.79 \\
2.83 \\
2.87 \\
2.91 \\
2.94 \\
2.98 \\
3.02 \\
3.06 \\
3.10 \\
3.14\end{array}$ & $\begin{array}{l}70.93 \\
71.93 \\
72.93 \\
73.93 \\
74.93 \\
75.93 \\
76.93 \\
77.93 \\
78.92 \\
79.92\end{array}$ & $\begin{array}{l}3.10 \\
3.14 \\
3.18 \\
3.23 \\
3.27 \\
3.32 \\
3.36 \\
3.40 \\
3.45 \\
3.49\end{array}$ & $\begin{array}{l}70.92 \\
71.92 \\
72.92 \\
73.91 \\
74.91 \\
75.91 \\
76.91 \\
77.91 \\
78.91 \\
79.91\end{array}$ & $\begin{array}{l}3.41 \\
3.45 \\
3.50 \\
3.55 \\
3.60 \\
3.65 \\
3.69 \\
3.74 \\
3.79 \\
3.84\end{array}$ & $\begin{array}{l}71 \\
72 \\
73 \\
74 \\
75 \\
76 \\
77 \\
78 \\
79 \\
80\end{array}$ \\
\hline $\begin{array}{l}81 \\
82 \\
83 \\
84 \\
85 \\
86 \\
87 \\
88 \\
89 \\
90\end{array}$ & $\begin{array}{l}80.95 \\
81.95 \\
82.95 \\
83.95 \\
84.95 \\
85.95 \\
86.95 \\
87.95 \\
88.95 \\
89.95\end{array}$ & $\begin{array}{l}2.83 \\
2.86 \\
2.90 \\
2.93 \\
2.97 \\
3.00 \\
3.04 \\
3.07 \\
3.11 \\
3.14\end{array}$ & $\begin{array}{l}80.94 \\
81.94 \\
82.94 \\
83.94 \\
84.93 \\
85.93 \\
86.93 \\
87.93 \\
88.93 \\
89.93\end{array}$ & $\begin{array}{l}3.18 \\
3.22 \\
3.26 \\
3.30 \\
3.34 \\
3.38 \\
3.42 \\
3.45 \\
3.49 \\
3.53\end{array}$ & $\begin{array}{l}80.92 \\
81.92 \\
82.92 \\
83.92 \\
84.92 \\
85.92 \\
86.92 \\
87.92 \\
88.92 \\
89.91\end{array}$ & $\begin{array}{l}3.53 \\
3.58 \\
3.62 \\
3.66 \\
3.71 \\
3.75 \\
3.79 \\
3.84 \\
3.88 \\
3.93\end{array}$ & $\begin{array}{l}80.91 \\
81.91 \\
82.90 \\
83.90 \\
84.90 \\
85.90 \\
86.90 \\
87.90 \\
88.90 \\
89.90\end{array}$ & $\begin{array}{l}3.89 \\
3.93 \\
3.98 \\
4.03 \\
4.08 \\
4.13 \\
4.17 \\
4.22 \\
4.27 \\
4.32\end{array}$ & $\begin{array}{l}81 \\
82 \\
83 \\
84 \\
85 \\
86 \\
87 \\
88 \\
89 \\
90\end{array}$ \\
\hline $\begin{array}{r}91 \\
92 \\
93 \\
94 \\
95 \\
96 \\
97 \\
98 \\
99 \\
100\end{array}$ & $\begin{array}{l}90.95 \\
91.94 \\
92.94 \\
93.94 \\
94.94 \\
95.94 \\
96.94 \\
97.94 \\
98.94 \\
99.94\end{array}$ & $\begin{array}{l}3.18 \\
3.21 \\
3.25 \\
3.28 \\
3.32 \\
3.35 \\
3.39 \\
3.42 \\
3.46 \\
3.49\end{array}$ & $\begin{array}{l}90.93 \\
91.93 \\
92.93 \\
93.93 \\
94.93 \\
95.93 \\
96.93 \\
97.92 \\
98.92 \\
99.92\end{array}$ & $\begin{array}{l}3.57 \\
3.61 \\
3.65 \\
3.69 \\
3.73 \\
3.77 \\
3.81 \\
3.85 \\
3.89 \\
3.93\end{array}$ & $\begin{array}{l}90.91 \\
91.91 \\
92.91 \\
93.91 \\
94.91 \\
95.91 \\
96.91 \\
97.91 \\
98.91 \\
99.90\end{array}$ & $\begin{array}{l}3.97 \\
4.01 \\
4.06 \\
4.10 \\
4.14 \\
4.19 \\
4.23 \\
4.27 \\
4.32 \\
4.36\end{array}$ & $\begin{array}{l}90.90 \\
91.89 \\
92.89 \\
93.89 \\
94.89 \\
95.89 \\
96.89 \\
97.89 \\
98.89 \\
99.88\end{array}$ & $\begin{array}{l}4.37 \\
4.41 \\
4.46 \\
4.51 \\
4.56 \\
4.61 \\
4.65 \\
4.70 \\
4.75 \\
4.80\end{array}$ & $\begin{array}{r}91 \\
92 \\
93 \\
94 \\
95 \\
96 \\
97 \\
98 \\
99 \\
100\end{array}$ \\
\hline \&ू & Dep. & Lat. & Dep. & Lat. & Dep. & Lat. & Dep. & Lat. & \&ี \\
\hline$\frac{n}{a}$ & \multicolumn{2}{|c|}{$85^{\circ}}$. & \multicolumn{2}{|c|}{$8734^{\circ}$. } & \multicolumn{2}{|c|}{$8715^{\circ}}$. & \multicolumn{2}{|c|}{$871 / 4^{\circ}}$. & \\
\hline
\end{tabular}


TABLE 4.-FPAVIRSE TABRE.

\begin{tabular}{|c|c|c|c|c|c|c|c|c|c|}
\hline \multirow{2}{*}{ 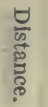 } & \multicolumn{2}{|c|}{$3^{\circ}$. } & \multicolumn{2}{|c|}{$31 / 4^{\circ}$. } & \multicolumn{2}{|c|}{$3 ! 2^{\circ}$. } & \multicolumn{2}{|c|}{$34^{\circ}$. } & \multirow{2}{*}{ 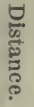 } \\
\hline & Lat. & Dep. & Lat. & Dep. & Lat. & Dep. & Lat. & Dep. & \\
\hline 1 & 1.00 & 0. 0.5 & 1.00 & 0.06 & 1.00 & 0.06 & 1.00 & 0.06 & 1 \\
\hline 2 & 2. 00 & 0.10 & 2.00 & 0.11 & 2.00 & 0.12 & 2.00 & 0.13 & 2 \\
\hline 3 & 3.00 & 0.16 & 3. 00 & 0.17 & 2. 99 & 0.19 & 2. 99 & 0.20 & 3 \\
\hline 4 & 3. 99 & 0.21 & 3. 99 & 0.23 & 3. 99 & 0.24 & 3. 99 & 0.26 & 4 \\
\hline 5 & 4. 99 & 0.26 & 4. 99 & 0.28 & 4. 99 & 0.31 & 4. 99 & 0.33 & 5 \\
\hline 6 & 5. 99 & 0.31 & 5.99 & 0.34 & 5. 99 & 0.37 & 5.99 & 0.39 & 6 \\
\hline 7 & 6.99 & 0.37 & 6. 99 & 0.40 & 6.99 & 0.43 & 6.99 & 0.46 & 7 \\
\hline 8 & 7.99 & 0.42 & 7.99 & 0.45 & 7. 99 & 0.49 & 7. 98 & 0.52 & 8 \\
\hline 9 & 8.99 & 0.47 & 8.99 & 0.51 & 8.98 & 0.55 & 8.98 & 0.59 & 9 \\
\hline 10 & 9.99 & 0.52 & 9.98 & 0.57 & 9.98 & 0.61 & 9.98 & 0.65 & 10 \\
\hline 11 & 10.98 & 0.58 & 10.98 & 0.62 & 10. 98 & 0.67 & 10.98 & 0.72 & 11 \\
\hline 12 & 11.98 & 0.63 & 11.98 & 0. 68 & 11. & 0.73 & 11.97 & 0.78 & 12 \\
\hline 13 & 12. 98 & 0.65 & 12.98 & 0.74 & 12. 98 & 0.79 & 12.97 & 0.85 & 13 \\
\hline 14 & 13. 98 & 0.73 & 13.98 & 0.79 & 13. 97 & 0.85 & 13. 97 & 0.92 & 14 \\
\hline 15 & 14. 98 & 0.79 & 14. 98 & 0.85 & 13.97 & 0.92 & 14.97 & 0.98 & 15 \\
\hline 16 & 15. 98 & 0.84 & 15.97 & 0.91 & 15. 97 & 0.98 & 15.97 & 1.05 & 16 \\
\hline 17 & 16.98 & 0.89 & 16.97 & 0.96 & 16. 97 & 1.04 & 16.96 & 1.11 & 17 \\
\hline 18 & 17.98 & 0.94 & 17.97 & 1. 02 & 17.97 & 1.10 & 17.96 & 1.18 & 18 \\
\hline 19 & 18. 97 & 0.99 & 18.97 & 1.08 & 18.96 & 1. 16 & 18. 96 & 1. 24 & 19 \\
\hline 20 & 19.97 & 1.05 & 19.97 & 1.13 & 19.96 & 1.22 & 19.96 & 1.31 & 20 \\
\hline 21 & 20.97 & 1. 10 & 20.97 & 1.19 & 20.96 & 1.28 & 20.96 & 1. 37 & 21 \\
\hline 22 & 21.97 & 1.15 & 21.96 & 1.25 & 21.96 & 1.34 & 21.95 & 1.44 & 22 \\
\hline 23 & 22.97 & 1.20 & 22.96 & 1.30 & 22.96 & 1.40 & 22.95 & 1.50 & 23 \\
\hline 24 & 23.97 & 1.26 & 23. 96 & 1.36 & 23.96 & 1.47 & 23.95 & 1.57 & 24 \\
\hline 25 & 24.97 & 1.31 & 24.96 & 1.42 & 24.95 & 1. 53 & 24.95 & 1.64 & 25 \\
\hline 26 & 25.96 & 1. 36 & 25.96 & 1.47 & 25.95 & 1. 59 & 25.94 & 1.70 & 26 \\
\hline 27 & 26.96 & 1.41 & 26. 96 & 1.53 & 26.95 & 1.65 & 26.94 & 1.77 & 27 \\
\hline 28 & 27.96 & 1.47 & 27.95 & 1.59 & 27.95 & 1.71 & 27.94 & 1.83 & 28 \\
\hline 29 & 28.96 & 1.52 & 28.95 & 1. 64 & 28.95 & 1.77 & 28.94 & 1.90 & 29 \\
\hline 30 & 29.96 & 1.57 & 29.95 & 1.70 & 29.94 & 1.83 & 29.94 & 1. 96 & 30 \\
\hline 31 & 30.96 & 1.62 & 30.95 & 1.76 & 30.94 & 1.8 & 30.93 & 2.0 & 31 \\
\hline 32 & 31.96 & 1.67 & 31.95 & 1.81 & 31.94 & 1.95 & 31. 93 & 2. 09 & 32 \\
\hline 33 & 32.95 & 1.73 & 32.95 & 1.87 & 32.94 & 2.01 & 32. 93 & 2. & 33 \\
\hline 34 & 33.95 & 1.78 & 33. 95 & 1.93 & 33.94 & 2. 08 & 33.93 & 2. 22 & 34 \\
\hline 35 & 34.95 & 1.83 & 34.94 & 1.98 & 34.93 & 2.14 & 34.93 & 2.29 & 35 \\
\hline 36 & 35.95 & 1.88 & 35.94 & 2.04 & 35. 93 & 2.20 & 35.92 & 2. 3.5 & 36 \\
\hline 37 & 36.95 & 1.94 & 36.94 & 2. 10 & 36. 93 & 2.26 & 36.92 & 2. 42 & 37 \\
\hline 38 & 37.95 & 1.99 & 37.94 & 2. 15 & 37.93 & 2.32 & 37.92 & 2.49 & 38 \\
\hline 39 & 39.95 & 2. 04 & 38.94 & 2. 21 & 38.93 & 2. 38 & 39.92 & 2.55 & 39 \\
\hline 40 & 39.95 & 2.09 & 39.94 & 2.27 & 39.93 & 2.44 & 39.91 & 2. 62 & 40 \\
\hline 41 & 40.94 & 2.15 & 40.93 & 2.32 & 40.92 & 2.5 & 40.91 & 2.6 & 41 \\
\hline 42 & 41.94 & 2. 20 & 41.93 & 2.38 & 41.92 & 2.5 & 41.91 & & 42 \\
\hline 43 & 42.94 & 2.25 & 42.93 & 2.44 & 42. 92 & 2.1 & 42.91 & 2.8 & 43 \\
\hline 44 & 43.94 & 2. 30 & 43.93 & 2.49 & 43.92 & 2.0 & 43.91 & 2.8 & 44 \\
\hline 45 & 44. 94 & 2.36 & 44. 93 & 2.55 & 44. 92 & 2.75 & 44. 90 & 2. 94 & 45 \\
\hline 46 & 45.94 & 2.41 & 45. 93 & 2.61 & 45.91 & 2.81 & 45.90 & 3.01 & 46 \\
\hline 47 & 46.94 & 2.46 & 46.92 & 2. 66 & 46.91 & 2.87 & 46. 90 & 3.07 & 47 \\
\hline 48 & 47. 93 & 2.51 & 47.92 & 2.72 & 47.91 & 2. 93 & 47.90 & 3.14 & 48 \\
\hline 49 & 48.93 & 2.56 & 48.92 & 2.78 & 4S. 91 & 2. 99 & 48.90 & 3. 20 & 49 \\
\hline 50 & 49.93 & 2. 62 & 19.92 & 2.83 & 49.91 & 3.05 & 49.89 & 3.27 & 50 \\
\hline \multirow{2}{*}{ 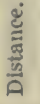 } & Dep. & Lat. & Dep. & Iat. & Dep. & Iat. & Dep. & Lat. & $\dot{\delta}$ \\
\hline & \multicolumn{2}{|c|}{$87^{\circ}}$. & \multicolumn{2}{|c|}{$566^{3} / 4^{\circ}$. } & \multicolumn{2}{|c|}{$S 61: 2^{\circ}}$. & \multicolumn{2}{|c|}{$81: 4^{\circ}}$. & \\
\hline
\end{tabular}


TABLE 4.-THAVEISE TABLE。

\begin{tabular}{|c|c|c|c|c|c|c|c|c|c|}
\hline \multirow{2}{*}{$\frac{\vec{D}}{\tilde{n}}$} & \multicolumn{2}{|c|}{$3^{\circ}$} & \multicolumn{2}{|c|}{$314^{\circ}$. } & \multicolumn{2}{|c|}{$31 / 2^{\circ}}$. & \multicolumn{2}{|c|}{$3 \% 4^{\circ}$. } & \multirow{2}{*}{ 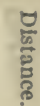 } \\
\hline & Lat. & Dep. & lat. & Dep. & Lat. & Dep. & Iat. & Dep. & \\
\hline $\begin{array}{l}51 \\
52 \\
53 \\
54 \\
55 \\
56 \\
57 \\
55 \\
59 \\
60\end{array}$ & $\begin{array}{l}50.93 \\
51.93 \\
52.93 \\
53.93 \\
54.92 \\
55.92 \\
56.92 \\
57.92 \\
58.92 \\
59.92\end{array}$ & $\begin{array}{l}2.67 \\
2.72 \\
2.77 \\
2.83 \\
2.85 \\
2.93 \\
2.95 \\
3.04 \\
3.09 \\
3.14\end{array}$ & $\begin{array}{l}50.92 \\
51.92 \\
52.91 \\
53.91 \\
54.91 \\
55.91 \\
56.91 \\
57.91 \\
58.91 \\
59.90\end{array}$ & $\begin{array}{l}2.89 \\
3.95 \\
3.00 \\
3.06 \\
3.12 \\
3.17 \\
3.23 \\
3.29 \\
3.34 \\
3.40\end{array}$ & $\begin{array}{l}50.90 \\
51.90 \\
52.90 \\
53.90 \\
54.90 \\
55.90 \\
56.89 \\
57.89 \\
54.89 \\
59.89\end{array}$ & $\begin{array}{l}3.11 \\
3.17 \\
3.24 \\
3.30 \\
3.36 \\
3.42 \\
3.48 \\
3.54 \\
3.60 \\
3.66\end{array}$ & $\begin{array}{l}50.89 \\
51.89 \\
52.89 \\
53.88 \\
54.85 \\
55.88 \\
56.88 \\
57.88 \\
58.87 \\
59.87\end{array}$ & $\begin{array}{l}3.34 \\
3.40 \\
3.47 \\
3.53 \\
3.60 \\
3.66 \\
3.73 \\
3.79 \\
3.86 \\
3.92\end{array}$ & $\begin{array}{l}51 \\
52 \\
53 \\
54 \\
55 \\
56 \\
57 \\
58 \\
59 \\
60\end{array}$ \\
\hline $\begin{array}{l}61 \\
62 \\
63 \\
64 \\
65 \\
65 \\
67 \\
68 \\
69 \\
70\end{array}$ & $\begin{array}{l}60.92 \\
61.92 \\
62.91 \\
63.91 \\
61.91 \\
65.91 \\
66.91 \\
67.91 \\
68.91 \\
69.90\end{array}$ & $\begin{array}{l}3.12 \\
3.24 \\
3.30 \\
3.35 \\
3.40 \\
3.45 \\
3.51 \\
3.56 \\
3.61 \\
3.66\end{array}$ & $\begin{array}{l}60.90 \\
61.90 \\
62.90 \\
63.90 \\
64.90 \\
65.89 \\
61.89 \\
67.89 \\
64.89 \\
69.89\end{array}$ & $\begin{array}{l}3.46 \\
3.51 \\
3.57 \\
3.63 \\
3.69 \\
3.74 \\
3.80 \\
3.86 \\
3.91 \\
3.97\end{array}$ & $\begin{array}{l}60.89 \\
61.88 \\
62.88 \\
63.88 \\
64.89 \\
65.88 \\
66.88 \\
67.87 \\
68.87 \\
69.87\end{array}$ & $\begin{array}{l}3.72 \\
3.79 \\
3.85 \\
3.91 \\
3.97 \\
4.03 \\
4.09 \\
4.15 \\
4.21 \\
4.27\end{array}$ & $\begin{array}{l}60.87 \\
61.87 \\
62.87 \\
63.86 \\
64.86 \\
65.86 \\
66.86 \\
67.85 \\
68.85 \\
69.85\end{array}$ & $\begin{array}{l}3.99 \\
4.05 \\
4.12 \\
4.19 \\
4.25 \\
4.32 \\
4.38 \\
4.45 \\
4.51 \\
4.58\end{array}$ & $\begin{array}{l}61 \\
62 \\
63 \\
64 \\
65 \\
66 \\
67 \\
68 \\
69 \\
70\end{array}$ \\
\hline $\begin{array}{l}71 \\
72 \\
73 \\
74 \\
75 \\
76 \\
77 \\
75 \\
79 \\
80\end{array}$ & $\begin{array}{l}70.90 \\
71.90 \\
72.90 \\
73.90 \\
74.90 \\
75.90 \\
76.59 \\
77.59 \\
78.89 \\
79.59\end{array}$ & $\begin{array}{l}3.72 \\
3.77 \\
3.82 \\
3.87 \\
3.93 \\
3.88 \\
4.03 \\
4.09 \\
4.13 \\
4.19\end{array}$ & $\begin{array}{l}70.89 \\
71.88 \\
72.88 \\
73.88 \\
74.89 \\
75.89 \\
76.88 \\
77.87 \\
78.87 \\
79.87\end{array}$ & $\begin{array}{l}4.03 \\
4.08 \\
4.14 \\
4.20 \\
4.25 \\
4.31 \\
4.37 \\
4.42 \\
4.48 \\
4.54\end{array}$ & $\begin{array}{l}70.87 \\
71.87 \\
72.86 \\
73.86 \\
74.86 \\
75.86 \\
76.86 \\
77.85 \\
78.85 \\
79.85\end{array}$ & $\begin{array}{l}4.33 \\
4.40 \\
4.46 \\
4.52 \\
4.58 \\
4.64 \\
4.70 \\
4.76 \\
4.82 \\
4.85\end{array}$ & $\begin{array}{l}70.85 \\
71.85 \\
72.84 \\
73.84 \\
74.81 \\
75.84 \\
76.84 \\
77.83 \\
78.83 \\
79.83\end{array}$ & $\begin{array}{l}4.64 \\
4.71 \\
4.77 \\
4.84 \\
4.91 \\
4.97 \\
5.04 \\
5.10 \\
5.17 \\
5.23\end{array}$ & $\begin{array}{l}71 \\
72 \\
73 \\
74 \\
75 \\
76 \\
77 \\
78 \\
79 \\
80\end{array}$ \\
\hline $\begin{array}{l}81 \\
82 \\
83 \\
84 \\
85 \\
86 \\
87 \\
89 \\
89 \\
90\end{array}$ & $\begin{array}{l}80.89 \\
81.89 \\
82.89 \\
83.85 \\
84.85 \\
85.85 \\
86.85 \\
87.85 \\
88.85 \\
89.88\end{array}$ & $\begin{array}{l}4.24 \\
4.29 \\
4.34 \\
4.40 \\
4.45 \\
4.50 \\
4.55 \\
4.61 \\
4.66 \\
4.71\end{array}$ & $\begin{array}{l}80.87 \\
81.87 \\
82.87 \\
83.86 \\
84.86 \\
85.86 \\
86.86 \\
87.86 \\
88.86 \\
89.86\end{array}$ & $\begin{array}{l}4.59 \\
4.65 \\
4.61 \\
4.76 \\
4.82 \\
4.89 \\
4.93 \\
4.99 \\
5.05 \\
5.10\end{array}$ & $\begin{array}{l}80.85 \\
81.85 \\
82.85 \\
83.84 \\
84.84 \\
85.84 \\
86.84 \\
87.84 \\
84.83 \\
89.83\end{array}$ & $\begin{array}{l}4.94 \\
5.01 \\
5.07 \\
5.13 \\
5.19 \\
5.25 \\
5.31 \\
5.37 \\
5.43 \\
5.43\end{array}$ & $\begin{array}{l}80.83 \\
81.82 \\
82.82 \\
83.82 \\
84.82 \\
85.82 \\
86.81 \\
87.81 \\
88.81 \\
89.81\end{array}$ & $\begin{array}{l}5.30 \\
5.36 \\
5.43 \\
5.49 \\
5.56 \\
5.62 \\
5.69 \\
5.76 \\
5.82 \\
5.89\end{array}$ & $\begin{array}{l}81 \\
82 \\
83 \\
84 \\
85 \\
86 \\
87 \\
88 \\
89 \\
90\end{array}$ \\
\hline $\begin{array}{r}91 \\
92 \\
93 \\
94 \\
95 \\
96 \\
97 \\
98 \\
99 \\
100\end{array}$ & $\begin{array}{l}90.88 \\
91.87 \\
92.87 \\
93.87 \\
94.87 \\
95.87 \\
96.87 \\
97.87 \\
98.86 \\
95.85\end{array}$ & $\begin{array}{l}\text { 4. } 73 \\
4.81 \\
4.87 \\
4.92 \\
4.97 \\
5.02 \\
5.08 \\
5.13 \\
5.18 \\
5.23\end{array}$ & $\begin{array}{l}90.85 \\
91.85 \\
92.85 \\
93.85 \\
94.85 \\
95.85 \\
96.84 \\
97.84 \\
98.84 \\
99.84\end{array}$ & $\begin{array}{l}\text { 5. } 16 \\
5.22 \\
5.27 \\
5.33 \\
5.39 \\
5.44 \\
5.50 \\
5.56 \\
5.61 \\
5.67\end{array}$ & $\begin{array}{l}90.83 \\
91.83 \\
92.83 \\
93.82 \\
94.82 \\
95.82 \\
93.82 \\
97.82 \\
98.82 \\
99.81\end{array}$ & $\begin{array}{l}5.56 \\
5.62 \\
5.68 \\
5.74 \\
5.80 \\
5.86 \\
5.92 \\
5.98 \\
6.04 \\
6.10\end{array}$ & $\begin{array}{l}90.81 \\
91.80 \\
92.80 \\
93.80 \\
94.80 \\
95.79 \\
96.79 \\
97.79 \\
91.79 \\
99.79\end{array}$ & $\begin{array}{l}5.95 \\
6.02 \\
6.08 \\
6.15 \\
6.21 \\
6.28 \\
6.34 \\
6.41 \\
6.47 \\
6.54\end{array}$ & $\begin{array}{r}91 \\
92 \\
93 \\
94 \\
95 \\
96 \\
97 \\
98 \\
99 \\
100\end{array}$ \\
\hline ๕ீं & Dep. & Lat. & Dep. & Lat. & Dep. & Lat. & Dep. & Isat. & ن \\
\hline 商 & \multicolumn{2}{|c|}{$87^{\circ}}$. & \multicolumn{2}{|c|}{$864^{3} 4^{\circ}$. } & \multicolumn{2}{|c|}{$8612^{\circ}$. } & \multicolumn{2}{|c|}{$861 / 4^{\circ}$. } & $\overrightarrow{0}$ \\
\hline
\end{tabular}


TABLE 4.-TRAVERSE TABLE.

\begin{tabular}{|c|c|c|c|c|c|c|c|c|c|}
\hline \multirow{2}{*}{ 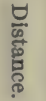 } & \multicolumn{2}{|c|}{$4^{\circ}}$. & \multicolumn{2}{|c|}{$41 / 4^{\circ}$. } & \multicolumn{2}{|c|}{$41 / 2^{\circ}}$. & \multicolumn{2}{|c|}{$4^{3} / 4^{\circ}$. } & \multirow{2}{*}{ 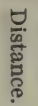 } \\
\hline & Lat. & Dep. & Iat. & Dep. & Lat. & Dep. & Iuat. & Dep. & \\
\hline $\begin{array}{r}1 \\
2 \\
3 \\
4 \\
5 \\
6 \\
7 \\
8 \\
9 \\
10\end{array}$ & $\begin{array}{l}1.00 \\
2.00 \\
2.99 \\
3.99 \\
4.99 \\
5.99 \\
6.98 \\
7.98 \\
8.98 \\
9.98\end{array}$ & $\begin{array}{l}0.07 \\
0.14 \\
0.21 \\
0.28 \\
0.35 \\
0.42 \\
0.49 \\
0.56 \\
0.63 \\
0.70\end{array}$ & $\begin{array}{l}1.00 \\
1.99 \\
2.99 \\
3.99 \\
4.99 \\
5.98 \\
6.98 \\
7.98 \\
8.98 \\
9.97\end{array}$ & $\begin{array}{l}0.07 \\
0.15 \\
0.22 \\
0.30 \\
0.37 \\
0.44 \\
0.52 \\
0.59 \\
0.67 \\
0.74\end{array}$ & $\begin{array}{l}1.00 \\
1.99 \\
2.99 \\
3.99 \\
4.95 \\
5.98 \\
6.98 \\
7.98 \\
8.97 \\
9.97\end{array}$ & $\begin{array}{l}0.08 \\
0.16 \\
0.24 \\
0.31 \\
0.39 \\
0.47 \\
0.55 \\
0.63 \\
0.71 \\
0.78\end{array}$ & $\begin{array}{l}1.00 \\
1.99 \\
2.99 \\
3.99 \\
4.98 \\
5.98 \\
6.98 \\
7.97 \\
8.97 \\
9.97\end{array}$ & $\begin{array}{l}0.08 \\
0.17 \\
0.25 \\
0.33 \\
0.41 \\
0.50 \\
0.58 \\
0.66 \\
0.75 \\
0.83\end{array}$ & $\begin{array}{r}1 \\
2 \\
3 \\
4 \\
5 \\
6 \\
7 \\
8 \\
9 \\
10\end{array}$ \\
\hline $\begin{array}{l}11 \\
12 \\
13 \\
14 \\
15 \\
16 \\
17 \\
18 \\
19 \\
20\end{array}$ & $\begin{array}{l}10.97 \\
11.97 \\
12.97 \\
13.97 \\
14.96 \\
15.96 \\
16.96 \\
17.96 \\
18.95 \\
19.95\end{array}$ & $\begin{array}{l}0.77 \\
0.84 \\
0.91 \\
0.98 \\
1.05 \\
1.12 \\
1.19 \\
1.26 \\
1.33 \\
1.40\end{array}$ & $\begin{array}{l}10.97 \\
11.97 \\
12.96 \\
13.96 \\
14.96 \\
15.96 \\
16.95 \\
17.95 \\
18.95 \\
19.95\end{array}$ & $\begin{array}{l}0.82 \\
0.89 \\
0.96 \\
1.04 \\
1.11 \\
1.19 \\
1.26 \\
1.33 \\
1.41 \\
1.48\end{array}$ & $\begin{array}{l}10.97 \\
11.96 \\
12.96 \\
13.96 \\
14.95 \\
15.95 \\
16.95 \\
17.94 \\
18.94 \\
19.94\end{array}$ & $\begin{array}{l}0.86 \\
0.94 \\
1.02 \\
1.10 \\
1.18 \\
1.26 \\
1.33 \\
1.41 \\
1.49 \\
1.57\end{array}$ & $\begin{array}{l}10.96 \\
11.96 \\
12.96 \\
13.95 \\
14.95 \\
15.95 \\
16.94 \\
17.94 \\
18.93 \\
19.93\end{array}$ & $\begin{array}{l}0.91 \\
0.99 \\
1.08 \\
1.16 \\
1.24 \\
1.32 \\
1.41 \\
1.49 \\
1.57 \\
1.66\end{array}$ & $\begin{array}{l}11 \\
12 \\
13 \\
14 \\
15 \\
16 \\
17 \\
18 \\
19 \\
20\end{array}$ \\
\hline $\begin{array}{l}21 \\
22 \\
23 \\
24 \\
25 \\
26 \\
27 \\
28 \\
29 \\
30\end{array}$ & $\begin{array}{l}20.95 \\
21.95 \\
22.94 \\
23.94 \\
24.94 \\
25.94 \\
26.93 \\
27.93 \\
28.93 \\
29.93\end{array}$ & $\begin{array}{l}1.46 \\
1.53 \\
1.60 \\
1.67 \\
1.74 \\
1.81 \\
1.88 \\
1.95 \\
2.02 \\
2.09\end{array}$ & $\begin{array}{l}20.94 \\
21.94 \\
22.94 \\
23.93 \\
24.93 \\
25.93 \\
26.93 \\
27.92 \\
28.92 \\
29.92\end{array}$ & $\begin{array}{l}1.56 \\
1.63 \\
1.70 \\
1.78 \\
1.85 \\
1.93 \\
2.00 \\
2.08 \\
2.15 \\
2.22\end{array}$ & $\begin{array}{l}20.94 \\
21.93 \\
22.93 \\
23.93 \\
24.92 \\
25.92 \\
26.92 \\
27.91 \\
28.91 \\
29.91\end{array}$ & $\begin{array}{l}1.65 \\
1.73 \\
1.80 \\
1.88 \\
1.96 \\
2.04 \\
2.12 \\
2.20 \\
2.28 \\
2.35\end{array}$ & $\begin{array}{l}20.93 \\
21.92 \\
22.92 \\
23.92 \\
24.91 \\
25.91 \\
26.91 \\
27.90 \\
28.90 \\
29.90\end{array}$ & $\begin{array}{l}1.74 \\
1.82 \\
1.90 \\
1.99 \\
2.07 \\
2.15 \\
2.24 \\
2.32 \\
2.40 \\
2.48\end{array}$ & $\begin{array}{l}21 \\
22 \\
23 \\
24 \\
25 \\
26 \\
27 \\
28 \\
29 \\
30\end{array}$ \\
\hline $\begin{array}{l}31 \\
32 \\
33 \\
34 \\
35 \\
36 \\
37 \\
38 \\
39 \\
10\end{array}$ & $\begin{array}{l}30.92 \\
31.92 \\
32.92 \\
33.92 \\
34.91 \\
35.91 \\
36.91 \\
37.91 \\
38.90 \\
39.90\end{array}$ & $\begin{array}{l}2.16 \\
2.23 \\
2.30 \\
2.37 \\
2.44 \\
2.51 \\
2.58 \\
2.65 \\
2.72 \\
2.79\end{array}$ & $\begin{array}{l}30.91 \\
31.91 \\
32.91 \\
33.91 \\
34.90 \\
35.90 \\
36.90 \\
37.90 \\
38.89 \\
39.89\end{array}$ & $\begin{array}{l}2.30 \\
2.37 \\
2.45 \\
2.52 \\
2.59 \\
2.67 \\
2.74 \\
2.82 \\
2.89 \\
2.96\end{array}$ & $\begin{array}{l}30.90 \\
31.90 \\
32.90 \\
33.90 \\
34.89 \\
35.89 \\
36.89 \\
37.88 \\
38.88 \\
39.88\end{array}$ & $\begin{array}{l}2.43 \\
2.51 \\
2.59 \\
2.67 \\
2.75 \\
2.82 \\
2.90 \\
2.98 \\
3.06 \\
3.14\end{array}$ & $\begin{array}{l}30.89 \\
31.89 \\
32.89 \\
33.88 \\
34.88 \\
35.88 \\
36.87 \\
37.87 \\
38.87 \\
39.86\end{array}$ & $\begin{array}{l}2.57 \\
2.65 \\
2.73 \\
2.82 \\
2.90 \\
2.98 \\
3.06 \\
3.15 \\
3.23 \\
3.31\end{array}$ & $\begin{array}{l}31 \\
32 \\
33 \\
34 \\
35 \\
36 \\
37 \\
38 \\
39 \\
40\end{array}$ \\
\hline $\begin{array}{l}41 \\
42 \\
43 \\
44 \\
45 \\
46 \\
47 \\
48 \\
49 \\
50\end{array}$ & $\begin{array}{l}40.90 \\
41.90 \\
42.90 \\
43.89 \\
44.89 \\
45.89 \\
46.89 \\
47.88 \\
49.88 \\
49.88\end{array}$ & $\begin{array}{l}2.86 \\
2.93 \\
3.00 \\
3.07 \\
3.14 \\
3.21 \\
3.28 \\
3.35 \\
3.42 \\
3.49\end{array}$ & $\begin{array}{l}40.89 \\
41.88 \\
42.88 \\
43.88 \\
44.88 \\
45.87 \\
46.87 \\
47.87 \\
48.87 \\
49.86\end{array}$ & $\begin{array}{l}3.04 \\
3.11 \\
3.19 \\
3.26 \\
3.33 \\
3.41 \\
3.48 \\
3.56 \\
3.63 \\
3.71\end{array}$ & $\begin{array}{l}40.87 \\
41.87 \\
42.87 \\
43.86 \\
44.86 \\
45.86 \\
46.86 \\
47.85 \\
48.85 \\
49.85\end{array}$ & $\begin{array}{l}3.22 \\
3.30 \\
3.37 \\
3.45 \\
3.53 \\
3.61 \\
3.69 \\
3.77 \\
3.84 \\
3.92\end{array}$ & $\begin{array}{l}40.86 \\
41.86 \\
42.85 \\
43.85 \\
44.85 \\
45.84 \\
46.84 \\
47.84 \\
48.83 \\
49.83\end{array}$ & $\begin{array}{l}3.40 \\
3.48 \\
3.56 \\
3.64 \\
3.73 \\
3.81 \\
3.89 \\
3.97 \\
4.06 \\
4.14\end{array}$ & $\begin{array}{l}41 \\
42 \\
43 \\
44 \\
45 \\
46 \\
47 \\
48 \\
49 \\
50\end{array}$ \\
\hline छ্छ & Dep. & Lat. & Dep. & Lat. & Dep. & Lat. & Dep. & Lat. & छ் \\
\hline & \multicolumn{2}{|c|}{$86^{\circ}$. } & \multicolumn{2}{|c|}{$85^{3} 4^{\circ}$. } & \multicolumn{2}{|c|}{$851 / 2^{\circ}$. } & \multicolumn{2}{|c|}{$851 / 4^{\circ}$. } & $\overline{\bar{A}}$ \\
\hline
\end{tabular}


TABLE 4.-TRAVERSE TABLE.

\begin{tabular}{|c|c|c|c|c|c|c|c|c|c|}
\hline \multirow{2}{*}{$\begin{array}{l}\frac{J}{n} \\
\frac{5}{5} \\
\frac{5}{0} \\
0\end{array}$} & \multicolumn{2}{|c|}{$4^{\circ}$} & \multicolumn{2}{|c|}{$41 / 4^{\circ}}$. & \multicolumn{2}{|c|}{$41 / 2^{\circ}$} & \multicolumn{2}{|c|}{$4^{3} / 4^{0}$} & \multirow{2}{*}{$\begin{array}{l}\frac{\theta}{5} \\
\frac{5}{5} \\
\frac{8}{\sigma}\end{array}$} \\
\hline & Lat. & Dep. & Lat. & Dep. & Lat. & Dep. & Lat. & Dep. & \\
\hline $\begin{array}{l}51 \\
52 \\
53 \\
54 \\
55 \\
56 \\
57 \\
58 \\
59 \\
60\end{array}$ & $\begin{array}{l}50.88 \\
51.87 \\
52.87 \\
53.87 \\
54.87 \\
55.86 \\
56.86 \\
57.86 \\
58.86 \\
59.85\end{array}$ & $\begin{array}{l}3.56 \\
\text { 3. } 63 \\
\text { 3. } 70 \\
\text { 3. } 77 \\
\text { 3. } 84 \\
\text { 3. } 91 \\
\text { 3. } 98 \\
4.05 \\
\text { 4. } 12 \\
\text { 4. } 19\end{array}$ & $\begin{array}{l}50.86 \\
51.86 \\
52.85 \\
53.85 \\
54.85 \\
55.85 \\
56.84 \\
57.84 \\
55.84 \\
59.84\end{array}$ & $\begin{array}{l}3.75 \\
3.85 \\
3.93 \\
4.00 \\
4.08 \\
4.15 \\
4.22 \\
4.30 \\
4.37 \\
4.45\end{array}$ & $\begin{array}{l}50.84 \\
51.84 \\
52.84 \\
53.83 \\
54.83 \\
54.83 \\
56.82 \\
57.82 \\
58.82 \\
59.82\end{array}$ & $\begin{array}{l}4.00 \\
4.08 \\
4.16 \\
4.24 \\
4.32 \\
4.39 \\
4.47 \\
4.55 \\
4.63 \\
4.71\end{array}$ & $\begin{array}{l}50.82 \\
51.82 \\
52.82 \\
53.81 \\
54.81 \\
55.81 \\
56.80 \\
57.80 \\
58.80 \\
59.79\end{array}$ & $\begin{array}{l}4.22 \\
4.31 \\
4.39 \\
4.47 \\
4.55 \\
4.64 \\
4.72 \\
4.80 \\
4.89 \\
4.97\end{array}$ & $\begin{array}{l}51 \\
52 \\
53 \\
54 \\
55 \\
56 \\
57 \\
58 \\
59 \\
60\end{array}$ \\
\hline $\begin{array}{l}61 \\
62 \\
63 \\
64 \\
65 \\
66 \\
67 \\
68 \\
69 \\
70\end{array}$ & $\begin{array}{l}60.85 \\
61.85 \\
62.85 \\
63.84 \\
64.84 \\
65.84 \\
60.84 \\
67.83 \\
68.83 \\
69.83\end{array}$ & $\begin{array}{l}4.26 \\
4.32 \\
4.39 \\
4.46 \\
4.53 \\
4.60 \\
4.67 \\
4.74 \\
4.81 \\
4.58\end{array}$ & $\begin{array}{l}60.83 \\
61.83 \\
62.83 \\
63.82 \\
64.82 \\
65.82 \\
66.82 \\
67.81 \\
68.81 \\
69.81\end{array}$ & $\begin{array}{l}4.52 \\
4.59 \\
4.67 \\
4.74 \\
4.82 \\
4.89 \\
4.97 \\
5.04 \\
5.11 \\
5.19\end{array}$ & $\begin{array}{l}60.81 \\
61.81 \\
62.81 \\
63.80 \\
64.80 \\
65.80 \\
66.79 \\
67.79 \\
68.79 \\
69.78\end{array}$ & $\begin{array}{l}\text { 4. } 79 \\
4.86 \\
4.94 \\
5.02 \\
5.10 \\
5.18 \\
5.26 \\
5.34 \\
5.41 \\
5.49\end{array}$ & $\begin{array}{l}60.79 \\
61.79 \\
62.78 \\
63.78 \\
64.78 \\
65.77 \\
66.77 \\
67.77 \\
68.76 \\
69.76\end{array}$ & $\begin{array}{l}\text { 5. } 05 \\
5.13 \\
5.22 \\
5.30 \\
5.38 \\
5.47 \\
5.55 \\
5.63 \\
5.71 \\
5.80\end{array}$ & $\begin{array}{l}61 \\
62 \\
63 \\
64 \\
65 \\
66 \\
67 \\
68 \\
69 \\
70\end{array}$ \\
\hline $\begin{array}{l}71 \\
72 \\
73 \\
74 \\
75 \\
76 \\
77 \\
78 \\
79 \\
80\end{array}$ & $\begin{array}{l}70.83 \\
71.82 \\
72.82 \\
73.82 \\
71.52 \\
75.81 \\
76.81 \\
77.81 \\
78.81 \\
79.81\end{array}$ & $\begin{array}{l}\text { 4. } 95 \\
5.02 \\
5.09 \\
5.16 \\
5.23 \\
5.30 \\
5.37 \\
5.44 \\
5.51 \\
5.58\end{array}$ & $\begin{array}{l}70.80 \\
71.80 \\
72.80 \\
73.80 \\
74.79 \\
75.79 \\
76.79 \\
77.79 \\
78.78 \\
79.78\end{array}$ & $\begin{array}{l}\text { 5. } 26 \\
5.34 \\
5.41 \\
5.48 \\
5.56 \\
5.63 \\
5.71 \\
5.78 \\
5.85 \\
5.93\end{array}$ & $\begin{array}{l}70.78 \\
71.78 \\
72.77 \\
73.77 \\
74.77 \\
75.77 \\
76.76 \\
77.76 \\
78.76 \\
79.75\end{array}$ & $\begin{array}{l}5.57 \\
5.65 \\
5.73 \\
5.81 \\
5.88 \\
5.96 \\
6.04 \\
6.12 \\
6.20 \\
6.28\end{array}$ & $\begin{array}{l}70.76 \\
71.75 \\
72.75 \\
73.75 \\
74.74 \\
75.74 \\
76.74 \\
77.73 \\
78.73 \\
79.73\end{array}$ & $\begin{array}{l}5.88 \\
5.96 \\
6.04 \\
6.13 \\
6.21 \\
6.29 \\
6.38 \\
6.46 \\
6.54 \\
6.62\end{array}$ & $\begin{array}{l}71 \\
72 \\
73 \\
74 \\
75 \\
76 \\
77 \\
78 \\
79 \\
80\end{array}$ \\
\hline $\begin{array}{l}81 \\
82 \\
83 \\
84 \\
85 \\
86 \\
87 \\
88 \\
89 \\
90\end{array}$ & $\begin{array}{l}80.80 \\
81.80 \\
82.80 \\
83.80 \\
81.79 \\
85.79 \\
86.79 \\
87.79 \\
89.78 \\
89.78\end{array}$ & $\begin{array}{l}5.65 \\
5.72 \\
5.79 \\
5.86 \\
5.93 \\
6.00 \\
6.07 \\
6.14 \\
6.21 \\
6.28\end{array}$ & $\begin{array}{l}80.78 \\
81.77 \\
82.77 \\
83.77 \\
84.77 \\
85.76 \\
86.76 \\
87.76 \\
88.76 \\
89.75\end{array}$ & $\begin{array}{l}6.00 \\
6.08 \\
6.15 \\
6.23 \\
6.30 \\
6.37 \\
6.45 \\
6.52 \\
6.60 \\
6.67\end{array}$ & $\begin{array}{l}80.75 \\
81.75 \\
82.74 \\
83.74 \\
84.74 \\
85.73 \\
80.73 \\
87.73 \\
85.73 \\
89.72\end{array}$ & $\begin{array}{l}6.36 \\
6.43 \\
6.51 \\
6.59 \\
6.67 \\
6.75 \\
6.83 \\
6.90 \\
6.98 \\
7.06\end{array}$ & $\begin{array}{l}80.72 \\
81.72 \\
82.71 \\
83.71 \\
84.71 \\
85.70 \\
86.70 \\
87.70 \\
88.69 \\
89.69\end{array}$ & $\begin{array}{l}6.71 \\
6.79 \\
6.87 \\
6.96 \\
7.04 \\
7.12 \\
7.20 \\
7.29 \\
7.37 \\
7.45\end{array}$ & $\begin{array}{l}81 \\
82 \\
83 \\
84 \\
85 \\
86 \\
87 \\
88 \\
89 \\
90\end{array}$ \\
\hline $\begin{array}{r}91 \\
92 \\
93 \\
94 \\
95 \\
96 \\
97 \\
98 \\
99 \\
100\end{array}$ & $\begin{array}{l}90.78 \\
91.75 \\
92.77 \\
93.77 \\
94.77 \\
95.77 \\
96.76 \\
97.76 \\
95.76 \\
99.76\end{array}$ & $\begin{array}{l}\text { 6. } 35 \\
\text { 6. } 42 \\
\text { G. } 49 \\
\text { G. } 56 \\
\text { 6. } 63 \\
\text { 6. } 70 \\
\text { 6. } 77 \\
\text { G. } 81 \\
\text { 6. } 91 \\
\text { 6. } 98\end{array}$ & $\begin{array}{l}90.75 \\
91.75 \\
92.74 \\
93.74 \\
94.74 \\
95.74 \\
96.73 \\
97.73 \\
9.73 \\
99.73\end{array}$ & $\begin{array}{l}\text { 6. } 74 \\
6.82 \\
\text { 6. } 89 \\
6.97 \\
7.04 \\
7.11 \\
7.19 \\
7.26 \\
7.34 \\
7.41\end{array}$ & $\begin{array}{l}90.72 \\
91.72 \\
92.71 \\
93.71 \\
94.71 \\
95.70 \\
96.70 \\
97.70 \\
9.69 \\
99.69\end{array}$ & $\begin{array}{l}7.14 \\
7.22 \\
7.30 \\
7.38 \\
7.45 \\
7.53 \\
7.61 \\
7.69 \\
7.77 \\
7.85\end{array}$ & $\begin{array}{l}90.69 \\
91.68 \\
92.68 \\
93.68 \\
94.67 \\
95.67 \\
96.67 \\
97.66 \\
98.66 \\
99.66\end{array}$ & $\begin{array}{l}7.54 \\
7.62 \\
7.70 \\
7.78 \\
7.87 \\
7.95 \\
8.03 \\
8.12 \\
8.20 \\
8.28\end{array}$ & $\begin{array}{r}91 \\
92 \\
93 \\
94 \\
95 \\
96 \\
97 \\
98 \\
99 \\
100\end{array}$ \\
\hline \multirow{2}{*}{ 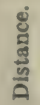 } & Dep. & Lat. & Dep. & Lat. & Dep. & Lat. & Dep. & Lat. & \multirow{2}{*}{ 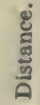 } \\
\hline & \multicolumn{2}{|c|}{$86^{\circ}}$. & \multicolumn{2}{|c|}{$8534^{\circ}}$. & \multicolumn{2}{|c|}{$851 / 2^{\circ}$. } & \multicolumn{2}{|c|}{$851 / 4^{\circ}$. } & \\
\hline
\end{tabular}




\begin{tabular}{|c|c|c|c|c|c|c|c|c|c|}
\hline \multirow{2}{*}{ 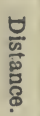 } & \multicolumn{2}{|c|}{$5^{\circ}}$. & \multicolumn{2}{|c|}{$51 / 44^{\circ}$. } & \multicolumn{2}{|c|}{$51 / 2^{\circ}$. } & \multicolumn{2}{|c|}{$584^{\circ}}$. & \multirow{2}{*}{ 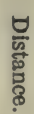 } \\
\hline & Lat. & Dep. & Lât. & Dep. & Lat. & Dep. & Lat. & Dep. & \\
\hline $\begin{array}{r}1 \\
2 \\
3 \\
4 \\
5 \\
6 \\
7 \\
8 \\
9 \\
10\end{array}$ & $\begin{array}{l}1.00 \\
1.99 \\
2.99 \\
3.98 \\
4.98 \\
5.95 \\
6.97 \\
7.97 \\
8.97 \\
9.96\end{array}$ & $\begin{array}{l}0.09 \\
0.17 \\
0.26 \\
0.35 \\
0.44 \\
0.52 \\
0.61 \\
0.70 \\
0.78 \\
0.87\end{array}$ & $\begin{array}{l}1.00 \\
1.99 \\
2.99 \\
3.98 \\
4.98 \\
5.97 \\
6.97 \\
7.97 \\
8.96 \\
9.96\end{array}$ & $\begin{array}{l}0.09 \\
0.18 \\
0.27 \\
0.37 \\
0.46 \\
0.55 \\
0.64 \\
0.73 \\
0.82 \\
0.92\end{array}$ & $\begin{array}{l}1.00 \\
1.99 \\
2.99 \\
3.98 \\
4.98 \\
5.97 \\
6.97 \\
7.96 \\
8.96 \\
9.95\end{array}$ & $\begin{array}{l}0.10 \\
0.19 \\
0.29 \\
0.38 \\
0.48 \\
0.58 \\
0.67 \\
0.77 \\
0.86 \\
0.96\end{array}$ & $\begin{array}{l}0.99 \\
1.99 \\
2.98 \\
3.98 \\
4.97 \\
5.97 \\
6.96 \\
7.96 \\
8.95 \\
9.95\end{array}$ & $\begin{array}{l}0.10 \\
0.20 \\
0.30 \\
0.40 \\
0.50 \\
0.60 \\
0.70 \\
0.80 \\
0.90 \\
1.00\end{array}$ & $\begin{array}{l}1 \\
2 \\
3 \\
4 \\
5 \\
6\end{array}$ \\
\hline $\begin{array}{l}11 \\
12 \\
13 \\
14 \\
15 \\
16 \\
17 \\
18 \\
19 \\
20\end{array}$ & $\begin{array}{l}10.96 \\
11.95 \\
12.95 \\
13.95 \\
14.94 \\
15.94 \\
16.94 \\
17.93 \\
18.93 \\
19.92\end{array}$ & $\begin{array}{l}0.96 \\
1.05 \\
1.13 \\
1.22 \\
1.31 \\
1.39 \\
1.48 \\
1.57 \\
1.66 \\
1.74\end{array}$ & $\begin{array}{l}10.95 \\
11.95 \\
12.95 \\
13.94 \\
14.94 \\
15.93 \\
16.93 \\
17.92 \\
18.92 \\
19.92\end{array}$ & $\begin{array}{l}1.01 \\
1.10 \\
1.19 \\
1.28 \\
1.37 \\
1.46 \\
1.56 \\
1.65 \\
1.74 \\
1.83\end{array}$ & $\begin{array}{l}10.95 \\
11.94 \\
12.94 \\
13.94 \\
14.93 \\
15.93 \\
16.92 \\
17.92 \\
18.91 \\
19.91\end{array}$ & $\begin{array}{l}1.05 \\
1.15 \\
1.25 \\
1.34 \\
1.44 \\
1.53 \\
1.63 \\
1.73 \\
1.82 \\
1.92\end{array}$ & $\begin{array}{l}10.94 \\
11.94 \\
12.93 \\
13.93 \\
14.92 \\
15.92 \\
16.91 \\
17.91 \\
18.90 \\
19.90\end{array}$ & $\begin{array}{l}1.10 \\
1.20 \\
1.30 \\
1.40 \\
1.50 \\
1.60 \\
1.70 \\
1.80 \\
1.90 \\
2.00\end{array}$ & $\begin{array}{l}11 \\
12 \\
13 \\
14 \\
15 \\
16 \\
17 \\
18 \\
19 \\
20\end{array}$ \\
\hline $\begin{array}{l}21 \\
22 \\
23 \\
24 \\
25 \\
26 \\
27 \\
28 \\
29 \\
30\end{array}$ & $\begin{array}{l}20.92 \\
21.92 \\
22.91 \\
23.91 \\
24.90 \\
25.90 \\
26.90 \\
27.89 \\
28.89 \\
29.89\end{array}$ & $\begin{array}{l}1.83 \\
1.92 \\
2.00 \\
2.09 \\
2.18 \\
2.27 \\
2.35 \\
2.44 \\
2.53 \\
2.61\end{array}$ & $\begin{array}{l}20.91 \\
21.91 \\
22.90 \\
23.90 \\
24.90 \\
25.89 \\
26.89 \\
27.88 \\
28.88 \\
29.87\end{array}$ & $\begin{array}{l}1.92 \\
2.01 \\
2.10 \\
2.20 \\
2.29 \\
2.3 S \\
2.47 \\
2.56 \\
2.65 \\
2.75\end{array}$ & $\begin{array}{l}20.90 \\
21.90 \\
22.89 \\
23.89 \\
24.89 \\
25.85 \\
26.88 \\
27.87 \\
28.87 \\
29.86\end{array}$ & $\begin{array}{l}2.01 \\
2.11 \\
2.20 \\
2.30 \\
2.40 \\
2.49 \\
2.59 \\
2.68 \\
2.78 \\
2.88\end{array}$ & $\begin{array}{l}20.89 \\
21.89 \\
22.88 \\
23.88 \\
24.87 \\
25.87 \\
26.86 \\
27.86 \\
28.85 \\
29.85\end{array}$ & $\begin{array}{l}2.10 \\
2.20 \\
2.30 \\
2.40 \\
2.50 \\
2.69 \\
2.71 \\
2.81 \\
2.91 \\
3.01\end{array}$ & $\begin{array}{l}21 \\
22 \\
23 \\
24 \\
25 \\
26 \\
27 \\
28 \\
29 \\
30\end{array}$ \\
\hline $\begin{array}{l}31 \\
32 \\
33 \\
34 \\
35 \\
36 \\
37 \\
38 \\
39 \\
40\end{array}$ & $\begin{array}{l}30.88 \\
31.88 \\
32.87 \\
33.87 \\
34.87 \\
35.86 \\
30.86 \\
37.86 \\
38.85 \\
39.85\end{array}$ & $\begin{array}{l}2.70 \\
2.79 \\
2.88 \\
2.96 \\
3.05 \\
3.14 \\
3.22 \\
3.31 \\
3.40 \\
3.49\end{array}$ & $\begin{array}{l}30.87 \\
31.87 \\
32.86 \\
33.86 \\
34.85 \\
35.85 \\
36.84 \\
37.84 \\
38.84 \\
39.83\end{array}$ & $\begin{array}{l}2.84 \\
2.93 \\
3.02 \\
3.11 \\
3.20 \\
3.29 \\
3.39 \\
3.48 \\
3.57 \\
3.66\end{array}$ & $\begin{array}{l}30.86 \\
31.85 \\
32.85 \\
33.84 \\
34.84 \\
35.83 \\
36.83 \\
37.83 \\
38.82 \\
39.82\end{array}$ & $\begin{array}{l}2.97 \\
3.07 \\
3.16 \\
3.26 \\
3.35 \\
3.45 \\
3.55 \\
3.64 \\
3.74 \\
3.83\end{array}$ & $\begin{array}{l}30.84 \\
31.84 \\
32.83 \\
33.83 \\
34.82 \\
35.82 \\
36.81 \\
37.81 \\
33.80 \\
39.80\end{array}$ & $\begin{array}{l}3.11 \\
3.21 \\
3.31 \\
3.4 ! \\
3.51 \\
3.61 \\
3.71 \\
3.81 \\
3.91 \\
4.01\end{array}$ & $\begin{array}{l}31 \\
32 \\
33 \\
34 \\
35 \\
36 \\
37 \\
38 \\
39 \\
40\end{array}$ \\
\hline $\begin{array}{l}41 \\
42 \\
43 \\
44 \\
45 \\
46 \\
47 \\
48 \\
49 \\
50\end{array}$ & $\begin{array}{l}40.84 \\
41.84 \\
42.84 \\
43.83 \\
44.82 \\
45.82 \\
46.82 \\
47.82 \\
48.81 \\
49.81\end{array}$ & $\begin{array}{l}3.57 \\
3.66 \\
3.75 \\
3.83 \\
3.92 \\
4.01 \\
4.10 \\
4.18 \\
4.27 \\
4.36\end{array}$ & $\begin{array}{l}40.83 \\
41.82 \\
42.82 \\
43.82 \\
44.81 \\
45.81 \\
46.80 \\
47.80 \\
48.79 \\
49.79\end{array}$ & $\begin{array}{l}3.75 \\
3.84 \\
3.93 \\
4.03 \\
4.12 \\
4.21 \\
4.30 \\
4.39 \\
4.48 \\
4.58\end{array}$ & $\begin{array}{l}40.81 \\
41.81 \\
42.80 \\
43.80 \\
44.79 \\
45.79 \\
46.78 \\
47.78 \\
48.77 \\
49.77\end{array}$ & $\begin{array}{l}3.93 \\
4.03 \\
4.12 \\
4.22 \\
4.31 \\
4.41 \\
4.50 \\
4.60 \\
4.70 \\
4.79\end{array}$ & $\begin{array}{l}40.79 \\
41.79 \\
42.78 \\
43.78 \\
44.77 \\
45.77 \\
46.76 \\
47.76 \\
48.75 \\
49.75\end{array}$ & $\begin{array}{l}4.11 \\
4.21 \\
4.31 \\
4.41 \\
4.51 \\
4.61 \\
4.71 \\
4.81 \\
4.91 \\
5.01\end{array}$ & $\begin{array}{l}41 \\
42 \\
43 \\
44 \\
45 \\
46 \\
47 \\
48 \\
49 \\
50\end{array}$ \\
\hline \&્ટ் & Dep. & Lat. & Dep. & Lat. & Dep. & Lat. & Dep. & Lat. & $\dot{8}$ \\
\hline คै & \multicolumn{2}{|c|}{$85^{\circ}}$. & \multicolumn{2}{|c|}{$8434^{\circ}}$. & \multicolumn{2}{|c|}{$841 / 2^{\circ}$. } & \multicolumn{2}{|c|}{$841 / 4^{\circ}$. } & \\
\hline
\end{tabular}


TABLE 4.-TRAVERSE TABLF.

\begin{tabular}{|c|c|c|c|c|c|c|c|c|c|}
\hline \multirow{2}{*}{ 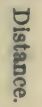 } & \multicolumn{2}{|c|}{$5^{\circ}$} & \multicolumn{2}{|c|}{$51 / 4^{\circ}$. } & \multicolumn{2}{|c|}{$51 / 2^{\circ}$. } & \multicolumn{2}{|c|}{$53 / 4^{\circ}$} & \multirow{2}{*}{ 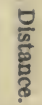 } \\
\hline & Lat. & Dep. & Lat. & Dep. & Lat. & Dep. & Lat. & Dep. & \\
\hline $\begin{array}{l}51 \\
52 \\
53 \\
54 \\
55 \\
56 \\
57 \\
58 \\
59 \\
60\end{array}$ & $\begin{array}{l}50.81 \\
51.80 \\
52.80 \\
53.79 \\
54.79 \\
55.79 \\
56.78 \\
57.78 \\
58.78 \\
59.77\end{array}$ & $\begin{array}{l}4.44 \\
4.53 \\
4.62 \\
4.71 \\
4.79 \\
4.88 \\
4.97 \\
5.06 \\
5.14 \\
5.23\end{array}$ & $\begin{array}{l}50.79 \\
51.78 \\
52.78 \\
53.77 \\
54.77 \\
55.77 \\
56.76 \\
57.76 \\
58.75 \\
59.75\end{array}$ & $\begin{array}{l}4.67 \\
4.76 \\
4.85 \\
4.94 \\
5.03 \\
5.12 \\
5.22 \\
5.31 \\
5.40 \\
5.49\end{array}$ & $\begin{array}{l}50.77 \\
51.76 \\
52.76 \\
53.75 \\
54.75 \\
55.74 \\
56.74 \\
57.73 \\
58.73 \\
59.72\end{array}$ & $\begin{array}{l}4.89 \\
4.98 \\
5.08 \\
5.18 \\
5.27 \\
5.37 \\
5.46 \\
5.56 \\
5.65 \\
5.75\end{array}$ & $\begin{array}{l}50.74 \\
51.74 \\
52.73 \\
53.73 \\
54.72 \\
55.72 \\
56.71 \\
57.71 \\
58.70 \\
59.70\end{array}$ & $\begin{array}{l}5.11 \\
5.21 \\
5.31 \\
5.41 \\
5.51 \\
5.61 \\
5.71 \\
5.81 \\
5.91 \\
6.01\end{array}$ & $\begin{array}{l}51 \\
52 \\
53 \\
54 \\
55 \\
56 \\
57 \\
58 \\
59 \\
60\end{array}$ \\
\hline $\begin{array}{l}61 \\
62 \\
63 \\
64 \\
65 \\
66 \\
67 \\
68 \\
69 \\
70\end{array}$ & $\begin{array}{l}60.77 \\
61.76 \\
62.76 \\
63.76 \\
64.75 \\
65.75 \\
66.75 \\
67.74 \\
68.74 \\
69.73\end{array}$ & $\begin{array}{l}5.32 \\
5.40 \\
5.49 \\
5.58 \\
5.67 \\
5.75 \\
5.84 \\
5.93 \\
6.01 \\
6.10\end{array}$ & $\begin{array}{l}60.74 \\
61.74 \\
62.74 \\
63.73 \\
64.73 \\
65.72 \\
66.72 \\
67.71 \\
68.71 \\
69.71\end{array}$ & $\begin{array}{l}5.58 \\
5.67 \\
5.76 \\
5.86 \\
5.95 \\
6.04 \\
6.13 \\
6.22 \\
6.31 \\
6.41\end{array}$ & $\begin{array}{l}60.72 \\
61.71 \\
62.71 \\
63.71 \\
64.70 \\
65.70 \\
66.69 \\
67.69 \\
68.68 \\
69.68\end{array}$ & $\begin{array}{l}5.85 \\
5.94 \\
6.04 \\
6.13 \\
6.23 \\
6.33 \\
6.42 \\
6.52 \\
6.61 \\
6.71\end{array}$ & $\begin{array}{l}60.69 \\
61.69 \\
62.68 \\
63.68 \\
64.67 \\
65.67 \\
66.66 \\
67.66 \\
68.65 \\
69.65\end{array}$ & $\begin{array}{l}6.11 \\
6.21 \\
6.31 \\
6.41 \\
6.51 \\
6.61 \\
6.71 \\
6.81 \\
6.91 \\
7.01\end{array}$ & $\begin{array}{l}61 \\
62 \\
63 \\
64 \\
65 \\
66 \\
67 \\
68 \\
69 \\
70\end{array}$ \\
\hline $\begin{array}{l}71 \\
72 \\
73 \\
74 \\
75 \\
76 \\
77 \\
78 \\
79 \\
80\end{array}$ & $\begin{array}{l}70.73 \\
71.73 \\
72.72 \\
73.72 \\
74.71 \\
75.71 \\
76.71 \\
77.70 \\
78.70 \\
79.70\end{array}$ & $\begin{array}{l}6.19 \\
6.28 \\
6.36 \\
6.45 \\
6.54 \\
6.62 \\
6.71 \\
6.80 \\
6.89 \\
6.97\end{array}$ & $\begin{array}{l}70.70 \\
71.70 \\
72.69 \\
73.69 \\
74.69 \\
75.68 \\
76.68 \\
77.67 \\
78.67 \\
79.66\end{array}$ & $\begin{array}{l}6.50 \\
6.59 \\
6.68 \\
6.77 \\
6.86 \\
6.95 \\
7.05 \\
7.14 \\
7.23 \\
7.32\end{array}$ & $\begin{array}{l}70.67 \\
71.67 \\
72.66 \\
73.66 \\
74.65 \\
75.65 \\
76.65 \\
77.64 \\
78.64 \\
79.63\end{array}$ & $\begin{array}{l}6.81 \\
6.90 \\
7.00 \\
7.09 \\
7.19 \\
7.28 \\
7.38 \\
7.18 \\
7.57 \\
7.67\end{array}$ & $\begin{array}{l}70.64 \\
71.64 \\
72.63 \\
73.63 \\
74.62 \\
75.62 \\
76.61 \\
77.61 \\
78.60 \\
79.60\end{array}$ & $\begin{array}{l}7.11 \\
7.21 \\
7.31 \\
7.41 \\
7.51 \\
7.61 \\
7.71 \\
7.81 \\
7.91 \\
8.02\end{array}$ & $\begin{array}{l}71 \\
72 \\
73 \\
74 \\
75 \\
76 \\
77 \\
78 \\
79 \\
80\end{array}$ \\
\hline $\begin{array}{l}81 \\
82 \\
83 \\
84 \\
85 \\
86 \\
87 \\
88 \\
89 \\
90\end{array}$ & $\begin{array}{l}80.69 \\
81.69 \\
82.68 \\
83.68 \\
84.68 \\
85.67 \\
86.67 \\
87.67 \\
88.66 \\
89.66\end{array}$ & $\begin{array}{l}7.06 \\
7.15 \\
7.23 \\
7.32 \\
7.41 \\
7.50 \\
7.58 \\
7.67 \\
7.76 \\
7.84\end{array}$ & $\begin{array}{l}80.66 \\
81.66 \\
82.65 \\
83.65 \\
84.64 \\
85.64 \\
86.64 \\
87.63 \\
88.63 \\
89.62\end{array}$ & $\begin{array}{l}7.41 \\
7.50 \\
7.59 \\
7.69 \\
7.78 \\
7.87 \\
7.96 \\
8.05 \\
8.14 \\
8.24\end{array}$ & $\begin{array}{l}80.63 \\
81.62 \\
82.62 \\
83.61 \\
84.61 . \\
85.60 \\
86.60 \\
87.59 \\
88.59 \\
89.59\end{array}$ & $\begin{array}{l}7.76 \\
7.86 \\
7.96 \\
8.05 \\
8.15 \\
8.24 \\
8.34 \\
8.43 \\
8.53 \\
8.63\end{array}$ & $\begin{array}{l}80.59 \\
81.59 \\
82.59 \\
83.58 \\
84.57 \\
85.57 \\
86.56 \\
87.56 \\
88.55 \\
89.55\end{array}$ & $\begin{array}{l}8.12 \\
8.22 \\
8.32 \\
8.42 \\
8.52 \\
8.62 \\
8.72 \\
8.82 \\
8.92 \\
9.02\end{array}$ & $\begin{array}{l}81 \\
82 \\
83 \\
84 \\
85 \\
86 \\
87 \\
88 \\
89 \\
90\end{array}$ \\
\hline $\begin{array}{r}91 \\
92 \\
93 \\
94 \\
95 \\
96 \\
97 \\
98 \\
99 \\
100\end{array}$ & $\begin{array}{l}90.65 \\
91.65 \\
92.65 \\
93.64 \\
94.64 \\
95.63 \\
96.63 \\
97.63 \\
98.62 \\
99.62\end{array}$ & $\begin{array}{l}7.93 \\
8.02 \\
8.11 \\
8.19 \\
8.28 \\
8.37 \\
8.45 \\
8.54 \\
8.63 \\
8.72\end{array}$ & $\begin{array}{l}90.62 \\
91.61 \\
92.61 \\
93.61 \\
94,60 \\
95.60 \\
96.59 \\
97.59 \\
93.58 \\
99.58\end{array}$ & $\begin{array}{l}8.33 \\
8.42 \\
8.51 \\
8.60 \\
8.69 \\
8.78 \\
8.89 \\
8.97 \\
9.06 \\
9.15\end{array}$ & $\begin{array}{l}90.58 \\
91.58 \\
92.57 \\
93.57 \\
94.56 \\
95.56 \\
96.55 \\
97.55 \\
98.54 \\
99.54\end{array}$ & $\begin{array}{l}8.72 \\
8.82 \\
8.91 \\
9.01 \\
9.11 \\
9.20 \\
9.30 \\
9.39 \\
9.49 \\
9.58\end{array}$ & $\begin{array}{l}90.54 \\
91.54 \\
92.53 \\
93.53 \\
94.52 \\
95.52 \\
96.51 \\
97.51 \\
98.50 \\
99.50\end{array}$ & $\begin{array}{r}9.12 \\
9.22 \\
9.32 \\
9.42 \\
9.52 \\
9.62 \\
9.72 \\
9.82 \\
9.92 \\
10.02\end{array}$ & $\begin{array}{r}91 \\
92 \\
93 \\
94 \\
95 \\
96 \\
97 \\
98 \\
99 \\
100\end{array}$ \\
\hline छ্口 & Dep. & Lat. & Dep. & Lat. & Dep. & Lat. & Dep. & Lat. & \&્g \\
\hline$\frac{\bar{n}}{a}$ & \multicolumn{2}{|c|}{$85^{\circ}}$. & \multicolumn{2}{|c|}{$84^{3} / 4^{\circ}}$. & \multicolumn{2}{|c|}{$841 / 5^{\circ}}$. & \multicolumn{2}{|c|}{$8414^{\circ}}$. & $\frac{\bar{n}}{\overline{0}}$ \\
\hline
\end{tabular}


TABLE 4.-TRAVEISE TABLE.

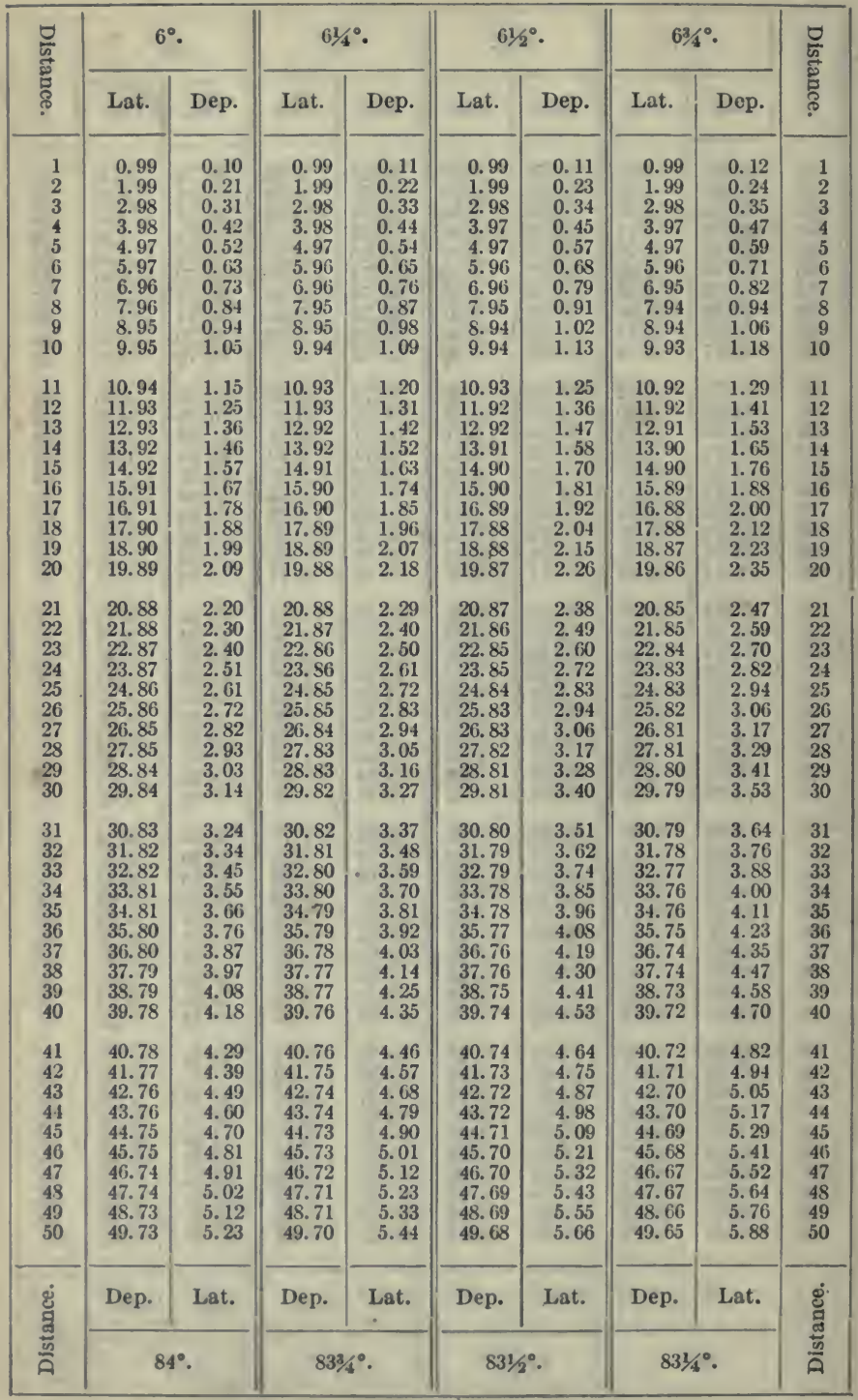


TABLE 4.-TRAVERSE TABLE.

\begin{tabular}{|c|c|c|c|c|c|c|c|c|c|}
\hline \multirow{2}{*}{ 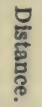 } & \multicolumn{2}{|c|}{$6^{\circ}$. } & \multicolumn{2}{|c|}{$614^{\circ}$. } & \multicolumn{2}{|c|}{$61 / 22^{\circ}$. } & \multicolumn{2}{|c|}{$634^{\circ}$. } & \multirow{2}{*}{ 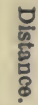 } \\
\hline & Lat. & Dep. & Lat. & Dep. & Lat. & Dep. & Lat. & Dep. & \\
\hline $\begin{array}{l}51 \\
52 \\
53 \\
54 \\
55 \\
56 \\
57 \\
58 \\
59 \\
60\end{array}$ & $\begin{array}{l}50.72 \\
51.72 \\
52.71 \\
53.70 \\
54.70 \\
55.69 \\
56.69 \\
57.68 \\
58.68 \\
59.67\end{array}$ & $\begin{array}{l}5.33 \\
5.44 \\
5.54 \\
5.64 \\
5.75 \\
5.85 \\
5.96 \\
6.06 \\
6.17 \\
6.27\end{array}$ & $\begin{array}{l}50.70 \\
51.69 \\
52.68 \\
53.68 \\
54.67 \\
55.67 \\
56.66 \\
57.66 \\
58.65 \\
59.64\end{array}$ & $\begin{array}{l}5.55 \\
5.66 \\
5.77 \\
5.88 \\
5.99 \\
6.10 \\
6.21 \\
6.31 \\
6.42 \\
6.53\end{array}$ & $\begin{array}{l}50.67 \\
51.67 \\
52.66 \\
53.65 \\
54.65 \\
55.64 \\
56.63 \\
57.63 \\
53.62 \\
59.61\end{array}$ & $\begin{array}{l}5.77 \\
5.89 \\
6.00 \\
6.11 \\
6.23 \\
6.34 \\
6.45 \\
6.57 \\
6.68 \\
6.79\end{array}$ & $\begin{array}{l}50.65 \\
51.64 \\
52.63 \\
53.63 \\
54.62 \\
55.61 \\
56.60 \\
57.60 \\
58.59 \\
59.58\end{array}$ & $\begin{array}{l}5.99 \\
6.11 \\
6.23 \\
6.35 \\
6.46 \\
6.58 \\
6.70 \\
6.82 \\
6.93 \\
7.05\end{array}$ & $\begin{array}{l}51 \\
52 \\
53 \\
54 \\
55 \\
56 \\
57 \\
58 \\
59 \\
60\end{array}$ \\
\hline $\begin{array}{l}61 \\
62 \\
63 \\
64 \\
65 \\
66 \\
67 \\
68 \\
69 \\
70\end{array}$ & $\begin{array}{l}60.67 \\
61.66 \\
62.65 \\
63.65 \\
64.64 \\
65.64 \\
66.63 \\
67.63 \\
68.62 \\
69.62\end{array}$ & $\begin{array}{l}6.38 \\
6.48 \\
6.59 \\
6.69 \\
6.79 \\
6.90 \\
7.00 \\
7.11 \\
7.21 \\
7.32\end{array}$ & $\begin{array}{l}60.64 \\
61.63 \\
62.63 \\
63.62 \\
64.61 \\
65.61 \\
66.60 \\
67.60 \\
68.59 \\
69.58\end{array}$ & $\begin{array}{l}6.64 \\
6.75 \\
6.86 \\
6.97 \\
7.08 \\
7.19 \\
7.29 \\
7.40 \\
7.51 \\
7.62\end{array}$ & $\begin{array}{l}60.61 \\
61.60 \\
62.60 \\
63.59 \\
64.58 \\
65.58 \\
66.57 \\
67.56 \\
68.56 \\
69.55\end{array}$ & $\begin{array}{l}6.91 \\
7.02 \\
7.13 \\
7.25 \\
7.36 \\
7.47 \\
7.58 \\
7.70 \\
7.81 \\
7.92\end{array}$ & $\begin{array}{l}60.58 \\
61.57 \\
62.56 \\
63.56 \\
64.55 \\
65.54 \\
66.54 \\
67.53 \\
68.52 \\
69.51\end{array}$ & $\begin{array}{l}7.17 \\
7.29 \\
7.40 \\
7.52 \\
7.64 \\
7.76 \\
7.88 \\
7.99 \\
8.11 \\
8.23\end{array}$ & $\begin{array}{l}61 \\
62 \\
63 \\
64 \\
65 \\
66 \\
67 \\
68 \\
69 \\
70\end{array}$ \\
\hline $\begin{array}{l}71 \\
72 \\
73 \\
74 \\
75 \\
76 \\
77 \\
78 \\
79 \\
80\end{array}$ & $\begin{array}{l}70.61 \\
71.61 \\
72.60 \\
73.59 \\
74.59 \\
75.59 \\
76.58 \\
77.57 \\
78.57 \\
79.56\end{array}$ & $\begin{array}{l}7.42 \\
7.53 \\
7.63 \\
7.74 \\
7.84 \\
7.94 \\
8.05 \\
8.15 \\
8.26 \\
8.36\end{array}$ & $\begin{array}{l}70.58 \\
71.57 \\
72.57 \\
73.56 \\
74.55 \\
75.55 \\
76.54 \\
77.54 \\
78.53 \\
79.53\end{array}$ & $\begin{array}{l}7.73 \\
7.84 \\
7.95 \\
8.06 \\
8.17 \\
8.27 \\
8.38 \\
8.49 \\
8.60 \\
8.71\end{array}$ & $\begin{array}{l}70.54 \\
71.54 \\
72.53 \\
73.52 \\
74.52 \\
75.51 \\
76.51 \\
77.50 \\
78.49 \\
79.49\end{array}$ & $\begin{array}{l}8.04 \\
8.15 \\
8.26 \\
8.38 \\
8.49 \\
8.60 \\
8.72 \\
8.83 \\
8.94 \\
9.06\end{array}$ & $\begin{array}{l}70.51 \\
71.50 \\
72.49 \\
73.49 \\
74.48 \\
75.47 \\
76.47 \\
77.46 \\
78.45 \\
79.45\end{array}$ & $\begin{array}{l}8.35 \\
8.46 \\
8.58 \\
8.70 \\
8.82 \\
8.93 \\
9.05 \\
9.17 \\
9.29 \\
9.40\end{array}$ & $\begin{array}{l}71 \\
72 \\
73 \\
74 \\
75 \\
76 \\
77 \\
78 \\
79 \\
80\end{array}$ \\
\hline $\begin{array}{l}81 \\
82 \\
83 \\
84 \\
85 \\
86 \\
87 \\
89 \\
89 \\
90\end{array}$ & $\begin{array}{l}80.56 \\
81.55 \\
82.55 \\
83.54 \\
84.53 \\
85.53 \\
86.52 \\
87.52 \\
89.51 \\
89.51\end{array}$ & $\begin{array}{l}8.47 \\
8.57 \\
8.68 \\
8.78 \\
8.83 \\
8.99 \\
9.09 \\
9.20 \\
9.30 \\
9.41\end{array}$ & $\begin{array}{l}80.52 \\
81.51 \\
82.51 \\
83.50 \\
84.50 \\
85.49 \\
86.48 \\
87.48 \\
88.47 \\
89.47\end{array}$ & $\begin{array}{l}8.82 \\
8.93 \\
9.04 \\
9.14 \\
9.25 \\
9.36 \\
9.47 \\
9.58 \\
9.69 \\
9.80\end{array}$ & $\begin{array}{l}80.48 \\
81.47 \\
82.47 \\
83.46 \\
84.45 \\
85.45 \\
86.44 \\
87.43 \\
88.43 \\
89.42\end{array}$ & $\begin{array}{r}9.17 \\
9.28 \\
9.40 \\
9.51 \\
9.62 \\
9.74 \\
9.85 \\
9.96 \\
10.08 \\
10.19\end{array}$ & $\begin{array}{l}80.44 \\
81.43 \\
82.42 \\
83.42 \\
84.41 \\
85.40 \\
86.40 \\
87.39 \\
88.38 \\
89.38\end{array}$ & $\begin{array}{r}9.52 \\
9.64 \\
9.76 \\
9.87 \\
9.99 \\
10.11 \\
10.23 \\
10.34 \\
10.46 \\
10.58\end{array}$ & $\begin{array}{l}81 \\
82 \\
83 \\
84 \\
85 \\
86 \\
87 \\
88 \\
89 \\
90\end{array}$ \\
\hline $\begin{array}{r}91 \\
92 \\
93 \\
94 \\
95 \\
96 \\
97 \\
99 \\
99 \\
100\end{array}$ & $\begin{array}{l}90.50 \\
91.50 \\
92.49 \\
93.49 \\
94.48 \\
95.47 \\
96.47 \\
97.46 \\
98.46 \\
99.45\end{array}$ & $\begin{array}{r}9.51 \\
9.62 \\
9.72 \\
9.83 \\
9.93 \\
10.03 \\
10.14 \\
10.24 \\
10.35 \\
10.45\end{array}$ & $\begin{array}{l}90.46 \\
91.45 \\
92.45 \\
93.44 \\
94.44 \\
95.43 \\
96.42 \\
97.42 \\
95.41 \\
99.41\end{array}$ & $\begin{array}{r}9.91 \\
10.02 \\
10.12 \\
10.23 \\
10.34 \\
10.45 \\
10.56 \\
10.67 \\
10.78 \\
10.89\end{array}$ & $\begin{array}{l}90.42 \\
91.41 \\
92.40 \\
93.40 \\
94.39 \\
95.38 \\
96.38 \\
97.37 \\
98.36 \\
99.36\end{array}$ & $\begin{array}{l}10.30 \\
10.41 \\
10.53 \\
10.64 \\
10.75 \\
10.87 \\
10.98 \\
11.09 \\
11.21 \\
11.32\end{array}$ & $\begin{array}{l}90.37 \\
91.36 \\
92.36 \\
93.35 \\
94.34 \\
95.33 \\
96.33 \\
97.32 \\
98.31 \\
99.31\end{array}$ & $\begin{array}{l}10.70 \\
10.81 \\
10.03 \\
11.05 \\
11.17 \\
11.28 \\
11.40 \\
11.52 \\
11.64 \\
11.75\end{array}$ & $\begin{array}{r}91 \\
92 \\
93 \\
94 \\
95 \\
96 \\
97 \\
98 \\
99 \\
100\end{array}$ \\
\hline 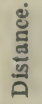 & Dep. & Lat. & Dep. & Lat. & Dep. & Lat. & Dep. & Lat. & 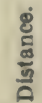 \\
\hline
\end{tabular}


TABLE 4.-TRAVERSE TABLE.

\begin{tabular}{|c|c|c|c|c|c|c|c|c|c|}
\hline \multirow{2}{*}{ 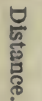 } & \multicolumn{2}{|c|}{$7^{\circ}}$. & \multicolumn{2}{|c|}{$714^{\circ}$. } & \multicolumn{2}{|c|}{$71 / 2^{\circ}$. } & \multicolumn{2}{|c|}{$73 / 4^{\circ}$. } & \multirow{2}{*}{$\begin{array}{l}\qquad \\
\frac{5}{2} \\
\frac{5}{0} \\
\frac{8}{8}\end{array}$} \\
\hline & Lat. & Dep. & Lat. & Dep. & Lat. & Dep. & Lat. & Dep. & \\
\hline $\begin{array}{r}1 \\
2 \\
3 \\
4 \\
5 \\
6 \\
7 \\
8 \\
9 \\
10\end{array}$ & $\begin{array}{l}0.99 \\
1.99 \\
2.98 \\
3.97 \\
4.96 \\
5.96 \\
6.95 \\
7.94 \\
8.93 \\
9.93\end{array}$ & $\begin{array}{l}0.12 \\
0.24 \\
0.37 \\
0.49 \\
0.61 \\
0.73 \\
0.85 \\
0.97 \\
1.10 \\
1.22\end{array}$ & $\begin{array}{l}0.99 \\
1.98 \\
2.98 \\
3.97 \\
4.96 \\
5.95 \\
6.94 \\
7.94 \\
8.98 \\
9.92\end{array}$ & $\begin{array}{l}0.13 \\
0.25 \\
0.38 \\
0.50 \\
0.63 \\
0.76 \\
0.88 \\
1.01 \\
1.14 \\
1.26\end{array}$ & $\begin{array}{l}0.99 \\
1.98 \\
2.97 \\
3.97 \\
4.96 \\
5.95 \\
6.94 \\
7.93 \\
8.92 \\
9.91\end{array}$ & $\begin{array}{l}0.13 \\
0.26 \\
0.39 \\
0.52 \\
0.65 \\
0.78 \\
0.91 \\
1.04 \\
1.17 \\
1.31\end{array}$ & $\begin{array}{l}0.99 \\
1.98 \\
2.97 \\
3.96 \\
4.95 \\
5.95 \\
6.94 \\
7.93 \\
8.92 \\
9.91\end{array}$ & $\begin{array}{l}0.13 \\
0.27 \\
0.40 \\
0.54 \\
0.67 \\
0.81 \\
0.94 \\
1.08 \\
1.21 \\
1.35\end{array}$ & $\begin{array}{r}1 \\
2 \\
3 \\
4 \\
5 \\
6 \\
7 \\
8 \\
9 \\
10\end{array}$ \\
\hline $\begin{array}{l}11 \\
12 \\
13 \\
14 \\
15 \\
16 \\
17 \\
18 \\
19 \\
20\end{array}$ & $\begin{array}{l}10.92 \\
11.91 \\
12.90 \\
13.90 \\
14.89 \\
15.88 \\
16.87 \\
17.87 \\
18.86 \\
19.85\end{array}$ & $\begin{array}{l}1.34 \\
1.46 \\
1.58 \\
1.71 \\
1.83 \\
1.95 \\
2.07 \\
2.19 \\
2.32 \\
2.44\end{array}$ & $\begin{array}{l}10.91 \\
11.90 \\
12.90 \\
13.89 \\
14.88 \\
15.87 \\
16.86 \\
17.86 \\
18.85 \\
19.84\end{array}$ & $\begin{array}{l}1.39 \\
1.51 \\
1.64 \\
1.77 \\
1.89 \\
2.02 \\
2.15 \\
2.27 \\
2.40 \\
2.52\end{array}$ & $\begin{array}{l}10.91 \\
11.90 \\
12.89 \\
13.88 \\
14.87 \\
15.86 \\
16.85 \\
17.85 \\
18.84 \\
19.83\end{array}$ & $\begin{array}{l}1.44 \\
1.57 \\
1.70 \\
1.83 \\
1.96 \\
2.09 \\
2.22 \\
2.35 \\
2.48 \\
2.61\end{array}$ & $\begin{array}{l}10.90 \\
11.89 \\
12.88 \\
13.87 \\
14.86 \\
15.85 \\
16.84 \\
17.84 \\
18.83 \\
19.82\end{array}$ & $\begin{array}{l}1.48 \\
1.62 \\
1.75 \\
1.89 \\
2.02 \\
2.16 \\
2.29 \\
2.43 \\
2.56 \\
2.70\end{array}$ & $\begin{array}{l}11 \\
12 \\
13 \\
14 \\
15 \\
16 \\
17 \\
18 \\
19 \\
20\end{array}$ \\
\hline $\begin{array}{l}21 \\
22 \\
23 \\
24 \\
25 \\
26 \\
27 \\
28 \\
29 \\
30\end{array}$ & $\begin{array}{l}20.84 \\
21.84 \\
22.83 \\
23.82 \\
24.81 \\
25.81 \\
26.80 \\
27.79 \\
28.78 \\
29.78\end{array}$ & $\begin{array}{l}2.56 \\
2.68 \\
2.80 \\
2.92 \\
3.05 \\
3.17 \\
3.29 \\
3.41 \\
3.53 \\
3.66\end{array}$ & $\begin{array}{l}20.83 \\
21.82 \\
22.82 \\
23.81 \\
24.80 \\
25.79 \\
26.78 \\
27.78 \\
28.77 \\
29.76\end{array}$ & $\begin{array}{l}2.65 \\
2.78 \\
2.90 \\
3.03 \\
3.15 \\
3.28 \\
3.41 \\
3.53 \\
3.66 \\
3.79\end{array}$ & $\begin{array}{l}20.82 \\
21.81 \\
22.80 \\
23.79 \\
24.79 \\
25.78 \\
26.77 \\
27.76 \\
28.75 \\
29.74\end{array}$ & $\begin{array}{l}2.74 \\
2.87 \\
3.00 \\
3.13 \\
3.26 \\
3.39 \\
3.52 \\
3.65 \\
3.79 \\
3.92\end{array}$ & $\begin{array}{l}20.81 \\
21.80 \\
22.79 \\
23.78 \\
24.77 \\
25.76 \\
26.75 \\
27.74 \\
28.74 \\
29.73\end{array}$ & $\begin{array}{l}2.83 \\
2.97 \\
3.10 \\
3.24 \\
3.37 \\
3.51 \\
3.64 \\
3.78 \\
3.91 \\
4.05\end{array}$ & $\begin{array}{l}21 \\
22 \\
23 \\
24 \\
25 \\
26 \\
27 \\
28 \\
29 \\
30\end{array}$ \\
\hline $\begin{array}{l}31 \\
32 \\
33 \\
34 \\
35 \\
36 \\
37 \\
38 \\
39 \\
40\end{array}$ & $\begin{array}{l}30.77 \\
31.76 \\
32.75 \\
33.75 \\
34.74 \\
35.73 \\
36.72 \\
37.72 \\
38.71 \\
39.70\end{array}$ & $\begin{array}{l}3.78 \\
3.90 \\
4.02 \\
4.14 \\
4.27 \\
4.39 \\
4.51 \\
4.63 \\
4.75 \\
4.87\end{array}$ & $\begin{array}{l}30.75 \\
31.74 \\
32.74 \\
33.73 \\
34.72 \\
35.71 \\
36.70 \\
37.70 \\
38.69 \\
39.68\end{array}$ & $\begin{array}{l}3.91 \\
4.04 \\
4.16 \\
4.29 \\
4.42 \\
4.54 \\
4.67 \\
4.80 \\
4.92 \\
5.05\end{array}$ & $\begin{array}{l}30.73 \\
31.73 \\
32.72 \\
33.71 \\
34.70 \\
35.69 \\
36.68 \\
37.67 \\
38.67 \\
39.66\end{array}$ & $\begin{array}{l}4.05 \\
4.18 \\
4.31 \\
4.44 \\
4.57 \\
4.70 \\
4.83 \\
4.96 \\
5.09 \\
5.22\end{array}$ & $\begin{array}{l}30.72 \\
31.71 \\
32.70 \\
33.69 \\
34.68 \\
35.67 \\
36.66 \\
37.65 \\
33.64 \\
39.63\end{array}$ & $\begin{array}{l}4.18 \\
4.32 \\
4.45 \\
4.58 \\
4.72 \\
4.85 \\
4.99 \\
5.12 \\
5.26 \\
5.39\end{array}$ & $\begin{array}{l}31 \\
32 \\
33 \\
34 \\
35 \\
36 \\
37 \\
38 \\
39 \\
40\end{array}$ \\
\hline $\begin{array}{l}41 \\
42 \\
43 \\
44 \\
45 \\
46 \\
47 \\
48 \\
49 \\
50\end{array}$ & $\begin{array}{l}40.69 \\
41.69 \\
42.68 \\
43.67 \\
44.67 \\
45.66 \\
46.65 \\
47.64 \\
48.63 \\
49.63\end{array}$ & $\begin{array}{l}5.00 \\
5.12 \\
5.24 \\
5.36 \\
5.48 \\
5.61 \\
5.73 \\
5.85 \\
5.97 \\
6.09\end{array}$ & $\begin{array}{l}40.67 \\
41.66 \\
42.66 \\
43.65 \\
44.64 \\
45.63 \\
46.62 \\
47.62 \\
48.61 \\
49.60\end{array}$ & $\begin{array}{l}5.17 \\
5.30 \\
5.43 \\
5.55 \\
5.68 \\
5.81 \\
5.93 \\
6.06 \\
6.18 \\
6.31\end{array}$ & $\begin{array}{l}40.65 \\
41.64 \\
42.63 \\
43.62 \\
44.62 \\
45.61 \\
46.60 \\
47.59 \\
48.58 \\
49.57\end{array}$ & $\begin{array}{l}5.35 \\
5.48 \\
5.61 \\
5.74 \\
5.87 \\
6.00 \\
6.13 \\
6.27 \\
6.40 \\
6.53\end{array}$ & $\begin{array}{l}40.63 \\
41.62 \\
42.61 \\
43.60 \\
44.59 \\
45.58 \\
46.57 \\
47.56 \\
48.55 \\
49.54\end{array}$ & $\begin{array}{l}5.53 \\
5.66 \\
5.80 \\
5.93 \\
6.07 \\
6.20 \\
6.34 \\
6.47 \\
6.61 \\
6.74\end{array}$ & $\begin{array}{l}41 \\
42 \\
43 \\
44 \\
45 \\
46 \\
47 \\
48 \\
49 \\
50\end{array}$ \\
\hline \&્ల & Dep. & Lat. & Dep. & Lat. & Dep. & Lat. & Dep. & Lat. & ष्ठ \\
\hline$\frac{n}{a}$ & \multicolumn{2}{|c|}{$83^{\circ}$. } & \multicolumn{2}{|c|}{$8234^{\circ}$. } & \multicolumn{2}{|c|}{$821 / 2^{\circ}$. } & \multicolumn{2}{|c|}{$821 / 4^{\circ}$. } & \\
\hline
\end{tabular}


TABLE 4.-TRAVERSE TABLE.

\begin{tabular}{|c|c|c|c|c|c|c|c|c|c|}
\hline \multirow{2}{*}{ 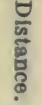 } & \multicolumn{2}{|c|}{$7^{\circ}}$. & \multicolumn{2}{|c|}{$71 / 4^{\circ}}$. & \multicolumn{2}{|c|}{$71 / 2^{\circ}$} & \multicolumn{2}{|c|}{$73 / 4^{\circ}$} & \multirow{2}{*}{$\begin{array}{l}\text { 븜 } \\
\frac{5}{5} \\
\text { 홍 }\end{array}$} \\
\hline & Lat. & Dep. & Lat. & Dep. & Lat. & Dep. & Lat. & Dep. & \\
\hline $\begin{array}{l}51 \\
52 \\
53 \\
54 \\
55 \\
56 \\
57 \\
55 \\
59 \\
60\end{array}$ & $\begin{array}{l}50.62 \\
51.61 \\
52.60 \\
53.60 \\
54.59 \\
55.58 \\
56.58 \\
57.57 \\
58.56 \\
59.55\end{array}$ & $\begin{array}{l}6.22 \\
6.34 \\
6.46 \\
6.58 \\
6.70 \\
6.82 \\
6.95 \\
7.07 \\
7.19 \\
7.31\end{array}$ & $\begin{array}{l}50.59 \\
51.58 \\
52.58 \\
53.57 \\
54.56 \\
55.55 \\
56.54 \\
57.54 \\
58.53 \\
59.52\end{array}$ & $\begin{array}{l}6.44 \\
6.56 \\
6.69 \\
6.81 \\
6.94 \\
7.07 \\
7.19 \\
7.32 \\
7.45 \\
7.57\end{array}$ & $\begin{array}{l}50.56 \\
51.56 \\
52.55 \\
53.54 \\
54.53 \\
55.52 \\
56.51 \\
57.50 \\
58.50 \\
59.49\end{array}$ & $\begin{array}{l}6.66 \\
6.79 \\
6.92 \\
7.05 \\
7.18 \\
7.31 \\
7.44 \\
7.57 \\
7.70 \\
7.83\end{array}$ & $\begin{array}{l}50.53 \\
51.53 \\
52.52 \\
53.51 \\
54.50 \\
55.49 \\
56.48 \\
57.47 \\
5.46 \\
59.45\end{array}$ & $\begin{array}{l}6.88 \\
7.01 \\
7.15 \\
7.28 \\
7.42 \\
7.55 \\
7.69 \\
7.82 \\
7.96 \\
8.09\end{array}$ & $\begin{array}{l}51 \\
52 \\
53 \\
54 \\
55 \\
56 \\
57 \\
58 \\
59 \\
60\end{array}$ \\
\hline $\begin{array}{l}61 \\
62 \\
63 \\
64 \\
65 \\
66 \\
67 \\
68 \\
69 \\
70\end{array}$ & $\begin{array}{l}60.55 \\
61.54 \\
62.53 \\
63.52 \\
64.52 \\
65.51 \\
66.50 \\
67.49 \\
68.49 \\
69.48\end{array}$ & $\begin{array}{l}7.43 \\
7.56 \\
7.68 \\
7.80 \\
7.92 \\
8.04 \\
8.17 \\
8.29 \\
8.41 \\
8.53\end{array}$ & $\begin{array}{l}60.51 \\
61.50 \\
62.50 \\
63.49 \\
64.45 \\
65.47 \\
66.46 \\
67.46 \\
68.45 \\
69.44\end{array}$ & $\begin{array}{l}7.70 \\
7.82 \\
7.95 \\
8.08 \\
8.20 \\
8.33 \\
8.46 \\
8.58 \\
8.71 \\
8.83\end{array}$ & $\begin{array}{l}60.48 \\
61.47 \\
62.46 \\
63.45 \\
64.44 \\
65.44 \\
66.43 \\
67.42 \\
68.41 \\
69.40\end{array}$ & $\begin{array}{l}7.96 \\
8.09 \\
8.22 \\
8.35 \\
8.48 \\
8.61 \\
8.75 \\
8.88 \\
9.01 \\
9.14\end{array}$ & $\begin{array}{l}60.44 \\
61.43 \\
62.42 \\
63.42 \\
64.41 \\
65.40 \\
66.39 \\
67.38 \\
68.37 \\
69.36\end{array}$ & $\begin{array}{l}8.23 \\
8.36 \\
8.50 \\
8.63 \\
8.77 \\
8.90 \\
9.04 \\
9.17 \\
9.30 \\
9.44\end{array}$ & $\begin{array}{l}61 \\
62 \\
63 \\
64 \\
65 \\
66 \\
67 \\
68 \\
69 \\
70\end{array}$ \\
\hline $\begin{array}{l}71 \\
72 \\
73 \\
74 \\
75 \\
76 \\
77 \\
78 \\
79 \\
80\end{array}$ & $\begin{array}{l}70.47 \\
71.46 \\
72.46 \\
73.45 \\
74.44 \\
75.43 \\
76.43 \\
77.42 \\
78.41 \\
79.40\end{array}$ & $\begin{array}{l}8.65 \\
8.77 \\
8.90 \\
9.02 \\
9.14 \\
9.26 \\
9.38 \\
9.51 \\
9.63 \\
9.75\end{array}$ & $\begin{array}{l}70.43 \\
71.42 \\
72.42 \\
73.41 \\
74.40 \\
75.39 \\
76.38 \\
77.38 \\
78.37 \\
79.36\end{array}$ & $\begin{array}{r}8.96 \\
9.09 \\
9.21 \\
9.34 \\
9.46 \\
9.59 \\
9.72 \\
9.81 \\
9.97 \\
10.10\end{array}$ & $\begin{array}{l}70.39 \\
71.38 \\
72.38 \\
73.37 \\
74.36 \\
75.35 \\
76.34 \\
77.33 \\
78.32 \\
79.32\end{array}$ & $\begin{array}{r}9.27 \\
9.40 \\
9.53 \\
9.66 \\
9.79 \\
9.92 \\
10.05 \\
10.18 \\
10.31 \\
10.44\end{array}$ & $\begin{array}{l}70.35 \\
71.34 \\
72.33 \\
73.32 \\
74.31 \\
75.31 \\
76.30 \\
77.29 \\
78.28 \\
79.27\end{array}$ & $\begin{array}{r}9.57 \\
9.71 \\
9.84 \\
9.98 \\
10.11 \\
10.25 \\
10.38 \\
10.52 \\
10.65 \\
10.79\end{array}$ & $\begin{array}{l}71 \\
72 \\
73 \\
74 \\
75 \\
76 \\
77 \\
78 \\
79 \\
80\end{array}$ \\
\hline $\begin{array}{l}81 \\
82 \\
83 \\
84 \\
85 \\
86 \\
87 \\
88 \\
89 \\
90\end{array}$ & $\begin{array}{l}80.40 \\
81.39 \\
82.38 \\
83.37 \\
84.37 \\
85.36 \\
86.35 \\
87.34 \\
88.34 \\
89.33\end{array}$ & $\begin{array}{r}9.87 \\
9.99 \\
10.12 \\
10.24 \\
10.36 \\
10.48 \\
10.60 \\
10.72 \\
10.85 \\
10.97\end{array}$ & $\begin{array}{l}80.35 \\
81.34 \\
82.34 \\
83.33 \\
84.32 \\
85.31 \\
86.30 \\
87.30 \\
85.29 \\
89.28\end{array}$ & $\begin{array}{l}10.22 \\
10.35 \\
10.47 \\
10.60 \\
10.73 \\
10.85 \\
10.98 \\
11.11 \\
11.23 \\
11.36\end{array}$ & $\begin{array}{l}80.31 \\
81.30 \\
82.29 \\
83.28 \\
81.27 \\
85.26 \\
86.26 \\
87.25 \\
88.24 \\
89.23\end{array}$ & $\begin{array}{l}10.57 \\
10.70 \\
10.83 \\
10.96 \\
11.09 \\
11.23 \\
11.36 \\
11.49 \\
11.62 \\
11.75\end{array}$ & $\begin{array}{l}80.26 \\
81.25 \\
82.24 \\
83.23 \\
84.22 \\
85.21 \\
86.21 \\
87.20 \\
88.19 \\
89.18\end{array}$ & $\begin{array}{l}10.92 \\
11.06 \\
11.19 \\
11.33 \\
11.46 \\
11.60 \\
11.73 \\
11.87 \\
12.00 \\
12.14\end{array}$ & $\begin{array}{l}81 \\
82 \\
83 \\
84 \\
85 \\
86 \\
87 \\
83 \\
89 \\
90\end{array}$ \\
\hline $\begin{array}{r}91 \\
92 \\
93 \\
91 \\
95 \\
96 \\
97 \\
98 \\
99 \\
100\end{array}$ & $\begin{array}{l}90.32 \\
91.31 \\
92.31 \\
93.30 \\
94.29 \\
95.28 \\
96.28 \\
97.27 \\
98.26 \\
99.25\end{array}$ & $\begin{array}{l}11.09 \\
11.21 \\
11.33 \\
11.46 \\
11.59 \\
11.70 \\
11.82 \\
11.94 \\
12.07 \\
12.19\end{array}$ & $\begin{array}{l}90.27 \\
91.26 \\
92.26 \\
93.25 \\
94.24 \\
95.23 \\
96.22 \\
97.22 \\
98.21 \\
99.20\end{array}$ & $\begin{array}{l}11.48 \\
11.61 \\
11.74 \\
11.86 \\
11.99 \\
12.12 \\
12.24 \\
12.37 \\
12.49 \\
12.62\end{array}$ & $\begin{array}{l}90.22 \\
91.21 \\
92.20 \\
93.20 \\
94.19 \\
95.18 \\
96.17 \\
97.16 \\
98.15 \\
99.14\end{array}$ & $\begin{array}{l}11.88 \\
12.01 \\
12.14 \\
12.27 \\
12.40 \\
12.53 \\
12.66 \\
12.79 \\
12.92 \\
13.05\end{array}$ & $\begin{array}{l}90.17 \\
91.16 \\
92.15 \\
93.14 \\
94.13 \\
95.12 \\
96.11 \\
97.10 \\
93.10 \\
99.09\end{array}$ & $\begin{array}{l}12.27 \\
12.41 \\
12.54 \\
12.68 \\
12.81 \\
12.95 \\
13.08 \\
13.22 \\
13.35 \\
13.49\end{array}$ & $\begin{array}{r}91 \\
92 \\
93 \\
94 \\
95 \\
96 \\
97 \\
98 \\
99 \\
100\end{array}$ \\
\hline$\ddot{\Xi}$ & Dep. & Lat. & Dep. & Lat. & Dep. & Lat. & Dep. & Lat. & $\dot{8}$ \\
\hline$\frac{m}{A}$ & \multicolumn{2}{|c|}{$83^{\circ}$. } & \multicolumn{2}{|c|}{$82 \% 4^{\circ}$. } & \multicolumn{2}{|c|}{$821 / 2^{\circ}$. } & \multicolumn{2}{|c|}{$8214^{\circ}$. } & $\bar{A}$ \\
\hline
\end{tabular}


TABLE 4.-TRAVERSE TABLE.

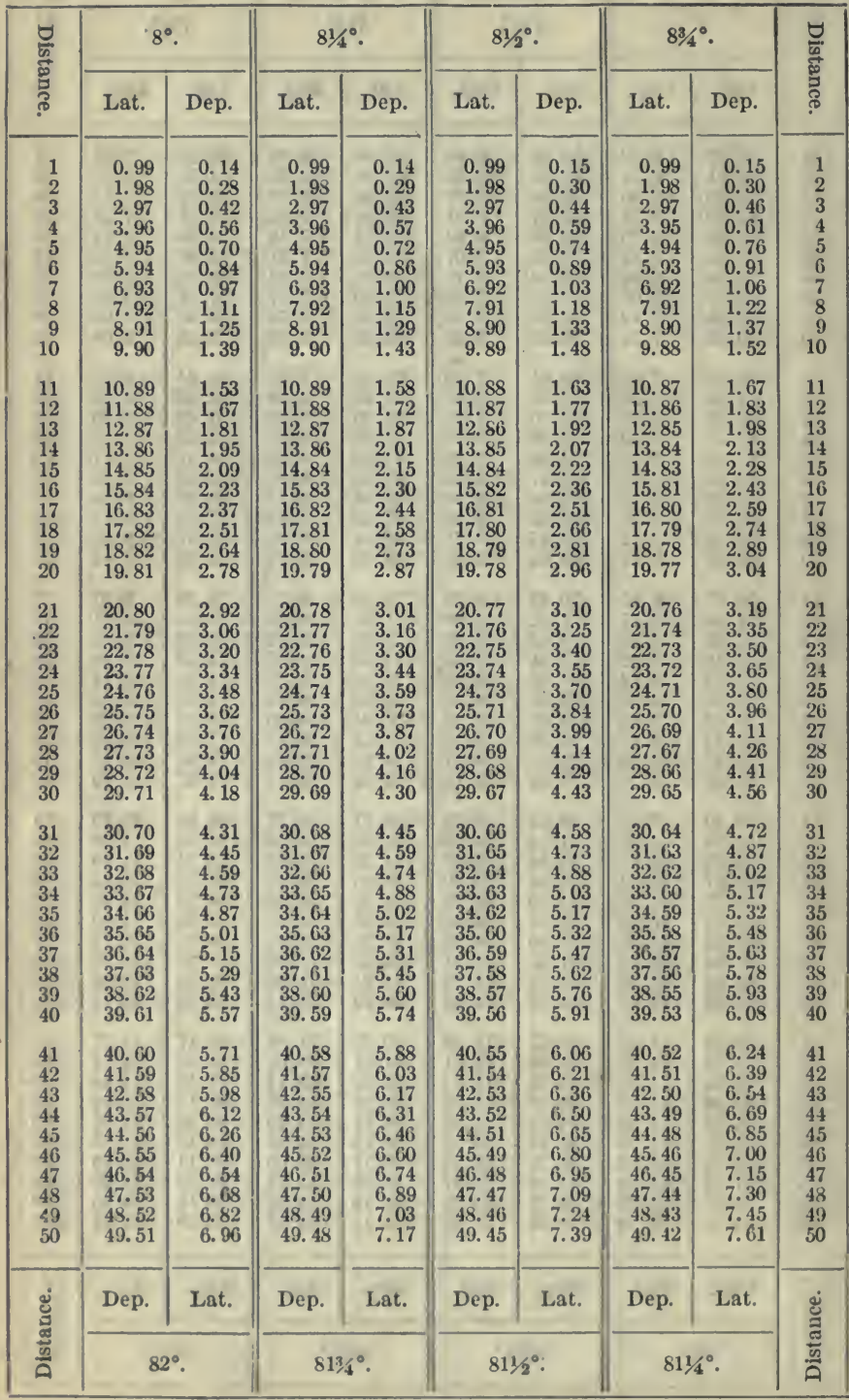


TABLE 4.-TRAVERSE TABLE.

\begin{tabular}{|c|c|c|c|c|c|c|c|c|c|}
\hline \multirow{2}{*}{ 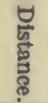 } & \multicolumn{2}{|c|}{$8^{\circ}}$. & \multicolumn{2}{|c|}{$81 / 4{ }^{\circ}}$. & \multicolumn{2}{|c|}{$81 / 2^{\circ}}$. & \multicolumn{2}{|c|}{$834^{\circ}}$. & \multirow{2}{*}{ 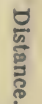 } \\
\hline & Lat. & Dep. & Lat. & Dep. & Lat. & Dep. & Lat. & Dep. & \\
\hline $\begin{array}{l}51 \\
52 \\
53 \\
54 \\
55 \\
56 \\
57 \\
58 \\
59 \\
60\end{array}$ & $\begin{array}{l}50.50 \\
51.49 \\
52.48 \\
53.47 \\
54.46 \\
55.46 \\
56.45 \\
57.44 \\
58.43 \\
59.42\end{array}$ & $\begin{array}{l}7.10 \\
7.24 \\
7.38 \\
7.52 \\
7.65 \\
7.79 \\
7.93 \\
8.07 \\
8.21 \\
8.35\end{array}$ & $\begin{array}{l}50.47 \\
51.46 \\
52.45 \\
53.44 \\
54.43 \\
55.42 \\
56.41 \\
57.40 \\
58.39 \\
59.38\end{array}$ & $\begin{array}{l}7.32 \\
7.46 \\
7.61 \\
7.75 \\
7.89 \\
8.04 \\
8.15 \\
8.32 \\
8.47 \\
8.61\end{array}$ & $\begin{array}{l}50.44 \\
51.43 \\
52.42 \\
53.41 \\
54.40 \\
55.39 \\
56.37 \\
57.36 \\
58.35 \\
59.34\end{array}$ & $\begin{array}{l}7.54 \\
7.69 \\
7.83 \\
7.98 \\
8.13 \\
8.28 \\
8.43 \\
8.57 \\
8.72 \\
8.87\end{array}$ & $\begin{array}{l}50.41 \\
51.39 \\
52.38 \\
53.37 \\
54.36 \\
55.35 \\
56.34 \\
57.32 \\
58.31 \\
59.30\end{array}$ & $\begin{array}{l}7.76 \\
7.91 \\
8.06 \\
8.21 \\
8.37 \\
8.52 \\
8.67 \\
8.82 \\
8.98 \\
9.13\end{array}$ & $\begin{array}{l}51 \\
52 \\
53 \\
54 \\
55 \\
56 \\
57 \\
58 \\
59 \\
60\end{array}$ \\
\hline $\begin{array}{l}61 \\
62 \\
63 \\
64 \\
65 \\
66 \\
67 \\
68 \\
69 \\
70\end{array}$ & $\begin{array}{l}60.41 \\
61.40 \\
62.39 \\
63.35 \\
64.37 \\
65.36 \\
66.35 \\
67.34 \\
68.33 \\
69.32\end{array}$ & $\begin{array}{l}8.49 \\
8.63 \\
8.77 \\
8.91 \\
9.05 \\
9.19 \\
9.32 \\
9.46 \\
9.60 \\
9.74\end{array}$ & $\begin{array}{l}60.37 \\
61.36 \\
62.35 \\
63.34 \\
61.33 \\
65.32 \\
66.31 \\
67.30 \\
68.29 \\
69.28\end{array}$ & $\begin{array}{r}8.75 \\
8.90 \\
9.04 \\
9.18 \\
9.33 \\
9.47 \\
9.61 \\
9.76 \\
9.90 \\
10.04\end{array}$ & $\begin{array}{l}60.33 \\
61.32 \\
62.31 \\
63.30 \\
64.29 \\
65.28 \\
66.21 \\
67.25 \\
68.24 \\
69.23\end{array}$ & $\begin{array}{r}9.02 \\
9.16 \\
9.31 \\
9.46 \\
9.61 \\
9.76 \\
9.90 \\
10.05 \\
10.20 \\
10.35\end{array}$ & $\begin{array}{l}60.29 \\
61.28 \\
62.27 \\
63.26 \\
64.24 \\
65.23 \\
66.22 \\
67.21 \\
68.20 \\
69.19\end{array}$ & $\begin{array}{r}9.28 \\
9.43 \\
9.58 \\
9.74 \\
9.89 \\
10.04 \\
10.19 \\
10.34 \\
10.50 \\
10.65\end{array}$ & $\begin{array}{l}61 \\
62 \\
63 \\
64 \\
65 \\
66 \\
67 \\
68 \\
69 \\
70\end{array}$ \\
\hline $\begin{array}{l}71 \\
72 \\
73 \\
74 \\
75 \\
76 \\
77 \\
78 \\
79 \\
80\end{array}$ & $\begin{array}{l}70.31 \\
71.30 \\
72.29 \\
73.28 \\
74.27 \\
75.26 \\
76.25 \\
77.24 \\
78.23 \\
79.22\end{array}$ & $\begin{array}{r}9.88 \\
10.02 \\
10.16 \\
10.30 \\
10.44 \\
10.58 \\
10.72 \\
10.86 \\
10.99 \\
11.13\end{array}$ & $\begin{array}{l}70.27 \\
71.25 \\
72.24 \\
73.23 \\
74.22 \\
75.21 \\
76.20 \\
77.19 \\
78.18 \\
79.17\end{array}$ & $\begin{array}{l}10.19 \\
10.33 \\
10.47 \\
10.62 \\
10.76 \\
10.91 \\
11.05 \\
11.19 \\
11.34 \\
11.48\end{array}$ & $\begin{array}{l}70.22 \\
71.21 \\
72.20 \\
73.19 \\
74.18 \\
75.17 \\
76.15 \\
77.14 \\
78.13 \\
79.12\end{array}$ & $\begin{array}{l}10.49 \\
10.64 \\
10.79 \\
10.94 \\
11.09 \\
11.23 \\
11.38 \\
11.53 \\
11.63 \\
11.82\end{array}$ & $\begin{array}{l}70.17 \\
71.16 \\
72.15 \\
73.14 \\
74.13 \\
75.12 \\
76.10 \\
77.09 \\
78.08 \\
79.07\end{array}$ & $\begin{array}{l}10.80 \\
10.95 \\
11.11 \\
11.26 \\
11.41 \\
11.56 \\
11.71 \\
11.87 \\
12.02 \\
12.17\end{array}$ & $\begin{array}{l}71 \\
72 \\
73 \\
74 \\
75 \\
76 \\
77 \\
78 \\
79 \\
80\end{array}$ \\
\hline $\begin{array}{l}81 \\
82 \\
83 \\
81 \\
85 \\
86 \\
87 \\
88 \\
89 \\
90\end{array}$ & $\begin{array}{l}80.21 \\
81.20 \\
82.19 \\
83.19 \\
84.17 \\
85.16 \\
80.15 \\
87.14 \\
89.13 \\
99.12\end{array}$ & $\begin{array}{l}11.27 \\
11.41 \\
11.55 \\
11.69 \\
11.83 \\
11.97 \\
12.11 \\
12.25 \\
12.39 \\
12.53\end{array}$ & $\begin{array}{l}80.16 \\
81.15 \\
82.14 \\
83.13 \\
84.12 \\
85.11 \\
86.10 \\
87.09 \\
88.08 \\
89.07\end{array}$ & $\begin{array}{l}11.62 \\
11.77 \\
11.91 \\
12.05 \\
12.20 \\
12.34 \\
12.48 \\
12.63 \\
12.77 \\
12.91\end{array}$ & $\begin{array}{l}80.11 \\
81.10 \\
82.09 \\
83.08 \\
84.07 \\
85.06 \\
86.04 \\
87.03 \\
88.02 \\
89.01\end{array}$ & $\begin{array}{l}11.97 \\
12.12 \\
12.27 \\
12.42 \\
12.56 \\
12.71 \\
12.86 \\
13.01 \\
13.16 \\
13.30\end{array}$ & $\begin{array}{l}80.06 \\
81.05 \\
82.03 \\
83.02 \\
84.01 \\
85.00 \\
85.99 \\
86.99 \\
87.96 \\
88.95\end{array}$ & $\begin{array}{l}12.32 \\
12.47 \\
12.63 \\
12.78 \\
12.93 \\
13.08 \\
13.23 \\
13.39 \\
13.54 \\
13.69\end{array}$ & $\begin{array}{l}81 \\
82 \\
83 \\
84 \\
85 \\
86 \\
87 \\
88 \\
89 \\
90\end{array}$ \\
\hline $\begin{array}{r}91 \\
92 \\
93 \\
94 \\
95 \\
96 \\
97 \\
98 \\
99 \\
100\end{array}$ & $\begin{array}{l}90.11 \\
91.10 \\
92.09 \\
93.09 \\
94.08 \\
95.07 \\
96.06 \\
97.05 \\
98.04 \\
99.03\end{array}$ & $\begin{array}{l}12.66 \\
12.80 \\
12.94 \\
13.08 \\
13.22 \\
13.36 \\
13.50 \\
13.94 \\
13.78 \\
13.92\end{array}$ & $\begin{array}{l}90.06 \\
91.05 \\
92.04 \\
93.03 \\
94.02 \\
95.01 \\
96.00 \\
96.99 \\
97.98 \\
98.97\end{array}$ & $\begin{array}{l}13.06 \\
13.20 \\
13.34 \\
13.49 \\
13.63 \\
13.78 \\
13.92 \\
14.06 \\
11.21 \\
14.35\end{array}$ & $\begin{array}{l}90.00 \\
90.99 \\
91.98 \\
92.97 \\
93.96 \\
94.95 \\
95.93 \\
96.92 \\
97.91 \\
98.90\end{array}$ & $\begin{array}{l}13.45 \\
13.60 \\
13.75 \\
13.89 \\
14.04 \\
14.19 \\
14.34 \\
14.49 \\
14.63 \\
14.78\end{array}$ & $\begin{array}{l}89.94 \\
90.03 \\
91.92 \\
92.91 \\
93.89 \\
94.88 \\
95.87 \\
96.86 \\
97.85 \\
98.84\end{array}$ & $\begin{array}{l}13.84 \\
14.00 \\
14.15 \\
14.30 \\
14.45 \\
14.60 \\
14.76 \\
14.91 \\
15.06 \\
15.21\end{array}$ & $\begin{array}{r}91 \\
92 \\
93 \\
94 \\
95 \\
913 \\
97 \\
98 \\
99 \\
100\end{array}$ \\
\hline छं & Den. & Iat. & Dep. & Lat. & Dep. & Lat. & Dep. & Lat. & : \\
\hline 믐 & \multicolumn{2}{|c|}{$83^{\circ}}$. & \multicolumn{2}{|c|}{$814^{\circ}}$. & \multicolumn{2}{|c|}{$81 / 22^{\circ}}$. & \multicolumn{2}{|c|}{$8114^{\circ}}$. & $\frac{\pi}{0}$ \\
\hline
\end{tabular}


TABLE 4.-TRAVERSE TABLE.

\begin{tabular}{|c|c|c|c|c|c|c|c|c|c|}
\hline \multirow{2}{*}{ 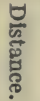 } & \multicolumn{2}{|c|}{$9^{\circ}$. } & \multicolumn{2}{|c|}{$91 / 4^{\circ}$. } & \multicolumn{2}{|c|}{$91 / 2^{\circ}}$. & \multicolumn{2}{|c|}{$93 / 4^{\circ}$. } & \multirow{2}{*}{ 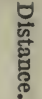 } \\
\hline & Lat. & Dep. & Lat. & Dep. & Lat. & Dep. & Lat. & Dep. & \\
\hline $\begin{array}{r}1 \\
2 \\
3 \\
4 \\
5 \\
6 \\
7 \\
8 \\
9 \\
10\end{array}$ & $\begin{array}{l}0.99 \\
1.98 \\
2.96 \\
3.95 \\
4.94 \\
5.93 \\
6.91 \\
7.90 \\
8.89 \\
9.88\end{array}$ & $\begin{array}{l}0.16 \\
0.31 \\
0.47 \\
0.63 \\
0.78 \\
0.94 \\
1.10 \\
1.25 \\
1.41 \\
1.56\end{array}$ & $\begin{array}{l}0.99 \\
1.97 \\
2.96 \\
3.95 \\
4.93 \\
5.92 \\
6.91 \\
7.90 \\
8.88 \\
9.87\end{array}$ & $\begin{array}{l}0.16 \\
0.32 \\
0.48 \\
0.64 \\
0.80 \\
0.96 \\
1.13 \\
1.29 \\
1.45 \\
1.61\end{array}$ & $\begin{array}{l}0.99 \\
1.97 \\
2.96 \\
3.95 \\
4.93 \\
5.92 \\
6.90 \\
7.89 \\
8.88 \\
9.86\end{array}$ & $\begin{array}{l}0.17 \\
0.33 \\
0.50 \\
0.66 \\
0.83 \\
0.99 \\
1.16 \\
1.32 \\
1.49 \\
1.65\end{array}$ & $\begin{array}{l}0.99 \\
1.97 \\
2.96 \\
3.94 \\
4.93 \\
5.91 \\
6.90 \\
7.88 \\
8.87 \\
9.86\end{array}$ & $\begin{array}{l}0.17 \\
0.34 \\
0.51 \\
0.68 \\
0.85 \\
1.02 \\
1.19 \\
1.35 \\
1.52 \\
1.69\end{array}$ & $\begin{array}{r}1 \\
2 \\
3 \\
4 \\
5 \\
6 \\
7 \\
8 \\
9 \\
10\end{array}$ \\
\hline $\begin{array}{l}11 \\
12 \\
13 \\
14 \\
15 \\
16 \\
17 \\
18 \\
19 \\
20\end{array}$ & $\begin{array}{l}10.86 \\
11.85 \\
12.84 \\
13.83 \\
14.82 \\
15.80 \\
16.79 \\
17.78 \\
18.77 \\
19.75\end{array}$ & $\begin{array}{l}1.72 \\
1.88 \\
2.03 \\
2.19 \\
2.35 \\
2.50 \\
2.66 \\
2.82 \\
2.97 \\
3.13\end{array}$ & $\begin{array}{l}10.86 \\
11.84 \\
12.83 \\
13.82 \\
14.80 \\
15.79 \\
16.78 \\
17.77 \\
18.75 \\
19.74\end{array}$ & $\begin{array}{l}1.77 \\
1.93 \\
2.09 \\
2.25 \\
2.41 \\
2.57 \\
2.73 \\
2.89 \\
3.05 \\
3.21\end{array}$ & $\begin{array}{l}10.85 \\
11.84 \\
12.82 \\
13.81 \\
14.79 \\
15.78 \\
16.77 \\
17.75 \\
18.74 \\
19.73\end{array}$ & $\begin{array}{l}1.82 \\
1.98 \\
2.15 \\
2.31 \\
2.48 \\
2.64 \\
2.81 \\
2.97 \\
3.14 \\
3.30\end{array}$ & $\begin{array}{l}10.84 \\
11.83 \\
12.81 \\
13.80 \\
14.78 \\
15.77 \\
16.75 \\
17.74 \\
18.73 \\
19.71\end{array}$ & $\begin{array}{l}1.86 \\
2.03 \\
2.20 \\
2.37 \\
2.54 \\
2.71 \\
2.88 \\
3.05 \\
3.22 \\
3.39\end{array}$ & $\begin{array}{l}11 \\
12 \\
13 \\
14 \\
15 \\
16 \\
17 \\
18 \\
19 \\
20\end{array}$ \\
\hline $\begin{array}{l}21 \\
22 \\
23 \\
24 \\
25 \\
26 \\
27 \\
28 \\
29 \\
30\end{array}$ & $\begin{array}{l}20.74 \\
21.73 \\
22.72 \\
23.70 \\
24.69 \\
25.68 \\
26.67 \\
27.66 \\
28.64 \\
29.63\end{array}$ & $\begin{array}{l}3.29 \\
3.44 \\
3.60 \\
3.75 \\
3.91 \\
4.07 \\
4.22 \\
4.38 \\
4.54 \\
4.69\end{array}$ & $\begin{array}{l}20.73 \\
21.71 \\
22.70 \\
23.69 \\
24.67 \\
25.66 \\
26.65 \\
27.64 \\
28.62 \\
29.61\end{array}$ & $\begin{array}{l}3.38 \\
3.54 \\
3.70 \\
3.86 \\
4.02 \\
4.18 \\
4.34 \\
4.50 \\
4.66 \\
4.82\end{array}$ & $\begin{array}{l}20.71 \\
21.70 \\
22.68 \\
23.67 \\
24.66 \\
25.64 \\
26.63 \\
27.62 \\
28.60 \\
29.59\end{array}$ & $\begin{array}{l}3.47 \\
3.63 \\
3.80 \\
3.96 \\
4.13 \\
4.29 \\
4.46 \\
4.62 \\
4.79 \\
4.95\end{array}$ & $\begin{array}{l}20.70 \\
21.68 \\
22.67 \\
23.65 \\
24.64 \\
25.62 \\
26.61 \\
27.60 \\
28.58 \\
29.57\end{array}$ & $\begin{array}{l}3.56 \\
3.73 \\
3.90 \\
4.06 \\
4.23 \\
4.40 \\
4.57 \\
4.74 \\
4.91 \\
5.08\end{array}$ & $\begin{array}{l}21 \\
22 \\
23 \\
24 \\
25 \\
26 \\
27 \\
28 \\
29 \\
30\end{array}$ \\
\hline $\begin{array}{l}31 \\
32 \\
33 \\
34 \\
35 \\
36 \\
37 \\
38 \\
39 \\
40\end{array}$ & $\begin{array}{l}30.62 \\
31.61 \\
32.59 \\
33.58 \\
34.57 \\
35.56 \\
36.54 \\
37.53 \\
35.52 \\
39.51\end{array}$ & $\begin{array}{l}4.85 \\
5.01 \\
5.16 \\
5.32 \\
5.48 \\
5.63 \\
5.79 \\
5.94 \\
6.10 \\
6.26\end{array}$ & $\begin{array}{l}30.60 \\
31.58 \\
32.57 \\
33.56 \\
34.54 \\
35.53 \\
36.52 \\
37.51 \\
35.49 \\
39.48\end{array}$ & $\begin{array}{l}4.98 \\
5.14 \\
5.30 \\
5.47 \\
5.63 \\
5.79 \\
5.95 \\
6.11 \\
6.27 \\
6.43\end{array}$ & $\begin{array}{l}30.57 \\
31.56 \\
32.55 \\
33.53 \\
34.52 \\
35.51 \\
36.49 \\
37.48 \\
38.47 \\
39.45\end{array}$ & $\begin{array}{l}5.12 \\
5.28 \\
5.45 \\
5.61 \\
5.78 \\
5.94 \\
6.11 \\
6.27 \\
6.44 \\
6.60\end{array}$ & $\begin{array}{l}30.55 \\
31.54 \\
32.52 \\
33.51 \\
34.49 \\
35.48 \\
36.47 \\
37.45 \\
38.44 \\
39.42\end{array}$ & $\begin{array}{l}5.25 \\
5.42 \\
5.59 \\
5.76 \\
5.93 \\
6.10 \\
6.27 \\
6.44 \\
6.60 \\
6.77\end{array}$ & $\begin{array}{l}31 \\
32 \\
33 \\
34 \\
35 \\
36 \\
37 \\
38 \\
39 \\
40\end{array}$ \\
\hline $\begin{array}{l}41 \\
42 \\
43 \\
44 \\
45 \\
46 \\
47 \\
48 \\
49 \\
50\end{array}$ & $\begin{array}{l}40.50 \\
41.48 \\
42.47 \\
43.46 \\
44.45 \\
45.43 \\
46.42 \\
47.41 \\
48.40 \\
49.38\end{array}$ & $\begin{array}{l}6.41 \\
6.57 \\
6.73 \\
6.88 \\
7.04 \\
7.20 \\
7.35 \\
7.51 \\
7.67 \\
7.82\end{array}$ & $\begin{array}{l}40.47 \\
41.45 \\
42.44 \\
43.43 \\
44.41 \\
45.40 \\
46.39 \\
47.38 \\
48.36 \\
49.35\end{array}$ & $\begin{array}{l}6.59 \\
6.75 \\
6.91 \\
7.07 \\
7.23 \\
7.39 \\
7.55 \\
7.72 \\
7.88 \\
8.04\end{array}$ & $\begin{array}{l}40.44 \\
41.42 \\
42.41 \\
43.40 \\
44.38 \\
45.37 \\
46.36 \\
47.34 \\
48.33 \\
49.32\end{array}$ & $\begin{array}{l}6.77 \\
6.92 \\
7.10 \\
7.26 \\
7.43 \\
7.59 \\
7.76 \\
7.92 \\
8.09 \\
8.25\end{array}$ & $\begin{array}{l}40.41 \\
41.39 \\
42.38 \\
43.36 \\
44.35 \\
45.34 \\
46.32 \\
47.31 \\
48.29 \\
49.28\end{array}$ & $\begin{array}{l}6.94 \\
7.11 \\
7.28 \\
7.45 \\
7.62 \\
7.79 \\
7.96 \\
8.13 \\
8.30 \\
8.47\end{array}$ & $\begin{array}{l}41 \\
42 \\
43 \\
44 \\
45 \\
46 \\
47 \\
48 \\
49 \\
50\end{array}$ \\
\hline ठृ & Dep. & Lat. & Dep. & Lat. & Dep. & Lat. & Dep. & Lat. & சृं \\
\hline$\frac{n}{a}$ & \multicolumn{2}{|c|}{$81^{\circ}$. } & \multicolumn{2}{|c|}{$8034^{\circ}$. } & \multicolumn{2}{|c|}{$801 / 2^{\circ}$. } & \multicolumn{2}{|c|}{$8034^{\circ}$. } & $\frac{a}{a}$ \\
\hline
\end{tabular}


TABLE 4.-TRAVERSE TABLE.

\begin{tabular}{|c|c|c|c|c|c|c|c|c|c|}
\hline \multirow{2}{*}{ 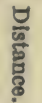 } & \multicolumn{2}{|c|}{$9^{\circ}$. } & \multicolumn{2}{|c|}{$914^{\circ}}$. & \multicolumn{2}{|c|}{$91 / 2^{\circ}$. } & \multicolumn{2}{|c|}{$934^{\circ}$} & \multirow{2}{*}{$\begin{array}{l}\frac{\forall}{\frac{n}{2}} \\
\frac{5}{5} \\
\frac{5}{8}\end{array}$} \\
\hline & Lat. & Dep. & Lat. & Dep. & Lat. & Dep. & Lat. & Dep. & \\
\hline $\begin{array}{l}51 \\
52 \\
53 \\
54 \\
55 \\
50 \\
57 \\
58 \\
59 \\
60\end{array}$ & $\begin{array}{l}50.37 \\
51.36 \\
52.35 \\
53.34 \\
54.32 \\
55.31 \\
56.30 \\
57.29 \\
58.27 \\
59.26\end{array}$ & $\begin{array}{l}7.98 \\
8.13 \\
8.29 \\
8.45 \\
8.60 \\
8.76 \\
8.92 \\
9.07 \\
9.23 \\
9.39\end{array}$ & $\begin{array}{l}50.34 \\
51.32 \\
52.31 \\
53.30 \\
54.28 \\
55.27 \\
56.26 \\
57.25 \\
58.23 \\
59.22\end{array}$ & $\begin{array}{l}8.20 \\
8.36 \\
8.52 \\
8.68 \\
8.84 \\
9.00 \\
9.16 \\
9.32 \\
9.48 \\
9.64\end{array}$ & $\begin{array}{l}50.30 \\
51.29 \\
52.27 \\
53.26 \\
54.25 \\
55.23 \\
56.22 \\
57.20 \\
58.19 \\
59.18\end{array}$ & $\begin{array}{l}8.42 \\
8.58 \\
8.75 \\
8.91 \\
9.08 \\
9.24 \\
9.41 \\
9.57 \\
9.74 \\
9.90\end{array}$ & $\begin{array}{l}50.26 \\
51.25 \\
52.23 \\
53.22 \\
54.21 \\
55.19 \\
56.18 \\
57.16 \\
58.15 \\
59.13\end{array}$ & $\begin{array}{r}8.64 \\
8.81 \\
8.98 \\
9.14 \\
9.31 \\
9.48 \\
9.65 \\
9.82 \\
9.99 \\
10.16\end{array}$ & $\begin{array}{l}51 \\
52 \\
53 \\
54 \\
55 \\
56 \\
57 \\
58 \\
59 \\
60\end{array}$ \\
\hline $\begin{array}{l}61 \\
62 \\
63 \\
64 \\
65 \\
66 \\
67 \\
68 \\
69 \\
70\end{array}$ & $\begin{array}{l}60.25 \\
61.24 \\
62.22 \\
63.21 \\
64.20 \\
65.19 \\
66.18 \\
67.16 \\
69.15 \\
69.14\end{array}$ & $\begin{array}{r}9.54 \\
9.70 \\
9.86 \\
10.01 \\
10.17 \\
10.32 \\
10.48 \\
10.64 \\
10.79 \\
10.95\end{array}$ & $\begin{array}{l}60.21 \\
61.19 \\
62.18 \\
63.17 \\
64.15 \\
65.14 \\
66.13 \\
67.12 \\
68.10 \\
69.09\end{array}$ & $\begin{array}{r}9.81 \\
9.97 \\
10.13 \\
10.29 \\
10.45 \\
10.61 \\
10.77 \\
10.93 \\
11.09 \\
11.25\end{array}$ & $\begin{array}{l}60.16 \\
61.15 \\
62.14 \\
63.12 \\
64.11 \\
65.09 \\
66.08 \\
67.07 \\
68.05 \\
69.04\end{array}$ & $\begin{array}{l}10.07 \\
10.23 \\
10.40 \\
10.56 \\
10.73 \\
10.89 \\
11.06 \\
11.22 \\
11.39 \\
11.55\end{array}$ & $\begin{array}{l}60.12 \\
61.10 \\
62.09 \\
63.08 \\
64.06 \\
65.05 \\
66.03 \\
67.02 \\
68.00 \\
68.99\end{array}$ & $\begin{array}{l}10.33 \\
10.50 \\
10.67 \\
10.84 \\
11.01 \\
11.18 \\
11.35 \\
11.52 \\
11.69 \\
11.85\end{array}$ & $\begin{array}{l}61 \\
62 \\
63 \\
64 \\
65 \\
66 \\
67 \\
68 \\
69 \\
70\end{array}$ \\
\hline $\begin{array}{l}71 \\
72 \\
73 \\
74 \\
75 \\
76 \\
77 \\
78 \\
79 \\
80\end{array}$ & $\begin{array}{l}70.13 \\
71.11 \\
72.10 \\
73.09 \\
74.08 \\
75.06 \\
76.05 \\
77.04 \\
78.03 \\
79.02\end{array}$ & $\begin{array}{l}11.11 \\
11.26 \\
11.42 \\
11.58 \\
11.73 \\
11.89 \\
12.05 \\
12.20 \\
12.36 \\
12.51\end{array}$ & $\begin{array}{l}70.08 \\
71.00 \\
72.05 \\
73.04 \\
74.02 \\
75.01 \\
76.00 \\
76.99 \\
77.97 \\
78.96\end{array}$ & $\begin{array}{l}11.41 \\
11.57 \\
11.73 \\
11.89 \\
12.06 \\
12.22 \\
12.38 \\
12.54 \\
12.70 \\
12.86\end{array}$ & $\begin{array}{l}70.03 \\
71.01 \\
72.00 \\
72.99 \\
73.97 \\
74.96 \\
75.94 \\
76.93 \\
77.92 \\
78.90\end{array}$ & $\begin{array}{l}11.72 \\
11.88 \\
12.05 \\
12.21 \\
12.38 \\
12.54 \\
12.71 \\
12.87 \\
13.04 \\
13.20\end{array}$ & $\begin{array}{l}69.97 \\
70.96 \\
71.95 \\
72.93 \\
73.92 \\
74.90 \\
75.89 \\
76.87 \\
77.86 \\
78.84\end{array}$ & $\begin{array}{l}12.02 \\
12.19 \\
12.36 \\
12.53 \\
12.70 \\
12.87 \\
13.04 \\
13.21 \\
13.38 \\
13.55\end{array}$ & $\begin{array}{l}71 \\
72 \\
73 \\
74 \\
75 \\
76 \\
77 \\
78 \\
79 \\
80\end{array}$ \\
\hline $\begin{array}{l}81 \\
82 \\
83 \\
84 \\
85 \\
86 \\
87 \\
89 \\
89 \\
90\end{array}$ & $\begin{array}{l}80.00 \\
80.99 \\
81.98 \\
82.97 \\
83.95 \\
84.94 \\
85.93 \\
86.92 \\
87.90 \\
88.89\end{array}$ & $\begin{array}{l}12.67 \\
12.83 \\
12.98 \\
13.14 \\
13.30 \\
13.45 \\
13.61 \\
13.77 \\
13.92 \\
14.08\end{array}$ & $\begin{array}{l}79.95 \\
80.93 \\
81.92 \\
82.91 \\
83.89 \\
81.88 \\
85.87 \\
86.86 \\
87.84 \\
88.83\end{array}$ & $\begin{array}{l}13.02 \\
13.18 \\
13.34 \\
13.50 \\
13.66 \\
13.82 \\
13.98 \\
14.15 \\
14.31 \\
14.47\end{array}$ & $\begin{array}{l}79.89 \\
80.88 \\
81.86 \\
82.85 \\
83.83 \\
81.82 \\
85.81 \\
86.79 \\
87.78 \\
88.77\end{array}$ & $\begin{array}{l}13.37 \\
13.53 \\
13.70 \\
13.86 \\
14.03 \\
14.19 \\
14.36 \\
14.52 \\
14.69 \\
14.85\end{array}$ & $\begin{array}{l}79.83 \\
80.82 \\
81.80 \\
82.79 \\
83.77 \\
81.76 \\
85.74 \\
86.73 \\
87.71 \\
88.70\end{array}$ & $\begin{array}{l}13.72 \\
13.89 \\
14.06 \\
14.23 \\
14.39 \\
14.56 \\
14.73 \\
14.90 \\
15.07 \\
15.24\end{array}$ & $\begin{array}{l}81 \\
82 \\
83 \\
81 \\
85 \\
86 \\
87 \\
88 \\
89 \\
90\end{array}$ \\
\hline $\begin{array}{r}91 \\
92 \\
93 \\
94 \\
95 \\
96 \\
97 \\
98 \\
99 \\
100\end{array}$ & $\begin{array}{l}89.88 \\
90.87 \\
91.86 \\
92.84 \\
93.83 \\
94.82 \\
95.81 \\
91.79 \\
97.78 \\
98.77\end{array}$ & $\begin{array}{l}14.24 \\
14.39 \\
14.55 \\
14.70 \\
14.86 \\
15.02 \\
15.17 \\
15.33 \\
15.49 \\
15.64\end{array}$ & $\begin{array}{l}89.82 \\
90.80 \\
91.79 \\
92.78 \\
93.76 \\
91.75 \\
95.74 \\
96.73 \\
97.71 \\
98.70\end{array}$ & $\begin{array}{l}14.63 \\
14.79 \\
14.95 \\
15.11 \\
15.27 \\
15.43 \\
15.59 \\
15.75 \\
15.91 \\
16.07\end{array}$ & $\begin{array}{l}89.75 \\
90.74 \\
91.72 \\
92.71 \\
93.70 \\
91.68 \\
95.67 \\
96.66 \\
97.64 \\
98.63\end{array}$ & $\begin{array}{l}15.02 \\
15.18 \\
15.35 \\
15.51 \\
15.68 \\
15.84 \\
16.01 \\
16.17 \\
16.34 \\
16.50\end{array}$ & $\begin{array}{l}89.69 \\
90.67 \\
91.66 \\
92.64 \\
93.63 \\
91.61 \\
95.60 \\
96.58 \\
97.57 \\
98.56\end{array}$ & $\begin{array}{l}15.41 \\
15.58 \\
15.75 \\
15.92 \\
16.09 \\
16.26 \\
16.43 \\
16.60 \\
16.77 \\
16.93\end{array}$ & $\begin{array}{r}91 \\
92 \\
93 \\
94 \\
95 \\
96 \\
97 \\
98 \\
99 \\
100\end{array}$ \\
\hline छீ & Dep. & Lat. & Dep. & Lat. & Dep. & Lat. & Dep. & Lat. & ष्ठ \\
\hline$\frac{5}{6}$ & \multicolumn{2}{|c|}{$81^{\circ}$} & \multicolumn{2}{|c|}{$803 / 4^{\circ}$. } & \multicolumn{2}{|c|}{$801 / 2^{\circ}$. } & \multicolumn{2}{|c|}{$801 / 4^{\circ}$. } & 蒙 \\
\hline
\end{tabular}


TABLE 4.-TRAVERSE TABLE.

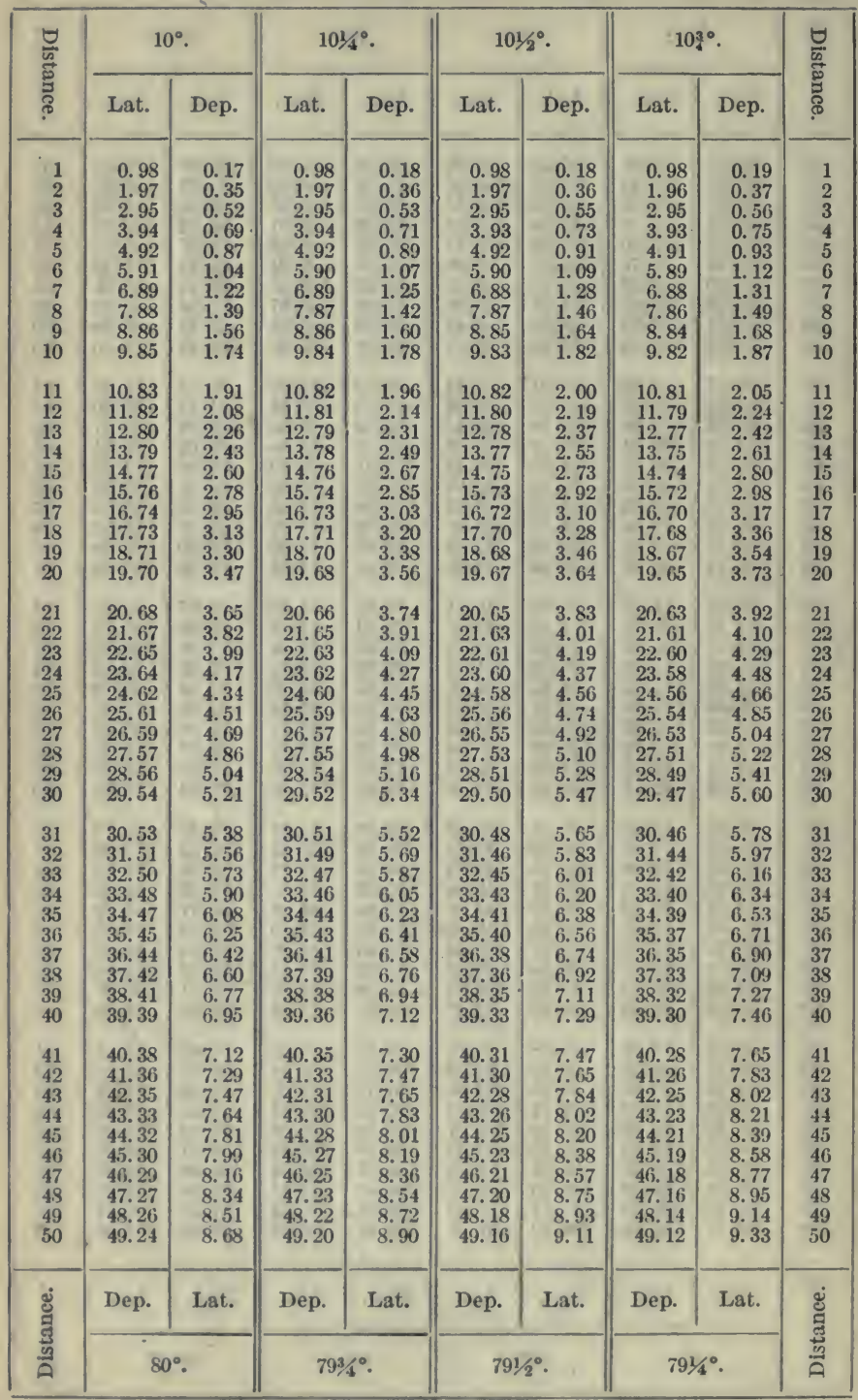


TABLE 4.-TRAVERSE TABLE.

\begin{tabular}{|c|c|c|c|c|c|c|c|c|c|}
\hline \multirow{2}{*}{ 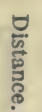 } & \multicolumn{2}{|c|}{$10^{\circ}$} & \multicolumn{2}{|c|}{$101 / 4^{\circ}$. } & \multicolumn{2}{|c|}{$101 / 2^{\circ}$. } & \multicolumn{2}{|c|}{$10 \% 4^{\circ}$. } & \multirow{2}{*}{ 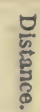 } \\
\hline & Isat. & Dep. & Lat. & Dep. & Lat. & Dep. & Lat. & Dep. & \\
\hline $\begin{array}{l}51 \\
52 \\
53 \\
54 \\
55 \\
56 \\
57 \\
58 \\
59 \\
60\end{array}$ & $\begin{array}{l}50.23 \\
51.21 \\
52.19 \\
53.18 \\
54.16 \\
55.15 \\
56.13 \\
57.12 \\
58.10 \\
59.09\end{array}$ & $\begin{array}{r}8.86 \\
9.03 \\
9.20 \\
9.38 \\
9.55 \\
9.72 \\
9.90 \\
10.07 \\
10.25 \\
10.42\end{array}$ & $\begin{array}{l}50.19 \\
51.17 \\
52.15 \\
53.14 \\
54.12 \\
55.11 \\
56.09 \\
57.07 \\
58.06 \\
59.04\end{array}$ & $\begin{array}{r}9.08 \\
9.25 \\
9.43 \\
9.61 \\
9.79 \\
9.96 \\
10.14 \\
10.32 \\
10.50 \\
10.68\end{array}$ & $\begin{array}{l}50.15 \\
51.13 \\
52.11 \\
53.10 \\
51.08 \\
55.06 \\
56.05 \\
57.03 \\
58.01 \\
59.00\end{array}$ & $\begin{array}{r}9.29 \\
9.48 \\
9.66 \\
9.84 \\
10.02 \\
10.21 \\
10.39 \\
10.57 \\
10.75 \\
10.93\end{array}$ & $\begin{array}{l}50.10 \\
51.09 \\
52.07 \\
53.05 \\
54.03 \\
55.02 \\
56.00 \\
56.98 \\
57.96 \\
58.95\end{array}$ & $\begin{array}{r}9.51 \\
9.70 \\
9.89 \\
10.07 \\
10.26 \\
10.45 \\
10.63 \\
10.82 \\
11.00 \\
11.19\end{array}$ & $\begin{array}{l}51 \\
52 \\
53 \\
54 \\
55 \\
50 \\
57 \\
58 \\
59 \\
60\end{array}$ \\
\hline $\begin{array}{l}61 \\
62 \\
63 \\
64 \\
65 \\
66 \\
67 \\
68 \\
69 \\
70\end{array}$ & $\begin{array}{l}60.07 \\
61.06 \\
62.04 \\
63.03 \\
64.01 \\
65.00 \\
65.98 \\
66.97 \\
67.95 \\
68.94\end{array}$ & $\begin{array}{l}10.59 \\
10.77 \\
10.94 \\
11.11 \\
11.29 \\
11.46 \\
11.63 \\
11.81 \\
11.98 \\
12.16\end{array}$ & $\begin{array}{l}60.03 \\
61.01 \\
61.99 \\
62.98 \\
63.96 \\
64.95 \\
65.93 \\
66.91 \\
67.90 \\
68.88\end{array}$ & $\begin{array}{l}10.85 \\
11.03 \\
11.21 \\
11.39 \\
11.57 \\
11.74 \\
11.92 \\
12.10 \\
12.28 \\
12.46\end{array}$ & $\begin{array}{l}59.98 \\
60.96 \\
61.95 \\
62.93 \\
63.91 \\
64.89 \\
65.88 \\
66.86 \\
67.84 \\
68.83\end{array}$ & $\begin{array}{l}11.12 \\
11.30 \\
11.48 \\
11.66 \\
11.85 \\
12.03 \\
12.21 \\
12.39 \\
12.57 \\
12.76\end{array}$ & $\begin{array}{l}59.93 \\
60.91 \\
61.89 \\
62.85 \\
63.86 \\
64.84 \\
65.82 \\
66.81 \\
67.79 \\
68.77\end{array}$ & $\begin{array}{l}11.35 \\
11.56 \\
11.75 \\
11.94 \\
12.12 \\
12.31 \\
12.50 \\
12.68 \\
12.87 \\
13.06\end{array}$ & $\begin{array}{l}61 \\
62 \\
63 \\
64 \\
65 \\
66 \\
67 \\
69 \\
69 \\
70\end{array}$ \\
\hline $\begin{array}{l}71 \\
72 \\
73 \\
74 \\
75 \\
76 \\
77 \\
78 \\
79 \\
80\end{array}$ & $\begin{array}{l}69.92 \\
70.91 \\
71.89 \\
72.88 \\
73.86 \\
74.85 \\
75.83 \\
76.82 \\
77.80 \\
78.78\end{array}$ & $\begin{array}{l}12.33 \\
12.50 \\
12.68 \\
12.85 \\
13.02 \\
13.20 \\
13.37 \\
13.54 \\
13.72 \\
13.89\end{array}$ & $\begin{array}{l}69.87 \\
70.85 \\
71.83 \\
72.82 \\
73.80 \\
74.79 \\
75.77 \\
76.76 \\
77.74 \\
78.72\end{array}$ & $\begin{array}{l}12.63 \\
12.81 \\
12.99 \\
13.17 \\
13.35 \\
13.52 \\
13.70 \\
13.89 \\
14.06 \\
14.24\end{array}$ & $\begin{array}{l}69.81 \\
70.79 \\
71.78 \\
72.76 \\
73.74 \\
74.73 \\
75.71 \\
76.69 \\
77.68 \\
78.66\end{array}$ & $\begin{array}{l}12.94 \\
13.12 \\
13.30 \\
13.49 \\
13.67 \\
13.85 \\
14.03 \\
14.21 \\
14.40 \\
14.58\end{array}$ & $\begin{array}{l}69.75 \\
70.74 \\
71.72 \\
72.70 \\
73.68 \\
74.67 \\
75.65 \\
76.63 \\
77.61 \\
78.60\end{array}$ & $\begin{array}{l}13.24 \\
13.43 \\
13.62 \\
13.80 \\
13.99 \\
14.18 \\
14.36 \\
14.55 \\
14.74 \\
14.92\end{array}$ & $\begin{array}{l}71 \\
72 \\
73 \\
74 \\
75 \\
76 \\
77 \\
78 \\
79 \\
80\end{array}$ \\
\hline $\begin{array}{l}81 \\
82 \\
83 \\
84 \\
85 \\
86 \\
87 \\
88 \\
89 \\
90\end{array}$ & $\begin{array}{l}79.77 \\
80.75 \\
81.74 \\
82.72 \\
83.71 \\
84.69 \\
85.68 \\
86.66 \\
87.65 \\
88.63\end{array}$ & $\begin{array}{l}14.07 \\
14.24 \\
14.41 \\
14.59 \\
14.76 \\
14.93 \\
15.11 \\
15.28 \\
15.45 \\
15.63\end{array}$ & $\begin{array}{l}79.71 \\
80.69 \\
81.68 \\
82.66 \\
83.64 \\
84.63 \\
85.61 \\
86.60 \\
87.59 \\
88.56\end{array}$ & $\begin{array}{l}14.41 \\
14.59 \\
14.77 \\
14.95 \\
15.13 \\
15.30 \\
15.48 \\
15.66 \\
15.84 \\
16.01\end{array}$ & $\begin{array}{r}79.64 \\
80.63 \\
81.61 \\
82.59 \\
83.58 \\
84.56 \\
85.54 \\
86.53 \\
87.51 \\
88.49\end{array}$ & $\begin{array}{l}14.76 \\
14.94 \\
15.13 \\
15.31 \\
15.49 \\
15.67 \\
15.85 \\
16.04 \\
16.22 \\
16.40\end{array}$ & $\begin{array}{l}79.58 \\
80.56 \\
81.54 \\
82.53 \\
83.51 \\
84.49 \\
85.47 \\
86.46 \\
87.44 \\
88.42\end{array}$ & $\begin{array}{l}15.11 \\
15.29 \\
15.48 \\
15.67 \\
15.85 \\
16.04 \\
16.23 \\
16.41 \\
16.60 \\
16.79\end{array}$ & $\begin{array}{l}81 \\
82 \\
83 \\
84 \\
85 \\
86 \\
87 \\
89 \\
99 \\
90\end{array}$ \\
\hline $\begin{array}{r}91 \\
92 \\
93 \\
94 \\
95 \\
96 \\
97 \\
98 \\
99 \\
100\end{array}$ & $\begin{array}{l}89.62 \\
90.60 \\
91.59 \\
92.57 \\
93.50 \\
94.54 \\
95.53 \\
96.51 \\
97.50 \\
98.48\end{array}$ & $\begin{array}{l}15.80 \\
15.98 \\
16.15 \\
16.32 \\
16.50 \\
16.67 \\
16.84 \\
17.02 \\
17.19 \\
17.36\end{array}$ & $\begin{array}{l}89.55 \\
90.53 \\
91.52 \\
92.50 \\
93.48 \\
94.47 \\
95.45 \\
96.44 \\
97.42 \\
98.40\end{array}$ & $\begin{array}{l}16.19 \\
16.37 \\
16.55 \\
16.73 \\
16.90 \\
17.08 \\
17.26 \\
17.44 \\
17.62 \\
17.79\end{array}$ & $\begin{array}{l}89.48 \\
90.46 \\
91.44 \\
92.43 \\
93.41 \\
94.39 \\
95.38 \\
96.36 \\
97.34 \\
98.33\end{array}$ & $\begin{array}{l}16.58 \\
16.77 \\
16.95 \\
17.13 \\
17.31 \\
17.49 \\
17.68 \\
17.86 \\
18.04 \\
18.22\end{array}$ & $\begin{array}{l}89.40 \\
90.39 \\
91.37 . \\
92.35 \\
93.33 \\
94.32 \\
95.30 \\
96.28 \\
97.26 \\
98.25\end{array}$ & $\begin{array}{l}16.97 \\
17.16 \\
17.35 \\
17.53 \\
17.72 \\
17.91 \\
18.09 \\
19.28 \\
18.47 \\
18.65\end{array}$ & $\begin{array}{r}91 \\
92 \\
93 \\
94 \\
95 \\
91 \\
97 \\
9.9 \\
99 \\
100\end{array}$ \\
\hline \multirow{2}{*}{ 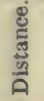 } & Dep. & Lat. & Dep. & Lat. & Dep. & Lat. & Dep. & Lat. & \multirow{2}{*}{ 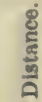 } \\
\hline & \multicolumn{2}{|c|}{$80^{\circ}$} & \multicolumn{2}{|c|}{$7934^{\circ}$. } & \multicolumn{2}{|c|}{$791 / 2^{\circ}$. } & \multicolumn{2}{|c|}{$7914^{\circ}$. } & \\
\hline
\end{tabular}


TABLE 4.-TRAVERSE TABLE.

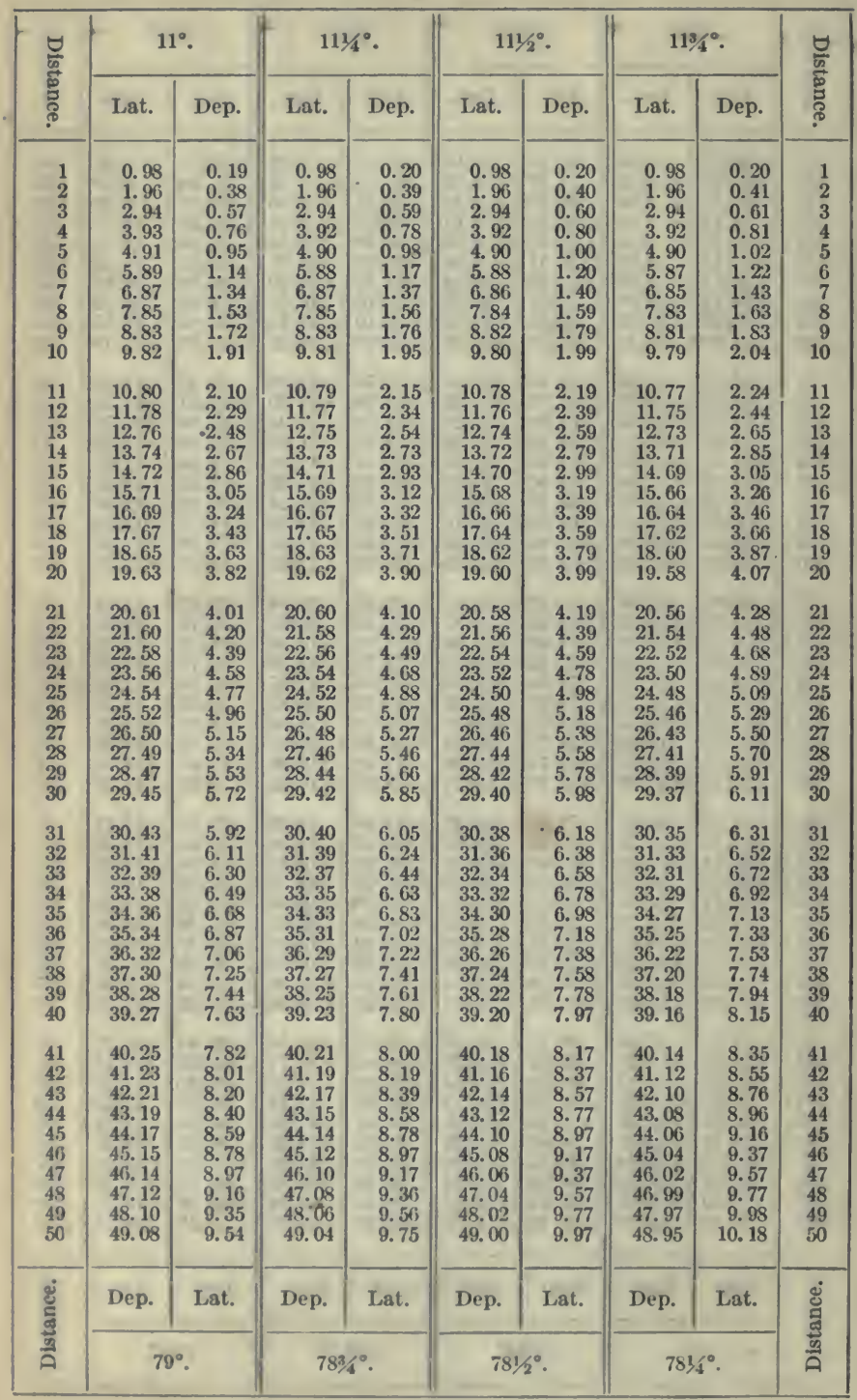


TABLE 4.-TRAVERSE TABLE.

\begin{tabular}{|c|c|c|c|c|c|c|c|c|c|}
\hline \multirow{2}{*}{ 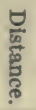 } & \multicolumn{2}{|c|}{$11^{\circ}$. } & \multicolumn{2}{|c|}{$111 / 4^{\circ}$. } & \multicolumn{2}{|c|}{$111 / 2^{\circ}$. } & \multicolumn{2}{|c|}{$11^{3} / 4^{\circ}$. } & \multirow{2}{*}{$\begin{array}{l}\text { ㅁ } \\
\text { है } \\
\text { है } \\
\text { है }\end{array}$} \\
\hline & Lat. & Dep. & Lat. & Dep. & Lat. & Dep. & Lat. & Dep. & \\
\hline $\begin{array}{l}51 \\
52 \\
53 \\
54 \\
55 \\
56 \\
57 \\
58 \\
59 \\
60\end{array}$ & $\begin{array}{l}50.06 \\
51.04 \\
52.03 \\
53.01 \\
53.99 \\
54.97 \\
55.95 \\
56.93 \\
57.92 \\
58.90\end{array}$ & $\begin{array}{r}9.73 \\
9.92 \\
10.11 \\
10.30 \\
10.49 \\
10.69 \\
10.88 \\
11.07 \\
11.26 \\
11.45\end{array}$ & $\begin{array}{l}50.02 \\
51.00 \\
51.98 \\
52.96 \\
53.94 \\
54.92 \\
55.90 \\
56.89 \\
57.87 \\
58.85\end{array}$ & $\begin{array}{r}9.95 \\
10.14 \\
10.34 \\
10.53 \\
10.73 \\
10.93 \\
11.12 \\
11.32 \\
11.51 \\
11.71\end{array}$ & $\begin{array}{l}49.98 \\
50.96 \\
51.94 \\
52.92 \\
53.90 \\
54.88 \\
55.86 \\
56.84 \\
57.82 \\
58.80\end{array}$ & $\begin{array}{l}10.17 \\
10.37 \\
10.57 \\
10.77 \\
10.97 \\
11.16 \\
11.36 \\
11.56 \\
11.76 \\
11.96\end{array}$ & $\begin{array}{l}49.93 \\
50.91 \\
51.89 \\
52.87 \\
53.85 \\
54.83 \\
55.81 \\
56.78 \\
57.76 \\
58.74\end{array}$ & $\begin{array}{l}10.39 \\
10.59 \\
10.79 \\
11.00 \\
11.20 \\
11.40 \\
11.61 \\
11.81 \\
12.01 \\
12.22\end{array}$ & $\begin{array}{l}51 \\
52 \\
53 \\
54 \\
55 \\
56 \\
57 \\
58 \\
59 \\
60\end{array}$ \\
\hline $\begin{array}{l}61 \\
62 \\
63 \\
64 \\
65 \\
66 \\
67 \\
68 \\
69 \\
70\end{array}$ & $\begin{array}{l}59.88 \\
60.86 \\
61.84 \\
62.82 \\
63.81 \\
64.79 \\
65.77 \\
66.75 \\
67.73 \\
68.71\end{array}$ & $\begin{array}{l}11.64 \\
11.83 \\
12.02 \\
12.21 \\
12.40 \\
12.59 \\
12.78 \\
12.98 \\
13.17 \\
13.36\end{array}$ & $\begin{array}{l}59.83 \\
60.81 \\
61.79 \\
62.77 \\
63.75 \\
64.73 \\
65.71 \\
66.69 \\
67.67 \\
68.65\end{array}$ & $\begin{array}{l}11.90 \\
12.10 \\
12.29 \\
12.49 \\
12.68 \\
12.88 \\
13.07 \\
13.27 \\
13.46 \\
13.66\end{array}$ & $\begin{array}{l}59.78 \\
60.76 \\
61.74 \\
62.72 \\
63.70 \\
64.68 \\
65.65 \\
66.63 \\
67.61 \\
68.59\end{array}$ & $\begin{array}{l}12.16 \\
12.36 \\
12.56 \\
12.76 \\
12.96 \\
13.16 \\
13.36 \\
13.56 \\
13.76 \\
13.96\end{array}$ & $\begin{array}{l}59.72 \\
60.70 \\
61.68 \\
62.66 \\
63.64 \\
64.62 \\
65.60 \\
66.58 \\
67.55 \\
68.53\end{array}$ & $\begin{array}{l}12.42 \\
12.63 \\
12.83 \\
13.03 \\
13.24 \\
13.44 \\
13.64 \\
13.85 \\
14.05 \\
14.25\end{array}$ & $\begin{array}{l}61 \\
62 \\
63 \\
64 \\
65 \\
66 \\
67 \\
68 \\
69 \\
70\end{array}$ \\
\hline $\begin{array}{l}71 \\
72 \\
73 \\
74 \\
75 \\
76 \\
77 \\
78 \\
79 \\
80\end{array}$ & $\begin{array}{l}69.70 \\
70.68 \\
71.66 \\
72.64 \\
73.62 \\
74.60 \\
75.59 \\
76.57 \\
77.55 \\
78.53\end{array}$ & $\begin{array}{l}13.55 \\
13.74 \\
13.93 \\
14.12 \\
14.31 \\
14.50 \\
14.69 \\
14.88 \\
15.07 \\
15.26\end{array}$ & $\begin{array}{l}69.64 \\
70.62 \\
71.60 \\
72.58^{4} \\
73.56 \\
74.54 \\
75.52 \\
76.50 \\
77.48 \\
78.46\end{array}$ & $\begin{array}{l}13.85 \\
14.05 \\
14.24 \\
14.44 \\
14.63 \\
14.83 \\
15.02 \\
15.22 \\
15.41 \\
15.61\end{array}$ & $\begin{array}{l}69.57 \\
70.55 \\
71.53 \\
72.51 \\
73.49 \\
74.47 \\
75.45 \\
76.43 \\
77.41 \\
78.39\end{array}$ & $\begin{array}{l}14.16 \\
14.35 \\
14.55 \\
14.75 \\
14.95 \\
15.15 \\
15.35 \\
15.55 \\
15.75 \\
15.95\end{array}$ & $\begin{array}{l}69.51 \\
70.49 \\
71.47 \\
72.45 \\
73.43 \\
74.41 \\
75.39 \\
76.37 \\
77.34 \\
78.32\end{array}$ & $\begin{array}{l}14.46 \\
14.66 \\
14.87 \\
15.07 \\
15.27 \\
15.48 \\
15.68 \\
15.88 \\
16.09 \\
16.29\end{array}$ & $\begin{array}{l}71 \\
72 \\
73 \\
74 \\
75 \\
76 \\
77 \\
78 \\
79 \\
80\end{array}$ \\
\hline $\begin{array}{l}81 \\
82 \\
83 \\
84 \\
85 \\
86 \\
87 \\
83 \\
89 \\
90\end{array}$ & $\begin{array}{l}79.51 \\
80.49 \\
81.48 \\
82.46 \\
83.44 \\
84.42 \\
85.40 \\
86.39 \\
87.36 \\
85.35\end{array}$ & $\begin{array}{l}15.46 \\
15.65 \\
15.84 \\
16.03 \\
16.22 \\
16.41 \\
16.60 \\
16.79 \\
16.98 \\
17.17\end{array}$ & $\begin{array}{l}79.44 \\
80.42 \\
81.41 \\
82.39 \\
83.37 \\
84.35 \\
85.33 \\
86.31 \\
87.29 \\
88.27\end{array}$ & $\begin{array}{l}15.80 \\
16.00 \\
16.19 \\
16.39 \\
16.58 \\
16.78 \\
16.97 \\
17.17 \\
17.36 \\
17.56\end{array}$ & $\begin{array}{l}79.37 \\
80.35 \\
81.33 \\
82.31 \\
83.29 \\
84.27 \\
85.25 \\
86.23 \\
87.21 \\
88.19\end{array}$ & $\begin{array}{l}16.15 \\
16.35 \\
16.55 \\
16.75 \\
16.95 \\
17.15 \\
17.35 \\
17.54 \\
17.74 \\
17.94\end{array}$ & $\begin{array}{l}79.30 \\
80.28 \\
81.26 \\
82.24 \\
83.22 \\
84.20 \\
85.18 \\
86.16 \\
87.14 \\
88.11\end{array}$ & $\begin{array}{l}16.49 \\
16.70 \\
16.90 \\
17.11 \\
17.31 \\
17.51 \\
17.72 \\
17.92 \\
18.12 \\
18.33\end{array}$ & $\begin{array}{l}81 \\
82 \\
83 \\
84 \\
85 \\
86 \\
87 \\
88 \\
89 \\
80\end{array}$ \\
\hline $\begin{array}{r}91 \\
92 \\
93 \\
94 \\
95 \\
96 \\
97 \\
98 \\
99 \\
100\end{array}$ & $\begin{array}{l}89.33 \\
90.31 \\
91.29 \\
92.27 \\
93.25 \\
94.24 \\
95.22 \\
96.20 \\
97.18 \\
98.16\end{array}$ & $\begin{array}{l}17.36 \\
17.55 \\
17.75 \\
17.94 \\
18.13 \\
18.32 \\
18.51 \\
18.70 \\
18.89 \\
19.08\end{array}$ & $\begin{array}{l}89.25 \\
90.23 \\
91.21 \\
92.19 \\
93.17 \\
94.16 \\
95.14 \\
96.12 \\
97.10 \\
98.08\end{array}$ & $\begin{array}{l}17.75 \\
17.95 \\
18.14 \\
18.34 \\
18.53 \\
18.73 \\
18.92 \\
19.12 \\
19.31 \\
19.51\end{array}$ & $\begin{array}{l}89.17 \\
90.15 \\
91.13 \\
9.11 \\
93.09 \\
94.07 \\
95.05 \\
96.03 \\
97.01 \\
97.99\end{array}$ & $\begin{array}{l}18.14 \\
18.34 \\
18.54 \\
18.74 \\
18.94 \\
19.14 \\
19.34 \\
19.54 \\
19.74 \\
19.94\end{array}$ & $\begin{array}{l}89.09 \\
90.07 \\
91.05 \\
92.03 \\
93.01 \\
93.99 \\
94.97 \\
95.95 \\
96.93 \\
97.90\end{array}$ & $\begin{array}{l}18.53 \\
18.74 \\
18.94 \\
19.14 \\
19.35 \\
19.55 \\
19.75 \\
19.96 \\
20.16 \\
20.36\end{array}$ & $\begin{array}{r}91 \\
92 \\
93 \\
94 \\
95 \\
96 \\
97 \\
98 \\
99 \\
100\end{array}$ \\
\hline \multirow{2}{*}{ 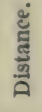 } & Dep. & Lat. & Dep. & Lat. & Dep. & Lat. & Dep. & Iat. & \\
\hline & \multicolumn{2}{|c|}{$79^{\circ}$. } & \multicolumn{2}{|c|}{$78 \% 4^{\circ}}$. & \multicolumn{2}{|c|}{$7 S 1 / 2^{\circ}$. } & \multicolumn{2}{|c|}{$7814^{\circ}}$. & $\frac{n}{a}$ \\
\hline
\end{tabular}


TABLE 4.-TRAVERSE TABLE.

\begin{tabular}{|c|c|c|c|c|c|c|c|c|c|}
\hline \multirow{2}{*}{ 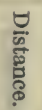 } & \multicolumn{2}{|c|}{$12^{\circ}$} & \multicolumn{2}{|c|}{$121 / 4^{\circ}$. } & \multicolumn{2}{|c|}{$121 / 2^{\circ}$. } & \multicolumn{2}{|c|}{$1234^{\circ}$. } & \multirow{2}{*}{ 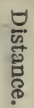 } \\
\hline & Lat. & Dep. & Lat. & Dep. & Lat. & Dep. & Lat. & Dep. & \\
\hline 1 & 0.98 & 0.21 & 0.98 & 0.21 & 0.98 & 0.22 & 0.98 & 0.22 & 1 \\
\hline 2 & 1.96 & 0.42 & 1.95 & 0.42 & 1.95 & 0.43 & 1.95 & 0.44 & 2 \\
\hline 3 & 2.93 & 0.62 & 2.93 & 0.64 & 2.93 & 0.65 & 2.93 & 0.66 & 3 \\
\hline 4 & 3.91 & 0.83 & 3.91 & 0.85 & 3.91 & 0.87 & 3.90 & 0.88 & 4 \\
\hline 5 & 4. 89 & 1.04 & 4. 89 & 1.06 & 4. 88 & 1.08 & 4.88 & 1.10 & 5 \\
\hline 6 & 5.87 & 1.25 & 5.86 & 1.27 & 5.86 & 1.30 & 5.85 & 1.32 & 6 \\
\hline 7 & 6.85 & 1.46 & 6.84 & 1.49 & 6.83 & 1.52 & 6.83 & 1.54 & 7 \\
\hline 8 & 7.83 & 1. 66 & 7.82 & 1.70 & 7.81 & 1.73 & 7.80 & 1.77 & 8 \\
\hline 9 & 8.80 & 1.87 & 8.80 & 1.91 & 8.79 & 1.95 & 8.78 & 1.99 & 9 \\
\hline 10 & 9.78 & 2.08 & 9.77 & 2.12 & 9.76 & 2.16 & 9.75 & 2.21 & 10 \\
\hline 11 & 10.76 & 2. 29 & 10.75 & 2.33 & 10.74 & 2. 38 & 10.73 & 2.43 & 11 \\
\hline 12 & 11.74 & 2.49 & 11.73 & 2.55 & 11.72 & 2. 60 & 11.70 & 2. 65 & 12 \\
\hline 13 & 12.72 & 2.70 & 12.70 & 2.76 & 12.69 & 2.81 & 12.68 & 2.87 & 13 \\
\hline 14 & 13. 69 & 2.91 & 13. 68 & 2.97 & 13.67 & 3.03 & 13.65 & 3.09 & 14 \\
\hline 15 & 14. 67 & 3. 12 & 14.66 & 3.18 & 14.64 & 3.25 & 14.63 & 3.31 & 15 \\
\hline 16 & 15. 65 & 3. 33 & 15. 64 & 3. 39 & 15.62 & 3.46 & 15. 61 & 3.53 & 16 \\
\hline 17 & 16. 63 & 3.53 & 16. 61 & 3.61 & 16. 60 & 3. 68 & 16.58 & 3.75 & 17 \\
\hline 18 & 17. 61 & 3.74 & 17. 59 & 3.82 & 17.57 & 3.90 & 17. 56 & 3. 97 & 18 \\
\hline 19 & 18.58 & 3.95 & 18.57 & 4.03 & 18.55 & 4. 11 & 18.53 & 4. 19 & 19 \\
\hline 20 & 19.56 & 4. 16 & 19.54 & 4.24 & 19.53 & 4. 33 & 19.51 & 4.41 & 20 \\
\hline 21 & 20.54 & 4. 37 & 20.52 & 4.46 & 20.50 & 4.55 & 20.48 & 4.63 & 21 \\
\hline 22 & 21.52 & 4.57 & 21.50 & 4. 67 & 21.48 & 4.76 & 21.46 & 4.86 & 22 \\
\hline 23 & 22.50 & 4.78 & 22.48 & 4. 88 & 22.45 & 4.98 & 22.43 & 5.08 & 23 \\
\hline 24 & 23.48 & 4.99 & 23.45 & 5.09 & 23.43 & 5.19 & 23.41 & 5.30 & 24 \\
\hline 25 & 24.45 & 5.20 & 24.43 & 5.30 & 24.41 & 5. 41 & 24.38 & 5.52 & 25 \\
\hline 26 & 25.43 & 5.41 & 25.41 & 5.52 & 25.38 & 5. 63 & 25.36 & 5.74 & 26 \\
\hline 27 & 26.41 & 5. 61 & 26.39 & 5.73 & 26.36 & 5.84 & 26. 33 & 5.96 & 27 \\
\hline 28 & 27. 39 & 5.82 & 27.36 & 5.94 & 27.34 & 6.06 & 27. 31 & 6.18 & 28 \\
\hline 29 & 28.37 & 6.03 & 28.34 & 6.15 & 28.31 & 6.28 & 28.28 & 6.40 & 29 \\
\hline 30 & 29.34 & 6.24 & 29.32 & 6.37 & 29.29 & 6.49 & 29.26 & 6.62 & 30 \\
\hline 31 & 30.32 & 6.45 & 30.29 & 6.58 & 30.27 & 6.71 & 30.24 & 6.84 & 31 \\
\hline 32 & 31.30 & 6.65 & 31.27 & 6.79 & 31.24 & 6.93 & 31.21 & 7.06 & 32 \\
\hline 33 & 32.28 & 6.86 & 32.25 & 7.00 & 32.22 & 7.14 & 32.19 & 7.28 & 33 \\
\hline 34 & 33.26 & 7.07 & 33.23 & 7.21 & 33. 19 & 7.36 & 33.16 & 7.50 & 34 \\
\hline 35 & 34.24 & 7.28 & 34.20 & 7.43 & 34.17 & 7.58 & 34.14 & 7.72 & 35 \\
\hline 36 & 35.21 & 7.48 & 35.18 & 7.64 & 35.15 & 7.79 & 35.11 & 7.95 & 36 \\
\hline 37 & 36.19 & 7.69 & 36.16 & 7.85 & 36.12 & 8.01 & 36.09 & 8.17 & 37 \\
\hline 38 & 37.17 & 7.90 & 37.13 & 8.06 & 37.10 & 8.22 & 37.06 & 8. 39 & 38 \\
\hline 39 & 38. 15 & 8.11 & 38.11 & 8.27 & 38.08 & 8.44 & 38.04 & 8. 61 & 39 \\
\hline 40 & 39.13 & 8.32 & 39.09 & 8.49 & 39.05 & 8.66 & 39.01 & 8. 83 & 40 \\
\hline 41 & 40.10 & 8.52 & 40.07 & 8. 70 & 40.03 & 8.87 & 39.99 & 9.05 & 41 \\
\hline 42 & 41.08 & 8.73 & 41.04 & 8.91 & 41.00 & 9. 09 & 40.96 & 9.27 & 42 \\
\hline 43 & 42.06 & 8.94 & 42.02 & 9.12 & 41.98 & 9.31 & 41.94 & 9.49 & 43 \\
\hline 44 & 43.04 & 9.15 & 43. 00 & 9.34 & 42.96 & 9.52 & 42.92 & 9.71 & 44 \\
\hline 45 & 44. 02 & 9.36 & 43. 98 & 9.55 & 43.93 & 9.74 & 43. 89 & 9.93 & 45 \\
\hline 46 & 44.99 & 9.56 & 44.95 & 9.76 & 44.91 & 9.96 & 44.87 & 10.15 & 46 \\
\hline 47 & 45.97 & 9.77 & 45.93 & 9.97 & 45.89 & 10. 17 & 45.84 & 10.37 & 47 \\
\hline 48 & 46.95 & 9.98 & 46.91 & 10.18 & 46.86 & 10. 39 & 46.82 & 10.59 & 48 \\
\hline 49 & 47.93 & 10. 19 & 47.88 & 10.40 & 47.84 & 10.61 & 47.79 & 10.81 & 49 \\
\hline 50 & 48.91 & 10.40 & 48.86 & 10.61 & 48.81 & 10.82 & 48. 77 & 11.03 & 50 \\
\hline \multirow{2}{*}{ 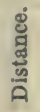 } & Dep. & Lat. & Dep. & Lat. & Dep. & Lat. & Dep. & Lat. & \multirow{2}{*}{ 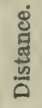 } \\
\hline & \multicolumn{2}{|c|}{$78^{\circ}}$. & \multicolumn{2}{|c|}{$77 \% 4^{\circ}$. } & \multicolumn{2}{|c|}{$771 / 2^{\circ}$. } & \multicolumn{2}{|c|}{$771 / 4^{\circ}$. } & \\
\hline
\end{tabular}


TABLE 4.-TRA VEIRE TABI.E.

\begin{tabular}{|c|c|c|c|c|c|c|c|c|c|}
\hline \multirow{2}{*}{ 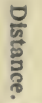 } & \multicolumn{2}{|c|}{$12^{\circ}$. } & \multicolumn{2}{|c|}{$121 / 4^{\circ}$. } & \multicolumn{2}{|c|}{$121 / 2^{\circ}$. } & \multicolumn{2}{|c|}{$123,4^{\circ}$} & \multirow{2}{*}{ 룰 } \\
\hline & Lat. & Dep. & Lat. & Dep. & Lat. & Dep. & Lat. & Dep. & \\
\hline $\begin{array}{l}51 \\
52 \\
53 \\
54 \\
55 \\
56 \\
57 \\
58 \\
59 \\
60\end{array}$ & $\begin{array}{l}49.89 \\
50.86 \\
51.84 \\
52.82 \\
53.80 \\
54.78 \\
55.75 \\
56.73 \\
57.71 \\
58.69\end{array}$ & $\begin{array}{l}10.60 \\
10.81 \\
11.02 \\
11.23 \\
11.44 \\
11.64 \\
11.85 \\
12.06 \\
12.27 \\
12.47\end{array}$ & $\begin{array}{l}49.84 \\
50.82 \\
51.79 \\
52.77 \\
53.75 \\
54.72 \\
55.70 \\
56.18 \\
57.66 \\
58.63\end{array}$ & $\begin{array}{l}10.82 \\
11.03 \\
11.25 \\
11.46 \\
11.67 \\
11.88 \\
12.09 \\
12.31 \\
12.52 \\
12.73\end{array}$ & $\begin{array}{l}49.79 \\
50.77 \\
51.74 \\
52.72 \\
53.70 \\
54.67 \\
55.65 \\
56.63 \\
57.60 \\
58.58\end{array}$ & $\begin{array}{l}11.04 \\
11.25 \\
11.47 \\
11.69 \\
11.90 \\
12.12 \\
12.34 \\
12.55 \\
12.77 \\
12.99\end{array}$ & $\begin{array}{l}49.74 \\
50.72 \\
51.69 \\
52.67 \\
53.64 \\
54.62 \\
55.59 \\
56.57 \\
57.55 \\
58.52\end{array}$ & $\begin{array}{l}11.26 \\
11.48 \\
11.70 \\
11.92 \\
12.14 \\
12.36 \\
12.58 \\
12.80 \\
13.02 \\
13.24\end{array}$ & $\begin{array}{l}51 \\
52 \\
53 \\
54 \\
55 \\
56 \\
57 \\
58 \\
59 \\
60\end{array}$ \\
\hline $\begin{array}{l}61 \\
62 \\
63 \\
64 \\
65 \\
66 \\
67 \\
68 \\
69 \\
70\end{array}$ & $\begin{array}{l}59.67 \\
60.65 \\
61.62 \\
62.60 \\
63.58 \\
64.56 \\
65.54 \\
66.51 \\
67.49 \\
68.47\end{array}$ & $\begin{array}{l}12.68 \\
12.89 \\
13.10 \\
13.31 \\
13.51 \\
13.72 \\
13.93 \\
14.14 \\
14.35 \\
14.55\end{array}$ & $\begin{array}{l}59.61 \\
60.59 \\
61.57 \\
62.54 \\
63.52 \\
64.50 \\
65.47 \\
66.45 \\
67.43 \\
68.41\end{array}$ & $\begin{array}{l}12.94 \\
13.16 \\
13.37 \\
13.58 \\
13.79 \\
14.00 \\
14.22 \\
14.43 \\
14.64 \\
14.85\end{array}$ & $\begin{array}{l}59.55 \\
60.53 \\
61.51 \\
62.48 \\
63.46 \\
64.44 \\
65.41 \\
66.39 \\
67.36 \\
68.34\end{array}$ & $\begin{array}{l}13.20 \\
13.42 \\
13.64 \\
13.85 \\
14.07 \\
14.29 \\
14.50 \\
14.72 \\
14.93 \\
15.15\end{array}$ & $\begin{array}{l}59.50 \\
60.47 \\
61.45 \\
62.42 \\
63.40 \\
64.37 \\
65.35 \\
66.32 \\
67.30 \\
68.27\end{array}$ & $\begin{array}{l}13.46 \\
13.68 \\
13.90 \\
14.12 \\
14.35 \\
14.57 \\
14.79 \\
15.01 \\
15.23 \\
15.45\end{array}$ & $\begin{array}{l}61 \\
62 \\
63 \\
64 \\
65 \\
66 \\
67 \\
68 \\
69 \\
70\end{array}$ \\
\hline $\begin{array}{l}71 \\
72 \\
73 \\
74 \\
75 \\
76 \\
77 \\
78 \\
79 \\
80\end{array}$ & $\begin{array}{l}69.45 \\
70.43 \\
71.40 \\
72.38 \\
73.36 \\
74.34 \\
75.32 \\
76.30 \\
77.27 \\
78.25\end{array}$ & $\begin{array}{l}14.76 \\
14.97 \\
15.18 \\
15.39 \\
15.59 \\
15.80 \\
16.01 \\
16.22 \\
16.43 \\
16.63\end{array}$ & $\begin{array}{l}69.38 \\
70.36 \\
71.34 \\
72.32 \\
73.29 \\
74.27 \\
75.25 \\
76.22 \\
77.20 \\
78.18\end{array}$ & $\begin{array}{l}15.06 \\
15.28 \\
15.49 \\
15.70 \\
15.91 \\
16.13 \\
16.34 \\
16.55 \\
16.76 \\
16.97\end{array}$ & $\begin{array}{l}69.32 \\
70.29 \\
71.27 \\
72.25 \\
73.22 \\
74.20 \\
75.17 \\
76.15 \\
77.13 \\
78.10\end{array}$ & $\begin{array}{l}15.37 \\
15.58 \\
15.80 \\
16.02 \\
16.23 \\
16.45 \\
16.67 \\
16.88 \\
17.10 \\
17.32\end{array}$ & $\begin{array}{l}69.25 \\
70.22 \\
71.20 \\
72.15 \\
73.15 \\
74.13 \\
75.10 \\
76.08 \\
77.05 \\
78.03\end{array}$ & $\begin{array}{l}15.67 \\
15.89 \\
16.11 \\
16.33 \\
16.55 \\
16.77 \\
16.99 \\
17.21 \\
17.44 \\
17.66\end{array}$ & $\begin{array}{l}71 \\
72 \\
73 \\
74 \\
75 \\
76 \\
77 \\
78 \\
79 \\
80\end{array}$ \\
\hline $\begin{array}{l}81 \\
82 \\
83 \\
84 \\
85 \\
86 \\
87 \\
89 \\
89 \\
90\end{array}$ & $\begin{array}{l}79.23 \\
80.21 \\
81.19 \\
82.16 \\
83.14 \\
84.12 \\
85.10 \\
81.08 \\
87.06 \\
88.03\end{array}$ & $\begin{array}{l}16.84 \\
17.05 \\
17.26 \\
17.46 \\
17.67 \\
17.88 \\
18.09 \\
18.30 \\
18.50 \\
18.71\end{array}$ & $\begin{array}{l}79.16 \\
80.13 \\
81.11 \\
82.09 \\
83.06 \\
84.04 \\
85.02 \\
86.00 \\
86.97 \\
87.95\end{array}$ & $\begin{array}{l}17.19 \\
17.40 \\
17.61 \\
17.82 \\
18.04 \\
18.25 \\
18.46 \\
18.67 \\
18.85 \\
19.10\end{array}$ & $\begin{array}{l}79.08 \\
80.06 \\
81.03 \\
82.01 \\
82.99 \\
83.96 \\
81.94 \\
85.91 \\
86.99 \\
87.87\end{array}$ & $\begin{array}{l}17.53 \\
17.75 \\
17.96 \\
18.18 \\
18.40 \\
18.41 \\
18.83 \\
19.05 \\
19.26 \\
19.48\end{array}$ & $\begin{array}{l}79.00 \\
79.918 \\
80.95 \\
81.93 \\
82.90 \\
83.88 \\
84.85 \\
85.83 \\
86.81 \\
87.78\end{array}$ & $\begin{array}{l}17.88 \\
18.10 \\
18.32 \\
18.54 \\
18.76 \\
18.98 \\
19.20 \\
19.42 \\
19.64 \\
19.86\end{array}$ & $\begin{array}{l}81 \\
82 \\
83 \\
81 \\
85 \\
86 \\
87 \\
88 \\
89 \\
90\end{array}$ \\
\hline $\begin{array}{r}91 \\
92 \\
93 \\
94 \\
95 \\
96 \\
97 \\
98 \\
99 \\
100\end{array}$ & $\begin{array}{l}89.01 \\
89.99 \\
90.97 \\
91.95 \\
92.92 \\
93.90 \\
94.88 \\
95.86 \\
96.84 \\
97.81\end{array}$ & $\begin{array}{l}18.92 \\
19.13 \\
19.34 \\
19.54 \\
19.75 \\
19.96 \\
20.17 \\
20.38 \\
20.58 \\
20.79\end{array}$ & $\begin{array}{l}85.93 \\
89.91 \\
90.85 \\
91.86 \\
92.84 \\
93.81 \\
94.79 \\
95.77 \\
96.75 \\
97.72\end{array}$ & $\begin{array}{l}19.31 \\
19.52 \\
19.73 \\
19.94 \\
20.16 \\
20.37 \\
20.58 \\
20.79 \\
21.01 \\
21.22\end{array}$ & $\begin{array}{l}83.84 \\
89.82 \\
90.80 \\
91.77 \\
92.75 \\
93.72 \\
94.70 \\
95.65 \\
96.65 \\
97.63\end{array}$ & $\begin{array}{l}19.70 \\
19.91 \\
20.13 \\
20.35 \\
20.56 \\
20.78 \\
20.99 \\
21.21 \\
21.43 \\
21.64\end{array}$ & $\begin{array}{l}88.76 \\
89.73 \\
90.71 \\
91.68 \\
92.66 \\
93.63 \\
94.61 \\
95.58 \\
96.56 \\
97.53\end{array}$ & $\begin{array}{l}20.08 \\
20.30 \\
20.52 \\
20.75 \\
20.07 \\
21.19 \\
21.41 \\
21.11 \\
21.85 \\
22.07\end{array}$ & $\begin{array}{r}91 \\
92 \\
93 \\
94 \\
95 \\
96 \\
97 \\
98 \\
99 \\
100\end{array}$ \\
\hline$\dot{8}$ & Dep. & Lat. & Dep. & Lat. & Dep. & Lat. & Dep. & Lat. & छ் \\
\hline & \multicolumn{2}{|c|}{$78^{\circ}}$. & \multicolumn{2}{|c|}{$773 / 3^{\circ}}$. & \multicolumn{2}{|c|}{$73: 2^{\circ}}$. & \multicolumn{2}{|c|}{$7734^{\circ}}$. & \\
\hline
\end{tabular}


TABLE 4.-TRAVERSE TABLE.

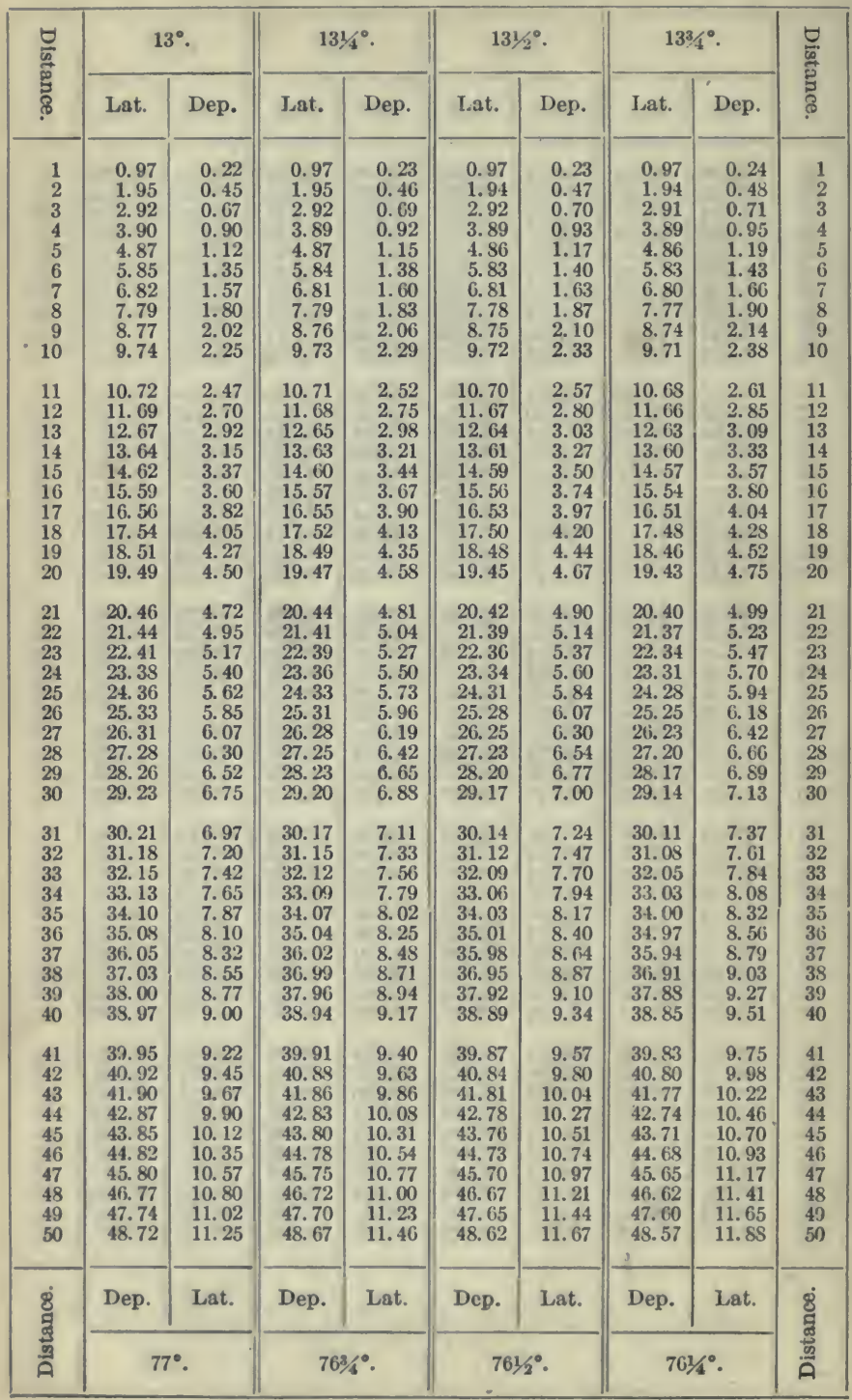


TABIE 4.-TIAVEIRSE TABLF。

\begin{tabular}{|c|c|c|c|c|c|c|c|c|c|}
\hline \multirow{2}{*}{ 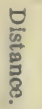 } & \multicolumn{2}{|c|}{$13^{\circ}$} & \multicolumn{2}{|c|}{$131 / 4^{\circ}$} & \multicolumn{2}{|c|}{$131 / 2^{\circ}$} & \multicolumn{2}{|c|}{$1334^{\circ}$} & \multirow{2}{*}{ 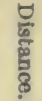 } \\
\hline & Lat. & Dep. & Ioat. & Dep. & Lat. & Dep. & Lat. & Dep. & \\
\hline $\begin{array}{l}51 \\
52 \\
53 \\
54 \\
55 \\
56 \\
57 \\
58 \\
59 \\
60\end{array}$ & $\begin{array}{l}49.69 \\
50.67 \\
51.64 \\
52.62 \\
53.59 \\
54.54 \\
55.54 \\
56.51 \\
57.49 \\
58.46\end{array}$ & $\begin{array}{l}11.47 \\
11.70 \\
11.92 \\
12.15 \\
12.37 \\
12.60 \\
12.82 \\
13.05 \\
13.27 \\
13.50\end{array}$ & $\begin{array}{l}40.64 \\
50.62 \\
51.59 \\
52.50 \\
53.54 \\
54.51 \\
55.48 \\
56.46 \\
57.43 \\
58.40\end{array}$ & $\begin{array}{l}11.69 \\
11.92 \\
12.15 \\
12.38 \\
12.61 \\
12.84 \\
13.06 \\
13.29 \\
13.52 \\
13.75\end{array}$ & $\begin{array}{l}49.59 \\
50.56 \\
51.34 \\
52.51 \\
53.48 \\
54.45 \\
55.43 \\
56.40 \\
57.37 \\
58.34\end{array}$ & $\begin{array}{l}11.91 \\
12.14 \\
12.37 \\
12.61 \\
12.84 \\
13.07 \\
13.31 \\
13.54 \\
13.77 \\
14.01\end{array}$ & $\begin{array}{l}49.54 \\
50.51 \\
51.49 \\
52.45 \\
53.42 \\
54.40 \\
55.37 \\
56.34 \\
57.31 \\
53.28\end{array}$ & $\begin{array}{l}12.12 \\
12.36 \\
12.60 \\
12.84 \\
13.07 \\
13.31 \\
13.55 \\
13.79 \\
14.02 \\
14.26\end{array}$ & $\begin{array}{l}51 \\
52 \\
53 \\
54 \\
55 \\
56 \\
57 \\
58 \\
59 \\
60\end{array}$ \\
\hline $\begin{array}{l}61 \\
62 \\
63 \\
64 \\
65 \\
66 \\
67 \\
68 \\
69 \\
70\end{array}$ & $\begin{array}{l}59.44 \\
60.41 \\
61.39 \\
62.36 \\
63.33 \\
64.31 \\
65.28 \\
66.26 \\
67.23 \\
68.21\end{array}$ & $\begin{array}{l}13.72 \\
13.95 \\
14.17 \\
14.40 \\
14.62 \\
14.85 \\
15.07 \\
15.30 \\
15.52 \\
1575\end{array}$ & $\begin{array}{l}59.39 \\
60.35 \\
61.32 \\
62.30 \\
63.27 \\
64.24 \\
65.22 \\
66.19 \\
67.16 \\
68.14\end{array}$ & $\begin{array}{l}13.98 \\
14.21 \\
14.44 \\
14.67 \\
14.90 \\
15.13 \\
15.36 \\
15.59 \\
15.81 \\
16.04\end{array}$ & $\begin{array}{l}59.31 \\
60.29 \\
61.26 \\
62.23 \\
63.20 \\
64.18 \\
65.15 \\
66.12 \\
67.09 \\
68.07\end{array}$ & $\begin{array}{l}14.24 \\
14.47 \\
14.71 \\
14.94 \\
15.17 \\
15.41 \\
15.64 \\
15.87 \\
16.11 \\
16.34\end{array}$ & $\begin{array}{l}59.25 \\
60.22 \\
61.19 \\
62.17 \\
63.14 \\
64.11 \\
65.08 \\
66.05 \\
67.02 \\
67.99\end{array}$ & $\begin{array}{l}14.50 \\
14.74 \\
14.97 \\
15.21 \\
15.45 \\
15.69 \\
15.92 \\
16.16 \\
16.40 \\
16.64\end{array}$ & $\begin{array}{l}61 \\
62 \\
63 \\
64 \\
65 \\
66 \\
67 \\
68 \\
69 \\
70\end{array}$ \\
\hline $\begin{array}{l}71 \\
72 \\
73 \\
74 \\
75 \\
76 \\
77 \\
78 \\
79 \\
80\end{array}$ & $\begin{array}{l}69.18 \\
70.15 \\
71.13 \\
72.10 \\
73.08 \\
74.05 \\
75.03 \\
76.00 \\
76.98 \\
77.95\end{array}$ & $\begin{array}{l}15.97 \\
16.20 \\
16.42 \\
16.65 \\
16.87 \\
17.10 \\
17.32 \\
17.55 \\
17.77 \\
18.00\end{array}$ & $\begin{array}{l}69.11 \\
70.08 \\
71.06 \\
72.03 \\
73.00 \\
73.99 \\
74.95 \\
75.92 \\
76.90 \\
77.87\end{array}$ & $\begin{array}{l}16.27 \\
16.50 \\
16.73 \\
16.96 \\
17.19 \\
17.42 \\
17.65 \\
17.83 \\
18.11 \\
18.34\end{array}$ & $\begin{array}{l}69.04 \\
70.01 \\
70.98 \\
71.96 \\
72.93 \\
73.90 \\
74.87 \\
75.84 \\
76.82 \\
77.79\end{array}$ & $\begin{array}{l}16.57 \\
16.81 \\
17.04 \\
17.27 \\
17.51 \\
17.74 \\
17.98 \\
18.21 \\
18.44 \\
18.68\end{array}$ & $\begin{array}{l}68.97 \\
69.94 \\
70.91 \\
71.88 \\
72.85 \\
73.82 \\
74.79 \\
75.76 \\
76.74 \\
77.71\end{array}$ & $\begin{array}{l}16.88 \\
17.11 \\
17.35 \\
17.59 \\
17.83 \\
18.06 \\
18.30 \\
18.54 \\
18.78 \\
19.01\end{array}$ & $\begin{array}{l}71 \\
72 \\
73 \\
74 \\
75 \\
76 \\
77 \\
78 \\
79 \\
80\end{array}$ \\
\hline $\begin{array}{l}81 \\
82 \\
83 \\
84 \\
85 \\
86 \\
87 \\
88 \\
89 \\
90\end{array}$ & $\begin{array}{l}78.92 \\
79.90 \\
80.87 \\
81.85 \\
82.82 \\
83.80 \\
84.77 \\
85.74 \\
81.72 \\
87.69\end{array}$ & $\begin{array}{l}18.22 \\
18.45 \\
18.67 \\
18.90 \\
19.12 \\
19.35 \\
19.57 \\
19.80 \\
20.02 \\
20.25\end{array}$ & $\begin{array}{l}78.84 \\
70.82 \\
80.79 \\
81.76 \\
82.74 \\
83.71 \\
81.68 \\
85.66 \\
86.63 \\
87.60\end{array}$ & $\begin{array}{l}18.57 \\
18.79 \\
19.02 \\
19.25 \\
19.48 \\
19.71 \\
19.94 \\
29.17 \\
20.40 \\
20.63\end{array}$ & $\begin{array}{l}78.76 \\
79.73 \\
80.71 \\
81.68 \\
82.65 \\
83.62 \\
84.60 \\
85.57 \\
86.54 \\
87.51\end{array}$ & $\begin{array}{l}18.91 \\
19.14 \\
19.38 \\
19.61 \\
19.84 \\
20.08 \\
20.31 \\
20.54 \\
20.78 \\
21.01\end{array}$ & $\begin{array}{l}78.68 \\
79.65 \\
80.62 \\
81.59 \\
82.56 \\
83.54 \\
84.51 \\
85.45 \\
86.45 \\
87.42\end{array}$ & $\begin{array}{l}19.25 \\
19.49 \\
19.73 \\
19.97 \\
20.20 \\
20.44 \\
20.68 \\
20.92 \\
21.15 \\
21.39\end{array}$ & $\begin{array}{l}81 \\
82 \\
83 \\
84 \\
85 \\
81 \\
87 \\
88 \\
89 \\
90\end{array}$ \\
\hline $\begin{array}{r}91 \\
92 \\
93 \\
94 \\
95 \\
963 \\
97 \\
98 \\
99 \\
100\end{array}$ & $\begin{array}{l}89.67 \\
89.64 \\
90.62 \\
91.59 \\
92.57 \\
93.54 \\
94.51 \\
95.49 \\
96.46 \\
97.44\end{array}$ & $\begin{array}{l}20.47 \\
20.70 \\
20.92 \\
21.15 \\
21.37 \\
21.60 \\
21.82 \\
22.05 \\
22.27 \\
22.50\end{array}$ & $\begin{array}{l}89.58 \\
89.55 \\
90.52 \\
91.5: \\
92.47 \\
93.44 \\
94.42 \\
95.39 \\
96.36 \\
97.34\end{array}$ & $\begin{array}{l}20.86 \\
21.09 \\
21.32 \\
21.54 \\
21.77 \\
22.00 \\
22.23 \\
22.46 \\
22.16 \\
22.92\end{array}$ & $\begin{array}{l}88.49 \\
89.46 \\
90.43 \\
91.40 \\
92.38 \\
93.35 \\
94.32 \\
95.20 \\
96.26 \\
97.24\end{array}$ & $\begin{array}{l}21.24 \\
21.48 \\
21.71 \\
21.94 \\
22.18 \\
22.41 \\
22.64 \\
22.89 \\
23.11 \\
23.34\end{array}$ & $\begin{array}{l}85.39 \\
89.36 \\
90.33 \\
91.31 \\
92.28 \\
93.25 \\
94.22 \\
95.19 \\
96.16 \\
97.13\end{array}$ & $\begin{array}{l}21.63 \\
21.87 \\
22.10 \\
22.34 \\
22.58 \\
22.82 \\
23.06 \\
23.29 \\
23.53 \\
23.77\end{array}$ & $\begin{array}{r}91 \\
92 \\
93 \\
94 \\
95 \\
96 \\
97 \\
98 \\
99 \\
100\end{array}$ \\
\hline \&્छ & Dep. & Iat. & Dep. & Lat. & Dep. & Lat. & Dep. & Lat. & $\ddot{B}$ \\
\hline$\frac{n}{a}$ & \multicolumn{2}{|c|}{$77^{\circ}$} & \multicolumn{2}{|c|}{$76: 4^{\circ}$} & \multicolumn{2}{|c|}{$761 / 2^{\circ}$} & \multicolumn{2}{|c|}{$764^{\circ}$} & A \\
\hline
\end{tabular}




\begin{tabular}{|c|c|c|c|c|c|c|c|c|c|}
\hline \multirow{2}{*}{ 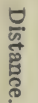 } & \multicolumn{2}{|c|}{$14^{\circ}$. } & \multicolumn{2}{|c|}{$141 / 4^{\circ}$. } & \multicolumn{2}{|c|}{$141 / 2^{\circ}$. } & \multicolumn{2}{|c|}{$1434^{\circ}$} & \multirow{2}{*}{ 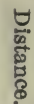 } \\
\hline & Lat. & Dep. & Lat. & Dep. & Lat. & Dep. & Iat. & Dep. & \\
\hline $\begin{array}{r}1 \\
2 \\
3 \\
4 \\
5 \\
6 \\
7 \\
8 \\
9 \\
10\end{array}$ & $\begin{array}{l}0.97 \\
1.94 \\
2.91 \\
3.88 \\
4.85 \\
5.82 \\
6.79 \\
7.76 \\
8.73 \\
9.70\end{array}$ & $\begin{array}{l}0.24 \\
0.48 \\
0.73 \\
0.97 \\
1.21 \\
1.45 \\
1.69 \\
1.94 \\
2.18 \\
2.42\end{array}$ & $\begin{array}{l}0.97 \\
1.94 \\
2.91 \\
3.88 \\
4.85 \\
5.82 \\
6.78 \\
7.75 \\
8.72 \\
9.69\end{array}$ & $\begin{array}{l}0.25 \\
0.49 \\
0.74 \\
0.98 \\
1.23 \\
1.48 \\
1.72 \\
1.97 \\
2.22 \\
2.46\end{array}$ & $\begin{array}{l}0.97 \\
1.94 \\
2.90 \\
3.87 \\
4.84 \\
5.81 \\
6.78 \\
7.75 \\
8.71 \\
9.68\end{array}$ & $\begin{array}{l}0.25 \\
0.50 \\
0.75 \\
1.00 \\
1.25 \\
1.50 \\
1.75 \\
2.00 \\
2.25 \\
2.50\end{array}$ & $\begin{array}{l}0.97 \\
1.93 \\
2.90 \\
3.87 \\
4.81 \\
5.80 \\
6.77 \\
7.74 \\
8.70 \\
9.67\end{array}$ & $\begin{array}{l}0.25 \\
0.51 \\
0.76 \\
1.02 \\
1.27 \\
1.53 \\
1.78 \\
2.04 \\
2.29 \\
2.55\end{array}$ & $\begin{array}{r}1 \\
2 \\
3 \\
4 \\
5 \\
6 \\
7 \\
8 \\
9 \\
10\end{array}$ \\
\hline $\begin{array}{l}11 \\
12 \\
13 \\
14 \\
15 \\
16 \\
17 \\
18 \\
19 \\
20\end{array}$ & $\begin{array}{l}10.67 \\
11.64 \\
12.61 \\
13.58 \\
14.55 \\
15.52 \\
16.50 \\
17.47 \\
18.44 \\
19.41\end{array}$ & $\begin{array}{l}2.66 \\
2.90 \\
3.14 \\
3.39 \\
3.63 \\
3.87 \\
4.11 \\
4.35 \\
4.60 \\
4.84\end{array}$ & $\begin{array}{l}10.66 \\
11.63 \\
12.60 \\
13.57 \\
14.54 \\
15.51 \\
16.48 \\
17.45 \\
18.42 \\
19.38\end{array}$ & $\begin{array}{l}2.71 \\
2.95 \\
3.20 \\
3.45 \\
3.69 \\
3.94 \\
4.18 \\
4.43 \\
4.68 \\
4.92\end{array}$ & $\begin{array}{l}10.65 \\
11.62 \\
12.59 \\
13.55 \\
14.52 \\
15.49 \\
16.46 \\
17.43 \\
18.39 \\
19.36\end{array}$ & $\begin{array}{l}2.75 \\
3.00 \\
3.25 \\
3.51 \\
3.76 \\
4.01 \\
4.26 \\
4.51 \\
4.76 \\
5.01\end{array}$ & $\begin{array}{l}10.64 \\
11.60 \\
12.57 \\
13.54 \\
14.51 \\
15.47 \\
16.44 \\
17.41 \\
18.37 \\
19.34\end{array}$ & $\begin{array}{l}2.80 \\
3.06 \\
3.31 \\
3.56 \\
3.82 \\
4.07 \\
4.33 \\
4.58 \\
4.84 \\
5.09\end{array}$ & $\begin{array}{l}11 \\
12 \\
13 \\
14 \\
15 \\
16 \\
17 \\
18 \\
19 \\
20\end{array}$ \\
\hline $\begin{array}{l}21 \\
22 \\
23 \\
24 \\
25 \\
26 \\
27 \\
28 \\
29 \\
30\end{array}$ & $\begin{array}{l}20.38 \\
21.35 \\
22.32 \\
23.29 \\
24.26 \\
25.23 \\
26.20 \\
27.17 \\
28.14 \\
29.11\end{array}$ & $\begin{array}{l}5.08 \\
5.32 \\
5.56 \\
5.81 \\
6.05 \\
6.29 \\
6.53 \\
6.77 \\
7.02 \\
7.26\end{array}$ & $\begin{array}{l}20.35 \\
21.32 \\
22.29 \\
23.26 \\
24.23 \\
25.20 \\
26.17 \\
27.14 \\
28.11 \\
29.08\end{array}$ & $\begin{array}{l}5.17 \\
5.42 \\
5.66 \\
5.91 \\
6.15 \\
6.40 \\
6.65 \\
6.89 \\
7.14 \\
7.38\end{array}$ & $\begin{array}{l}20.33 \\
21.30 \\
22.27 \\
23.24 \\
24.20 \\
25.17 \\
26.14 \\
27.11 \\
28.08 \\
29.04\end{array}$ & $\begin{array}{l}5.26 \\
5.51 \\
5.76 \\
6.01 \\
6.26 \\
6.51 \\
6.76 \\
7.01 \\
7.26 \\
7.51\end{array}$ & $\begin{array}{l}20.31 \\
21.28 \\
22.24 \\
23.21 \\
24.18 \\
25.14 \\
26.11 \\
27.08 \\
28.04 \\
29.01\end{array}$ & $\begin{array}{l}5.35 \\
5.60 \\
5.86 \\
6.11 \\
6.37 \\
6.62 \\
6.87 \\
7.13 \\
7.38 \\
7.64\end{array}$ & $\begin{array}{l}21 \\
22 \\
23 \\
24 \\
25 \\
26 \\
27 \\
28 \\
29 \\
30\end{array}$ \\
\hline $\begin{array}{l}31 \\
32 \\
33 \\
34 \\
35 \\
36 \\
37 \\
38 \\
39 \\
40\end{array}$ & $\begin{array}{l}30.08 \\
31.05 \\
32.02 \\
32.99 \\
33.96 \\
34.93 \\
35.90 \\
36.87 \\
37.84 \\
38.81\end{array}$ & $\begin{array}{l}7.50 \\
7.74 \\
7.98 \\
8.23 \\
8.47 \\
8.71 \\
8.95 \\
9.19 \\
9.43 \\
9.68\end{array}$ & $\begin{array}{l}30.05 \\
31.02 \\
31.98 \\
32.95 \\
33.92 \\
34.89 \\
35.86 \\
36.83 \\
37.80 \\
38.77\end{array}$ & $\begin{array}{l}7.63 \\
7.88 \\
8.12 \\
8.37 \\
8.62 \\
8.86 \\
9.11 \\
9.35 \\
9.60 \\
9.85\end{array}$ & $\begin{array}{l}30.01 \\
30.98 \\
31.95 \\
32.92 \\
33.89 \\
34.85 \\
35.82 \\
36.79 \\
37.76 \\
38.73\end{array}$ & $\begin{array}{r}7.76 \\
8.01 \\
8.26 \\
8.51 \\
8.76 \\
9.01 \\
9.26 \\
9.51 \\
9.76 \\
10.02\end{array}$ & $\begin{array}{l}29.98 \\
30.95 \\
31.91 \\
32.88 \\
33.85 \\
34.81 \\
35.78 \\
36.75 \\
37.71 \\
38.68\end{array}$ & $\begin{array}{r}7.89 \\
8.15 \\
8.40 \\
8.66 \\
8.91 \\
9.17 \\
9.42 \\
9.67 \\
9.93 \\
10.18\end{array}$ & $\begin{array}{l}31 \\
32 \\
33 \\
34 \\
35 \\
36 \\
37 \\
38 \\
39 \\
40\end{array}$ \\
\hline $\begin{array}{l}41 \\
42 \\
43 \\
44 \\
45 \\
46 \\
47 \\
48 \\
49 \\
50\end{array}$ & $\begin{array}{l}39.78 \\
40.75 \\
41.72 \\
42.69 \\
43.66 \\
44.63 \\
45.60 \\
46.57 \\
47.54 \\
48.51\end{array}$ & $\begin{array}{r}9.92 \\
10.16 \\
10.40 \\
10.64 \\
10.89 \\
11.13 \\
11.37 \\
11.61 \\
11.85 \\
12.10\end{array}$ & $\begin{array}{l}39.74 \\
40.71 \\
41.68 \\
42.65 \\
43.62 \\
44.58 \\
45.55 \\
46.52 \\
47.49 \\
48.46\end{array}$ & $\begin{array}{l}10.09 \\
10.34 \\
10.58 \\
10.83 \\
11.08 \\
11.32 \\
11.57 \\
11.82 \\
12.06 \\
12.31\end{array}$ & $\begin{array}{l}39.69 \\
40.66 \\
41.63 \\
42.60 \\
43.57 \\
44.53 \\
45.50 \\
46.47 \\
47.44 \\
48.41\end{array}$ & $\begin{array}{l}10.27 \\
10.52 \\
10.77 \\
11.02 \\
11.27 \\
11.52 \\
11.77 \\
12.02 \\
12.27 \\
12.52\end{array}$ & $\begin{array}{l}39.65 \\
40.62 \\
41.58 \\
42.55 \\
43.52 \\
44.48 \\
45.45 \\
46.42 \\
47.39 \\
48.35\end{array}$ & $\begin{array}{l}10.44 \\
10.69 \\
10.95 \\
11.20 \\
11.46 \\
11.71 \\
11.97 \\
12.22 \\
12.48 \\
12.73\end{array}$ & $\begin{array}{l}41 \\
42 \\
43 \\
44 \\
45 \\
46 \\
47 \\
48 \\
49 \\
50\end{array}$ \\
\hline छீ & Dep. & Lat. & Dep. & Lat. & Dep. & Lat. & Dep. & Lat. & $8^{\circ}$ \\
\hline$\frac{\vec{n}}{a}$ & \multicolumn{2}{|c|}{$76^{\circ}$. } & \multicolumn{2}{|c|}{$75^{3} \div 4^{\circ}$. } & \multicolumn{2}{|c|}{$751 / 2^{\circ}}$. & \multicolumn{2}{|c|}{$751 / 4^{\circ}$. } & $\frac{m}{a}$ \\
\hline
\end{tabular}


TABLE 4.-TRAVERSE TABI.F.

\begin{tabular}{|c|c|c|c|c|c|c|c|c|c|}
\hline \multirow{2}{*}{ 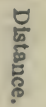 } & \multicolumn{2}{|c|}{$14^{\circ}$. } & \multicolumn{2}{|c|}{$141 / 4^{\circ}$. } & \multicolumn{2}{|c|}{$111 / 2^{\circ}$. } & \multicolumn{2}{|c|}{$144^{2}$. } & \multirow{2}{*}{ 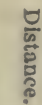 } \\
\hline & Lat. & Dep. & Lat. & Dep. & Lat. & Dep. & Lat. & Dep. & \\
\hline $\begin{array}{l}51 \\
52 \\
53 \\
54 \\
55 \\
56 \\
57 \\
58 \\
59 \\
60\end{array}$ & $\begin{array}{l}49.49 \\
50.46 \\
51.43 \\
52.40 \\
53.37 \\
54.34 \\
55.31 \\
56.28 \\
57.25 \\
58.22\end{array}$ & $\begin{array}{l}12.34 \\
12.58 \\
12.82 \\
13.06 \\
13.31 \\
13.55 \\
13.79 \\
14.03 \\
14.27 \\
14.52\end{array}$ & $\begin{array}{l}49.43 \\
50.40 \\
51.37 \\
52.34 \\
53.31 \\
54.28 \\
55.25 \\
56.22 \\
57.18 \\
58.15\end{array}$ & $\begin{array}{l}12.55 \\
12.80 \\
13.05 \\
13.29 \\
13.54 \\
13.78 \\
14.03 \\
14.28 \\
14.52 \\
14.77\end{array}$ & $\begin{array}{l}49.38 \\
50.34 \\
51.31 \\
52.28 \\
53.25 \\
54.22 \\
55.18 \\
56.15 \\
57.12 \\
58.09\end{array}$ & $\begin{array}{l}12.77 \\
13.02 \\
13.27 \\
13.52 \\
13.77 \\
14.02 \\
14.27 \\
14.52 \\
14.77 \\
15.02\end{array}$ & $\begin{array}{l}49.32 \\
50.29 \\
51.25 \\
52.22 \\
53.19 \\
54.15 \\
55.12 \\
56.09 \\
57.06 \\
53.02\end{array}$ & $\begin{array}{l}12.98 \\
13.24 \\
13.49 \\
13.75 \\
14.00 \\
14.26 \\
14.51 \\
14.77 \\
15.02 \\
15.28\end{array}$ & $\begin{array}{l}51 \\
52 \\
53 \\
54 \\
55 \\
56 \\
57 \\
59 \\
59 \\
60\end{array}$ \\
\hline $\begin{array}{l}61 \\
62 \\
63 \\
64 \\
65 \\
66 \\
67 \\
68 \\
69 \\
70\end{array}$ & $\begin{array}{l}59.19 \\
60.16 \\
61.13 \\
62.10 \\
63.07 \\
64.04 \\
65.01 \\
65.08 \\
66.95 \\
67.92\end{array}$ & $\begin{array}{l}14.76 \\
15.00 \\
15.24 \\
15.48 \\
15.72 \\
15.97 \\
16.21 \\
16.45 \\
16.69 \\
16.93\end{array}$ & $\begin{array}{l}59.12 \\
60.09 \\
61.06 \\
62.03 \\
63.00 \\
63.97 \\
64.94 \\
65.91 \\
66.88 \\
67.85\end{array}$ & $\begin{array}{l}15.02 \\
15.26 \\
15.51 \\
15.75 \\
16.00 \\
16.25 \\
16.49 \\
16.74 \\
16.98 \\
17.23\end{array}$ & $\begin{array}{l}59.06 \\
60.03 \\
60.99 \\
61.96 \\
62.93 \\
63.90 \\
64.87 \\
65.83 \\
66.80 \\
67.77\end{array}$ & $\begin{array}{l}15.27 \\
15.52 \\
15.77 \\
16.02 \\
16.27 \\
16.53 \\
16.78 \\
17.03 \\
17.28 \\
17.53\end{array}$ & $\begin{array}{l}58.99 \\
59.96 \\
60.92 \\
61.89 \\
62.86 \\
63.83 \\
64.79 \\
65.76 \\
66.73 \\
67.69\end{array}$ & $\begin{array}{l}15.53 \\
15.79 \\
16.04 \\
16.29 \\
16.55 \\
16.80 \\
17.06 \\
17.31 \\
17.57 \\
17.82\end{array}$ & $\begin{array}{l}61 \\
62 \\
63 \\
64 \\
65 \\
66 \\
67 \\
68 \\
69 \\
70\end{array}$ \\
\hline $\begin{array}{l}71 \\
72 \\
73 \\
74 \\
75 \\
76 \\
77 \\
78 \\
79 \\
80\end{array}$ & $\begin{array}{l}68.89 \\
69.86 \\
70.83 \\
71.80 \\
72.77 \\
73.74 \\
74.71 \\
75.68 \\
76.65 \\
77.62\end{array}$ & $\begin{array}{l}17.18 \\
17.42 \\
17.66 \\
17.90 \\
18.14 \\
18.39 \\
18.63 \\
18.87 \\
19.11 \\
19.35\end{array}$ & $\begin{array}{l}68.82 \\
69.78 \\
70.75 \\
71.72 \\
72.69 \\
73.66 \\
74.63 \\
75.60 \\
76.57 \\
77.54\end{array}$ & $\begin{array}{l}17.48 \\
17.72 \\
17.97 \\
18.22 \\
18.46 \\
18.71 \\
18.95 \\
19.20 \\
19.45 \\
19.69\end{array}$ & $\begin{array}{l}68.74 \\
69.71 \\
70.67 \\
71.64 \\
72.61 \\
73.58 \\
74.55 \\
75.52 \\
76.44 \\
77.45\end{array}$ & $\begin{array}{l}17.78 \\
18.03 \\
18.28 \\
18.53 \\
18.78 \\
19.03 \\
19.28 \\
19.53 \\
19.78 \\
20.03\end{array}$ & $\begin{array}{l}\text { G8. } 66 \\
69.63 \\
70.59 \\
71.56 \\
72.53 \\
73.50 \\
74.46 \\
75.43 \\
76.40 \\
77.36\end{array}$ & $\begin{array}{l}18.08 \\
18.33 \\
18.59 \\
18.84 \\
19.10 \\
19.35 \\
19.60 \\
19.86 \\
20.11 \\
20.37\end{array}$ & $\begin{array}{l}71 \\
72 \\
73 \\
74 \\
75 \\
76 \\
77 \\
78 \\
79 \\
80\end{array}$ \\
\hline $\begin{array}{l}81 \\
82 \\
83 \\
81 \\
85 \\
86 \\
87 \\
89 \\
89 \\
90\end{array}$ & $\begin{array}{l}78.59 \\
79.56 \\
80.53 \\
81.50 \\
82.48 \\
83.45 \\
81.42 \\
85.39 \\
86.36 \\
87.33\end{array}$ & $\begin{array}{l}19.60 \\
19.84 \\
20.09 \\
20.32 \\
20.56 \\
20.81 \\
21.05 \\
21.29 \\
21.53 \\
21.77\end{array}$ & $\begin{array}{l}78.51 \\
79.48 \\
80.45 \\
81.42 \\
82.38 \\
83.35 \\
84.32 \\
85.29 \\
86.26 \\
87.23\end{array}$ & $\begin{array}{l}19.94 \\
20.18 \\
20.43 \\
20.68 \\
20.92 \\
21.17 \\
21.42 \\
21.66 \\
21.91 \\
22.15\end{array}$ & $\begin{array}{l}78.42 \\
79.39 \\
80.36 \\
81.32 \\
82.29 \\
83.26 \\
81.23 \\
85.20 \\
86.17 \\
87.13\end{array}$ & $\begin{array}{l}20.28 \\
20.53 \\
20.78 \\
21.03 \\
21.28 \\
21.53 \\
21.75 \\
22.03 \\
22.28 \\
22.53\end{array}$ & $\begin{array}{l}73.33 \\
79.30 \\
80.26 \\
81.23 \\
82.20 \\
83.17 \\
84.13 \\
85.10 \\
88.07 \\
87.03\end{array}$ & $\begin{array}{l}20.62 \\
20.85 \\
21.13 \\
21.39 \\
21.64 \\
21.90 \\
22.15 \\
22.40 \\
22.66 \\
22.91\end{array}$ & $\begin{array}{l}81 \\
82 \\
83 \\
84 \\
85 \\
86 \\
87 \\
85 \\
89 \\
90\end{array}$ \\
\hline $\begin{array}{r}91 \\
92 \\
93 \\
94 \\
95 \\
96 \\
97 \\
98 \\
99 \\
100\end{array}$ & $\begin{array}{l}88.30 \\
89.27 \\
90.24 \\
91.21 \\
92.18 \\
93.15 \\
91.12 \\
95.09 \\
96.06 \\
97.03\end{array}$ & $\begin{array}{l}22.01 \\
22.26 \\
22.50 \\
22.74 \\
22.99 \\
23.22 \\
23.47 \\
23.71 \\
23.95 \\
24.19\end{array}$ & $\begin{array}{l}85.20 \\
89.17 \\
90.14 \\
91.11 \\
92.08 \\
93.05 \\
94.02 \\
94.38 \\
95.95 \\
96.92\end{array}$ & $\begin{array}{l}22.40 \\
22.65 \\
22.89 \\
23.14 \\
23.38 \\
23.63 \\
23.88 \\
24.12 \\
24.37 \\
24.62\end{array}$ & $\begin{array}{l}88.10 \\
89.07 \\
90.04 \\
91.01 \\
91.97 \\
92.94 \\
93.91 \\
94.88 \\
95.85 \\
96.81\end{array}$ & $\begin{array}{l}22.78 \\
23.03 \\
23.29 \\
23.54 \\
23.79 \\
24.04 \\
24.29 \\
24.54 \\
24.79 \\
25.04\end{array}$ & $\begin{array}{l}88.10 \\
88.97 \\
89.94 \\
90.90 \\
91.87 \\
92.81 \\
93.80 \\
94.77 \\
95.74 \\
96.70\end{array}$ & $\begin{array}{l}23.17 \\
23.42 \\
23.08 \\
23.93 \\
24.19 \\
21.44 \\
21.70 \\
24.95 \\
25.21 \\
25.46\end{array}$ & $\begin{array}{r}91 \\
92 \\
93 \\
94 \\
95 \\
96 \\
97 \\
98 \\
99 \\
100\end{array}$ \\
\hline 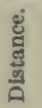 & Dep. & Iat. & Dep. & Iat. & Dep. & Lat. & Dep. & Lat. & 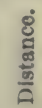 \\
\hline
\end{tabular}




\begin{tabular}{|c|c|c|c|c|c|c|c|c|c|}
\hline \multirow{2}{*}{ 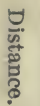 } & \multicolumn{2}{|c|}{$15^{\circ}$. } & \multicolumn{2}{|c|}{$151 / 4^{\circ}$. } & \multicolumn{2}{|c|}{$151 / 2^{\circ}$. } & \multicolumn{2}{|c|}{$15^{3} 4^{\circ}$. } & \multirow{2}{*}{ 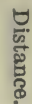 } \\
\hline & Lat. & Dep. & Lat. & Dep. & Lat. & Dep. & Lat. & Dep. & \\
\hline $\begin{array}{r}1 \\
2 \\
3 \\
4 \\
5 \\
6 \\
7 \\
8 \\
9 \\
10\end{array}$ & $\begin{array}{l}0.97 \\
1.93 \\
2.90 \\
3.86 \\
4.83 \\
5.80 \\
6.76 \\
7.73 \\
8.69 \\
9.66\end{array}$ & $\begin{array}{l}0.26 \\
0.52 \\
0.78 \\
1.04 \\
1.29 \\
1.55 \\
1.81 \\
2.07 \\
2.33 \\
2.59\end{array}$ & $\begin{array}{l}0.96 \\
1.93 \\
2.89 \\
3.86 \\
4.82 \\
5.79 \\
6.75 \\
7.72 \\
8.68 \\
9.65\end{array}$ & $\begin{array}{l}0.26 \\
0.53 \\
0.79 \\
1.05 \\
1.32 \\
1.58 \\
1.84 \\
2.10 \\
2.37 \\
2.63\end{array}$ & $\begin{array}{l}0.96 \\
1.93 \\
2.89 \\
3.85 \\
4.82 \\
5.78 \\
6.75 \\
7.71 \\
8.67 \\
9.64\end{array}$ & $\begin{array}{l}0.27 \\
0.53 \\
0.80 \\
1.07 \\
1.34 \\
1.60 \\
1.87 \\
2.14 \\
2.41 \\
2.67\end{array}$ & $\begin{array}{l}0.96 \\
1.92 \\
2.89 \\
3.85 \\
4.81 \\
5.77 \\
6.74 \\
7.70 \\
8.66 \\
9.62\end{array}$ & $\begin{array}{l}0.27 \\
0.54 \\
0.81 \\
1.09 \\
1.36 \\
1.63 \\
1.90 \\
2.17 \\
2.44 \\
2.71\end{array}$ & $\begin{array}{r}1 \\
2 \\
3 \\
4 \\
5 \\
6 \\
7 \\
8 \\
9 \\
10\end{array}$ \\
\hline $\begin{array}{l}11 \\
12 \\
13 \\
14 \\
15 \\
16 \\
17 \\
18 \\
19 \\
20\end{array}$ & $\begin{array}{l}10.63 \\
11.59 \\
12.56 \\
13.52 \\
14.49 \\
15.45 \\
16.42 \\
17.39 \\
18.35 \\
19.32\end{array}$ & $\begin{array}{l}2.85 \\
3.11 \\
3.36 \\
3.62 \\
3.88 \\
4.14 \\
4.40 \\
4.66 \\
4.92 \\
5.18\end{array}$ & $\begin{array}{l}10.61 \\
11.58 \\
12.54 \\
13.51 \\
14.47 \\
15.44 \\
16.40 \\
17.37 \\
18.33 \\
19.30\end{array}$ & $\begin{array}{l}2.89 \\
3.16 \\
3.42 \\
3.68 \\
3.95 \\
4.21 \\
4.47 \\
4.73 \\
5.00 \\
5.26\end{array}$ & $\begin{array}{l}10.60 \\
11.56 \\
12.53 \\
13.49 \\
14.45 \\
15.42 \\
16.38 \\
17.35 \\
18.31 \\
19.27\end{array}$ & $\begin{array}{l}2.94 \\
3.21 \\
3.47 \\
3.74 \\
4.01 \\
4.28 \\
4.54 \\
4.81 \\
5.08 \\
5.34\end{array}$ & $\begin{array}{l}10.59 \\
11.55 \\
12.51 \\
13.47 \\
14.44 \\
15.40 \\
16.36 \\
17.32 \\
18.29 \\
19.25\end{array}$ & $\begin{array}{l}2.99 \\
3.26 \\
3.53 \\
3.80 \\
4.07 . \\
4.34 \\
4.61 \\
4.89 \\
5.16 \\
5.43\end{array}$ & $\begin{array}{l}11 \\
12 \\
13 \\
14 \\
15 \\
16 \\
17 \\
18 \\
19 \\
20\end{array}$ \\
\hline $\begin{array}{l}21 \\
22 \\
23 \\
24 \\
25 \\
26 \\
27 \\
28 \\
29 \\
30\end{array}$ & $\begin{array}{l}20.28 \\
21.25 \\
22.22 \\
23.18 \\
24.15 \\
25.11 \\
26.08 \\
27.05 \\
28.01 \\
28.98\end{array}$ & $\begin{array}{l}5.44 \\
5.69 \\
5.95 \\
6.21 \\
6.47 \\
6.73 \\
6.99 \\
7.25 \\
7.51 \\
7.76\end{array}$ & $\begin{array}{l}20.26 \\
21.23 \\
22.19 \\
23.15 \\
24.12 \\
25.08 \\
26.05 \\
27.01 \\
27.98 \\
28.94\end{array}$ & $\begin{array}{l}5.52 \\
5.79 \\
6.05 \\
6.31 \\
6.58 \\
6.84 \\
7.10 \\
7.36 \\
7.63 \\
7.89\end{array}$ & $\begin{array}{l}20.24 \\
21.20 \\
22.16 \\
23.13 \\
24.09 \\
25.05 \\
26.02 \\
26.98 \\
27.95 \\
25.91\end{array}$ & $\begin{array}{l}5.61 \\
5.88 \\
6.15 \\
6.41 \\
6.68 \\
6.95 \\
7.22 \\
7.48 \\
7.75 \\
8.02\end{array}$ & $\begin{array}{l}20.21 \\
21.17 \\
22.14 \\
23.10 \\
24.06 \\
25.02 \\
25.99 \\
26.95 \\
27.91 \\
28.87\end{array}$ & $\begin{array}{l}5.70 \\
5.97 \\
6.24 \\
6.51 \\
6.79 \\
7.06 \\
7.33 \\
7.60 \\
7.87 \\
8.14\end{array}$ & $\begin{array}{l}21 \\
22 \\
23 \\
24 \\
25 \\
26 \\
27 \\
28 \\
29 \\
30\end{array}$ \\
\hline $\begin{array}{l}31 \\
32 \\
33 \\
34 \\
35 \\
36 \\
37 \\
38 \\
39 \\
40\end{array}$ & $\begin{array}{l}29.94 \\
30.91 \\
31.88 \\
32.84 \\
33.81 \\
34.77 \\
35.74 \\
36.71 \\
37.67 \\
38.64\end{array}$ & $\begin{array}{r}8.02 \\
8.28 \\
8.54 \\
8.80 \\
9.06 \\
9.32 \\
9.58 \\
9.84 \\
10.09 \\
10.35\end{array}$ & $\begin{array}{l}29.91 \\
30.87 \\
31.84 \\
32.80 \\
33.77 \\
34.73 \\
35.70 \\
36.66 \\
37.63 \\
38.59\end{array}$ & $\begin{array}{r}8.15 \\
8.42 \\
8.68 \\
8.94 \\
9.21 \\
9.47 \\
9.73 \\
10.00 \\
10.26 \\
10.52\end{array}$ & $\begin{array}{l}29.87 \\
30.84 \\
31.80 \\
32.76 \\
33.73 \\
34.69 \\
35.65 \\
36.62 \\
37.58 \\
38.55\end{array}$ & $\begin{array}{r}8.28 \\
8.55 \\
8.82 \\
9.09 \\
9.35 \\
9.62 \\
9.89 \\
10.16 \\
10.42 \\
10.69\end{array}$ & $\begin{array}{l}29.84 \\
30.80 \\
31.76 \\
32.72 \\
33.69 \\
34.65 \\
35.61 \\
36.57 \\
37.54 \\
38.50\end{array}$ & $\begin{array}{r}8.41 \\
8.69 \\
8.96 \\
9.23 \\
9.50 \\
9.77 \\
10.04 \\
10.31 \\
10.59 \\
10.86\end{array}$ & $\begin{array}{l}31 \\
32 \\
33 \\
34 \\
35 \\
36 \\
37 \\
38 \\
39 \\
40\end{array}$ \\
\hline $\begin{array}{l}41 \\
42 \\
43 \\
44 \\
45 \\
46 \\
47 \\
48 \\
49 \\
50\end{array}$ & $\begin{array}{l}39.60 \\
40.57 \\
41.53 \\
42.50 \\
43.47 \\
41.43 \\
45.40 \\
46.36 \\
47.33 \\
48.30\end{array}$ & $\begin{array}{r}10.61 \\
10.87 \\
11.13 \\
11.39 \\
11.65 \\
11.91 \\
12.16 \\
12.42 \\
12.68 \\
12.94\end{array}$ & $\begin{array}{l}39.56 \\
40.52 \\
41.49 \\
42.45 \\
43.42 \\
44.38 \\
45.35 \\
46.31 \\
47.27 \\
48.24\end{array}$ & $\begin{array}{l}10.78 \\
11.05 \\
11.31 \\
11.57 \\
11.84 \\
12.10 \\
12.36 \\
12.63 \\
12.89 \\
13.15\end{array}$ & $\begin{array}{l}39.51 \\
40.47 \\
41.44 \\
42.40 \\
43.36 \\
44.33 \\
45.29 \\
46.25 \\
47.22 \\
48.18\end{array}$ & $\begin{array}{l}10.96 \\
11.22 \\
11.49 \\
11.70 \\
12.03 \\
12.29 \\
12.56 \\
12.83 \\
13.09 \\
13.36\end{array}$ & $\begin{array}{l}39.46 \\
40.42 \\
41.39 \\
42.35 \\
43.31 \\
44.27 \\
45.24 \\
46.20 \\
47.16 \\
48.12\end{array}$ & $\begin{array}{l}11.13 \\
11.40 \\
11.67 \\
11.94 \\
12.21 \\
12.49 \\
12.76 \\
13.03 \\
13.30 \\
13.57\end{array}$ & $\begin{array}{l}41 \\
42 \\
43 \\
44 \\
45 \\
46 \\
47 \\
48 \\
49 \\
50\end{array}$ \\
\hline 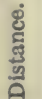 & Dep. & Lat. & Dep. & Lat. & Dep. & Lat. & Dep. & Iat. & 8 \\
\hline
\end{tabular}


TABIE 4.-TRA VERSE TABLE.

\begin{tabular}{|c|c|c|c|c|c|c|c|c|c|}
\hline \multirow{2}{*}{ 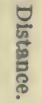 } & \multicolumn{2}{|c|}{$15^{\circ}$. } & \multicolumn{2}{|c|}{$151 / 4^{\circ}$. } & \multicolumn{2}{|c|}{$151 / 2^{\circ}$. } & \multicolumn{2}{|c|}{$15^{3} 4^{\circ}$. } & \multirow{2}{*}{ 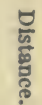 } \\
\hline & Lat. & Dep. & Lat. & Dep. & lat. & Dep. & Lat. & Dep. & \\
\hline $\begin{array}{l}51 \\
52 \\
53 \\
54 \\
55 \\
56 \\
57 \\
58 \\
59 \\
60\end{array}$ & $\begin{array}{l}49.26 \\
50.23 \\
51.19 \\
52.16 \\
53.13 \\
54.09 \\
55.06 \\
56.02 \\
56.99 \\
57.96\end{array}$ & $\begin{array}{l}13.20 \\
13.46 \\
13.72 \\
13.98 \\
14.24 \\
14.49 \\
14.75 \\
15.01 \\
15.27 \\
15.53\end{array}$ & $\begin{array}{l}49.20 \\
50.17 \\
51.13 \\
52.10 \\
53.06 \\
54.03 \\
54.99 \\
55.96 \\
56.92 \\
57.89\end{array}$ & $\begin{array}{l}13.41 \\
13.68 \\
13.94 \\
14.20 \\
14.47 \\
14.73 \\
14.99 \\
15.26 \\
15.52 \\
15.78\end{array}$ & $\begin{array}{l}49.15 \\
50.11 \\
51.07 \\
52.04 \\
53.00 \\
53.96 \\
54.93 \\
55.89 \\
56.85 \\
57.82\end{array}$ & $\begin{array}{l}13.63 \\
13.90 \\
14.16 \\
14.43 \\
14.70 \\
14.97 \\
15.23 \\
15.50 \\
15.77 \\
16.03\end{array}$ & $\begin{array}{l}49.09 \\
50.05 \\
51.01 \\
51.97 \\
52.94 \\
53.90 \\
54.86 \\
55.82 \\
56.78 \\
57.75\end{array}$ & $\begin{array}{l}13.84 \\
14.11 \\
14.39 \\
14.66 \\
14.93 \\
15.20 \\
15.47 \\
15.74 \\
16.01 \\
16.29\end{array}$ & $\begin{array}{l}51 \\
52 \\
53 \\
54 \\
55 \\
56 \\
57 \\
59 \\
59 \\
60\end{array}$ \\
\hline $\begin{array}{l}61 \\
62 \\
63 \\
64 \\
65 \\
66 \\
67 \\
68 \\
69 \\
70\end{array}$ & $\begin{array}{l}58.92 \\
59.89 \\
60.85 \\
61.82 \\
62.79 \\
63.75 \\
64.72 \\
65.68 \\
66.65 \\
67.61\end{array}$ & $\begin{array}{l}15.79 \\
16.05 \\
16.31 \\
16.56 \\
16.82 \\
17.08 \\
17.34 \\
17.60 \\
17.86 \\
18.12\end{array}$ & $\begin{array}{l}58.85 \\
59.82 \\
60.78 \\
61.75 \\
62.71 \\
63.68 \\
64.64 \\
65.61 \\
6657 \\
67.54\end{array}$ & $\begin{array}{l}16.04 \\
16.31 \\
16.57 \\
16.83 \\
17.10 \\
17.36 \\
17.62 \\
17.89 \\
18.15 \\
18.41\end{array}$ & $\begin{array}{l}58.78 \\
59.75 \\
60.71 \\
61.67 \\
62.64 \\
63.60 \\
64.56 \\
65.53 \\
66.49 \\
67.45\end{array}$ & $\begin{array}{l}16.30 \\
16.57 \\
16.84 \\
17.10 \\
17.37 \\
17.64 \\
17.90 \\
18.17 \\
18.44 \\
18.71\end{array}$ & $\begin{array}{l}58.71 \\
59.67 \\
60.63 \\
61.60 \\
62.56 \\
63.52 \\
64.48 \\
65.45 \\
66.41 \\
67.37\end{array}$ & $\begin{array}{l}16.56 \\
16.83 \\
17.10 \\
17.37 \\
17.64 \\
17.92 \\
18.19 \\
18.46 \\
18.73 \\
19.00\end{array}$ & $\begin{array}{l}61 \\
62 \\
63 \\
64 \\
65 \\
66 \\
67 \\
68 \\
69 \\
70\end{array}$ \\
\hline $\begin{array}{l}71 \\
72 \\
73 \\
74 \\
75 \\
76 \\
77 \\
78 \\
79 \\
80\end{array}$ & $\begin{array}{l}68.58 \\
69.55 \\
70.51 \\
71.48 \\
72.44 \\
73.41 \\
74.39 \\
75.34 \\
76.31 \\
77.27\end{array}$ & $\begin{array}{l}18.38 \\
18.63 \\
18.89 \\
19.15 \\
19.41 \\
19.67 \\
19.93 \\
20.19 \\
20.45 \\
20.71\end{array}$ & $\begin{array}{l}68.50 \\
69.46 \\
70.43 \\
71.39 \\
72.36 \\
73.32 \\
74.29 \\
75.25 \\
76.22 \\
77.18\end{array}$ & $\begin{array}{l}18.68 \\
18.94 \\
19.20 \\
19.46 \\
19.73 \\
19.99 \\
20.25 \\
20.52 \\
20.78 \\
21.04\end{array}$ & $\begin{array}{l}68.42 \\
69.38 \\
70.35 \\
71.31 \\
72.27 \\
73.24 \\
74.20 \\
75.16 \\
76.13 \\
77.09\end{array}$ & $\begin{array}{l}18.97 \\
19.24 \\
19.51 \\
19.78 \\
20.04 \\
20.31 \\
20.58 \\
20.84 \\
21.11 \\
21.38\end{array}$ & $\begin{array}{l}68.33 \\
69.30 \\
70.26 \\
71.22 \\
72.18 \\
73.15 \\
74.11 \\
75.07 \\
76.03 \\
77.00\end{array}$ & $\begin{array}{l}19.27 \\
19.54 \\
19.82 \\
20.09 \\
20.36 \\
20.63 \\
20.90 \\
21.17 \\
21.44 \\
21.72\end{array}$ & $\begin{array}{l}71 \\
72 \\
73 \\
74 \\
75 \\
76 \\
77 \\
78 \\
79 \\
80\end{array}$ \\
\hline $\begin{array}{l}81 \\
82 \\
83 \\
84 \\
85 \\
86 \\
87 \\
88 \\
89 \\
00\end{array}$ & $\begin{array}{l}78.24 \\
79.21 \\
80.17 \\
81.14 \\
82.10 \\
83.07 \\
84.04 \\
85.00 \\
85.97 \\
86.93\end{array}$ & $\begin{array}{l}20.96 \\
21.22 \\
21.48 \\
21.74 \\
22.00 \\
22.26 \\
22.54 \\
22.78 \\
23.03 \\
23.29\end{array}$ & $\begin{array}{l}78.15 \\
79.11 \\
80.08 \\
81.04 \\
82.01 \\
82.97 \\
83.94 \\
84.90 \\
85.87 \\
86.83\end{array}$ & $\begin{array}{l}21.31 \\
21.57 \\
21.83 \\
22.09 \\
22.36 \\
22.62 \\
22.89 \\
23.15 \\
23.41 \\
23.67\end{array}$ & $\begin{array}{l}78.05 \\
79.02 \\
79.98 \\
80.94 \\
81.91 \\
82.87 \\
83.84 \\
84.80 \\
85.76 \\
86.73\end{array}$ & $\begin{array}{l}21.65 \\
21.91 \\
22.18 \\
22.45 \\
22.72 \\
22.98 \\
23.25 \\
23.52 \\
23.78 \\
24.05\end{array}$ & $\begin{array}{l}77.96 \\
78.92 \\
79.88 \\
80.85 \\
81.81 \\
82.77 \\
83.73 \\
84.70 \\
85.66 \\
86.62\end{array}$ & $\begin{array}{l}21.99 \\
22.26 \\
22.53 \\
22.80 \\
23.07 \\
23.34 \\
23.62 \\
23.89 \\
24.16 \\
24.43\end{array}$ & $\begin{array}{l}81 \\
82 \\
83 \\
84 \\
85 \\
86 \\
87 \\
88 \\
89 \\
90\end{array}$ \\
\hline $\begin{array}{r}91 \\
92 \\
93 \\
91 \\
8.5 \\
96 \\
97 \\
9.9 \\
99 \\
100\end{array}$ & $\begin{array}{l}87.90 \\
85.87 \\
89.83 \\
90.80 \\
91.76 \\
92.73 \\
93.69 \\
91.66 \\
95.63 \\
96.59\end{array}$ & $\begin{array}{l}23.55 \\
23.81 \\
24.07 \\
24.33 \\
24.59 \\
24.85 \\
25.11 \\
25.36 \\
25.62 \\
25.88\end{array}$ & $\begin{array}{l}87.80 \\
88.76 \\
83.73 \\
90.69 \\
91.65 \\
92.62 \\
93.58 \\
94.55 \\
95.51 \\
96.48\end{array}$ & $\begin{array}{l}23.94 \\
24.20 \\
24.46 \\
24.72 \\
24.99 \\
25.25 \\
25.51 \\
25.78 \\
26.04 \\
26.30\end{array}$ & $\begin{array}{l}87.69 \\
88.65 \\
89.62 \\
90.58 \\
91.54 \\
92.51 \\
93.47 \\
94.44 \\
95.40 \\
96.36\end{array}$ & $\begin{array}{l}24.32 \\
24.59 \\
24.85 \\
25.12 \\
25.39 \\
25.65 \\
25.92 \\
26.19 \\
26.16 \\
26.72\end{array}$ & $\begin{array}{r}87.58 \\
88.55 \\
89.51 \\
90.47 \\
91.43 \\
92.40 \\
93.36 \\
94.32 \\
9.7 .24 \\
96.25\end{array}$ & $\begin{array}{l}24.70 \\
24.97 \\
25.24 \\
25.52 \\
25.79 \\
26.06 \\
26.33 \\
26.60 \\
26.87 \\
27.14\end{array}$ & $\begin{array}{r}91 \\
92 \\
93 \\
94 \\
95 \\
96 \\
97 \\
98 \\
99 \\
100\end{array}$ \\
\hline ஓृं & Dep. & Lat. & Dep. & Lat. & Dep. & Lat. & Dep. & Lat. & छू \\
\hline$\frac{m}{a}$ & \multicolumn{2}{|c|}{$75^{\circ}$} & \multicolumn{2}{|c|}{$74^{2} 4^{\circ}}$. & \multicolumn{2}{|c|}{$711 / 2^{\circ}}$. & \multicolumn{2}{|c|}{$744^{\circ}}$. & \\
\hline
\end{tabular}


TABLE 4.-TRAVERSE TABLE.

\begin{tabular}{|c|c|c|c|c|c|c|c|c|c|}
\hline \multirow{2}{*}{ 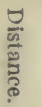 } & \multicolumn{2}{|c|}{$16^{\circ}$. } & \multicolumn{2}{|c|}{$161 / 4^{\circ}$. } & \multicolumn{2}{|c|}{$161 / 2^{\circ}$. } & \multicolumn{2}{|c|}{$16^{3} 4^{\circ}$. } & \multirow{2}{*}{ 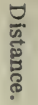 } \\
\hline & Lat. & Dep. & Lat. & Dep. & Lat. & Dep. & Lat. & Dep. & \\
\hline $\begin{array}{r}1 \\
2 \\
3 \\
4 \\
5 \\
6 \\
7 \\
8 \\
9 \\
10\end{array}$ & $\begin{array}{l}0.96 \\
1.92 \\
2.88 \\
3.85 \\
4.81 \\
5.77 \\
6.73 \\
7.69 \\
8.65 \\
9.61\end{array}$ & $\begin{array}{l}0.28 \\
0.55 \\
0.83 \\
1.10 \\
1.38 \\
1.65 \\
1.93 \\
2.21 \\
2.48 \\
2.76\end{array}$ & $\begin{array}{l}0.96 \\
1.92 \\
2.88 \\
3.84 \\
4.80 \\
5.76 \\
6.72 \\
7.68 \\
8.64 \\
9.60\end{array}$ & $\begin{array}{l}0.28 \\
0.56 \\
0.84 \\
1.12 \\
1.40 \\
1.68 \\
1.96 \\
2.24 \\
2.52 \\
2.80\end{array}$ & $\begin{array}{l}0.96 \\
1.92 \\
2.88 \\
3.84 \\
4.79 \\
5.75 \\
6.71 \\
7.67 \\
8.63 \\
9.59\end{array}$ & $\begin{array}{l}0.28 \\
0.57 \\
0.85 \\
1.14 \\
1.42 \\
1.70 \\
1.99 \\
2.27 \\
2.56 \\
2.84\end{array}$ & $\begin{array}{l}0.96 \\
1.92 \\
2.87 \\
3.83 \\
4.79 \\
5.75 \\
6.70 \\
7.66 \\
8.62 \\
9.58\end{array}$ & $\begin{array}{l}0.29 \\
0.58 \\
0.86 \\
1.15 \\
1.44 \\
1.73 \\
2.02 \\
2.31 \\
2.59 \\
2.88\end{array}$ & $\begin{array}{r}1 \\
2 \\
3 \\
4 \\
5 \\
6 \\
7 \\
8 \\
9 \\
10\end{array}$ \\
\hline $\begin{array}{l}11 \\
12 \\
13 \\
14 \\
15 \\
16 \\
17 \\
18 \\
19 \\
20\end{array}$ & $\begin{array}{l}10.57 \\
11.54 \\
12.50 \\
13.46 \\
14.42 \\
15.38 \\
16.34 \\
17.30 \\
18.26 \\
19.23\end{array}$ & $\begin{array}{l}3.03 \\
3.31 \\
3.58 \\
3.86 \\
4.13 \\
4.41 \\
4.69 \\
4.96 \\
5.24 \\
5.51\end{array}$ & $\begin{array}{l}10.56 \\
11.52 \\
12.48 \\
13.44 \\
14.40 \\
15.36 \\
16.32 \\
17.28 \\
18.24 \\
19.20\end{array}$ & $\begin{array}{l}3.08 \\
3.36 \\
3.64 \\
3.92 \\
4.20 \\
4.48 \\
4.76 \\
5.04 \\
5.32 \\
5.60\end{array}$ & $\begin{array}{l}10.55 \\
11.51 \\
12.46 \\
13.42 \\
14.38 \\
15.34 \\
16.30 \\
17.26 \\
18.22 \\
19.18\end{array}$ & $\begin{array}{l}3.12 \\
3.41 \\
3.69 \\
3.98 \\
4.26 \\
4.54 \\
4.83 \\
5.11 \\
5.40 \\
5.68\end{array}$ & $\begin{array}{l}10.53 \\
11.49 \\
12.45 \\
13.41 \\
14.36 \\
15.32 \\
16.28 \\
17.24 \\
18.19 \\
19.15\end{array}$ & $\begin{array}{l}3.17 \\
3.46 \\
3.75 \\
4.03 \\
4.32 \\
4.61 \\
4.90 \\
5.19 \\
5.48 \\
5.76\end{array}$ & $\begin{array}{l}11 \\
12 \\
13 \\
14 \\
15 \\
16 \\
17 \\
18 \\
19 \\
20\end{array}$ \\
\hline $\begin{array}{l}21 \\
22 \\
23 \\
24 \\
25 \\
26 \\
27 \\
28 \\
29 \\
30\end{array}$ & $\begin{array}{l}20.19 \\
21.15 \\
22.11 \\
23.07 \\
24.03 \\
24.99 \\
25.95 \\
26.92 \\
27.89 \\
28.84\end{array}$ & $\begin{array}{l}5.79 \\
6.06 \\
6.34 \\
6.62 \\
6.89 \\
7.17 \\
7.44 \\
7.72 \\
7.99 \\
8.27\end{array}$ & $\begin{array}{l}20.16 \\
21.12 \\
22.08 \\
23.04 \\
24.00 \\
24.96 \\
25.92 \\
26.83 \\
27.84 \\
28.80\end{array}$ & $\begin{array}{l}5.88 \\
6.16 \\
6.44 \\
6.72 \\
7.00 \\
7.29 \\
7.56 \\
7.84 \\
8.12 \\
8.39\end{array}$ & $\begin{array}{l}20.14 \\
21.09 \\
22.05 \\
23.01 \\
23.97 \\
24.93 \\
25.89 \\
26.85 \\
27.81 \\
28.76\end{array}$ & $\begin{array}{l}5.96 \\
6.25 \\
6.53 \\
6.82 \\
7.10 \\
7.38 \\
7.67 \\
7.95 \\
8.24 \\
8.52\end{array}$ & $\begin{array}{l}20.11 \\
21.07 \\
22.02 \\
22.98 \\
23.94 \\
24.90 \\
25.85 \\
26.81 \\
27.77 \\
28.73\end{array}$ & $\begin{array}{l}6.05 \\
6.34 \\
6.63 \\
6.92 \\
7.20 \\
7.49 \\
7.78 \\
8.07 \\
8.36 \\
8.65\end{array}$ & $\begin{array}{l}21 \\
22 \\
23 \\
24 \\
25 \\
26 \\
27 \\
28 \\
29 \\
30\end{array}$ \\
\hline $\begin{array}{l}31 \\
32 \\
33 \\
34 \\
35 \\
36 \\
37 \\
38 \\
39 \\
40\end{array}$ & $\begin{array}{l}29.80 \\
30.76 \\
31.72 \\
32.68 \\
33.64 \\
34.61 \\
35.57 \\
36.53 \\
37.49 \\
38.45\end{array}$ & $\begin{array}{r}8.54 \\
8.82 \\
9.10 \\
9.37 \\
9.65 \\
9.92 \\
10.20 \\
10.47 \\
10.75 \\
11.03\end{array}$ & $\begin{array}{l}29.76 \\
30.72 \\
31.68 \\
32.64 \\
33.60 \\
34.56 \\
35.52 \\
36.48 \\
37.44 \\
38.40\end{array}$ & $\begin{array}{r}8.67 \\
8.95 \\
9.23 \\
9.51 \\
9.79 \\
10.07 \\
10.35 \\
10.63 \\
10.91 \\
11.19\end{array}$ & $\begin{array}{r}29.72 \\
30.68 \\
31.64 \\
32.60 \\
33.56 \\
34.52 \\
35.48 \\
36.44 \\
37.39 \\
38.35\end{array}$ & $\begin{array}{r}8.80 \\
9.09 \\
9.37 \\
9.66 \\
9.94 \\
10.22 \\
10.51 \\
10.79 \\
11.08 \\
11.36\end{array}$ & $\begin{array}{l}29.68 \\
30.64 \\
31.60 \\
32.56 \\
33.51 \\
34.47 \\
35.43 \\
36.39 \\
37.35 \\
38.30\end{array}$ & $\begin{array}{r}8.93 \\
9.22 \\
9.51 \\
9.80 \\
10.09 \\
10.38 \\
10.66 \\
10.95 \\
11.24 \\
11.53\end{array}$ & $\begin{array}{l}31 \\
32 \\
33 \\
34 \\
35 \\
36 \\
37 \\
39 \\
39 \\
40\end{array}$ \\
\hline $\begin{array}{l}41 \\
42 \\
43 \\
44 \\
45 \\
46 \\
47 \\
49 \\
49 \\
50\end{array}$ & $\begin{array}{l}39.41 \\
40.37 \\
41.33 \\
42.30 \\
43.26 \\
44.22 \\
45.18 \\
46.14 \\
47.10 \\
48.06\end{array}$ & $\begin{array}{l}11.30 \\
11.58 \\
11.85 \\
12.13 \\
12.40 \\
12.68 \\
12.95 \\
13.23 \\
13.51 \\
13.78\end{array}$ & $\begin{array}{l}39.36 \\
40.32 \\
41.28 \\
42.24 \\
43.21 \\
44.16 \\
45.12 \\
46.08 \\
47.04 \\
48.00\end{array}$ & $\begin{array}{l}11.47 \\
11.75 \\
12.03 \\
12.31 \\
12.59 \\
12.87 \\
13.15 \\
13.43 \\
13.71 \\
13.99\end{array}$ & $\begin{array}{l}39.31 \\
40.27 \\
41.23 \\
42.19 \\
43.15 \\
44.11 \\
45.06 \\
46.02 \\
46.98 \\
47.94\end{array}$ & $\begin{array}{l}11.64 \\
11.93 \\
12.21 \\
12.50 \\
12.78 \\
13.06 \\
13.35 \\
13.63 \\
13.92 \\
14.20\end{array}$ & $\begin{array}{l}39.26 \\
40.22 \\
41.18 \\
42.13 \\
43.09 \\
44.05 \\
45.01 \\
45.96 \\
46.92 \\
47.88\end{array}$ & $\begin{array}{l}11.82 \\
12.10 \\
12.39 \\
12.69 \\
12.97 \\
13.26 \\
13.55 \\
13.83 \\
14.12 \\
14.41\end{array}$ & $\begin{array}{l}41 \\
42 \\
43 \\
44 \\
45 \\
46 \\
47 \\
48 \\
49 \\
50\end{array}$ \\
\hline \multirow{2}{*}{$\begin{array}{l}\dot{8} \\
\text { : } \\
\frac{5}{5} \\
\frac{0}{0}\end{array}$} & Drp. & Lat. & Dep. & Lat. & Dep. & Lat. & Dep. & Lat. & \multirow{2}{*}{ 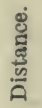 } \\
\hline & \multicolumn{2}{|c|}{$74^{\circ}$. } & \multicolumn{2}{|c|}{$7334^{\circ}$. } & \multicolumn{2}{|c|}{$731 / 2^{\circ}$. } & \multicolumn{2}{|c|}{$731 / 4^{\circ}$. } & \\
\hline
\end{tabular}


TABLE 4.-TRAVERSE TABLE.

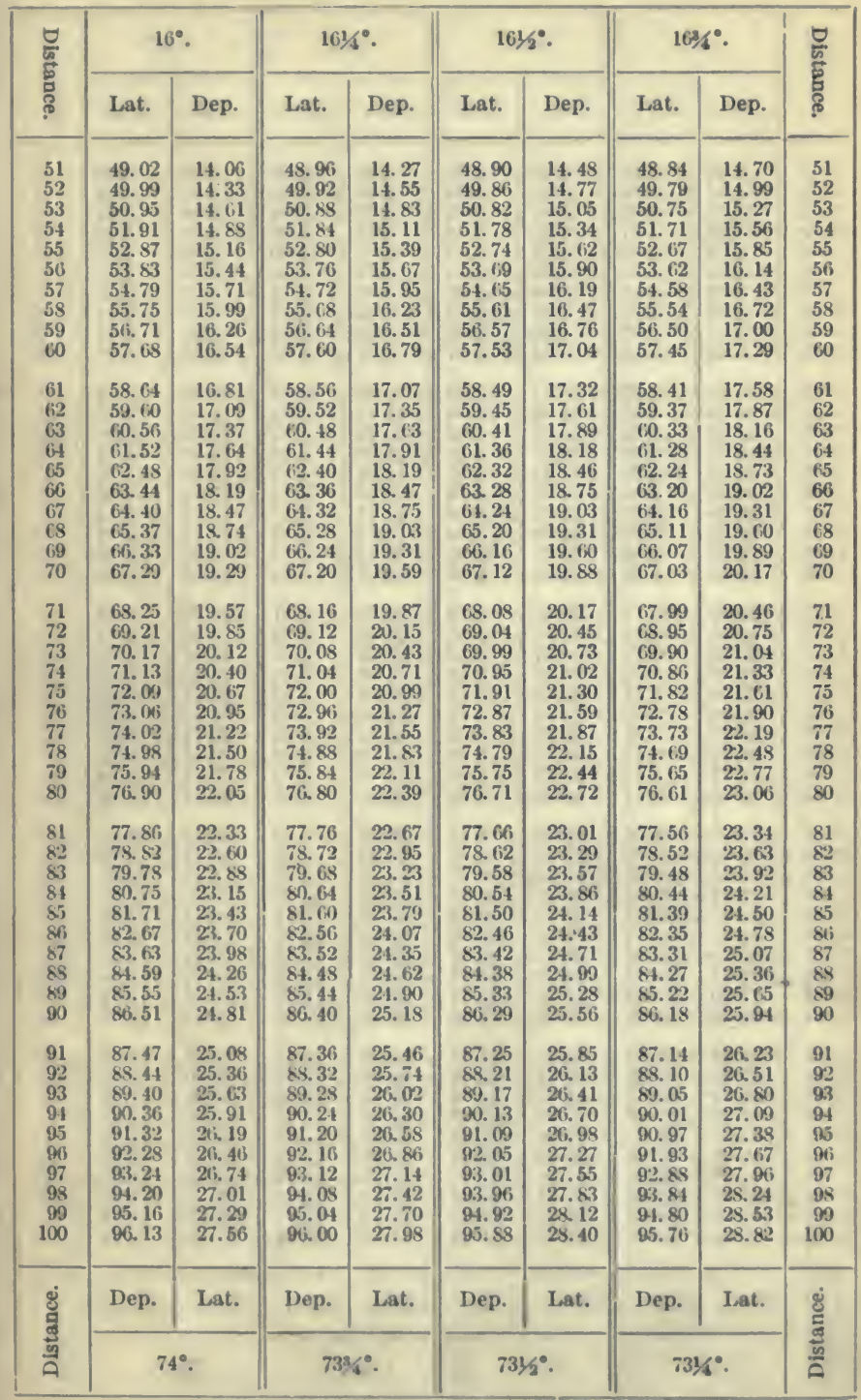


TABLE 4.-TRAVERSE TABLE.

\begin{tabular}{|c|c|c|c|c|c|c|c|c|c|}
\hline \multirow{2}{*}{$\frac{\emptyset}{\frac{\sigma}{n}}$} & \multicolumn{2}{|c|}{$17^{\circ}}$. & \multicolumn{2}{|c|}{$1714^{\circ}$. } & \multicolumn{2}{|c|}{$171 / 2^{\circ}$. } & \multicolumn{2}{|c|}{$1734^{\circ}$. } & \multirow{2}{*}{ 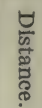 } \\
\hline & Lat. & Dep. & Lat. & Dep. & Lat. & Dep. & Lat. & Dep. & \\
\hline $\begin{array}{r}1 \\
2 \\
3 \\
4 \\
5 \\
6 \\
7 \\
8 \\
9 \\
10\end{array}$ & $\begin{array}{l}0.96 \\
1.91 \\
2.87 \\
3.83 \\
4.78 \\
5.74 \\
6.69 \\
7.65 \\
8.61 \\
9.56\end{array}$ & $\begin{array}{l}0.29 \\
0.58 \\
0.88 \\
1.17 \\
1.46 \\
1.75 \\
2.05 \\
2.34 \\
2.63 \\
2.92\end{array}$ & $\begin{array}{l}0.96 \\
1.91 \\
2.87 \\
3.82 \\
4.78 \\
5.73 \\
6.69 \\
7.64 \\
8.60 \\
9.55\end{array}$ & $\begin{array}{l}0.30 \\
0.59 \\
0.89 \\
1.19 \\
1.43 \\
1.78 \\
2.08 \\
2.37 \\
2.67 \\
2.97\end{array}$ & $\begin{array}{l}0.95 \\
1.91 \\
2.86 \\
3.81 \\
4.77 \\
5.72 \\
6.68 \\
7.63 \\
8.58 \\
9.54\end{array}$ & $\begin{array}{l}0.30 \\
0.60 \\
0.90 \\
1.20 \\
1.50 \\
1.80 \\
2.10 \\
2.41 \\
2.71 \\
3.01\end{array}$ & $\begin{array}{l}0.95 \\
1.90 \\
2.86 \\
3.81 \\
4.76 \\
5.71 \\
6.67 \\
7.62 \\
8.57 \\
9.52\end{array}$ & $\begin{array}{l}0.30 \\
0.61 \\
0.91 \\
1.22 \\
1.52 \\
1.83 \\
2.13 \\
2.44 \\
2.74 \\
3.05\end{array}$ & $\begin{array}{r}1 \\
2 \\
3 \\
4 \\
5 \\
6 \\
7 \\
8 \\
9 \\
10\end{array}$ \\
\hline $\begin{array}{l}11 \\
12 \\
13 \\
14 \\
15 \\
16 \\
17 \\
18 \\
19 \\
20\end{array}$ & $\begin{array}{l}10.52 \\
11.48 \\
12.43 \\
13.39 \\
14.34 \\
15.30 \\
16.26 \\
17.21 \\
18.17 \\
19.13\end{array}$ & $\begin{array}{l}3.22 \\
3.51 \\
3.80 \\
4.09 \\
4.39 \\
4.68 \\
4.97 \\
5.26 \\
5.56 \\
5.85\end{array}$ & $\begin{array}{l}10.51 \\
11.46 \\
12.42 \\
13.37 \\
14.33 \\
15.28 \\
16.24 \\
17.19 \\
18.15 \\
19.10\end{array}$ & $\begin{array}{l}3.26 \\
3.56 \\
3.86 \\
4.15 \\
4.45 \\
4.74 \\
5.04 \\
5.34 \\
5.63 \\
5.93\end{array}$ & $\begin{array}{l}10.49 \\
11.44 \\
12.40 \\
13.35 \\
14.31 \\
15.26 \\
16.21 \\
17.17 \\
18.12 \\
19.07\end{array}$ & $\begin{array}{l}3.31 \\
3.61 \\
3.91 \\
4.21 \\
4.51 \\
4.81 \\
5.11 \\
5.41 \\
5.71 \\
6.01\end{array}$ & $\begin{array}{l}10.48 \\
11.43 \\
12.38 \\
13.33 \\
14.29 \\
15.24 \\
16.19 \\
17.14 \\
18.10 \\
19.05\end{array}$ & $\begin{array}{l}3.35 \\
3.66 \\
3.96 \\
4.27 \\
4.57 \\
4.88 \\
5.18 \\
5.49 \\
5.79 \\
6.10\end{array}$ & $\begin{array}{l}11 \\
12 \\
13 \\
14 \\
15 \\
16 \\
17 \\
18 \\
19 \\
20\end{array}$ \\
\hline $\begin{array}{l}21 \\
22 \\
23 \\
24 \\
25 \\
26 \\
27 \\
28 \\
29 \\
30\end{array}$ & $\begin{array}{l}20.08 \\
21.04 \\
22.00 \\
22.95 \\
23.91 \\
24.86 \\
25.82 \\
26.78 \\
27.73 \\
28.69\end{array}$ & $\begin{array}{l}6.14 \\
6.43 \\
6.72 \\
7.02 \\
7.31 \\
7.60 \\
7.89 \\
8.19 \\
8.48 \\
8.77\end{array}$ & $\begin{array}{l}20.06 \\
21.01 \\
21.97 \\
22.92 \\
23.88 \\
24.83 \\
25.79 \\
26.74 \\
27.70 \\
28.65\end{array}$ & $\begin{array}{l}6.23 \\
6.52 \\
6.82 \\
7.12 \\
7.41 \\
7.71 \\
8.01 \\
8.30 \\
8.60 \\
8.90\end{array}$ & $\begin{array}{l}20.03 \\
20.98 \\
21.94 \\
22.89 \\
23.84 \\
24.80 \\
25.75 \\
26.70 \\
27.66 \\
28.61\end{array}$ & $\begin{array}{l}6.31 \\
6.62 \\
6.92 \\
7.22 \\
7.52 \\
7.82 \\
8.12 \\
8.42 \\
8.72 \\
9.02\end{array}$ & $\begin{array}{l}20.00 \\
20.95 \\
21.91 \\
22.86 \\
23.81 \\
24.76 \\
25.71 \\
26.67 \\
27.62 \\
29.57\end{array}$ & $\begin{array}{l}6.40 \\
6.71 \\
7.01 \\
7.32 \\
7.62 \\
7.93 \\
8.23 \\
8.54 \\
8.84 \\
9.15\end{array}$ & $\begin{array}{l}21 \\
22 \\
23 \\
24 \\
25 \\
26 \\
27 \\
28 \\
29 \\
30\end{array}$ \\
\hline $\begin{array}{l}31 \\
32 \\
33 \\
34 \\
35 \\
36 \\
37 \\
38 \\
39 \\
40\end{array}$ & $\begin{array}{l}29.65 \\
30.60 \\
31.56 \\
32.51 \\
33.47 \\
34.43 \\
35.38 \\
36.34 \\
37.30 \\
38.25\end{array}$ & $\begin{array}{r}9.06 \\
9.36 \\
9.65 \\
9.94 \\
10.23 \\
10.53 \\
10.82 \\
11.11 \\
11.40 \\
11.69\end{array}$ & $\begin{array}{l}29.61 \\
30.56 \\
31.52 \\
32.47 \\
33.43 \\
34.38 \\
35.34 \\
36.29 \\
37.25 \\
38.20\end{array}$ & $\begin{array}{r}9.19 \\
9.49 \\
9.79 \\
10.08 \\
10.38 \\
10.68 \\
10.97 \\
11.27 \\
11.57 \\
11.86\end{array}$ & $\begin{array}{l}29.57 \\
30.52 \\
31.47 \\
32.43 \\
33.38 \\
34.33 \\
35.29 \\
36.24 \\
37.19 \\
35.15\end{array}$ & $\begin{array}{r}9.32 \\
9.62 \\
9.92 \\
10.22 \\
10.52 \\
10.83 \\
11.13 \\
11.43 \\
11.73 \\
12.03\end{array}$ & $\begin{array}{l}29.52 \\
30.48 \\
31.43 \\
32.38 \\
33.33 \\
34.29 \\
35.24 \\
36.19 \\
37.14 \\
38.10\end{array}$ & $\begin{array}{r}9.45 \\
9.76 \\
10.06 \\
10.37 \\
10.67 \\
10.98 \\
11.24 \\
11.58 \\
11.89 \\
12.19\end{array}$ & $\begin{array}{l}31 \\
32 \\
33 \\
34 \\
35 \\
36 \\
37 \\
38 \\
39 \\
40\end{array}$ \\
\hline $\begin{array}{l}41 \\
42 \\
43 \\
44 \\
45 \\
46 \\
47 \\
48 \\
49 \\
50\end{array}$ & $\begin{array}{l}39.21 \\
40.16 \\
41.12 \\
42.08 \\
43.03 \\
43.99 \\
44.95 \\
45.90 \\
46.86 \\
47.82\end{array}$ & $\begin{array}{l}11.99 \\
12.28 \\
12.57 \\
12.86 \\
13.16 \\
13.45 \\
13.74 \\
14.03 \\
14.33 \\
14.62\end{array}$ & $\begin{array}{l}39.16 \\
40.11 \\
41.07 \\
42.02 \\
42.98 \\
43.93 \\
44.89 \\
45.84 \\
46.80 \\
47.75\end{array}$ & $\begin{array}{l}12.16 \\
12.45 \\
12.75 \\
13.05 \\
13.34 \\
13.64 \\
13.94 \\
14.23 \\
14.53 \\
14.83\end{array}$ & $\begin{array}{l}39.10 \\
40.06 \\
41.01 \\
41.96 \\
42.92 \\
43.87 \\
44.82 \\
45.78 \\
46.73 \\
47.69\end{array}$ & $\begin{array}{l}12.33 \\
12.63 \\
12.93 \\
13.23 \\
13.53 \\
13.83 \\
14.13 \\
14.43 \\
14.73 \\
15.04\end{array}$ & $\begin{array}{l}39.05 \\
40.00 \\
40.95 \\
41.91 \\
42.86 \\
43.81 \\
44.76 \\
45.71 \\
46.67 \\
47.62\end{array}$ & $\begin{array}{l}12.50 \\
12.80 \\
13.11 \\
13.41 \\
13.72 \\
14.02 \\
14.33 \\
14.63 \\
14.94 \\
15.24\end{array}$ & $\begin{array}{l}41 \\
42 \\
43 \\
44 \\
45 \\
46 \\
47 \\
48 \\
49 \\
50\end{array}$ \\
\hline 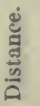 & $73^{\circ}$. & Lat. & Dep. & Lat. & Dep. & Lat. & Dep. & Iat. & 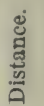 \\
\hline
\end{tabular}


TABLE 4.-TRAVEIREE TABLE.

\begin{tabular}{|c|c|c|c|c|c|c|c|c|c|}
\hline \multirow{2}{*}{ 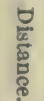 } & \multicolumn{2}{|c|}{$17^{\circ}$. } & \multicolumn{2}{|c|}{$171 / 4^{\circ}}$. & \multicolumn{2}{|c|}{$171 / 2^{\circ}$. } & \multicolumn{2}{|c|}{$177^{3} 4^{\circ}}$. & \multirow{2}{*}{ 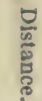 } \\
\hline & Lat. & Dep. & Lat. & Dep. & Lat. & Dep. & Lat. & Dep. & \\
\hline $\begin{array}{l}51 \\
52 \\
53 \\
54 \\
55 \\
56 \\
57 \\
58 \\
59 \\
60\end{array}$ & $\begin{array}{l}48.77 \\
49.73 \\
50.68 \\
51.64 \\
52.60 \\
53.55 \\
54.51 \\
55.47 \\
56.42 \\
57.38\end{array}$ & $\begin{array}{l}14.91 \\
15.20 \\
15.50 \\
15.79 \\
16.08 \\
16.37 \\
16.67 \\
16.96 \\
17.25 \\
17.54\end{array}$ & $\begin{array}{l}48.71 \\
49.66 \\
50.62 \\
51.57 \\
52.53 \\
53.48 \\
54.44 \\
55.39 \\
56.35 \\
57.30\end{array}$ & $\begin{array}{l}15.12 \\
15.42 \\
15.72 \\
16.01 \\
16.31 \\
16.61 \\
16.90 \\
17.20 \\
17.50 \\
17.79\end{array}$ & $\begin{array}{l}48.64 \\
49.59 \\
50.55 \\
51.50 \\
52.45 \\
53.41 \\
54.36 \\
55.32 \\
56.27 \\
57.22\end{array}$ & $\begin{array}{l}15.34 \\
15.64 \\
15.94 \\
16.24 \\
16.54 \\
16.84 \\
17.14 \\
17.44 \\
17.74 \\
18.04\end{array}$ & $\begin{array}{l}48.57 \\
49.52 \\
50.48 \\
51.43 \\
52.38 \\
53.33 \\
54.29 \\
55.24 \\
56.19 \\
57.14\end{array}$ & $\begin{array}{l}15.55 \\
15.85 \\
16.16 \\
16.46 \\
16.77 \\
17.07 \\
17.38 \\
17.68 \\
17.99 \\
18.29\end{array}$ & $\begin{array}{l}51 \\
52 \\
53 \\
54 \\
55 \\
56 \\
57 \\
58 \\
59 \\
60\end{array}$ \\
\hline $\begin{array}{l}61 \\
62 \\
63 \\
64 \\
65 \\
66 \\
67 \\
68 \\
69 \\
70\end{array}$ & $\begin{array}{l}58.33 \\
59.29 \\
60.25 \\
61.20 \\
62.16 \\
63.12 \\
64.07 \\
65.03 \\
65.99 \\
66.94\end{array}$ & $\begin{array}{l}17.83 \\
18.13 \\
18.42 \\
18.71 \\
19.00 \\
19.30 \\
19.59 \\
19.88 \\
20.17 \\
20.47\end{array}$ & $\begin{array}{l}55.26 \\
59.21 \\
60.17 \\
61.12 \\
62.08 \\
63.03 \\
63.99 \\
64.94 \\
65.90 \\
66.85\end{array}$ & $\begin{array}{l}15.09 \\
18.39 \\
18.68 \\
18.98 \\
19.29 \\
19.57 \\
19.87 \\
20.16 \\
20.46 \\
20.76\end{array}$ & $\begin{array}{l}58.18 \\
59.13 \\
60.08 \\
61.04 \\
61.99 \\
62.95 \\
63.90 \\
64.85 \\
65.81 \\
66.76\end{array}$ & $\begin{array}{l}18.34 \\
13.64 \\
18.94 \\
19.25 \\
19.55 \\
19.85 \\
20.15 \\
20.45 \\
20.75 \\
21.05\end{array}$ & $\begin{array}{l}58.10 \\
59.05 \\
60.00 \\
60.95 \\
61.91 \\
62.86 \\
63.81 \\
64.76 \\
65.72 \\
66.67\end{array}$ & $\begin{array}{l}18.60 \\
18.90 \\
19.21 \\
19.51 \\
19.82 \\
20.12 \\
20.43 \\
20.73 \\
21.04 \\
21.34\end{array}$ & $\begin{array}{l}61 \\
62 \\
63 \\
64 \\
65 \\
66 \\
67 \\
68 \\
69 \\
70\end{array}$ \\
\hline $\begin{array}{l}71 \\
72 \\
73 \\
74 \\
75 \\
76 \\
77 \\
78 \\
79 \\
80\end{array}$ & $\begin{array}{l}67.90 \\
68.85 \\
69.81 \\
70.77 \\
71.72 \\
72.88 \\
73.64 \\
74.59 \\
75.55 \\
76.50\end{array}$ & $\begin{array}{l}20.76 \\
21.05 \\
21.34 \\
21.64 \\
21.93 \\
22.22 \\
22.51 \\
22.80 \\
23.10 \\
23.39\end{array}$ & $\begin{array}{l}67.81 \\
68.76 \\
69.72 \\
70.67 \\
71.63 \\
72.58 \\
73.54 \\
74.49 \\
75.45 \\
76.40\end{array}$ & $\begin{array}{l}21.05 \\
21.35 \\
21.65 \\
21.94 \\
22.24 \\
22.54 \\
22.83 \\
23.13 \\
23.43 \\
23.72\end{array}$ & $\begin{array}{l}67.71 \\
68.67 \\
69.62 \\
70.58 \\
71.53 \\
72.48 \\
73.44 \\
74.39 \\
75.34 \\
76.30\end{array}$ & $\begin{array}{l}21.35 \\
21.65 \\
21.95 \\
22.25 \\
22.55 \\
22.85 \\
23.15 \\
23.46 \\
23.76 \\
24.06\end{array}$ & $\begin{array}{l}67.62 \\
68.57 \\
69.52 \\
70.48 \\
71.43 \\
72.38 \\
73.33 \\
74.29 \\
75.24 \\
76.19\end{array}$ & $\begin{array}{l}21.65 \\
21.95 \\
22.26 \\
22.56 \\
22.86 \\
23.17 \\
23.47 \\
23.78 \\
24.08 \\
24.39\end{array}$ & $\begin{array}{l}71 \\
72 \\
73 \\
74 \\
75 \\
76 \\
77 \\
78 \\
79 \\
80\end{array}$ \\
\hline $\begin{array}{l}81 \\
82 \\
83 \\
81 \\
85 \\
86 \\
87 \\
88 \\
89 \\
90\end{array}$ & $\begin{array}{l}77.46 \\
78.42 \\
79.37 \\
80.33 \\
81.29 \\
82.24 \\
83.20 \\
84.15 \\
85.11 \\
86.07\end{array}$ & $\begin{array}{l}23.68 \\
23.97 \\
24.27 \\
24.56 \\
24.85 \\
25.14 \\
25.41 \\
25.73 \\
26.02 \\
36.31\end{array}$ & $\begin{array}{l}77.36 \\
78.31 \\
79.27 \\
80.22 \\
81.18 \\
83.13 \\
83.09 \\
84.04 \\
85.00 \\
85.95\end{array}$ & $\begin{array}{l}24.02 \\
24.32 \\
24.61 \\
24.91 \\
25.21 \\
25.50 \\
25.80 \\
26.10 \\
26.39 \\
26.69\end{array}$ & $\begin{array}{l}77.25 \\
78.20 \\
79.16 \\
80.11 \\
81.07 \\
82.02 \\
82.97 \\
83.93 \\
84.88 \\
85.83\end{array}$ & $\begin{array}{l}24.36 \\
24.66 \\
24.96 \\
25.26 \\
25.56 \\
25.86 \\
26.16 \\
26.46 \\
26.76 \\
27.06\end{array}$ & $\begin{array}{l}77.14 \\
78.10 \\
79.05 \\
80.00 \\
80.95 \\
81.91 \\
82.56 \\
83.81 \\
84.76 \\
85.72\end{array}$ & $\begin{array}{l}24.69 \\
25.00 \\
25.30 \\
25.61 \\
25.91 \\
26.22 \\
26.52 \\
26.83 \\
27.13 \\
27.44\end{array}$ & $\begin{array}{l}81 \\
82 \\
83 \\
84 \\
85 \\
86 \\
87 \\
89 \\
89 \\
90\end{array}$ \\
\hline $\begin{array}{r}91 \\
92 \\
93 \\
94 \\
95 \\
96 \\
97 \\
98 \\
99 \\
100\end{array}$ & $\begin{array}{l}87.02 \\
87.98 \\
88.94 \\
89.80 \\
90.85 \\
91.81 \\
92.76 \\
93.72 \\
94.67 \\
95.63\end{array}$ & $\begin{array}{l}26.61 \\
26.90 \\
27.19 \\
27.48 \\
27.78 \\
28.07 \\
28.36 \\
28.65 \\
28.94 \\
29.24\end{array}$ & $\begin{array}{l}86.91 \\
87.86 \\
88.82 \\
89.77 \\
90.73 \\
91.68 \\
92.64 \\
93.59 \\
94.55 \\
95.50\end{array}$ & $\begin{array}{l}26.99 \\
27.28 \\
27.58 \\
27.87 \\
25.17 \\
25.47 \\
29.76 \\
29.06 \\
29.36 \\
29.65\end{array}$ & $\begin{array}{l}86.79 \\
87.74 \\
88.70 \\
89.65 \\
90.60 \\
91.56 \\
92.51 \\
93.46 \\
94.42 \\
95.37\end{array}$ & $\begin{array}{l}27.36 \\
27.66 \\
27.97 \\
28.27 \\
28.57 \\
28.87 \\
39.17 \\
29.47 \\
29.77 \\
30.07\end{array}$ & $\begin{array}{l}86.67 \\
87.62 \\
83.57 \\
83.53 \\
90.48 \\
91.43 \\
92.38 \\
93.33 \\
94.29 \\
95.24\end{array}$ & $\begin{array}{l}27.74 \\
25.05 \\
28.35 \\
28.66 \\
28.96 \\
29.27 \\
29.57 \\
29.88 \\
30.18 \\
30.49\end{array}$ & $\begin{array}{r}91 \\
92 \\
93 \\
94 \\
95 \\
96 \\
97 \\
98 \\
99 \\
100\end{array}$ \\
\hline \multirow{2}{*}{ 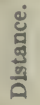 } & Dep. & Lat. & Dep. & Lat. & Dep. & Lat. & lep. & Lat. & \multirow{2}{*}{ 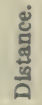 } \\
\hline & \multicolumn{2}{|c|}{$73^{\circ}$. } & \multicolumn{2}{|c|}{$7234^{\circ}}$. & \multicolumn{2}{|c|}{$721 / 2^{\circ}$. } & \multicolumn{2}{|c|}{$721 / 4^{\circ}$. } & \\
\hline
\end{tabular}


TABLE 4.-TRIVERSE TABLE.

\begin{tabular}{|c|c|c|c|c|c|c|c|c|c|}
\hline \multirow{2}{*}{ 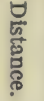 } & \multicolumn{2}{|c|}{$18^{\circ}$. } & \multicolumn{2}{|c|}{$181 / 4^{\circ}$. } & \multicolumn{2}{|c|}{$181 / 2^{\circ}}$. & \multicolumn{2}{|c|}{$18^{3} 4^{\circ}}$. & \multirow{2}{*}{ 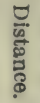 } \\
\hline & Lat. & Dep. & Lat. & Dep. & Lat. & Dep. & Lat. & Dep. & \\
\hline $\begin{array}{r}1 \\
2 \\
3 \\
4 \\
5 \\
6 \\
7 \\
8 \\
9 \\
10\end{array}$ & $\begin{array}{l}0.95 \\
1.90 \\
2.85 \\
3.80 \\
4.76 \\
5.71 \\
6.66 \\
7.61 \\
8.56 \\
9.51\end{array}$ & $\begin{array}{l}0.31 \\
0.62 \\
0.93 \\
1.24 \\
1.55 \\
1.85 \\
2.16 \\
2.47 \\
2.78 \\
3.09\end{array}$ & $\begin{array}{l}0.95 \\
1.90 \\
2.85 \\
3.80 \\
4.75 \\
5.70 \\
6.65 \\
7.60 \\
8.55 \\
9.50\end{array}$ & $\begin{array}{l}0.31 \\
0.63 \\
0.94 \\
1.25 \\
1.57 \\
1.88 \\
2.19 \\
2.51 \\
2.82 \\
3.13\end{array}$ & $\begin{array}{l}0.95 \\
1.90 \\
2.84 \\
3.79 \\
4.74 \\
5.69 \\
6.64 \\
7.59 \\
8.53 \\
9.48\end{array}$ & $\begin{array}{l}0.32 \\
0.63 \\
0.95 \\
1.27 \\
1.59 \\
1.90 \\
2.22 \\
2.54 \\
2.86 \\
3.17\end{array}$ & $\begin{array}{l}0.95 \\
1.89 \\
2.84 \\
3.79 \\
4.73 \\
5.68 \\
6.63 \\
7.58 \\
8.52 \\
9.47\end{array}$ & $\begin{array}{l}0.32 \\
0.64 \\
0.96 \\
1.29 \\
1.61 \\
1.93 \\
2.25 \\
2.57 \\
2.89 \\
3.21\end{array}$ & $\begin{array}{r}1 \\
2 \\
3 \\
4 \\
5 \\
6 \\
7 \\
8 \\
9 \\
10\end{array}$ \\
\hline $\begin{array}{l}11 \\
12 \\
13 \\
14 \\
15 \\
16 \\
17 \\
18 \\
19 \\
20\end{array}$ & $\begin{array}{l}10.46 \\
11.41 \\
12.36 \\
13.31 \\
14.27 \\
15.22 \\
16.17 \\
17.12 \\
18.07 \\
19.02\end{array}$ & $\begin{array}{l}3.40 \\
3.71 \\
4.02 \\
4.33 \\
4.64 \\
4.94 \\
5.25 \\
5.56 \\
5.87 \\
6.18\end{array}$ & $\begin{array}{l}10.45 \\
11.40 \\
12.35 \\
13.30 \\
14.25 \\
15.20 \\
16.14 \\
17.09 \\
18.04 \\
18.99\end{array}$ & $\begin{array}{l}3.44 \\
3.76 \\
4.07 \\
4.38 \\
4.70 \\
5.01 \\
5.32 \\
5.64 \\
5.95 \\
6.26\end{array}$ & $\begin{array}{l}10.43 \\
11.38 \\
12.33 \\
13.28 \\
14.22 \\
15.17 \\
16.12 \\
17.07 \\
18.02 \\
18.97\end{array}$ & $\begin{array}{l}3.49 \\
3.81 \\
4.12 \\
4.44 \\
4.76 \\
5.08 \\
5.39 \\
5.71 \\
6.03 \\
6.35\end{array}$ & $\begin{array}{l}10.42 \\
11.36 \\
12.31 \\
13.26 \\
14.20 \\
15.15 \\
16.10 \\
17.04 \\
17.99 \\
18.94\end{array}$ & $\begin{array}{l}3.54 \\
3.86 \\
4.18 \\
4.50 \\
4.82 \\
5.14 \\
5.46 \\
5.79 \\
6.11 \\
6.43\end{array}$ & $\begin{array}{l}11 \\
12 \\
13 \\
14 \\
15 \\
16 \\
17 \\
18 \\
19 \\
20\end{array}$ \\
\hline $\begin{array}{l}21 \\
22 \\
23 \\
24 \\
25 \\
26 \\
27 \\
28 \\
29 \\
30\end{array}$ & $\begin{array}{l}19.97 \\
20.92 \\
21.87 \\
22.83 \\
23.78 \\
24.73 \\
25.68 \\
26.63 \\
27.58 \\
28.53\end{array}$ & $\begin{array}{l}6.49 \\
6.80 \\
7.11 \\
7.42 \\
7.73 \\
8.03 \\
8.34 \\
8.65 \\
8.96 \\
9.27\end{array}$ & $\begin{array}{l}19.94 \\
20.89 \\
21.84 \\
22.79 \\
23.74 \\
24.69 \\
25.64 \\
26.59 \\
27.54 \\
28.49\end{array}$ & $\begin{array}{l}6.58 \\
6.89 \\
7.20 \\
7.52 \\
7.83 \\
8.14 \\
8.46 \\
8.77 \\
9.08 \\
9.39\end{array}$ & $\begin{array}{l}19.91 \\
20.86 \\
21.81 \\
22.76 \\
23.71 \\
24.66 \\
25.60 \\
26.55 \\
27.50 \\
28.45\end{array}$ & $\begin{array}{l}6.66 \\
6.98 \\
7.30 \\
7.62 \\
7.93 \\
8.25 \\
8.57 \\
8.88 \\
9.20 \\
9.52\end{array}$ & $\begin{array}{l}19.89 \\
20.83 \\
21.78 \\
22.73 \\
23.67 \\
24.62 \\
25.57 \\
26.51 \\
27.46 \\
28.41\end{array}$ & $\begin{array}{l}6.75 \\
7.07 \\
7.39 \\
7.71 \\
8.04 \\
8.36 \\
8.68 \\
9.00 \\
9.32 \\
9.64\end{array}$ & $\begin{array}{l}21 \\
22 \\
23 \\
24 \\
25 \\
26 \\
27 \\
28 \\
29 \\
30\end{array}$ \\
\hline $\begin{array}{l}31 \\
32 \\
33 \\
34 \\
35 \\
36 \\
37 \\
38 \\
39 \\
40\end{array}$ & $\begin{array}{l}29.48 \\
30.43 \\
31.38 \\
32.34 \\
33.29 \\
34.24 \\
35.19 \\
36.14 \\
37.09 \\
33.04\end{array}$ & $\begin{array}{r}9.58 \\
9.89 \\
10.20 \\
10.51 \\
10.82 \\
11.12 \\
11.43 \\
11.74 \\
12.05 \\
12.36\end{array}$ & $\begin{array}{l}29.44 \\
30.39 \\
31.34 \\
32.29 \\
33.24 \\
34.19 \\
35.14 \\
36.09 \\
37.04 \\
37.99\end{array}$ & $\begin{array}{r}9.71 \\
10.02 \\
10.33 \\
10.65 \\
10.96 \\
11.27 \\
11.59 \\
11.90 \\
12.21 \\
12.53\end{array}$ & $\begin{array}{l}29.40 \\
30.35 \\
31.29 \\
32.24 \\
33.19 \\
34.14 \\
35.09 \\
36.04 \\
36.99 \\
37.93\end{array}$ & $\begin{array}{r}9.84 \\
10.15 \\
10.47 \\
10.79 \\
11.11 \\
11.42 \\
11.74 \\
12.06 \\
12.37 \\
12.69\end{array}$ & $\begin{array}{l}29.35 \\
30.30 \\
31.25 \\
32.20 \\
33.14 \\
34.09 \\
35.04 \\
3.59 \\
36.93 \\
37.88\end{array}$ & $\begin{array}{r}9.96 \\
10.29 \\
10.61 \\
10.93 \\
11.25 \\
11.57 \\
11.59 \\
12.21 \\
12.54 \\
12.86\end{array}$ & $\begin{array}{l}31 \\
32 \\
33 \\
34 \\
35 \\
36 \\
37 \\
38 \\
39 \\
40\end{array}$ \\
\hline $\begin{array}{l}41 \\
42 \\
43 \\
41 \\
45 \\
46 \\
47 \\
48 \\
49 \\
50\end{array}$ & $\begin{array}{l}38.99 \\
39.94 \\
40.90 \\
41.85 \\
42.80 \\
43.75 \\
44.70 \\
45.65 \\
46.60 \\
47.55\end{array}$ & $\begin{array}{l}12.67 \\
12.98 \\
13.29 \\
13.60 \\
13.91 \\
14.21 \\
14.52 \\
14.83 \\
15.14 \\
15.45\end{array}$ & $\begin{array}{l}33.94 \\
39.89 \\
40.84 \\
41.79 \\
42.74 \\
43.69 \\
44.64 \\
45.59 \\
46.54 \\
47.48\end{array}$ & $\begin{array}{l}12.84 \\
13.15 \\
13.47 \\
13.78 \\
14.09 \\
14.41 \\
14.72 \\
15.03 \\
15.35 \\
15.66\end{array}$ & $\begin{array}{l}38.88 \\
39.83 \\
40.78 \\
41.73 \\
42.67 \\
43.62 \\
44.57 \\
45.52 \\
46.47 \\
47.42\end{array}$ & $\begin{array}{l}13.01 \\
13.33 \\
13.64 \\
13.96 \\
14.28 \\
14.60 \\
14.91 \\
15.23 \\
15.55 \\
15.87\end{array}$ & $\begin{array}{l}38.82 \\
39.77 \\
40.72 \\
41.66 \\
42.61 \\
43.56 \\
44.51 \\
45.45 \\
46.40 \\
47.35\end{array}$ & $\begin{array}{l}13.18 \\
13.50 \\
13.82 \\
14.14 \\
14.46 \\
14.79 \\
15.11 \\
15.43 \\
15.75 \\
16.07\end{array}$ & $\begin{array}{l}41 \\
42 \\
43 \\
44 \\
45 \\
40 \\
47 \\
48 \\
49 \\
50\end{array}$ \\
\hline \&్ & Dep. & Lat. . & Dep. & Lat. & Dep. & Lat. & Dep. & Lat. & \&் \\
\hline $\bar{A}$ & & & & & & & & & $\frac{\vec{n}}{A}$ \\
\hline
\end{tabular}


TABLE 4.-TIAVERSE TABLE.

\begin{tabular}{|c|c|c|c|c|c|c|c|c|c|}
\hline \multirow{2}{*}{ 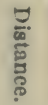 } & \multicolumn{2}{|c|}{$15^{\circ}$. } & \multicolumn{2}{|c|}{$151 / 4^{\circ}$. } & \multicolumn{2}{|c|}{$181 / 2^{\circ}$. } & \multicolumn{2}{|c|}{$18^{3} 4^{\circ}}$. & \multirow{2}{*}{ 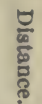 } \\
\hline & Lat. & Dep. & Lat. & Dep. & Lat. & Dep. & Lat. & Dep. & \\
\hline $\begin{array}{l}51 \\
52 \\
53 \\
54 \\
55 \\
56 \\
57 \\
58 \\
59 \\
60\end{array}$ & $\begin{array}{l}48.50 \\
49.45 \\
50.41 \\
51.36 \\
52.31 \\
53.26 \\
54.21 \\
55.16 \\
51.11 \\
57.06\end{array}$ & $\begin{array}{l}15.76 \\
16.07 \\
16.38 \\
16.69 \\
17.00 \\
17.30 \\
17.61 \\
17.92 \\
18.23 \\
18.54\end{array}$ & $\begin{array}{l}48.43 \\
49.38 \\
50.33 \\
51.28 \\
52.23 \\
53.18 \\
54.13 \\
55.08 \\
56.03 \\
56.98\end{array}$ & $\begin{array}{l}15.97 \\
16.28 \\
16.60 \\
16.91 \\
17.22 \\
17.54 \\
17.85 \\
18.16 \\
18.48 \\
18.79\end{array}$ & $\begin{array}{l}48.36 \\
49.31 \\
50.26 \\
51.21 \\
52.16 \\
53.11 \\
54.05 \\
55.00 \\
55.95 \\
56.90\end{array}$ & $\begin{array}{l}16.18 \\
16.50 \\
16.82 \\
17.13 \\
17.45 \\
17.77 \\
18.09 \\
18.40 \\
18.72 \\
19.04\end{array}$ & $\begin{array}{l}48.29 \\
49.24 \\
50.19 \\
51.13 \\
52.08 \\
53.03 \\
53.98 \\
54.92 \\
55.87 \\
56.82\end{array}$ & $\begin{array}{l}16.39 \\
16.71 \\
17.04 \\
17.36 \\
17.68 \\
18.00 \\
18.32 \\
18.64 \\
18.96 \\
19.29\end{array}$ & $\begin{array}{l}51 \\
52 \\
53 \\
54 \\
55 \\
56 \\
57 \\
58 \\
59 \\
60\end{array}$ \\
\hline $\begin{array}{l}61 \\
62 \\
63 \\
64 \\
65 \\
65 \\
67 \\
69 \\
69 \\
70\end{array}$ & $\begin{array}{l}58.01 \\
58.97 \\
59.92 \\
60.87 \\
61.82 \\
62.77 \\
63.72 \\
64.67 \\
65.62 \\
66.57\end{array}$ & $\begin{array}{l}18.85 \\
19.16 \\
19.47 \\
19.78 \\
20.09 \\
20.49 \\
20.70 \\
21.01 \\
21.32 \\
21.63\end{array}$ & $\begin{array}{l}57.93 \\
58.88 \\
59.83 \\
60.78 \\
61.73 \\
62.68 \\
63.63 \\
64.59 \\
65.53 \\
66.48\end{array}$ & $\begin{array}{l}19.10 \\
19.42 \\
19.73 \\
20.04 \\
20.36 \\
20.67 \\
20.98 \\
21.30 \\
21.61 \\
21.92\end{array}$ & $\begin{array}{l}57.85 \\
55.80 \\
59.74 \\
60.69 \\
61.64 \\
62.59 \\
63.54 \\
64.49 \\
65.43 \\
66.38\end{array}$ & $\begin{array}{l}19.36 \\
19.67 \\
19.99 \\
20.31 \\
20.62 \\
20.94 \\
21.26 \\
21.55 \\
21.89 \\
22.21\end{array}$ & $\begin{array}{l}57.76 \\
58.71 \\
59.66 \\
60.60 \\
61.55 \\
62.50 \\
63.44 \\
64.39 \\
65.34 \\
66.29\end{array}$ & $\begin{array}{l}19.61 \\
19.93 \\
20.25 \\
20.57 \\
20.89 \\
21.22 \\
21.54 \\
21.86 \\
22.18 \\
22.50\end{array}$ & $\begin{array}{l}61 \\
62 \\
63 \\
64 \\
65 \\
66 \\
67 \\
68 \\
69 \\
70\end{array}$ \\
\hline $\begin{array}{l}71 \\
72 \\
73 \\
74 \\
75 \\
76 \\
77 \\
78 \\
79 \\
80\end{array}$ & $\begin{array}{l}67.53 \\
68.45 \\
69.43 \\
70.38 \\
71.33 \\
72.28 \\
73.23 \\
74.18 \\
75.13 \\
76.08\end{array}$ & $\begin{array}{l}21.94 \\
22.25 \\
22.56 \\
22.87 \\
23.18 \\
23.49 \\
23.79 \\
24.10 \\
24.41 \\
24.72\end{array}$ & $\begin{array}{l}67.43 \\
68.38 \\
69.33 \\
70.28 \\
71.23 \\
72.18 \\
73.13 \\
74.08 \\
75.03 \\
75.98\end{array}$ & $\begin{array}{l}22.23 \\
22.55 \\
22.86 \\
23.17 \\
23.49 \\
23.80 \\
24.11 \\
24.43 \\
24.74 \\
25.05\end{array}$ & $\begin{array}{l}67.33 \\
68.28 \\
69.23 \\
70.13 \\
71.12 \\
72.07 \\
73.02 \\
73.97 \\
74.92 \\
75.87\end{array}$ & $\begin{array}{l}22.53 \\
22.85 \\
23.16 \\
23.48 \\
23.80 \\
24.12 \\
24.43 \\
24.75 \\
25.07 \\
25.38\end{array}$ & $\begin{array}{l}67.23 \\
68.18 \\
69.13 \\
70.07 \\
71.02 \\
71.97 \\
72.91 \\
73.86 \\
74.81 \\
75.75\end{array}$ & $\begin{array}{l}22.82 \\
23.14 \\
23.47 \\
23.79 \\
24.11 \\
24.43 \\
24.75 \\
25.07 \\
25.39 \\
25.72\end{array}$ & $\begin{array}{l}71 \\
72 \\
73 \\
74 \\
75 \\
76 \\
77 \\
78 \\
79 \\
80\end{array}$ \\
\hline $\begin{array}{l}81 \\
82 \\
83 \\
81 \\
85 \\
86 \\
87 \\
89 \\
89 \\
90\end{array}$ & $\begin{array}{l}77.04 \\
77.93 \\
78.94 \\
79.89 \\
80.84 \\
81.79 \\
82.74 \\
83.69 \\
84.64 \\
85.60\end{array}$ & $\begin{array}{l}25.03 \\
25.34 \\
25.65 \\
25.96 \\
26.27 \\
26.58 \\
26.88 \\
27.19 \\
27.50 \\
27.81\end{array}$ & $\begin{array}{l}76.93 \\
77.85 \\
78.83 \\
79.77 \\
80.72 \\
81.67 \\
82.62 \\
83.57 \\
84.52 \\
85.47\end{array}$ & $\begin{array}{l}25.37 \\
25.68 \\
25.99 \\
26.31 \\
26.62 \\
26.93 \\
27.25 \\
27.54 \\
27.87 \\
28.18\end{array}$ & $\begin{array}{l}76.81 \\
77.76 \\
78.71 \\
79.66 \\
80.61 \\
81.54 \\
82.50 \\
83.45 \\
84.40 \\
85.35\end{array}$ & $\begin{array}{l}25.70 \\
26.02 \\
26.34 \\
26.65 \\
26.97 \\
27.29 \\
27.61 \\
27.92 \\
28.24 \\
28.56\end{array}$ & $\begin{array}{l}76.70 \\
77.65 \\
78.60 \\
79.54 \\
80.49 \\
81.44 \\
82.39 \\
83.33 \\
84.28 \\
85.22\end{array}$ & $\begin{array}{l}26.04 \\
26.36 \\
26.68 \\
27.00 \\
27.32 \\
27.64 \\
27.97 \\
23.29 \\
28.61 \\
28.93\end{array}$ & $\begin{array}{l}81 \\
82 \\
83 \\
84 \\
85 \\
86 \\
87 \\
89 \\
89 \\
90\end{array}$ \\
\hline $\begin{array}{r}91 \\
92 \\
83 \\
94 \\
95 \\
96 \\
97 \\
98 \\
99 \\
100\end{array}$ & $\begin{array}{l}86.55 \\
87.50 \\
88.45 \\
89.40 \\
90.35 \\
91.30 \\
92.25 \\
93.20 \\
94.15 \\
95.11\end{array}$ & $\begin{array}{l}29.12 \\
29.4 .3 \\
29.74 \\
29.05 \\
29.36 \\
29.67 \\
29.97 \\
30.28 \\
30.59 \\
30.90\end{array}$ & $\begin{array}{l}86.42 \\
87.37 \\
83.32 \\
83.27 \\
90.22 \\
91.17 \\
92.12 \\
93.07 \\
94.02 \\
94.97\end{array}$ & $\begin{array}{l}28.50 \\
28.81 \\
29.12 \\
29.44 \\
29.75 \\
30.06 \\
30.38 \\
30.69 \\
31.00 \\
31.32\end{array}$ & $\begin{array}{l}86.30 \\
87.25 \\
88.19 \\
89.14 \\
90.09 \\
91.04 \\
91.99 \\
92.94 \\
93.88 \\
94.83\end{array}$ & $\begin{array}{l}29.87 \\
29.19 \\
29.51 \\
29.83 \\
30.14 \\
30.46 \\
30.78 \\
31.10 \\
31.41 \\
31.73\end{array}$ & $\begin{array}{l}86.17 \\
87.12 \\
89.06 \\
89.01 \\
89.96 \\
90.91 \\
91.85 \\
92.80 \\
83.75 \\
94.69\end{array}$ & $\begin{array}{l}29.25 \\
29.57 \\
29.89 \\
30.22 \\
30.54 \\
30.86 \\
31.18 \\
31.50 \\
31.82 \\
32.14\end{array}$ & $\begin{array}{r}91 \\
92 \\
93 \\
94 \\
95 \\
96 \\
97 \\
98 \\
99 \\
100\end{array}$ \\
\hline \multirow{2}{*}{ 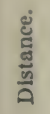 } & Dep. & Lat. & Dep. & Lat. & Dep. & Lat. & Dep. & Lat. & \multirow{2}{*}{ 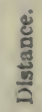 } \\
\hline & \multicolumn{2}{|c|}{$72^{\circ}$. } & \multicolumn{2}{|c|}{$713 \%$} & \multicolumn{2}{|c|}{$7132^{\circ}$. } & \multicolumn{2}{|c|}{$7116^{\circ}$. } & \\
\hline
\end{tabular}


TABLE 4.-TRAVERSE TABLE.

\begin{tabular}{|c|c|c|c|c|c|c|c|c|c|}
\hline \multirow{2}{*}{ 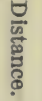 } & \multicolumn{2}{|c|}{$19^{\circ}$. } & \multicolumn{2}{|c|}{$191 / 4^{\circ}$. } & \multicolumn{2}{|c|}{$191 / 2^{\circ}$. } & \multicolumn{2}{|c|}{$1934^{\circ}$. } & \multirow{2}{*}{$\frac{\vec{\sigma}}{\frac{\vec{\omega}}{\tilde{E}}}$} \\
\hline & Lat. & Dep. & Lat. & Dep. & Lat. & Dep. & Lat. & Dep. & \\
\hline $\begin{array}{r}1 \\
2 \\
3 \\
4 \\
5 \\
6 \\
7 \\
8 \\
9 \\
10\end{array}$ & $\begin{array}{l}0.95 \\
1.89 \\
2.84 \\
3.78 \\
4.73 \\
5.67 \\
6.62 \\
7.56 \\
8.51 \\
9.46\end{array}$ & $\begin{array}{l}0.33 \\
0.65 \\
0.98 \\
1.30 \\
1.63 \\
1.95 \\
2.28 \\
2.60 \\
2.93 \\
3.26\end{array}$ & $\begin{array}{l}0.94 \\
1.89 \\
2.83 \\
3.78 \\
4.72 \\
5.66 \\
6.61 \\
7.55 \\
8.50 \\
9.44\end{array}$ & $\begin{array}{l}0.33 \\
0.66 \\
0.99 \\
1.32 \\
1.65 \\
1.98 \\
2.31 \\
2.64 \\
2.97 \\
3.30\end{array}$ & $\begin{array}{l}0.94 \\
1.89 \\
2.83 \\
3.77 \\
4.71 \\
5.66 \\
6.60 \\
7.54 \\
8.48 \\
9.43\end{array}$ & $\begin{array}{l}0.33 \\
0.67 \\
1.00 \\
1.34 \\
1.67 \\
2.00 \\
2.34 \\
2.67 \\
3.00 \\
3.34\end{array}$ & $\begin{array}{l}0.94 \\
1.88 \\
2.82 \\
3.76 \\
4.71 \\
5.65 \\
6.59 \\
7.53 \\
8.47 \\
9.41\end{array}$ & $\begin{array}{l}0.34 \\
0.68 \\
1.01 \\
1.35 \\
1.69 \\
2.03 \\
2.37 \\
2.70 \\
3.04 \\
3.38\end{array}$ & $\begin{array}{r}1 \\
2 \\
3 \\
4 \\
5 \\
6 \\
7 \\
8 \\
9 \\
10\end{array}$ \\
\hline $\begin{array}{l}11 \\
12 \\
13 \\
14 \\
15 \\
16 \\
17 \\
18 \\
19 \\
20\end{array}$ & $\begin{array}{l}10.40 \\
11.35 \\
12.29 \\
13.24 \\
14.18 \\
15.13 \\
16.07 \\
17.02 \\
17.90 \\
18.91\end{array}$ & $\begin{array}{l}3.58 \\
3.91 \\
4.23 \\
4.56 \\
4.88 \\
5.21 \\
5.53 \\
5.86 \\
6.19 \\
6.51\end{array}$ & $\begin{array}{l}10.38 \\
11.33 \\
12.27 \\
13.22 \\
14.16 \\
15.11 \\
16.05 \\
16.99 \\
17.94 \\
18.88\end{array}$ & $\begin{array}{l}3.63 \\
3.96 \\
4.29 \\
4.62 \\
4.95 \\
5.28 \\
5.60 \\
5.93 \\
6.26 \\
6.59\end{array}$ & $\begin{array}{l}10.37 \\
11.31 \\
12.25 \\
13.20 \\
14.14 \\
15.08 \\
16.02 \\
16.97 \\
17.91 \\
18.85\end{array}$ & $\begin{array}{l}3.67 \\
4.01 \\
4.34 \\
4.67 \\
5.01 \\
5.34 \\
5.67 \\
6.01 \\
6.34 \\
6.68\end{array}$ & $\begin{array}{l}10.35 \\
11.29 \\
12.24 \\
13.18 \\
14.12 \\
15.06 \\
16.00 \\
16.94 \\
17.88 \\
18.82\end{array}$ & $\begin{array}{l}3.72 \\
4.06 \\
4.39 \\
4.73 \\
5.07 \\
5.41 \\
5.74 \\
6.08 \\
6.42 \\
6.76\end{array}$ & $\begin{array}{l}11 \\
12 \\
13 \\
14 \\
15 \\
16 \\
17 \\
18 \\
19 \\
20\end{array}$ \\
\hline $\begin{array}{l}21 \\
22 \\
23 \\
24 \\
25 \\
26 \\
27 \\
28 \\
29 \\
30\end{array}$ & $\begin{array}{l}19.86 \\
20.80 \\
21.75 \\
22.69 \\
23.64 \\
24.58 \\
25.53 \\
21.47 \\
27.42 \\
28.37\end{array}$ & $\begin{array}{l}6.84 \\
7.16 \\
7.49 \\
7.81 \\
8.14 \\
8.46 \\
8.79 \\
9.12 \\
9.44 \\
9.77\end{array}$ & $\begin{array}{l}19.83 \\
20.77 \\
21.71 \\
22.66 \\
23.60 \\
24.55 \\
25.49 \\
21.43 \\
27.38 \\
23.32\end{array}$ & $\begin{array}{l}6.92 \\
7.25 \\
7.58 \\
7.91 \\
8.24 \\
8.57 \\
8.90 \\
9.23 \\
9.56 \\
9.89\end{array}$ & $\begin{array}{l}19.80 \\
20.74 \\
21.68 \\
22.62 \\
23.57 \\
24.51 \\
25.45 \\
21.39 \\
27.34 \\
28.28\end{array}$ & $\begin{array}{r}7.01 \\
7.34 \\
7.68 \\
8.01 \\
8.35 \\
8.68 \\
9.01 \\
9.35 \\
9.68 \\
10.01\end{array}$ & $\begin{array}{l}19.76 \\
20.71 \\
21.65 \\
22.59 \\
23.53 \\
21.47 \\
25.41 \\
26.35 \\
27.29 \\
28.24\end{array}$ & $\begin{array}{r}7.10 \\
7.43 \\
7.77 \\
8.11 \\
8.45 \\
8.79 \\
9.12 \\
9.46 \\
9.80 \\
10.14\end{array}$ & $\begin{array}{l}21 \\
22 \\
23 \\
24 \\
25 \\
26 \\
27 \\
28 \\
29 \\
30\end{array}$ \\
\hline $\begin{array}{l}31 \\
32 \\
33 \\
34 \\
35 \\
36 \\
37 \\
38 \\
39 \\
40\end{array}$ & $\begin{array}{l}29.31 \\
30.26 \\
31.20 \\
32.15 \\
33.09 \\
34.04 \\
34.98 \\
35.93 \\
36.88 \\
37.82\end{array}$ & $\begin{array}{l}10.09 \\
10.42 \\
10.74 \\
11.07 \\
11.39 \\
11.72 \\
12.05 \\
12.37 \\
12.70 \\
13.02\end{array}$ & $\begin{array}{l}29.27 \\
3 ! .21 \\
31.15 \\
32.10 \\
33.01 \\
33.99 \\
34.93 \\
35.88 \\
36.82 \\
37.76\end{array}$ & $\begin{array}{l}10.22 \\
10.55 \\
10.88 \\
11.21 \\
11.54 \\
11.87 \\
12.20 \\
12.53 \\
12.86 \\
13.19\end{array}$ & $\begin{array}{l}29.22 \\
30.16 \\
31.11 \\
32.05 \\
32.99 \\
33.94 \\
34.88 \\
35.82 \\
36.76 \\
37.71\end{array}$ & $\begin{array}{l}10.35 \\
10.68 \\
11.02 \\
11.35 \\
11.68 \\
12.02 \\
12.35 \\
12.68 \\
13.02 \\
13.35\end{array}$ & $\begin{array}{l}29.18 \\
30.12 \\
31.06 \\
32.00 \\
32.94 \\
33.88 \\
34.82 \\
35.76 \\
36.71 \\
37.65\end{array}$ & $\begin{array}{l}10.48 \\
10.81 \\
11.15 \\
11.49 \\
11.83 \\
12.17 \\
12.50 \\
12.84 \\
13.18 \\
13.52\end{array}$ & $\begin{array}{l}31 \\
32 \\
33 \\
34 \\
35 \\
36 \\
37 \\
38 \\
39 \\
40\end{array}$ \\
\hline $\begin{array}{l}41 \\
42 \\
43 \\
44 \\
45 \\
46 \\
47 \\
48 \\
49 \\
50\end{array}$ & $\begin{array}{l}38.77 \\
39.71 \\
40.66 \\
41.60 \\
42.55 \\
43.49 \\
44.44 \\
45.38 \\
46.33 \\
47.28\end{array}$ & $\begin{array}{l}13.35 \\
13.67 \\
14.00 \\
14.33 \\
14.65 \\
14.98 \\
15.30 \\
15.63 \\
15.95 \\
16.28\end{array}$ & $\begin{array}{l}38.71 \\
39.65 \\
40.60 \\
41.54 \\
42.48 \\
43.43 \\
44.37 \\
45.32 \\
46.213 \\
47.20\end{array}$ & $\begin{array}{l}13.52 \\
13.85 \\
14.18 \\
14.51 \\
14.84 \\
15.17 \\
15.50 \\
15.83 \\
16.15 \\
16.48\end{array}$ & $\begin{array}{l}38.65 \\
39.59 \\
40.53 \\
41.48 \\
42.42 \\
43.36 \\
44.30 \\
45.25 \\
46.19 \\
47.13\end{array}$ & $\begin{array}{l}13.69 \\
14.02 \\
14.35 \\
14.69 \\
15.02 \\
15.36 \\
15.69 \\
16.02 \\
16.36 \\
16.69\end{array}$ & $\begin{array}{l}38.59 \\
39.53 \\
40.47 \\
41.41 \\
42.35 \\
43.29 \\
44.24 \\
45.18 \\
46.12 \\
47.06\end{array}$ & $\begin{array}{l}13.85 \\
14.19 \\
14.53 \\
14.87 \\
15.21 \\
15.54 \\
15.88 \\
16.22 \\
16.56 \\
16.90\end{array}$ & $\begin{array}{l}41 \\
42 \\
43 \\
44 \\
45 \\
46 \\
47 \\
48 \\
49 \\
50\end{array}$ \\
\hline छ & Dep. & Lat. & Dep. & Lat. & Dep. & Lat. & Dep. & Lat. & \\
\hline & \multicolumn{2}{|c|}{$71^{\circ}$. } & \multicolumn{2}{|c|}{$7034^{\circ}$. } & \multicolumn{2}{|c|}{$701 / 2^{\circ}$. } & \multicolumn{2}{|c|}{$701 / 4^{\circ}$. } & 7 \\
\hline
\end{tabular}


TABLE 4.-TBAVEISE TABLE.

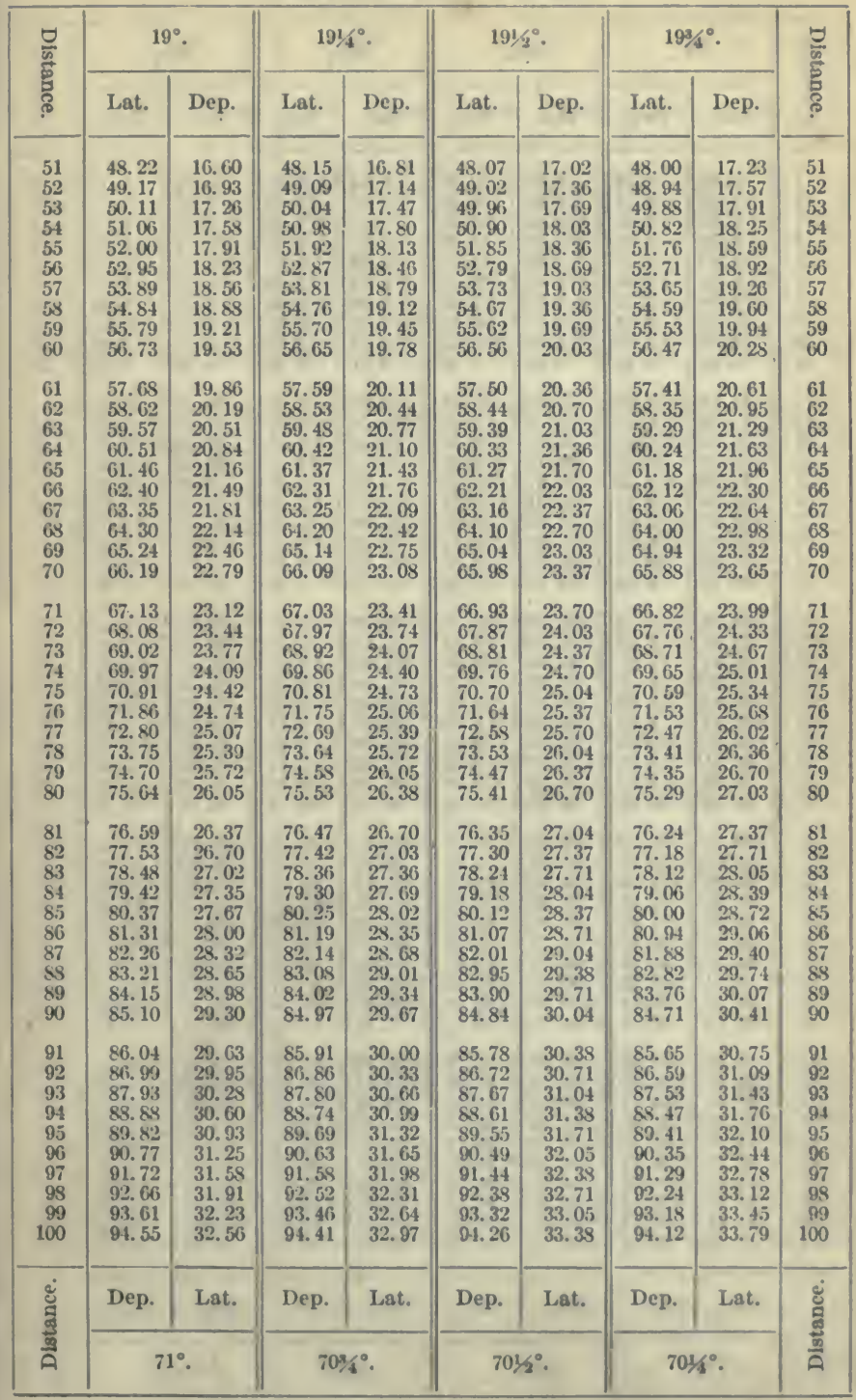


TABLE 4.-TRA VERSE TABLE.

\begin{tabular}{|c|c|c|c|c|c|c|c|c|c|}
\hline \multirow{2}{*}{ 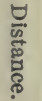 } & \multicolumn{2}{|c|}{$20^{\circ}$. } & \multicolumn{2}{|c|}{$201 / 4^{\circ}$. } & \multicolumn{2}{|c|}{$201 / 2^{\circ}$. } & \multicolumn{2}{|c|}{$20^{3} 4^{\circ}$. } & \multirow{2}{*}{ 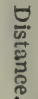 } \\
\hline & Lat. & Dep. & Lat. & Dep. & Lat. & Dep. & Lat. & Dep. & \\
\hline $\begin{array}{r}1 \\
2 \\
3 \\
4 \\
5 \\
6 \\
7 \\
8 \\
9 \\
10\end{array}$ & $\begin{array}{l}0.94 \\
1.88 \\
2.82 \\
3.76 \\
4.70 \\
5.64 \\
6.58 \\
7.52 \\
8.46 \\
9.40\end{array}$ & $\begin{array}{l}0.34 \\
0.68 \\
1.03 \\
1.37 \\
1.71 \\
2.05 \\
2.39 \\
2.74 \\
3.08 \\
3.42\end{array}$ & $\begin{array}{l}0.94 \\
1.88 \\
2.81 \\
3.75 \\
4.69 \\
5.63 \\
6.57 \\
7.51 \\
8.44 \\
9.38\end{array}$ & $\begin{array}{l}0.35 \\
0.69 \\
1.04 \\
1.38 \\
1.73 \\
2.08 \\
2.42 \\
2.77 \\
3.12 \\
3.46\end{array}$ & $\begin{array}{l}0.94 \\
1.87 \\
2.81 \\
3.75 \\
4.68 \\
5.62 \\
6.56 \\
7.49 \\
8.43 \\
9.37\end{array}$ & $\begin{array}{l}0.35 \\
0.70 \\
1.05 \\
1.40 \\
1.75 \\
2.10 \\
2.45 \\
2.80 \\
3.15 \\
3.50\end{array}$ & $\begin{array}{l}0.94 \\
1.87 \\
2.81 \\
3.74 \\
4 .(8 \\
5.61 \\
6.55 \\
7.48 \\
8.42 \\
9.35\end{array}$ & $\begin{array}{l}0.35 \\
0.71 \\
1.06 \\
1.42 \\
1.77 \\
2.13 \\
2.48 \\
2.83 \\
3.19 \\
3.54\end{array}$ & $\begin{array}{r}1 \\
2 \\
3 \\
4 \\
5 \\
6 \\
7 \\
8 \\
9 \\
10\end{array}$ \\
\hline $\begin{array}{l}11 \\
12 \\
13 \\
14 \\
15 \\
16 \\
17 \\
18 \\
19 \\
20\end{array}$ & $\begin{array}{l}10.34 \\
11.28 \\
12.22 \\
13.16 \\
14.10 \\
15.04 \\
15.97 \\
16.91 \\
17.85 \\
18.79\end{array}$ & $\begin{array}{l}3.76 \\
4.10 \\
4.45 \\
4.79 \\
5.13 \\
5.47 \\
5.81 \\
6.16 \\
6.50 \\
6.81\end{array}$ & $\begin{array}{l}10.32 \\
11.26 \\
12.20 \\
13.13 \\
14.07 \\
15.01 \\
15.95 \\
16.89 \\
17.83 \\
18.76\end{array}$ & $\begin{array}{l}3.81 \\
4.15 \\
4.50 \\
4.85 \\
5.19 \\
5.54 \\
5.88 \\
6.23 \\
6.58 \\
6.92\end{array}$ & $\begin{array}{l}10.30 \\
11.24 \\
12.18 \\
13.11 \\
14.05 \\
14.99 \\
15.92 \\
16.86 \\
17.80 \\
18.73\end{array}$ & $\begin{array}{l}3.85 \\
4.20 \\
4.55 \\
4.90 \\
5.25 \\
5.60 \\
5.95 \\
6.30 \\
6.65 \\
7.00\end{array}$ & $\begin{array}{l}10.29 \\
11.22 \\
12.16 \\
13.09 \\
14.03 \\
14.96 \\
15.90 \\
16.83 \\
17.77 \\
18.70\end{array}$ & $\begin{array}{l}3.90 \\
4.25 \\
4.61 \\
4.96 \\
5.31 \\
5.67 \\
6.02 \\
6.38 \\
6.73 \\
7.09\end{array}$ & $\begin{array}{l}11 \\
12 \\
13 \\
14 \\
15 \\
16 \\
17 \\
18 \\
19 \\
20\end{array}$ \\
\hline $\begin{array}{l}21 \\
22 \\
23 \\
24 \\
25 \\
26 \\
27 \\
28 \\
29 \\
30\end{array}$ & $\begin{array}{l}19.73 \\
20.67 \\
21.61 \\
22.55 \\
23.49 \\
24.43 \\
25.37 \\
26.31 \\
27.25 \\
28.19\end{array}$ & $\begin{array}{r}7.18 \\
7.52 \\
7.87 \\
8.21 \\
8.55 \\
8.59 \\
9.23 \\
9.58 \\
9.92 \\
10.26\end{array}$ & $\begin{array}{l}19.70 \\
20.64 \\
21.58 \\
22.52 \\
23.45 \\
24.39 \\
25.33 \\
26.27 \\
27.21 \\
28.15\end{array}$ & $\begin{array}{r}7.27 \\
7.61 \\
7.96 \\
8.31 \\
8.65 \\
9.00 \\
9.35 \\
9.69 \\
10.04 \\
10.38\end{array}$ & $\begin{array}{l}19.67 \\
20.61 \\
21.54 \\
22.48 \\
23.42 \\
24.35 \\
25.29 \\
26.23 \\
27.16 \\
28.10\end{array}$ & $\begin{array}{r}7.35 \\
7.70 \\
8.05 \\
8.40 \\
8.76 \\
9.11 \\
9.46 \\
9.81 \\
10.16 \\
10.51\end{array}$ & $\begin{array}{l}19.64 \\
20.57 \\
21.51 \\
22.44 \\
23.38 \\
24.31 \\
25.25 \\
26.18 \\
27.12 \\
28.05\end{array}$ & $\begin{array}{r}7.44 \\
7.79 \\
8.15 \\
8.50 \\
8.86 \\
9.21 \\
9.57 \\
9.92 \\
10.27 \\
10.63\end{array}$ & $\begin{array}{l}21 \\
22 \\
23 \\
24 \\
25 \\
26 \\
27 \\
28 \\
29 \\
30\end{array}$ \\
\hline $\begin{array}{l}31 \\
32 \\
33 \\
34 \\
35 \\
36 \\
37 \\
38 \\
39 \\
40\end{array}$ & $\begin{array}{l}29.13 \\
30.07 \\
31.01 \\
31.95 \\
32.89 \\
33.83 \\
34.77 \\
35.71 \\
36.65 \\
37.59\end{array}$ & $\begin{array}{l}10.60 \\
10.94 \\
11.29 \\
11.63 \\
11.97 \\
12.31 \\
12.65 \\
13.00 \\
13.34 \\
13.68\end{array}$ & $\begin{array}{l}29.08 \\
30.02 \\
30.96 \\
31.90 \\
32.84 \\
33.77 \\
34.71 \\
35.65 \\
36.59 \\
37.53\end{array}$ & $\begin{array}{l}10.73 \\
11.08 \\
11.42 \\
11.77 \\
12.11 \\
12.46 \\
12.81 \\
13.15 \\
13.50 \\
13.84\end{array}$ & $\begin{array}{l}29.04 \\
29.97 \\
30.91 \\
31.85 \\
32.78 \\
33.72 \\
34.66 \\
35.59 \\
36.53 \\
37.47\end{array}$ & $\begin{array}{l}10.86 \\
11.21 \\
11.56 \\
11.91 \\
12.26 \\
12.61 \\
12.96 \\
13.31 \\
13.66 \\
14.01\end{array}$ & $\begin{array}{l}28.99 \\
29.92 \\
30.86 \\
31.79 \\
32.73 \\
33.66 \\
34.60 \\
35.54 \\
36.47 \\
37.41\end{array}$ & $\begin{array}{l}10.98 \\
11.34 \\
11.69 \\
12.05 \\
12.40 \\
12.75 \\
13.11 \\
13.46 \\
13.82 \\
14.17\end{array}$ & $\begin{array}{l}31 \\
32 \\
33 \\
34 \\
35 \\
36 \\
37 \\
38 \\
39 \\
40\end{array}$ \\
\hline $\begin{array}{l}41 \\
42 \\
43 \\
44 \\
45 \\
46 \\
47 \\
48 \\
49 \\
50\end{array}$ & $\begin{array}{l}38.53 \\
39.47 \\
40.41 \\
41.35 \\
42.29 \\
43.23 \\
44.17 \\
45.11 \\
46.04 \\
46.98\end{array}$ & $\begin{array}{l}14.02 \\
14.36 \\
14.71 \\
15.05 \\
15.39 \\
15.73 \\
16.07 \\
16.42 \\
16.76 \\
17.10\end{array}$ & $\begin{array}{l}38.47 \\
39.40 \\
40.34 \\
41.28 \\
42.22 \\
43.16 \\
44.09 \\
45.03 \\
45.97 \\
46.91\end{array}$ & $\begin{array}{l}14.19 \\
14.54 \\
14.88 \\
15.23 \\
15.58 \\
15.92 \\
10.27 \\
16.61 \\
16.96 \\
17.31\end{array}$ & $\begin{array}{l}38.40 \\
39.34 \\
40.25 \\
41.21 \\
42.15 \\
43.09 \\
44.02 \\
44.96 \\
45.90 \\
46.83\end{array}$ & $\begin{array}{l}14.36 \\
14.71 \\
15.06 \\
15.41 \\
15.76 \\
16.11 \\
16.46 \\
16.81 \\
17.16 \\
17.51\end{array}$ & $\begin{array}{l}38.34 \\
39.28 \\
40.21 \\
41.15 \\
42.08 \\
43.02 \\
43.95 \\
44.89 \\
45.82 \\
46.76\end{array}$ & $\begin{array}{l}14.53 \\
14.88 \\
15.23 \\
15.59 \\
15.94 \\
16.30 \\
16.65 \\
17.01 \\
17.36 \\
17.71\end{array}$ & $\begin{array}{l}41 \\
42 \\
43 \\
44 \\
45 \\
46 \\
47 \\
48 \\
49 \\
50\end{array}$ \\
\hline త్ర & Dep. & Lat. & Dep. & Lat. & Dep. & Lat. & Dep. & Lat. & తீं \\
\hline$\frac{n}{a}$ & \multicolumn{2}{|c|}{$70^{\circ}$. } & \multicolumn{2}{|c|}{$6934^{\circ}$. } & \multicolumn{2}{|c|}{ C91/2 $2^{\circ}$. } & \multicolumn{2}{|c|}{$691 / 4^{\circ}$. } & $\frac{\vec{n}}{a}$ \\
\hline
\end{tabular}


TABLE 4.-TRAVERSE TABLE.

\begin{tabular}{|c|c|c|c|c|c|c|c|c|c|}
\hline \multirow{2}{*}{ 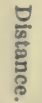 } & \multicolumn{2}{|c|}{$20^{\circ}$. } & \multicolumn{2}{|c|}{$201 / 4^{\circ}$. } & \multicolumn{2}{|c|}{$201 / 2^{\circ}$. } & \multicolumn{2}{|c|}{$20^{3} / 4^{\circ}$. } & \multirow{2}{*}{$\begin{array}{l}\frac{\overrightarrow{5}}{5} \\
\frac{5}{5} \\
\overrightarrow{5}\end{array}$} \\
\hline & Lat. & Dep. & Lat. & Dep. & Lat. & Dep. & Lat. & Dep. & \\
\hline $\begin{array}{l}51 \\
52 \\
53 \\
54 \\
55 \\
56 \\
57 \\
58 \\
59 \\
60\end{array}$ & $\begin{array}{l}47.92 \\
48.86 \\
49.50 \\
50.74 \\
51.68 \\
52.62 \\
53.56 \\
54.50 \\
55.44 \\
56.38\end{array}$ & $\begin{array}{l}17.44 \\
17.79 \\
18.13 \\
18.47 \\
18.81 \\
19.15 \\
19.50 \\
19.84 \\
20.18 \\
20.52\end{array}$ & $\begin{array}{l}47.85 \\
48.79 \\
49.72 \\
50.66 \\
51.60 \\
52.54 \\
53.48 \\
54.42 \\
55.35 \\
56.29\end{array}$ & $\begin{array}{l}17.65 \\
18.00 \\
18.34 \\
18.69 \\
19.04 \\
19.38 \\
19.73 \\
20.07 \\
20.42 \\
20.77\end{array}$ & $\begin{array}{l}47.77 \\
48.71 \\
49.64 \\
50.58 \\
51.52 \\
52.45 \\
53.39 \\
54.33 \\
55.26 \\
56.20\end{array}$ & $\begin{array}{l}17.86 \\
18.21 \\
18.56 \\
18.91 \\
19.26 \\
19.61 \\
19.96 \\
20.31 \\
20.66 \\
21.01\end{array}$ & $\begin{array}{l}47.69 \\
48.63 \\
49.55 \\
50.50 \\
51.43 \\
52.37 \\
53.30 \\
54.24 \\
55.17 \\
56.11\end{array}$ & $\begin{array}{l}18.07 \\
18.42 \\
18.78 \\
19.13 \\
19.49 \\
19.84 \\
20.19 \\
20.55 \\
20.90 \\
21.26\end{array}$ & $\begin{array}{l}51 \\
52 \\
53 \\
54 \\
55 \\
56 \\
57 \\
58 \\
59 \\
60\end{array}$ \\
\hline $\begin{array}{l}61 \\
62 \\
63 \\
64 \\
65 \\
66 \\
67 \\
68 \\
69 \\
70\end{array}$ & $\begin{array}{l}57.32 \\
58.20 \\
59.20 \\
60.14 \\
61.08 \\
62.02 \\
62.96 \\
63.91 \\
64.84 \\
65.78\end{array}$ & $\begin{array}{l}20.86 \\
21.21 \\
21.55 \\
21.89 \\
22.23 \\
22.57 \\
22.92 \\
23.26 \\
23.60 \\
23.94\end{array}$ & $\begin{array}{l}57.23 \\
58.17 \\
59.11 \\
60.04 \\
60.98 \\
61.92 \\
62.86 \\
63.80 \\
64.74 \\
65.67\end{array}$ & $\begin{array}{l}21.11 \\
21.46 \\
21.81 \\
22.15 \\
22.50 \\
22.84 \\
23.19 \\
23.54 \\
23.83 \\
24.23\end{array}$ & $\begin{array}{l}57.14 \\
58.07 \\
59.01 \\
59.95 \\
60.88 \\
61.82 \\
62.76 \\
63.69 \\
64.63 \\
65.57\end{array}$ & $\begin{array}{l}21.36 \\
21.71 \\
22.06 \\
22.41 \\
22.76 \\
23.11 \\
23.46 \\
23.81 \\
24.16 \\
24.51\end{array}$ & $\begin{array}{l}57.04 \\
57.98 \\
58.91 \\
59.85 \\
60.78 \\
61.72 \\
62.65 \\
63.59 \\
64.52 \\
65.46\end{array}$ & $\begin{array}{l}21.61 \\
21.97 \\
22.32 \\
22.67 \\
23.03 \\
23.38 \\
23.74 \\
24.09 \\
24.45 \\
24.80\end{array}$ & $\begin{array}{l}61 \\
62 \\
63 \\
64 \\
65 \\
66 \\
67 \\
68 \\
69 \\
70\end{array}$ \\
\hline $\begin{array}{l}71 \\
72 \\
73 \\
74 \\
75 \\
76 \\
77 \\
78 \\
79 \\
80\end{array}$ & $\begin{array}{l}66.72 \\
67.66 \\
68.60 \\
69.54 \\
70.48 \\
71.42 \\
72.36 \\
73.30 \\
74.24 \\
75.18\end{array}$ & $\begin{array}{l}24.28 \\
24.63 \\
24.97 \\
25.31 \\
25.65 \\
25.99 \\
26.34 \\
26.68 \\
27.02 \\
27.36\end{array}$ & $\begin{array}{l}66.61 \\
67.55 \\
68.49 \\
69.43 \\
70.36 \\
71.30 \\
72.24 \\
73.18 \\
74.12 \\
75.06\end{array}$ & $\begin{array}{l}24.57 \\
24.92 \\
25.27 \\
25.61 \\
25.96 \\
26.30 \\
26.65 \\
27.00 \\
27.34 \\
27.69\end{array}$ & $\begin{array}{l}66.50 \\
67.44 \\
68.38 \\
69.31 \\
70.25 \\
71.19 \\
72.12 \\
73.06 \\
74.00 \\
74.93\end{array}$ & $\begin{array}{l}24.86 \\
25.21 \\
25.57 \\
25.92 \\
26.27 \\
26.62 \\
26.97 \\
27.32 \\
27.67 \\
28.02\end{array}$ & $\begin{array}{l}66.39 \\
67.33 \\
68.26 \\
69.20 \\
70.14 \\
71.07 \\
72.01 \\
72.94 \\
73.88 \\
74.81\end{array}$ & $\begin{array}{l}25.15 \\
25.51 \\
25.86 \\
26.22 \\
26.57 \\
26.93 \\
27.28 \\
27.63 \\
27.99 \\
28.34\end{array}$ & $\begin{array}{l}71 \\
72 \\
73 \\
74 \\
75 \\
76 \\
77 \\
78 \\
79 \\
80\end{array}$ \\
\hline $\begin{array}{l}81 \\
82 \\
83 \\
84 \\
85 \\
86 \\
87 \\
89 \\
89 \\
90\end{array}$ & $\begin{array}{l}76.12 \\
77.05 \\
77.99 \\
78.93 \\
79.87 \\
80.81 \\
81.75 \\
82.69 \\
83.63 \\
84.57\end{array}$ & $\begin{array}{l}27.70 \\
28.05 \\
28.39 \\
28.73 \\
29.07 \\
29.41 \\
29.76 \\
30.10 \\
30.44 \\
30.78\end{array}$ & $\begin{array}{l}75.99 \\
76.93 \\
77.87 \\
78.81 \\
79.75 \\
80.68 \\
81.62 \\
82.56 \\
83.50 \\
84.44\end{array}$ & $\begin{array}{l}28.04 \\
28.38 \\
28.73 \\
29.07 \\
29.42 \\
29.77 \\
30.11 \\
30.46 \\
30.80 \\
31.15\end{array}$ & $\begin{array}{l}75.87 \\
76.81 \\
77.74 \\
78.68 \\
79.62 \\
80.55 \\
81.49 \\
82.43 \\
83.36 \\
84.30\end{array}$ & $\begin{array}{l}28.37 \\
28.72 \\
29.07 \\
29.42 \\
29.77 \\
30.12 \\
30.47 \\
30.82 \\
31.17 \\
31.52\end{array}$ & $\begin{array}{l}75.75 \\
76.68 \\
77.62 \\
78.55 \\
79.49 \\
80.42 \\
81.36 \\
82.29 \\
83.23 \\
84.16\end{array}$ & $\begin{array}{l}28.70 \\
29.05 \\
29.41 \\
29.76 \\
30.11 \\
30.47 \\
30.82 \\
31.15 \\
31.53 \\
31.89\end{array}$ & $\begin{array}{l}81 \\
82 \\
83 \\
84 \\
85 \\
86 \\
87 \\
88 \\
89 \\
90\end{array}$ \\
\hline $\begin{array}{r}91 \\
92 \\
93 \\
94 \\
95 \\
96 \\
97 \\
98 \\
99 \\
100\end{array}$ & $\begin{array}{l}85.51 \\
86.45 \\
87.39 \\
85.33 \\
89.27 \\
90.21 \\
91.15 \\
92.19 \\
93.03 \\
03.97\end{array}$ & $\begin{array}{l}31.12 \\
31.47 \\
31.81 \\
32.15 \\
32.49 \\
32.83 \\
33.18 \\
33.52 \\
33.86 \\
34.20\end{array}$ & $\begin{array}{l}85.38 \\
86.31 \\
57.25 \\
89.19 \\
89.13 \\
90.07 \\
91.00 \\
91.94 \\
92.88 \\
93.82\end{array}$ & $\begin{array}{l}31.50 \\
31.84 \\
32.19 \\
32.54 \\
32.88 \\
33.23 \\
33.57 \\
33.92 \\
34.27 \\
34.61\end{array}$ & $\begin{array}{l}85.24 \\
86.17 \\
87.11 \\
89.05 \\
88.98 \\
89.92 \\
90.86 \\
91.79 \\
92.73 \\
93.67\end{array}$ & $\begin{array}{l}31.87 \\
32.22 \\
32.57 \\
32.92 \\
33.27 \\
33.62 \\
33.97 \\
34.32 \\
34.67 \\
35.02\end{array}$ & $\begin{array}{l}85.10 \\
86.03 \\
86.97 \\
87.90 \\
88.84 \\
89.77 \\
90.71 \\
91.64 \\
92.78 \\
93.51\end{array}$ & $\begin{array}{l}32.24 \\
32.59 \\
32.95 \\
33.30 \\
33.66 \\
34.01 \\
34.37 \\
34.72 \\
35.07 \\
35.43\end{array}$ & $\begin{array}{r}91 \\
92 \\
93 \\
94 \\
95 \\
96 \\
97 \\
98 \\
99 \\
100\end{array}$ \\
\hline غु & Dep. & Lat. & Dep. & Lat. & Dep. & Lat. & Dep. & Lat. & छ \\
\hline$\frac{\square}{a}$ & \multicolumn{2}{|c|}{$70^{\circ}}$. & \multicolumn{2}{|c|}{$6934^{\circ}$. } & \multicolumn{2}{|c|}{$0912^{\circ}$. } & \multicolumn{2}{|c|}{$691 / 4^{\circ}}$. & $\frac{a}{a}$ \\
\hline
\end{tabular}


TABLE 4.-TIRAVERSE TABLE.

\begin{tabular}{|c|c|c|c|c|c|c|c|c|c|}
\hline \multirow{2}{*}{ 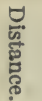 } & \multicolumn{2}{|c|}{$21^{\circ}$. } & \multicolumn{2}{|c|}{$211 / 4^{\circ}$. } & \multicolumn{2}{|c|}{$211 / 2^{\circ}$. } & \multicolumn{2}{|c|}{$2134^{\circ}$. } & \multirow{2}{*}{ 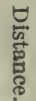 } \\
\hline & Lat. & Dep. & Lat. & Dep. & Lat. & Dep. & Lat. & Dep. & \\
\hline $\begin{array}{r}1 \\
2 \\
3 \\
4 \\
5 \\
6 \\
7 \\
8 \\
9 \\
10\end{array}$ & $\begin{array}{l}0.93 \\
1.87 \\
2.80 \\
3.73 \\
4.67 \\
5.60 \\
6.54 \\
7.47 \\
8.40 \\
9.34\end{array}$ & $\begin{array}{l}0.36 \\
0.72 \\
1.08 \\
1.43 \\
1.79 \\
2.15 \\
2.51 \\
2.87 \\
3.23 \\
3.58\end{array}$ & $\begin{array}{l}0.93 \\
1.86 \\
2.80 \\
3.73 \\
4.66 \\
5.59 \\
6.52 \\
7.46 \\
8.39 \\
9.32\end{array}$ & $\begin{array}{l}0.36 \\
0.72 \\
1.09 \\
1.45 \\
1.81 \\
2.17 \\
2.54 \\
2.90 \\
3.26 \\
3.62\end{array}$ & $\begin{array}{l}0.93 \\
1.86 \\
2.79 \\
3.72 \\
4.65 \\
5.58 \\
6.51 \\
7.44 \\
8.37 \\
9.30\end{array}$ & $\begin{array}{l}0.37 \\
0.73 \\
1.10 \\
1.47 \\
1.83 \\
2.20 \\
2.57 \\
2.93 \\
3.30 \\
3.67\end{array}$ & $\begin{array}{l}0.93 \\
1.86 \\
2.79 \\
3.72 \\
4.64 \\
5.57 \\
6.50 \\
7.43 \\
8.36 \\
9.29\end{array}$ & $\begin{array}{l}0.37 \\
0.74 \\
1.11 \\
1.48 \\
1.85 \\
2.22 \\
2.59 \\
2.96 \\
3.34 \\
3.71\end{array}$ & $\begin{array}{r}1 \\
2 \\
3 \\
4 \\
5 \\
6 \\
7 \\
8 \\
9 \\
10\end{array}$ \\
\hline $\begin{array}{l}11 \\
12 \\
13 \\
14 \\
15 \\
16 \\
17 \\
18 \\
19 \\
20\end{array}$ & $\begin{array}{l}10.27 \\
11.20 \\
12.14 \\
13.07 \\
14.00 \\
14.94 \\
15.87 \\
16.80 \\
17.74 \\
18.67\end{array}$ & $\begin{array}{l}3.94 \\
4.30 \\
4.66 \\
5.02 \\
5.38 \\
5.73 \\
6.09 \\
6.45 \\
6.81 \\
7.17\end{array}$ & $\begin{array}{l}10.25 \\
11.18 \\
12.12 \\
13.05 \\
13.98 \\
14.91 \\
15.84 \\
16.78 \\
17.71 \\
18.64\end{array}$ & $\begin{array}{l}3.99 \\
4.35 \\
4.71 \\
5.07 \\
5.44 \\
5.80 \\
6.16 \\
6.52 \\
6.89 \\
7.25\end{array}$ & $\begin{array}{l}10.23 \\
11.17 \\
12.10 \\
13.03 \\
13.96 \\
14.89 \\
15.82 \\
16.75 \\
17.68 \\
18.61\end{array}$ & $\begin{array}{l}4.03 \\
4.40 \\
4.76 \\
5.13 \\
5.50 \\
5.86 \\
6.23 \\
6.60 \\
6.96 \\
7.33\end{array}$ & $\begin{array}{l}10.22 \\
11.15 \\
12.07 \\
13.00 \\
13.93 \\
14.86 \\
15.79 \\
16.72 \\
17.65 \\
18.58\end{array}$ & $\begin{array}{l}4.08 \\
4.45 \\
4.82 \\
5.19 \\
5.56 \\
5.93 \\
6.30 \\
6.67 \\
7.04 \\
7.41\end{array}$ & $\begin{array}{l}11 \\
12 \\
13 \\
14 \\
15 \\
16 \\
17 \\
18 \\
19 \\
20\end{array}$ \\
\hline $\begin{array}{l}21 \\
22 \\
23 \\
24 \\
25 \\
26 \\
27 \\
28 \\
29 \\
30\end{array}$ & $\begin{array}{l}19.61 \\
20.54 \\
21.47 \\
22.41 \\
23.34 \\
24.27 \\
25.21 \\
26.14 \\
27.07 \\
28.01\end{array}$ & $\begin{array}{r}7.53 \\
7.88 \\
8.24 \\
8.60 \\
8.96 \\
9.32 \\
9.68 \\
10.03 \\
10.39 \\
10.75\end{array}$ & $\begin{array}{l}19.57 \\
20.50 \\
21.44 \\
22.37 \\
23.30 \\
24.23 \\
25.16 \\
26.10 \\
27.03 \\
27.96\end{array}$ & $\begin{array}{r}7.61 \\
7.97 \\
8.34 \\
8.70 \\
9.06 \\
9.42 \\
9.79 \\
10.15 \\
10.51 \\
10.87\end{array}$ & $\begin{array}{l}19.54 \\
20.47 \\
21.40 \\
22.33 \\
23.26 \\
24.19 \\
25.12 \\
26.05 \\
26.98 \\
27.91\end{array}$ & $\begin{array}{r}7.70 \\
8.06 \\
8.43 \\
8.80 \\
9.16 \\
9.53 \\
9.90 \\
10.26 \\
10.63 \\
11.00\end{array}$ & $\begin{array}{l}19.51 \\
20.43 \\
21.36 \\
22.29 \\
23.22 \\
24.15 \\
25.08 \\
26.01 \\
26.94 \\
27.86\end{array}$ & $\begin{array}{r}7.78 \\
8.15 \\
8.52 \\
8.89 \\
9.26 \\
9.63 \\
10.01 \\
10.38 \\
10.75 \\
11.12\end{array}$ & $\begin{array}{l}21 \\
22 \\
23 \\
24 \\
25 \\
26 \\
27 \\
28 \\
29 \\
30\end{array}$ \\
\hline $\begin{array}{l}31 \\
32 \\
33 \\
34 \\
35 \\
36 \\
37 \\
38 \\
39 \\
40\end{array}$ & $\begin{array}{l}28.94 \\
29.87 \\
30.81 \\
31.74 \\
32.68 \\
33.61 \\
34.54 \\
35.48 \\
36.41 \\
37.34\end{array}$ & $\begin{array}{l}11.11 \\
11.47 \\
11.83 \\
12.18 \\
12.54 \\
12.90 \\
13.26 \\
13.62 \\
13.98 \\
14.33\end{array}$ & $\begin{array}{l}28.89 \\
29.82 \\
30.76 \\
31.69 \\
32.62 \\
33.55 \\
34.48 \\
35.42 \\
36.35 \\
37.28\end{array}$ & $\begin{array}{l}11.24 \\
11.60 \\
11.96 \\
12.32 \\
12.69 \\
13.05 \\
13.41 \\
13.77 \\
14.14 \\
14.50\end{array}$ & $\begin{array}{l}28.84 \\
29.77 \\
30.70 \\
31.63 \\
32.56 \\
33.50 \\
34.43 \\
35.36 \\
36.29 \\
37.22\end{array}$ & $\begin{array}{l}11.36 \\
11.73 \\
12.09 \\
12.46 \\
12.83 \\
13.19 \\
13.56 \\
13.93 \\
14.29 \\
14.66\end{array}$ & $\begin{array}{l}28.79 \\
29.72 \\
30.65 \\
31.58 \\
32.51 \\
33.44 \\
34.37 \\
35.29 \\
36.22 \\
37.15\end{array}$ & $\begin{array}{l}11.49 \\
11.86 \\
12.23 \\
12.60 \\
12.97 \\
13.34 \\
13.71 \\
14.08 \\
14.45 \\
14.82\end{array}$ & $\begin{array}{l}31 \\
32 \\
33 \\
34 \\
35 \\
36 \\
37 \\
38 \\
39 \\
40\end{array}$ \\
\hline $\begin{array}{l}41 \\
42 \\
43 \\
44 \\
45 \\
46 \\
47 \\
48 \\
49 \\
50\end{array}$ & $\begin{array}{l}38.28 \\
39.21 \\
40.14 \\
41.08 \\
42.01 \\
42.94 \\
43.88 \\
44.81 \\
45.75 \\
46.68\end{array}$ & $\begin{array}{l}14.69 \\
15.05 \\
15.41 \\
15.77 \\
16.13 \\
16.48 \\
16.84 \\
17.20 \\
17.56 \\
17.92\end{array}$ & $\begin{array}{l}38.21 \\
39.14 \\
40.08 \\
41.01 \\
41.94 \\
42.87 \\
43.80 \\
44.74 \\
45.67 \\
46.60\end{array}$ & $\begin{array}{l}14.86 \\
15.22 \\
15.58 \\
15.95 \\
16.31 \\
16.67 \\
17.03 \\
17.40 \\
17.76 \\
18.12\end{array}$ & $\begin{array}{l}38.15 \\
39.08 \\
40.01 \\
40.94 \\
41.87 \\
42.80 \\
43.73 \\
44.66 \\
45.59 \\
46.52\end{array}$ & $\begin{array}{l}15.03 \\
15.39 \\
15.76 \\
16.13 \\
16.49 \\
16.86 \\
17.23 \\
17.59 \\
17.96 \\
18.33\end{array}$ & $\begin{array}{l}38.08 \\
39.01 \\
39.94 \\
40.87 \\
41.80 \\
42.73 \\
43.65 \\
44.58 \\
45.51 \\
46.44\end{array}$ & $\begin{array}{l}15.19 \\
15.56 \\
15.93 \\
16.30 \\
16.68 \\
17.05 \\
17.42 \\
17.79 \\
18.16 \\
18.53\end{array}$ & $\begin{array}{l}41 \\
42 \\
43 \\
44 \\
45 \\
46 \\
47 \\
48 \\
49 \\
50\end{array}$ \\
\hline छ் & Dep. & Lat. & Dep. & Lat. & Dep. & Lat. & Dep. & Lat. & غं \\
\hline A & \multicolumn{2}{|c|}{$69^{\circ}$. } & \multicolumn{2}{|c|}{$6834^{\circ}$. } & \multicolumn{2}{|c|}{$681 / 2^{\circ}$. } & \multicolumn{2}{|c|}{$681 / 4^{\circ}$. } & $\vec{A}$ \\
\hline
\end{tabular}


TABLE 4.-TRAVERSE TABLE.

\begin{tabular}{|c|c|c|c|c|c|c|c|c|c|}
\hline \multirow{2}{*}{$\begin{array}{l}\frac{0}{5} \\
\frac{5}{5} \\
\frac{5}{5} \\
\frac{5}{8}\end{array}$} & \multicolumn{2}{|c|}{$21^{\circ}$. } & \multicolumn{2}{|c|}{$211 / 4^{\circ}$. } & \multicolumn{2}{|c|}{$211 / 2^{\circ}$. } & \multicolumn{2}{|c|}{$2134^{\circ}$. } & \multirow{2}{*}{$\begin{array}{l}\text { b } \\
\text { ह5 } \\
\text { है } \\
\text { है }\end{array}$} \\
\hline & Lat. & Dep. & Lat. & Dep. & Lat. & Dep. & Lat. & Dep. & \\
\hline $\begin{array}{l}51 \\
52 \\
53 \\
54 \\
55 \\
56 \\
57 \\
55 \\
59 \\
60\end{array}$ & $\begin{array}{l}47.61 \\
48.55 \\
49.48 \\
50.41 \\
51.35 \\
52.28 \\
53.21 \\
54.15 \\
55.08 \\
56.01\end{array}$ & $\begin{array}{l}18.28 \\
18.64 \\
18.99 \\
19.35 \\
19.71 \\
20.07 \\
20.43 \\
20.79 \\
21.14 \\
21.50\end{array}$ & $\begin{array}{l}47.53 \\
48.46 \\
49.40 \\
50.33 \\
51.26 \\
52.19 \\
53.12 \\
54.06 \\
54.99 \\
55.92\end{array}$ & $\begin{array}{l}18.48 \\
18.85 \\
19.21 \\
19.57 \\
19.93 \\
20.30 \\
20.66 \\
21.02 \\
21.38 \\
21.75\end{array}$ & $\begin{array}{l}47.45 \\
43.38 \\
49.31 \\
50.24 \\
51.17 \\
52.10 \\
53.03 \\
53.96 \\
54.89 \\
55.83\end{array}$ & $\begin{array}{l}18.69 \\
19.06 \\
19.42 \\
19.79 \\
20.16 \\
20.52 \\
20.89 \\
21.26 \\
21.62 \\
21.99\end{array}$ & $\begin{array}{l}47.37 \\
48.30 \\
49.23 \\
50.16 \\
51.08 \\
52.01 \\
52.94 \\
53.87 \\
54.80 \\
55.73\end{array}$ & $\begin{array}{l}18.90 \\
19.27 \\
19.64 \\
20.01 \\
20.38 \\
20.75 \\
21.12 \\
21.49 \\
21.86 \\
22.23\end{array}$ & $\begin{array}{l}51 \\
52 \\
53 \\
54 \\
55 \\
56 \\
57 \\
58 \\
59 \\
60\end{array}$ \\
\hline $\begin{array}{l}61 \\
62 \\
63 \\
64 \\
65 \\
66 \\
67 \\
68 \\
69 \\
70\end{array}$ & $\begin{array}{l}56.95 \\
57.83 \\
58.82 \\
59.75 \\
60.68 \\
61.62 \\
62.55 \\
63.48 \\
64.42 \\
65.35\end{array}$ & $\begin{array}{l}21.86 \\
22.22 \\
22.58 \\
22.94 \\
23.29 \\
23.65 \\
24.01 \\
24.37 \\
24.73 \\
25.09\end{array}$ & $\begin{array}{l}56.85 \\
57.78 \\
58.72 \\
59.65 \\
60.58 \\
61.51 \\
62.44 \\
63.38 \\
64.31 \\
65.24\end{array}$ & $\begin{array}{l}22.11 \\
22.47 \\
22.83 \\
23.20 \\
23.56 \\
23.92 \\
24.29 \\
24.65 \\
25.01 \\
25.37\end{array}$ & $\begin{array}{l}56.76 \\
57.69 \\
58.62 \\
59.55 \\
60.48 \\
61.41 \\
62.34 \\
63.27 \\
64.20 \\
65.13\end{array}$ & $\begin{array}{l}22.36 \\
22.72 \\
23.09 \\
23.46 \\
23.82 \\
24.19 \\
24.56 \\
24.92 \\
25.29 \\
25.66\end{array}$ & $\begin{array}{l}56.66 \\
57.59 \\
58.52 \\
59.44 \\
60.37 \\
61.30 \\
62.23 \\
63.16 \\
64.09 \\
65.02\end{array}$ & $\begin{array}{l}22.60 \\
22.97 \\
23.35 \\
23.72 \\
24.09 \\
24.46 \\
24.53 \\
25.20 \\
25.57 \\
25.94\end{array}$ & $\begin{array}{l}61 \\
62 \\
63 \\
64 \\
65 \\
66 \\
67 \\
68 \\
69 \\
70\end{array}$ \\
\hline $\begin{array}{l}71 \\
72 \\
73 \\
74 \\
75 \\
76 \\
77 \\
78 \\
79 \\
80\end{array}$ & $\begin{array}{l}66.28 \\
67.22 \\
68.15 \\
69.08 \\
70.02 \\
70.95 \\
71.89 \\
72.82 \\
73.75 \\
74.69\end{array}$ & $\begin{array}{l}25.44 \\
25.80 \\
26.16 \\
26.52 \\
26.88 \\
27.24 \\
27.59 \\
27.95 \\
28.31 \\
28.67\end{array}$ & $\begin{array}{l}66.17 \\
67.10 \\
68.04 \\
68.97 \\
69.90 \\
70.83 \\
71.76 \\
72.70 \\
73.63 \\
74.56\end{array}$ & $\begin{array}{l}25.73 \\
26.10 \\
26.46 \\
26.82 \\
27.18 \\
27.55 \\
27.91 \\
28.27 \\
28.63 \\
29.00\end{array}$ & $\begin{array}{l}66.06 \\
66.99 \\
67.92 \\
68.85 \\
69.75 \\
70.71 \\
71.64 \\
72.57 \\
73.50 \\
74.43\end{array}$ & $\begin{array}{l}26.02 \\
26.39 \\
26.75 \\
27.12 \\
27.49 \\
27.85 \\
28.22 \\
28.59 \\
28.95 \\
29.32\end{array}$ & $\begin{array}{l}65.95 \\
66.87 \\
67.80 \\
68.73 \\
69.66 \\
70.59 \\
71.52 \\
72.45 \\
73.38 \\
74.30\end{array}$ & $\begin{array}{l}26.31 \\
26.68 \\
27.05 \\
27.42 \\
27.79 \\
28.16 \\
28.53 \\
28.90 \\
29.27 \\
29.64\end{array}$ & $\begin{array}{l}71 \\
72 \\
73 \\
74 \\
75 \\
76 \\
77 \\
78 \\
79 \\
80\end{array}$ \\
\hline $\begin{array}{l}81 \\
82 \\
83 \\
84 \\
85 \\
86 \\
87 \\
83 \\
89 \\
90\end{array}$ & $\begin{array}{l}75.62 \\
76.55 \\
77.49 \\
73.42 \\
79.35 \\
80.29 \\
81.22 \\
82.16 \\
83.09 \\
84.02\end{array}$ & $\begin{array}{l}29.03 \\
29.39 \\
29.74 \\
30.10 \\
30.46 \\
30.82 \\
31.18 \\
31.54 \\
31.89 \\
32.25\end{array}$ & $\begin{array}{l}75.49 \\
76.42 \\
77.36 \\
78.29 \\
79.22 \\
80.15 \\
81.08 \\
82.02 \\
82.95 \\
83.88\end{array}$ & $\begin{array}{l}29.36 \\
29.72 \\
30.08 \\
30.44 \\
30.81 \\
31.17 \\
31.53 \\
31.89 \\
32.26 \\
32.62\end{array}$ & $\begin{array}{l}75.36 \\
76.29 \\
77.22 \\
78.16 \\
79.09 \\
80.02 \\
80.95 \\
81.88 \\
82.81 \\
83.74\end{array}$ & $\begin{array}{l}29.69 \\
30.05 \\
30.42 \\
30.79 \\
31.15 \\
31.52 \\
31.89 \\
32.25 \\
32.62 \\
32.99\end{array}$ & $\begin{array}{l}75.23 \\
76.16 \\
77.09 \\
78.02 \\
78.95 \\
79.88 \\
89.81 \\
81.74 \\
82.66 \\
83.59\end{array}$ & $\begin{array}{l}30.02 \\
30.39 \\
30.76 \\
31.13 \\
31.50 \\
31.87 \\
32.24 \\
32.61 \\
32.98 \\
33.35\end{array}$ & $\begin{array}{l}81 \\
82 \\
83 \\
84 \\
85 \\
86 \\
87 \\
88 \\
89 \\
90\end{array}$ \\
\hline $\begin{array}{r}91 \\
92 \\
93 \\
94 \\
95 \\
96 \\
97 \\
98 \\
99 \\
100\end{array}$ & $\begin{array}{l}84.96 \\
85.89 \\
86.82 \\
87.76 \\
88.69 \\
89.62 \\
90.56 \\
91.49 \\
92.42 \\
93.36\end{array}$ & $\begin{array}{l}32.61 \\
32.97 \\
33.33 \\
33.69 \\
34.04 \\
34.40 \\
34.76 \\
35.12 \\
35.48 \\
35.84\end{array}$ & $\begin{array}{l}84.81 \\
85.74 \\
86.68 \\
87.61 \\
88.54 \\
89.47 \\
90.40 \\
91.34 \\
92.27 \\
93.20\end{array}$ & $\begin{array}{l}32.98 \\
33.34 \\
33.71 \\
34.07 \\
34.43 \\
34.79 \\
35.16 \\
35.52 \\
35.88 \\
36.24\end{array}$ & $\begin{array}{l}84.67 \\
85.60 \\
86.53 \\
87.46 \\
88.39 \\
89.32 \\
90.25 \\
91.18 \\
92.11 \\
93.04\end{array}$ & $\begin{array}{l}33.35 \\
33.72 \\
34.08 \\
34.45 \\
34.82 \\
35.18 \\
35.55 \\
35.92 \\
36.2 \\
36.65\end{array}$ & $\begin{array}{l}84.52 \\
85.45 \\
86.38 \\
87.31 \\
88.21 \\
89.17 \\
90.09 \\
91.02 \\
91.95 \\
92.85\end{array}$ & $\begin{array}{l}33.72 \\
34.09 \\
34.46 \\
34.83 \\
35.20 \\
35.57 \\
35.94 \\
36.31 \\
36.69 \\
37.06\end{array}$ & $\begin{array}{r}91 \\
92 \\
93 \\
94 \\
95 \\
96 \\
97 \\
98 \\
99 \\
100\end{array}$ \\
\hline \multirow{2}{*}{$\begin{array}{l}\text { சू } \\
\text { 용 } \\
\frac{3}{0}\end{array}$} & Dep. & Lat. & Dep. & Lat. & Dep. & Lat. & Dep. & Lat. & \multirow{2}{*}{ 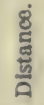 } \\
\hline & & & & & & & & & \\
\hline
\end{tabular}


TABLE 4.-TRAVERSE TABLE.

\begin{tabular}{|c|c|c|c|c|c|c|c|c|c|}
\hline \multirow{2}{*}{ 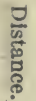 } & \multicolumn{2}{|c|}{$22^{\circ}$. } & \multicolumn{2}{|c|}{$221 / 4^{\circ}$. } & \multicolumn{2}{|c|}{$221 / 2^{\circ}$. } & \multicolumn{2}{|c|}{$2234^{\circ}$. } & \multirow{2}{*}{ 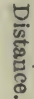 } \\
\hline & Lat. & Dep. & Lat. & Dep. & Lat. & Dep. & Lat. & Dep. & \\
\hline $\begin{array}{r}1 \\
2 \\
3 \\
3 \\
4 \\
5 \\
6 \\
6 \\
7 \\
8 \\
8 \\
9 \\
10\end{array}$ & $\begin{array}{l}0.93 \\
1.85 \\
2.78 \\
3.71 \\
4.61 \\
5.56 \\
6.49 \\
7.42 \\
8.34 \\
9.27\end{array}$ & $\begin{array}{l}0.37 \\
0.75 \\
1.12 \\
1.50 \\
1.87 \\
2.25 \\
2.62 \\
3.00 \\
3.37 \\
3.75\end{array}$ & $\begin{array}{l}0.93 \\
1.85 \\
2.78 \\
3.70 \\
4.63 \\
5.55 \\
6.48 \\
7.40 \\
8.33 \\
9.26\end{array}$ & $\begin{array}{l}0.38 \\
0.76 \\
1.14 \\
1.51 \\
1.89 \\
2.27 \\
2.65 \\
3.03 \\
3.41 \\
3.79\end{array}$ & $\begin{array}{l}0.92 \\
1.85 \\
2.77 \\
3.70 \\
4.62 \\
5.54 \\
6.47 \\
7.39 \\
8.31 \\
9.24\end{array}$ & $\begin{array}{l}0.38 \\
0.77 \\
1.15 \\
1.53 \\
1.91 \\
2.30 \\
2.68 \\
3.06 \\
3.44 \\
3.83\end{array}$ & $\begin{array}{l}0.92 . \\
1.84 \\
2.77 \\
3.69 \\
4.61 \\
5.53 \\
6.46 \\
7.35 \\
8.30 \\
9.22\end{array}$ & $\begin{array}{l}0.39 \\
0.77 \\
1.16 \\
1.55 \\
1.93 \\
2.32 \\
2.71 \\
3.09 \\
3.49 \\
3.87\end{array}$ & $\begin{array}{r}7 \\
8 \\
9 \\
10\end{array}$ \\
\hline $\begin{array}{l}11 \\
12 \\
13 \\
14 \\
15 \\
16 \\
17 \\
18 \\
18 \\
19 \\
20\end{array}$ & $\begin{array}{l}10.20 \\
11.13 \\
12.05 \\
12.98 \\
13.91 \\
14.83 \\
15.76 \\
16.69 \\
17.62 \\
18.54\end{array}$ & $\begin{array}{l}4.12 \\
4.50 \\
4.87 \\
5.24 \\
5.62 \\
5.99 \\
6.37 \\
6.74 \\
7.12 \\
7.49\end{array}$ & $\begin{array}{l}10.18 \\
11.11 \\
12.03 \\
12.96 \\
13.88 \\
14.81 \\
15.73 \\
16.66 \\
17.59 \\
18.51\end{array}$ & $\begin{array}{l}4.17 \\
4.54 \\
4.92 \\
5.30 \\
5.68 \\
6.06 \\
6.44 \\
6.82 \\
7.19 \\
7.57\end{array}$ & $\begin{array}{l}10.16 \\
11.09 \\
12.01 \\
12.93 \\
13.86 \\
14.78 \\
15.71 \\
16.63 \\
17.55 \\
18.48\end{array}$ & $\begin{array}{l}4.21 \\
4.59 \\
4.97 \\
5.36 \\
5.74 \\
6.12 \\
6.51 \\
6.89 \\
7.27 \\
7.65\end{array}$ & $\begin{array}{l}10.14 \\
11.07 \\
11.99 \\
12.91 \\
13.83 \\
14.76 \\
15.68 \\
16.60 \\
17.52 \\
18.44\end{array}$ & $\begin{array}{l}4.25 \\
4.64 \\
5.03 \\
5.41 \\
5.80 \\
6.19 \\
6.57 \\
6.96 \\
7.35 \\
7.73\end{array}$ & $\begin{array}{l}11 \\
12 \\
13 \\
14 \\
15 \\
16 \\
17 \\
18 \\
19 \\
20\end{array}$ \\
\hline $\begin{array}{l}21 \\
22 \\
23 \\
24 \\
24 \\
25 \\
26 \\
27 \\
27 \\
28 \\
29 \\
-30\end{array}$ & $\begin{array}{l}19.47 \\
20.40 \\
21.33 \\
22.25 \\
23.18 \\
24.11 \\
25.03 \\
25.96 \\
26.89 \\
27.82\end{array}$ & $\begin{array}{r}7.87 \\
8.24 \\
8.62 \\
8.99 \\
9.37 \\
9.74 \\
10.11 \\
10.49 \\
10.86 \\
11.24\end{array}$ & $\begin{array}{l}19.44 \\
20.36 \\
21.29 \\
22.21 \\
23.14 \\
24.06 \\
24.99 \\
25.92 \\
26.84 \\
27.77\end{array}$ & $\begin{array}{r}7.95 \\
8.33 \\
8.71 \\
9.09 \\
9.47 \\
9.84 \\
10.22 \\
10.100 \\
10.98 \\
11.36\end{array}$ & $\begin{array}{l}19.40 \\
20.33 \\
21.25 \\
22.17 \\
23.10 \\
24.02 \\
24.94 \\
25.87 \\
26.79 \\
27.72\end{array}$ & $\begin{array}{r}8.04 \\
8.42 \\
8.80 \\
9.18 \\
9.57 \\
9.95 \\
10.33 \\
10.72 \\
11.10 \\
11.48\end{array}$ & $\begin{array}{l}19.37 \\
20.29 \\
21.21 \\
22.13 \\
23.06 \\
23.98 \\
24.90 \\
25.82 \\
26.74 \\
27.67\end{array}$ & $\begin{array}{r}8.12 \\
8.51 \\
8.89 \\
9.28 \\
9.67 \\
10.05 \\
10.44 \\
10.83 \\
11.21 \\
11.60\end{array}$ & $\begin{array}{l}21 \\
22 \\
23 \\
24 \\
25 \\
26 \\
27 \\
28 \\
29 \\
30\end{array}$ \\
\hline $\begin{array}{l}31 \\
32 \\
33 \\
34 \\
35 \\
36 \\
37 \\
38 \\
39 \\
40\end{array}$ & $\begin{array}{l}28.74 \\
29.67 \\
30.60 \\
31.52 \\
32.45 \\
33.38 \\
34.31 \\
35.23 \\
36.16 \\
37.09\end{array}$ & $\begin{array}{l}11.61 \\
11.99 \\
12.36 \\
12.74 \\
13.11 \\
13.49 \\
13.86 \\
14.24 \\
14.61 \\
14.98\end{array}$ & $\begin{array}{l}28.69 \\
29.62 \\
30.54 \\
31.47 \\
32.39 \\
33.32 \\
34.24 \\
35.17 \\
36.10 \\
37.02\end{array}$ & $\begin{array}{l}11.74 \\
12.12 \\
12.50 \\
12.87 \\
13.25 \\
13.63 \\
14.01 \\
14.39 \\
14.77 \\
15.15\end{array}$ & $\begin{array}{l}28.64 \\
29.56 \\
30.49 \\
31.41 \\
32.34 \\
33.26 \\
34.18 \\
35.11 \\
36.03 \\
36.96\end{array}$ & $\begin{array}{l}11.86 \\
12.25 \\
12.63 \\
13.01 \\
13.39 \\
13.78 \\
14.16 \\
14.54 \\
14.92 \\
15.31\end{array}$ & $\begin{array}{l}28.59 \\
29.51 \\
30.43 \\
31.35 \\
32.28 \\
33.20 \\
34.12 \\
35.04 \\
35.97 \\
36.89\end{array}$ & $\begin{array}{l}11.99 \\
12.37 \\
12.76 \\
13.15 \\
13.53 \\
13.92 \\
14.31 \\
14.70 \\
15.08 \\
15.47\end{array}$ & $\begin{array}{l}31 \\
32 \\
33 \\
34 \\
35 \\
36 \\
37 \\
35 \\
39 \\
40\end{array}$ \\
\hline $\begin{array}{l}41 \\
42 \\
43 \\
44 \\
45 \\
46 \\
47 \\
48 \\
49 \\
50\end{array}$ & $\begin{array}{l}38.01 \\
38.94 \\
39.87 \\
40.80 \\
41.72 \\
42.65 \\
43.58 \\
44.50 \\
45.43 \\
46.36\end{array}$ & $\begin{array}{l}15.36 \\
15.73 \\
16.11 \\
16.48 \\
16.86 \\
17.23 \\
17.61 \\
17.98 \\
18.36 \\
18.73\end{array}$ & $\begin{array}{l}37.95 \\
38.87 \\
39.80 \\
40.72 \\
41.65 \\
42.57 \\
43.50 \\
44.43 \\
45.35 \\
46.28\end{array}$ & $\begin{array}{l}15.52 \\
15.90 \\
16.28 \\
16.66 \\
17.04 \\
17.42 \\
17.80 \\
18.18 \\
18.55 \\
18.93\end{array}$ & $\begin{array}{l}37.88 \\
38.80 \\
39.73 \\
40.65 \\
41.57 \\
42.50 \\
43.42 \\
44.35 \\
45.27 \\
46.19\end{array}$ & $\begin{array}{l}15.69 \\
16.07 \\
16.46 \\
16.84 \\
17.22 \\
17.60 \\
17.99 \\
18.37 \\
18.75 \\
19.13\end{array}$ & $\begin{array}{l}37.81 \\
38.73 \\
39.65 \\
40.58 \\
41.50 \\
42.42 \\
43.34 \\
44.27 \\
45.19 \\
46.11\end{array}$ & $\begin{array}{l}15.86 \\
16.24 \\
16.63 \\
17.02 \\
17.40 \\
17.79 \\
18.18 \\
18.56 \\
18.95 \\
19.34\end{array}$ & $\begin{array}{l}41 \\
42 \\
43 \\
44 \\
45 \\
46 \\
47 \\
48 \\
49 \\
50\end{array}$ \\
\hline త్ & Dep. & Lat. & Dep. & Lat. & Dep. & Lat. & Dep. & Lat. & $\bigotimes^{\circ}$ \\
\hline$\stackrel{\text { ติ }}{\circ}$ & \multicolumn{2}{|c|}{$65^{\circ}$. } & \multicolumn{2}{|c|}{$6 \pi^{3} / 4^{\circ}}$. & \multicolumn{2}{|c|}{ (i) $1 / 2^{\circ}$. } & \multicolumn{2}{|c|}{$6734^{\circ}}$. & \\
\hline
\end{tabular}


TAIBLE 4.-TRAVERSE: TABLF.

\begin{tabular}{|c|c|c|c|c|c|c|c|c|c|}
\hline \multirow{2}{*}{ 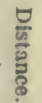 } & \multicolumn{2}{|c|}{$22^{\circ}$. } & \multicolumn{2}{|c|}{$221 / 4^{\circ}$. } & \multicolumn{2}{|c|}{$221 / 3^{\circ}$. } & \multicolumn{2}{|c|}{$2234^{\circ}$. } & \multirow{2}{*}{$\frac{\frac{\theta}{n}}{\frac{0}{6}}$} \\
\hline & Lat. & Dep. & Lat. & Dep. & Lat. & Dep. & Lat. & Dep. & \\
\hline 51 & 47.29 & 19.10 & 47.20 & 19. 31 & 47. 12 & 19.52 & 47.03 & 19. 72 & 51 \\
\hline 52 & 48. 21 & 19.48 & 48. 13 & 19. 69 & 48. 04 & 19.90 & 47.95 & 20.11 & 52 \\
\hline 53 & 49.14 & 19. S5 & 49.05 & 20.07 & 48.97 & 20.28 & 48. 58 & 20.50 & 53 \\
\hline 54 & 50.07 & 20.23 & 49.98 & 20.45 & 49. 89 & 20.66 & 49. 80 & 20.88 & 54 \\
\hline 55 & 51.00 & 20.60 & 50.90 & 20.83 & 50.81 & 21.05 & 50.72 & 21.27 & 55 \\
\hline 56 & 51.92 & 20 . 9 & 51.83 & 21.20 & 51.74 & 21.43 & 51.64 & 21.66 & 56 \\
\hline 57 & 52.85 & 21.35 & 52.76 & 21.58 & 52.66 & 21.81 & 52.57 & 22.04 & 57 \\
\hline is & 53.78 & 21.73 & 53.68 & 21.98 & 53.59 & 22.20 & 53. 49 & 22.43 & 58 \\
\hline 59 & 54.70 & 22.10 & 54.61 & 22.34 & 54.51 & 22.58 & 54.41 & 22.82 & 59 \\
\hline 60 & 55.63 & 22.48 & 55.53 & 22.72 & 55. 43 & 22.96 & 55.33 & 23. 20 & 60 \\
\hline 61 & 56.56 & 22. 85 & 56. 46 & 23.10 & 56.36 & 23. 34 & 56.25 & 23.59 & 61 \\
\hline 62 & 57.49 & 23. 23 & 57.38 & 23. 48 & 57.28 & 23.73 & 57.18 & 23.98 & 62 \\
\hline 63 & 58.11 & 23.60 & 55. 31 & 23.85 & 58. 20 & 24.11 & 5. 10 & 24. 36 & 63 \\
\hline 64 & $5 ! 34$ & 23. .77 & 59. 23 & 24.23 & 59. 13 & 24.49 & 59.02 & 24.75 & 64 \\
\hline 65 & 60. 27 & 24.35 & (50. 16 & 24.61 & 60.05 & 24.87 & 59.94 & 25.14 & 65 \\
\hline 66 & 61.19 & 24.72 & 61.09 & 24.99 & 60.98 & 25.26 & 60.87 & 25.52 & 66 \\
\hline 67 & 62.12 & 25.10 & 62.01 & 25.37 & 61.90 & 25.64 & (61).79 & 25.91 & 67 \\
\hline 68 & 63. 05 & 25.47 & 62.94 & 25.75 & 62.82 & 26.02 & 62.71 & 26.30 & 68 \\
\hline 69 & 63. 98 & 25.85 & 63. 86 & 26.13 & 63. 75 & 26.41 & 63. 63 & 23. fis & 69 \\
\hline 70 & 64.90 & 26.22 & 64.79 & 26.51 & 64.67 & 26.79 & 64.55 & 27.07 & 70 \\
\hline 71 & 6.5. 83 & 26.60 & 65. 71 & 26. 88 & 65.60 & 27.17 & 65.48 & 27. 46 & 71 \\
\hline 72 & (56. 76 & 26.97 & 66.64 & 27.26 & 66. 52 & 27.55 & 66. 40 & 27.81 & 72 \\
\hline 73 & 67.68 & 27.35 & 67.56 & 27.64 & 67.41 & 27.94 & 67.32 & 28. 23 & 73 \\
\hline 74 & 68.61 & 27.72 & 68. 49 & 28.02 & 68. 37 & 28.32 & 68.21 & 28. 62 & 74 \\
\hline 75 & 69.54 & 28.10 & 6.9. 42 & 28.40 & 69.29 & 28.70 & 69.17 & 29.00 & 75 \\
\hline 76 & 70.47 & 28. 47 & 70.34 & 28. 78 & 70.21 & 29.08 & 70.09 & 29. 39 & 76 \\
\hline 77 & 71.39 & 28.84 & 71.27 & 29.16 & 71.14 & 29.47 & 71.01 & 29. 78 & 77 \\
\hline 78 & 72.32 & 29. 22 & 72.19 & 29.53 & 72.06 & 29.85 & 71.93 & 30.16 & 78 \\
\hline 79 & 73.25 & 29.59 & 73. 12 & 29.91 & 72.99 & 30. 23 & 72. 55 & 30.55 & 79 \\
\hline 80 & 74.17 & 29.97 & 74.04 & 30.29 & 73. 91 & 30.61 & 73.78 & 30.94 & 80 \\
\hline 81 & 75.10 & 39. 34 & 74.97 & 30. 67 & 74. 83 & 31.00 & 74.70 & 31. 32 & 81 \\
\hline 82 & 76.03 & 30.72 & 75. 89 & 31.05 & 75.76 & 31.38 & 75.62 & 31.71 & 82 \\
\hline 83 & 76.96 & 31.09 & 76.82 & 31. 43 & 76.68 & 31.76 & 76.54 & 32. 10 & 83 \\
\hline 84 & 77. 88 & 31.47 & 77.75 & 31.81 & 77.61 & 32.15 & 77.46 & 32. is & 81 \\
\hline 85 & 78.81 & 31.84 & 78.67 & 32. 19 & 78.53 & 32.53 & 78.39 & 32. 87 & 85 \\
\hline $8 i$ & 79.71 & 32.22 & 79.60 & 32.56 & 79.45 & 32.91 & 79. 31 & 33.26 & 86 \\
\hline 87 & 80. fi & 32.59 & 80.52 & 32.94 & 80. 38 & 33. 29 & S0. 23 & 33. 64 & 87 \\
\hline 8.8 & 81.59 & 32.97 & 81.45 & 33. 32 & 81.30 & 33. 18 & 81.15 & 34.03 & 88 \\
\hline 89 & 83.52 & 33. 34 & 82.37 & 33.70 & 82. 23 & 34.065 & 82.08 & 34. 42 & 89 \\
\hline 90 & 83.45 & 33.71 & 83. 30 & 34.08 & 83.15 & 34.44 & 83.00 & 34.80 & 90 \\
\hline 91 & S1. 37 & 34.09 & 81.22 & 34.46 & 81.07 & 34.82 & 83.92 & 35. 19 & 91 \\
\hline 92 & 85.30 & 34. 46 & 85.15 & 34.84 & 85.00 & 35.21 & 84.84 & 35. 58 & 02 \\
\hline 93 & S6. 23 & 34.84 & 86.08 & 35. 21 & 85. 92 & 3.5. 59 & 85.76 & 35. 96 & 93 \\
\hline 94 & 87.16 & $3.5,21$ & 87.00 & 35. 59 & 8ti. 84 & 35. 97 & 81. 69 & 36. 35 & 94 \\
\hline 95 & 88. 08 & 35. 59 & 87.93 & 35.97 & 87.77 & 36. 35 & 87.61 & 36.71 & 95 \\
\hline 93 & 89. 01 & 35.96 & 8. 85 & 36.35 & 88.69 & 33. 74 & 8R. 53 & 37.12 & 96 \\
\hline $9 i$ & 83.94 & 36. 34 & 89. 78 & 36. 73 & 89.62 & 37.12 & 80. 45 & 37.51 & 97 \\
\hline 08 & 99. 86 & 36. $7 i$ & 90. 70 & 37.11 & 90.54 & 37.50 & 90.35 & 37.90 & 98 \\
\hline 99 & 91.79 & 37.09 & 91.63 & 37.49 & 91.46 & 37.89 & 91. 30 & 35. 28 & 9 \\
\hline 100 & 02.72 & 37.46 & 92.55 & 37.86 & 92. 39 & 38. 27 & 92.22 & 38.67 & 100 \\
\hline \multirow{2}{*}{ 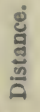 } & Dep. & Lat. & Dep. & Lat. & Dep. & Lat. & Dep. & Lat. & \multirow{2}{*}{ 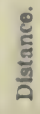 } \\
\hline & \multicolumn{2}{|c|}{$68^{\circ}}$. & \multicolumn{2}{|c|}{$6.34^{\circ}}$. & \multicolumn{2}{|c|}{$6715^{\circ}}$. & \multicolumn{2}{|c|}{$671 / 4^{\circ}}$. & \\
\hline
\end{tabular}


TABLE 4.-TRAVERSE TABIF.

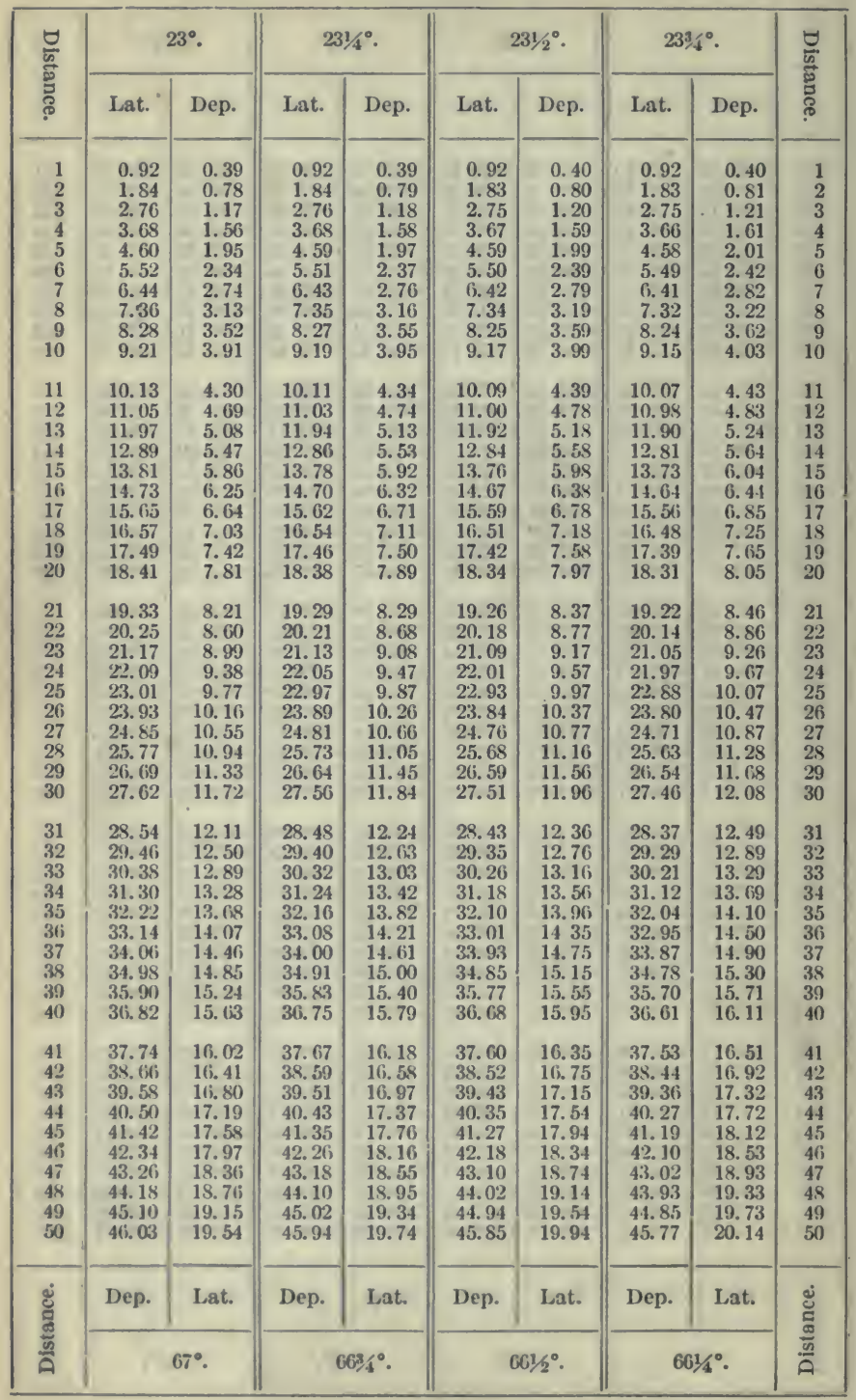


TABLE 4.-TRAVERSE TABIE.

\begin{tabular}{|c|c|c|c|c|c|c|c|c|c|}
\hline \multirow{2}{*}{ 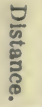 } & \multicolumn{2}{|c|}{$23^{\circ}$. } & \multicolumn{2}{|c|}{$231 / 4^{\circ}$. } & \multicolumn{2}{|c|}{$231 / 2^{\circ}$. } & \multicolumn{2}{|c|}{$234^{\circ}$. } & \multirow{2}{*}{ 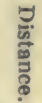 } \\
\hline & Lat. & Dep. & Lat. & Dep. & Lat. & Dep. & Lat. & Dep. & \\
\hline $\begin{array}{l}51 \\
52 \\
53 \\
54 \\
55 \\
56 \\
57 \\
58 \\
59 \\
60\end{array}$ & $\begin{array}{l}46.95 \\
47.87 \\
48.79 \\
49.71 \\
50.63 \\
51.55 \\
52.47 \\
53.39 \\
54.31 \\
55.23\end{array}$ & $\begin{array}{l}19.93 \\
20.32 \\
20.71 \\
21.10 \\
21.49 \\
21.88 \\
22.27 \\
22.66 \\
23.05 \\
23.44\end{array}$ & $\begin{array}{l}46.86 \\
47.78 \\
48.70 \\
49.61 \\
50.53 \\
51.45 \\
52.37 \\
53.29 \\
54.21 \\
55.13\end{array}$ & $\begin{array}{l}20.13 \\
20.53 \\
20.92 \\
21.32 \\
21.71 \\
22.11 \\
22.50 \\
22.90 \\
23.29 \\
23.68\end{array}$ & $\begin{array}{l}46.77 \\
47.69 \\
48.60 \\
49.52 \\
50.44 \\
51.36 \\
52.27 \\
53.19 \\
54.11 \\
55.02\end{array}$ & $\begin{array}{l}20.34 \\
20.73 \\
21.13 \\
21.53 \\
21.93 \\
22.33 \\
22.73 \\
23.13 \\
23.53 \\
23.92\end{array}$ & $\begin{array}{l}46.68 \\
47.60 \\
43.51 \\
49.43 \\
50.34 \\
51.26 \\
52.17 \\
53.09 \\
54.00 \\
54.92\end{array}$ & $\begin{array}{l}20.54 \\
20.94 \\
21.35 \\
21.75 \\
22.15 \\
22.55 \\
22.96 \\
23.36 \\
23.76 \\
24.16\end{array}$ & $\begin{array}{l}51 \\
52 \\
53 \\
54 \\
55 \\
56 \\
57 \\
58 \\
59 \\
60\end{array}$ \\
\hline $\begin{array}{l}61 \\
62 \\
63 \\
64 \\
65 \\
66 \\
67 \\
68 \\
619 \\
70\end{array}$ & $\begin{array}{l}56.15 \\
57.07 \\
57.99 \\
58.91 \\
59.83 \\
60.75 \\
61.67 \\
62.59 \\
63.51 \\
64.44\end{array}$ & $\begin{array}{l}23.83 \\
24.23 \\
24.62 \\
25.01 \\
25.40 \\
25.79 \\
26.18 \\
26.57 \\
26.96 \\
27.35\end{array}$ & $\begin{array}{l}56.05 \\
56.97 \\
57.88 \\
58.80 \\
59.72 \\
60.64 \\
61.56 \\
62.48 \\
63.40 \\
64.32\end{array}$ & $\begin{array}{l}24.08 \\
24.47 \\
24.87 \\
25.26 \\
25.66 \\
26.05 \\
21.45 \\
26.84 \\
27.24 \\
27.63\end{array}$ & $\begin{array}{l}55.94 \\
56.86 \\
57.77 \\
58.69 \\
59.61 \\
60.53 \\
61.44 \\
62.36 \\
63.28 \\
64.19\end{array}$ & $\begin{array}{l}21.32 \\
24.72 \\
25.12 \\
25.52 \\
25.92 \\
26.32 \\
26.72 \\
27.11 \\
27.51 \\
27.91\end{array}$ & $\begin{array}{l}55.83 \\
56.75 \\
57.66 \\
58.58 \\
59.50 \\
60.41 \\
61.33 \\
62.24 \\
63.16 \\
64.07\end{array}$ & $\begin{array}{l}24.57 \\
24.97 \\
25.37 \\
25.78 \\
26.18 \\
26.58 \\
26.98 \\
27.39 \\
27.79 \\
28.19\end{array}$ & $\begin{array}{l}61 \\
62 \\
63 \\
64 \\
65 \\
66 \\
67 \\
68 \\
69 \\
70\end{array}$ \\
\hline $\begin{array}{l}71 \\
72 \\
73 \\
74 \\
75 \\
76 \\
77 \\
78 \\
79 \\
80\end{array}$ & $\begin{array}{l}65.36 \\
66.28 \\
67.21 \\
68.12 \\
69.04 \\
69.96 \\
70.88 \\
71.80 \\
72.72 \\
73.64\end{array}$ & $\begin{array}{l}27.74 \\
29.13 \\
28.52 \\
29.91 \\
29.30 \\
29.70 \\
30.09 \\
30.48 \\
30.87 \\
31.26\end{array}$ & $\begin{array}{l}55.23 \\
66.15 \\
67.07 \\
67.99 \\
68.91 \\
69.83 \\
70.75 \\
71.67 \\
72.58 \\
73.50\end{array}$ & $\begin{array}{l}28.03 \\
28.42 \\
28.82 \\
29.21 \\
29.61 \\
30.00 \\
30.40 \\
30.79 \\
31.18 \\
31.58\end{array}$ & $\begin{array}{l}65.11 \\
66.03 \\
66.95 \\
67.85 \\
68.78 \\
69.70 \\
70.61 \\
71.53 \\
72.45 \\
73.36\end{array}$ & $\begin{array}{l}28.31 \\
28.71 \\
29.11 \\
29.51 \\
29.91 \\
30.30 \\
30.70 \\
31.10 \\
31.50 \\
31.90\end{array}$ & $\begin{array}{l}64.99 \\
65.90 \\
66.82 \\
67.73 \\
68.65 \\
69.56 \\
70.48 \\
71.39 \\
72.31 \\
73.22\end{array}$ & $\begin{array}{l}28.60 \\
29.00 \\
29.40 \\
29.80 \\
30.21 \\
30.61 \\
31.01 \\
31.41 \\
31.82 \\
32.22\end{array}$ & $\begin{array}{l}71 \\
72 \\
73 \\
74 \\
75 \\
76 \\
77 \\
79 \\
79 \\
80\end{array}$ \\
\hline $\begin{array}{l}81 \\
82 \\
83 \\
84 \\
85 \\
81 \\
87 \\
88 \\
83 \\
90\end{array}$ & $\begin{array}{l}74.56 \\
75.48 \\
76.40 \\
77.32 \\
78.24 \\
79.16 \\
80.08 \\
81.00 \\
81.92 \\
82.85\end{array}$ & $\begin{array}{l}31.65 \\
32.04 \\
32.43 \\
32.82 \\
33.21 \\
33.60 \\
33.99 \\
34.38 \\
34.78 \\
35.17\end{array}$ & $\begin{array}{l}74.42 \\
75.34 \\
76.26 \\
77.18 \\
73.10 \\
79.02 \\
79.93 \\
80.85 \\
81.77 \\
82.69\end{array}$ & $\begin{array}{l}31.97 \\
32.37 \\
32.76 \\
33.16 \\
33.55 \\
33.95 \\
34.34 \\
34.74 \\
35.13 \\
35.53\end{array}$ & $\begin{array}{l}74.28 \\
75.20 \\
76.12 \\
77.03 \\
77.95 \\
78.87 \\
79.78 \\
80.70 \\
81.62 \\
82.54\end{array}$ & $\begin{array}{l}32.30 \\
32.70 \\
33.10 \\
33.49 \\
33.89 \\
34.29 \\
34.69 \\
35.09 \\
3.54 \\
35.89\end{array}$ & $\begin{array}{l}74.14 \\
75.06 \\
75.97 \\
76.89 \\
77.80 \\
78.72 \\
79.63 \\
80.55 \\
81.46 \\
82.38\end{array}$ & $\begin{array}{l}32.62 \\
33.03 \\
33.43 \\
33.83 \\
34.23 \\
34.64 \\
35.04 \\
35.44 \\
35.84 \\
36.25\end{array}$ & $\begin{array}{l}81 \\
82 \\
83 \\
84 \\
85 \\
86 \\
87 \\
89 \\
89 \\
90\end{array}$ \\
\hline $\begin{array}{c}91 \\
92 \\
93 \\
94 \\
95 \\
96 \\
97 \\
98 \\
99 \\
100\end{array}$ & $\begin{array}{l}83.77 \\
84.69 \\
85.61 \\
86.53 \\
87.45 \\
88.37 \\
89.29 \\
90.21 \\
91.13 \\
92.05\end{array}$ & $\begin{array}{l}35.56 \\
35.95 \\
36.34 \\
36.73 \\
37.12 \\
37.51 \\
37.90 \\
3 . .29 \\
38.68 \\
39.07\end{array}$ & $\begin{array}{l}83.61 \\
84.53 \\
85.45 \\
86.37 \\
87.29 \\
88.20 \\
89.12 \\
90.04 \\
90.96 \\
91.89\end{array}$ & $\begin{array}{l}35.92 \\
36.32 \\
36.71 \\
37.11 \\
37.50 \\
37.90 \\
38.29 \\
35.69 \\
39.08 \\
39.47\end{array}$ & $\begin{array}{l}83.45 \\
84.37 \\
85.29 \\
86.20 \\
87.12 \\
89.04 \\
89.95 \\
89.87 \\
90.79 \\
91.71\end{array}$ & $\begin{array}{l}36.29 \\
36.68 \\
37.08 \\
37.48 \\
37.88 \\
38.28 \\
39.68 \\
39.08 \\
39.48 \\
39.87\end{array}$ & $\begin{array}{l}83.29 \\
84.21 \\
85.12 \\
86.04 \\
86.95 \\
87.87 \\
88.79 \\
89.70 \\
90.62 \\
91.53\end{array}$ & $\begin{array}{l}36.65 \\
37.05 \\
37.46 \\
37.86 \\
35.26 \\
38.66 \\
39.07 \\
39.47 \\
39.87 \\
40.27\end{array}$ & $\begin{array}{r}91 \\
92 \\
93 \\
94 \\
95 \\
91 \\
97 \\
98 \\
99 \\
100\end{array}$ \\
\hline 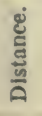 & Dep. & Lat. & $68 ?$ & Ist. & Dep. & Lat. & Dep. & Lat. & 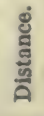 \\
\hline
\end{tabular}


TABLE 4.-TRAVERSE TABLE.

\begin{tabular}{|c|c|c|c|c|c|c|c|c|c|}
\hline \multirow{2}{*}{ 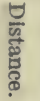 } & \multicolumn{2}{|c|}{$24^{\circ}$. } & \multicolumn{2}{|c|}{$241 / 4^{\circ}$. } & \multicolumn{2}{|c|}{$241 / 2^{\circ}$. } & \multicolumn{2}{|c|}{$21^{3} 4^{\circ}$. } & \multirow{2}{*}{ 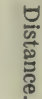 } \\
\hline & Lat. & Dep. & Lat. & Dep. & Lat. & Dep. & Lat. & Dep. & \\
\hline $\begin{array}{r}1 \\
2 \\
3 \\
4 \\
5 \\
6 \\
7 \\
8 \\
9 \\
10\end{array}$ & $\begin{array}{l}0.91 \\
1.83 \\
2.74 \\
3.65 \\
4.57 \\
5.48 \\
6.39 \\
7.31 \\
8.22 \\
9.14\end{array}$ & $\begin{array}{l}0.41 \\
0.81 \\
1.22 \\
1.63 \\
2.03 \\
2.44 \\
2.85 \\
3.25 \\
3.66 \\
4.07\end{array}$ & $\begin{array}{l}0.91 \\
1.82 \\
2.74 \\
3.65 \\
4.56 \\
5.47 \\
6.38 \\
7.29 \\
8.21 \\
9.12\end{array}$ & $\begin{array}{l}0.41 \\
0.82 \\
1.23 \\
1.64 \\
2.05 \\
2.46 \\
2.88 \\
3.29 \\
3.70 \\
4.11\end{array}$ & $\begin{array}{l}0.91 \\
1.82 \\
2.73 \\
3.64 \\
4.55 \\
5.46 \\
6.37 \\
7.28 \\
8.19 \\
9.10\end{array}$ & $\begin{array}{l}0.41 \\
0.83 \\
1.24 \\
1.66 \\
2.07 \\
2.49 \\
2.90 \\
3.32 \\
3.73 \\
4.15\end{array}$ & $\begin{array}{l}0.91 \\
1.82 \\
2.72 \\
3.63 \\
4.54 \\
5.45 \\
6.36 \\
7.27 \\
8.17 \\
9.08\end{array}$ & $\begin{array}{l}0.42 \\
0.84 \\
1.26 \\
1.67 \\
2.09 \\
2.51 \\
2.93 \\
3.35 \\
3.77 \\
4.19\end{array}$ & $\begin{array}{r}1 \\
2 \\
3 \\
4 \\
5 \\
6 \\
7 \\
8 \\
9 \\
10\end{array}$ \\
\hline $\begin{array}{l}11 \\
12 \\
13 \\
14 \\
15 \\
16 \\
17 \\
18 \\
19 \\
20\end{array}$ & $\begin{array}{l}10.05 \\
10.96 \\
11.88 \\
12.79 \\
13.70 \\
14.62 \\
15.53 \\
16.44 \\
17.36 \\
18.27\end{array}$ & $\begin{array}{l}4.47 \\
4.83 \\
5.29 \\
5.69 \\
6.10 \\
6.51 \\
6.91 \\
7.32 \\
7.73 \\
8.13\end{array}$ & $\begin{array}{l}10.03 \\
10.94 \\
11.85 \\
12.76 \\
13.68 \\
14.59 \\
15.50 \\
16.41 \\
17.32 \\
18.24\end{array}$ & $\begin{array}{l}4.52 \\
4.93 \\
5.34 \\
5.75 \\
6.16 \\
6.57 \\
6.98 \\
7.39 \\
7.80 \\
8.21\end{array}$ & $\begin{array}{l}10.01 \\
10.92 \\
11.83 \\
12.74 \\
13.65 \\
14.56 \\
15.47 \\
16.38 \\
17.29 \\
18.20\end{array}$ & $\begin{array}{l}4.56 \\
4.98 \\
5.39 \\
5.81 \\
6.22 \\
6.64 \\
7.05 \\
7.46 \\
7.88 \\
8.29\end{array}$ & $\begin{array}{r}9.99 \\
10.90 \\
11.81 \\
12.71 \\
13.62 \\
14.53 \\
15.44 \\
16.35 \\
17.25 \\
18.16\end{array}$ & $\begin{array}{l}4.61 \\
5.02 \\
5.44 \\
5.86 \\
6.28 \\
6.70 \\
7.12 \\
7.54 \\
7.95 \\
8.37\end{array}$ & $\begin{array}{l}11 \\
12 \\
13 \\
14 \\
15 \\
16 \\
17 \\
18 \\
19 \\
20\end{array}$ \\
\hline $\begin{array}{l}21 \\
22 \\
23 \\
24 \\
25 \\
26 \\
27 \\
28 \\
29 \\
30\end{array}$ & $\begin{array}{l}19.18 \\
20.10 \\
21.01 \\
21.93 \\
22.84 \\
23.75 \\
24.67 \\
25.58 \\
26.49 \\
27.41\end{array}$ & $\begin{array}{r}8.54 \\
8.95 \\
9.35 \\
9.76 \\
10.17 \\
10.58 \\
10.98 \\
11.39 \\
11.80 \\
12.20\end{array}$ & $\begin{array}{l}19.15 \\
20.06 \\
20.97 \\
21.88 \\
22.79 \\
23.71 \\
24.62 \\
25.53 \\
26.41 \\
27.35\end{array}$ & $\begin{array}{r}8.63 \\
9.04 \\
9.45 \\
9.86 \\
10.27 \\
10.68 \\
11.09 \\
11.50 \\
11.91 \\
12.32\end{array}$ & $\begin{array}{l}19.11 \\
20.02 \\
20.93 \\
21.84 \\
22.75 \\
23.66 \\
24.57 \\
25.48 \\
26.39 \\
27.30\end{array}$ & $\begin{array}{r}8.71 \\
9.12 \\
9.54 \\
9.95 \\
10.37 \\
10.78 \\
11.20 \\
11.61 \\
12.03 \\
12.44\end{array}$ & $\begin{array}{l}19.07 \\
19.98 \\
20.89 \\
21.80 \\
22.70 \\
23.61 \\
24.52 \\
25.43 \\
26.34 \\
27.24\end{array}$ & $\begin{array}{r}8.79 \\
9.21 \\
9.63 \\
10.05 \\
10.47 \\
10.89 \\
11.30 \\
11.72 \\
12.14 \\
12.56\end{array}$ & $\begin{array}{l}21 \\
22 \\
23 \\
24 \\
25 \\
26 \\
27 \\
25 \\
29 \\
30\end{array}$ \\
\hline $\begin{array}{l}31 \\
32 \\
33 \\
34 \\
35 \\
36 \\
37 \\
38 \\
39 \\
40\end{array}$ & $\begin{array}{l}28.32 \\
29.23 \\
30.15 \\
31.06 \\
31.97 \\
32.89 \\
33.80 \\
34.71 \\
35.63 \\
36.54\end{array}$ & $\begin{array}{l}12.61 \\
13.02 \\
13.42 \\
13.83 \\
14.24 \\
14.64 \\
15.05 \\
15.46 \\
15.86 \\
16.27\end{array}$ & $\begin{array}{l}28.26 \\
29.18 \\
30.09 \\
31.00 \\
31.91 \\
32.82 \\
33.74 \\
34.65 \\
35.56 \\
36.47\end{array}$ & $\begin{array}{l}12.73 \\
13.14 \\
13.55 \\
13.96 \\
14.38 \\
14.79 \\
15.20 \\
15.61 \\
16.02 \\
16.43\end{array}$ & $\begin{array}{l}28.21 \\
29.12 \\
30.03 \\
30.94 \\
31.85 \\
32.76 \\
33.67 \\
31.58 \\
35.49 \\
36.40\end{array}$ & $\begin{array}{l}12.86 \\
13.27 \\
13.68 \\
14.10 \\
14.51 \\
14.93 \\
15.34 \\
15.76 \\
16.17 \\
16.59\end{array}$ & $\begin{array}{l}28.15 \\
29.06 \\
29.97 \\
30.88 \\
31.79 \\
32.69 \\
33.60 \\
34.51 \\
35.42 \\
36.33\end{array}$ & $\begin{array}{l}12.98 \\
13.40 \\
13.82 \\
14.23 \\
14.65 \\
15.07 \\
15.49 \\
15.91 \\
16.33 \\
16.75\end{array}$ & $\begin{array}{l}31 \\
32 \\
33 \\
34 \\
35 \\
36 \\
37 \\
38 \\
39 \\
40\end{array}$ \\
\hline $\begin{array}{l}41 \\
42 \\
43 \\
44 \\
45 \\
46 \\
47 \\
48 \\
49 \\
50\end{array}$ & $\begin{array}{l}37.46 \\
38.37 \\
39.28 \\
40.20 \\
41.11 \\
42.02 \\
42.94 \\
43.85 \\
44.76 \\
45.68\end{array}$ & $\begin{array}{l}16.68 \\
17.08 \\
17.49 \\
17.90 \\
18.30 \\
18.71 \\
19.12 \\
19.52 \\
19.93 \\
20.34\end{array}$ & $\begin{array}{l}37.38 \\
38.29 \\
39.21 \\
40.12 \\
41.03 \\
41.94 \\
42.85 \\
43.76 \\
44.68 \\
45.59\end{array}$ & $\begin{array}{l}16.84 \\
17.25 \\
17.66 \\
18.07 \\
18.48 \\
18.89 \\
19.30 \\
19.71 \\
20.13 \\
20.54\end{array}$ & $\begin{array}{l}37.31 \\
33.22 \\
39.13 \\
40.04 \\
40.95 \\
41.86 \\
42.77 \\
43.68 \\
44.59 \\
45.50\end{array}$ & $\begin{array}{l}17.00 \\
17.42 \\
17.83 \\
18.25 \\
18.665 \\
19.08 \\
19.49 \\
19.91 \\
20.32 \\
20.73\end{array}$ & $\begin{array}{l}37.23 \\
38.14 \\
39.05 \\
39.96 \\
40.87 \\
41.77 \\
42.68 \\
43.59 \\
44.50 \\
45.41\end{array}$ & $\begin{array}{l}17.17 \\
17.58 \\
18.00 \\
18.42 \\
18.84 \\
19.26 \\
19.68 \\
20.10 \\
20.51 \\
20.93\end{array}$ & $\begin{array}{l}41 \\
42 \\
43 \\
44 \\
45 \\
46 \\
47 \\
48 \\
49 \\
50\end{array}$ \\
\hline 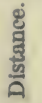 & Dep. & Lat. & Dep. & Lat. & Dep. & Lat. & Dep. & Lat. & 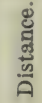 \\
\hline
\end{tabular}


TABLE 4.-TRAVERSE TABLE.

\begin{tabular}{|c|c|c|c|c|c|c|c|c|c|}
\hline \multirow{2}{*}{ 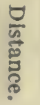 } & \multicolumn{2}{|c|}{$24^{\circ}$. } & \multicolumn{2}{|c|}{$241 / 4^{\circ}$. } & \multicolumn{2}{|c|}{$241 / 2^{\circ}$. } & \multicolumn{2}{|c|}{$2434^{\circ}$. } & \multirow{2}{*}{ 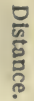 } \\
\hline & Lat. & Dep. & Lat. & Dep. & Lat. & Dep. & Lat. & Dep. & \\
\hline 51 & 46. 59 & 20.74 & 46. 50 & 20.95 & 46.41 & 21.15 & 46. 32 & 21.35 & 51 \\
\hline 52 & 47.50 & 21.15 & 47.41 & 21.36 & 47.32 & 21.56 & 47.22 & 21.77 & 52 \\
\hline 53 & 48.42 & 21.56 & 48.32 & 21.77 & 48.23 & 21.98 & 48.13 & 22.19 & 53 \\
\hline 54 & 49.33 & 21.96 & 49.24 & 22.18 & 49.14 & 22.39 & 49.04 & 22.61 & 54 \\
\hline 55 & 50.25 & 22.37 & 50.15 & 22.59 & 50.05 & 22.81 & 49.95 & 23.03 & 55 \\
\hline 56 & 51.16 & 22.78 & 51.06 & 23.00 & 50.96 & 23.22 & 50.86 & 23.44 & 56 \\
\hline 57 & 52.07 & 23. 18 & 51.97 & 23. 41 & 51.87 & 23. 64 & 51.76 & 23.86 & 57 \\
\hline 58 & 52.99 & 23.59 & 52.88 & 23.82 & 52.78 & 24. 05 & 52.67 & 24.28 & 58 \\
\hline 59 & 53.90 & 24.00 & 53.79 & 24.23 & 53. 69 & 24.47 & 53.58 & 24.70 & 59 \\
\hline 60 & 54.81 & 24.40 & 54.71 & 24.64 & 54.60 & 24.88 & 54.49 & 25.12 & 60 \\
\hline 61 & 55.73 & 24.81 & 55.62 & 25.05 & 55.51 & 25.30 & 55.40 & 25.54 & 61 \\
\hline 62 & 56.64 & 25.22 & 56.53 & 25.46 & 56.42 & 25.71 & 56.30 & 25. 96 & 62 \\
\hline 63 & 57.55 & 25.62 & 57.44 & 25.88 & 57.33 & 26.13 & 57. 21 & 26.38 & 63 \\
\hline 64 & 58.47 & 26.03 & 58.35 & 26.29 & 58.24 & 26.54 & 58.12 & 26.79 & 64 \\
\hline 65 & 39.38 & 26.44 & 59.26 & 26. 70 & 59.15 & 26.96 & 59.03 & 27.21 & 65 \\
\hline 66 & Co. 29 & 26.84 & 60.18 & 27.11 & 60.06 & 27.37 & 59.94 & 27.63 & 66 \\
\hline 67 & 61.21 & 27.25 & 61.09 & 27.52 & 60.97 & 27.78 & 60.85 & 28.05 & 67 \\
\hline 68 & 62.12 & 27.66 & 62.00 & 27.93 & 61.88 & 28.20 & 61.75 & 28.47 & 68 \\
\hline 69 & 63. 03 & 28.06 & 62.91 & 28.34 & 62.79 & 28.61 & 62.66 & 28.89 & 69 \\
\hline 70 & 63.95 & 28.47 & 63.82 & 28.75 & 63.70 & 29.03 & 63.57 & 29.31 & 70 \\
\hline 71 & 64.86 & 28. 85 & 64.74 & 29.16 & 64.61 & 29.44 & 64.48 & 29.72 & 71 \\
\hline 72 & 65.78 & 29.29 & 65.65 & 29.57 & 65.52 & 29.86 & 65.39 & 30.14 & 72 \\
\hline 73 & 66.69 & 29.69 & 66.56 & 29.98 & 66.43 & 30.27 & 66. 29 & 30.56 & 73 \\
\hline 74 & 67.60 & 30.10 & 67.47 & 30.39 & 67.34 & 30.69 & 67.20 & 30.98 & 74 \\
\hline 75 & 68.52 & 30.51 & 68.38 & 30.80 & 68.25 & 31.10 & 68.11 . & 31.40 & 75 \\
\hline 76 & 69.43 & 30.91 & 69. 39 & 31.21 & 69. 16 & 31.52 & 69.02 & 31.82 & 76 \\
\hline 77 & 70.34 & 31.32 & 70.21 & 31. 63 & 70.07 & 31.93 & 69.93 & 32.24 & 77 \\
\hline 78 & 71.26 & 31.73 & 71.12 & 32.04 & 70.98 & 32.35 & 70.84 & 32.66 & 78 \\
\hline 79 & 72.17 & 32.13 & 72.03 & 32.45 & 71.89 & 32.76 & 71.74 & 33.07 & 79 \\
\hline 80 & 73.08 & 32.54 & 72.94 & 32.86 & 72.80 & 33.18 & 72.65 & 33.49 & 80 \\
\hline 81 & 74.00 & 32.95 & 73.85 & 33. 27 & 73.71 & 33.59 & 73.56 & 33.91 & 81 \\
\hline 82 & 74.91 & 33.35 & 74.76 & 33. 68 & 74.62 & 34.00 & 74.47 & 34.33 & 82 \\
\hline 8.3 & 75.82 & 33.76 & 75.188 & 34.09 & 75.53 & 34.42 & 75.38 & 34.75 & 83 \\
\hline 84 & 76.74 & 34.17 & 76.59 & 34.50 & 76.44 & 34.83 & 76.28 & 35.17 & 84 \\
\hline 85 & 77.65 & 34.57 & 77.50 & 34.91 & 77.35 & 35.25 & 77.19 & 35.59 & 85 \\
\hline 86 & 78.56 & 34.98 & 78.41 & 35.32 & 78.26 & 35.66 & 78.10 & 36.00 & 86 \\
\hline 87 & 79.48 & 35. 39 & 79.32 & 35.73 & 79.17 & 36.08 & 79.01 & 36.42 & 87 \\
\hline 83 & 80.39 & 35.79 & 80.24 & 36. 14 & 80.08 & 36.49 & 79.92 & 36.84 & 88 \\
\hline 89 & 81.31 & 36.20 & 81.15 & 36.55 & 80.99 & 36.91 & 80.82 & 37.26 & 89 \\
\hline 90 & 82.22 & 36.61 & 82.06 & 36.96 & 81.90 & 37.32 & 81.73 & 37.68 & 90 \\
\hline 91 & 83.13 & 37.01 & 82.97 & 37.38 & 82.81 & 37.74 & 82.64 & 35. 10 & 91 \\
\hline 92 & 84.05 & 37.42 & 83.88 & 37.79 & 83.72 & 38.15 & 83.55 & 38.52 & 92 \\
\hline 93 & 84. 96 & 37.83 & 84.79 & 38.20 & 84.63 & 38.57 & 84. 46 & 38.94 & 93 \\
\hline 94 & 85.87 & 38.23 & 85.71 & 38.61 & 85.54 & 38.98 & 85.37 & 39.35 & 94 \\
\hline 95 & 86.79 & 38. (i4 & 86. 62 & 39.02 & 86.45 & 39.40 & 86.27 & 39.77 & 95 \\
\hline 96 & 87.70 & 39.05 & 87.53 & 39.43 & 87.36 & 39.81 & 87.18 & 40.19 & 96 \\
\hline 97 & 88.61 & 39.45 & 89.44 & 39.84 & 88.27 & 40.23 & 88.09 & 40.61 & 97 \\
\hline 98 & 89.53 & 39.86 & 89.35 & 40.25 & 89.18 & 40. (5) & 89.00 & 41.03 & 98 \\
\hline 99 & 90.44 & 40.27 & 90.26 & 40.66 & 90.09 & 41.05 & 89.91 & 41.45 & 99 \\
\hline 100 & 91.35 & 40.67 & 91.18 & 41.07 & 91.00 & 41.47 & 90.81 & 41.87 & 100 \\
\hline \multirow{2}{*}{ 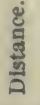 } & Dep. & Lat. & Dep. & Iat. & Dep. & Lat. & Dep. & Lat. & \multirow{2}{*}{$\begin{array}{l}\text { ष्ठ } \\
\text { క్ } \\
\frac{3}{0}\end{array}$} \\
\hline & \multicolumn{2}{|c|}{$66^{\circ}}$. & \multicolumn{2}{|c|}{$65 \% 4^{\circ}}$. & \multicolumn{2}{|c|}{$652 / 22^{\circ}$. } & \multicolumn{2}{|c|}{$\cos 14^{\circ}}$. & \\
\hline
\end{tabular}


TABLE 4.-TRAVERSE TABLE.

\begin{tabular}{|c|c|c|c|c|c|c|c|c|c|}
\hline \multirow{2}{*}{ 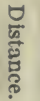 } & \multicolumn{2}{|c|}{$25^{\circ}$. } & \multicolumn{2}{|c|}{$251 / 4^{\circ}$. } & \multicolumn{2}{|c|}{$251 / 2^{\circ}$. } & \multicolumn{2}{|c|}{$2534^{\circ}$. } & \multirow{2}{*}{$\begin{array}{l}\forall \\
\frac{\vec{n}}{0} \\
\frac{0}{0} \\
\frac{0}{8}\end{array}$} \\
\hline & Lat. & Dep. & Lat. & Dep. & Lat. & Dep. & Lat. & Dep. & \\
\hline 1 & 0.91 & 0.42 & 0.90 & 0.43 & 0.90 & 0.43 & 0.90 & 0.43 & 1 \\
\hline 2 & 1.81 & 0.85 & 1.81 & 0.85 & 1.81 & 0.86 & 1.80 & 0.87 & 2 \\
\hline 3 & 2.72 & 1. 27 & 2.71 & 1.28 & 2.71 & 1. 29 & 2.70 & 1.30 & 3 \\
\hline 4 & 3. 63 & 1. 69 & 3. 62 & 1.71 & 3.61 & 1.72 & 3. 60 & 1. 74 & 4 \\
\hline 5 & 4.53 & 2.11 & 4.52 & 2.13 & 4.51 & 2.15 & 4.50 & 2.17 & 5 \\
\hline 6 & 5. 44 & 2.54 & 5.43 & 2.56 & 5. 42 & 2.58 & 5.40 & 2.61 & 6 \\
\hline 7 & 6.34 & 2.96 & 6.33 & 2.99 & 6.32 & 3.01 & 6. 30 & 3.04 & 7 \\
\hline 8 & 7.25 & 3. 38 & 7.24 & 3.41 & 7.22 & 3.44 & 7.21 & 3. 48 & 8 \\
\hline 9 & 8.16 & 3.80 & 8.14 & 3.84 & 8.12 & 3.87 & 8.11 & 3. 91 & 9 \\
\hline 10 & 9.06 & 4. 23 & 9.04 & 4.27 & 9.03 & 4.31 & 9.01 & 4. 34 & 10 \\
\hline 11 & 9.97 & 4. 65 & 9.95 & 4. 69 & 9.93 & 4.74 & 9.91 & 4. 78 & 11 \\
\hline 12 & 10.88 & 5.07 & 10.85 & 5. 12 & 10.83 & 5.17 & 10.81 & 5. 21 & 12 \\
\hline 13 & 11.78 & 5. 49 & 11.76 & 5. 55 & 11.73 & 5. 60 & 11.71 & 5.65 & 13 \\
\hline 14 & 12. 69 & 5. 92 & 12.66 & 5.97 & 12.64 & 6. 03 & 12.61 & 6.08 & 14 \\
\hline 15 & 13. 59 & 6.34 & 13.57 & 6.40 & 13.54 & 6.46 & 13.51 & 6.52 & 15 \\
\hline 16 & 14.50 & 6.76 & 14.47 & 6. 83 & 14.44 & 6.89 & 14.41 & 6.95 & 16 \\
\hline 17 & 15. 41 & 7.18 & 15. 38 & 7.25 & 15. 34 & 7.32 & 15.31 & 7.39 & 17 \\
\hline 18 & 16.31 & 7.61 & 16.28 & 7.68 & 16.25 & 7.75 & 16.21 & 7.82 & 18 \\
\hline 19 & 17.22 & 8.03 & 17.18 & 8.10 & 17.15 & 8.18 & 17.11 & 8.25 & 19 \\
\hline 20 & 18.13 & 8.45 & 18.09 & 8.53 & 18.05 & 8.61 & 18.01 & 8.69 & 20 \\
\hline 21 & 19.03 & 8.87 & 18.99 & 8.96 & 18.95 & 9.04 & 18.91 & 9.12 & 21 \\
\hline 22 & 19.94 & 9.30 & 19.90 & 9.38 & 19.86 & 9.47 & 19.82 & 9.56 & 22 \\
\hline 23 & 20.85 & 9.72 & 20.80 & 9.81 & 20,76 & 9.90 & 20.72 & 9.99 & 23 \\
\hline 24 & 21.75 & 10.14 & 21.71 & 10.24 & 21.66 & 10.33 & 21.62 & 10.43 & 24 \\
\hline 25 & 22.66 & 10.57 & 22.61 & 10.66 & 22.56 & 10.76 & 22.52 & 10.86 & 25 \\
\hline 26 & 23.56 & 10.99 & 23. 52 & 11.09 & 23.47 & 11. 19 & 23.42 & 11.30 & 26 \\
\hline 27 & 24.47 & 11.41 & 24.42 & 11.52 & 24.37 & 11. 62 & 24.32 & 11.73 & 27 \\
\hline 28 & 25. 38 & 11.83 & 25. 32 & 11.94 & 25.27 & 12.05 & 25.22 & 12.16 & 28 \\
\hline 29 & 26.28 & 12. 26 & 26.23 & 12.37 & 26.17 & 12.48 & 26.12 & 12.60 & 29 \\
\hline 30 & 27.19 & 12.68 & 27.13 & 12.80 & 27.08 & 12.92 & 27.02 & 13.03 & 30 \\
\hline 31 & 28.10 & 13.10 & 28.04 & 13.22 & 27.98 & 13. 35 & 27.92 & 13.47 & 31 \\
\hline 32 & 29.00 & 13. 52 & 28.94 & 13.65 & 28.88 & 13. 78 & 28.82 & 13.90 & 32 \\
\hline 33 & 29.91 & 13.95 & 29.85 & 14. 08 & 29.79 & 14.21 & 29.72 & 14.34 & 33 \\
\hline 34 & 30.81 & 14.37 & 30.75 & 14.50 & 30.69 & 14.64 & 30.62 & 14.77 & 34 \\
\hline 35 & 31.72 & 14.79 & 31.66 & 14.93 & 31.59 & 15.07 & 31.52 & 15. 21 & 35 \\
\hline 36 & 32.63 & 15. 21 & 32.56 & 15. 36 & 32.49 & 15. 50 & 32.43 & 15.64 & 36 \\
\hline 37 & 33. 53 & 15.64 & 33.46 & 15.78 & 33.40 & 15.93 & 33.33 & 16.07 & 37 \\
\hline 38 & 34.44 & 16.06 & 34.37 & 16.21 & 34.30 & 16.36 & 34.23 & 16. 51 & 38 \\
\hline 39 & 35.35 & 16.48 & 35.27 & 16.64 & 35. 20 & 16. 79 & 35. 13 & 16. 94 & 39 \\
\hline 40 & 36.25 & 16.90 & 36.18 & 17.06 & 36.10 & 17.22 & 36. 03 & 17.38 & 40 \\
\hline 41 & 37.16 & 17. 33 & 37.08 & 17.49 & 37.01 & 17.65 & 36.93 & 17.81 & 41 \\
\hline 42 & 38.06 & 17.75 & 37.99 & 17.92 & 37.91 & 18. 08 & 37.83 & 18.25 & 42 \\
\hline 43 & 38.97 & 18. 17 & 38.89 & 18.34 & 38.81 & 18. 51 & 38.73 & 18. 68 & 43 \\
\hline 44 & 39.88 & 18. 60 & 39.80 & 18. 77 & 39.71 & 18. 94 & 39.63 & 19.12 & 44 \\
\hline 45 & 40.78 & 19.02 & 40.70 & 19.20 & 40. 62 & 19. 37 & 40.53 & 19.55 & 45 \\
\hline 46 & 41. (i9 & 19.44 & 41.60 & 19.62 & 41.52 & 19.80 & 41,43 & 19.98 & 46 \\
\hline 47 & 42.60 & 19.86 & 42.51 & 20.05 & 42.42 & 20.23 & 42.33 & 20.42 & 47 \\
\hline 48 & 43.50 & 20.29 & 43.41 & 20.48 & 43. 32 & 20.66 & 43.23 & 20.85 & 48 \\
\hline 49 & 44.41 & 20.71 & 44. 32 & 20.90 & 44.23 & 21.10 & 44.13 & 21.29 & 49 \\
\hline 50 & 45.32 & 21.13 & 45.22 & 21.33 & 45.13 & 21.53 & 45.03 & 21.72 & 50 \\
\hline \multirow{2}{*}{ 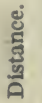 } & Dep. & Lat. & Dep. & Lat. & Dep. & Lat. & Dep. & Lat. & \multirow{2}{*}{$\begin{array}{l}\dot{8} \\
\text { d̆ } \\
\frac{\text { gै }}{0}\end{array}$} \\
\hline & \multicolumn{2}{|c|}{$65^{\circ}}$. & \multicolumn{2}{|c|}{$6434^{\circ}$. } & \multicolumn{2}{|c|}{$641 / 2^{\circ}}$. & \multicolumn{2}{|c|}{$641 / 4^{\circ}$. } & \\
\hline
\end{tabular}


TAILE 4.-TRAVERSE TABLE.

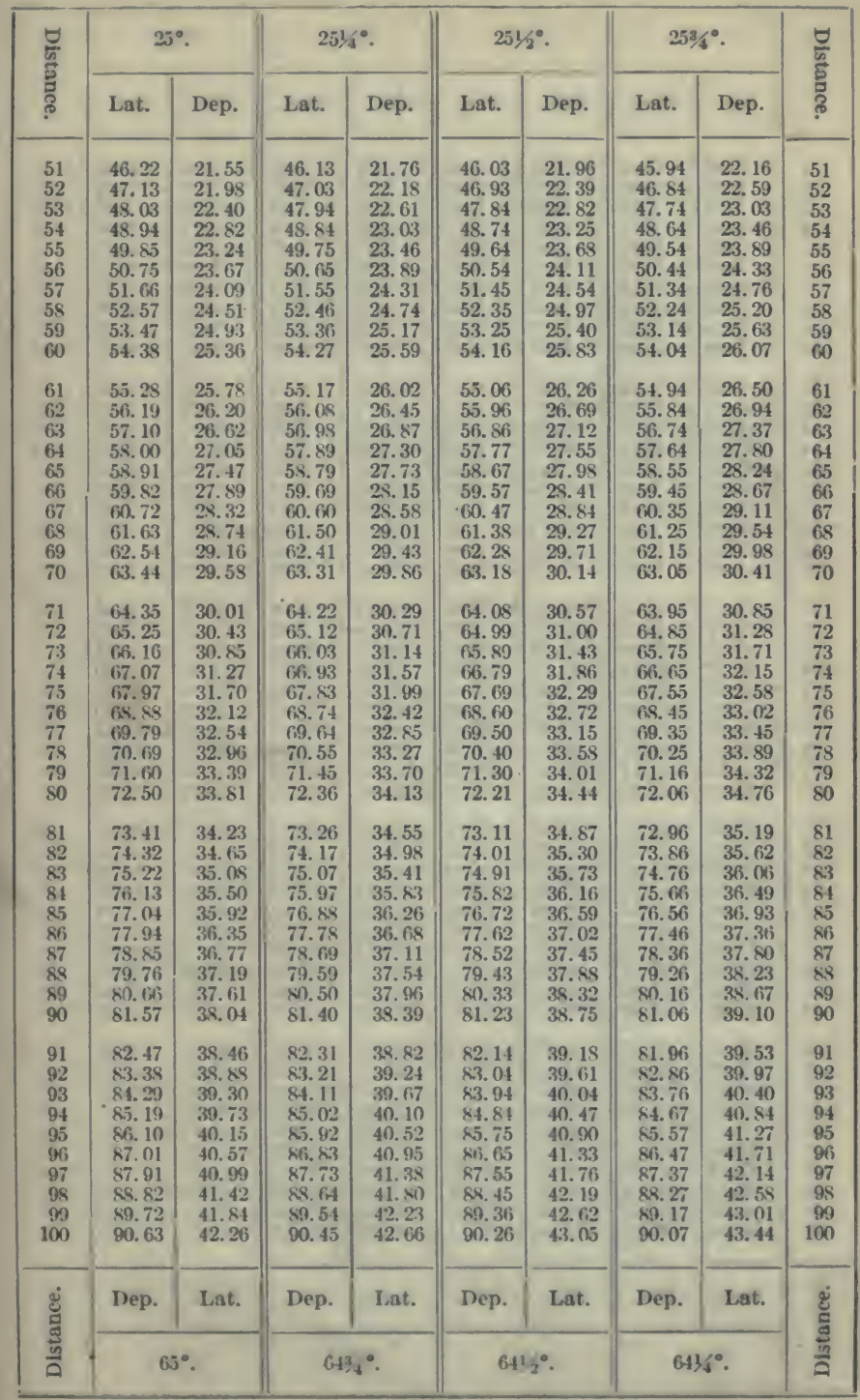


TABLE 4.-TRAVERSE TABLE.

\begin{tabular}{|c|c|c|c|c|c|c|c|c|c|}
\hline \multirow{2}{*}{ 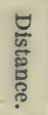 } & \multicolumn{2}{|c|}{$26^{\circ}$. } & \multicolumn{2}{|c|}{$26 \frac{1}{4^{\circ}}$. } & \multicolumn{2}{|c|}{$261 / 2^{\circ}$. } & \multicolumn{2}{|c|}{$26^{3} 4^{\circ}$. } & \multirow{2}{*}{ 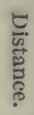 } \\
\hline & Lat. & Dep. & Lat. & Dep. & Lat. & Dep. & Lat. & Dep. & \\
\hline 1 & 0.90 & 0.44 & 0.90 & 0.44 & 0.89 & 0.45 & 0.89 & 0.45 & 1 \\
\hline 2 & $\begin{array}{l}0.90 \\
1.80\end{array}$ & $\begin{array}{l}0.47 \\
0.88\end{array}$ & $\begin{array}{l}0.70 \\
1.79\end{array}$ & 0.88 & $\begin{array}{l}0.03 \\
1.79\end{array}$ & 0.89 & 1.79 & 0.90 & 2 \\
\hline 3 & 2.70 & 1.32 & 2. 69 & 1.33 & 2.68 & 1.34 & 2.68 & 1.35 & 3 \\
\hline 4 & 3.60 & 1.75 & 3.59 & 1.77 & 3.58 & 1.78 & 3.57 & 1.80 & 4 \\
\hline 5 & 4. 49 & 2. 19 & 4. 48 & 2.21 & 4. 47 & 2.23 & 4.46 & 2.25 & 5 \\
\hline 6 & 5. 39 & 2. 63 & 5.38 & 2.65 & 5.37 & 2.68 & 5.36 & 2.70 & 6 \\
\hline 7 & 6.29 & 3.07 & 6.28 & 3.10 & 6. 26 & 3.12 & 6.25 & 3.15 & 7 \\
\hline 8 & 7. 19 & 3.51 & 7.17 & 3.54 & 7.16 & 3.57 & 7.14 & 3. 60 & 8 \\
\hline 9 & 8.09 & 3.95 & 8.07 & 3.98 & 8.05 & 4.02 & 8.04 & 4.05 & 9 \\
\hline 10 & 8.99 & 4. 38 & 8.97 & 4. 42 & 8.95 & 4. 46 & 8.93 & 4.50 & 10 \\
\hline 11 & 9.89 & 4. 82 & 9.87 & 4.87 & 9.84 & 4.91 & 9.82 & 4.95 & 11 \\
\hline 12 & 10.79 & 5.26 & 10.76 & 5. 31 & 10.74 & 5.35 & 10.72 & 5.40 & 12 \\
\hline 13 & 11.68 & 5.70 & 11. 66 & 5.75 & 11. 63 & 5.80 & 11.61 & 5.85 & 13 \\
\hline 14 & 12.58 & 6. 14 & 12.56 & 6. 19 & 12.53 & 6.25 & 12.50 & 6. 30 & 14 \\
\hline 15 & 13. 48 & 6.58 & 13. 45 & 6.63 & 13. 42 & 6. 69 & 13. 39 & 6.75 & 15 \\
\hline 16 & 14.38 & 7.01 & 14.35 & 7.08 & 14.32 & 7.14 & 14. 29 & 7.20 & 16 \\
\hline 17 & 15. 28 & 7.45 & 15. 25 & $7.5^{\circ}$ & 15. 21 & 7.59 & 15.18 & 7.65 & 17 \\
\hline 18 & 16. 18 & 7.89 & 16. 14 & 7.96 & 16. 11 & 8.03 & 16.07 & 8.10 & 18 \\
\hline 19 & 17.08 & 8.33 & 17.04 & 8.40 & 17.00 & 8.48 & 16.97 & 8.55 & 19 \\
\hline 20 & 17.98 & 8.77 & 17.94 & 8.85 & 17.90 & 8.92 & 17.86 & 9.00 & 20 \\
\hline 21 & 18.87 & 9.21 & 18.83 & 9.29 & 18. 79 & 9.37 & 18.75 & 9. 45 & 21 \\
\hline 22 & 19.77 & 9.64 & 19. 73 & 9.73 & 19. 69 & 9.82 & 19.65 & 9.90 & 22 \\
\hline 23 & 20.67 & 10.08 & 20. & 10.17 & 20.58 & 10.26 & 20.54 & 10.35 & 23 \\
\hline 24 & 21.57 & 10.52 & 21.52 & 10. 61 & 21.48 & 10.71 & 21.43 & 10.80 & 24 \\
\hline 25 & 22.47 & 10.96 & 22.42 & 11.06 & 22.37 & 11.15 & 22.32 & 11.25 & 25 \\
\hline 26 & 23.37 & 11.40 & 23. 32 & 11.50 & 23.27 & 11.60 & 23.22 & 11. 70 & 26 \\
\hline 27 & 24.27 & 11.84 & 24. 22 & 11.94 & 24.16 & 12.05 & 24.11 & 12. 15 & 27 \\
\hline 28 & 25.17 & 12.27 & 25.11 & 12.38 & 25.06 & 12.49 & 25.00 & 12. 60 & 28 \\
\hline 29 & 26.07 & 12.71 & 26.01 & 12. 83 & 25.95 & 12.94 & 25.90 & 13.05 & 29 \\
\hline 30 & 26.96 & 13.15 & 26.91 & 13. 27 & 26.85 & 13.39 & 26.79 & 13.50 & 30 \\
\hline 31 & 27.86 & 13.59 & 27.80 & 13. 71 & 27.74 & 13. 83 & 27.68 & 13.95 & 31 \\
\hline 32 & 28.76 & 14.03 & 28.70 & 14.15 & 28.64 & 14.28 & 28.59 & 14.40 & 32 \\
\hline 33 & $29 .(66)$ & 14.47 & 29.60 & 14.60 & 29.53 & 14.72 & 29.47 & 14.85 & 33 \\
\hline 34 & 30.56 & 14.90 & 30.49 & 15.04 & 30.43 & 15.17 & 30.36 & 15.30 & 34 \\
\hline 35 & 31.46 & 15.34 & 31.39 & 15.48 & 31.32 & 15.62 & 31.25 & 15.75 & 35 \\
\hline 36 & 32.36 & 15.78 & 32.29 & 15.92 & 32.22 & 16.06 & 32.15 & 16.20 & 36 \\
\hline 37 & 33.26 & 16.22 & 33.18 & 16.36 & 33.11 & 16.51 & 33.04 & 16. 65 & 37 \\
\hline 38 & 34.15 & 16.66 & 34.08 & 16.81 & 34.01 & 16.96 & 33.93 & 17.10 & 38 \\
\hline 39 & 35.05 & 17.10 & 34.98 & 17.25 & 34.90 & 17.40 & 34.83 & 17.55 & 39 \\
\hline 40 & 35.95 & 17.53 & 35.87 & 17.69 & 35.80 & 17.85 & 35.72 & 18.00 & 40 \\
\hline 41 & 36.85 & 17.97 & 36.77 & 18. 13 & 36.69 & 18.29 & 36.61 & 18.45 & 41 \\
\hline 42 & 37.75 & 18.41 & 37.67 & 18. 58 & 37.59 & 18. 74 & 37.51 & 18. 90 & 42 \\
\hline 43 & 38. 65 & 18. 85 & 38.57 & 19.02 & 38. 48 & 19.19 & 38.40 & 19.35 & 43 \\
\hline 44 & 39.55 & 19.29 & 39.46 & 19. 46 & 39. 38 & 19. 63 & 39.29 & 19.80 & 44 \\
\hline 45 & 40.45 & 19.73 & 40.36 & 19.90 & 40.27 & 20.08 & 40.18 & 20.25 & 45 \\
\hline 46 & 41.34 & 20.17 & 41. 26 & 20.35 & 41.17 & 20.53 & 41. Os & 20.70 & 46 \\
\hline 47 & 42.24 & 20.60 & 42.15 & 20.79 & 42.06 & 20.97 & 41.97 & 21.15 & 47 \\
\hline 48 & 43.14 & 21.04 & 43.05 & 21.23 & 42.96 & 21.42 & 42.86 & 21.60 & 48 \\
\hline 49 & 44.04 & 21.48 & 43.95 & 21.67 & 43. 85 & 21.86 & 43. 76 & 22.05 & 49 \\
\hline 50 & 44.94 & 21.92 & 44.84 & 22.11 & 44.75 & 22.31 & 44.65 & 22.50 & 50 \\
\hline \multirow{2}{*}{ 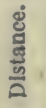 } & Dep. & Lat. & Dep. & Lat. & Dep. & Lat. & Dep. & Lat. & \multirow{2}{*}{ 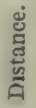 } \\
\hline & \multicolumn{2}{|c|}{$64^{\circ}$. } & \multicolumn{2}{|c|}{$633 / 4^{\circ}$. } & \multicolumn{2}{|c|}{$631 / 2^{\circ}$. } & \multicolumn{2}{|c|}{$631 / 4^{\circ}}$. & \\
\hline
\end{tabular}


TABLE 4.-TRAVERSE TABLE.

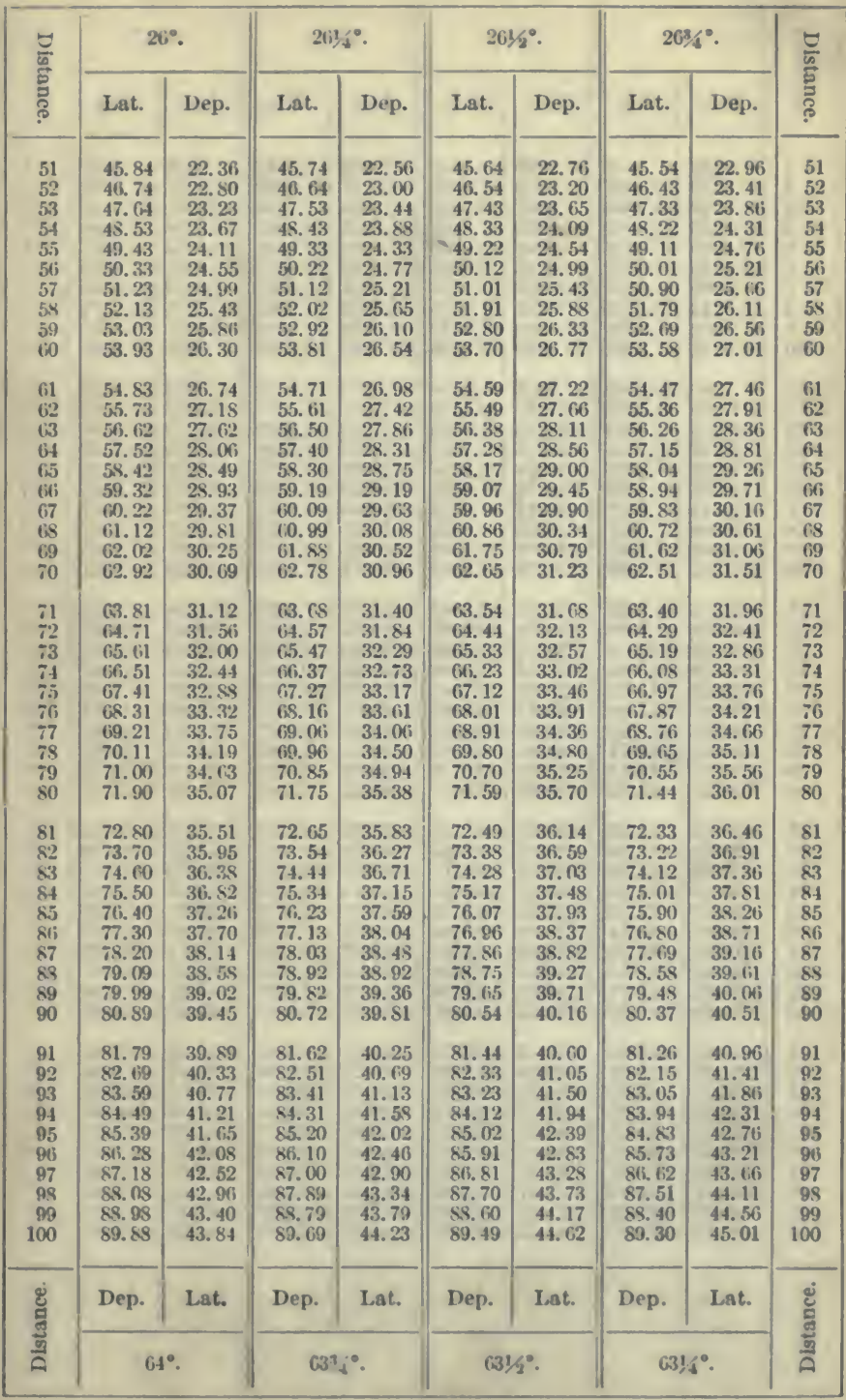


TABLE 4.-TRAVERSE TABLE.

\begin{tabular}{|c|c|c|c|c|c|c|c|c|c|}
\hline \multirow{2}{*}{ 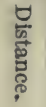 } & \multicolumn{2}{|c|}{$27^{\circ}$. } & \multicolumn{2}{|c|}{$271 / 4^{\circ}$. } & \multicolumn{2}{|c|}{$271 / 2^{\circ}$. } & \multicolumn{2}{|c|}{$27^{3} / 4^{\circ}$. } & \multirow{2}{*}{ 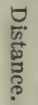 } \\
\hline & Lat. & Dep. & Lat. & Dep. & Lat. & Dep. & Lat. & Dep. & \\
\hline $\begin{array}{r}1 \\
2 \\
3 \\
4 \\
5 \\
6 \\
7 \\
8 \\
9 \\
10\end{array}$ & $\begin{array}{l}0.89 \\
1.78 \\
2.67 \\
3.56 \\
4.46 \\
5.35 \\
6.24 \\
7.13 \\
8.02 \\
8.91\end{array}$ & $\begin{array}{l}0.45 \\
0.91 \\
1.36 \\
1.82 \\
2.27 \\
2.72 \\
3.18 \\
3.63 \\
4.09 \\
4.54\end{array}$ & $\begin{array}{r}0.89 \\
1.78 \\
2.67 \\
3.56 \\
4.45 \\
5.33 \\
6.22 \\
7.11 \\
8.00 \\
8.89\end{array}$ & $\begin{array}{l}0.46 \\
0.92 \\
1.37 \\
1.83 \\
2.29 \\
2.75 \\
3.21 \\
3.66 \\
4.12 \\
4.58\end{array}$ & $\begin{array}{l}0.89 \\
1.77 \\
2.66 \\
3.55 \\
4.44 \\
5.32 \\
6.21 \\
7.10 \\
7.98 \\
8.87\end{array}$ & $\begin{array}{l}0.46 \\
0.92 \\
1.39 \\
1.85 \\
2.31 \\
2.77 \\
3.23 \\
3.69 \\
4.16 \\
4.62\end{array}$ & $\begin{array}{l}0.88 \\
1.77 \\
2.65 \\
3.54 \\
4.42 \\
5.31 \\
6.19 \\
7.08 \\
7.96 \\
8.85\end{array}$ & $\begin{array}{l}0.47 \\
0.93 \\
1.40 \\
1.86 \\
2.33 \\
2.79 \\
3.26 \\
3.72 \\
4.19 \\
4.66\end{array}$ & $\begin{array}{r}1 \\
2 \\
3 \\
4 \\
5 \\
6 \\
7 \\
8 \\
9 \\
10\end{array}$ \\
\hline $\begin{array}{l}11 \\
12 \\
13 \\
14 \\
15 \\
16 \\
17 \\
18 \\
19 \\
20\end{array}$ & $\begin{array}{r}9.80 \\
10.69 \\
11.58 \\
12.47 \\
13.37 \\
14.26 \\
15.15 \\
16.04 \\
16.93 \\
17.82\end{array}$ & $\begin{array}{l}4.99 \\
5.45 \\
5.90 \\
6.36 \\
6.81 \\
7.26 \\
7.72 \\
8.17 \\
8.63 \\
9.08\end{array}$ & $\begin{array}{r}9.78 \\
10.67 \\
11.56 \\
12.45 \\
13.34 \\
14.22 \\
15.11 \\
16.00 \\
16.89 \\
17.78\end{array}$ & $\begin{array}{l}5.04 \\
5.49 \\
5.95 \\
6.41 \\
6.87 \\
7.33 \\
7.78 \\
8.24 \\
8.70 \\
9.16\end{array}$ & $\begin{array}{r}9.76 \\
10.64 \\
11.53 \\
12.42 \\
13.31 \\
14.19 \\
15.08 \\
15.97 \\
16.85 \\
17.74\end{array}$ & $\begin{array}{l}5.08 \\
5.54 \\
6.00 \\
6.46 \\
6.93 \\
7.39 \\
7.85 \\
8.31 \\
8.77 \\
9.23\end{array}$ & $\begin{array}{r}9.73 \\
10.62 \\
11.50 \\
12.39 \\
13.27 \\
14.16 \\
15.04 \\
15.93 \\
16.81 \\
17.70\end{array}$ & $\begin{array}{l}5.12 \\
5.59 \\
6.05 \\
6.52 \\
6.98 \\
7.45 \\
7.92 \\
8.38 \\
8.85 \\
9.31\end{array}$ & $\begin{array}{l}11 \\
12 \\
13 \\
14 \\
15 \\
16 \\
17 \\
18 \\
19 \\
20\end{array}$ \\
\hline $\begin{array}{l}21 \\
22 \\
23 \\
24 \\
25 \\
26 \\
27 \\
28 \\
29 \\
30\end{array}$ & $\begin{array}{l}18.71 \\
19.60 \\
20.49 \\
21.38 \\
22.28 \\
23.17 \\
24.06 \\
24.95 \\
25.84 \\
26.73\end{array}$ & $\begin{array}{r}9.53 \\
9.99 \\
10.44 \\
10.90 \\
11.35 \\
11.80 \\
12.26 \\
12.71 \\
13.17 \\
13.62\end{array}$ & $\begin{array}{l}18.67 \\
19.56 \\
20.45 \\
21.34 \\
22.23 \\
23.11 \\
24.00 \\
24.89 \\
25.78 \\
26.67\end{array}$ & $\begin{array}{r}9.62 \\
10.07 \\
10.53 \\
10.99 \\
11.45 \\
11.90 \\
12.36 \\
12.82 \\
13.28 \\
13.74\end{array}$ & $\begin{array}{l}18.63 \\
19.51 \\
20.40 \\
21.29 \\
22.18 \\
23.06 \\
23.95 \\
24.84 \\
25.72 \\
26.61\end{array}$ & $\begin{array}{r}9.70 \\
10.16 \\
10.62 \\
11.08 \\
11.54 \\
12.01 \\
12.47 \\
12.93 \\
13.39 \\
13.85\end{array}$ & $\begin{array}{l}18.58 \\
19.47 \\
20.35 \\
21.24 \\
22.12 \\
23.01 \\
23.89 \\
24.78 \\
25.66 \\
26.55\end{array}$ & $\begin{array}{r}9.78 \\
10.24 \\
10.71 \\
11.17 \\
11.64 \\
12.11 \\
12.57 \\
13.04 \\
13.50 \\
13.97\end{array}$ & $\begin{array}{l}21 \\
22 \\
23 \\
24 \\
25 \\
26 \\
27 \\
28 \\
29 \\
30\end{array}$ \\
\hline $\begin{array}{l}31 \\
32 \\
33 \\
34 \\
35 \\
36 \\
37 \\
38 \\
39 \\
40\end{array}$ & $\begin{array}{l}27.62 \\
28.51 \\
29.40 \\
30.29 \\
31.19 \\
32.08 \\
32.97 \\
33.86 \\
34.75 \\
35.64\end{array}$ & $\begin{array}{l}14.07 \\
14.53 \\
14.98 \\
15.44 \\
15.89 \\
16.34 \\
16.80 \\
17.25 \\
17.71 \\
18.16\end{array}$ & $\begin{array}{l}27.56 \\
28.45 \\
29.34 \\
30.23 \\
31.12 \\
32.00 \\
32.89 \\
33.78 \\
34.67 \\
35.56\end{array}$ & $\begin{array}{l}14.19 \\
14.65 \\
15.11 \\
15.57 \\
16.03 \\
16.48 \\
16.94 \\
17.40 \\
17.86 \\
18.31\end{array}$ & $\begin{array}{l}27.50 \\
28.38 \\
29.27 \\
30.16 \\
31.05 \\
31.93 \\
32.82 \\
33.71 \\
34.59 \\
35.48\end{array}$ & $\begin{array}{l}14.31 \\
14.78 \\
15.24 \\
15.70 \\
16.16 \\
16.62 \\
17.08 \\
17.55 \\
18.01 \\
18.47\end{array}$ & $\begin{array}{l}27.43 \\
28.32 \\
29.20 \\
30.09 \\
30.97 \\
31.86 \\
32.74 \\
33.643 \\
34.51 \\
35.40\end{array}$ & $\begin{array}{l}14.43 \\
14.90 \\
15.37 \\
15.83 \\
16.30 \\
16.76 \\
17.23 \\
17.69 \\
18.16 \\
18.62\end{array}$ & $\begin{array}{l}31 \\
32 \\
33 \\
34 \\
35 \\
36 \\
37 \\
38 \\
39 \\
40\end{array}$ \\
\hline $\begin{array}{l}41 \\
42 \\
43 \\
44 \\
45 \\
46 \\
47 \\
48 \\
43 \\
50\end{array}$ & $\begin{array}{l}36.53 \\
37.42 \\
38.31 \\
39.20 \\
40.10 \\
40.99 \\
41.88 \\
42.77 \\
43.66 \\
44.55\end{array}$ & $\begin{array}{l}18.61 \\
19.07 \\
19.52 \\
19.98 \\
20.43 \\
20.88 \\
21.34 \\
21.79 \\
22.25 \\
22.70\end{array}$ & $\begin{array}{l}36.45 \\
37.34 \\
38.23 \\
39.12 \\
40.01 \\
40.89 \\
41.78 \\
42.67 \\
43.56 \\
44.45\end{array}$ & $\begin{array}{l}18.77 \\
19.23 \\
19.69 \\
20.15 \\
20.60 \\
21.06 \\
21.52 \\
21.98 \\
22.44 \\
22.89\end{array}$ & $\begin{array}{l}36.37 \\
37.25 \\
38.14 \\
39.03 \\
39.92 \\
40.80 \\
41.69 \\
42.58 \\
43.46 \\
44.35\end{array}$ & $\begin{array}{l}18.93 \\
19.39 \\
19.86 \\
20.32 \\
20.78 \\
21.24 \\
21.70 \\
22.16 \\
22.63 \\
23.09\end{array}$ & $\begin{array}{l}36.28 \\
37.17 \\
38.05 \\
38.94 \\
39.82 \\
40.71 \\
41.59 \\
42.49 \\
43.36 \\
44.25\end{array}$ & $\begin{array}{l}19.09 \\
19.56 \\
20.02 \\
20.49 \\
20.95 \\
21.42 \\
21.88 \\
22.35 \\
22.82 \\
23.28\end{array}$ & $\begin{array}{l}41 \\
42 \\
43 \\
44 \\
45 \\
46 \\
47 \\
48 \\
49 \\
50\end{array}$ \\
\hline 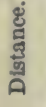 & Dep. & Lat. & Dep. & Lat. & Dep. & Lat. & Dep. & Lat. & 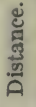 \\
\hline
\end{tabular}


TABLE 4.-TRAVERSE TABLE.

\begin{tabular}{|c|c|c|c|c|c|c|c|c|c|}
\hline \multirow{2}{*}{ 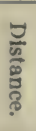 } & \multicolumn{2}{|c|}{$27^{\circ}$. } & \multicolumn{2}{|c|}{$271 / 4^{\circ}$. } & \multicolumn{2}{|c|}{$271 / 2^{\circ}$. } & \multicolumn{2}{|c|}{$27^{3} / 4^{\circ}$. } & \multirow{2}{*}{ 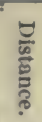 } \\
\hline & Lat. & Dep. & Lat. & Dep. & Lat. & Dep. & Lat. & Dep. & \\
\hline $\begin{array}{l}51 \\
52 \\
53 \\
54 \\
55 \\
56 \\
57 \\
58 \\
59 \\
60\end{array}$ & $\begin{array}{l}45.44 \\
46.33 \\
47.22 \\
48.11 \\
49.01 \\
49.90 \\
50.79 \\
51.68 \\
52.57 \\
53.46\end{array}$ & $\begin{array}{l}23.15 \\
23.61 \\
24.06 \\
24.52 \\
24.97 \\
25.42 \\
25.88 \\
26.33 \\
26.79 \\
27.24\end{array}$ & $\begin{array}{l}45.34 \\
46.23 \\
47.12 \\
43.01 \\
48.90 \\
43.75 \\
50.67 \\
51.56 \\
52.45 \\
53.34\end{array}$ & $\begin{array}{l}23.35 \\
23.81 \\
24.27 \\
24.73 \\
25.18 \\
25.64 \\
26.10 \\
26.56 \\
27.01 \\
27.47\end{array}$ & $\begin{array}{l}45.24 \\
46.12 \\
47.01 \\
47.90 \\
43.79 \\
49.67 \\
50.56 \\
51.45 \\
52.33 \\
53.22\end{array}$ & $\begin{array}{l}23.55 \\
24.01 \\
24.47 \\
24.93 \\
25.40 \\
25.86 \\
26.32 \\
26.78 \\
27.24 \\
27.70\end{array}$ & $\begin{array}{l}45.13 \\
46.02 \\
46.90 \\
47.79 \\
48.67 \\
49.56 \\
50.44 \\
51.33 \\
52.21 \\
53.10\end{array}$ & $\begin{array}{l}23.75 \\
24.21 \\
24.68 \\
25.14 \\
25.61 \\
26.07 \\
26.54 \\
27.01 \\
27.47 \\
27.94\end{array}$ & $\begin{array}{l}51 \\
52 \\
53 \\
54 \\
55 \\
56 \\
57 \\
58 \\
59 \\
60\end{array}$ \\
\hline $\begin{array}{l}61 \\
62 \\
63 \\
64 \\
65 \\
66 \\
67 \\
65 \\
69 \\
70\end{array}$ & $\begin{array}{l}54.35 \\
55.24 \\
56.13 \\
57.02 \\
57.92 \\
58.81 \\
59.70 \\
60.59 \\
61.48 \\
62.37\end{array}$ & $\begin{array}{l}27.69 \\
28.15 \\
28.60 \\
29.06 \\
29.51 \\
29.96 \\
30.42 \\
30.87 \\
31.33 \\
31.78\end{array}$ & $\begin{array}{l}54.23 \\
55.12 \\
56.01 \\
56.90 \\
57.79 \\
58.68 \\
59.56 \\
60.45 \\
61.34 \\
62.23\end{array}$ & $\begin{array}{l}27.93 \\
28.39 \\
28.85 \\
29.30 \\
29.76 \\
30.22 \\
30.68 \\
31.14 \\
31.59 \\
32.05\end{array}$ & $\begin{array}{l}54.11 \\
54.99 \\
55.88 \\
56.77 \\
57.66 \\
58.54 \\
59.43 \\
60.32 \\
61.20 \\
62.09\end{array}$ & $\begin{array}{l}28.17 \\
28.63 \\
29.09 \\
29.55 \\
30.01 \\
30.48 \\
30.94 \\
31.40 \\
31.86 \\
32.32\end{array}$ & $\begin{array}{l}53.98 \\
54.87 \\
55.75 \\
56.64 \\
57.52 \\
58.41 \\
59.29 \\
60.18 \\
61.06 \\
61.95\end{array}$ & $\begin{array}{l}28.40 \\
28.87 \\
29.33 \\
29.80 \\
30.26 \\
30.73 \\
31.20 \\
31.66 \\
32.13 \\
32.59\end{array}$ & $\begin{array}{l}61 \\
62 \\
63 \\
64 \\
65 \\
66 \\
67 \\
68 \\
69 \\
70\end{array}$ \\
\hline $\begin{array}{l}71 \\
72 \\
73 \\
74 \\
75 \\
76 \\
77 \\
78 \\
79 \\
80\end{array}$ & $\begin{array}{l}63.26 \\
64.15 \\
65.04 \\
65.93 \\
66.83 \\
67.72 \\
68.61 \\
69.50 \\
70.39 \\
71.29\end{array}$ & $\begin{array}{l}32.23 \\
32.69 \\
33.14 \\
33.60 \\
34.05 \\
34.50 \\
34.96 \\
35.41 \\
35.57 \\
36.32\end{array}$ & $\begin{array}{l}63.12 \\
64.01 \\
64.90 \\
65.79 \\
66.68 \\
67.57 \\
65.45 \\
69.34 \\
70.23 \\
71.12\end{array}$ & $\begin{array}{l}32.51 \\
32.97 \\
33.42 \\
33.88 \\
34.34 \\
34.80 \\
35.26 \\
35.71 \\
36.17 \\
36.63\end{array}$ & $\begin{array}{l}62.98 \\
63.86 \\
64.75 \\
65.64 \\
66.53 \\
67.41 \\
68.30 \\
69.19 \\
70.07 \\
70.96\end{array}$ & $\begin{array}{l}32.78 \\
33.25 \\
33.71 \\
34.17 \\
34.63 \\
35.09 \\
35.55 \\
36.02 \\
36.48 \\
36.94\end{array}$ & $\begin{array}{l}62.83 \\
63.72 \\
64.60 \\
65.49 \\
66.37 \\
67.26 \\
68.14 \\
69.03 \\
69.91 \\
70.80\end{array}$ & $\begin{array}{l}33.06 \\
33.52 \\
33.99 \\
34.46 \\
34.92 \\
35.39 \\
35.85 \\
36.32 \\
36.78 \\
37.25\end{array}$ & $\begin{array}{l}71 \\
72 \\
73 \\
74 \\
75 \\
76 \\
77 \\
78 \\
79 \\
80\end{array}$ \\
\hline $\begin{array}{l}81 \\
82 \\
83 \\
84 \\
85 \\
86 \\
87 \\
85 \\
89 \\
90\end{array}$ & $\begin{array}{l}72.17 \\
73.06 \\
73.95 \\
74.84 \\
75.74 \\
76.63 \\
77.52 \\
78.41 \\
79.30 \\
80.19\end{array}$ & $\begin{array}{l}36.77 \\
37.23 \\
37.68 \\
39.14 \\
35.59 \\
39.04 \\
39.50 \\
39.95 \\
40.41 \\
40.86\end{array}$ & $\begin{array}{l}72.01 \\
72.90 \\
73.79 \\
74.68 \\
75.57 \\
76.46 \\
77.34 \\
78.23 \\
79.12 \\
80.01\end{array}$ & $\begin{array}{l}37.09 \\
37.55 \\
35.00 \\
35.46 \\
35.92 \\
39.38 \\
39.84 \\
40.29 \\
40.75 \\
41.21\end{array}$ & $\begin{array}{l}71.85 \\
72.73 \\
73.62 \\
74.51 \\
75.40 \\
76.29 \\
77.17 \\
78.06 \\
78.94 \\
79.83\end{array}$ & $\begin{array}{l}37.40 \\
37.86 \\
38.33 \\
38.79 \\
39.25 \\
39.71 \\
40.17 \\
40.63 \\
41.10 \\
41.56\end{array}$ & $\begin{array}{l}71.68 \\
72.57 \\
73.45 \\
74.34 \\
75.22 \\
76.11 \\
76.99 \\
77.88 \\
78.76 \\
79.65\end{array}$ & $\begin{array}{l}37.71 \\
38.18 \\
38.65 \\
39.11 \\
39.58 \\
40.04 \\
40.51 \\
40.97 \\
41.44 \\
41.91\end{array}$ & $\begin{array}{l}81 \\
82 \\
83 \\
84 \\
85 \\
86 \\
87 \\
85 \\
89 \\
90\end{array}$ \\
\hline $\begin{array}{r}91 \\
92 \\
93 \\
94 \\
95 \\
96 \\
97 \\
98 \\
99 \\
100\end{array}$ & $\begin{array}{l}81.08 \\
81.97 \\
82.86 \\
83.75 \\
84.65 \\
85.54 \\
86.43 \\
87.32 \\
83.21 \\
89.10\end{array}$ & $\begin{array}{l}41.31 \\
41,77 \\
42.22 \\
42.68 \\
43.13 \\
43.58 \\
44.04 \\
44.49 \\
44.95 \\
45.40\end{array}$ & $\begin{array}{l}80.90 \\
81.79 \\
82.68 \\
83.57 \\
84.46 \\
85.35 \\
86.23 \\
87.12 \\
83.01 \\
88.90\end{array}$ & $\begin{array}{l}41.67 \\
42.12 \\
42.58 \\
43.04 \\
43.50 \\
43.96 \\
44.41 \\
44.87 \\
45.33 \\
45.79\end{array}$ & $\begin{array}{l}80.72 \\
81.60 \\
82.49 \\
83.38 \\
84.27 \\
85.15 \\
86.04 \\
86.93 \\
87.81 \\
85.70\end{array}$ & $\begin{array}{l}42.02 \\
42.48 \\
42.94 \\
43.40 \\
43.87 \\
44.33 \\
44.79 \\
45.25 \\
45.71 \\
46.17\end{array}$ & $\begin{array}{l}80.53 \\
81.42 \\
82.30 \\
83.19 \\
84.07 \\
84.96 \\
85.84 \\
86.73 \\
87.61 \\
88.50\end{array}$ & $\begin{array}{l}42.37 \\
42.84 \\
43.30 \\
43.77 \\
44.23 \\
44.70 \\
45.16 \\
45.63 \\
46.10 \\
46.56\end{array}$ & $\begin{array}{r}91 \\
92 \\
93 \\
94 \\
95 \\
96 \\
97 \\
98 \\
99 \\
100\end{array}$ \\
\hline \multirow{2}{*}{ 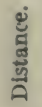 } & Dep. & Lat. & Dep. & Lat. & Dep. & Lat. & Dep. & Lat. & \multirow{2}{*}{ 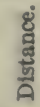 } \\
\hline & \multicolumn{2}{|c|}{$63^{\circ}}$. & \multicolumn{2}{|c|}{$624^{\circ}}$. & \multicolumn{2}{|c|}{$621 / 2^{\circ}$. } & \multicolumn{2}{|c|}{$62 Y^{\circ}}$. & \\
\hline
\end{tabular}


TABLE 4.-TRAVEISE TABLF.

\begin{tabular}{|c|c|c|c|c|c|c|c|c|c|}
\hline \multirow{2}{*}{ 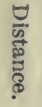 } & \multicolumn{2}{|c|}{$28^{\circ}$. } & \multicolumn{2}{|c|}{$281 / 4^{\circ}$. } & \multicolumn{2}{|c|}{$281 / 2^{\circ}$. } & \multicolumn{2}{|c|}{$2834^{\circ}$. } & \multirow{2}{*}{ 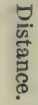 } \\
\hline & Lat. & Dep. & Lat. & Dep. & Lat. & Dep. & Lat. & Dep. & \\
\hline $\begin{array}{r}1 \\
2 \\
3 \\
4 \\
5 \\
6 \\
7 \\
8 \\
9 \\
10\end{array}$ & $\begin{array}{l}0.88 \\
1.77 \\
2.65 \\
3.53 \\
4.41 \\
5.30 \\
6.18 \\
7.06 \\
7.95 \\
8.83\end{array}$ & $\begin{array}{l}0.47 \\
0.94 \\
1.41 \\
1.88 \\
2.35 \\
2.82 \\
3.29 \\
3.76 \\
4.23 \\
4.69\end{array}$ & $\begin{array}{l}0.88 \\
1.76 \\
2.64 \\
3.52 \\
4.40 \\
5.29 \\
6.17 \\
7.05 \\
7.93 \\
8.81\end{array}$ & $\begin{array}{l}0.47 \\
0.95 \\
1.42 \\
1.89 \\
2.37 \\
2.84 \\
3.31 \\
3.79 \\
4.26 \\
4.73\end{array}$ & $\begin{array}{l}0.88 \\
1.76 \\
2.64 \\
3.52 \\
4.39 \\
5.27 \\
6.15 \\
7.03 \\
7.91 \\
8.79\end{array}$ & $\begin{array}{l}0.48 \\
0.95 \\
1.43 \\
1.91 \\
2.39 \\
2.86 \\
3.34 \\
3.82 \\
4.29 \\
4.77\end{array}$ & $\begin{array}{l}0.88 \\
1.75 \\
2.63 \\
3.51 \\
4.38 \\
5.26 \\
6.14 \\
7.01 \\
7.89 \\
8.77\end{array}$ & $\begin{array}{l}0.48 \\
0.96 \\
1.44 \\
1.92 \\
2.40 \\
2.89 \\
3.37 \\
3.85 \\
4.33 \\
4.81\end{array}$ & $\begin{array}{r}1 \\
2 \\
3 \\
4 \\
5 \\
6 \\
7 \\
8 \\
9 \\
10\end{array}$ \\
\hline $\begin{array}{l}11 \\
12 \\
13 \\
14 \\
15 \\
16 \\
17 \\
18 \\
19 \\
20\end{array}$ & $\begin{array}{r}9.71 \\
10.60 \\
11.48 \\
12.36 \\
13.24 \\
14.13 \\
15.01 \\
15.89 \\
16.78 \\
17.66\end{array}$ & $\begin{array}{l}5.16 \\
5.63 \\
6.10 \\
6.57 \\
7.04 \\
7.51 \\
7.98 \\
8.45 \\
8.92 \\
9.39\end{array}$ & $\begin{array}{r}9.69 \\
10.57 \\
11.45 \\
12.33 \\
13.21 \\
14.09 \\
14.98 \\
15.86 \\
16.74 \\
17.62\end{array}$ & $\begin{array}{l}5.21 \\
5.68 \\
6.15 \\
6.63 \\
7.10 \\
7.57 \\
8.05 \\
8.52 \\
8.99 \\
9.47\end{array}$ & $\begin{array}{r}9.67 \\
10.55 \\
11.42 \\
12.30 \\
13.18 \\
14.06 \\
14.94 \\
15.82 \\
16.70 \\
17.58\end{array}$ & $\begin{array}{l}5.25 \\
5.73 \\
6.20 \\
6.68 \\
7.16 \\
7.63 \\
8.11 \\
8.59 \\
9.07 \\
9.54\end{array}$ & $\begin{array}{r}9.64 \\
10.52 \\
11.40 \\
12.27 \\
13.15 \\
14.03 \\
14.90 \\
15.78 \\
16.66 \\
17.53\end{array}$ & $\begin{array}{l}5.29 \\
5.77 \\
6.25 \\
6.73 \\
7.21 \\
7.70 \\
8.18 \\
8.66 \\
9.14 \\
9.62\end{array}$ & $\begin{array}{l}11 \\
12 \\
13 \\
14 \\
15 \\
16 \\
17 \\
18 \\
19 \\
20\end{array}$ \\
\hline $\begin{array}{l}21 \\
22 \\
23 \\
24 \\
25 \\
26 \\
27 \\
28 \\
29 \\
30\end{array}$ & $\begin{array}{l}18.54 \\
19.42 \\
20.31 \\
21.19 \\
22.07 \\
22.96 \\
23.84 \\
24.72 \\
25.61 \\
26.49\end{array}$ & $\begin{array}{r}9.86 \\
10.33 \\
10.80 \\
11.27 \\
11.74 \\
12.21 \\
12.68 \\
13.15 \\
13.61 \\
14.08\end{array}$ & $\begin{array}{l}18.50 \\
19.38 \\
20.26 \\
21.14 \\
22.02 \\
22.90 \\
23.78 \\
24.66 \\
25.55 \\
26.43\end{array}$ & $\begin{array}{r}9.94 \\
10.41 \\
10.89 \\
11.36 \\
11.83 \\
12.31 \\
12.78 \\
13.25 \\
13.73 \\
14.20\end{array}$ & $\begin{array}{l}18.46 \\
19.33 \\
20.21 \\
21.09 \\
21.97 \\
22.85 \\
23.73 \\
24.61 \\
25.49 \\
26.36\end{array}$ & $\begin{array}{l}10.02 \\
10.50 \\
10.97 \\
11.45 \\
11.93 \\
12.41 \\
12.88 \\
13.36 \\
13.84 \\
14.31\end{array}$ & $\begin{array}{l}18.41 \\
19.29 \\
20.16 \\
21.04 \\
21.92 \\
22.79 \\
23.67 \\
24.55 \\
25.43 \\
26.30\end{array}$ & $\begin{array}{l}10.10 \\
10.58 \\
11.06 \\
11.54 \\
12.02 \\
12.51 \\
12.99 \\
13.47 \\
13.95 \\
14.43\end{array}$ & $\begin{array}{l}21 \\
22 \\
23 \\
24 \\
25 \\
26 \\
27 \\
28 \\
29 \\
30\end{array}$ \\
\hline $\begin{array}{l}31 \\
32 \\
33 \\
34 \\
35 \\
36 \\
37 \\
38 \\
39 \\
40\end{array}$ & $\begin{array}{l}27.37 \\
28.25 \\
29.14 \\
30.02 \\
30.90 \\
31.79 \\
32.67 \\
33.55 \\
34.43 \\
35.32\end{array}$ & $\begin{array}{l}14.55 \\
15.02 \\
15.49 \\
15.96 \\
16.43 \\
16.90 \\
17.37 \\
17.84 \\
18.31 \\
18.78\end{array}$ & $\begin{array}{l}27.31 \\
28.19 \\
29.07 \\
29.95 \\
30.83 \\
31.71 \\
32.59 \\
33.47 \\
34.35 \\
35.24\end{array}$ & $\begin{array}{l}14.67 \\
15.15 \\
15.62 \\
16.09 \\
16.57 \\
17.04 \\
17.51 \\
17.99 \\
18.46 \\
18.93\end{array}$ & $\begin{array}{l}27.24 \\
25.12 \\
29.00 \\
29.88 \\
30.76 \\
31.64 \\
32.52 \\
33.40 \\
34.27 \\
35.15\end{array}$ & $\begin{array}{l}14.79 \\
15.27 \\
15.75 \\
16.22 \\
16.70 \\
17.18 \\
17.65 \\
18.13 \\
18.61 \\
19.09\end{array}$ & $\begin{array}{l}27.18 \\
28.06 \\
28.93 \\
29.81 \\
30.69 \\
31.56 \\
32.44 \\
33.32 \\
34.19 \\
35.07\end{array}$ & $\begin{array}{l}14.91 \\
15.39 \\
15.87 \\
16.35 \\
16.83 \\
17.32 \\
17.80 \\
18.28 \\
18.76 \\
19.24\end{array}$ & $\begin{array}{l}31 \\
32 \\
33 \\
34 \\
35 \\
36 \\
37 \\
38 \\
39 \\
40\end{array}$ \\
\hline $\begin{array}{l}41 \\
42 \\
43 \\
44 \\
45 \\
46 \\
47 \\
48 \\
49 \\
50\end{array}$ & $\begin{array}{l}36.20 \\
37.08 \\
37.97 \\
38.85 \\
39.73 \\
40.62 \\
41.50 \\
42.38 \\
43.26 \\
44.15\end{array}$ & $\begin{array}{l}19.25 \\
19.72 \\
20.19 \\
20.66 \\
21.13 \\
21.60 \\
22.07 \\
22.53 \\
23.00 \\
23.47\end{array}$ & $\begin{array}{l}36.12 \\
37.00 \\
37.88 \\
38.76 \\
39.64 \\
40.52 \\
41.40 \\
42.28 \\
43.16 \\
44.04\end{array}$ & $\begin{array}{l}19.41 \\
19.88 \\
20.35 \\
20.83 \\
21.30 \\
21.77 \\
22.25 \\
22.72 \\
23.19 \\
23.67\end{array}$ & $\begin{array}{l}36.03 \\
36.91 \\
37.79 \\
38.67 \\
39.55 \\
40.43 \\
41.30 \\
42.18 \\
43.06 \\
43.94\end{array}$ & $\begin{array}{l}19.56 \\
20.04 \\
20.52 \\
20.99 \\
21.47 \\
21.95 \\
22.43 \\
22.90 \\
23.38 \\
23.86\end{array}$ & $\begin{array}{l}35.95 \\
36.82 \\
37.70 \\
35.58 \\
39.45 \\
40.33 \\
41.21 \\
42.08 \\
42.96 \\
43.84\end{array}$ & $\begin{array}{l}19.72 \\
20.20 \\
20.78 \\
21.16 \\
21.64 \\
22.13 \\
22.61 \\
23.09 \\
23.57 \\
24.05\end{array}$ & $\begin{array}{l}41 \\
42 \\
43 \\
44 \\
45 \\
46 \\
47 \\
48 \\
49 \\
50\end{array}$ \\
\hline 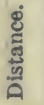 & Dep. & Lat. & Dep. & Lat. & Dep. & Lat. & Dep. & Lat. & 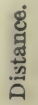 \\
\hline
\end{tabular}


TABLE 4.-TRAVERSE TABLE.

\begin{tabular}{|c|c|c|c|c|c|c|c|c|c|}
\hline \multirow{2}{*}{ 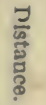 } & \multicolumn{2}{|c|}{$2 S^{\circ}$. } & \multicolumn{2}{|c|}{$2514^{\circ}$. } & \multicolumn{2}{|c|}{$281 / 2^{\circ}$. } & \multicolumn{2}{|c|}{$28 \% 4^{\circ}$. } & \multirow{2}{*}{ 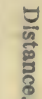 } \\
\hline & Lat. & Dep. & Lat. & Dep. & Lat. & Dep. & Lat. & Dep. & \\
\hline $\begin{array}{l}51 \\
52 \\
53 \\
54 \\
55 \\
56 \\
57 \\
58 \\
59 \\
60\end{array}$ & $\begin{array}{l}45.03 \\
45.91 \\
46.80 \\
47.68 \\
48.56 \\
49.45 \\
50.33 \\
51.21 \\
52.09 \\
52.98\end{array}$ & $\begin{array}{l}23.94 \\
24.41 \\
24.88 \\
25.35 \\
25.83 \\
36.29 \\
26.76 \\
27.23 \\
27.70 \\
25.17\end{array}$ & $\begin{array}{l}44.93 \\
45.81 \\
46.69 \\
47.57 \\
48.45 \\
49.33 \\
50.21 \\
51.09 \\
51.97 \\
52.85\end{array}$ & $\begin{array}{l}24.14 \\
24.61 \\
25.09 \\
25.56 \\
26.03 \\
26.51 \\
26.98 \\
27.45 \\
27.93 \\
28.40\end{array}$ & $\begin{array}{l}44.82 \\
45.70 \\
46.58 \\
47.40 \\
43.33 \\
49.21 \\
50.09 \\
50.97 \\
51.85 \\
52.73\end{array}$ & $\begin{array}{l}24.34 \\
24.81 \\
25.29 \\
25.77 \\
26.24 \\
26.72 \\
27.20 \\
27.18 \\
28.15 \\
28.63\end{array}$ & $\begin{array}{l}44.71 \\
45.59 \\
46.47 \\
47.34 \\
48.22 \\
49.10 \\
49.97 \\
50.85 \\
51.73 \\
52.60\end{array}$ & $\begin{array}{l}24.53 \\
25.01 \\
25.49 \\
25.97 \\
26.45 \\
26.94 \\
27.42 \\
27.90 \\
28.38 \\
28.86\end{array}$ & $\begin{array}{l}51 \\
52 \\
53 \\
54 \\
55 \\
56 \\
57 \\
58 \\
59 \\
60\end{array}$ \\
\hline $\begin{array}{l}61 \\
62 \\
63 \\
64 \\
65 \\
66 \\
67 \\
68 \\
69 \\
70\end{array}$ & $\begin{array}{l}53.86 \\
54.74 \\
55 .(43 \\
56.51 \\
57.39 \\
58.27 \\
59.16 \\
60.01 \\
60.92 \\
61.81\end{array}$ & $\begin{array}{l}23.64 \\
29.11 \\
29.58 \\
30.05 \\
30.52 \\
30.99 \\
31.45 \\
31.92 \\
32.39 \\
32.86\end{array}$ & $\begin{array}{l}53.73 \\
54.62 \\
55.50 \\
56.38 \\
57.26 \\
58.14 \\
59.02 \\
59.90 \\
60.78 \\
61.66\end{array}$ & $\begin{array}{l}28.87 \\
29.35 \\
29.82 \\
30.29 \\
30.77 \\
31.24 \\
31.71 \\
32.19 \\
32.66 \\
33.13\end{array}$ & $\begin{array}{l}53.61 \\
54.49 \\
55.37 \\
56.24 \\
57.12 \\
58.00 \\
58.88 \\
59.76 \\
60.64 \\
61.52\end{array}$ & $\begin{array}{l}29.11 \\
29.58 \\
30.06 \\
30.54 \\
31.02 \\
31.49 \\
31.97 \\
32.45 \\
32.92 \\
33.40\end{array}$ & $\begin{array}{l}53.48 \\
54.36 \\
55.23 \\
56.11 \\
56.99 \\
57.86 \\
58.74 \\
59.62 \\
60.49 \\
61.37\end{array}$ & $\begin{array}{l}29.34 \\
29.82 \\
30.30 \\
30.78 \\
31.26 \\
31.75 \\
32.23 \\
32.71 \\
33.19 \\
33.67\end{array}$ & $\begin{array}{l}61 \\
62 \\
63 \\
64 \\
65 \\
66 \\
67 \\
68 \\
69 \\
70\end{array}$ \\
\hline $\begin{array}{l}71 \\
72 \\
73 \\
74 \\
75 \\
76 \\
77 \\
78 \\
79 \\
80\end{array}$ & $\begin{array}{l}62.69 \\
63.57 \\
64.46 \\
65.34 \\
66.22 \\
67.10 \\
67.99 \\
68.87 \\
69.75 \\
70.64\end{array}$ & $\begin{array}{l}33.33 \\
33.80 \\
34.27 \\
34.74 \\
35.21 \\
35.68 \\
36.15 \\
36.62 \\
37.09 \\
37.56\end{array}$ & $\begin{array}{l}62.54 \\
63.42 \\
64.31 \\
65.19 \\
66.07 \\
66.95 \\
67.83 \\
68.71 \\
69.59 \\
70.47\end{array}$ & $\begin{array}{l}33.61 \\
34.08 \\
34.55 \\
35.03 \\
35.50 \\
35.97 \\
36.45 \\
36.92 \\
37.39 \\
37.87\end{array}$ & $\begin{array}{l}62.40 \\
63.27 \\
64.15 \\
65.03 \\
65.91 \\
66.79 \\
67.67 \\
68.55 \\
69.43 \\
70.31\end{array}$ & $\begin{array}{l}33.88 \\
34.36 \\
34.83 \\
35.31 \\
35.79 \\
36.26 \\
36.74 \\
37.22 \\
37.70 \\
38.17\end{array}$ & $\begin{array}{l}62.25 \\
63.12 \\
64.00 \\
64.88 \\
65.75 \\
66.63 \\
67.51 \\
68.38 \\
69.26 \\
70.14\end{array}$ & $\begin{array}{l}34.15 \\
34.03 \\
35.11 \\
35.59 \\
36.07 \\
36.56 \\
37.04 \\
37.52 \\
38.00 \\
38.48\end{array}$ & $\begin{array}{l}71 \\
72 \\
73 \\
74 \\
75 \\
76 \\
77 \\
78 \\
79 \\
80\end{array}$ \\
\hline $\begin{array}{l}81 \\
82 \\
83 \\
84 \\
85 \\
83 \\
87 \\
83 \\
87 \\
90\end{array}$ & $\begin{array}{l}71.52 \\
72.40 \\
73.28 \\
74.17 \\
75.05 \\
75.9 .3 \\
76.82 \\
77.70 \\
78.58 \\
79.47\end{array}$ & $\begin{array}{l}38.03 \\
38.50 \\
38.97 \\
39.44 \\
39.91 \\
40.37 \\
40.84 \\
41.31 \\
41.78 \\
42.25\end{array}$ & $\begin{array}{l}71.35 \\
72.23 \\
73.11 \\
73.99 \\
74.88 \\
75.76 \\
76.64 \\
77.52 \\
78.40 \\
79.28\end{array}$ & $\begin{array}{l}38.34 \\
38.81 \\
39.29 \\
39.76 \\
40.23 \\
40.71 \\
41.18 \\
41.65 \\
42.13 \\
42.60\end{array}$ & $\begin{array}{l}71.18 \\
72.06 \\
72.94 \\
73.82 \\
74.70 \\
75.58 \\
76.46 \\
77.34 \\
78.21 \\
79.09\end{array}$ & $\begin{array}{l}38.65 \\
39.13 \\
39.60 \\
40.08 \\
40.56 \\
41.04 \\
41.51 \\
41.99 \\
42.47 \\
42.94\end{array}$ & $\begin{array}{l}71.01 \\
71.89 \\
72.77 \\
73.65 \\
74.52 \\
75.40 \\
76.28 \\
77.15 \\
78.03 \\
78.91\end{array}$ & $\begin{array}{l}38.96 \\
39.44 \\
39.92 \\
40.40 \\
40.88 \\
41.37 \\
41.85 \\
42.33 \\
42.81 \\
43.29\end{array}$ & $\begin{array}{l}81 \\
82 \\
83 \\
84 \\
85 \\
86 \\
87 \\
88 \\
89 \\
90\end{array}$ \\
\hline $\begin{array}{r}91 \\
92 \\
93 \\
94 \\
95 \\
96 \\
97 \\
98 \\
99 \\
100\end{array}$ & $\begin{array}{l}80.35 \\
81.23 \\
82.11 \\
83.00 \\
83.85 \\
81.70 \\
85.65 \\
83.53 \\
87.41 \\
88.29\end{array}$ & $\begin{array}{l}42.72 \\
43.19 \\
43.66 \\
44.13 \\
44.60 \\
45.07 \\
45.54 \\
46.01 \\
46.48 \\
46.95\end{array}$ & $\begin{array}{l}80.16 \\
81.04 \\
81.92 \\
82.80 \\
83.68 \\
81.57 \\
85.45 \\
81.33 \\
87.21 \\
88.09\end{array}$ & $\begin{array}{l}43.07 \\
43.55 \\
44.02 \\
44.49 \\
44.97 \\
45.44 \\
45.91 \\
46.39 \\
46.86 \\
47.33\end{array}$ & $\begin{array}{l}79.97 \\
89.85 \\
81.73 \\
82.61 \\
83.49 \\
84.37 \\
85.25 \\
85.12 \\
87.00 \\
87.83\end{array}$ & $\begin{array}{l}43.42 \\
43.90 \\
44.38 \\
44.85 \\
45.33 \\
45.81 \\
46.28 \\
46.76 \\
47.24 \\
47.72\end{array}$ & $\begin{array}{l}79.78 \\
80.66 \\
81.54 \\
82.41 \\
83.29 \\
84.17 \\
85.04 \\
85.92 \\
86.80 \\
87.67\end{array}$ & $\begin{array}{l}43.77 \\
44.25 \\
44.73 \\
45.21 \\
45.69 \\
46.17 \\
46.66 \\
47.14 \\
47.62 \\
48.10\end{array}$ & $\begin{array}{r}91 \\
92 \\
93 \\
94 \\
85 \\
96 \\
97 \\
98 \\
99 \\
100\end{array}$ \\
\hline \multirow{2}{*}{ 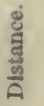 } & Dep. & Lat. & Dep. & Lat. & Depp. & Lat. & Dep. & Lat. & \multirow{2}{*}{$\begin{array}{l}\text { हूँ } \\
\text { दू } \\
\frac{\text { nू }}{0}\end{array}$} \\
\hline & \multicolumn{2}{|c|}{$62^{\circ}$. } & \multicolumn{2}{|c|}{$613 \%^{\circ}}$. & \multicolumn{2}{|c|}{$61: 2^{\circ}$. } & \multicolumn{2}{|c|}{$61: 4^{\circ}$. } & \\
\hline
\end{tabular}


TABLE 4.-TRAVERSE TABLE.

\begin{tabular}{|c|c|c|c|c|c|c|c|c|c|}
\hline \multirow{2}{*}{ 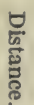 } & \multicolumn{2}{|c|}{$29^{\circ}$. } & \multicolumn{2}{|c|}{$291 / 4^{\circ}$. } & \multicolumn{2}{|c|}{$291 / 2^{\circ}$. } & \multicolumn{2}{|c|}{$29^{3} 4^{\circ}$. } & \multirow{2}{*}{ 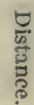 } \\
\hline & Lat. & Dep. & Lat. & Dep. & Lat. & Dep. & Lat. & Dep. & \\
\hline $\begin{array}{r}1 \\
2 \\
3 \\
4 \\
5 \\
6 \\
7 \\
8 \\
9 \\
10\end{array}$ & $\begin{array}{l}0.87 \\
1.75 \\
2.62 \\
3.50 \\
4.37 \\
5.25 \\
6.12 \\
7.00 \\
7.87 \\
8.75\end{array}$ & $\begin{array}{l}0.48 \\
0.97 \\
1.45 \\
1.94 \\
2.42 \\
2.91 \\
3.39 \\
3.88 \\
4.36 \\
4.85\end{array}$ & $\begin{array}{l}0.87 \\
1.74 \\
2.62 \\
3.49 \\
4.36 \\
5.23 \\
6.11 \\
6.98 \\
7.85 \\
8.72\end{array}$ & $\begin{array}{l}0.49 \\
0.98 \\
1.47 \\
1.95 \\
2.44 \\
2.93 \\
3.42 \\
3.91 \\
4.40 \\
4.89\end{array}$ & $\begin{array}{l}0.87 \\
1.74 \\
2.61 \\
3.48 \\
4.35 \\
5.22 \\
6.09 \\
6.96 \\
7.83 \\
8.70\end{array}$ & $\begin{array}{l}0.49 \\
0.98 \\
1.48 \\
1.97 \\
2.46 \\
2.95 \\
3.45 \\
3.94 \\
4.43 \\
4.92\end{array}$ & $\begin{array}{l}0.87 \\
1.74 \\
2.60 \\
3.47 \\
4.34 \\
5.21 \\
6.08 \\
6.95 \\
7.81 \\
8.68\end{array}$ & $\begin{array}{l}0.50 \\
0.99 \\
1.49 \\
1.98 \\
2.48 \\
2.98 \\
3.47 \\
3.97 \\
4.47 \\
4.96\end{array}$ & $\begin{array}{r}1 \\
2 \\
3 \\
4 \\
5 \\
6 \\
7 \\
8 \\
9 \\
10\end{array}$ \\
\hline $\begin{array}{l}11 \\
12 \\
13 \\
14 \\
15 \\
16 \\
17 \\
18 \\
19 \\
20\end{array}$ & $\begin{array}{r}9.62 \\
10.50 \\
11.37 \\
12.24 \\
13.12 \\
13.99 \\
14.87 \\
15.74 \\
16.62 \\
17.49\end{array}$ & $\begin{array}{l}5.33 \\
5.82 \\
6.30 \\
6.79 \\
7.27 \\
7.76 \\
8.24 \\
8.73 \\
9.21 \\
9.70\end{array}$ & $\begin{array}{r}9.60 \\
10.47 \\
11.34 \\
12.21 \\
13.09 \\
13.96 \\
14.83 \\
15.70 \\
16.58 \\
17.45\end{array}$ & $\begin{array}{l}5.37 \\
5.86 \\
6.35 \\
6.84 \\
7.33 \\
7.82 \\
8.31 \\
8.80 \\
9.28 \\
9.77\end{array}$ & $\begin{array}{r}9.57 \\
10.44 \\
11.31 \\
12.18 \\
13.06 \\
13.93 \\
14.80 \\
15.67 \\
16.54 \\
17.41\end{array}$ & $\begin{array}{l}5.42 \\
5.91 \\
6.40 \\
6.89 \\
7.39 \\
7.88 \\
8.37 \\
8.86 \\
9.36 \\
9.85\end{array}$ & $\begin{array}{r}9.55 \\
10.42 \\
11.29 \\
12.15 \\
13.02 \\
13.89 \\
14.76 \\
15.63 \\
16.50 \\
17.36\end{array}$ & $\begin{array}{l}5.46 \\
5.95 \\
6.45 \\
6.95 \\
7.44 \\
7.94 \\
8.44 \\
8.93 \\
9.43 \\
9.92\end{array}$ & $\begin{array}{l}11 \\
12 \\
13 \\
14 \\
15 \\
16 \\
17 \\
18 \\
19 \\
20\end{array}$ \\
\hline $\begin{array}{l}21 \\
22 \\
23 \\
24 \\
25 \\
26 \\
27 \\
28 \\
29 \\
30\end{array}$ & $\begin{array}{l}18.37 \\
19.24 \\
20.12 \\
20.99 \\
21.87 \\
22.74 \\
23.61 \\
24.49 \\
25.36 \\
26.24\end{array}$ & $\begin{array}{l}10.18 \\
10.67 \\
11.15 \\
11.64 \\
12.12 \\
12.61 \\
13.09 \\
13.57 \\
14.06 \\
14.54\end{array}$ & $\begin{array}{l}18.32 \\
19.19 \\
20.07 \\
20.94 \\
21.81 \\
22.68 \\
23.56 \\
24.43 \\
25.30 \\
26.17\end{array}$ & $\begin{array}{l}10.26 \\
10.75 \\
11.24 \\
11.73 \\
12.22 \\
12.70 \\
13.19 \\
13.68 \\
14.17 \\
14.66\end{array}$ & $\begin{array}{l}18.28 \\
19.15 \\
20.02 \\
20.89 \\
21.76 \\
22.63 \\
23.50 \\
24.37 \\
25.24 \\
26.11\end{array}$ & $\begin{array}{l}10.34 \\
10.83 \\
11.33 \\
11.82 \\
12.31 \\
12.80 \\
13.30 \\
13.79 \\
14.28 \\
14.77\end{array}$ & $\begin{array}{l}18.23 \\
19.10 \\
19.97 \\
20.84 \\
21.70 \\
22.57 \\
23.44 \\
24.31 \\
25.18 \\
26.05\end{array}$ & $\begin{array}{l}10.42 \\
10.92 \\
11.41 \\
11.91 \\
12.41 \\
12.90 \\
13.40 \\
13.89 \\
14.39 \\
14.89\end{array}$ & $\begin{array}{l}21 \\
22 \\
23 \\
24 \\
25 \\
20 \\
27 \\
28 \\
29 \\
30\end{array}$ \\
\hline $\begin{array}{l}31 \\
32 \\
33 \\
34 \\
35 \\
36 \\
37 \\
35 \\
39 \\
40\end{array}$ & $\begin{array}{l}27.11 \\
27.99 \\
28.86 \\
29.74 \\
30.61 \\
31.49 \\
32.36 \\
33.24 \\
34.11 \\
34.98\end{array}$ & $\begin{array}{l}15.03 \\
15.51 \\
16.00 \\
16.48 \\
16.97 \\
17.45 \\
17.94 \\
18.42 \\
18.91 \\
19.39\end{array}$ & $\begin{array}{l}27.05 \\
27.92 \\
28.79 \\
29.66 \\
30.54 \\
31.41 \\
32.28 \\
33.15 \\
34.03 \\
34.90\end{array}$ & $\begin{array}{l}15.15 \\
15.64 \\
16.12 \\
16.61 \\
17.10 \\
17.59 \\
18.08 \\
18.57 \\
19.06 \\
19.54\end{array}$ & $\begin{array}{l}26.98 \\
27.85 \\
28.72 \\
29.59 \\
30.46 \\
31.33 \\
32.20 \\
33.07 \\
33.94 \\
34.81\end{array}$ & $\begin{array}{l}15.27 \\
15.76 \\
16.25 \\
16.74 \\
17.23 \\
17.73 \\
18.22 \\
18.71 \\
19.20 \\
19.70\end{array}$ & $\begin{array}{l}26.91 \\
27.78 \\
28.65 \\
29.52 \\
30.39 \\
31.26 \\
32.12 \\
32.99 \\
33.86 \\
34.73\end{array}$ & $\begin{array}{l}15.38 \\
15.88 \\
16.38 \\
16.87 \\
17.37 \\
17.86 \\
18.36 \\
18.86 \\
19.35 \\
19.85\end{array}$ & $\begin{array}{l}31 \\
32 \\
33 \\
34 \\
35 \\
36 \\
37 \\
38 \\
39 \\
40\end{array}$ \\
\hline $\begin{array}{l}41 \\
42 \\
43 \\
44 \\
45 \\
46 \\
47 \\
48 \\
49 \\
50\end{array}$ & $\begin{array}{l}35.86 \\
36.73 \\
37.61 \\
38.48 \\
39.36 \\
40.23 \\
41.11 \\
41.98 \\
42.86 \\
43.73\end{array}$ & $\begin{array}{l}19.88 \\
20.36 \\
20.85 \\
21.33 \\
21.82 \\
22.30 \\
22.79 \\
23.27 \\
23.76 \\
24.24\end{array}$ & $\begin{array}{l}35.77 \\
36.64 \\
37.52 \\
38.39 \\
39.26 \\
40.13 \\
41.01 \\
41.88 \\
42.75 \\
43.62\end{array}$ & $\begin{array}{l}20.03 \\
20.52 \\
21.01 \\
21.50 \\
21.99 \\
22.48 \\
22.97 \\
23.45 \\
23.94 \\
24.43\end{array}$ & $\begin{array}{l}35.68 \\
36.55 \\
37.43 \\
38.30 \\
39.17 \\
40.04 \\
40.91 \\
41.78 \\
42.65 \\
43.52\end{array}$ & $\begin{array}{l}20.19 \\
20.68 \\
21.17 \\
21.67 \\
22.16 \\
22.65 \\
23.14 \\
23.64 \\
24.13 \\
24.62\end{array}$ & $\begin{array}{l}35.60 \\
36.46 \\
37.33 \\
38.20 \\
39.07 \\
39.94 \\
40.81 \\
41.67 \\
42.54 \\
43.41\end{array}$ & $\begin{array}{l}20.34 \\
20.84 \\
21.34 \\
21.83 \\
22.33 \\
22.83 \\
23.32 \\
23.82 \\
24.31 \\
24.81\end{array}$ & $\begin{array}{l}41 \\
42 \\
43 \\
44 \\
45 \\
46 \\
47 \\
48 \\
49 \\
50\end{array}$ \\
\hline ङू & Dep. & Lat. & Dep. & Lat. & Dep. & Lat. & Dep. & Lat. & हृ \\
\hline â & \multicolumn{2}{|c|}{$61^{\circ}$. } & \multicolumn{2}{|c|}{$6034^{\circ}$. } & \multicolumn{2}{|c|}{$001 / 2^{\circ}$. } & \multicolumn{2}{|c|}{$601 / 4^{\circ}$. } & $\ddot{A}$ \\
\hline
\end{tabular}


TABLE 4.-TRAVERSF TABLF.

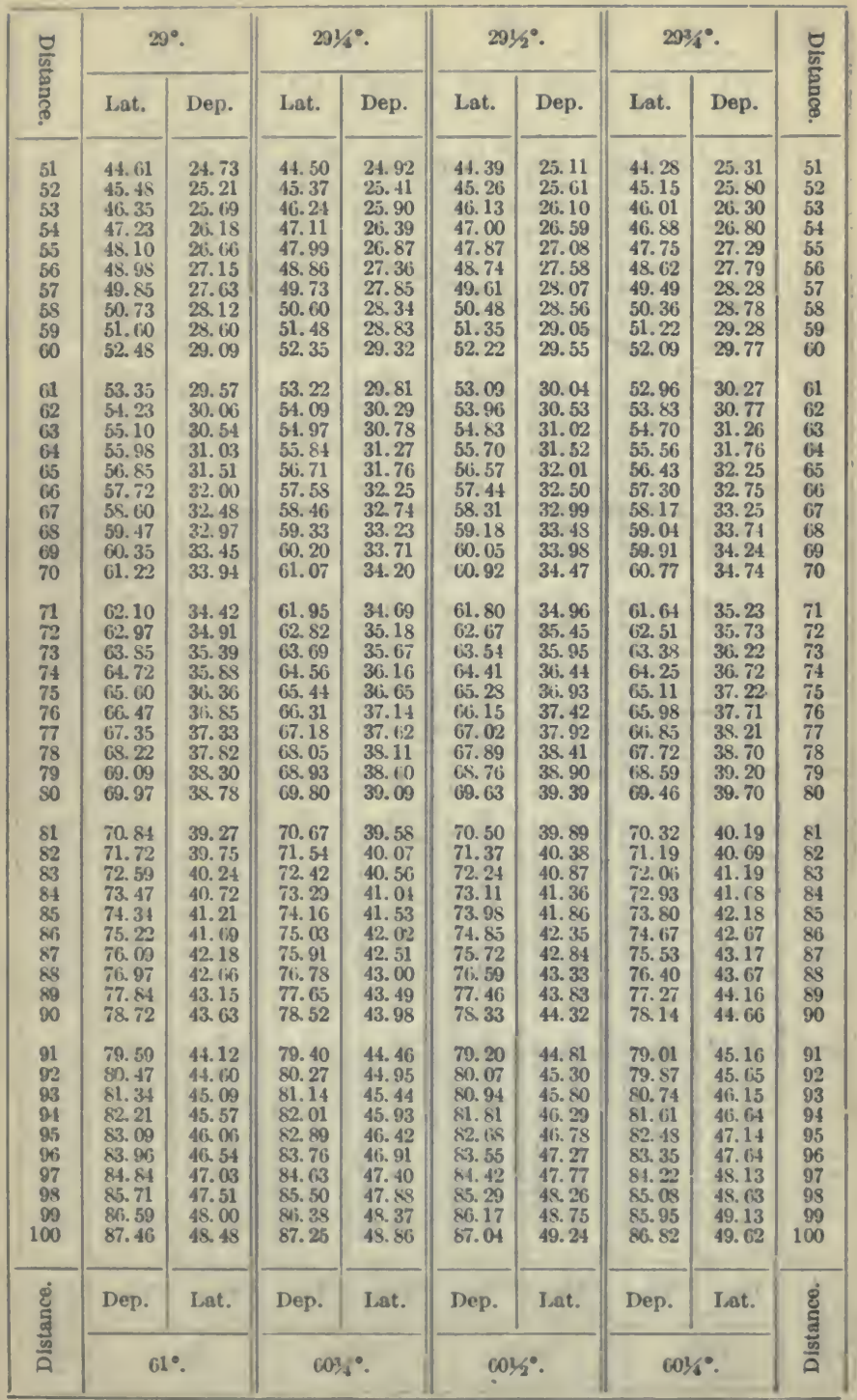


TABLF 4.-TRAVERSE TABLE.

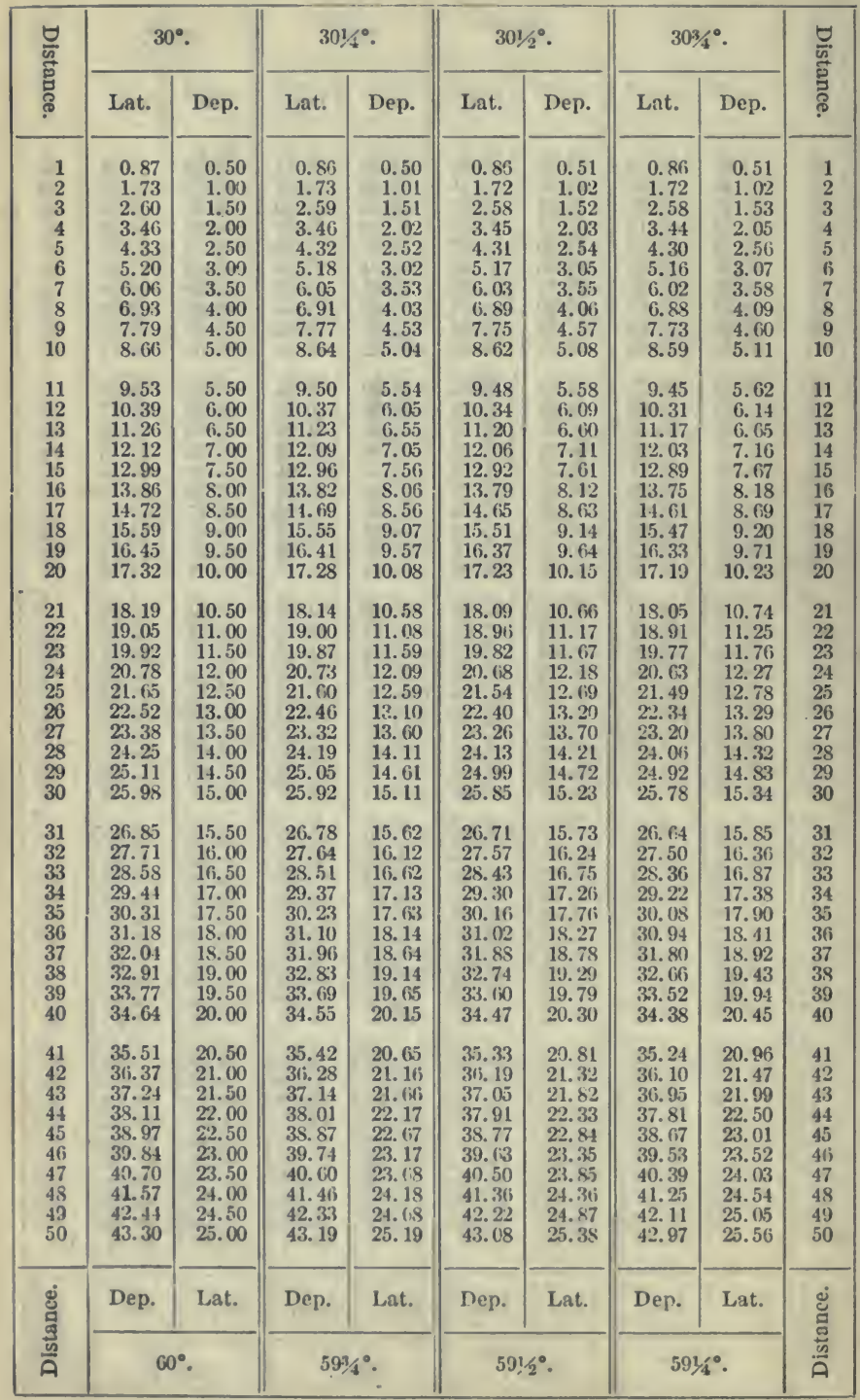


TABLE 4.-TRA VERSE TABLE.

\begin{tabular}{|c|c|c|c|c|c|c|c|c|c|}
\hline \multirow{2}{*}{ 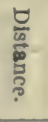 } & \multicolumn{2}{|c|}{$30^{\circ}$. } & \multicolumn{2}{|c|}{$3014^{\circ}$. } & \multicolumn{2}{|c|}{$301 / 2^{\circ}$. } & \multicolumn{2}{|c|}{$3034^{\circ}$. } & \multirow{2}{*}{ 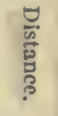 } \\
\hline & Lat. & Dep. & Lat. & Dep. & Lat. & Dep. & Lat. & Dep. & \\
\hline $\begin{array}{l}51 \\
52 \\
53 \\
54 \\
55 \\
56 \\
57 \\
58 \\
59 \\
60\end{array}$ & $\begin{array}{l}44.17 \\
45.03 \\
45.90 \\
46.77 \\
47.63 \\
48.50 \\
49.36 \\
50.23 \\
51.10 \\
51.96\end{array}$ & $\begin{array}{l}25.50 \\
26.00 \\
26.50 \\
27.00 \\
27.50 \\
28.00 \\
28.50 \\
29.00 \\
29.50 \\
30.00\end{array}$ & $\begin{array}{l}44.06 \\
44.92 \\
45.78 \\
46.65 \\
47.51 \\
48.37 \\
49.24 \\
50.10 \\
50.97 \\
51.83\end{array}$ & $\begin{array}{l}25.69 \\
26.20 \\
26.70 \\
27.20 \\
27.71 \\
28.21 \\
28.72 \\
29.22 \\
29.72 \\
30.23\end{array}$ & $\begin{array}{l}43.94 \\
44.80 \\
45.67 \\
46.53 \\
47.39 \\
48.25 \\
49.11 \\
49.97 \\
50.84 \\
51.70\end{array}$ & $\begin{array}{l}25.88 \\
26.39 \\
26.90 \\
27.41 \\
27.91 \\
28.42 \\
28.93 \\
29.44 \\
29.94 \\
30.45\end{array}$ & $\begin{array}{l}43.83 \\
44.69 \\
45.55 \\
46.41 \\
47.27 \\
48.13 \\
48.99 \\
49.85 \\
50.70 \\
51.56\end{array}$ & $\begin{array}{l}26.08 \\
26.59 \\
27.10 \\
27.61 \\
28.12 \\
28.63 \\
29.14 \\
29.65 \\
30.17 \\
30.68\end{array}$ & $\begin{array}{l}51 \\
52 \\
53 \\
54 \\
55 \\
56 \\
57 \\
58 \\
59 \\
60\end{array}$ \\
\hline $\begin{array}{l}61 \\
62 \\
63 \\
64 \\
65 \\
66 \\
67 \\
68 \\
69 \\
70\end{array}$ & $\begin{array}{l}52.83 \\
53.69 \\
54.56 \\
55.43 \\
56.29 \\
57.16 \\
58.02 \\
58.89 \\
59.76 \\
60.62\end{array}$ & $\begin{array}{l}30.50 \\
31.00 \\
31.50 \\
32.00 \\
32.50 \\
33.00 \\
33.50 \\
34.00 \\
34.50 \\
35.00\end{array}$ & $\begin{array}{l}52.69 \\
53.56 \\
54.42 \\
55.29 \\
56.15 \\
57.01 \\
57.89 \\
58.74 \\
59.60 \\
60.47\end{array}$ & $\begin{array}{r}30.73 \\
31.23 \\
31.74 \\
32.24 \\
32.75 \\
33.25 \\
33.75 \\
34.26 \\
34.76 \\
35.26\end{array}$ & $\begin{array}{l}52.56 \\
53.42 \\
54.28 \\
55.14 \\
56.01 \\
56.87 \\
57.73 \\
58.59 \\
59.45 \\
60.31\end{array}$ & $\begin{array}{l}30.96 \\
31.47 \\
31.97 \\
32.48 \\
32.99 \\
33.50 \\
34.01 \\
34.51 \\
35.02 \\
35.53\end{array}$ & $\begin{array}{l}52.42 \\
53.28 \\
54.14 \\
55.00 \\
55.86 \\
56.72 \\
57.58 \\
58.44 \\
59.30 \\
60.16\end{array}$ & $\begin{array}{l}31.19 \\
31.70 \\
32.21 \\
32.72 \\
33.23 \\
33.75 \\
34.26 \\
34.77 \\
35.28 \\
35.79\end{array}$ & $\begin{array}{l}61 \\
62 \\
63 \\
64 \\
65 \\
66 \\
67 \\
68 \\
69 \\
70\end{array}$ \\
\hline $\begin{array}{l}71 \\
72 \\
73 \\
74 \\
75 \\
76 \\
77 \\
78 \\
79 \\
80\end{array}$ & $\begin{array}{l}61.49 \\
62.35 \\
63.22 \\
64.09 \\
64.95 \\
65.82 \\
66.68 \\
67.55 \\
69.42 \\
69.28\end{array}$ & $\begin{array}{l}35.50 \\
36.00 \\
36.50 \\
37.00 \\
37.50 \\
38.00 \\
38.50 \\
39.00 \\
39.50 \\
40.00\end{array}$ & $\begin{array}{l}61.33 \\
62.20 \\
63.06 \\
63.92 \\
64.79 \\
65.65 \\
66.52 \\
67.38 \\
69.24 \\
69.11\end{array}$ & $\begin{array}{l}35.77 \\
36.27 \\
36.78 \\
37.28 \\
37.78 \\
35.29 \\
38.79 \\
39.29 \\
39.80 \\
40.30\end{array}$ & $\begin{array}{l}61.18 \\
62.04 \\
62.90 \\
63.76 \\
64.62 \\
65.48 \\
66.35 \\
67.21 \\
65.07 \\
68.93\end{array}$ & $\begin{array}{l}36.04 \\
36.54 \\
37.05 \\
37.56 \\
38.07 \\
35.57 \\
39.08 \\
39.59 \\
40.10 \\
40.60\end{array}$ & $\begin{array}{l}61.02 \\
61.88 \\
62.74 \\
63.60 \\
64.46 \\
65.31 \\
66.17 \\
67.03 \\
67.89 \\
68.75\end{array}$ & $\begin{array}{l}36.30 \\
36.81 \\
37.32 \\
37.84 \\
38.35 \\
38.86 \\
39.37 \\
39.88 \\
40.39 \\
40.90\end{array}$ & $\begin{array}{l}71- \\
72 \\
73 \\
74 \\
75 \\
76 \\
77 \\
78 \\
79 \\
80\end{array}$ \\
\hline $\begin{array}{l}81 \\
82 \\
83 \\
84 \\
85 \\
86 \\
87 \\
88 \\
89 \\
90\end{array}$ & $\begin{array}{l}70.15 \\
71.01 \\
71.88 \\
72.75 \\
73.61 \\
74.48 \\
75.34 \\
76.21 \\
77.08 \\
77.94\end{array}$ & $\begin{array}{l}40.50 \\
41.00 \\
41.50 \\
42.00 \\
42.50 \\
43.00 \\
43.50 \\
44.00 \\
44.50 \\
45.00\end{array}$ & $\begin{array}{l}69.97 \\
70.83 \\
71.70 \\
72.56 \\
73.43 \\
74.29 \\
75.15 \\
76.02 \\
76.89 \\
77.75\end{array}$ & $\begin{array}{l}40.81 \\
41.31 \\
41.81 \\
42.32 \\
42.82 \\
43.32 \\
43.83 \\
44.33 \\
44.84 \\
45.34\end{array}$ & $\begin{array}{l}69.79 \\
70.65 \\
71.52 \\
72.38 \\
73.24 \\
74.10 \\
74.96 \\
75.82 \\
76.68 \\
77.55\end{array}$ & $\begin{array}{l}41.11 \\
41.62 \\
42.13 \\
42.63 \\
43.14 \\
43.65 \\
44.16 \\
44.66 \\
45.17 \\
45.68\end{array}$ & $\begin{array}{l}69.61 \\
70.47 \\
71.33 \\
72.19 \\
73.05 \\
73.91 \\
74.77 \\
75.63 \\
76.49 \\
77.35\end{array}$ & $\begin{array}{l}41.41 \\
41.93 \\
42.44 \\
42.95 \\
43.46 \\
43.97 \\
44.48 \\
44.99 \\
45.51 \\
46.02\end{array}$ & $\begin{array}{l}81 \\
82 \\
\$ 3 \\
84 \\
85 \\
86 \\
87 \\
88 \\
89 \\
90\end{array}$ \\
\hline $\begin{array}{r}91 \\
92 \\
93 \\
94 \\
95 \\
96 \\
97 \\
98 \\
99 \\
100\end{array}$ & $\begin{array}{l}78.81 \\
79.67 \\
80.54 \\
81.41 \\
82.27 \\
83.14 \\
81.00 \\
84.87 \\
85.74 \\
86.60\end{array}$ & $\begin{array}{l}45.50 \\
46.00 \\
46.50 \\
47.00 \\
47.50 \\
48.00 \\
48.50 \\
49.00 \\
49.50 \\
50.00\end{array}$ & $\begin{array}{l}78.61 \\
79.47 \\
80.34 \\
81.20 \\
82.06 \\
82.93 \\
83.79 \\
84.06 \\
85.52 \\
86.38\end{array}$ & $\begin{array}{l}45.84 \\
46.35 \\
46.85 \\
47.35 \\
47.86 \\
48.36 \\
48.87 \\
49.37 \\
49.87 \\
50.38\end{array}$ & $\begin{array}{l}78.41 \\
79.27 \\
80.13 \\
80.99 \\
81.85 \\
82.72 \\
83.58 \\
84.44 \\
85.30 \\
86.16\end{array}$ & $\begin{array}{l}46.19 \\
46.69 \\
47.20 \\
47.71 \\
48.22 \\
48.72 \\
49.23 \\
49.74 \\
50.25 \\
50.75\end{array}$ & $\begin{array}{l}78.21 \\
79.07 \\
79.92 \\
80.78 \\
81.64 \\
82.50 \\
83.36 \\
84.22 \\
85.08 \\
85.94\end{array}$ & $\begin{array}{l}46.53 \\
47.04 \\
47.55 \\
48.06 \\
48.57 \\
49.08 \\
49.60 \\
50.11 \\
50.012 \\
51.13\end{array}$ & $\begin{array}{r}91 \\
92 \\
93 \\
94 \\
95 \\
96 \\
97 \\
99 \\
99 \\
100\end{array}$ \\
\hline \multirow{2}{*}{ 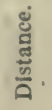 } & Dep. & Lat. & Dep. & Lat. & Dep. & lat. & Dep. & Lat. & \multirow{2}{*}{ 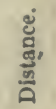 } \\
\hline & \multicolumn{2}{|c|}{$60^{\circ}}$. & \multicolumn{2}{|c|}{$5934^{\circ}}$. & \multicolumn{2}{|c|}{$591 / 2^{\circ}$. } & \multicolumn{2}{|c|}{$5213^{\circ}}$. & \\
\hline
\end{tabular}


TABLE 4.-TRAVERSE TABLE.

\begin{tabular}{|c|c|c|c|c|c|c|c|c|c|}
\hline \multirow{2}{*}{ 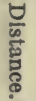 } & \multicolumn{2}{|c|}{$31^{\circ}$. } & \multicolumn{2}{|c|}{$311 / 4^{\circ}$. } & \multicolumn{2}{|c|}{$311 / 2^{\circ}$. } & \multicolumn{2}{|c|}{$3134^{\circ}$. } & \multirow{2}{*}{ 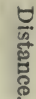 } \\
\hline & Lat. & Dep. & Lat. & Dep. & Lat. & Dep. & Lat. & Dep. & \\
\hline $\begin{array}{r}1 \\
2 \\
3 \\
4 \\
5 \\
6 \\
7 \\
8 \\
9 \\
10\end{array}$ & $\begin{array}{l}0.86 \\
1.71 \\
2.57 \\
3.43 \\
4.29 \\
5.14 \\
6.00 \\
6.86 \\
7.71 \\
8.57\end{array}$ & $\begin{array}{l}0.52 \\
1.03 \\
1.55 \\
2.06 \\
2.58 \\
3.09 \\
3.61 \\
4.12 \\
4.04 \\
5.15\end{array}$ & $\begin{array}{l}0.85 \\
1.71 \\
2.56 \\
3.42 \\
4.27 \\
5.13 \\
5.98 \\
6.84 \\
7.69 \\
8.55\end{array}$ & $\begin{array}{l}0.52 \\
1.04 \\
1.56 \\
2.08 \\
2.59 \\
3.11 \\
3.63 \\
4.15 \\
4.67 \\
5.19\end{array}$ & $\begin{array}{l}0.85 \\
1.71 \\
2.56 \\
3.41 \\
4.26 \\
5.12 \\
5.97 \\
6.82 \\
7.67 \\
8.53\end{array}$ & $\begin{array}{l}0.52 \\
1.04 \\
1.57 \\
2.09 \\
2.61 \\
3.13 \\
3.66 \\
4.18 \\
4.70 \\
5.22\end{array}$ & $\begin{array}{l}0.85 \\
1.70 \\
2.55 \\
3.40 \\
4.25 \\
5.10 \\
5.95 \\
6.80 \\
7.65 \\
8.50\end{array}$ & $\begin{array}{l}0.53 \\
1.05 \\
1.58 \\
2.10 \\
2.63 \\
3.16 \\
3.68 \\
4.21 \\
4.74 \\
5.26\end{array}$ & $\begin{array}{r}1 \\
2 \\
3 \\
4 \\
5 \\
-6 \\
7 \\
8 \\
9 \\
10\end{array}$ \\
\hline $\begin{array}{l}11 \\
12 \\
13 \\
14 \\
15 \\
16 \\
17 \\
18 \\
19 \\
20\end{array}$ & $\begin{array}{r}9.43 \\
10.29 \\
11.14 \\
12.00 \\
12.86 \\
13.71 \\
14.57 \\
15.43 \\
16.29 \\
17.14\end{array}$ & $\begin{array}{r}5.67 \\
6.18 \\
6.70 \\
7.21 \\
7.73 \\
8.24 \\
8.76 \\
9.27 \\
9.79 \\
10.30\end{array}$ & $\begin{array}{r}9.40 \\
10.26 \\
11.11 \\
11.97 \\
12.82 \\
13.68 \\
14.53 \\
15.39 \\
16.24 \\
17.10\end{array}$ & $\begin{array}{r}5.71 \\
6.23 \\
6.74 \\
7.26 \\
7.78 \\
8.30 \\
8.82 \\
9.34 \\
9.86 \\
10.38\end{array}$ & $\begin{array}{r}9.38 \\
10.23 \\
11.08 \\
11.94 \\
12.79 \\
13.64 \\
14.49 \\
15.35 \\
16.30 \\
17.05\end{array}$ & $\begin{array}{r}5.75 \\
6.27 \\
6.79 \\
7.31 \\
7.84 \\
8.36 \\
8.88 \\
9.40 \\
9.93 \\
10.45\end{array}$ & $\begin{array}{r}9.35 \\
10.20 \\
11.05 \\
11.90 \\
12.76 \\
13.61 \\
14.46 \\
15.31 \\
16.16 \\
17.01\end{array}$ & $\begin{array}{r}5.79 \\
6.31 \\
6.84 \\
7.37 \\
7.89 \\
8.42 \\
8.95 \\
9.47 \\
10.00 \\
10.52\end{array}$ & $\begin{array}{l}11 \\
12 \\
13 \\
14 \\
15 \\
16 \\
17 \\
18 \\
19 \\
20\end{array}$ \\
\hline $\begin{array}{l}21 \\
22 \\
23 \\
24 \\
25 \\
26 \\
27 \\
29 \\
29 \\
30\end{array}$ & $\begin{array}{l}18.00 \\
18.86 \\
19.71 \\
20.57 \\
21.43 \\
22.29 \\
23.14 \\
24.00 \\
24.86 \\
25.72\end{array}$ & $\begin{array}{l}10.82 \\
11.33 \\
11.85 \\
12.36 \\
12.89 \\
13.39 \\
13.91 \\
14.42 \\
14.94 \\
15.45\end{array}$ & $\begin{array}{l}17.95 \\
18.81 \\
19.66 \\
20.52 \\
21.37 \\
22.23 \\
23.08 \\
23.94 \\
24.79 \\
25.65\end{array}$ & $\begin{array}{l}10.89 \\
11.41 \\
11.93 \\
12.45 \\
12.97 \\
13.49 \\
14.01 \\
14.53 \\
15.04 \\
15.56\end{array}$ & $\begin{array}{l}17.91 \\
18.76 \\
19.61 \\
20.46 \\
21.32 \\
22.17 \\
23.02 \\
23.87 \\
24.73 \\
25.58\end{array}$ & $\begin{array}{l}10.97 \\
11.49 \\
12.02 \\
12.54 \\
13.06 \\
13.58 \\
14.11 \\
14.63 \\
15.15 \\
15.67\end{array}$ & $\begin{array}{l}17.86 \\
18.71 \\
19.56 \\
20.41 \\
21.26 \\
22.11 \\
22.96 \\
23.81 \\
24.66 \\
25.51\end{array}$ & $\begin{array}{l}11.05 \\
11.58 \\
12.10 \\
12.63 \\
13.16 \\
13.68 \\
14.21 \\
14.73 \\
15.26 \\
15.79\end{array}$ & $\begin{array}{l}21 \\
22 \\
23 \\
24 \\
25 \\
26 \\
27 \\
28 \\
29 \\
30\end{array}$ \\
\hline $\begin{array}{l}31 \\
32 \\
33 \\
34 \\
35 \\
36 \\
37 \\
39 \\
39 \\
40\end{array}$ & $\begin{array}{l}26.57 \\
27.43 \\
28.29 \\
29.14 \\
30.00 \\
30.86 \\
31.72 \\
32.57 \\
33.43 \\
34.29\end{array}$ & $\begin{array}{l}15.97 \\
16.48 \\
17.00 \\
17.51 \\
18.03 \\
18.54 \\
19.06 \\
19.57 \\
20.09 \\
20.60\end{array}$ & $\begin{array}{l}26.50 \\
27.36 \\
28.21 \\
29.07 \\
29.92 \\
30.78 \\
31.63 \\
32.49 \\
33.34 \\
34.20\end{array}$ & $\begin{array}{l}16.09 \\
16.60 \\
17.12 \\
17.64 \\
18.16 \\
18.68 \\
19.19 \\
19.71 \\
20.23 \\
20.75\end{array}$ & $\begin{array}{l}26.43 \\
27.28 \\
28.14 \\
28.99 \\
29.84 \\
30.70 \\
31.55 \\
32.40 \\
33.25 \\
34.11\end{array}$ & $\begin{array}{l}16.20 \\
16.72 \\
17.24 \\
17.76 \\
18.29 \\
18.81 \\
19.33 \\
19.85 \\
20.38 \\
20.90\end{array}$ & $\begin{array}{l}26.36 \\
27.21 \\
28.06 \\
28.91 \\
29.76 \\
30.61 \\
31.46 \\
32.31 \\
33.16 \\
34.01\end{array}$ & $\begin{array}{l}16.31 \\
16.84 \\
17.37 \\
17.89 \\
18.42 \\
18.91 \\
19.47 \\
20.00 \\
20.52 \\
21.05\end{array}$ & $\begin{array}{l}31 \\
32 \\
33 \\
34 \\
35 \\
30 \\
37 \\
38 \\
39 \\
40\end{array}$ \\
\hline $\begin{array}{l}41 \\
42 \\
43 \\
44 \\
45 \\
46 \\
47 \\
48 \\
49 \\
50\end{array}$ & $\begin{array}{l}35.14 \\
36.00 \\
36.86 \\
37.72 \\
33.57 \\
39.43 \\
40.29 \\
41.14 \\
42.00 \\
42.86\end{array}$ & $\begin{array}{l}21.12 \\
21.63 \\
22.15 \\
22.66 \\
23.18 \\
23.69 \\
24.21 \\
24.72 \\
25.24 \\
25.75\end{array}$ & $\begin{array}{l}35.05 \\
35.91 \\
36.76 \\
37.62 \\
38.47 \\
39.33 \\
40.18 \\
41.04 \\
41.89 \\
42.75\end{array}$ & $\begin{array}{l}21.27 \\
21.79 \\
22.31 \\
22.83 \\
23.34 \\
23.86 \\
24.38 \\
24.90 \\
25.42 \\
25.94\end{array}$ & $\begin{array}{l}34.96 \\
35.81 \\
36.66 \\
37.52 \\
38.37 \\
39.22 \\
40.07 \\
40.93 \\
41.79 \\
42.63\end{array}$ & $\begin{array}{l}21.42 \\
21.94 \\
22.47 \\
22.99 \\
23.51 \\
24.03 \\
24.56 \\
25.08 \\
25.60 \\
26.12\end{array}$ & $\begin{array}{l}34.86 \\
35.71 \\
36.57 \\
37.42 \\
39.27 \\
39.12 \\
39.97 \\
40.82 \\
41.67 \\
42.52\end{array}$ & $\begin{array}{l}21.57 \\
22.10 \\
22.63 \\
23.15 \\
23.68 \\
24.21 \\
24.73 \\
25.26 \\
25.78 \\
26.31\end{array}$ & $\begin{array}{l}41 \\
42 \\
43 \\
44 \\
45 \\
46 \\
47 \\
49 \\
49 \\
50\end{array}$ \\
\hline छ & Dep. & Lat. & Dep. & Lat. & Dep. & Lat. & Dep. & Lat. & ச் \\
\hline$\frac{n}{A}$ & \multicolumn{2}{|c|}{$59^{\circ}$} & \multicolumn{2}{|c|}{$55^{3} / 4^{\circ}$. } & \multicolumn{2}{|c|}{$581 / 2^{\circ}}$. & \multicolumn{2}{|c|}{$581 / 4^{\circ}$. } & $\frac{n}{0}$ \\
\hline
\end{tabular}


TABLE 4.-TRAVERSE TABLE.

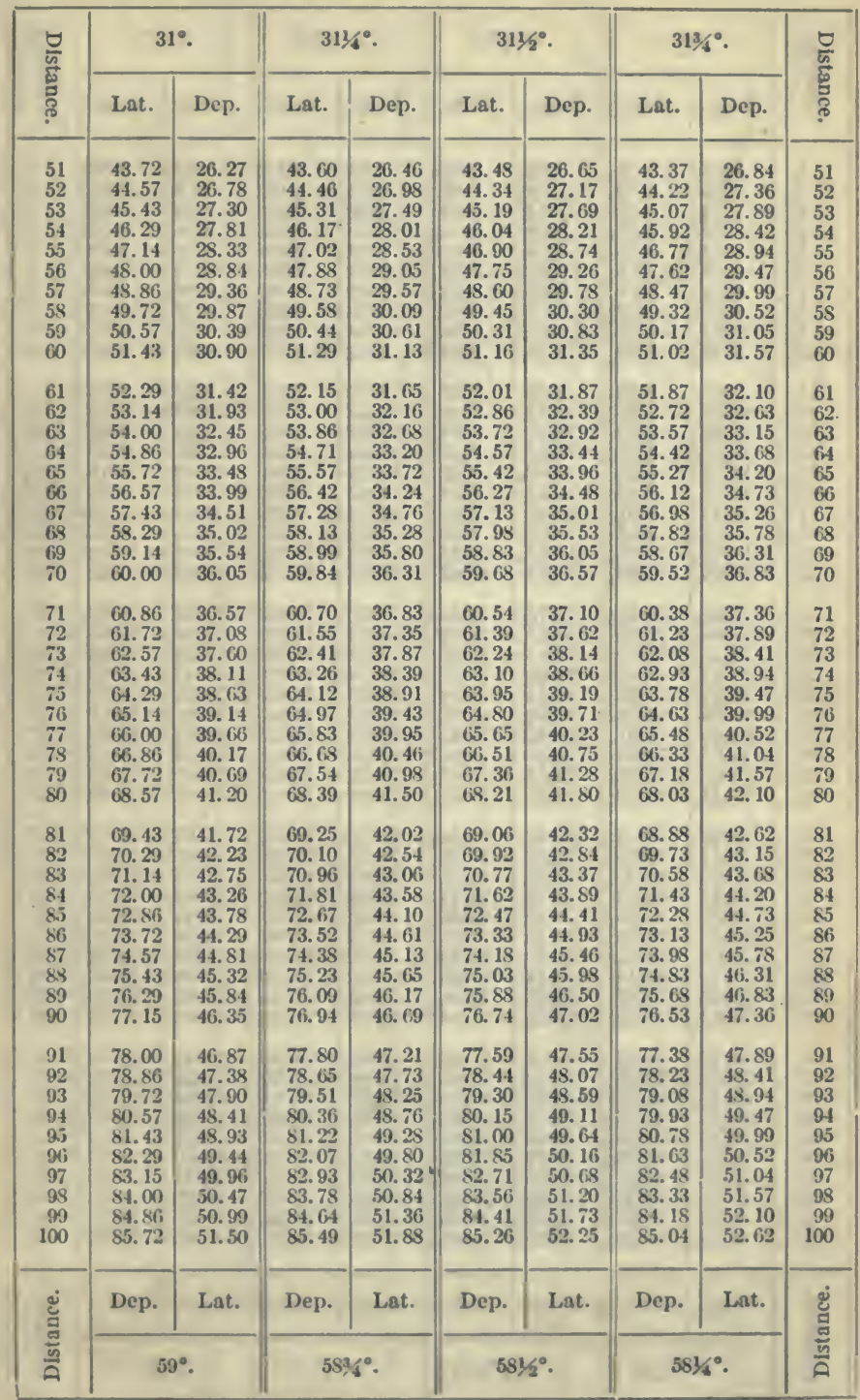


TABLE 4. -TRAVERSE TABLE.

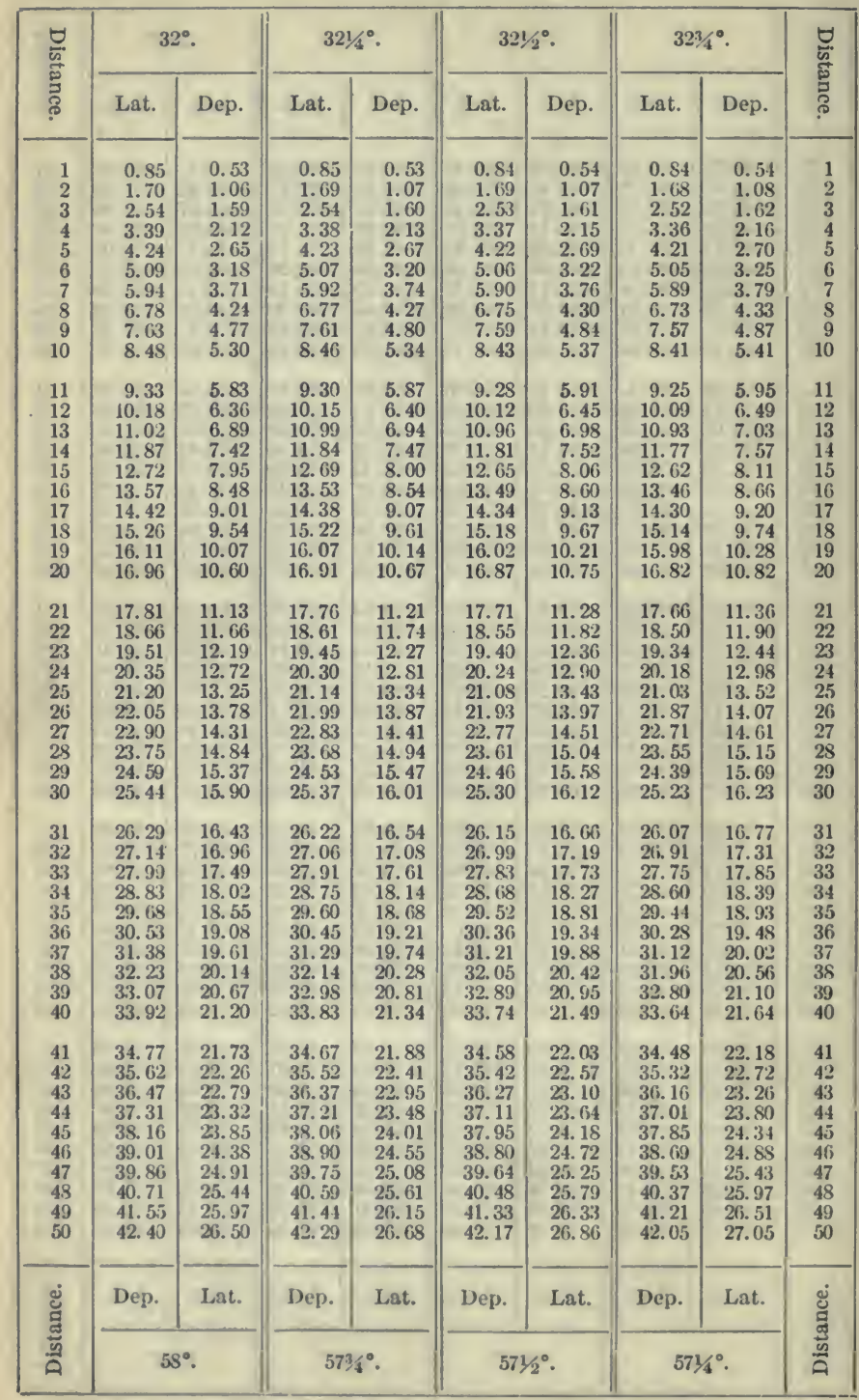


TABI.E 4.-TIIVERSE TABLE.

\begin{tabular}{|c|c|c|c|c|c|c|c|c|c|}
\hline \multirow{2}{*}{ 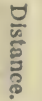 } & \multicolumn{2}{|c|}{$32^{\circ}$. } & \multicolumn{2}{|c|}{$3214^{\circ}$. } & \multicolumn{2}{|c|}{$321 / 2^{\circ}$. } & \multicolumn{2}{|c|}{$3234^{\circ}$. } & \multirow{2}{*}{$\frac{\vec{\theta}}{\frac{\vec{\omega}}{\vec{\omega}}}$} \\
\hline & Lat. & Dep. & Lat. & Dep. & Lat. & Dep. & Lat. & Dep. & \\
\hline $\begin{array}{l}51 \\
52 \\
53 \\
54 \\
55 \\
56 \\
57 \\
59 \\
59 \\
60\end{array}$ & $\begin{array}{l}43.25 \\
44.10 \\
44.95 \\
45.79 \\
46.64 \\
47.49 \\
48.34 \\
49.19 \\
50.03 \\
50.85\end{array}$ & $\begin{array}{l}27.03 \\
27.56 \\
38.09 \\
25.62 \\
29.15 \\
29.68 \\
30.21 \\
30.74 \\
31.27 \\
31.80\end{array}$ & $\begin{array}{l}43.13 \\
43.98 \\
44.82 \\
45.67 \\
46.52 \\
47.36 \\
48.21 \\
49.05 \\
49.90 \\
50.74\end{array}$ & $\begin{array}{l}27.21 \\
27.75 \\
28.28 \\
28.82 \\
29.35 \\
29.88 \\
30.42 \\
30.95 \\
31.48 \\
32.02\end{array}$ & $\begin{array}{l}43.01 \\
43.86 \\
44.70 \\
45.54 \\
46.39 \\
47.23 \\
48.07 \\
48.92 \\
49.76 \\
50.60\end{array}$ & $\begin{array}{l}27.40 \\
27.94 \\
28.48 \\
29.01 \\
29.55 \\
30.09 \\
30.63 \\
31.16 \\
31.70 \\
32.24\end{array}$ & $\begin{array}{l}42.89 \\
43.73 \\
44.55 \\
45.42 \\
46.26 \\
47.10 \\
47.94 \\
48.78 \\
49.62 \\
50.46\end{array}$ & $\begin{array}{l}27.59 \\
23.13 \\
28.67 \\
29.21 \\
29.75 \\
30.29 \\
30.84 \\
31.38 \\
31.92 \\
32.46\end{array}$ & $\begin{array}{l}51 \\
52 \\
53 \\
54 \\
55 \\
56 \\
57 \\
58 \\
59 \\
60\end{array}$ \\
\hline $\begin{array}{l}61 \\
62 \\
63 \\
64 \\
65 \\
66 \\
67 \\
68 \\
69 \\
70\end{array}$ & $\begin{array}{l}51.73 \\
52.58 \\
53.43 \\
54.28 \\
55.12 \\
55.97 \\
56.82 \\
57.67 \\
58.52 \\
59.36\end{array}$ & $\begin{array}{l}32.33 \\
32.85 \\
33.38 \\
33.91 \\
34.44 \\
34.97 \\
35.50 \\
36.03 \\
36.56 \\
37.09\end{array}$ & $\begin{array}{l}51.59 \\
52.44 \\
53.25 \\
54.13 \\
54.97 \\
55.82 \\
56.66 \\
57.51 \\
58.36 \\
59.20\end{array}$ & $\begin{array}{l}32.55 \\
33.68 \\
33.62 \\
34.15 \\
34.68 \\
35.22 \\
35.75 \\
36.29 \\
36.82 \\
37.35\end{array}$ & $\begin{array}{l}51.45 \\
52.29 \\
53.13 \\
53.95 \\
54.82 \\
55.66 \\
56.51 \\
57.35 \\
58.19 \\
59.04\end{array}$ & $\begin{array}{l}32.78 \\
33.31 \\
33.85 \\
34.39 \\
34.92 \\
35.46 \\
36.00 \\
36.54 \\
37.07 \\
37.61\end{array}$ & $\begin{array}{l}51.30 \\
52.14 \\
52.99 \\
53.83 \\
54.67 \\
55.51 \\
56.35 \\
57.19 \\
58.03 \\
58.87\end{array}$ & $\begin{array}{l}33.00 \\
33.54 \\
34.08 \\
34.62 \\
35.16 \\
35.70 \\
36.25 \\
36.79 \\
37.33 \\
37.87\end{array}$ & $\begin{array}{l}61 \\
62 \\
63 \\
64 \\
65 \\
66 \\
67 \\
68 \\
69 \\
70\end{array}$ \\
\hline $\begin{array}{l}71 \\
72 \\
73 \\
74 \\
75 \\
76 \\
77 \\
78 \\
79 \\
80\end{array}$ & $\begin{array}{l}60.21 \\
61.06 \\
61.91 \\
62.711 \\
63.10 \\
64.45 \\
65.30 \\
66.15 \\
67.00 \\
67.84\end{array}$ & $\begin{array}{l}37.62 \\
38.15 \\
38.68 \\
39.21 \\
39.74 \\
40.27 \\
40.80 \\
41.33 \\
41.86 \\
42.39\end{array}$ & $\begin{array}{l}60.05 \\
60.59 \\
61.74 \\
62.58 \\
63.43 \\
64.28 \\
65.12 \\
65.97 \\
66.81 \\
67.66\end{array}$ & $\begin{array}{l}37.89 \\
38.42 \\
38.95 \\
39.49 \\
40.02 \\
40.55 \\
41.09 \\
41.62 \\
42.16 \\
42.69\end{array}$ & $\begin{array}{l}59.88 \\
60.72 \\
61.57 \\
62.41 \\
63.25 \\
64.10 \\
64.94 \\
65.78 \\
66.63 \\
67.47\end{array}$ & $\begin{array}{l}35.15 \\
35.69 \\
39.22 \\
39.76 \\
40.30 \\
40.83 \\
41.37 \\
41.91 \\
42.45 \\
42.98\end{array}$ & $\begin{array}{l}59.71 \\
60.55 \\
61.40 \\
62.24 \\
63.08 \\
63.92 \\
64.76 \\
65.60 \\
66.44 \\
67.28\end{array}$ & $\begin{array}{l}38.41 \\
38.95 \\
39.49 \\
40.03 \\
40.57 \\
41.11 \\
41.66 \\
42.20 \\
42.74 \\
43.28\end{array}$ & $\begin{array}{l}71 \\
72 \\
73 \\
74 \\
75 \\
76 \\
77 \\
78 \\
79 \\
80\end{array}$ \\
\hline $\begin{array}{l}81 \\
82 \\
83 \\
84 \\
85 \\
86 \\
87 \\
85 \\
89 \\
90\end{array}$ & $\begin{array}{l}65.69 \\
69.54 \\
70.39 \\
71.24 \\
72.08 \\
72.93 \\
73.78 \\
74.63 \\
75.44 \\
76.32\end{array}$ & $\begin{array}{l}42.92 \\
43.45 \\
43.98 \\
44.51 \\
45.04 \\
45.57 \\
46.10 \\
46.16 \\
47.16 \\
47.69\end{array}$ & $\begin{array}{l}68.50 \\
69.35 \\
70.20 \\
71.04 \\
71.89 \\
72.73 \\
73.58 \\
74.42 \\
75.27 \\
76.12\end{array}$ & $\begin{array}{r}43.22 \\
43.76 \\
44.29 \\
44.82 \\
45.36 \\
45.89 \\
46.42 \\
46.96 \\
47.49 \\
48.03\end{array}$ & $\begin{array}{l}68.31 \\
69.16 \\
70.00 \\
70.84 \\
71.69 \\
72.53 \\
73.38 \\
74.22 \\
75.06 \\
75.91\end{array}$ & $\begin{array}{l}43.52 \\
44.06 \\
41.60 \\
45.13 \\
45.67 \\
46.21 \\
46.75 \\
47.28 \\
47.82 \\
48.36\end{array}$ & $\begin{array}{l}68.12 \\
68.97 \\
69.81 \\
70.65 \\
71.49 \\
72.33 \\
73.17 \\
74.01 \\
74.85 \\
75.69\end{array}$ & $\begin{array}{l}43.82 \\
44.36 \\
44.90 \\
45.44 \\
45.98 \\
46.52 \\
47.06 \\
47.61 \\
48.15 \\
48.69\end{array}$ & $\begin{array}{l}81 \\
82 \\
83 \\
84 \\
85 \\
86 \\
87 \\
88 \\
89 \\
90\end{array}$ \\
\hline $\begin{array}{r}91 \\
92 \\
93 \\
94 \\
95 \\
965 \\
97 \\
93 \\
99 \\
100\end{array}$ & $\begin{array}{l}77.17 \\
78.02 \\
78.87 \\
79.72 \\
80.51 \\
81.41 \\
82.26 \\
83.11 \\
83.96 \\
84.80\end{array}$ & $\begin{array}{l}48.22 \\
48.75 \\
49.28 \\
49.81 \\
50.34 \\
50.87 \\
51.40 \\
51.93 \\
52.46 \\
52.99\end{array}$ & $\begin{array}{l}76.96 \\
77.81 \\
78.65 \\
79.50 \\
80.34 \\
81.19 \\
82.04 \\
82.88 \\
83.73 \\
84.57\end{array}$ & $\begin{array}{l}48.56 \\
49.09 \\
49.63 \\
50.16 \\
50.69 \\
51.23 \\
51.76 \\
52.29 \\
52.83 \\
53.36\end{array}$ & $\begin{array}{l}76.75 \\
77.59 \\
78.44 \\
79.28 \\
80.12 \\
80.97 \\
81.81 \\
82.615 \\
83.50 \\
84.34\end{array}$ & $\begin{array}{l}48.89 \\
49.43 \\
49.97 \\
50.51 \\
51.04 \\
51.58 \\
52.12 \\
52.66 \\
53.19 \\
53.73\end{array}$ & $\begin{array}{l}76.53 \\
77.38 \\
78.22 \\
79.06 \\
79.90 \\
80.74 \\
81.58 \\
82.42 \\
83.26 \\
81.10\end{array}$ & $\begin{array}{l}49.23 \\
49.77 \\
50.31 \\
50.85 \\
51.39 \\
51.93 \\
52.47 \\
53.02 \\
53.54 \\
54.10\end{array}$ & $\begin{array}{r}91 \\
92 \\
93 \\
94 \\
95 \\
96 \\
97 \\
98 \\
99 \\
100\end{array}$ \\
\hline छ் & Dep. & Lat. & Dep. & Lat. & Dep. & Lat. & Dep. & Lat. & ¿ू் \\
\hline$\frac{n}{a}$ & \multicolumn{2}{|c|}{$5 S^{\circ}}$. & \multicolumn{2}{|c|}{$5 i^{3} / 4^{\circ}$. } & \multicolumn{2}{|c|}{$571 / 2^{\circ}$. } & \multicolumn{2}{|c|}{$57 Y^{\circ}$. } & $\bar{D}$ \\
\hline
\end{tabular}


TABLE 4.-TRAVERSE TABLE.

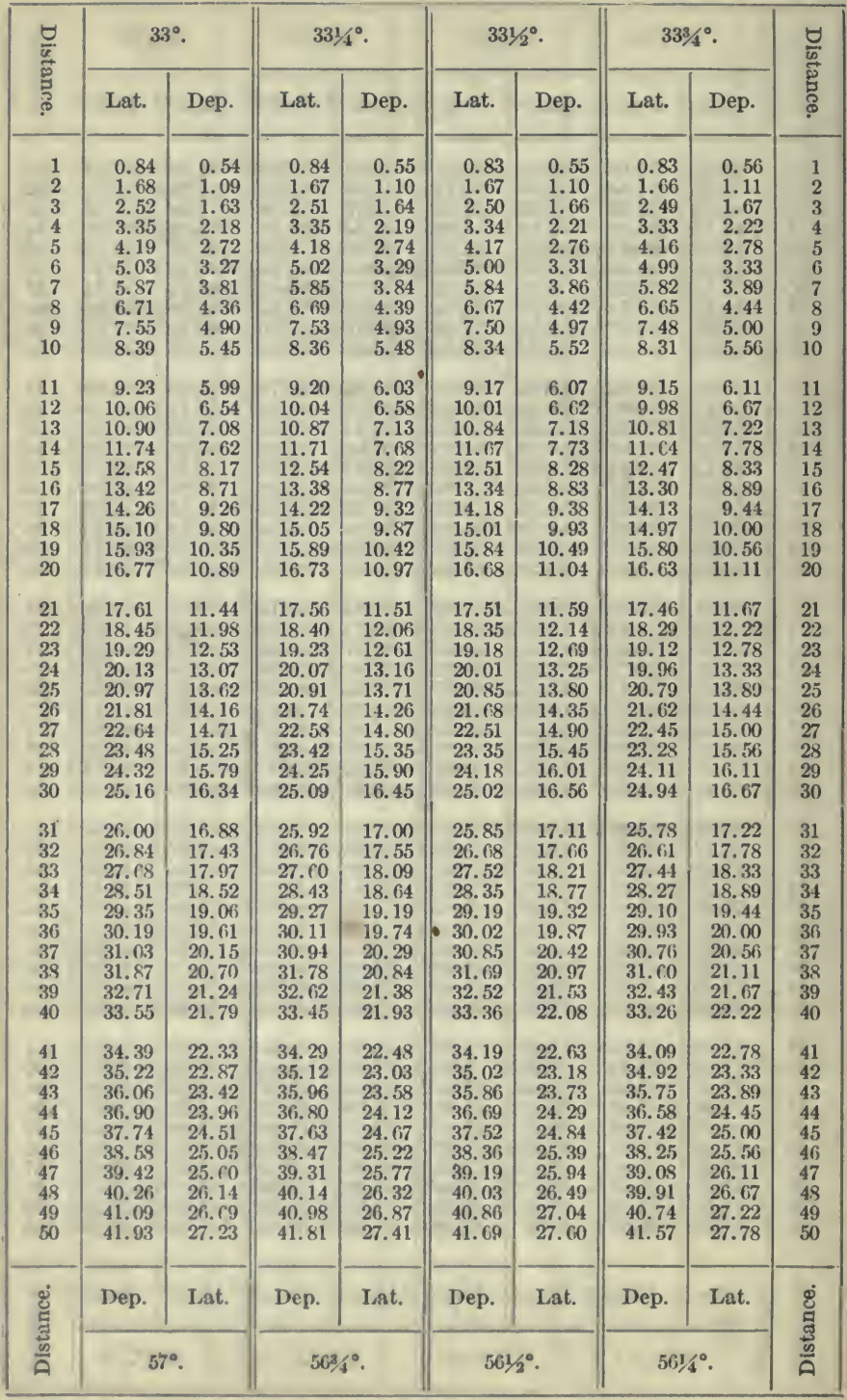


TABLE 4.-TRA YERSE TABIE.

\begin{tabular}{|c|c|c|c|c|c|c|c|c|c|}
\hline \multirow{2}{*}{ 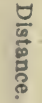 } & \multicolumn{2}{|c|}{$33^{\circ}$. } & \multicolumn{2}{|c|}{$331 / 4^{\circ}$. } & \multicolumn{2}{|c|}{$331 / 2^{\circ}$. } & \multicolumn{2}{|c|}{$33 /^{\circ}$. } & \multirow{2}{*}{ 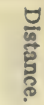 } \\
\hline & Lat. & Dep. & Lat. & Dep. & Lat. & Dep. & Lat. & Dep. & \\
\hline $\begin{array}{l}51 \\
52 \\
53 \\
54 \\
55 \\
56 \\
57 \\
58 \\
59 \\
60\end{array}$ & $\begin{array}{l}42.77 \\
43.61 \\
44.45 \\
45.29 \\
46.13 \\
46.97 \\
47.80 \\
48.64 \\
49.48 \\
50.32\end{array}$ & $\begin{array}{l}27.78 \\
28.32 \\
28.87 \\
29.41 \\
29.96 \\
30.50 \\
31.04 \\
31.59 \\
32.13 \\
32.68\end{array}$ & $\begin{array}{l}42.65 \\
43.40 \\
44.32 \\
45.16 \\
46.00 \\
46.83 \\
47.67 \\
48.50 \\
49.34 \\
50.18\end{array}$ & $\begin{array}{l}27.96 \\
28.51 \\
29.06 \\
29.61 \\
30.16 \\
30.70 \\
31.25 \\
31.80 \\
32.35 \\
32.90\end{array}$ & $\begin{array}{l}42.53 \\
43.36 \\
44.20 \\
45.03 \\
45.86 \\
46.70 \\
47.53 \\
48.37 \\
49.20 \\
50.03\end{array}$ & $\begin{array}{l}28.15 \\
28.70 \\
29.25 \\
29.80 \\
30.36 \\
30.91 \\
31.46 \\
32.01 \\
32.56 \\
33.12\end{array}$ & $\begin{array}{l}42.40 \\
43.24 \\
44.07 \\
44.90 \\
45.73 \\
46.56 \\
47.39 \\
48.23 \\
49.06 \\
49.89\end{array}$ & $\begin{array}{l}28.33 \\
28.89 \\
29.45 \\
30.00 \\
30.56 \\
31.11 \\
31.67 \\
32.22 \\
32.78 \\
33.33\end{array}$ & $\begin{array}{l}51 \\
52 \\
53 \\
54 \\
55 \\
56 \\
57 \\
58 \\
59 \\
60\end{array}$ \\
\hline $\begin{array}{l}61 \\
62 \\
63 \\
64 \\
65 \\
66 \\
67 \\
68 \\
69 \\
70\end{array}$ & $\begin{array}{l}51.16 \\
52.00 \\
52.84 \\
53.67 \\
54.51 \\
55.35 \\
56.19 \\
57.03 \\
57.87 \\
58.71\end{array}$ & $\begin{array}{l}33.22 \\
33.77 \\
34.31 \\
34.86 \\
35.40 \\
35.95 \\
36.49 \\
37.04 \\
37.58 \\
38.12\end{array}$ & $\begin{array}{l}51.01 \\
51.85 \\
52.69 \\
53.52 \\
54.36 \\
55.19 \\
56.03 \\
56.87 \\
57.70 \\
58.54\end{array}$ & $\begin{array}{l}33.45 \\
33.99 \\
34.54 \\
35.09 \\
35.64 \\
36.19 \\
36.74 \\
37.28 \\
37.83 \\
38.38\end{array}$ & $\begin{array}{l}50.87 \\
51.70 \\
52.53 \\
53.37 \\
54.20 \\
55.04 \\
55.87 \\
56.70 \\
57.54 \\
58.37\end{array}$ & $\begin{array}{l}33.67 \\
34.22 \\
34.77 \\
35.32 \\
35.88 \\
36.43 \\
36.98 \\
37.53 \\
38.08 \\
38.64\end{array}$ & $\begin{array}{l}50.72 \\
51.55 \\
52.38 \\
53.21 \\
54.05 \\
54.88 \\
55.71 \\
56.54 \\
57.37 \\
58.20\end{array}$ & $\begin{array}{l}33.89 \\
34.45 \\
35.00 \\
35.56 \\
36.11 \\
36.67 \\
37.22 \\
37.78 \\
38.33 \\
38.89\end{array}$ & $\begin{array}{l}61 \\
62 \\
63 \\
64 \\
65 \\
66 \\
67 \\
68 \\
69 \\
70\end{array}$ \\
\hline $\begin{array}{l}71 \\
72 \\
73 \\
74 \\
75 \\
76 \\
77 \\
78 \\
79 \\
80\end{array}$ & $\begin{array}{l}59.55 \\
60.38 \\
61.22 \\
62.06 \\
62.90 \\
63.74 \\
64.58 \\
65.42 \\
66.25 \\
67.09\end{array}$ & $\begin{array}{l}38.67 \\
39.21 \\
39.76 \\
40.30 \\
40.85 \\
41.39 \\
41.94 \\
42.48 \\
43.03 \\
43.57\end{array}$ & $\begin{array}{l}59.38 \\
60.21 \\
61.05 \\
61.89 \\
62.72 \\
63.56 \\
64.39 \\
65.23 \\
66.07 \\
66.90\end{array}$ & $\begin{array}{l}38.93 \\
39.48 \\
40.03 \\
40.57 \\
41.12 \\
41.67 \\
42.22 \\
42.77 \\
43.32 \\
43.86\end{array}$ & $\begin{array}{l}59.21 \\
60.04 \\
60.87 \\
61.71 \\
62.54 \\
63.38 \\
64.21 \\
65.04 \\
65.88 \\
66.71\end{array}$ & $\begin{array}{l}39.19 \\
39.74 \\
40.29 \\
40.84 \\
41.40 \\
41.95 \\
42.50 \\
43.05 \\
43.60 \\
44.15\end{array}$ & $\begin{array}{l}59.03 \\
59.87 \\
60.70 \\
61.53 \\
62.36 \\
63.19 \\
64.02 \\
64.85 \\
65.69 \\
66.52\end{array}$ & $\begin{array}{l}39.45 \\
40.00 \\
40.56 \\
41.11 \\
41.67 \\
42.22 \\
42.78 \\
43.33 \\
43.89 \\
44.45\end{array}$ & $\begin{array}{l}71 \\
72 \\
73 \\
74 \\
75 \\
76 \\
77 \\
78 \\
79 \\
80\end{array}$ \\
\hline $\begin{array}{l}81 \\
82 \\
83 \\
84 \\
85 \\
86 \\
87 \\
89 \\
89 \\
90\end{array}$ & $\begin{array}{l}67.93 \\
68.77 \\
69.61 \\
70.45 \\
71.29 \\
72.13 \\
72.96 \\
73.80 \\
74.64 \\
75.48\end{array}$ & $\begin{array}{l}44.12 \\
44.66 \\
45.21 \\
45.75 \\
46.29 \\
46.84 \\
47.38 \\
47.93 \\
48.47 \\
49.02\end{array}$ & $\begin{array}{l}67.74 \\
68.59 \\
69.41 \\
70.25 \\
71.08 \\
71.92 \\
72.76 \\
73.59 \\
74.43 \\
75.27\end{array}$ & $\begin{array}{l}44.41 \\
44.96 \\
45.51 \\
46.06 \\
46.60 \\
47.15 \\
47.70 \\
48.25 \\
48.80 \\
49.35\end{array}$ & $\begin{array}{l}67.54 \\
68.38 \\
69.21 \\
70.05 \\
70.88 \\
71.71 \\
72.55 \\
73.38 \\
74.22 \\
75.05\end{array}$ & $\begin{array}{l}44.71 \\
45.26 \\
45.81 \\
46.36 \\
46.91 \\
47.47 \\
48.02 \\
48.57 \\
49.12 \\
49.67\end{array}$ & $\begin{array}{l}67.35 \\
68.18 \\
69.01 \\
69.84 \\
70.67 \\
71.51 \\
72.34 \\
73.17 \\
74.00 \\
74.83\end{array}$ & $\begin{array}{l}45.00 \\
45.56 \\
46.11 \\
46.67 \\
47.22 \\
47.78 \\
48.33 \\
48.89 \\
49.45 \\
50.00\end{array}$ & $\begin{array}{l}81 \\
82 \\
83 \\
84 \\
85 \\
86 \\
87 \\
88 \\
89 \\
90\end{array}$ \\
\hline $\begin{array}{r}91 \\
92 \\
93 \\
94 \\
95 \\
96 \\
97 \\
98 \\
99 \\
100\end{array}$ & $\begin{array}{l}76.32 \\
77.16 \\
78.00 \\
78.84 \\
79.67 \\
80.51 \\
81.35 \\
82.19 \\
83.03 \\
83.87\end{array}$ & $\begin{array}{l}49.56 \\
50.11 \\
50.65 \\
51.20 \\
51.74 \\
52.29 \\
52.83 \\
53.37 \\
53.92 \\
54.46\end{array}$ & $\begin{array}{l}76.10 \\
76.94 \\
77.77 \\
78.61 \\
79.45 \\
80.28 \\
81.12 \\
81.96 \\
82.79 \\
83.63\end{array}$ & $\begin{array}{l}49.89 \\
50.44 \\
50.99 \\
51.54 \\
52.09 \\
52.64 \\
53.18 \\
53.73 \\
54.28 \\
54.83\end{array}$ & $\begin{array}{l}75.88 \\
76.72 \\
77.55 \\
78.39 \\
79.22 \\
80.05 \\
80.89 \\
81.73 \\
82.55 \\
83.39\end{array}$ & $\begin{array}{l}50.23 \\
50.78 \\
51.33 \\
51.88 \\
52.43 \\
52.99 \\
53.54 \\
54.09 \\
54.04 \\
55.19\end{array}$ & $\begin{array}{l}75.66 \\
76.50 \\
77.33 \\
78.16 \\
78.99 \\
79.82 \\
80.65 \\
81.48 \\
82.32 \\
83.15\end{array}$ & $\begin{array}{l}50.56 \\
51.11 \\
51.67 \\
52.22 \\
52.78 \\
53.33 \\
53.89 \\
54.45 \\
55.00 \\
55.56\end{array}$ & $\begin{array}{r}91 \\
92 \\
93 \\
94 \\
95 \\
96 \\
97 \\
98 \\
99 \\
100\end{array}$ \\
\hline 巳 & Dep. & Iat. & Dep. & Lat. & Dep. & Lat. & Dep. & Lat. & छீ \\
\hline$\frac{m}{a}$ & \multicolumn{2}{|c|}{$57^{\circ}$} & \multicolumn{2}{|c|}{$56 \% 4^{\circ}$. } & \multicolumn{2}{|c|}{$561 / 2^{\circ}$. } & \multicolumn{2}{|c|}{$561 / 4^{\circ}}$. & $\frac{5}{0}$ \\
\hline
\end{tabular}


TABLE 4.-TRAVERSE TABLE.

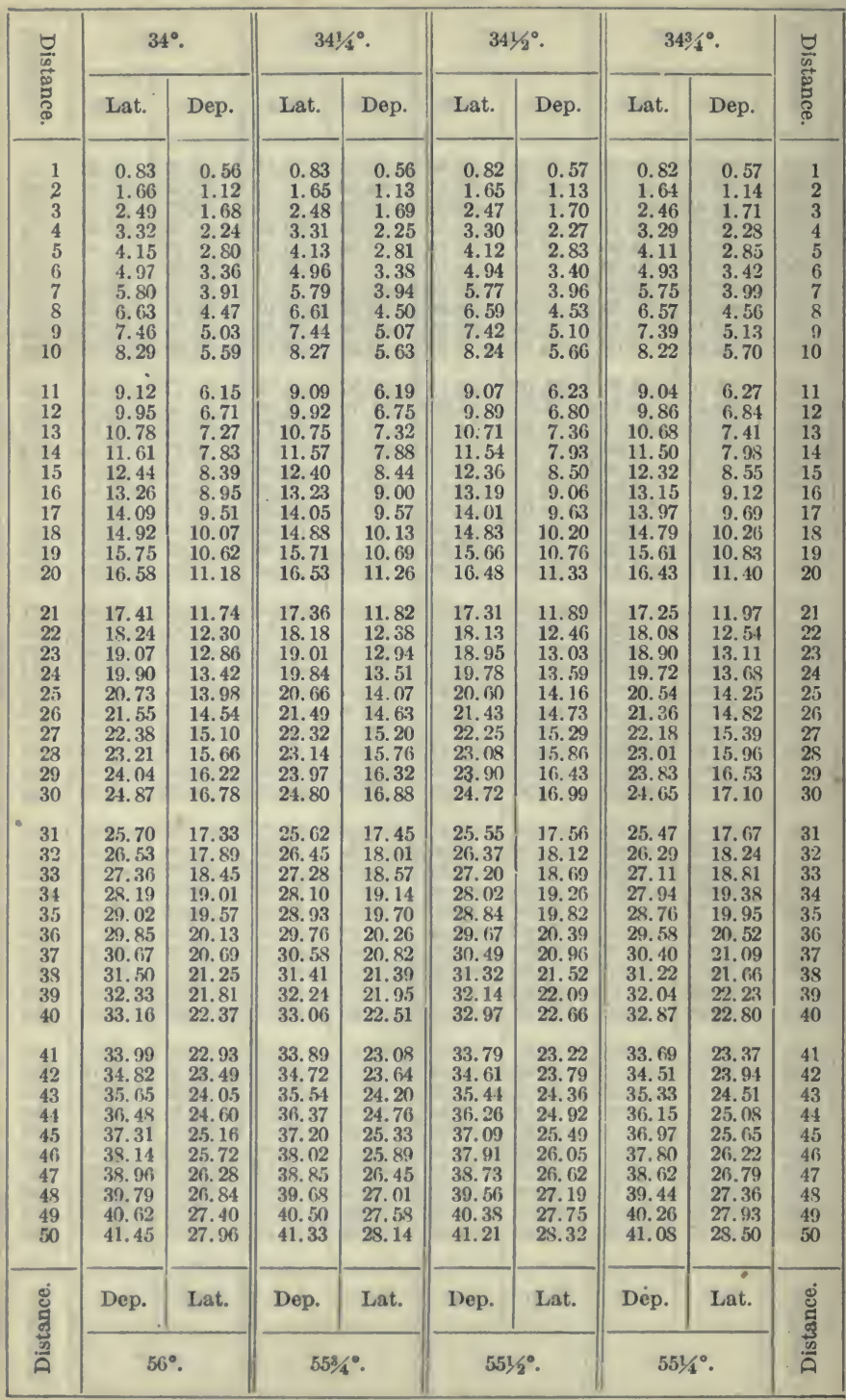


TABLE 4.-TRIVERSE TABLE.

\begin{tabular}{|c|c|c|c|c|c|c|c|c|c|}
\hline \multirow{2}{*}{ 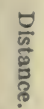 } & \multicolumn{2}{|c|}{$34^{\circ}}$. & \multicolumn{2}{|c|}{$34^{1} / 4^{\circ}$. } & \multicolumn{2}{|c|}{$342 / 5^{\circ}$. } & \multicolumn{2}{|c|}{$344^{\circ}$. } & \multirow{2}{*}{ 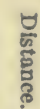 } \\
\hline & Lat. & Dep. & Lat. & Dep. & Lat. & Dep. & Lat. & Dep. & \\
\hline $\begin{array}{l}51 \\
52 \\
53 \\
54 \\
55 \\
56 \\
57 \\
58 \\
59 \\
60\end{array}$ & $\begin{array}{l}42.29 \\
43.11 \\
43.94 \\
44.77 \\
45.60 \\
46.43 \\
47.25 \\
48.08 \\
48.91 \\
49.74\end{array}$ & $\begin{array}{l}28.52 \\
29.08 \\
29.64 \\
30.20 \\
30.76 \\
31.31 \\
31.87 \\
32.43 \\
32.99 \\
33.55\end{array}$ & $\begin{array}{l}42.16 \\
42.98 \\
43.81 \\
44.64 \\
45.46 \\
46.29 \\
47.12 \\
47.94 \\
48.77 \\
49.60\end{array}$ & $\begin{array}{l}28.70 \\
29.27 \\
29.83 \\
30.39 \\
30.95 \\
31.52 \\
32.08 \\
32.64 \\
33.21 \\
33.77\end{array}$ & $\begin{array}{l}42.03 \\
42.85 \\
43.68 \\
44.50 \\
45.33 \\
46.15 \\
46.98 \\
47.80 \\
48.62 \\
49.45\end{array}$ & $\begin{array}{l}28.89 \\
29.45 \\
30.02 \\
30.59 \\
31.15 \\
31.72 \\
32.29 \\
32.85 \\
33.42 \\
33.98\end{array}$ & $\begin{array}{l}41.90 \\
42.73 \\
43.55 \\
44.37 \\
45.19 \\
46.01 \\
46.83 \\
47.66 \\
48.48 \\
49.30\end{array}$ & $\begin{array}{l}29.07 \\
29.64 \\
30.21 \\
30.78 \\
31.35 \\
31.92 \\
32.49 \\
33.06 \\
33.63 \\
34.20\end{array}$ & $\begin{array}{l}51 \\
52 \\
53 \\
54 \\
55 \\
56 \\
57 \\
58 \\
59 \\
60\end{array}$ \\
\hline $\begin{array}{l}61 \\
62 \\
63 \\
64 \\
65 \\
66 \\
67 \\
68 \\
69 \\
70\end{array}$ & $\begin{array}{l}50.57 \\
51.40 \\
52.23 \\
53.06 \\
53.39 \\
54.72 \\
55.55 \\
56.37 \\
57.20 \\
58.03\end{array}$ & $\begin{array}{l}34.11 \\
34.67 \\
35.23 \\
35.79 \\
36.35 \\
36.91 \\
37.47 \\
38.03 \\
38.58 \\
39.14\end{array}$ & $\begin{array}{l}50.42 \\
51.25 \\
52.08 \\
52.90 \\
53.73 \\
54.55 \\
55.38 \\
56.21 \\
57.03 \\
57.86\end{array}$ & $\begin{array}{l}34.33 \\
34.89 \\
35.46 \\
36.02 \\
36.58 \\
37.15 \\
37.71 \\
38.27 \\
38.83 \\
39.40\end{array}$ & $\begin{array}{l}50.27 \\
51.10 \\
51.92 \\
52.74 \\
53.57 \\
54.39 \\
55.22 \\
56.04 \\
56.86 \\
57.69\end{array}$ & $\begin{array}{l}34.55 \\
35.12 \\
35.68 \\
36.25 \\
36.82 \\
37.38 \\
37.95 \\
38.52 \\
39.08 \\
39.65\end{array}$ & $\begin{array}{l}50.12 \\
50.94 \\
51.76 \\
52.59 \\
53.41 \\
54.23 \\
55.05 \\
55.87 \\
56.69 \\
57.52\end{array}$ & $\begin{array}{l}34.77 \\
35.34 \\
35.91 \\
36.48 \\
37.05 \\
37.62 \\
38.19 \\
38.76 \\
39.33 \\
39.90\end{array}$ & $\begin{array}{l}61 \\
62 \\
63 \\
64 \\
615 \\
60 \\
67 \\
68 \\
69 \\
70\end{array}$ \\
\hline $\begin{array}{l}71 \\
72 \\
73 \\
74 \\
75 \\
76 \\
77 \\
78 \\
79 \\
80\end{array}$ & $\begin{array}{l}68.86 \\
59.69 \\
60.52 \\
61.35 \\
62.18 \\
63.01 \\
63.84 \\
64.66 \\
65.49 \\
66.32\end{array}$ & $\begin{array}{l}39.70 \\
40.26 \\
40.82 \\
41.38 \\
41.94 \\
42.50 \\
43.06 \\
43.62 \\
44.18 \\
44.74\end{array}$ & $\begin{array}{l}58.69 \\
59.51 \\
60.34 \\
61.17 \\
61.99 \\
62.82 \\
63.65 \\
64.47 \\
65.30 \\
66.13\end{array}$ & $\begin{array}{l}39.96 \\
40.52 \\
41.08 \\
41.65 \\
42.21 \\
42.77 \\
43.34 \\
43.90 \\
44.46 \\
45.02\end{array}$ & $\begin{array}{l}58.51 \\
59.34 \\
60.16 \\
60.99 \\
61.81 \\
62.63 \\
63.46 \\
64.28 \\
65.11 \\
65.93\end{array}$ & $\begin{array}{l}40.21 \\
40.78 \\
41.35 \\
41.91 \\
42.48 \\
43.05 \\
43.61 \\
44.18 \\
44.75 \\
45.31\end{array}$ & $\begin{array}{l}58.34 \\
59.16 \\
59.98 \\
60.80 \\
61.62 \\
62.45 \\
63.27 \\
34.09 \\
64.91 \\
65.73\end{array}$ & $\begin{array}{l}40.47 \\
41.04 \\
41.61 \\
42.18 \\
42.75 \\
43.32 \\
43.89 \\
44.46 \\
45.03 \\
45.60\end{array}$ & $\begin{array}{l}71 \\
72 \\
73 \\
74 \\
75 \\
76 \\
77 \\
78 \\
79 \\
80\end{array}$ \\
\hline $\begin{array}{l}81 \\
82 \\
83 \\
84 \\
85 \\
86 \\
87 \\
88 \\
89 \\
90\end{array}$ & $\begin{array}{l}67.15 \\
67.98 \\
68.81 \\
69.64 \\
70.47 \\
71.30 \\
72.13 \\
72.96 \\
73.78 \\
74.61\end{array}$ & $\begin{array}{l}45.29 \\
45.85 \\
46.41 \\
46.97 \\
47.53 \\
45.09 \\
48.65 \\
49.21 \\
49.77 \\
50.33\end{array}$ & $\begin{array}{l}66.95 \\
67.78 \\
68.61 \\
69.43 \\
70.26 \\
71.09 \\
71.91 \\
72.74 \\
73.57 \\
74.39\end{array}$ & $\begin{array}{l}45.59 \\
46.15 \\
46.71 \\
47.23 \\
47.84 \\
48.40 \\
48.96 \\
49.53 \\
50.09 \\
50.65\end{array}$ & $\begin{array}{l}66.75 \\
67.58 \\
68.40 \\
69.23 \\
70.05 \\
70.87 \\
71.70 \\
72.52 \\
73.35 \\
74.17\end{array}$ & $\begin{array}{l}45.88 \\
46.45 \\
47.01 \\
47.58 \\
48.14 \\
48.71 \\
49.28 \\
49.84 \\
50.41 \\
50.93\end{array}$ & $\begin{array}{l}66.55 \\
67.38 \\
68.20 \\
69.02 \\
69.84 \\
70.66 \\
71.48 \\
72.30 \\
73.13 \\
73.95\end{array}$ & $\begin{array}{l}46.17 \\
46.74 \\
47.31 \\
47.88 \\
48.45 \\
49.02 \\
49.59 \\
50.16 \\
50.73 \\
51.30\end{array}$ & $\begin{array}{l}81 \\
82 \\
83 \\
84 \\
85 \\
86 \\
87 \\
88 \\
89 \\
90\end{array}$ \\
\hline $\begin{array}{r}91 \\
92 \\
93 \\
94 \\
95 \\
96 \\
97 \\
96 \\
99 \\
100\end{array}$ & $\begin{array}{l}75.44 \\
76.27 \\
77.10 \\
77.93 \\
78.76 \\
79.59 \\
80.42 \\
81.25 \\
82.07 \\
82.00\end{array}$ & $\begin{array}{l}50.89 \\
51.45 \\
52.00 \\
52.56 \\
53.12 \\
53.68 \\
51.24 \\
54.80 \\
5.56 \\
55.92\end{array}$ & $\begin{array}{l}75.22 \\
76.05 \\
76.87 \\
77.70 \\
78.53 \\
79.35 \\
80.19 \\
81.01 \\
81.83 \\
82.60\end{array}$ & $\begin{array}{l}51.22 \\
51.78 \\
52.34 \\
52.90 \\
53.47 \\
54.03 \\
54.59 \\
55.15 \\
55.72 \\
56.23\end{array}$ & $\begin{array}{l}75.00 \\
75.82 \\
76.64 \\
77.47 \\
78.29 \\
79.12 \\
79.94 \\
80.76 \\
81.59 \\
82.41\end{array}$ & $\begin{array}{l}51.54 \\
52.11 \\
52.68 \\
53.24 \\
53.81 \\
54.37 \\
54.94 \\
55.51 \\
54.07 \\
56.64\end{array}$ & $\begin{array}{l}74.77 \\
75.59 \\
76.41 \\
77.23 \\
73.06 \\
78.89 \\
79.70 \\
80.52 \\
81.34 \\
82.16\end{array}$ & $\begin{array}{l}51.87 \\
52.44 \\
53.01 \\
53.58 \\
54.15 \\
54.72 \\
55.29 \\
55.80 \\
56.43 \\
57.00\end{array}$ & $\begin{array}{r}91 \\
92 \\
93 \\
94 \\
95 \\
90 \\
97 \\
98 \\
99 \\
100\end{array}$ \\
\hline 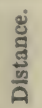 & Dep. & Iat. & Dep. & Lat. & Dep. & Lat. & Dep. & Lat. & 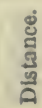 \\
\hline
\end{tabular}


TABLE 4.-TRAVERSE TABLE.

\begin{tabular}{|c|c|c|c|c|c|c|c|c|c|}
\hline \multirow{2}{*}{ 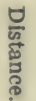 } & \multicolumn{2}{|c|}{$35^{\circ}$. } & \multicolumn{2}{|c|}{$351 / 4^{\circ}$. } & \multicolumn{2}{|c|}{$351 / 2^{\circ}$. } & \multicolumn{2}{|c|}{$35^{3} / 4^{\circ}$. } & \multirow{2}{*}{ 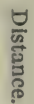 } \\
\hline & Lat. & Dep. & Lat. & Dep. & Lat. & Dep. & Lat. & Dep. & \\
\hline 1 & 0.82 & 0.57 & 0.82 & 0.59 & 0.81 & 0.58 & 0.81 & 0.58 & 1 \\
\hline 2 & 1.64 & 1.15 & 1.63 & 1.15 & 1.63 & 1.16 & 1.62 & 1.17 & 2 \\
\hline 3 & 2.46 & 1.72 & 2.45 & 1.73 & 2.44 & 1.74 & 2.43 & 1.75 & 3 \\
\hline 4 & 3.28 & 2.29 & 3.27 & 2.31 & 3.26 & 2.32 & 3.25 & 2.34 & 4 \\
\hline 5 & 4.10 & 2.87 & 4.08 & 2.89 & 4. 07 & 2.90 & 4.06 & 2.92 & 5 \\
\hline 6 & 4.91 & 3.44 & 4.90 & 3.46 & 4.88 & 3.48 & 4.87 & 3.51 & 6 \\
\hline 7 & 5.73 & 4.02 & 5.72 & 4.04 & 5.70 & 4.06 & 5.68 & 4.09 & 7 \\
\hline 8 & 6.55 & 4.59 & 6.53 & 4.62 & 6.51 & 4.65 & 6.49 & 4. 177 & 8 \\
\hline 9 & 7. 37 & 5.16 & 7.35 & 5.19 & 7.33 & 5.23 & 7.30 & 5.26 & 9 \\
\hline 10 & 8. 19 & 5.74 & 8.17 & 5.77 & 8.14 & 5.81 & 8.12 & 5.84 & 10 \\
\hline 11 & 9.01 & 6.31 & 8.98 & 6.35 & 8.96 & 6. 39 & 8.93 & 6. 43 & 11 \\
\hline 12 & 9.83 & 6.88 & 9.80 & 6.93 & 9.77 & 6.97 & 9.74 & 7.01 & 12 \\
\hline 13 & 10.65 & 7.46 & 10.62 & 7.50 & 10.58 & 7.55 & 10.55 & 7. (u) & 13 \\
\hline 14 & 11.47 & 8.03 & 11.43 & 8.08 & 11.40 & 8.13 & 11.36 & 8.18 & 14 \\
\hline 15 & 12.29 & 8.60 & 12.25 & 8.66 & 12.21 & 8.71 & 12.17 & 8.76 & 15 \\
\hline 16 & 13.11 & 9.18 & 13.07 & 9.23 & 13.03 & 9.29 & 12.99 & 9.35 & 16 \\
\hline 17 & 13.93 & 9.75 & 13.88 & 9.81 & 13.84 & 9.87 & 13.80 & 9.93 & 17 \\
\hline 18 & 14.74 & 10.32 & 14. 70 & 10.39 & 14.65 & 10.45 & 14.61 & 10.52 & 18 \\
\hline 19 & 15. 56 & 10.90 & 15. 52 & 10.97 & 15.47 & 11.03 & 15.42 & 11.10 & 19 \\
\hline 20 & 16.38 & 11.47 & 16.33 & 11.54 & 16.28 & 11.61 & 16.23 & 11. 68 & 20 \\
\hline 21 & 17.20 & 12.05 & 17.15 & 12.12 & 17.10 & 12.19 & 17.04 & 12.27 & 21 \\
\hline 22 & 18.02 & 12. 62 & 17.97 & 12.70 & 17.91 & 12.78 & 17.85 & 12.85 & 22 \\
\hline 23 & 18.84 & 13.19 & 18.78 & 13. 27 & 18.72 & 13. 36 & 18.67 & 13. 44 & 23 \\
\hline 24 & 19.66 & 13. 77 & 19. 60 & 13.85 & 19.54 & 13.94 & 19.48 & 14.02 & 24 \\
\hline 25 & 20.48 & 14.34 & 20.42 & 14.43 & 20.35 & 14.52 & 20.29 & 14. 61 & 25 \\
\hline 26 & 21.30 & 14.91 & 21.23 & 15.01 & 21. 27 & 15.10 & 21.10 & 15. 19 & 26 \\
\hline 27 & 22.12 & 15. 49 & 22.05 & 15. 58 & 21.98 & 15.68 & 21.91 & 15.77 & 27 \\
\hline 28 & 22.94 & 16.06 & 22.87 & 16.16 & 22.80 & 16.26 & 22.72 & 16.36 & 28 \\
\hline 29 & 23.76 & 16.63 & 23. is & 16.74 & 23.61 & 16.84 & 23.54 & 16.94 & 29 \\
\hline 30 & 24.57 & 17.21 & 24.50 & 17.31 & 24.42 & 17.42 & 24.35 & 17.53 & 30 \\
\hline 31 & 25. 39 & 17.78 & 25.32 & 17.89 & 25.24 & 18.00 & 25.16 & 18.11 & 31 \\
\hline 32 & 26.21 & 18.35 & 26.13 & 18.47 & 26.05 & 18.58 & 25.97 & 18.70 & 32 \\
\hline 33 & 27.03 & 18.93 & 26.95 & 19.05 & 26.87 & 19. & 26.78 & 19.28 & 33 \\
\hline 34 & 27.85 & 19.50 & 27.77 & 19.62 & 27.68 & 19.74 & 27.59 & 19.86 & 34 \\
\hline 35 & 28.67 & 20.08 & 28.58 & 20. & 28.49 & 20.32 & 28.41 & 20.45 & 35 \\
\hline 36 & 29. 49 & 20.65 & 29.40 & 20.78 & 29.31 & 20.91 & 29.22 & 21.03 & 36 \\
\hline 37 & 30.31 & 21.22 & 30.22 & 21 . & 30. & 21 . & 30.03 & 21.62 & 37 \\
\hline 38 & 31.13 & 21.80 & 31.03 & 21.93 & 30.94 & 22.07 & 30.84 & 22.20 & 38 \\
\hline 39 & 31.95 & 22.37 & 31.85 & 22.51 & 31. & 22.65 & 31.65 & 22.79 & 39 \\
\hline 40 & 32.77 & 22.94 & 32.67 & 23.09 & 32.56 & 23.23 & 32.46 & 23.37 & 40 \\
\hline 41 & 33. 59 & 23. 52 & 33.48 & $23 . \mathrm{r}$ & 33.38 & 23.8 & 33.27 & 23.95 & 41 \\
\hline 42 & 34.40 & 24.09 & 31 & 24. & 34.19 & 24. 39 & 34.09 & 24. & 42 \\
\hline 43 & 35. 22 & 24.68 & 35.12 & 24.82 & 35.01 & 24.97 & 34.90 & 25.12 & 43 \\
\hline 44 & 36.04 & 25.24 & 35.93 & 25.39 & 35.82 & 25.55 & 35.71 & 25.71 & 44 \\
\hline 45 & 36.86 & 25.81 & 36.75 & 25.97 & 36.64 & 26.13 & 36.52 & 26. 29 & 45 \\
\hline 46 & 37.68 & 26.38 & 37.57 & 26.55 & 37.45 & 26.71 & 37.33 & 26.88 & 46 \\
\hline 47 & 38.50 & 26.96 & 38.38 & 27.13 & 38.26 & 27.29 & 38.14 & 27.46 & 47 \\
\hline 48 & 39.32 & 27.53 & 39.20 & 27.70 & 39.08 & 27.87 & 38.96 & 28.04 & 48 \\
\hline 49 & 40.14 & 28.11 & 40.02 & 28.28 & 39.89 & 28.45 & 39.77 & 28.63 & 49 \\
\hline 50 & 40.96 & 28.68 & 40.83 & 28.86 & 40.71 & 29.04 & 40.58 & 29.21 & 50 \\
\hline \multirow{2}{*}{ 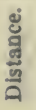 } & Dep. & Lat. & Dep. & Lat. & Dep. & Lat. & Dep. & Lat. & \multirow{2}{*}{ 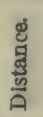 } \\
\hline & \multicolumn{2}{|c|}{$65^{\circ}}$. & \multicolumn{2}{|c|}{$54^{3} 4^{\circ}$. } & \multicolumn{2}{|c|}{$541 / 2^{\circ}}$. & \multicolumn{2}{|c|}{$541 / 4^{\circ}$. } & \\
\hline
\end{tabular}


TABLE 4.-TRAVERSE TABLE.

\begin{tabular}{|c|c|c|c|c|c|c|c|c|c|}
\hline \multirow{2}{*}{$\begin{array}{l}\text { Ð } \\
\text { है } \\
\text { है } \\
\text { है }\end{array}$} & \multicolumn{2}{|c|}{$35^{\circ}$. } & \multicolumn{2}{|c|}{$351 / 4^{\circ}$. } & \multicolumn{2}{|c|}{$351 / 2^{\circ}$. } & \multicolumn{2}{|c|}{$35 \% 4^{\circ}$. } & \multirow{2}{*}{ 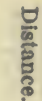 } \\
\hline & Lat. & Dep. & Lat. & Dep. & Lat. & Dep. & Lat. & Dess. & \\
\hline 51 & 41. 78 & 29.25 & 41.65 & 29.43 & 41.52 & 29.62 & 41.39 & 29.80 & 51 \\
\hline 52 & 42. 60 & 29.83 & 42.47 & 30.01 & 42.33 & 30.20 & 42.20 & 30.38 & 52 \\
\hline 53 & 43.42 & 30.40 & 43.28 & 30.59 & 43.15 & 30.78 & 43.01 & 30.97 & 53 \\
\hline 54 & 44.23 & 30.97 & 44.10 & 31.17 & 43.96 & 31.36 & 43.82 & 31.55 & 54 \\
\hline 55 & 45.05 & 31.55 & 44.92 & 31.74 & 44.78 & 31.94 & 44.64 & 32.13 & 55 \\
\hline 56 & 45. 87 & 32.12 & 45.73 & 32.32 & 45.59 & 32.52 & 45.45 & 32.72 & 56 \\
\hline 57 & 46. 69 & 32.69 & 46.55 & 32.90 & 46. 40 & 33.10 & 46. 26 & 33.30 & 57 \\
\hline 58 & 47.51 & 33.27 & 47.37 & 33.47 & 47.22 & 33. 68 & 47.07 & 33.89 & 58 \\
\hline 59 & 48. 33 & 33.84 & 48. 18 & 34.05 & 48.03 & 34.26 & 47.88 & 34.47 & 59 \\
\hline 60 & 49.15 & 34.41 & 49.00 & 34.63 & 48.85 & 34.84 & 48.69 & 35.05 & 60 \\
\hline 61 & 49.97 & 34.99 & 49.82 & 35. 21 & 49. 66 & 35.42 & 49.51 & 35.64 & 61 \\
\hline 62 & 50.79 & 35.56 & 50.63 & 35.78 & 50.48 & 36.00 & 50.32 & 36.22 & 62 \\
\hline 63 & 51.61 & 36.14 & 51.45 & 36.36 & 51.29 & 36. 58 & 51.13 & 36.81 & 63 \\
\hline 64 & 52.43 & 36.71 & 52.27 & 36.94 & 52.10 & 37.16 & 51.94 & 37.39 & 64 \\
\hline 65 & 53.24 & 37.28 & 53.08 & 37.51 & 52.92 & 37.75 & 52.75 & 37.98 & 65 \\
\hline 66 & 54.06 & 37.86 & 53.90 & 38.09 & 53.73 & 38. 33 & 53.56 & 38.56 & 66 \\
\hline 67 & 54.88 & 38.43 & 54.71 & 38. 67 & 54.55 & 38.91 & 54.38 & 39.14 & 67 \\
\hline 68 & 55.70 & 39.00 & 55.53 & 39.25 & 55. 36 & 39.49 & 55.19 & 39.73 & 68 \\
\hline 69 & 56.52 & 39.58 & 56.35 & 39.82 & 56.17 & 40.07 & 56.00 & 40.31 & 69 \\
\hline 70 & 57.34 & 40.15 & 57.16 & 40.40 & 56.99 & 40.65 & 56.81 & 40.90 & 70 \\
\hline 71 & 53.16 & 40.72 & 57.88 & 40.98 & 57.80 & 41.23 & 57.62 & 41.48 & 71 \\
\hline 72 & 58.98 & 41.30 & 58.80 & 41.55 & 59.62 & 41.81 & 58.43 & 42.07 & 72 \\
\hline 73 & 59.80 & 41.87 & 59.61 & 42.13 & 59.43 & 42.39 & 59.24 & 42.65 & 73 \\
\hline 74 & 60.62 & 42.44 & 60.43 & 42.71 & 60.24 & 42.97 & 60.06 & 43.23 & 74 \\
\hline 75 & 61.44 & 43. 02 & 61.25 & 43.29 & 61.06 & 43.55 & 60.87 & 43. 82 & 75 \\
\hline 76 & C2.26 & 43.59 & 62.06 & 43.86 & 61.87 & 44.13 & 61.68 & 44.40 & 76 \\
\hline 77 & 63.07 . & 44. 17 & 62.88 & 44.44 & 62.69 & 44.71 & 62.49 & 44.99 & 77 \\
\hline 78 & 63.89 & 44.74 & 63.70 & 45.02 & 63.50 & 45.29 & 63.30 & 45.57 & 78 \\
\hline 79 & 64.71 & 45.31 & 64.51 & 45.59 & 64.32 & 45.88 & 64.11 & 46.16 & 79 \\
\hline 80 & 65.53 & 45.89 & 65.33 & 46. 17 & 65.13 & 46.46 & 64.93 & 46.74 & 80 \\
\hline 81 & 66.35 & 46.46 & 66.15 & 46.75 & 65.94 & 47.04 & 65.74 & 47.32 & 81 \\
\hline 82 & 67.17 & 47.03 & 66.96 & 47.33 & 66.76 & 47. 62 & 66. 55 & 47.91 & 82 \\
\hline 83 & 67.99 . & 47.61 & 67.78 & 47.90 & 67.57 & 48.20 & 67.36 & 48.49 & 83 \\
\hline 84 & 68. 81 & 49.18 & 68.60 & 48.48 & 68.39 & 48.78 & 68.17 & 49.08 & 84 \\
\hline 85 & 69.63 & 48.75 & 69.41 & 49.06 & 69.20 & 49.36 & 68.98 & 49. 66 & 8.5 \\
\hline 86 & 70.45 & 49.33 & 70.23 & 49. & 70.01 & 49.94 & 69.80 & 50.25 & 86 \\
\hline 87 & 71.27 & 49.90 & 71.05 & 50.21 & 70.83 & 50.52 & 70. 61 & 50.83 & 87 \\
\hline 88 & 72.09 & 50.47 & 71.86 & 50.79 & 71.64 & 51.10 & 71.42 & 51.41 & S8 \\
\hline 89 & 72.90 & 51.05 & 72.68 & 51.37 & 72.46 & 51. 68 & 72.23 & 52.00 & 89 \\
\hline 90 & 73.72 & 51.62 & 73.50 & 51.94 & 73.27 & 52.26 & 73.04 & 52.58 & 00 \\
\hline & 74.54 & 52.20 & 74.31 & 52.5 & 74.08 & 52.84 & 73.85 & 33.17 & 91 \\
\hline 92 & 75.36 & 52.77 & 75.13 & 53.10 & 74.90 & 53.42 & 74.66 & 53.75 & 92 \\
\hline 03 & 7 ti. 18 & 53. 34 & 75.95 & 53.67 & 75.71 & 54.01 & 75.48 & 54.34 & 93 \\
\hline 94 & 77.00 & 53.92 & 76.76 & 54.25 & 76.53 & 54.59 & 76.29 & 54.92 & 94 \\
\hline 95 & 77.82 & 54.49 & 77.58 & 54.83 & 77.34 & 55.17 & 77.10 & 55.50 & 85 \\
\hline 96 & 78.64 & 55.06 & 78.40 & 55.41 & 78.16 & 55. 75 & 77.91 & 56.09 & 96 \\
\hline 97 & 79.46 & 55.64 & 79.21 & 55. 98 & 78.97 & 56.33 & 78.72 & 56.67 & 97 \\
\hline 98 & 80.28 & 56.21 & 80.03 & 56.56 & 79.78 & 56.91 & 79.53 & 57.26 & 98 \\
\hline 99 & 81.10 & 56.78 & 80.85 & 57.14 & 80.60 & 57.49 & 80.35 & 57.84 & 99 \\
\hline 100 & 81.92 & 57.36 & 81.66 & 57.71 & 81.41 & 58.07 & 81.16 & 58.42 & 100 \\
\hline \multirow{2}{*}{ 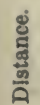 } & Dep. & Lat. & Dep. & Lat. & Dep. & Lat. & Dep. & Lat. & : \\
\hline & \multicolumn{2}{|c|}{$55^{\circ}$. } & \multicolumn{2}{|c|}{$511^{3} / 4^{\circ}$. } & \multicolumn{2}{|c|}{$541 / 2^{\circ}}$. & \multicolumn{2}{|c|}{$511 / 4^{\circ}}$. & $\frac{n}{2}$ \\
\hline
\end{tabular}


TABLE A-TRAVERSE TABLE.

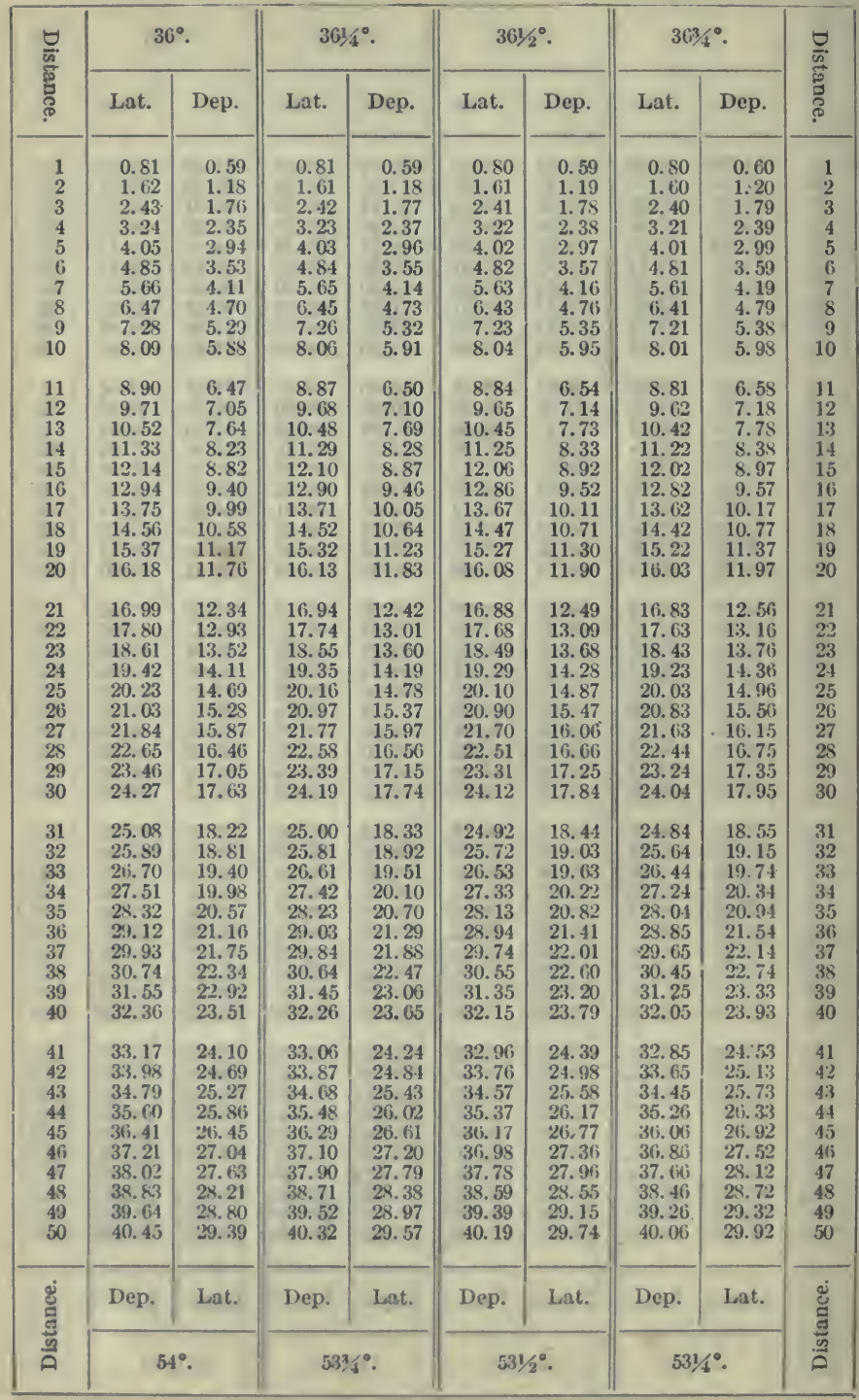


TABLE 4.-TRAVERSE TABLE.

\begin{tabular}{|c|c|c|c|c|c|c|c|c|c|}
\hline \multirow{2}{*}{$\begin{array}{l}\frac{\theta}{\infty} \\
\text { है } \\
\text { है }\end{array}$} & \multicolumn{2}{|c|}{$36^{\circ}$. } & \multicolumn{2}{|c|}{$361 / 4^{\circ}}$. & \multicolumn{2}{|c|}{$361 / 2^{\circ}$. } & \multicolumn{2}{|c|}{$36^{3} \%^{\circ}$. } & \multirow{2}{*}{ 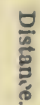 } \\
\hline & Lat. & Dẹ. & Lat. & Dep. & Lat. & Dep. & Lat. & Dep. & \\
\hline $\begin{array}{l}51 \\
52 \\
53 \\
54 \\
55 \\
56 \\
57 \\
58 \\
59 \\
60\end{array}$ & $\begin{array}{l}41.26 \\
42.07 \\
42.88 \\
43.69 \\
44.50 \\
45.30 \\
46.11 \\
46.92 \\
47.73 \\
48.54\end{array}$ & $\begin{array}{l}29.98 \\
30.54 \\
31.15 \\
31.74 \\
32.33 \\
32.92 \\
33.50 \\
34.09 \\
34.68 \\
35.27\end{array}$ & $\begin{array}{l}41.13 \\
41.94 \\
42.74 \\
43.55 \\
44.35 \\
45.16 \\
45.97 \\
46.77 \\
47.58 \\
48.39\end{array}$ & $\begin{array}{l}30.16 \\
30.75 \\
31.34 \\
31.93 \\
32.52 \\
33.11 \\
33.70 \\
34.30 \\
34.89 \\
35.48\end{array}$ & $\begin{array}{l}41.00 \\
41.80 \\
42.60 \\
43.41 \\
44.21 \\
45.02 \\
45.82 \\
46.62 \\
47.43 \\
48.23\end{array}$ & $\begin{array}{l}30.34 \\
30.93 \\
31.53 \\
32.12 \\
32.72 \\
33.31 \\
33.90 \\
34.50 \\
35.09 \\
35.69\end{array}$ & $\begin{array}{l}40.86 \\
41.67 \\
42.47 \\
43.27 \\
44.07 \\
44.87 \\
45.67 \\
46.47 \\
47.27 \\
48.08\end{array}$ & $\begin{array}{l}30.51 \\
31.11 \\
31.71 \\
32.31 \\
32.91 \\
33.51 \\
34.10 \\
34.70 \\
35.30 \\
35.90\end{array}$ & $\begin{array}{l}51 \\
52 \\
53 \\
54 \\
55 \\
56 \\
57 \\
58 \\
59 \\
60\end{array}$ \\
\hline $\begin{array}{l}61 \\
62 \\
63 \\
64 \\
65 \\
66 \\
67 \\
68 \\
69 \\
70\end{array}$ & $\begin{array}{l}49.35 \\
50.16 \\
50.97 \\
51.78 \\
52.59 \\
53.40 \\
54.20 \\
55.01 \\
55.82 \\
56.63\end{array}$ & $\begin{array}{l}35.85 \\
36.44 \\
37.03 \\
37.62 \\
38.21 \\
38.79 \\
39.38 \\
39.97 \\
40.56 \\
41.14\end{array}$ & $\begin{array}{l}49.19 \\
50.00 \\
50.81 \\
51.61 \\
52.42 \\
53.23 \\
54.03 \\
54.84 \\
54.64 \\
56.45\end{array}$ & $\begin{array}{l}36.07 \\
36.66 \\
37.25 \\
37.84 \\
38.44 \\
39.03 \\
39.62 \\
40.21 \\
40.80 \\
41.39\end{array}$ & $\begin{array}{l}49.04 \\
49.84 \\
50.64 \\
51.45 \\
52.25 \\
53.05 \\
53.86 \\
54.66 \\
55.47 \\
56.27\end{array}$ & $\begin{array}{l}36.28 \\
36.88 \\
37.47 \\
38.07 \\
38.66 \\
39.26 \\
39.85 \\
40.45 \\
41.04 \\
41.64\end{array}$ & $\begin{array}{l}48.88 \\
49.68 \\
50.48 \\
51.28 \\
52.08 \\
52.88 \\
53.68 \\
54.49 \\
55.29 \\
56.09\end{array}$ & $\begin{array}{l}36.50 \\
37.10 \\
37.69 \\
38.29 \\
38.89 \\
39.49 \\
40.09 \\
40.69 \\
41.28 \\
41.88\end{array}$ & $\begin{array}{l}61 \\
62 \\
63 \\
64 \\
65 \\
66 \\
67 \\
68 \\
69 \\
70\end{array}$ \\
\hline $\begin{array}{l}71 \\
72 \\
73 \\
74 \\
75 \\
76 \\
77 \\
78 \\
79 \\
80\end{array}$ & $\begin{array}{l}57.44 \\
58.25 \\
59.01 \\
59.87 \\
60.18 \\
61.49 \\
62.29 \\
63.10 \\
63.91 \\
64.72\end{array}$ & $\begin{array}{l}41.73 \\
42.32 \\
42.91 \\
43.50 \\
44.08 \\
44.67 \\
45.26 \\
45.85 \\
46.44 \\
47.02\end{array}$ & $\begin{array}{l}57.26 \\
55.06 \\
58.87 \\
59.68 \\
60.48 \\
61.29 \\
62.10 \\
62.90 \\
63.71 \\
64.52\end{array}$ & $\begin{array}{l}41.98 \\
42.57 \\
43.17 \\
43.76 \\
44.35 \\
44.94 \\
45.53 \\
46.12 \\
46.71 \\
4730\end{array}$ & $\begin{array}{l}57.07 \\
57.88 \\
58.68 \\
59.49 \\
60.29 \\
61.09 \\
61.90 \\
62.70 \\
63.50 \\
64.31\end{array}$ & $\begin{array}{l}42.23 \\
42.83 \\
43.42 \\
44.02 \\
44.61 \\
45.21 \\
45.80 \\
46.40 \\
46.99 \\
47.59\end{array}$ & $\begin{array}{l}56.89 \\
57.69 \\
58.49 \\
59.29 \\
60.09 \\
60.90 \\
61.70 \\
62.50 \\
63.30 \\
64.10\end{array}$ & $\begin{array}{l}42.48 \\
43.08 \\
43.68 \\
44.28 \\
44.87 \\
45.47 \\
46.07 \\
46.67 \\
47.27 \\
47.87\end{array}$ & $\begin{array}{l}71 \\
72 \\
73 \\
74 \\
75 \\
76 \\
77 \\
78 \\
79 \\
80\end{array}$ \\
\hline $\begin{array}{l}81 \\
82 \\
83 \\
54 \\
85 \\
86 \\
87 \\
89 \\
89 \\
90\end{array}$ & $\begin{array}{l}65.53 \\
66.34 \\
67.15 \\
67.96 \\
68.77 \\
69.58 \\
70.38 \\
71.19 \\
72.00 \\
72.81\end{array}$ & $\begin{array}{l}47.61 \\
48.20 \\
48.79 \\
49.37 \\
49.96 \\
50.55 \\
51.14 \\
51.73 \\
52.31 \\
52.90\end{array}$ & $\begin{array}{l}65.32 \\
66.13 \\
66.93 \\
67.74 \\
68.55 \\
69.35 \\
70.16 \\
70.97 \\
71.77 \\
72.58\end{array}$ & $\begin{array}{l}47.90 \\
48.49 \\
49.08 \\
49.67 \\
50.26 \\
50.85 \\
51.44 \\
52.04 \\
52.63 \\
53.22\end{array}$ & $\begin{array}{l}65.11 \\
65.92 \\
66.72 \\
67.52 \\
68.33 \\
69.13 \\
69.94 \\
70.74 \\
71.54 \\
72.35\end{array}$ & $\begin{array}{l}48.18 \\
48.78 \\
49.37 \\
49.97 \\
50.56 \\
51.15 \\
51.75 \\
52.34 \\
52.94 \\
53.53\end{array}$ & $\begin{array}{l}64.90 \\
65.70 \\
66.50 \\
67.31 \\
68.11 \\
68.91 \\
69.71 \\
70.51 \\
71.31 \\
72.11\end{array}$ & $\begin{array}{l}48.46 \\
49.06 \\
49.66 \\
50.26 \\
50.86 \\
51.46 \\
52.05 \\
52.65 \\
53.25 \\
53.85\end{array}$ & $\begin{array}{l}81 \\
82 \\
83 \\
84 \\
85 \\
86 \\
87 \\
88 \\
89 \\
90\end{array}$ \\
\hline $\begin{array}{r}91 \\
92 \\
93 \\
94 \\
95 \\
96 \\
97 \\
98 \\
99 \\
100\end{array}$ & $\begin{array}{l}73.62 \\
74.43 \\
75.24 \\
76.05 \\
76.86 \\
77.67 \\
78.47 \\
79.28 \\
80.03 \\
80.90\end{array}$ & $\begin{array}{l}53.49 \\
54.08 \\
54.66 \\
55.25 \\
55.84 \\
51.43 \\
57.02 \\
57.60 \\
58.19 \\
58.78\end{array}$ & $\begin{array}{l}73.39 \\
74.19 \\
75.00 \\
75.81 \\
76.01 \\
77.42 \\
78.23 \\
79.03 \\
79.84 \\
80.64\end{array}$ & $\begin{array}{l}53.81 \\
54.40 \\
54.99 \\
55.58 \\
56.17 \\
56.77 \\
57.36 \\
57.95 \\
58.54 \\
59.13\end{array}$ & $\begin{array}{l}73.15 \\
73.95 \\
74.76 \\
75.56 \\
76.37 \\
77.17 \\
77.97 \\
78.78 \\
79.58 \\
80.39\end{array}$ & $\begin{array}{l}54.13 \\
54.72 \\
55.32 \\
55.91 \\
56.51 \\
57.10 \\
57.70 \\
58.29 \\
58.89 \\
59.48\end{array}$ & $\begin{array}{l}72.91 \\
73.72 \\
74.52 \\
75.32 \\
76.12 \\
76.92 \\
77.72 \\
78.52 \\
79.32 \\
80.13\end{array}$ & $\begin{array}{l}54.45 \\
55.05 \\
55.64 \\
56.24 \\
51.84 \\
57.44 \\
58.04 \\
58.64 \\
59.23 \\
59.83\end{array}$ & $\begin{array}{r}91 \\
92 \\
93 \\
94 \\
95 \\
96 \\
97 \\
98 \\
99 \\
100\end{array}$ \\
\hline \multirow{2}{*}{ 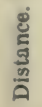 } & Dep. & Lat. & Dep. & Lat. & Dep. & Lat. & Dep. & Lat. & \multirow{2}{*}{ 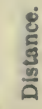 } \\
\hline & \multicolumn{2}{|c|}{$54^{\circ}$. } & \multicolumn{2}{|c|}{$53 \% 4^{\circ}}$. & \multicolumn{2}{|c|}{$531 / 2^{\circ}$} & \multicolumn{2}{|c|}{$5314^{\circ}$. } & \\
\hline
\end{tabular}


TABLE 4. -TIIVEISE TABLE.

\begin{tabular}{|c|c|c|c|c|c|c|c|c|c|}
\hline \multirow{2}{*}{ 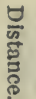 } & \multicolumn{2}{|c|}{$37^{\circ}$. } & \multicolumn{2}{|c|}{$371 / 4^{\circ}$. } & \multicolumn{2}{|c|}{$371 / 2^{\circ}$. } & \multicolumn{2}{|c|}{$3734^{\circ}$. } & \multirow{2}{*}{ 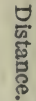 } \\
\hline & Lat. & Dep. & Lat. & Dep. & Lat. & Dep. & Lat. & Dep. & \\
\hline $\begin{array}{r}1 \\
2 \\
3 \\
4 \\
5 \\
6 \\
7 \\
8 \\
9 \\
10\end{array}$ & $\begin{array}{l}0.80 \\
1.60 \\
2.40 \\
3.19 \\
3.99 \\
4.79 \\
5.59 \\
6.39 \\
7.19 \\
7.99\end{array}$ & $\begin{array}{l}0.60 \\
1.20 \\
1.81 \\
2.41 \\
3.01 \\
3.61 \\
4.21 \\
4.81 \\
5.42 \\
6.02\end{array}$ & $\begin{array}{l}0.80 \\
1.59 \\
2.39 \\
3.18 \\
3.98 \\
4.78 \\
5.57 \\
6.37 \\
7.16 \\
7.96\end{array}$ & $\begin{array}{l}0.61 \\
1.21 \\
1.82 \\
2.42 \\
3.03 \\
3.63 \\
4.24 \\
4.84 \\
5.45 \\
6.05\end{array}$ & $\begin{array}{l}0.79 \\
1.59 \\
2.38 \\
3.17 \\
3.97 \\
4.76 \\
5.55 \\
6.35 \\
7.14 \\
7.93\end{array}$ & $\begin{array}{l}0.61 \\
1.22 \\
1.83 \\
2.44 \\
3.04 \\
3.65 \\
4.26 \\
4.87 \\
5.48 \\
6.09\end{array}$ & $\begin{array}{l}0.79 \\
1.58 \\
2.37 \\
3.16 \\
3.95 \\
4.74 \\
5.53 \\
6.33 \\
7.12 \\
7.91\end{array}$ & $\begin{array}{l}0.61 \\
1.22 \\
1.84 \\
2.45 \\
3.06 \\
3.67 \\
4.29 \\
4.90 \\
5.51 \\
6.12\end{array}$ & $\begin{array}{r}1 \\
2 \\
3 \\
4 \\
5 \\
6 \\
7 \\
8 \\
9 \\
10\end{array}$ \\
\hline $\begin{array}{l}11 \\
12 \\
13 \\
14 \\
15 \\
16 \\
17 \\
18 \\
19 \\
20\end{array}$ & $\begin{array}{r}8.78 \\
9.58 \\
10.38 \\
11.18 \\
11.98 \\
12.78 \\
13.58 \\
14.38 \\
15.17 \\
15.97\end{array}$ & $\begin{array}{r}6.62 \\
7.22 \\
7.82 \\
8.43 \\
9.03 \\
9.63 \\
10.23 \\
10.83 \\
11.43 \\
12.04\end{array}$ & $\begin{array}{r}8.76 \\
9.55 \\
10.35 \\
11.14 \\
11.94 \\
12.74 \\
13.53 \\
14.33 \\
15.12 \\
15.92\end{array}$ & $\begin{array}{r}6.66 \\
7.26 \\
7.87 \\
8.47 \\
9.08 \\
9.68 \\
10.29 \\
10.90 \\
11.50 \\
12.11\end{array}$ & $\begin{array}{r}8.73 \\
9.52 \\
10.31 \\
11.11 \\
11.90 \\
12.69 \\
13.49 \\
14.28 \\
15.07 \\
15.87\end{array}$ & $\begin{array}{r}6.70 \\
7.31 \\
7.91 \\
8.52 \\
9.13 \\
9.74 \\
10.35 \\
10.96 \\
11.57 \\
12.18\end{array}$ & $\begin{array}{r}8.70 \\
9.49 \\
10.28 \\
11.07 \\
11.86 \\
12.65 \\
13.44 \\
14.23 \\
15.02 \\
15.81\end{array}$ & $\begin{array}{r}6.73 \\
7.35 \\
7.96 \\
8.57 \\
9.18 \\
9.80 \\
10.41 \\
11.02 \\
11.63 \\
12.24\end{array}$ & $\begin{array}{l}11 \\
12 \\
13 \\
14 \\
15 \\
16 \\
17 \\
18 \\
19 \\
20\end{array}$ \\
\hline $\begin{array}{l}21 \\
22 \\
23 \\
24 \\
25 \\
26 \\
27 \\
28 \\
29 \\
30\end{array}$ & $\begin{array}{l}16.77 \\
17.57 \\
18.37 \\
19.17 \\
19.97 \\
20.76 \\
21.56 \\
22.36 \\
23.16 \\
23.96\end{array}$ & $\begin{array}{l}12.64 \\
13.24 \\
13.84 \\
14.44 \\
15.05 \\
15.65 \\
16.25 \\
16.85 \\
17.45 \\
18.05\end{array}$ & $\begin{array}{l}16.72 \\
17.51 \\
18.31 \\
19.10 \\
19.90 \\
20.70 \\
21.49 \\
22.29 \\
23.08 \\
23.88\end{array}$ & $\begin{array}{l}12.71 \\
13.32 \\
13.92 \\
14.53 \\
15.13 \\
15.74 \\
16.34 \\
16.95 \\
17.55 \\
18.16\end{array}$ & $\begin{array}{l}16.66 \\
17.45 \\
18.25 \\
19.04 \\
19.83 \\
20.63 \\
21.42 \\
22.21 \\
23.01 \\
23.80\end{array}$ & $\begin{array}{l}12.78 \\
13.39 \\
14.00 \\
14.61 \\
15.22 \\
15.83 \\
16.44 \\
17.05 \\
17.65 \\
18.26\end{array}$ & $\begin{array}{l}16.60 \\
17.40 \\
18.19 \\
18.98 \\
19.77 \\
20.56 \\
21.35 \\
22.14 \\
22.93 \\
23.72\end{array}$ & $\begin{array}{l}12.86 \\
13.47 \\
14.05 \\
14.69 \\
15.31 \\
15.92 \\
16.53 \\
17.14 \\
17.75 \\
18.37\end{array}$ & $\begin{array}{l}21 \\
22 \\
23 \\
24 \\
25 \\
26 \\
27 \\
28 \\
29 \\
30\end{array}$ \\
\hline $\begin{array}{l}31 \\
32 \\
33 \\
34 \\
35 \\
36 \\
37 \\
38 \\
39 \\
40\end{array}$ & $\begin{array}{l}24.76 \\
25.56 \\
26.35 \\
27.15 \\
27.95 \\
28.75 \\
29.55 \\
30.35 \\
31.15 \\
31.95\end{array}$ & $\begin{array}{l}18.66 \\
19.26 \\
19.86 \\
20.46 \\
21.06 \\
21.67 \\
22.27 \\
22.87 \\
23.47 \\
24.07\end{array}$ & $\begin{array}{l}24.68 \\
25.47 \\
26.27 \\
27.06 \\
27.86 \\
28.66 \\
29.45 \\
30.25 \\
31.04 \\
31.84\end{array}$ & $\begin{array}{l}18.76 \\
19.37 \\
19.97 \\
20.58 \\
21.19 \\
21.79 \\
22.40 \\
23.00 \\
23.61 \\
24.21\end{array}$ & $\begin{array}{l}24.59 \\
25.39 \\
26.18 \\
26.97 \\
27.77 \\
28.56 \\
29.35 \\
30.15 \\
30.94 \\
31.73\end{array}$ & $\begin{array}{l}18.87 \\
19.44 \\
20.09 \\
20.70 \\
21.31 \\
21.92 \\
22.52 \\
23.13 \\
23.74 \\
24.35\end{array}$ & $\begin{array}{l}24.51 \\
25.30 \\
26.09 \\
26.88 \\
27.67 \\
28.46 \\
29.26 \\
30.05 \\
30.84 \\
31.63\end{array}$ & $\begin{array}{l}18.98 \\
19.59 \\
20.20 \\
20.82 \\
21.43 \\
22.04 \\
22.65 \\
23.26 \\
23.88 \\
24.49\end{array}$ & $\begin{array}{l}31 \\
32 \\
33 \\
34 \\
35 \\
36 \\
37 \\
38 \\
39 \\
40\end{array}$ \\
\hline $\begin{array}{l}41 \\
42 \\
43 \\
44 \\
45 \\
46 \\
47 \\
48 \\
49 \\
50\end{array}$ & $\begin{array}{l}32.74 \\
33.54 \\
34.34 \\
35.14 \\
35.94 \\
36.74 \\
37.54 \\
38.33 \\
39.13 \\
39.93\end{array}$ & $\begin{array}{l}24.67 \\
25.28 \\
25.88 \\
26.48 \\
27.08 \\
27.68 \\
28.29 \\
28.89 \\
29.49 \\
30.09\end{array}$ & $\begin{array}{l}32.64 \\
33.43 \\
34.23 \\
35.02 \\
35.82 \\
36.62 \\
37.41 \\
38.21 \\
39.00 \\
39.80\end{array}$ & $\begin{array}{l}24.82 \\
25.42 \\
26.03 \\
26.63 \\
27.24 \\
27.84 \\
28.45 \\
29.05 \\
29.66 \\
30.26\end{array}$ & $\begin{array}{l}32.53 \\
33.32 \\
34.11 \\
34.91 \\
35.70 \\
36.49 \\
37.29 \\
38.09 \\
38.87 \\
39.67\end{array}$ & $\begin{array}{l}24.96 \\
25.57 \\
26.18 \\
26.79 \\
27.39 \\
28.00 \\
28.61 \\
29.22 \\
29.83 \\
30.44\end{array}$ & $\begin{array}{l}32.42 \\
33.21 \\
34.00 \\
34.79 \\
35.58 \\
36.37 \\
37.16 \\
37.95 \\
38.74 \\
39.53\end{array}$ & $\begin{array}{l}25.10 \\
25.71 \\
26.33 \\
26.94 \\
27.55 \\
28.16 \\
28.77 \\
29.39 \\
30.00 \\
30.61\end{array}$ & $\begin{array}{l}41 \\
42 \\
43 \\
44 \\
45 \\
46 \\
47 \\
48 \\
49 \\
50\end{array}$ \\
\hline$\dot{\mathscr{E}}$ & Dep. & Lat. & Dep. & - Lat. & Dep. & Lat. & Dep. & Lat. & త્ટ \\
\hline & \multicolumn{2}{|c|}{$53^{\circ}$. } & \multicolumn{2}{|c|}{$5234^{\circ}$. } & \multicolumn{2}{|c|}{$521 / 2^{\circ}$. } & \multicolumn{2}{|c|}{$521 / 4^{\circ}$. } & A \\
\hline
\end{tabular}


TABLE 4.-TRAVERSE TABLE.

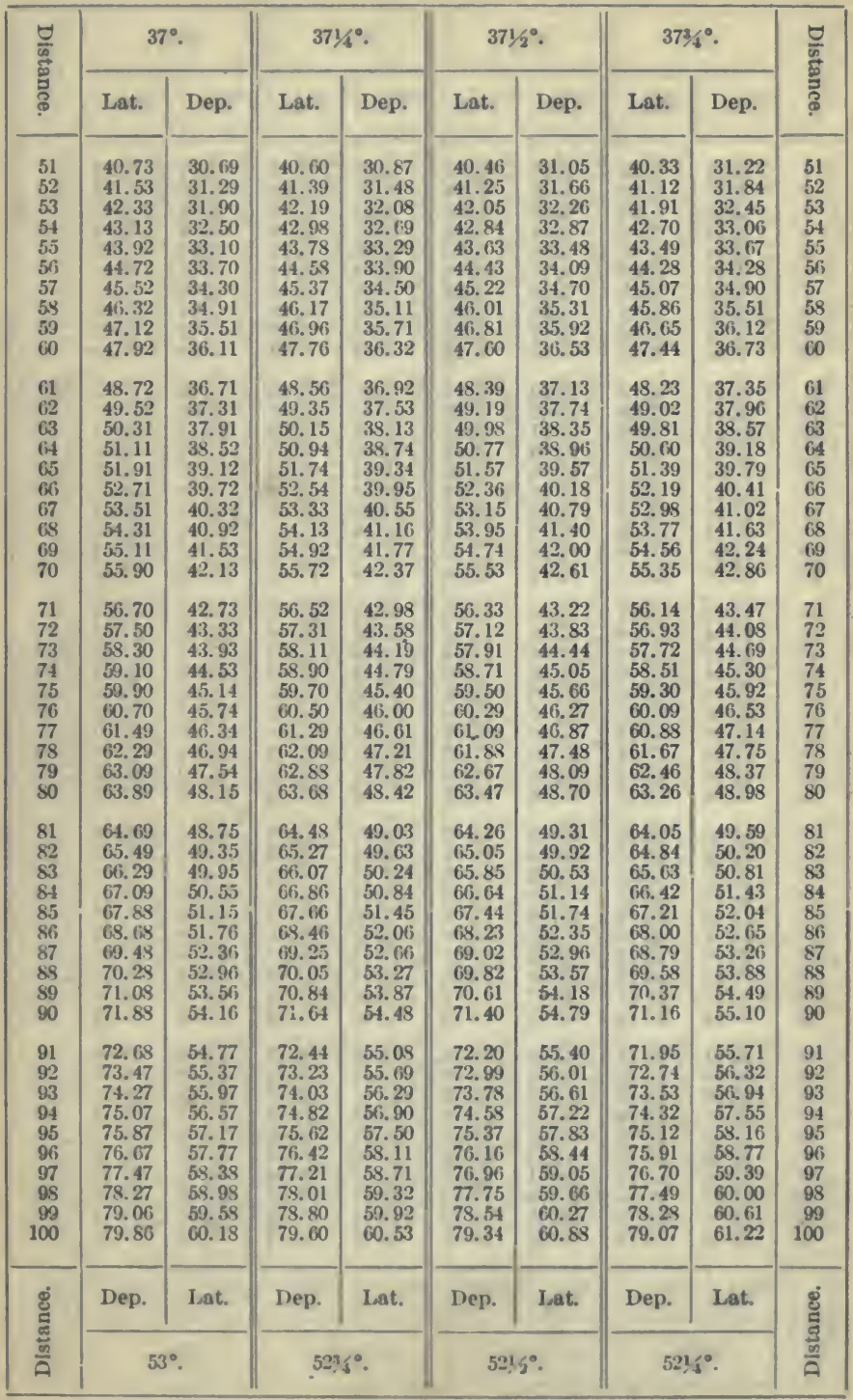


TABLE 4.-TRAVERSE TABLF.

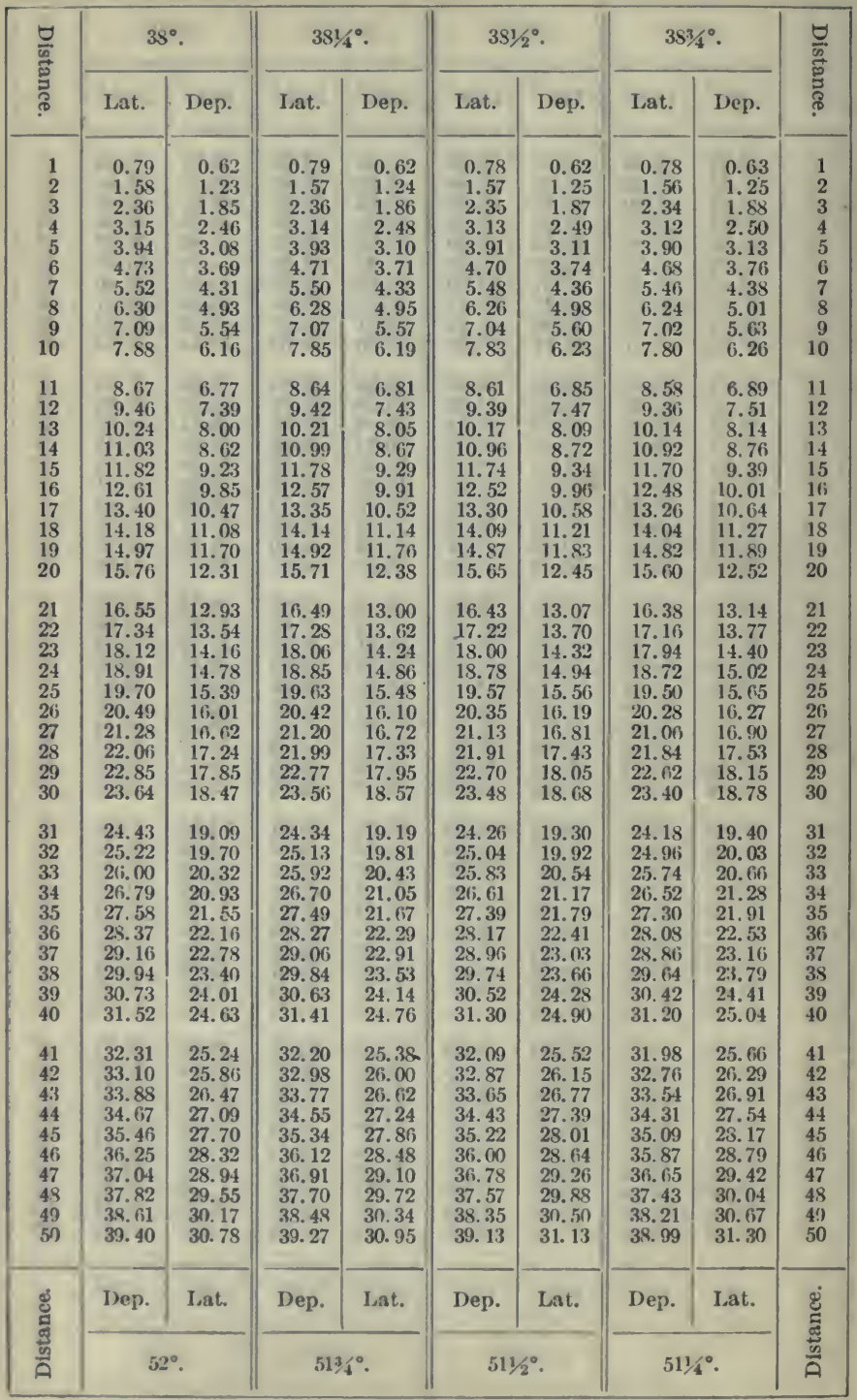


TABLE 4.-TRAVERSE TABLE.

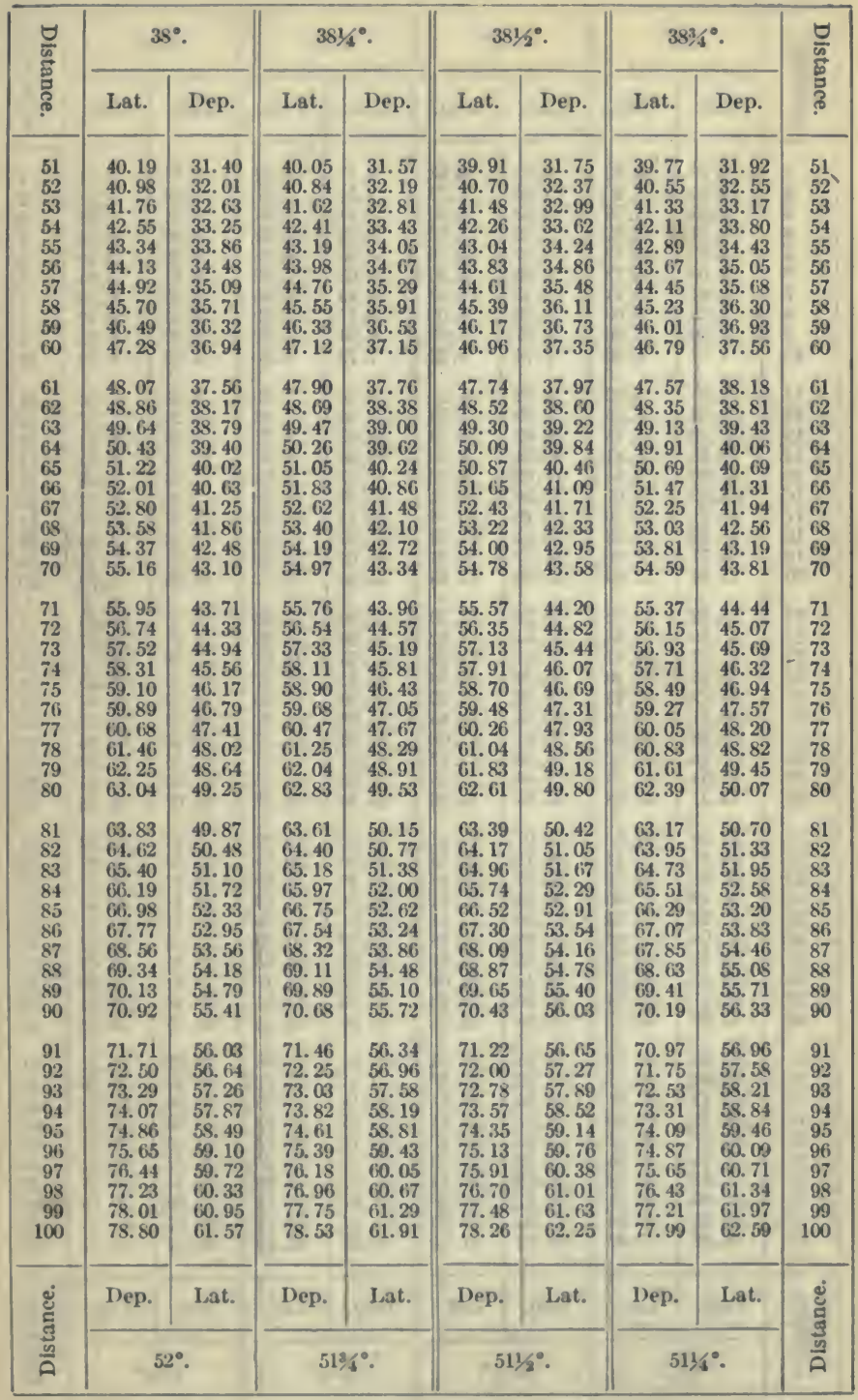


TABLE 4.-TRAVERSE TABLE.

\begin{tabular}{|c|c|c|c|c|c|c|c|c|c|}
\hline \multirow{2}{*}{$\frac{\sigma}{\tilde{w}}$} & \multicolumn{2}{|c|}{$39^{\circ}$. } & \multicolumn{2}{|c|}{$391 / 4^{\circ}$. } & \multicolumn{2}{|c|}{$391 / 2^{\circ}$. } & \multicolumn{2}{|c|}{$3934^{\circ}$. } & \multirow{2}{*}{ 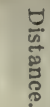 } \\
\hline & Lat. & Dep. & Lat. & Dep. & Lat. & Dep. & Lat. & Dep. & \\
\hline $\begin{array}{r}1 \\
2 \\
3 \\
4 \\
5 \\
6 \\
7 \\
8 \\
9 \\
10\end{array}$ & $\begin{array}{l}0.78 \\
1.55 \\
2.33 \\
3.11 \\
3.89 \\
4.66 \\
5.44 \\
6.22 \\
6.99 \\
7.77\end{array}$ & $\begin{array}{l}0.63 \\
1.26 \\
1.89 \\
2.52 \\
3.15 \\
3.78 \\
4.41 \\
5.03 \\
5.66 \\
6.29\end{array}$ & $\begin{array}{l}0.77 \\
1.55 \\
2.32 \\
3.10 \\
3.87 \\
4.65 \\
5.42 \\
6.20 \\
6.97 \\
7.74\end{array}$ & $\begin{array}{l}0.63 \\
1.27 \\
1.90 \\
2.53 \\
3.16 \\
3.80 \\
4.43 \\
5.06 \\
5.69 \\
6.33\end{array}$ & $\begin{array}{l}0.77 \\
1.54 \\
2.31 \\
3.09 \\
3.86 \\
4.63 \\
5.40 \\
6.17 \\
6.94 \\
7.72\end{array}$ & $\begin{array}{l}0.64 \\
1.27 \\
1.91 \\
2.54 \\
3.18 \\
3.82 \\
4.45 \\
5.09 \\
5.72 \\
6.36\end{array}$ & $\begin{array}{l}0.77 \\
1.54 \\
2.31 \\
3.08 \\
3.84 \\
4.61 \\
5.38 \\
6.15 \\
6.92 \\
7.69\end{array}$ & $\begin{array}{l}0.64 \\
1.28 \\
1.92 \\
2.56 \\
3.20 \\
3.84 \\
4.48 \\
5.12 \\
5.75 \\
6.39\end{array}$ & $\begin{array}{r}1 \\
2 \\
3 \\
4 \\
5 \\
6 \\
7 \\
8 \\
9 \\
10\end{array}$ \\
\hline $\begin{array}{l}11 \\
12 \\
13 \\
14 \\
15 \\
16 \\
17 \\
18 \\
19 \\
20\end{array}$ & $\begin{array}{r}8.55 \\
9.33 \\
10.10 \\
10.88 \\
11.66 \\
12.43 \\
13.21 \\
13.99 \\
14.77 \\
15.54\end{array}$ & $\begin{array}{r}6.92 \\
7.55 \\
8.18 \\
8.81 \\
9.44 \\
10.07 \\
10.70 \\
11.33 \\
11.96 \\
12.59\end{array}$ & $\begin{array}{r}8.52 \\
9.29 \\
10.07 \\
10.84 \\
11.62 \\
12.39 \\
13.16 \\
13.94 \\
14.71 \\
15.49\end{array}$ & $\begin{array}{r}6.96 \\
7.59 \\
8.23 \\
8.86 \\
9.49 \\
10.12 \\
10.76 \\
11.39 \\
12.02 \\
12.65\end{array}$ & $\begin{array}{r}8.49 \\
9.26 \\
10.03 \\
10.80 \\
11.57 \\
12.35 \\
13.12 \\
13.89 \\
14.66 \\
15.43\end{array}$ & $\begin{array}{r}7.00 \\
7.63 \\
8.27 \\
8.91 \\
9.54 \\
10.18 \\
10.81 \\
11.45 \\
12.09 \\
12.72\end{array}$ & $\begin{array}{r}8.46 \\
9.23 \\
9.99 \\
10.76 \\
11.53 \\
12.30 \\
13.07 \\
13.84 \\
14.61 \\
15.38\end{array}$ & $\begin{array}{r}7.03 \\
7.67 \\
8.31 \\
8.95 \\
9.59 \\
10.23 \\
10.87 \\
11.51 \\
12.15 \\
12.79\end{array}$ & $\begin{array}{l}11 \\
12 \\
13 \\
14 \\
15 \\
16 \\
17 \\
18 \\
19 \\
20\end{array}$ \\
\hline $\begin{array}{l}21 \\
22 \\
23 \\
24 \\
25 \\
26 \\
27 \\
28 \\
29 \\
30\end{array}$ & $\begin{array}{l}16.32 \\
17.10 \\
17.87 \\
18.65 \\
19.43 \\
20.21 \\
20.98 \\
21.76 \\
22.54 \\
23.31\end{array}$ & $\begin{array}{l}13.22 \\
13.85 \\
14.47 \\
15.10 \\
15.73 \\
16.36 \\
16.99 \\
17.62 \\
18.25 \\
18.88\end{array}$ & $\begin{array}{l}16.26 \\
17.04 \\
17.81 \\
18.59 \\
19.36 \\
20.13 \\
20.91 \\
21.68 \\
22.46 \\
23.23\end{array}$ & $\begin{array}{l}13.29 \\
13.92 \\
14.55 \\
15.18 \\
15.82 \\
16.45 \\
17.08 \\
17.72 \\
18.35 \\
18.98\end{array}$ & $\begin{array}{l}16.20 \\
16.98 \\
17.75 \\
18.52 \\
19.29 \\
20.06 \\
20.83 \\
21.61 \\
22.38 \\
23.15\end{array}$ & $\begin{array}{l}13.36 \\
13.99 \\
14.63 \\
15.27 \\
15.90 \\
16.54 \\
17.17 \\
17.81 \\
18.45 \\
19.08\end{array}$ & $\begin{array}{l}16.15 \\
16.91 \\
17.68 \\
18.45 \\
19.22 \\
19.99 \\
20.76 \\
21.53 \\
22.30 \\
23.07\end{array}$ & $\begin{array}{l}13.43 \\
14.07 \\
14.71 \\
15.35 \\
15.99 \\
16.63 \\
17.26 \\
17.90 \\
18.54 \\
19.18\end{array}$ & $\begin{array}{l}21 \\
22 \\
23 \\
24 \\
25 \\
26 \\
27 \\
25 \\
29 \\
30\end{array}$ \\
\hline $\begin{array}{l}31 \\
32 \\
33 \\
31 \\
35 \\
36 \\
37 \\
38 \\
39 \\
40\end{array}$ & $\begin{array}{l}24.09 \\
24.87 \\
25.65 \\
26.42 \\
27.20 \\
27.98 \\
23.75 \\
29.53 \\
30.31 \\
31.09\end{array}$ & $\begin{array}{l}19.51 \\
20.14 \\
20.77 \\
21.40 \\
22.03 \\
22.66 \\
23.28 \\
23.91 \\
24.54 \\
25.17\end{array}$ & $\begin{array}{l}24.01 \\
24.78 \\
25.55 \\
26.33 \\
27.10 \\
27.88 \\
28.65 \\
29.43 \\
30.20 \\
30.98\end{array}$ & $\begin{array}{l}19.61 \\
20.25 \\
20.88 \\
21.51 \\
22.14 \\
22.78 \\
23.41 \\
24.04 \\
24.68 \\
25.31\end{array}$ & $\begin{array}{l}23.92 \\
24.69 \\
25.46 \\
26.24 \\
27.01 \\
27.78 \\
28.55 \\
29.32 \\
30.09 \\
30.86\end{array}$ & $\begin{array}{l}19.72 \\
20.35 \\
20.99 \\
21.63 \\
22.26 \\
22.90 \\
23.53 \\
24.17 \\
24.81 \\
25.44\end{array}$ & $\begin{array}{l}23.83 \\
24.60 \\
25.37 \\
26.14 \\
26.91 \\
27.68 \\
28.45 \\
29.22 \\
29.98 \\
30.75\end{array}$ & $\begin{array}{l}19.82 \\
20.46 \\
21.10 \\
21.74 \\
22.38 \\
23.02 \\
23.66 \\
24.30 \\
24.94 \\
25.58\end{array}$ & $\begin{array}{l}31 \\
32 \\
33 \\
34 \\
35 \\
36 \\
37 \\
38 \\
39 \\
40\end{array}$ \\
\hline $\begin{array}{l}41 \\
42 \\
43 \\
44 \\
45 \\
46 \\
47 \\
48 \\
49 \\
50\end{array}$ & $\begin{array}{l}31.86 \\
32.64 \\
33.42 \\
34.19 \\
34.97 \\
35.75 \\
36.53 \\
37.30 \\
38.08 \\
38.86\end{array}$ & $\begin{array}{l}25.80 \\
26.43 \\
27.06 \\
27.69 \\
28.32 \\
28.95 \\
29.58 \\
30.21 \\
30.84 \\
31.47\end{array}$ & $\begin{array}{l}31.75 \\
32.52 \\
33.30 \\
34.07 \\
34.85 \\
35.62 \\
36.40 \\
37.17 \\
37.95 \\
38.72\end{array}$ & $\begin{array}{l}25.94 \\
26.57 \\
27.21 \\
27.84 \\
28.47 \\
29.10 \\
29.74 \\
30.37 \\
31.00 \\
31.64\end{array}$ & $\begin{array}{l}31.64 \\
32.41 \\
33.18 \\
33.95 \\
34.72 \\
35.49 \\
31.27 \\
37.04 \\
37.81 \\
38.58\end{array}$ & $\begin{array}{l}26.08 \\
26.72 \\
27.35 \\
27.99 \\
28.6 .2 \\
29.26 \\
29.90 \\
30.53 \\
31.17 \\
31.80\end{array}$ & $\begin{array}{r}31.52 \\
32.29 \\
33.06 \\
33.83 \\
34.60 \\
35.37 \\
36.14 \\
36.90 \\
37.67 \\
33.44\end{array}$ & $\begin{array}{l}26.22 \\
26.86 \\
27.50 \\
28.14 \\
25.77 \\
29.41 \\
30.05 \\
30.19 \\
31.33 \\
31.97\end{array}$ & $\begin{array}{r}41 \\
42 \\
43 \\
44 \\
45 \\
46 \\
47 \\
-48 \\
49 \\
50\end{array}$ \\
\hline छூ & Dep. & Lat. & Dep. & Lat. & Dep. & Lat. & Dep. & Lat. & 8 \\
\hline$\frac{n}{a}$ & \multicolumn{2}{|c|}{$51^{\circ}$. } & \multicolumn{2}{|c|}{$5034^{\circ}$. } & \multicolumn{2}{|c|}{$501 / 2^{\circ}$. } & \multicolumn{2}{|c|}{$501 / 4^{\circ}$} & $\frac{\sigma}{A}$ \\
\hline
\end{tabular}


TABLE 4.-TRAVERSE TABLE.

\begin{tabular}{|c|c|c|c|c|c|c|c|c|c|}
\hline \multirow{2}{*}{$\frac{\forall}{\frac{\theta}{5}}$} & \multicolumn{2}{|c|}{$39^{\circ}$. } & \multicolumn{2}{|c|}{$391 / 4^{\circ}$. } & \multicolumn{2}{|c|}{$391 / 2^{\circ}$. } & \multicolumn{2}{|c|}{$3934^{\circ}$. } & \multirow{2}{*}{ 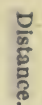 } \\
\hline & Lat. & Dep. & Lat. & Dep. & Lat. & Dep. & Lat. & Dep. & \\
\hline $\begin{array}{l}51 \\
52 \\
53 \\
54 \\
55 \\
56 \\
57 \\
58 \\
59 \\
60\end{array}$ & $\begin{array}{l}39.63 \\
40.41 \\
41.19 \\
41.97 \\
42.74 \\
43.52 \\
44.30 \\
45.07 \\
45.85 \\
46.63\end{array}$ & $\begin{array}{l}32.10 \\
32.72 \\
33.35 \\
33.98 \\
34.61 \\
35.24 \\
35.87 \\
36.50 \\
37.13 \\
37.76\end{array}$ & $\begin{array}{l}39.49 \\
40.27 \\
41.04 \\
41.82 \\
42.59 \\
43.37 \\
44.14 \\
44.91 \\
45.69 \\
46.46\end{array}$ & $\begin{array}{l}32.27 \\
32.90 \\
33.53 \\
34.17 \\
34.80 \\
35.43 \\
36.06 \\
36.70 \\
37.33 \\
37.96\end{array}$ & $\begin{array}{l}39.35 \\
40.12 \\
40.90 \\
41.67 \\
42.44 \\
43.21 \\
43.98 \\
44.75 \\
45.53 \\
46.30\end{array}$ & $\begin{array}{l}32.44 \\
33.08 \\
33.71 \\
34.35 \\
34.98 \\
35.62 \\
36.26 \\
36.89 \\
37.53 \\
38.16\end{array}$ & $\begin{array}{l}39.21 \\
39.98 \\
40.75 \\
41.52 \\
42.29 \\
43.06 \\
43.82 \\
44.59 \\
45.36 \\
46.13\end{array}$ & $\begin{array}{l}32.61 \\
33.25 \\
33.89 \\
34.53 \\
35.17 \\
35.81 \\
36.45 \\
37.09 \\
37.73 \\
38.37\end{array}$ & $\begin{array}{l}51 \\
52 \\
53 \\
54 \\
55 \\
56 \\
57 \\
58 \\
59 \\
60\end{array}$ \\
\hline $\begin{array}{l}61 \\
62 \\
63 \\
64 \\
65 \\
66 \\
67 \\
68 \\
69 \\
70\end{array}$ & $\begin{array}{l}47.41 \\
48.18 \\
48.96 \\
49.74 \\
50.51 \\
51.29 \\
52.07 \\
52.85 \\
53.62 \\
54.40\end{array}$ & $\begin{array}{l}38.39 \\
39.02 \\
39.65 \\
40.28 \\
40.91 \\
41.54 \\
42.16 \\
42.79 \\
43.42 \\
44.05\end{array}$ & $\begin{array}{l}47.24 \\
48.01 \\
48.79 \\
49.56 \\
50.34 \\
51.11 \\
51.88 \\
52.66 \\
53.43 \\
54.21\end{array}$ & $\begin{array}{l}38.60 \\
39.23 \\
39.86 \\
40.49 \\
41.13 \\
41.76 \\
42.39 \\
43.02 \\
43.66 \\
44.29\end{array}$ & $\begin{array}{l}47.07 \\
47.84 \\
48.61 \\
49.38 \\
50.16 \\
50.93 \\
51.70 \\
52.47 \\
53.24 \\
54.01\end{array}$ & $\begin{array}{l}38.80 \\
39.44 \\
40.07 \\
40.71 \\
41.35 \\
41.98 \\
42.62 \\
43.25 \\
43.89 \\
44.53\end{array}$ & $\begin{array}{l}46.90 \\
47.67 \\
48.44 \\
49.21 \\
49.97 \\
50.74 \\
51.51 \\
52.28 \\
53.05 \\
53.82\end{array}$ & $\begin{array}{l}39.01 \\
39.65 \\
40.28 \\
40.92 \\
41.56 \\
42.20 \\
42.84 \\
43.48 \\
44.12 \\
44.76\end{array}$ & $\begin{array}{l}61 \\
62 \\
63 \\
64 \\
65 \\
66 \\
67 \\
68 \\
69 \\
70\end{array}$ \\
\hline $\begin{array}{l}71 \\
72 \\
73 \\
74 \\
75 \\
76 \\
77 \\
78 \\
79 \\
80\end{array}$ & $\begin{array}{l}55.18 \\
55.95 \\
56.73 \\
57.51 \\
58.29 \\
59.06 \\
59.84 \\
60.62 \\
61.39 \\
62.17\end{array}$ & $\begin{array}{l}44.68 \\
45.31 \\
45.94 \\
46.57 \\
47.20 \\
47.83 \\
48.46 \\
49.09 \\
49.72 \\
50.35\end{array}$ & $\begin{array}{l}54.98 \\
55.76 \\
56.53 \\
57.31 \\
58.08 \\
58.85 \\
59.63 \\
60.40 \\
61.18 \\
61.95\end{array}$ & $\begin{array}{l}44.92 \\
45.55 \\
46.19 \\
46.82 \\
47.45 \\
48.09 \\
48.72 \\
49.35 \\
49.98 \\
50.62\end{array}$ & $\begin{array}{l}54.79 \\
55.56 \\
56.33 \\
57.10 \\
57.87 \\
58.64 \\
59.42 \\
60.19 \\
60.96 \\
61.73\end{array}$ & $\begin{array}{l}45.16 \\
45.80 \\
46.43 \\
47.07 \\
47.71 \\
48.34 \\
48.98 \\
49.61 \\
50.25 \\
50.89\end{array}$ & $\begin{array}{l}54.59 \\
55.36 \\
56.13 \\
56.89 \\
57.66 \\
58.43 \\
59.20 \\
59.97 \\
60.74 \\
61.51\end{array}$ & $\begin{array}{l}45.40 \\
46.04 \\
46.68 \\
47.32 \\
47.96 \\
48.60 \\
49.24 \\
49.88 \\
50.52 \\
51.16\end{array}$ & $\begin{array}{l}71 \\
72 \\
73 \\
74 \\
75 \\
76 \\
77 \\
78 \\
79 \\
80\end{array}$ \\
\hline $\begin{array}{l}81 \\
82 \\
83 \\
84 \\
85 \\
86 \\
87 \\
85 \\
89 \\
90\end{array}$ & $\begin{array}{l}62.95 \\
63.73 \\
64.50 \\
65.28 \\
66.06 \\
66.83 \\
67.61 \\
65.39 \\
69.17 \\
69.94\end{array}$ & $\begin{array}{l}50.97 \\
51.60 \\
52.23 \\
52.86 \\
53.49 \\
54.12 \\
54.75 \\
55.38 \\
56.01 \\
56.64\end{array}$ & $\begin{array}{l}62.73 \\
63.50 \\
64.27 \\
65.05 \\
65.82 \\
66.60 \\
67.37 \\
68.15 \\
68.92 \\
69.70\end{array}$ & $\begin{array}{l}51.25 \\
51.88 \\
52.51 \\
53.15 \\
53.78 \\
54.41 \\
55.05 \\
55.68 \\
56.31 \\
56.94\end{array}$ & $\begin{array}{l}62.50 \\
63.27 \\
64.04 \\
64.82 \\
65.59 \\
66.36 \\
67.13 \\
67.90 \\
65.67 \\
69.45\end{array}$ & $\begin{array}{l}51.52 \\
52.16 \\
52.79 \\
53.43 \\
54.07 \\
54.70 \\
55.34 \\
55.97 \\
56.61 \\
57.25\end{array}$ & $\begin{array}{l}62.28 \\
63.04 \\
63.81 \\
64.58 \\
65.35 \\
66.12 \\
66.89 \\
67.66 \\
68.43 \\
69.20\end{array}$ & $\begin{array}{l}51.79 \\
52.43 \\
53.07 \\
53.71 \\
54.35 \\
54.99 \\
55.63 \\
56.27 \\
56.91 \\
57.55\end{array}$ & $\begin{array}{l}81 \\
82 \\
83 \\
84 \\
85 \\
86 \\
87 \\
88 \\
89 \\
90\end{array}$ \\
\hline $\begin{array}{l}91 \\
92 \\
93 \\
94 \\
95 \\
96 \\
97 \\
98 \\
699 \\
100\end{array}$ & $\begin{array}{l}70.72 \\
71.50 \\
72.27 \\
73.05 \\
73.83 \\
74.61 \\
75.38 \\
76.16 \\
76.04 \\
77.71\end{array}$ & $\begin{array}{l}57.27 \\
57.90 \\
59.53 \\
59.16 \\
59.79 \\
60.41 \\
61.04 \\
61.67 \\
62.30 \\
62.93\end{array}$ & $\begin{array}{l}70.47 \\
71.24 \\
72.02 \\
72.79 \\
73.37 \\
74.34 \\
75.12 \\
75.89 \\
76.66 \\
77.44\end{array}$ & $\begin{array}{l}57.58 \\
58.21 \\
58.84 \\
59.47 \\
60.11 \\
60.74 \\
61.37 \\
62.01 \\
62.64 \\
63.27\end{array}$ & $\begin{array}{l}70.22 \\
70.99 \\
71.76 \\
72.53 \\
73.30 \\
74.08 \\
74.85 \\
75.62 \\
76.39 \\
77.16\end{array}$ & $\begin{array}{l}57.88 \\
58.52 \\
59.16 \\
59.79 \\
60.43 \\
61.06 \\
61.70 \\
62.34 \\
62.97 \\
63.61\end{array}$ & $\begin{array}{l}69.96 \\
70.73 \\
71.50 \\
72.27 \\
73.04 \\
73.81 \\
74.58 \\
75.35 \\
76.12 \\
76.88\end{array}$ & $\begin{array}{l}58.19 \\
58.83 \\
59.47 \\
60.11 \\
60.75 \\
61.39 \\
62.03 \\
62.67 \\
63.30 \\
63.94\end{array}$ & $\begin{array}{r}91 \\
92 \\
93 \\
94 \\
95 \\
96 \\
97 \\
98 \\
99 \\
100\end{array}$ \\
\hline 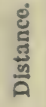 & Dep. & Lat. & Dep. & Lat. & Dep. & Lat. & Dep. & Lat. & 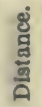 \\
\hline
\end{tabular}


TABLE 4.-TRAVERSE TABLE.

\begin{tabular}{|c|c|c|c|c|c|c|c|c|c|}
\hline \multirow{2}{*}{ 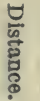 } & \multicolumn{2}{|c|}{$40^{\circ}$. } & \multicolumn{2}{|c|}{$40^{1} / 4^{\circ}$. } & \multicolumn{2}{|c|}{$401 / 2^{\circ}$. } & \multicolumn{2}{|c|}{$40^{3} 4^{\circ}$. } & \multirow{2}{*}{$\begin{array}{l}\forall \\
\forall \\
\vec{E} \\
\vec{E} \\
\text { है }\end{array}$} \\
\hline & Lat. & Dep. & Lat. & Dep. & Lat. & Dep. & Lat. & Dep. & \\
\hline $\begin{array}{r}1 \\
2 \\
3 \\
4 \\
5 \\
6 \\
7 \\
8 \\
9 \\
10\end{array}$ & $\begin{array}{l}0.77 \\
1.53 \\
2.30 \\
3.06 \\
3.83 \\
4.60 \\
5.36 \\
6.13 \\
6.89 \\
7.66\end{array}$ & $\begin{array}{l}0.64 \\
1.29 \\
1.93 \\
2.57 \\
3.21 \\
3.86 \\
4.50 \\
5.14 \\
5.79 \\
6.43\end{array}$ & $\begin{array}{l}0.76 \\
1.53 \\
2.29 \\
3.05 \\
3.82 \\
4.58 \\
5.34 \\
6.11 \\
6.87 \\
7.63\end{array}$ & $\begin{array}{l}0.65 \\
1.29 \\
1.94 \\
2.58 \\
3.23 \\
3.88 \\
4.52 \\
5.17 \\
5.82 \\
6.46\end{array}$ & $\begin{array}{l}0.76 \\
1.52 \\
2.28 \\
3.04 \\
3.80 \\
4.56 \\
5.32 \\
6.08 \\
6.84 \\
7.60\end{array}$ & $\begin{array}{l}0.65 \\
1.30 \\
1.95 \\
2.60 \\
3.25 \\
3.90 \\
4.55 \\
5.20 \\
5.85 \\
6.49\end{array}$ & $\begin{array}{l}0.76 \\
1.52 \\
2.27 \\
3.03 \\
3.79 \\
4.55 \\
5.30 \\
6.06 \\
6.82 \\
7.58\end{array}$ & $\begin{array}{l}0.65 \\
1.31 \\
1.96 \\
2.61 \\
3.26 \\
3.92 \\
4.57 \\
5.22 \\
5.87 \\
6.53\end{array}$ & $\begin{array}{r}1 \\
2 \\
3 \\
4 \\
5 \\
6 \\
7 \\
8 \\
9 \\
10\end{array}$ \\
\hline $\begin{array}{l}11 \\
12 \\
13 \\
14 \\
15 \\
16 \\
17 \\
18 \\
19 \\
20\end{array}$ & $\begin{array}{r}8.43 \\
9.19 \\
9.96 \\
10.72 \\
11.49 \\
12.26 \\
13.02 \\
13.79 \\
14.55 \\
15.32\end{array}$ & $\begin{array}{r}7.07 \\
7.71 \\
8.36 \\
9.00 \\
9.04 \\
10.28 \\
10.93 \\
11.57 \\
12.21 \\
12.86\end{array}$ & $\begin{array}{r}8.40 \\
9.16 \\
9.92 \\
10.69 \\
11.45 \\
12.21 \\
12.97 \\
13.74 \\
14.50 \\
15.26\end{array}$ & $\begin{array}{r}7.11 \\
7.75 \\
8.40 \\
9.05 \\
9.69 \\
10.34 \\
10.98 \\
11.63 \\
12.28 \\
12.92\end{array}$ & $\begin{array}{r}8.36 \\
9.12 \\
9.89 \\
10.65 \\
11.41 \\
12.17 \\
12.93 \\
13.69 \\
14.45 \\
15.21\end{array}$ & $\begin{array}{r}7.14 \\
7.79 \\
8.44 \\
9.09 \\
9.74 \\
10.39 \\
11.04 \\
11.69 \\
12.34 \\
12.99\end{array}$ & $\begin{array}{r}8.33 \\
9.09 \\
9.85 \\
10.61 \\
11.36 \\
12.12 \\
12.88 \\
13.64 \\
14.39 \\
15.15\end{array}$ & $\begin{array}{r}7.18 \\
7.83 \\
8.49 \\
9.14 \\
9.79 \\
10.44 \\
11.10 \\
11.75 \\
12.40 \\
13.06\end{array}$ & $\begin{array}{l}11 \\
12 \\
13 \\
14 \\
15 \\
16 \\
17 \\
18 \\
19 \\
20\end{array}$ \\
\hline $\begin{array}{l}21 \\
22 \\
23 \\
24 \\
25 \\
26 \\
27 \\
23 \\
29 \\
30\end{array}$ & $\begin{array}{l}16.09 \\
16.85 \\
17.62 \\
18.39 \\
19.15 \\
19.92 \\
20.68 \\
21.45 \\
22.22 \\
22.98\end{array}$ & $\begin{array}{l}13.50 \\
14.14 \\
14.78 \\
15.43 \\
16.07 \\
16.71 \\
17.36 \\
18.00 \\
18.64 \\
19.28\end{array}$ & $\begin{array}{l}16.03 \\
16.79 \\
17.55 \\
18.32 \\
19.08 \\
19.84 \\
20.61 \\
21.37 \\
22.13 \\
22.90\end{array}$ & $\begin{array}{l}13.57 \\
14.21 \\
14.86 \\
15.51 \\
16.15 \\
16.80 \\
17.45 \\
18.09 \\
18.74 \\
19.38\end{array}$ & $\begin{array}{l}15.97 \\
16.73 \\
17.49 \\
18.25 \\
19.01 \\
19.77 \\
20.53 \\
21.29 \\
22.05 \\
22.81\end{array}$ & $\begin{array}{l}13.64 \\
14.29 \\
14.94 \\
15.59 \\
16.24 \\
16.83 \\
17.54 \\
18.18 \\
18.83 \\
19.48\end{array}$ & $\begin{array}{l}15.91 \\
16.67 \\
17.42 \\
18.18 \\
18.94 \\
19.70 \\
20.45 \\
21.21 \\
21.97 \\
22.73\end{array}$ & $\begin{array}{l}13.71 \\
14.36 \\
15.01 \\
15.67 \\
16.32 \\
16.97 \\
17.62 \\
18.28 \\
18.93 \\
19.58\end{array}$ & $\begin{array}{l}21 \\
22 \\
23 \\
24 \\
25 \\
26 \\
27 \\
28 \\
29 \\
30\end{array}$ \\
\hline $\begin{array}{l}31 \\
32 \\
33 \\
34 \\
35 \\
36 \\
37 \\
38 \\
39 \\
40\end{array}$ & $\begin{array}{l}23.75 \\
24.51 \\
25.28 \\
26.05 \\
26.81 \\
27.58 \\
28.34 \\
29.11 \\
29.88 \\
30.64\end{array}$ & $\begin{array}{l}19.93 \\
20.57 \\
21.21 \\
21.85 \\
22.50 \\
23.14 \\
23.78 \\
24.43 \\
25.07 \\
25.71\end{array}$ & $\begin{array}{l}23.66 \\
24.42 \\
25.19 \\
25.95 \\
26.71 \\
27.48 \\
28.24 \\
29.00 \\
29.77 \\
30.53\end{array}$ & $\begin{array}{l}20.03 \\
20.68 \\
21.32 \\
21.97 \\
22.61 \\
23.26 \\
23.91 \\
24.55 \\
25.20 \\
25.84\end{array}$ & $\begin{array}{l}23.57 \\
24.33 \\
25.09 \\
25.85 \\
26.61 \\
27.37 \\
28.14 \\
28.90 \\
29.66 \\
30.42\end{array}$ & $\begin{array}{l}20.13 \\
20.78 \\
21.43 \\
22.08 \\
22.73 \\
23.38 \\
24.03 \\
24.68 \\
25.33 \\
25.98\end{array}$ & $\begin{array}{l}23.48 \\
24.24 \\
25.00 \\
25.76 \\
26.51 \\
27.27 \\
28.03 \\
28.79 \\
29.55 \\
30.30\end{array}$ & $\begin{array}{l}20.24 \\
20.89 \\
21.54 \\
22.19 \\
22.85 \\
23.50 \\
24.15 \\
24.80 \\
25.46 \\
26.11\end{array}$ & $\begin{array}{l}31 \\
32 \\
33 \\
34 \\
35 \\
36 \\
37 \\
38 \\
39 \\
40\end{array}$ \\
\hline $\begin{array}{l}41 \\
42 \\
43 \\
44 \\
45 \\
46 \\
47 \\
48 \\
49 \\
50\end{array}$ & $\begin{array}{l}31.41 \\
32.17 \\
33.94 \\
33.71 \\
34.47 \\
35.24 \\
36.00 \\
36.77 \\
37.54 \\
38.30\end{array}$ & $\begin{array}{l}26.35 \\
27.00 \\
27.64 \\
28.28 \\
28.93 \\
29.57 \\
30.21 \\
30.85 \\
31.50 \\
32.14\end{array}$ & $\begin{array}{l}31.29 \\
32.06 \\
32.82 \\
33.58 \\
34.35 \\
35.11 \\
35.87 \\
36.64 \\
37.40 \\
38.16\end{array}$ & $\begin{array}{l}26.49 \\
27.14 \\
27.78 \\
28.43 \\
29.08 \\
29.72 \\
30.37 \\
31.01 \\
31.60 \\
32.31\end{array}$ & $\begin{array}{l}31.18 \\
31.94 \\
32.70 \\
33.40 \\
34.22 \\
34.98 \\
35.74 \\
36.50 \\
37.26 \\
38.02\end{array}$ & $\begin{array}{l}26.63 \\
27.25 \\
27.93 \\
28.58 \\
29.23 \\
29.87 \\
30.52 \\
31.17 \\
31.82 \\
32.47\end{array}$ & $\begin{array}{l}31.06 \\
31.82 \\
32.58 \\
33.33 \\
34.09 \\
34.85 \\
35.61 \\
36.36 \\
37.12 \\
37.88\end{array}$ & $\begin{array}{l}26.76 \\
27.42 \\
28.07 \\
25.72 \\
29.37 \\
30.03 \\
30.68 \\
31.33 \\
31.99 \\
32.64\end{array}$ & $\begin{array}{l}41 \\
42 \\
43 \\
44 \\
45 \\
46 \\
47 \\
48 \\
49 \\
50\end{array}$ \\
\hline 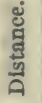 & $50^{\circ}$. & Lat. & Dep. & Lat. & Dep. & Lat. & Dep. & Lat. & 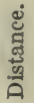 \\
\hline
\end{tabular}


TABLE 4.-TRA TERSE TABIE.

\begin{tabular}{|c|c|c|c|c|c|c|c|c|c|}
\hline \multirow{2}{*}{ 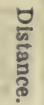 } & \multicolumn{2}{|c|}{$40^{\circ}$. } & \multicolumn{2}{|c|}{$401 / 4^{\circ}$. } & \multicolumn{2}{|c|}{$401 / 2^{\circ}}$. & \multicolumn{2}{|c|}{$40 \% 4^{\circ}$. } & \multirow{2}{*}{ 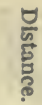 } \\
\hline & Lat. & Dep. & Lat. & Dep. & Lat. & Dep. & Lat. & Dep. & \\
\hline $\begin{array}{l}51 \\
52 \\
53 \\
54 \\
55 \\
56 \\
57 \\
58 \\
59 \\
60\end{array}$ & $\begin{array}{l}39.07 \\
39.83 \\
40.60 \\
41.37 \\
42.13 \\
42.90 \\
43.66 \\
44.43 \\
45.20 \\
45.96\end{array}$ & $\begin{array}{l}32.78 \\
33.42 \\
34.07 \\
34.71 \\
35.35 \\
36.00 \\
36.64 \\
37.28 \\
37.92 \\
38.57\end{array}$ & $\begin{array}{l}38.92 \\
39.69 \\
40.45 \\
41.21 \\
41.98 \\
42.74 \\
43.50 \\
44.27 \\
45.03 \\
45.79\end{array}$ & $\begin{array}{l}32.95 \\
33.60 \\
34.24 \\
34.89 \\
35.54 \\
36.18 \\
36.83 \\
37.48 \\
38.12 \\
38.77\end{array}$ & $\begin{array}{l}38.78 \\
39.54 \\
40.30 \\
41.06 \\
41.82 \\
42.58 \\
43.34 \\
44.10 \\
44.86 \\
45.62\end{array}$ & $\begin{array}{l}33.12 \\
33.77 \\
34.42 \\
35.07 \\
35.72 \\
36.37 \\
37.02 \\
37.67 \\
38.32 \\
38.97\end{array}$ & $\begin{array}{l}38.64 \\
39.39 \\
40.15 \\
40.91 \\
41.67 \\
42.42 \\
43.18 \\
43.94 \\
44.70 \\
45.45\end{array}$ & $\begin{array}{l}33.29 \\
33.94 \\
34.60 \\
35.25 \\
35.90 \\
3.55 \\
37.21 \\
37.86 \\
33.51 \\
39.17\end{array}$ & $\begin{array}{l}51 \\
52 \\
53 \\
54 \\
55 \\
56 \\
57 \\
68 \\
59 \\
60\end{array}$ \\
\hline $\begin{array}{l}61 \\
62 \\
63 \\
64 \\
65 \\
66 \\
67 \\
68 \\
69 \\
70\end{array}$ & $\begin{array}{l}46.73 \\
47.49 \\
48.26 \\
49.03 \\
49.79 \\
50.56 \\
51.32 \\
52.09 \\
52.86 \\
53.62\end{array}$ & $\begin{array}{l}39.21 \\
39.85 \\
40.50 \\
41.14 \\
41.78 \\
42.42 \\
43.07 \\
43.71 \\
44.35 \\
45.00\end{array}$ & $\begin{array}{l}46.56 \\
47.32 \\
48.08 \\
48.85 \\
49.61 \\
50.37 \\
51.14 \\
51.90 \\
52.66 \\
53.43\end{array}$ & $\begin{array}{l}39.41 \\
40.06 \\
40.71 \\
41.35 \\
42.00 \\
42.64 \\
43.29 \\
43.94 \\
44.58 \\
45.23\end{array}$ & $\begin{array}{l}46.38 \\
47.15 \\
47.91 \\
48.67 \\
49.43 \\
50.19 \\
50.95 \\
51.71 \\
52.47 \\
53.23\end{array}$ & $\begin{array}{l}39.62 \\
40.27 \\
40.92 \\
41.56 \\
42.21 \\
42.86 \\
43.51 \\
44.16 \\
44.81 \\
45.46\end{array}$ & $\begin{array}{l}46.21 \\
46.97 \\
47.73 \\
48.48 \\
49.24 \\
50.00 \\
50.76 \\
51.51 \\
52.27 \\
53.03\end{array}$ & $\begin{array}{l}39.82 \\
40.47 \\
41.12 \\
41.78 \\
42.43 \\
43.08 \\
43.73 \\
44.39 \\
45.04 \\
45.69\end{array}$ & $\begin{array}{l}61 \\
62 \\
63 \\
64 \\
65 \\
66 \\
67 \\
68 \\
69 \\
70\end{array}$ \\
\hline $\begin{array}{l}71 \\
72 \\
73 \\
74 \\
75 \\
76 \\
77 \\
78 \\
79 \\
80\end{array}$ & $\begin{array}{l}54.39 \\
55.16 \\
55.92 \\
56.69 \\
57.45 \\
58.22 \\
58.99 \\
59.75 \\
60.52 \\
61.28\end{array}$ & $\begin{array}{l}45.64 \\
46.28 \\
46.92 \\
47.57 \\
48.21 \\
48.85 \\
49.49 \\
50.14 \\
50.78 \\
51.42\end{array}$ & $\begin{array}{l}54.19 \\
54.95 \\
55.72 \\
56.48 \\
57.24 \\
58.01 \\
58.77 \\
59.53 \\
60.30 \\
61.06\end{array}$ & $\begin{array}{l}45.87 \\
46.52 \\
47.17 \\
47.81 \\
48.46 \\
49.11 \\
49.75 \\
50.40 \\
51.04 \\
51.69\end{array}$ & $\begin{array}{l}53.99 \\
54.75 \\
55.51 \\
56.27 \\
57.03 \\
57.79 \\
55.55 \\
59.31 \\
60.07 \\
60.83\end{array}$ & $\begin{array}{l}46.11 \\
46.76 \\
47.41 \\
48.06 \\
48.71 \\
49.36 \\
50.01 \\
50.66 \\
51.31 \\
51.96\end{array}$ & $\begin{array}{l}53.79 \\
54.54 \\
55.30 \\
56.06 \\
56.82 \\
57.57 \\
58.33 \\
59.09 \\
59.85 \\
60.61\end{array}$ & $\begin{array}{l}46.35 \\
47.00 \\
47.65 \\
48.30 \\
48.96 \\
49.61 \\
50.26 \\
50.92 \\
51.57 \\
52.22\end{array}$ & $\begin{array}{l}71 \\
72 \\
73 \\
74 \\
75 \\
76 \\
77 \\
78 \\
79 \\
80\end{array}$ \\
\hline $\begin{array}{l}81 \\
82 \\
83 \\
84 \\
85 \\
86 \\
87 \\
83 \\
89 \\
90\end{array}$ & $\begin{array}{l}62.05 \\
62.82 \\
63.53 \\
64.35 \\
65.11 \\
65.88 \\
66.65 \\
67.41 \\
68.18 \\
68.94\end{array}$ & $\begin{array}{l}52.07 \\
52.71 \\
53.35 \\
53.99 \\
54.64 \\
55.28 \\
55.92 \\
56.57 \\
57.21 \\
57.85\end{array}$ & $\begin{array}{l}61.82 \\
62.59 \\
63.35 \\
64.11 \\
64.87 \\
65.64 \\
66.40 \\
67.16 \\
67.93 \\
68.69\end{array}$ & $\begin{array}{l}52.34 \\
52.98 \\
53.63 \\
54.27 \\
54.92 \\
55.57 \\
56.21 \\
56.86 \\
57.51 \\
58.15\end{array}$ & $\begin{array}{l}61.59 \\
62.35 \\
63.11 \\
63.87 \\
64.63 \\
65.39 \\
66.16 \\
66.92 \\
67.68 \\
68.44\end{array}$ & $\begin{array}{l}52.61 \\
53.25 \\
53.90 \\
54.55 \\
55.20 \\
55.85 \\
56.50 \\
57.15 \\
57.80 \\
58.45\end{array}$ & $\begin{array}{l}61.36 \\
62.12 \\
62.88 \\
63.64 \\
64.39 \\
65.15 \\
65.91 \\
66.67 \\
67.42 \\
68.18\end{array}$ & $\begin{array}{l}52.87 \\
53.53 \\
54.18 \\
54.23 \\
55.48 \\
56.14 \\
56.79 \\
57.44 \\
58.10 \\
58.75\end{array}$ & $\begin{array}{l}81 \\
82 \\
83 \\
84 \\
85 \\
86 \\
87 \\
85 \\
89 \\
90\end{array}$ \\
\hline $\begin{array}{r}91 \\
92 \\
93 \\
94 \\
95 \\
96 \\
97 \\
98 \\
99 \\
100\end{array}$ & $\begin{array}{l}69.71 \\
70.48 \\
71.24 \\
72.01 \\
72.77 \\
73.54 \\
74.31 \\
75.07 \\
75.84 \\
76.60\end{array}$ & $\begin{array}{l}59.49 \\
59.14 \\
59.78 \\
60.42 \\
61.06 \\
61.71 \\
62.35 \\
62.99 \\
63.64 \\
64.25\end{array}$ & $\begin{array}{l}69.45 \\
70.22 \\
70.98 \\
71.74 \\
72.51 \\
73.27 \\
74.03 \\
74.80 \\
75.56 \\
76.32\end{array}$ & $\begin{array}{l}58.80 \\
59.44 \\
60.09 \\
60.74 \\
61.38 \\
62.03 \\
62.67 \\
63.32 \\
63.97 \\
64.61\end{array}$ & $\begin{array}{l}69.20 \\
69.96 \\
70.72 \\
71.48 \\
72.24 \\
73.00 \\
73.76 \\
74.52 \\
75.28 \\
76.04\end{array}$ & $\begin{array}{l}59.10 \\
59.75 \\
60.40 \\
61.05 \\
61.70 \\
62.35 \\
63.00 \\
63.65 \\
64.30 \\
64.94\end{array}$ & $\begin{array}{l}68.94 \\
69.70 \\
70.45 \\
71.21 \\
71.97 \\
72.73 \\
73.48 \\
74.24 \\
75.00 \\
75.76\end{array}$ & $\begin{array}{l}59.40 \\
60.05 \\
60.71 \\
61.36 \\
62.01 \\
62.66 \\
63.32 \\
63.97 \\
64.62 \\
65.28\end{array}$ & $\begin{array}{r}91 \\
92 \\
93 \\
94 \\
95 \\
96 \\
97 \\
95 \\
99 \\
100\end{array}$ \\
\hline \multirow{2}{*}{ 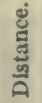 } & Dep. & Lat. & Dep. & Lat. & Dep. & Lat. & Dep. & Lat. & \multirow{2}{*}{ 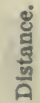 } \\
\hline & \multicolumn{2}{|c|}{$50^{\circ}$. } & \multicolumn{2}{|c|}{$4934^{\circ}}$. & \multicolumn{2}{|c|}{$491 / 2^{\circ}}$. & \multicolumn{2}{|c|}{$491 / 4^{\circ}$. } & \\
\hline
\end{tabular}


TABLE 4.-TRAVERSE TABLE.

\begin{tabular}{|c|c|c|c|c|c|c|c|c|c|}
\hline \multirow{2}{*}{ 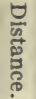 } & \multicolumn{2}{|c|}{$41^{\circ}$} & \multicolumn{2}{|c|}{$411 / 4^{\circ}$. } & \multicolumn{2}{|c|}{$411 / 2^{\circ}$. } & \multicolumn{2}{|c|}{$413 / 4^{\circ}$. } & \multirow{2}{*}{ 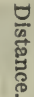 } \\
\hline & Lat. & Dep. & Lat. & Dep. & Lat. & Dep. & Lat. & Dep. & \\
\hline 1 & 0.75 & 0.66 & 0.75 & 0.66 & 0.75 & 0.66 & 0.75 & 0.67 & 1 \\
\hline 2 & 1.51 & 1.31 & 1. 50 & 1.32 & 1.50 & 1. 33 & 1.49 & 1.33 & 2 \\
\hline 3 & 2.26 & 1.97 & 2. 26 & 1.98 & 2.25 & 1.99 & 2.24 & 2.00 & 3 \\
\hline 4 & 3.02 & 2.62 & 3.01 & 2.64 & 3.00 & 2.65 & 2.98 & 2.66 & 4 \\
\hline 5 & 3.77 & 3.28 & 3.76 & 3.30 & 3.74 & 3.31 & 3.73 & 3.33 & 5 \\
\hline 6 & 4.53 & 3.94 & 4.51 & 3.96 & 4. 49 & 3.98 & 4.48 & 4.00 & 6 \\
\hline 7 & 5.28 & 4.59 & 5.26 & 4. 62 & 5.24 & 4. 64 & 5.22 & 4. 66 & 7 \\
\hline 8 & 6.04 & 5.25 & 6.01 & 5.27 & 5.99 & 5.30 & 5.97 & 5. 33 & 8 \\
\hline 9 & 6.79 & 5.90 & 6.77 & 5. 93 & 6.74 & 5. 96 & 6.71 & 5.99 & 9 \\
\hline 10 & 7.55 & 6.56 & 7.52 & 6. 59 & 7.49 & 6. 63 & 7.46 & 6. 66 & 10 \\
\hline 11 & 8.30 & 7.22 & 8.27 & 7.25 & 8. 24 & 7. 29 & 8. 21 & 7. 32 & 11 \\
\hline 12 & 9.06 & 7.87 & 9.02 & 7.91 & 8.99 & 7.95 & 8.95 & 7.99 & 12 \\
\hline 13 & 9.81 & 8.53 & 9.77 & 8. 57 & 9.74 & 8. 61 & 9.70 & 8. $f, 6$ & 13 \\
\hline 14 & 10.57 & 9.18 & 10.53 & 9.23 & 10.49 & 9.28 & 10.44 & 9.32 & 14 \\
\hline 15 & 11.32 & 9.84 & 11.28 & 9.89 & 11.23 & 9.94 & 11.19 & 9.99 & 15 \\
\hline 16 & 12.08 & 10.50 & 12.03 & 10.55 & 11.98 & 10.60 & 11.94 & 10.65 & 16 \\
\hline 17 & 12.83 & 11.15 & 12.78 & 11.21 & 12.73 & 11.26 & 12.68 & 11.32 & 17 \\
\hline 18 & 13.58 & 11.81 & 13.53 & 11.87 & 13.48 & 11.93 & 13.43 & 11.99 & 18 \\
\hline 19 & 14.34 & 12.47 & 14.28 & 12.53 & 14.23 & 12.59 & 14.18 & 12.65 & 19 \\
\hline 20 & 15. 09 & 13. 12 & 15. 04 & 13.19 & 14.98 & 13.25 & 14.92 & 13. 32 & 20 \\
\hline 21 & 15. 85 & 13.78 & 15. 79 & 13.85 & 15. 73 & 13.92 & 15.67 & 13.98 & 21 \\
\hline 22 & 16. 60 & 14.43 & 16.54 & 14.51 & 16.48 & 14.58 & 16.41 & 14.65 & 22 \\
\hline 23 & 17.36 & 15.09 & 17.29 & 15. 16 & 17.23 & 15. 24 & 17.16 & 15.32 & 23 \\
\hline 24 & 18.11 & 15.75 & 18. 04 & 15. 82 & 17.97 & 15.90 & 17.91 & 15.98 & 24 \\
\hline 25 & 18.87 & 16.40 & 18.80 & 16.48 & 18. 72 & 16.57 & 18.65 & 16.65 & 25 \\
\hline 26 & 19. 62 & 17.06 & 19.55 & 17.14 & 19.47 & 17.23 & 19.40 & 17.31 & 26 \\
\hline 27 & 20.38 & 17.71 & 20.30 & 17.80 & 20.22 & 17.89 & 20.14 & 17.98 & 27 \\
\hline 28 & 21.13 & 18.37 & 21.05 & 18.46 & 20.97 & 18.55 & 20.89 & 18. 64 & 28 \\
\hline 29 & 21.89 & 19.03 & 21.80 & 19.12 & 21.72 & 19.22 & 21.64 & 19.31 & 29 \\
\hline 30 & 22.64 & 19.68 & 22.56 & 19.78 & 22.47 & 19.88 & 22.38 & 19.98 & 30 \\
\hline 31 & 23.40 & 20.34 & 23.31 & 20.44 & 23. 22 & 20.54 & 23.13 & 20.64 & 31 \\
\hline 32 & 24.15 & 20.99 & 24.06 & 21.10 & 23.97 & 21.20 & 23.87 & 21.31 & 32 \\
\hline 33 & 24.91 & 21.65 & 24.81 & 21.76 & 24.72 & 21.87 & 24. 62 & 21.97 & 33 \\
\hline 34 & 25.66 & 22.31 & 25.56 & 22.42 & 25.46 & 22.53 & 25.37 & 22.64 & 34 \\
\hline 35 & 26.41 & 22.96 & 26.31 & 23. 08 & 26.21 & 23. 19 & 26.11 & 23. 31 & 35 \\
\hline 36 & 27.17 & 23. (2) & 27.07 & 23.74 & 26.96 & 23.85 & 26.86 & 23.97 & 36 \\
\hline 37 & 27.92 & 24.27 & 27.82 & 24.40 & 27.71 & 24.52 & 27.60 & 24.64 & 37 \\
\hline 38 & 28.68 & 24.93 & 28.57 & 25.06 & 28.46 & 25.18 & 28.35 & 25.30 & 38 \\
\hline 39 & 29.43 & 25.59 & 29.32 & 25.71 & 29.21 & 25.84 & 29.10 & 25.97 & 39 \\
\hline 40 & 30.19 & 26.24 & 30.07 & 26.37 & 29.96 & 26. 50 & 29.84 & 26.64 & 40 \\
\hline 41 & 30.94 & 26.90 & 30. 83 & 27.03 & 30.71 & 27.17 & 30.59 & 27.30 & 41 \\
\hline 42 & 31.70 & 27.55 & 31.58 & 27.69 & 31.46 & 27.83 & 31.33 & 27.97 & 42 \\
\hline 43 & 32.45 & 23.21 & 32.33 & 28. 35 & 32.21 & 28.49 & 32.08 & 28. 63 & 43 \\
\hline 44 & 33. 21 & 28.87 & 33.08 & 29.01 & 32.95 & 29.16 & 32.83 & 29.30 & 44 \\
\hline 45 & 33.96 & 29.52 & 33. 83 & 29.67 & 33.70 & 29.82 & 33.57 & 29.97 & 45 \\
\hline 46 & 34.72 & 30.18 & 34.58 & 30.33 & 34.45 & 30.48 & 34.32 & 30.63 & 46 \\
\hline 47 & 35.47 & 30.83 & 35.34 & 30.99 & 35.20 & 31.14 & 35.06 & 31.30 & 47 \\
\hline 48 & 36. 23 & 31.49 & 36.09 & 31.65 & 35.95 & 31.81 & 35.81 & 31.96 & 48 \\
\hline 49 & 36.98 & 32.15 & 30.84 & 32.31 & 36.70 & 32.47 & 36.56 & 32. 63 & 49 \\
\hline 50 & 37.74 & 32.80 & 37.59 & 32.97 & 37.45 & 33.13 & 37.30 & 33. 29 & 50 \\
\hline \multirow{2}{*}{ 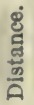 } & Dep. & Lat. & Dep. & Lat. & Dep. & Lat. & Dep. & Lat. & \multirow{2}{*}{ 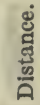 } \\
\hline & \multicolumn{2}{|c|}{$49^{\circ}$. } & \multicolumn{2}{|c|}{$4834^{\circ}$. } & \multicolumn{2}{|c|}{$4 S 1 / 2^{\circ}}$. & \multicolumn{2}{|c|}{$481 / 4^{\circ}$. } & \\
\hline
\end{tabular}


TABLE 4.-TIBAVERSE TABLE.

\begin{tabular}{|c|c|c|c|c|c|c|c|c|c|}
\hline \multirow{2}{*}{ 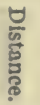 } & \multicolumn{2}{|c|}{$41^{\circ}$. } & \multicolumn{2}{|c|}{$411 / 8^{\circ}$. } & \multicolumn{2}{|c|}{$4115^{\circ}$. } & \multicolumn{2}{|c|}{$413 / 4^{\circ}$. } & \multirow{2}{*}{$\begin{array}{l}\text { అ } \\
\text { है } \\
\text { है }\end{array}$} \\
\hline & Lat. & Dep. & Lat. & Dep. & Lat. & Dep. & Lat. & Dep. & \\
\hline $\begin{array}{l}51 \\
52 \\
53 \\
54 \\
55 \\
56 \\
57 \\
58 \\
59 \\
60\end{array}$ & $\begin{array}{l}38.49 \\
39.24 \\
40.00 \\
40.75 \\
41.51 \\
42.26 \\
43.02 \\
43.77 \\
44.53 \\
45.28\end{array}$ & $\begin{array}{l}33.46 \\
34.12 \\
34.77 \\
35.43 \\
36.08 \\
36.74 \\
37.40 \\
33.05 \\
39.71 \\
39.36\end{array}$ & $\begin{array}{l}38.34 \\
39.10 \\
39.85 \\
40.60 \\
41.35 \\
42.10 \\
42.85 \\
43.61 \\
44.36 \\
45.11\end{array}$ & $\begin{array}{l}33.63 \\
34.29 \\
34.95 \\
35.60 \\
36.26 \\
36.92 \\
37.58 \\
38.24 \\
38.90 \\
39.56\end{array}$ & $\begin{array}{l}38.20 \\
38.95 \\
39.69 \\
40.44 \\
41.19 \\
41.94 \\
42.69 \\
43.44 \\
44.19 \\
44.94\end{array}$ & $\begin{array}{l}33.79 \\
34.46 \\
35.12 \\
35.78 \\
36.44 \\
37.11 \\
37.77 \\
38.43 \\
39.09 \\
39.76\end{array}$ & $\begin{array}{l}38.05 \\
38.79 \\
39.54 \\
40.29 \\
41.03 \\
41.78 \\
42.53 \\
43.27 \\
44.02 \\
44.76\end{array}$ & $\begin{array}{l}33.96 \\
34.63 \\
35.29 \\
35.96 \\
36.62 \\
37.29 \\
37.96 \\
38.62 \\
39.29 \\
39.95\end{array}$ & $\begin{array}{l}51 \\
52 \\
53 \\
54 \\
55 \\
56 \\
57 \\
58 \\
59 \\
60\end{array}$ \\
\hline $\begin{array}{l}61 \\
62 \\
63 \\
64 \\
65 \\
66 \\
67 \\
68 \\
69 \\
70\end{array}$ & $\begin{array}{l}46.04 \\
46.79 \\
47.55 \\
48.30 \\
49.06 \\
49.81 \\
50.57 \\
51.32 \\
52.07 \\
52.83\end{array}$ & $\begin{array}{l}40.02 \\
40.68 \\
41.33 \\
41.99 \\
42.64 \\
43.30 \\
43.96 \\
44.61 \\
45.27 \\
45.92\end{array}$ & $\begin{array}{l}45.86 \\
46.61 \\
47.37 \\
48.12 \\
48.87 \\
49.62 \\
50.37 \\
51.13 \\
51.88 \\
52.63\end{array}$ & $\begin{array}{l}40.22 \\
40.89 \\
41.54 \\
42.20 \\
42.86 \\
43.52 \\
44.18 \\
44.84 \\
45.49 \\
46.15\end{array}$ & $\begin{array}{l}45.69 \\
46.44 \\
47.18 \\
47.93 \\
48.68 \\
49.43 \\
50.18 \\
50.93 \\
51.68 \\
52.43\end{array}$ & $\begin{array}{l}40.42 \\
41.08 \\
41.75 \\
42.41 \\
43.07 \\
43.73 \\
44.40 \\
45.06 \\
45.72 \\
46.38\end{array}$ & $\begin{array}{l}45.51 \\
46.26 \\
47.00 \\
47.75 \\
48.49 \\
49.21 \\
49.99 \\
50.73 \\
51.48 \\
52.22\end{array}$ & $\begin{array}{l}40.62 \\
41.28 \\
41.95 \\
42.62 \\
43.28 \\
43.95 \\
44.61 \\
45.28 \\
45.95 \\
46.61\end{array}$ & $\begin{array}{l}61 \\
62 \\
63 \\
64 \\
65 \\
66 \\
67 \\
68 \\
69 \\
70\end{array}$ \\
\hline $\begin{array}{l}71 \\
72 \\
73 \\
74 \\
75 \\
76 \\
77 \\
78 \\
79 \\
80\end{array}$ & $\begin{array}{l}53.58 \\
54.34 \\
55.09 \\
55.85 \\
56.60 \\
57.36 \\
58.11 \\
58.87 \\
59.62 \\
60.38\end{array}$ & $\begin{array}{l}46.58 \\
47.24 \\
47.89 \\
48.55 \\
49.20 \\
49.86 \\
50.52 \\
51.17 \\
51.83 \\
52.48\end{array}$ & $\begin{array}{l}53.38 \\
54.13 \\
54.88 \\
55.64 \\
56.39 \\
57.14 \\
57.89 \\
58.64 \\
59.40 \\
60.15\end{array}$ & $\begin{array}{l}46.81 \\
47.47 \\
48.13 \\
48.79 \\
49.45 \\
50.11 \\
50.77 \\
51.43 \\
52.09 \\
52.75\end{array}$ & $\begin{array}{l}53.18 \\
53.92 \\
54.67 \\
55.42 \\
56.17 \\
56.92 \\
57.67 \\
58.42 \\
59.17 \\
59.92\end{array}$ & $\begin{array}{l}47.05 \\
47.71 \\
48.37 \\
49.03 \\
49.70 \\
50.36 \\
51.02 \\
51.68 \\
52.35 \\
53.01\end{array}$ & $\begin{array}{l}52.97 \\
53.72 \\
54.46 \\
55.21 \\
55.95 \\
56.70 \\
57.45 \\
58.19 \\
58.94 \\
59.68\end{array}$ & $\begin{array}{l}47.28 \\
47.91 \\
48.61 \\
49.28 \\
49.94 \\
50.61 \\
51.27 \\
51.94 \\
52.60 \\
53.27\end{array}$ & $\begin{array}{l}71 \\
72 \\
73 \\
74 \\
75 \\
76 \\
77 \\
78 \\
79 \\
80\end{array}$ \\
\hline $\begin{array}{l}81 \\
82 \\
83 \\
81 \\
85 \\
86 \\
87 \\
88 \\
89 \\
90\end{array}$ & $\begin{array}{l}61.13 \\
61.89 \\
62.64 \\
63.40 \\
64.15 \\
64.91 \\
65.66 \\
66.41 \\
67.17 \\
67.92\end{array}$ & $\begin{array}{l}53.14 \\
53.80 \\
54.45 \\
55.11 \\
55.77 \\
56.42 \\
57.08 \\
57.73 \\
58.39 \\
59.05\end{array}$ & $\begin{array}{l}60.90 \\
61.65 \\
62.40 \\
63.15 \\
63.91 \\
64.66 \\
65.41 \\
64.16 \\
66.91 \\
67.67\end{array}$ & $\begin{array}{l}53.41 \\
54.07 \\
54.73 \\
55.39 \\
56.04 \\
56.70 \\
57.36 \\
58.02 \\
58.68 \\
59.34\end{array}$ & $\begin{array}{l}60.67 \\
61.41 \\
62.16 \\
62.91 \\
63.66 \\
64.41 \\
65.16 \\
65.91 \\
66.66 \\
67.41\end{array}$ & $\begin{array}{l}53.67 \\
54.33 \\
55.00 \\
55.66 \\
56.32 \\
56.99 \\
57.65 \\
58.31 \\
58.97 \\
59.64\end{array}$ & $\begin{array}{l}60.43 \\
61.18 \\
61.92 \\
62.67 \\
63.41 \\
64.16 \\
64.91 \\
65.65 \\
66.40 \\
67.15\end{array}$ & $\begin{array}{l}53.94 \\
54.60 \\
55.27 \\
55.93 \\
56.60 \\
57.27 \\
57.93 \\
58.10 \\
59.26 \\
59.93\end{array}$ & $\begin{array}{l}81 \\
82 \\
83 \\
84 \\
85 \\
86 \\
87 \\
58 \\
89 \\
90\end{array}$ \\
\hline $\begin{array}{r}91 \\
92 \\
93 \\
94 \\
95 \\
96 \\
97 \\
98 \\
99 \\
100\end{array}$ & $\begin{array}{l}68.68 \\
69.43 \\
70.19 \\
70.94 \\
71.70 \\
72.45 \\
73.21 \\
73.96 \\
74.72 \\
75.47\end{array}$ & $\begin{array}{l}59.70 \\
60.36 \\
61.01 \\
61.67 \\
62.33 \\
62.08 \\
63.64 \\
64.29 \\
64.95 \\
65.61\end{array}$ & $\begin{array}{l}68.42 \\
69.17 \\
69.92 \\
70.67 \\
71.43 \\
72.18 \\
72.93 \\
73.68 \\
74.43 \\
75.18\end{array}$ & $\begin{array}{l}60.00 \\
60.66 \\
61.32 \\
61.93 \\
62.64 \\
63.30 \\
63.96 \\
64.62 \\
65.23 \\
65.03\end{array}$ & $\begin{array}{l}68.15 \\
68.90 \\
69.65 \\
70.40 \\
71.15 \\
71.90 \\
72.65 \\
73.40 \\
74.15 \\
74.90\end{array}$ & $\begin{array}{l}60.30 \\
60.96 \\
91.62 \\
62.29 \\
62.95 \\
63.61 \\
64.27 \\
64.94 \\
65.10 \\
66.26\end{array}$ & $\begin{array}{l}67.89 \\
68.64 \\
69.38 \\
70.13 \\
70.88 \\
71.62 \\
72.37 \\
73.11 \\
73.813 \\
74.61\end{array}$ & $\begin{array}{l}60.60 \\
61.26 \\
61.93 \\
6.2 .59 \\
63.26 \\
63.92 \\
64.59 \\
65.20 \\
65.93 \\
66.59\end{array}$ & $\begin{array}{r}91 \\
92 \\
93 \\
94 \\
05 \\
96 \\
97 \\
98 \\
99 \\
100\end{array}$ \\
\hline \multirow{2}{*}{ 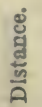 } & Dep. & Lat. & Dep. & Lat. & Dep. & Lat. & Dep. & Iat. & \multirow{2}{*}{$\begin{array}{l}\text { ¿ूँ } \\
\frac{3}{0} \\
\frac{5}{0}\end{array}$} \\
\hline & \multicolumn{2}{|c|}{$49^{\circ}$} & \multicolumn{2}{|c|}{$4834^{\circ}$. } & \multicolumn{2}{|c|}{$4832^{\circ}$. } & \multicolumn{2}{|c|}{$4514^{\circ}$. } & \\
\hline
\end{tabular}


TABLE 4.-TRAVERSE TABLE.

\begin{tabular}{|c|c|c|c|c|c|c|c|c|c|}
\hline \multirow{2}{*}{ 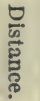 } & \multicolumn{2}{|c|}{$42^{\circ}$. } & \multicolumn{2}{|c|}{$421 / 4^{\circ}$. } & \multicolumn{2}{|c|}{$421 / 2^{\circ}$. } & \multicolumn{2}{|c|}{$4234^{\circ}$. } & \multirow{2}{*}{ 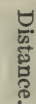 } \\
\hline & Lat. & Dep. & Lat. & Dep. & Lat. & Dep. & Lat. & Dep. & \\
\hline $\begin{array}{r}1 \\
2 \\
3 \\
4 \\
5 \\
6 \\
7 \\
8 \\
9 \\
10\end{array}$ & $\begin{array}{l}0.74 \\
1.49 \\
2.23 \\
2.97 \\
3.72 \\
4.46 \\
5.20 \\
5.95 \\
6.69 \\
7.43\end{array}$ & $\begin{array}{l}0.67 \\
1.34 \\
2.01 \\
2.68 \\
3.35 \\
4.01 \\
4.68 \\
5.35 \\
6.02 \\
6.69\end{array}$ & $\begin{array}{l}0.74 \\
1.48 \\
2.22 \\
2.96 \\
3.70 \\
4.44 \\
5.18 \\
5.92 \\
6.66 \\
7.40\end{array}$ & $\begin{array}{l}0.67 \\
1.34 \\
2.02 \\
2.69 \\
3.36 \\
4.03 \\
4.71 \\
5.38 \\
6.05 \\
6.72\end{array}$ & $\begin{array}{l}0.74 \\
1.47 \\
2.21 \\
2.95 \\
3.69 \\
4.42 \\
5.16 \\
5.90 \\
6.64 \\
7.37\end{array}$ & $\begin{array}{l}0.68 \\
1.35 \\
2.03 \\
2.70 \\
3.38 \\
4.05 \\
4.73 \\
5.40 \\
6.08 \\
6.76\end{array}$ & $\begin{array}{l}0.73 \\
1.47 \\
2.20 \\
2.94 \\
3.67 \\
4.41 \\
5.14 \\
5.87 \\
6.61 \\
7.34\end{array}$ & $\begin{array}{l}0.68 \\
1.36 \\
2.04 \\
2.72 \\
3.39 \\
4.07 \\
4.75 \\
5.43 \\
6.11 \\
6.79\end{array}$ & $\begin{array}{r}1 \\
2 \\
3 \\
4 \\
5 \\
6 \\
7 \\
8 \\
9 \\
10\end{array}$ \\
\hline $\begin{array}{l}11 \\
12 \\
13 \\
14 \\
15 \\
16 \\
17 \\
18 \\
19 \\
20\end{array}$ & $\begin{array}{r}8.17 \\
8.92 \\
9.66 \\
10.40 \\
11.15 \\
11.89 \\
12.63 \\
13.38 \\
14.12 \\
14.86\end{array}$ & $\begin{array}{r}7.36 \\
8.03 \\
8.70 \\
9.37 \\
10.04 \\
10.71 \\
11.38 \\
12.04 \\
12.71 \\
13.38\end{array}$ & $\begin{array}{r}8.14 \\
8.88 \\
9.62 \\
10.36 \\
11.10 \\
11.84 \\
12.55 \\
13.32 \\
14.06 \\
14.80\end{array}$ & $\begin{array}{r}7.40 \\
8.07 \\
8.74 \\
9.41 \\
10.09 \\
10.76 \\
11.43 \\
12.10 \\
12.77 \\
13.45\end{array}$ & $\begin{array}{r}8.11 \\
8.85 \\
9.55 \\
10.32 \\
11.06 \\
11.80 \\
12.53 \\
13.27 \\
14.01 \\
14.75\end{array}$ & $\begin{array}{r}7.43 \\
8.11 \\
8.78 \\
9.46 \\
10.13 \\
10.81 \\
11.49 \\
12.16 \\
12.84 \\
13.51\end{array}$ & $\begin{array}{r}8.08 \\
8.81 \\
9.55 \\
10.28 \\
11.01 \\
11.75 \\
12.48 \\
13.22 \\
13.95 \\
14.69\end{array}$ & $\begin{array}{r}7.47 \\
8.15 \\
8.82 \\
9.50 \\
10.18 \\
10.86 \\
11.54 \\
12.22 \\
12.90 \\
13.58\end{array}$ & $\begin{array}{l}11 \\
12 \\
13 \\
14 \\
15 \\
16 \\
17 \\
18 \\
19 \\
20\end{array}$ \\
\hline $\begin{array}{l}21 \\
22 \\
23 \\
24 \\
25 \\
26 \\
27 \\
28 \\
29 \\
30\end{array}$ & $\begin{array}{l}15.61 \\
16.35 \\
17.09 \\
17.84 \\
18.58 \\
19.32 \\
20.06 \\
20.81 \\
21.55 \\
22.29\end{array}$ & $\begin{array}{l}14.05 \\
14.72 \\
15.39 \\
16.06 \\
16.73 \\
17.40 \\
18.07 \\
18.74 \\
19.40 \\
20.07\end{array}$ & $\begin{array}{l}15.54 \\
16.28 \\
17.03 \\
17.77 \\
18.51 \\
19.25 \\
19.99 \\
20.73 \\
21.47 \\
22.21\end{array}$ & $\begin{array}{l}14.12 \\
14.79 \\
15.46 \\
16.14 \\
16.81 \\
17.48 \\
18.15 \\
18.83 \\
19.50 \\
20.17\end{array}$ & $\begin{array}{l}15.48 \\
16.22 \\
16.96 \\
17.69 \\
18.43 \\
19.17 \\
19.91 \\
20.64 \\
21.38 \\
22.12\end{array}$ & $\begin{array}{l}14.19 \\
14.86 \\
15.54 \\
16.21 \\
16.89 \\
17.57 \\
18.24 \\
18.92 \\
19.59 \\
20.27\end{array}$ & $\begin{array}{l}15.42 \\
16.16 \\
16.89 \\
17.62 \\
18.36 \\
19.09 \\
19.83 \\
20.56 \\
21.30 \\
22.03\end{array}$ & $\begin{array}{l}14.25 \\
14.93 \\
15.61 \\
16.29 \\
16.97 \\
17.65 \\
18.33 \\
19.01 \\
19.69 \\
20.36\end{array}$ & $\begin{array}{l}21 \\
22 \\
23 \\
24 \\
25 \\
26 \\
27 \\
28 \\
29 \\
30\end{array}$ \\
\hline $\begin{array}{l}31 \\
32 \\
33 \\
34 \\
35 \\
36 \\
37 \\
38 \\
39 \\
40\end{array}$ & $\begin{array}{l}23.04 \\
23.78 \\
24.52 \\
25.27 \\
26.01 \\
26.75 \\
27.50 \\
28.24 \\
28.98 \\
29.73\end{array}$ & $\begin{array}{l}20.74 \\
21.41 \\
22.08 \\
22.75 \\
23.42 \\
24.09 \\
24.76 \\
25.43 \\
26.10 \\
26.77\end{array}$ & $\begin{array}{l}22.95 \\
23.19 \\
24.43 \\
25.17 \\
25.91 \\
26.65 \\
27.39 \\
28.13 \\
28.87 \\
29.61\end{array}$ & $\begin{array}{l}20.84 \\
21.52 \\
22.19 \\
22.86 \\
23.53 \\
24.21 \\
24.88 \\
25.55 \\
26.22 \\
26.89\end{array}$ & $\begin{array}{l}22.86 \\
23.59 \\
24.33 \\
25.07 \\
25.80 \\
26.54 \\
27.28 \\
28.02 \\
28.75 \\
29.49\end{array}$ & $\begin{array}{l}20.94 \\
21.62 \\
22.29 \\
22.97 \\
23.65 \\
24.32 \\
25.00 \\
25.67 \\
26.35 \\
27.02\end{array}$ & $\begin{array}{l}22.76 \\
23.50 \\
24.23 \\
24.97 \\
25.70 \\
26.44 \\
27.17 \\
27.90 \\
28.64 \\
29.37\end{array}$ & $\begin{array}{l}21.04 \\
21.72 \\
22.40 \\
23.08 \\
23.76 \\
24.44 \\
25.12 \\
25.79 \\
26.47 \\
27.15\end{array}$ & $\begin{array}{l}31 \\
32 \\
33 \\
34 \\
35 \\
36 \\
37 \\
38 \\
39 \\
40\end{array}$ \\
\hline $\begin{array}{l}41 \\
42 \\
43 \\
44 \\
45 \\
46 \\
47 \\
48 \\
49 \\
50\end{array}$ & $\begin{array}{l}30.47 \\
31.21 \\
31.96 \\
32.70 \\
33.44 \\
34.18 \\
34.93 \\
3.5 .67 \\
36.41 \\
37.16\end{array}$ & $\begin{array}{l}27.43 \\
28.10 \\
28.77 \\
29.44 \\
30.11 \\
30.78 \\
31.45 \\
32.12 \\
32.79 \\
33.46\end{array}$ & $\begin{array}{l}30.35 \\
31.09 \\
31.83 \\
32.57 \\
33.31 \\
34.05 \\
34.79 \\
35.53 \\
36.27 \\
37.01\end{array}$ & $\begin{array}{l}27.57 \\
23.24 \\
28.91 \\
29.58 \\
30.26 \\
30.93 \\
31.60 \\
32.27 \\
32.95 \\
33.62\end{array}$ & $\begin{array}{l}30.23 \\
30.97 \\
31.70 \\
32.44 \\
33.18 \\
33.91 \\
34.65 \\
35.39 \\
36.13 \\
36.86\end{array}$ & $\begin{array}{l}27.70 \\
23.37 \\
29.05 \\
29.73 \\
30.40 \\
31.08 \\
31.75 \\
32.43 \\
33.10 \\
33.78\end{array}$ & $\begin{array}{l}30.11 \\
30.84 \\
31.58 \\
32.31 \\
33.04 \\
33.78 \\
34.51 \\
35.25 \\
35.98 \\
36.72\end{array}$ & $\begin{array}{l}27.83 \\
28.51 \\
29.19 \\
29.87 \\
30.55 \\
31.22 \\
31.90 \\
32.58 \\
33.26 \\
33.94\end{array}$ & $\begin{array}{l}41 \\
42 \\
43 \\
44 \\
45 \\
46 \\
47 \\
48 \\
49 \\
50\end{array}$ \\
\hline 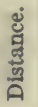 & Dep. & Lat. & Dep. & Lat. & Dep. & Lat. & Dep. & Lat. & 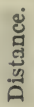 \\
\hline
\end{tabular}


TABIA 4.-TRAVERSE TABLE.

\begin{tabular}{|c|c|c|c|c|c|c|c|c|c|}
\hline \multirow{2}{*}{ 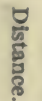 } & \multicolumn{2}{|c|}{$42^{\circ}$} & \multicolumn{2}{|c|}{$421 / 4^{\circ}$. } & \multicolumn{2}{|c|}{$421 / 2^{\circ}$. } & \multicolumn{2}{|c|}{$4234^{\circ}$. } & \multirow{2}{*}{ 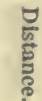 } \\
\hline & Lat. & Dep. & Lat. & Dep. & Lat. & Dep. & Lat. & Dep. & \\
\hline 51 & 37.90 & 34.13 & 37.75 & 34,29 & 37.00 & 34.46 & 37.45 & 34,62 & 51 \\
\hline 52 & 38.64 & 34.79 & 38.49 & 34.96 & 38.34 & 35.13 & 38 . is & 35.30 & 52 \\
\hline 53 & 39. 39 & 35.46 & 39.23 & 35.64 & 39.08 & 35.81 & 38.92 & 35.98 & 53 \\
\hline 54 & 40.13 & 36.13 & 39.97 & 36.31 & 39.81 & 36.48 & 39.65 & 36.66 & 54 \\
\hline 55 & 40.87 & 36.80 & 40.71 & 36.98 & 40.55 & 37.16 & 40.39 & 37.23 & 55 \\
\hline 56 & 41.62 & 37.47 & 41.45 & 37.65 & 41.29 & 37.83 & 41.12 & 38.01 & 56 \\
\hline 57 & 42.36 & 38.14 & 42.19 & 38.32 & 42.02 & 38.51 & 41.86 & 38.69 & 57 \\
\hline 58 & 43. 10 & 38.81 & 42.93 & 39.00 & 42.76 & 39.18 & 42.59 & 39.37 & 58 \\
\hline 59 & 43.85 & 39.48 & 43. 67 & 39.67 & 43.50 & 39.86 & 43. 33 & 40.05 & 59 \\
\hline 60 & 44.59 & 40.15 & 44.41 & 40.34 & 44.24 & 40.54 & 44.06 & 40.73 & 60 \\
\hline 61 & 45.33 & 40.82 & 45.15 & 41.01 & 44.97 & 41.21 & 44. 79 & 41.41 & 61 \\
\hline 62 & 46.07 & 41.49 & 45. 89 & 41.69 & 45.71 & 41.89 & 45. 53 & 42.09 & 62 \\
\hline 63 & 46.82 & $4^{2} .16$ & 46. 63 & 42.36 & 46.45 & 42.56 & 46.26 & 42.76 & 63 \\
\hline 64 & $47.5 k$ & 42.82 & 47.37 & 43.03 & 47.19 & 43. 24 & 47.00 & 43.44 & 64 \\
\hline 65 & 48.30 & 43. 49 & 48.11 & 43.70 & 47.92 & 43.91 & 47.73 & 44.12 & 65 \\
\hline 66 & 49.05 & 44.16 & 48. 85 & 44. 38 & 48.66 & 44. 59 & 48.47 & 44.80 & 66 \\
\hline 67 & 49.79 & 44.83 & 49.59 & 45.05 & 49.40 & 45.26 & 49.20 & 45.48 & 67 \\
\hline (is & 50.53 & 45. 50 & 50.33 & 45.72 & 50.13 & 45.94 & 49.93 & 46.16 & 68 \\
\hline 69 & 51.28 & 46. 17 & 51.08 & 46.39 & 50.87 & 46.62 & 50.67 & 46.84 & 69 \\
\hline 70 & 52.02 & 46.84 & 51.82 & 47.07 & 51.61 & $\begin{array}{l}40.02 \\
47.29\end{array}$ & 51.40 & $\begin{array}{l}40.87 \\
47.52\end{array}$ & 70 \\
\hline 71 & 52.76 & 47.51 & 52.56 & 47.74 & 52.35 & 47.97 & 52.14 & 48.19 & 71 \\
\hline 72 & 53.51 & 48.18 & 53.30 & 48.41 & 53.08 & 48.64 & 52.87 & 48.87 & 72 \\
\hline 73 & 54.25 & 48.85 & 54.04 & 49.08 & 53.82 & 49. 32 & 53.61 & 49.55 & 73 \\
\hline 74 & 54.99 & $\begin{array}{l}49.52 \\
\end{array}$ & 54.78 & 49. 76 & 54.56 & 49.99 & 54.34 & 50.23 & 74 \\
\hline 75 & 55.74 & 50.18 & 55.52 & 50.43 & 55.30 & 50.67 & 55.07 & 50.91 & 75 \\
\hline 76 & 56.48 & 50.85 & 56.26 & 51.10 & 56.03 & 51.34 & 55.81 & 51.59 & 76 \\
\hline 77 & 57.22 & 51.52 & 57.00 & 51.77 & 56.77 & 52.02 & 56.54 & 52.27 & 77 \\
\hline 78 & 57.97 & 52.19 & 57.74 & 52.44 & 57.51 & 52.70 & 57.28 & 52.95 & 78 \\
\hline 79 & 58.71 & 52.866 & 58. 48 & 53.12 & 58.24 & 53.37 & 58.01 & 53.63 & 79 \\
\hline 80 & 59.45 & 53.53 & 59.22 & 53.79 & 58.98 & 54.05 & 58.75 & 54.30 & 80 \\
\hline 81 & 60.19 & 54.20 & 59.96 & 54.46 & 59.72 & 54.72 & 59.48 & 54.98 & 81 \\
\hline 82 & 60.94 & 54.87 & 60.70 & 55.13 & 60.46 & 55.40 & 60.21 & 55.66 & 82 \\
\hline 83 & 61. es & 55. 54 & 61.41 & 55.81 & 61.19 & 56.07 & 60.95 & 56.34 & 83 \\
\hline 84 & 62.42 & 56.21 & 62.18 & 56.48 & 61.93 & 56.75 & 61.68 & 57.02 & 84 \\
\hline 85 & 63.17 & 56.88 & 62.92 & 57.15 & 62.67 & 57,43 & 62.42 & 57.70 & 85 \\
\hline S6 & 63.91 & 57.55 & 63.66 & 57.82 & 63.41 & 58.10 & 63.15 & 58.38 & 86 \\
\hline 87 & 64.65 & 58.21 & 64.40 & 58.50 & 64.14 & 58.78 & 63.89 & 59.06 & 87 \\
\hline 88 & 65.40 & 58. 83 & 65.14 & 59.17 & 64.88 & 59.45 & 64.62 & 59.73 & 89 \\
\hline 89 & 66.14 & 59.55 & 65.58 & 59.84 & 65.62 & 60.13 & 65.35 & 60.41 & 89 \\
\hline 90 & 66. 88 & 60.22 & 66.62 & 60.51 & 66.35 & 60.80 & 66.09 & 61.09 & $\infty$ \\
\hline 91 & 67.63 & 60.89 & 67.36 & 61.19 & 67.09 & 61.48 & 66.82 & 61.77 & 91 \\
\hline 92 & GS. 37 & 61.56 & 68.10 & 61.86 & 67.83 & 62.15 & 67.56 & 62.45 & 92 \\
\hline 93 & 69.11 & 62.23 & 68.84 & 62.53 & 68.57 & 62.83 & 68. 29 & 63.13 & 93 \\
\hline 94 & 69.86 & 62.90 & 69.58 & 63.20 & 69.30 & 63.51 & 69.03 & 63. 81 & 94 \\
\hline 95 & 70.60 & 63.57 & 70.32 & 63.87 & 70.04 & 64.18 & 69.76 & 64.49 & 95 \\
\hline 96 & 71.34 & 64.24 & 71.06 & 64. 55 & 70.78 & 64.86 & 70.49 & 65.16 & 96 \\
\hline 97 & 72.09 & 64.91 & 71.80 & 65.22 & 71.52 & 65.53 & 71.23 & 65.84 & 97 \\
\hline 98 & 72.83 & 65.57 & 72.54 & 65.89 & 72.25 & 66. 21 & 71.06 & 66.52 & 98 \\
\hline 99 & 73.57 & 66.24 & 73.28 & 66.56 & 72.99 & 66.88 & 72.70 & 67.20 & 99 \\
\hline 100 & 74.31 & 66.91 & 74.02 & 67.24 & 73.73 & 67.56 & 73.43 & 67.88 & 100 \\
\hline \multirow{2}{*}{ 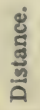 } & Dep. & Lat. & Dep. & Lat. & Dep. & Lat. & Dep. & Lat. & \multirow{2}{*}{ 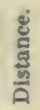 } \\
\hline & \multicolumn{2}{|c|}{$48^{\circ}}$. & \multicolumn{2}{|c|}{$47^{3} 4^{\circ}$} & \multicolumn{2}{|c|}{$47 L_{2}^{\circ}}$. & \multicolumn{2}{|c|}{$471 / 4^{\circ}}$. & \\
\hline
\end{tabular}


TABLE 4.-TRAVERSE TABLE.

\begin{tabular}{|c|c|c|c|c|c|c|c|c|c|}
\hline \multirow{2}{*}{ 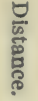 } & \multicolumn{2}{|c|}{$43^{\circ}$} & \multicolumn{2}{|c|}{$431 / 4^{\circ}$. } & \multicolumn{2}{|c|}{$431 / 2^{\circ}$. } & \multicolumn{2}{|c|}{$4334^{\circ}$. } & \multirow{2}{*}{ 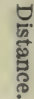 } \\
\hline & Lat. & Dep. & Lat. & Dep. & Lat. & Dep. & Lat. & Dep. & \\
\hline $\begin{array}{r}1 \\
2 \\
3 \\
4 \\
5 \\
6 \\
7 \\
8 \\
9 \\
10\end{array}$ & $\begin{array}{l}0.73 \\
1.46 \\
2.19 \\
2.93 \\
3.66 \\
4.39 \\
5.12 \\
5.85 \\
6.58 \\
7.31\end{array}$ & $\begin{array}{r}0.68 \\
1.36 \\
2.05 \\
2.73 \\
3.41 \\
4.09 \\
4.77 \\
5.46 \\
6.14 \\
6.82\end{array}$ & $\begin{array}{l}0.73 \\
1.46 \\
2.19 \\
2.91 \\
3.64 \\
4.37 \\
5.10 \\
5.83 \\
6.56 \\
7.28\end{array}$ & $\begin{array}{l}0.69 \\
1.37 \\
2.06 \\
2.74 \\
3.43 \\
4.11 \\
4.80 \\
5.48 \\
6.17 \\
6.85\end{array}$ & $\begin{array}{l}0.73 \\
1.45 \\
2.18 \\
2.90 \\
3.63 \\
4.35 \\
5.08 \\
5.80 \\
6.53 \\
7.25\end{array}$ & $\begin{array}{l}0.69 \\
1.38 \\
2.07 \\
2.75 \\
3.44 \\
4.13 \\
4.82 \\
5.51 \\
6.20 \\
6.88\end{array}$ & $\begin{array}{l}0.72 \\
1.44 \\
2.17 \\
2.89 \\
3.61 \\
4.33 \\
5.06 \\
5.78 \\
6.50 \\
7.22\end{array}$ & $\begin{array}{l}0.69 \\
1.38 \\
2.07 \\
2.77 \\
3.46 \\
4.15 \\
4.84 \\
5.53 \\
6.22 \\
6.92\end{array}$ & $\begin{array}{r}1 \\
2 \\
3 \\
4 \\
5 \\
6 \\
7 \\
8 \\
9 \\
10\end{array}$ \\
\hline $\begin{array}{l}11 \\
12 \\
13 \\
14 \\
15 \\
16 \\
17 \\
18 \\
19 \\
20\end{array}$ & $\begin{array}{r}8.04 \\
8.78 \\
9.51 \\
10.24 \\
10.97 \\
11.70 \\
12.43 \\
13.16 \\
13.90 \\
14.63\end{array}$ & $\begin{array}{r}7.50 \\
8.18 \\
8.87 \\
9.55 \\
10.23 \\
10.91 \\
11.59 \\
12.28 \\
12.96 \\
13.64\end{array}$ & $\begin{array}{r}8.01 \\
8.74 \\
9.47 \\
10.20 \\
10.93 \\
11.65 \\
12.38 \\
13.11 \\
13.84 \\
14.57\end{array}$ & $\begin{array}{r}7.54 \\
8.22 \\
8.91 \\
9.59 \\
10.28 \\
10.96 \\
11.65 \\
12.33 \\
13.02 \\
13.70\end{array}$ & $\begin{array}{r}7.98 \\
8.70 \\
9.43 \\
10.16 \\
10.88 \\
11.61 \\
12.33 \\
13.06 \\
13.78 \\
14.51\end{array}$ & $\begin{array}{r}7.57 \\
8.26 \\
8.95 \\
9.64 \\
10.33 \\
11.01 \\
11.70 \\
12.39 \\
13.08 \\
13.77\end{array}$ & $\begin{array}{r}7.95 \\
8.67 \\
9.39 \\
10.11 \\
10.84 \\
11.56 \\
12.28 \\
13.00 \\
13.72 \\
14.45\end{array}$ & $\begin{array}{r}7.61 \\
3.30 \\
8.99 \\
9.68 \\
10.37 \\
11.06 \\
11.76 \\
12.45 \\
13.14 \\
13.83\end{array}$ & $\begin{array}{l}11 \\
12 \\
13 \\
14 \\
15 \\
16 \\
17 \\
18 \\
19 \\
20\end{array}$ \\
\hline $\begin{array}{l}21 \\
22 \\
23 \\
24 \\
25 \\
26 \\
27 \\
28 \\
29 \\
20\end{array}$ & $\begin{array}{l}15.36 \\
16.09 \\
16.82 \\
17.55 \\
18.28 \\
19.02 \\
19.75 \\
20.48 \\
21.21 \\
21.94\end{array}$ & $\begin{array}{l}14.32 \\
15.00 \\
15.69 \\
16.37 \\
17.05 \\
17.73 \\
18.41 \\
19.10 \\
19.78 \\
20.46\end{array}$ & $\begin{array}{l}15.30 \\
16.02 \\
16.75 \\
17.48 \\
18.21 \\
18.94 \\
19.67 \\
20.39 \\
21.12 \\
21.85\end{array}$ & $\begin{array}{l}14.39 \\
15.07 \\
15.76 \\
16.44 \\
17.13 \\
17.81 \\
18.50 \\
19.19 \\
19.87 \\
20.56\end{array}$ & $\begin{array}{l}15.23 \\
15.96 \\
16.68 \\
17.41 \\
18.13 \\
18.86 \\
19.59 \\
20.31 \\
21.04 \\
21.76\end{array}$ & $\begin{array}{l}14.46 \\
15.14 \\
15.83 \\
16.52 \\
17.21 \\
17.90 \\
18.59 \\
19.27 \\
19.96 \\
20.65\end{array}$ & $\begin{array}{l}15.17 \\
15.89 \\
16.51 \\
17.34 \\
18.06 \\
18.78 \\
19.50 \\
20.23 \\
20.95 \\
21.67\end{array}$ & $\begin{array}{l}14.52 \\
15.21 \\
15.90 \\
16.60 \\
17.29 \\
17.98 \\
18.67 \\
19.36 \\
20.05 \\
20.75\end{array}$ & $\begin{array}{l}21 \\
22 \\
23 \\
24 \\
25 \\
26 \\
27 \\
28 \\
29 \\
30\end{array}$ \\
\hline $\begin{array}{l}31 \\
32 \\
33 \\
34 \\
35 \\
31 \\
37 \\
38 \\
39 \\
40\end{array}$ & $\begin{array}{l}22.67 \\
23.40 \\
24.13 \\
24.87 \\
25.10 \\
26.33 \\
27.06 \\
27.79 \\
28.52 \\
29.25\end{array}$ & $\begin{array}{l}21.14 \\
21.82 \\
22.51 \\
23.19 \\
23.87 \\
24.55 \\
25.23 \\
25.92 \\
26.60 \\
27.28\end{array}$ & $\begin{array}{l}22.58 \\
23.31 \\
24.04 \\
21.76 \\
25.49 \\
26.22 \\
26.95 \\
27.68 \\
28.41 \\
29.13\end{array}$ & $\begin{array}{l}21.24 \\
21.93 \\
22.61 \\
23.30 \\
23.98 \\
24.67 \\
25.35 \\
26.04 \\
26.72 \\
27.41\end{array}$ & $\begin{array}{l}22.49 \\
23.21 \\
23.94 \\
24.66 \\
25.39 \\
26.11 \\
26.84 \\
27.56 \\
28.29 \\
29.01\end{array}$ & $\begin{array}{l}21.34 \\
22.03 \\
22.72 \\
23.40 \\
24.09 \\
24.78 \\
25.47 \\
26.16 \\
26.85 \\
27.53\end{array}$ & $\begin{array}{l}22.39 \\
23.12 \\
23.84 \\
24.56 \\
25.28 \\
26.01 \\
26.73 \\
27.45 \\
28.17 \\
28.89\end{array}$ & $\begin{array}{l}21.44 \\
22.13 \\
22.82 \\
23.51 \\
24.20 \\
24.89 \\
25.59 \\
26.28 \\
26.97 \\
27.66\end{array}$ & $\begin{array}{l}31 \\
32 \\
33 \\
34 \\
35 \\
36 \\
37 \\
38 \\
39 \\
40\end{array}$ \\
\hline $\begin{array}{l}41 \\
42 \\
43 \\
44 \\
45 \\
46 \\
47 \\
49 \\
49 \\
50\end{array}$ & $\begin{array}{l}29.99 \\
30.72 \\
31.45 \\
32.18 \\
32.91 \\
33 .(14 \\
34.37 \\
35.10 \\
35.84 \\
36.57\end{array}$ & $\begin{array}{l}27.96 \\
28.64 \\
29.33 \\
30.01 \\
30.69 \\
31.37 \\
32.05 \\
32.74 \\
33.42 \\
34.10\end{array}$ & $\begin{array}{l}29.86 \\
30.59 \\
31.32 \\
32.05 \\
32.78 \\
33.51 \\
34.23 \\
34.96 \\
35.69 \\
36.42\end{array}$ & $\begin{array}{l}28.09 \\
28.78 \\
29.46 \\
30.15 \\
30.83 \\
31.52 \\
32.20 \\
32.89 \\
33.57 \\
34.26\end{array}$ & $\begin{array}{l}29.74 \\
30.47 \\
31.19 \\
31.92 \\
32.64 \\
33.37 \\
34.09 \\
34.82 \\
35.54 \\
36.27\end{array}$ & $\begin{array}{l}28.22 \\
28.91 \\
29.60 \\
30.29 \\
30.98 \\
31.66 \\
32.35 \\
33.04 \\
33.73 \\
34.42\end{array}$ & $\begin{array}{l}29.62 \\
30.34 \\
31.06 \\
31.78 \\
32.51 \\
33.23 \\
33.9 .5 \\
34.67 \\
35.40 \\
36.12\end{array}$ & $\begin{array}{l}25.35 \\
29.04 \\
29.74 \\
30.43 \\
31.12 \\
31.81 \\
32.50 \\
33.19 \\
33.88 \\
34.58\end{array}$ & $\begin{array}{l}41 \\
42 \\
43 \\
44 \\
45 \\
46 \\
47 \\
48 \\
49 \\
50\end{array}$ \\
\hline छू & Dep. & Lat. & Dep. & Lat. & Dep. & Iat. & Dep. & Lat. & ठํ \\
\hline$\frac{m}{a}$ & \multicolumn{2}{|c|}{$47^{\circ}$. } & \multicolumn{2}{|c|}{$4634^{\circ}$. } & \multicolumn{2}{|c|}{$461 / 2^{\circ}$. } & \multicolumn{2}{|c|}{$461 / 4^{\circ}$. } & A \\
\hline
\end{tabular}


TABLE 4.-TRAVERSE TABLE.

\begin{tabular}{|c|c|c|c|c|c|c|c|c|c|}
\hline \multirow{2}{*}{ 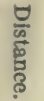 } & \multicolumn{2}{|c|}{$43^{\circ}$. } & \multicolumn{2}{|c|}{$431 / 4^{\circ}$. } & \multicolumn{2}{|c|}{$431 / 2^{\circ}$. } & \multicolumn{2}{|c|}{$433 / s^{\circ}$. } & \multirow{2}{*}{ 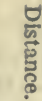 } \\
\hline & Lat. & Dep: & Lat. & Dep. & Lat. & Dep. & Lat. & Dep. & \\
\hline $\begin{array}{l}51 \\
52 \\
53 \\
54 \\
55 \\
56 \\
57 \\
55 \\
59 \\
60\end{array}$ & $\begin{array}{l}37.30 \\
38.03 \\
38.76 \\
39.49 \\
40.22 \\
40.96 \\
41.69 \\
42.42 \\
43.15 \\
43.88\end{array}$ & $\begin{array}{l}34.78 \\
35.46 \\
36.15 \\
36.83 \\
37.51 \\
38.19 \\
38.37 \\
39.56 \\
40.24 \\
40.92\end{array}$ & $\begin{array}{l}37.15 \\
37.88 \\
38.60 \\
39.33 \\
40.06 \\
40.79 \\
41.52 \\
42.25 \\
42.97 \\
43.70\end{array}$ & $\begin{array}{l}34.94 \\
35.63 \\
36.31 \\
37.00 \\
37.69 \\
38.37 \\
39.06 \\
39.74 \\
40.43 \\
41.11\end{array}$ & $\begin{array}{l}36.99 \\
37.72 \\
33.44 \\
39.17 \\
39.90 \\
40.62 \\
41.35 \\
42.07 \\
42.80 \\
43.52\end{array}$ & $\begin{array}{l}35.11 \\
35.79 \\
36.48 \\
37.17 \\
37.86 \\
38.55 \\
39.24 \\
39.92 \\
40.61 \\
41.30\end{array}$ & $\begin{array}{l}36.84 \\
37.56 \\
38.29 \\
39.01 \\
39.73 \\
40.45 \\
41.17 \\
41.90 \\
42.62 \\
43.34\end{array}$ & $\begin{array}{l}35.27 \\
35.96 \\
36.65 \\
37.34 \\
38.03 \\
38.72 \\
39.42 \\
40.11 \\
40.80 \\
41.49\end{array}$ & $\begin{array}{l}51 \\
52 \\
53 \\
54 \\
55 \\
56 \\
57 \\
58 \\
59 \\
60\end{array}$ \\
\hline $\begin{array}{l}61 \\
62 \\
63 \\
64 \\
65 \\
66 \\
67 \\
68 \\
69 \\
70\end{array}$ & $\begin{array}{l}44.61 \\
45.34 \\
46.08 \\
46.81 \\
47.54 \\
48.27 \\
49.00 \\
49.73 \\
50.46 \\
51.19\end{array}$ & $\begin{array}{r}41.60 \\
42.28 \\
42.97 \\
43.65 \\
44.33 \\
45.01 \\
45.69 \\
46.38 \\
47.06 \\
47.74\end{array}$ & $\begin{array}{l}44.43 \\
45.16 \\
45.89 \\
46.62 \\
47.34 \\
48.07 \\
48.80 \\
49.53 \\
50.26 \\
50.99\end{array}$ & $\begin{array}{l}41.80 \\
42.48 \\
43.17 \\
43.85 \\
44.54 \\
45.22 \\
45.91 \\
46.59 \\
47.28 \\
47.96\end{array}$ & $\begin{array}{l}44.25 \\
44.97 \\
45.70 \\
46.42 \\
47.15 \\
47.87 \\
48.60 \\
49.33 \\
50.05 \\
50.78\end{array}$ & $\begin{array}{l}41.99 \\
42.68 \\
43.37 \\
44.05 \\
44.74 \\
45.43 \\
46.12 \\
46.81 \\
47.50 \\
43.18\end{array}$ & $\begin{array}{l}44.06 \\
44.79 \\
45.51 \\
46.23 \\
46.95 \\
47.65 \\
48.40 \\
49.12 \\
49.84 \\
50.57\end{array}$ & $\begin{array}{l}42.18 \\
42.87 \\
43.57 \\
44.26 \\
44.95 \\
45.64 \\
46.33 \\
47.02 \\
47.71 \\
48.41\end{array}$ & $\begin{array}{l}61 \\
62 \\
63 \\
64 \\
65 \\
60 \\
67 \\
68 \\
69 \\
70\end{array}$ \\
\hline $\begin{array}{l}71 \\
72 \\
73 \\
74 \\
75 \\
76 \\
77 \\
78 \\
79 \\
80\end{array}$ & $\begin{array}{l}51.93 \\
52.66 \\
53.39 \\
54.12 \\
54.85 \\
55.58 \\
56.31 \\
57.05 \\
57.78 \\
58.51\end{array}$ & $\begin{array}{l}48.42 \\
49.10 \\
49.79 \\
50.47 \\
51.15 \\
51.83 \\
52.51 \\
53.20 \\
53.88 \\
54.56\end{array}$ & $\begin{array}{l}51.71 \\
52.44 \\
53.17 \\
53.90 \\
54.63 \\
55.36 \\
56.08 \\
56.81 \\
57.54 \\
58.27\end{array}$ & $\begin{array}{l}48.65 \\
49.33 \\
50.02 \\
50.70 \\
51.39 \\
52.07 \\
52.76 \\
53.44 \\
54.13 \\
54.81\end{array}$ & $\begin{array}{l}51.50 \\
52.23 \\
52.95 \\
53.68 \\
54.40 \\
55.13 \\
55.85 \\
56.58 \\
57.30 \\
58.03\end{array}$ & $\begin{array}{l}48.87 \\
49.56 \\
50.25 \\
50.94 \\
51.63 \\
52.31 \\
53.00 \\
53.69 \\
54.38 \\
55.07\end{array}$ & $\begin{array}{l}51.29 \\
52.01 \\
52.73 \\
53.45 \\
54.18 \\
54.90 \\
55.62 \\
56.34 \\
57.07 \\
57.79\end{array}$ & $\begin{array}{l}49.10 \\
49.79 \\
50.48 \\
51.17 \\
51.86 \\
52.55 \\
53.25 \\
53.94 \\
54.63 \\
55.32\end{array}$ & $\begin{array}{l}71 \\
72 \\
73 \\
74 \\
75 \\
76 \\
77 \\
78 \\
79 \\
80\end{array}$ \\
\hline $\begin{array}{l}81 \\
82 \\
83 \\
84 \\
85 \\
86 \\
87 \\
88 \\
89 \\
90\end{array}$ & $\begin{array}{l}59.24 \\
59.97 \\
60.70 \\
61.43 \\
62.17 \\
62.90 \\
63.63 \\
64.36 \\
65.09 \\
65.82\end{array}$ & $\begin{array}{l}55.24 \\
55.92 \\
56.61 \\
57.29 \\
57.97 \\
58.65 \\
59.33 \\
60.02 \\
60.70 \\
61.38\end{array}$ & $\begin{array}{l}59.00 \\
59.73 \\
60.45 \\
61.18 \\
61.91 \\
62.64 \\
63.37 \\
64.10 \\
64.82 \\
65.55\end{array}$ & $\begin{array}{l}55.50 \\
56.19 \\
56.87 \\
57.56 \\
58.24 \\
58.93 \\
59.61 \\
60.30 \\
60.98 \\
61.67\end{array}$ & $\begin{array}{l}58.76 \\
59.48 \\
60.21 \\
60.93 \\
61.66 \\
62.38 \\
63.11 \\
63.83 \\
64.56 \\
65.28\end{array}$ & $\begin{array}{l}55.76 \\
56.45 \\
57.13 \\
57.82 \\
58.51 \\
59.20 \\
59.89 \\
60.58 \\
61.26 \\
61.95\end{array}$ & $\begin{array}{l}58.51 \\
59.23 \\
59.96 \\
60.68 \\
61.40 \\
62.12 \\
62.85 \\
63.57 \\
64.29 \\
65.01\end{array}$ & $\begin{array}{l}56.01 \\
56.70 \\
57.40 \\
58.09 \\
58.78 \\
59.47 \\
60.16 \\
60.85 \\
61.54 \\
62.24\end{array}$ & $\begin{array}{l}81 \\
82 \\
83 \\
84 \\
85 \\
86 \\
87 \\
85 \\
89 \\
90\end{array}$ \\
\hline $\begin{array}{r}91 \\
92 \\
93 \\
94 \\
95 \\
96 \\
97 \\
98 \\
99 \\
100\end{array}$ & $\begin{array}{l}66.55 \\
67.28 \\
68.02 \\
68.75 \\
69.48 \\
70.21 \\
70.94 \\
71.67 \\
72.40 \\
73.14\end{array}$ & $\begin{array}{l}62.06 \\
62.74 \\
63.43 \\
64.11 \\
64.79 \\
65.47 \\
66.15 \\
66.84 \\
67.52 \\
68.20\end{array}$ & $\begin{array}{l}66.28 \\
67.01 \\
67.74 \\
68.47 \\
69.20 \\
69.92 \\
70.65 \\
71.38 \\
72.11 \\
72.84\end{array}$ & $\begin{array}{l}62.35 \\
63.04 \\
63.72 \\
64.41 \\
65.09 \\
65.78 \\
66.46 \\
67.15 \\
67.83 \\
68.52\end{array}$ & $\begin{array}{l}66.01 \\
66.73 \\
67.46 \\
68.19 \\
68.91 \\
69.64 \\
70.36 \\
71.09 \\
71.81 \\
72.54\end{array}$ & $\begin{array}{l}62.64 \\
63.33 \\
64.02 \\
64.71 \\
65.39 \\
66.08 \\
66.77 \\
67.46 \\
68.15 \\
68.84\end{array}$ & $\begin{array}{l}65.74 \\
66.46 \\
67.18 \\
67.90 \\
68.62 \\
69.35 \\
70.07 \\
70.79 \\
71.51 \\
72.24\end{array}$ & $\begin{array}{l}62.03 \\
63.62 \\
64.31 \\
65.00 \\
65.69 \\
66.39 \\
67.08 \\
67.77 \\
68.46 \\
69.15\end{array}$ & $\begin{array}{r}91 \\
92 \\
93 \\
94 \\
95 \\
90 \\
97 \\
98 \\
99 \\
100\end{array}$ \\
\hline \multirow{2}{*}{ 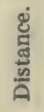 } & Dep. & Lat. & Dep. & Lat. & Dep. & Lat. & Dep. & Lat. & \multirow{2}{*}{$\begin{array}{l}\stackrel{\mathscr{g}}{\mathrm{g}} \\
\frac{\mathrm{g}}{0} \\
\end{array}$} \\
\hline & \multicolumn{2}{|c|}{$47^{\circ}}$. & \multicolumn{2}{|c|}{$46 \% 4^{\circ}$} & \multicolumn{2}{|c|}{$461 / 2^{\circ}}$. & \multicolumn{2}{|c|}{$461 / 4^{\circ}$. } & \\
\hline
\end{tabular}


TABLE 4.-TRAVERSE TABLE.

\begin{tabular}{|c|c|c|c|c|c|c|c|c|c|c|c|}
\hline \multirow{2}{*}{ 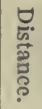 } & \multicolumn{2}{|c|}{$44^{\circ}$. } & \multicolumn{2}{|c|}{$441 / 4^{\circ}$. } & \multicolumn{2}{|c|}{$441 / 2^{\circ}$. } & \multicolumn{2}{|c|}{$44^{3} / 4^{\circ}$. } & \multicolumn{2}{|c|}{$45^{\circ}$. } & \multirow{2}{*}{ 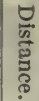 } \\
\hline & Lat. & Dep. & Lat. & Dep. & Lat. & Dep. & Lat. & Dep. & Lat. & Dep. & \\
\hline 1 & 0.72 & 0.69 & 0.72 & 0.70 & 0.71 & 0.70 & 0.71 & 0.70 & 0.71 & 0.71 & 1 \\
\hline 2 & $1.4 \overline{4}$ & 1.39 & 1. 43 & & 1. 43 & 1. 40 & 1.42 & & 1.41 & 1.41 & \\
\hline 3 & 2.16 & 2.08 & 2.15 & 2.09 & 2.14 & 2.10 & 2.13 & & 2.12 & 2.12 & \\
\hline 4 & 2.83 & 2.78 & 2.87 & 2. 79 & 2.85 & 2. 80 & 2.84 & 2.82 & 2.83 & 2.83 & \\
\hline 5 & 3.60 & 3.47 & 3.58 & 3. 49 & 3.57 & 3.50 & 3.55 & 3. 52 & 3. 54 & 3.54 & \\
\hline 6 & 4.32 & 4.17 & 4. 30 & 4. 19 & 4.28 & 4.21 & 4.26 & 4.22 & 4.24 & 4.24 & 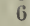 \\
\hline 7 & 5.04 & 4.86 & 5.01 & 4.88 & 4.99 & 4.91 & 4.97 & 4.93 & 4.95 & 4.95 & 7 \\
\hline 8 & 5.75 & 5. 56 & 5.73 & 5. 58 & 5. 71 & 5.61 & 5. 68 & 5. 63 & 5. 66 & 5. 66 & \\
\hline 9 & 6.47 & 6.25 & 6.45 & 6.28 & 6.42 & 6.31 & 6.39 & 6.34 & 6.36 & 6.36 & \\
\hline 10 & 7.19 & 6.95 & 7.16 & 6.98 & 7.13 & 7.01 & 7.10 & 7.04 & 7.07 & 7.07 & 10 \\
\hline 11 & 7.91 & 7. & 7.88 & $7 . C$ & 7.85 & 7.71 & 7.81 & 7.74 & 7.78 & 7. 78 & 11 \\
\hline 12 & 8.63 & 8.34 & 8.60 & 8. & 8.56 & 8.41 & 8. & 8. & 8.49 & 8.49 & 12 \\
\hline 13 & 9.35 & 9.03 & 9.31 & 9.07 & 9.27 & 9.11 & 9.23 & 9.15 & 9.19 & 9.19 & 13 \\
\hline 14 & 10.07 & 9.73 & 10.03 & 9. 77 & 9.99 & 9.81 & 9.94 & 9.86 & 9.90 & 9.90 & . \\
\hline 15 & 10.79 & 10.42 & 10.74 & 10.47 & 10.70 & 10.51 & 10.65 & 10.56 & 10.61 & 10.61 & 15 \\
\hline 16 & 11.51 & 11.11 & 11.46 & 11.16 & 11.41 & 11.21 & 11.36 & 11. & 11.31 & 11.31 & 16 \\
\hline 17 & 12.23 & 11.81 & 12.18 & 11.86 & 12.13 & 11.92 & 12.07 & 11.97 & 12.02 & 2.02 & 17 \\
\hline 18 & 12. 95 & 12.50 & 12.89 & 12.56 & 12.84 & 12. 62 & 12.78 & 7 & 12.73 & 2. 73 & 10 \\
\hline 19 & 13. 67 & 13.20 & 13. 61 & $13.26 \mathrm{i}$ & 13.55 & 13.32 & 13. 49 & 13.38 & 13.44 & 13. 44 & 19 \\
\hline 20 & 14.39 & 13. $\$ 9$ & 14.33 & 13.96 & 14. 27 . & 14.02 & 14.20 & 14.08 & 14.14 & 14.14 & 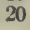 \\
\hline 21 & 15.11 & 14. 59 & 15.04 & 14. & 14.98 & 1 & 1 & & 14.85 & 14.85 & -1 \\
\hline 22 & 15. 83 & 15. 28 & 15. 76 & 15. & 15. 69 & 1 & & & 15. 56 & 15.56 & 22 \\
\hline 23 & 16.54 & 15. 98 & 16.47 & 10 & 16. & 1 & 3 & & 16. 26 & 16.26 & 23 \\
\hline 24 & 17.26 & 16.67 & 17.19 & 16. & 17.12 & 16. 82 & 17.04 & 1 & 16.97 & 16.97 & 24 \\
\hline 25 & 17.98 & 17.37 & 17.91 & 17.44 & 17.83 & 17.52 & 17.75 & 0 & 17. 08 & 17. GS & 25 \\
\hline 26 & 18.70 & 18. 06 & 18. 62 & 18.14 & 18. 54 & 1 & 18. 46 & 18. & 18.38 & 18.38 & 26 \\
\hline 27 & 19.42 & 18. 76 & 19. 34 & 18. & 19.26 & 2 & 19.18 & 1 & 19.09 & 19.09 & 27 \\
\hline 28 & 20.14 & 19.45 & 20.06 & 19. & 19.97 & 1 & 19. 89 & 1 & 19. 80 & 19.80 & 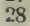 \\
\hline 29 & 20.86 & 20.15 & 20.77 & 20.24 & 20.68 & 20.33 & 20.60 & 2 & 20.51 & 20.51 & 29 \\
\hline 30 & 21.58 & 20.84 & 21.49 & 20.93 & 21.40 & 21.03 & 21.31 & 21.12 & 21.21 & 21.21 & 30 \\
\hline 31 & 22.30 & 21.53 & 32.21 & 2 & 22. & 21.73 & 2 & 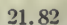 & 2 & 1.92 & 31 \\
\hline 32 & 23.02 & 22.23 & 22.92 & 22 . & 22.82 & 2 & & & 22 & 2.63 & 32 \\
\hline 33 & 23.74 & 22.92 & 23.64 & 23. & 23.54 & 3 & 4 & 3 & 23. & 23.33 & 33 \\
\hline 34 & 24.46 & 23.62 & 24.35 & 23.7 & 24.25 & 23. 83 & 24.15 & 23.94 & 24.04 & 24.04 & 34 \\
\hline 35 & 25.18 & 24.31 & 25.07 & 2 & 24.96 & 3 & 6 & 4 & 24.75 & 24.75 & 35 \\
\hline 36 & 25.90 & 25.01 & 25.79 & 2 & 25. & 2 & 25.57 & 4 & 25.46 & 25.46 & 36 \\
\hline 37 & 26.62 & 25.70 & 26.50 & & 2 & & 26.28 & & 26.16 & 26.16 & 37 \\
\hline 38 & 27.33 & 26.40 & 27.22 & 26. & 7 & 2 & 9 & 2 & 7 & 26.87 & 30 \\
\hline 39 & 28.05 & 27.09 & 27.94 & 27:? & 27.82 & 27.34 & .70 & 6 & 58 & 27.58 & 39 \\
\hline 40 & 28.77 & 27.79 & 28.65 & 27.91 & 28.53 & 28.04 & 28.41 & 28.16 & 28.28 & 28.28 & 40 \\
\hline 41 & $29.4 \mathrm{~S}$ & 48 & 7 & 2 & 29.2 & 2 & 2 & & 9 & 9 & 41 \\
\hline 42 & 30.21 & 29.18 & .08 & & & & & & 29.70 & 9.70 & 42 \\
\hline 43 & 30.93 & 29.87 & 30.80 & 30 . & & & & & 30.41 & 30.41 & 43 \\
\hline 44 & 31.65 & 30.56 & 31.52 & 30. & 31 & 3 & 5 & 3 & 31.11 & 31.11 & 44 \\
\hline 45 & 32.37 & 31.26 & 32.23 & 31. & 32 & & 3106 & & 31.82 & 31.82 & 45 \\
\hline 46 & 33.09 & 31.95 & 32.95 & 3 & 32.81 & 3 & 7 & 3 & 32.53 & 32.53 & 46 \\
\hline 47 & 33.81 & 32.65 & 33.67 & & 33. 52 & 32.94 & & & 33.23 & 33.23 & 7 \\
\hline 48 & 34.53 & 33.34 & 34.38 & 33. & 34.24 & 33. & 34.09 & 33. & 33.94 & 33.94 & 8 \\
\hline 49 & 35.25 & 34.04 & 35.10 & 34. & 34.8 & 34. & 34.80 & & 34.65 & 34. 65 & 9 \\
\hline 50 & 35.97 & 34.73 & 35.82 & 34.89 & 35.66 & 35.05 & 35.51 & 35.20 & 35.36 & 35.36 & 50 \\
\hline \multirow{2}{*}{ 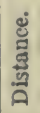 } & Dep. & I at. & Dep. & Lat. & Dep. & Lat. & Dep. & Lat. & Dep. & Lat. & \multirow{2}{*}{ 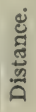 } \\
\hline & \multicolumn{2}{|c|}{$46^{\circ}$. } & \multicolumn{2}{|c|}{$4534^{\circ}$. } & \multicolumn{2}{|c|}{$451 / 2^{\circ}}$. & \multicolumn{2}{|c|}{$451 / 4^{\circ}$. } & \multicolumn{2}{|c|}{$45^{\circ}}$. & \\
\hline
\end{tabular}


TABLE 4.-TRAVERSE TABLF.

\begin{tabular}{|c|c|c|c|c|c|c|c|c|c|c|c|}
\hline \multirow{2}{*}{ 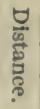 } & \multicolumn{2}{|c|}{$44^{\circ}$} & \multicolumn{2}{|c|}{$441 / 4^{\circ}}$. & \multicolumn{2}{|c|}{$441 / 2^{\circ}$. } & \multicolumn{2}{|c|}{$44^{\circ} / 4^{\circ}$. } & \multicolumn{2}{|c|}{$45^{\circ}$} & \multirow{2}{*}{$\frac{\vec{b}}{\frac{5}{n}}$} \\
\hline & Lat. & Dep. & Lat. & Dep. & Lat. & Dep. & Lat. & De & Lat. & Dep. & \\
\hline 51 & 36.69 & 35.43 & 36.53 & 35. 59 & 36. 38 & 35. 75 & 36.22 & 35.90 & 36.06 & 36.06 & 01 \\
\hline 52 & 37.41 & 36.12 & 37.25 & & & & 36.93 & & & & 52 \\
\hline 53 & $3 \mathrm{~S} .13$ & 36.82 & 37.96 & 36.98 & .80 & 37.15 & 37.64 & & 37.48 & 48 & 53 \\
\hline 54 & 35.84 & 37.51 & $38 . \mathrm{cs}$ & 37.68 & 38.52 & 37.85 & 38.35 & 35.02 & 38.18 & 38.18 & 54 \\
\hline 55 & 39.56 & 38.21 & 39.40 & 38.38 & .23 & 38.55 & 39.06 & 72 & 38.39 & 38.89 & \\
\hline 56 & 40. 28 & 38.90 & 40.11 & 39.08 & 39.94 & 39.25 & 39.77 & 39.42 & 39.60 & $39 . \mathrm{Co}$ & 56 \\
\hline 57 & 41.00 & 39. 10 & 40.83 & 39.77 & .66 & 39.95 & 40.48 & $40 . \overline{13}$ & 40.31 & 40.31 & 57 \\
\hline 58 & 41.72 & 40.29 & 41.55 & 40. 47 & 1.37 & 40.65 & 41.19 & 40.83 & 41.01 & 41.01 & 58 \\
\hline 59 & 42.44 & 40.98 & 42.20 & 41.17 & 2.08 & 41.35 & 41.90 & 41.54 & 41.72 & 41.72 & 59 \\
\hline $\mathrm{CO}$ & 43.16 & 41.68 & 42.98 & 4 & 42.80 & 42.05 & 42.61 & 42 & 43 & .43 & 0 \\
\hline 61 & 43.88 & 42.37 & 43.69 & 42.57 & 43.51 & 42.76 & 43.32 & 42.94 & 43.13 & 3.13 & \\
\hline 62 & 44. 60 & 43.07 & 44.41 & & .22 & & 44.03 & & 84 & .84 & 62 \\
\hline $\mathrm{C} 3$ & 45.32 & 43.76 & 45.13 & 43.96 & .93 & 44.16 & 44.74 & & & 4.55 & 63 \\
\hline 64 & 46.04 & 44. 46 & 45.84 & 44. 66 & 5.65 & 44.86 & 45.45 & 45.06 & 45.25 & 5.25 & \\
\hline 65 & 46.76 & 15 & 46.56 & & 66 & 45.56 & 16 & 45 & 66 & & \\
\hline 66 & 47.49 & 45.85 & 47.28 & 4 & .07 & 6 & 46.87 & 46.46 & 46.67 & 67 & \\
\hline 6.7 & 48.20 & 46.54 & 47.99 & 46.75 & & 4 & & 47.17 & & & \\
\hline cis & 48.92 & 47.24 & 48.71 & & 0 & 4 & 29 & 7 & 08 & 08 & \\
\hline 1.9 & 49. (33 & 47.93 & 49.42 & 15 & 21 & 6 & 49.00 & 48.58 & 48. 79 & 79 & \\
\hline 70 & 50.35 & 3 & 50.14 & 4. & 9.93 & 49.06 & 49.71 & 49.28 & 49.50 & 49.50 & \\
\hline$\pi 1$ & 51.07 & 32 & 86 & & 64 & 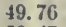 & 2 & .99 & .20 & & 11 \\
\hline 72 & 51.79 & & 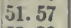 & & 3 & & & & & & \\
\hline 73 & 52.51 & 50.71 & 52.29 & 50.94 & 07 & 51. & 51.84 & & 51.62 & & 73 \\
\hline 74 & 53.23 & 51.40 & 53.01 & 51.64 & 8 & 51. & 52.55 & 0 & 52.33 & 33 & 74 \\
\hline 75 & 53.95 & 52.10 & 53.72 & 52. & 19 & 5 & 53.26 & & 53.03 & & 7 \\
\hline 76 & 54.67 & 52.79 & 54.44 & 53.03 & 21 & 5 & 53.97 & 5 & 53.74 & 84 & 76 \\
\hline 77 & 55.39 & 53.49 & 55.16 & & & 5 & 5 & 5. & 5 & 15 & 77 \\
\hline 78 & 56.11 & 54.18 & 55. & 54 & 3 & 5 & 9 & 1 & 5 & 15 & 78 \\
\hline 79 & 56.83 & 54.88 & 56.59 & 55.13 & 35 & 55 & 56. & 5 & 6 & 5. 86 & 79 \\
\hline 80 & 57.55 & 55.57 & 57.30 & 55.82 & 96 & 5 & 1 & $5 t$ & 56.57 & 6.57 & 80 \\
\hline 81 & 58.27 & .27 & 58.02 & 56 & .77 & 5 & 3 & & 8 & & 81 \\
\hline 82 & 58.9 & 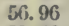 & & & & & 4 & & & & \\
\hline 83 & 59.71 & 6 & 59.45 & 5 & & & 5 & & & & 8 \\
\hline 84 & 60.42 & 5 & & & & & & & 0 & & 8 \\
\hline 85 & 61.14 & 05 & 60.89 & & 0 & & 60. 37 & 4 & 0 & 10 & 8 \\
\hline 86 & 61.86 & 59.74 & 61.60 & & & & 61.08 & & & & 80 \\
\hline 87 & 6.2. 58 & c & 2 & & & & 9 & & & 2 & 87 \\
\hline 88 & 63.30 & 61.13 & 3 & & & & & & & & 80 \\
\hline 89 & 64.02 & 61.82 & 63.75 & & & & 63.21 & & 62.93 & 3 & ח \\
\hline 90 & 64.74 & 62.52 & 64.47 & 62.80 & 64.19 & 63. & 63.92 & 63 & 63.64 & 63.64 & 90 \\
\hline 91 & 65. & G & 8 & 0 & 64.91 & & 3 & 64.07 & 5 & 5 & 91 \\
\hline 92 & 66 & & & & & & & & & & 9 \\
\hline 93 & 66.9 & 6 & 66. 62 & & 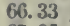 & & 5 & & & & 93 \\
\hline 94 & 67 & 6 & 6 & & & & & & & & 94 \\
\hline 95 & 8.34 & 65.9 & 68.05 & & & & & & & & 8 \\
\hline 96 & 199.0 & 66.6 & 68.76 & & & & & & 8 & & \\
\hline 07 & 69.78 & 67.3 & 69.48 & & & & 68.89 & & 9 & 68.59 & 97 \\
\hline 98 & & 68.0 & 70.20 & & & & & & 69.30 & & \\
\hline 99 & $\pi 1.21$ & 68.7 & 70.91 & & & & 70.31 & & 70.00 & 70.00 & \\
\hline 100 & 71.93 & 69.47 & 71.63 & 69. 78 & 71.33 & 70.09 & 71.02 & 70.40 & 70.71 & 70.71 & 100 \\
\hline \multirow{2}{*}{ 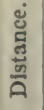 } & Dep. & Lat. & Dep. & Lat. & Dep. & Lat. & Dep. & Lat. & Dep. & Iat. & ¿ \\
\hline & \multicolumn{2}{|c|}{$46^{\circ}}$. & \multicolumn{2}{|c|}{$45^{8} 4^{\circ}$} & \multicolumn{2}{|c|}{$451 / 22^{\circ}$. } & \multicolumn{2}{|c|}{ y } & \multicolumn{2}{|c|}{20.} & \\
\hline
\end{tabular}


TABLE 5.-CORRECTION OF ERROR IN STADIA WIRE INTERVAL.

\begin{tabular}{|c|c|c|c|c|c|c|c|c|c|c|}
\hline \multicolumn{11}{|c|}{ Distance in chains. } \\
\hline $\mathbf{0}$ & 1 & 2 & 3 & 4 & 5 & 6 & 7 & 8 & 9 & 10 \\
\hline \multicolumn{11}{|c|}{ Error in Links. } \\
\hline 0.0 & 0.1 & 0.2 & 0.3 & 0.4 & 0.5 & 0.6 & 0.7 & 0.8 & 0.9 & 1.0 \\
\hline 0.0 & 0.2 & 0.4 & 0.6 & 0.8 & 1.0 & 1.2 & 1.4 & 1.6 & 1.8 & 2. 0 \\
\hline 0.0 & 0.3 & 0.6 & 0.9 & 1.2 & 1.5 & 1.8 & 2.1 & 2.4 & 2. 7 & 3. 0 \\
\hline 0.0 & 0.4 & 0.8 & 1.2 & 1.6 & 2.0 & 2.4 & 2.8 & 3.2 & 3.6 & 4.0 \\
\hline 0.0 & 0.5 & 1.0 & 1.5 & 2. 0 & 2.5 & 3.0 & 3.5 & 4. 0 & 4.5 & 5.0 \\
\hline 0.0 & 0.6 & 1.2 & 1.8 & 2.4 & 3. 0 & 3.6 & 4. 2 & 4.8 & 5.4 & 6. 0 \\
\hline 0.0 & 0.7 & 1.4 & 2.1 & 2.8 & 3.5 & 4.2 & 4. 9 & 5.6 & 6.3 & 7.0 \\
\hline 0.0 & 0.8 & 1.6 & 2.4 & 3. 2 & 4. 0 & 4.8 & 5.6 & 6.4 & 7.2 & 8. 0 \\
\hline 0.0 & 0.9 & 1.8 & 2.7 & 3.6 & 4.5 & 5.4 & 6. 3 & 7.2 & 8.1 & 9.0 \\
\hline 0.0 & 1.0 & 2.0 & 3.0 & 4. 0 & 5.0 & 6.0 & 7.0 & 8.0 & 9.0 & 10.0 \\
\hline-0.0 & 1.1 & 2.2 & 3.3 & 4. 4 & 5.5 & 6.6 & 7.7 & 8.8 & 9.9 & 11.0 \\
\hline 0.0 & 1.2 & 2.4 & 3. 6 & 4.8 & 6.0 & 7.2 & 8.4 & 9. 6 & 10.8 & 12.0 \\
\hline 0.0 & 1.3 & 2.6 & 3.9 & 5.2 & 6.5 & 7.8 & 9.1 & 10.4 & 11.7 & 13.0 \\
\hline 0.0 & 1.4 & 2.8 & 4.2 & 5.6 & 7.0 & 8.4 & 9.8 & 11.2 & 12. 6 & 14. 0 \\
\hline 0.0 & 1.5 & 3.0 & 4.5 & 6.0 & 7.5 & 9.0 & 10.5 & 12. 0 & 13.5 & 15. 0 \\
\hline 0.0 & 1.6 & 3.2 & 4.8 & 6.4 & 8.0 & 9.6 & 11.2 & 12.8 & 14.4 & 16. 0 \\
\hline 0.0 & 1. 7 & 3.4 & 5.1 & 6.8 & 8.5 & 10.2 & 11.9 & 13. 6 & 15. 3 & 17. 0 \\
\hline 0.0 & 1.8 & 3. 6 & 5.4 & 7.2 & 9.0 & 10.8 & 12.6 & 14.4 & 16. 2 & 18. 0 \\
\hline 0.0 & 1.9 & 3.8 & 5.7 & 7. 6 & 9.5 & 11.4 & 13. 3 & 15. 2 & 17.1 & 19.0 \\
\hline 0.0 & 2.0 & 4.0 & 6.0 & 8.0 & 10.0 & 12.0 & 14.0 & 16.0 & 18.0 & 20.0 \\
\hline 0.0 & 2.1 & 4.2 & 6. 3 & 8.4 & 10.5 & 12.6 & 14. 7 & 16. 8 & 18.9 & 21.0 \\
\hline 0.0 & 2.2 & 4.4 & 6.6 & 8.8 & 11.0 & 13.2 & 15.4 & 17.6 & 19.8 & 22.0 \\
\hline 0.0 & 2.3 & 4. 6 & 6.9 & 9.2 & 11.5 & 13.8 & 16.1 & 18.4 & 20.7 & 23.0 \\
\hline 0.0 & 2.4 & 4. 8 & 7.2 & 9.6 & 12.0 & 14.4 & 16.8 & 19.2 & 21.6 & 24.0 \\
\hline 0.0 & 2. 5 & 5.0 & 7.5 & 10.0 & 12.5 & 15. 0 & 17.5 & 20.0 & 22.5 & 25.0 \\
\hline 0.0 & 2.6 & 5.2 & 7.8 & 10.4 & 13.0 & 15.6 & 18. 2 & 20.8 & 23.4 & 26.0 \\
\hline 0.0 & 2. 7 & 5.4 & 8.1 & 10.8 & 13.5 & 16.2 & 18.9 & 21.6 & 24.3 & 27.0 \\
\hline 0.0 & 2.8 & 5.6 & 8.4 & 11.2 & 14.0 & 16.8 & 19.6 & 22.4 & 25.2 & 28.0 \\
\hline 0.0 & 2.9 & 5.8 & 8.7 & 11.6 & 14.5 & 17.4 & 20.3 & 23.2 & 26.1 & 29.0 \\
\hline 0.0 & 3.0 & 6.0 & 9.0 & 12.0 & 15. 0 & 18.0 & 21.0 & 21.0 & 27.0 & 30.0 \\
\hline \multicolumn{11}{|c|}{ Distance in chains. } \\
\hline 10 & 11 & 12 & 13 & 14 & 15 & 16 & 17 & 18 & 19 & 20 \\
\hline \multicolumn{11}{|c|}{ Error in links. } \\
\hline 1.0 & 1.1 & 1.2 & 1.3 & 1.4 & 1.5 & 1.6 & 1. 7 & 1.8 & 1.9 & 2.0 \\
\hline 2.0 & 2.2 & 2.4 & 2.6 & 2.8 & 3. 0 & 3.2 & 3.4 & 3. 6 & 3.8 & 4. 0 \\
\hline 3.0 & 3.3 & 3.6 & 3. 9 & 4.2 & 4.5 & 4.8 & 5.1 & 5.4 & 5.7 & 6.0 \\
\hline 4.0 & 4.4 & 4.8 & 5.2 & 5.6 & 6.0 & 6.4 & 6.8 & 7.2 & 7.6 & 8. 0 \\
\hline 5.0 & 5.5 & 6.0 & 6.5 & 7.0 & 7.5 & 8.0 & 8.5 & 9.0 & 9.5 & 10.0 \\
\hline 6.0 & 6. 6 & 7.2 & 7.8 & 8.4 & 9.0 & 9.6 & 10.2 & 10.8 & 11.4 & 12.0 \\
\hline 7.0 & 7.7 & 8.4 & 9.1 & 9.8 & 10.5 & 11.2 & 11.9 & 12.6 & 13. 3 & 14.0 \\
\hline 8.0 & 8.8 & 9.6 & 10.4 & 11.2 & 12.0 & 12.8 & 13. 6 & 14.4 & 15. 2 & 16. 0 \\
\hline 9.0 & 9.9 & 10.8 & 11.7 & 12.6 & 13.5 & 14.4 & 15.3 & 16.2 & 17.1 & 18.0 \\
\hline 10.0 & 11.0 & 12.0 & 13.0 & 14. 0 & 15. 0 & 16.0 & 17.0 & 18. 0 & 19.0 & 20.0 \\
\hline 11.0 & 12.1 & 13.2 & 14. 3 & 15.4 & 16. 5 & 17.6 & 18.7 & 19.8 & 20.9 & 22.0 \\
\hline 12.0 & 13. 2 & 14. 4 & 15. 6 & 16. 8 & 18.0 & 19.2 & 20.4 & 21.6 & 22.8 & 24.0 \\
\hline 13.0 & 14. 3 & 15. 6 & 16.9 & 18. 2 & 19. 5 & 20.8 & 22.1 & 23.4 & 24.7 & 26.0 \\
\hline 14.0 & 15.4 & 16.8 & 18. 2 & 19.6 & 21.0 & 22.4 & 23.8 & 25.2 & 26.6 & 28.0 \\
\hline 15.0 & 16.5 & 18. 0 & 19.5 & 21.0 & 22.5 & 24.0 & 25.5 & 27.0 & 28.5 & 30.0 \\
\hline 16.0 & 17.6 & 19.2 & 20.8 & 22.4 & 24.0 & 25.6 & 27.2 & 28.8 & 30.4 & 32.0 \\
\hline 17.0 & 18. 7 & 20. 4 & 22.1 & 23. 8 & 25.5 & 27.2 & 28.9 & 30.6 & 32.3 & 34.0 \\
\hline 18.0 & 19.8 & 21.6 & 23.4 & 25. 2 & 27.0 & 28.8 & 30.6 & 32.4 & 34.2 & 36.0 \\
\hline 19.0 & 20.9 & 22.8 & 24.7 & 26.6 & 28.5 & 30.4 & 32.3 & 34.2 & 36.1 & 38.0 \\
\hline 20.0 & 22.0 & 24.0 & 26.0 & 28.0 & 30.0 & 32.0 & 34.0 & 36.0 & 38.0 & 40.0 \\
\hline 21.0 & 23. 1 & 25.2 & 27.3 & 29.4 & 31.5 & 33.6 & 35.7 & 37.8 & 39.9 & 42.0 \\
\hline 22.0 & 24.2 & 26. 4 & 28.6 & 30.8 & 33.0 & 35.2 & 37.4 & 39. 6 & 41.8 & 44.0 \\
\hline 23.0 & 25. 3 & 27.6 & 29.9 & 32. 2 & 34.5 & 36.8 & 39.1 & 41.4 & 43.7 & 46.0 \\
\hline 24.0 & 26.4 & 28. 8 & 31.2 & 33. 8 & 36.0 & 38.4 & 40.8 & 43.2 & 45.6 & 48.0 \\
\hline 25.0 & 27.5 & 30. 0 & 32.5 & 35.0 & 37.5 & 40.0 & 42.5 & 45.0 & 47.5 & 50.0 \\
\hline 26.0 & $2 x .6$ & 31.2 & 33.8 & 36.4 & 39.0 & 41.6 & 44.2 & 46.8 & 49.4 & 52.0 \\
\hline 27.0 & 29. 7 & 32.4 & 35.1 & 37.8 & 40.5 & 43.2 & 45.9 & 48.6 & 51.3 & 54.0 \\
\hline 28.0 & 30.8 & 33.6 & 36.4 & 39.2 & 42,0 & 44.8 & 47.6 & 50.4 & 53.2 & 56.0 \\
\hline 29.0 & 31.9 & 34.8 & 37.7 & 40.6 & 43.5 & 46.4 & 49. 3 & 52.2 & 55.1 & 58.0 \\
\hline 30.0 & 33. 0 & 36.0 & 39.0 & 42.0 & 45. 0 & 48.0 & 51.0 & 54.0 & 57.0 & 60.0 \\
\hline
\end{tabular}


TABLF 6. STADIA COFFFICIENTS, VERTICAL ROD.

$\cos ^{2} v$ and $1 / 2 \sin 2 v$.

\begin{tabular}{|c|c|c|c|c|c|c|c|c|}
\hline \multirow[b]{2}{*}{$\begin{array}{l}7 . n \\
x\end{array}$} & \multicolumn{2}{|c|}{$0^{\circ}$} & \multicolumn{2}{|c|}{$1^{\circ}$} & \multicolumn{2}{|c|}{$2^{\circ}$} & \multicolumn{2}{|c|}{$3^{\circ}$} \\
\hline & $\begin{array}{l}\text { Hor. } \\
\text { dist. }\end{array}$ & $\begin{array}{l}\text { Diff. } \\
\text { elev. }\end{array}$ & $\begin{array}{l}\text { Hor. } \\
\text { dist. }\end{array}$ & $\begin{array}{l}\text { Diff. } \\
\text { elev. }\end{array}$ & $\begin{array}{l}\text { IIor. } \\
\text { dist. }\end{array}$ & $\begin{array}{l}\text { Diff. } \\
\text { elev. }\end{array}$ & $\begin{array}{l}\text { Hor. } \\
\text { dist. }\end{array}$ & $\begin{array}{l}\text { Difr. } \\
\text { elev. }\end{array}$ \\
\hline , & & & & & & & & 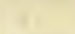 \\
\hline 0 & 1.0000 & .0000 & .9997 & .0174 & .9988 & .0349 & .9973 & .0523 \\
\hline 2 & 1.0000 & .0006 & .9997 & .0180 & .9987 & .0355 & .9972 & .0528 \\
\hline 4 & 1.0000 & .0012 & .9997 & .0186 & .9987 & .0360 & .9971 & .0534 \\
\hline 6 & 1.0000 & .0017 & .9996 & .0192 & .9987 & .0366 & .9971 & .0540 \\
\hline 8 & 1.0000 & .0023 & .9996 & .0198 & .9986 & .0372 & .9970 & .0546 \\
\hline 10 & 1.0000 & .0029 & .9996 & .0204 & .9986 & .0378 & .9969 & .0552 \\
\hline 12 & 1. 0000 & .0035 & .9996 & .0209 & .9985 & .0354 & .9969 & .0557 \\
\hline 14 & 1.0000 & .0041 & .9905 & .0215 & .9985 & .0390 & .9968 & .0563 \\
\hline 16 & 1.0000 & .0047 & .9995 & .0221 & .9984 & .0395 & .9968 & .0569 \\
\hline 18 & 1. 0000 & .0052 & .9995 & .0227 & .9984 & .0401 & .9967 & .0575 \\
\hline 20 & 1.0000 & .0058 & .9995 & .0233 & .9983 & .0407 & .9966 & .0580 \\
\hline 22 & 1.0000 & .0064 & .9994 & .0238 & .9983 & .0413 & .9966 & .0586 \\
\hline 24 & 1.0000 & .0070 & .9994 & .0244 & .9982 & .0418 & .9965 & .0592 \\
\hline 26 & .9999 & .0076 & .9994 & .0250 & .9982 & .0424 & .9964 & .0598 \\
\hline 28 & .9999 & .0081 & .9993 & .0256 & .9981 & .0430 & .9963 & .0604 \\
\hline 30 & .9999 & .0087 & .9993 & .0262 & .9981 & .0436 & .9963 & .0609 \\
\hline 32 & .9999 & .0093 & .9993 & .0267 & .9980 & .0442 & .9962 & .0615 \\
\hline 34 & .9999 & .0099 & .9993 & .0273 & .9980 & .0448 & .9962 & .0621 \\
\hline 36 & .9999 & .0105 & .9992 & .0279 & .9979 & .0453 & .9961 & .0627 \\
\hline 38 & .9909 & .0111 & .9992 & .0285 & .9979 & .0459 & .9960 & .0633 \\
\hline 40 & .9999 & .0116 & .9992 & .0291 & .9978 & .0465 & .9959 & .0638 \\
\hline 42 & .0999 & .0122 & .9991 & .0297 & .9978 & .0471 & .9959 & .0644 \\
\hline 44 & .9998 & .0128 & .9991 & .0302 & .9977 & .0476 & .9958 & .0650 \\
\hline 46 & .9998 & .0134 & .9990 & .0308 & .9977 & .0482 & .9957 & .0656 \\
\hline 48 & .9998 & .0140 & .9990 & .0314 & .9976 & .0488 & .9956 & .0061 \\
\hline 50 & .9998 & .0145 & .9990 & .0320 & .9976 & .0494 & .9956 & .0667 \\
\hline & .9998 & .0151 & .9989 & .0326 & .9975 & .0499 & .9955 & .0673 \\
\hline 54 & .9998 & .0157 & .9989 & .0331 & .9974 & .0505 & .9954 & .0678 \\
\hline 56 & .9997 & .0163 & .9959 & .0337 & .9974 & .0511 & .9953 & .0654 \\
\hline 58 & .9997 & .0169 & .9958 & .0343 & .9973 & .0517 & .9952 & .0690 \\
\hline 60 & .9997 & .0174 & .9958 & .0349 & .9973 & .0523 & .9951 & .0690 \\
\hline$c+f$ & & & & & & & & \\
\hline 0.75 & 0.75 & 0.01 & 0.75 & 0.02 & 0.75 & 0.03 & 0.75 & 0.05 \\
\hline 1.00 & 1.00 & 0.01 & 1.00 & 0.03 & 1.00 & 0.04 & 1.00 & 0.06 \\
\hline 1.25 & 1.25 & 0.02 & 1.25 & 0.03 & 1.25 & 0.05 & 1.25 & 0.08 \\
\hline
\end{tabular}

$(c+f) \cos v$ and $(c+f) \sin v$.

Natural functions 
TABLE 6.-STADIA COEFFICIENTS, VERTICAL ROD.

$\operatorname{Cos}^{2} v$ and $1 / 2 \sin 2 v$.

\begin{tabular}{|c|c|c|c|c|c|c|c|c|}
\hline \multirow[b]{2}{*}{$=$} & \multicolumn{2}{|c|}{$4^{\circ}$} & \multicolumn{2}{|c|}{$5^{\circ}$} & \multicolumn{2}{|c|}{$6^{\circ}$} & \multicolumn{2}{|c|}{$7^{\circ}$} \\
\hline & $\begin{array}{l}\text { Hor. } \\
\text { dist. }\end{array}$ & $\begin{array}{l}\text { Diff. } \\
\text { elev. }\end{array}$ & $\begin{array}{l}\text { IIor. } \\
\text { dist. }\end{array}$ & $\begin{array}{l}\text { Diff. } \\
\text { elev. }\end{array}$ & $\begin{array}{l}\text { Hor. } \\
\text { dist. }\end{array}$ & $\begin{array}{l}\text { Diff. } \\
\text { elev. }\end{array}$ & $\begin{array}{l}\text { Hor. } \\
\text { dist. }\end{array}$ & $\begin{array}{l}\text { Diff. } \\
\text { elev. }\end{array}$ \\
\hline & & & & & & $=$ & & \\
\hline & .9951 & .0696 & .9924 & .0868 & .9891 & .1040 & .9851 & .1210 \\
\hline & .9951 & .0702 & .9923 & .0874 & .0890 & .1045 & .9850 & .1215 \\
\hline & .9950 & .0707 & .9922 & .0880 & .9588 & .1051 & 9848 & .1221 \\
\hline & .9949 & .0713 & .9921 & .0885 & .9857 & .1057 & .9847 & .1226 \\
\hline & .9948 & .0719 & .9920 & .0891 & .9886 & .1062 & .9846 & .1232 \\
\hline 10 & .9947 & .0725 & .9919 & .0597 & .9885 & .1068 & .9844 & .1238 \\
\hline 12 & .9946 & .0730 & .9918 & .0903 & .9883 & .1074 & .9843 & .1243 \\
\hline 14 & $.99+6$ & .0736 & .9917 & .0908 & .9882 & .1079 & .9841 & .1249 \\
\hline 16 & .9945 & .0742 & .9916 & .0914 & .9881 & .1085 & .9810 & .1255 \\
\hline 18 & .9944 & .0748 & .9915 & .0920 & .9880 & .1091 & .9839 & .1260 \\
\hline 20 & .9943 & .0753 & .9914 & .0925 & .9878 & .1096 & .9837 & .1266 \\
\hline 22 & .9942 & .0759 & .9913 & .0931 & .9877 & .1102 & 9836 & .1272 \\
\hline 24 & .9941 & .0765 & .9911 & .0937 & .9876 & .1108 & .9834 & .1277 \\
\hline 26 & .9940 & .0771 & .9910 & .0943 & .9874 & .1113 & .9833 & $.12 S 3$ \\
\hline 28 & .9939 & .0776 & .9909 & .0948 & .9873 & .1119 & .9831 & .1288 \\
\hline 30 & .9938 & .0782 & .9908 & .0954 & .9872 & .1125 & 9829 & - . 1294 \\
\hline 32 & .9938 & .0788 & .9907 & .0960 & .9871 & .1130 & .9828 & .1300 \\
\hline 34 & .9937 & .0794 & .9906 & .0965 & .9869 & .1136 & .9827 & .1305 \\
\hline 36 & .9936 & .0799 & .0905 & .0971 & .9868 & .1142 & .9825 & .1311 \\
\hline 38 & .9935 & .0805 & .9904 & .0977 & .9867 & .1147 & .9824 & .1317 \\
\hline 40 & .9934 & .0811 & .9903 & .0953 & .9865 & .1153 & .9822 & .1322 \\
\hline 42 & .9933 & .0817 & .9901 & .0988 & .9864 & .1159 & .9820 & .1328 \\
\hline 44 & .9932 & .0822 & .9900 & .0994 & .9863 & .1164 & .9819 & .1333 \\
\hline 46 & .9931 & .0828 & .9899 & .1000 & .0861 & .1170 & .9817 & .1339 \\
\hline 48 & .9930 & .0834 & .9898 & .1005 & .9860 & .1176 & .9816 & .1345 \\
\hline 50 & .9929 & .0840 & .9897 & .1011 & .9858 & .1181 & .9814 & .1350 \\
\hline 52 & .9928 & .0845 & .9896 & .1017 & .9857 & .1187 & .9813 & .1356 \\
\hline 54 & .9927 & .0851 & .9894 & .1022 & .9856 & .1193 & .9811 & .1361 \\
\hline 56 & .9926 & .0857 & .9893 & .1028 & .9854 & .1198 & .9810 & .1367 \\
\hline 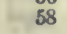 & .9925 & .0863 & .9892 & .1034 & .9853 & .1204 & .9508 & .1373 \\
\hline 60 & .9924 & .0868 & .9891 & .1040 & .9851 & .1210 & .9806 & .1378 \\
\hline$c+f$ & & & & & & & & \\
\hline 1.00 & 1.00 & $\begin{array}{l}0.00 \\
0.08\end{array}$ & $\begin{array}{l}0.75 \\
0.99\end{array}$ & $\begin{array}{l}0.07 \\
0.09\end{array}$ & $\begin{array}{l}0.75 \\
0.09\end{array}$ & 0.11 & $\begin{array}{l}0.74 \\
0.99\end{array}$ & $\begin{array}{l}0.10 \\
0.13\end{array}$ \\
\hline 1.25 . & 1.25 & 0.10 & 1.24 . & 0.11 & 1.24 & 0.14 & 1.24 & 0.16 \\
\hline
\end{tabular}

$(c+f) \cos v$ and $(c+f) \sin v$.

Natural functions. 
TABLF 6. - STADIA COEFFICIENTS, VERTICAL ROD.

$\operatorname{Cos}^{2} v$ and $1 / 2 \sin 2 v$.

\begin{tabular}{|c|c|c|c|c|c|c|c|c|}
\hline \multirow[b]{2}{*}{9} & \multicolumn{2}{|c|}{$8^{\circ}$} & \multicolumn{2}{|c|}{$9^{\circ}$} & \multicolumn{2}{|c|}{$10^{\circ}$} & \multicolumn{2}{|c|}{$11^{\circ}$} \\
\hline & $\begin{array}{l}\text { Hor. } \\
\text { dist. }\end{array}$ & $\begin{array}{l}\text { Diff. } \\
\text { elev. }\end{array}$ & $\begin{array}{l}\text { Hor. } \\
\text { dist. }\end{array}$ & $\begin{array}{l}\text { Diff. } \\
\text { elev. }\end{array}$ & $\begin{array}{l}\text { Hor. } \\
\text { dist. }\end{array}$ & $\begin{array}{l}\text { Diff. } \\
\text { elev. }\end{array}$ & $\begin{array}{l}\text { Mor. } \\
\text { dist. }\end{array}$ & $\begin{array}{l}\text { Diff. } \\
\text { elev. }\end{array}$ \\
\hline $\begin{array}{l}\text { ' } \\
0 \\
2 \\
4 \\
6 \\
8\end{array}$ & $\begin{array}{l}.9506 \\
.9805 \\
.9503 \\
.9501 \\
.9500\end{array}$ & $\begin{array}{l}.1378 \\
.1384 \\
.1359 \\
.1395 \\
.1401\end{array}$ & $\begin{array}{l}.9755 \\
.9753 \\
.9752 \\
.9750 \\
.9743\end{array}$ & $\begin{array}{l}.1545 \\
.1551 \\
.1556 \\
.1562 \\
.1567\end{array}$ & $\begin{array}{l}.9698 \\
.9696 \\
.9694 \\
.9692 \\
.9690\end{array}$ & $\begin{array}{r}.1710 \\
.1716 \\
.1721 \\
.1726 \\
.1732\end{array}$ & $\begin{array}{l}.9636 \\
.9634 \\
.9632 \\
.9629 \\
.9627\end{array}$ & $\begin{array}{l}.1873 \\
.1878 \\
.1854 \\
.1859 \\
.1895\end{array}$ \\
\hline $\begin{array}{l}10 \\
12 \\
14 \\
16 \\
18\end{array}$ & $\begin{array}{l}.9793 \\
.9797 \\
.9795 \\
.9793 \\
.9792\end{array}$ & $\begin{array}{l}.1406 \\
.1412 \\
.1417 \\
.1423 \\
.1428\end{array}$ & $\begin{array}{l}.9746 \\
.9744 \\
.9743 \\
.9741 \\
.9739\end{array}$ & $\begin{array}{l}.1573 \\
.1578 \\
.1554 \\
.1589 \\
.1595\end{array}$ & $\begin{array}{l}.9688 \\
.9656 \\
.9684 \\
.9682 \\
.9650\end{array}$ & $\begin{array}{l}.1737 \\
.1743 \\
.1748 \\
.1754 \\
.1759\end{array}$ & $\begin{array}{l}.0625 \\
.9623 \\
.9621 \\
.9618 \\
.9616\end{array}$ & $\begin{array}{l}.1900 \\
.1905 \\
.1911 \\
.1916 \\
.1921\end{array}$ \\
\hline $\begin{array}{l}20 \\
22 \\
24 \\
26 \\
29\end{array}$ & $\begin{array}{l}.9790 \\
.9788 \\
.9787 \\
.9785 \\
.9783\end{array}$ & $\begin{array}{l}.1434 \\
.1410 \\
.1445 \\
.1451 \\
.1456\end{array}$ & $\begin{array}{l}.9737 \\
.9735 \\
.9733 \\
.9731 \\
.9729\end{array}$ & $\begin{array}{l}.1600 \\
.1606 \\
.1611 \\
.1617 \\
.1622\end{array}$ & $\begin{array}{l}.9678 \\
.9676 \\
.9674 \\
.9672 \\
.9670\end{array}$ & $\begin{array}{l}.1765 \\
.1770 \\
.1776 \\
.1781 \\
.1786\end{array}$ & $\begin{array}{l}.9614 \\
.9612 \\
.9609 \\
.9607 \\
.9605\end{array}$ & $\begin{array}{l}.1927 \\
.1932 \\
.193 S \\
.1943 \\
.1948\end{array}$ \\
\hline $\begin{array}{l}30 \\
32 \\
34 \\
36 \\
34\end{array}$ & $\begin{array}{l}.9782 \\
.9780 \\
.9778 \\
.9776 \\
.9775\end{array}$ & $\begin{array}{l}.1462 \\
.1467 \\
.1473 \\
.1479 \\
.1484\end{array}$ & $\begin{array}{l}.9728 \\
.9726 \\
.9724 \\
.9722 \\
.9720\end{array}$ & $\begin{array}{l}.1628 \\
.1633 \\
.1639 \\
.1644 \\
.1650\end{array}$ & $\begin{array}{l}.9668 \\
.9666 \\
.9664 \\
.9662 \\
.9660\end{array}$ & $\begin{array}{l}.1792 \\
.1797 \\
.1803 \\
.1808 \\
.1814\end{array}$ & $\begin{array}{l}.9603 \\
.9600 \\
.9598 \\
.9596 \\
.9593\end{array}$ & $\begin{array}{l}.1954 \\
.1959 \\
.1964 \\
.1970 \\
.1975\end{array}$ \\
\hline $\begin{array}{l}40 \\
42 \\
44 \\
46 \\
48\end{array}$ & $\begin{array}{l}.9773 \\
.9771 \\
.9769 \\
.9768 \\
.9766\end{array}$ & $\begin{array}{l}.1490 \\
.1495 \\
.1501 \\
.1506 \\
.1512\end{array}$ & $\begin{array}{l}.9718 \\
.9716 \\
.9714 \\
.9712 \\
.9710\end{array}$ & $\begin{array}{l}.1655 \\
.1661 \\
.1666 \\
.1672 \\
.1677\end{array}$ & $\begin{array}{l}.9657 \\
.9655 \\
.9653 \\
.9651 \\
.9649\end{array}$ & $\begin{array}{l}.1819 \\
.1824 \\
.1830 \\
.1835 \\
.1841\end{array}$ & $\begin{array}{l}.9591 \\
.9589 \\
.9586 \\
.9584 \\
.9582\end{array}$ & $\begin{array}{l}.1950 \\
.1946 \\
.1991 \\
.1996 \\
.2002\end{array}$ \\
\hline $\begin{array}{l}50 \\
52 \\
54 \\
56 \\
58\end{array}$ & $\begin{array}{l}.9764 \\
.9762 \\
.9761 \\
.9759 \\
.9757\end{array}$ & $\begin{array}{l}.1517 \\
.1523 \\
.1528 \\
.1534 \\
.1540\end{array}$ & $\begin{array}{l}.9708 \\
.9706 \\
.9704 \\
.9702 \\
.9700\end{array}$ & $\begin{array}{l}.1683 \\
.1688 \\
.1694 \\
.1699 \\
.1705\end{array}$ & $\begin{array}{l}.9647 \\
.9645 \\
.9642 \\
.9640 \\
.9638\end{array}$ & $\begin{array}{l}.1846 \\
.1851 \\
.1857 \\
.1862 \\
.18648\end{array}$ & $\begin{array}{l}.9579 \\
.9577 \\
.9575 \\
.9572 \\
.9570\end{array}$ & $\begin{array}{l}.2007 \\
.2012 \\
.2018 \\
.2023 \\
.2028\end{array}$ \\
\hline 60 & .9755 & .1545 & .9698 & .1710 & .9636 & .1573 & .9568 & .2034 \\
\hline $\begin{array}{l}c+f \\
0.75 \\
1.00 \\
1.25\end{array}$ & $\begin{array}{l}0.74 \\
0.99 \\
1.23\end{array}$ & $\begin{array}{l}0.11 \\
0.15 \\
0.18\end{array}$ & $\begin{array}{l}0.74 \\
0.99 \\
1.23\end{array}$ & $\begin{array}{l}0.12 \\
0.16 \\
0.21\end{array}$ & $\begin{array}{l}0.74 \\
0.98 \\
1.23\end{array}$ & $\begin{array}{l}0.14 \\
0.18 \\
0.23\end{array}$ & $\begin{array}{l}0.73 \\
0.98 \\
1.22\end{array}$ & $\begin{array}{l}0.15 \\
0.20 \\
0.25\end{array}$ \\
\hline
\end{tabular}

$(c+\Omega \cos v$ and $(c+\Omega) \sin v$.

Natural funetions. 
TABLE 6.-STADIA COEFFICIENTS, VERTICAL ROD.

$\cos ^{2} v$ and $1 / 2 \sin 2 v$.

\begin{tabular}{|c|c|c|c|c|c|c|c|c|}
\hline & \multicolumn{2}{|c|}{$12^{\circ}$} & \multicolumn{2}{|c|}{$13^{\circ}$} & \multicolumn{2}{|c|}{$14^{\circ}$} & \multicolumn{2}{|c|}{$15^{\circ}$} \\
\hline$=$ & $\begin{array}{l}\text { Hor. } \\
\text { dist. }\end{array}$ & $\begin{array}{l}\text { Diff. } \\
\text { elev. }\end{array}$ & $\begin{array}{l}\text { Hor. } \\
\text { dist. }\end{array}$ & $\begin{array}{l}\text { Diff. } \\
\text { elev. }\end{array}$ & $\begin{array}{l}\text { Hor. } \\
\text { dist. }\end{array}$ & $\begin{array}{l}\text { Diff. } \\
\text { elev. }\end{array}$ & $\begin{array}{l}\text { Hor. } \\
\text { dist. }\end{array}$ & $\begin{array}{l}\text { Diff. } \\
\text { elev. }\end{array}$ \\
\hline , & & & & & & & & \\
\hline 0 & .9568 & .2034 & .9494 & .2192 & .9415 & .2347 & .9330 & .2500 \\
\hline 2 & .9565 & .2039 & .9491 & .2197 & .9412 & .2352 & .9327 & .2505 \\
\hline 4 & .9563 & .2044 & .9489 & .2202 & .9409 & .2358 & .9324 & .2510 \\
\hline 6 & .9561 & .2050 & .9486 & .2208 & .9407 & .2363 & .9321 & .2515 \\
\hline 8 & .9558 & .2055 & .9484 & .2213 & .9404 & .2368 & .9318 & .2520 \\
\hline 10 & .9556 & .2060 & .9481 & .2218 & .9401 & .2373 & .9316 & .2525 \\
\hline 12 & .9553 & 2066 & .9479 & .2223 & .9398 & .2378 & .9313 & .2530 \\
\hline 14 & .9551 & .2071 & .9476 & .2228 & .9395 & .2393 & .9310 & .2535 \\
\hline 16 & .9549 & .2076 & .9473 & .2234 & .9393 & .2388 & .9307 & .2540 \\
\hline 18 & .9546 & .2081 & .9471 & .2239 & .9390 & .2393 & .9304 & .2545 \\
\hline 20 & .9544 & .2087 & .9468 & .2244 & .9387 & . 2399 & .9301 & .2550 \\
\hline 22 & .9541 & .2092 & .9466 & .2249 & .9384 & .2404 & .9298 & .2555 \\
\hline 24 & .9539 & .2097 & .9463 & .2254 & .9381 & .2400 & .9295 & .2560 \\
\hline 26 & .9536 & .2103 & .9460 & .2260 & .9379 & .2414 & .9292 & .2565 \\
\hline 28 & .9534 & .2108 & .9458 & .2265 & .9376 & .2419 & .9289 & .2570 \\
\hline 30 & .9532 & .2113 & .9455 & .2270 & .9373 & .2424 & .9256 & .2575 \\
\hline 32 & .9529 & .2118 & .9452 & .2275 & .9370 & .2429 & .9283 & .2550 \\
\hline 34 & .9527 & .2124 & .9450 & .2280 & .9367 & .2434 & .9280 & $.25 \$ 5$ \\
\hline 36 & .9524 & .2129 & .9447 & .2285 & .9365 & .2439 & .9277 & .2590 \\
\hline 38 & .9522 & .2134 & .9444 & .2291 & .9362 & .2444 & $.92 \pi 4$ & .2595 \\
\hline 40 & .9519 & .2139 & .9442 & .2296 & .9359 & .2449 & .9271 & .2600 \\
\hline 42 & .9517 & .2145 & .9439 & .2301 & .9356 & .2455 & .9268 & .2605 \\
\hline 44 & .9514 & .2150 & .9436 & .2306 & .9353 & .2460 & .9265 & .2610 \\
\hline 46 & .9512 & .2155 & .9434 & .2311 & .9350 & .2465 & .9262 & .2615 \\
\hline 48 & .9509 & .2160 & .9431 & .2316 & .9347 & .2470 & .9259 & .2620 \\
\hline 50 & .9507 & .2166 & .9428 & .2322 & .9345 & .2475 & .9256 & .2625 \\
\hline 52 & .9504 & .2171 & .9426 & .2327 & .9342 & .2480 & .9253 & .2630 \\
\hline 54 & .9502 & .2176 & .9423 & .2332 & .9339 & .2485 & .9249 & .2635 \\
\hline 56 & .9499 & .2181 & .9420 & .2337 & .9336 & .2490 & .9246 & .2640 \\
\hline 58 & .9497 & .2187 & .9417 & .2312 & .9333 & .2495 & .9243 & $.26+15$ \\
\hline 60 & .9494 & .2192 & .9415 & .2347 & .9330 & .2500 & .9240 & .2650 \\
\hline & & & & & & & & \\
\hline 0.75 & 0.73 & 0.16 & 0. 73 & 0.17 & 0.73 & 0.19 & 0.72 & 0.20 \\
\hline 1.00 & 0.98 & 0. 22 & 0.97 & 0.23 & 0.97 & 0.25 & 0.96 & 0.27 \\
\hline 1.25 & 1.22 & 0.27 & 1. 21 & 0.29 & 1. 21 & 0.31 & 1.20 & 0.34 \\
\hline
\end{tabular}

$(c+f) \cos v$ and $(c+f) \sin v$.

Natural functions. 
TABLE 6.-STADIA COEFFICIENTS, VERTICAL ROD.

$\operatorname{Cos}^{2} v$ and $1 / 2 \sin 2 v$.

\begin{tabular}{|c|c|c|c|c|c|c|c|c|}
\hline \multirow[b]{2}{*}{$=$} & \multicolumn{2}{|c|}{$16^{\circ}$} & \multicolumn{2}{|c|}{$17^{\circ}$} & \multicolumn{2}{|c|}{$18^{\circ}$} & \multicolumn{2}{|c|}{$19^{\circ}$} \\
\hline & $\begin{array}{l}\text { Hor. } \\
\text { dist. }\end{array}$ & $\begin{array}{l}\text { Diff. } \\
\text { elev. }\end{array}$ & $\begin{array}{l}\text { IIor. } \\
\text { dist. }\end{array}$ & $\begin{array}{l}\text { Diff. } \\
\text { elev. }\end{array}$ & $\begin{array}{l}\text { Hor. } \\
\text { dist. }\end{array}$ & $\begin{array}{l}\text { Diff. } \\
\text { elev. }\end{array}$ & $\begin{array}{l}\text { Hor. } \\
\text { dist. }\end{array}$ & $\begin{array}{l}\text { Diff. } \\
\text { elev. }\end{array}$ \\
\hline , & & & & & & & & \\
\hline & .9240 & .2650 & .9145 & .2796 & .9045 & .2939 & .8940 & .3078 \\
\hline & .9237 & .2655 & .9142 & 2801 & .9042 & .2944 & 8936 & .3083 \\
\hline & .9234 & .2659 & .9139 & .2806 & .9038 & .29 & .8933 & .3087 \\
\hline & .9231 & .2664 & .9135 & .2810 & - 9035 & .2953 & 8929 & .3092 \\
\hline & .9228 & .2669 & .9132 & .2815 & .9031 & .2958 & .8926 & .3097 \\
\hline 10 & 9225 & .2674 & .9129 & $.2 S 20$ & .9028 & .2962 & 8922 & .3101 \\
\hline 12 & .9222 & .2679 & .9126 & .2825 & .9024 & .2967 & .8918 & .3106 \\
\hline 14 & .9219 & .2684 & .9122 & .2830 & .9021 & .29 & .8915 & .3110 \\
\hline 16 & .9215 & .2689 & .9119 & .2834 & .9018 & .2976 & 8911 & .3115 \\
\hline 18 & .9212 & .2694 & .9116 & .2839 & .9014 & .2981 & .8908 & .3119 \\
\hline 20 & .9209 & .2699 & .9112 & .2844 & 9011 & .2986 & .8904 & .3124 \\
\hline 22 & 9206 & .2704 & 9109 & 2849 & 9007 & .2990 & 8900 & .3128 \\
\hline 24 & .9203 & $.27(19$ & .9106 & .2854 & .9004 & .2995 & .8896 & .3133 \\
\hline 26 & .92100 & .2713 & .9102 & .2858 & $.9000)$ & .3000 & .8893 & .3138 \\
\hline 28 & .9197 & .2718 & .9099 & .2863 & .8997 & .3004 & .8889 & .3142 \\
\hline 30 & .9193 & .2723 & .9096 & .2868 & .8993 & .3009 & .8886 & .3147 \\
\hline 32 & .9190 & .2728 & .9092 & .2873 & .8990 & .30 & .8582 & .3151 \\
\hline 34 & .9187 & .2733 & .9089 & .2877 & .8956 & .30 & .8878 & .3156 \\
\hline 36 & .9184 & .2738 & .9086 & .2882 & .8983 & .30 & .8875 & .3160 \\
\hline 38 & .9181 & .2743 & .9052 & .2887 & .8979 & .3028 & .8871 & .3165 \\
\hline 40 & .9177 & .2748 & .9079 & .2892 & .8976 & .3032 & .8867 & .3169 \\
\hline 42 & .9174 & .2752 & .9076 & .2896 & .8972 & .3037 & .8564 & .3174 \\
\hline 44 & .9171 & .2757 & .9072 & .2901 & .8969 & .3041 & .8840 & .3178 \\
\hline 46 & .91688 & .2702 & .9069 & .2906 & .8965 & $.3046^{\circ}$ & .8856 & .3183 \\
\hline 48 & .9165 & .2767 & .9066 & .2911 & .8901 & .3051 & .8853 & .3157 \\
\hline 50 & .9161 & .2772 & .9062 & .2915 & .8958 & .30.55 & .8849 & .3192 \\
\hline 52 & .0158 & .2777 & .90599 & .2920 & .8954 & .3060 & .8845 & .3196 \\
\hline 54 & .9155 & .2781 & .90555 & .2925 & .8951 & .30655 & .8841 & .3201 \\
\hline 56 & .9152 & .2786 & .9052 & .2930 & .8947 & .3069 & .8838 & .3205 \\
\hline 58 & .9148 & .2791 & .9048 & .2934 & .8944 & .3074 & .8834 & .3209 \\
\hline 60 & .9145 & .2796 & .9045 & .2939 & .8940 & .3078 & $.8 S 30$ & .3214 \\
\hline $\begin{array}{c}c+f \\
0.75\end{array}$ & 0.72 & 0.21 & 0.7 & 0.23 & 0.7 & 0.2 & 0.7 & 0.25 \\
\hline 1.00 & 0.96 & $\begin{array}{l}0.21 \\
0.28\end{array}$ & $\begin{array}{l}0.82 \\
0.95\end{array}$ & $\begin{array}{l}0.23 \\
0.30\end{array}$ & 0.95 & $\begin{array}{l}0.24 \\
0.32\end{array}$ & 0.94 & $\begin{array}{l}0.23 \\
0.33\end{array}$ \\
\hline 1.25 & 1.20 & 0.36 & 1.19 & 0.38 & 1.19 & 0.40 & 1.18 & 0.42 \\
\hline
\end{tabular}

$(c+f) \cos v$ and $(c+f) \sin v$.

Natural functions. 
TABLE 6.-STADIA COEFFICIENTS, VERTICAL ROD.

$\operatorname{Cos}^{2} v$ and $1 / 2 \sin 2 v$.

\begin{tabular}{|c|c|c|c|c|c|c|c|c|}
\hline \multirow[b]{2}{*}{$\begin{array}{l}n+1 \\
4=1\end{array}$} & \multicolumn{2}{|c|}{$20^{\circ}$} & \multicolumn{2}{|c|}{$21^{\circ}$} & \multicolumn{2}{|c|}{$22^{\circ}$} & \multicolumn{2}{|c|}{$23^{\circ}$} \\
\hline & $\begin{array}{l}\text { Hor. } \\
\text { dist. }\end{array}$ & $\begin{array}{l}\text { Diff. } \\
\text { elev, }\end{array}$ & $\begin{array}{l}\text { Hor. } \\
\text { dist. }\end{array}$ & $\begin{array}{l}\text { Diff. } \\
\text { elev. }\end{array}$ & $\begin{array}{l}\text { HIor. } \\
\text { dist. }\end{array}$ & $\begin{array}{l}\text { Ditf. } \\
\text { elev. }\end{array}$ & $\begin{array}{l}\text { IIor. } \\
\text { dist. }\end{array}$ & $\begin{array}{l}\text { Diff. } \\
\text { elev. }\end{array}$ \\
\hline $\begin{array}{l}\text { ' } \\
0 \\
2 \\
4 \\
6 \\
8\end{array}$ & $\begin{array}{l}.8530 \\
.8826 \\
.8823 \\
.8819 \\
.8815\end{array}$ & $\begin{array}{l}.3214 \\
.3218 \\
.3223 \\
.3227 \\
.3232\end{array}$ & $\begin{array}{r}.8716 \\
.8712 \\
.8708 \\
.8704 \\
.8700\end{array}$ & $\begin{array}{l}.3346 \\
.3350 \\
.3354 \\
.3359 \\
.3363\end{array}$ & $\begin{array}{l}.8597 \\
.8593 \\
.8589 \\
.8585 \\
.8580\end{array}$ & $\begin{array}{l}.3473 \\
.3477 \\
.3482 \\
.3486 \\
.3490\end{array}$ & $\begin{array}{l}.8473 \\
.8469 \\
.8465 \\
.8461 \\
.8457\end{array}$ & $\begin{array}{r}.3597 \\
.3601 \\
.3605 \\
.3609 \\
.3613\end{array}$ \\
\hline $\begin{array}{r}10 \\
12 \\
14 \\
16 \\
18\end{array}$ & $\begin{array}{l}.8811 \\
.8508 \\
.8504 \\
.8500 \\
.8796\end{array}$ & $\begin{array}{r}.3236 \\
.3241 \\
.3245 \\
.3249 \\
.3254\end{array}$ & $\begin{array}{l}.8696 \\
.8692 \\
.8688 \\
.8684 \\
.8680\end{array}$ & $\begin{array}{l}.3367 \\
.3372 \\
.3376 \\
.3380 \\
.3384\end{array}$ & $\begin{array}{r}.8576 \\
.8572 \\
.8568 \\
.8564 \\
.8560\end{array}$ & $\begin{array}{l}.3494 \\
.3498 \\
.3502 \\
.3507 \\
.3511\end{array}$ & $\begin{array}{l}.8452 \\
.8448 \\
.8444 \\
.8440 \\
.8435\end{array}$ & $\begin{array}{r}.3617 \\
.3621 \\
.3625 \\
.3629 \\
.3633\end{array}$ \\
\hline $\begin{array}{l}20 \\
22 \\
24 \\
26 \\
28\end{array}$ & $\begin{array}{r}.8793 \\
.8789 \\
.8785 \\
.8781 \\
.8777\end{array}$ & $\begin{array}{l}.3258 \\
.3263 \\
.3267 \\
.3272 \\
.3276\end{array}$ & $\begin{array}{l}.8677 \\
.8673 \\
.8669 \\
.8665 \\
.8661\end{array}$ & $\begin{array}{l}.3389 \\
.3393 \\
.3397 \\
.3401 \\
.3406\end{array}$ & $\begin{array}{l}.8556 \\
.8552 \\
.8548 \\
.8544 \\
.8540\end{array}$ & $\begin{array}{r}.3515 \\
.3519 \\
.3523 \\
.3527 \\
.3531\end{array}$ & $\begin{array}{l}.8431 \\
.8427 \\
.8423 \\
.8418 \\
.8414\end{array}$ & $\begin{array}{r}.3637 \\
.3641 \\
.3645 \\
.3649 \\
.3653\end{array}$ \\
\hline $\begin{array}{l}30 \\
32 \\
34 \\
36 \\
38\end{array}$ & $\begin{array}{r}.8774 \\
.8770 \\
.8766 \\
.8762 \\
.8758\end{array}$ & $\begin{array}{l}.3280 \\
.3285 \\
.3289 \\
.3293 \\
.3298\end{array}$ & $\begin{array}{r}.8657 \\
.8653 \\
.8649 \\
.8645 \\
.8641\end{array}$ & $\begin{array}{l}.3410 \\
.3414 \\
.3418 \\
.3423 \\
.3427\end{array}$ & $\begin{array}{r}.8536 \\
.8531 \\
.8527 \\
.8523 \\
.8519\end{array}$ & $\begin{array}{l}.3536 \\
.3540 \\
.3544 \\
.3548 \\
.3552\end{array}$ & $\begin{array}{l}.8410 \\
.8406 \\
.8401 \\
.8397 \\
.8393\end{array}$ & $\begin{array}{r}.3657 \\
.3661 \\
.3665 \\
.3669 \\
.3673\end{array}$ \\
\hline $\begin{array}{r}40 \\
42 \\
44 \\
46 \\
48\end{array}$ & $\begin{array}{l}.8754 \\
.8751 \\
.8747 \\
.8743 \\
.8739\end{array}$ & $\begin{array}{l}.3302 \\
.3307 \\
.3311 \\
.3315 \\
.3320\end{array}$ & $\begin{array}{l}.8637 \\
.8633 \\
.8629 \\
.8625 \\
.8621\end{array}$ & $\begin{array}{l}.3431 \\
.3435 \\
.3440 \\
.3444 \\
.3448\end{array}$ & $\begin{array}{r}.8515 \\
.8511 \\
.8507 \\
.8502 \\
.8498\end{array}$ & $\begin{array}{r}.3556 \\
.3560 \\
.3564 \\
.3568 \\
.3572\end{array}$ & $\begin{array}{l}.8389 \\
.8384 \\
.8380 \\
.8376 \\
.8372\end{array}$ & $\begin{array}{l}.3677 \\
.3680 \\
.36884 \\
.3688 \\
.3692\end{array}$ \\
\hline $\begin{array}{l}50 \\
52 \\
54 \\
56 \\
58\end{array}$ & $\begin{array}{l}.8735 \\
.8731 \\
.8727 \\
.8724 \\
.8720\end{array}$ & $\begin{array}{l}.3324 \\
.3328 \\
.3333 \\
.3337 \\
.3341\end{array}$ & $\begin{array}{l}.8617 \\
.8613 \\
.8609 \\
.8605 \\
.8601\end{array}$ & $\begin{array}{l}.3452 \\
.3457 \\
.3461 \\
.3465 \\
.3469\end{array}$ & $\begin{array}{l}.8494 \\
.8490 \\
.8456 \\
.8482 \\
.8477\end{array}$ & $\begin{array}{l}.3576 \\
.3580 \\
.3585 \\
.3589 \\
.3593\end{array}$ & $\begin{array}{l}.8367 \\
.8363 \\
.8359 \\
.8354 \\
.8350\end{array}$ & $\begin{array}{l}.3696 \\
.3700 \\
.3704 \\
.3708 \\
.3712\end{array}$ \\
\hline 60 & .8716 & . 3340 & .8597 & .3473 & .8473 & .3597 & .8346 & .3716 \\
\hline $\begin{array}{l}c+f \\
0.75 \\
1.00 \\
1.25\end{array}$ & $\begin{array}{l}0.70 \\
0.94 \\
1.17\end{array}$ & $\begin{array}{l}0.26 \\
0.35 \\
0.44\end{array}$ & $\begin{array}{l}0.70 \\
0.93 \\
1.16\end{array}$ & $\begin{array}{l}0.27 \\
0.37 \\
0.46\end{array}$ & $\begin{array}{l}0.69 \\
0.92 \\
1.15\end{array}$ & $\begin{array}{l}0.29 \\
0.38 \\
0.48\end{array}$ & $\begin{array}{l}0.69 \\
0.92 \\
1.15\end{array}$ & $\begin{array}{l}0.30 \\
0.40 \\
0.50\end{array}$ \\
\hline
\end{tabular}

$(c+f) \cos v$ and $(c+f) \sin v$.

Natural functions. 
TABLE 6.-STADIA COEFFICIENTS, VERTICAL ROD.

$\cos ^{2} v$ and $1 / 3 \sin 2 v$.

\begin{tabular}{|c|c|c|c|c|c|c|c|c|}
\hline$=$ & $\omega$ & & - & $5^{\circ}$ & & & 2 & $7^{\circ}$ \\
\hline 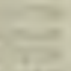 & $\begin{array}{l}\text { Hor. } \\
\text { dist. }\end{array}$ & $\begin{array}{l}\text { Diff. } \\
\text { elev. }\end{array}$ & $\begin{array}{l}\text { Hor. } \\
\text { dist. }\end{array}$ & $\begin{array}{l}\text { Difr. } \\
\text { elev. }\end{array}$ & $\begin{array}{l}\text { Hor. } \\
\text { dist. }\end{array}$ & $\begin{array}{l}\text { Didr. } \\
\text { elev. }\end{array}$ & $\begin{array}{l}\text { Hor. } \\
\text { dist. }\end{array}$ & $\begin{array}{l}\text { Diff. } \\
\text { elev. }\end{array}$ \\
\hline , & $y=$ & & & & & & & \\
\hline $\begin{array}{l}0 \\
2 \\
4 \\
6 \\
8\end{array}$ & $\begin{array}{l}.8346 \\
.8341 \\
.8337 \\
.8333 \\
.8328\end{array}$ & $\begin{array}{l}.3716 \\
.3720 \\
.3723 \\
.3727 \\
.3731\end{array}$ & $\begin{array}{l}.8214 \\
.8209 \\
.8205 \\
.8201 \\
.8196\end{array}$ & $\begin{array}{l}.3530 \\
.3534 \\
.3538 \\
.3541 \\
.3545\end{array}$ & $\begin{array}{l}.8078 \\
.8074 \\
.8069 \\
.80655 \\
.8060\end{array}$ & $\begin{array}{l}.3940 \\
.3944 \\
.3947 \\
.3951 \\
.3954\end{array}$ & $\begin{array}{l}.7939 \\
.7934 \\
.7930 \\
.7925 \\
.7920\end{array}$ & $\begin{array}{r}.4045 \\
.4049 \\
.4052 \\
.4055 \\
.4059\end{array}$ \\
\hline $\begin{array}{l}10 \\
12 \\
14 \\
16 \\
18\end{array}$ & $\begin{array}{l}.8324 \\
.8320 \\
.8315 \\
.8311 \\
.8307\end{array}$ & $\begin{array}{r}.3735 \\
.3739 \\
.3743 \\
.3747 \\
.3751\end{array}$ & $\begin{array}{l}.8192 \\
.8187 \\
.8183 \\
.8178 \\
.8174\end{array}$ & $\begin{array}{l}.3849 \\
.3853 \\
.3856 \\
.3860 \\
.3864\end{array}$ & $\begin{array}{l}.8055 \\
.8051 \\
.8046 \\
.8041 \\
.8037\end{array}$ & $\begin{array}{l}.3958 \\
.3961 \\
.3965 \\
.3969 \\
.3972\end{array}$ & $\begin{array}{l}.7915 \\
.7911 \\
.7906 \\
.7901 \\
.7896\end{array}$ & $\begin{array}{r}.4062 \\
.4066 \\
.4069 \\
.4072 \\
.4076\end{array}$ \\
\hline $\begin{array}{l}20 \\
22 \\
24 \\
26 \\
25\end{array}$ & $\begin{array}{l}.8302 \\
.8298 \\
.8293 \\
.8259 \\
.8255\end{array}$ & $\begin{array}{l}.3754 \\
.3758 \\
.3762 \\
.3766 \\
.3770\end{array}$ & $\begin{array}{l}.8169 \\
.8165 \\
.8160 \\
.8156 \\
.8151\end{array}$ & $\begin{array}{l}.3967 \\
.3571 \\
.3575 \\
.3578 \\
.3552\end{array}$ & $\begin{array}{l}.8032 \\
.8029 \\
.8023 \\
.8018 \\
.8014\end{array}$ & $\begin{array}{r}.3976 \\
.3979 \\
.3983 \\
.3986 \\
.3990\end{array}$ & $\begin{array}{l}.7892 \\
.7887 \\
.7882 \\
.7877 \\
.7873\end{array}$ & $\begin{array}{r}.4079 \\
.4052 \\
.4086 \\
.4089 \\
.4092\end{array}$ \\
\hline $\begin{array}{l}30 \\
32 \\
34 \\
36 \\
39\end{array}$ & $\begin{array}{l}.8280 \\
.8276 \\
.8272 \\
.8267 \\
.8263\end{array}$ & $\begin{array}{l}.3774 \\
.3777 \\
.3781 \\
.3785 \\
.3789\end{array}$ & $\begin{array}{l}.8147 \\
.8142 \\
.8139 \\
.8133 \\
.8128\end{array}$ & $\begin{array}{l}.3586 \\
.3589 \\
.3893 \\
.3897 \\
.3900\end{array}$ & $\begin{array}{l}.8009 \\
.8004 \\
.8000 \\
.7995 \\
.7990\end{array}$ & $\begin{array}{l}.3993 \\
.3997 \\
.4000 \\
.4004 \\
.4007\end{array}$ & $\begin{array}{r}.7864 \\
.7863 \\
.7858 \\
.7854 \\
.7849\end{array}$ & $\begin{array}{r}.4096 \\
.4099 \\
.4102 \\
.4106 \\
.4109\end{array}$ \\
\hline $\begin{array}{l}40 \\
42 \\
44 \\
413 \\
43\end{array}$ & $\begin{array}{l}.8259 \\
.8254 \\
.8249 \\
.8245 \\
.8241\end{array}$ & $\begin{array}{l}.3793 \\
.3796 \\
.3500 \\
.3504 \\
.3508\end{array}$ & $\begin{array}{l}.8124 \\
.8119 \\
.8115 \\
.8110 \\
.8106\end{array}$ & $\begin{array}{l}.3904 \\
.3908 \\
.3911 \\
.3915 \\
.3918\end{array}$ & $\begin{array}{l}.7956 \\
.7951 \\
.7976 \\
.7972 \\
.7967\end{array}$ & $\begin{array}{l}.4011 \\
.4014 \\
.4018 \\
.4021 \\
.4024\end{array}$ & $\begin{array}{l}.7844 \\
.7839 \\
.7834 \\
.7830 \\
.7825\end{array}$ & $\begin{array}{r}.4112 \\
.4116 \\
.4119 \\
.4122 \\
.4126\end{array}$ \\
\hline $\begin{array}{l}50 \\
52 \\
54 \\
56 \\
58\end{array}$ & $\begin{array}{l}.8236 \\
.8232 \\
.8227 \\
.8223 \\
.8215\end{array}$ & $\begin{array}{l}.3511 \\
.3515 \\
.3519 \\
.3523 \\
.3526\end{array}$ & $\begin{array}{l}.8101 \\
.8097 \\
.8092 \\
.8057 \\
.8083\end{array}$ & $\begin{array}{l}.3922 \\
.3926 \\
.3929 \\
.3933 \\
.3936\end{array}$ & $\begin{array}{l}.7962 \\
.7958 \\
.7953 \\
.7948 \\
.7944\end{array}$ & $\begin{array}{l}.4028 \\
.4031 \\
.4035 \\
.4038 \\
.4042\end{array}$ & $\begin{array}{l}.7820 \\
.7815 \\
.7810 \\
.7806 \\
.7801\end{array}$ & $\begin{array}{r}.4129 \\
.4132 \\
.4135 \\
.4139 \\
.4142\end{array}$ \\
\hline 60 & .8214 & .3330 & .8078 & . 3940 & .7039 & .4045 & .7796 & .4145 \\
\hline $\begin{array}{l}c+f \\
0.75 \\
1.00 \\
1.25\end{array}$ & $\begin{array}{l}0.68 \\
0.91 \\
1.14\end{array}$ & $\begin{array}{l}0.31 \\
0.41 \\
0 \quad 52\end{array}$ & $\begin{array}{l}0.68 \\
0.90 \\
1.13\end{array}$ & $\begin{array}{l}0.32 \\
0.43 \\
0.54\end{array}$ & $\begin{array}{l}0.67 \\
0.89 \\
1.12\end{array}$ & $\begin{array}{l}0.33 \\
0.45 \\
0.56 j\end{array}$ & $\begin{array}{l}0.66 \\
0.89 \\
1.11\end{array}$ & $\begin{array}{l}0.35 \\
0.46 \\
0.58\end{array}$ \\
\hline
\end{tabular}

$(c+f) \cos v$ and $(c+f) \sin v$.

Natural functions. 
TABLE 7.-NATURAL SINES AND COSINES.

\begin{tabular}{|c|c|c|c|c|c|c|c|c|c|c|c|}
\hline \multirow{2}{*}{ I } & \multicolumn{2}{|c|}{$0^{\circ}$} & \multicolumn{2}{|c|}{$1^{\circ}$} & \multicolumn{2}{|c|}{$2^{\circ}$} & \multicolumn{2}{|c|}{$3^{\circ}$} & \multicolumn{2}{|c|}{$4^{\circ}$} & \\
\hline & Sine. & Cosin. & Sine. & Cosin. & Sine. & Cosin. & Sine. & Cosin. & Sine. & Cosin. & \\
\hline 0 & .00030 & One. & .01745 & .99985 & .03490 & $\overline{.99939}$ & .05234 & .99863 & .06976 & \multicolumn{2}{|c|}{.9975660} \\
\hline 1) & .00029 & One. & .01774 & .99984 & .03519 & .99938 & .05263 & .99561 & .07005 & \multicolumn{2}{|c|}{.9975459} \\
\hline 2 & .00058 & One. & .01803 & .99984 & .03548 & .99937 & .05292 & .99860 & .07034 & \multicolumn{2}{|c|}{.9975258} \\
\hline 3 & .00087 & One. & .01832 & .99953 & .03577 & .99336 & .05321 & .99858 & .07063 & \multicolumn{2}{|c|}{.9975057} \\
\hline 4 & .00116 & One. & .01862 & .99983 & .03600 & .99935 & .05350 & .99857 & .07092 & 99748 & 56 \\
\hline 5 & .00145 & One. & .01891 & .99982 & .03635 & .99934 & .05379 & .99855 & .07121 & .99746 & 55 \\
\hline 6 & .00175 & One. & .01920 & .99982 & .03664 & .99933 & .05408 & .99854 & .07150 & .99744 & 54 \\
\hline 7 & .00204 & One. & .01949 & .93981 & .03693 & .93932 & .05437 & .99352 & .07179 & .99742 & 53 \\
\hline 8 & .00233 & One. & .01978 & .99980 & .03723 & .99931 & .05466 & .99851 & .07208 & .99740 & 52 \\
\hline 9 & .00262 & One. & .02007 & .93380 & .03752 & .99930 & .05495 & .99849 & .07237 & .99738 & 51 \\
\hline 10 & .00291 & One. & .02036 & .99979 & .03781 & .99929 & .05524 & .99847 & .07266 & .99736 & 50 \\
\hline 11 & .00320 & .99999 & .02065 & .99979 & .03810 & .99927 & .05553 & .99846 & .07295 & .99734 & 49 \\
\hline 12 & .00349 & .93999 & .02094 & .99378 & .03839 & .99926 & .05582 & .99844 & .07324 & 31 & 8 \\
\hline 13 & .00378 & .93399 & .02123 & .99977 & .03868 & .93925 & .05611 & .99842 & .07353 & 29 & \\
\hline 14 & .00407 & 99999 & .02152 & .93977 & .03897 & .99924 & .05640 & .99841 & .07382 & .99727 & 46 \\
\hline 15 & .00436 & .99939 & .02181 & .99976 & . 03926 & .99923 & .05669 & . 99839 & .07411 & 725 & 45 \\
\hline 16 & .00465 & .99999 & .02211 & . 93976 & .03955 & .99922 & .05698 & . 99838 & .07440 & .99723 & 44 \\
\hline 17 & .00495 & .99999 & .02240 & .99975 & .03984 & .99921 & .05727 & .99836 & .07469 & .99721 & 43 \\
\hline 18 & .00524 & .99999 & .02269 & .99974 & .04013 & .99919 & .05756 & .99834 & .07498 & .99719 & 42 \\
\hline 19 & .00553 & .99998 & .02298 & .99974 & .04042 & .99918 & .05785 & .99833 & .07527 & 716 & 41 \\
\hline 20 & .00582 & .99998 & .02327 & .99973 & .04071 & .99917 & .05814 & .99831 & .07556 & 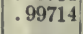 & 40 \\
\hline 21 & .00611 & .99998 & .02356 & .99972 & .04100 & .99916 & .05844 & .99829 & $.075 S 5$ & .99712 & 39 \\
\hline 22 & .00640 & .9 & 385 & .99972 & .04129 & 915 & 5873 & 827 & .07614 & 10 & \\
\hline 23 & .00669 & .99998 & .02414 & .99971 & .04159 & .99913 & .05902 & 99826 & .07643 & 08 & 37 \\
\hline 24 & .00698 & .99998 & .02443 & .99970 & .04188 & .99912 & .05931 & .99824 & .07672 & 05 & 36 \\
\hline 25 & .00727 & .99997 & .02472 & .99969 & .04217 & .99911 & .05960 & .99822 & .07701 & 03 & 35 \\
\hline 26 & .00756 & .99997 & .02501 & .99969 & .04246 & .99910 & .05989 & .99521 & .07730 & .99701 & 34 \\
\hline 27 & .00785 & .99997 & .02530 & $.993 t 8$ & .04275 & .99909 & .06018 & .99819 & .07759 & $99 t i 93$ & 33 \\
\hline 28 & .00814 & .99397 & .02560 & .99967 & .04304 & .99907 & .06047 & .99817 & .07788 & 86 & 32 \\
\hline 29 & .00844 & .99996 & .02589 & .99966 & .04333 & .99906 & .06076 & .99815 & .07817 & .99694 & 31 \\
\hline 30 & .00873 & .99996 & .02618 & .99966 & .04362 & .99905 & .06105 & .99813 & .07846 & .9 & 30 \\
\hline 31 & .00302 & .99996 & .02647 & .99965 & .04391 & .99901 & .06134 & .99812 & .07875 & 89 & 29 \\
\hline 32 & .00931 & .99996 & .02676 & .99964 & .04420 & .99902 & .06163 & .99810 & .07904 & & 28 \\
\hline 33 & .00360 & .93995 & .02705 & .99963 & .04449 & .99901 & .06192 & $.9980 \mathrm{~S}$ & .07933 & 65 & 27 \\
\hline 34 & .00989 & .99395 & .02734 & .99963 & .04478 & .99900 & .06221 & .99806 & .07962 & 83 & 26 \\
\hline 35 & .01018 & .99995 & .02763 & .99962 & .04507 & .9 & .06250 & 04 & .07991 & 80 & 25 \\
\hline 30 & .01047 & .99995 & .02792 & .99961 & .04536 & .99897 & .06279 & .99803 & .08020 & 78 & 24 \\
\hline 37 & .01076 & .99394 & .02821 & .99360 & .04565 & .99896 & .06308 & .99801 & .08049 & 76 & 23 \\
\hline 38 & .01105 & .99394 & .02850 & .99959 & .04594 & .99894 & 37 & .99799 & 88078 & & 22 \\
\hline 39 & .01134 & .93994 & .02879 & .99950 & .04623 & .99893 & .06366 & .99797 & .08107 & .99671 & 21 \\
\hline 40 & .01164 & .99993 & .02908 & .99958 & .01653 & .99892 & .06395 & .99795 & .08136 & .99668 & 20 \\
\hline 41 & .01193 & .99933 & .02938 & .99957 & .04682 & .99890 & .06421 & .99793 & .08165 & .99666 & 19 \\
\hline 42 & .01222 & & .02967 & .99956 & .04711 & .99889 & .06453 & .99792 & .08194 & & 18 \\
\hline 43 & . 01251 & .9 & .02996 & .99955 & .04740 & & .0 & .9 & . 08223 & 9 & 17 \\
\hline 41 & .01280 & .99392 & .03025 & .99954 & .04769 & .9 & .06511 & .99788 & 8252 & 39 & 16 \\
\hline 45 & .01309 & .99991 & .03054 & .99953 & .04798 & .9 & 40 & .99786 & .08281 & & 15 \\
\hline 46 & .01338 & .99991 & .03083 & .99952 & . 04827 & .99883 & .06569 & .99784 & .08310 & & 14 \\
\hline 47 & .01367 & .99991 & .03112 & .93952 & .04856 & .99882 & $.0659 \mathrm{~S}$ & .99782 & .08339 & & \\
\hline 48 & .01396 & .99 & 141 & .99951 & .04885 & .99881 & .06627 & .99780 & . 05368 & & 12 \\
\hline 49 & .01425 & .99390 & .03170 & .99950 & .04914 & . .99879 & .06056 & .99778 & .08397 & & \\
\hline 50 & .01454 & .99989 & .03199 & 99949 & .04943 & .99878 & .06685 & 99776 & .08426 & .99044 & 10 \\
\hline 51 & .01483 & .99989 & .03228 & .99948 & .04972 & .99876 & .06714 & .99774 & .08455 & .99642 & 9 \\
\hline 52 & & & & & & & & & & & \\
\hline 53 & .01542 & .99388 & .03286 & .99946 & .05030 & .99573 & .0 & .99770 & .08513 & & 7 \\
\hline 51 & .01571 & & .03316 & .99945 & .05059 & & .06802 & & .08542 & & 6 \\
\hline 55 & .01600 & .99987 & .03345 & .99944 & .05088 & .99870 & .06831 & .99766 & .08571 & .99632 & 5 \\
\hline 56 & & & .03374 & .9 .2943 & .05117 & .99869 & .06860 & .99764 & .08600 & & \\
\hline 57 & .01658 & .99986 & .03403 & .99342 & .05146 & .99567 & . OCR899 & .99762 & .08629 & .99627 & 3 \\
\hline 58 & .01687 & .39986 & .03432 & .93941 & & & .06918 & .99760 & .08658 & & 2 \\
\hline 59 & .01716 & .99955 & .03461 & .99940 & .05205 & .99 & .06947 & .99758 & .08687 & .99622 & 1 \\
\hline 60 & .01745 & .99955 & .03190 & .99939 & .05234 & .99863 & .06976 & .99756 & .08716 & .99619 & 0 \\
\hline & Cosin. & Sine. & Cosin. & Sine. & $\operatorname{Cosin}$ & Sine. & $\overline{\text { Cosin. }}$ & Sine. & Cosin. & Sine. & \\
\hline & & & 88 & $8^{\circ}$ & & & & 86 & & 85 & \\
\hline
\end{tabular}


TABLE 7.-NATURAI, SINES AND COSINES.

\begin{tabular}{|c|c|c|c|c|c|c|c|c|c|c|c|}
\hline \multirow[t]{2}{*}{, } & \multicolumn{2}{|c|}{$5^{\circ}$} & \multicolumn{2}{|c|}{$6^{\circ}$} & \multicolumn{2}{|c|}{$7^{\circ}$} & \multicolumn{2}{|c|}{$8^{\circ}$} & \multicolumn{2}{|l|}{9} & \\
\hline & c. & & Sine. & in. & ine. & osin. & ine. & osin. & ine. & osin. & \\
\hline $\begin{array}{r}0 \\
1 \\
2 \\
3 \\
4 \\
5 \\
6 \\
7 \\
8 \\
9 \\
10\end{array}$ & $\begin{array}{l}.08716 \\
.08745 \\
.08774 \\
.08503 \\
.08831 \\
.08560 \\
.08889 \\
.05918 \\
.08947 \\
.08976 \\
.09005\end{array}$ & $\begin{array}{l}.99619 \\
.99617 \\
.99614 \\
.99612 \\
.99609 \\
.99607 \\
.99604 \\
.99602 \\
.99599 \\
.99596 \\
.99594\end{array}$ & $\begin{array}{l}.10453 \\
.10482 \\
.10511 \\
.10540 \\
.10569 \\
.10597 \\
.10626 \\
.10655 \\
.10684 \\
.10713 \\
.10742\end{array}$ & $\begin{array}{l}.99452 \\
.99449 \\
.99446 \\
.99443 \\
.99440 \\
.99437 \\
.99434 \\
.99431 \\
.99428 \\
.99424 \\
.99421\end{array}$ & $\begin{array}{l}.12187 \\
.12216 \\
.12245 \\
.12274 \\
.12302 \\
.12331 \\
.12300 \\
.12389 \\
.12418 \\
.12447 \\
.12476\end{array}$ & $\begin{array}{l}.99255 \\
.93251 \\
.99248 \\
.93244 \\
.99240 \\
.99237 \\
.99233 \\
.99230 \\
.99226 \\
.99222 \\
.99219\end{array}$ & $\begin{array}{l}.13917 \\
.13946 \\
.13975 \\
.14004 \\
.14033 \\
.14061 \\
.14090 \\
.14119 \\
.14148 \\
.14177 \\
.14205\end{array}$ & $\begin{array}{l}.99027 \\
.99023 \\
.99019 \\
.99015 \\
.99011 \\
.99006 \\
.99002 \\
.98998 \\
.98994 \\
.98990 \\
.98986\end{array}$ & $\begin{array}{l}.15643 \\
.15672 \\
.15701 \\
.15730 \\
.15758 \\
.15787 \\
.15816 \\
.15545 \\
.15873 \\
.15902 \\
.15931\end{array}$ & .081 & \\
\hline $\begin{array}{l}11 \\
12 \\
13 \\
14 \\
1.5 \\
16 \\
17 \\
18 \\
19 \\
20\end{array}$ & $\begin{array}{l}.09034 \\
.09063 \\
.09092 \\
.09121 \\
.09150 \\
.09179 \\
.09208 \\
.09237 \\
.09266 \\
.09295\end{array}$ & $\begin{array}{l}.99591 \\
.99588 \\
.99566 \\
.99553 \\
.99580 \\
.99578 \\
.99575 \\
.99572 \\
.99570 \\
.99567\end{array}$ & & & & & $\begin{array}{l}.14234 \\
.14263 \\
.14292 \\
.14320 \\
.14349 \\
14378 \\
.14407 \\
14436 \\
14464 \\
.14493\end{array}$ & & & & \\
\hline $\begin{array}{l}21 \\
22 \\
23 \\
24 \\
25 \\
26 \\
27 \\
28 \\
29 \\
30\end{array}$ & & & & & & $\begin{array}{l}.92178 \\
.99175 \\
.93171 \\
.99167 \\
.99163 \\
.99160 \\
.99156 \\
.99152 \\
.99148 \\
.99144\end{array}$ & & & & & \\
\hline $\begin{array}{l}31 \\
32 \\
33 \\
34\end{array}$ & & & & & & & & $\begin{array}{l}.95897 \\
.95593 \\
.95859 \\
.95884 \\
.95550 \\
.98576 \\
.95871 \\
.95567 \\
.95563 \\
.98555\end{array}$ & & & \\
\hline $\begin{array}{l}41 \\
42\end{array}$ & & & & & & $\begin{array}{l}.99102 \\
.99095 \\
.99194 \\
.99091 \\
.99087 \\
.990<3 \\
.99079 \\
.99075 \\
99071\end{array}$ & & & & & \\
\hline $\begin{array}{l}51 \\
52 \\
53\end{array}$ & $\begin{array}{l}10192 \\
.10221 \\
.10250 \\
.10279 \\
.10305 \\
10337 \\
10360 \\
10395 \\
.10424 \\
10453\end{array}$ & $m=0$ & $\begin{array}{l}.11927 \\
.11956 \\
.11985 \\
.12014 \\
.12043 \\
12071 \\
.12100 \\
.12129 \\
12158\end{array}$ & 9258 & & & $\begin{array}{l}15385 \\
.15414 \\
15442 \\
.15471 \\
15500 \\
.15529\end{array}$ & $\begin{array}{l}.95809 \\
.98405 \\
.95800 \\
.98796 \\
.95791 \\
.95787 \\
.98752 \\
.95775 \\
.95773 \\
.98769\end{array}$ & $\begin{array}{l}.17107 \\
.17136 \\
.17164 \\
.17193 \\
.17222 \\
.17250 \\
.17279 \\
.17305 \\
.17336 \\
.17365\end{array}$ & $\begin{array}{l}.98484 \\
.98481\end{array}$ & $\begin{array}{l}7 \\
6 \\
5 \\
4 \\
3 \\
2 \\
1 \\
0\end{array}$ \\
\hline & $\sin$. & - & . & & 10. & & n. & sit & n. & Norite. & \\
\hline & \multicolumn{2}{|c|}{$81^{\circ}$} & \multicolumn{2}{|c|}{8.3} & \multicolumn{2}{|c|}{$82^{\circ}$} & \multicolumn{2}{|c|}{$81^{\circ}$} & \multicolumn{2}{|c|}{$80^{\circ}$} & \\
\hline
\end{tabular}


TABLF 7.-NATURAL SINES AND COSINES.

\begin{tabular}{|c|c|c|c|c|c|c|c|c|c|c|c|}
\hline \multirow{2}{*}{, } & \multicolumn{2}{|c|}{$10^{\circ}$} & \multicolumn{2}{|c|}{$11^{\circ}$} & \multicolumn{2}{|c|}{$12^{\circ}$} & \multicolumn{2}{|c|}{$13^{\circ}$} & \multicolumn{2}{|c|}{$14^{\circ}$} & \\
\hline & Sine. & Cosin. & Sine. & Cosin. & Sine. & Cosin. & Sine. & Cosin. & Sine. & Cosin. & \\
\hline 0 & .17365 & .98181 & .19081 & .98163 & .20791 & .97815 & .22495 & .97437 & .24192 & .97030 & 60 \\
\hline 1 & .17393 & .98476 & .19109 & .98157 & .20820 & .97809 & .22523 & .97430 & .24220 & & 59 \\
\hline 2 & .17422 & .98471 & .19138 & .98152 & .20848 & .97803 & .22552 & .97424 & .24249 & .97015 & \\
\hline 3 & .17451 & .98466 & .19167 & .98146 & .20877 & .97797 & .22580 & .97417 & .24277 & .97008 & 57 \\
\hline 4 & .17479 & .98461 & .19195 & .98140 & .20905 & .97791 & .22608 & .97411 & .24305 & .97001 & 56 \\
\hline 5 & .17508 & .98155 & .19224 & .98135 & .20933 & .97781 & .22637 & .97404 & .24333 & .96994 & 55 \\
\hline 6 & .17537 & .98450 & .19252 & .98129 & .20962 & .97778 & .22665 & .97398 & .24362 & .96987 & 54 \\
\hline 7 & .17565 & .98445 & .19281 & .98124 & .20990 & .97772 & .22693 & .97391 & .24390 & .96950 & \\
\hline 8 & .17594 & .98440 & .19309 & .98118 & .21019 & .97766 & .22722 & .97384 & .24418 & .96973 & 52 \\
\hline 9 & .17623 & .98435 & .19338 & .98112 & .21047 & .97760 & .22750 & .97378 & .24446 & & \\
\hline 10 & .17651 & .98430 & .19366 & .98107 & .21076 & .97754 & . 22778 & .97371 & .24474 & .96959 & 50 \\
\hline 11 & $.176 S 0$ & 425 & .19395 & .98101 & .21104 & .97748 & .22807 & .97365 & .24503 & .96952 & 49 \\
\hline 12 & .17708 & & 4i23 & & .21132 & & -22835 & .9 & .24531 & & 48 \\
\hline $\begin{array}{l}13 \\
14\end{array}$ & $\begin{array}{l}17737 \\
17766\end{array}$ & 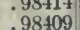 & $\begin{array}{r}.19+52 \\
.19481\end{array}$ & .98090 & $\begin{array}{r}.21101 \\
.21189\end{array}$ & .97735 & $\begin{array}{r}.22503 \\
.22592\end{array}$ & .97351 & $\begin{array}{r}.24559 \\
.24587\end{array}$ & .96930 & 47 \\
\hline 15 & 17794 & .98404 & .19509 & .95079 & .21218 & .97723 & .22920 & .97338 & .24615 & .96923 & 45 \\
\hline 16 & .17823 & .98399 & . 19538 & .98073 & .21246 & .97717 & .22948 & .97331 & .24644 & .96916 & 44 \\
\hline 17 & .17852 & .98394 & . 19566 & .98067 & .21275 & .97711 & . 22977 & .97325 & .24672 & .96909 & 43 \\
\hline 18 & .17880 & . 933S9 & . 19595 & .95061 & .21303 & .97705 & .23005 & .97318 & .24700 & . 96902 & 42 \\
\hline 19 & . 17909 & .98383 & . 19623 & .98056 & .21331 & .97698 & . 23033 & .97311 & .24728 & .96894 & 41 \\
\hline 20 & .17937 & .98378 & .19652 & .98050 & .21360 & .97692 & .23062 & .97304 & .24756 & | 96887 & 40 \\
\hline 21 & .17966 & .98373 & .19680 & .95044 & .21388 & .97686 & .23090 & .97298 & .24784 & .96880 & 39 \\
\hline 22 & .17995 & .98368 & .19709 & .98039 & .21417 & .97650 & .23118 & .97291 & .24813 & 8873 & 38 \\
\hline 23 & .18023 & .98362 & . 19737 & .98033 & .21445 & .97673 & .23146 & .97284 & .24841 & & 37 \\
\hline 24 & .18052 & . 98357 & .19766 & .98027 & .21474 & .97667 & .23175 & .97278 & .24869 & .96858 & 36 \\
\hline 25 & . 18081 & .98352 & . 19794 & .95021 & .21502 & .97661 & .23203 & .97271 & .24897 & .96851 & 35 \\
\hline 26 & . 18109 & . 98347 & .19823 & .98016 & .21530 & .97655 & .23231 & .97264 & .24925 & .96844 & 34 \\
\hline 27 & . 18138 & . 98341 & . 19851 & .98010 & .21559 & .97618 & .23260 & .97257 & .24954 & .96837 & 33 \\
\hline 28 & .18166 & .98336 & . 19880 & .98004 & .21587 & .97012 & .23288 & .97251 & .24982 & & 32 \\
\hline 29 & .18195 & .98331 & . 19908 & .97998 & .21616 & .97636 & .23316 & .97244 & .25010 & .90 & 31 \\
\hline 30 & 224 & .9 & 37 & 92 & 14 & 30 & .23345 & .97237 & .25038 & .9 & 30 \\
\hline 31 & .18252 & .98320 & . 19965 & .97987 & .21672 & .97623 & .23373 & .97230 & .25066 & .9 & 29 \\
\hline 32 & .18281 & .9 & 994 & .9 & 701 & 17 & .23401 & 23 & 994 & 00 & 28 \\
\hline 33 & . 18309 & .98310 & .20022 & .97975 & .21729 & .9 & .23429 & . 97217 & .25122 & & 27 \\
\hline 34 & . 18338 & . 98304 & .20051 & .97969 & .21758 & .97604 & .23458 & .97210 & .25151 & .9 & 26 \\
\hline 35 & . 18367 & . 98299 & . 20079 & .97963 & 786 & .9 & .23186 & .97203 & . 25179 & .9 & 25 \\
\hline 36 & . 18395 & .98294 & . 20108 & .97958 & .21814 & .97592 & .23514 & .97196 & .25207 & .96771 & 24 \\
\hline 37 & . 18424 & .95288 & .20136 & .97952 & 1843 & .97585 & .23542 & .97189 & .25235 & & 23 \\
\hline 38 & .18452 & .98283 & . 20165 & .97946 & .21871 & .97579 & .23571 & .97182 & .25263 & .96756 & 22 \\
\hline 39 & .18481 & .99277 & .20193 & .97940 & 1899 & .97573 & .23599 & .97176 & .25291 & .96749 & 21 \\
\hline 40 & . 18509 & 272 & & & & .97566 & .23627 & .97169 & 25320 & .967 & 0 \\
\hline 41 & .18538 & .98267 & .20250 & .97928 & .21956 & .97500 & .23656 & .97162 & .25348 & .96734 & 19 \\
\hline 42 & & 61 & & .8 & & & & & 376 & & \\
\hline 43 & . 18595 & .98256 & .20307 & .97916 & .22013 & .97547 & .23712 & .97148 & .25404 & .96719 & 17 \\
\hline 44 & . 18624 & .9 & .20336 & .97910 & 011 & 1 & .23740 & 141 & .25432 & & 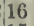 \\
\hline 45 & . 18652 & .98245 & .20364 & .97905 & .22070 & .97534 & .23769 & .97134 & .25460 & .96705 & 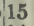 \\
\hline 46 & . 18681 & .98240 & .20393 & .97899 & 2098 & & .23797 & & 188 & .96697 & 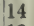 \\
\hline 47 & . 18710 & .98234 & .20421 & .97893 & .22126 & .97521 & .23825 & .97120 & 516 & .96690 & 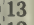 \\
\hline 48 & . 18738 & 229 & .20450 & & 155 & & .23853 & .97113 & 515 & & \\
\hline 49 & . 18767 & .98223 & 478 & .9 & 2183 & .97508 & .23882 & 06 & 773 & 5 & 11 \\
\hline 50 & . 18795 & .98218 & . 20507 & . 97875 & 22212 & .97502 & 10 & 100 & .25601 & .96667 & 10 \\
\hline 51 & .18824 & .98212 & .20535 & .97869 & 22240 & .97496 & .23938 & .97093 & . 25629 & .96660 & 9 \\
\hline 52 & & & & & & & & & 5657 & & 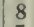 \\
\hline 53 & . 18851 & - 98201 & .20592 & .97857 & .22297 & .97483 & & .97079 & .25685 & & 7 \\
\hline 54 & . 18910 & .98196 & .20620 & .97851 & 22325 & .97476 & .24023 & .97072 & .25713 & .96638 & 6 \\
\hline 55 & . 18938 & .98190 & .20649 & .97845 & .22353 & .97470 & .24051 & .97065 & .25741 & & 5 \\
\hline 56 & . 18367 & .98185 & .20677 & .97839 & .22382 & .97463 & . 24079 & $.97 \mathrm{C}$ & . 25769 & .96623 & 4 \\
\hline 57 & . 18995 & .98179 & 20706 & . 97833 & .22410 & .97457 & . 24108 & .97051 & . 25798 & & 3 \\
\hline 58 & . 19024 & . 98174 & 20734 & . 97827 & 2438 & .97450 & . 24136 & 44 & .25826 & .96 & 2 \\
\hline 59 & .19052 & . 98168 & 20763 & .97821 & 2467 & .97444 & . 24164 & .97037 & . 25854 & . 96600 & 1 \\
\hline 60 & . 19081 & .98163 & . 20791 & .97815 & .22495 & .97437 & . 24192 & .97030 & .25852 & .96593 & 0 \\
\hline \multirow{2}{*}{ ' } & Cosin. & Sine. & Cosin. & Sine. & CDSTI. & Sine. & Cosin. & Sine. & Cosin. & Sine. & \\
\hline & \multicolumn{2}{|c|}{$79^{\circ}$} & \multicolumn{2}{|c|}{$78^{\circ}$} & 78 & & 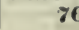 & & 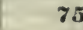 & & \\
\hline
\end{tabular}


TABLE 7.-NATURAL SINES AND COSINES.

\begin{tabular}{|c|c|c|c|c|c|c|c|c|c|c|c|}
\hline \multirow{2}{*}{, } & \multicolumn{2}{|c|}{$15^{\circ}$} & \multicolumn{2}{|c|}{$16^{\circ}$} & \multicolumn{2}{|c|}{$17^{\circ}$} & \multicolumn{2}{|c|}{$18^{\circ}$} & \multicolumn{2}{|c|}{$10^{\circ}$} & \\
\hline & Sine. & n. & Sine. & $\mathrm{Co}$ & Sine. & Cosin. & Sine. & Cosin. & Sine. & Cosin. & \\
\hline 0 & $.25 \times 82$ & 393 & . 27564 & 96126 & .29237 & 630 & .30902 & 95106 & .32557 & $1552 \mathrm{C}$ & \\
\hline 1 & & & & & & & & & & & \\
\hline 2 & 938 & & .27620 & & & & .30957 & & 612 & & \\
\hline 3 & & & $.2764 S$ & & 321 & & .30985 & & .32639 & & \\
\hline 4 & & & .27676 & & & & .31012 & 070 & .32667 & & \\
\hline 5 & .26022 & & .27704 & & & & & & . 32694 & & \\
\hline 6 & & & .27731 & & & & .31068 & & .32722 & & \\
\hline 7 & .26079 & & .27759 & & & & & & .32749 & & \\
\hline 8 & & & .27787 & & & & .31123 & 5033 & . 32777 & & \\
\hline 9 & & & & & & & & & . 32s04 & & \\
\hline 10 & .26 & & .27843 & 16 & 15 & & .3 & 015 & .32832 & & \\
\hline 11 & .26191 & & .27871 & $-\frac{9}{g}$ & 543 & 6 & .31206 & 006 & . 32859 & & \\
\hline $\begin{array}{l}12 \\
13\end{array}$ & & & & & & & & & & & \\
\hline $\begin{array}{l}13 \\
14\end{array}$ & 47. & & & & 96 & & $\cdot 3$ & & 32914 & & \\
\hline 15 & .26303 & 79 & .27983 & & .29654 & & .31316 & 70 & $\begin{array}{l}32942 \\
32969\end{array}$ & & \\
\hline 16 & & & & & 32 & & & & .32997 & & \\
\hline 17 & 59 & 103 & $.2 \$ 039$ & & 10 & & 72 & 52 & .33024 & & \\
\hline 18 & .26 & & .25067 & & 0.0 & & & & .33051 & & \\
\hline 19 & .26415 & & . 28095 & & .29765 & 67 & & 33 & .33079 & & \\
\hline 20 & .26443 & 10 & .28123 & 4 & .29 & 39 & 54 & 24 & .33106 & & 40 \\
\hline 21 & $.264 \pi 1$ & 433 & .28150 & .95956 & .29921 & 5450 & .31482 & 4915 & .33134 & & \\
\hline 22 & & & 78 & & 49 & & & & 161 & & \\
\hline 23 & .26 & & .2 & & .29876 & & & & . 33189 & & \\
\hline 24 & 56 & & .28234 & & .29904 & & & & . 33216 & & \\
\hline 25 & & & .23262 & & .29932 & & 93 & & .33244 & & \\
\hline 20 & & & .28290 & & .29960 & & & & . 33271 & & \\
\hline 27 & & & .28 & & .29 & & & & 298 & 293 & \\
\hline 28 & & & & & .30 & & & & & & \\
\hline 29. & & & & & 33 & & & & 53 & & \\
\hline 30 & . & & .2 & & 71 & & & & 81 & & \\
\hline 31 & .26752 & .9 & .2 & .8 & .3 & 63 & $5 S$ & 23 & 3408 & & \\
\hline & & & & & 6 & & & & & & \\
\hline 33 & & & & & 44 & & & & & & \\
\hline 34 & & & & & & & & & 90 & & \\
\hline 35 & & & & & & & & & & & \\
\hline 3 & .2 & & & & .3 & & & & 45 & & \\
\hline ? & & & & & & & & & & & \\
\hline 38 & 48 & & & & 2 & & & & 00 & & \\
\hline 39 & 2 & & .28 & & & & & & & & \\
\hline 40 & - 2 & 80. & .28650 & & & 84 & 06 & 40 & 55 & & \\
\hline 41 & .27032 & .9 & .2 & .8 & 6 & & . 32034 & & 82 & & 19 \\
\hline 42 & & & & & & & & & & & \\
\hline 43 & & & & & & & & & 37 & & \\
\hline 44 & & & & & & & & & & & \\
\hline 45 & & & & & & & & & & & \\
\hline 46 & & & & & & & & & & & \\
\hline 4i & & & & & & & & & & & \\
\hline & & & & & & & & & & & \\
\hline 49 & & & & & 7 & & & & & & \\
\hline 50 & & & & & & & & & 29 & & \\
\hline 51 & .27312 & .8 & 387 & .8 & 33 & .95186 & 309 & & 56 & .94058 & \\
\hline & & & & & & & & & & & \\
\hline & & & & & & & & & & & \\
\hline 54 & & & & & & & & & & & \\
\hline 55 & & & & & & & & & & & \\
\hline $56 i$ & & & & & & & & & & & \\
\hline & & & & & & & & & & & \\
\hline & & & & & & & & & & & \\
\hline 70 & & & & & & & & & & & \\
\hline w & .27064 & & & & & & 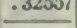 & & & & \\
\hline \multirow{2}{*}{ 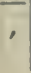 } & Cosin. & Sine. & Cosin. & Sine. & Cosin. & Sine. & Cosin. & Sine. & Cosin. & Sine. & \\
\hline & \multicolumn{2}{|c|}{$74^{\circ}$} & \multicolumn{2}{|c|}{$73^{\circ}$} & \multicolumn{2}{|c|}{$72^{\circ}$} & 7 & $1^{\circ}$ & & & \\
\hline
\end{tabular}


TABLE 7.-NATURAL SINES AND COSINES.

\begin{tabular}{|c|c|c|c|c|c|c|c|c|c|c|c|}
\hline \multirow[t]{2}{*}{1} & \multicolumn{2}{|c|}{$20^{\circ}$} & \multicolumn{2}{|c|}{$21^{\circ}$} & \multicolumn{2}{|c|}{$22^{\circ}$} & \multicolumn{2}{|c|}{$23^{\circ}$} & \multicolumn{2}{|c|}{$21^{\circ}$} & \\
\hline & Sine. & osin. & Sine. & osin. & ine. & Cosin. & ine. & osin. & ine. & $\sin$. & \\
\hline $\begin{array}{r}0 \\
1 \\
2 \\
3 \\
4 \\
5 \\
6 \\
7 \\
8 \\
9 \\
10\end{array}$ & $\begin{array}{l}.34202 \\
.34229 \\
.34257 \\
.34284 \\
.34311 \\
.34339 \\
.34360 \\
.34393 \\
.34421 \\
.34448 \\
.34475\end{array}$ & $\begin{array}{r}.93899 \\
.93889 \\
.93879 \\
.93869\end{array}$ & $\begin{array}{l}.35837 \\
.35864 \\
.35891 \\
.35918 \\
.35945 \\
.35973 \\
.36000 \\
.36027 \\
.36054 \\
.36081 \\
.36108\end{array}$ & $\begin{array}{l}.93295 \\
.93285 \\
.93274 \\
.93264 \\
.93253\end{array}$ & $\begin{array}{l}.37461 \\
.37488 \\
.37515 \\
.37542 \\
.37569 \\
.37595 \\
.37622 \\
.37649 \\
.37676 \\
.37703 \\
.37730\end{array}$ & $\begin{array}{l}.92718 \\
.92707 \\
.92697 \\
.92686 \\
.92675 \\
.92664 \\
.92653 \\
.92642 \\
.92631 \\
.92620 \\
.92609\end{array}$ & $\begin{array}{l}.39073 \\
.39100 \\
.39127 \\
.39153 \\
.39180 \\
.39207 \\
.39234 \\
.39260 \\
.39287 \\
.39314 \\
.39341\end{array}$ & $\begin{array}{l}.92050 \\
.92039 \\
.92028 \\
.92016 \\
.92005 \\
.91994 \\
.91952 \\
.91971 \\
.91959 \\
.91948 \\
.91936\end{array}$ & $\begin{array}{r}.40674 \\
.40700 \\
.40727 \\
.40753 \\
.40780 \\
.40806 \\
.40833 \\
.40860 \\
.40886 \\
.40913 \\
.40939\end{array}$ & .01200 & \\
\hline $\begin{array}{l}11 \\
12 \\
13 \\
14 \\
15 \\
16 \\
17 \\
18 \\
19 \\
20\end{array}$ & $\begin{array}{r}.34639 \\
.34666 \\
.34694 \\
.34721 \\
.34748\end{array}$ & $\begin{array}{l}.93859 \\
.93849 \\
.93839 \\
.93829 \\
.93819 \\
.93809 \\
.93799 \\
.93789 \\
.93779 \\
.93769\end{array}$ & & $\begin{array}{r}93243 \\
.93232 \\
.93222\end{array}$ & $\begin{array}{l}.37757 \\
.37784 \\
.37811 \\
.37838 \\
.37865 \\
.37892 \\
.37919 \\
.37946 \\
.37973 \\
.37999\end{array}$ & $\begin{array}{r}.92598 \\
.92587 \\
.92576 \\
.92565 \\
.92554 \\
.92543 \\
.92532 \\
.92521 \\
.92510 \\
.92499\end{array}$ & & & & & \\
\hline $\begin{array}{l}21 \\
22 \\
23 \\
24 \\
25 \\
26 \\
27 \\
28 \\
29 \\
30\end{array}$ & $\begin{array}{l}.34775 \\
.34803 \\
.34830 \\
.34857 \\
.34884 \\
.34912 \\
.34939 \\
.34966 \\
.34993 \\
.35021\end{array}$ & $\begin{array}{l}\cdot \\
. \\
. \\
. \\
. \\
. \\
.8\end{array}$ & & $\begin{array}{r}93052 \\
.93042\end{array}$ & $\begin{array}{l}.38026 \\
.38053 \\
.38080 \\
.38107 \\
.38134 \\
.38161 \\
.38188 \\
.38215 \\
.38241 \\
.38268\end{array}$ & $\begin{array}{r}.92488 \\
.92477 \\
.92466 \\
.92455 \\
.92444 \\
.92432 \\
.92421 \\
.92410 \\
.92399 \\
.92388\end{array}$ & & & & & ) \\
\hline $\begin{array}{l}31 \\
32 \\
33\end{array}$ & $\begin{array}{l}.35048 \\
.35075 \\
.35102 \\
.35130 \\
.35157 \\
.35184 \\
.35211 \\
.35239 \\
.35266 \\
.35293\end{array}$ & & & & & $\begin{array}{l}.92377 \\
.92366 \\
.92355 \\
.92343 \\
.92332 \\
.92321 \\
.92310 \\
.92299 \\
.92287 \\
.92276\end{array}$ & & & & & 20 \\
\hline $\begin{array}{l}41 \\
42\end{array}$ & $\begin{array}{l}.35320 \\
.35347 \\
.35375 \\
.35402 \\
.35429 \\
.35456 \\
.35484 \\
35511\end{array}$ & & & $\begin{array}{l}.92924 \\
.92913 \\
.92902 \\
.92892 \\
.92881 \\
.92870 \\
.92859 \\
92849\end{array}$ & $\begin{array}{l}.38564 \\
.38591 \\
.38617 \\
.38644 \\
.38671 \\
.38698 \\
.38725 \\
.38752 \\
.38778 \\
.38505\end{array}$ & $\begin{array}{r}.92265 \\
.92254 \\
.92243 \\
.92231 \\
.92220 \\
.92209 \\
.92195 \\
.92186 \\
.92175 \\
.92164\end{array}$ & & & & & \\
\hline & $\begin{array}{r}.35592 \\
.35619 \\
.35647 \\
.35674 \\
.35701 \\
.35728 \\
.35755 \\
.35782\end{array}$ & $\begin{array}{l}.934 \\
.94 \\
.934 \\
.934 \\
.934 \\
.934 \\
.933 \\
.933\end{array}$ & $\begin{array}{l}.37218 \\
.37245 \\
.37272 \\
.37299 \\
.37326 \\
.37353 \\
.37380 \\
.37407 \\
37434\end{array}$ & $\begin{array}{l}.92816 \\
.92305 \\
.92794 \\
.92784 \\
.92773 \\
.92762 \\
.92751 \\
.92740 \\
.92729 \\
.92718 \\
\end{array}$ & $\begin{array}{l}.38832 \\
.38559 \\
.35586 \\
.38912 \\
.38939 \\
.35966 \\
.35993 \\
.39020 \\
.39046 \\
.39073\end{array}$ & $\begin{array}{l}.92152 \\
.92141 \\
.92130 \\
.92119 \\
.92107 \\
.92096 \\
.92085 \\
.92073 \\
.92062 \\
.92050\end{array}$ & $\begin{array}{l}.40434 \\
.40461 \\
.40488 \\
.40514 \\
.40541 \\
.40567 \\
.40594 \\
.40621 \\
.40647 \\
.40674\end{array}$ & & $\begin{array}{l}.42024 \\
.42051 \\
.42077 \\
.42104 \\
.42130 \\
.42156 \\
.42183 \\
.42209 \\
.42235 \\
.42262 \\
\end{array}$ & $\begin{array}{r}.90717 \\
.90704 \\
.90692 \\
.90680 \\
.90668 \\
.90655 \\
.90643 \\
.90631 \\
\end{array}$ & \\
\hline & Cosin. & Sine. & osin. & $\mathrm{Si}$ & osin. & Sine. & osin. & Slue. & Cosin. & Sine. & \\
\hline & \multicolumn{2}{|c|}{$69^{\circ}$} & \multicolumn{2}{|c|}{$68^{\circ}$} & \multicolumn{2}{|c|}{$67^{\circ}$} & \multicolumn{2}{|c|}{66} & \multicolumn{2}{|c|}{65} & \\
\hline
\end{tabular}


TABLE 7.-NATURAL SINES AND COSIXES.

\begin{tabular}{|c|c|c|c|c|c|c|c|c|c|c|c|}
\hline \multirow{2}{*}{ ' } & \multicolumn{2}{|c|}{$25^{\circ}$} & \multicolumn{2}{|c|}{$26^{\circ}$} & \multicolumn{2}{|c|}{$27^{\circ}$} & \multicolumn{2}{|c|}{$28^{\circ}$} & \multicolumn{2}{|c|}{$29^{\circ}$} & \multirow{2}{*}{ ' } \\
\hline & Sine. & Cosin. & Sine. & Cosin. & Sine. & Cosin. & Sine. & Cosin. & Siue. & Cosin. & \\
\hline 0 & .42262 & .90631 & .43837 & .89579 & .45399 & 89101 & .46947 & $.8 \times 295$ & .48451 & .87462 & 60 \\
\hline 1 & .42285 & .90618 & .43863 & .89567 & $.4 j+25$ & 3087 & .46973 & .85251 & .48506 & 7448 & \\
\hline 2 & .42315 & .90606 & .43859 & .824854 & .4 .5451 & .89074 & . 46999 & .85267 & .485332 & .87434 & \\
\hline 3 & .42341 & .90594 & .43916 & .89841 & .45477 & .89061 & .47024 & .88254 & . 48555 & .87420 & \\
\hline 4 & .42367 & .90582 & . 43942 & .89828 & . 45503 & 9048 & . 47050 & .88240 & $.485 \times 3$ & & \\
\hline 5 & .42394 & .90569 & . 43968 & .89816 & .45529 & .89035 & .47976 & .88226 & .48600 & 87391 & \\
\hline 6 & .42420 & $.9055 \pi$ & . 43994 & .89803 & . 45554 & .89021 & .47101 & .88213 & . 48634 & .87377 & \\
\hline 7 & .42446 & .90545 & .44020 & .89790 & .45580 & .89008 & .47127 & .88199 & .48659 & .87363 & 53 \\
\hline 8 & .42473 & .00532 & .44046 & .89777 & .45606 & . 88995 & .47153 & .88185 & .45684 & .87349 & \\
\hline 9 & .42499 & .90520 & .44072 & .89764 & .45632 & . 88981 & . 47178 & .88172 & .48710 & .873335 & 51 \\
\hline 10 & .42525 & .90507 & .44 & .89752 & . 45058 & . 88968 & .47204 & .88158 & .48735 & .87321 & 9 \\
\hline 11 & .42552 & .90495 & . 44124 & .89739 & .45684 & . 88955 & .47229 & .88144 & .48761 & .87306 & 49 \\
\hline 12 & .42578 & .90483 & .44151 & 726 & .45710 & 88942 & .47255 & .88130 & .48786 & 7292 & \\
\hline 13 & .42604 & .90470 & . 44177 & .89713 & .45736 & .88928 & .47281 & .88117 & . 48811 & .87278 & \\
\hline 14 & .42631 & .90455 & . 44203 & .89700 & .45762 & .85915 & .47306 & .88103 & .48837 & .87264 & \\
\hline 15 & .42657 & .90446 & .44229 & . 89687 & .45787 & .88902 & . 47332 & .88089 & . 488662 & .87250 & \\
\hline 16 & .42683 & .90433 & . 44255 & .89674 & . 45813 & .88588 & .47358 & .88075 & .48858 & .87235 & 44 \\
\hline 17 & .42709 & .90421 & .44281 & .89662 & $.45 \$ 39$ & .88875 & .47383 & .88062 & .48913 & .87221 & \\
\hline 18 & .42736 & .90408 & :44307 & .89649 & .458615 & .88862 & .47409 & .88048 & .45938 & .87207 & 12 \\
\hline 19 & .42762 & .90396 & . 44333 & .89636 & .45891 & .88545 & .47434 & .85034 & .45904 & .87193 & \\
\hline 20 & .42788 & .90383 & . 44359 & .89623 & 17) & 35 & .47460 & .88020 & . 48989 & .8 & 40 \\
\hline 21 & .42815 & .90371 & .44385 & .89610 & .45942 & .83822 & .47456 & .88006 & . 49014 & .87 & 39 \\
\hline 22 & .42841 & & & & & & .47511 & 993 & .49040 & & \\
\hline 23. & .42567 & .90346 & . 44437 & .89584 & . 45994 & 8795 & .47537 & .87979 & .49065 & 36 & 37 \\
\hline 24 & .42894 & .90334 & .44464 & .89571 & .46020 & .88782 & .47562 & .87965 & .49090 & .87121 & 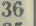 \\
\hline 25 & .42920 & .90321 & 14490 & .89558 & .46046 & .88768 & .47588 & .87951 & .49116 & .87107 & \\
\hline 26 & .42946 & .90309 & .44516 & .89545 & .46072 & .88755 & .47614 & .87937 & .49141 & .87093 & 3 \\
\hline 27 & .42972 & .90296 & .44542 & .89532 & . 46097 & .88741 & .47639 & .87923 & .49166 & .87 & \\
\hline 28 & .42999 & .90284 & . 445lis & .89519 & .46123 & .88728 & .47665 & .87909 & .49192 & .87064 & 3 \\
\hline 29 & .43025 & .90271 & . 44594 & .89506 & .46149 & .88715 & . 47690 & .87896 & .49217 & 50 & 31 \\
\hline 30 & .43051 & .90259 & 620 & .89493 & .46175 & .88701 & .47716 & .87882 & .49242 & .87036 & 0 \\
\hline 31 & .43077 & .90246 & .44646 & .89480 & .46201 & .88688 & .47741 & .87868 & .49268 & 87021 & 29 \\
\hline 32 & .43104 & 233 & 672 & 467 & 6226 & 74 & 767 & 54 & . 49293 & & \\
\hline 33 & .43130 & .90221 & . 44698 & $.894 i \mathrm{~A}$ & .46252 & .886661 & .47793 & .87840 & .49318 & 86993 & \\
\hline 34 & .43156 & .90208 & .44724 & .89441 & .46278 & .85647 & . 47818 & .87820 & . 49344 & & \\
\hline 35 & .43182 & .90196 & . 44750 & .89428 & . 4630-4 & .83634 & .47844 & .87812 & . 43369 & 86 & 25 \\
\hline 36 & .43209 & .90183 & .44776 & .89415 & .46330 & .89620 & .47869 & .87798 & .49394 & & \\
\hline 37 & .43235 & .90171 & .44502 & .89402 & . $4(i 355$ & .88607 & .47 & .87784 & .49419 & 5 & 23 \\
\hline 38 & .43261 & .90158 & $.44 \$ 25$ & .89389 & .46381 & .88593 & .47920 & .87770 & .49445 & 86921 & 2 \\
\hline 39 & .432287 & .90146 & .44854 & $.893 \% 6$ & .46407 & so & .47 & .87756 & .49470 & 81 & 21 \\
\hline 40 & .43313 & .90133 & .44880 & .89363 & . 46433 & 566 & .47971 & .87743 & .49495 & 86892 & 20 \\
\hline 41 & .43340 & .90120 & .44906 & .89350 & .46458 & .88553 & .47997 & .87729 & .49521 & 86878 & 19 \\
\hline 42 & 366 & 108 & 2) & 7 & & & 2 & 15 & 46. & & \\
\hline 43 & .43392 & .90095 & . 44958 & .89324 & .46510 & .88526 & 48045 & .87701 & .49571 & & 17 \\
\hline 44 & .43418 & .9 & .4 & .8 & 6 & 88512 & $.4 \times 073$ & .8 & .49596 & & \\
\hline 45 & .43445 & .90070 & . 45010 & $.8929 \mathrm{~B}$ & . 46561 & .88499 & . 48099 & .87673 & .49622 & 20 & 15 \\
\hline 46 & .43471 & .90057 & . 45036 & .8 & .46587 & $.8848^{-1}$ & .48124 & .87659 & .49647 & & \\
\hline 47 & .43497 & .90045 & .45062 & .89272 & .46613 & .88472 & .48150 & .87645 & $496 \pi 2$ & 91 & 3 \\
\hline 48 & .4 & .90032 & .45058 & .8 & . 46639 & & & .8 & 49697 & & \\
\hline 49 & . 43549 & .90019 & .45114 & .89245 & .460604 & 88445 & . 4S201 & .87617 & . 49723 & 2 & 11 \\
\hline 50 & .43575 & .90007 & .45140 & .89232 & .46690 & .85431 & .48226 & .87603 & .49748 & .86748 & 0 \\
\hline 51 & .43602 & .89994 & .45160 & .89219 & . 46716 & .88417 & .45252 & .87559 & .49773 & 86733 & 9 \\
\hline 52 & & .89981 & & .89206 & .46742 & .88404 & $.4527 \%$ & & .49798 & 19 & $\gamma$ \\
\hline 53 & .43654 & .89968 & .4521s & .89193 & .46767 & .88390 & $.4 \times 303$ & 61 & .49524 & .86704 & 7 \\
\hline 54 & .43680 & .89956 & .45243 & .89180 & . 46793 & .88377 & . $4 \times 328$ & .87546 & .49849 & $.80 \times 000$ & 6 \\
\hline 55 & 43706 & .89943 & . 45269 & & . 46819 & $.8 \times 363$ & .48354 & .87532 & $.498 \% 4$ & .886675 & 5 \\
\hline 56 & .43733 & .89930 & .45295 & .89153 & .46844 & .88349 & .48379 & $.8751 \mathrm{~s}$ & .49599 & .80661 & 4 \\
\hline 57 & . 43759 & .80918 & . 45321 & .89140 & . 46:870 & .89336 & .48405 & .87504 & . 49924 & $.86640^{\prime}$ & \\
\hline 58 & 43785 & .89905 & $.4534 \pi$ & 89127 & .46996 & $.8 \times 322$ & .45430 & .87 .400 & .49950 & . 84632 & 2 \\
\hline 59 & 43811 & .89592 & .452373 & .89114 & . 46:921 & $.8 \times 30 \mathrm{~S}$ & . AS456i & .87476 & . 499975 & .86617 & 1 \\
\hline 60 & $.4353 \pi$ & .89879 & . 45399 & .89101 & .46947 & .88295 & . 48481 & .87462 & .50000 & .86603 & 0 \\
\hline \multirow{2}{*}{, } & Cosin. & Sine. & Cosin. & Sine. & Cosin. & Sine. & Cosin. & Sine. & Cosin. & Sine. & \\
\hline & \multicolumn{2}{|c|}{$64^{\circ}$} & \multicolumn{2}{|c|}{$63^{\circ}$} & & & & & & & \\
\hline
\end{tabular}


TABLE 7.-NATURAL SINES AND COSINES.

\begin{tabular}{|c|c|c|c|c|c|c|c|c|c|c|c|}
\hline \multirow{2}{*}{ ' } & \multicolumn{2}{|c|}{$30^{\circ}$} & \multicolumn{2}{|c|}{$31^{\circ}$} & \multicolumn{2}{|c|}{$32^{\circ}$} & \multicolumn{2}{|c|}{$33^{\circ}$} & \multicolumn{2}{|c|}{$31^{\circ}$} & \\
\hline & Sine. & Cosin. & Sine. & Cosin. & Sine. & Cosin. & Sine. & Cosin. & Sine. & Cosin. & \\
\hline 0 & .50000 & & 604 & 17 & 992 & & 1464 & & 55919 & & 00 \\
\hline 1 & & & & & & & & & & & \\
\hline 2 & .50050 & & & 87 & 041 & & 513 & & $.5596 \mathrm{~S}$ & & \\
\hline 3 & .50076 & & & & & & 537 & & 55992 & & \\
\hline 4 & .50101 & & & 657 & 091 & & .54561 & & .56016 & & \\
\hline 5 & .50126 & & & & 3115 & & & & .56040 & & \\
\hline 6 & .50151 & & & & & & .54610 & 3772 & .56064 & & \\
\hline 7 & .50176 & & & & .53164 & & & & & & \\
\hline 8 & .50201 & & & 597 & .53189 & & 659 & 740 & .56112 & & \\
\hline 9 & .5 & & & & & & & & & & \\
\hline 10 & .50 & & & & 8 & & & & 80 & & \\
\hline 11 & .5 & - & 78 & 51 & 63 & & 732 & & 184 & & \\
\hline 13 & & & & & & & & & & & \\
\hline 14 & $\begin{array}{r}.00027 \\
.50352\end{array}$ & & & & 3312 & & & & & & \\
\hline 15 & .50377 & 841 & 77 & 491 & .53361 & & 54829 & .8 & .56250 & & 45 \\
\hline 16. & 50403 & & & & .53386 & & & & & & \\
\hline 17 & & .8 & & 161 & .53411 & & & & 29 & & \\
\hline 18 & .5 & & & & 3435 & & 02 & & & & \\
\hline 19 & .504 & & & 31 & .53460 & & 27 & & 377 & & \\
\hline 20 & .50503 & & & 116 & 84 & 5 & 51 & .8 & .56101 & 77. & \\
\hline 21 & .50528 & 295 & 2026 & 401 & .53509 & 0 & 54975 & 33 & 56425 & & 39 \\
\hline 22 & .50553 & & & & & & & & 49 & & \\
\hline 23 & .5 & 6 & s. & & 8 & & 24 & 1 & 73 & & \\
\hline 24 & .5 & & & & & & & & & & \\
\hline 2 & .5 & .8 & & & 07 . & & & & & & \\
\hline 27 & .50679 & & & & & & & & 45 & & \\
\hline 28 & 04 & & & & & & & & & & \\
\hline 29 & 0729 & 8 & & 9 & 5 & & & & & & \\
\hline 30 & .50754 & 63 & 0 & & 30 & a & 94 & .83 & .56641 & & 30 \\
\hline 31 & .50779 & 48 & 75 & 249 & .53754 & \# & 18 & 73 & 65 & & 2 \\
\hline & & & & & & & & & & & \\
\hline 33 & .5 & & & & 4 & & & & & & \\
\hline 34 & .50854 & 4 & 9 & & .5 & 7 & 01 & & 36 & 7 & \\
\hline 3.5 & .5 & & & & & & & & & & \\
\hline 36 & .5 & 4 & 9 & 173 & 77 & & 39 & & 84 & 4 & 2 \\
\hline $\begin{array}{l}37 \\
38\end{array}$ & .5 & 0 & & & 2 & & 3 & & & & \\
\hline & & & & & 66 & & & & & & 2 \\
\hline 40 & & & & & & & 12 & & 66 & 8221 it & 2 \\
\hline 41 & 29 & 000 & 2 & mat & & & & & & & \\
\hline 42 & & & & & & & 4460 & 12 & 04 & 2231 & \\
\hline 43 & & & & & & & & & & & \\
\hline 44 & & & & & & & & & & & \\
\hline 45 & .51 & & i & & & & 37 & & & & \\
\hline 40 & & & & & & & & & & & \\
\hline 47 & & 1 & 1 & & & & & & & & \\
\hline & & & & & & & & & & & $i$ \\
\hline 48 & & & & & & & & & & & \\
\hline 50 & & & & & & & & & & & 10 \\
\hline 51 & 279 & 851 & 80 & & 4 & .8 & .55702 & & & & 9 \\
\hline & & & & & & & & & & & \\
\hline 53 & & & & & & & 75 & & & & \\
\hline & & & 4 & & 7 & & 5 & & 15 & & \\
\hline & & 2 & & & & & & & & & \\
\hline 56 & & & 3 & & 66 & & 23 & & 62 & & \\
\hline & & & & & & & & & & & \\
\hline & & & & & & & & & & & \\
\hline 59 & & & & & & & & & & & \\
\hline 60 & 04 & 00117 & 52992 & 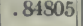 & 54464 & .83867 & 55919 & .82904 & . 57358 & 81915 & 0 \\
\hline & Cosin. & Sine. & Cosin. & Sine. & Cosin. & Sine. & Cosin. & Sine & Cosin. & Sine. & \\
\hline & \multicolumn{2}{|c|}{$59^{\circ}$} & & & \multicolumn{2}{|c|}{56} & 55 & & \\
\hline
\end{tabular}


TABLE 7.-NATIRAL SINES AND COSINES.

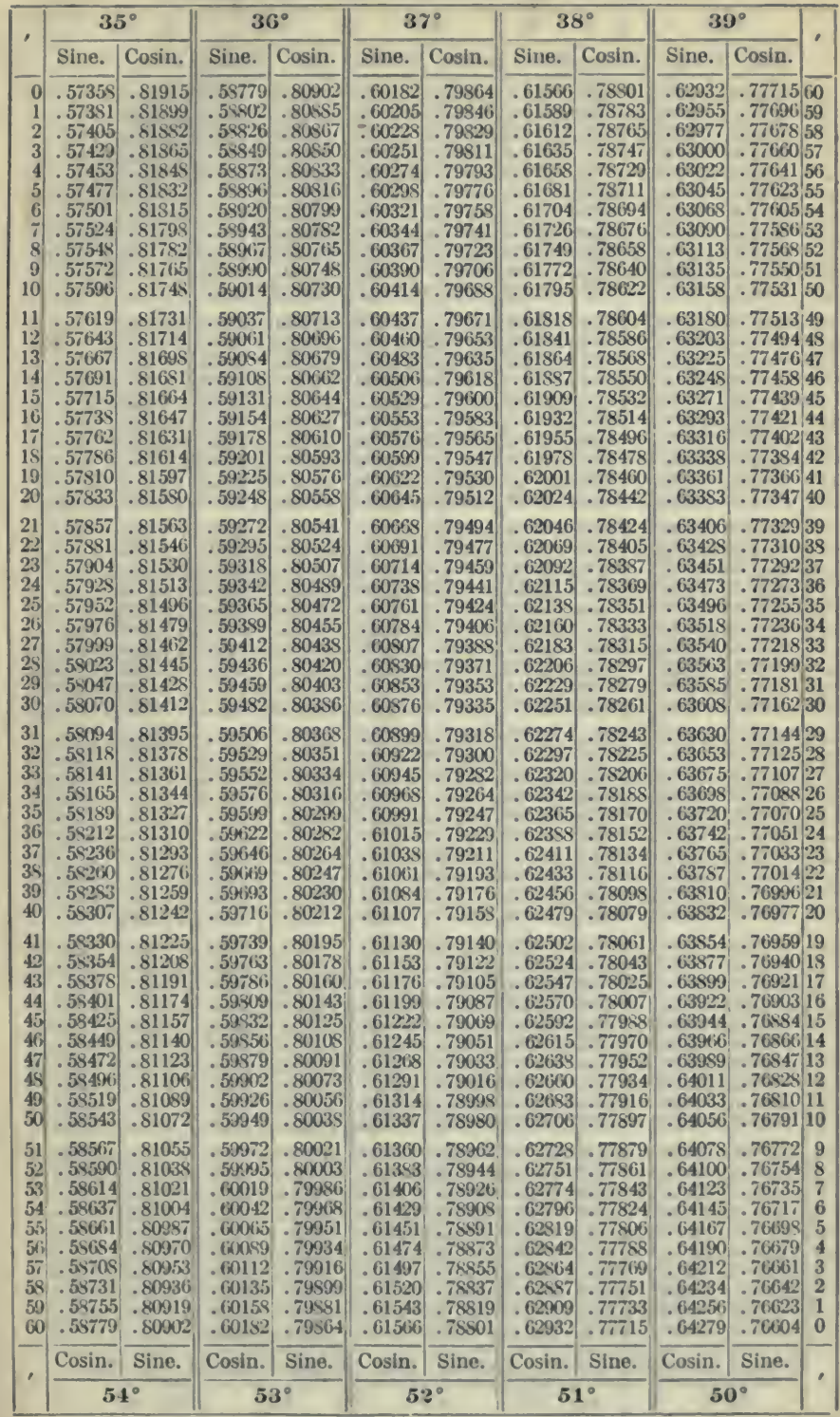


TABLE 7.-NATURAI SINES AND COSINES.

\begin{tabular}{|c|c|c|c|c|c|c|c|c|c|c|c|}
\hline \multirow{2}{*}{, } & \multicolumn{2}{|c|}{$40^{\circ}$} & \multicolumn{2}{|c|}{$41^{\circ}$} & \multicolumn{2}{|c|}{$42^{\circ}$} & \multicolumn{2}{|c|}{$43^{\circ}$} & \multicolumn{2}{|c|}{$44^{\circ}$} & \\
\hline & ne. & Cosin. & ine. & Cosin. & ne. & in. & ine. & Cosin. & ine. & Cosin. & \\
\hline 0 & 279 & 6604 & 606 & 1 & 913 & 4314 & .68200 & . 73135 & .69466 & 71934 & 00 \\
\hline 1 & & & & & & & & & & & \\
\hline 2 & 323 & & 650 & & 956 & & 8242 & 096 & 9508 & & \\
\hline 3 & & & & & & & & & 529 & & \\
\hline 4 & $36 s$ & 530 & 694 & 395 & 6999 & 37 & .68285 & .73056 & .69549 & & \\
\hline 5 & 390 & & 716 & & 7021 & & 5306 & 36 & .69570 & & \\
\hline 6 & & & & & 7043 & 198 & . 68327 & .73016 & .69591 & & \\
\hline 7 & 35 & & 59 & & 0064 & & 8349 & 96 & 612 & & \\
\hline 8 & 157 & & 781 & & 7086 & & . 68370 & & .69633 & & \\
\hline 9 & 479 & & & & 107 & & & & .69654 & & \\
\hline 10 & .64501 & 417 & & & 7129 & 20 & 8412 & & .69675 & & \\
\hline 11 & 524 & & 847 & 5261 & 7151 & 4100 & 5434 & 17 & 69696 & 71 & \\
\hline 12 & & & & & & & & & & & \\
\hline 13 & & 61 & & & 7194 & & 8476 & 77 & 69737 & & 4 \\
\hline 14 & & & & & 215 & & & & 69758 & & \\
\hline 15 & 612 & 323 & 935 & & 7237 & 4022 & $851 \mathrm{~S}$ & 37 & .69779 & & 4. \\
\hline 16 & &. & & & $25 \mathrm{~S}$ & & & & .69800 & & \\
\hline 17 & 657 & & 78 & & 7280 & 83 & $\$ 561$ & 97 & . 69821 & & \\
\hline 18 & 679 & 37 & 000 & & 301 & & & 77 & 69542 & & \\
\hline 19 & & & & & & & 03 & & 362 & & 4 \\
\hline 20 & 723 & 229 & & 88 & 7344 & 24 & 224 & 37 & 83 & & 4 \\
\hline 21 & 4746 & 210 & 6066 & 5069 & 7366 & 3904 & $\$ 645$ & 2717 & 69904 & 71 & 3 \\
\hline 22 & & & & & 87 & & & & 25 & & \\
\hline 23 & & & & & & & & & 16 & & 3 \\
\hline 24 & 12 & & & & 30 & & 09 & & & & \\
\hline 25 & & 35 & & & 452 & & & & . 69987 & & \\
\hline 26 & 6 & & & & & & & & & & \\
\hline 27 & & & & & 95 & & & & 29 & & \\
\hline 28 & & & & & & & & & & & \\
\hline 29 & & & & & & & & & 70 & & 3 \\
\hline 30 & & & & & & & & & 91 & & \\
\hline 31 & 967 & 022 & 284 & 76 & 7580 & 3708 & 857 & 17 & .70112 & & 29 \\
\hline 32 & & & & & & & & & & & \\
\hline 33 & & & & & & & & & & & \\
\hline 34 & & & & & 15 & & & & 74 & & \\
\hline 35 & & & & & & & & & & & 2 \\
\hline 36 & 7 & & & & & & & & & & \\
\hline 37 & & & & & & & & & & & \\
\hline $3 \mathrm{~s}$ & & & & & & & & & & & \\
\hline 39 & & & & & & & & & & & 2 \\
\hline 40 & .6 & & & & 7773 & 1 & & .7 & 98 & & 0 \\
\hline 41 & .05188 & 832 & 501 &. & 95 & 11 & 37 & .72317 & .70319 & .71 & 19 \\
\hline 42 & & & & & & & & & & & \\
\hline 43 & & & & & & & & & & & \\
\hline 44 & & & & & & & & & & & \\
\hline 45 & & & & & & & & & & & \\
\hline 46 & & & & & & & & & & & \\
\hline 47 & & & & & & & & & & & \\
\hline 48 & & & & & & & & & & & \\
\hline 49 & & & & & & & & & & & \\
\hline 50 & & & & & & & & & (j) & 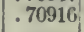 & \\
\hline 51 & 408 & 34 & 8 & . & 008 & 4 & & & 25 & & 9 \\
\hline & & & & & & & & & & & \\
\hline 53 & & & & & & & & & & & \\
\hline 54 & & & & & & & & & & & \\
\hline 55 & & & & & & & & & & & \\
\hline 56$]$ & & & & & & & & & & & \\
\hline 57 & & & & & & & & & 49 & & \\
\hline & & & & & & & & & & & \\
\hline 59 & & & & & & & & & & & \\
\hline 60 & .00 & .015471 & & .74314 & 68200 & .10 & 69466 & .71934 & 70711 & 70711 & 0 \\
\hline & . & Sine. & - & Sine. & osin. & Sine. & Cosin. & Sine. & Cosin. & Sinc. & \\
\hline & \multicolumn{2}{|c|}{$49^{\circ}$} & \multicolumn{2}{|c|}{48} & & & & & & & \\
\hline
\end{tabular}


TABLE 8.-NATURAL TANGENTS AND COTANGENTS.

\begin{tabular}{|c|c|c|c|c|c|c|c|c|c|}
\hline \multirow{2}{*}{ - } & \multicolumn{2}{|c|}{$0^{\circ}$} & \multicolumn{2}{|c|}{$1^{\circ}$} & \multicolumn{2}{|c|}{$2^{\circ}$} & \multicolumn{2}{|c|}{$3^{\circ}$} & \multirow{2}{*}{, } \\
\hline & Tang. & Cotang. & Tang. & Cotang. & Tang. & Cotang. & Tang. & Cotang. & \\
\hline 0 & .00000 & Infinite. & .01746 & 57.2900 & .03492 & 28.6363 & .05241 & 19.0811 & 60 \\
\hline 1 & & & & & & & .05270 & & 59 \\
\hline 2 & .00058 & 1718.87 & .01804 & 55.4415 & .03550 & 28. 1664 & .05299 & $18.87 \mathrm{H}$ & 58 \\
\hline 3 & .00087 & 1145.92 & .01833 & 54.5613 & .03579 & 27.9372 & & 7678 & 57 \\
\hline 4 & .00116 & 859.436 & .01862 & 53. 7056 & .03609 & 27.7117 & .05357 & 18. 6656 & 56 \\
\hline 5 & .00145 & 687 & & & & & .05387 & 18. 5645 & 55 \\
\hline 6 & .00175 & $.5 \% 2.957$ & .01920 & 52.0807 & .03667 & 27.2715 & .05416 & 18.4645 & 54 \\
\hline 7 & .00204 & 491.106 & .01949 & 51. 3032 & .03696 & 27.0566 & .05445 & 18. 3655 & . \\
\hline 8 & .00233 & 429.718 & .01978 & 50.5485 & $.037^{2} 25$ & 26.8450 & .05474 & 18. 2677 & 52 \\
\hline 9 & 262 & 381.971 & .02007 & & .03754 & 26. & .05503 & 18. 1708 & 51 \\
\hline 10 & .00291 & 343.774 & .03036 & 49. 1039 & .03783 & 26.4316 & .05533 & 18.0750 & 50 \\
\hline 11 & $.003: 20$ & 312.521 & .02066 & 48.4121 & .03812 & 26. 2296 & .05502 & 17.9802 & 49 \\
\hline 12 & & & & & .03842 & & & & 48 \\
\hline 13 & .00378 & 264.441 & .02124 & 47. & .03571 & 25.8348 & .05620 & 17.7934 & 47 \\
\hline 14 & 407 & 245. 552 & .02153 & 4 & .03900 & 25.6418 & .05649 & 17 & 46 \\
\hline 15 & .00436 & 229.152 & .02182 & 45. 8:94 & .03929 & 25.4517 & .05678 & 17.6106 & 45 \\
\hline 16 & 465 & 214.8 & .02211 & 261 & .03958 & 25.2 & .05708 & & 44 \\
\hline 17 & .00495 & 202.219 & .02240 & 44. 6356 & .03987 & 25.0798 & .05737 & 17.4314 & 43 \\
\hline 18 & .00524 & & .02269 & & .04016 & & & 17 & 42 \\
\hline 19 & .00553 & 180. & .02298 & & .04046 & 24.7185 & .05795 & & 41 \\
\hline 20 & .00582 & 171.885 & .02328 & 42. & .04075 & 24.5418 & .05524 & 17.1693 & 40 \\
\hline 21 & 6011 & 63.700 & .02357 & 335 & .04104 & 24.3675 & .05854 & 17. & 39 \\
\hline 22 & & & .02386 & & 33 & & 83 & & 38 \\
\hline 23 & .00669 & & .02415 & & & & & & 37 \\
\hline 24 & .00698 & 143. 237 & .02444 & & 91 & 593 & .05941 & & 0 \\
\hline 25 & 27 & & .02473 & & & & & 6 & 35 \\
\hline 26 & 56 & 1 & .02502 & & 60 & & 99 & & 34 \\
\hline 27 & 85 & 321 & .02531 & & & & .06029 & & 33 \\
\hline 28 & 815 & 1. & .02560 & & 08 & & .06058 & & 32 \\
\hline 29 & 844 & & .02583 & & & & 87 & & 31 \\
\hline 30 & 73 & 1 & .02619 & & 6 & & .06116 & 99 & 30 \\
\hline 31 & .00902 & 892 & .02648 & 3 & .04395 & 19 & .06145 & 722 & 29 \\
\hline 32 & & & 7 & & & & & & 28 \\
\hline 33 & & & 6 & & & & & & 27 \\
\hline 34 & & & .0 & & & & & & 26 \\
\hline 35 & 18 & 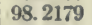 & .02764 & & 2 & & .06262 & & 25 \\
\hline 36 & & & .02793 & & & & & & 24 \\
\hline 37 & & 9 & .02822 & & 0 & & 21 & & 2 \\
\hline 38 & & & .02851 & & & & & & 2 \\
\hline 39 & & & .02581 & & .04 & & & & 21 \\
\hline 40 & .0 & 85.9398 & .02910 & 34. & .04658 & & .06408 & 48 & 20 \\
\hline 41 & 193 & 8435 & .02939 & 3 & .04657 & 9 & .06437 & 1 & 19 \\
\hline 42 & & & & & & & & & 18 \\
\hline 43 & & & & & & & & & 17 \\
\hline 44 & & & 6 & & & & & & 16 \\
\hline 45 & & & & & & & & & 15 \\
\hline 40 & & & & & & & & & 14 \\
\hline 47 & & & & & & & & & 13 \\
\hline 48 & & & & & & & & & 12 \\
\hline 49 & & & & & & & & & 11 \\
\hline 50 & & & & & 49 & & .06700 & 244 & 10 \\
\hline 51 & . & 6 & 0 & 3 & 8 & & 30 & 6 & 9 \\
\hline 70 & & & & & & & & & \\
\hline 53 & & & & & 7 & & & & 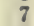 \\
\hline 54 & & & & & & & & & \\
\hline 55 & & & & & .0 & & & & 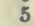 \\
\hline 50 & & & & & & & & & 4 \\
\hline 57 & & & .1 & & & & .00 & & 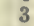 \\
\hline 5 & & & & & & & & & 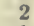 \\
\hline 59 & & & .0 & & .05 & & .00 & & 1 \\
\hline 60 & .01746 & 57.2900 & .03492 & 25. 6303 & 05241 & 19. 0S11 & .06093 & 14. 3007 & 0 \\
\hline & Cotang. & Tang. & Cotang. & Tang. & Cotang. & Tang. & Cotang. & Tang. & \multirow[t]{2}{*}{ 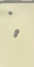 } \\
\hline & \multicolumn{2}{|c|}{$89^{\circ}$} & \multicolumn{2}{|c|}{$88^{\circ}$} & \multicolumn{2}{|c|}{$87^{\circ}$} & \multicolumn{2}{|c|}{86} & \\
\hline
\end{tabular}


TABLE 8.-NATURAI TANGENTS AND COTANGENTS.

\begin{tabular}{|c|c|c|c|c|c|c|c|c|c|}
\hline \multirow{2}{*}{ ' } & \multicolumn{2}{|c|}{$4^{\circ}$} & \multicolumn{2}{|c|}{$5^{\circ}$} & \multicolumn{2}{|c|}{$6^{\circ}$} & \multicolumn{2}{|c|}{$7^{\circ}$} & \\
\hline & Tang. & Cotang. & Tang. & Cotang. & Tang. & Cotang. & Tang. & Cotang. & \\
\hline 0 & .06993 & 14. 3007 & .08749 & 11. 4301 & .10510 & 9.51436 & .12278 & 8.14435 & 60 \\
\hline 1 & .07022 & & .08778 & & .10540 & & . 12308 & 8.12481 & 59 \\
\hline 2 & .07051 & 14.1821 & .08807 & 11.3540 & . 10569 & 9.46141 & .12338 & 8. 10536 & 8 \\
\hline 3 & .07080 & 14.1235 & .08537 & 11.3163 & . 10599 & 9.43515 & .12367 & 8.086000 & 7 \\
\hline 4 & .07110 & 14.0655 & .08566 & 11.2789 & .10628 & 9.40904 & .12397 & 8.06674 & 6 \\
\hline 5 & .07139 & 14.0079 & .08395 & 11.2417 & .10657 & 9.38307 & .12426 & 8.04756 & 55 \\
\hline 6 & .07168 & 13. 9507 & .08925 & 11.2043 & .10687 & 9.35724 & .12456 & $8.02 S 48$ & 54 \\
\hline 7 & .07197 & 13. 8940 & .08954 & 11.1681 & .10716 & 9. 33155 & $.124 \$ 5$ & 8.00948 & 53 \\
\hline 8 & .07227 & 13.8378 & .0S983 & 11.1316 & .10746 & 9.30599 & .12515 & 7.99558 & 52 \\
\hline 9 & .07256 & 13. 7821 & .09013 & 11.0954 & .10775 & 9.28058 & .12544 & 7.97176 & 1 \\
\hline 10 & .07285 & 13. & .09042 & 94 & 305 & 5530 & .12574 & 5302 & 0 \\
\hline 11 & .07314 & 13.6719 & .09071 & 11.0237 & .10834 & 9.23016 & .12603 & 7.93438 & 49 \\
\hline 12 & .07344 & 13.6174 & .09101 & & .10863 & 9.20516 & .12633 & 7.91582 & 48 \\
\hline 13 & .07373 & 13. 5634 & .09130 & 10.9529 & .10893 & 9.18028 & .12662 & 7.89734 & 47 \\
\hline 14 & .07402 & 13. 5098 & .09159 & 10.9178 & . 10922 & 9.15554 & . 12692 & 7.87595 & 46 \\
\hline 15 & .07431 & 13.4566 & .09189 & 10.8829 & . 10952 & 9.13093 & . 12722 & 7.8(20)4 & 45 \\
\hline 16 & .07461 & 13. 4039 & .09218 & 10.8483 & 10981 & 9.10646 & .12751 & 7. 84242 & 44 \\
\hline 17 & .07490 & 13. 3515 & .09247 & 10. & .11011 & 9. & .12781 & 7.82429 & 43 \\
\hline 18 & .07519 & 13.2996 & .09277 & 10.7797 & .11040 & 9.05789 & .12810 & 7.80622 & 42 \\
\hline 19 & .07548 & 13.2480 & .09306 & 10. & .11070 & 379 & .12540 & 7.78825 & 41 \\
\hline 20 & .07 & 13. & .09335 & 10 & .11099 & 83 & 869 & 35 & 0 \\
\hline 21 & .07607 & 13. & 365 & 10.6783 & .11128 & 5993 & .12899 & 54 & 39 \\
\hline 22 & .07636 & 13.0958 & .09394 & & & 3227 & . 12929 & 7.73480 & 8 \\
\hline 23 & 665 & 13.0458 & .09423 & 10. & .11187 & 8.93867 & .12958 & 7.71715 & 37 \\
\hline 24 & 695 & 12.9962 & .09453 & 10. & .11217 & 8.91520 & .12988 & 7. 69957 & 36 \\
\hline 25 & .07724 & 12:9469 & .09482 & 10. & .11246 & 9185 & .13017 & 7. 65208 & 35 \\
\hline 20 & .07753 & 12.8981 & .09511 & 10. & .11276 & 62 & .13047 & 7.66466 & 4 \\
\hline 27 & .07782 & 12.8496 & .09541 & 13 & .11305 & 551 & . 13076 & 7. 64732 & 3 \\
\hline 28 & 812 & 12.8014 & .09570 & 10. & .11335 & 8. 82252 & .13106 & 7.63005 & 2 \\
\hline 29 & .07841 & 12. & .09600 & & .11 & 964 & .13136 & & 31 \\
\hline 30 & .07870 & 12.7062 & .09629 & S54 & . 11394 & 8.77689 & . 13165 & 7.59575 & 30 \\
\hline 31 & .07899 & 12. 6591 & .09658 & 10. & .11423 & 5 & .13195 & 7. & 29 \\
\hline 3 & 29. & 12. & 088 & & & & & 76 & \\
\hline 33 & 958 & 12. & .09717 & 10. & 2 & 31 & . 13254 & 7.54487 & 7 \\
\hline 3. & 987 & & .09746 & & 1 & & 84 & 7. 52806 & 6 \\
\hline 35 & 17 & 12. & 9776 & 4 & 41 & 8. & 13 & 7.51132 & 5 \\
\hline 36 & .05046 & 12 & .09805 & 10 & 70 & 8. & .13343 & 9465 & 4 \\
\hline 3 & 975 & 12.3 & 4 & & 0 & 8. & 2 & 7. & 3 \\
\hline 3 & .08104 & 12.339 & .09864 & 10 & .11 & 8. 59893 & .13402 & 54 & 22 \\
\hline 3 & .08134 & 12. & .09893 & & 99 & 18 & 32 & 09 & 21 \\
\hline 40 & .08163 & 12. & 923 & 10. & 38 & 55 & & 71 & 0 \\
\hline 41 & .08192 & 12. 2067 & .09952 & 10. & 11718 & 402 & .13 & 240 & 19 \\
\hline 4 & & & & & & & & & 8 \\
\hline 43 & .51 & 12.1201 & .10011 & 9. & 7 & 28 & 60 & 7999 & 17 \\
\hline 44 & & 12.077 & & & 6 & & & 89 & 6 \\
\hline 45 & 309 & 12 & .10069 & 9.8 & .11 & 96 & .13609 & 7. & 15 \\
\hline 46 & & & & & & & & & 4 \\
\hline 47 & 363 & 11. & .10128 & 9. & .11 & 8. & .13669 & 7. & 13 \\
\hline 48 & & & & & & & & & 2 \\
\hline 49 & & & 187 & & 4 & & & & 11 \\
\hline 50 & .08456 & 11. & .10216 & 9.78517 & .11983 & 8.34496 & .13758 & 7.26873 & 10 \\
\hline 51 & .08485 & 11.7853 & .10246 & 8.7 & .12013 & 8.3 & .13787 & 7. & 9 \\
\hline 52 & & & 10275 & & .12 & & & & 8 \\
\hline 53 & & & & & & & & & 7 \\
\hline 54 & 573 & 11. & .10334 & 9.6 & . 12101 & 8. 2633.55 & .13876 & 7.20661 & 6 \\
\hline 55 & & & .10363 & & & & & & 5 \\
\hline 50 & 632 & 11. & 10393 & 9.6 & .12160 & 8.2 & . 13935 & 7.17594 & 4 \\
\hline 57 & & & & & & & & & 3 \\
\hline 5. & 590 & 11.5 & 2 & & .12219 & & & 7.1 & 2 \\
\hline 59 & .0 & & .10 & & 12249 & & & & 1 \\
\hline 6 & .08749 & 11.4301 & .10510 & 9. 51436 & .12278 & 8.14435 & .14054 & 7.11537 & 0 \\
\hline \multirow{2}{*}{ 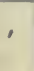 } & Cotang. & Tang. & Cotang. & Tang. & Cotang. & Tang. & Cotang. & Tang. & \\
\hline & \multicolumn{2}{|c|}{$85^{\circ}$} & \multicolumn{2}{|c|}{$84^{\circ}$} & \multicolumn{2}{|l|}{8} & \multicolumn{2}{|c|}{$82^{\circ}$} & \\
\hline
\end{tabular}


TABLE 8.-NATURAI TANGENTS AND COTANGENTS.

\begin{tabular}{|c|c|c|c|c|c|c|c|c|c|}
\hline \multirow{2}{*}{, } & \multicolumn{2}{|c|}{$8^{\circ}$} & \multicolumn{2}{|c|}{$9^{\circ}$} & \multicolumn{2}{|c|}{$10^{\circ}$} & \multicolumn{2}{|c|}{$11^{\circ}$} & \\
\hline & Tang. & Cotang. & Tang. & Cotang. & Tang. & Cotang. & Tang. & Cotang. & \\
\hline $\begin{array}{r}0 \\
1 \\
2 \\
3 \\
4 \\
5 \\
6 \\
7 \\
8 \\
9 \\
10\end{array}$ & $\begin{array}{l}.14054 \\
.14084 \\
.14113 \\
.14143 \\
.14173 \\
.14202 \\
.14232 \\
.14262 \\
.14291 \\
.14321 \\
.14351\end{array}$ & $\begin{array}{l}7.11537 \\
7.10038 \\
7.08546 \\
7.07059 \\
7.05579 \\
7.04105 \\
7.02637 \\
7.01174 \\
6.99718 \\
6.93268 \\
6.96823\end{array}$ & $\begin{array}{l}.15838 \\
.15368 \\
.15998 \\
.15928 \\
15958 \\
.15938 \\
16017 \\
16047 \\
16077 \\
16107 \\
.16137\end{array}$ & $\begin{array}{l}6.31375 \\
6.30189 \\
6.29007 \\
6.27829 \\
6.26055 \\
6.25486 \\
6.24321 \\
6.23100 \\
6.22003 \\
6.20851 \\
\text { 6. } 19703\end{array}$ & $\begin{array}{l}.17633 \\
.17663 \\
.17693 \\
.17723 \\
.17753 \\
.17783 \\
.17813 \\
.17843 \\
.17873 \\
.17903 \\
.17933\end{array}$ & $\begin{array}{l}5.67128 \\
\text { 5. } 66165 \\
5.65205 \\
\text { 5. } 64248 \\
5.63295 \\
5.62344 \\
\text { 5. } 61397 \\
\text { 5. } 60452 \\
\text { 5. } 59511 \\
\text { 5. } 55573 \\
\text { 5. } 57638\end{array}$ & $\begin{array}{l}.19435 \\
.13465 \\
.19498 \\
.19529 \\
.19559 \\
.19589 \\
.10619 \\
.19649 \\
.19680 \\
.19710 \\
.19740\end{array}$ & $\begin{array}{l}\text { 5. } 14455 \\
5.13659 \\
5.12862 \\
5.12069 \\
5.11279 \\
5.10490 \\
5.09704 \\
5.05921 \\
5.08139 \\
5.07360 \\
\text { 5. } 06584\end{array}$ & $\begin{array}{l}60 \\
59 \\
59 \\
57 \\
56 \\
55 \\
54 \\
53 \\
52 \\
51 \\
50\end{array}$ \\
\hline $\begin{array}{l}11 \\
12 \\
13 \\
14 \\
15 \\
16 \\
17 \\
18 \\
19 \\
20\end{array}$ & $\begin{array}{l}.14381 \\
.14410 \\
.14440 \\
.14470 \\
.14499 \\
.14529 \\
.14559 \\
.14589 \\
.14618 \\
.14649\end{array}$ & $\begin{array}{l}\text { 6. } 95385 \\
\text { G. } 93952 \\
\text { 6. } 92525 \\
\text { 6. } 91104 \\
\text { 6. } 89688 \\
\text { 6. } 85278 \\
\text { 6. } 86874 \\
\text { 6. } 85475 \\
\text { 6. } 84052 \\
\text { 6. } \$ 2694\end{array}$ & $\begin{array}{l}.16167 \\
.16196 \\
.16226 \\
.16256 \\
.16256 \\
.16316 \\
.16346 \\
.16376 \\
.16405 \\
.16435\end{array}$ & $\begin{array}{l}\text { 6. } 15559 \\
6.17419 \\
6.16243 \\
6.15151 \\
6.14023 \\
6.12599 \\
6.11779 \\
6.10664 \\
6.09552 \\
6.08444\end{array}$ & $\begin{array}{l}.17963 \\
.17993 \\
.18023 \\
.15053 \\
.18053 \\
.18113 \\
.18143 \\
.18173 \\
.15203 \\
.18233\end{array}$ & $\begin{array}{l}\text { 5. } 56706 \\
\text { 5. } 5.5777 \\
\text { 5. } 54851 \\
\text { 5. } 53927 \\
\text { 5. } 53007 \\
\text { 5. } 52090 \\
\text { 5. } 51176 \\
\text { 5. } 50264 \\
\text { 5. } 49356 \\
\text { 5. } 48451\end{array}$ & $\begin{array}{l}.19770 \\
.19 S 01 \\
.19531 \\
.19 S 61 \\
.19391 \\
.19021 \\
.19952 \\
.19952 \\
.20012 \\
.20042\end{array}$ & $\begin{array}{l}\text { 5. } 05809 \\
\text { 5. } 05037 \\
\text { 5. } 04267 \\
\text { 5. } 03499 \\
\text { 5. } 02734 \\
5.01971 \\
5.01210 \\
5.00451 \\
\text { 4. } 99695 \\
4.95940\end{array}$ & $\begin{array}{l}49 \\
43 \\
47 \\
46 \\
45 \\
44 \\
43 \\
42 \\
41 \\
40\end{array}$ \\
\hline $\begin{array}{l}21 \\
22 \\
23 \\
24 \\
25 \\
26 \\
27 \\
23 \\
29 \\
30\end{array}$ & $\begin{array}{l}.14678 \\
.14707 \\
.14737 \\
.14767 \\
.14736 \\
.14526 \\
.14556 \\
.14586 \\
.14915 \\
.14945\end{array}$ & $\begin{array}{l}\text { 6. } 81312 \\
\text { 6. } 79936 \\
6.78564 \\
\text { 6. } 77199 \\
\text { 6. } 75535 \\
\text { 6. } 74483 \\
\text { 6. } 73133 \\
\text { 6. } 71789 \\
\text { 6. } 70450 \\
\text { 6. } 69116\end{array}$ & $\begin{array}{l}.16465 \\
.16495 \\
.16525 \\
.16555 \\
.16555 \\
.16615 \\
.16645 \\
.16674 \\
.16704 \\
.16734\end{array}$ & $\begin{array}{l}\text { 6. } 07340 \\
6.06240 \\
6.05143 \\
6.04051 \\
\text { 6. } 02962 \\
6.01878 \\
6.00797 \\
\text { 5. } 99720 \\
\text { 5. } 95646 \\
\text { 5. } 97576\end{array}$ & $\begin{array}{l}.18263 \\
.1 S 293 \\
.1 S 323 \\
.1 S 353 \\
.18354 \\
.18414 \\
.18444 \\
.18474 \\
.18504 \\
.18534\end{array}$ & $\begin{array}{l}\text { 5. } 47549 \\
\text { 5. } 46649 \\
\text { 5. } 45751 \\
\text { 5. } 44557 \\
\text { 5. } 43366 \\
\text { 5. } 43077 \\
\text { 5. } 42192 \\
\text { 5. } 41309 \\
\text { 5. } 40429 \\
\text { 5. } 39552\end{array}$ & $\begin{array}{l}.20073 \\
.20103 \\
.20133 \\
.20164 \\
.20194 \\
.20224 \\
.20254 \\
.20255 \\
.20315 \\
.20345\end{array}$ & $\begin{array}{l}\text { 4. } 95158 \\
4.97439 \\
4.96690 \\
4.95945 \\
4.95201 \\
4.94460 \\
4.93721 \\
4.92254 \\
4.92249 \\
4.91516\end{array}$ & $\begin{array}{l}39 \\
39 \\
37 \\
36 \\
35 \\
34 \\
33 \\
32 \\
31 \\
30\end{array}$ \\
\hline $\begin{array}{l}31 \\
32 \\
33 \\
34 \\
35 \\
36 \\
37 \\
38 \\
39 \\
40\end{array}$ & $\begin{array}{l}.14975 \\
.15005 \\
.15034 \\
.15064 \\
.15094 \\
.15124 \\
.15153 \\
.151 .3 \\
.15213 \\
.15243\end{array}$ & $\begin{array}{l}\text { 6. } 67787 \\
\text { 6. } 66463 \\
\text { 6. } 65144 \\
\text { 6. } 63531 \\
\text { 6. } 62523 \\
\text { 6. } 61219 \\
\text { 6. } 59921 \\
\text { 6. } 55627 \\
\text { 6. } 57339 \\
\text { 6. } 56055\end{array}$ & $\begin{array}{l}.16764 \\
.16794 \\
.16524 \\
.16854 \\
.16584 \\
.16914 \\
.16944 \\
.16974 \\
.17004 \\
.17033\end{array}$ & $\begin{array}{l}\text { 5. } 96510 \\
\text { 5. } 95448 \\
\text { 5. } 94330 \\
\text { 5. } 93335 \\
\text { 5. } 92283 \\
\text { 5. } 91236 \\
\text { 5. } 90191 \\
\text { 5. } 89151 \\
\text { 5. } 85114 \\
\text { 5. } 87080\end{array}$ & $\begin{array}{l}.18564 \\
.18594 \\
.18624 \\
.18654 \\
.18654 \\
.18714 \\
.18745 \\
.18775 \\
.18505 \\
.18535\end{array}$ & $\begin{array}{l}\text { 5. } 35677 \\
\text { 5. } 37805 \\
\text { 5. } 36936 \\
\text { 5. } 36070 \\
\text { 5. } 35206 \\
\text { 5. } 34345 \\
\text { 5. } 33457 \\
\text { 5. } 32631 \\
\text { 5. } 31778 \\
\text { 5. } 30923\end{array}$ & $\begin{array}{l}.20376 \\
.20406 \\
.20436 \\
.20466 \\
.20497 \\
.20527 \\
.20557 \\
.20548 \\
.20615 \\
.20648\end{array}$ & $\begin{array}{l}\text { 4. } 90785 \\
\text { 4. } 90056 \\
\text { 4. } 89330 \\
\text { 4. } 85605 \\
\text { 4. } 85882 \\
\text { 4. } 87162 \\
\text { 4. } 86444 \\
\text { 4. } 85727 \\
\text { 4. } 85013 \\
\text { 4. } 84300\end{array}$ & $\begin{array}{l}29 \\
29 \\
27 \\
26 \\
25 \\
24 \\
23 \\
22 \\
21 \\
20\end{array}$ \\
\hline $\begin{array}{l}41 \\
42 \\
43 \\
44 \\
45 \\
46 \\
47 \\
48 \\
49 \\
50\end{array}$ & $\begin{array}{l}.15272 \\
.15302 \\
.15332 \\
.15362 \\
.15391 \\
.15421 \\
.15451 \\
.15481 \\
.15511 \\
.15540\end{array}$ & $\begin{array}{l}\text { 6. } 54777 \\
\text { 6. } 5: 3503 \\
\text { 6. } 52234 \\
\text { 6. } 50970 \\
\text { 6. } 49710 \\
\text { 6. } 44456 \\
\text { 6. } 47206 \\
\text { 6. } 45.961 \\
\text { 6. } 44720 \\
\text { 6. } 43434\end{array}$ & $\begin{array}{l}.17003 \\
.17093 \\
.17123 \\
.171 \% 3 \\
.171 \mathrm{~S} 3 \\
.17213 \\
.17243 \\
.17273 \\
.17303 \\
.17333\end{array}$ & $\begin{array}{l}\text { 5. } 86051 \\
\text { 5. } 85024 \\
\text { 5. } 84001 \\
\text { 5. } 82952 \\
\text { 5. } 81966 \\
\text { 5. } 80953 \\
\text { 5. } 79944 \\
\text { 5. } 78938 \\
\text { 5. } 79936 \\
\text { 5. } 76937\end{array}$ & $\begin{array}{l}.15865 \\
.15895 \\
.18925 \\
.15955 \\
.18956 \\
.19016 \\
.19046 \\
.19076 \\
.19106 \\
.19136\end{array}$ & $\begin{array}{l}\text { 5. } 30050 \\
\text { 5. } 23235 \\
\text { 5. } 25393 \\
\text { 5. } 27553 \\
\text { 5. } 26715 \\
\text { 5. } 25580 \\
\text { 5. } 25043 \\
\text { 5. } 24218 \\
\text { 5. } 23391 \\
\text { 5. } 22566\end{array}$ & $\begin{array}{l}.20679 \\
.20709 \\
.20739 \\
.20770 \\
.20500 \\
.20530 \\
.20561 \\
.20591 \\
.20921 \\
.20952\end{array}$ & $\begin{array}{l}\text { 4. } 83530 \\
\text { 4. } 82583 \\
\text { 4. } 82175 \\
\text { 4. } 81471 \\
\text { 4. } 80769 \\
\text { 4. } 80069 \\
\text { 4. } 79370 \\
\text { 4. } 78673 \\
\text { 4. } 77978 \\
\text { 4. } 77256\end{array}$ & $\begin{array}{l}19 \\
18 \\
17 \\
16 \\
15 \\
14 \\
13 \\
12 \\
11 \\
10\end{array}$ \\
\hline $\begin{array}{l}51 \\
52 \\
53 \\
54 \\
55 \\
56 \\
57 \\
58 \\
59 \\
60\end{array}$ & $\begin{array}{l}.15570 \\
.1500 \\
.15630 \\
.15660 \\
.15689 \\
.15719 \\
.15749 \\
.15779 \\
.15509 \\
.15538\end{array}$ & $\begin{array}{l}\text { 6. } 42253 \\
\text { 6. } 41026 \\
\text { 6. } 39904 \\
\text { 6. } 35587 \\
\text { 6. } 37374 \\
\text { 6. } 36165 \\
\text { 6. } 34991 \\
\text { 6. } 33761 \\
\text { 6. } 32576 \\
\text { G. } 31375\end{array}$ & $\begin{array}{l}.17363 \\
.17393 \\
.17423 \\
.174 .3 \\
.17443 \\
.17513 \\
.17543 \\
.17573 \\
.17603 \\
.17633\end{array}$ & $\begin{array}{l}\text { 5. } 75941 \\
\text { 5. } 74949 \\
\text { 5. } 73960 \\
\text { 5. } 72974 \\
\text { 5. } 71992 \\
\text { 5. } 71013 \\
\text { 5. } 70037 \\
\text { 5. } 60064 \\
\text { 5. } 69094 \\
\text { 5. } 67123\end{array}$ & $\begin{array}{l}.19166 \\
.19197 \\
.19227 \\
.19257 \\
.19287 \\
.19317 \\
.19347 \\
.19378 \\
.19409 \\
.19433\end{array}$ & $\begin{array}{l}\text { 5. } 21744 \\
\text { 5. } 20925 \\
\text { 5. } 20107 \\
\text { 5. } 19293 \\
\text { 5. } 18450 \\
\text { 5. } 17671 \\
\text { 5. } 16503 \\
\text { 5. } 160598 \\
\text { 5. } 1.5256 \\
\text { 5. } 144.25\end{array}$ & $\begin{array}{l}.20952 \\
.21013 \\
.21043 \\
.21073 \\
.21104 \\
.21134 \\
.21164 \\
.21195 \\
.21225 \\
.21250\end{array}$ & $\begin{array}{l}\text { 4. } 76595 \\
\text { 4. } 75906 \\
\text { 4. } 75219 \\
\text { 4. } 74534 \\
\text { 4. } 73651 \\
\text { 4. } 731 \% 0 \\
\text { 4. } 72400 \\
\text { 4. } 71813 \\
\text { 4. } 71137 \\
\text { 4. } 70463\end{array}$ & $\begin{array}{l}9 \\
8 \\
7 \\
6 \\
5 \\
4 \\
3 \\
2 \\
1 \\
0\end{array}$ \\
\hline & Cotang. & Tang. & Cotang. & Tang. & Cotang. & Taug. & Cotang. & Tang. & \\
\hline & \multicolumn{2}{|c|}{$81^{\circ}$} & \multicolumn{2}{|c|}{$80^{\circ}$} & \multicolumn{2}{|c|}{$89^{\circ}$} & \multicolumn{2}{|c|}{$78^{\circ}$} & \\
\hline
\end{tabular}


TABLE 8.-NATURAL TANGENTS AND COTANGENTS.

\begin{tabular}{|c|c|c|c|c|c|c|c|c|c|}
\hline \multirow{2}{*}{, } & \multicolumn{2}{|c|}{$12^{\circ}$} & \multicolumn{2}{|c|}{$13^{\circ}$} & \multicolumn{2}{|c|}{$14^{\circ}$} & \multicolumn{2}{|c|}{$15^{\circ}$} & \\
\hline & Tang. & Cotang. & Tang. & Cotang. & Tang. & Cotang. & Tang. & Cotang. & \\
\hline 0 & .21256 & 4. 70463 & .23087 & 18 & .24933 & 4.01078 & .26795 & 3.73205 & 0 \\
\hline 1 & .21286 & & .23117 & & & 4.00582 & & 3. 72771 & 59 \\
\hline 2 & .21316 & 4. 69121 & .23148 & 4. 32001 & .24995 & 4. 00086 & .26857 & 3. 72339 & 58 \\
\hline 3 & .21347 & 4. 68452 & .23179 & 4. 31430 & .25026 & 3. 99592 & .26888 & 3. 71907 & 57 \\
\hline 4 & .21377 & & .23209 & 4. 30860 & .25056 & 3. 99099 & .26920 & 3. 71476 & 56 \\
\hline 5 & .21408 & 4. 67121 & .23240 & 4. 30291 & .25087 & 3.98607 & .26951 & 3. 71046 & 55 \\
\hline 6 & .21438 & 4. 66458 & .23271 & 4. 29724 & .25118 & 3. 98117 & .26982 & 3. 70616 & 54 \\
\hline 7 & .21469 & 4. 65797 & .23301 & 4. 29159 & .25149 & 3.97627 & .27013 & 3. 70188 & 53 \\
\hline 8 & & & .23332 & & .25 & 3.97139 & .27044 & 3. 69761 & 52 \\
\hline 9 & $\cdot 2$ & 4.6 & 363 & 4.2 & .25211 & 3. 96651 & .27076 & 3. 69335 & 51 \\
\hline 10 & .21560 & 5 & .23393 & 4. 27471 & .25242 & 3.96165 & .27107 & 3. 68909 & 50 \\
\hline 11 & .21590 & 171 & .23424 & 6911 & .25273 & 3. 95680 & .27138 & 3. 65485 & 49 \\
\hline 12 & & & 4455 & & & & & 3061 & 48 \\
\hline 13 & .21651 & 4. 61868 & .23485 & 4.2 & .25335 & 3.9 & .27201 & 3. 67638 & 47 \\
\hline 14 & .21682 & 4. 61219 & .23516 & 4.25239 & .25366 & 3.94232 & .27232 & 3. 67217 & 46 \\
\hline 15 & .21712 & 4.60572 & 3547 & 4. 24685 & .25397 & 3. 93751 & .27263 & 3. 66796 & 45 \\
\hline 16 & .21743 & 4. 59927 & .23578 & 4. 24132 & .25428 & 3. 93271 & .27294 & 3. 66376 & 44 \\
\hline 17 & .21773 & 4. 59283 & .2360 .8 & 4.23 & .25459 & 3.9 & .27326 & 3. 65957 & 43 \\
\hline 18 & .21804 & 4. 58641 & .23639 & 4. 23030 & .25490 & 3.92316 & . 27357 & 3.65538 & 42 \\
\hline 19 & .21834 & 4 & .23670 & 4. & 21 & & & 121 & 41 \\
\hline 20 & .2 & & 0 & & & & & 05 & 0 \\
\hline 21 & $\begin{array}{r}21895 \\
21925\end{array}$ & $4.2^{2}$ & .23731 & 4 & 3 & & 1 & 59 & 39 \\
\hline & $\begin{array}{r}21925 \\
.21956\end{array}$ & 4. & & $\begin{array}{l}2 \\
8\end{array}$ & & & & & 8 \\
\hline 24 & .21986 & $\begin{array}{l}458 \\
826\end{array}$ & $\begin{array}{l}7793 \\
823\end{array}$ & 4. 1 & 76 & & .27 & $\begin{array}{l}61 \\
48\end{array}$ & 17 \\
\hline 25 & .22017 & 4. 51196 & .23854 & 4. 19215 & .25707 & 3. 89004 & .27576 & 3. $6^{2} 636$ & 5 \\
\hline 26 & .22047 & 568 & .23885 & 4.1 & .25738 & & .27607 & 3. 62224 & 34 \\
\hline 27 & .22078 & 4. & .23916 & 4. 18137 & .25769 & 3.8 & .27638 & 3.61814 & 33 \\
\hline 28 & .22108 & 316 & .23946 & 4.176 & .25800 & 3.8 & .27670 & 3.61405 & 32 \\
\hline 0 & .22139 & 4. & .23977 & 4. & .25831 & 3.8 & .27701 & & 31 \\
\hline 30 & .22169 & 71 & .24008 & 4 & 25862 & 3.8 & 32 & 88 & 30 \\
\hline 31 & .22200 & 4. 50451 & .24 & 4. 15997 & .25893 & 3. 86208 & .27764 & 181 & 29 \\
\hline 32 & .22231 & & .2 & & .25924 & 3. & .27 & & 8 \\
\hline 33 & .22261 & 4 & .24 & & .25955 & & .27826 & & 27 \\
\hline 34 & .22292 & 4 & .24 & & .25986 & 3. & .27858 & 3. & 26 \\
\hline 35 & .22322 & 86 & 22 & 4. & .26017 & 3. & .27889 & 3. & 25 \\
\hline 36 & .22353 & & 3 & 4 & .20 & & .27921 & 3. & 4 \\
\hline 37. & .22383 & 4. & .24223 & 4. 12825 & .26079 & 3. & .27952 & 3. & 23 \\
\hline 38 & .22414 & & .24254 & 4. & .26110 & & .27983 & 357 & 22 \\
\hline 39 & .22444 & 4. & .24285 & 4. & .26141 & 3.8 & .28 & 57 & 21 \\
\hline 40 & .22475 & 942 & 316 & & .26172 & 3.82083 & 16 & & 20 \\
\hline 41 & .22505 & 4. 44338 & .24347 & 4. 10 & .26203 & $3.81 \mathrm{C}$ & .28077 & 3. & 19 \\
\hline 32 & .22536 & & & & & & & & 8 \\
\hline 43 & .22567 & & .24 & & .26 & & .28 & 3. & 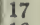 \\
\hline 44 & .22597 & 4 & .2443 & 4. & .26297 & 3. & .28172 & & 16 \\
\hline & .22623 & & .24 & & .26 & & .28 & & 5 \\
\hline 46 & .22658 & 4. & .2 & 4. & .26359 & & .28234 & & 14 \\
\hline 47 & .22689 & & .21 & & .20 & & .28266 & & 13 \\
\hline 48 & .22719 & 4. & .2 & & .26421 & & 28297 & & 12 \\
\hline 49 & .22750 & & .24 & & & & & & 11 \\
\hline 50 & .22781 & 4 & & 7 & 3 & 5 & 30 & 09 & 10 \\
\hline 51 & .22311 & 4. 38381 & .21655 & 4. 05599 & .26515 & 3. 77152 & . 28391 & 3. & 9 \\
\hline & .2 & & & & & & & & 8 \\
\hline 53 & .22872 & & .24 & & .2 & & 54 & & 7 \\
\hline $5-1$ & .22903 & & .24 & & & & & & 6 \\
\hline 55 & .22931 & & .2 & & .26 & & 17 & & 5 \\
\hline 5 & .22964 & & .24 & & & & & & 4 \\
\hline 57 & .22995 & 4. & .24840 & 4.0 & .26 & & .28580 & & 3 \\
\hline & .23026 & & 248 & & .26733 & & 28612 & & 2 \\
\hline & .23056 & 4. & .24902 & & .26764 & & .28643 & 3.4 & 1 \\
\hline 6 & .23087 & 4. 33148 & .21933 & 4.01078 & .26795 & 3. 73205 & .28675 & 3.48741 & 0 \\
\hline \multirow{2}{*}{ ' } & Cotang. & 'Tang. & Cotang. & Tang. & Cotang. & Tang. & Cotang. & Tang. & \\
\hline & \multicolumn{2}{|c|}{$77^{\circ}$} & \multicolumn{2}{|c|}{$76^{\circ}$} & \multicolumn{2}{|c|}{$75^{\circ}$} & \multicolumn{2}{|c|}{$74^{\circ}$} & \\
\hline
\end{tabular}


TABLE 8. -NATERAL TANGENTS AND COTANGENTS.

\begin{tabular}{|c|c|c|c|c|c|c|c|c|c|}
\hline \multirow[b]{2}{*}{, } & \multicolumn{2}{|c|}{$16^{\circ}$} & \multicolumn{2}{|c|}{$17^{\circ}$} & \multicolumn{2}{|c|}{$18^{\circ}$} & \multicolumn{2}{|c|}{$19^{\circ}$} & \multirow{2}{*}{, } \\
\hline & Tang. & Cotang. & Tang. & Cotang. & Tang. & Cotang. & Tang. & Cotang. & \\
\hline $\begin{array}{r}0 \\
1 \\
2 \\
3 \\
4 \\
5 \\
6 \\
7 \\
8 \\
9 \\
10\end{array}$ & $\begin{array}{l}.25675 \\
.28706 \\
.28738 \\
.28769 \\
.28800 \\
.25832 \\
.28904 \\
.25995 \\
.25927 \\
.25958 \\
.25990\end{array}$ & $\begin{array}{l}\text { 3. } 48741 \\
\text { 3. } 48359 \\
\text { 3. } 47977 \\
\text { 3. } 47596 \\
\text { 3. } 47216 \\
\text { 3. } 46837 \\
\text { 3. } 46458 \\
\text { 3. } 46080 \\
\text { 3. } 45703 \\
\text { 3. } 45327 \\
\text { 3. } 44951\end{array}$ & $\begin{array}{l}.30573 \\
.30605 \\
.30637 \\
.30669 \\
.30700 \\
.30732 \\
.30764 \\
.30796 \\
.30828 \\
.30860 \\
.30591\end{array}$ & $\begin{array}{l}\text { 3. } 27085 \\
\text { 3. } 26745 \\
\text { 3. } 26406 \\
\text { 3. } 26067 \\
\text { 3. } 25729 \\
\text { 3. } 25392 \\
\text { 3. } 25055 \\
\text { 3. } 24719 \\
\text { 3. } 24383 \\
\text { 3. } 24049 \\
\text { 3. } 23714\end{array}$ & $\begin{array}{l}.32492 \\
.32524 \\
.32556 \\
.32588 \\
.32621 \\
.32653 \\
.32655 \\
.32717 \\
.32749 \\
.32782 \\
.32814\end{array}$ & $\begin{array}{l}\text { 3. } 07768 \\
3.07464 \\
3.07160 \\
3.06857 \\
3.06554 \\
\text { 3. } 06252 \\
\text { 3. } 05950 \\
\text { 3. } 05649 \\
\text { 3. } 05349 \\
\text { 3. } 05049 \\
\text { 3. } 04749\end{array}$ & $\begin{array}{l}.34433 \\
.34465 \\
.34498 \\
.34530 \\
.34563 \\
.34590 \\
.34628 \\
.34661 \\
.34693 \\
.34726 \\
.34758\end{array}$ & $\begin{array}{l}2.90421 \\
2.90147 \\
2.89873 \\
2.89600 \\
2.89327 \\
2.89055 \\
2.88783 \\
2.85511 \\
2.85240 \\
2.87970 \\
2.87700\end{array}$ & $\begin{array}{l}60 \\
59 \\
58 \\
57 \\
56 \\
55 \\
54 \\
53 \\
52 \\
51 \\
50\end{array}$ \\
\hline $\begin{array}{l}11 \\
12 \\
13 \\
14 \\
15 \\
16 \\
17 \\
18 \\
19 \\
20\end{array}$ & $\begin{array}{l}.29021 \\
.29053 \\
.29084 \\
.29116 \\
.29147 \\
.29179 \\
.29210 \\
.29242 \\
.29274 \\
.29305\end{array}$ & $\begin{array}{l}\text { 3. } 44576 \\
\text { 3. } 44202 \\
\text { 3. } 43329 \\
\text { 3. } 43456 \\
3.43084 \\
\text { 3. } 42713 \\
\text { 3. } 42343 \\
\text { 3. } 41973 \\
\text { 3. } 41604 \\
\text { 3. } 41236\end{array}$ & $\begin{array}{l}.30923 \\
.30955 \\
.30957 \\
.31019 \\
.31051 \\
.31083 \\
.31115 \\
.31147 \\
.31178 \\
.31210\end{array}$ & $\begin{array}{l}\text { 3. } 23331 \\
\text { 3. } 23048 \\
\text { 3. } 22715 \\
\text { 3. } 22384 \\
\text { 3. } 22053 \\
\text { 3. } 21722 \\
\text { 3. } 21392 \\
\text { 3. } 21063 \\
\text { 3. } 20734 \\
\text { 3. } 20406\end{array}$ & $\begin{array}{l}.32546 \\
.32878 \\
.32911 \\
.32943 \\
.32975 \\
.33007 \\
.33040 \\
.33072 \\
.33104 \\
.33136\end{array}$ & $\begin{array}{l}\text { 3. } 04450 \\
3.04152 \\
3.03554 \\
3.03556 \\
3.03200 \\
3.02903 \\
3.026017 \\
3.02372 \\
3.02077 \\
3.01783\end{array}$ & $\begin{array}{l}.34791 \\
.34824 \\
.34856 \\
.34859 \\
.34922 \\
.34954 \\
.34987 \\
.35020 \\
.35052 \\
.35085\end{array}$ & $\begin{array}{l}2.87430 \\
2.87161 \\
2.86592 \\
2.86624 \\
2.86356 \\
2.86089 \\
2.85822 \\
2.85555 \\
2.85289 \\
2.85023\end{array}$ & $\begin{array}{l}49 \\
48 \\
47 \\
46 \\
45 \\
44 \\
43 \\
42 \\
41 \\
40\end{array}$ \\
\hline $\begin{array}{l}21 \\
22 \\
23 \\
24 \\
25 \\
26 \\
27 \\
28 \\
29 \\
30\end{array}$ & $\begin{array}{l}.29337 \\
.29368 \\
.29400 \\
.29432 \\
.29463 \\
.29495 \\
.29526 \\
.2955 .8 \\
.29590 \\
.29621\end{array}$ & $\begin{array}{l}\text { 3. } 40869 \\
\text { 3. } 40502 \\
\text { 3. } 40136 \\
\text { 3. } 39771 \\
\text { 3. } 39106 \\
\text { 3. } 39042 \\
\text { 3. } 38679 \\
\text { 3. } 38317 \\
\text { 3. } 37955 \\
\text { 3. } 37594\end{array}$ & $\begin{array}{l}.31242 \\
.31274 \\
.31306 \\
.31338 \\
.31370 \\
.31402 \\
.31434 \\
.31466 \\
.31498 \\
.31530\end{array}$ & $\begin{array}{l}\text { 3. } 20079 \\
\text { 3. } 19752 \\
\text { 3. } 19426 \\
\text { 3. } 19100 \\
\text { 3. } 19775 \\
\text { 3. } 18451 \\
\text { 3. } 18127 \\
\text { 3. } 17804 \\
\text { 3. } 17481 \\
\text { 3. } 17159\end{array}$ & $\begin{array}{l}.33169 \\
.33201 \\
.33233 \\
.33266 \\
.33298 \\
.33330 \\
.33363 \\
.33395 \\
.33427 \\
.33460\end{array}$ & $\begin{array}{l}\text { 3. } 01489 \\
\text { 3. } 01196 \\
3.00903 \\
3.00611 \\
3.00319 \\
3.00028 \\
2.99738 \\
2.99447 \\
2.9915 .8 \\
2.95868\end{array}$ & $\begin{array}{l}.35118 \\
.35150 \\
.35183 \\
.35216 \\
.35248 \\
.35281 \\
.35314 \\
.35346 \\
.35379 \\
.35412\end{array}$ & $\begin{array}{l}2.84758 \\
2.84494 \\
2.84229 \\
2.83965 \\
2.83702 \\
2.83439 \\
2.83176 \\
2.82914 \\
2.82653 \\
2.82391\end{array}$ & $\begin{array}{l}39 \\
38 \\
37 \\
36 \\
35 \\
34 \\
33 \\
32 \\
31 \\
30\end{array}$ \\
\hline $\begin{array}{l}31 \\
32 \\
33 \\
34 \\
35 \\
36 \\
37 \\
38 \\
39 \\
40\end{array}$ & $\begin{array}{l}.29653 \\
.29685 \\
.29716 \\
.29748 \\
.29780 \\
.29811 \\
.29513 \\
.29875 \\
.29906 \\
.29938\end{array}$ & $\begin{array}{l}\text { 3. } 37234 \\
\text { 3. } 36875 \\
\text { 3. } 36516 \\
\text { 3. } 36158 \\
\text { 3. } 35800 \\
\text { 3. } 35443 \\
\text { 3. } 35087 \\
\text { 3. } 34732 \\
\text { 3. } 34377 \\
\text { 3. } 34023\end{array}$ & $\begin{array}{l}.31562 \\
.31594 \\
.31626 \\
.31658 \\
.31690 \\
.31722 \\
.31754 \\
.31786 \\
.31818 \\
.31850\end{array}$ & $\begin{array}{l}\text { 3. } 16838 \\
\text { 3. } 16517 \\
\text { 3. } 16197 \\
\text { 3. } 15877 \\
\text { 3. } 15558 \\
\text { 3. } 15240 \\
\text { 3. } 11922 \\
3.14605 \\
\text { 3. } 14288 \\
\text { 3. } 13972\end{array}$ & $\begin{array}{l}.33492 \\
.33524 \\
.33557 \\
.33589 \\
.33621 \\
.33654 \\
.33686 \\
.33718 \\
.33751 \\
.33783\end{array}$ & $\begin{array}{l}2.98550 \\
2.98292 \\
2.98004 \\
2.97717 \\
2.97430 \\
2.97144 \\
2.96558 \\
2.96573 \\
2.96288 \\
2.96004\end{array}$ & $\begin{array}{l}.35445 \\
.35477 \\
.35510 \\
.35543 \\
.35576 \\
.35608 \\
.35641 \\
.35674 \\
.35707 \\
.35740\end{array}$ & $\begin{array}{l}2.82130 \\
2.81870 \\
2.81610 \\
2.81350 \\
2.81091 \\
2.80533 \\
2.80574 \\
2.80316 \\
2.80059 \\
2.79802\end{array}$ & $\begin{array}{l}29 \\
28 \\
27 \\
26 \\
25 \\
24 \\
23 \\
22 \\
21 \\
20\end{array}$ \\
\hline $\begin{array}{l}41 \\
42 \\
43 \\
44 \\
45 \\
46 \\
47 \\
48 \\
49 \\
50\end{array}$ & $\begin{array}{l}.29970 \\
.30001 \\
.30033 \\
.300625 \\
.30097 \\
.30128 \\
.30160 \\
.30192 \\
.30224 \\
.30255\end{array}$ & $\begin{array}{l}\text { 3. } 33670 \\
\text { 3. } 33317 \\
\text { 3. } 32965 \\
\text { 3. } 32614 \\
\text { 3. } 32264 \\
\text { 3. } 31914 \\
\text { 3. } 315135 \\
\text { 3. } 31216 \\
\text { 3. } 30868 \\
\text { 3. } 30521\end{array}$ & $\begin{array}{l}.31852 \\
.31914 \\
.31946 \\
.31978 \\
.32010 \\
.32042 \\
.32074 \\
.32106 \\
.32139 \\
.32171\end{array}$ & $\begin{array}{l}\text { 3. } 13656 \\
\text { 3. } 13341 \\
\text { 3. } 13027 \\
\text { 3. } 12713 \\
\text { 3. } 12400 \\
\text { 3. } 12087 \\
\text { 3. } 11775 \\
\text { 3. } 11464 \\
\text { 3. } 11153 \\
\text { 3. } 10842\end{array}$ & $\begin{array}{l}.33516 \\
.33848 \\
.33581 \\
.33913 \\
.33945 \\
.33978 \\
.34010 \\
.34043 \\
.34075 \\
.34108\end{array}$ & $\begin{array}{l}2.95721 \\
2.95437 \\
2.9515 .5 \\
2.94872 \\
2.94591 \\
2.94309 \\
2.94028 \\
2.93748 \\
2.93468 \\
2.93189\end{array}$ & $\begin{array}{l}.35772 \\
.35805 \\
.35838 \\
.35871 \\
.35904 \\
.35937 \\
.35969 \\
.36002 \\
.36035 \\
.36068\end{array}$ & $\begin{array}{l}\text { 2. } 79545 \\
2.79289 \\
2.79033 \\
2.75778 \\
2.73523 \\
2.78269 \\
2.75014 \\
2.77761 \\
2.77507 \\
2.77254\end{array}$ & $\begin{array}{l}19 \\
18 \\
17 \\
16 \\
15 \\
14 \\
13 \\
12 \\
11 \\
10\end{array}$ \\
\hline $\begin{array}{l}51 \\
52 \\
53 \\
54 \\
55 \\
56 \\
57 \\
58 \\
59 \\
60\end{array}$ & $\begin{array}{l}.30257 \\
.30319 \\
.30351 \\
.30352 \\
.30411 \\
.30146 \\
.30478 \\
.30509 \\
.30541 \\
.30573\end{array}$ & $\begin{array}{l}\text { 3. } 30174 \\
\text { 3. } 29529 \\
\text { 3. } 29483 \\
\text { 3. } 29139 \\
\text { 3. } 2879.5 \\
\text { 3. } 28452 \\
\text { 3. } 28109 \\
\text { 3. } 27767 \\
\text { 3. } 27426 \\
\text { 3. } 27055\end{array}$ & $\begin{array}{l}.32203 \\
.32235 \\
.32267 \\
.32299 \\
.32331 \\
.32363 \\
.32396 \\
.32429 \\
.32460 \\
.32192\end{array}$ & $\begin{array}{l}\text { 3. } 10532 \\
\text { 3. } 10223 \\
\text { 3. } 09914 \\
\text { 3. } 09406 \\
\text { 3. } 09299 \\
\text { 3. } 05991 \\
\text { 3. } 05645 \\
\text { 3. } 05379 \\
\text { 3. } 05073 \\
\text { 3. } 07668\end{array}$ & $\begin{array}{l}.34140 \\
.34173 \\
.34205 \\
.34238 \\
.34270 \\
.34303 \\
.34335 \\
.34345 \\
.34400 \\
.34433\end{array}$ & $\begin{array}{l}\text { 2. } 92910 \\
2.92632 \\
2.92354 \\
2.92076 \\
2.91799 \\
2.91523 \\
2.91246 \\
2.90971 \\
2.90696 \\
2.90421\end{array}$ & $\begin{array}{l}.36101 \\
.36134 \\
.36167 \\
.36199 \\
.36232 \\
.36265 \\
.36295 \\
.36331 \\
.36384 \\
.36397\end{array}$ & $\begin{array}{l}\text { 2. } 77002 \\
2.76750 \\
\text { 2. } 76498 \\
2.76247 \\
2.75906 \\
2.75746 \\
\text { 2. } 75496 \\
\text { 2.75.246 } \\
2.74997 \\
2.74748\end{array}$ & $\begin{array}{l}9 \\
8 \\
7 \\
6 \\
5 \\
4 \\
3 \\
2 \\
1 \\
0\end{array}$ \\
\hline & tang. & Tang. & otang. & Tang. & otang. & $18 \mathrm{~g}$. & Cotang. & Tang. & \\
\hline & \multicolumn{2}{|c|}{$73^{\circ}$} & \multicolumn{2}{|c|}{$72^{\circ}$} & \multicolumn{2}{|c|}{$71^{\circ}$} & \multicolumn{2}{|c|}{$80^{\circ}$} & \\
\hline
\end{tabular}


TABLE 8.-NATURAL TANGENTS AND COTANGENTS.

\begin{tabular}{|c|c|c|c|c|c|c|c|c|c|}
\hline \multirow{2}{*}{ ' } & \multicolumn{2}{|c|}{$20^{\circ}$} & \multicolumn{2}{|c|}{$21^{\circ}$} & \multicolumn{2}{|c|}{$22^{\circ}$} & \multicolumn{2}{|c|}{$23^{\circ}$} & \multirow{2}{*}{, } \\
\hline & Tang. & Cotang. & Tang. & Cotang. & Tang. & Cotang. & Tang. & Cotang. & \\
\hline $\begin{array}{r}0 \\
1 \\
2 \\
3 \\
4 \\
5 \\
6 \\
7 \\
8 \\
9 \\
10\end{array}$ & $\begin{array}{l}.36397 \\
.36430 \\
.36463 \\
.36496 \\
.36529 \\
.36562 \\
.36595 \\
.36628 \\
.36661 \\
.36694 \\
.36727\end{array}$ & $\begin{array}{l}2.74748 \\
2.74499 \\
2.74251 \\
2.74004 \\
2.73756 \\
2.73509 \\
2.73263 \\
2.73017 \\
2.72771 \\
2.72526 \\
2.72281\end{array}$ & $\begin{array}{l}.38386 \\
.38120 \\
.38153 \\
.38187 \\
.38520 \\
.38553 \\
.38587 \\
.38620 \\
.38654 \\
.38687 \\
.38721\end{array}$ & $\begin{array}{l}2.60509 \\
2.60283 \\
2.60057 \\
2.59831 \\
2.59606 \\
2.59381 \\
2.5915 t \\
2.58932 \\
2.58708 \\
2.58481 \\
2.58261\end{array}$ & $\begin{array}{l}.40403 \\
.40436 \\
.40470 \\
.40504 \\
.40538 \\
.40572 \\
.40606 \\
.40640 \\
.40674 \\
.40707 \\
.40741\end{array}$ & $\begin{array}{l}2.47509 \\
2.47302 \\
2.47095 \\
2.46858 \\
2.46682 \\
2.46476 \\
2.46270 \\
2.46065 \\
2.45860 \\
2.45655 \\
2.45451\end{array}$ & $\begin{array}{l}.42447 \\
.42482 \\
.42516 \\
.42551 \\
.42585 \\
.42619 \\
.42654 \\
.42685 \\
.42722 \\
.42757 \\
.42791\end{array}$ & $\begin{array}{l}2.35585 \\
2.35395 \\
2.35205 \\
2.35015 \\
2.34825 \\
2.34 c 36 \\
2.34447 \\
2.34258 \\
2.340 \mathrm{C} 9 \\
2.33851 \\
2.33693\end{array}$ & $\begin{array}{l}0 \\
59 \\
58 \\
57 \\
56 \\
55 \\
54 \\
53 \\
52 \\
51 \\
50\end{array}$ \\
\hline $\begin{array}{l}11 \\
12 \\
13 \\
14 \\
15 \\
16 \\
17 \\
18 \\
19 \\
20\end{array}$ & $\begin{array}{l}.36760 \\
.36793 \\
.36826 \\
.36859 \\
.36892 \\
.36925 \\
.36958 \\
.36991 \\
.37024 \\
.37057\end{array}$ & $\begin{array}{l}2.72036 \\
2.71792 \\
2.71548 \\
2.71305 \\
2.71062 \\
2.70819 \\
2.70577 \\
2.70335 \\
2.70094 \\
2.69853\end{array}$ & $\begin{array}{l}.38754 \\
.38787 \\
.38821 \\
.38854 \\
.38888 \\
.38921 \\
.38955 \\
.38985 \\
.39022 \\
.39055\end{array}$ & $\begin{array}{l}2.58038 \\
2.57815 \\
2.57593 \\
2.57371 \\
2.57150 \\
2.56928 \\
2.56707 \\
2.56487 \\
2.56266 \\
2.56046\end{array}$ & $\begin{array}{l}.40775 \\
.40809 \\
.40843 \\
.40877 \\
.40911 \\
.40945 \\
.40979 \\
.41013 \\
.41047 \\
.41081\end{array}$ & $\begin{array}{l}2.45246 \\
2.45043 \\
2.44839 \\
2.44636 \\
2.44433 \\
2.44230 \\
2.44027 \\
2.43825 \\
2.43623 \\
2.43422\end{array}$ & $\begin{array}{l}.42826 \\
.42860 \\
.42894 \\
.42929 \\
.42963 \\
.42998 \\
.43032 \\
.43067 \\
.43101 \\
.43136\end{array}$ & $\begin{array}{l}2.33505 \\
2.33317 \\
2.33130 \\
2.32943 \\
2.32756 \\
2.32570 \\
2.32383 \\
2.32197 \\
2.32012 \\
2.31526\end{array}$ & $\begin{array}{l}49 \\
48 \\
47 \\
46 \\
45 \\
44 \\
43 \\
42 \\
41 \\
40\end{array}$ \\
\hline $\begin{array}{l}21 \\
22 \\
23 \\
24 \\
25 \\
26 \\
27 \\
28 \\
29 \\
30\end{array}$ & $\begin{array}{l}.37090 \\
.37123 \\
.37157 \\
.37190 \\
.37223 \\
.37256 \\
.37289 \\
.37322 \\
.37355 \\
.37388\end{array}$ & $\begin{array}{l}2.69612 \\
2.69371 \\
2.69131 \\
2.68892 \\
2.68653 \\
2.68414 \\
2.68175 \\
2.67937 \\
2.67700 \\
2.67462\end{array}$ & \begin{tabular}{|}
.39089 \\
.39122 \\
.39156 \\
.39190 \\
.39223 \\
.39257 \\
.39290 \\
.39324 \\
.39357 \\
.39391
\end{tabular} & & $\begin{array}{l}.41115 \\
.41149 \\
.41183 \\
.41217 \\
.41251 \\
.41285 \\
.41319 \\
.41353 \\
.41387 \\
.41421\end{array}$ & & $\begin{array}{l}.43170 \\
.43205 \\
.43239 \\
.43274 \\
.43308 \\
.43343 \\
.43378 \\
.43412 \\
.43447 \\
.43481\end{array}$ & $\begin{array}{l}2.31641 \\
2.31456 \\
2.31271 \\
2.31086 \\
2.30902 \\
2.30718 \\
2.30534 \\
2.30351 \\
2.30167 \\
2.29954\end{array}$ & $\begin{array}{l}39 \\
38 \\
37 \\
36 \\
35 \\
34 \\
33 \\
32 \\
31 \\
30\end{array}$ \\
\hline $\begin{array}{l}31 \\
32 \\
33 \\
34 \\
35 \\
36 \\
37 \\
38 \\
39 \\
40\end{array}$ & $\begin{array}{l}.37422 \\
.37455 \\
.37488 \\
.37521 \\
.37554 \\
.37588 \\
.37621 \\
.37654 \\
.37687 \\
.37720\end{array}$ & $\begin{array}{l}2.67225 \\
2.66989 \\
2.66752 \\
2.66516 \\
2.66281 \\
2.66046 \\
2.65811 \\
2.65576 \\
2.65342 \\
2.65109\end{array}$ & $\begin{array}{l}.39425 \\
.39458 \\
.39492 \\
.39526 \\
.39559 \\
.39593 \\
.39626 \\
.39660 \\
.39694 \\
.39727\end{array}$ & & $\begin{array}{l}.41455 \\
.41490 \\
.41524 \\
.41558 \\
.41592 \\
.41626 \\
.41660 \\
.41694 \\
.41728 \\
.41763\end{array}$ & & $\begin{array}{l}.43516 \\
.43550 \\
.43585 \\
.43620 \\
.43654 \\
.43089 \\
.43724 \\
.43758 \\
.43793 \\
.43828\end{array}$ & $\begin{array}{l}2.29 .801 \\
2.29619 \\
2.29437 \\
2.29254 \\
2.29073 \\
2.28891 \\
2.28710 \\
2.28528 \\
2.28348 \\
2.28167\end{array}$ & $\begin{array}{l}29 \\
28 \\
27 \\
26 \\
25 \\
24 \\
23 \\
22 \\
21 \\
20\end{array}$ \\
\hline $\begin{array}{l}41 \\
42 \\
43 \\
44 \\
45 \\
46 \\
47 \\
48 \\
49 \\
50\end{array}$ & $\begin{array}{l}.37754 \\
.37787 \\
.37820 \\
.37853 \\
.37887 \\
.37920 \\
.37953 \\
.37986 \\
.38020 \\
.38053\end{array}$ & $\begin{array}{l}2.64875 \\
2.64642 \\
2.64410 \\
2.64177 \\
2.633945 \\
2 .(03714 \\
2.03383 \\
2.63252 \\
2.63021 \\
2.62791\end{array}$ & $\begin{array}{l}.39761 \\
.39795 \\
.39529 \\
.39862 \\
.39596 \\
.39930 \\
.39963 \\
.39997 \\
.40031 \\
.40065\end{array}$ & $\begin{array}{l}2.51502 \\
2.51289 \\
2.51076 \\
2.50864 \\
2.50652 \\
2.50440 \\
2.50229 \\
2.50018 \\
2.49807 \\
2.49597\end{array}$ & $\begin{array}{l}.41797 \\
.41831 \\
.41865 \\
.41899 \\
.41933 \\
.41968 \\
.42002 \\
.42036 \\
.42070 \\
.42105\end{array}$ & & $\begin{array}{l}.438622 \\
.43597 \\
.43932 \\
.43966 \\
.44001 \\
.44036 \\
.44071 \\
.44105 \\
.44140 \\
.44175\end{array}$ & $\begin{array}{l}2.27987 \\
2.27806 \\
2.27626 \\
2.27447 \\
2.27267 \\
2.27088 \\
2.26909 \\
2.26730 \\
2.26552 \\
2.26374\end{array}$ & $\begin{array}{l}19 \\
18 \\
17 \\
16 \\
15 \\
14 \\
13 \\
12 \\
11 \\
10\end{array}$ \\
\hline $\begin{array}{l}\mathbf{5 1} \\
\mathbf{5 2} \\
\mathbf{5 3} \\
\mathbf{5 4} \\
\mathbf{5 5} \\
\mathbf{5 6} \\
\mathbf{5 7} \\
58 \\
\mathbf{5 9} \\
\mathbf{6 0}\end{array}$ & $\begin{array}{r}.38086 \\
.38120 \\
.38153 \\
.38186 \\
.38220 \\
.38253 \\
.38286 \\
.38320 \\
.38353 \\
.38386\end{array}$ & $\begin{array}{l}2.62561 \\
2.62332 \\
2.62103 \\
2.61874 \\
2.61646 \\
2.61418 \\
2.61190 \\
2.60963 \\
2.60736 \\
2.60509\end{array}$ & $\begin{array}{l}.40098 \\
.40132 \\
.40166 \\
.40200 \\
.40234 \\
.40267 \\
.40301 \\
.40335 \\
.412369 \\
.40403\end{array}$ & $\begin{array}{l}2.49386 \\
2.49177 \\
2.48967 \\
2.48758 \\
2.48549 \\
2.48340 \\
2.48132 \\
2.47924 \\
2.47716 \\
2.47509\end{array}$ & $\begin{array}{l}.42139 \\
.42173 \\
.42207 \\
.42212 \\
.42276 \\
.42310 \\
.42345 \\
.42379 \\
.42413 \\
.42447\end{array}$ & $\begin{array}{l}2.37311 \\
2.37118 \\
2.36925 \\
2.36733 \\
2.36541 \\
2.36349 \\
2.36158 \\
2.35367 \\
2.35776 \\
2.35585\end{array}$ & $\begin{array}{l}.44210 \\
.44244 \\
.44279 \\
.44314 \\
.44349 \\
.44381 \\
.44418 \\
.44453 \\
.44488 \\
.44523\end{array}$ & $\begin{array}{l}2.26196 \\
2.21018 \\
2.25840 \\
2.25663 \\
2.25486 \\
2.25309 \\
2.25132 \\
2.24956 \\
2.24780 \\
2.24604\end{array}$ & $\begin{array}{l}9 \\
8 \\
7 \\
6 \\
5 \\
4 \\
3 \\
2 \\
1 \\
0\end{array}$ \\
\hline & Lotang. & Tang. & Cotang. & Tang. & Cotang. & Tang. & constap & Tang. & \\
\hline & \multicolumn{2}{|c|}{$69^{\circ}$} & \multicolumn{2}{|c|}{$65^{\circ}$} & \multicolumn{2}{|c|}{$67^{\circ}$} & \multicolumn{2}{|c|}{$66^{\circ}$} & \\
\hline
\end{tabular}


TABLF 8. - NATURAL TANGENTS AND COTANGENT.

\begin{tabular}{|c|c|c|c|c|c|c|c|c|c|}
\hline \multirow{2}{*}{ ' } & \multicolumn{2}{|c|}{$21^{\circ}$} & \multicolumn{2}{|c|}{$25^{\circ}$} & \multicolumn{2}{|c|}{$26^{\circ}$} & \multicolumn{2}{|c|}{$27^{\circ}$} & \multirow{2}{*}{, } \\
\hline & Tang. & Cotang. & Tang. & Cotang. & Tang. & Cotang. & Tang. & Cotang. & \\
\hline 0 & .44523 & 2.24004 & .46631 & 2.14451 & $.4 \$ 773$ & 2.05030 & .50953 & 1. 96261 & 60 \\
\hline 1 & .44558 & 2.24428 & .46666 & 2.14259 & $4 S 809$ & 2. 04879 & .50989 & 1.96120 & 59 \\
\hline 2 & .44593 & 2. 24252 & .46702 & 2.14125 & .48845 & 2. 04728 & .51026 & 1. 95979 & 58 \\
\hline 3 & .44627 & 2.24077 & . 46737 & 2. 13963 & .48881 & 2.04577 & .51063 & 1. 95838 & 57 \\
\hline 4 & .44662 & 2. 23902 & .46772 & 2. 13801 & .48917 & 2. 04426 & .51099 & 1.95698 & 56 \\
\hline 5 & .44697 & 2.23727 & .46808 & 2. 13639 & .48953 & 2. 04276 & .51136 & 1. 95557 & 55 \\
\hline 6 & .44732 & 2. 23553 & .46843 & 2.13477 & .48989 & 2.04125 & .51173 & 1.95417 & 54 \\
\hline 7 & .44767 & 2. 23378 & .46879 & 2. 13316 & .49026 & 2. 03975 & .51209 & 1.95277 & 53 \\
\hline 8 & .44802 & 2. 23204 & .46914 & 2. 13154 & .49062 & 2. 03825 & .51246 & 1.95137 & 52 \\
\hline 9 & .44537 & 2. 23030 & .46950 & 2. 12993 & .49098 & 2. 03675 & .51283 & 1. 94997 & 51 \\
\hline 10 & .44872 & 2. 22857 & .46985 & 2. $12 \varsigma 32$ & .49134 & 2. 03526 & .51319 & 1. 948.58 & 50 \\
\hline 11 & .44907 & 2. 22683 & .47021 & 2. 12671 & .49170 & 2. 03376 & .51356 & 1.94718 & 49 \\
\hline 12 & .44942 & 2. 22510 & .47056 & 2.12511 & .49206 & 2. 03227 & .51393 & 1.94579 & 48 \\
\hline 13 & .44977 & 2.22337 & .47092 & 2.12350 & .49242 & 2. $0307 \mathrm{~s}$ & .51430 & 1.94440 & 47 \\
\hline 14 & .45012 & 2. 22164 & .47128 & 2.12190 & .49278 & 2. 02929 & .51467 & 1. 94301 & 46 \\
\hline 15 & .45047 & 2. 21992 & .47163 & 2. 12030 & .49315 & 2.02780 & .51503 & 1.94162 & 45 \\
\hline 16 & .45082 & 2. 21819 & .47199 & 2.11871 & .49351 & 2. 02631 & .51540 & 1.94023 & 44 \\
\hline 17 & .45117 & 2.21647 & .47234 & 2.11711 & .49387 & 2.02483 & .51577 & 1.938855 & 43 \\
\hline 18 & .45152 & 2.21475 & .47270 & 2.11552 & .43423 & 2. 02335 & .51614 & 1. 93746 & 42 \\
\hline 19 & .45187 & 2. 21304 & .47305 & 2.11392 & .49459 & 2. 02187 & .51651 & 1. 93008 & 41 \\
\hline 20 & .45222 & 2.21132 & .47341 & 2.11233 & .49495 & 2. 02039 & .51688 & 1.93470 & 40 \\
\hline 21 & .45257 & 2. 20961 & .47377 & 2.11075 & .49532 & 2. 01891 & .51724 & 1. 93332 & 39 \\
\hline 22 & .45292 & 2. 20790 & .47412 & 2.10916 & .49568 & 2.01743 & .51761 & 1.93195 & 38 \\
\hline 23 & .45327 & 2.20619 & .47448 & 2.10758 & .49604 & 2. 01596 & .51798 & 1.93057 & 37 \\
\hline 24 & .45362 & 2. 20449 & .47483 & 2.10600 & .49640 & 2. 01449 & .51835 & 1. 92920 & 36 \\
\hline 25 & .45397 & 2.20278 & .47519 & 2. 10442 & .49677 & 2. 01302 & .51872 & 1. 92782 & 35 \\
\hline 26 & .45432 & 2.20108 & .47555 & 2.10281 & .49713 & 2.01155 & .51909 & 1. 92645 & 34 \\
\hline 27 & .45467 & 2. 19038 & .47590 & 2.10126 & .49749 & 2. 01008 & .51946 & 1. 92508 & 33 \\
\hline 28 & .45502 & 2.19769 & .47626 & 2. 09969 & .49786 & 2. 00562 & .51983 & 1.92371 & 32 \\
\hline 29 & .45538 & 2. 19599 & .47682 & 2.09811 & .43822 & 2.00715 & .52020 & 1.92235 & 31 \\
\hline 30 & .45573 & 2.19430 & .47698 & $2.0965 t$ & .49555 & 2. 00569 & .52057 & 1. 92098 & 30 \\
\hline 31 & .45608 & 2. 19261 & . 47733 & 2.09498 & .49894 & 2. 00423 & .52094 & 1.91962 & 29 \\
\hline 32 & .45643 & 2. 13092 & .47769 & 2. 09341 & .49931 & 2.00277 & .52131 & 1.91826 & 28 \\
\hline 33 & .45678 & 2. 18923 & .47805 & 2.09184 & .49967 & 2. 00131 & .52168 & 1.91690 & 27 \\
\hline 34 & .45713 & 2.18755 & .47840 & 2.09028 & .50004 & 1.99986 & .52205 & 1.91554 & 26 \\
\hline 35 & .45748 & 2. 18587 & .47576 & 2.08872 & .50040 & 1.99841 & 52242 & 1. 91418 & 25 \\
\hline 36 & .45784 & 2. 18419 & .47912 & 2.08716 & .50076 & 1. 99695 & .52279 & 1. 91282 & 24 \\
\hline 37 & .45819 & 2.18251 & .47948 & 2.08560 & .50113 & 1.99550 & .52316 & 1. 91147 & 23 \\
\hline 38 & .45854 & 2. 18084 & .47984 & 2. 08405 & .50149 & 1. 99406 & .52353 & 1.91012 & 22 \\
\hline 39 & .45889 & 2.17916 & .48019 & 2.08250 & .50185 & 1.99261 & .52390 & 1.90876 & 21 \\
\hline 40 & .45924 & 2. 17749 & .48055 & 2.08094 & .50222 & 1.99116 & .52427 & 1.90741 & 20 \\
\hline 41 & .45960 & 2.17552 & .48091 & 2. 07939 & .50258 & 1. 98972 & .52464 & 1.90607 & 19 \\
\hline 42 & .45995 & 2.17416 & .48127 & 2.07785 & .50295 & 1. 08828 & .52501 & 1.90472 & 18 \\
\hline 43 & .46030 & 2.17249 & .48163 & 2.07630 & .50331 & 1.08684 & .52538 & 1. 90337 & 17 \\
\hline 44 & .46065 & 2.17083 & .48198 & 2.07476 & .50368 & 1. 95540 & .52575 & 1. 90203 & 16 \\
\hline 45 & .46101 & 2.1 (f917 & .48234 & 2. 07321 & .50404 & 1. 98396 & .52613 & 1.90069 & 15 \\
\hline 46 & .46136 & 2.16751 & .45270 & 2.07167 & .50441 & 1. 95253 & .52650 & 1. 89035 & 14 \\
\hline 47 & .46171 & 2. 10585 & $.4 \times 306$ & 2.07014 & .50477 & 1. 95110 & .52657 & 1.89801 & 13 \\
\hline 48 & .46206 & 2. $16+20$ & $.4 \$ 342$ & 2.06860 & .50514 & 1.97966 & .52724 & 1. 89667 & 12 \\
\hline 49 & .46242 & 2.16255 & .48378 & 2.06706 & .50550 & 1.97823 & .52761 & 1.89533 & 11 \\
\hline 50 & .46277 & 2. 16090 & $.4 S 414$ & 2.06553 & .50587 & 1. 97681 & .52798 & 1.89400 & 10 \\
\hline 51 & .46312 & 2.15925 & .48450 & 2. 06400 & .50623 & 1.97538 & $.52 \times 36$ & 1.89366 & 9 \\
\hline 52 & .46348 & 2.15760 & 48486 & 2.06247 & .50660 & 1. 97395 & & 1.89133 & 8 \\
\hline 53 & .46383 & 2.15596 & .48521 & 2.06094 & .50696 & 1. 97253 & .52910 & 1. 89000 & 7 \\
\hline 54 & .46418 & 2.15432 & .48557 & 2.05942 & .50733 & 1.97111 & & 1. 885667 & 6 \\
\hline 55 & .46454 & 2.15263 & .48593 & 2.05790 & .50769 & 1. 96969 & .52985 & 1. 88734 & 5 \\
\hline 50 & .46489 & 2.15104 & .48629 & 2. $05 \times 37$ & .50806 & 1. 96527 & .53022 & 1. .83002 & 4 \\
\hline 57 & .46525 & 2. 14940 & .48665 & 2.05485 & .50843 & 1. meris5 & .53059 & 1. 84469 & 3 \\
\hline 58 & $.465(0)$ & 2.14777 & .4S701 & 2. 05333 & .50879 & 1. 95544 & .53096 & 1. 85337 & 2 \\
\hline 59 & .46595 & 2. 14614 & .48737 & 2.05182 & .50916 & 1. 96402 & .53134 & 1.85205 & i \\
\hline 60 & .46631 & 2. 14451 & .48773 & 2.05030 & .50953 & 1. 96261 & .53171 & 1.85073 & 0 \\
\hline \multirow{2}{*}{, } & $\overline{\text { Cotang. }}$ & Tang. & Cotang. & Tang. & Cotang. & Tang. & $\overline{\text { Cotang. }}$ & Tang. & \\
\hline & \multicolumn{2}{|c|}{$65^{\circ}$} & \multicolumn{2}{|c|}{$61^{\circ}$} & \multicolumn{2}{|c|}{$6.3^{\circ}$} & \multicolumn{2}{|c|}{$62^{\circ}$} & \\
\hline
\end{tabular}


TABLE 8.-NATURAL TANGENTS AND COTANGENTS.

\begin{tabular}{|c|c|c|c|c|c|c|c|c|c|}
\hline \multirow{2}{*}{, } & \multicolumn{2}{|c|}{$28^{\circ}$} & \multicolumn{2}{|c|}{$29^{\circ}$} & \multicolumn{2}{|c|}{$30^{\circ}$} & \multicolumn{2}{|c|}{$31^{\circ}$} & \\
\hline & Tang. & Cotang. & Tang. & Cotang. & Tang. & Cot & Tang. & Cotang. & \\
\hline 0 & .53171 & 1. 88073 & .55431 & $1.8 \cup 405$ & .57735 & 1. 73205 & .60086 & 1. 66428 & 60 \\
\hline 1 & & & & & .57774 & & .60126 & & 59 \\
\hline 2 & .53246 & 1. 87809 & .55507 & 1. 80158 & .57813 & 1. 72973 & .60165 & 1. 66209 & 58 \\
\hline 3 & .53283 & 1.87677 & .55545 & 1.80034 & .57851 & 1.72857 & . 60205 & 1. 66099 & 57 \\
\hline 4 & .53320 & 1.87546 & .55583 & 1.79911 & .57890 & 1.72741 & . 60245 & 1. 65990 & 56 \\
\hline 5 & .53358 & 1.87415 & .55621 & 1.79788 & .57929 & 1.72625 & .60284 & 1. 65881 & 55 \\
\hline 6 & .53395 & 1. 87283 & .55659 & 1.79665 & .57968 & 1.72509 & .60324 & 1. 65772 & 54 \\
\hline 7 & .534 .32 & 1.87152 & .55697 & 1.79542 & .58007 & 1.72393 & . 60304 & 1. 65663 & 53 \\
\hline 8 & .53470 & 1.87021 & .55736 & 1.79419 & .58016 & 1.72278 & .60403 & 1. 65554 & 52 \\
\hline 9 & .53507 & 891 & .55774 & 1. & .58085 & 1. & & 445 & 51 \\
\hline 10 & .53545 & 1.8 & 812 & 174 & .58124 & 047 & .60483 & 337 & 50 \\
\hline 11 & .52582 & 1.8 & .55850 & 51 & $.581 \mathrm{fi2}$ & 32 & .60522 & 28 & 49 \\
\hline 12 & .53620 & 1.8 & 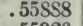 & 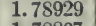 & .58201 & 17 & . 60562 & 5120 & 48 \\
\hline 13 & .53657 & 1. 86369 & .55926 & 1.78807 & .58240 & 1.71702 & .60602 & 1. 65011 & 47 \\
\hline 14 & .53694 & 1.86239 & .55964 & 1.78685 & .58279 & 1.715 & . 60642 & 1. 64903 & 46 \\
\hline 15 & .53732 & 1. 86109 & .56003 & 178563 & .58318 & 1. 71473 & .60681 & 1. 64795 & 45 \\
\hline 16 & .53769 & 979 & 041 & 441 & .58357 & & .60721 & 688 & 44 \\
\hline 17 & .53807 & 1.85850 & .56079 & 1.78319 & .58396 & 1.71244 & . 60761 & 1. 64579 & 43 \\
\hline 18 & .53844 & 720 & 117 & 1. & . 58435 & & & 1. 64471 & 42 \\
\hline 19 & .53882 & 1. 85591 & .56156 & 077 & $.5 S 474$ & 1.7 & . 60841 & 1. 64363 & 41 \\
\hline 20 & .53920 & 1.85462 & .56194 & 955 & .58513 & 1.7 & .60881 & 1. 64256 & 40 \\
\hline 21 & .53957 & 1. 85333 & .56232 & 1. 77834 & .58552 & 1.70787 & .60921 & 1. 64148 & 39 \\
\hline 22 & .53995 & & .56270 & 1. & .58591 & & 360 & 41 & 38 \\
\hline 2 & .54032 & 1.85075 & .56309 & 1.7 & .58631 & 1. & & & 37 \\
\hline 2 & .54070 & 1.84946 & .56347 & 1.7 & .58670 & 2446 & 40 & 3826 & 36 \\
\hline 2 & & 1.8 & .56385 & & . 58709 & & & & 35 \\
\hline 2 & .5 & 1.8 & .56424 & 1.7 & .58748 & 1. & 20 & 3612 & 34 \\
\hline 2 & & 1.8 & .56462 & 1.7 & .58787 & & 160 & 1. 63505 & 33 \\
\hline 2 & .5 & 1.8 & 01 & & $.58 \$ 26$ & & & 3398 & 32 \\
\hline 2 & .54258 & 1.8 & .56539 & 1.7 & .58865 & 1. & 40 & 3292 & 31 \\
\hline 30 & .54296 & 77 & .56577 & 1. & 05 & 1. & 80 & 85 & 30 \\
\hline 31 & .543333 & 1.8 & .56616 & 1. & 44 & 1. & .61320 & 3079 & 29 \\
\hline 3 & .5 & & .5 & & & & & & 28 \\
\hline & & 1.8 & .5 & & 22 & & 00 & 2866 & 27 \\
\hline 3 & & 1.8 & .56731 & & 61 & & & 2760 & 26 \\
\hline 3 & & & .56769 & & & & & 2654 & 25 \\
\hline 3 & & 1.8 & .56808 & & 0 & & 20 & 2548 & 21 \\
\hline 3 & & 1. & .56846 & 1. 7 & & & 61 & 2442 & 23 \\
\hline 3. & .5 & 1.8 & .56885 & & & & & 36 & 22 \\
\hline 39 & .54635 & 033 & .56923 & & .59258 & & 11 & 1. 62230 & 21 \\
\hline 40 & .54673 & 1.82906 & .56962 & 1.75556 & .59297 & 1. 68643 & .61681 & 1. 62125 & 20 \\
\hline 41 & .54711 & 1.8 & .57000 & 1.7 & .59336 & $1 . t$ & .61721 & 19 & 19 \\
\hline & & & & & & & & & 18 \\
\hline 43 & & & .5 & & & & & & 17 \\
\hline 44 & & & .57116 & & & & & & 16 \\
\hline 4 & & & & & & & & & 15 \\
\hline 40 & & & .57193 & & & & . 61922 & 493 & 14 \\
\hline 47 & & & .57232 & & & & & & 13 \\
\hline 48 & & 1. & .57271 & & & & & & 12 \\
\hline 41 & .55013 & & & & & & & & 11 \\
\hline 0 & 00 & 649 & .57348 & 1.743 & .59691 & 1. 67530 & 83 & 74 & 10 \\
\hline 51 & $.550 \$ 99$ & 1.81524 & .57386 & 1.7 & .59730 & & .62124 & & 9 \\
\hline 52 & & & & & & & & & 8 \\
\hline 53 & & & .57 & & & & & 61 & 7 \\
\hline 54 & & & .57503 & & & & & 1. $60(57)$ & 6 \\
\hline 55 & & & & & & & & 1. 60553 & 5 \\
\hline 56 & & 1. & .57 & & & & . 62325 & 1. 60449 & 4 \\
\hline 57 & & & .57619 & & .59967 & & .62366 & 1. 60345 & 3 \\
\hline 5. & & & & & .60007 & & & & 2 \\
\hline 59 & & & .5 & & & & .62446 & 1. 60137 & 1 \\
\hline & 31 & 1.80405 & .57735 & 1.73205 & .60086 & 1. 66428 & .62487 & 1. 60033 & 0 \\
\hline \multirow{2}{*}{ - } & Cotang. & Tang. & Cotang. & Tang. & Cotang. & Tang. & Cotang. & Tang. & \\
\hline & \multicolumn{2}{|c|}{$61^{\circ}$} & \multicolumn{2}{|c|}{$60^{\circ}$} & \multicolumn{2}{|c|}{59} & \multicolumn{2}{|c|}{$58^{\circ}$} & \\
\hline
\end{tabular}


TABLE 8.-NATURAL TANGENTS AND COTANGENTS.

\begin{tabular}{|c|c|c|c|c|c|c|c|c|c|}
\hline \multirow{2}{*}{, } & \multicolumn{2}{|c|}{$32^{\circ}$} & \multicolumn{2}{|c|}{$33^{\circ}$} & \multicolumn{2}{|c|}{$34^{\circ}$} & \multicolumn{2}{|c|}{$35^{\circ}$} & \multirow{2}{*}{ - } \\
\hline & Tang. & Cotang. & Tang. & Cotang. & Tang. & Cotang. & Tang. & Cotang. & \\
\hline 0 & .62457 & 1. 60033 & .64941 & 1.53986 & .67451 & 1.48256 & .70021 & 1.42815 & 60 \\
\hline 1 & .62527 & 1. 59930 & .64982 & 1. 53588 & .67493 & 1.48163 & .70064 & 1.42726 & 59 \\
\hline 2 & .62508 & 1. 59826 & .65024 & 1.53791 & .67536 & 1.48070 & .70107 & 1.42638 & 58 \\
\hline 3 & .62608 & 1. 59723 & .65065 & 1.53693 & .67578 & 1.47977 & .70151 & 1.42550 & 57 \\
\hline 4 & .62649 & 1. 59620 & .65106 & 1. 53595 & .67620 & 1.47885 & .70194 & 1.42462 & 56 \\
\hline 5 & .62659 & 1. 59517 & .65148 & 1. 53497 & .67663 & 1.47792 & .70238 & 1.42374 & 55 \\
\hline 6 & .62730 & 1. 59414 & .65189 & 1.53400 & .67705 & 1.47699 & .70281 & 1.42286 & 54 \\
\hline 7 & .62770 & 1. 59311 & .65231 & 1.53302 & .67748 & 1.47607 & .70325 & 1.42198 & 53 \\
\hline 8 & .62811 & 1. 59208 & .65272 & 1. 53205 & .67790 & 1.47514 & .70368 & 1.42110 & 52 \\
\hline 9 & .62852 & 1.59105 & .65314 & 1.53107 & .67832 & 1.47422 & .70412 & 1.42022 & 51 \\
\hline 10 & .62592 & 1.59002 & .65355 & 1. 53010 & .67875 & 1.47330 & .70455 & 1.41934 & 50 \\
\hline 11 & .62933 & 1.59900 & .65397 & 1. 52913 & .67917 & 1. 47238 & .70499 & 1.41847 & 49 \\
\hline 12 & .62973 & 1.55797 & .65438 & 1.52816 & .67960 & 1.47146 & .70542 & 1.41759 & 48 \\
\hline 13 & .63014 & 1.58695 & .65480 & 1. 52719 & .65002 & 1.47053 & .70586 & 1.41672 & 47 \\
\hline 14 & .63055 & 1. 58593 & .65521 & 1.52622 & .68045 & 1.46962 & .70629 & 1.41584 & 46 \\
\hline 15 & .63095 & 1.58490 & .65563 & 1.52525 & .65058 & 1.46870 & .70673 & 1.41497 & 45 \\
\hline 16 & .63136 & 1.58358 & .65604 & 1. 52429 & .68130 & 1.46778 & .70717 & 1.41409 & 44 \\
\hline 17 & .63177 & 1. 58286 & .65646 & 1.52332 & .68173 & 1.46686 & .70760 & 1.41322 & 43 \\
\hline 18 & .63217 & 1. 58184 & .65688 & 1.52235 & .68215 & 1.46595 & .70804 & 1.41235 & 42 \\
\hline 19 & .63258 & 1. 58083 & .65729 & 1.52139 & .68258 & 1.46503 & .70848 & 1.41148 & 41 \\
\hline 20 & .63299 & 1.57981 & .65771 & 1. 52043 & .68301 & 1.46411 & .70891 & 1.41061 & 40 \\
\hline 21 & .63340 & 1. 57879 & .65813 & 1. 51946 & .68343 & 1. 46320 & .70935 & 1. 40974 & 39 \\
\hline 22 & .63380 & 1.57778 & .65554 & 1.51850 & .68386 & 1.46229 & .70979 & 1.40587 & 38 \\
\hline 23 & .63421 & 1.57676 & .65896 & 1.51754 & .68429 & 1.46137 & .71023 & 1.40800 & 37 \\
\hline 24 & .63462 & 1. 57575 & .65938 & 1.51658 & .68471 & 1.46046 & .71066 & 1.40714 & 36 \\
\hline 25 & .63503 & 1. 57474 & . 65980 & 1.51562 & .68514 & 1.45955 & .71110 & 1.40627 & 35 \\
\hline 26 & .63544 & 1. 57372 & .66021 & 1.51466 & .68557 & 1.45864 & .71154 & 1.40540 & 34 \\
\hline 27 & .63584 & 1. 57271 & .66063 & 1.51370 & .68600 & 1.45773 & .71198 & 1.40454 & 33 \\
\hline 28 & .63625 & 1. 57170 & . 66105 & 1.51275 & .68642 & 1.45682 & .71242 & 1.40367 & 32 \\
\hline 29 & .63606 & 1.57069 & .66147 & 1.51179 & .68685 & 1.45592 & .71285 & 1.40281 & 31 \\
\hline 30 & .63707 & 1. 56969 & .66189 & 1.51084 & .68728 & 1.45501 & .71329 & 1.40195 & 30 \\
\hline 31 & .63748 & 1. 56868 & .66230 & 1. 50988 & .68771 & 1.45410 & .71373 & 1. 40109 & 29 \\
\hline 32 & .63789 & $1.56 \% 67$ & .66272 & 93 & 814 & 1.45320 & .71417 & 1. 40022 & 28 \\
\hline 33 & .63830 & 1. 56667 & .66314 & 797 & .68857 & 1.45229 & .71461 & 1.39936 & 27 \\
\hline 34 & .63871 & 1. 56566 & .66356 & $1.50 \% 02$ & .68900 & 1.45139 & .71505 & 1. 39550 & 26 \\
\hline 3.5 & .63912 & 1.56466 & .66398 & 1. 50607 & .68942 & 1. 45049 & 49 & 1. $39 \div 64$ & 25 \\
\hline 36 & .63953 & 1. 56366 & .66440 & 1. 50512 & .68985 & 1.44958 & .71593 & 1. 39679 & 24 \\
\hline 37 & .63994 & 1. 56265 & .60482 & 1.50417 & .69028 & 1.44868 & .71637 & 1. 39593 & 23 \\
\hline 38 & .64035 & 1.56165 & .66524 & 1. 50322 & .69071 & 1.44778 & .71681 & 1.39507 . & 22 \\
\hline 39 & $.640 \pi 6$ & 1. $5 \times 065$ & .66566 & 1.50228 & .69114 & $1.446 \mathrm{GS}$ & .71725 & 1. 39421 & 21 \\
\hline 40 & .64117 & 1. 55966 & .66608 & 1.50133 & .69157 & 1.44508 & .71769 & 1. 39336 & 20 \\
\hline 41 & .64158 & 1. 55866 & .66650 & 1. 50039 & .69200 & 1.44508 & $.71 S 13$ & 1. 39250 & 19 \\
\hline 42 & .64199 & 1.55766 & .66602 & 1. 49944 & .69243 & 1.44418 & .71857 & 1.39165 & 18 \\
\hline 43 & .64240 & 1. 556066 & .66734 & 1. 49849 & .69286 & 1. 44329 & .719001 & 1. 39079 & 17 \\
\hline 44 & .64381 & 1.55567 & .66776 & 1.49755 & .69329 & 1. 44239 & .71946 & 1. 35994 & 16 \\
\hline 45 & .64322 & 1. 55467 & .66818 & 1.49661 & .69372 & 1.44149 & .71990 & 1.38909 & 15 \\
\hline 46 & .64363 & 1. 55368 & .60860 & 1.49566 & .69416 & $1.440<0$ & .72034 & 1. 38824 & 14 \\
\hline 47 & .64404 & 1. 55269 & .66902 & $1.494 \pi 2$ & .69459 & 1. $439 \% 0$ & .72078 & 1.35738 & 13 \\
\hline 49 & .64446 & 1. 55170 & .66944 & 1. 49378 & .69502 & 1. 43651 & .72122 & 1. 38653 & 12 \\
\hline 49 & .64487 & 1. 55071 & . 6rias6 & 1.49254 & .69545 & $1.43 \pi 92$ & .72167 & 1. 35568 & 11 \\
\hline 50 & .64528 & 1. 54972 & .67028 & 1.49190 & .69588 & 1.43703 & .72211 & 1.38454 & 10 \\
\hline 51 & .64569 & 1.54873 & .67071 & 1. 49097 & .69631 & 1. 43614 & .72255 & 1. 36399 & 9 \\
\hline 52 & .64610 & 1.54774 & .67113 & 1.49003 & .69675 & 1. 43525 & .72290 & 1.38314 & 8 \\
\hline 53 & .64652 & 1.51675 & .67155 & 1.48909 & .69718 & 1. 43436 & .72344 & 1.38229 & \\
\hline 54 & .64693 & 1. 54576 & .67197 & 1.48816 & .69761 & 1. 43347 & .72358 & 1. 35145 & 6 \\
\hline 55 & .64734 & 1. 54478 & .67239 & 1.48722 & .69504 & 1.43258 & .72432 & 1.35060 & 5 \\
\hline 56 & .64775 & 1.54379 & .67252 & 1.48629 & .68847 & 1.43169 & .72477 & 1.37976 & 4 \\
\hline 57 & .64817 & 1. 54281 & .67324 & 1.48536 & .69591 & 1.43050 & .72521 & 1.37591 & 3 \\
\hline 58 & .64858 & 1. 54183 & .07366 & 1.48442 & .69934 & 1. 42992 & .72505 & 1. 37507 & 2 \\
\hline 53 & . 641599 & 1. 54085 & .67409 & 1. 48349 & . 69977 & 1.42903 & .72610 & 1.37722 & 1 \\
\hline 60 & .64941 & 1. 53956 & .67451 & 1. 45256 & .70021 & 1.42515 & .72654 & 1.3\%638 & 0 \\
\hline & Cotang. & Tang. & Cotang. & Tang. & Cotang. & Tang. & $\overline{\text { Cotang. }}$ & Tang. & \\
\hline & \multicolumn{2}{|c|}{$57^{\circ}$} & \multicolumn{2}{|c|}{$56^{\circ}$} & \multicolumn{2}{|c|}{$55^{\circ}$} & \multicolumn{2}{|c|}{$54^{\circ}$} & \\
\hline
\end{tabular}


TABLE 8.-NATURAL TANGENTS AND COTANGENTS.

\begin{tabular}{|c|c|c|c|c|c|c|c|c|c|}
\hline \multirow{2}{*}{, } & \multicolumn{2}{|c|}{$36^{\circ}$} & \multicolumn{2}{|c|}{$37^{\circ}$} & \multicolumn{2}{|c|}{$38^{\circ}$} & \multicolumn{2}{|c|}{$39^{\circ}$} & \\
\hline & Tang. & Cotang. & Tang. & Cotang. & Tang. & Cotang. & ang. & Cotang. & \\
\hline 0 & .72654 & 1.37638 & .75355 & 1.32704 & .78129 & 1. 27994 & .80978 & 1. 23490 & 60 \\
\hline 1 & & & & & .78175 & & 027 & & 50 \\
\hline 2 & .72743 & 1. 37470 & .75447 & 1. 32544 & .78222 & 1. 27841 & .81075 & 1. 23343 & 58 \\
\hline 3 & .72788 & 1. 37386 & .75492 & 1. 32464 & .78269 & 1.27764 & 81123 & 1. 23270 & 57 \\
\hline 4 & .72832 & 1. 37302 & .75538 & 1.32384 & .78316 & 1.27688 & .81171 & 1. 23196 & 56 \\
\hline 5 & 877 & 218 & 84 & 1. & .78363 & 1.27611 & 1220 & 1.23123 & 55 \\
\hline 6 & .72921 & 1. 37134 & .75629 & 1.32224 & .78410 & 1. 27535 & .81268 & 1. 23050 & 54 \\
\hline 7 & & & & 1. 32144 & .78457 & 1. 27458 & 81316 & 1.22977 & \\
\hline 8 & 010 & 1.36967 & .75721 & 1. 32064 & .78504 & 1. 27382 & .81364 & 1. 22904 & 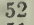 \\
\hline 9 & 055 & 1. 36883 & .75767 & 1.31984 & .78551 & 1. 27306 & 81413 & 1. 22831 & \\
\hline 10 & .73100 & 1. 36800 & .75 & 1. 31904 & .78598 & 1. 27230 & 81461 & 1. 22758 & 50 \\
\hline 11 & .73144 & 1. 36716 & .75858 & 1. 31825 & .78645 & 1. 27153 & 81510 & 1. 22685 & 49 \\
\hline 12 & & & & & & & & 2612 & 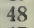 \\
\hline 13 & .73234 & 6549 & .75950 & 1.310 & .78739 & 1. 27001 & 31606 & 1. 22539 & 47 \\
\hline 14 & 278 & & .75996 & 1. & .78786 & 1. 26925 & 31655 & 1. 22467 & 40 \\
\hline 15 & .73323 & 1. 36383 & .76042 & 1. 31507 & .78834 & 1. 26849 & 81703 & 1. 22394 & 45 \\
\hline 16 & & & .76088 & 1. 31427 & $.788 S 1$ & 1. 26774 & 1752 & 1.22321 & 44 \\
\hline 17 & .73413 & 1.3 & .76134 & 1. 31348 & .78928 & 1.26 & 81800 & 1. 22249 & 43 \\
\hline 18 & & & & & & & .81849 & 1.22176 & 42 \\
\hline 19 & 502 & 1.3 & .76226 & 1.2 & .79022 & 1. 26546 & 81898 & 1. 22104 & 41 \\
\hline 20 & .73547 & 1.35968 & .76272 & 110 & .79070 & 1.26471 & .81946 & 1.22031 & 40 \\
\hline 21 & .73592 & 1.3 & .76318 & 1031 & .79117 & 1. 26395 & 81995 & 21959 & 39 \\
\hline 22 & C37 & & .76364 & 2 & .79164 & 1. & 2044 & SSO & 8 \\
\hline 23 & & & & & & & 2092 & 14 & 37 \\
\hline 24 & 726 & & 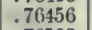 & 5 & 59 & & 2141 & 1. 21742 & 36 \\
\hline 25 & & & & & & & & 1.21670 & \\
\hline 20 & .7 & 1. & 8 & & 54 & 1. & 2238 & 1598 & 4 \\
\hline 27 & & & & & & & 2287 & 1.21526 & \\
\hline 2 & 306 & 1. & 0 & & 19 & & 2336 & 154 & 2 \\
\hline 2 & & & & & & & .82385 & 382 & 31 \\
\hline 30 & .73996 & 1. 35142 & .76733 & 1. 30323 & .79544 & 1. 25717 & .82434 & 1.21310 & 30 \\
\hline 31 & .74041 & 1. 35060 & .76779 & 1. 30244 & .79591 & 1. 25642 & .82483 & 1. 21238 & 29 \\
\hline 32 & & & & & & & 31 & & \\
\hline 3 & 31 & & 1 & 7 & 36 & 2 & 80 & 94 & 27 \\
\hline 34 & 176 & & & 1. & 34 & 1. & 29 & 23 & 6 \\
\hline 35 & 221 & & .76964 & 1. & 81 & & 82678 & 51 & 5 \\
\hline $3 f$ & & & & & 29 & & 2727 & 79 & 4 \\
\hline 3 & 312 & 1. & 57 & 1. & 67 & 93 & 76 & 08 & 3 \\
\hline 3 & & & & & 24 & & 2825 & 736 & 22 \\
\hline 3 & 102 & & & 1. & 79972 & 1.2 & 82874 & 85 & 21 \\
\hline 40 & .74447 & 1. 34323 & .77196 & 1.29541 & .80020 & 1. 24969 & .82923 & 1. 20593 & 20 \\
\hline 41 & 492 & 1. 34242 & .77242 & 1. & .80067 & 1.245 & .82972 & 0522 & 19 \\
\hline 42 & & & & & & & 3022 & 51 & 8 \\
\hline 43 & .7 & & & & & & 71 & 79 & 17 \\
\hline 4. & 28 & & .7 & & & & 120 & & 16 \\
\hline 4 & .74674 & & & 1. & & & 89 & 237 & 15 \\
\hline 46 & 19 & & & & & & 18 & 66 & 14 \\
\hline 47 & 7 & & & & & & 6.8 & & 13 \\
\hline 4 & & & & & & & & & 2 \\
\hline 49 & & & & & & & & & 11 \\
\hline 50 & .74 & 1.3 & .77661 & 4 & 8 & 1. 24227 & .83415 & 1. 19882 & 10 \\
\hline 51 & .74946 & 1.3 & .77 & & 46 & 1. 24153 & .83465 & 1. 19811 & 9 \\
\hline 5. & & & & & & & & & 8 \\
\hline & & & & & & & & & 7 \\
\hline 5 & & 1.3 & & & & & 13 & & 6 \\
\hline 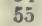 & 128 & & & & & & & & 5 \\
\hline 5 & 173 & 1.3 & & & & 1.2 & 12 & 157 & 4 \\
\hline 5 & .75219 & 1.3 & .77 & & .80834 & & & & 3 \\
\hline 5 & & & & & & 1.2 & .83811 & & 2 \\
\hline 59 & & & & & .80930 & & 0 & & 1 \\
\hline & .75355 & 1. 32704 & .78129 & 1. 27994 & .89978 & 1. 23490 & .83910 & 1. 19175 & 0 \\
\hline & Cotang. & Tang. & Cotang: & Tang. & Cotang. & Tang. & Cotan & Tang. & \\
\hline & \multicolumn{2}{|c|}{$53^{\circ}$} & \multicolumn{2}{|c|}{$52^{\circ}$} & \multicolumn{2}{|c|}{$51^{\circ}$} & \multicolumn{2}{|c|}{$50^{\circ}$} & \\
\hline
\end{tabular}


TABLE 8. -NATURAL TANGENTS AND COTANGENTS.

\begin{tabular}{|c|c|c|c|c|c|c|c|c|c|}
\hline \multirow{2}{*}{ ' } & \multicolumn{2}{|c|}{$40^{\circ}$} & \multicolumn{2}{|c|}{$41^{\circ}$} & \multicolumn{2}{|c|}{$42^{\circ}$} & \multicolumn{2}{|c|}{$43^{\circ}$} & \multirow{2}{*}{ ' } \\
\hline & Tang. & Cotang. & Tang. & Cotang. & Tang. & Cotang. & Tang. & Cotang. & \\
\hline 0 & .83910 & 1.19175 & .86929 & 1.15037 & .90040 & 1.11061 & .93252 & 1.07237 & 60 \\
\hline 1 & .83910 & 1.19105 & .80980 & 1.14909 & .90093 & 1.10996 & .93300 & 97174 & 59 \\
\hline 2 & .84009 & 1.19035 & .87031 & 1.14902 & .90146 & 1.10931 & .93360 & 1.07112 & 58 \\
\hline 3 & .84059 & 1. 18964 & .87052 & 1.14534 & .90199 & 1.10867 & .93415 & 1. 07049 & 57 \\
\hline 4 & .84108 & 1.15s94 & .87133 & 1. 14767 & .90251 & $1.10 \times 02$ & .93469 & 1. 06987 & 56 \\
\hline 5 & .84158 & 1. 18524 & .87184 & 1.14699 & .90304 & 1.10737 & .93524 & 1.06925 & 55 \\
\hline 6 & .84208 & 1.18754 & .87236 & 1.14632 & .90357 & 1.10672 & .93578 & 1. 06862 & 54 \\
\hline 7 & .84258 & 1.15684 & .87287 & 1. 14565 & .90410 & 1.10607 & .93633 & 1.06500 & 53 \\
\hline 8 & .84307 & 1.18614 & .87338 & 1.14498 & .90463 & 1.10543 & .93658 & 1.06738 & 52 \\
\hline 9 & .84357 & 1.18544 & .87359 & 1.14430 & .90516 & 1.10478 & .93742 & 1. 06676 & 51 \\
\hline 10 & .84107 & 1.18474 &.$\$ 7441$ & 1.14363 & .90569 & 1.10414 & .93797 & 1.06613 & 50 \\
\hline 11 & .84457 & 1.18404 & .87492 & 1.14296 & .90621 & 1. 10349 & .93552 & 1. 06551 & 49 \\
\hline 12 & .84507 & 1.15334 & .87543 & 1.14229 & $.90 \mathrm{i} 74$ & 1.10255 & .93906 & 1. 0 ints9 & 48 \\
\hline 13 & .84556 & 1.15264 & .87595 & 1.14162 & .90727 & 1.10220 & .93961 & 1.06427 & 47 \\
\hline 14 & .84606 & 1.18194 & .87646 & 1.14095 & .90781 & 1.10156 & .94016 & 1. 04365 & 46 \\
\hline 15 & $.84 t 56$ & 1.15125 & .87698 & 1.14028 & .90834 & 1.10091 & .94071 & 1.06303 & 45 \\
\hline 16 & .84700 & 1.18055 & .87749 & 1.13961 & .90887 & 1.10027 & .94125 & 1. 06241 & 44 \\
\hline 17 & .84756 & 1.17986 & .87801 & 1. 13594 & .90910 & 1. 09963 & .94180 & 1. 06179 & 43 \\
\hline 18 & .84800 & 1.17916 & .87852 & 1.13828 & .90993 & 1.09599 & .94235 & 1.06117 & 42 \\
\hline 19 & .81556 & 1.17846 & .87904 & 1.13761 & .91046 & 1. 02534 & .94290 & 1. 06056 & 41 \\
\hline 20 & .84906 & 1.17777 & .87955 & 1.13694 & .91099 & 1.09770 & .94345 & 1. 05994 & 40 \\
\hline 21 & .84956 & 1.17708 & .88007 & 1.13627 & .91153 & 1.09706 & .94400 & 1. 0.5932 & 39 \\
\hline 22 & .85006 & 1.17638 & .88059 & 1.13561 & .91206 & 1. 09642 & .94455 & 1.05870 & 38 \\
\hline 23 & .85057 & 1.17569 & .88110 & 1.13494 & .91259 & $1.095 \pi 8$ & .94510 & 1. 05809 & 37 \\
\hline 24 & .85107 & 1.17500 & .88162 & $1.13+28$ & .91313 & 1. 09514 & .94565 & 1. 05747 & 36 \\
\hline 25 & .85157 & 1.17430 & .88214 & 1.13361 & .91366 & 1.09450 & .94620 & 1. 05685 & 35 \\
\hline $2 \dot{ }$ & .85207 & 1. 17361 & .85265 & 1.13295 & .91419 & 1. 09386 & .94676 & 1. 05624 & 34 \\
\hline 27 & .85257 & 1.17292 & .88317 & 1.13228 & .91473 & 1.00322 & .94731 & 1. 05562 & 33 \\
\hline 29 & .85308 & 1.17223 & .85369 & 1. 13162 & .91526 & 1.00258 & .94786 & 1. 05501 & 32 \\
\hline 23 & .85358 & 1.17154 & .85421 & 1. 13096 & .91580 & 1. 09195 & .94841 & 1. 05439 & 31 \\
\hline 30 & $.85: 08$ & 1. 17085 & .85473 & 1.13029 & .91633 & 1. 03131 & .94896 & 1. 05378 & 30 \\
\hline 31 & .85458 & 1.17016 & .88524 & 1.12963 & .91687 & 1. 09067 & .94952 & 1. 05317 & 29 \\
\hline 32 & .85509 & 1.16947 & 576 & 1.12597 & .91740 & 1.09003 & .95007 & 1. 05255 & 23 \\
\hline 33 & .85559 & 1.16878 & .85628 & 1.12831 & .91794 & 1. 05940 & .95062 & 1.05194 & 27 \\
\hline 34 & .85609 & 1.16809 & .88650 & 1.12765 & .91847 & 1. $08 \times 76$ & .95118 & 1. 05133 & 26 \\
\hline 35 & $.856 t i 0$ & 1.16741 & .89732 & 1.12699 & .91901 & 1. 08513 & .95173 & 1. 05072 & 25 \\
\hline 36 & .85710 & 1. 16672 & .85784 & 1.12633 & .91955 & 1. 08749 & .95229 & 1. 05010 & 24 \\
\hline 37 & .85761 & $1.16 ; 03$ & . 58536 & 1.12567 & .92008 & 1. 08686 & .95284 & 1. 04949 & 23 \\
\hline 33 & .85811 & 1.16535 & .88588 & 1.12501 & .92062 & 1. 08622 & .95340 & 1.04888 & 22 \\
\hline 39 & .85862 & 1.16466 & .85940 & 1.12435 & .92116 & 1. 08559 & .95395 & 1. 04827 & 21 \\
\hline 40 & .85912 & 1.16398 & .85992 & 1.12369 & .92170 & 1.08496 & .95451 & 1.04766 & 20 \\
\hline 41 & .85963 & 1.16329 & .89045 & 1.12303 & .92224 & 1. 08432 & .95506 & 1. 04705 & 19 \\
\hline 42 & .86014 & 1.16261 & 097 & 1.12238 & .92277 & 1. & 562 & 44 & 18 \\
\hline 43 & .860044 & 1.16192 & .89149 & 1.12172 & . 92331 & 1.08306 & .95618 & 1. 04583 & 17 \\
\hline 44 & .861115 & 1.16124 & .89201 & 1.12106 & .92345 & 1. 05243 & .95673 & 1. 04522 & 16 \\
\hline 45 & .86166 & 1. 16056 & .89253 & 1.12041 & .92439 & 1. 08179 & .95729 & 1. 04461 & 15 \\
\hline 46 & .86216 & 1.15987 & .89306 & 1.11975 & .92493 & 1.08116 & .95785 & 1.04401 & 14 \\
\hline 47 & .86267 & 1.15919 & .89358 & 1.11909 & .92547 & 1. 05053 & .95841 & 1. $0 \$ 340$ & 13 \\
\hline 43 & .86318 & 1.15651 & .89410 & $1.118+4$ & .92601 & 1.07990 & .95897 & 1. 04279 & 12 \\
\hline 49 & $.8636 \mathrm{is}$ & 783 & .89463 & 1.1 & .92655 & 1.07927 & .95952 & 1. 04218 & 11 \\
\hline 50 & .86419 & 1.15715 & .89515 & 1.11713 & .92709 & 1.07864 & .96008 & 1.04158 & 10 \\
\hline 51 & .86470 & 1.15047 & .89567 & 1. 11648 & .92763 & 1.07801 & .96064 & 1.04097 & 9 \\
\hline 52 & .806521 & 1.15579 & .89620 & 1.115. 2 & .92817 & 1.07738 & .96120 & 1. 04036 & 8 \\
\hline 53 & .86572 & 1.15511 & .89672 & 1.11517 & .92872 & 1.07676 & .96176 & 1.03976 & 7 \\
\hline 54 & .86623 & 1.15443 & .89725 & 1. 11452 & .92926 & 1.07613 & .96232 & 1. 03915 & 6 \\
\hline 55 & $.8667 t$ & 1.15375 & .89777 & & .02880 & 1.07530 & .96288 & 1. 03855 & 5 \\
\hline 56 & .86725 & 1.1 .5308 & .89530 & 1.11321 & .93034 & 1. 07487 & .96344 & 1. 03794 & 4 \\
\hline 57 & .86776 & 1.15210 & .89583 & & .93058 & 1.07425 & .96400 & 1. 03734 & 3 \\
\hline 58 & .86527 & 1.15172 & .89935 & 1.11191 & .93143 & 1. 07362 & .96457 & 1.03674 & 2 \\
\hline 59 & . Sikis & 1.15104 & .89958 & 1.11126 & .93197 & 1.07209 & .96513 & $1.03+13$ & 1 \\
\hline 60 & .86929 & 1.15037 & .90010 & 1.11001 & .93252 & 1.07237 & .96560 & 1. 03553 & 0 \\
\hline \multirow{2}{*}{ ' } & Cotang. & Tang. & Cotang. & Tang. & Cotang. & Tang. & Cotang. & Tang. & \multirow{2}{*}{ ' } \\
\hline & \multicolumn{2}{|c|}{$49^{\circ}$} & \multicolumn{2}{|c|}{$48^{\circ}$} & \multicolumn{2}{|c|}{$47^{\circ}$} & \multicolumn{2}{|c|}{$46^{\circ}$} & \\
\hline
\end{tabular}


TABLE 8.-NATURAL TANGENTS AND COTANGENTS.

\begin{tabular}{|c|c|c|c|c|c|c|c|c|c|c|c|}
\hline \multirow{2}{*}{, } & \multicolumn{2}{|c|}{$44^{\circ}$} & \multirow{2}{*}{ I } & \multirow{2}{*}{, } & \multicolumn{2}{|c|}{$44^{\circ}$} & \multirow{2}{*}{, } & \multirow{2}{*}{1} & \multicolumn{2}{|c|}{$44^{\circ}$} & \\
\hline & Tang. & Cotang. & & & Tang. & Cotang. & & & Tang. & Cotang. & \\
\hline 0 & .96569 & 1. 03553 & 60 & 20 & .97700 & 1. 02355 & 40 & 40 & .98843 & 1.01170 & 20 \\
\hline 1 & & & 5 & 21 & & & 39 & 41 & 01 & & 19 \\
\hline 2 & & 1. 03433 & 58 & 22 & & 1. 02236 & 38 & 42 & .98958 & 1. 01053 & 18 \\
\hline 3 & 738 & 3372 & 57 & 23 & .97870 & 1.02176 & 37 & 43 & .99016 & 1. 00994 & 17 \\
\hline 4 & & 312 & 56 & 24 & 927 & 1.02117 & 36 & 44 & .99073 & & 16 \\
\hline 5 & & 252 & 55 & 25 & .97984 & 1.02057 & 35 & 45 & .99131 & 1. 00876 & 15 \\
\hline 6 & & & 54 & 26 & 8041 & 1. & 34 & 46 & .99189 & & 4 \\
\hline 7 & & 32 & 53 & 27 & .98098 & 1.0 & 33 & 47 & . 99247 & 1. & 13 \\
\hline 8 & & 72 & 52 & 28 & 155 & & 32 & 48 & .99304 & & 12 \\
\hline 9 & & 1. & 51 & 29 & 213 & 1. & 31 & 49 & .99362 & 1. & 11 \\
\hline 10 & & 1.1 & 50 & 30 & 0 & & 30 & 50 & 420 & 83 & 10 \\
\hline 11 & .97189 & 1.0 & 49 & 31 & .98327 & $1 . c$ & 29 & 51 & .99478 & & 9 \\
\hline 12 & & & 48 & 3 & & & 28 & 52 & & & 0 \\
\hline 13 & & 1. ( & 47 & 33 & 1 & & 27 & 53 & 94 & & 7 \\
\hline 14 & & 1. & 46 & 34 & .9 & 1. & 26 & 54 & .99652 & 1. & 6 \\
\hline 15 & & $1 .($ & 45 & 35 & & & 25 & 5.5 & 10 & & 5 \\
\hline 16 & & 1.0 & 44 & 36 & .98 & & 24 & 56 & . 99768 & 33 & 4 \\
\hline 17 & & $1 .($ & 43 & 3 & .9 & & 23 & 57 & 26 & 1. & 3 \\
\hline 18 & & 1.0 & 42 & 38 & . 98728 & & 22 & 58 & .99884 & $1 .($ & 2 \\
\hline 19 & & & 41 & 39 & & & 21 & 59 & 942 & & 1 \\
\hline 20 & .97700 & 1.02355 & 40 & 40 & .98843 & 1.01170 & 20 & 60 & 1.00000 & 1.00000 & 0 \\
\hline & Cotang. & Tang. & \multirow[t]{2}{*}{, } & \multirow[b]{2}{*}{ 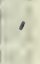 } & Cotang. & $\mathrm{T}$ & \multirow{2}{*}{1} & \multirow{2}{*}{, } & - & $\mathrm{T} 2$ & \\
\hline & \multicolumn{2}{|c|}{$45^{\circ}$} & & & 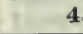 & & & & & \\
\hline
\end{tabular}


TABLE 9.-LOGARITHMIC SINES, COSINES, ETC.

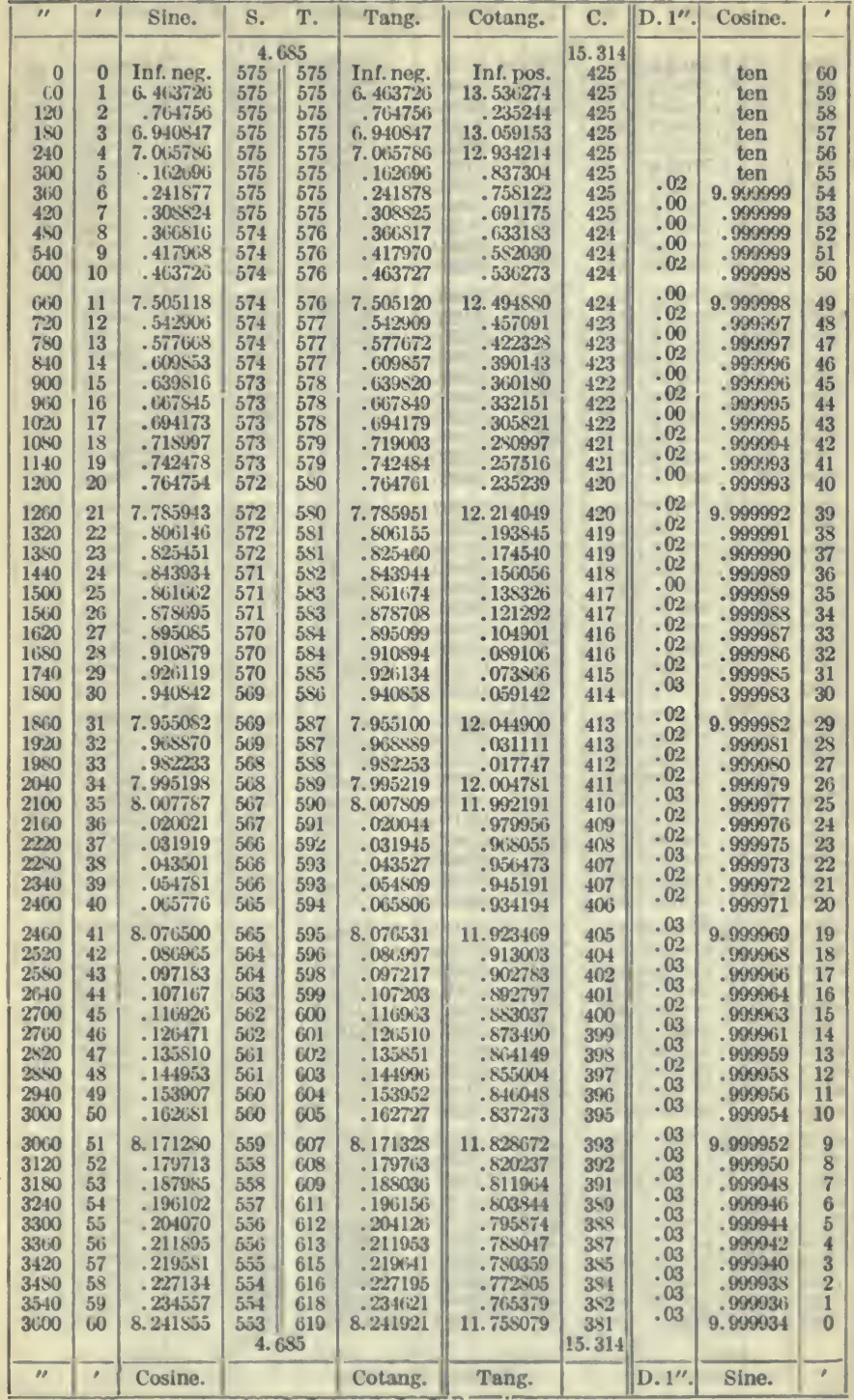




\begin{tabular}{|c|c|c|c|c|c|c|c|c|c|c|}
\hline " & ' & Sine. & \multicolumn{2}{|c|}{ S. T. } & Tang. & Cotang. & C. & D.1". & Cosine. & ' \\
\hline & & & \multicolumn{2}{|c|}{ 4. 685} & & & 15.314 & \multirow[b]{2}{*}{.03} & \multirow[b]{2}{*}{ 9. 999934} & \multirow[b]{2}{*}{60} \\
\hline 3600 & 0 & 8.241855 & 553 & 619 & 8. 241521 & 11. $75 S 079$ & 381 & & & \\
\hline & 1 & .249 & 552 & 620 & .249102 & .750898 & $3 \triangleleft 0$ & & . 999432 & 59 \\
\hline 3 & 2 & .256094 & 551 & 622 & .256165 & .743535 & 378 & & .999929 & 58 \\
\hline & 3 & .263042 & 551 & 623 & .263115 & .736885 & 377 & & .999927 & 57 \\
\hline 3 & 4 & .269581 & 550 & 625 & .269956 & .730044 & 375 & & .993925 & 56 \\
\hline 3 & 5 & .276614 & 549 & 627 & .276691 & .723309 & 373 & & .999922 & 55 \\
\hline 3 & 6 & .253243 & 548 & 628 & .283323 & .716677 & 372 & & $.9999 \geq 0$ & 54 \\
\hline 4 & 7 & .289773 & 547 & 630 & .289856 & 0144 & 370 & & .999918 & 53 \\
\hline 408 & 8 & .296207 & 546 & 632 & .296292 & 3703 & 368 & & . 999915 & 52 \\
\hline 41 & 9 & .302546 & 546 & 633 & .302634 & & 367 & & . 999913 & 51 \\
\hline 4200 & 10 & . 308794 & 545 & 635 & .308584 & .691116 & 365 & & .999910 & 50 \\
\hline 4260 & 11 & 8. 314954 & 544 & 637 & 8. 315046 & 11. 684954 & 363 & & 9. 999907 & 49 \\
\hline 4320 & 12 & .321 & 543 & 638 & 321122 & 378 & $3 t$ & & & 48 \\
\hline 438 & 13 & .327 & 542 & 640 & .327114 & 72886 & 360 & & .999902 & 47 \\
\hline 4440 & 14 & .332924 & 541 & 642 & .333025 & 36975 & 358 & & .999899 & 46 \\
\hline 4500 & 15 & .338753 & 540 & 644 & .338856 & 11144 & 356 & & .999897 & 45 \\
\hline 45 & 16 & .344504 & 539 & 646 & .344610 & & 3 & & .990894 & 44 \\
\hline 4620 & 17 & .350181 & 539 & 648 & .350289 & 11 & 3 & & .999891 & 43 \\
\hline & 18 & .355 & 538 & 649 & .355 & & 3 & & .999888 & 42 \\
\hline 4740 & 19 & .361315 & 537 & 651 & . 361430 & 70 & 349 & & .9998855 & 41 \\
\hline 480 & 20 & .36 & 536 & 653 & 5 & 05 & 347 & & 82 & 40 \\
\hline 4860 & 21 & 8. 372 & 535 & 655 & 8. 372292 & 11. 627708 & 345 & .05 & 9.999879 & 39 \\
\hline 492 & 22 & .377 & 534 & 657 & .37 & 78 & 34 & & 76 & 38 \\
\hline 4 ? & 23 & .382 & 533 & 659 & .38 & & 34 & & .95 & 37 \\
\hline 504 & 24 & .387 & 532 & 661 & .388 & & 33 & & .999870 & 36 \\
\hline 51 & 25 & .393 & 531 & 663 & .393 & & 33 & & .999867 & 35 \\
\hline 51 & 26 & .3 & 530 & 666 & .39 & & 33 & & .990864 & 34 \\
\hline & 27 & .40 & 529 & 668 & .40 & & 33 & & .999861 & 3 \\
\hline 5 & 28 & .4 & 527 & 67 & & & 330 & & .999858 & 32 \\
\hline 53 & 29 & .413063 & 526 & 67 & .41 & & 328 & & .99 & 31 \\
\hline 5400 & 30 & .417919 & 525 & 674 & .4180 & 32 & 326 & & .99 & 30 \\
\hline 5460 & 31 & 8.42 & 524 & 676 & 8. 42 & 1 & 324 & 05 & 9.9 & 29 \\
\hline & 32 & & 52 & & & & 3 & & .9 & 28 \\
\hline 5 & 33 & .43 & 522 & & .43 & & 31 & & & 27 \\
\hline & 34 & .43 & 521 & 6 & .43 & & 31 & & .9 & 26 \\
\hline & 35 & .4 & 520 & & & & & & & 25 \\
\hline & 36 & .4 & 518 & 0 & .4 & & 31 & & .99 & 24 \\
\hline & 37 & & 51 & & & & 31 & & & 23 \\
\hline & 38 & 4 & 51 & 69 & & & 30 & & .99 & 22 \\
\hline & 39 & 1 & 515 & & & & 30 & & & 21 \\
\hline 600 & 40 & 5 & 514 & 6 & & & 303 & .07 & 816 & 20 \\
\hline 6060 & 41 & 8.46 & 512 & 700 & 8.4 & 28 & 30 & & 9. & 19 \\
\hline & 42 & & 5 & & & & & & & 18 \\
\hline & 43 & & 51 & & & & & & & 17 \\
\hline & 44 & & 5 & & & & & & & 16 \\
\hline & 45 & & 50 & 7 & & & 2 & & & 15 \\
\hline & $46^{\circ}$ & & 50 & & & & 28 & & & 14 \\
\hline & 47 & & 50 & & & & 2 & & & 13 \\
\hline & 48 & & 50 & 71 & & & & & & 12 \\
\hline & 49 & & 50 & & & & 2 & & & 11 \\
\hline 660 & 50 & 45 & 501 & 723 & & & 277 & & 78 & 10 \\
\hline 6660 & 51 & 8. & 49 & 72 & 8. 509200 & 11. $490 \$ 00$ & 27 & 7 & 9.9 & 9 \\
\hline & 52 & & 45 & 7 & & & 27 & & & 8 \\
\hline & 53 & 5 & 49 & 73 & & & 20 & & & 7 \\
\hline 68 & 54 & & 4 ? & 73 & & 0 & 2 & & & 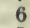 \\
\hline & 55 & 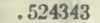 & 49 & 73 & &. & 26 & & & 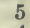 \\
\hline 68 & 56 & & 49 & 74 & & .4 & 20 & & 53 & 4 \\
\hline & 57 & & 49 & 74 & & & 25 & & & 3 \\
\hline 7 & 58 & & 49 & 74 & 5 & .4 & 25 & 07 & 44 & 2 \\
\hline & 59 & 53 & 488 & 748 & & & 252 & & .99 & 1 \\
\hline 720 & 60 & 8. 542819 & $\begin{array}{r}487 \\
4 .\end{array}$ & 751 & 8.54 & 11. 456916 & $\begin{array}{r}249 \\
15.314\end{array}$ & & 9.999735 & 0 \\
\hline " & ' & Cosine. & & & Cotang. & Tar & & $.1^{\prime \prime}$ & Sine & \\
\hline
\end{tabular}


TANGENTS, AND COTANGENTS.

\begin{tabular}{|c|c|c|c|c|c|c|c|c|}
\hline , & Sine. & D. $1^{\prime \prime}$ & Cosine. & D. $1^{\prime \prime}$ & Tang. & D. $1^{\prime \prime}$ & Cotang. & , \\
\hline $\begin{array}{r}0 \\
1 \\
2 \\
3 \\
4 \\
5 \\
6 \\
7 \\
8 \\
9 \\
10\end{array}$ & $\begin{array}{r}8.542919 \\
.546422 \\
.549995 \\
.553539 \\
.557054 \\
.560540 \\
.563999 \\
.567431 \\
.570836 \\
.574214 \\
.577566\end{array}$ & $\begin{array}{l}60.05 \\
59.55 \\
59.07 \\
58.58 \\
58.10 \\
57.65 \\
57.20 \\
56.75 \\
56.30 \\
55.87 \\
55.43\end{array}$ & $\begin{array}{r}9.999735 \\
.999731 \\
.999726 \\
.999722 \\
.999717 \\
.999713 \\
.999708 \\
.999704 \\
.999699 \\
.999694 \\
.999659\end{array}$ & $\begin{array}{l}.07 \\
.08 \\
.07 \\
.08 \\
.07 \\
.08 \\
.07 \\
.08 \\
.08 \\
.08 \\
.07\end{array}$ & $\begin{array}{r}8.543084 \\
.546691 \\
.550268 \\
.553817 \\
.557336 \\
.560828 \\
.564291 \\
.567727 \\
.571137 \\
.574520 \\
.577877\end{array}$ & $\begin{array}{l}60.12 \\
59.62 \\
59.15 \\
58.65 \\
58.20 \\
57.72 \\
57.27 \\
56.83 \\
56.38 \\
55.95 \\
55.52\end{array}$ & $\begin{array}{r}11.456916 \\
.453309 \\
.449732 \\
.446183 \\
.442664 \\
.439172 \\
.435709 \\
.432273 \\
.428863 \\
.425480 \\
.422123\end{array}$ & $\begin{array}{l}60 \\
59 \\
58 \\
57 \\
56 \\
55 \\
54 \\
53 \\
52 \\
51 \\
50\end{array}$ \\
\hline $\begin{array}{l}11 \\
12 \\
13 \\
14 \\
15 \\
16 \\
17 \\
18 \\
19 \\
20\end{array}$ & $\begin{array}{r}8.580892 \\
.584193 \\
.587469 \\
.590721 \\
.593948 \\
.597152 \\
.600332 \\
.603459 \\
.606623 \\
.609734\end{array}$ & $\begin{array}{l}55.02 \\
54.60 \\
54.20 \\
53.78 \\
53.40 \\
53.00 \\
52.62 \\
52.23 \\
51.85 \\
51.48\end{array}$ & $\begin{array}{r}9.999685 \\
.999680 \\
.999675 \\
.999670 \\
.999665 \\
.999660 \\
.999655 \\
.999650 \\
.999645 \\
.999640\end{array}$ & $\begin{array}{l}.08 \\
.08 \\
.08 \\
.08 \\
.08 \\
.08 \\
.08 \\
.08 \\
.08 \\
.08\end{array}$ & $\begin{array}{r}8.581208 \\
.584514 \\
.587795 \\
.591051 \\
.594283 \\
.597492 \\
.600677 \\
.603839 \\
.606978 \\
.610094\end{array}$ & $\begin{array}{l}55.10 \\
54.68 \\
54.27 \\
53.87 \\
53.48 \\
53.08 \\
52.70 \\
52.32 \\
51.93 \\
51.58\end{array}$ & $\begin{array}{r}11.418792 \\
.415486 \\
.412205 \\
.408949 \\
.405717 \\
.402508 \\
.399323 \\
.396161 \\
.393022 \\
.389906\end{array}$ & $\begin{array}{l}49 \\
48 \\
47 \\
46 \\
45 \\
44 \\
43 \\
42 \\
41 \\
40\end{array}$ \\
\hline $\begin{array}{l}21 \\
22 \\
23 \\
24 \\
25 \\
26 \\
27 \\
28 \\
29 \\
30\end{array}$ & $\begin{array}{r}8.612523 \\
.615891 \\
.618937 \\
.621962 \\
.624965 \\
.627948 \\
.630911 \\
.633854 \\
.636776 \\
.639680\end{array}$ & $\begin{array}{l}51.13 \\
50.77 \\
50.42 \\
50.05 \\
49.72 \\
49.38 \\
49.05 \\
48.70 \\
48.40 \\
48.05\end{array}$ & $\begin{array}{r}9.999635 \\
.999629 \\
.999624 \\
.999619 \\
.999614 \\
.999608 \\
.999603 \\
.999597 \\
.999592 \\
.999586\end{array}$ & $\begin{array}{l}.10 \\
.08 \\
.08 \\
.08 \\
.10 \\
.08 \\
.10 \\
.08 \\
.10 \\
.08\end{array}$ & $\begin{array}{r}8.613189 \\
.616262 \\
.619313 \\
.622343 \\
.625352 \\
.628340 \\
.631308 \\
.634256 \\
.637184 \\
.640093\end{array}$ & $\begin{array}{l}51.22 \\
50.85 \\
50.50 \\
50.15 \\
49.80 \\
49.47 \\
49.13 \\
48.80 \\
48.48 \\
48.15\end{array}$ & $\begin{array}{r}11.386811 \\
.383738 \\
.350687 \\
.377657 \\
.374648 \\
.371660 \\
.368692 \\
.365744 \\
.362816 \\
.359907\end{array}$ & $\begin{array}{l}39 \\
38 \\
37 \\
36 \\
35 \\
34 \\
33 \\
32 \\
31 \\
30\end{array}$ \\
\hline $\begin{array}{l}31 \\
32 \\
33 \\
34 \\
35 \\
36 \\
37 \\
38 \\
39 \\
40\end{array}$ & $\begin{array}{r}8.642563 \\
.645428 \\
.618274 \\
.651102 \\
.653911 \\
.656702 \\
.659475 \\
.662230 \\
.664968 \\
.667689\end{array}$ & $\begin{array}{l}47.75 \\
47.43 \\
47.13 \\
46.82 \\
46.52 \\
46.22 \\
45.92 \\
45.63 \\
45.35 \\
45.07\end{array}$ & $\begin{array}{r}9.999581 \\
.999575 \\
.999570 \\
.999564 \\
.999558 \\
.999553 \\
.999547 \\
.999541 \\
.999535 \\
.999529\end{array}$ & $\begin{array}{l}.10 \\
.08 \\
.10 \\
.10 \\
.08 \\
.10 \\
.10 \\
.10 \\
.10 \\
.08\end{array}$ & $\begin{array}{r}8.642982 \\
.645853 \\
.648704 \\
.651537 \\
.654352 \\
.657149 \\
.659928 \\
.662689 \\
.665433 \\
.668160\end{array}$ & $\begin{array}{l}47.85 \\
47.52 \\
47.22 \\
46.92 \\
46.62 \\
46.32 \\
46.02 \\
45.73 \\
45.45 \\
45.17\end{array}$ & $\begin{array}{r}11.357018 \\
.354147 \\
.351296 \\
.348463 \\
.345648 \\
.342851 \\
.340072 \\
.337311 \\
.334567 \\
.331840\end{array}$ & $\begin{array}{l}29 \\
28 \\
27 \\
26 \\
25 \\
24 \\
23 \\
22 \\
21 \\
20\end{array}$ \\
\hline $\begin{array}{l}41 \\
42 \\
43 \\
44 \\
45 \\
46 \\
47 \\
48 \\
49 \\
50\end{array}$ & $\begin{array}{r}8.670393 \\
.673050 \\
.675751 \\
.678405 \\
.651043 \\
.683665 \\
.656272 \\
.655863 \\
.691438 \\
.693998\end{array}$ & $\begin{array}{l}44.78 \\
44.52 \\
44.23 \\
43.97 \\
43.70 \\
43.45 \\
43.18 \\
42.92 \\
42.67 \\
42.42\end{array}$ & $\begin{array}{r}9.999524 \\
.999518 \\
.999512 \\
.999506 \\
.999500 \\
.999493 \\
.999487 \\
.999481 \\
.999475 \\
.999469\end{array}$ & $\begin{array}{l}.10 \\
.10 \\
.10 \\
.10 \\
.12 \\
.10 \\
.10 \\
.10 \\
.10 \\
.10\end{array}$ & $\begin{array}{r}8.670870 \\
.673563 \\
.676239 \\
.678900 \\
.681544 \\
.684172 \\
.686784 \\
.689381 \\
.691963 \\
.694529\end{array}$ & $\begin{array}{l}44.88 \\
44.60 \\
44.35 \\
44.07 \\
43.80 \\
43.53 \\
43.28 \\
43.03 \\
42.77 \\
42.53\end{array}$ & $\begin{array}{r}11.329130 \\
.326437 \\
.323761 \\
.321100 \\
.318456 \\
.315828 \\
.313216 \\
.310619 \\
.305037 \\
.305471\end{array}$ & $\begin{array}{l}19 \\
18 \\
17 \\
16 \\
15 \\
14 \\
13 \\
12 \\
11 \\
10\end{array}$ \\
\hline $\begin{array}{l}51 \\
52 \\
53 \\
54 \\
55 \\
56 \\
57 \\
58 \\
59 \\
60\end{array}$ & $\begin{array}{r}8.696543 \\
.699073 \\
.701589 \\
.704090 \\
.706577 \\
.709049 \\
.711507 \\
.713952 \\
.716383 \\
8.715800\end{array}$ & $\begin{array}{l}42.17 \\
41.93 \\
41.68 \\
41.45 \\
41.20 \\
40.97 \\
40.75 \\
40.52 \\
40.28\end{array}$ & $\begin{array}{r}9.999463 \\
.999456 \\
.999450 \\
.999443 \\
.999437 \\
.999431 \\
.999424 \\
.999418 \\
.999411 \\
9.999404\end{array}$ & $\begin{array}{l}.12 \\
.10 \\
.12 \\
.10 \\
.10 \\
.12 \\
.10 \\
.12 \\
.12\end{array}$ & $\begin{array}{r}8.697081 \\
.699617 \\
.702139 \\
.704646 \\
.707140 \\
.709618 \\
.712083 \\
.714534 \\
.716972 \\
8.719396\end{array}$ & $\begin{array}{l}42.27 \\
42.03 \\
41.78 \\
41.57 \\
41.30 \\
41.08 \\
40.85 \\
40.63 \\
40.40\end{array}$ & $\begin{array}{r}11.302919 \\
.300383 \\
.297861 \\
.295354 \\
.292860 \\
.290382 \\
.287917 \\
.255466 \\
.253028 \\
11.250604\end{array}$ & $\begin{array}{l}9 \\
8 \\
7 \\
6 \\
5 \\
4 \\
3 \\
2 \\
1 \\
0\end{array}$ \\
\hline - & Cosine. & D. 1". & Sino. & D. $1^{\prime \prime}$. & Cotang. & D. $1^{\prime \prime}$. & Tang. & e \\
\hline
\end{tabular}




\begin{tabular}{|c|c|c|c|c|c|c|c|c|}
\hline ' & Sine. & D. $1^{\prime \prime}$. & Cosine. & D. $1^{\prime \prime}$. & Tang. & D. $1^{\prime \prime}$. & Cotang. & ' \\
\hline $\begin{array}{r}0 \\
1 \\
2 \\
3 \\
4 \\
5 \\
6 \\
7 \\
8 \\
9 \\
10\end{array}$ & $\begin{array}{r}8.718800 \\
.721204 \\
.723595 \\
.725972 \\
.728337 \\
.730658 \\
.733027 \\
.735354 \\
.737667 \\
.739969 \\
.742259\end{array}$ & $\begin{array}{l}40.07 \\
39.85 \\
39.62 \\
39.42 \\
39.18 \\
38.98 \\
38.78 \\
38.55 \\
38.37 \\
38.17 \\
37.95\end{array}$ & $\begin{array}{r}9.999404 \\
.999398 \\
.999391 \\
.999354 \\
.999378 \\
.999371 \\
.999364 \\
.999357 \\
.999350 \\
.993343 \\
.999336\end{array}$ & $\begin{array}{l}.10 \\
.12 \\
.12 \\
.10 \\
.12 \\
.12 \\
.12 \\
.12 \\
.12 \\
.12 \\
.12\end{array}$ & $\begin{array}{r}8.719396 \\
.721806 \\
.724204 \\
.726588 \\
.725959 \\
.731317 \\
.733663 \\
.735996 \\
.738317 \\
.740626 \\
.742922\end{array}$ & $\begin{array}{l}40.17 \\
39.97 \\
39.73 \\
39.52 \\
39.30 \\
39.10 \\
38.88 \\
38.68 \\
38.48 \\
38.27 \\
38.08\end{array}$ & $\begin{array}{r}11.280604 \\
.278194 \\
.275796 \\
.273412 \\
.271041 \\
.268683 \\
.266337 \\
.264004 \\
.261683 \\
.259374 \\
.257078\end{array}$ & 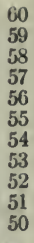 \\
\hline $\begin{array}{l}11 \\
12 \\
13 \\
14 \\
15 \\
16 \\
17 \\
18 \\
19 \\
20\end{array}$ & $\begin{array}{r}8.744536 \\
.746802 \\
.749055 \\
.751297 \\
.753528 \\
.7555747 \\
.757955 \\
.760151 \\
.762337 \\
.764511\end{array}$ & $\begin{array}{l}37.77 \\
37.55 \\
37.37 \\
37.18 \\
36.98 \\
36.80 \\
36.60 \\
36.43 \\
36.23 \\
36.07\end{array}$ & $\begin{array}{r}9.999329 \\
.999322 \\
.999315 \\
.999308 \\
.999301 \\
.999294 \\
.999287 \\
.999279 \\
.999272 \\
.999265\end{array}$ & $\begin{array}{l}.12 \\
.12 \\
.12 \\
.12 \\
.12 \\
.12 \\
.13 \\
.12 \\
.12 \\
.13\end{array}$ & $\begin{array}{r}8.745207 \\
.747479 \\
.749740 \\
.751989 \\
.754227 \\
.756453 \\
.758668 \\
.760872 \\
.763065 \\
.765246\end{array}$ & $\begin{array}{l}37.87 \\
37.68 \\
37.48 \\
37.30 \\
37.10 \\
36.92 \\
36.73 \\
36.55 \\
36.35 \\
36.18\end{array}$ & $\begin{array}{r}11.254793 \\
.252521 \\
.250260 \\
.248011 \\
.245773 \\
.243547 \\
.241332 \\
.239128 \\
.236935 \\
.234754\end{array}$ & 49 \\
\hline $\begin{array}{l}21 \\
22 \\
23 \\
24 \\
25 \\
26 \\
27 \\
28 \\
29 \\
30\end{array}$ & $\begin{array}{r}8.766675 \\
.768528 \\
.770970 \\
.773101 \\
.775223 \\
.777333 \\
.779434 \\
.781524 \\
.783605 \\
.785675\end{array}$ & $\begin{array}{l}35.88 \\
35.70 \\
35.52 \\
35.37 \\
35.17 \\
35.02 \\
34.83 \\
34.68 \\
34.50 \\
34.35\end{array}$ & $\begin{array}{r}9.999257 \\
.999250 \\
.999242 \\
.999235 \\
.999227 \\
.999220 \\
.999212 \\
.999205 \\
.999197 \\
.999189\end{array}$ & $\begin{array}{l}.12 \\
.13 \\
.12 \\
.13 \\
.12 \\
.13 \\
.12 \\
.13 \\
.13 \\
.13\end{array}$ & $\begin{array}{r}8.767417 \\
.769578 \\
.771727 \\
.773866 \\
.775995 \\
.778114 \\
.780222 \\
.782320 \\
.784408 \\
.7864 S 6\end{array}$ & $\begin{array}{l}36.02 \\
35.82 \\
35.65 \\
35.48 \\
35.32 \\
35.13 \\
34.97 \\
34.80 \\
34.63 \\
34.47\end{array}$ & $\begin{array}{r}11.232583 \\
.230422 \\
.228273 \\
.226134 \\
.224005 \\
.221886 \\
.219778 \\
.217680 \\
.215592 \\
.213514\end{array}$ & 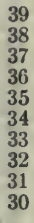 \\
\hline $\begin{array}{l}31 \\
32 \\
33 \\
34 \\
35 \\
36 \\
37 \\
38 \\
39 \\
40\end{array}$ & $\begin{array}{r}8.787736 \\
.789787 \\
.791828 \\
.793859 \\
.795881 \\
.797894 \\
.799897 \\
.801892 \\
.803876 \\
.805852\end{array}$ & $\begin{array}{l}34.18 \\
34.02 \\
33.85 \\
33.70 \\
33.55 \\
33.38 \\
33.25 \\
33.07 \\
32.93 \\
32.78\end{array}$ & $\begin{array}{r}9.999181 \\
.999174 \\
.999166 \\
.999158 \\
.999150 \\
.999142 \\
.939134 \\
.999126 \\
.999118 \\
.999110\end{array}$ & $\begin{array}{l}.12 \\
.13 \\
.13 \\
.13 \\
.13 \\
.13 \\
.13 \\
.13 \\
.13 \\
.13\end{array}$ & $\begin{array}{r}8.788554 \\
.790613 \\
.792662 \\
.794701 \\
.796731 \\
.798752 \\
.800763 \\
.802765 \\
.804758 \\
.806742\end{array}$ & $\begin{array}{l}34.32 \\
34.15 \\
33.98 \\
33.83 \\
33.68 \\
33.52 \\
33.37 \\
33.22 \\
33.07 \\
32.92\end{array}$ & $\begin{array}{r}11.211446 \\
.209387 \\
.207338 \\
.205299 \\
.203269 \\
.201248 \\
.199237 \\
.197235 \\
.195242 \\
.193258\end{array}$ & $\begin{array}{l}29 \\
28 \\
27 \\
26 \\
25 \\
24 \\
23 \\
22 \\
21 \\
20\end{array}$ \\
\hline $\begin{array}{l}41 \\
42 \\
43 \\
44 \\
45 \\
46 \\
47 \\
48 \\
49 \\
50\end{array}$ & $\begin{array}{r}8.807819 \\
.809777 \\
.811726 \\
.813667 \\
.815599 \\
.817522 \\
.819436 \\
.821343 \\
.823240 \\
.825130\end{array}$ & $\begin{array}{l}32.63 \\
32.48 \\
32.35 \\
32.20 \\
32.05 \\
31.90 \\
31.78 \\
31.62 \\
31.50 \\
31.35\end{array}$ & $\begin{array}{r}9.999102 \\
.999094 \\
.999086 \\
.999077 \\
.999069 \\
.999061 \\
.999053 \\
.999044 \\
.999036 \\
.999027\end{array}$ & $\begin{array}{l}.13 \\
.13 \\
.15 \\
.13 \\
.13 \\
.13 \\
.15 \\
.13 \\
.15 \\
.13\end{array}$ & $\begin{array}{r}8.808717 \\
.810683 \\
.812641 \\
.814589 \\
.816529 \\
.818461 \\
.820384 \\
.822298 \\
.824205 \\
.826103\end{array}$ & $\begin{array}{l}32.77 \\
32.63 \\
32.47 \\
32.33 \\
32.20 \\
32.05 \\
31.90 \\
31.78 \\
31.63 \\
31.48\end{array}$ & $\begin{array}{r}11.191283 \\
.189317 \\
.187359 \\
.185411 \\
.183471 \\
.181539 \\
.179616 \\
.177702 \\
.175795 \\
.173897\end{array}$ & $\begin{array}{l}7 \\
6 \\
5\end{array}$ \\
\hline $\begin{array}{l}51 \\
52 \\
53 \\
54 \\
55 \\
56 \\
57 \\
58 \\
59 \\
60\end{array}$ & $\begin{array}{r}8.827011 \\
.828884 \\
.830749 \\
.832607 \\
.834456 \\
.836297 \\
.838130 \\
.839956 \\
.841774 \\
8.843585\end{array}$ & $\begin{array}{l}31.22 \\
31.08 \\
30.97 \\
30.82 \\
30.68 \\
30.55 \\
30.43 \\
30.30 \\
30.18\end{array}$ & $\begin{array}{r}9.999019 \\
.999010 \\
.999002 \\
.998993 \\
.998984 \\
.998976 \\
.998967 \\
.998958 \\
.998950 \\
9.998941\end{array}$ & $\begin{array}{l}.15 \\
.13 \\
.15 \\
.15 \\
.13 \\
.15 \\
.15 \\
.13 \\
.15\end{array}$ & $\begin{array}{r}8.827992 \\
.829874 \\
.831748 \\
.833613 \\
.835471 \\
.837321 \\
.839163 \\
.840998 \\
.842525 \\
8.844644\end{array}$ & $\begin{array}{l}31.37 \\
31.23 \\
31.08 \\
30.97 \\
30.83 \\
30.70 \\
30.58 \\
30.45 \\
30.32\end{array}$ & $\begin{array}{r}11.172008 \\
.170126 \\
.168252 \\
.166387 \\
.164529 \\
.162679 \\
.160837 \\
.159002 \\
.157175 \\
11.155356\end{array}$ & $\begin{array}{l}9 \\
8 \\
7 \\
6\end{array}$ \\
\hline ' & Cosine. & D. $1^{\prime \prime}$. & EIne. & D. $1^{\prime \prime}$. & Cotang. & D. $1^{\prime \prime}$. & Tang. & ' \\
\hline
\end{tabular}


TANGENTS, AND COTANGENTS.

\begin{tabular}{|c|c|c|c|c|c|c|c|c|}
\hline , & Sine. & D. 1"'. & Cosine. & D. 1" & Tang. & D. 1". & Cotang. & , \\
\hline $\begin{array}{r}0 \\
1 \\
2 \\
3 \\
4 \\
5 \\
6 \\
7 \\
8 \\
9 \\
10\end{array}$ & $\begin{array}{r}8.843585 \\
.845357 \\
.847183 \\
.845971 \\
.850751 \\
.852525 \\
.854291 \\
.856049 \\
.857801 \\
.859546 \\
.861253\end{array}$ & $\begin{array}{l}30.03 \\
29.93 \\
29.80 \\
29.67 \\
29.57 \\
29.43 \\
29.30 \\
29.20 \\
29.08 \\
23.95 \\
28.85\end{array}$ & $\begin{array}{r}9.998941 \\
.995932 \\
.995923 \\
.998914 \\
.998905 \\
.998896 \\
.998587 \\
.998578 \\
.998569 \\
.995560 \\
.995851\end{array}$ & $\begin{array}{l}.15 \\
.15 \\
.15 \\
.15 \\
.15 \\
.15 \\
.15 \\
.15 \\
.15 \\
.15 \\
.17\end{array}$ & $\begin{array}{r}8.841644 \\
.846455 \\
.848260 \\
.850057 \\
.851846 \\
.853628 \\
.855403 \\
.857171 \\
.858932 \\
.860686 \\
.862433\end{array}$ & $\begin{array}{l}30.18 \\
30.09 \\
29.95 \\
29.82 \\
29.70 \\
29.58 \\
29.47 \\
29.35 \\
29.23 \\
29.12 \\
29.00\end{array}$ & $\begin{array}{r}11.155356 \\
.153545 \\
.151740 \\
.149943 \\
.148154 \\
.146372 \\
.144597 \\
.142829 \\
.141068 \\
.139314 \\
.137567\end{array}$ & $\begin{array}{l}60 \\
59 \\
58 \\
57 \\
56 \\
55 \\
54 \\
53 \\
52 \\
51 \\
50\end{array}$ \\
\hline $\begin{array}{l}11 \\
12 \\
13 \\
14 \\
15 \\
16 \\
17 \\
18 \\
19 \\
20\end{array}$ & $\begin{array}{r}8.863014 \\
.864738 \\
.866455 \\
.868165 \\
.869868 \\
.871565 \\
.873255 \\
.874938 \\
.876615 \\
.875255\end{array}$ & $\begin{array}{l}28.73 \\
28.62 \\
28.50 \\
28.38 \\
28.28 \\
28.17 \\
28.05 \\
27.95 \\
27.83 \\
27.73\end{array}$ & $\begin{array}{r}9.998841 \\
.998532 \\
.998823 \\
.998813 \\
.998804 \\
.998795 \\
.998785 \\
.998776 \\
.995766 \\
.998757\end{array}$ & $\begin{array}{l}.15 \\
.15 \\
.17 \\
.15 \\
.15 \\
.17 \\
.15 \\
.17 \\
.15 \\
.17\end{array}$ & $\begin{array}{r}8.864173 \\
.865906 \\
.867632 \\
.869351 \\
.871064 \\
.872770 \\
.874469 \\
.876162 \\
.877849 \\
.879529\end{array}$ & $\begin{array}{l}28.88 \\
28.77 \\
28.65 \\
28.55 \\
28.43 \\
28.32 \\
28.22 \\
28.12 \\
28.00 \\
27.88\end{array}$ & $\begin{array}{r}11.135827 \\
.134094 \\
.132368 \\
.130649 \\
.125936 \\
.127230 \\
.125531 \\
.123538 \\
.122151 \\
.120471\end{array}$ & $\begin{array}{l}49 \\
48 \\
47 \\
46 \\
45 \\
44 \\
43 \\
42 \\
41 \\
40\end{array}$ \\
\hline $\begin{array}{l}21 \\
22 \\
23 \\
24 \\
25 \\
26 \\
27 \\
28 \\
29 \\
30\end{array}$ & $\begin{array}{r}8.879949 \\
.851607 \\
.883258 \\
.884003 \\
.886542 \\
.888174 \\
.889801 \\
.891421 \\
.893035 \\
.891643\end{array}$ & $\begin{array}{l}27.63 \\
27.52 \\
27.42 \\
27.32 \\
27.20 \\
27.12 \\
27.00 \\
26.90 \\
26.80 \\
26.72\end{array}$ & $\begin{array}{r}9.938747 \\
.998738 \\
.998728 \\
.998718 \\
.998708 \\
.998699 \\
.998689 \\
.998679 \\
.998669 \\
.998659\end{array}$ & $\begin{array}{l}.15 \\
.17 \\
.17 \\
.17 \\
.15 \\
.17 \\
.17 \\
.17 \\
.17 \\
.17\end{array}$ & $\begin{array}{r}8.881202 \\
.882869 \\
.884530 \\
.886185 \\
.887833 \\
.889476 \\
.891112 \\
.892742 \\
.894366 \\
.895984\end{array}$ & \begin{tabular}{l|}
27.78 \\
27.68 \\
27.58 \\
27.47 \\
27.38 \\
27.27 \\
27.17 \\
27.07 \\
26.97 \\
26.87
\end{tabular} & $\begin{array}{r}11.118798 \\
.117131 \\
.115470 \\
.113815 \\
.112167 \\
.110524 \\
.108585 \\
.107258 \\
.105634 \\
.104016\end{array}$ & $\begin{array}{l}39 \\
38 \\
37 \\
36 \\
35 \\
34 \\
33 \\
32 \\
31 \\
30\end{array}$ \\
\hline $\begin{array}{l}31 \\
32 \\
33 \\
34 \\
35 \\
36 \\
37 \\
38 \\
39 \\
40\end{array}$ & $\begin{array}{r}8.896246 \\
.897542 \\
.899432 \\
.901017 \\
.902596 \\
.904169 \\
.905736 \\
.907297 \\
.908853 \\
.910404\end{array}$ & $\begin{array}{l}26.60 \\
26.50 \\
26.42 \\
26.32 \\
26.22 \\
26.12 \\
26.02 \\
25.93 \\
25.85 \\
25.75\end{array}$ & $\begin{array}{r}9.998649 \\
.998639 \\
.998629 \\
.998619 \\
.995609 \\
.998599 \\
.998589 \\
.998578 \\
.998564 \\
.998558\end{array}$ & $\begin{array}{l}.17 \\
.17 \\
.17 \\
.17 \\
.17 \\
.17 \\
.18 \\
.17 \\
.17 \\
.17\end{array}$ & $\begin{array}{r}8.897596 \\
.899303 \\
.900503 \\
.902398 \\
.903987 \\
.905570 \\
.907147 \\
.908719 \\
.910285 \\
.911846\end{array}$ & $\begin{array}{l}26.78 \\
26.67 \\
26.58 \\
26.48 \\
26.38 \\
26.28 \\
26.20 \\
26.10 \\
26.02 \\
25.92\end{array}$ & $\begin{array}{r}11.102404 \\
.100797 \\
.099197 \\
.097602 \\
.096013 \\
.094430 \\
.092853 \\
.091281 \\
.089715 \\
.085154\end{array}$ & $\begin{array}{l}29 \\
28 \\
27 \\
26 \\
25 \\
24 \\
23 \\
22 \\
21 \\
20\end{array}$ \\
\hline $\begin{array}{l}41 \\
42 \\
43 \\
44 \\
45 \\
46 \\
47 \\
48 \\
49 \\
50\end{array}$ & $\begin{array}{r}8.911949 \\
.913458 \\
.915022 \\
.916550 \\
.918073 \\
.919591 \\
.921103 \\
.922610 \\
.924112 \\
.925609\end{array}$ & $\begin{array}{l}25.65 \\
25.57 \\
25.47 \\
25.38 \\
25.30 \\
25.30 \\
25.12 \\
25.03 \\
24.95 \\
24.85\end{array}$ & $\begin{array}{r}9.998548 \\
.998537 \\
.998527 \\
.998516 \\
.998506 \\
.998195 \\
.998485 \\
.998474 \\
.998464 \\
.998453\end{array}$ & $\begin{array}{l}.18 \\
.17 \\
.18 \\
.17 \\
.18 \\
.17 \\
.18 \\
.17 \\
.18 \\
.18\end{array}$ & $\begin{array}{r}8.913401 \\
.914951 \\
.916495 \\
.918034 \\
.919568 \\
.921006 \\
.922319 \\
.924136 \\
.925049 \\
.927156\end{array}$ & \begin{tabular}{l|}
25.83 \\
25.73 \\
25.63 \\
25.57 \\
25.47 \\
25.33 \\
25.28 \\
25.22 \\
25.12 \\
25.03
\end{tabular} & $\begin{array}{r}11.086599 \\
.085049 \\
.053505 \\
.081966 \\
.080432 \\
.078904 \\
.077381 \\
.075564 \\
.074351 \\
.072844\end{array}$ & $\begin{array}{l}19 \\
19 \\
17 \\
16 \\
15 \\
14 \\
13 \\
12 \\
11 \\
10\end{array}$ \\
\hline $\begin{array}{l}51 \\
52 \\
53 \\
54 \\
55 \\
56 \\
57 \\
58 \\
59 \\
60\end{array}$ & $\begin{array}{r}8.927100 \\
.925557 \\
.930068 \\
.931544 \\
.933015 \\
.934451 \\
.935942 \\
.937398 \\
.938550 \\
8.940296\end{array}$ & $\begin{array}{l}24.78 \\
24.68 \\
24.60 \\
24.52 \\
24.43 \\
24.35 \\
24.27 \\
24.20 \\
24.10\end{array}$ & $\begin{array}{r}9.998442 \\
.998431 \\
.998421 \\
.998410 \\
.998399 \\
.998383 \\
.998377 \\
.998366 \\
.998355 \\
9.998344\end{array}$ & $\begin{array}{l}.18 \\
.17 \\
.18 \\
.18 \\
.18 \\
.18 \\
.18 \\
.18\end{array}$ & $\begin{array}{r}8.928655 \\
.930155 \\
.931647 \\
.933134 \\
.931616 \\
.936093 \\
.937565 \\
.939032 \\
.940494 \\
8.941952\end{array}$ & $\begin{array}{l}24.95 \\
24.87 \\
24.78 \\
24.70 \\
24.62 \\
24.53 \\
24.45 \\
24.37 \\
24.30\end{array}$ & $\begin{array}{r}11.071342 \\
.069845 \\
.068353 \\
.066866 \\
.065384 \\
.063907 \\
.062435 \\
.060968 \\
.059506 \\
11.05 \times 048\end{array}$ & $\begin{array}{l}9 \\
8 \\
7 \\
6 \\
5 \\
4 \\
3 \\
2 \\
1 \\
0\end{array}$ \\
\hline , & Cosine. & D. $1^{\prime \prime}$. & sue. & D. 1 . & Cotang. & D. $1^{\prime \prime}$. & 1 ang. & , \\
\hline
\end{tabular}


TABLE 9.-LOGARITHMIC SINES, COSINES,

\begin{tabular}{|c|c|c|c|c|c|c|c|c|}
\hline , & Sine. & D. 1"'. & Cosine. & D. $1^{\prime \prime}$ & Tang. & D. $1^{\prime \prime}$ & Cotang. & , \\
\hline $\begin{array}{r}0 \\
1 \\
2 \\
3 \\
4 \\
5 \\
6 \\
7 \\
8 \\
9 \\
10\end{array}$ & $\begin{array}{r}8.940296 \\
.941738 \\
.943174 \\
.944606 \\
.946034 \\
.947456 \\
.948874 \\
.950257 \\
.951696 \\
.953100 \\
.954499\end{array}$ & $\begin{array}{l}24.03 \\
23.93 \\
23.87 \\
23.80 \\
23.70 \\
23.63 \\
23.55 \\
23.48 \\
23.40 \\
23.32 \\
23.25\end{array}$ & $\begin{array}{r}9.998344 \\
.998333 \\
.998322 \\
.998311 \\
.998300 \\
.998289 \\
.998277 \\
.998266 \\
.998255 \\
.998243 \\
.998232\end{array}$ & $\begin{array}{l}.18 \\
.18 \\
.18 \\
.18 \\
.18 \\
.20 \\
.18 \\
.18 \\
.20 \\
.18 \\
.20\end{array}$ & $\begin{array}{r}8.941952 \\
.943404 \\
.944852 \\
.946295 \\
.947734 \\
.949168 \\
.950597 \\
.952021 \\
.953441 \\
.954856 \\
.956267\end{array}$ & $\begin{array}{l}24.20 \\
24.13 \\
24.05 \\
23.98 \\
23.90 \\
23.82 \\
23.73 \\
23.67 \\
23.58 \\
23.52 \\
23.45\end{array}$ & $\begin{array}{r}11.058048 \\
.056596 \\
.055148 \\
.053705 \\
.052266 \\
.050832 \\
.049403 \\
.047979 \\
.046559 \\
.045144 \\
.043733\end{array}$ & $\begin{array}{l}60 \\
59 \\
58 \\
57 \\
56 \\
55 \\
54 \\
53 \\
52 \\
51 \\
50\end{array}$ \\
\hline $\begin{array}{l}11 \\
12 \\
13 \\
14 \\
15 \\
16 \\
17 \\
18 \\
19 \\
20\end{array}$ & $\begin{array}{r}8.955894 \\
.957254 \\
.958670 \\
.960052 \\
.961429 \\
.962801 \\
.964170 \\
.965534 \\
.966593 \\
.968249\end{array}$ & $\begin{array}{l}23.17 \\
23.10 \\
23.03 \\
22.95 \\
22.87 \\
22.82 \\
22.73 \\
22.65 \\
22.60 \\
22.52\end{array}$ & $\begin{array}{r}9.998220 \\
.998209 \\
.998197 \\
.998186 \\
.998174 \\
.998163 \\
.998151 \\
.998139 \\
.998128 \\
.998116\end{array}$ & $\begin{array}{l}.18 \\
.20 \\
.18 \\
.20 \\
.18 \\
.20 \\
.20 \\
.18 \\
.20 \\
.20\end{array}$ & $\begin{array}{r}8.957674 \\
.959075 \\
.960473 \\
.961866 \\
.963255 \\
.964639 \\
.966019 \\
.967394 \\
.968766 \\
.970133\end{array}$ & $\begin{array}{l}23.35 \\
23.30 \\
23.22 \\
23.15 \\
23.07 \\
23.00 \\
22.92 \\
22.87 \\
22.78 \\
22.72\end{array}$ & $\begin{array}{r}11.042326 \\
.040925 \\
.039527 \\
.038134 \\
.036745 \\
.035361 \\
.033981 \\
.032606 \\
.031234 \\
.029867\end{array}$ & $\begin{array}{l}49 \\
48 \\
47 \\
46 \\
45 \\
44 \\
43 \\
42 \\
41 \\
40\end{array}$ \\
\hline $\begin{array}{l}21 \\
22 \\
23 \\
24 \\
25 \\
26 \\
27 \\
28 \\
29 \\
30\end{array}$ & $\begin{array}{r}8.969600 \\
.970947 \\
.972289 \\
.973628 \\
.974962 \\
.976293 \\
.977619 \\
.978941 \\
.980259 \\
.981573\end{array}$ & $\begin{array}{l}22.45 \\
22.37 \\
22.32 \\
22.23 \\
22.18 \\
22.10 \\
22.03 \\
21.97 \\
21.90 \\
21.83\end{array}$ & $\begin{array}{r}9.998104 \\
.998092 \\
.998080 \\
.998068 \\
.998056 \\
.998044 \\
.998032 \\
.998020 \\
.998008 \\
.997996\end{array}$ & $\begin{array}{l}.20 \\
.20 \\
.20 \\
.20 \\
.20 \\
.20 \\
.20 \\
.20 \\
.20 \\
.20\end{array}$ & $\begin{array}{r}8.971496 \\
.972855 \\
.974209 \\
.975560 \\
.976906 \\
.978248 \\
.979586 \\
.980921 \\
.982251 \\
.983577\end{array}$ & $\begin{array}{l}22.65 \\
22.57 \\
22.52 \\
22.43 \\
22.37 \\
22.30 \\
22.25 \\
22.17 \\
22.10 \\
22.03\end{array}$ & $\begin{array}{r}11.028504 \\
.027145 \\
.025791 \\
.024440 \\
.023094 \\
.021752 \\
.020414 \\
.019079 \\
.017749 \\
.016423\end{array}$ & $\begin{array}{l}39 \\
38 \\
37 \\
36 \\
35 \\
34 \\
33 \\
32 \\
31 \\
30\end{array}$ \\
\hline $\begin{array}{l}31 \\
32 \\
33 \\
34 \\
35 \\
36 \\
37 \\
38 \\
39 \\
40\end{array}$ & $\begin{array}{r}8.982883 \\
.984189 \\
.985491 \\
.986789 \\
.988083 \\
.989374 \\
.990660 \\
.991943 \\
.993222 \\
.994497\end{array}$ & $\begin{array}{l}21.77 \\
21.72 \\
21.63 \\
21.57 \\
21.52 \\
21.43 \\
21.38 \\
21.32 \\
21.25 \\
21.18\end{array}$ & $\begin{array}{r}9.997984 \\
.997972 \\
.997959 \\
.997947 \\
.997935 \\
.997922 \\
.997910 \\
.997897 \\
.997885 \\
.997872\end{array}$ & $\begin{array}{l}.20 \\
.22 \\
.20 \\
.20 \\
.22 \\
.20 \\
.22 \\
.20 \\
.22 \\
.20\end{array}$ & $\begin{array}{r}8.984899 \\
.986217 \\
. .987532 \\
.988842 \\
.990149 \\
.991451 \\
.992750 \\
. .994045 \\
.995337 \\
.996624\end{array}$ & $\begin{array}{l}21.97 \\
21.92 \\
21.83 \\
21.78 \\
21.70 \\
21.65 \\
21.58 \\
21.53 \\
21.45 \\
21.40\end{array}$ & $\begin{array}{r}11.015101 \\
.013783 \\
.012468 \\
.011158 \\
.009851 \\
.008549 \\
.007250 \\
.005955 \\
.004663 \\
.003376\end{array}$ & $\begin{array}{l}29 \\
28 \\
27 \\
26 \\
25 \\
24 \\
23 \\
22 \\
21 \\
20\end{array}$ \\
\hline $\begin{array}{l}41 \\
42 \\
43 \\
44 \\
45 \\
40 \\
47 \\
48 \\
49 \\
50\end{array}$ & $\begin{array}{r}8.995768 \\
.997036 \\
.998299 \\
8.999560 \\
9.000816 \\
.002069 \\
.003318 \\
.004563 \\
.005805 \\
.007044\end{array}$ & $\begin{array}{l}21.13 \\
21.05 \\
21.02 \\
20.93 \\
20.88 \\
20.82 \\
20.75 \\
20.70 \\
20.65 \\
20.57\end{array}$ & $\begin{array}{r}9.997860 \\
.997847 \\
.997835 \\
.997822 \\
.997809 \\
.997797 \\
.997784 \\
.997771 \\
.997758 \\
.997745\end{array}$ & $\begin{array}{l}.22 \\
.20 \\
.22 \\
.22 \\
.20 \\
.22 \\
.22 \\
.22 \\
.22 \\
.22\end{array}$ & $\begin{array}{r}8.997908 \\
8.999188 \\
9.000465 \\
.001738 \\
.003007 \\
.004272 \\
.005534 \\
.006792 \\
.008047 \\
.009298\end{array}$ & $\begin{array}{l}21.33 \\
21.28 \\
21.22 \\
21.15 \\
21.08 \\
21.03 \\
20.97 \\
20.92 \\
20.85 \\
20.80\end{array}$ & $\begin{array}{r}11.002092 \\
11.000812 \\
10.999535 \\
.998262 \\
.996993 \\
.995728 \\
.9944 C 6 \\
.993208 \\
.991953 \\
.990702\end{array}$ & $\begin{array}{l}19 \\
18 \\
17 \\
16 \\
15 \\
14 \\
13 \\
12 \\
11 \\
10\end{array}$ \\
\hline $\begin{array}{l}51 \\
52 \\
53 \\
54 \\
55 \\
56 \\
57 \\
58 \\
59 \\
60\end{array}$ & $\begin{array}{r}9.008278 \\
.009510 \\
.010737 \\
.011962 \\
.013182 \\
.014400 \\
.015613 \\
.016824 \\
.018031 \\
9.019235\end{array}$ & $\begin{array}{l}20.53 \\
20.45 \\
20.42 \\
20.33 \\
20.30 \\
20.22 \\
20.18 \\
20.12 \\
20.07\end{array}$ & $\begin{array}{r}9.997732 \\
.997719 \\
.997706 \\
.997693 \\
.997680 \\
.997667 \\
.997654 \\
.997641 \\
.997628 \\
9.997614\end{array}$ & $\begin{array}{l}.22 \\
.22 \\
.22 \\
.22 \\
.22 \\
.22 \\
.22 \\
.22 \\
.23\end{array}$ & $\begin{array}{r}9.010546 \\
.011790 \\
.013031 \\
.014268 \\
.015502 \\
.016732 \\
.017959 \\
.019183 \\
.020403 \\
9.021620\end{array}$ & $\begin{array}{l}20.73 \\
20.68 \\
20.62 \\
20.57 \\
20.50 \\
20.45 \\
20.40 \\
20.33 \\
20.28\end{array}$ & $\begin{array}{r}10.989454 \\
.988210 \\
.986969 \\
.985732 \\
.984498 \\
.983268 \\
.982041 \\
.980817 \\
.979597 \\
10.978380\end{array}$ & $\begin{array}{l}9 \\
8 \\
7 \\
6 \\
5 \\
4 \\
3 \\
2 \\
1 \\
0\end{array}$ \\
\hline ' & Cosine. & D. $1^{\prime \prime}$ & Sine. & D. 1 . & Cotang. & D. $1^{\prime \prime}$ & Tang. & , \\
\hline
\end{tabular}




\begin{tabular}{|c|c|c|c|c|c|c|c|c|}
\hline , & Sine. & D. $1^{\prime \prime}$. & Cosine. & D. $1^{\prime \prime}$ & Tang. & D. $1^{\prime \prime}$. & Cotang. & , \\
\hline $\begin{array}{r}0 \\
1 \\
2 \\
3 \\
4 \\
5 \\
6 \\
7 \\
8 \\
9 \\
10\end{array}$ & $\begin{array}{r}9.019235 \\
.020435 \\
.021632 \\
.022825 \\
.024016 \\
.025203 \\
.026386 \\
.027567 \\
.028744 \\
.029918 \\
.031059\end{array}$ & $\begin{array}{l}20.00 \\
19.95 \\
19.88 \\
19.85 \\
19.78 \\
19.72 \\
19.68 \\
19.62 \\
19.57 \\
19.52 \\
19.47\end{array}$ & $\begin{array}{r}9.997614 \\
.997601 \\
.997588 \\
.997574 \\
.997561 \\
.997547 \\
.997534 \\
.997520 \\
.997507 \\
.997493 \\
.997480\end{array}$ & $\begin{array}{l}.22 \\
.22 \\
.23 \\
.22 \\
.23 \\
.22 \\
.23 \\
.22 \\
.23 \\
.22 \\
.23\end{array}$ & $\begin{array}{r}9.021620 \\
.022834 \\
.024044 \\
.025251 \\
.026455 \\
.027655 \\
.028552 \\
.030046 \\
.031237 \\
.032425 \\
.033609\end{array}$ & $\begin{array}{l}20.23 \\
20.17 \\
20.12 \\
20.07 \\
20.00 \\
19.95 \\
19.90 \\
19.85 \\
19.80 \\
19.73 \\
19.70\end{array}$ & $\begin{array}{r}10.97 \AA 350 \\
.977166 \\
.975956 \\
.974749 \\
.973545 \\
.972345 \\
.971148 \\
.969954 \\
.965763 \\
.967575 \\
.966391\end{array}$ & $\begin{array}{l}60 \\
59 \\
58 \\
57 \\
56 \\
55 \\
54 \\
53 \\
52 \\
51 \\
50\end{array}$ \\
\hline $\begin{array}{l}11 \\
12 \\
13 \\
14 \\
15 \\
16 \\
17 \\
18 \\
19 \\
20\end{array}$ & $\begin{array}{r}9.032257 \\
.033421 \\
.034582 \\
.035741 \\
.036896 \\
.035048 \\
.039197 \\
.040342 \\
.041455 \\
.042625\end{array}$ & $\begin{array}{l}19.40 \\
19.35 \\
19.32 \\
19.25 \\
19.20 \\
19.15 \\
19.08 \\
19.05 \\
19.00 \\
18.95\end{array}$ & $\begin{array}{r}9.997466 \\
.997452 \\
.997439 \\
.997425 \\
.997411 \\
.997397 \\
.997383 \\
.997369 \\
.997355 \\
.997341\end{array}$ & $\begin{array}{l}.23 \\
.22 \\
.23 \\
.23 \\
.23 \\
.23 \\
.23 \\
.23 \\
.23 \\
.23\end{array}$ & $\begin{array}{l}9.034791 \\
.035969 \\
.037144 \\
.038316 \\
.039485 \\
.040651 \\
.041813 \\
.042973 \\
.044130 \\
.045284\end{array}$ & $\begin{array}{l}19.63 \\
19.58 \\
19.53 \\
19.48 \\
19.43 \\
19.37 \\
19.33 \\
19.28 \\
19.23 \\
19.17\end{array}$ & $\begin{array}{r}10.965209 \\
.964031 \\
.962856 \\
.961654 \\
.960515 \\
.959349 \\
.958187 \\
.957027 \\
.955870 \\
.954716\end{array}$ & $\begin{array}{l}49 \\
48 \\
47 \\
46 \\
45 \\
44 \\
43 \\
42 \\
41 \\
40\end{array}$ \\
\hline $\begin{array}{l}21 \\
22 \\
23 \\
24 \\
25 \\
26 \\
27 \\
28 \\
29 \\
30\end{array}$ & $\begin{array}{r}9.043762 \\
.044595 \\
.046026 \\
.047154 \\
.045279 \\
.049400 \\
.050519 \\
.051635 \\
.052749 \\
.053859\end{array}$ & $\begin{array}{l}18.88 \\
18.85 \\
18.80 \\
18.75 \\
18.68 \\
18.65 \\
18.60 \\
18.57 \\
18.50 \\
18.45\end{array}$ & $\begin{array}{r}9.997327 \\
.997313 \\
.997299 \\
.997285 \\
.997271 \\
.997257 \\
.997242 \\
.997228 \\
.997214 \\
.997199\end{array}$ & $\begin{array}{l}.23 \\
.23 \\
.23 \\
.23 \\
.23 \\
.25 \\
.23 \\
.23 \\
.25 \\
.23\end{array}$ & $\begin{array}{r}9.046434 \\
.047582 \\
.048727 \\
.049869 \\
.051008 \\
.052144 \\
.053277 \\
.054407 \\
.0555535 \\
.056659\end{array}$ & $\begin{array}{l}19.13 \\
19.08 \\
19.03 \\
18.93 \\
18.93 \\
18.88 \\
18.83 \\
18.80 \\
18.73 \\
18.70\end{array}$ & $\begin{array}{r}10.953566 \\
.952418 \\
.951273 \\
.950131 \\
.948992 \\
.947856 \\
.946723 \\
.945593 \\
.944465 \\
.943341\end{array}$ & $\begin{array}{l}39 \\
38 \\
37 \\
36 \\
35 \\
34 \\
33 \\
32 \\
31 \\
30\end{array}$ \\
\hline $\begin{array}{l}31 \\
32 \\
33 \\
34 \\
35 \\
36 \\
37 \\
38 \\
39 \\
40\end{array}$ & $\begin{array}{r}9.054966 \\
.056071 \\
.057172 \\
.058271 \\
.059367 \\
.060460 \\
.061551 \\
.062639 \\
.063724 \\
.064806\end{array}$ & $\begin{array}{l}18.42 \\
18.35 \\
18.32 \\
18.27 \\
18.22 \\
18.18 \\
18.13 \\
18.03 \\
18.03 \\
17.98\end{array}$ & $\begin{array}{r}9.997185 \\
.997170 \\
.997156 \\
.997141 \\
.997127 \\
.997112 \\
.997093 \\
.997083 \\
.997068 \\
.997053\end{array}$ & $\begin{array}{l}.25 \\
.23 \\
.25 \\
.23 \\
.25 \\
.23 \\
.25 \\
.25 \\
.25 \\
.23\end{array}$ & $\begin{array}{r}9.057781 \\
.058900 \\
.060016 \\
.061130 \\
.062240 \\
.063348 \\
.064453 \\
.0655556 \\
.0666555 \\
.067752\end{array}$ & $\begin{array}{l}18.65 \\
18.60 \\
18.57 \\
18.50 \\
18.47 \\
18.42 \\
18.38 \\
18.32 \\
18.28 \\
18.25\end{array}$ & $\begin{array}{r}10.942219 \\
.941100 \\
.939984 \\
.938870 \\
.937760 \\
.936652 \\
.935547 \\
.934444 \\
.933345 \\
.932248\end{array}$ & $\begin{array}{l}29 \\
28 \\
27 \\
26 \\
25 \\
24 \\
23 \\
22 \\
21 \\
20\end{array}$ \\
\hline $\begin{array}{l}41 \\
42 \\
43 \\
44 \\
45 \\
46 \\
47 \\
4.3 \\
49 \\
50\end{array}$ & $\begin{array}{r}9.065885 \\
.066962 \\
.068036 \\
.069107 \\
.070176 \\
.071242 \\
.072306 \\
.073366 \\
.074424 \\
.0754 \times 0\end{array}$ & $\begin{array}{l}17.95 \\
17.90 \\
17.85 \\
17.82 \\
17.77 \\
17.73 \\
17.67 \\
17.63 \\
17.60 \\
17.55\end{array}$ & $\begin{array}{r}9.997039 \\
.997024 \\
.997009 \\
.926994 \\
.936979 \\
.996964 \\
.996949 \\
.996934 \\
.996919 \\
.996904\end{array}$ & $\begin{array}{l}.25 \\
.25 \\
.25 \\
.25 \\
.25 \\
.25 \\
.25 \\
.25 \\
.25 \\
.25\end{array}$ & $\begin{array}{r}9.0688846 \\
.069938 \\
.071027 \\
.072113 \\
.073197 \\
.074278 \\
.075356 \\
.076432 \\
.077505 \\
.078576\end{array}$ & $\begin{array}{l}18.20 \\
18.15 \\
18.10 \\
18.07 \\
18.02 \\
17.97 \\
17.93 \\
17.88 \\
17.85 \\
17.80\end{array}$ & $\begin{array}{r}10.931154 \\
.930062 \\
.928973 \\
.927887 \\
.926803 \\
.925722 \\
.924644 \\
.923568 \\
.922405 \\
.921424\end{array}$ & $\begin{array}{l}19 \\
18 \\
17 \\
16 \\
15 \\
14 \\
13 \\
12 \\
11 \\
10\end{array}$ \\
\hline $\begin{array}{l}51 \\
52 \\
53 \\
54 \\
55 \\
56 \\
57 \\
58 \\
59 \\
60\end{array}$ & $\begin{array}{r}9.076533 \\
.077543 \\
.078631 \\
.079676 \\
.050719 \\
.081759 \\
.082797 \\
.083832 \\
.084864 \\
9.085594\end{array}$ & $\begin{array}{l}17.50 \\
17.47 \\
17.42 \\
17.38 \\
17.33 \\
17.30 \\
17.25 \\
17.20 \\
17.17\end{array}$ & $\begin{array}{r}9.906889 \\
.996874 \\
.996558 \\
.906543 \\
.906628 \\
.996812 \\
.906797 \\
.996782 \\
.996766 \\
9.906751\end{array}$ & $\begin{array}{l}.25 \\
.27 \\
.25 \\
.27 \\
.27 \\
.25 \\
.25 \\
.27 \\
.25\end{array}$ & $\begin{array}{r}9.079644 \\
.050710 \\
.081773 \\
.082833 \\
.083591 \\
.084947 \\
.084000 \\
.087050 \\
.0850643 \\
9.089144\end{array}$ & $\begin{array}{l}17.00 \\
17.77 \\
17.72 \\
17.67 \\
17.63 \\
17.60 \\
17.55 \\
17.50 \\
17.47 \\
17.43\end{array}$ & $\begin{array}{r}10.920356 \\
.919290 \\
.918227 \\
.917167 \\
.916109 \\
.915053 \\
.914000 \\
.912250 \\
.911902 \\
10.910556\end{array}$ & $\begin{array}{l}9 \\
8 \\
7 \\
7 \\
6 \\
5 \\
4 \\
3 \\
2 \\
1 \\
0\end{array}$ \\
\hline ' & Cosine. & 1). $1^{\prime \prime}$. & Sine. & D. $1^{\prime \prime}$. & Cotang. & D. $1 \%$ & Tang. & ' \\
\hline
\end{tabular}




\begin{tabular}{|c|c|c|c|c|c|c|c|c|}
\hline , & Sine. & D. 1". & Cosine. & D. $1^{\prime \prime}$. & Tang. & D. 1". & Cotang. & , \\
\hline $\begin{array}{r}0 \\
1 \\
2 \\
3 \\
4 \\
5 \\
6 \\
7 \\
8 \\
9 \\
10\end{array}$ & $\begin{array}{r}9.085894 \\
.086922 \\
.087947 \\
.088970 \\
.089990 \\
.091008 \\
.092024 \\
.093037 \\
.094047 \\
.095056 \\
.096062\end{array}$ & $\begin{array}{l}17.13 \\
17.08 \\
17.05 \\
17.00 \\
16.97 \\
16.93 \\
16.88 \\
16.83 \\
16.82 \\
16.77 \\
16.72\end{array}$ & $\begin{array}{r}9.996751 \\
.996735 \\
.996720 \\
.996704 \\
.996688 \\
.996673 \\
.996657 \\
.996641 \\
.996625 \\
.996610 \\
.996594\end{array}$ & $\begin{array}{l}.27 \\
.25 \\
.27 \\
.27 \\
.25 \\
.27 \\
.27 \\
.27 \\
.25 \\
.27 \\
.27\end{array}$ & $\begin{array}{r}9.089144 \\
.090187 \\
.091228 \\
.092266 \\
.093302 \\
.094336 \\
.095367 \\
.096395 \\
.097422 \\
.098446 \\
.099468\end{array}$ & $\begin{array}{l}17.35 \\
17.35 \\
17.30 \\
17.27 \\
17.23 \\
17.18 \\
17.13 \\
17.12 \\
17.07 \\
17.03 \\
16.98\end{array}$ & $\begin{array}{r}10.910856 \\
.909813 \\
.905772 \\
.907734 \\
.906698 \\
.905664 \\
.904633 \\
.903605 \\
.902578 \\
.901554 \\
.900532\end{array}$ & $\begin{array}{l}60 \\
59 \\
58 \\
57 \\
56 \\
55 \\
54 \\
53 \\
32 \\
51 \\
50\end{array}$ \\
\hline $\begin{array}{l}11 \\
12 \\
13 \\
14 \\
15 \\
16 \\
17 \\
18 \\
19 \\
20\end{array}$ & $\begin{array}{r}9.097065 \\
.098066 \\
.099065 \\
.100062 \\
.101056 \\
.102048 \\
.103037 \\
.104025 \\
.105010 \\
.105992\end{array}$ & $\begin{array}{l}16.68 \\
16.65 \\
16.62 \\
16.57 \\
16.53 \\
16.48 \\
16.47 \\
16.42 \\
16.37 \\
16.35\end{array}$ & $\begin{array}{r}9.996578 \\
.996562 \\
.996546 \\
.996530 \\
.996514 \\
.996498 \\
.996482 \\
.996465 \\
.996449 \\
.996433\end{array}$ & $\begin{array}{l}.27 \\
.27 \\
.27 \\
.27 \\
.27 \\
.27 \\
.28 \\
.27 \\
.27 \\
.27\end{array}$ & $\begin{array}{r}9.100487 \\
.101504 \\
.102519 \\
.103532 \\
.104542 \\
.105550 \\
.106556 \\
.107559 \\
.108560 \\
.109559\end{array}$ & $\begin{array}{l}16.95 \\
16.92 \\
16.88 \\
16.83 \\
16.80 \\
16.77 \\
16.72 \\
16.68 \\
16.65 \\
16.62\end{array}$ & $\begin{array}{r}10.899513 \\
.898496 \\
.897481 \\
.896468 \\
.895458 \\
.894450 \\
.893444 \\
.892441 \\
.891440 \\
.890441\end{array}$ & $\begin{array}{l}49 \\
48 \\
47 \\
46 \\
45 \\
44 \\
43 \\
42 \\
41 \\
40\end{array}$ \\
\hline $\begin{array}{l}21 \\
22 \\
23 \\
24 \\
25 \\
26 \\
27 \\
28 \\
29 \\
30\end{array}$ & $\begin{array}{r}9.106973 \\
.107951 \\
.108927 \\
.109901 \\
.110873 \\
.111842 \\
.112809 \\
.113774 \\
.114737 \\
.115698\end{array}$ & $\begin{array}{l}16.30 \\
16.27 \\
16.23 \\
16.20 \\
16.15 \\
16.12 \\
16.08 \\
16.05 \\
16.02 \\
15.97\end{array}$ & $\begin{array}{r}9.996417 \\
.996400 \\
.996384 \\
.996368 \\
.996351 \\
.996335 \\
.996318 \\
.996302 \\
.996285 \\
.996269\end{array}$ & $\begin{array}{l}.28 \\
.27 \\
.27 \\
.28 \\
.27 \\
.28 \\
.27 \\
.28 \\
.27 \\
.28\end{array}$ & $\begin{array}{r}9.110556 \\
.111551 \\
.112543 \\
.113533 \\
.114521 \\
.115507 \\
.116491 \\
.117472 \\
.118452 \\
.119429\end{array}$ & $\begin{array}{l}16.53 \\
16.53 \\
16.50 \\
16.47 \\
16.43 \\
16.40 \\
16.35 \\
16.33 \\
16.28 \\
16.25\end{array}$ & $\begin{array}{r}10.889444 \\
.888449 \\
.887457 \\
.886467 \\
.885479 \\
.884493 \\
.883509 \\
.882528 \\
.881548 \\
.880571\end{array}$ & $\begin{array}{l}39 \\
38 \\
37 \\
36 \\
35 \\
34 \\
33 \\
32 \\
31 \\
30\end{array}$ \\
\hline $\begin{array}{l}31 \\
32 \\
33 \\
34 \\
35 \\
36 \\
37 \\
38 \\
39 \\
40\end{array}$ & $\begin{array}{r}9.116656 \\
.117613 \\
.118567 \\
.119519 \\
.120469 \\
.121417 \\
.122362 \\
.123306 \\
.124248 \\
.125187\end{array}$ & $\begin{array}{l}15.95 \\
15.90 \\
15.87 \\
15.83 \\
15.80 \\
15.75 \\
15.73 \\
15.70 \\
15.65 \\
15.63\end{array}$ & $\begin{array}{r}9.996252 \\
.996235 \\
.996219 \\
.996202 \\
.996185 \\
.996168 \\
.996151 \\
.996134 \\
.996117 \\
.996100\end{array}$ & $\begin{array}{l}.28 \\
.27 \\
.28 \\
.28 \\
.28 \\
.28 \\
.28 \\
.28 \\
.28 \\
.28\end{array}$ & $\begin{array}{r}9.120404 \\
.121377 \\
.122348 \\
.123317 \\
.124284 \\
.125249 \\
.126211 \\
.127172 \\
.128130 \\
.129087\end{array}$ & $\begin{array}{l}16.22 \\
16.18 \\
16.15 \\
16.12 \\
16.08 \\
16.03 \\
16.02 \\
15.97 \\
15.95 \\
15.90\end{array}$ & $\begin{array}{r}10.879596 \\
.878623 \\
.877652 \\
.876683 \\
.875716 \\
.874751 \\
.873789 \\
.872828 \\
.871870 \\
.870913\end{array}$ & $\begin{array}{l}29 \\
28 \\
27 \\
26 \\
25 \\
24 \\
23 \\
22 \\
21 \\
20\end{array}$ \\
\hline $\begin{array}{l}41 \\
42 \\
43 \\
44 \\
45 \\
46 \\
47 \\
48 \\
49 \\
50\end{array}$ & $\begin{array}{r}9.126125 \\
.127060 \\
.127993 \\
.128925 \\
.129854 \\
.130781 \\
.131706 \\
.132630 \\
.133551 \\
.134470\end{array}$ & $\begin{array}{l}15.58 \\
15.55 \\
15.53 \\
15.48 \\
15.45 \\
15.42 \\
15.40 \\
15.35 \\
15.32 \\
15.28\end{array}$ & $\begin{array}{r}9.996083 \\
.996066 \\
.996049 \\
.996032 \\
.996015 \\
.995998 \\
.995980 \\
.995963 \\
.995946 \\
.995928\end{array}$ & $\begin{array}{l}.28 \\
.28 \\
.28 \\
.28 \\
.28 \\
.30 \\
.28 \\
.28 \\
.30 \\
.28\end{array}$ & $\begin{array}{r}9.130041 \\
.130994 \\
.131944 \\
.132893 \\
.133839 \\
.134784 \\
.135726 \\
.1366667 \\
.137605 \\
.138542\end{array}$ & $\begin{array}{l}15.88 \\
15.83 \\
15.82 \\
15.77 \\
15.75 \\
15.70 \\
15.68 \\
15.63 \\
15.62 \\
15.57\end{array}$ & $\begin{array}{r}10.869959 \\
.869006 \\
.868056 \\
.88^{7} 107 \\
.866161 \\
.865216 \\
.864274 \\
.8633333 \\
.862395 \\
.861458\end{array}$ & $\begin{array}{l}19 \\
18 \\
17 \\
16 \\
15 \\
14 \\
13 \\
12 \\
11 \\
10\end{array}$ \\
\hline $\begin{array}{l}51 \\
52 \\
53 \\
54 \\
55 \\
56 \\
57 \\
58 \\
59 \\
60\end{array}$ & $\begin{array}{r}9.135387 \\
.136303 \\
.137216 \\
.138128 \\
.139037 \\
.139944 \\
.140850 \\
.141754 \\
.142655 \\
9.143555\end{array}$ & $\begin{array}{l}15.27 \\
15.22 \\
15.20 \\
15.15 \\
15.12 \\
15.10 \\
15.07 \\
15.02 \\
15.00\end{array}$ & $\begin{array}{r}9.995911 \\
.995894 \\
.995876 \\
.995859 \\
.995841 \\
.995823 \\
.995806 \\
.995788 \\
.995771 \\
9.995753\end{array}$ & $\begin{array}{l}.28 \\
.30 \\
.28 \\
.30 \\
.30 \\
.28 \\
.30 \\
.28 \\
.30\end{array}$ & $\begin{array}{r}9.139476 \\
.140409 \\
.141340 \\
.142269 \\
.143196 \\
.144121 \\
.145044 \\
.145966 \\
.146865 \\
9.147803\end{array}$ & $\begin{array}{l}15.55 \\
15.52 \\
15.48 \\
15.45 \\
15.42 \\
15.38 \\
15.37 \\
15.32 \\
15.30\end{array}$ & $\begin{array}{r}10.860524 \\
.859591 \\
.858660 \\
.857731 \\
.856804 \\
.855879 \\
.854956 \\
.854034 \\
.853115 \\
10.852197\end{array}$ & $\begin{array}{l}9 \\
8 \\
7 \\
6 \\
5 \\
4 \\
3 \\
2 \\
1 \\
0\end{array}$ \\
\hline ' & Cosine. & D. 1". & $\infty$ & $\cdot \cdot$ & Cotang. & D. $1^{\prime \prime}$. & 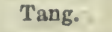 & \\
\hline
\end{tabular}




\begin{tabular}{|c|c|c|c|c|c|c|c|c|}
\hline , & Sine. & D. $1^{\prime \prime}$ & Cosine. & D. 1". & Tang. & D. 1"' & Cotang. & I \\
\hline $\begin{array}{r}0 \\
1 \\
2 \\
3 \\
4 \\
5 \\
6 \\
7 \\
8 \\
9 \\
10\end{array}$ & $\begin{array}{r}9.143555 \\
.144453 \\
.145349 \\
.146243 \\
.147136 \\
.148026 \\
.145915 \\
.149802 \\
.150686 \\
.151569 \\
.152451\end{array}$ & $\begin{array}{l}14.97 \\
14.93 \\
14.90 \\
14.88 \\
14.83 \\
14.82 \\
14.78 \\
14.73 \\
14.72 \\
14.70 \\
14.65\end{array}$ & $\begin{array}{r}9.995753 \\
.995735 \\
.995717 \\
.995699 \\
.995651 \\
.995664 \\
.995646 \\
.995628 \\
.995610 \\
.995591 \\
.995573\end{array}$ & $\begin{array}{l}.30 \\
.30 \\
.30 \\
.30 \\
.23 \\
.30 \\
.30 \\
.30 \\
.32 \\
.30 \\
.30\end{array}$ & $\begin{array}{r}9.147803 \\
.148718 \\
.149632 \\
.150544 \\
.151454 \\
.152363 \\
.153269 \\
.154174 \\
.155077 \\
.155978 \\
.156877\end{array}$ & $\begin{array}{l}15.25 \\
15.23 \\
15.20 \\
15.17 \\
15.15 \\
15.10 \\
15.08 \\
15.05 \\
15.02 \\
14.93 \\
14.97\end{array}$ & $\begin{array}{r}10.852197 \\
.851252 \\
.850368 \\
.849456 \\
.848546 \\
.847637 \\
.846731 \\
.845826 \\
.844923 \\
.844022 \\
.843123\end{array}$ & $\begin{array}{l}60 \\
59 \\
58 \\
57 \\
56 \\
55 \\
54 \\
53 \\
52 \\
51 \\
50\end{array}$ \\
\hline $\begin{array}{l}11 \\
12 \\
13 \\
14 \\
15 \\
16 \\
17 \\
18 \\
19 \\
20\end{array}$ & $\begin{array}{r}9.153330 \\
.154208 \\
.155083 \\
.155957 \\
.156830 \\
.157700 \\
.158569 \\
.159435 \\
.160301 \\
.161164\end{array}$ & $\begin{array}{l}14.63 \\
14.58 \\
14.57 \\
14.55 \\
14.50 \\
14.48 \\
14.43 \\
14.43 \\
14.38 \\
14.35\end{array}$ & $\begin{array}{r}9.995555 \\
.995537 \\
.995519 \\
.995501 \\
.995482 \\
.995464 \\
.995446 \\
.995427 \\
.995409 \\
.995390\end{array}$ & $\begin{array}{l}.30 \\
.30 \\
.30 \\
.32 \\
.30 \\
.30 \\
.32 \\
.30 \\
.32 \\
.30\end{array}$ & $\begin{array}{r}9.157775 \\
.158671 \\
.159565 \\
.160457 \\
.161347 \\
.162236 \\
.163123 \\
.164008 \\
.164892 \\
.165774\end{array}$ & $\begin{array}{l}14.93 \\
14.90 \\
14.87 \\
14.83 \\
14.82 \\
14.78 \\
14.75 \\
14.73 \\
14.70 \\
14.67\end{array}$ & $\begin{array}{r}10.842225 \\
.841329 \\
.840435 \\
.839543 \\
.838653 \\
.837764 \\
.836877 \\
.835992 \\
.835108 \\
.834226\end{array}$ & $\begin{array}{l}49 \\
48 \\
47 \\
46 \\
45 \\
44 \\
43 \\
42 \\
41 \\
40\end{array}$ \\
\hline $\begin{array}{l}21 \\
22 \\
23 \\
24 \\
25 \\
26 \\
27 \\
28 \\
29 \\
30\end{array}$ & $\begin{array}{r}9.162025 \\
.162885 \\
.163743 \\
.164600 \\
.165454 \\
.166307 \\
.167159 \\
.168008 \\
.168856 \\
.169702\end{array}$ & $\begin{array}{l}14.33 \\
14.30 \\
14.28 \\
14.23 \\
14.22 \\
14.20 \\
14.15 \\
14.13 \\
14.10 \\
14.08\end{array}$ & $\begin{array}{r}9.995372 \\
.995353 \\
.995334 \\
.995316 \\
.995297 \\
.995278 \\
.995260 \\
.995241 \\
.995222 \\
.995203\end{array}$ & $\begin{array}{l}.32 \\
.32 \\
.30 \\
.32 \\
.32 \\
.30 \\
.32 \\
.32 \\
.32 \\
.32\end{array}$ & $\begin{array}{r}9.166654 \\
.167532 \\
.168409 \\
.169284 \\
.170157 \\
.171029 \\
.171899 \\
.172767 \\
.173634 \\
.174499\end{array}$ & $\begin{array}{l}14.63 \\
14.62 \\
14.58 \\
14.55 \\
14.53 \\
14.50 \\
14.47 \\
14.45 \\
14.42 \\
14.38\end{array}$ & $\begin{array}{r}10.833346 \\
.832468 \\
.831591 \\
.830716 \\
.829843 \\
.828971 \\
.828101 \\
.827233 \\
.826366 \\
.825501\end{array}$ & $\begin{array}{l}39 \\
38 \\
37 \\
36 \\
35 \\
34 \\
33 \\
32 \\
31 \\
30\end{array}$ \\
\hline $\begin{array}{l}31 \\
32 \\
33 \\
34 \\
35 \\
36 \\
37 \\
33 \\
39 \\
40\end{array}$ & $\begin{array}{r}9.170547 \\
.171359 \\
.172230 \\
.173070 \\
.173905 \\
.174744 \\
.175578 \\
.176411 \\
.177242 \\
.178072\end{array}$ & $\begin{array}{l}14.03 \\
14.02 \\
14.00 \\
13.97 \\
13.93 \\
13.90 \\
13.88 \\
13.85 \\
13.83 \\
13.80\end{array}$ & $\begin{array}{r}9.995184 \\
.995165 \\
.995146 \\
.995127 \\
.995108 \\
.995089 \\
.995070 \\
.995051 \\
.995032 \\
.995013\end{array}$ & $\begin{array}{l}.32 \\
.32 \\
.32 \\
.32 \\
.32 \\
.32 \\
.32 \\
.32 \\
.32 \\
.33\end{array}$ & $\begin{array}{r}9.175362 \\
.176224 \\
.177084 \\
.177942 \\
.178799 \\
.179655 \\
.180508 \\
.181360 \\
.182211 \\
.183059\end{array}$ & $\begin{array}{l}14.37 \\
14.33 \\
14.30 \\
14.28 \\
14.27 \\
14.22 \\
14.20 \\
14.18 \\
14.13 \\
14.13\end{array}$ & $\begin{array}{r}10.824638 \\
.823776 \\
.822916 \\
.822058 \\
.821201 \\
.820345 \\
.819492 \\
.818640 \\
.817789 \\
.816941\end{array}$ & $\begin{array}{l}29 \\
28 \\
27 \\
26 \\
25 \\
24 \\
23 \\
22 \\
21 \\
20\end{array}$ \\
\hline $\begin{array}{l}41 \\
42 \\
43 \\
44 \\
4.5 \\
46 \\
47 \\
48 \\
49 \\
50\end{array}$ & $\begin{array}{r}9.178900 \\
.179726 \\
.180551 \\
.181374 \\
.182196 \\
.183016 \\
.183834 \\
.184651 \\
.185466 \\
.186280\end{array}$ & $\begin{array}{l}13.77 \\
13.75 \\
13.72 \\
13.70 \\
13.67 \\
13.63 \\
13.62 \\
13.58 \\
13.57 \\
13.53\end{array}$ & $\begin{array}{r}9.994993 \\
.994974 \\
.994955 \\
.994935 \\
.994916 \\
.994896 \\
.994877 \\
.994857 \\
.994538 \\
.994518\end{array}$ & $\begin{array}{l}.32 \\
.32 \\
.33 \\
.32 \\
.33 \\
.32 \\
.33 \\
.32 \\
.33 \\
.33\end{array}$ & $\begin{array}{r}9.183907 \\
.184752 \\
.185597 \\
.186439 \\
.187280 \\
.188120 \\
.188958 \\
.189794 \\
.190629 \\
.191462\end{array}$ & $\begin{array}{l}14.08 \\
14.08 \\
14.03 \\
14.02 \\
14.00 \\
13.97 \\
13.93 \\
13.92 \\
13.88 \\
13.87\end{array}$ & $\begin{array}{r}10.816093 \\
.815248 \\
.814403 \\
.813561 \\
.812720 \\
.811850 \\
.811042 \\
.810206 \\
.809371 \\
.808538\end{array}$ & $\begin{array}{l}19 \\
18 \\
17 \\
16 \\
15 \\
14 \\
13 \\
12 \\
11 \\
10\end{array}$ \\
\hline $\begin{array}{l}51 \\
52 \\
53 \\
54 \\
55 \\
56 \\
57 \\
58 \\
59 \\
60\end{array}$ & $\begin{array}{r}9.187092 \\
.187903 \\
.183712 \\
.189519 \\
.190325 \\
.191130 \\
.191933 \\
.192734 \\
.193534 \\
9.194332\end{array}$ & $\begin{array}{l}13.52 \\
13.48 \\
13.45 \\
13.43 \\
13.42 \\
13.38 \\
13.35 \\
13.33 \\
13.30\end{array}$ & $\begin{array}{r}9.994798 \\
.994779 \\
.994759 \\
.994739 \\
.994720 \\
.994700 \\
.994680 \\
.994600 \\
.994640 \\
9.994620\end{array}$ & $\begin{array}{r}.32 \\
.33 \\
.33 \\
.32 \\
.33 \\
.33 \\
.33 \\
.33 \\
.33\end{array}$ & $\begin{array}{r}9.192294 \\
.193124 \\
.193953 \\
.194780 \\
.195606 \\
.196430 \\
.197253 \\
.193074 \\
.198594 \\
9.199713\end{array}$ & $\begin{array}{l}13.83 \\
13.82 \\
13.78 \\
13.77 \\
13.73 \\
13.72 \\
13.69 \\
13.67 \\
13.65\end{array}$ & $\begin{array}{r}10.807706 \\
.800876 \\
.840047 \\
.845220 \\
.804394 \\
.80 .5570 \\
.802747 \\
.801926 \\
.801106 \\
10.800257\end{array}$ & $\begin{array}{l}9 \\
8 \\
7 \\
6 \\
5 \\
4 \\
3 \\
2 \\
1 \\
0\end{array}$ \\
\hline - & Cosine. & D. $1^{\prime \prime}$. & Sine. & D. $1^{\prime \prime}$. & Cotang. & D. $1^{\prime \prime}$. & Tang. & ? \\
\hline
\end{tabular}


TABLE 9. LOGARITHMiC SINES, COSINES,

\begin{tabular}{|c|c|c|c|c|c|c|c|c|}
\hline , & Sine. & D. $1^{\prime \prime}$. & Cosine. & D. 1". & Tang. & D. $1^{\prime \prime}$. & Cotang. & ' \\
\hline $\begin{array}{r}0 \\
1 \\
2 \\
3 \\
4 \\
5 \\
6 \\
7 \\
8 \\
9 \\
10\end{array}$ & $\begin{array}{r}9.194332 \\
.195129 \\
.195925 \\
.196719 \\
.197511 \\
.195302 \\
.199091 \\
.199879 \\
.200666 \\
.201451 \\
.202234\end{array}$ & $\begin{array}{l}13.28 \\
13.27 \\
13.23 \\
13.20 \\
13.18 \\
13.15 \\
13.13 \\
13.12 \\
13.08 \\
13.05 \\
13.05\end{array}$ & $\begin{array}{r}9.994620 \\
.994600 \\
.994580 \\
.994560 \\
.994540 \\
.994519 \\
.994499 \\
.994479 \\
.994459 \\
.994438 \\
.994418\end{array}$ & $\begin{array}{l}.33 \\
.33 \\
.33 \\
.33 \\
.35 \\
.33 \\
.33 \\
.33 \\
.35 \\
.33 \\
.33\end{array}$ & $\begin{array}{r}9.199713 \\
.200529 \\
.201345 \\
.202159 \\
.202971 \\
.203782 \\
.204592 \\
.205400 \\
.206207 \\
.207013 \\
.207817\end{array}$ & $\begin{array}{l}13.60 \\
13.60 \\
13.57 \\
13.53 \\
13.52 \\
13.50 \\
13.47 \\
13.45 \\
13.43 \\
13.40 \\
13.37\end{array}$ & $\begin{array}{r}10.800287 \\
.799471 \\
.798655 \\
.797841 \\
.797029 \\
.796218 \\
.795408 \\
.794600 \\
.793793 \\
.792987 \\
.792183\end{array}$ & $\begin{array}{l}60 \\
59 \\
58 \\
57 \\
56 \\
55 \\
54 \\
53 \\
52 \\
51 \\
50\end{array}$ \\
\hline $\begin{array}{l}11 \\
12 \\
13 \\
14 \\
15 \\
16 \\
17 \\
18 \\
19 \\
20\end{array}$ & $\begin{array}{r}9.203017 \\
.203797 \\
.204577 \\
.205354 \\
.206131 \\
.201906 \\
.207679 \\
.208452 \\
.209222 \\
.209992\end{array}$ & $\begin{array}{l}13.00 \\
13.00 \\
12.95 \\
12.95 \\
12.92 \\
12.88 \\
12.88 \\
12.83 \\
12.83 \\
12.80\end{array}$ & $\begin{array}{r}9.994398 \\
.994377 \\
.994357 \\
.994336 \\
.994316 \\
.994295 \\
.994274 \\
.994254 \\
.994233 \\
.994212\end{array}$ & $\begin{array}{l}.35 \\
.33 \\
.35 \\
.33 \\
.35 \\
.35 \\
.33 \\
.35 \\
.35 \\
.35\end{array}$ & $\begin{array}{r}9.208619 \\
.209420 \\
.210220 \\
.211018 \\
.211815 \\
.212611 \\
.213405 \\
.214198 \\
.214989 \\
.215780\end{array}$ & $\begin{array}{l}13.35 \\
13.33 \\
13.30 \\
13.28 \\
13.27 \\
13.23 \\
13.22 \\
13.18 \\
13.18 \\
13.13\end{array}$ & $\begin{array}{r}10.791381 \\
.790580 \\
.789780 \\
.785982 \\
.788185 \\
.787389 \\
.786595 \\
.785802 \\
.785011 \\
.784220\end{array}$ & $\begin{array}{l}49 \\
48 \\
47 \\
46 \\
45 \\
44 \\
43 \\
42 \\
41 \\
40\end{array}$ \\
\hline $\begin{array}{l}21 \\
22 \\
23 \\
24 \\
25 \\
26 \\
27 \\
28 \\
29 \\
30\end{array}$ & $\begin{array}{r}9.210760 \\
.211526 \\
.212291 \\
.213055 \\
.213818 \\
.214579 \\
.215338 \\
.216097 \\
.216854 \\
.217609\end{array}$ & $\begin{array}{l}12.77 \\
12.75 \\
12.73 \\
12.72 \\
12.68 \\
12.65 \\
12.65 \\
12.62 \\
12.58 \\
12.57\end{array}$ & $\begin{array}{r}9.994191 \\
.994171 \\
.994150 \\
.994129 \\
.994108 \\
.994087 \\
.994066 \\
.994045 \\
.994024 \\
.994003\end{array}$ & $\begin{array}{l}.33 \\
.35 \\
.35 \\
.35 \\
.35 \\
.35 \\
.35 \\
.35 \\
.35 \\
.35\end{array}$ & $\begin{array}{r}9.216568 \\
.217356 \\
.218142 \\
.218926 \\
.219710 \\
.220492 \\
.221272 \\
.222052 \\
.222830 \\
.223607\end{array}$ & $\begin{array}{l}13.13 \\
13.10 \\
13.07 \\
13.07 \\
13.03 \\
13.00 \\
13.00 \\
12.97 \\
12.95 \\
12.92\end{array}$ & $\begin{array}{r}10.783432 \\
.782644 \\
.781858 \\
.781074 \\
.780290 \\
.779508 \\
.778728 \\
.777948 \\
.777170 \\
.776393\end{array}$ & $\begin{array}{l}39 \\
38 \\
37 \\
36 \\
35 \\
34 \\
33 \\
32 \\
31 \\
30\end{array}$ \\
\hline $\begin{array}{l}31 \\
32 \\
33 \\
34 \\
35 \\
36 \\
37 \\
38 \\
39 \\
40\end{array}$ & $\begin{array}{r}9.218363 \\
.219116 \\
.219868 \\
.220618 \\
.221367 \\
.222115 \\
.222861 \\
.223606 \\
.224349 \\
.225092\end{array}$ & $\begin{array}{l}12.55 \\
12.53 \\
12.50 \\
12.48 \\
12.47 \\
12.43 \\
12.42 \\
12.38 \\
12.38 \\
12.35\end{array}$ & $\begin{array}{r}9.993982 \\
.993960 \\
.993939 \\
.993918 \\
.993897 \\
.993875 \\
.993854 \\
.993832 \\
.993811 \\
.993789\end{array}$ & $\begin{array}{l}.37 \\
.35 \\
.35 \\
.35 \\
.37 \\
.35 \\
.37 \\
.35 \\
.37 \\
.35\end{array}$ & $\begin{array}{r}9.224382 \\
.225156 \\
.225929 \\
.226700 \\
.227471 \\
.228239 \\
.229007 \\
.229773 \\
.230539 \\
.231302\end{array}$ & $\begin{array}{l}12.90 \\
12.88 \\
12.85 \\
12.85 \\
12.80 \\
12.80 \\
12.77 \\
12.77 \\
12.72 \\
12.72\end{array}$ & $\begin{array}{r}10.775618 \\
.774844 \\
.774071 \\
.773300 \\
.772529 \\
.771761 \\
.770993 \\
.770227 \\
.769461 \\
.765698\end{array}$ & $\begin{array}{l}29 \\
28 \\
27 \\
26 \\
25 \\
24 \\
23 \\
22 \\
21 \\
20\end{array}$ \\
\hline $\begin{array}{l}41 \\
42 \\
43 \\
44 \\
45 \\
46 \\
47 \\
48 \\
49 \\
50\end{array}$ & $\begin{array}{r}0.225833 \\
.226573 \\
.227311 \\
.228048 \\
.228784 \\
.229518 \\
.230252 \\
.230984 \\
.231715 \\
.232444\end{array}$ & $\begin{array}{l}12.33 \\
12.30 \\
12.28 \\
12.27 \\
12.23 \\
12.23 \\
12.20 \\
12.18 \\
12.15 \\
12.13\end{array}$ & $\begin{array}{r}9.993768 \\
.993746 \\
.993725 \\
.993703 \\
.993681 \\
.993660 \\
.993638 \\
.993616 \\
.993594 \\
.993572\end{array}$ & $\begin{array}{l}.37 \\
.35 \\
.37 \\
.37 \\
.35 \\
.37 \\
.37 \\
.37 \\
.37 \\
.37\end{array}$ & $\begin{array}{r}9.232065 \\
.232826 \\
.233586 \\
.234345 \\
.235103 \\
.235859 \\
.236614 \\
.237368 \\
.238120 \\
.238872\end{array}$ & $\begin{array}{l}12.68 \\
12.67 \\
12.65 \\
12.63 \\
12.60 \\
12.58 \\
12.57 \\
12.53 \\
12.53 \\
12.50\end{array}$ & $\begin{array}{r}10.767935 \\
.767174 \\
.766414 \\
.765655 \\
.764897 \\
.764141 \\
.763386 \\
.762632 \\
.761880 \\
.761128\end{array}$ & $\begin{array}{l}19 \\
18 \\
17 \\
16 \\
15 \\
14 \\
13 \\
12 \\
11 \\
10\end{array}$ \\
\hline $\begin{array}{l}51 \\
52 \\
53 \\
54 \\
55 \\
56 \\
57 \\
58 \\
59 \\
60\end{array}$ & $\begin{array}{r}9.233172 \\
.233899 \\
.234625 \\
.235349 \\
.236073 \\
.236795 \\
.237515 \\
.238235 \\
.238953 \\
9.239670\end{array}$ & $\begin{array}{l}12.12 \\
12.10 \\
12.07 \\
12.07 \\
12.03 \\
12.00 \\
12.00 \\
11.97 \\
11.95\end{array}$ & $\begin{array}{r}9.993550 \\
.993528 \\
.993506 \\
.993484 \\
.993462 \\
.993440 \\
.993418 \\
.993396 \\
.993374 \\
9.993351\end{array}$ & $\begin{array}{l}.37 \\
.37 \\
.37 \\
.37 \\
.37 \\
.37 \\
.37 \\
.37 \\
.38\end{array}$ & $\begin{array}{r}9.239622 \\
.240371 \\
.241118 \\
.241865 \\
.242610 \\
.243354 \\
.244097 \\
.244839 \\
.245579 \\
9.24 t 319\end{array}$ & $\begin{array}{l}12.48 \\
12.45 \\
12.45 \\
12.42 \\
12.40 \\
12.38 \\
12.37 \\
12.33 \\
12.33\end{array}$ & $\begin{array}{r}10.760378 \\
.759629 \\
.758882 \\
.758135 \\
.757390 \\
.756646 \\
.755903 \\
.755161 \\
.754421 \\
10.753681\end{array}$ & $\begin{array}{l}9 \\
8 \\
7 \\
6 \\
5 \\
4 \\
3 \\
2 \\
1 \\
0\end{array}$ \\
\hline ' & Cosine. & D. $1^{\prime \prime}$. & Sine. & D. $1^{\prime \prime}$. & Cotang. & D. $1^{\prime \prime}$. & Tang. & ' \\
\hline
\end{tabular}


TANGENTS, AND CoTANaENTS.

\begin{tabular}{|c|c|c|c|c|c|c|c|c|}
\hline , & Slne. & D. 1". & Cosine. & D. 1". & Tang. & D. 1". & Cotang. & , \\
\hline $\begin{array}{r}0 \\
1 \\
2 \\
3 \\
4 \\
5 \\
6 \\
7 \\
8 \\
9 \\
10\end{array}$ & $\begin{array}{r}9.239070 \\
.240386 \\
.241101 \\
.241814 \\
.242526 \\
.243237 \\
.243947 \\
.244656 \\
.245363 \\
.246069 \\
.246775\end{array}$ & $\begin{array}{l}11.93 \\
11.92 \\
11.83 \\
11.87 \\
11.85 \\
11.83 \\
11.82 \\
11.73 \\
11.77 \\
11.77 \\
11.72\end{array}$ & $\begin{array}{r}9.993351 \\
.993329 \\
.993307 \\
.993284 \\
.993262 \\
.993240 \\
.993217 \\
.993195 \\
.993172 \\
.993149 \\
.993127\end{array}$ & $\begin{array}{l}37 \\
.37 \\
.38 \\
.37 \\
.37 \\
.38 \\
.37 \\
.38 \\
.38 \\
.37 \\
.38 \\
.38\end{array}$ & $\begin{array}{r}9.246319 \\
.247057 \\
.247794 \\
.248530 \\
.249264 \\
.249998 \\
.250730 \\
.251461 \\
.252191 \\
.252920 \\
.253648\end{array}$ & $\begin{array}{l}12.30 \\
12.23 \\
12.27 \\
12.23 \\
12.23 \\
12.20 \\
12.18 \\
12.17 \\
12.15 \\
12.13 \\
12.10\end{array}$ & $\begin{array}{r}10.753681 \\
.752943 \\
.752206 \\
.751470 \\
.750736 \\
.750002 \\
.749270 \\
.748539 \\
.747809 \\
.747080 \\
.746352\end{array}$ & $\begin{array}{l}60 \\
59 \\
58 \\
57 \\
56 \\
55 \\
54 \\
53 \\
52 \\
51 \\
50\end{array}$ \\
\hline $\begin{array}{l}11 \\
12 \\
13 \\
14 \\
15 \\
16 \\
17 \\
18 \\
19 \\
20\end{array}$ & $\begin{array}{r}9.247478 \\
.248181 \\
.248583 \\
.249583 \\
.250282 \\
.250950 \\
.251677 \\
.252373 \\
.253067 \\
.253761\end{array}$ & $\begin{array}{l}11.72 \\
11.70 \\
11.67 \\
11.65 \\
11.63 \\
11.62 \\
11.60 \\
11.57 \\
11.57 \\
11.53\end{array}$ & $\begin{array}{r}9.993104 \\
.993081 \\
.993059 \\
.993036 \\
.993013 \\
.992990 \\
.992967 \\
.992944 \\
.922921 \\
.992598\end{array}$ & $\begin{array}{l}.38 \\
.38 \\
.37 \\
.38 \\
.38 \\
.38 \\
.38 \\
.38 \\
.38 \\
.38 \\
.38\end{array}$ & $\begin{array}{r}9.254374 \\
.255100 \\
.2558^{4} 4 \\
.256547 \\
.257269 \\
.257990 \\
.258710 \\
.259429 \\
.260146 \\
.260863\end{array}$ & $\begin{array}{l}12.10 \\
12.07 \\
12.05 \\
12.03 \\
12.02 \\
12.00 \\
11.98 \\
11.95 \\
11.95 \\
11.92\end{array}$ & $\begin{array}{r}10.745626 \\
.744900 \\
.744176 \\
.743453 \\
.742731 \\
.742010 \\
.741290 \\
.740571 \\
.739854 \\
.739137\end{array}$ & $\begin{array}{l}49 \\
48 \\
47 \\
46 \\
45 \\
44 \\
43 \\
42 \\
41 \\
40\end{array}$ \\
\hline $\begin{array}{l}21 \\
22 \\
23 \\
24 \\
25 \\
26 \\
27 \\
28 \\
29 \\
30\end{array}$ & $\begin{array}{r}9.254453 \\
.255144 \\
.255834 \\
.256523 \\
.257211 \\
257898 \\
.258583 \\
.259263 \\
.259951 \\
.260633\end{array}$ & $\begin{array}{l}11.52 \\
11.50 \\
11.48 \\
11.47 \\
11.45 \\
11.42 \\
11.42 \\
11.38 \\
11.37 \\
11.35\end{array}$ & $\begin{array}{r}9.992575 \\
.992852 \\
.992829 \\
.992806 \\
.992783 \\
.992759 \\
.992736 \\
.992713 \\
.992690 \\
.992666\end{array}$ & $\begin{array}{l}.38 \\
.38 \\
.38 \\
.38 \\
.40 \\
.38 \\
.38 \\
.38 \\
.40 \\
.38\end{array}$ & $\begin{array}{r}9.261578 \\
.262292 \\
.263005 \\
.263717 \\
.264428 \\
.265138 \\
.265847 \\
.266555 \\
.267261 \\
.267967\end{array}$ & $\begin{array}{l}11.90 \\
11.88 \\
11.87 \\
11.85 \\
11.83 \\
11.82 \\
11.80 \\
11.77 \\
11.77 \\
11.73\end{array}$ & $\begin{array}{r}10.738422 \\
.737708 \\
.736995 \\
.736283 \\
.735572 \\
.734862 \\
.734153 \\
.733445 \\
.732739 \\
.732033\end{array}$ & $\begin{array}{l}39 \\
38 \\
37 \\
36 \\
35 \\
34 \\
33 \\
32 \\
31 \\
30\end{array}$ \\
\hline $\begin{array}{l}31 \\
32 \\
33 \\
34 \\
35 \\
36 \\
37 \\
38 \\
39 \\
40\end{array}$ & $\begin{array}{r}9.261314 \\
.261994 \\
.262673 \\
.263351 \\
.264027 \\
.264703 \\
.265377 \\
.266051 \\
.266723 \\
.267325\end{array}$ & $\begin{array}{l}11.33 \\
11.32 \\
11.30 \\
11.27 \\
11.27 \\
11.23 \\
11.23 \\
11.20 \\
11.20 \\
11.17\end{array}$ & $\begin{array}{r}9.992643 \\
.992619 \\
.992596 \\
.992572 \\
.992549 \\
.992525 \\
.992501 \\
.992478 \\
.992454 \\
.992430\end{array}$ & $\begin{array}{l}.40 \\
.40 \\
.38 \\
.40 \\
.38 \\
.40 \\
.40 \\
.38 \\
.40 \\
.40 \\
.40\end{array}$ & $\begin{array}{r}9.268671 \\
.269375 \\
.270077 \\
.270779 \\
.271479 \\
.272178 \\
.272876 \\
.273573 \\
.274269 \\
.274964\end{array}$ & $\begin{array}{l}11.73 \\
11.70 \\
11.70 \\
11.67 \\
11.65 \\
11.63 \\
11.62 \\
11.60 \\
11.58 \\
11.57\end{array}$ & $\begin{array}{r}10.731329 \\
.730625 \\
.729923 \\
.729221 \\
.728521 \\
.727822 \\
.727124 \\
.726427 \\
.725731 \\
.725036\end{array}$ & $\begin{array}{l}29 \\
28 \\
27 \\
26 \\
25 \\
24 \\
23 \\
22 \\
21 \\
20\end{array}$ \\
\hline $\begin{array}{l}41 \\
42 \\
43 \\
44 \\
45 \\
46 \\
47 \\
48 \\
49 \\
50\end{array}$ & $\begin{array}{r}9268065 \\
.268734 \\
.269402 \\
270069 \\
.270735 \\
.271400 \\
.272064 \\
.272726 \\
.273388 \\
.274049\end{array}$ & $\begin{array}{l}11.15 \\
11.13 \\
11.12 \\
11.10 \\
11.08 \\
11.07 \\
11.03 \\
11.03 \\
11.02 \\
10.98\end{array}$ & $\begin{array}{r}9.992406 \\
.992382 \\
.992359 \\
.992335 \\
.902311 \\
.992287 \\
.922263 \\
.992239 \\
.992214 \\
.992190\end{array}$ & $\begin{array}{l}.40 \\
.38 \\
.40 \\
.40 \\
.40 \\
.40 \\
.40 \\
.42 \\
.40 \\
.40\end{array}$ & $\begin{array}{r}9.275655 \\
.276351 \\
.277043 \\
.277734 \\
.278424 \\
.279113 \\
.279501 \\
.280488 \\
.281174 \\
.281858\end{array}$ & $\begin{array}{l}11.55 \\
11.53 \\
11.52 \\
11.50 \\
11.44 \\
11.47 \\
11.45 \\
11.43 \\
11.40 \\
11.40\end{array}$ & $\begin{array}{r}10.724342 \\
.723649 \\
.722957 \\
.722266 \\
.721576 \\
.720687 \\
.720199 \\
.719512 \\
.715526 \\
.718142\end{array}$ & $\begin{array}{l}19 \\
18 \\
17 \\
16 \\
15 \\
14 \\
13 \\
12 \\
11 \\
10\end{array}$ \\
\hline $\begin{array}{l}51 \\
52 \\
53 \\
54 \\
55 \\
56 \\
57 \\
58 \\
59 \\
60\end{array}$ & $\begin{array}{r}9.274705 \\
.275367 \\
.276025 \\
.276051 \\
.277337 \\
.277991 \\
.275645 \\
.279297 \\
.279948 \\
9.230599\end{array}$ & $\begin{array}{l}10.95 \\
10.97 \\
10.93 \\
10.93 \\
10.90 \\
10.90 \\
10.87 \\
10.85 \\
10.85\end{array}$ & $\begin{array}{r}9.992166 \\
.992142 \\
.992118 \\
.992093 \\
.992069 \\
.992044 \\
.992020 \\
.991996 \\
.991971 \\
9.901947\end{array}$ & $\begin{array}{l}.40 \\
.40 \\
.42 \\
.40 \\
.42 \\
.40 \\
.40 \\
.42 \\
.40\end{array}$ & $\begin{array}{r}9.252542 \\
.233225 \\
.283907 \\
.284589 \\
.255268 \\
.255947 \\
.256624 \\
.287301 \\
.257977 \\
9.258652\end{array}$ & $\begin{array}{l}11.38 \\
11.37 \\
11.35 \\
11.33 \\
11.32 \\
11.28 \\
11.28 \\
11.27 \\
11.25\end{array}$ & $\begin{array}{r}10.717458 \\
.716775 \\
.716093 \\
.715412 \\
.714732 \\
.714053 \\
.713376 \\
.712699 \\
.712023 \\
10.711348\end{array}$ & $\begin{array}{l}9 \\
8 \\
7 \\
6 \\
5 \\
4 \\
3 \\
2 \\
1 \\
0\end{array}$ \\
\hline ' & Cosine. & D. $1^{\prime \prime}$. & Sine. & D. 1". & Cotang. & D. 1" & Tang. & 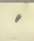 \\
\hline
\end{tabular}




\begin{tabular}{|c|c|c|c|c|c|c|c|c|}
\hline , & Sine. & D. $1^{\prime \prime}$. & Cosine. & D. $1^{\prime \prime}$. & Tang. & D. $1^{\prime \prime}$, & Cotang. & ' \\
\hline $\begin{array}{r}0 \\
1 \\
2 \\
3 \\
4 \\
5 \\
6 \\
7 \\
8 \\
9 \\
10\end{array}$ & $\begin{array}{r}9.280599 \\
.281248 \\
.281897 \\
.282544 \\
.283190 \\
.283836 \\
.284480 \\
.285124 \\
.285766 \\
.286408 \\
.287048\end{array}$ & $\begin{array}{l}10.82 \\
10.82 \\
10.78 \\
10.77 \\
10.77 \\
10.73 \\
10.73 \\
10.70 \\
10.70 \\
10.67 \\
10.67\end{array}$ & $\begin{array}{r}9.991947 \\
.991922 \\
.991897 \\
.991873 \\
.991848 \\
.991823 \\
.991799 \\
.991774 \\
.991749 \\
.991724 \\
.991699\end{array}$ & $\begin{array}{l}.42 \\
.42 \\
.40 \\
.42 \\
.42 \\
.40 \\
.42 \\
.42 \\
.42 \\
.42 \\
.42\end{array}$ & $\begin{array}{r}9.288652 \\
.289326 \\
.289999 \\
.290671 \\
.291342 \\
.292013 \\
.292652 \\
.293350 \\
.294017 \\
.294684 \\
.295349\end{array}$ & $\begin{array}{l}11.23 \\
11.22 \\
11.20 \\
11.18 \\
11.18 \\
11.15 \\
11.13 \\
11.12 \\
11.12 \\
11.08 \\
11.07\end{array}$ & $\begin{array}{r}10.711348 \\
.710674 \\
.710001 \\
.709329 \\
.708658 \\
.707987 \\
.707318 \\
.706650 \\
.705983 \\
.705316 \\
.704651\end{array}$ & $\begin{array}{l}60 \\
59 \\
58 \\
57 \\
56 \\
55 \\
54 \\
53 \\
52 \\
51 \\
50\end{array}$ \\
\hline $\begin{array}{l}11 \\
12 \\
13 \\
14 \\
15 \\
16 \\
17 \\
18 \\
19 \\
20\end{array}$ & $\begin{array}{r}9.287688 \\
.288326 \\
.288964 \\
.289600 \\
.290236 \\
.290870 \\
.291504 \\
.292137 \\
.292768 \\
.293399\end{array}$ & $\begin{array}{l}10.63 \\
10.63 \\
10.60 \\
10.60 \\
10.57 \\
10.57 \\
10.55 \\
10.52 \\
10.52 \\
10.50\end{array}$ & $\begin{array}{r}9.991674 \\
.991649 \\
.991624 \\
.991599 \\
.991574 \\
.991549 \\
.991524 \\
.991498 \\
.991473 \\
.991448\end{array}$ & $\begin{array}{l}.42 \\
.42 \\
.42 \\
.42 \\
.42 \\
.42 \\
.43 \\
.42 \\
.42 \\
.43\end{array}$ & $\begin{array}{r}9.296013 \\
.296677 \\
.297339 \\
.298001 \\
.298662 \\
.299322 \\
.299980 \\
.300638 \\
.301295 \\
.301951\end{array}$ & $\begin{array}{l}11.07 \\
11.03 \\
11.03 \\
11.02 \\
11.00 \\
10.97 \\
10.97 \\
10.95 \\
10.93 \\
10.93\end{array}$ & $\begin{array}{r}10.703987 \\
.703323 \\
.702661 \\
.701999 \\
.701338 \\
.700678 \\
.700020 \\
.699362 \\
.695705 \\
.698049\end{array}$ & $\begin{array}{l}49 \\
48 \\
47 \\
46 \\
45 \\
44 \\
43 \\
42 \\
41 \\
40\end{array}$ \\
\hline $\begin{array}{l}21 \\
22 \\
23 \\
24 \\
25 \\
26 \\
27 \\
28 \\
29 \\
30\end{array}$ & $\begin{array}{r}9.294029 \\
.294658 \\
.295286 \\
.295913 \\
.296539 \\
.297164 \\
.297788 \\
.298412 \\
.299034 \\
.299655\end{array}$ & $\begin{array}{l}10.48 \\
10.47 \\
10.45 \\
10.43 \\
10.42 \\
10.40 \\
10.40 \\
10.37 \\
10.35 \\
10.35\end{array}$ & $\begin{array}{r}9.991422 \\
.991397 \\
.991372 \\
.991346 \\
.991321 \\
.991295 \\
.991270 \\
.991244 \\
.991218 \\
.991193\end{array}$ & $\begin{array}{l}.42 \\
.42 \\
.43 \\
.42 \\
.43 \\
.42 \\
.43 \\
.43 \\
.42 \\
.43\end{array}$ & $\begin{array}{r}9.302607 \\
.303261 \\
.303914 \\
.304567 \\
.305218 \\
.305869 \\
.306519 \\
.307168 \\
.307816 \\
.308463\end{array}$ & $\begin{array}{l}10.90 \\
10.88 \\
10.88 \\
10.85 \\
10.85 \\
10.83 \\
10.82 \\
10.80 \\
10.78 \\
10.77\end{array}$ & $\begin{array}{r}10.697393 \\
.696739 \\
.696086 \\
.695433 \\
.694782 \\
.694131 \\
.693481 \\
.692832 \\
.692184 \\
.691537\end{array}$ & $\begin{array}{l}39 \\
38 \\
37 \\
36 \\
35 \\
34 \\
33 \\
32 \\
31 \\
30\end{array}$ \\
\hline $\begin{array}{l}31 \\
32 \\
33 \\
34 \\
35 \\
36 \\
37 \\
38 \\
39 \\
40\end{array}$ & $\begin{array}{r}9.300276 \\
.300895 \\
.301514 \\
.302132 \\
.302748 \\
.303364 \\
.303979 \\
.304593 \\
.305207 \\
.305819\end{array}$ & \begin{tabular}{l|}
10.32 \\
10.32 \\
10.30 \\
10.27 \\
10.27 \\
10.25 \\
10.23 \\
10.23 \\
10.20 \\
10.18
\end{tabular} & $\begin{array}{r}9.991167 \\
.991141 \\
.991115 \\
.991090 \\
.991064 \\
.991038 \\
.991012 \\
.990986 \\
.990960 \\
.990934\end{array}$ & $\begin{array}{l}.43 \\
.43 \\
.42 \\
.43 \\
.43 \\
.43 \\
.43 \\
.43 \\
.43 \\
.43\end{array}$ & $\begin{array}{r}9.309109 \\
.309754 \\
.310399 \\
.311042 \\
.311685 \\
.312327 \\
.312968 \\
.313608 \\
.314247 \\
.314885\end{array}$ & $\begin{array}{l}10.75 \\
10.75 \\
10.72 \\
10.72 \\
10.70 \\
10.68 \\
10.67 \\
10.65 \\
10.63 \\
10.63\end{array}$ & $\begin{array}{r}10.690891 \\
.690246 \\
.689601 \\
.685958 \\
.688315 \\
.687673 \\
.687032 \\
.686392 \\
.685753 \\
.685115\end{array}$ & $\begin{array}{l}29 \\
28 \\
27 \\
26 \\
25 \\
24 \\
23 \\
22 \\
21 \\
20\end{array}$ \\
\hline $\begin{array}{l}41 \\
42 \\
43 \\
44 \\
45 \\
46 \\
47 \\
48 \\
49 \\
50\end{array}$ & $\begin{array}{r}9.306430 \\
.307041 \\
.307650 \\
.305259 \\
.305867 \\
.309474 \\
.310 n 50 \\
.310655 \\
.311289 \\
.311893\end{array}$ & $\begin{array}{l}10.18 \\
10.15 \\
10.15 \\
10.13 \\
10.12 \\
10.10 \\
10.08 \\
10.07 \\
10.07 \\
10.03\end{array}$ & $\begin{array}{r}9.990908 \\
.990882 \\
.990855 \\
.990829 \\
.990803 \\
.990777 \\
.990750 \\
.990724 \\
.990697 \\
.990671\end{array}$ & $\begin{array}{l}.43 \\
.45 \\
.43 \\
.43 \\
.43 \\
.45 \\
.43 \\
.45 \\
.43 \\
.43\end{array}$ & $\begin{array}{r}9.315523 \\
.316159 \\
.316795 \\
.317430 \\
.318064 \\
.318697 \\
.319330 \\
.319961 \\
.320592 \\
.321222\end{array}$ & $\begin{array}{l}10.60 \\
10.60 \\
10.58 \\
10.57 \\
10.55 \\
10.55 \\
10.52 \\
10.52 \\
10.50 \\
10.48\end{array}$ & $\begin{array}{r}10.684477 \\
.683841 \\
.683205 \\
.682570 \\
.681936 \\
.681303 \\
.680670 \\
.680039 \\
.679408 \\
.678778\end{array}$ & $\begin{array}{l}19 \\
18 \\
17 \\
16 \\
15 \\
14 \\
13 \\
12 \\
11 \\
10\end{array}$ \\
\hline $\begin{array}{l}51 \\
52 \\
53 \\
54 \\
55 \\
56 \\
57 \\
58 \\
59 \\
60\end{array}$ & $\begin{array}{r}9.312495 \\
.313097 \\
.313698 \\
.314297 \\
.314897 \\
.315495 \\
.316092 \\
.316659 \\
.317284 \\
9.317879\end{array}$ & $\begin{array}{r}10.03 \\
10.02 \\
9.98 \\
10.00 \\
9.97 \\
9.95 \\
9.95 \\
9.92 \\
9.92\end{array}$ & $\begin{array}{r}9.990645 \\
.990618 \\
.990591 \\
.990565 \\
.990538 \\
.990511 \\
.990485 \\
.990458 \\
.990131 \\
9.990404\end{array}$ & $\begin{array}{l}.45 \\
.45 \\
.43 \\
.45 \\
.45 \\
.43 \\
.45 \\
.45 \\
.45\end{array}$ & $\begin{array}{r}9.321851 \\
.322479 \\
.323106 \\
.323733 \\
.324358 \\
.324983 \\
.325607 \\
.326231 \\
.326853 \\
9.327475\end{array}$ & $\begin{array}{l}10.47 \\
10.45 \\
10.45 \\
10.42 \\
10.42 \\
10.40 \\
10.40 \\
10.37 \\
10.37\end{array}$ & $\begin{array}{r}10.678149 \\
.677521 \\
.676894 \\
.676267 \\
.675642 \\
.675017 \\
.674393 \\
.673769 \\
.673147 \\
10.672525\end{array}$ & $\begin{array}{l}9 \\
8 \\
7 \\
6 \\
5 \\
4 \\
3 \\
2 \\
1 \\
0\end{array}$ \\
\hline ' & Cosine. & D. $1^{\prime \prime}$. & Sine. & D. $1^{\prime \prime}$. & Cotang. & D. $1^{\prime \prime}$. & Tang. & ' \\
\hline
\end{tabular}


TANGENTS, AND COTANGENTS.

\begin{tabular}{|c|c|c|c|c|c|c|c|c|}
\hline , & Sine. & D. $1^{\prime \prime}$. & Cosine. & D. $1^{\prime \prime}$. & Tang. & D. $1^{\prime \prime}$. & Cotang. & , \\
\hline $\begin{array}{r}0 \\
1 \\
2 \\
3 \\
4 \\
5 \\
6 \\
7 \\
8 \\
9 \\
10\end{array}$ & $\begin{array}{r}9.317879 \\
.318473 \\
.319046 \\
.319658 \\
.320249 \\
.320840 \\
.321430 \\
.322019 \\
.322607 \\
.323194 \\
.323780\end{array}$ & $\begin{array}{l}9.90 \\
9.88 \\
9.87 \\
9.85 \\
9.85 \\
9.83 \\
9.82 \\
9.80 \\
9.78 \\
9.77 \\
9.77\end{array}$ & $\begin{array}{r}9.990404 \\
.990378 \\
.990351 \\
.990324 \\
.990297 \\
.990270 \\
.990243 \\
.990215 \\
.990188 \\
.990161 \\
.990134\end{array}$ & $\begin{array}{l}.43 \\
.45 \\
.45 \\
.45 \\
.45 \\
.45 \\
.47 \\
.45 \\
.45 \\
.45 \\
.45\end{array}$ & $\begin{array}{r}9.327475 \\
.328095 \\
.328715 \\
.329334 \\
.329953 \\
.330570 \\
.331187 \\
.331803 \\
.332418 \\
.333033 \\
.333646\end{array}$ & $\begin{array}{l}10.33 \\
10.33 \\
10.32 \\
10.32 \\
10.28 \\
10.28 \\
10.27 \\
10.25 \\
10.25 \\
10.22 \\
10.22\end{array}$ & $\begin{array}{r}10.672525 \\
.671905 \\
.671285 \\
.670666 \\
.670047 \\
.669430 \\
.665813 \\
.668197 \\
.667582 \\
.666967 \\
.666354\end{array}$ & $\begin{array}{l}60 \\
59 \\
58 \\
57 \\
56 \\
55 \\
54 \\
53 \\
52 \\
51 \\
50\end{array}$ \\
\hline $\begin{array}{l}11 \\
12 \\
13 \\
14 \\
15 \\
16 \\
17 \\
18 \\
19 \\
20\end{array}$ & $\begin{array}{r}9.324366 \\
.324950 \\
.325534 \\
.326117 \\
.326700 \\
.327281 \\
.327862 \\
.328442 \\
.329021 \\
.329599\end{array}$ & $\begin{array}{l}9.73 \\
9.73 \\
9.72 \\
9.72 \\
9.68 \\
9.68 \\
9.67 \\
9.65 \\
9.63 \\
9.62\end{array}$ & $\begin{array}{r}9.990107 \\
.990079 \\
.990052 \\
.990025 \\
.989997 \\
.989970 \\
.989942 \\
.959915 \\
.989887 \\
.989860\end{array}$ & $\begin{array}{l}.47 \\
.45 \\
.45 \\
.47 \\
.45 \\
.47 \\
.45 \\
.47 \\
.45 \\
.47\end{array}$ & $\begin{array}{r}9.334259 \\
.334871 \\
.335482 \\
.336093 \\
.336702 \\
.337311 \\
.337919 \\
.338527 \\
.339133 \\
.339739\end{array}$ & $\begin{array}{l}10.20 \\
10.18 \\
10.18 \\
10.15 \\
10.15 \\
10.13 \\
10.13 \\
10.10 \\
10.10 \\
10.08\end{array}$ & $\begin{array}{r}10.665741 \\
.665129 \\
.664518 \\
.663907 \\
.663298 \\
.662689 \\
.662081 \\
.661473 \\
.660867 \\
.660261\end{array}$ & $\begin{array}{l}49 \\
48 \\
47 \\
46 \\
45 \\
44 \\
43 \\
42 \\
41 \\
40\end{array}$ \\
\hline $\begin{array}{l}21 \\
22 \\
23 \\
24 \\
25 \\
26 \\
27 \\
28 \\
29 \\
30\end{array}$ & $\begin{array}{r}9.330176 \\
.330753 \\
.331329 \\
.331903 \\
.332478 \\
.333051 \\
.333624 \\
.334195 \\
.334767 \\
.335337\end{array}$ & $\begin{array}{l}9.62 \\
9.60 \\
9.57 \\
9.58 \\
9.55 \\
9.55 \\
9.52 \\
9.53 \\
9.50 \\
9.48\end{array}$ & $\begin{array}{r}9.989832 \\
.989804 \\
.989777 \\
.989749 \\
.989721 \\
.989693 \\
.989665 \\
.989637 \\
.989610 \\
.989582\end{array}$ & $\begin{array}{l}.47 \\
.45 \\
.47 \\
.47 \\
.47 \\
.47 \\
.47 \\
.45 \\
.47 \\
.48\end{array}$ & $\begin{array}{r}9.340344 \\
.340948 \\
.341552 \\
.342155 \\
.342757 \\
.343358 \\
.343958 \\
.344558 \\
.345157 \\
.345755\end{array}$ & $\begin{array}{r}10.07 \\
10.07 \\
10.05 \\
10.03 \\
10.02 \\
10.00 \\
10.00 \\
9.98 \\
9.97 \\
9.97\end{array}$ & $\begin{array}{r}10.659656 \\
.659052 \\
.658448 \\
.657845 \\
.657243 \\
.656642 \\
.656042 \\
.655442 \\
.654843 \\
.654245\end{array}$ & $\begin{array}{l}39 \\
38 \\
37 \\
36 \\
35 \\
34 \\
33 \\
32 \\
31 \\
30\end{array}$ \\
\hline $\begin{array}{l}31 \\
32 \\
33 \\
34 \\
35 \\
36 \\
37 \\
38 \\
39 \\
40\end{array}$ & $\begin{array}{r}9.335906 \\
.336475 \\
.337043 \\
.337610 \\
.338176 \\
.335742 \\
.339307 \\
.339871 \\
.340434 \\
.340996\end{array}$ & $\begin{array}{l}9.48 \\
9.47 \\
9.45 \\
9.43 \\
9.43 \\
9.42 \\
9.40 \\
9.38 \\
9.37 \\
9.37\end{array}$ & $\begin{array}{r}9.989553 \\
.989525 \\
.989497 \\
.989469 \\
.989441 \\
.989413 \\
.989385 \\
.989356 \\
.989328 \\
.989300\end{array}$ & $\begin{array}{l}.47 \\
.47 \\
.47 \\
.47 \\
.47 \\
.47 \\
.48 \\
.47 \\
.47 \\
.48\end{array}$ & $\begin{array}{r}9.346353 \\
.346949 \\
.347545 \\
.348141 \\
.348735 \\
.349329 \\
.349922 \\
.350514 \\
.351106 \\
.351697\end{array}$ & $\begin{array}{l}9.93 \\
9.93 \\
9.93 \\
9.90 \\
9.90 \\
9.88 \\
9.87 \\
9.87 \\
9.85 \\
9.83\end{array}$ & $\begin{array}{r}10.653647 \\
.653051 \\
.652455 \\
.651859 \\
.651265 \\
.650671 \\
.650078 \\
.649486 \\
.648594 \\
.648303\end{array}$ & $\begin{array}{l}29 \\
28 \\
27 \\
26 \\
25 \\
24 \\
23 \\
22 \\
21 \\
20\end{array}$ \\
\hline $\begin{array}{l}41 \\
42 \\
43 \\
44 \\
45 \\
46 \\
47 \\
48 \\
49 \\
50\end{array}$ & $\begin{array}{r}9.341558 \\
.342119 \\
.342679 \\
.343239 \\
.343797 \\
.344355 \\
.344912 \\
.345469 \\
.346024 \\
.346579\end{array}$ & $\begin{array}{l}9.35 \\
9.33 \\
9.33 \\
9.30 \\
9.30 \\
9.28 \\
9.28 \\
9.25 \\
9.25 \\
9.25\end{array}$ & $\begin{array}{r}9.989271 \\
.989243 \\
.989214 \\
.989186 \\
.989157 \\
.989128 \\
.989100 \\
.989071 \\
.989042 \\
.989014\end{array}$ & $\begin{array}{l}.47 \\
.48 \\
.47 \\
.48 \\
.48 \\
.47 \\
.48 \\
.48 \\
.47 \\
.45\end{array}$ & $\begin{array}{r}9.352287 \\
.352876 \\
.353465 \\
.354053 \\
.354640 \\
.355227 \\
.355813 \\
.356398 \\
.356952 \\
.357566\end{array}$ & $\begin{array}{l}9.82 \\
9.82 \\
9.80 \\
9.78 \\
9.78 \\
9.77 \\
9.75 \\
9.73 \\
9.73 \\
9.72\end{array}$ & $\begin{array}{r}10.647713 \\
.647124 \\
.646535 \\
.645947 \\
.645360 \\
.644773 \\
.644187 \\
.643602 \\
.643018 \\
.642434\end{array}$ & $\begin{array}{l}19 \\
18 \\
17 \\
16 \\
15 \\
14 \\
13 \\
12 \\
11 \\
10\end{array}$ \\
\hline $\begin{array}{l}51 \\
52 \\
53 \\
54 \\
55 \\
56 \\
57 \\
58 \\
59 \\
60\end{array}$ & $\begin{array}{r}9.347134 \\
.347657 \\
.345240 \\
.348792 \\
.349343 \\
.349893 \\
.350443 \\
.350902 \\
.351540 \\
9.352058\end{array}$ & $\begin{array}{l}9.22 \\
9.22 \\
9.20 \\
9.18 \\
9.17 \\
9.17 \\
9.15 \\
9.13 \\
9.13\end{array}$ & $\begin{array}{r}9.988985 \\
.988956 \\
.985927 \\
.988595 \\
.858869 \\
.985540 \\
.985511 \\
.985752 \\
.985753 \\
9.985724\end{array}$ & $\begin{array}{l}.40 \\
.48 \\
.49 \\
.48 \\
.48 \\
.49 \\
.48 \\
.48 \\
.48 \\
.48\end{array}$ & $\begin{array}{r}9.358149 \\
.358731 \\
.359313 \\
.359593 \\
.360474 \\
.361053 \\
.361632 \\
.362210 \\
.362787 \\
9.363364\end{array}$ & $\begin{array}{l}9.70 \\
9.70 \\
9.67 \\
9.68 \\
9.65 \\
9.65 \\
9.63 \\
9.62 \\
9.62\end{array}$ & $\begin{array}{r}10.641851 \\
.641269 \\
.6406897 \\
.640107 \\
.639526 \\
.635947 \\
.633368 \\
.637790 \\
.637213 \\
10.636636\end{array}$ & $\begin{array}{l}9 \\
8 \\
7 \\
6 \\
5 \\
4 \\
3 \\
2 \\
1 \\
0\end{array}$ \\
\hline$\theta$ & Cosine. & D. !". & sine. & D. 1". & Cotang. & D. $1^{\prime \prime}$. & Tang. & ' \\
\hline
\end{tabular}


TABLE 9.-LOGARITHMIC SINES, COSINES,

\begin{tabular}{|c|c|c|c|c|c|c|c|c|}
\hline , & Sine. & D. $1^{\prime \prime}$ & Cosine. & D. $1^{\prime \prime}$ & Tang. & D. $1^{\prime \prime}$ & Cotang. & , \\
\hline $\begin{array}{r}0 \\
1 \\
2 \\
3 \\
4 \\
5 \\
6 \\
7 \\
8 \\
9 \\
10\end{array}$ & $\begin{array}{r}9.352088 \\
.352635 \\
.353181 \\
.353726 \\
.354271 \\
.354815 \\
.355358 \\
.355901 \\
.356443 \\
.356984 \\
.357524\end{array}$ & $\begin{array}{l}9.12 \\
9.10 \\
9.08 \\
9.08 \\
9.07 \\
9.05 \\
9.05 \\
9.03 \\
9.02 \\
9.00 \\
9.00\end{array}$ & $\begin{array}{r}9.988724 \\
.988695 \\
.988666 \\
.988636 \\
.988607 \\
.988578 \\
.938548 \\
.988519 \\
.988489 \\
.988460 \\
.988430\end{array}$ & $\begin{array}{r}.48 \\
.48 \\
.50 \\
.48 \\
.48 \\
.50 \\
.48 \\
.50 \\
.48 \\
.50 \\
.48\end{array}$ & $\begin{array}{r}9.363364 \\
.363940 \\
.364515 \\
.365090 \\
.365664 \\
.366237 \\
.366810 \\
.367382 \\
.367953 \\
.368524 \\
.369094\end{array}$ & $\begin{array}{l}9.60 \\
9.58 \\
9.58 \\
9.57 \\
9.55 \\
9.55 \\
9.53 \\
9.52 \\
9.52 \\
9.50 \\
9.48\end{array}$ & $\begin{array}{r}10.636636 \\
.636060 \\
.635485 \\
.634910 \\
.634336 \\
.633763 \\
.633190 \\
.632618 \\
.632047 \\
.631476 \\
.630560\end{array}$ & $\begin{array}{l}60 \\
59 \\
58 \\
57 \\
56 \\
55 \\
54 \\
53 \\
52 \\
51 \\
50\end{array}$ \\
\hline $\begin{array}{l}11 \\
12 \\
13 \\
14 \\
15 \\
16 \\
17 \\
18 \\
19 \\
20\end{array}$ & $\begin{array}{r}9.358064 \\
.358603 \\
.359141 \\
.359678 \\
.360215 \\
.360752 \\
.361287 \\
.361822 \\
.362356 \\
.362889\end{array}$ & $\begin{array}{l}8.98 \\
8.97 \\
8.95 \\
8.95 \\
8.95 \\
8.92 \\
8.92 \\
8.90 \\
8.88 \\
8.88\end{array}$ & $\begin{array}{r}9.988401 \\
.988371 \\
.988342 \\
.988312 \\
.988282 \\
.988252 \\
.988223 \\
.988193 \\
.988163 \\
.988133\end{array}$ & $\begin{array}{l}.50 \\
.48 \\
.50 \\
.50 \\
.50 \\
.48 \\
.50 \\
.50 \\
.50 \\
.50\end{array}$ & $\begin{array}{r}9.369663 \\
.370232 \\
.370799 \\
.371367 \\
.371933 \\
.372499 \\
.373064 \\
.373629 \\
.374193 \\
.374756\end{array}$ & $\begin{array}{l}9.40 \\
9.48 \\
9.45 \\
9.47 \\
9.43 \\
9.43 \\
9.42 \\
9.42 \\
9.40 \\
9.38 \\
9.38\end{array}$ & $\begin{array}{r}10.630337 \\
.629768 \\
.629201 \\
.628633 \\
.628067 \\
.627501 \\
.626936 \\
.626371 \\
.625807 \\
.625244\end{array}$ & $\begin{array}{l}49 \\
48 \\
47 \\
46 \\
45 \\
44 \\
43 \\
42 \\
41 \\
40\end{array}$ \\
\hline $\begin{array}{l}21 \\
22 \\
23 \\
24 \\
25 \\
26 \\
27 \\
28 \\
29 \\
30\end{array}$ & $\begin{array}{r}9.363422 \\
.363954 \\
.364485 \\
.365016 \\
.365546 \\
.366075 \\
.366604 \\
.367131 \\
.367659 \\
.368185\end{array}$ & $\begin{array}{l}8.87 \\
8.85 \\
8.85 \\
8.83 \\
8.82 \\
8.82 \\
8.78 \\
8.80 \\
8.77 \\
8.77\end{array}$ & $\begin{array}{r}9.988103 \\
.988073 \\
.988043 \\
.988013 \\
.987983 \\
.987953 \\
.987922 \\
.987892 \\
.987862 \\
.987832\end{array}$ & $\begin{array}{l}.50 \\
.50 \\
.50 \\
.50 \\
50 \\
.52 \\
.50 \\
.50 \\
.50 \\
.52\end{array}$ & $\begin{array}{r}9.375319 \\
.375881 \\
.376442 \\
377003 \\
.377563 \\
.378122 \\
.378681 \\
.379239 \\
.379797 \\
.380354\end{array}$ & $\begin{array}{l}9.37 \\
9.35 \\
9.35 \\
9.33 \\
9.32 \\
9.32 \\
9.30 \\
9.30 \\
9.28 \\
9.27\end{array}$ & $\begin{array}{r}10.624681 \\
.624119 \\
.623558 \\
.622997 \\
.622437 \\
.621878 \\
.621319 \\
.620761 \\
.620203 \\
.619646\end{array}$ & $\begin{array}{l}39 \\
38 \\
37 \\
36 \\
35 \\
34 \\
33 \\
32 \\
31 \\
30\end{array}$ \\
\hline $\begin{array}{l}31 \\
32 \\
33 \\
34 \\
35 \\
36 \\
37 \\
38 \\
39 \\
40\end{array}$ & $\begin{array}{r}9.368711 \\
.369236 \\
.369761 \\
.370285 \\
.370808 \\
.371330 \\
.371852 \\
.372373 \\
.372894 \\
.373414\end{array}$ & $\begin{array}{l}8.75 \\
8.75 \\
8.72 \\
8.72 \\
8.70 \\
8.70 \\
8.68 \\
8.68 \\
8.67 \\
8.65\end{array}$ & $\begin{array}{r}9.987801 \\
.987771 \\
.987740 \\
.987710 \\
.987679 \\
.987649 \\
.987618 \\
.987588 \\
.987557 \\
.987526\end{array}$ & $\begin{array}{l}.50 \\
.52 \\
.50 \\
.52 \\
.50 \\
.52 \\
.50 \\
.52 \\
.52 \\
.50\end{array}$ & $\begin{array}{r}9.380910 \\
.381466 \\
.382020 \\
.382575 \\
.383129 \\
.383682 \\
.384234 \\
.384786 \\
.385337 \\
.385888\end{array}$ & $\begin{array}{l}9.27 \\
9.23 \\
9.25 \\
9.23 \\
9.22 \\
9.20 \\
9.20 \\
9.18 \\
9.18 \\
9.17\end{array}$ & $\begin{array}{r}10.619090 \\
.618534 \\
.617980 \\
.617425 \\
.616871 \\
.616318 \\
.615766 \\
.615214 \\
.614663 \\
.614112\end{array}$ & $\begin{array}{l}29 \\
28 \\
27 \\
26 \\
25 \\
24 \\
23 \\
22 \\
21 \\
20\end{array}$ \\
\hline $\begin{array}{l}41 \\
42 \\
43 \\
44 \\
45 \\
46 \\
47 \\
48 \\
49 \\
50\end{array}$ & $\begin{array}{r}9.373933 \\
.374452 \\
.374970 \\
.375487 \\
.376003 \\
.376519 \\
.377035 \\
.377549 \\
.378063 \\
.378577\end{array}$ & $\begin{array}{l}8.65 \\
8.63 \\
8.62 \\
8.60 \\
8.60 \\
8.60 \\
8.57 \\
8.57 \\
8.57 \\
8.53\end{array}$ & $\begin{array}{r}9.987496 \\
.987465 \\
.987434 \\
.987403 \\
.987372 \\
.987341 \\
.987310 \\
.987279 \\
.987248 \\
.987217\end{array}$ & $\begin{array}{l}.52 \\
.52 \\
.52 \\
.52 \\
.52 \\
.52 \\
.52 \\
.52 \\
.52 \\
.52\end{array}$ & $\begin{array}{r}9.386438 \\
.386987 \\
.387536 \\
.388054 \\
.388631 \\
.389178 \\
.389724 \\
.390270 \\
.390815 \\
.391360\end{array}$ & $\begin{array}{l}9.15 \\
9.15 \\
9.13 \\
9.12 \\
9.12 \\
9.10 \\
9.10 \\
9.08 \\
9.08 \\
9.05\end{array}$ & $\begin{array}{r}10.613562 \\
.613013 \\
.612464 \\
.611916 \\
.611369 \\
.610822 \\
.610276 \\
.609730 \\
.609185 \\
.605640\end{array}$ & $\begin{array}{l}19 \\
18 \\
17 \\
16 \\
15 \\
14 \\
13 \\
12 \\
11 \\
10\end{array}$ \\
\hline $\begin{array}{l}51 \\
52 \\
53 \\
54 \\
55 \\
56 \\
57 \\
58 \\
59 \\
60\end{array}$ & $\begin{array}{r}9.379089 \\
.379601 \\
.380113 \\
.380624 \\
.381134 \\
.381643 \\
.382152 \\
.382661 \\
.383168 \\
9.383675\end{array}$ & $\begin{array}{l}8.53 \\
8.53 \\
8.52 \\
8.50 \\
8.48 \\
8.48 \\
8.48 \\
8.45 \\
8.45\end{array}$ & $\begin{array}{r}9.987186 \\
.987155 \\
.987124 \\
.987092 \\
.987061 \\
.987030 \\
.986998 \\
.986967 \\
.986936 \\
0.986004\end{array}$ & $\begin{array}{r}.52 \\
.52 \\
.53 \\
.52 \\
.52 \\
.53 \\
.52 \\
.52 \\
.53\end{array}$ & $\begin{array}{r}9.391903 \\
.392447 \\
.392989 \\
.393531 \\
.394073 \\
.394614 \\
.395154 \\
.395694 \\
.396233 \\
9.396771\end{array}$ & $\begin{array}{l}9.07 \\
9.03 \\
9.03 \\
9.03 \\
9.02 \\
9.00 \\
9.00 \\
8.98 \\
8.97\end{array}$ & $\begin{array}{r}10.605097 \\
.607553 \\
.607011 \\
.606469 \\
.605927 \\
.605386 \\
.604846 \\
.604306 \\
.603767 \\
10.603229\end{array}$ & $\begin{array}{l}9 \\
8 \\
7 \\
6 \\
5 \\
4 \\
3 \\
2 \\
1 \\
0\end{array}$ \\
\hline , & Cosine. & D. $1^{\prime \prime}$. & Sine. & D. $1^{\prime \prime}$. & Cotang. & D. $1^{\prime \prime}$. & Tang. & , \\
\hline
\end{tabular}


TAYGEXTS, AND COTANGENTS.

\begin{tabular}{|c|c|c|c|c|c|c|c|c|}
\hline , & Sine. & D. $1^{\prime \prime}$ & Cosine. & D. $1^{\prime \prime}$ & Tang. & D. $1^{\prime \prime}$. & Cotang. & , \\
\hline $\begin{array}{r}0 \\
1 \\
2 \\
3 \\
4 \\
5 \\
6 \\
7 \\
8 \\
9 \\
10\end{array}$ & $\begin{array}{r}9.383675 \\
.384182 \\
.384687 \\
.385192 \\
.385697 \\
.380201 \\
.386704 \\
.387207 \\
.387709 \\
.358210 \\
.385711\end{array}$ & $\begin{array}{l}8.45 \\
8.42 \\
8.42 \\
8.42 \\
8.40 \\
8.38 \\
8.38 \\
8.37 \\
8.35 \\
8.35 \\
8.33\end{array}$ & $\begin{array}{r}9.986904 \\
.986873 \\
.986841 \\
.986809 \\
.986778 \\
.986746 \\
.986714 \\
.986683 \\
.986651 \\
.986619 \\
.986557\end{array}$ & $\begin{array}{r}.52 \\
.53 \\
.53 \\
.52 \\
.53 \\
.53 \\
.52 \\
.53 \\
.53 \\
.53 \\
.53\end{array}$ & $\begin{array}{r}9.396771 \\
.397309 \\
.397846 \\
.398383 \\
.398919 \\
.399455 \\
.399990 \\
.400524 \\
.401058 \\
.401591 \\
.402124\end{array}$ & $\begin{array}{l}8.97 \\
8.95 \\
8.95 \\
8.93 \\
8.93 \\
8.92 \\
8.90 \\
8.90 \\
8.88 \\
8.89 \\
8.87\end{array}$ & $\begin{array}{r}10.603229 \\
.602691 \\
.602154 \\
.601617 \\
.601081 \\
.600545 \\
.600010 \\
.599476 \\
.598942 \\
.598409 \\
.597876\end{array}$ & $\begin{array}{l}60 \\
59 \\
58 \\
57 \\
56 \\
55 \\
54 \\
53 \\
52 \\
51 \\
50\end{array}$ \\
\hline $\begin{array}{l}11 \\
12 \\
13 \\
14 \\
15 \\
15 \\
16 \\
17 \\
18 \\
19 \\
20 \\
\end{array}$ & $\begin{array}{r}9.389211 \\
.389711 \\
.390210 \\
.390708 \\
.391206 \\
.391703 \\
.392199 \\
.392695 \\
.393191 \\
.393655\end{array}$ & $\begin{array}{l}8.33 \\
8.32 \\
8.30 \\
8.30 \\
8.28 \\
8.27 \\
8.27 \\
8.27 \\
8.23 \\
8.23\end{array}$ & $\begin{array}{r}9.986555 \\
.986523 \\
.986491 \\
.986459 \\
.986427 \\
.986395 \\
.986363 \\
.986331 \\
.986299 \\
.986266\end{array}$ & $\begin{array}{l}.53 \\
.53 \\
.53 \\
.53 \\
.53 \\
.53 \\
.53 \\
.53 \\
.55 \\
.53\end{array}$ & $\begin{array}{r}9.402656 \\
.403187 \\
.403718 \\
.404249 \\
.404778 \\
.405308 \\
.405836 \\
.406364 \\
.406892 \\
.407419\end{array}$ & $\begin{array}{l}8.85 \\
8.85 \\
8.85 \\
8.82 \\
8.83 \\
8.80 \\
8.80 \\
8.80 \\
8.78 \\
8.77\end{array}$ & $\begin{array}{r}10.597344 \\
.596813 \\
.596282 \\
.595751 \\
.595222 \\
.594692 \\
.594164 \\
.593636 \\
.593108 \\
.599581\end{array}$ & $\begin{array}{l}49 \\
48 \\
47 \\
46 \\
45 \\
44 \\
43 \\
42 \\
41 \\
40\end{array}$ \\
\hline $\begin{array}{l}21 \\
22 \\
23 \\
24 \\
25 \\
26 \\
27 \\
28 \\
29 \\
30\end{array}$ & $\begin{array}{r}9.394179 \\
.394673 \\
.395166 \\
.395658 \\
.396150 \\
.396641 \\
.397132 \\
.397621 \\
.398111 \\
.398600\end{array}$ & $\begin{array}{l}8.23 \\
8.22 \\
8.20 \\
8.20 \\
8.18 \\
8.18 \\
8.15 \\
8.17 \\
8.15 \\
8.13\end{array}$ & $\begin{array}{r}9.986234 \\
.986202 \\
.986169 \\
.986137 \\
.986104 \\
.986072 \\
.986039 \\
.986007 \\
.955974 \\
.955942\end{array}$ & $\begin{array}{l}.53 \\
.55 \\
.53 \\
.55 \\
.53 \\
.55 \\
.53 \\
.55 \\
.53 \\
.55\end{array}$ & $\begin{array}{r}9.407945 \\
.405471 \\
.405996 \\
.409521 \\
.410045 \\
.410569 \\
.411092 \\
.411615 \\
.412137 \\
.412658\end{array}$ & $\begin{array}{l}8.77 \\
8.75 \\
8.75 \\
8.73 \\
8.73 \\
8.72 \\
8.72 \\
8.70 \\
8.68 \\
8.68\end{array}$ & $\begin{array}{r}10.592055 \\
.591529 \\
.591004 \\
.590479 \\
.589955 \\
.589431 \\
.585908 \\
.588355 \\
.587863 \\
.587342\end{array}$ & $\begin{array}{l}39 \\
38 \\
37 \\
36 \\
35 \\
34 \\
33 \\
32 \\
31 \\
30\end{array}$ \\
\hline $\begin{array}{l}31 \\
32 \\
33 \\
34 \\
35 \\
36 \\
37 \\
38 \\
39 \\
40\end{array}$ & $\begin{array}{r}9.399088 \\
.399575 \\
.400062 \\
.400549 \\
.401035 \\
.401520 \\
.402005 \\
.402489 \\
.402972 \\
.403455\end{array}$ & $\begin{array}{l}8.12 \\
8.12 \\
8.12 \\
8.10 \\
8.08 \\
8.08 \\
8.07 \\
8.05 \\
8.05 \\
8.05\end{array}$ & $\begin{array}{r}9.985909 \\
.985876 \\
.985843 \\
.985811 \\
.985778 \\
.985745 \\
.985712 \\
.985679 \\
.985646 \\
.985613\end{array}$ & $\begin{array}{l}.55 \\
.55 \\
.53 \\
.55 \\
.55 \\
.55 \\
.55 \\
.55 \\
.55 \\
.55\end{array}$ & $\begin{array}{r}9.413179 \\
.413699 \\
.414219 \\
.414738 \\
.415257 \\
.415775 \\
.416293 \\
.416810 \\
.417326 \\
.417842\end{array}$ & $\begin{array}{l}8.67 \\
8.67 \\
8.65 \\
8.65 \\
8.63 \\
8.63 \\
8.62 \\
8.60 \\
8.60 \\
8.60\end{array}$ & $\begin{array}{r}10.586821 \\
.586301 \\
.585781 \\
.585262 \\
.584743 \\
.584225 \\
.583707 \\
.583190 \\
.582674 \\
.582158\end{array}$ & $\begin{array}{l}29 \\
28 \\
27 \\
26 \\
25 \\
24 \\
23 \\
22 \\
21 \\
20\end{array}$ \\
\hline $\begin{array}{l}41 \\
42 \\
43 \\
44 \\
45 \\
46 \\
47 \\
49 \\
49 \\
50\end{array}$ & $\begin{array}{r}9.403938 \\
.404420 \\
.404901 \\
.405382 \\
.405862 \\
.406341 \\
.406820 \\
.407299 \\
.407777 \\
.405254\end{array}$ & $\begin{array}{l}8.03 \\
8.02 \\
8.02 \\
8.00 \\
7.98 \\
7.98 \\
7.99 \\
7.97 \\
7.95 \\
7.95\end{array}$ & $\begin{array}{r}9.985580 \\
.985547 \\
.985514 \\
.955450 \\
.985447 \\
.985414 \\
.985381 \\
.955347 \\
.985314 \\
.985280\end{array}$ & $\begin{array}{l}.55 \\
.55 \\
.57 \\
.55 \\
.55 \\
.55 \\
.57 \\
.55 \\
.57 \\
.55\end{array}$ & $\begin{array}{r}9.418358 \\
.418873 \\
.419387 \\
.419901 \\
.420415 \\
.420927 \\
.421440 \\
.421952 \\
.422463 \\
.422974\end{array}$ & $\begin{array}{l}8.58 \\
8.57 \\
8.57 \\
8.57 \\
8.55 \\
8.55 \\
8.53 \\
8.52 \\
8.52 \\
8.50\end{array}$ & $\begin{array}{r}10.581642 \\
.581127 \\
.580613 \\
.580099 \\
.579555 \\
.579073 \\
.578560 \\
.578048 \\
.577537 \\
.577026\end{array}$ & $\begin{array}{l}19 \\
18 \\
17 \\
16 \\
15 \\
14 \\
13 \\
12 \\
11 \\
10\end{array}$ \\
\hline $\begin{array}{l}51 \\
52 \\
53 \\
54 \\
55 \\
56 \\
57 \\
58 \\
59 \\
60\end{array}$ & $\begin{array}{r}9.408731 \\
.409207 \\
.409652 \\
.410157 \\
.410632 \\
.411106 \\
.411579 \\
.412052 \\
.412524 \\
9.412996\end{array}$ & $\begin{array}{l}7.93 \\
7.93 \\
7.92 \\
7.92 \\
7.90 \\
7.88 \\
7.88 \\
7.87 \\
7.87\end{array}$ & $\begin{array}{r}9.985247 \\
.985213 \\
.985180 \\
.985146 \\
.985113 \\
.985079 \\
.985045 \\
.985011 \\
.984978 \\
9.984944\end{array}$ & $\begin{array}{l}.57 \\
.55 \\
.57 \\
.55 \\
.57 \\
.57 \\
.57 \\
.55 \\
.57\end{array}$ & $\begin{array}{r}9.423484 \\
.423993 \\
.424503 \\
.425011 \\
.425519 \\
.426027 \\
.426534 \\
.427041 \\
.427547 \\
9.428052\end{array}$ & $\begin{array}{l}8.48 \\
8.50 \\
8.47 \\
8.47 \\
8.47 \\
8.45 \\
8.45 \\
8.43 \\
8.42\end{array}$ & $\begin{array}{r}10.576516 \\
.576007 \\
.575497 \\
.574989 \\
.574481 \\
.573973 \\
.573466 \\
.572959 \\
.572453 \\
10.571948\end{array}$ & $\begin{array}{l}9 \\
8 \\
7 \\
6 \\
5 \\
4 \\
3 \\
2 \\
1 \\
0\end{array}$ \\
\hline , & Cosine. & D. $1^{\prime \prime}$ & Sine. & D. $1^{\prime \prime}$. & Cotang. & D. $1^{\prime \prime}$. & Tang. & 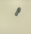 \\
\hline
\end{tabular}




\begin{tabular}{|c|c|c|c|c|c|c|c|c|}
\hline , & Sine. & D. 1". & Cosine. & D. $1^{\prime \prime}$. & Tang. & D. $1^{\prime \prime}$. & Cotang. & , \\
\hline $\begin{array}{r}0 \\
1 \\
2 \\
3 \\
4 \\
4 \\
5 \\
6 \\
7 \\
8 \\
9 \\
10\end{array}$ & $\begin{array}{r}9.412996 \\
.413467 \\
.413938 \\
.414408 \\
.414878 \\
.415347 \\
.415815 \\
.416283 \\
.416751 \\
.417217 \\
.417684\end{array}$ & $\begin{array}{l}7.85 \\
7.85 \\
7.83 \\
7.83 \\
7.82 \\
7.80 \\
7.80 \\
7.80 \\
7.77 \\
7.78 \\
7.77\end{array}$ & $\begin{array}{r}9.984944 \\
.984910 \\
.984876 \\
.984842 \\
.984808 \\
984774 \\
.984740 \\
.984706 \\
.984672 \\
.984638 \\
.984603\end{array}$ & $\begin{array}{l}.57 \\
.57 \\
.57 \\
.57 \\
.57 \\
.57 \\
.57 \\
.57 \\
.57 \\
.58 \\
.57\end{array}$ & $\begin{array}{r}9.428052 \\
.428558 \\
.429062 \\
.429566 \\
.430070 \\
.430573 \\
.431075 \\
.431577 \\
.432079 \\
.432580 \\
.433080\end{array}$ & $\begin{array}{l}8.43 \\
8.40 \\
8.40 \\
8.40 \\
8.38 \\
8.37 \\
8.37 \\
8.37 \\
8.35 \\
8.33 \\
8.33\end{array}$ & $\begin{array}{r}10.571948 \\
.571442 \\
.570938 \\
.570434 \\
569930 \\
.569427 \\
.568925 \\
.568423 \\
.567921 \\
.567420 \\
.566920\end{array}$ & 60 \\
\hline $\begin{array}{l}11 \\
12 \\
13 \\
14 \\
15 \\
16 \\
17 \\
18 \\
19 \\
20\end{array}$ & $\begin{array}{r}9.418150 \\
.418615 \\
.419079 \\
.419544 \\
.420007 \\
420470 \\
.420933 \\
.421395 \\
.421857 \\
.422318\end{array}$ & $\begin{array}{l}7.75 \\
7.73 \\
7.75 \\
7.72 \\
7.72 \\
7.72 \\
7.70 \\
7.70 \\
7.68 \\
7.67\end{array}$ & $\begin{array}{r}9.984569 \\
.984535 \\
.984500 \\
.984466 \\
.984432 \\
.984397 \\
.984363 \\
.984328 \\
.984294 \\
.984259\end{array}$ & $\begin{array}{l}.57 \\
.58 \\
.58 \\
.67 \\
.57 \\
.58 \\
.57 \\
.58 \\
.57 \\
.58 \\
.58\end{array}$ & $\begin{array}{r}9.433580 \\
.434080 \\
.434579 \\
.435078 \\
435576 \\
.436073 \\
.436570 \\
.437067 \\
.437563 \\
.438059\end{array}$ & $\begin{array}{l}8.33 \\
8.33 \\
8.32 \\
8.32 \\
8.30 \\
8.28 \\
8.28 \\
8.28 \\
8.27 \\
8.27 \\
8.25\end{array}$ & $\begin{array}{r}10.566420 \\
.565920 \\
.565421 \\
.564922 \\
.564424 \\
.563927 \\
.563430 \\
.562933 \\
.562437 \\
.561941\end{array}$ & 49 \\
\hline $\begin{array}{l}21 \\
22 \\
23 \\
24 \\
25 \\
26 \\
27 \\
28 \\
29 \\
30\end{array}$ & $\begin{array}{r}9.422778 \\
.423238 \\
.423697 \\
.424156 \\
.424615 \\
.425073 \\
.425530 \\
.425987 \\
.426443 \\
.426899\end{array}$ & $\begin{array}{l}7.67 \\
7.65 \\
7.65 \\
7.65 \\
7.63 \\
7.62 \\
7.62 \\
7.60 \\
7.60 \\
7.58\end{array}$ & $\begin{array}{l}.984224 \\
.984190 \\
.984155 \\
.984120 \\
.984085 \\
.984050 \\
.984015 \\
.983981 \\
.983946 \\
.983911\end{array}$ & $\begin{array}{l}.57 \\
.58 \\
.58 \\
.58 \\
.58 \\
.58 \\
.57 \\
.58 \\
.58 \\
.60\end{array}$ & $\begin{array}{r}9.438554 \\
.439048 \\
.439543 \\
.440036 \\
.440529 \\
.441022 \\
.441514 \\
.442006 \\
.442497 \\
.442988\end{array}$ & $\begin{array}{l}8.23 \\
8.25 \\
8.22 \\
8.22 \\
8.22 \\
8.20 \\
8.20 \\
8.18 \\
8.18 \\
8.18\end{array}$ & $\begin{array}{r}10.561446 \\
.560952 \\
.560457 \\
.559964 \\
.559471 \\
.558978 \\
.558486 \\
.557994 \\
.557503 \\
.557012\end{array}$ & $\begin{array}{l}39 \\
38 \\
37 \\
36 \\
35 \\
34 \\
33 \\
32 \\
31 \\
30\end{array}$ \\
\hline $\begin{array}{l}31 \\
32 \\
33 \\
34 \\
35 \\
36 \\
37 \\
38 \\
39 \\
40\end{array}$ & $\begin{array}{r}9.427354 \\
.427809 \\
.428263 \\
.428717 \\
.429170 \\
.429623 \\
.430075 \\
.430527 \\
.430978 \\
.431429\end{array}$ & $\begin{array}{l}7.58 \\
7.57 \\
7.57 \\
7.55 \\
7.55 \\
7.53 \\
7.53 \\
7.52 \\
7.52 \\
7.50\end{array}$ & $\begin{array}{r}9.983875 \\
.983840 \\
.983805 \\
.983770 \\
.983735 \\
.983700 \\
.983664 \\
.983629 \\
.983594 \\
.983558\end{array}$ & $\begin{array}{l}.58 \\
.58 \\
.58 \\
.58 \\
.58 \\
.60 \\
.58 \\
.58 \\
.60 \\
.58\end{array}$ & $\begin{array}{r}9.443479 \\
.443968 \\
.444458 \\
.444947 \\
.445435 \\
.445923 \\
.446411 \\
.446898 \\
.447384 \\
.447870\end{array}$ & $\begin{array}{l}8.15 \\
8.17 \\
8.15 \\
8.13 \\
8.13 \\
8.13 \\
8.12 \\
8.10 \\
8.10 \\
8.10\end{array}$ & $\begin{array}{r}10.556521 \\
.556032 \\
.555542 \\
.555053 \\
.554565 \\
.554077 \\
.553589 \\
.553102 \\
.552616 \\
.552130\end{array}$ & 29 \\
\hline $\begin{array}{l}41 \\
42 \\
43 \\
44 \\
45 \\
46 \\
47 \\
48 \\
49 \\
50\end{array}$ & $\begin{array}{r}9.431879 \\
.432329 \\
.432778 \\
.433226 \\
.433675 \\
.434122 \\
.434569 \\
.435016 \\
.435462 \\
.435908\end{array}$ & $\begin{array}{l}7.50 \\
7.48 \\
7.47 \\
7.48 \\
7.45 \\
7.45 \\
7.45 \\
7.43 \\
7.43 \\
7.42\end{array}$ & $\begin{array}{r}9.983523 \\
.983487 \\
.983452 \\
.983416 \\
.983381 \\
.983345 \\
.983309 \\
.983273 \\
.983238 \\
.983202\end{array}$ & $\begin{array}{l}.60 \\
.58 \\
.60 \\
.58 \\
.60 \\
.60 \\
.60 \\
.58 \\
.60 \\
.60\end{array}$ & $\begin{array}{r}9.448356 \\
.448841 \\
.449326 \\
.449810 \\
.450994 \\
.450777 \\
.451260 \\
.451743 \\
.452225 \\
.452706\end{array}$ & $\begin{array}{l}8.08 \\
8.08 \\
8.07 \\
8.07 \\
8.05 \\
8.05 \\
8.05 \\
8.03 \\
8.02 \\
8.02\end{array}$ & $\begin{array}{r}10551644 \\
.551159 \\
.550674 \\
.550190 \\
.549706 \\
.549223 \\
.548740 \\
.548257 \\
.547775 \\
.547294\end{array}$ & 19 \\
\hline $\begin{array}{l}51 \\
52 \\
53 \\
54 \\
55 \\
56 \\
57 \\
58 \\
59 \\
60\end{array}$ & $\begin{array}{r}9.436353 \\
.436798 \\
.437242 \\
.437686 \\
.438129 \\
.438572 \\
.439014 \\
.439456 \\
.439897 \\
9.440338\end{array}$ & $\begin{array}{l}7.42 \\
7.40 \\
7.40 \\
7.38 \\
7.38 \\
7.37 \\
7.37 \\
7.35 \\
7.35\end{array}$ & $\begin{array}{r}9.983166 \\
.983130 \\
.983094 \\
.983058 \\
.983022 \\
.982986 \\
.982950 \\
.982914 \\
.982878 \\
9.982842\end{array}$ & $\begin{array}{l}.60 \\
.60 \\
.60 \\
.60 \\
.60 \\
.60 \\
.60 \\
.60 \\
.60\end{array}$ & $\begin{array}{r}9.453187 \\
.453668 \\
.454148 \\
.454628 \\
.455107 \\
.455586 \\
.456064 \\
.4565442 \\
.457019 \\
9.457496\end{array}$ & $\begin{array}{l}8.02 \\
8.00 \\
8.00 \\
7.98 \\
7.98 \\
7.97 \\
7.97 \\
7.95 \\
7.95\end{array}$ & $\begin{array}{r}10.546813 \\
.546332 \\
.545552 \\
.545372 \\
.544893 \\
.544414 \\
.543936 \\
.543458 \\
.542981 \\
10.542404\end{array}$ & $\begin{array}{l}7 \\
6 \\
5 \\
4 \\
3 \\
2 \\
1 \\
0\end{array}$ \\
\hline ' & Cosine. & D. 1". & Sine. & $D_{i} 1^{\prime \prime}$ 。 & Cotang. & D. $1^{\prime \prime}$ & Tang. & ' \\
\hline
\end{tabular}


TANGENTS, AND COTANGENTS.

\begin{tabular}{|c|c|c|c|c|c|c|c|c|}
\hline , & Sine. & D. $1^{\prime \prime}$ 。 & Cosine. & D. $1^{\prime \prime}$. & Tang. & D. $1^{\prime \prime}$. & Cotang. & , \\
\hline $\begin{array}{r}0 \\
1 \\
2 \\
3 \\
4 \\
5 \\
6 \\
7 \\
8 \\
9 \\
10\end{array}$ & $\begin{array}{r}9.440338 \\
.440778 \\
.441218 \\
.441658 \\
.442096 \\
.442535 \\
.442973 \\
.443410 \\
.443847 \\
.444254 \\
.444720\end{array}$ & $\begin{array}{l}7.33 \\
7.33 \\
7.33 \\
7.30 \\
7.32 \\
7.30 \\
7.28 \\
7.28 \\
7.29 \\
7.27 \\
7.25\end{array}$ & $\begin{array}{r}9.952842 \\
.952505 \\
.982769 \\
.982733 \\
.982696 \\
.982660 \\
.982624 \\
.982587 \\
.952551 \\
.982514 \\
.952477\end{array}$ & $\begin{array}{l}.62 \\
.60 \\
.60 \\
.62 \\
.60 \\
.60 \\
.62 \\
.60 \\
.62 \\
.62 \\
.60\end{array}$ & $\begin{array}{r}9.457496 \\
.457973 \\
.458449 \\
.458925 \\
.459400 \\
.459875 \\
.460349 \\
.460523 \\
.461297 \\
.461770 \\
.462242\end{array}$ & $\begin{array}{l}7.95 \\
7.93 \\
7.93 \\
7.92 \\
7.92 \\
7.90 \\
7.90 \\
7.90 \\
7.88 \\
7.87 \\
7.88\end{array}$ & $\begin{array}{r}10.542504 \\
.542027 \\
.541551 \\
.541075 \\
.540600 \\
.540125 \\
.539651 \\
.539177 \\
.538703 \\
.538230 \\
.537758\end{array}$ & $\begin{array}{l}60 \\
59 \\
58 \\
57 \\
56 \\
55 \\
54 \\
53 \\
52 \\
51 \\
50\end{array}$ \\
\hline $\begin{array}{l}11 \\
12 \\
13 \\
14 \\
15 \\
16 \\
17 \\
18 \\
19 \\
20\end{array}$ & $\begin{array}{r}9.445155 \\
.445590 \\
.446025 \\
.446459 \\
.446893 \\
.447326 \\
.447759 \\
.448191 \\
.448623 \\
.449054\end{array}$ & $\begin{array}{l}7.25 \\
7.25 \\
7.23 \\
7.23 \\
7.22 \\
7.22 \\
7.20 \\
7.20 \\
7.18 \\
7.18\end{array}$ & $\begin{array}{r}9.952441 \\
.952404 \\
.952367 \\
.952331 \\
.952294 \\
.952257 \\
.952220 \\
.982183 \\
.952146 \\
.952109\end{array}$ & $\begin{array}{l}.62 \\
.62 \\
.60 \\
.62 \\
.62 \\
.62 \\
.62 \\
.62 \\
.62 \\
.62\end{array}$ & $\begin{array}{r}9.462715 \\
.463186 \\
.463658 \\
.464128 \\
.464599 \\
.465069 \\
.465539 \\
.466008 \\
.466477 \\
.466945\end{array}$ & $\begin{array}{l}7.85 \\
7.87 \\
7.83 \\
7.85 \\
7.83 \\
7.83 \\
7.82 \\
7.82 \\
7.80 \\
7.80\end{array}$ & $\begin{array}{r}10.537285 \\
.536814 \\
.536342 \\
.535872 \\
.535401 \\
.534931 \\
.534461 \\
.533992 \\
.533523 \\
.533055\end{array}$ & $\begin{array}{l}49 \\
48 \\
47 \\
46 \\
45 \\
44 \\
43 \\
42 \\
41 \\
40\end{array}$ \\
\hline $\begin{array}{l}21 \\
22 \\
23 \\
24 \\
25 \\
26 \\
27 \\
28 \\
29 \\
30\end{array}$ & $\begin{array}{r}9.449485 \\
.449915 \\
.450345 \\
.450775 \\
.451204 \\
.451632 \\
.452060 \\
.452488 \\
.452915 \\
.453342\end{array}$ & $\begin{array}{l}7.17 \\
7.17 \\
7.17 \\
7.15 \\
7.13 \\
7.13 \\
7.13 \\
7.12 \\
7.12 \\
7.10\end{array}$ & $\begin{array}{r}9.982072 \\
.982035 \\
.981998 \\
.981961 \\
.981924 \\
.981886 \\
.981849 \\
.981812 \\
.981774 \\
.981737\end{array}$ & $\begin{array}{l}.62 \\
.62 \\
.62 \\
.62 \\
.63 \\
.62 \\
.62 \\
.63 \\
.62 \\
.62\end{array}$ & $\begin{array}{r}9.467413 \\
.467880 \\
.468347 \\
.468814 \\
.469280 \\
.469746 \\
.470211 \\
.470676 \\
.471141 \\
.471605\end{array}$ & $\begin{array}{l}7.78 \\
7.78 \\
7.78 \\
7.77 \\
7.77 \\
7.75 \\
7.75 \\
7.75 \\
7.73 \\
7.73\end{array}$ & $\begin{array}{r}10.532587 \\
.532120 \\
.531653 \\
.531186 \\
.530720 \\
.530254 \\
.529789 \\
.529324 \\
.528859 \\
.528395\end{array}$ & $\begin{array}{l}39 \\
38 \\
37 \\
36 \\
35 \\
34 \\
33 \\
32 \\
31 \\
30\end{array}$ \\
\hline $\begin{array}{l}31 \\
32 \\
33 \\
34 \\
35 \\
36 \\
37 \\
38 \\
39 \\
40\end{array}$ & $\begin{array}{r}9.453768 \\
454194 \\
454619 \\
.455044 \\
.455469 \\
.455593 \\
.456316 \\
.456739 \\
.457162 \\
.457584\end{array}$ & $\begin{array}{l}7.10 \\
7.08 \\
7.08 \\
7.08 \\
7.07 \\
7.05 \\
7.05 \\
7.05 \\
7.03 \\
7.03\end{array}$ & $\begin{array}{r}9.981700 \\
.981662 \\
.981625 \\
.981587 \\
.981549 \\
.981512 \\
.981474 \\
.981436 \\
.981399 \\
.981361\end{array}$ & $\begin{array}{l}.63 \\
.62 \\
.63 \\
.63 \\
.62 \\
.63 \\
.63 \\
.62 \\
.63 \\
.63\end{array}$ & $\begin{array}{r}9.472069 \\
.472532 \\
.472995 \\
.473457 \\
.473919 \\
.474381 \\
.474842 \\
.475303 \\
.475763 \\
.476223\end{array}$ & $\begin{array}{l}7.72 \\
7.72 \\
7.70 \\
7.70 \\
7.70 \\
7.68 \\
7.68 \\
7.67 \\
7.67 \\
7.67\end{array}$ & $\begin{array}{r}10.527931 \\
.527468 \\
.527005 \\
.526543 \\
.526081 \\
.525619 \\
.525158 \\
.524697 \\
.524237 \\
.523777\end{array}$ & $\begin{array}{l}29 \\
28 \\
27 \\
26 \\
25 \\
24 \\
23 \\
22 \\
21 \\
20\end{array}$ \\
\hline $\begin{array}{l}41 \\
42 \\
43 \\
44 \\
45 \\
46 \\
47 \\
48 \\
49 \\
50\end{array}$ & $\begin{array}{r}9.458006 \\
.458427 \\
.458848 \\
.459268 \\
.459688 \\
.460108 \\
.460527 \\
.460946 \\
.461364 \\
.461782\end{array}$ & $\begin{array}{l}7.02 \\
7.02 \\
7.00 \\
7.00 \\
7.00 \\
6.98 \\
6.98 \\
6.97 \\
6.97 \\
6.95\end{array}$ & $\begin{array}{r}9.981323 \\
.981285 \\
.981247 \\
.981209 \\
.981171 \\
.981133 \\
.981095 \\
.981057 \\
.981019 \\
.980381\end{array}$ & $\begin{array}{l}.63 \\
.63 \\
.63 \\
.63 \\
.63 \\
.63 \\
.63 \\
.63 \\
.63 \\
.65\end{array}$ & $\begin{array}{r}9.476683 \\
.477142 \\
.477601 \\
.478059 \\
.478517 \\
.478975 \\
.479432 \\
.479859 \\
.480345 \\
.480801\end{array}$ & $\begin{array}{l}7.65 \\
7.65 \\
7.63 \\
7.63 \\
7.63 \\
7.62 \\
7.62 \\
7.60 \\
7.60 \\
7.60\end{array}$ & $\begin{array}{r}10.523317 \\
.522858 \\
.522399 \\
.521941 \\
.521483 \\
.521025 \\
.5205 t i 8 \\
.520111 \\
.519655 \\
.519199\end{array}$ & $\begin{array}{l}19 \\
18 \\
17 \\
16 \\
15 \\
14 \\
13 \\
12 \\
11 \\
10\end{array}$ \\
\hline $\begin{array}{l}51 \\
52 \\
53 \\
54 \\
55 \\
56 \\
57 \\
58 \\
59 \\
60\end{array}$ & $\begin{array}{r}9.462199 \\
.462616 \\
.463032 \\
.463448 \\
.463864 \\
.464279 \\
.464694 \\
.465108 \\
.465522 \\
9.465935\end{array}$ & $\begin{array}{l}\text { 6. } 95 \\
6.93 \\
6.93 \\
6.93 \\
6.92 \\
6.92 \\
6.90 \\
6.90 \\
6.88\end{array}$ & $\begin{array}{r}9.980942 \\
.980904 \\
.980866 \\
.980627 \\
.980789 \\
.950750 \\
.980712 \\
.980673 \\
.9906355 \\
9.950596\end{array}$ & $\begin{array}{l}.63 \\
.63 \\
.63 \\
.65 \\
.63 \\
.65 \\
63 \\
.65 \\
.63 \\
.65\end{array}$ & $\begin{array}{r}9.481257 \\
.481712 \\
.482167 \\
.482621 \\
.483075 \\
.483529 \\
.483982 \\
.484435 \\
.484857 \\
9.485339\end{array}$ & $\begin{array}{l}7.50 \\
7.58 \\
7.58 \\
7.57 \\
7.57 \\
7.57 \\
7.55 \\
7.55 \\
7.53 \\
7.53\end{array}$ & $\begin{array}{r}10.518743 \\
.518288 \\
.517833 \\
.517379 \\
.516925 \\
.516471 \\
.516018 \\
.515565 \\
.515113 \\
10.514661\end{array}$ & $\begin{array}{l}9 \\
8 \\
7 \\
6 \\
5 \\
1 \\
3 \\
3 \\
2 \\
1 \\
0\end{array}$ \\
\hline ' & Cosine. & D. $1^{\prime \prime}$. & Sine. & D. $1^{\prime \prime}$. & Cotang. & D. $1^{\prime \prime}$. & rang. & 1 \\
\hline
\end{tabular}


TABLE 9.-LOGARITHMIC SINES, COSINES,

\begin{tabular}{|c|c|c|c|c|c|c|c|c|}
\hline , & Sine. & D. $1^{\prime \prime}$. & Cosine. & D. $1^{\prime \prime}$. & Tang. & D. $1^{\prime \prime}$. & Cotang. & , \\
\hline $\begin{array}{r}0 \\
1 \\
2 \\
3 \\
4 \\
5 \\
6 \\
7 \\
8 \\
9 \\
10\end{array}$ & $\begin{array}{r}9.465935 \\
.466348 \\
.466761 \\
.467173 \\
.467585 \\
.467996 \\
.468407 \\
.468817 \\
.469227 \\
.469637 \\
.470046\end{array}$ & $\begin{array}{l}6.88 \\
6.88 \\
6.87 \\
6.87 \\
6.85 \\
6.85 \\
6.83 \\
6.83 \\
6.83 \\
6.82 \\
6.82\end{array}$ & $\begin{array}{r}9.980596 \\
.980558 \\
.980519 \\
.980480 \\
.980442 \\
.980403 \\
.980364 \\
.980325 \\
.980286 \\
.980247 \\
.980208\end{array}$ & $\begin{array}{l}.63 \\
.65 \\
.65 \\
.63 \\
.65 \\
.65 \\
.65 \\
.65 \\
.65 \\
.65 \\
.65\end{array}$ & $\begin{array}{r}9.485339 \\
.485791 \\
.486242 \\
.486693 \\
.487143 \\
.487593 \\
.488043 \\
.488492 \\
.488941 \\
.489390 \\
.489838\end{array}$ & $\begin{array}{l}7.53 \\
7.52 \\
7.52 \\
7.50 \\
7.50 \\
7.50 \\
7.48 \\
7.48 \\
7.48 \\
7.47 \\
7.47\end{array}$ & $\begin{array}{r}10.514661 \\
.514209 \\
.513758 \\
.513307 \\
.512857 \\
.512407 \\
.511957 \\
.511508 \\
.511059 \\
.510610 \\
.510162\end{array}$ & $\begin{array}{l}60 \\
59 \\
58 \\
57 \\
56 \\
55 \\
54 \\
53 \\
52 \\
51 \\
50\end{array}$ \\
\hline $\begin{array}{l}11 \\
12 \\
13 \\
14 \\
15 \\
16 \\
17 \\
18 \\
18 \\
19 \\
20\end{array}$ & $\begin{array}{r}9.470455 \\
.470863 \\
.471271 \\
.471679 \\
.472086 \\
.472492 \\
.472898 \\
.473304 \\
.473710 \\
.474115\end{array}$ & $\begin{array}{l}6.80 \\
6.80 \\
6.80 \\
6.78 \\
6.77 \\
6.77 \\
6.77 \\
6.77 \\
6.75 \\
6.73\end{array}$ & $\begin{array}{r}9.980169 \\
.980130 \\
.980091 \\
.980052 \\
.980012 \\
.979973 \\
.979934 \\
.979895 \\
.979855 \\
.979816\end{array}$ & $\begin{array}{l}.65 \\
.65 \\
.65 \\
.67 \\
.65 \\
.65 \\
.65 \\
.67 \\
.65 \\
.67\end{array}$ & $\begin{array}{r}9.490286 \\
.490733 \\
.491180 \\
.491627 \\
.492073 \\
.492519 \\
.492965 \\
.493410 \\
.493854 \\
.494299\end{array}$ & $\begin{array}{l}7.45 \\
7.45 \\
7.45 \\
7.43 \\
7.43 \\
7.43 \\
7.42 \\
7.40 \\
7.42 \\
7.40\end{array}$ & $\begin{array}{r}10.509714 \\
.509267 \\
.508820 \\
.508373 \\
.507927 \\
.507481 \\
.507035 \\
.506590 \\
.506146 \\
.505701\end{array}$ & $\begin{array}{l}49 \\
48 \\
47 \\
46 \\
45 \\
44 \\
43 \\
42 \\
41 \\
40\end{array}$ \\
\hline $\begin{array}{l}21 \\
22 \\
23 \\
24 \\
25 \\
26 \\
27 \\
28 \\
29 \\
30\end{array}$ & $\begin{array}{r}9.474519 \\
.474923 \\
.475327 \\
.475730 \\
.476133 \\
.476536 \\
.476938 \\
.477340 \\
.477741 \\
.478142\end{array}$ & $\begin{array}{l}6.73 \\
6.73 \\
6.72 \\
6.72 \\
6.72 \\
6.70 \\
6.70 \\
6.68 \\
6.68 \\
6.67\end{array}$ & $\begin{array}{r}9.979776 \\
.979737 \\
.979697 \\
.979658 \\
.979618 \\
.979579 \\
.979539 \\
.979499 \\
.979459 \\
.979420\end{array}$ & $\begin{array}{l}.65 \\
.67 \\
.65 \\
.67 \\
.65 \\
.67 \\
.67 \\
.67 \\
.65 \\
.67\end{array}$ & $\begin{array}{r}9.494743 \\
.495186 \\
.495630 \\
.496073 \\
.496515 \\
496957 \\
.497399 \\
.497841 \\
.498282 \\
.498722\end{array}$ & $\begin{array}{l}7.38 \\
7.40 \\
7.38 \\
7.37 \\
7.37 \\
7.37 \\
7.37 \\
7.35 \\
7.33 \\
7.35\end{array}$ & $\begin{array}{r}10.505257 \\
.504814 \\
.504370 \\
.503927 \\
.503485 \\
.503043 \\
.502601 \\
.502159 \\
.501718 \\
.501278\end{array}$ & $\begin{array}{l}39 \\
38 \\
37 \\
36 \\
35 \\
34 \\
33 \\
32 \\
31 \\
30\end{array}$ \\
\hline $\begin{array}{l}31 \\
32 \\
33 \\
34 \\
35 \\
36 \\
37 \\
38 \\
39 \\
40\end{array}$ & $\begin{array}{r}9.478542 \\
.478942 \\
.479342 \\
.479741 \\
.480140 \\
.480539 \\
.480937 \\
.481334 \\
.481731 \\
.482128\end{array}$ & $\begin{array}{l}6.67 \\
6.67 \\
6.65 \\
6.65 \\
6.65 \\
6.63 \\
662 \\
6.62 \\
6.62 \\
6.62\end{array}$ & $\begin{array}{r}9.979380 \\
.979340 \\
.979300 \\
.979260 \\
.979220 \\
.979180 \\
.979140 \\
.979100 \\
.979059 \\
.979019\end{array}$ & $\begin{array}{l}.67 \\
.67 \\
.67 \\
.67 \\
.67 \\
.67 \\
.67 \\
.68 \\
.67 \\
.67\end{array}$ & $\begin{array}{r}9.499163 \\
.499603 \\
.500042 \\
.500481 \\
.500920 \\
.501359 \\
.501797 \\
.502235 \\
.502672 \\
.503109\end{array}$ & $\begin{array}{l}7.33 \\
7.32 \\
7.32 \\
7.32 \\
7.32 \\
7.30 \\
7.30 \\
7.28 \\
7.28 \\
7.28\end{array}$ & $\begin{array}{r}10.500837 \\
.500397 \\
.499958 \\
.499519 \\
.499080 \\
.498641 \\
.498203 \\
.497765 \\
.497328 \\
.496891\end{array}$ & $\begin{array}{l}29 \\
28 \\
27 \\
26 \\
25 \\
24 \\
23 \\
22 \\
21 \\
20\end{array}$ \\
\hline $\begin{array}{l}41 \\
42 \\
43 \\
44 \\
45 \\
46 \\
47 \\
48 \\
49 \\
50\end{array}$ & $\begin{array}{r}9.482525 \\
.482921 \\
.483316 \\
.483712 \\
.484107 \\
.484501 \\
.484895 \\
.485289 \\
.485682 \\
.486075\end{array}$ & $\begin{array}{l}6.60 \\
6.58 \\
6.60 \\
6.58 \\
6.57 \\
6.57 \\
6.57 \\
6.55 \\
6.55 \\
6.53\end{array}$ & $\begin{array}{r}9.978979 \\
.978939 \\
.978898 \\
.978858 \\
.978817 \\
.978777 \\
.978737 \\
.978696 \\
.978655 \\
.978615\end{array}$ & $\begin{array}{l}.67 \\
.68 \\
.67 \\
.68 \\
.67 \\
.67 \\
.68 \\
.68 \\
.67 \\
.68\end{array}$ & $\begin{array}{r}9.503546 \\
.503982 \\
.504418 \\
.504854 \\
.505289 \\
.505724 \\
.506159 \\
.506593 \\
.507027 \\
.507460\end{array}$ & $\begin{array}{l}7.27 \\
7.27 \\
7.27 \\
7.25 \\
7.25 \\
7.25 \\
7.23 \\
7.23 \\
7.23 \\
7.22\end{array}$ & $\begin{array}{r}10.496454 \\
.496018 \\
.495582 \\
.495146 \\
.494711 \\
.494276 \\
.493841 \\
.493407 \\
.492973 \\
.492540\end{array}$ & $\begin{array}{l}19 \\
18 \\
17 \\
16 \\
15 \\
14 \\
13 \\
12 \\
11 \\
10\end{array}$ \\
\hline $\begin{array}{l}51 \\
52 \\
53 \\
54 \\
55 \\
56 \\
57 \\
58 \\
59 \\
60\end{array}$ & $\begin{array}{r}9.486467 \\
.486860 \\
.487251 \\
.487643 \\
.488034 \\
.488424 \\
.488814 \\
.489204 \\
.489593 \\
9.489982\end{array}$ & $\begin{array}{l}6.55 \\
6.52 \\
6.53 \\
6.52 \\
6.50 \\
6.50 \\
6.50 \\
6.48 \\
6.48\end{array}$ & $\begin{array}{r}9.978574 \\
.978533 \\
.978493 \\
.978452 \\
.978411 \\
.978370 \\
.978329 \\
.978288 \\
.978247 \\
9.978206\end{array}$ & $\begin{array}{l}.68 \\
.68 \\
.67 \\
.68 \\
.68 \\
.68 \\
.68 \\
.68 \\
.68 \\
.68\end{array}$ & $\begin{array}{r}9.507893 \\
.508326 \\
.508759 \\
.509191 \\
.509622 \\
.510054 \\
.510485 \\
.510916 \\
.511346 \\
9.511776\end{array}$ & $\begin{array}{l}7.22 \\
7.22 \\
7.20 \\
7.18 \\
7.20 \\
7.18 \\
7.18 \\
7.17 \\
7.17\end{array}$ & $\begin{array}{r}10.492107 \\
.491674 \\
.491241 \\
.490809 \\
.490378 \\
.489946 \\
.489515 \\
.489084 \\
.488654 \\
10.488224\end{array}$ & $\begin{array}{l}9 \\
8 \\
7 \\
6 \\
5 \\
4 \\
3 \\
2 \\
1 \\
0\end{array}$ \\
\hline , & Cosine. & D. $1^{\prime \prime}$. & Sine. & D. $1^{\prime \prime}$. & Cotang. & D. $1^{\prime \prime}$. & Tang. & , \\
\hline
\end{tabular}


TANGENTS, AND COTANGENTS.

\begin{tabular}{|c|c|c|c|c|c|c|c|c|}
\hline , & Sine. & D. $1^{\prime \prime}$ 。 & Cosine. & D. 1". & Tang. & D. 1". & Cotang. & , \\
\hline $\begin{array}{r}0 \\
1 \\
2 \\
3 \\
4 \\
5 \\
6 \\
7 \\
8 \\
9 \\
10\end{array}$ & $\begin{array}{r}9.489982 \\
.490371 \\
.490759 \\
.491147 \\
.491535 \\
.491922 \\
.492308 \\
.492695 \\
.493081 \\
.493466 \\
.493851\end{array}$ & $\begin{array}{l}6.48 \\
6.47 \\
6.47 \\
6.47 \\
6.45 \\
6.43 \\
6.45 \\
6.43 \\
6.42 \\
6.42 \\
6.42\end{array}$ & $\begin{array}{r}9.978206 \\
.978165 \\
.978124 \\
.978083 \\
.978042 \\
.978001 \\
.977959 \\
.977918 \\
.977877 \\
.977835 \\
.977794\end{array}$ & $\begin{array}{l}.68 \\
.68 \\
.68 \\
.68 \\
.68 \\
.70 \\
.68 \\
.68 \\
.70 \\
.68 \\
.70\end{array}$ & $\begin{array}{r}9.511776 \\
.512206 \\
512635 \\
.513064 \\
.513493 \\
.513921 \\
.514349 \\
.514777 \\
.515204 \\
.515631 \\
.516057\end{array}$ & $\begin{array}{l}7.17 \\
7.15 \\
7.15 \\
7.15 \\
7.13 \\
7.13 \\
7.13 \\
7.12 \\
7.12 \\
7.10 \\
7\end{array}$ & $\begin{array}{r}10.488824 \\
.487794 \\
.487365 \\
.486936 \\
.486507 \\
.486079 \\
.485651 \\
.485223 \\
.484796 \\
.484369 \\
.483943\end{array}$ & $\begin{array}{l}60 \\
59 \\
58 \\
57 \\
56 \\
55 \\
54 \\
53 \\
52 \\
51 \\
50\end{array}$ \\
\hline $\begin{array}{l}11 \\
12 \\
13 \\
14 \\
15 \\
16 \\
17 \\
18 \\
19 \\
20\end{array}$ & $\begin{array}{r}9.494236 \\
.494621 \\
.495005 \\
.495388 \\
.495772 \\
.496154 \\
.496537 \\
.496919 \\
.497301 \\
.497682\end{array}$ & $\begin{array}{l}6.42 \\
6.40 \\
6.38 \\
6.40 \\
6.37 \\
6.38 \\
6.37 \\
6.37 \\
6.35 \\
6.35\end{array}$ & $\begin{array}{r}9.977752 \\
.977711 \\
.977669 \\
.977628 \\
977586 \\
.977544 \\
.977503 \\
.977461 \\
.977419 \\
.977377\end{array}$ & $\begin{array}{l}.68 \\
.70 \\
.68 \\
.70 \\
.70 \\
.68 \\
.70 \\
.70 \\
.70 \\
.70\end{array}$ & $\begin{array}{r}9.516484 \\
.516910 \\
.517335 \\
.517761 \\
.518186 \\
.518610 \\
.519034 \\
.519458 \\
.519882 \\
.520305\end{array}$ & $\begin{array}{l}7.10 \\
7.08 \\
7.10 \\
7.08 \\
7.07 \\
7.07 \\
7.07 \\
7.07 \\
7.05 \\
7.05\end{array}$ & $\begin{array}{r}10.483516 \\
.483090 \\
.482665 \\
.482239 \\
.481814 \\
.481390 \\
.480966 \\
.480542 \\
.480118 \\
.479695\end{array}$ & $\begin{array}{l}49 \\
48 \\
47 \\
46 \\
45 \\
44 \\
43 \\
42 \\
41 \\
40\end{array}$ \\
\hline $\begin{array}{l}21 \\
22 \\
23 \\
24 \\
25 \\
26 \\
27 \\
28 \\
29 \\
30\end{array}$ & $\begin{array}{r}9.498064 \\
.498444 \\
.498525 \\
.499204 \\
.499584 \\
.499963 \\
.500342 \\
.500721 \\
.501099 \\
.501476\end{array}$ & $\begin{array}{l}6.33 \\
6.35 \\
6.32 \\
6.33 \\
6.32 \\
6.32 \\
6.32 . \\
6.30 \\
6.28 \\
6.30\end{array}$ & $\begin{array}{r}9.977335 \\
.977293 \\
.977251 \\
.977209 \\
.977167 \\
.977125 \\
.977083 \\
.977041 \\
.976999 \\
.976957\end{array}$ & $\begin{array}{l}.70 \\
.70 \\
.70 \\
.70 \\
.70 \\
.70 \\
.70 \\
.70 \\
.70 \\
.72\end{array}$ & $\begin{array}{r}9.520728 \\
.521151 \\
.521573 \\
.521995 \\
.522417 \\
.522838 \\
.523259 \\
.523680 \\
.524100 \\
.524520\end{array}$ & $\begin{array}{l}7.05 \\
7.03 \\
7.03 \\
7.03 \\
7.02 \\
7.02 \\
7.02 \\
7.00 \\
7.00 \\
7.00\end{array}$ & $\begin{array}{r}10479272 \\
.478849 \\
.478427 \\
.478005 \\
.477583 \\
.477162 \\
.476741 \\
.476320 \\
.475900 \\
.475480\end{array}$ & $\begin{array}{l}39 \\
38 \\
37 \\
36 \\
35 \\
34 \\
33 \\
32 \\
31 \\
30\end{array}$ \\
\hline $\begin{array}{l}31 \\
32 \\
33 \\
34 \\
35 \\
36 \\
37 \\
38 \\
39 \\
40\end{array}$ & $\begin{array}{r}9.501854 \\
.502231 \\
.502607 \\
.502984 \\
.503360 \\
.503735 \\
.504110 \\
.504485 \\
.504860 \\
.505234\end{array}$ & $\begin{array}{l}6.28 \\
6.27 \\
6.28 \\
6.27 \\
6.25 \\
6.25 \\
6.25 \\
6.25 \\
6.23 \\
6.23\end{array}$ & $\begin{array}{r}9.976914 \\
.976872 \\
.976830 \\
.976787 \\
.976745 \\
.976702 \\
.976660 \\
.976617 \\
.976574 \\
.976532\end{array}$ & $\begin{array}{l}.70 \\
.70 \\
.72 \\
.70 \\
.72 \\
.70 \\
.72 \\
.72 \\
.70 \\
.72\end{array}$ & $\begin{array}{r}9.524940 \\
.525359 \\
.525778 \\
.526197 \\
.526615 \\
.527033 \\
.527451 \\
.527868 \\
.528285 \\
.525702\end{array}$ & $\begin{array}{l}6.98 \\
6.98 \\
6.98 \\
6.97 \\
6.97 \\
6.97 \\
6.95 \\
6.95 \\
6.95 \\
6.95\end{array}$ & $\begin{array}{r}10.475060 \\
.474641 \\
.474222 \\
.473803 \\
.473385 \\
.472967 \\
.472549 \\
.472132 \\
.471715 \\
.471298\end{array}$ & $\begin{array}{l}29 \\
28 \\
27 \\
26 \\
25 \\
24 \\
23 \\
22 \\
21 \\
20\end{array}$ \\
\hline $\begin{array}{l}41 \\
42 \\
43 \\
44 \\
45 \\
46 \\
47 \\
48 \\
49 \\
50\end{array}$ & $\begin{array}{r}9.505608 \\
.505951 \\
.506354 \\
.506727 \\
.507099 \\
.507471 \\
.507843 \\
.508214 \\
.508585 \\
.508956\end{array}$ & $\begin{array}{l}6.22 \\
6.22 \\
6.22 \\
6.20 \\
6.20 \\
6.20 \\
6.18 \\
6.18 \\
6.18 \\
6.17\end{array}$ & $\begin{array}{r}9.976489 \\
.976446 \\
.976404 \\
.976361 \\
.976318 \\
.976275 \\
.976232 \\
.976189 \\
.976146 \\
.976103\end{array}$ & $\begin{array}{l}.72 \\
.70 \\
.72 \\
.72 \\
.72 \\
.72 \\
.72 \\
.72 \\
.72 \\
.72\end{array}$ & $\begin{array}{r}9.529119 \\
.529535 \\
.529251 \\
.530360 \\
.530781 \\
.531196 \\
531611 \\
.532025 \\
.532439 \\
.532553\end{array}$ & $\begin{array}{l}6.93 \\
6.93 \\
6.92 \\
6.92 \\
6.92 \\
6.92 \\
6.90 \\
6.90 \\
6.90 \\
6.88\end{array}$ & $\begin{array}{r}10.470881 \\
.470465 \\
.470049 \\
.469634 \\
.469219 \\
.468504 \\
.468389 \\
.467975 \\
.467561 \\
.467147\end{array}$ & $\begin{array}{l}19 \\
18 \\
17 \\
16 \\
15 \\
14 \\
13 \\
12 \\
11 \\
10\end{array}$ \\
\hline $\begin{array}{l}51 \\
52 \\
53 \\
54 \\
55 \\
56 \\
57 \\
58 \\
59 \\
60\end{array}$ & $\begin{array}{r}9.509326 \\
.509696 \\
.510065 \\
.510434 \\
.510803 \\
.511172 \\
.511540 \\
.511907 \\
.512275 \\
9.512642\end{array}$ & $\begin{array}{l}6.17 \\
6.15 \\
6.15 \\
6.15 \\
6.15 \\
6.13 \\
6.12 \\
6.13 \\
6.12\end{array}$ & $\begin{array}{r}9.976060 \\
.976017 \\
.975974 \\
.975930 \\
.975857 \\
.975844 \\
.975800 \\
.975757 \\
.975714 \\
9.975670\end{array}$ & $\begin{array}{l}.72 \\
.72 \\
.73 \\
.72 \\
.72 \\
.73 \\
.72 \\
.72 \\
.73\end{array}$ & $\begin{array}{r}9.533266 \\
.533679 \\
.534092 \\
.534504 \\
.534916 \\
.535328 \\
.535739 \\
.536150 \\
.536561 \\
9.536972\end{array}$ & $\begin{array}{l}6.88 \\
6.88 \\
6.87 \\
6.87 \\
6.87 \\
6.85 \\
6.85 \\
6.85 \\
6.85\end{array}$ & $\begin{array}{r}10.466734 \\
.466321 \\
.465908 \\
.465406 \\
.465084 \\
.464672 \\
.464261 \\
.463550 \\
.463439 \\
10.163028\end{array}$ & $\begin{array}{l}9 \\
8 \\
7 \\
6 \\
5 \\
4 \\
3 \\
2 \\
1 \\
0\end{array}$ \\
\hline & Cosine. & D. 1". & Sine. & D. $1^{\prime \prime}$ & Cotang. & D. $1^{\prime \prime}$. & Tang. & ' \\
\hline
\end{tabular}




\begin{tabular}{|c|c|c|c|c|c|c|c|c|}
\hline , & Sine. & D. $1^{\prime \prime}$. & Cosine. & D. $1^{\prime \prime}$. & Tang. & D. $1^{\prime \prime}$. & Cotang. & , \\
\hline $\begin{array}{r}0 \\
1 \\
2 \\
3 \\
4 \\
5 \\
6 \\
7 \\
8 \\
9 \\
10\end{array}$ & $\begin{array}{r}9.512642 \\
.513009 \\
.513375 \\
.513741 \\
.514107 \\
.514472 \\
.514837 \\
.515202 \\
.515566 \\
.515930 \\
.516294\end{array}$ & $\begin{array}{l}6.12 \\
6.10 \\
6.10 \\
6.10 \\
6.08 \\
6.08 \\
6.08 \\
6.07 \\
6.07 \\
6.07 \\
6.05\end{array}$ & $\begin{array}{r}9.975670 \\
.975627 \\
.975583 \\
.975539 \\
.975496 \\
.975452 \\
.975408 \\
.975365 \\
.975321 \\
.975277 \\
.975233\end{array}$ & $\begin{array}{l}.72 \\
.73 \\
.73 \\
.72 \\
.73 \\
.73 \\
.72 \\
.73 \\
.73 \\
.73 \\
.73\end{array}$ & $\begin{array}{r}9.536972 \\
.537382 \\
.537792 \\
.538202 \\
.538611 \\
.539020 \\
.539429 \\
.539837 \\
.540245 \\
.540663 \\
.541061\end{array}$ & $\begin{array}{l}6.83 \\
6.83 \\
6.83 \\
6.82 \\
6.82 \\
6.82 \\
6.80 \\
6.80 \\
6.80 \\
6.80 \\
6.78\end{array}$ & $\begin{array}{r}10.463028 \\
.462618 \\
.462208 \\
.461798 \\
.461389 \\
.460980 \\
.460571 \\
.460163 \\
.459755 \\
.459347 \\
.455939\end{array}$ & $\begin{array}{l}60 \\
59 \\
58 \\
57 \\
56 \\
55 \\
54 \\
53 \\
52 \\
51 \\
50\end{array}$ \\
\hline $\begin{array}{l}11 \\
12 \\
13 \\
14 \\
15 \\
16 \\
17 \\
18 \\
19 \\
20\end{array}$ & $\begin{array}{r}9.516657 \\
.517020 \\
.517382 \\
.517745 \\
.518107 \\
.518468 \\
.518829 \\
.519190 \\
.519551 \\
.519911\end{array}$ & $\begin{array}{l}6.05 \\
6.03 \\
6.05 \\
6.03 \\
6.02 \\
6.02 \\
6.02 \\
6.02 \\
6.00 \\
6.00\end{array}$ & $\begin{array}{r}9.975189 \\
.975145 \\
.975101 \\
.975057 \\
.975013 \\
.974969 \\
.974925 \\
.974880 \\
.974836 \\
.974792\end{array}$ & $\begin{array}{l}.73 \\
.73 \\
.73 \\
.73 \\
.73 \\
.73 \\
.75 \\
.73 \\
.73 \\
.73\end{array}$ & $\begin{array}{r}9.541468 \\
.541875 \\
.542281 \\
.542688 \\
.543094 \\
.543499 \\
.543905 \\
.544310 \\
.544715 \\
.545119\end{array}$ & \begin{tabular}{l|}
6.78 \\
6.77 \\
6.78 \\
6.77 \\
6.75 \\
6.77 \\
6.75 \\
6.75 \\
6.73 \\
6.75
\end{tabular} & $\begin{array}{r}10.458532 \\
.458125 \\
.457719 \\
.457312 \\
.456906 \\
.456501 \\
.456095 \\
.455690 \\
.455285 \\
.454881\end{array}$ & $\begin{array}{l}49 \\
48 \\
47 \\
46 \\
45 \\
44 \\
43 \\
42 \\
41 \\
40\end{array}$ \\
\hline $\begin{array}{l}21 \\
22 \\
23 \\
24 \\
25 \\
26 \\
27 \\
28 \\
29 \\
30\end{array}$ & $\begin{array}{r}9.520271 \\
.520631 \\
.520990 \\
.521349 \\
.521707 \\
.522066 \\
.522424 \\
.522781 \\
.523138 \\
.523495\end{array}$ & $\begin{array}{l}6.00 \\
5.98 \\
5.98 \\
5.97 \\
5.98 \\
5.97 \\
5.95 \\
5.95 \\
5.95 \\
5.95\end{array}$ & $\begin{array}{r}9.974748 \\
.974703 \\
.974659 \\
.974614 \\
.974570 \\
.974525 \\
.974481 \\
.974436 \\
.974391 \\
.974347\end{array}$ & $\begin{array}{l}.75 \\
.73 \\
.75 \\
.73 \\
.75 \\
.73 \\
.75 \\
.75 \\
.73 \\
.75\end{array}$ & $\begin{array}{r}\mathbf{9 . 5 4 5 5 2 4} \\
.545928 \\
.546331 \\
.546735 \\
.547138 \\
.547540 \\
.547943 \\
.548345 \\
.548747 \\
.549149\end{array}$ & $\begin{array}{l}6.73 \\
6.72 \\
6.73 \\
6.72 \\
6.70 \\
6.72 \\
6.70 \\
6.70 \\
6.70 \\
6.68\end{array}$ & $\begin{array}{r}10.454476 \\
.454072 \\
.453669 \\
.453265 \\
.452862 \\
.452460 \\
.452057 \\
.451655 \\
.451253 \\
.450851\end{array}$ & $\begin{array}{l}39 \\
38 \\
37 \\
30 \\
35 \\
34 \\
33 \\
32 \\
31 \\
30\end{array}$ \\
\hline $\begin{array}{l}31 \\
32 \\
33 \\
34 \\
35 \\
36 \\
37 \\
38 \\
39 \\
40\end{array}$ & $\begin{array}{r}9.523852 \\
.524208 \\
.524564 \\
.524920 \\
.525275 \\
.525630 \\
.525984 \\
.526339 \\
.526693 \\
.527046\end{array}$ & $\begin{array}{l}5.93 \\
5.93 \\
5.93 \\
5.92 \\
5.92 \\
5.90 \\
5.92 \\
5.90 \\
5.88 \\
5.90\end{array}$ & $\begin{array}{r}9.974302 \\
.974257 \\
.974212 \\
.974167 \\
.974122 \\
.974077 \\
.974032 \\
.973987 \\
.973942 \\
.973897\end{array}$ & $\begin{array}{l}.75 \\
.75 \\
.75 \\
.75 \\
.75 \\
.75 \\
.75 \\
.75 \\
.75 \\
.75\end{array}$ & $\begin{array}{r}9.549550 \\
.549951 \\
.550352 \\
.550752 \\
.551153 \\
.551552 \\
.551952 \\
.552351 \\
.552750 \\
.553149\end{array}$ & $\begin{array}{l}6.68 \\
6.68 \\
6.67 \\
6.68 \\
6.65 \\
6.67 \\
6.65 \\
6.65 \\
6.65 \\
6.65\end{array}$ & $\begin{array}{r}10.450450 \\
.450049 \\
.449648 \\
.449248 \\
.448847 \\
.448448 \\
.448048 \\
.447649 \\
.447250 \\
.446851\end{array}$ & $\begin{array}{l}29 \\
28 \\
27 \\
26 \\
25 \\
24 \\
23 \\
22 \\
21\end{array}$ \\
\hline $\begin{array}{l}41 \\
42 \\
43 \\
44 \\
45 \\
46 \\
47 \\
48 \\
49 \\
50\end{array}$ & $\begin{array}{r}9.527400 \\
.527753 \\
.528105 \\
.528458 \\
.528810 \\
.529161 \\
.529513 \\
.529864 \\
.530215 \\
.530565\end{array}$ & $\begin{array}{l}5.88 \\
5.87 \\
5.88 \\
5.87 \\
5.85 \\
5.87 \\
5.85 \\
5.85 \\
5.83 \\
5.83\end{array}$ & $\begin{array}{r}9.973852 \\
.973807 \\
.973761 \\
.973716 \\
.973671 \\
.973625 \\
.973580 \\
.973535 \\
.973489 \\
.973444\end{array}$ & $\begin{array}{l}.75 \\
.77 \\
.75 \\
.75 \\
.77 \\
.75 \\
.75 \\
.77 \\
.75 \\
.77\end{array}$ & $\begin{array}{r}9.553548 \\
.553946 \\
.554344 \\
.554741 \\
.555139 \\
.555536 \\
.555933 \\
.556329 \\
.556725 \\
.557121\end{array}$ & $\begin{array}{l}6.63 \\
6.63 \\
6.62 \\
6.63 \\
6.62 \\
6.62 \\
6.60 \\
6.60 \\
6.60 \\
6.60\end{array}$ & $\begin{array}{r}10.446452 \\
.446054 \\
.445656 \\
.445259 \\
.444861 \\
.444464 \\
.444067 \\
.443671 \\
.443275 \\
.442879\end{array}$ & $\begin{array}{l}19 \\
18 \\
17 \\
16 \\
15 \\
14 \\
13 \\
12\end{array}$ \\
\hline $\begin{array}{l}51 \\
52 \\
53 \\
54 \\
55 \\
56 \\
57 \\
58 \\
59 \\
60\end{array}$ & $\begin{array}{r}9.530915 \\
.531265 \\
.531614 \\
.531903 \\
.532312 \\
.532661 \\
.533009 \\
.533357 \\
.533704 \\
9.534052\end{array}$ & $\begin{array}{l}5.83 \\
5.82 \\
5.82 \\
5.82 \\
5.82 \\
5.80 \\
5.80 \\
5.78 \\
5.80\end{array}$ & $\begin{array}{r}9.973398 \\
.973352 \\
.973307 \\
.973261 \\
.973215 \\
.973169 \\
.973124 \\
.973078 \\
.973032 \\
9.972986\end{array}$ & $\begin{array}{l}.77 \\
.75 \\
.77 \\
.77 \\
.77 \\
.75 \\
.77 \\
.77 \\
.77\end{array}$ & $\begin{array}{r}9.557517 \\
.557913 \\
.558308 \\
.558703 \\
.559097 \\
.559491 \\
.559885 \\
.560279 \\
.560673 \\
9.561066\end{array}$ & $\begin{array}{l}6.60 \\
6.58 \\
6.58 \\
6.57 \\
6.57 \\
6.57 \\
6.57 \\
6.57 \\
6.55\end{array}$ & $\begin{array}{r}10.442483 \\
.442087 \\
.441692 \\
.441297 \\
.440903 \\
.440509 \\
.440115 \\
.439721 \\
.439327 \\
10.438934\end{array}$ & $\begin{array}{l}9 \\
8 \\
7 \\
6 \\
5 \\
4 \\
3 \\
2 \\
1 \\
0\end{array}$ \\
\hline ' & Cosine. & D. $1^{\prime \prime}$. & Sine. & D. $1^{\prime \prime}$. & Cotang. & D. $1^{\prime \prime}$. & Tang. & ' \\
\hline
\end{tabular}


TANGENTS, AND COTANGENTS.

\begin{tabular}{|c|c|c|c|c|c|c|c|c|}
\hline , & Sine. & D. $1^{\prime \prime}$. & Cosine. & D. $1^{\prime \prime}$. & Tang. & D. $1^{\prime \prime}$. & Cotang. & , \\
\hline $\begin{array}{r}0 \\
1 \\
2 \\
3 \\
4 \\
5 \\
6 \\
7 \\
8 \\
9 \\
10\end{array}$ & $\begin{array}{r}9.534052 \\
.534399 \\
.534745 \\
.535092 \\
.535438 \\
.535783 \\
.536129 \\
.536474 \\
.536818 \\
.537163 \\
.537507\end{array}$ & $\begin{array}{l}5.78 \\
5.77 \\
5.78 \\
5.77 \\
5.75 \\
5.77 \\
5.75 \\
5.73 \\
5.75 \\
5.73 \\
5.73\end{array}$ & $\begin{array}{r}9.972986 \\
.972940 \\
.972894 \\
.972848 \\
.972 \times 02 \\
.972755 \\
.972709 \\
.972603 \\
.972617 \\
.972570 \\
.972524\end{array}$ & $\begin{array}{l}.77 \\
.77 \\
.77 \\
.77 \\
.78 \\
.77 \\
.77 \\
.77 \\
.78 \\
.77 \\
.77\end{array}$ & $\begin{array}{r}9.561066 \\
.561459 \\
.561551 \\
.562244 \\
.562636 \\
.563028 \\
.563419 \\
.563511 \\
.564202 \\
.564593 \\
.564983\end{array}$ & $\begin{array}{l}6.55 \\
6.53 \\
6.55 \\
6.53 \\
6.53 \\
6.52 \\
6.53 \\
6.52 \\
6.52 \\
6.50\end{array}$ & $\begin{array}{r}10.435934 \\
.435541 \\
.438149 \\
.437756 \\
.437364 \\
.436972 \\
.436581 \\
.436189 \\
.435798 \\
.435407 \\
.435017\end{array}$ & $\begin{array}{l}60 \\
59 \\
58 \\
57 \\
56 \\
55 \\
54 \\
53 \\
52 \\
51 \\
50\end{array}$ \\
\hline $\begin{array}{l}11 \\
12 \\
13 \\
14 \\
15 \\
16 \\
17 \\
18 \\
19 \\
20\end{array}$ & $\begin{array}{r}9.537851 \\
.538194 \\
.535538 \\
.535550 \\
.539223 \\
.539565 \\
.539907 \\
.540249 \\
.540590 \\
.540931\end{array}$ & $\begin{array}{l}5.72 \\
5.73 \\
5.70 \\
5.72 \\
5.70 \\
5.70 \\
5.68 \\
5.68 \\
5.68 \\
5.68\end{array}$ & $\begin{array}{r}9.972478 \\
.972: 31 \\
.972385 \\
.972338 \\
.972291 \\
.972245 \\
.972198 \\
.972151 \\
.972105 \\
.972058\end{array}$ & $\begin{array}{l}.78 \\
.77 \\
.78 \\
.78 \\
.77 \\
.78 \\
.78 \\
.77 \\
.78 \\
.78\end{array}$ & $\begin{array}{r}9.565373 \\
.565763 \\
.566153 \\
.566542 \\
.566932 \\
.567320 \\
.567709 \\
.568098 \\
.568486 \\
.568873\end{array}$ & $\begin{array}{l}6.50 \\
6.50 \\
6.48 \\
6.50 \\
6.47 \\
6.48 \\
6.48 \\
6.47 \\
6.45 \\
6.47\end{array}$ & $\begin{array}{r}10.434627 \\
.434237 \\
.433847 \\
.433458 \\
.433018 \\
.432680 \\
.432291 \\
.431902 \\
.431514 \\
.431127\end{array}$ & $\begin{array}{l}49 \\
48 \\
47 \\
46 \\
45 \\
44 \\
43 \\
42 \\
41 \\
40\end{array}$ \\
\hline $\begin{array}{l}21 \\
22 \\
23 \\
24 \\
25 \\
26 \\
27 \\
28 \\
29 \\
30\end{array}$ & $\begin{array}{r}9.541272 \\
.541613 \\
.541953 \\
.542293 \\
.542632 \\
.542971 \\
.543310 \\
.543649 \\
.543987 \\
.544325\end{array}$ & $\begin{array}{l}5.68 \\
5.67 \\
5.67 \\
5.65 \\
5.65 \\
5.65 \\
5.65 \\
5.63 \\
5.63 \\
5.63\end{array}$ & $\begin{array}{r}9.972011 \\
.971964 \\
.971917 \\
.971870 \\
.971823 \\
.971776 \\
.971729 \\
.971682 \\
.971635 \\
.971588\end{array}$ & $\begin{array}{l}.78 \\
.78 \\
.78 \\
.78 \\
.78 \\
.78 \\
.78 \\
.80\end{array}$ & $\begin{array}{r}9.569261 \\
.569648 \\
.570035 \\
.570422 \\
.570809 \\
.571195 \\
.571581 \\
.571967 \\
.572352 \\
.572738\end{array}$ & $\begin{array}{l}6.45 \\
6.45 \\
6.45 \\
6.45 \\
6.43 \\
6.43 \\
6.43 \\
6.42 \\
6.43 \\
6.42\end{array}$ & $\begin{array}{r}10.430739 \\
.430352 \\
.429965 \\
.429578 \\
.429191 \\
.425805 \\
.425419 \\
.425033 \\
.427648 \\
.427262\end{array}$ & $\begin{array}{l}39 \\
38 \\
37 \\
36 \\
35 \\
34 \\
33 \\
32 \\
31 \\
30\end{array}$ \\
\hline $\begin{array}{l}31 \\
32 \\
33 \\
34 \\
35 \\
36 \\
37 \\
38 \\
39 \\
40\end{array}$ & $\begin{array}{r}9.544063 \\
.545000 \\
.545338 \\
.545074 \\
.546011 \\
.546347 \\
.546683 \\
.547019 \\
.547354 \\
.547689\end{array}$ & $\begin{array}{l}5.62 \\
5.63 \\
5.60 \\
5.62 \\
5.60 \\
5.60 \\
5.60 \\
5.58 \\
5.58 \\
5.58\end{array}$ & $\begin{array}{r}9.971540 \\
.971493 \\
.971446 \\
.971398 \\
.971351 \\
.971303 \\
.971256 \\
.971208 \\
.971161 \\
.971113\end{array}$ & $\begin{array}{r}.80 \\
.78 \\
.80 \\
.78 \\
.80 \\
.78\end{array}$ & $\begin{array}{r}9.573123 \\
.573507 \\
.573592 \\
.574276 \\
.574660 \\
.575044 \\
.575427 \\
.575810 \\
.576193 \\
.576576\end{array}$ & $\begin{array}{l}6.40 \\
6.42 \\
6.40 \\
6.40 \\
6.40 \\
6.33 \\
6.38 \\
6.35 \\
6.39\end{array}$ & $\begin{array}{r}10.426877 \\
.426493 \\
.426105 \\
.425724 \\
.425340 \\
.424956 \\
.424573 \\
.424190 \\
.423807 \\
.423424\end{array}$ & $\begin{array}{l}29 \\
28 \\
27 \\
26 \\
25 \\
24 \\
23 \\
22 \\
21 \\
20\end{array}$ \\
\hline $\begin{array}{l}41 \\
42 \\
43 \\
44 \\
45 \\
46 \\
47 \\
48 \\
49 \\
50\end{array}$ & $\begin{array}{r}9.545024 \\
.548359 \\
.545693 \\
.549027 \\
.549360 \\
.549693 \\
.550026 \\
.550359 \\
.550692 \\
.551024\end{array}$ & $\begin{array}{l}5.58 \\
5.57 \\
5.57 \\
5.55 \\
5.55 \\
5.55 \\
5.55 \\
5.55 \\
5.53 \\
5.53\end{array}$ & $\begin{array}{r}9.971066 \\
.971018 \\
.970970 \\
.970922 \\
.970874 \\
.970527 \\
.970779 \\
.970731 \\
.970653 \\
.970635\end{array}$ & $\begin{array}{r}.80 \\
.80 \\
.80 \\
.80 \\
.78 \\
.80\end{array}$ & $\begin{array}{r}9.576959 \\
.577341 \\
.577723 \\
.578104 \\
.578486 \\
.578867 \\
.579248 \\
.579629 \\
.550009 \\
.550359\end{array}$ & $\begin{array}{l}6.37 \\
6.37 \\
6.35 \\
6.37 \\
6.35 \\
6.35 \\
6.35 \\
6.33\end{array}$ & $\begin{array}{r}10.423041 \\
.422059 \\
.422277 \\
.421896 \\
.421514 \\
.421133 \\
.420752 \\
.420371 \\
.419991 \\
.4190611\end{array}$ & $\begin{array}{l}19 \\
18 \\
17 \\
16 \\
15 \\
14 \\
13 \\
12 \\
11 \\
10\end{array}$ \\
\hline $\begin{array}{l}51 \\
52 \\
53 \\
54 \\
55 \\
56 \\
57 \\
58 \\
59 \\
60\end{array}$ & $\begin{array}{r}9.551356 \\
.551687 \\
.552018 \\
.552349 \\
.552680 \\
.553010 \\
.553341 \\
.553670 \\
.554000 \\
9.554329\end{array}$ & $\begin{array}{l}5.52 \\
5.52 \\
5.52 \\
5.52 \\
5.50 \\
5.52 \\
5.48 \\
5.50 \\
5.48\end{array}$ & $\begin{array}{r}9.970580 \\
.970538 \\
.970490 \\
.970442 \\
.970394 \\
.970345 \\
.970297 \\
.970249 \\
.970200 \\
9.970152\end{array}$ & $\begin{array}{l}.80 \\
.80 \\
.80 \\
.80 \\
.82 \\
.80 \\
.80 \\
.82 \\
.80\end{array}$ & $\begin{array}{r}9.580769 \\
.581149 \\
.581528 \\
.581207 \\
.582286 \\
.582665 \\
.583044 \\
.583422 \\
.583800 \\
9.584177\end{array}$ & $\begin{array}{l}\text { 6. } 33 \\
6.32 \\
6.32 \\
6.32 \\
6.32 \\
6.32 \\
6.30 \\
6.30 \\
6.35\end{array}$ & $\begin{array}{r}10.419231 \\
.418851 \\
.418472 \\
.418093 \\
.417714 \\
.417335 \\
.416956 \\
.416578 \\
.41 \% 200 \\
10.415623\end{array}$ & $\begin{array}{l}9 \\
8 \\
7 \\
6 \\
5 \\
4 \\
3 \\
2 \\
1 \\
0\end{array}$ \\
\hline ' & Cosine. & $\mathrm{D} 1^{\prime \prime}$ & Sine. & D. 1". & Cotang. & D. 1 & Tang. & \\
\hline
\end{tabular}




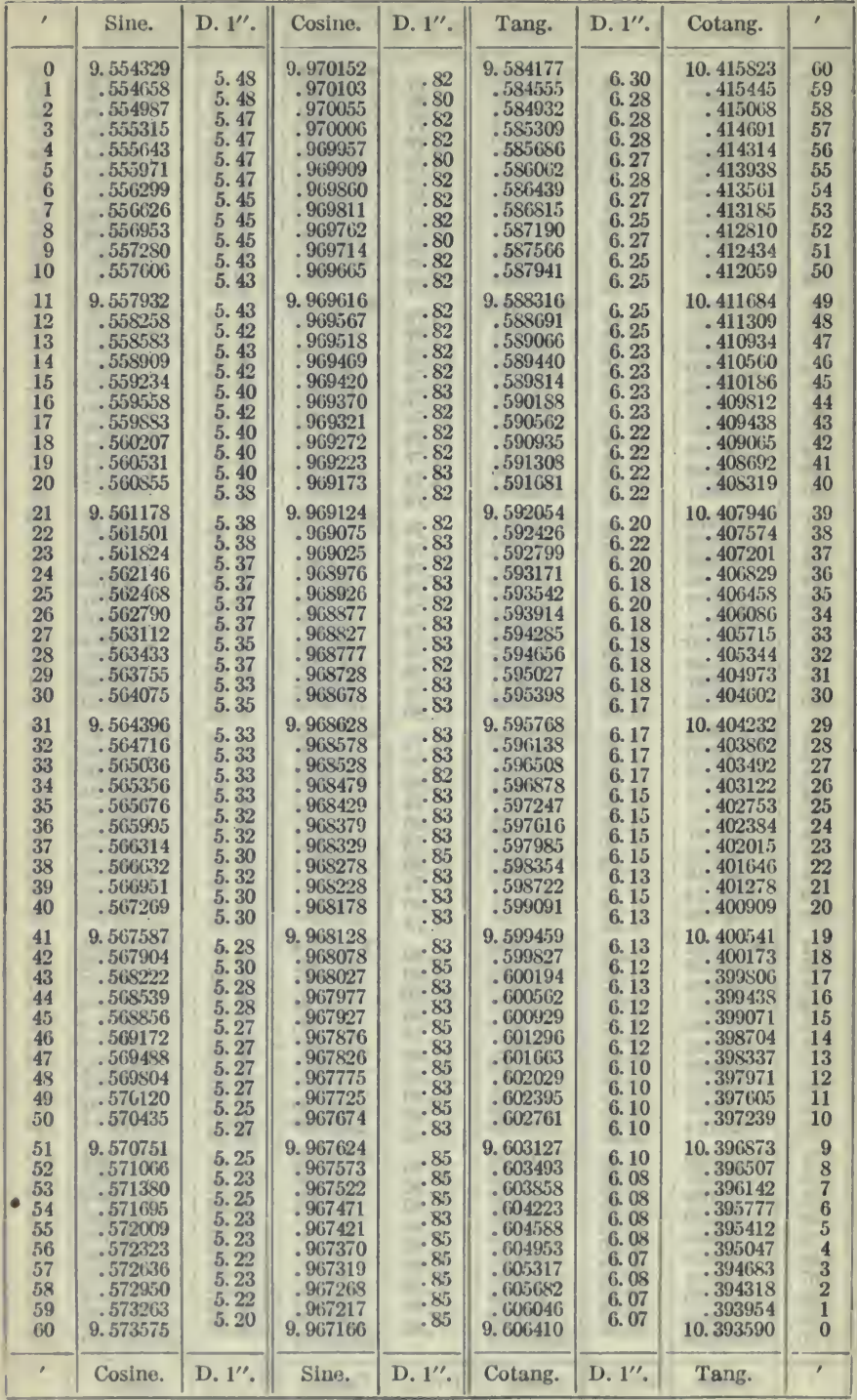




\begin{tabular}{|c|c|c|c|c|c|c|c|c|}
\hline ' & Sine. & D. $1^{\prime \prime}$. & Cosine. & D. $1^{\prime \prime}$ 。 & Tang. & D. $1^{\prime \prime}$. & Cotang. & , \\
\hline $\begin{array}{r}0 \\
1 \\
2 \\
3 \\
4 \\
5 \\
6 \\
7 \\
8 \\
9 \\
10\end{array}$ & $\begin{array}{r}9.573575 \\
.573888 \\
.574200 \\
.574512 \\
.574824 \\
.575136 \\
.575447 \\
.575758 \\
.576069 \\
.576379 \\
.576059\end{array}$ & $\begin{array}{l}5.22 \\
5.20 \\
5.20 \\
5.20 \\
5.20 \\
5.18 \\
5.18 \\
5.18 \\
5.17 \\
5.17 \\
5.17\end{array}$ & $\begin{array}{r}9.967160 \\
.967115 \\
.967064 \\
.967013 \\
.966961 \\
.966910 \\
.966859 \\
.966808 \\
.966756 \\
.966705 \\
.966653\end{array}$ & $\begin{array}{l}.85 \\
.85 \\
.85 \\
.87 \\
.85 \\
.85 \\
.85 \\
.87 \\
.85 \\
.87 \\
.85\end{array}$ & $\begin{array}{r}9.606410 \\
.606773 \\
.607137 \\
.607500 \\
.607863 \\
.608225 \\
.608583 \\
.603950 \\
.609312 \\
.609674 \\
.610036\end{array}$ & $\begin{array}{l}6.05 \\
6.07 \\
6.05 \\
6.05 \\
6.03 \\
6.05 \\
6.03 \\
6.03 \\
6.03 \\
6.03 \\
6.02\end{array}$ & $\begin{array}{r}10393590 \\
.393227 \\
.392863 \\
.392500 \\
.392137 \\
.391775 \\
.391412 \\
.391050 \\
.390688 \\
.390326 \\
.389964\end{array}$ & $\begin{array}{l}60 \\
59 \\
58 \\
57 \\
56 \\
55 \\
54 \\
53 \\
52 \\
51 \\
50\end{array}$ \\
\hline $\begin{array}{l}11 \\
12 \\
13 \\
14 \\
15 \\
16 \\
17 \\
18 \\
19 \\
20\end{array}$ & $\begin{array}{r}9.576999 \\
.577309 \\
.577618 \\
.577927 \\
.578236 \\
.578545 \\
.578853 \\
.579162 \\
.579470 \\
.579777\end{array}$ & $\begin{array}{l}5.17 \\
5.15 \\
5.15 \\
5.15 \\
5.15 \\
5.13 \\
5.15 \\
5.13 \\
5.12 \\
5.13\end{array}$ & $\begin{array}{r}9.966602 \\
.9665550 \\
.966499 \\
.966147 \\
.966395 \\
.966344 \\
.966292 \\
.966240 \\
.966188 \\
.966136\end{array}$ & $\begin{array}{l}.87 \\
.85 \\
.87 \\
.87 \\
.85 \\
.87 \\
.87 \\
.87 \\
.87 \\
.85\end{array}$ & $\begin{array}{r}9.610397 \\
.610759 \\
.611120 \\
.611480 \\
.611841 \\
.612201 \\
.612561 \\
.612921 \\
.613281 \\
.613641\end{array}$ & $\begin{array}{l}6.03 \\
6.02 \\
6.00 \\
6.02 \\
6.00 \\
6.00 \\
6.00 \\
6.00 \\
6.00 \\
5.98\end{array}$ & $\begin{array}{r}10.389603 \\
.389241 \\
.388850 \\
.388520 \\
.388159 \\
.387799 \\
.387439 \\
.387079 \\
.386719 \\
.386359\end{array}$ & $\begin{array}{l}49 \\
48 \\
47 \\
46 \\
45 \\
44 \\
43 \\
42 \\
41 \\
40\end{array}$ \\
\hline $\begin{array}{l}21 \\
22 \\
23 \\
24 \\
25 \\
26 \\
27 \\
28 \\
29 \\
30\end{array}$ & $\begin{array}{r}9.580085 \\
.580392 \\
.580699 \\
.581005 \\
.581312 \\
.581618 \\
.581924 \\
.552229 \\
.582535 \\
.582840\end{array}$ & $\begin{array}{l}5.12 \\
5.12 \\
5.10 \\
5.12 \\
5.10 \\
5.10 \\
5.08 \\
5.10 \\
5.08 \\
5.08\end{array}$ & $\begin{array}{r}9.966085 \\
.966033 \\
.9655981 \\
.9659929 \\
.965876 \\
.965824 \\
.9657772 \\
.965720 \\
.965668 \\
.965615\end{array}$ & $\begin{array}{l}.87 \\
.87 \\
.87 \\
.88 \\
.87 \\
.87 \\
.87 \\
.87 \\
.88 \\
.87\end{array}$ & $\begin{array}{r}9.614000 \\
.614359 \\
.614718 \\
.615077 \\
.615435 \\
.615793 \\
.616151 \\
.616509 \\
.616867 \\
.617224\end{array}$ & $\begin{array}{l}5.98 \\
5.98 \\
5.98 \\
5.97 \\
5.97 \\
5.97 \\
5.97 \\
5.97 \\
5.95 \\
5.97\end{array}$ & $\begin{array}{r}10.386000 \\
.385641 \\
.385282 \\
.384923 \\
.384565 \\
.384207 \\
.383849 \\
.383491 \\
.383133 \\
.382776\end{array}$ & $\begin{array}{l}39 \\
38 \\
37 \\
36 \\
35 \\
34 \\
33 \\
32 \\
31 \\
30\end{array}$ \\
\hline $\begin{array}{l}31 \\
32 \\
33 \\
34 \\
35 \\
36 \\
37 \\
38 \\
39 \\
40\end{array}$ & $\begin{array}{r}9.583145 \\
.583449 \\
.583754 \\
.584058 \\
.584361 \\
.584665 \\
.584968 \\
.585272 \\
.585574 \\
.585877\end{array}$ & $\begin{array}{l}5.07 \\
5.08 \\
5.07 \\
5.05 \\
5.07 \\
5.05 \\
5.07 \\
5.03 \\
5.05 \\
5.03\end{array}$ & $\begin{array}{r}9.965563 \\
.965511 \\
.965458 \\
.965406 \\
.965353 \\
.965301 \\
.965248 \\
.965195 \\
.965143 \\
.965090\end{array}$ & $\begin{array}{l}.87 \\
.88 \\
.87 \\
.88 \\
.89 \\
.87\end{array}$ & $\begin{array}{r}9.617582 \\
.617939 \\
.618295 \\
.618152 \\
.619008 \\
.619364 \\
.619720 \\
.620076 \\
.620432 \\
.620787\end{array}$ & $\begin{array}{l}5.95 \\
5.93 \\
5.95 \\
5.93 \\
5.93 \\
5.93 \\
5.93 \\
5.93 \\
5.92 \\
5.92\end{array}$ & $\begin{array}{r}10.382418 \\
.382061 \\
.381705 \\
.381348 \\
.380992 \\
.330636 \\
.380250 \\
.379924 \\
.3795 t i 8 \\
.379213\end{array}$ & $\begin{array}{l}29 \\
23 \\
27 \\
26 \\
25 \\
24 \\
23 \\
22 \\
21 \\
20\end{array}$ \\
\hline $\begin{array}{l}41 \\
42 \\
43 \\
44 \\
45 \\
46 \\
47 \\
48 \\
49 \\
50\end{array}$ & $\begin{array}{r}9.586179 \\
.586482 \\
.586783 \\
.587085 \\
.587356 \\
.587688 \\
.587959 \\
.588289 \\
.588590 \\
.588590\end{array}$ & $\begin{array}{l}5.05 \\
5.02 \\
5.03 \\
5.02 \\
5.03 \\
5.02 \\
5.00 \\
5.02 \\
5.00 \\
5.00\end{array}$ & $\begin{array}{r}9.965037 \\
.964984 \\
.964931 \\
.964879 \\
.964526 \\
.964773 \\
.964720 \\
.964666 \\
.964613 \\
.964560\end{array}$ & .88 & $\begin{array}{r}9.621142 \\
.621497 \\
.621852 \\
.622207 \\
.622561 \\
.622915 \\
.623269 \\
.623023 \\
.623976 \\
.624330\end{array}$ & $\begin{array}{l}5.92 \\
5.92 \\
5.92 \\
5.90 \\
5.90 \\
5.90 \\
5.90 \\
5.89 \\
5.90 \\
5.89\end{array}$ & $\begin{array}{r}10.378858 \\
.378503 \\
.378145 \\
.377793 \\
.377439 \\
.377085 \\
.376731 \\
.376377 \\
.376024 \\
.375670\end{array}$ & $\begin{array}{l}19 \\
18 \\
17 \\
16 \\
15 \\
14 \\
13 \\
12 \\
11 \\
10\end{array}$ \\
\hline $\begin{array}{l}51 \\
52 \\
53 \\
54 \\
55 \\
56 \\
57 \\
58 \\
59 \\
60\end{array}$ & $\begin{array}{r}9.559190 \\
.589489 \\
.589759 \\
.5900<8 \\
.590387 \\
.5904 i 86 \\
.5904384 \\
.591282 \\
.591580 \\
9.591878\end{array}$ & $\begin{array}{l}4.98 \\
5.00 \\
4.98 \\
4.98 \\
4.98 \\
4.97 \\
4.97 \\
4.97 \\
4.97\end{array}$ & $\begin{array}{r}9.964507 \\
.964454 \\
.964400 \\
.964347 \\
.964294 \\
.964240 \\
.964187 \\
.964133 \\
.9134080 \\
9.964023\end{array}$ & $\begin{array}{l}.88 \\
.90 \\
.88 \\
.88 \\
.90 \\
.88 \\
.90 \\
.89 \\
.90\end{array}$ & $\begin{array}{r}9.624683 \\
.625036 \\
.625358 \\
.625741 \\
.626093 \\
.626445 \\
.626797 \\
.627149 \\
.627501 \\
9.627852\end{array}$ & $\begin{array}{l}5.83 \\
5.88 \\
5.87 \\
5.88 \\
5.87 \\
5.87 \\
5.87 \\
5.87 \\
5.87 \\
5.85\end{array}$ & $\begin{array}{r}10.375317 \\
.374964 \\
.374612 \\
.374259 \\
.373907 \\
.373555 \\
.373203 \\
.372651 \\
.372499 \\
10.372148\end{array}$ & $\begin{array}{l}9 \\
8 \\
7 \\
6 \\
5 \\
4 \\
3 \\
2 \\
1 \\
0\end{array}$ \\
\hline , & Cosine. & D. 1 & sine. & D. $1^{\prime \prime}$. & Cotang. & $1^{\prime \prime}$ & Tang. & ' \\
\hline
\end{tabular}




\begin{tabular}{|c|c|c|c|c|c|c|c|c|}
\hline , & Sine. & D. $1^{\prime \prime}$. & Cosine. & D. $1^{\prime \prime}$. & Tang. & D. $1^{\prime \prime}$ & Cotang. & , \\
\hline $\begin{array}{r}0 \\
1 \\
2 \\
3 \\
4 \\
5 \\
6 \\
7 \\
8 \\
9 \\
10\end{array}$ & $\begin{array}{r}9.591878 \\
.592176 \\
.592473 \\
.592770 \\
.593067 \\
.593363 \\
.593659 \\
.593955 \\
.594251 \\
.594547 \\
.594842\end{array}$ & $\begin{array}{l}4.97 \\
4.95 \\
4.95 \\
4.95 \\
4.93 \\
4.93 \\
4.93 \\
4.93 \\
4.93 \\
4.92 \\
4.92\end{array}$ & $\begin{array}{r}9.964026 \\
.963972 \\
.963919 \\
.863865 \\
.963811 \\
.963757 \\
.963704 \\
.963650 \\
.963596 \\
.963542 \\
.963488\end{array}$ & $\begin{array}{l}.90 \\
.88 \\
.90 \\
.90 \\
.90 \\
.88 \\
.90 \\
.90 \\
.90 \\
.90 \\
.90\end{array}$ & $\begin{array}{r}9.627852 \\
.628203 \\
.628554 \\
.628905 \\
.629255 \\
.629606 \\
.629956 \\
.630306 \\
.630656 \\
.631005 \\
.631355\end{array}$ & $\begin{array}{l}5.85 \\
5.85 \\
5.85 \\
5.83 \\
5.85 \\
5.83 \\
5.83 \\
5.83 \\
5.82 \\
5.83 \\
5.82\end{array}$ & $\begin{array}{r}10.372148 \\
.371797 \\
.371446 \\
.371095 \\
.370745 \\
.370394 \\
.370044 \\
.369694 \\
.369344 \\
.368995 \\
.368645\end{array}$ & $\begin{array}{l}60 \\
59 \\
58 \\
57 \\
56 \\
55 \\
54 \\
53 \\
52 \\
51 \\
50\end{array}$ \\
\hline $\begin{array}{l}11 \\
12 \\
13 \\
14 \\
15 \\
16 \\
17 \\
18 \\
19 \\
20\end{array}$ & $\begin{array}{r}9.595137 \\
.595432 \\
.595727 \\
.596021 \\
.596315 \\
.596609 \\
.596903 \\
.597196 \\
.597490 \\
.597783\end{array}$ & $\begin{array}{l}4.92 \\
4.92 \\
4.90 \\
4.90 \\
4.90 \\
4.90 \\
4.88 \\
4.90 \\
4.88 \\
4.87\end{array}$ & $\begin{array}{r}9.963434 \\
.963379 \\
.963325 \\
.963271 \\
.963217 \\
.963163 \\
.963108 \\
.963054 \\
.962999 \\
.962945\end{array}$ & $\begin{array}{l}.92 \\
.90 \\
.90 \\
.90 \\
.90 \\
.92 \\
.90 \\
.92 \\
.90 \\
.92\end{array}$ & $\begin{array}{r}9.631704 \\
.632053 \\
.632402 \\
.632750 \\
.633099 \\
.633447 \\
.633795 \\
.634143 \\
.634490 \\
.634838\end{array}$ & $\begin{array}{l}\text { 5. } 82 \\
5.82 \\
5.80 \\
5.82 \\
5.80 \\
5.80 \\
5.80 \\
5.78 \\
5.80 \\
5.78\end{array}$ & $\begin{array}{r}10.368296 \\
.367947 \\
.367598 \\
.367250 \\
.366901 \\
.366553 \\
.366205 \\
.365857 \\
.365510 \\
.365162\end{array}$ & $\begin{array}{l}49 \\
48 \\
47 \\
46 \\
45 \\
44 \\
43 \\
42 \\
41 \\
40\end{array}$ \\
\hline $\begin{array}{l}21 \\
22 \\
23 \\
24 \\
25 \\
26 \\
27 \\
28 \\
29 \\
30\end{array}$ & $\begin{array}{r}9.598075 \\
.598368 \\
.598660 \\
.598952 \\
.599244 \\
.599536 \\
.599827 \\
.600118 \\
.600409 \\
.600700\end{array}$ & $\begin{array}{l}4.88 \\
4.87 \\
4.87 \\
4.87 \\
4.87 \\
4.85 \\
4.85 \\
4.85 \\
4.85 \\
4.83\end{array}$ & $\begin{array}{r}9.962890 \\
.962836 \\
.962781 \\
.962727 \\
.962672 \\
.962617 \\
.962562 \\
.962508 \\
.962453 \\
.962398\end{array}$ & $\begin{array}{l}.90 \\
.92 \\
.90 \\
.92 \\
.92 \\
.92 \\
.90 \\
.92 \\
.92 \\
.92\end{array}$ & $\begin{array}{r}9.635185 \\
.635532 \\
.635879 \\
.636226 \\
.636572 \\
.636919 \\
.637265 \\
.637611 \\
.637956 \\
.638302\end{array}$ & $\begin{array}{l}5.78 \\
5.78 \\
5.78 \\
5.77 \\
5.78 \\
5.77 \\
5.77 \\
5.75 \\
5.77 \\
5.75\end{array}$ & $\begin{array}{r}10.364815 \\
.364468 \\
.364121 \\
.363774 \\
.363428 \\
.363081 \\
.362735 \\
.362389 \\
.362044 \\
.361698\end{array}$ & $\begin{array}{l}39 \\
38 \\
37 \\
36 \\
35 \\
34 \\
33 \\
32 \\
31 \\
30\end{array}$ \\
\hline $\begin{array}{l}31 \\
32 \\
33 \\
34 \\
35 \\
36 \\
37 \\
38 \\
39 \\
40\end{array}$ & $\begin{array}{r}9.600990 \\
.601280 \\
.601570 \\
.601860 \\
.602150 \\
.602439 \\
.602728 \\
.603017 \\
.603305 \\
.603594\end{array}$ & $\begin{array}{l}4.83 \\
4.83 \\
4.83 \\
4.83 \\
4.82 \\
4.82 \\
4.82 \\
4.80 \\
4.82 \\
4.80\end{array}$ & $\begin{array}{r}9.962343 \\
.962288 \\
.962233 \\
.962178 \\
.962123 \\
.962067 \\
.962012 \\
.961957 \\
.961902 \\
.961846\end{array}$ & $\begin{array}{l}.92 \\
.92 \\
.92 \\
.92 \\
.93 \\
.92 \\
.92 \\
.92 \\
.93 \\
.92\end{array}$ & $\begin{array}{r}9.638647 \\
.638992 \\
.639337 \\
.639 t i 82 \\
.640027 \\
.640371 \\
.640716 \\
.641060 \\
.641404 \\
.641747\end{array}$ & $\begin{array}{l}\text { 5. } 75 \\
5.75 \\
5.75 \\
5.75 \\
5.73 \\
5.75 \\
\text { 5. } 73 \\
5.73 \\
5.72 \\
5.73\end{array}$ & $\begin{array}{r}\text { 10. } 361353 \\
.361008 \\
.360663 \\
.360318 \\
.359973 \\
.359629 \\
.359284 \\
.358940 \\
.358596 \\
.358253\end{array}$ & $\begin{array}{l}29 \\
28 \\
27 \\
26 \\
25 \\
24 \\
23 \\
22 \\
21 \\
20\end{array}$ \\
\hline $\begin{array}{l}41 \\
42 \\
43 \\
44 \\
45 \\
46 \\
47 \\
48 \\
49 \\
50\end{array}$ & $\begin{array}{r}9.603882 \\
.604170 \\
.604457 \\
.604745 \\
.605032 \\
.605319 \\
.605606 \\
.605892 \\
.606179 \\
.606465\end{array}$ & $\begin{array}{l}4.80 \\
4.78 \\
4.80 \\
4.78 \\
4.78 \\
4.78 \\
4.77 \\
4.78 \\
4.77 \\
4.77\end{array}$ & $\begin{array}{r}9.961791 \\
.961735 \\
.961680 \\
.961624 \\
.961569 \\
.961513 \\
.961458 \\
.961402 \\
.961346 \\
.961290\end{array}$ & $\begin{array}{l}.93 \\
.92 \\
.93 \\
.92 \\
.93 \\
.92 \\
.93 \\
.93 \\
.93 \\
.92\end{array}$ & $\begin{array}{r}9.642091 \\
.642434 \\
.642777 \\
.643120 \\
.643463 \\
.643806 \\
.644148 \\
.644490 \\
.644832 \\
.645174\end{array}$ & $\begin{array}{l}\text { 5. } 72 \\
\text { 5. } 72 \\
\text { 5. } 72 \\
\text { 5. } 72 \\
\text { 5. } 72 \\
\text { 5. } 70 \\
\text { 5. } 70 \\
\text { 5. } 70 \\
\text { 5. } 70 \\
\text { 5. } 70\end{array}$ & $\begin{array}{r}10.357909 \\
.357566 \\
.357223 \\
.356880 \\
.356537 \\
.356194 \\
.355852 \\
.355510 \\
.355168 \\
.354826\end{array}$ & $\begin{array}{l}19 \\
18 \\
17 \\
16 \\
15 \\
14 \\
13 \\
12 \\
11 \\
10\end{array}$ \\
\hline $\begin{array}{l}51 \\
52 \\
53 \\
54 \\
55 \\
56 \\
57 \\
58 \\
59 \\
60\end{array}$ & $\begin{array}{r}9.606751 \\
.607036 \\
.607322 \\
.607607 \\
.607892 \\
.608177 \\
.608461 \\
.608745 \\
.609029 \\
9.600313\end{array}$ & $\begin{array}{l}4.75 \\
4.77 \\
4.75 \\
4.75 \\
4.75 \\
4.73 \\
4.73 \\
4.73 \\
4.73\end{array}$ & $\begin{array}{r}9.961235 \\
.961179 \\
.961123 \\
.961067 \\
.961011 \\
.960955 \\
.960899 \\
.960843 \\
.960786 \\
9.960730\end{array}$ & $\begin{array}{r}.93 \\
.93 \\
.93 \\
.93 \\
.93 \\
.93 \\
.93 \\
.95 \\
.93\end{array}$ & $\begin{array}{r}9.645516 \\
.645857 \\
.646199 \\
.646540 \\
.646881 \\
.647222 \\
.647562 \\
.647903 \\
.648243 \\
9.648583\end{array}$ & $\begin{array}{l}5.68 \\
5.70 \\
5.68 \\
5.68 \\
5.68 \\
5.67 \\
5.68 \\
5.67 \\
5.67\end{array}$ & $\begin{array}{r}10.354484 \\
.354143 \\
.353501 \\
.353460 \\
.353119 \\
.352778 \\
.352438 \\
.352097 \\
.351757 \\
10.351417\end{array}$ & $\begin{array}{l}9 \\
8 \\
7 \\
6 \\
5 \\
4 \\
3 \\
2 \\
1 \\
0\end{array}$ \\
\hline ' & Cosine. & D. $1^{\prime \prime}$. & Sine. & D. $1^{\prime \prime}$. & Cotang & D. $1^{\prime \prime}$ & Tang. & , \\
\hline
\end{tabular}


TANGENTS AND COTANGESTS.

\begin{tabular}{|c|c|c|c|c|c|c|c|c|}
\hline , & Sine. & D. $1^{\prime \prime}$. & Cosine. & D. $1^{\prime \prime}$. & Tang. & D. $1 "$ 。 & Cotang. & ' \\
\hline $\begin{array}{r}0 \\
1 \\
2 \\
3 \\
4 \\
5 \\
6 \\
7 \\
8 \\
9 \\
10\end{array}$ & $\begin{array}{r}9.609313 \\
.609597 \\
.609880 \\
.610164 \\
.610447 \\
.610729 \\
.611012 \\
.611294 \\
.611576 \\
.611858 \\
.612140\end{array}$ & $\begin{array}{l}4.73 \\
4.72 \\
4.73 \\
4.72 \\
4.70 \\
4.72 \\
4.70 \\
4.70 \\
4.70 \\
4.70 \\
4.68\end{array}$ & $\begin{array}{r}9.960730 \\
.960674 \\
.960618 \\
.960561 \\
.960505 \\
.960448 \\
.960392 \\
.960335 \\
.960279 \\
.960222 \\
.960165\end{array}$ & $\begin{array}{l}.93 \\
.93 \\
.95 \\
93 \\
.95 \\
.93 \\
.95 \\
.93 \\
.95 \\
.95 \\
.93\end{array}$ & $\begin{array}{r}9.648583 \\
.648923 \\
.649263 \\
.649602 \\
.649942 \\
.650281 \\
.650620 \\
.650959 \\
.651297 \\
.651636 \\
.651974\end{array}$ & $\begin{array}{l}5.67 \\
5.67 \\
5.65 \\
5.67 \\
5.65 \\
5.65 \\
5.65 \\
5.63 \\
5.65 \\
5.63 \\
5.63\end{array}$ & $\begin{array}{r}10.351417 \\
.351077 \\
.350737 \\
.350398 \\
.350058 \\
.349719 \\
.349380 \\
.349041 \\
.348703 \\
.348364 \\
.348026\end{array}$ & $\begin{array}{l}60 \\
59 \\
58 \\
57 \\
56 \\
55 \\
b 4 \\
53 \\
52 \\
51 \\
50\end{array}$ \\
\hline $\begin{array}{l}11 \\
12 \\
13 \\
14 \\
15 \\
16 \\
17 \\
18 \\
19 \\
20\end{array}$ & $\begin{array}{r}9.612421 \\
.612702 \\
.612983 \\
.613264 \\
.613545 \\
.613525 \\
.614105 \\
.614385 \\
.614665 \\
.614944\end{array}$ & $\begin{array}{l}4.68 \\
4.68 \\
4.68 \\
4.68 \\
4.67 \\
4.67 \\
4.67 \\
4.67 \\
4.65 \\
4.65\end{array}$ & $\begin{array}{r}9.960109 \\
.960052 \\
.959905 \\
.959938 \\
.959582 \\
.959525 \\
.959768 \\
.959711 \\
.959654 \\
.959596\end{array}$ & $\begin{array}{l}.95 \\
.95 \\
.95 \\
.93 \\
.95 \\
.95 \\
.95 \\
.95 \\
.97 \\
.95\end{array}$ & $\begin{array}{r}9.652312 \\
.652650 \\
.652988 \\
.653326 \\
.653663 \\
.654000 \\
.654337 \\
.654674 \\
.655011 \\
.655348\end{array}$ & $\begin{array}{l}5.63 \\
5.63 \\
5.63 \\
5.62 \\
5.62 \\
5.62 \\
5.62 \\
5.62 \\
5.62 \\
5.60\end{array}$ & $\begin{array}{r}10.347688 \\
.347350 \\
.347012 \\
.346674 \\
.346337 \\
.346000 \\
.345663 \\
.345326 \\
.344989 \\
.344652\end{array}$ & $\begin{array}{l}49 \\
48 \\
47 \\
46 \\
45 \\
44 \\
43 \\
42 \\
41 \\
40\end{array}$ \\
\hline $\begin{array}{l}21 \\
22 \\
23 \\
24 \\
25 \\
26 \\
27 \\
28 \\
29 \\
30\end{array}$ & $\begin{array}{r}9.615223 \\
.615502 \\
.615781 \\
.616060 \\
.616338 \\
.616616 \\
.616894 \\
.617172 \\
.617450 \\
.617727\end{array}$ & $\begin{array}{l}4.65 \\
4.65 \\
4.65 \\
4.63 \\
4.63 \\
4.63 \\
4.63 \\
4.63 \\
4.62 \\
4.62\end{array}$ & $\begin{array}{r}9.959539 \\
.959482 \\
.959425 \\
.959368 \\
.959310 \\
.959253 \\
.959195 \\
.959138 \\
.959080 \\
.959023\end{array}$ & $\begin{array}{l}.95 \\
.95 \\
.95 \\
.97 \\
.95 \\
.97 \\
.95 \\
.97 \\
.95 \\
.97\end{array}$ & $\begin{array}{r}9.655684 \\
.656020 \\
.656356 \\
.656692 \\
.657028 \\
.657364 \\
.657699 \\
.658034 \\
.658369 \\
.658704\end{array}$ & $\begin{array}{l}5.60 \\
5.60 \\
5.60 \\
5.60 \\
5.60 \\
5.58 \\
5.58 \\
5.58 \\
5.58 \\
5.58\end{array}$ & $\begin{array}{r}10.344316 \\
.343980 \\
.343644 \\
.343308 \\
.342972 \\
.342636 \\
.342301 \\
.341966 \\
.341631 \\
.341296\end{array}$ & $\begin{array}{l}39 \\
38 \\
37 \\
36 \\
35 \\
34 \\
33 \\
32 \\
31 \\
30\end{array}$ \\
\hline $\begin{array}{l}31 \\
32 \\
33 \\
34 \\
35 \\
36 \\
37 \\
38 \\
39 \\
40\end{array}$ & $\begin{array}{r}9.618004 \\
.618281 \\
.618558 \\
.618534 \\
.619110 \\
.619386 \\
.619662 \\
.619938 \\
.620213 \\
.620483\end{array}$ & $\begin{array}{l}4.62 \\
4.62 \\
4.60 \\
4.60 \\
4.60 \\
4.60 \\
4.60 \\
4.58 \\
4.58 \\
4.58\end{array}$ & $\begin{array}{r}9.958965 \\
.958908 \\
.958550 \\
.958792 \\
.958734 \\
.958677 \\
.958619 \\
.958561 \\
.958503 \\
.958445\end{array}$ & $\begin{array}{l}.95 \\
.97 \\
.97 \\
.97 \\
.95 \\
.97 \\
.97 \\
.97 \\
.97 \\
.97\end{array}$ & $\begin{array}{r}9.659039 \\
.659373 \\
.659708 \\
.660042 \\
.660376 \\
.660710 \\
.661043 \\
.661377 \\
.661710 \\
.662043\end{array}$ & $\begin{array}{l}5.00 \\
5.57 \\
5.58 \\
5.57 \\
5.57 \\
5.57 \\
5.55 \\
5.57 \\
5.55 \\
5.55 \\
5.55\end{array}$ & $\begin{array}{r}10.340961 \\
.340627 \\
.340292 \\
.339958 \\
.339624 \\
.339290 \\
.335957 \\
.335623 \\
.335290 \\
.337957\end{array}$ & $\begin{array}{l}29 \\
28 \\
27 \\
26 \\
25 \\
24 \\
23 \\
22 \\
21 \\
20\end{array}$ \\
\hline $\begin{array}{l}41 \\
42 \\
43 \\
44 \\
45 \\
46 \\
47 \\
48 \\
49 \\
50\end{array}$ & $\begin{array}{r}9.620763 \\
.621038 \\
.621313 \\
.621587 \\
.621861 \\
.622135 \\
.622409 \\
.622682 \\
.622956 \\
.623229\end{array}$ & $\begin{array}{l}4.58 \\
4.58 \\
4.57 \\
4.57 \\
4.57 \\
4.57 \\
4.55 \\
4.57 \\
4.55 \\
4.55\end{array}$ & $\begin{array}{r}9.958387 \\
.958329 \\
.958271 \\
.958213 \\
.958154 \\
.958096 \\
.958038 \\
.957979 \\
.957921 \\
.957863\end{array}$ & $\begin{array}{l}.97 \\
.97 \\
97 \\
.98 \\
.97 \\
.97 \\
.98 \\
.97 \\
.97 \\
.98\end{array}$ & $\begin{array}{r}9.662376 \\
.662709 \\
.663042 \\
.663375 \\
.663707 \\
.664039 \\
.664371 \\
.664703 \\
.665035 \\
.665366\end{array}$ & $\begin{array}{l}5.55 \\
5.55 \\
5.55 \\
5.53 \\
5.53 \\
5.53 \\
5.53 \\
5.53 \\
5.52 \\
5.53\end{array}$ & $\begin{array}{r}10.337624 \\
.337291 \\
.336958 \\
.336625 \\
.336293 \\
.335961 \\
.335629 \\
.335297 \\
.334965 \\
.334634\end{array}$ & $\begin{array}{l}19 \\
18 \\
17 \\
16 \\
15 \\
14 \\
13 \\
12 \\
11 \\
10\end{array}$ \\
\hline $\begin{array}{l}51 \\
52 \\
53 \\
54 \\
55 \\
56 \\
57 \\
58 \\
59 \\
60\end{array}$ & $\begin{array}{r}9.623502 \\
.623774 \\
.624047 \\
.624319 \\
.624591 \\
.624863 \\
.625135 \\
.625406 \\
.625677 \\
9.625948\end{array}$ & $\begin{array}{l}4.53 \\
4.55 \\
4.53 \\
4.53 \\
4.53 \\
4.53 \\
4.52 \\
4.52 \\
4.52\end{array}$ & $\begin{array}{r}9.957504 \\
.957746 \\
.957657 \\
.957628 \\
.957570 \\
.957511 \\
.957452 \\
.957393 \\
.957335 \\
0.957276\end{array}$ & $\begin{array}{l}.97 \\
.98 \\
.98 \\
.97 \\
.98 \\
.98 \\
.98 \\
.97 \\
.98\end{array}$ & $\begin{array}{r}9.665698 \\
.666029 \\
.666360 \\
.6666991 \\
.667021 \\
.6673 .52 \\
.667652 \\
.665013 \\
.665343 \\
9.665673\end{array}$ & $\begin{array}{l}5.52 \\
5.52 \\
5.52 \\
5.52 \\
5.50 \\
5.52 \\
5.50 \\
5.52 \\
5.50 \\
5.50\end{array}$ & $\begin{array}{r}10.334302 \\
.333971 \\
.333640 \\
.333309 \\
.332979 \\
.332648 \\
.332318 \\
.331987 \\
.331657 \\
10.331327\end{array}$ & $\begin{array}{l}9 \\
8 \\
7 \\
6 \\
5 \\
4 \\
3 \\
2 \\
1 \\
0\end{array}$ \\
\hline ' & Cosine. & D. $1^{\prime \prime}$. & Sinc. & D. $1^{\prime \prime}$. & Cotang. & D. $\boldsymbol{1}^{\prime \prime}$. & Tang. & ' \\
\hline
\end{tabular}


TABLE 9.-LOGARITIMIC SINES, COSINES,

\begin{tabular}{|c|c|c|c|c|c|c|c|c|}
\hline , & Sine. & D. $1^{\prime \prime}$. & Cosine. & D. $1^{\prime \prime}$. & Tang. & D. $1^{\prime \prime}$. & Cotang. & , \\
\hline $\begin{array}{r}0 \\
1 \\
2 \\
3 \\
4 \\
5 \\
6 \\
7 \\
8 \\
9 \\
10\end{array}$ & $\begin{array}{r}9.625948 \\
.626219 \\
.626490 \\
.626760 \\
.627030 \\
.627300 \\
.627570 \\
.627840 \\
.628109 \\
.628378 \\
.628647\end{array}$ & $\begin{array}{l}4.52 \\
4.52 \\
4.50 \\
4.50 \\
4.50 \\
4.50 \\
4.50 \\
4.48 \\
4.48 \\
4.48 \\
4.48\end{array}$ & $\begin{array}{r}9.957276 \\
.957217 \\
.957158 \\
.957099 \\
.957040 \\
.956981 \\
.956921 \\
.956862 \\
.956803 \\
.956744 \\
.956684\end{array}$ & $\begin{array}{r}.98 \\
.98 \\
.98 \\
.98 \\
.98 \\
1.00 \\
.98 \\
.98 \\
.98 \\
1.00 \\
.98\end{array}$ & $\begin{array}{r}9.668673 \\
.669002 \\
.669332 \\
.669661 \\
.669991 \\
.670320 \\
.6706 i 49 \\
.670977 \\
.671306 \\
.671635 \\
.671963\end{array}$ & $\begin{array}{l}5.48 \\
5.50 \\
5.48 \\
5.50 \\
5.48 \\
5.48 \\
5.47 \\
5.48 \\
5.48 \\
5.47 \\
5.47\end{array}$ & $\begin{array}{r}10.331327 \\
.330998 \\
.330668 \\
.330339 \\
.330009 \\
.329080 \\
.329351 \\
.329023 \\
.328694 \\
.328365 \\
.328037\end{array}$ & $\begin{array}{l}60 \\
59 \\
58 \\
57 \\
56 \\
55 \\
54 \\
53 \\
52 \\
51 \\
50\end{array}$ \\
\hline $\begin{array}{l}11 \\
12 \\
13 \\
14 \\
15 \\
16 \\
17 \\
18 \\
19 \\
20\end{array}$ & $\begin{array}{r}9.628916 \\
.629185 \\
.629453 \\
.629721 \\
.629989 \\
.630257 \\
.630524 \\
.630792 \\
.631059 \\
.631326\end{array}$ & $\begin{array}{l}4.48 \\
4.47 \\
4.47 \\
4.47 \\
4.47 \\
4.45 \\
4.47 \\
4.45 \\
4.45 \\
4.45\end{array}$ & $\begin{array}{r}9.956625 \\
.956566 \\
.956506 \\
.956447 \\
.956387 \\
.956327 \\
.956268 \\
.956208 \\
.956148 \\
.956089\end{array}$ & $\begin{array}{r}.98 \\
1.00 \\
.98 \\
1.00 \\
1.00 \\
.98 \\
1.00 \\
1.00 \\
.98 \\
1.00\end{array}$ & $\begin{array}{r}9.672291 \\
.672619 \\
.672947 \\
.673274 \\
.673602 \\
.673929 \\
.674257 \\
.674584 \\
.674911 \\
.675237\end{array}$ & $\begin{array}{l}5.47 \\
5.47 \\
5.45 \\
5.47 \\
5.45 \\
5.47 \\
5.45 \\
5.45 \\
5.43 \\
5.45\end{array}$ & $\begin{array}{r}10.327709 \\
.327381 \\
.327053 \\
.326726 \\
.326398 \\
.326071 \\
.325743 \\
.325416 \\
.325089 \\
.324763\end{array}$ & $\begin{array}{l}49 \\
48 \\
47 \\
46 \\
45 \\
44 \\
43 \\
42 \\
41 \\
40\end{array}$ \\
\hline $\begin{array}{l}21 \\
22 \\
23 \\
24 \\
25 \\
26 \\
27 \\
28 \\
29 \\
30\end{array}$ & $\begin{array}{r}9.631593 \\
.631859 \\
.632125 \\
.632392 \\
.632658 \\
.632923 \\
.633189 \\
.633454 \\
.633719 \\
.633984\end{array}$ & $\begin{array}{l}4.43 \\
4.43 \\
4.45 \\
4.43 \\
4.42 \\
4.43 \\
4.42 \\
4.42 \\
4.42 \\
4.42\end{array}$ & $\begin{array}{r}9.956029 \\
.955969 \\
.955909 \\
.955849 \\
.955789 \\
.955729 \\
.955669 \\
.955609 \\
.955548 \\
.955488\end{array}$ & $\begin{array}{l}1.00 \\
1.00 \\
1.00 \\
1.00 \\
1.00 \\
1.00 \\
1.00 \\
1.98 \\
1.00 \\
1.00\end{array}$ & $\begin{array}{r}9.675564 \\
.675890 \\
.676217 \\
.676543 \\
.676869 \\
.677194 \\
.677520 \\
.677846 \\
.678171 \\
.678496\end{array}$ & $\begin{array}{l}5.43 \\
5.45 \\
5.43 \\
5.43 \\
5.42 \\
5.43 \\
5.43 \\
5.42 \\
5.42 \\
5.42\end{array}$ & $\begin{array}{r}10.324436 \\
.324110 \\
.323783 \\
.323457 \\
.323131 \\
.322806 \\
.322480 \\
.322154 \\
.321829 \\
.321504\end{array}$ & $\begin{array}{l}39 \\
39 \\
37 \\
36 \\
35 \\
34 \\
33 \\
32 \\
31 \\
30\end{array}$ \\
\hline $\begin{array}{l}31 \\
32 \\
33 \\
34 \\
35 \\
36 \\
37 \\
38 \\
39 \\
40\end{array}$ & $\begin{array}{r}9.634249 \\
.634514 \\
.634778 \\
.635042 \\
.635306 \\
.635570 \\
.635834 \\
.636097 \\
.636360 \\
.636623\end{array}$ & $\begin{array}{l}4.42 \\
4.40 \\
4.40 \\
4.40 \\
4.40 \\
4.40 \\
4.38 \\
4.38 \\
4.38 \\
4.38\end{array}$ & $\begin{array}{r}9.955428 \\
.955368 \\
.955307 \\
.955247 \\
.955186 \\
.955126 \\
.955065 \\
.955005 \\
.954944 \\
.954883\end{array}$ & $\begin{array}{l}1.00 \\
1.02 \\
1.00 \\
1.02 \\
1.00 \\
1.02 \\
1.00 \\
1.02 \\
1.02 \\
1.00\end{array}$ & $\begin{array}{r}9.678821 \\
.679146 \\
.679471 \\
.679795 \\
.680120 \\
.680444 \\
.680768 \\
.681092 \\
.681416 \\
.681740\end{array}$ & $\begin{array}{l}\text { 5. } 42 \\
5.42 \\
5.40 \\
5.42 \\
5.40 \\
5.40 \\
5.40 \\
5.40 \\
5.40 \\
5.38\end{array}$ & $\begin{array}{r}10.321179 \\
.320554 \\
.320529 \\
.320205 \\
.319880 \\
.319556 \\
.319232 \\
.318908 \\
.318584 \\
.318260\end{array}$ & $\begin{array}{l}29 \\
28 \\
27 \\
26 \\
25 \\
24 \\
23 \\
22 \\
21 \\
20\end{array}$ \\
\hline $\begin{array}{l}41 \\
42 \\
43 \\
44 \\
45 \\
46 \\
47 \\
48 \\
49 \\
50\end{array}$ & $\begin{array}{r}9.636886 \\
.637148 \\
.637411 \\
-637673 \\
.637935 \\
.638197 \\
.638458 \\
.638720 \\
.638981 \\
.639242\end{array}$ & $\begin{array}{l}4.37 \\
4.38 \\
4.37 \\
4.37 \\
4.37 \\
4.35 \\
4.37 \\
4.35 \\
4.35 \\
4.35\end{array}$ & $\begin{array}{r}9.954823 \\
.954762 \\
.954701 \\
.954640 \\
.954579 \\
.954518 \\
.954457 \\
.954396 \\
.954335 \\
.954274\end{array}$ & $\begin{array}{l}1.02 \\
1.02 \\
1.02 \\
1.02 \\
1.02 \\
1.02 \\
1.02 \\
1.02 \\
1.02 \\
1.02\end{array}$ & $\begin{array}{r}9.682063 \\
.682387 \\
.682710 \\
.683033 \\
.683356 \\
.683679 \\
.684001 \\
.684324 \\
.684646 \\
.6849618\end{array}$ & $\begin{array}{l}\text { 5. } 40 \\
5.38 \\
5.38 \\
5.38 \\
5.38 \\
5.37 \\
5.38 \\
5.37 \\
5.37 \\
5.37\end{array}$ & $\begin{array}{r}10.317937 \\
.317613 \\
.317290 \\
.316967 \\
.316644 \\
.316321 \\
.315999 \\
.315676 \\
.315354 \\
.315032\end{array}$ & $\begin{array}{l}19 \\
18 \\
17 \\
16 \\
15 \\
14 \\
13 \\
12 \\
11 \\
10\end{array}$ \\
\hline $\begin{array}{l}51 \\
52 \\
53 \\
54 \\
55 \\
56 \\
57 \\
58 \\
59 \\
60\end{array}$ & $\begin{array}{r}9.639503 \\
.639764 \\
.640024 \\
.640284 \\
.640544 \\
.640804 \\
.641064 \\
.641324 \\
.641583 \\
9.641842\end{array}$ & $\begin{array}{l}4.35 \\
4.33 \\
4.33 \\
4.33 \\
4.33 \\
4.33 \\
4.33 \\
4.32 \\
4.32\end{array}$ & $\begin{array}{r}9.954213 \\
.954152 \\
.954090 \\
.954029 \\
.953968 \\
.953966 \\
.953845 \\
.953783 \\
.953722 \\
9.953660\end{array}$ & $\begin{array}{l}1.02 \\
1.02 \\
1.03 \\
1.02 \\
1.02 \\
1.03 \\
1.02 \\
1.03 \\
1.02 \\
1.03\end{array}$ & $\begin{array}{r}9.685290 \\
.685612 \\
.685934 \\
.686255 \\
.686577 \\
.686898 \\
.687219 \\
.687540 \\
.687861 \\
9.688182\end{array}$ & $\begin{array}{l}5.37 \\
5.37 \\
5.35 \\
5.37 \\
5.35 \\
5.35 \\
5.35 \\
5.35 \\
5.35\end{array}$ & $\begin{array}{r}10.314710 \\
.314388 \\
.314066 \\
.313745 \\
.313423 \\
.313102 \\
.312781 \\
.312460 \\
.312139 \\
10.311818\end{array}$ & $\begin{array}{l}9 \\
8 \\
7 \\
6 \\
5 \\
4 \\
3 \\
2 \\
1 \\
0\end{array}$ \\
\hline ' & Cosine. & D. $1^{\prime \prime}$. & Sine. & D. $1^{\prime \prime}$ & Cotang. & D. $1^{\prime \prime}$. & Tang. & ' \\
\hline
\end{tabular}


TANGENTS, AND COTANGENTS.

\begin{tabular}{|c|c|c|c|c|c|c|c|c|}
\hline , & Sine. & D. $1^{\prime \prime}$. & Cosine. & D. $1^{\prime \prime}$. & Tang. & D. $1^{\prime \prime}$. & Cotang. & , \\
\hline $\begin{array}{r}0 \\
1 \\
2 \\
3 \\
4 \\
5 \\
6 \\
7 \\
8 \\
9 \\
10\end{array}$ & $\begin{array}{r}9.641842 \\
.642101 \\
.642360 \\
.642618 \\
.642877 \\
.643135 \\
.643393 \\
.643650 \\
.643908 \\
.644165 \\
.644423\end{array}$ & $\begin{array}{l}\text { 4. } 32 \\
4.32 \\
4.30 \\
4.32 \\
4.30 \\
4.30 \\
4.28 \\
4.30 \\
4.28 \\
4.30 \\
4.28\end{array}$ & $\begin{array}{r}9.953000 \\
.953 i 99 \\
.953537 \\
.953475 \\
.953413 \\
.953352 \\
.953290 \\
.953228 \\
.953166 \\
.953104 \\
.953042\end{array}$ & $\begin{array}{l}1.02 \\
1.03 \\
1.03 \\
1.03 \\
1.02 \\
1.03 \\
1.03 \\
1.03 \\
1.03 \\
1.03 \\
1.03\end{array}$ & $\begin{array}{r}9.685182 \\
.685502 \\
.658523 \\
.689143 \\
.689463 \\
.689783 \\
.690103 \\
.690423 \\
.690742 \\
.691062 \\
.691381\end{array}$ & $\begin{array}{l}\text { 5. } 33 \\
5.32 \\
5.33 \\
5.33 \\
5.33 \\
5.33 \\
5.33 \\
5.32 \\
5.33 \\
5.32 \\
5.32\end{array}$ & $\begin{array}{r}10.311818 \\
.311498 \\
.311177 \\
.310857 \\
.310537 \\
.310217 \\
.309897 \\
.309577 \\
.309258 \\
.308938 \\
.308619\end{array}$ & $\begin{array}{l}60 \\
59 \\
58 \\
57 \\
56 \\
55 \\
54 \\
53 \\
52 \\
51 \\
50\end{array}$ \\
\hline $\begin{array}{l}11 \\
12 \\
13 \\
14 \\
15 \\
16 \\
17 \\
18 \\
19 \\
20\end{array}$ & $\begin{array}{r}9.644680 \\
.644936 \\
.645193 \\
.645450 \\
.645706 \\
.645962 \\
.646218 \\
.646474 \\
.646729 \\
.646984\end{array}$ & $\begin{array}{l}4.27 \\
4.28 \\
4.28 \\
4.27 \\
4.27 \\
4.27 \\
4.27 \\
4.25 \\
4.25 \\
4.27\end{array}$ & $\begin{array}{r}9.952350 \\
.952918 \\
.952855 \\
.952793 \\
.952731 \\
.952669 \\
.952606 \\
.952544 \\
.952481 \\
.952419\end{array}$ & $\begin{array}{l}1.03 \\
1.05 \\
1.03 \\
1.03 \\
1.03 \\
1.05 \\
1.03 \\
1.05 \\
1.03 \\
1.05\end{array}$ & $\begin{array}{r}9.691700 \\
.692019 \\
.692338 \\
.692656 \\
.692975 \\
.693293 \\
.693612 \\
.693930 \\
.694248 \\
.694566\end{array}$ & $\begin{array}{l}5.32 \\
5.32 \\
5.30 \\
5.32 \\
5.30 \\
5.32 \\
5.30 \\
5.30 \\
5.30 \\
5.29\end{array}$ & $\begin{array}{r}10.308300 \\
.307981 \\
.307662 \\
.307344 \\
.307025 \\
.306707 \\
.306388 \\
.306070 \\
.305752 \\
.305434\end{array}$ & $\begin{array}{l}49 \\
48 \\
47 \\
46 \\
45 \\
44 \\
43 \\
42 \\
41 \\
40\end{array}$ \\
\hline $\begin{array}{l}21 \\
22 \\
23 \\
24 \\
25 \\
26 \\
27 \\
28 \\
29 \\
30\end{array}$ & $\begin{array}{r}9.647240 \\
.647494 \\
.647749 \\
.645004 \\
.649258 \\
.648512 \\
.648766 \\
.649020 \\
.649274 \\
.649527\end{array}$ & $\begin{array}{l}4.23 \\
4.25 \\
4.25 \\
4.23 \\
4.23 \\
4.23 \\
4.23 \\
4.23 \\
4.22 \\
4.23\end{array}$ & $\begin{array}{r}9.952356 \\
.952394 \\
.952231 \\
.952168 \\
.952106 \\
.952043 \\
.951950 \\
.951917 \\
.951854 \\
.951791\end{array}$ & $\begin{array}{l}1.03 \\
1.05 \\
1.05 \\
1.03 \\
1.05 \\
1.05 \\
1.05 \\
1.05 \\
1.05 \\
1.05\end{array}$ & $\begin{array}{r}9.694883 \\
.695201 \\
.695518 \\
.695836 \\
.696153 \\
.696470 \\
.696787 \\
.697103 \\
.697420 \\
.697736\end{array}$ & $\begin{array}{l}5.30 \\
5.28 \\
5.30 \\
5.28 \\
5.28 \\
5.28 \\
5.27 \\
5.28 \\
5.27 \\
5.28\end{array}$ & $\begin{array}{r}10.305117 \\
.304799 \\
.304482 \\
.304164 \\
.303847 \\
.303530 \\
.303213 \\
.302897 \\
.302580 \\
.302264\end{array}$ & $\begin{array}{l}39 \\
38 \\
37 \\
36 \\
35 \\
34 \\
33 \\
32 \\
31 \\
30\end{array}$ \\
\hline $\begin{array}{l}31 \\
32 \\
33 \\
34 \\
35 \\
36 \\
37 \\
38 \\
39 \\
40\end{array}$ & $\begin{array}{r}9.649781 \\
.650034 \\
.650287 \\
.650539 \\
.650792 \\
.651044 \\
.651297 \\
.651549 \\
.651800 \\
.652052\end{array}$ & $\begin{array}{l}4.22 \\
4.22 \\
4.20 \\
4.22 \\
4.20 \\
4.22 \\
4.20 \\
4.18 \\
4.20 \\
4.20\end{array}$ & $\begin{array}{r}9.951728 \\
.951665 \\
.951602 \\
.951539 \\
.951476 \\
.951412 \\
.951349 \\
.951286 \\
.951222 \\
.951159\end{array}$ & $\begin{array}{l}1.05 \\
1.05 \\
1.05 \\
1.05 \\
1.07 \\
1.05 \\
1.05 \\
1.07 \\
1.05 \\
1.05\end{array}$ & $\begin{array}{r}9.695053 \\
.695369 \\
.695685 \\
.699001 \\
.699316 \\
.699632 \\
.699947 \\
.700263 \\
.700578 \\
.700893\end{array}$ & $\begin{array}{l}5.27 \\
5.27 \\
5.27 \\
5.25 \\
5.27 \\
5.25 \\
5.27 \\
5.25 \\
5.25 \\
5.25\end{array}$ & $\begin{array}{r}10.301947 \\
.301631 \\
.301315 \\
.300999 \\
.300684 \\
.300368 \\
.300053 \\
.299737 \\
.299422 \\
.299107\end{array}$ & $\begin{array}{l}29 \\
28 \\
27 \\
26 \\
25 \\
24 \\
23 \\
22 \\
21 \\
20\end{array}$ \\
\hline $\begin{array}{l}41 \\
42 \\
43 \\
44 \\
45 \\
46 \\
47 \\
48 \\
49 \\
50\end{array}$ & $\begin{array}{r}9.652304 \\
.652555 \\
.652806 \\
.653057 \\
.653308 \\
.653558 \\
.653908 \\
.654059 \\
.654309 \\
.654558\end{array}$ & $\begin{array}{l}4.18 \\
4.18 \\
4.18 \\
4.18 \\
4.17 \\
4.17 \\
4.18 \\
4.17 \\
4.15 \\
4.17\end{array}$ & $\begin{array}{r}9.951096 \\
.951032 \\
.950368 \\
.950305 \\
.950841 \\
.950778 \\
.950714 \\
.950650 \\
.950586 \\
.950522\end{array}$ & $\begin{array}{l}1.07 \\
1.07 \\
1.05 \\
1.07 \\
1.05 \\
1.07 \\
1.07 \\
1.07 \\
1.07 \\
1.07\end{array}$ & $\begin{array}{r}9.701205 \\
.701523 \\
.701837 \\
.702152 \\
.702466 \\
.702781 \\
.703095 \\
.703409 \\
.703722 \\
.704036\end{array}$ & $\begin{array}{l}5.25 \\
5.23 \\
5.25 \\
5.23 \\
5.25 \\
5.23 \\
5.23 \\
5.22 \\
5.23 \\
5.23\end{array}$ & $\begin{array}{r}10.295792 \\
.298477 \\
.298163 \\
.297848 \\
.297534 \\
.297219 \\
.296905 \\
.296591 \\
.296278 \\
.295964\end{array}$ & $\begin{array}{l}19 \\
18 \\
17 \\
16 \\
15 \\
14 \\
13 \\
12 \\
11 \\
10\end{array}$ \\
\hline $\begin{array}{l}\mathbf{5 1} \\
\mathbf{5 2} \\
\mathbf{5 3} \\
\mathbf{5 4} \\
\mathbf{5 5} \\
\mathbf{5 6} \\
\mathbf{5 7} \\
\mathbf{5} \\
\mathbf{5 9} \\
\mathbf{6 0}\end{array}$ & $\begin{array}{r}9.654808 \\
.655058 \\
.655307 \\
.655556 \\
.655805 \\
.656054 \\
.656302 \\
.656551 \\
.656799 \\
9.657047\end{array}$ & $\begin{array}{l}4.17 \\
4.15 \\
4.15 \\
4.15 \\
4.15 \\
4.13 \\
4.15 \\
4.13 \\
4.13\end{array}$ & $\begin{array}{r}9.950458 \\
.950394 \\
.950330 \\
.950266 \\
.950202 \\
.950139 \\
.950074 \\
.950010 \\
.949945 \\
9.943851\end{array}$ & $\begin{array}{l}1.07 \\
1.07 \\
1.07 \\
1.07 \\
1.07 \\
1.07 \\
1.07 \\
1.08 \\
1.07\end{array}$ & $\begin{array}{r}9.704350 \\
.704663 \\
.701976 \\
.705290 \\
.705603 \\
.705916 \\
.706229 \\
.706541 \\
.705854 \\
9.707166\end{array}$ & $\begin{array}{l}5.22 \\
5.22 \\
5.23 \\
5.22 \\
5.22 \\
5.20 \\
5.22 \\
5.22 \\
5.20\end{array}$ & $\begin{array}{r}10.295650 \\
.295337 \\
.295024 \\
.291710 \\
.294397 \\
.294084 \\
.293772 \\
.293459 \\
.393146 \\
10.292834\end{array}$ & $\begin{array}{l}9 \\
8 \\
7 \\
6 \\
5 \\
4 \\
3 \\
2 \\
1 \\
0\end{array}$ \\
\hline , & Cosine. & D. $1^{\prime \prime}$. & Sine. & D. $1^{\prime \prime}$. & Cotang. & D. $1^{\prime \prime}$ & Tang. & 1 \\
\hline
\end{tabular}


TABLE 9.-LOGARITIMIC SINES, COSINES,

\begin{tabular}{|c|c|c|c|c|c|c|c|c|}
\hline , & Sine. & D. $1^{\prime \prime}$. & Cosine. & D. $1^{\prime \prime}$. & Tang. & D. $1^{\prime \prime}$. & Cotang. & , \\
\hline $\begin{array}{r}0 \\
1 \\
2 \\
3 \\
4 \\
5 \\
6 \\
7 \\
8 \\
9 \\
10\end{array}$ & $\begin{array}{r}9.657047 \\
.657295 \\
.657542 \\
.657790 \\
.658037 \\
.658284 \\
.658531 \\
.658778 \\
.659025 \\
.659271 \\
.659517\end{array}$ & $\begin{array}{l}4.13 \\
4.12 \\
4.13 \\
4.12 \\
4.12 \\
4.12 \\
4.12 \\
4.12 \\
4.10 \\
4.10 \\
4.10\end{array}$ & $\begin{array}{r}9.949881 \\
.949816 \\
.949752 \\
.949688 \\
.949623 \\
.949558 \\
.949494 \\
.949429 \\
.949364 \\
.949300 \\
.949235\end{array}$ & $\begin{array}{l}1.08 \\
1.07 \\
1.07 \\
1.08 \\
1.08 \\
1.07 \\
1.08 \\
1.08 \\
1.07 \\
1.08 \\
1.08\end{array}$ & $\begin{array}{r}9.707166 \\
.707478 \\
.707790 \\
.708102 \\
.708414 \\
.708726 \\
.709037 \\
.709349 \\
.709660 \\
.709971 \\
.710282\end{array}$ & $\begin{array}{l}5.20 \\
5.20 \\
5.20 \\
5.20 \\
5.20 \\
5.18 \\
5.20 \\
5.18 \\
5.18 \\
5.18 \\
5.18\end{array}$ & $\begin{array}{r}10.292834 \\
.292522 \\
.292210 \\
.291898 \\
.291586 \\
.291274 \\
.290963 \\
.290651 \\
.290340 \\
.290029 \\
.289718\end{array}$ & $\begin{array}{l}60 \\
59 \\
58 \\
57 \\
56 \\
55 \\
54 \\
53 \\
52 \\
51 \\
50\end{array}$ \\
\hline $\begin{array}{l}11 \\
12 \\
13 \\
14 \\
15 \\
16 \\
17 \\
18 \\
19 \\
20 \\
\end{array}$ & $\begin{array}{r}9.659763 \\
.660009 \\
.660255 \\
.660501 \\
.660746 \\
.660991 \\
.661236 \\
.661481 \\
.661726 \\
.661970\end{array}$ & $\begin{array}{l}4.10 \\
4.10 \\
4.10 \\
4.08 \\
4.08 \\
4.08 \\
4.08 \\
4.08 \\
4.07 \\
4.07\end{array}$ & $\begin{array}{r}9.949170 \\
.949105 \\
.949040 \\
.948975 \\
.948910 \\
.948845 \\
.948780 \\
.948715 \\
.948650 \\
.948584\end{array}$ & $\begin{array}{l}1.08 \\
1.08 \\
1.09 \\
1.08 \\
1.08 \\
1.08 \\
1.08 \\
1.08 \\
1.10 \\
1.08\end{array}$ & $\begin{array}{r}9.710593 \\
.710904 \\
.711215 \\
.711525 \\
.711836 \\
.712146 \\
.712456 \\
.712766 \\
.713076 \\
.713356\end{array}$ & $\begin{array}{l}5.18 \\
5.18 \\
5.17 \\
5.18 \\
5.17 \\
5.17 \\
5.17 \\
5.17 \\
5.17 \\
5.17\end{array}$ & $\begin{array}{r}10.289407 \\
.289096 \\
.288785 \\
.288475 \\
.288164 \\
.287854 \\
.287544 \\
.287234 \\
.286924 \\
.286614\end{array}$ & $\begin{array}{l}49 \\
48 \\
47 \\
46 \\
45 \\
44 \\
43 \\
42 \\
41 \\
10\end{array}$ \\
\hline $\begin{array}{l}21 \\
22 \\
23 \\
24 \\
25 \\
26 \\
27 \\
28 \\
29 \\
30\end{array}$ & $\begin{array}{r}9.662214 \\
.662459 \\
.662703 \\
.662946 \\
.663190 \\
.663433 \\
.663077 \\
.663920 \\
.664163 \\
.664406\end{array}$ & $\begin{array}{l}4.08 \\
4.07 \\
4.05 \\
4.07 \\
4.05 \\
4.07 \\
4.05 \\
4.05 \\
4.05 \\
4.03\end{array}$ & $\begin{array}{r}9.948519 \\
.948454 \\
.948388 \\
.948323 \\
.948257 \\
.945192 \\
.948126 \\
.945060 \\
.947995 \\
.947929\end{array}$ & $\begin{array}{l}1.08 \\
1.10 \\
1.08 \\
1.10 \\
1.08 \\
1.10 \\
1.10 \\
1.08 \\
1.10 \\
1.10\end{array}$ & $\begin{array}{r}9.713696 \\
.714005 \\
.714314 \\
.714624 \\
.714933 \\
.715242 \\
.715551 \\
.715860 \\
.716168 \\
.716477\end{array}$ & $\begin{array}{l}5.15 \\
5.15 \\
5.17 \\
5.15 \\
5.15 \\
5.15 \\
5.15 \\
5.13 \\
5.15 \\
5.13\end{array}$ & $\begin{array}{r}10.286304 \\
.285995 \\
.285686 \\
.285376 \\
.285067 \\
.284758 \\
.284449 \\
.284140 \\
.283832 \\
.283523\end{array}$ & $\begin{array}{l}39 \\
38 \\
37 \\
36 \\
35 \\
34 \\
33 \\
32 \\
31 \\
30\end{array}$ \\
\hline $\begin{array}{l}31 \\
32 \\
33 \\
34 \\
35 \\
36 \\
37 \\
38 \\
39 \\
40\end{array}$ & $\begin{array}{r}9.664648 \\
.664891 \\
.665133 \\
.665375 \\
.665617 \\
.665859 \\
.666100 \\
.666342 \\
.666583 \\
.666824\end{array}$ & $\begin{array}{l}4.05 \\
4.03 \\
4.03 \\
4.03 \\
4.03 \\
4.02 \\
4.03 \\
4.02 \\
4.02 \\
4.02\end{array}$ & $\begin{array}{r}9.947863 \\
.947797 \\
.947731 \\
.947665 \\
.947600 \\
.947533 \\
.947467 \\
.947401 \\
.947335 \\
.947269\end{array}$ & $\begin{array}{l}1.10 \\
1.10 \\
1.10 \\
1.08 \\
1.12 \\
1.10 \\
1.10 \\
1.10 \\
1.10 \\
1.10\end{array}$ & $\begin{array}{r}9.716785 \\
.717093 \\
.717401 \\
.717709 \\
.718017 \\
.718325 \\
.718633 \\
.718940 \\
.719248 \\
.719555\end{array}$ & $\begin{array}{l}5.13 \\
5.13 \\
5.13 \\
5.13 \\
5.13 \\
5.13 \\
5.12 \\
5.13 \\
5.12 \\
5.12\end{array}$ & $\begin{array}{r}10.283215 \\
.282907 \\
.282599 \\
.282291 \\
.281983 \\
.281675 \\
.281367 \\
.281060 \\
.280752 \\
.280445\end{array}$ & $\begin{array}{l}29 \\
28 \\
27 \\
26 \\
25 \\
24 \\
23 \\
22 \\
21 \\
20\end{array}$ \\
\hline $\begin{array}{l}41 \\
42 \\
43 \\
44 \\
45 \\
46 \\
47 \\
48 \\
49 \\
50\end{array}$ & $\begin{array}{r}9.667065 \\
.667305 \\
.667546 \\
.667786 \\
.668027 \\
.668267 \\
.665506 \\
.6688746 \\
.668986 \\
.669225\end{array}$ & $\begin{array}{l}4.00 \\
4.02 \\
4.00 \\
4.02 \\
4.00 \\
3.98 \\
4.00 \\
4.00 \\
3.98 \\
3.98\end{array}$ & $\begin{array}{r}9.947203 \\
.947136 \\
.947070 \\
.947004 \\
.946937 \\
.946871 \\
.946504 \\
.946738 \\
.9466671 \\
.946604\end{array}$ & $\begin{array}{l}1.12 \\
1.10 \\
1.10 \\
1.12 \\
1.10 \\
1.12 \\
1.10 \\
1.12 \\
1.12 \\
1.10\end{array}$ & $\begin{array}{r}9.719862 \\
.720169 \\
.720476 \\
.720783 \\
.721059 \\
.721396 \\
.721702 \\
.722009 \\
.722315 \\
.722621\end{array}$ & $\begin{array}{l}5.12 \\
5.12 \\
5.12 \\
5.10 \\
5.12 \\
5.10 \\
5.12 \\
5.10 \\
5.10 \\
5.10\end{array}$ & $\begin{array}{r}10.280138 \\
.279831 \\
.279524 \\
.279217 \\
.278911 \\
.278604 \\
.278298 \\
.277991 \\
.277685 \\
.277379\end{array}$ & $\begin{array}{l}19 \\
18 \\
17 \\
16 \\
15 \\
14 \\
13 \\
12 \\
11 \\
10\end{array}$ \\
\hline $\begin{array}{l}51 \\
52 \\
53 \\
54 \\
55 \\
56 \\
57 \\
58 \\
59 \\
60\end{array}$ & $\begin{array}{r}9.669464 \\
.669703 \\
.6699942 \\
.670181 \\
.670419 \\
.6706558 \\
.670596 \\
.671134 \\
.671372 \\
9.671009\end{array}$ & $\begin{array}{l}3.98 \\
3.98 \\
3.98 \\
3.97 \\
3.98 \\
3.97 \\
3.97 \\
3.97 \\
3.95\end{array}$ & $\begin{array}{r}9.946538 \\
.946471 \\
.946404 \\
.946337 \\
.946270 \\
.946203 \\
.946136 \\
.946069 \\
.946002 \\
9.945935\end{array}$ & \begin{tabular}{l|l|}
1.12 \\
1.12 \\
1.12 \\
1.12 \\
1.12 \\
1.12 \\
1.12 \\
1.12 \\
1.12
\end{tabular} & $\begin{array}{r}9.722927 \\
.723232 \\
.723538 \\
.723844 \\
.724149 \\
.724454 \\
.724760 \\
.725065 \\
.725370 \\
9.725674\end{array}$ & $\begin{array}{l}5.08 \\
5.10 \\
5.10 \\
5.08 \\
5.08 \\
5.10 \\
5.08 \\
5.08 \\
5.07\end{array}$ & $\begin{array}{r}10.277073 \\
.276765 \\
.276462 \\
.276156 \\
.275851 \\
.275546 \\
.275240 \\
.274935 \\
.274630 \\
10.274326\end{array}$ & $\begin{array}{l}9 \\
8 \\
7 \\
6 \\
5 \\
4 \\
3 \\
2 \\
1 \\
0\end{array}$ \\
\hline te & Cosine. & D. $1^{\prime \prime}$. & Sine. & D. $1^{\prime \prime}$. & Cotang. & 10.1. & Tang. & ' \\
\hline
\end{tabular}


TANGENTS, AND COTANGENTS.

\begin{tabular}{|c|c|c|c|c|c|c|c|c|}
\hline , & Sine. & D. $1^{\prime \prime}$. & Cosine. & D. 1". & Tang. & D. 1". & Cotang. & , \\
\hline $\begin{array}{r}0 \\
1 \\
2 \\
3 \\
4 \\
5 \\
6 \\
7 \\
8 \\
9 \\
10\end{array}$ & $\begin{array}{r}9.671609 \\
.671847 \\
.672084 \\
.672321 \\
.672558 \\
.672795 \\
.673032 \\
.673208 \\
.673505 \\
.673741 \\
.673977\end{array}$ & $\begin{array}{l}3.97 \\
3.95 \\
3.95 \\
3.95 \\
3.95 \\
3.95 \\
3.93 \\
3.95 \\
3.93 \\
3.93 \\
3.93\end{array}$ & $\begin{array}{r}9.945935 \\
.945568 \\
.945800 \\
.945733 \\
.945666 \\
.945598 \\
.945531 \\
.945464 \\
.945396 \\
.945328 \\
.945261\end{array}$ & $\begin{array}{l}1.12 \\
1.13 \\
1.12 \\
1.12 \\
1.13 \\
1.12 \\
1.12 \\
1.13 \\
1.13 \\
1.12 \\
1.13\end{array}$ & $\begin{array}{r}9.725674 \\
.725979 \\
.726254 \\
.726589 \\
.726892 \\
.727197 \\
.727501 \\
.727805 \\
.728109 \\
.728412 \\
.728716\end{array}$ & $\begin{array}{l}5.08 \\
5.08 \\
5.07 \\
5.07 \\
5.05 \\
5.07 \\
5.07 \\
5.07 \\
5.05 \\
5.07 \\
5.07\end{array}$ & $\begin{array}{r}10.274326 \\
.274021 \\
.273716 \\
.273412 \\
.273108 \\
.272503 \\
.272499 \\
.272195 \\
.271891 \\
.271589 \\
.271254\end{array}$ & $\begin{array}{l}60 \\
59 \\
58 \\
57 \\
56 \\
55 \\
54 \\
53 \\
52 \\
51 \\
50\end{array}$ \\
\hline $\begin{array}{l}11 \\
12 \\
13 \\
14 \\
15 \\
16 \\
17 \\
18 \\
19 \\
20\end{array}$ & $\begin{array}{r}9.674213 \\
.674448 \\
.674684 \\
.674919 \\
.675155 \\
.675390 \\
.675024 \\
.675859 \\
.676094 \\
.676328\end{array}$ & $\begin{array}{l}3.92 \\
3.93 \\
3.92 \\
3.93 \\
3.92 \\
3.90 \\
3.92 \\
3.92 \\
3.90 \\
3.90\end{array}$ & $\begin{array}{r}9.945193 \\
.945125 \\
.945058 \\
.944990 \\
.944922 \\
.944554 \\
.944786 \\
.944718 \\
.944650 \\
.944582\end{array}$ & $\begin{array}{l}1.13 \\
1.12 \\
1.13 \\
1.13 \\
1.13 \\
1.13 \\
1.13 \\
1.13 \\
1.13 \\
1.13\end{array}$ & $\begin{array}{r}9.729020 \\
.729323 \\
.729626 \\
.729929 \\
.730233 \\
.730535 \\
.730835 \\
.731141 \\
.731444 \\
.731746\end{array}$ & $\begin{array}{l}5.05 \\
5.05 \\
5.05 \\
5.07 \\
5.03 \\
5.05 \\
5.05 \\
5.05 \\
5.03 \\
5.03\end{array}$ & $\begin{array}{r}10.270980 \\
.270677 \\
.270374 \\
.270071 \\
.269767 \\
.269465 \\
.269162 \\
.265859 \\
.268556 \\
.265254\end{array}$ & $\begin{array}{l}49 \\
48 \\
47 \\
46 \\
45 \\
44 \\
43 \\
42 \\
41 \\
40\end{array}$ \\
\hline $\begin{array}{l}21 \\
22 \\
23 \\
24 \\
25 \\
26 \\
27 \\
28 \\
29 \\
30\end{array}$ & $\begin{array}{r}9.676562 \\
.676796 \\
.677030 \\
.677264 \\
.677493 \\
.677731 \\
.677964 \\
.678197 \\
.678430 \\
.678663\end{array}$ & $\begin{array}{l}3.90 \\
3.90 \\
3.90 \\
3.90 \\
3.88 \\
3.89 \\
3.89 \\
3.89 \\
3.89 \\
3.87\end{array}$ & $\begin{array}{r}9.944514 \\
.944446 \\
.944377 \\
.944309 \\
.944241 \\
.944172 \\
.944104 \\
.944036 \\
.943967 \\
.943899\end{array}$ & $\begin{array}{l}1.13 \\
1.15 \\
1.13 \\
1.13 \\
1.15 \\
1.13 \\
1.13 \\
1.15 \\
1.13 \\
1.15\end{array}$ & $\begin{array}{r}9.732048 \\
.732351 \\
.732653 \\
.732955 \\
.733257 \\
.733558 \\
.733860 \\
.734162 \\
.734463 \\
.734764\end{array}$ & $\begin{array}{l}5.05 \\
5.03 \\
5.03 \\
5.03 \\
5.02 \\
5.03 \\
5.03 \\
5.02 \\
5.02 \\
5.03\end{array}$ & $\begin{array}{r}10.267952 \\
.267649 \\
.267347 \\
.267045 \\
.266743 \\
.266442 \\
.266140 \\
.265839 \\
.265537 \\
.265236\end{array}$ & $\begin{array}{l}39 \\
38 \\
37 \\
36 \\
35 \\
34 \\
33 \\
32 \\
31 \\
30\end{array}$ \\
\hline $\begin{array}{l}31 \\
32 \\
33 \\
34 \\
35 \\
36 \\
37 \\
38 \\
39 \\
40\end{array}$ & $\begin{array}{r}9.678595 \\
.679128 \\
.679360 \\
.679592 \\
.679824 \\
.680056 \\
.680288 \\
.680519 \\
.680750 \\
.680982\end{array}$ & $\begin{array}{l}3.89 \\
3.87 \\
3.87 \\
3.87 \\
3.87 \\
3.87 \\
3.85 \\
3.85 \\
3.87 \\
3.85\end{array}$ & $\begin{array}{r}9.943530 \\
.943761 \\
.943693 \\
.943624 \\
.943555 \\
.943486 \\
.943417 \\
.943348 \\
.943279 \\
.943210\end{array}$ & $\begin{array}{l}1.15 \\
1.13 \\
1.15 \\
1.15 \\
1.15 \\
1.15 \\
1.15 \\
1.15 \\
1.15 \\
1.15\end{array}$ & $\begin{array}{r}9.735066 \\
.735367 \\
.735668 \\
.735969 \\
.736269 \\
.736570 \\
.736870 \\
.737171 \\
.737471 \\
.737771\end{array}$ & $\begin{array}{l}5.02 \\
5.02 \\
5.02 \\
5.00 \\
5.02 \\
5.00 \\
5.02 \\
5.00 \\
5.00 \\
5.00\end{array}$ & $\begin{array}{r}10.264934 \\
.264633 \\
.264332 \\
.264031 \\
.263731 \\
.263430 \\
.263130 \\
.262529 \\
.262529 \\
.262229\end{array}$ & $\begin{array}{l}29 \\
28 \\
27 \\
26 \\
25 \\
24 \\
23 \\
22 \\
21 \\
20\end{array}$ \\
\hline $\begin{array}{l}41 \\
42 \\
43 \\
44 \\
45 \\
46 \\
47 \\
48 \\
49 \\
50\end{array}$ & $\begin{array}{l}9.681213 \\
.681443 \\
.681674 \\
.681905 \\
.652135 \\
.682365 \\
.682535 \\
.682825 \\
.683055 \\
.683284\end{array}$ & $\begin{array}{l}3.83 \\
3.85 \\
3.85 \\
3.83 \\
3.83 \\
3.83 \\
3.83 \\
3.83 \\
3.82 \\
3.83\end{array}$ & $\begin{array}{r}9.943141 \\
.943072 \\
.943003 \\
.942934 \\
.942864 \\
.942795 \\
.942726 \\
.942656 \\
.942587 \\
.942517\end{array}$ & $\begin{array}{l}1.15 \\
1.15 \\
1.15 \\
1.17 \\
1.15 \\
1.15 \\
1.17 \\
1.15 \\
1.17 \\
1.15\end{array}$ & $\begin{array}{r}9.738071 \\
.738371 \\
.738671 \\
.738971 \\
.739271 \\
.739570 \\
.739870 \\
.740160 \\
.740468 \\
.740767\end{array}$ & $\begin{array}{l}5.00 \\
5.00 \\
5.00 \\
5.00 \\
4.98 \\
5.00 \\
4.98 \\
4.98 \\
4.98 \\
4.98\end{array}$ & $\begin{array}{r}10.261929 \\
.261629 \\
.261329 \\
.261029 \\
.260729 \\
.260430 \\
.260130 \\
.250831 \\
.259532 \\
.259233\end{array}$ & $\begin{array}{l}19 \\
18 \\
17 \\
16 \\
15 \\
14 \\
13 \\
12 \\
11 \\
10\end{array}$ \\
\hline $\begin{array}{l}\mathbf{5 1} \\
\mathbf{5 2} \\
\mathbf{5 3} \\
\mathbf{5 4} \\
\mathbf{5 5} \\
\mathbf{5 6} \\
\mathbf{5 7} \\
\mathbf{5} 8 \\
\mathbf{5 9} \\
\mathrm{C0}\end{array}$ & $\begin{array}{r}9.683514 \\
.683743 \\
.683972 \\
.684201 \\
.634430 \\
.684058 \\
.684887 \\
.655115 \\
.685343 \\
9.655571\end{array}$ & $\begin{array}{l}3.82 \\
3.82 \\
3.82 \\
3.82 \\
3.80 \\
3.82 \\
3.80 \\
3.80 \\
3.80\end{array}$ & $\begin{array}{r}9.942448 \\
.942378 \\
.942308 \\
.042239 \\
.942169 \\
.942099 \\
.942029 \\
.941959 \\
.941559 \\
9.941819\end{array}$ & $\begin{array}{l}1.17 \\
1.17 \\
1.15 \\
1.17 \\
1.17 \\
1.17 \\
1.17 \\
1.17 \\
1.17\end{array}$ & $\begin{array}{r}9.741066 \\
.741365 \\
.741664 \\
.741962 \\
.742261 \\
.742559 \\
.742558 \\
.743154 \\
.743454 \\
9.743752\end{array}$ & $\begin{array}{l}\text { 4. } 98 \\
\text { 4. } 98 \\
\text { 4. } 97 \\
\text { 4. } 98 \\
\text { 4. } 97 \\
\text { 4. } 98 \\
4.97 \\
4.97 \\
\text { 4. } 97\end{array}$ & $\begin{array}{r}10.258934 \\
.258635 \\
.25 \times 336 \\
.255038 \\
.257739 \\
.257441 \\
.257142 \\
.256844 \\
.256546 \\
10.256248\end{array}$ & $\begin{array}{l}9 \\
8 \\
7 \\
6 \\
5 \\
4 \\
3 \\
2 \\
1 \\
0\end{array}$ \\
\hline , & Cosine. & D. $1^{\prime \prime}$. & Sine. & D. $1^{\prime \prime}$. & Cotang. & D. $1^{\prime \prime}$. & Tang. & , \\
\hline
\end{tabular}




\begin{tabular}{|c|c|c|c|c|c|c|c|c|}
\hline , & Sine. & D. $1^{\prime \prime}$. & Cosine. & D. $1^{\prime \prime}$. & T'ang. & D. $1^{\prime \prime}$. & Cotang. & , \\
\hline $\begin{array}{r}0 \\
1 \\
2 \\
3 \\
4 \\
5 \\
6 \\
7 \\
8 \\
9 \\
10\end{array}$ & $\begin{array}{l}9.685571 \\
.685799 \\
.686027 \\
.686254 \\
.686482 \\
.686709 \\
.686936 \\
.687163 \\
.687389 \\
.687616 \\
.687843\end{array}$ & $\begin{array}{l}3.80 \\
3.80 \\
3.78 \\
3.80 \\
3.78 \\
3.78 \\
3.78 \\
3.77 \\
3.78 \\
3.78 \\
3.77\end{array}$ & $\begin{array}{r}9.941819 \\
.941749 \\
.941679 \\
.941609 \\
.941539 \\
.941469 \\
.941398 \\
.941328 \\
.941258 \\
.941187 \\
.941117\end{array}$ & $\begin{array}{l}1.17 \\
1.17 \\
1.17 \\
1.17 \\
1.17 \\
1.18 \\
1.17 \\
1.17 \\
1.18 \\
1.17 \\
1.18\end{array}$ & $\begin{array}{r}9.743752 \\
.744050 \\
.744348 \\
.744645 \\
.744943 \\
.745240 \\
.745538 \\
.745835 \\
.746132 \\
.746429 \\
.746726\end{array}$ & $\begin{array}{l}4.97 \\
4.97 \\
4.95 \\
4.97 \\
4.95 \\
4.97 \\
4.95 \\
4.95 \\
4.95 \\
4.95 \\
4.95\end{array}$ & $\begin{array}{r}10.256248 \\
.255950 \\
.255652 \\
.255355 \\
.255057 \\
.254760 \\
.254462 \\
.254165 \\
.253868 \\
.253571 \\
.253274\end{array}$ & $\begin{array}{l}60 \\
59 \\
58 \\
57 \\
56 \\
55 \\
54 \\
53 \\
52 \\
51 \\
50\end{array}$ \\
\hline $\begin{array}{l}11 \\
12 \\
13 \\
14 \\
15 \\
16 \\
17 \\
18 \\
19 \\
20\end{array}$ & $\begin{array}{r}9.688069 \\
.688295 \\
.688521 \\
.688747 \\
.688972 \\
.689198 \\
.689423 \\
.689648 \\
.689873 \\
.690098\end{array}$ & $\begin{array}{l}3.77 \\
3.77 \\
3.77 \\
3.75 \\
3.77 \\
3.75 \\
3.75 \\
3.75 \\
3.75 \\
3.75\end{array}$ & $\begin{array}{r}9.941046 \\
.940975 \\
.940905 \\
.940834 \\
.940763 \\
.940693 \\
.940622 \\
.940551 \\
.940480 \\
.940409\end{array}$ & $\begin{array}{l}1.18 \\
1.17 \\
1.18 \\
1.18 \\
1.17 \\
1.18 \\
1.18 \\
1.18 \\
1.18 \\
1.18\end{array}$ & $\begin{array}{r}9.747023 \\
.747319 \\
.747616 \\
.747913 \\
.748209 \\
.748505 \\
.748801 \\
.749097 \\
.749393 \\
.749689\end{array}$ & $\begin{array}{l}4.93 \\
4.95 \\
4.95 \\
4.93 \\
4.93 \\
4.93 \\
4.93 \\
4.93 \\
4.93 \\
4.93\end{array}$ & $\begin{array}{r}10.252977 \\
.252681 \\
.252384 \\
.252087 \\
.251791 \\
.251495 \\
.251199 \\
.250903 \\
.250607 \\
.250311\end{array}$ & $\begin{array}{l}49 \\
48 \\
47 \\
46 \\
45 \\
44 \\
43 \\
42 \\
41 \\
40\end{array}$ \\
\hline $\begin{array}{l}21 \\
22 \\
23 \\
24 \\
25 \\
26 \\
27 \\
28 \\
29 \\
30 \\
\end{array}$ & $\begin{array}{r}9.690323 \\
.690548 \\
.690772 \\
.690996 \\
.691220 \\
.691444 \\
.691668 \\
.691892 \\
.692115 \\
.692339\end{array}$ & \begin{tabular}{l|}
3.75 \\
3.73 \\
3.73 \\
3.73 \\
3.73 \\
3.73 \\
3.73 \\
3.72 \\
3.73 \\
3.72 \\
\end{tabular} & $\begin{array}{r}9.940338 \\
.940267 \\
.940196 \\
.940125 \\
.940054 \\
.939982 \\
.939911 \\
.939840 \\
.939768 \\
.939697\end{array}$ & $\begin{array}{l}1.18 \\
1.18 \\
1.18 \\
1.18 \\
1.20 \\
1.18 \\
1.18 \\
1.20 \\
1.18 \\
1.20\end{array}$ & $\begin{array}{r}9.749985 \\
.750281 \\
.750576 \\
.750872 \\
.751167 \\
.751462 \\
.751757 \\
.752052 \\
.752347 \\
.752642\end{array}$ & $\begin{array}{l}4.93 \\
4.92 \\
4.93 \\
4.92 \\
4.92 \\
4.92 \\
4.92 \\
4.92 \\
4.92 \\
4.92\end{array}$ & $\begin{array}{r}10.250015 \\
.249719 \\
.249424 \\
.249128 \\
.248833 \\
.248538 \\
.248243 \\
.247948 \\
.247653 \\
.247358\end{array}$ & $\begin{array}{l}39 \\
38 \\
37 \\
36 \\
35 \\
34 \\
33 \\
32 \\
31 \\
30\end{array}$ \\
\hline $\begin{array}{l}31 \\
32 \\
33 \\
34 \\
35 \\
36 \\
37 \\
38 \\
39 \\
40\end{array}$ & $\begin{array}{r}9.692562 \\
.692785 \\
.693008 \\
.693231 \\
.693453 \\
.693676 \\
.693998 \\
.694120 \\
.694342 \\
.694564\end{array}$ & $\begin{array}{l}3.72 \\
3.72 \\
3.72 \\
3.70 \\
3.72 \\
3.70 \\
3.70 \\
3.70 \\
3.70 \\
3.70\end{array}$ & $\begin{array}{r}9.939625 \\
.939554 \\
.939482 \\
.939410 \\
.939339 \\
.939267 \\
.939195 \\
.939123 \\
.939052 \\
.938980\end{array}$ & $\begin{array}{l}1.18 \\
1.20 \\
1.20 \\
1.18 \\
1.20 \\
1.20 \\
1.20 \\
1.18 \\
1.20 \\
1.20\end{array}$ & $\begin{array}{r}9.752937 \\
.753231 \\
.753526 \\
.753820 \\
.754115 \\
.754409 \\
.754703 \\
.754997 \\
.755291 \\
.755585\end{array}$ & $\begin{array}{l}4.90 \\
4.92 \\
4.90 \\
4.92 \\
4.90 \\
4.90 \\
4.90 \\
4.90 \\
4.90 \\
4.88\end{array}$ & $\begin{array}{r}10.247063 \\
.246769 \\
.246474 \\
.246180 \\
.245885 \\
.245591 \\
.245297 \\
.245003 \\
.244709 \\
.244415\end{array}$ & $\begin{array}{l}29 \\
28 \\
27 \\
26 \\
25 \\
24 \\
23 \\
22 \\
21 \\
20\end{array}$ \\
\hline $\begin{array}{l}41 \\
42 \\
43 \\
44 \\
45 \\
46 \\
47 \\
48 \\
49 \\
50\end{array}$ & $\begin{array}{r}9.694786 \\
.695007 \\
.695229 \\
.695450 \\
.695671 \\
.695892 \\
.696113 \\
.696334 \\
.6965154 \\
.696775\end{array}$ & $\begin{array}{l}3.68 \\
3.70 \\
3.68 \\
3.68 \\
3.68 \\
3.68 \\
3.68 \\
3.67 \\
3.68 \\
3.67\end{array}$ & $\begin{array}{r}9.938908 \\
.938836 \\
.938763 \\
.938691 \\
.938619 \\
.938547 \\
.935475 \\
.938402 \\
.938330 \\
.938258\end{array}$ & $\begin{array}{l}1.20 \\
1.22 \\
1.20 \\
1.20 \\
1.20 \\
1.20 \\
1.22 \\
1.20 \\
1.20 \\
1.22\end{array}$ & $\begin{array}{r}9.755878 \\
.756172 \\
.756465 \\
.756759 \\
.757052 \\
.757345 \\
.757638 \\
.757931 \\
.758224 \\
.758517\end{array}$ & $\begin{array}{l}4.90 \\
4.85 \\
4.90 \\
4.88 \\
4.88 \\
4.88 \\
4.88 \\
4.88 \\
4.88 \\
4.88\end{array}$ & $\begin{array}{r}10.244122 \\
.243828 \\
.243535 \\
.243241 \\
.242948 \\
.242655 \\
.242362 \\
.242069 \\
.241776 \\
.241483\end{array}$ & $\begin{array}{l}19 \\
18 \\
17 \\
16 \\
15 \\
14 \\
13 \\
12 \\
11 \\
10\end{array}$ \\
\hline $\begin{array}{l}51 \\
52 \\
53 \\
54 \\
55 \\
56 \\
57 \\
58 \\
59 \\
60\end{array}$ & $\begin{array}{r}9.696925 \\
.697215 \\
.697435 \\
.697654 \\
.697874 \\
.695094 \\
.698313 \\
.698532 \\
.698751 \\
9.698970\end{array}$ & $\begin{array}{l}3.67 \\
3.67 \\
3.65 \\
3.67 \\
3.67 \\
3.65 \\
3.65 \\
3.65 \\
3.65\end{array}$ & $\begin{array}{r}9.938185 \\
.938113 \\
.938040 \\
.937967 \\
.937895 \\
.937822 \\
.937749 \\
.937676 \\
.937604 \\
9.937531\end{array}$ & $\begin{array}{l}1.20 \\
1.22 \\
1.22 \\
1.20 \\
1.22 \\
1.22 \\
1.22 \\
1.20 \\
1.22\end{array}$ & $\begin{array}{r}9.758810 \\
.759102 \\
.759395 \\
.759687 \\
.759979 \\
.760272 \\
.760564 \\
.760856 \\
.761148 \\
9.761439\end{array}$ & $\begin{array}{l}4.87 \\
4.88 \\
4.87 \\
4.87 \\
4.89 \\
4.87 \\
4.87 \\
4.87 \\
4.85\end{array}$ & $\begin{array}{r}10.241190 \\
.240898 \\
.240605 \\
.240313 \\
.240021 \\
.239728 \\
.239436 \\
.239144 \\
.238852 \\
10.238561\end{array}$ & $\begin{array}{l}9 \\
8 \\
7 \\
6 \\
5 \\
4 \\
3 \\
2 \\
1 \\
0\end{array}$ \\
\hline ' & Cosine. & D. $1^{\prime \prime}$. & Sine. & D. $1^{\prime \prime}$. & Cotang. & D. $1^{\prime \prime}$. & Tang. & ' \\
\hline
\end{tabular}




\begin{tabular}{|c|c|c|c|c|c|c|c|c|}
\hline , & Sine. & D. $1^{\prime \prime}$. & Cosine. & D. $1^{\prime \prime}$. & Tang. & D. $1^{\prime \prime}$. & Cotang. & , \\
\hline $\begin{array}{r}0 \\
1 \\
2 \\
3 \\
4 \\
5 \\
6 \\
7 \\
8 \\
9 \\
10\end{array}$ & $\begin{array}{r}9.695970 \\
.699159 \\
.699407 \\
.699626 \\
.699544 \\
.700062 \\
.700250 \\
.700498 \\
.700716 \\
.700933 \\
.701151\end{array}$ & $\begin{array}{l}3.65 \\
3.63 \\
3.65 \\
3.63 \\
3.63 \\
3.63 \\
3.63 \\
3.63 \\
3.62 \\
3.63 \\
3.62\end{array}$ & $\begin{array}{r}9.937531 \\
.937458 \\
.937385 \\
.937312 \\
.937238 \\
.937165 \\
.937092 \\
.937019 \\
.936946 \\
.936872 \\
.936799\end{array}$ & $\begin{array}{l}1.22 \\
1.22 \\
1.22 \\
1.23 \\
1.22 \\
1.22 \\
1.22 \\
1.22 \\
1.23 \\
1.22 \\
1.23\end{array}$ & $\begin{array}{r}9.761439 \\
.761731 \\
.762023 \\
.762314 \\
.762606 \\
.762597 \\
.763188 \\
.763479 \\
.763770 \\
.764061 \\
.764352\end{array}$ & $\begin{array}{l}4.87 \\
4.87 \\
4.85 \\
4.87 \\
4.85 \\
4.85 \\
4.85 \\
4.85 \\
4.85 \\
4.85 \\
4.85\end{array}$ & $\begin{array}{r}10.238561 \\
.238269 \\
.237977 \\
.237686 \\
.237394 \\
.237103 \\
.236812 \\
.236521 \\
.236230 \\
.235939 \\
.235648\end{array}$ & $\begin{array}{l}60 \\
59 \\
58 \\
57 \\
56 \\
55 \\
54 \\
53 \\
52 \\
51 \\
50\end{array}$ \\
\hline $\begin{array}{l}11 \\
12 \\
13 \\
14 \\
15 \\
16 \\
17 \\
18 \\
19 \\
20\end{array}$ & $\begin{array}{r}9.701368 \\
.701585 \\
.701802 \\
.702019 \\
.702236 \\
.702452 \\
.702669 \\
.702885 \\
.703101 \\
.703317\end{array}$ & \begin{tabular}{l|l|}
3.62 & \\
3.62 \\
3.62 \\
3.62 \\
3.60 \\
3.62 \\
3.60 \\
3.60 \\
3.60 \\
3.60
\end{tabular} & $\begin{array}{r}9.936725 \\
.936652 \\
.936578 \\
.936505 \\
.936431 \\
.936357 \\
.936284 \\
.936210 \\
.936136 \\
.936062\end{array}$ & $\begin{array}{l}1.22 \\
1.23 \\
1.22 \\
1.23 \\
1.23 \\
1.22 \\
1.23 \\
1.23 \\
1.23 \\
1.23\end{array}$ & $\begin{array}{r}9.764643 \\
.764933 \\
.765224 \\
.765514 \\
.765805 \\
.766095 \\
.766385 \\
.766675 \\
.766965 \\
.767255\end{array}$ & $\begin{array}{l}4.83 \\
4.85 \\
4.83 \\
4.85 \\
4.83 \\
4.83 \\
4.83 \\
4.83 \\
4.83 \\
4.83\end{array}$ & $\begin{array}{r}10.235357 \\
.235067 \\
.234776 \\
.234486 \\
.234195 \\
.233905 \\
.233615 \\
.233325 \\
.233035 \\
.232745\end{array}$ & $\begin{array}{l}49 \\
48 \\
47 \\
46 \\
45 \\
44 \\
43 \\
42 \\
41 \\
40\end{array}$ \\
\hline $\begin{array}{l}21 \\
22 \\
23 \\
24 \\
25 \\
26 \\
27 \\
28 \\
29 \\
30\end{array}$ & $\begin{array}{r}9.703533 \\
.703749 \\
.703964 \\
.704179 \\
.704395 \\
.704610 \\
.704825 \\
.705040 \\
.705254 \\
.705469\end{array}$ & $\begin{array}{l}3.60 \\
3.58 \\
3.58 \\
3.60 \\
3.58 \\
3.58 \\
3.58 \\
3.57 \\
3.58 \\
3.57\end{array}$ & $\begin{array}{r}9.935988 \\
.935914 \\
.935840 \\
.935766 \\
.935692 \\
.935618 \\
.935543 \\
.935469 \\
.935395 \\
.935320\end{array}$ & $\begin{array}{l}1.23 \\
1.23 \\
1.23 \\
1.23 \\
1.23 \\
1.25 \\
1.23 \\
1.23 \\
1.25 \\
1.23\end{array}$ & $\begin{array}{r}9.767545 \\
.767834 \\
.768124 \\
.768414 \\
.768703 \\
.768992 \\
.769281 \\
.769571 \\
.769860 \\
.770148\end{array}$ & $\begin{array}{l}4.82 \\
4.83 \\
4.83 \\
4.82 \\
4.82 \\
4.82 \\
4.83 \\
4.82 \\
4.80 \\
4.82\end{array}$ & $\begin{array}{r}10.232455 \\
.232166 \\
.231876 \\
.231586 \\
.231297 \\
.231008 \\
.230719 \\
.230429 \\
.230140 \\
.229852\end{array}$ & $\begin{array}{l}39 \\
38 \\
37 \\
36 \\
35 \\
34 \\
33 \\
32 \\
31 \\
30\end{array}$ \\
\hline $\begin{array}{l}31 \\
32 \\
33 \\
34 \\
35 \\
36 \\
37 \\
39 \\
39 \\
40\end{array}$ & $\begin{array}{r}9.705683 \\
.705998 \\
.706112 \\
.706326 \\
.706539 \\
.706753 \\
.706967 \\
.707180 \\
.707393 \\
.707606\end{array}$ & $\begin{array}{l}3.58 \\
3.57 \\
3.57 \\
3.55 \\
3.57 \\
3.57 \\
3.55 \\
3.55 \\
3.55 \\
3.55\end{array}$ & $\begin{array}{r}9.935246 \\
.935171 \\
.935097 \\
.935022 \\
.934948 \\
.934873 \\
.934798 \\
.934723 \\
.934649 \\
.934574\end{array}$ & $\begin{array}{l}1.25 \\
1.23 \\
1.25 \\
1.23 \\
1.25 \\
1.25 \\
1.25 \\
1.23 \\
1.25 \\
1.25\end{array}$ & $\begin{array}{r}9.770437 \\
.770726 \\
.771015 \\
.771303 \\
.771592 \\
.771880 \\
.772168 \\
.772457 \\
.772745 \\
.773033\end{array}$ & $\begin{array}{l}4.82 \\
4.82 \\
4.80 \\
4.82 \\
4.80 \\
4.80 \\
4.82 \\
4.80 \\
4.80 \\
4.80\end{array}$ & $\begin{array}{r}10.229563 \\
.229274 \\
.225985 \\
.228697 \\
.228408 \\
.228120 \\
.227832 \\
.227543 \\
.227255 \\
.226967\end{array}$ & $\begin{array}{l}29 \\
28 \\
27 \\
26 \\
25 \\
24 \\
23 \\
22 \\
21 \\
20\end{array}$ \\
\hline $\begin{array}{l}41 \\
42 \\
43 \\
44 \\
45 \\
46 \\
47 \\
49 \\
49 \\
50\end{array}$ & $\begin{array}{r}9.707819 \\
.70 \times 032 \\
.708245 \\
.708458 \\
.708670 \\
.708582 \\
.709094 \\
.709306 \\
.709518 \\
.709730\end{array}$ & $\begin{array}{l}3.53 \\
3.53 \\
3.53 \\
3.53 \\
3.52\end{array}$ & $\begin{array}{r}9.934499 \\
.934424 \\
.934349 \\
.934274 \\
.934199 \\
.934123 \\
.934048 \\
.933973 \\
.933898 \\
.933522\end{array}$ & $\begin{array}{l}1.25 \\
1.25 \\
1.25 \\
1.25 \\
1.27 \\
1.25 \\
1.25 \\
1.25 \\
1.27 \\
1.25\end{array}$ & $\begin{array}{r}9.773321 \\
.773608 \\
.773896 \\
.774184 \\
.774471 \\
.774759 \\
.775046 \\
.775333 \\
.775621 \\
.775908\end{array}$ & $\begin{array}{l}4.78 \\
4.80 \\
4.80 \\
4.78 \\
4.80 \\
4.78 \\
4.78 \\
4.80 \\
4.78 \\
4.78\end{array}$ & $\begin{array}{r}10.226679 \\
.226392 \\
.226104 \\
.225816 \\
.225529 \\
.225241 \\
.224954 \\
.224667 \\
.224379 \\
.224092\end{array}$ & $\begin{array}{l}19 \\
18 \\
17 \\
16 \\
15 \\
14 \\
13 \\
12 \\
12 \\
11 \\
10\end{array}$ \\
\hline $\begin{array}{l}51 \\
52 \\
53 \\
54 \\
55 \\
56 \\
57 \\
58 \\
59 \\
60\end{array}$ & $\begin{array}{r}9.709941 \\
.710153 \\
.710364 \\
.710575 \\
.710786 \\
.710997 \\
.711208 \\
.711419 \\
.711629 \\
9.711839\end{array}$ & \begin{tabular}{l|}
3.53 \\
3.52 \\
3.52 \\
3.52 \\
3.52 \\
3.52 \\
3.52 \\
3.50 \\
3.50
\end{tabular} & $\begin{array}{r}9.933747 \\
.933671 \\
.933596 \\
.933520 \\
.933445 \\
.933369 \\
.933293 \\
.933217 \\
.933141 \\
9.933066\end{array}$ & $\begin{array}{l}1.27 \\
1.25 \\
1.27 \\
1.25 \\
1.27 \\
1.27 \\
1.27 \\
1.27 \\
1.25\end{array}$ & $\begin{array}{r}9.776195 \\
.776482 \\
.776768 \\
.777055 \\
.777342 \\
.777628 \\
.777915 \\
.778201 \\
.778485 \\
9.778774\end{array}$ & $\begin{array}{l}4.78 \\
4.77 \\
4.78 \\
4.78 \\
4.77 \\
4.78 \\
4.77 \\
4.78 \\
4.77 \\
4.77\end{array}$ & $\begin{array}{r}10.223805 \\
.223518 \\
.223232 \\
.222945 \\
.222658 \\
.222372 \\
.222055 \\
.221799 \\
.221512 \\
10.221226\end{array}$ & $\begin{array}{l}9 \\
8 \\
7 \\
6 \\
5 \\
5 \\
4 \\
3 \\
2 \\
1 \\
0\end{array}$ \\
\hline ' & toside. & 6.8 & 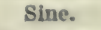 & 20 & Wrang. & 10.1 & $200 \mathrm{~s}$ & 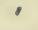 \\
\hline
\end{tabular}




\begin{tabular}{|c|c|c|c|c|c|c|c|c|}
\hline , & Sine. & D. $1^{\prime \prime}$. & Cosine. & D. $1^{\prime \prime}$. & Tang. & D. $1^{\prime \prime}$. & Cotang. & ' \\
\hline $\begin{array}{r}0 \\
1 \\
2 \\
3 \\
4 \\
5 \\
6 \\
7 \\
8 \\
9 \\
10\end{array}$ & $\begin{array}{r}9.711839 \\
.712050 \\
.712260 \\
.712469 \\
.712679 \\
.712889 \\
.713098 \\
.713308 \\
.713517 \\
.713726 \\
.713935\end{array}$ & $\begin{array}{l}3.52 \\
3.50 \\
3.48 \\
3.50 \\
3.50 \\
3.48 \\
3.50 \\
3.48 \\
3.48 \\
3.48 \\
3.48\end{array}$ & $\begin{array}{r}9.933066 \\
.932990 \\
.932914 \\
.932838 \\
.932762 \\
.932685 \\
.932609 \\
.932533 \\
.932457 \\
.932380 \\
.932304\end{array}$ & $\begin{array}{l}1.27 \\
1.27 \\
1.27 \\
1.27 \\
1.28 \\
1.27 \\
1.27 \\
1.27 \\
1.28 \\
1.27 \\
1.27\end{array}$ & $\begin{array}{r}9.778774 \\
.779060 \\
.779346 \\
.779632 \\
.779918 \\
.780203 \\
.780489 \\
.780775 \\
.781060 \\
.781346 \\
.781631\end{array}$ & $\begin{array}{l}4.77 \\
4.77 \\
4.77 \\
4.77 \\
4.75 \\
4.77 \\
4.77 \\
4.75 \\
4.77 \\
4.75 \\
4.75\end{array}$ & $\begin{array}{r}10.221226 \\
.220940 \\
.220654 \\
.220368 \\
.220082 \\
.219797 \\
.219511 \\
.219225 \\
.218940 \\
.218654 \\
.218369\end{array}$ & $\begin{array}{l}60 \\
59 \\
58 \\
57 \\
56 \\
55 \\
54 \\
53 \\
52 \\
51 \\
50\end{array}$ \\
\hline $\begin{array}{l}11 \\
12 \\
13 \\
14 \\
15 \\
16 \\
17 \\
18 \\
19 \\
20\end{array}$ & $\begin{array}{r}9.714144 \\
.714352 \\
.714561 \\
.714769 \\
.714978 \\
.715186 \\
.715394 \\
.715602 \\
.715809 \\
.716017\end{array}$ & $\begin{array}{l}3.47 \\
3.48 \\
3.47 \\
3.48 \\
3.47 \\
3.47 \\
3.47 \\
3.45 \\
3.47 \\
3.45\end{array}$ & $\begin{array}{r}9.932228 \\
.932151 \\
.932075 \\
.931998 \\
.931921 \\
.931845 \\
.931768 \\
.931691 \\
.931614 \\
.931537\end{array}$ & $\begin{array}{l}1.28 \\
1.27 \\
1.28 \\
1.28 \\
1.27 \\
1.28 \\
1.28 \\
1.28 \\
1.28 \\
1.28\end{array}$ & $\begin{array}{r}9.781916 \\
.782201 \\
.782486 \\
.782771 \\
.783056 \\
.783341 \\
.783626 \\
.783910 \\
.784195 \\
.784479\end{array}$ & $\begin{array}{l}4.75 \\
4.75 \\
4.75 \\
4.75 \\
4.75 \\
4.75 \\
4.75 \\
4.73 \\
4.75 \\
4.73 \\
4.75\end{array}$ & $\begin{array}{r}10.218084 \\
.217799 \\
.217514 \\
.217229 \\
.216944 \\
.216659 \\
.216374 \\
.216090 \\
.215805 \\
.215521\end{array}$ & $\begin{array}{l}49 \\
48 \\
47 \\
46 \\
45 \\
44 \\
43 \\
42 \\
41 \\
40\end{array}$ \\
\hline $\begin{array}{l}21 \\
22 \\
23 \\
24 \\
25 \\
26 \\
27 \\
28 \\
29 \\
30\end{array}$ & $\begin{array}{r}9.716224 \\
.716432 \\
.716639 \\
.716846 \\
.717053 \\
.717259 \\
.717466 \\
.717673 \\
.717879 \\
.718085\end{array}$ & $\begin{array}{l}3.47 \\
3.45 \\
3.45 \\
3.45 \\
3.43 \\
3.45 \\
3.45 \\
3.43 \\
3.43 \\
3.43\end{array}$ & $\begin{array}{r}9.931460 \\
.931383 \\
.931306 \\
.931229 \\
.931152 \\
.931075 \\
.930998 \\
.930921 \\
.930843 \\
.930766\end{array}$ & $\begin{array}{l}1.28 \\
1.28 \\
1.28 \\
1.28 \\
1.28 \\
1.28 \\
1.28 \\
1.30 \\
1.28 \\
1.30\end{array}$ & $\begin{array}{r}9.784764 \\
.785048 \\
.785332 \\
.785616 \\
.785900 \\
.786184 \\
.786468 \\
.786752 \\
.787036 \\
.787319\end{array}$ & $\begin{array}{l}4.73 \\
4.73 \\
4.73 \\
4.73 \\
4.73 \\
4.73 \\
4.73 \\
4.73 \\
4.72 \\
4.73\end{array}$ & $\begin{array}{r}10.215236 \\
.214952 \\
.214668 \\
.214384 \\
.214100 \\
.213816 \\
.213532 \\
.213248 \\
.212964 \\
.212681\end{array}$ & $\begin{array}{l}39 \\
38 \\
37 \\
36 \\
35 \\
34 \\
33 \\
32 \\
31 \\
30\end{array}$ \\
\hline $\begin{array}{l}31 \\
32 \\
33 \\
34 \\
35 \\
36 \\
37 \\
38 \\
39 \\
40\end{array}$ & $\begin{array}{r}9.718291 \\
.718497 \\
.718703 \\
.718909 \\
.719114 \\
.719320 \\
.719525 \\
.719730 \\
.719935 \\
.720140\end{array}$ & $\begin{array}{l}3.43 \\
3.43 \\
3.43 \\
3.42 \\
3.43 \\
3.42 \\
3.42 \\
3.42 \\
3.42 \\
3.42\end{array}$ & $\begin{array}{r}9.930688 \\
.930611 \\
.930533 \\
.930456 \\
.930378 \\
.930300 \\
.930223 \\
.930145 \\
.930067 \\
.929989\end{array}$ & $\begin{array}{l}1.28 \\
1.30 \\
1.28 \\
1.30 \\
1.30 \\
1.28 \\
1.30 \\
1.30 \\
1.30 \\
1.30\end{array}$ & $\begin{array}{r}9.787603 \\
.787886 \\
.788170 \\
.788453 \\
.788736 \\
.789019 \\
.789302 \\
.789585 \\
.789868 \\
.790151\end{array}$ & $\begin{array}{l}4.72 \\
4.73 \\
4.72 \\
4.72 \\
4.72 \\
4.72 \\
4.72 \\
4.72 \\
4.72 \\
4.72\end{array}$ & $\begin{array}{r}10.212397 \\
.212114 \\
.211830 \\
.211547 \\
.211264 \\
.210981 \\
.210698 \\
.210415 \\
.210132 \\
.209849\end{array}$ & $\begin{array}{l}29 \\
28 \\
27 \\
26 \\
25 \\
24 \\
23 \\
22 \\
21 \\
20\end{array}$ \\
\hline $\begin{array}{l}41 \\
42 \\
43 \\
44 \\
45 \\
46 \\
47 \\
48 \\
49 \\
50\end{array}$ & $\begin{array}{r}9.720345 \\
.720549 \\
.720754 \\
.720958 \\
.721162 \\
.721366 \\
.721570 \\
.721774 \\
.721978 \\
.722181\end{array}$ & $\begin{array}{l}3.40 \\
3.42 \\
3.40 \\
3.40 \\
3.40 \\
3.40 \\
3.40 \\
3.40 \\
3.38 \\
3.40\end{array}$ & $\begin{array}{r}9.929911 \\
.929833 \\
.929755 \\
.929677 \\
.929599 \\
.929521 \\
.929442 \\
.939364 \\
.929286 \\
.929207\end{array}$ & $\begin{array}{l}1.30 \\
1.30 \\
1.30 \\
1.30 \\
1.30 \\
1.32 \\
1.30 \\
1.30 \\
1.32 \\
1.30\end{array}$ & $\begin{array}{r}9.790434 \\
.790716 \\
.790999 \\
.791281 \\
.7915(63 \\
.791846 \\
.792128 \\
.792410 \\
.792692 \\
.792974\end{array}$ & $\begin{array}{l}4.72 \\
4.70 \\
4.72 \\
4.70 \\
4.70 \\
4.72 \\
4.70 \\
4.70 \\
4.70 \\
4.70 \\
4.70\end{array}$ & $\begin{array}{r}10.209566 \\
.209284 \\
.209001 \\
.208719 \\
.208437 \\
.208154 \\
.207872 \\
.207590 \\
.207308 \\
.207026\end{array}$ & $\begin{array}{l}19 \\
18 \\
17 \\
16 \\
15 \\
14 \\
13 \\
12 \\
11 \\
10\end{array}$ \\
\hline $\begin{array}{l}51 \\
52 \\
53 \\
54 \\
55 \\
56 \\
57 \\
58 \\
59 \\
60\end{array}$ & $\begin{array}{r}9.722385 \\
.722588 \\
.722791 \\
.722994 \\
.723197 \\
.723400 \\
.723603 \\
.723805 \\
.724007 \\
9.724210\end{array}$ & $\begin{array}{l}\text { 3. } 38 \\
3.38 \\
3.38 \\
3.38 \\
3.38 \\
3.38 \\
3.37 \\
3.37 \\
3.38\end{array}$ & $\begin{array}{r}9.929129 \\
.929050 \\
.928972 \\
.928893 \\
.928815 \\
.928736 \\
.928657 \\
.928578 \\
.928499 \\
9.928420\end{array}$ & $\begin{array}{l}1.32 \\
1.30 \\
1.32 \\
1.30 \\
1.32 \\
1.32 \\
1.32 \\
1.32 \\
1.32\end{array}$ & $\begin{array}{r}9.793256 \\
.793538 \\
.793819 \\
.794101 \\
.794383 \\
.794664 \\
.794946 \\
.795227 \\
.795508 \\
9.795789\end{array}$ & $\begin{array}{l}4.70 \\
4.68 \\
4.70 \\
4.70 \\
4.68 \\
4.70 \\
4.68 \\
4.68 \\
4.68\end{array}$ & $\begin{array}{r}10.206744 \\
.206462 \\
.206181 \\
.205899 \\
.205617 \\
.205336 \\
.205054 \\
.204773 \\
.204492 \\
10.204211\end{array}$ & $\begin{array}{l}9 \\
8 \\
7 \\
6 \\
6 \\
5 \\
4 \\
3 \\
2 \\
1 \\
0\end{array}$ \\
\hline & Cosine. & D. $1^{\prime \prime}$. & Sine. & D. $1^{\prime \prime}$. & Cotang. & D. $1^{\prime \prime}$. & 1 ants. & ' \\
\hline
\end{tabular}




\begin{tabular}{|c|c|c|c|c|c|c|c|c|}
\hline , & Sine. & D. $1^{\prime \prime}$. & Cosine. & D. $1^{\prime \prime}$. & Tang. & D. $1^{\prime \prime}$. & Cotang. & , \\
\hline $\begin{array}{r}0 \\
1 \\
2 \\
3 \\
4 \\
5 \\
6 \\
7 \\
8 \\
9 \\
10\end{array}$ & $\begin{array}{r}9.724210 \\
.724412 \\
.724614 \\
.724816 \\
.725017 \\
.725219 \\
.725420 \\
.725622 \\
.725523 \\
.726024 \\
.726225\end{array}$ & $\begin{array}{l}\text { 3. } 37 \\
\text { 3. } 37 \\
\text { 3. } 37 \\
\text { 3. } 35 \\
\text { 3. } 37 \\
\text { 3. } 35 \\
\text { 3. } 37 \\
\text { 3. } 35 \\
3.35 \\
3.35 \\
3.35\end{array}$ & $\begin{array}{r}9.928420 \\
.928342 \\
.925263 \\
.928183 \\
.928104 \\
.925025 \\
.927946 \\
.927867 \\
.927787 \\
.927708 \\
.927629\end{array}$ & $\begin{array}{l}1.30 \\
1.32 \\
1.33 \\
1.32 \\
1.32 \\
1.32 \\
1.32 \\
1.33 \\
1.32 \\
1.32 \\
1.33\end{array}$ & $\begin{array}{r}9.795789 \\
.796070 \\
.796351 \\
.7966332 \\
.796913 \\
.797194 \\
.797474 \\
.797755 \\
.798036 \\
.798316 \\
.795596\end{array}$ & $\begin{array}{l}4.68 \\
4.68 \\
4.68 \\
4.68 \\
4.68 \\
4.67 \\
4.68 \\
4.68 \\
4.67 \\
4.67 \\
4.68\end{array}$ & $\begin{array}{r}10.204211 \\
.203930 \\
.203649 \\
.203368 \\
.203087 \\
.202506 \\
.202326 \\
.202245 \\
.201964 \\
.201684 \\
.201404\end{array}$ & $\begin{array}{l}60 \\
59 \\
58 \\
57 \\
56 \\
55 \\
54 \\
53 \\
52 \\
51 \\
50\end{array}$ \\
\hline $\begin{array}{l}11 \\
12 \\
13 \\
14 \\
14 \\
15 \\
16 \\
17 \\
18 \\
19 \\
20 \\
\end{array}$ & $\begin{array}{r}9.726426 \\
.726626 \\
.726 \times 27 \\
.727027 \\
.727228 \\
.727428 \\
.727628 \\
.727528 \\
.728027 \\
.728227\end{array}$ & $\begin{array}{l}\text { 3. } 33 \\
\text { 3. } 35 \\
\text { 3. } 33 \\
\text { 3. } 35 \\
\text { 3. } 33 \\
\text { 3. } 33 \\
\text { 3. } 33 \\
\text { 3. } 32 \\
\text { 3. } 33 \\
\text { 3. } 33\end{array}$ & $\begin{array}{r}9.927549 \\
.927470 \\
.927390 \\
.927310 \\
.927231 \\
.927151 \\
.927071 \\
.926991 \\
.926911 \\
.926831\end{array}$ & $\begin{array}{l}1.32 \\
1.33 \\
1.33 \\
1.32 \\
1.33 \\
1.33 \\
1.33 \\
1.33 \\
1.33 \\
1.33\end{array}$ & $\begin{array}{r}9.798877 \\
.799157 \\
.799437 \\
.799717 \\
.799997 \\
.800277 \\
.800557 \\
.800836 \\
.801116 \\
.801396\end{array}$ & $\begin{array}{l}4.67 \\
4.67 \\
4.67 \\
4.67 \\
4.67 \\
4.67 \\
4.65 \\
4.67 \\
4.67 \\
4.65\end{array}$ & $\begin{array}{r}10.201123 \\
.200843 \\
.200563 \\
.200283 \\
.200003 \\
.199723 \\
.199443 \\
.199164 \\
.198584 \\
.198604\end{array}$ & $\begin{array}{l}49 \\
49 \\
47 \\
46 \\
45 \\
44 \\
43 \\
42 \\
41 \\
40\end{array}$ \\
\hline $\begin{array}{l}21 \\
22 \\
23 \\
24 \\
25 \\
26 \\
27 \\
29 \\
29 \\
30\end{array}$ & $\begin{array}{r}9.728427 \\
.728626 \\
.728825 \\
.729024 \\
.729223 \\
.729422 \\
.729621 \\
.729820 \\
.730018 \\
.730217\end{array}$ & $\begin{array}{l}\text { 3. } 32 \\
\text { 3. } 32 \\
\text { 3. } 32 \\
\text { 3. } 32 \\
\text { 3. } 32 \\
\text { 3. } 32 \\
\text { 3. } 32 \\
\text { 3. } 30 \\
\text { 3. } 32 \\
\text { 3. } 30\end{array}$ & $\begin{array}{r}9.926751 \\
.926671 \\
.926591 \\
.926511 \\
.926431 \\
.926351 \\
.926270 \\
.926190 \\
.926110 \\
.926029\end{array}$ & $\begin{array}{l}1.33 \\
1.33 \\
1.33 \\
1.33 \\
1.33 \\
1.35 \\
1.33 \\
1.33 \\
1.35 \\
1.33\end{array}$ & $\begin{array}{r}9.801675 \\
.801955 \\
.802234 \\
.802513 \\
.802793 \\
.803072 \\
.803351 \\
.803630 \\
.803909 \\
.804187\end{array}$ & $\begin{array}{l}4.67 \\
4.65 \\
4.65 \\
4.65 \\
4.67 \\
4.65 \\
4.65 \\
4.65 \\
4.63 \\
4.65\end{array}$ & $\begin{array}{r}10.198325 \\
.193045 \\
.197766 \\
.197487 \\
.197208 \\
.196928 \\
.196649 \\
.196370 \\
.196091 \\
.195813\end{array}$ & $\begin{array}{l}39 \\
38 \\
37 \\
36 \\
35 \\
34 \\
33 \\
32 \\
31 \\
30\end{array}$ \\
\hline $\begin{array}{l}31 \\
32 \\
33 \\
34 \\
35 \\
36 \\
37 \\
35 \\
39 \\
40\end{array}$ & $\begin{array}{r}9.730415 \\
.730613 \\
.730811 \\
.731009 \\
.731206 \\
.731404 \\
.731602 \\
.731799 \\
.731996 \\
.732193\end{array}$ & $\begin{array}{l}3.30 \\
3.30 \\
3.30 \\
3.28 \\
3.30 \\
3.30 \\
3.28 \\
3.28 \\
3.28 \\
3.28\end{array}$ & $\begin{array}{r}9.925949 \\
.925868 \\
.925788 \\
.925707 \\
.925626 \\
.925545 \\
.925465 \\
.925384 \\
.925303 \\
.925222\end{array}$ & $\begin{array}{l}1.35 \\
1.33 \\
1.35 \\
1.35 \\
1.35 \\
1.33 \\
1.35 \\
1.35 \\
1.35 \\
1.35\end{array}$ & $\begin{array}{r}9.804466 \\
.804745 \\
.805023 \\
.805302 \\
.805580 \\
.805859 \\
.806137 \\
.806415 \\
.806693 \\
.806971\end{array}$ & $\begin{array}{l}4.65 \\
\text { 4. } 63 \\
\text { 4. } 65 \\
\text { 4. } 63 \\
\text { 4. } 65 \\
\text { 4. } 63 \\
\text { 4. } 63 \\
\text { 4. } 63 \\
\text { 4. } 63 \\
\text { 4. } 63\end{array}$ & $\begin{array}{r}10.195534 \\
.195255 \\
.194977 \\
.194699 \\
.194420 \\
.194141 \\
.193863 \\
.193585 \\
.193307 \\
.193029\end{array}$ & $\begin{array}{l}29 \\
28 \\
27 \\
26 \\
25 \\
24 \\
23 \\
22 \\
21 \\
20\end{array}$ \\
\hline $\begin{array}{l}41 \\
42 \\
43 \\
44 \\
45 \\
46 \\
47 \\
48 \\
49 \\
50\end{array}$ & $\begin{array}{r}9.732390 \\
.732587 \\
.732784 \\
.732950 \\
.733177 \\
.733373 \\
.733569 \\
.733765 \\
.733961 \\
.734157\end{array}$ & $\begin{array}{l}3.28 \\
3.28 \\
3.27 \\
3.28 \\
3.27 \\
3.27 \\
3.27 \\
3.27 \\
3.27 \\
3.27\end{array}$ & $\begin{array}{r}9.925141 \\
.925060 \\
.924979 \\
.924897 \\
.924316 \\
.924735 \\
.924654 \\
.924572 \\
.924491 \\
.924409\end{array}$ & $\begin{array}{l}1.35 \\
1.35 \\
1.37 \\
1.35 \\
1.35 \\
1.35 \\
1.37 \\
1.35 \\
1.37 \\
1.35\end{array}$ & $\begin{array}{r}9.807249 \\
.807527 \\
.807805 \\
.808083 \\
.805361 \\
.803638 \\
.808916 \\
.809193 \\
.809471 \\
.809745\end{array}$ & $\begin{array}{l}4.63 \\
4.63 \\
4.63 \\
4.63 \\
4.62 \\
4.63 \\
4.62 \\
4.63 \\
4.62 \\
4.62\end{array}$ & $\begin{array}{r}10.192751 \\
.192473 \\
.192195 \\
.191917 \\
.191639 \\
.191362 \\
.191084 \\
.190507 \\
.190529 \\
.190252\end{array}$ & $\begin{array}{l}19 \\
18 \\
17 \\
16 \\
15 \\
14 \\
13 \\
12 \\
11 \\
10\end{array}$ \\
\hline $\begin{array}{l}51 \\
52 \\
53 \\
54 \\
55 \\
56 \\
57 \\
58 \\
59 \\
60\end{array}$ & $\begin{array}{r}9.734353 \\
.734549 \\
.734744 \\
.734939 \\
.735135 \\
.735330 \\
.735525 \\
.735719 \\
.735914 \\
9.736109\end{array}$ & $\begin{array}{l}3.27 \\
3.25 \\
3.25 \\
3.27 \\
3.25 \\
3.25 \\
3.23 \\
3.25 \\
3.25\end{array}$ & $\begin{array}{r}9.924328 \\
.924246 \\
.924164 \\
.924083 \\
.924001 \\
.923919 \\
.923537 \\
.923735 \\
.923073 \\
9.923591\end{array}$ & $\begin{array}{l}1.37 \\
1.37 \\
1.35 \\
1.37 \\
1.37 \\
1.37 \\
1.37 \\
1.37 \\
1.37\end{array}$ & $\begin{array}{r}9.810025 \\
.810302 \\
.810580 \\
.810557 \\
.811134 \\
.811410 \\
.811687 \\
.811934 \\
.812241 \\
9.812517\end{array}$ & $\begin{array}{l}\text { 4. } 62 \\
4.63 \\
\text { 4. } 62 \\
\text { 4. } 62 \\
\text { 4. } 60 \\
\text { 4. } 62 \\
\text { 4. } 62 \\
\text { 4. } 62 \\
\text { 4. } 60\end{array}$ & $\begin{array}{r}10.189975 \\
.189698 \\
.189420 \\
.189143 \\
.188866 \\
.188590 \\
.188313 \\
.188036 \\
.157759 \\
10.187483\end{array}$ & $\begin{array}{l}9 \\
8 \\
7 \\
6 \\
5 \\
4 \\
3 \\
2 \\
1 \\
0\end{array}$ \\
\hline , & Cosine. & D. $1^{\prime \prime}$ & Sine. & D. 1"' & Cotang. & D. 1". & 1 ang. & 1 \\
\hline
\end{tabular}


TABLE 9.-LOGARITHMIC SINES, COSINES,

\begin{tabular}{|c|c|c|c|c|c|c|c|c|}
\hline , & Sine. & D. $1^{\prime \prime}$. & Cosine. & D. $1^{\prime \prime}$. & Tang. & D. $1^{\prime \prime}$. & Cotang. & , \\
\hline $\begin{array}{r}0 \\
1 \\
2 \\
3 \\
4 \\
5 \\
6 \\
7 \\
8 \\
9 \\
10\end{array}$ & $\begin{array}{r}9.736109 \\
.736303 \\
.736498 \\
.736692 \\
.736886 \\
.737050 \\
.737274 \\
.737467 \\
.737661 \\
.737855 \\
.738048\end{array}$ & $\begin{array}{l}3.23 \\
3.25 \\
3.23 \\
3.23 \\
3.23 \\
3.23 \\
3.22 \\
3.23 \\
3.23 \\
3.22 \\
3.22\end{array}$ & $\begin{array}{r}9.923591 \\
.923509 \\
.923427 \\
.923345 \\
.923263 \\
.923181 \\
.923098 \\
.923016 \\
.922933 \\
.922851 \\
.922768\end{array}$ & $\begin{array}{l}1.37 \\
1.37 \\
1.37 \\
1.37 \\
1.37 \\
1.38 \\
1.37 \\
1.38 \\
1.37 \\
1.38 \\
1.37\end{array}$ & $\begin{array}{r}9.812517 \\
.812794 \\
.813070 \\
.813347 \\
.813623 \\
.813899 \\
.814176 \\
.814452 \\
.814728 \\
.815004 \\
.815280\end{array}$ & $\begin{array}{l}4.62 \\
4.60 \\
4.62 \\
4.60 \\
4.60 \\
4.62 \\
4.60 \\
4.60 \\
4.60 \\
4.60\end{array}$ & $\begin{array}{r}10.187483 \\
.187206 \\
.186930 \\
.186653 \\
.186377 \\
.186101 \\
.185824 \\
.185548 \\
.185272 \\
.184996 \\
.184720\end{array}$ & $\begin{array}{l}60 \\
59 \\
58 \\
57 \\
56 \\
55 \\
54 \\
53 \\
52 \\
51 \\
50\end{array}$ \\
\hline $\begin{array}{l}11 \\
12 \\
13 \\
14 \\
15 \\
16 \\
17 \\
18 \\
19 \\
20\end{array}$ & $\begin{array}{r}9.738241 \\
.735434 \\
.735627 \\
.738820 \\
.739013 \\
.739206 \\
.739398 \\
.739590 \\
.739783 \\
.739975\end{array}$ & $\begin{array}{l}3.22 \\
3.22 \\
3.22 \\
3.22 \\
3.22 \\
3.20 \\
3.20 \\
3.22 \\
3.20 \\
3.20\end{array}$ & $\begin{array}{r}9.922686 \\
.922603 \\
.922520 \\
.922438 \\
.922355 \\
.922272 \\
.922189 \\
.922106 \\
.922023 \\
.921940\end{array}$ & $\begin{array}{l}1.38 \\
1.38 \\
1.37 \\
1.38 \\
1.38 \\
1.38 \\
1.38 \\
1.38 \\
1.38 \\
1.38\end{array}$ & $\begin{array}{r}9.815555 \\
.815831 \\
.816107 \\
.816382 \\
.816658 \\
.816933 \\
.817209 \\
.817484 \\
.817759 \\
.818035\end{array}$ & $\begin{array}{l}4.60 \\
4.60 \\
4.58 \\
4.60 \\
4.58 \\
4.60 \\
4.58 \\
4.58 \\
4.60 \\
4.58\end{array}$ & $\begin{array}{r}10.184445 \\
.184169 \\
.183893 \\
.183618 \\
.183342 \\
.183067 \\
.182791 \\
.182516 \\
.182241 \\
.181965\end{array}$ & $\begin{array}{l}49 \\
48 \\
47 \\
46 \\
45 \\
44 \\
43 \\
42 \\
41 \\
40\end{array}$ \\
\hline $\begin{array}{l}21 \\
22 \\
23 \\
24 \\
25 \\
26 \\
27 \\
28 \\
29 \\
30\end{array}$ & $\begin{array}{r}9.740167 \\
.740359 \\
.740550 \\
.740742 \\
.740934 \\
.741125 \\
.741316 \\
.741508 \\
.741699 \\
.741889\end{array}$ & $\begin{array}{l}3.20 \\
3.18 \\
3.20 \\
3.20 \\
3.18 \\
3.18 \\
3.20 \\
3.18 \\
3.17 \\
3.18\end{array}$ & $\begin{array}{r}9.921857 \\
.921774 \\
.921691 \\
.921607 \\
.921524 \\
.921441 \\
.921357 \\
.921274 \\
.921190 \\
.921107\end{array}$ & $\begin{array}{l}1.38 \\
1.38 \\
1.40 \\
1.38 \\
1.38 \\
1.40 \\
1.38 \\
1.40 \\
1.38 \\
1.40\end{array}$ & $\begin{array}{r}9.818310 \\
.818585 \\
.818860 \\
.819135 \\
.819410 \\
.819684 \\
.819959 \\
.820234 \\
.820508 \\
.820783\end{array}$ & $\begin{array}{l}4.58 \\
4.58 \\
4.58 \\
4.58 \\
4.57 \\
4.58 \\
4.58 \\
4.57 \\
4.58 \\
4.57\end{array}$ & $\begin{array}{r}10.181690 \\
.181415 \\
.181140 \\
.180865 \\
.180590 \\
.180316 \\
.180041 \\
.179766 \\
.179492 \\
.179217\end{array}$ & $\begin{array}{l}39 \\
38 \\
37 \\
36 \\
35 \\
34 \\
33 \\
32 \\
31 \\
30\end{array}$ \\
\hline $\begin{array}{l}31 \\
32 \\
33 \\
34 \\
35 \\
36 \\
37 \\
38 \\
39 \\
40 \\
\end{array}$ & $\begin{array}{r}9.742080 \\
.742271 \\
.742462 \\
.742652 \\
.742842 \\
.743033 \\
.743223 \\
.743413 \\
.743602 \\
.743792\end{array}$ & $\begin{array}{l}3.18 \\
3.18 \\
3.17 \\
3.17 \\
3.18 \\
3.17 \\
3.17 \\
3.15 \\
3.17 \\
3.17\end{array}$ & $\begin{array}{r}9.921023 \\
.920939 \\
.920856 \\
.920772 \\
.920688 \\
.920604 \\
.920520 \\
.920436 \\
.920352 \\
.920268\end{array}$ & $\begin{array}{l}1.40 \\
1.38 \\
1.40 \\
1.40 \\
1.40 \\
1.40 \\
1.40 \\
1.40 \\
1.40 \\
1.40\end{array}$ & $\begin{array}{r}9.821057 \\
.821332 \\
.821606 \\
.821880 \\
.822154 \\
.822429 \\
.822703 \\
.822977 \\
.823251 \\
.823524\end{array}$ & $\begin{array}{l}4.58 \\
4.57 \\
4.57 \\
4.57 \\
4.58 \\
4.57 \\
4.57 \\
4.57 \\
4.55 \\
4.57\end{array}$ & $\begin{array}{r}10.178943 \\
.178668 \\
.178394 \\
.178120 \\
.177846 \\
.177571 \\
.177297 \\
.177023 \\
.176749 \\
.176476\end{array}$ & $\begin{array}{l}29 \\
28 \\
27 \\
26 \\
25 \\
24 \\
23 \\
22 \\
21 \\
20\end{array}$ \\
\hline $\begin{array}{l}41 \\
42 \\
43 \\
44 \\
45 \\
46 \\
47 \\
48 \\
49 \\
50\end{array}$ & $\begin{array}{r}9.743982 \\
.744171 \\
.744361 \\
.744550 \\
.744739 \\
.744928 \\
.745117 \\
.745306 \\
.745494 \\
.745683\end{array}$ & $\begin{array}{l}3.15 \\
3.17 \\
3.15 \\
3.15 \\
3.15 \\
3.15 \\
3.15 \\
3.13 \\
3.15 \\
3.13\end{array}$ & $\begin{array}{r}9.920184 \\
.920099 \\
.920015 \\
.919931 \\
.919846 \\
.919762 \\
.919677 \\
.919593 \\
.919508 \\
.919424\end{array}$ & $\begin{array}{l}1.30 \\
1.42 \\
1.40 \\
1.40 \\
1.42 \\
1.40 \\
1.42 \\
1.40 \\
1.42 \\
1.40 \\
1.42\end{array}$ & $\begin{array}{r}8.823798 \\
.824072 \\
.824345 \\
.824619 \\
.824893 \\
.825166 \\
.825439 \\
.825713 \\
.825986 \\
.826259\end{array}$ & $\begin{array}{l}4.57 \\
4.55 \\
4.57 \\
4.57 \\
4.55 \\
4.55 \\
4.57 \\
4.55 \\
4.55 \\
4.55\end{array}$ & $\begin{array}{r}10.176202 \\
.175928 \\
.175655 \\
.175381 \\
.175107 \\
.174834 \\
.174561 \\
.174287 \\
.174014 \\
.173741\end{array}$ & $\begin{array}{l}19 \\
18 \\
17 \\
16 \\
15 \\
14 \\
13 \\
12 \\
11 \\
10\end{array}$ \\
\hline $\begin{array}{l}51 \\
52 \\
53 \\
54 \\
55 \\
56 \\
57 \\
58 \\
59 \\
69\end{array}$ & $\begin{array}{r}9.745871 \\
.74060 \\
.746248 \\
.746436 \\
.746624 \\
.746812 \\
.746999 \\
.747187 \\
.747374 \\
9.747562\end{array}$ & $\begin{array}{l}3.15 \\
3.13 \\
3.13 \\
3.13 \\
3.13 \\
3.12 \\
3.13 \\
3.12 \\
3.13\end{array}$ & $\begin{array}{r}9.919339 \\
.919254 \\
.919169 \\
.919085 \\
.919000 \\
.918915 \\
.918830 \\
918745 \\
.918659 \\
9.018574\end{array}$ & $\begin{array}{l}1.72 \\
1.42 \\
1.42 \\
1.40 \\
1.42 \\
1.42 \\
1.42 \\
1.42 \\
1.43 \\
1.42\end{array}$ & $\begin{array}{r}9.826532 \\
.826505 \\
.827078 \\
.827351 \\
.827624 \\
.827897 \\
.828170 \\
.828442 \\
.828715 \\
9.828957\end{array}$ & $\begin{array}{l}4.55 \\
4.55 \\
4.55 \\
4.55 \\
4.55 \\
4.55 \\
4.53 \\
4.55 \\
4.53\end{array}$ & $\begin{array}{r}10.173468 \\
.173195 \\
.172922 \\
.172649 \\
.172376 \\
.172103 \\
.171830 \\
.171558 \\
.171285 \\
10.171013\end{array}$ & $\begin{array}{l}9 \\
8 \\
7 \\
6 \\
5 \\
4 \\
3 \\
2 \\
1 \\
0\end{array}$ \\
\hline ' & Cosine. & D. $1^{\prime \prime}$. & Sine. & D. 1". & Cotang. & D. $1^{\prime \prime}$. & Tang. & , \\
\hline
\end{tabular}




\begin{tabular}{|c|c|c|c|c|c|c|c|c|}
\hline , & Sine. & D. $1^{\prime \prime}$. & Cosine. & D. $1 "$. & Tang. & D. $1^{\prime \prime}$. & Cotsng. & , \\
\hline $\begin{array}{r}0 \\
1 \\
2 \\
3 \\
4 \\
5 \\
6 \\
7 \\
8 \\
9 \\
10\end{array}$ & $\begin{array}{r}9.747562 \\
.747749 \\
.747936 \\
.748123 \\
.748310 \\
.748497 \\
.748683 \\
.748870 \\
.749056 \\
.749243 \\
.749429\end{array}$ & \begin{tabular}{l|}
3.12 \\
3.12 \\
3.12 \\
3.12 \\
3.12 \\
3.10 \\
3.12 \\
3.10 \\
3.12 \\
3.10 \\
3.10
\end{tabular} & $\begin{array}{r}9.918574 \\
.918489 \\
.918404 \\
.918318 \\
.918233 \\
.918147 \\
.918062 \\
.917976 \\
.917891 \\
.917805 \\
.917719 \\
9.917634\end{array}$ & $\begin{array}{l}1.42 \\
1.42 \\
1.43 \\
1.42 \\
1.43 \\
1.42 \\
1.43 \\
1.42 \\
1.43 \\
1.43 \\
1.42\end{array}$ & $\begin{array}{r}9.825987 \\
.839260 \\
.829532 \\
.829505 \\
.830077 \\
.830349 \\
.830621 \\
.830593 \\
.831165 \\
.831437 \\
.831709\end{array}$ & $\begin{array}{l}4.55 \\
4.53 \\
4.55 \\
4.53 \\
4.53 \\
4.53 \\
4.53 \\
4.53 \\
4.53 \\
4.53 \\
4.53\end{array}$ & $\begin{array}{r}10.171013 \\
.170740 \\
.170465 \\
.170195 \\
.169923 \\
.169651 \\
.169379 \\
.169107 \\
.168535 \\
.165563 \\
.168291\end{array}$ & $\begin{array}{l}57 \\
56 \\
55 \\
54 \\
53 \\
52 \\
51 \\
50\end{array}$ \\
\hline $\begin{array}{l}11 \\
12 \\
13 \\
14 \\
15 \\
16 \\
17 \\
18 \\
19 \\
20\end{array}$ & $\begin{array}{r}9.749615 \\
.749801 \\
.749987 \\
.750172 \\
.750358 \\
.750543 \\
.750729 \\
.750914 \\
.751099 \\
.751284\end{array}$ & $\begin{array}{l}3.10 \\
3.10 \\
3.08 \\
3.10 \\
3.08 \\
3.10 \\
3.08 \\
3.08 \\
3.08 \\
3.08\end{array}$ & $\begin{array}{r}9.917634 \\
.917548 \\
.917462 \\
.917376 \\
.917290 \\
.917204 \\
.917118 \\
.917032 \\
.916946 \\
.916859\end{array}$ & $\begin{array}{l}1.43 \\
1.43 \\
1.43 \\
1.43 \\
1.43 \\
1.43 \\
1.43 \\
1.43 \\
1.45 \\
1.43\end{array}$ & $\begin{array}{l}9.831981 \\
.832253 \\
.832525 \\
.832796 \\
.833068 \\
.833339 \\
.833611 \\
.833552 \\
.834154 \\
.834425\end{array}$ & $\begin{array}{l}4.53 \\
4.53 \\
4.52 \\
4.53 \\
4.52 \\
4.53 \\
4.52 \\
4.53 \\
4.52 \\
4.52\end{array}$ & $\begin{array}{r}10.168019 \\
.167747 \\
.167475 \\
.167204 \\
.166932 \\
.166661 \\
.166389 \\
.166118 \\
.165846 \\
.165575\end{array}$ & $\begin{array}{l}49 \\
48 \\
47 \\
46 \\
45 \\
44 \\
43 \\
42 \\
41 \\
40\end{array}$ \\
\hline $\begin{array}{l}21 \\
22 \\
23 \\
24 \\
25 \\
26 \\
27 \\
28 \\
29 \\
30\end{array}$ & $\begin{array}{r}9.751469 \\
.751654 \\
.751839 \\
.752023 \\
.752208 \\
.752392 \\
.752576 \\
.752760 \\
.752944 \\
.753128\end{array}$ & $\begin{array}{l}3.08 \\
3.08 \\
3.07 \\
3.08 \\
3.07 \\
3.07 \\
3.07 \\
3.07 \\
3.07 \\
3.07\end{array}$ & $\begin{array}{r}9.916773 \\
.916687 \\
.916600 \\
.916514 \\
.916427 \\
.916341 \\
.916254 \\
.916167 \\
.916081 \\
.915994\end{array}$ & $\begin{array}{l}1.43 \\
1.45 \\
1.43 \\
1.45 \\
1.43 \\
1.45 \\
1.45 \\
1.43 \\
1.45 \\
1.45\end{array}$ & $\begin{array}{r}9.834696 \\
.834967 \\
.835238 \\
.835509 \\
.835780 \\
.836051 \\
.836322 \\
.836593 \\
.836864 \\
.837134\end{array}$ & $\begin{array}{l}4.52 \\
4.52 \\
4.52 \\
4.52 \\
4.52 \\
4.52 \\
4.52 \\
4.52 \\
4.52 \\
4.50 \\
4.52\end{array}$ & $\begin{array}{r}10.165304 \\
.165033 \\
.164762 \\
.164491 \\
.164220 \\
.163949 \\
.163678 \\
.163407 \\
.163136 \\
.162566\end{array}$ & $\begin{array}{l}39 \\
38 \\
37 \\
36 \\
35 \\
34 \\
33 \\
32 \\
31 \\
30\end{array}$ \\
\hline $\begin{array}{l}31 \\
32 \\
33 \\
34 \\
35 \\
36 \\
37 \\
35 \\
39 \\
40\end{array}$ & $\begin{array}{r}9.753312 \\
.753495 \\
.753679 \\
.753862 \\
.754046 \\
.754229 \\
.754412 \\
.754595 \\
.754778 \\
.754960\end{array}$ & $\begin{array}{l}\text { 3. } 05 \\
\text { 3. } 07 \\
\text { 3. } 07 \\
\text { 3. } 07 \\
\text { 3.05 } \\
\text { 3. } 05 \\
3.05 \\
\text { 3. } 05 \\
\text { 3. } 03 \\
\text { 3. } 05\end{array}$ & $\begin{array}{r}9.915907 \\
.915520 \\
.915733 \\
.915646 \\
.915559 \\
.915472 \\
.915385 \\
.915297 \\
.915210 \\
.915123\end{array}$ & $\begin{array}{l}1.45 \\
1.45 \\
1.45 \\
1.45 \\
1.45 \\
1.45 \\
1.47 \\
1.45 \\
1.45 \\
1.47\end{array}$ & $\begin{array}{r}9.837405 \\
.837675 \\
.837946 \\
.838216 \\
.838457 \\
.838757 \\
.839027 \\
.839297 \\
.8395 t 8 \\
.839838\end{array}$ & $\begin{array}{l}4.50 \\
4.52 \\
4.50 \\
4.52 \\
4.50 \\
4.50 . \\
4.50 \\
4.52 \\
4.50 \\
4.50\end{array}$ & $\begin{array}{r}10.162595 \\
.162325 \\
.162054 \\
.161784 \\
.161513 \\
.161243 \\
.160973 \\
.160703 \\
.160432 \\
.160162\end{array}$ & $\begin{array}{l}29 \\
28 \\
27 \\
26 \\
25 \\
24 \\
23 \\
22 \\
21 \\
20\end{array}$ \\
\hline $\begin{array}{l}41 \\
42 \\
43 \\
44 \\
45 \\
46 \\
47 \\
48 \\
49 \\
50\end{array}$ & $\begin{array}{r}9.755148 \\
.755326 \\
.755508 \\
.755690 \\
.755872 \\
.756054 \\
.756236 \\
.756418 \\
.756600 \\
.756782\end{array}$ & $\begin{array}{l}\text { 3. } 05 \\
3.03 \\
3.03 \\
3.03 \\
3.03 \\
3.03 \\
3.03 \\
3.03 \\
3.03 \\
3.02\end{array}$ & $\begin{array}{r}9.915035 \\
.914948 \\
.914560 \\
.914773 \\
.914685 \\
.914598 \\
.914510 \\
.914422 \\
.914334 \\
.914246\end{array}$ & $\begin{array}{l}1.4 \\
1.4 \\
1.4 \\
1.4 \\
1.4 \\
1.4 \\
1.4 \\
1.4 \\
1.4 \\
1.4\end{array}$ & $\begin{array}{r}9.840108 \\
.840378 \\
.840648 \\
.840917 \\
.841187 \\
.841457 \\
.841727 \\
.841906 \\
.842666 \\
.842535\end{array}$ & $\begin{array}{l}4.50 \\
4.50 \\
4.48 \\
4.50 \\
4.50 \\
4.50 \\
4.48 \\
4.50 \\
4.48\end{array}$ & $\begin{array}{r}10.159892 \\
.1596222 \\
.159352 \\
.159083 \\
.158513 \\
.158543 \\
.158273 \\
.158004 \\
.157734 \\
.157465\end{array}$ & $\begin{array}{l}19 \\
18 \\
17 \\
16 \\
15 \\
14 \\
13 \\
12 \\
11 \\
10\end{array}$ \\
\hline $\begin{array}{l}51 \\
52 \\
53 \\
54 \\
55 \\
56 \\
57 \\
58 \\
59 \\
60\end{array}$ & $\begin{array}{r}9.756963 \\
.757144 \\
.757326 \\
.757507 \\
.757688 \\
.757869 \\
.755050 \\
.758230 \\
.758411 \\
9.758591\end{array}$ & $\begin{array}{l}3.02 \\
3.03 \\
3.02 \\
3.02 \\
3.02 \\
3.02 \\
3.00 \\
3.02 \\
3.00\end{array}$ & $\begin{array}{r}9.914158 \\
.914070 \\
.913982 \\
.913894 \\
.913806 \\
.913718 \\
.913630 \\
.913541 \\
.913453 \\
9.913305\end{array}$ & $\begin{array}{l}1.47 \\
1.47 \\
1.47 \\
1.47 \\
1.47 \\
1.47 \\
1.48 \\
1.47 \\
1.47\end{array}$ & $\begin{array}{r}9.842505 \\
.843074 \\
.843343 \\
.843612 \\
.843552 \\
.844151 \\
.844420 \\
.844659 \\
.844958 \\
9.845227\end{array}$ & $\begin{array}{l}4.48 \\
148 \\
1.48 \\
4.50 \\
1.48 \\
4.48 \\
4.48 \\
4.48 \\
4.48\end{array}$ & $\begin{array}{r}10.157185 \\
.1569926 \\
.156657 \\
.1563888 \\
.156118 \\
.155549 \\
.155580 \\
.155311 \\
.155042 \\
10.154773\end{array}$ & $\begin{array}{l}9 \\
8 \\
7 \\
6 \\
5 \\
4 \\
3 \\
2 \\
1 \\
0\end{array}$ \\
\hline & Cosine. & D. 1 ". & Sine. & D. $1^{\prime \prime}$. & Cotang. & D. $1^{\prime \prime}$. & Tang. & 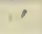 \\
\hline
\end{tabular}


TABLE 9.-LOGARITHMIC SINES, COSINES,

\begin{tabular}{|c|c|c|c|c|c|c|c|c|}
\hline ' & Sine. & D. $1^{\prime \prime}$. & Cosine. & D. $1^{\prime \prime}$. & Tang. & D. $1^{\prime \prime}$. & Cotang. & , \\
\hline $\begin{array}{r}0 \\
1 \\
2 \\
3 \\
4 \\
5 \\
6 \\
7 \\
8 \\
9 \\
10\end{array}$ & $\begin{array}{r}9.758591 \\
.758772 \\
.758952 \\
.759132 \\
.759312 \\
.759492 \\
.759672 \\
.759852 \\
.760031 \\
.760211 \\
.760390\end{array}$ & $\begin{array}{l}3.02 \\
3.00 \\
3.00 \\
3.00 \\
3.00 \\
3.00 \\
3.00 \\
2.98 \\
3.00 \\
2.98 \\
2.98\end{array}$ & $\begin{array}{r}9.913365 \\
.913276 \\
.913187 \\
.913099 \\
.913010 \\
.912922 \\
.912833 \\
.912744 \\
.912655 \\
.912566 \\
.912477\end{array}$ & $\begin{array}{l}1.48 \\
1.48 \\
1.47 \\
1.48 \\
1.47 \\
1.48 \\
1.48 \\
1.48 \\
1.48 \\
1.48 \\
1.49\end{array}$ & $\begin{array}{r}9.845227 \\
.845496 \\
.845764 \\
.846033 \\
.846302 \\
.846570 \\
.846839 \\
.847108 \\
.847376 \\
.847644 \\
.847913\end{array}$ & $\begin{array}{l}4.48 \\
4.47 \\
4.48 \\
4.48 \\
4.47 \\
4.48 \\
4.48 \\
4.47 \\
4.47 \\
4.48 \\
4.47\end{array}$ & $\begin{array}{r}10.154773 \\
.154504 \\
.154236 \\
.153967 \\
.153698 \\
.153430 \\
.153161 \\
.152892 \\
.152624 \\
.152356 \\
.152087\end{array}$ & $\begin{array}{l}60 \\
59 \\
58 \\
57 \\
56 \\
55 \\
54 \\
53 \\
52 \\
51 \\
50\end{array}$ \\
\hline $\begin{array}{l}11 \\
12 \\
13 \\
14 \\
15 \\
16 \\
17 \\
18 \\
19 \\
20\end{array}$ & $\begin{array}{r}9.760569 \\
.760748 \\
.760927 \\
.761106 \\
.761285 \\
.761464 \\
.761642 \\
.761821 \\
.761999 \\
.762177\end{array}$ & $\begin{array}{l}2.98 \\
2.98 \\
2.98 \\
2.98 \\
2.98 \\
2.97 \\
2.98 \\
2.97 \\
2.97 \\
2.98\end{array}$ & $\begin{array}{r}9.912388 \\
.912299 \\
.912210 \\
.912121 \\
.912031 \\
.911942 \\
.911853 \\
.911763 \\
.911674 \\
.911584\end{array}$ & $\begin{array}{l}1.40 \\
1.48 \\
1.48 \\
1.48 \\
1.50 \\
1.48 \\
1.48 \\
1.50 \\
1.48 \\
1.50 \\
1.48\end{array}$ & $\begin{array}{r}9.848181 \\
.848449 \\
.848717 \\
.848986 \\
.849254 \\
.849522 \\
.849790 \\
.850057 \\
.850325 \\
.850593\end{array}$ & $\begin{array}{l}4.47 \\
4.47 \\
4.47 \\
4.48 \\
4.47 \\
4.47 \\
4.47 \\
4.45 \\
4.47 \\
4.47 \\
4.47\end{array}$ & $\begin{array}{r}10.151819 \\
.151551 \\
.151283 \\
.151014 \\
.150746 \\
.150478 \\
.150210 \\
.149943 \\
.149675 \\
.149407\end{array}$ & $\begin{array}{l}49 \\
48 \\
47 \\
46 \\
45 \\
44 \\
43 \\
42 \\
41 \\
40\end{array}$ \\
\hline $\begin{array}{l}21 \\
22 \\
23 \\
24 \\
25 \\
26 \\
27 \\
28 \\
29 \\
30\end{array}$ & $\begin{array}{r}9.762356 \\
.762534 \\
.762712 \\
.762889 \\
.763067 \\
.763245 \\
.763422 \\
.763600 \\
.763777 \\
.763954\end{array}$ & $\begin{array}{l}2.97 \\
2.97 \\
2.95 \\
2.97 \\
2.97 \\
2.95 \\
2.97 \\
2.95 \\
2.95 \\
2.95\end{array}$ & $\begin{array}{r}9.911495 \\
.911405 \\
.911315 \\
.911226 \\
.911136 \\
.911046 \\
.910956 \\
.910866 \\
.910776 \\
.910686\end{array}$ & $\begin{array}{l}1.50 \\
1.50 \\
1.50 \\
1.48 \\
1.50 \\
1.50 \\
1.50 \\
1.50 \\
1.50 \\
1.50 \\
1.50\end{array}$ & $\begin{array}{r}9.850861 \\
.851129 \\
.851396 \\
.851664 \\
.851931 \\
.852199 \\
.852466 \\
.852733 \\
.853001 \\
.853265\end{array}$ & $\begin{array}{l}4.47 \\
4.45 \\
4.47 \\
4.45 \\
4.47 \\
4.45 \\
4.45 \\
4.47 \\
4.45 \\
4.45\end{array}$ & $\begin{array}{r}10.149139 \\
.148871 \\
.145604 \\
.148336 \\
.148069 \\
.147801 \\
.147534 \\
.147267 \\
.146999 \\
.146732\end{array}$ & $\begin{array}{l}39 \\
38 \\
37 \\
36 \\
35 \\
34 \\
33 \\
32 \\
31 \\
30\end{array}$ \\
\hline $\begin{array}{l}31 \\
32 \\
33 \\
34 \\
35 \\
36 \\
37 \\
38 \\
39 \\
40\end{array}$ & $\begin{array}{r}9.764131 \\
.764308 \\
.764485 \\
.764662 \\
.764838 \\
.765015 \\
.765191 \\
.765367 \\
.765544 \\
.765720\end{array}$ & $\begin{array}{l}2.95 \\
2.95 \\
2.95 \\
2.93 \\
2.95 \\
2.93 \\
2.93 \\
2.95 \\
2.93 \\
2.93\end{array}$ & $\begin{array}{r}9.910596 \\
.910506 \\
.910415 \\
.910325 \\
.910235 \\
.910144 \\
.910054 \\
.909963 \\
.909873 \\
.909782\end{array}$ & $\begin{array}{l}1.50 \\
1.52 \\
1.50 \\
1.50 \\
1.52 \\
1.50 \\
1.52 \\
1.50 \\
1.52 \\
1.52\end{array}$ & $\begin{array}{r}9.853535 \\
.853802 \\
.854069 \\
.854336 \\
.854603 \\
.854870 \\
.855137 \\
.855404 \\
.855671 \\
.855938\end{array}$ & $\begin{array}{l}4.45 \\
4.45 \\
4.45 \\
4.45 \\
4.45 \\
4.45 \\
4.45 \\
4.45 \\
4.45 \\
4.43\end{array}$ & $\begin{array}{r}10.146465 \\
.146198 \\
.145931 \\
.145664 \\
.145397 \\
.145130 \\
.144563 \\
.144596 \\
.144329 \\
.144062\end{array}$ & $\begin{array}{l}29 \\
28 \\
27 \\
20 \\
25 \\
24 \\
23 \\
22 \\
21 \\
20\end{array}$ \\
\hline $\begin{array}{l}41 \\
42 \\
43 \\
44 \\
45 \\
46 \\
47 \\
48 \\
49 \\
50\end{array}$ & $\begin{array}{r}9.765596 \\
.766072 \\
.766247 \\
.766423 \\
.766598 \\
.766774 \\
.766949 \\
.767124 \\
.767300 \\
.767475\end{array}$ & $\begin{array}{l}2.93 \\
2.92 \\
2.93 \\
2.92 \\
2.93 \\
2.92 \\
2.92 \\
2.93 \\
2.92 \\
2.90\end{array}$ & $\begin{array}{r}9.909691 \\
.909601 \\
.909510 \\
.909419 \\
.909328 \\
.909237 \\
.909146 \\
.909055 \\
.908964 \\
.908873\end{array}$ & \begin{tabular}{l|}
1.52 \\
1.50 \\
1.52 \\
1.52 \\
1.52 \\
1.52 \\
1.52 \\
1.52 \\
1.52 \\
1.52 \\
1.53
\end{tabular} & $\begin{array}{r}9.856204 \\
.856471 \\
.856737 \\
.857004 \\
.857270 \\
.857537 \\
.857803 \\
.858069 \\
.858336 \\
.858602\end{array}$ & \begin{tabular}{l|}
4.45 \\
4.43 \\
4.45 \\
4.43 \\
4.45 \\
4.43 \\
4.43 \\
4.45 \\
4.43 \\
4.43
\end{tabular} & $\begin{array}{r}10.143796 \\
.143529 \\
.143263 \\
.142996 \\
.142730 \\
.142463 \\
.142197 \\
.141931 \\
.141664 \\
.141398\end{array}$ & $\begin{array}{l}19 \\
18 \\
17 \\
16 \\
15 \\
14 \\
13 \\
12 \\
11 \\
10\end{array}$ \\
\hline $\begin{array}{l}51 \\
52 \\
53 \\
54 \\
55 \\
56 \\
57 \\
58 \\
59 \\
60\end{array}$ & $\begin{array}{r}9.767649 \\
.767824 \\
.767999 \\
.768173 \\
.768348 \\
.768522 \\
.768697 . \\
.768871 \\
.769045 \\
0.769219\end{array}$ & $\begin{array}{l}2.90 \\
2.92 \\
2.90 \\
2.92 \\
2.90 \\
2.92 \\
2.90 \\
2.90 \\
2.90\end{array}$ & $\begin{array}{r}9.908781 \\
.908690 \\
.908599 \\
.908507 \\
.908416 \\
.908324 \\
.908233 \\
.908141 \\
.908049 \\
9.907958\end{array}$ & $\begin{array}{l}1.53 \\
1.52 \\
1.52 \\
1.53 \\
1.52 \\
1.53 \\
1.52 \\
1.53 \\
1.53 \\
1.52\end{array}$ & $\begin{array}{r}9.858868 \\
.859134 \\
.859400 \\
.859666 \\
.859932 \\
.860198 \\
.860464 \\
.860730 \\
.860995 \\
9.861261\end{array}$ & $\begin{array}{l}4.43 \\
4.43 \\
4.43 \\
4.43 \\
4.43 \\
4.43 \\
4.43 \\
4.43 \\
4.42 \\
4.43\end{array}$ & $\begin{array}{r}10.141132 \\
.140866 \\
.140600 \\
.140334 \\
.140068 \\
.139802 \\
.139536 \\
.139270 \\
.139005 \\
10.138739\end{array}$ & $\begin{array}{l}9 \\
8 \\
7 \\
6 \\
5 \\
4 \\
3 \\
2 \\
1 \\
0\end{array}$ \\
\hline I & Cosine. & D. $1^{\prime \prime}$. & Sine. & D. $1^{\prime \prime}$. & Cotang. & D. $1^{\prime \prime}$. & Tang. & ' \\
\hline
\end{tabular}




\begin{tabular}{|c|c|c|c|c|c|c|c|c|}
\hline ' & Sine. & D. 1". & Cosine. & D. $1^{\prime \prime}$. & Tang. & D. $1^{\prime \prime}$. & Cotang. & ' \\
\hline $\begin{array}{r}0 \\
1 \\
2 \\
3 \\
4 \\
5 \\
6 \\
7 \\
8 \\
9 \\
10\end{array}$ & $\begin{array}{r}9.769219 \\
.760393 \\
.769566 \\
.769740 \\
.769913 \\
.770087 \\
.770260 \\
.770433 \\
.770606 \\
.770779 \\
.770952\end{array}$ & $\begin{array}{l}2.90 \\
2.88 \\
2.90 \\
2.85 \\
2.90 \\
2.88 \\
2.88 \\
2.88 \\
2.88 \\
2.88 \\
2.88\end{array}$ & $\begin{array}{r}9.907958 \\
.907866 \\
.907774 \\
.907682 \\
.907590 \\
.907498 \\
.907406 \\
.907314 \\
.907222 \\
.907129 \\
.907037\end{array}$ & \begin{tabular}{l|}
1.53 \\
1.53 \\
1.53 \\
1.53 \\
1.53 \\
1.53 \\
1.53 \\
1.53 \\
1.55 \\
1.53 \\
1.53
\end{tabular} & $\begin{array}{r}9.861261 \\
.861527 \\
.861792 \\
.862058 \\
.862323 \\
.862589 \\
.862854 \\
.863119 \\
.863385 \\
.863650 \\
.863915\end{array}$ & $\begin{array}{l}4.43 \\
4.42 \\
4.43 \\
4.42 \\
4.43 \\
4.42 \\
4.42 \\
4.43 \\
4.42 \\
4.42 \\
4.42\end{array}$ & $\begin{array}{r}10.138739 \\
.138473 \\
.138208 \\
.137942 \\
.137677 \\
.137411 \\
.137146 \\
.136851 \\
.136615 \\
.136350 \\
.136085\end{array}$ & 60 \\
\hline $\begin{array}{l}11 \\
12 \\
13 \\
14 \\
15 \\
16 \\
17 \\
18 \\
19 \\
20\end{array}$ & $\begin{array}{r}9.771125 \\
.771298 \\
.771470 \\
.771643 \\
.771815 \\
.771957 \\
.772159 \\
.772331 \\
.772503 \\
.772675\end{array}$ & $\begin{array}{l}2.88 \\
2.87 \\
2.88 \\
2.87 \\
2.87 \\
2.87 \\
2.87 \\
2.87 \\
2.87 \\
2.87\end{array}$ & $\begin{array}{r}9.906945 \\
.906852 \\
.906760 \\
.9066667 \\
.906575 \\
.9064182 \\
.906389 \\
.906296 \\
.906204 \\
.906111\end{array}$ & \begin{tabular}{l|}
1.55 \\
1.53 \\
1.55 \\
1.53 \\
1.55 \\
1.55 \\
1.55 \\
1.53 \\
1.55 \\
1.55
\end{tabular} & $\begin{array}{r}9.864180 \\
.864445 \\
.864710 \\
.864975 \\
.865240 \\
.865505 \\
.865770 \\
.866035 \\
.866300 \\
.866564\end{array}$ & $\begin{array}{l}\text { 4. } 42 \\
\text { 4. } 42 \\
\text { 4. } 42 \\
\text { 4. } 42 \\
4.42 \\
4.42 \\
4.42 \\
\text { 4. } 42 \\
4.40 \\
4.42\end{array}$ & $\begin{array}{r}10.135820 \\
.135555 \\
.135290 \\
.135025 \\
.134760 \\
.134495 \\
.134230 \\
.133965 \\
.133700 \\
.133436\end{array}$ & 41 \\
\hline $\begin{array}{l}21 \\
22 \\
23 \\
24 \\
25 \\
26 \\
27 \\
28 \\
29 \\
30\end{array}$ & $\begin{array}{r}9.772847 \\
.773018 \\
.773190 \\
.773361 \\
.7735333 \\
.773704 \\
.773375 \\
.774046 \\
.774217 \\
.774388\end{array}$ & $\begin{array}{l}2.85 \\
2.87 \\
2.85 \\
2.87 \\
2.85 \\
2.85 \\
2.85 \\
2.85 \\
2.85 \\
2.83\end{array}$ & $\begin{array}{r}9.906018 \\
.905925 \\
.905832 \\
.905739 \\
.905645 \\
.905552 \\
.905459 \\
.905306 \\
.905272 \\
.905179\end{array}$ & $\begin{array}{l}1.55 \\
1.55 \\
1.55 \\
1.57 \\
1.55 \\
1.55 \\
1.55 \\
1.57 \\
1.55 \\
1.57\end{array}$ & $\begin{array}{r}9.866529 \\
.867094 \\
.867358 \\
.867623 \\
.867897 \\
.868152 \\
.868416 \\
.865650 \\
.865945 \\
.869209\end{array}$ & $\begin{array}{l}4.42 \\
4.40 \\
4.42 \\
4.40 \\
4.42 \\
4.40 \\
4.40 \\
4.42 \\
4.40 \\
4.40\end{array}$ & $\begin{array}{r}10.133171 \\
.132906 \\
.132642 \\
.132377 \\
.132113 \\
.131849 \\
.131584 \\
.131320 \\
.131055 \\
.130791\end{array}$ & $\begin{array}{l}39 \\
39 \\
37 \\
36 \\
35 \\
34 \\
33 \\
32 \\
31 \\
30\end{array}$ \\
\hline $\begin{array}{l}31 \\
32 \\
33 \\
34 \\
35 \\
36 \\
37 \\
39 \\
39 \\
40\end{array}$ & $\begin{array}{r}9.774558 \\
.774729 \\
.774599 \\
.775070 \\
.775240 \\
.775410 \\
.775550 \\
.775750 \\
.775920 \\
.776090\end{array}$ & $\begin{array}{l}2.85 \\
2.83 \\
2.85 \\
2.83 \\
2.83 \\
2.83 \\
2.83 \\
2.83 \\
2.83 \\
2.82\end{array}$ & $\begin{array}{r}9.905085 \\
.904992 \\
.904898 \\
.904804 \\
.904711 \\
.904617 \\
.904523 \\
.904429 \\
.904335 \\
.904241\end{array}$ & $\begin{array}{l}1.55 \\
1.57 \\
1.57 \\
1.55 \\
1.57 \\
1.57 \\
1.57 \\
1.57 \\
1.57 \\
1.57\end{array}$ & $\begin{array}{r}9.869473 \\
.869737 \\
.870001 \\
.870265 \\
.870529 \\
.870793 \\
.871057 \\
.871321 \\
.871585 \\
.871849\end{array}$ & $\begin{array}{l}4.40 \\
4.40 \\
4.40 \\
4.40 \\
4.40 \\
4.40 \\
4.40 \\
4.40 \\
4.40 \\
4.38\end{array}$ & $\begin{array}{r}10.130527 \\
.130263 \\
.129999 \\
.129730 \\
.129471 \\
.129207 \\
.125943 \\
.128679 \\
.128415 \\
.128151\end{array}$ & 29 \\
\hline $\begin{array}{l}41 \\
42 \\
43 \\
44 \\
45 \\
46 \\
47 \\
48 \\
49 \\
50\end{array}$ & $\begin{array}{r}9.776259 \\
.776429 \\
.776598 \\
.776768 \\
.776937 \\
.777106 \\
.777275 \\
.777444 \\
.777613 \\
.777781\end{array}$ & $\begin{array}{l}2.83 \\
2.82 \\
2.83 \\
2.82 \\
2.82 \\
2.82 \\
2.82 \\
2.82 \\
2.80 \\
2.82\end{array}$ & $\begin{array}{r}9.904147 \\
.904053 \\
.903959 \\
.903864 \\
.003770 \\
.903676 \\
.903581 \\
.903487 \\
.903392 \\
.903298\end{array}$ & $\begin{array}{l}1.57 \\
1.57 \\
1.58 \\
1.57 \\
1.57 \\
1.58 \\
1.57 \\
1.58 \\
1.57 \\
1.58\end{array}$ & $\begin{array}{r}9.872112 \\
.872376 \\
.873640 \\
.872903 \\
.873167 \\
.873430 \\
.873694 \\
.873957 \\
.874220 \\
.874484\end{array}$ & $\begin{array}{l}\text { 4. } 40 \\
4.40 \\
\text { 4. 38 } \\
4.40 \\
\text { 4. } 38 \\
4.40 \\
4.39 \\
4.38 \\
4.40 \\
4.38\end{array}$ & $\begin{array}{r}10.127858 \\
.127624 \\
.127360 \\
.127097 \\
.126333 \\
.126570 \\
.126306 \\
.126043 \\
.125780 \\
.125516\end{array}$ & 3 \\
\hline $\begin{array}{l}51 \\
52 \\
53 \\
54 \\
5.5 \\
56 \\
57 \\
5 . \\
59 \\
60\end{array}$ & $\begin{array}{r}9.777950 \\
.778119 \\
.778287 \\
.778455 \\
.778624 \\
.778792 \\
.778960 \\
.779128 \\
.779295 \\
9.779463\end{array}$ & $\begin{array}{l}2.82 \\
2.80 \\
2.80 \\
2.82 \\
2.80 \\
2.80 \\
2.80 \\
2.78 \\
2.80\end{array}$ & $\begin{array}{r}9.903203 \\
.903103 \\
.903014 \\
.902919 \\
.902924 \\
.902729 \\
.902634 \\
.902539 \\
.902444 \\
9.902349\end{array}$ & $\begin{array}{l}1.58 \\
1.57 \\
1.58 \\
1.58 \\
1.58 \\
1.58 \\
1.58 \\
1.58 \\
1.58\end{array}$ & $\begin{array}{r}9.874747 \\
.875010 \\
.875273 \\
.875537 \\
.875500 \\
.876003 \\
.876324 \\
.870689 \\
.876552 \\
9.877114\end{array}$ & $\begin{array}{l}4.38 \\
4.38 \\
4.40 \\
4.38 \\
4.38 \\
4.38 \\
4.38 \\
4.38 \\
4.37\end{array}$ & $\begin{array}{r}10.125253 \\
.124990 \\
.124727 \\
.124463 \\
.124200 \\
.123937 \\
.123674 \\
.123411 \\
.123148 \\
10.122856\end{array}$ & $\begin{array}{l}6 \\
5 \\
4 \\
3 \\
2 \\
1 \\
0\end{array}$ \\
\hline ' & Cosine. & D. $1^{\prime \prime}$. & Sine. & D. $1^{\prime \prime}$. & Cotang. & D. $1^{\prime \prime}$. & Tang. & 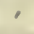 \\
\hline
\end{tabular}


TABLE 9,-LOGARITHMIC SINES, COSINES,

\begin{tabular}{|c|c|c|c|c|c|c|c|c|}
\hline , & Sine. & D. $1^{\prime \prime}$. & Cosine. & D. $1^{\prime \prime}$. & Tang. & D. $1^{\prime \prime}$. & Cotang. & , \\
\hline $\begin{array}{r}0 \\
1 \\
2 \\
3 \\
4 \\
5 \\
6 \\
7 \\
8 \\
9 \\
10\end{array}$ & $\begin{array}{r}9.779463 \\
.779631 \\
.779798 \\
.779966 \\
.780133 \\
.780300 \\
.780467 \\
.780634 \\
.780801 \\
.780968 \\
.781134\end{array}$ & $\begin{array}{l}2.80 \\
2.78 \\
2.80 \\
2.78 \\
2.78 \\
2.78 \\
2.78 \\
2.78 \\
2.78 \\
2.77 \\
2.78\end{array}$ & $\begin{array}{r}9.902349 \\
.902253 \\
.902158 \\
.902063 \\
.901967 \\
.901872 \\
.901776 \\
.901681 \\
.901585 \\
.901490 \\
.901394\end{array}$ & $\begin{array}{l}1.60 \\
1.58 \\
1.58 \\
1.60 \\
1.58 \\
1.60 \\
1.58 \\
1.60 \\
1.58 \\
1.60\end{array}$ & $\begin{array}{r}9.877114 \\
.877377 \\
.877640 \\
.877903 \\
.878165 \\
.878428 \\
.878691 \\
.878953 \\
.879216 \\
.879478 \\
.879741\end{array}$ & $\begin{array}{l}4.38 \\
4.38 \\
4.38 \\
4.37 \\
4.38 \\
4.38 \\
4.37 \\
4.38 \\
4.37 \\
4.38 \\
4.37\end{array}$ & $\begin{array}{r}10.122586 \\
.122623 \\
.122360 \\
.122097 \\
.121835 \\
.121572 \\
.121309 \\
.121047 \\
.120784 \\
.120522 \\
.120259\end{array}$ & $\begin{array}{l}52 \\
51 \\
50\end{array}$ \\
\hline $\begin{array}{l}11 \\
12 \\
13 \\
14 \\
15 \\
16 \\
17 \\
18 \\
19 \\
20\end{array}$ & $\begin{array}{r}9.781301 \\
.781468 \\
.781634 \\
.781800 \\
.781966 \\
.782132 \\
.782298 \\
.782464 \\
.783630 \\
.782796\end{array}$ & $\begin{array}{l}2.78 \\
2.77 \\
2.77 \\
2.77 \\
2.77 \\
2.77 \\
2.77 \\
2.77 \\
2.77 \\
2.75\end{array}$ & $\begin{array}{r}9.901298 \\
.901202 \\
.901106 \\
.901010 \\
.900914 \\
.900818 \\
.900722 \\
.900626 \\
.900529 \\
.900433\end{array}$ & $\begin{array}{l}1.60 \\
1.60 \\
1.60 \\
1.60 \\
1.60 \\
1.60 \\
1.60 \\
1.62 \\
1.60 \\
1.60\end{array}$ & $\begin{array}{r}9.880003 \\
.880265 \\
.880528 \\
.880790 \\
.881052 \\
.881314 \\
.881577 \\
.881839 \\
.882101 \\
.852363\end{array}$ & $\begin{array}{l}\text { 4. } 37 \\
\text { 4. } 38 \\
4.37 \\
4.37 \\
4.37 \\
4.38 \\
4.37 \\
4.37 \\
4.37 \\
4.37\end{array}$ & $\begin{array}{r}10.119997 \\
.119735 \\
.119472 \\
.119210 \\
.118948 \\
.118686 \\
.118423 \\
.118161 \\
.117899 \\
.117637\end{array}$ & $\begin{array}{l}49 \\
48 \\
47 \\
46 \\
45 \\
44 \\
43 \\
42 \\
41 \\
40\end{array}$ \\
\hline $\begin{array}{l}21 \\
22 \\
23 \\
24 \\
25 \\
26 \\
27 \\
28 \\
29 \\
30\end{array}$ & $\begin{array}{r}8.782961 \\
.783127 \\
.783292 \\
.783458 \\
.783623 \\
.783788 \\
.783953 \\
.784118 \\
.784282 \\
.784447\end{array}$ & $\begin{array}{l}2.77 \\
2.75 \\
2.77 \\
2.75 \\
2.75 \\
2.75 \\
2.75 \\
2.73 \\
2.75 \\
2.75\end{array}$ & $\begin{array}{r}9.900337 \\
.900240 \\
.900144 \\
.900047 \\
.899951 \\
.899854 \\
.899757 \\
.899660 \\
.899564 \\
.899467\end{array}$ & $\begin{array}{l}1.62 \\
1.60 \\
1.62 \\
1.60 \\
1.62 \\
1.62 \\
1.62 \\
1.60 \\
1.62 \\
1.62\end{array}$ & $\begin{array}{r}9.882625 \\
.882887 \\
.883148 \\
.883410 \\
.883672 \\
.883934 \\
.884196 \\
.884457 \\
.884719 \\
.884980\end{array}$ & $\begin{array}{l}4.07 \\
4.37 \\
4.35 \\
4.37 \\
4.37 \\
4.37 \\
4.37 \\
4.35 \\
4.37 \\
4.35 \\
4.37\end{array}$ & $\begin{array}{r}10.117375 \\
.117113 \\
.116552 \\
.116590 \\
.116328 \\
.116066 \\
.115804 \\
.115543 \\
.115281 \\
.115020\end{array}$ & $\begin{array}{l}39 \\
38 \\
37 \\
36 \\
35 \\
34 \\
33 \\
32 \\
31 \\
30\end{array}$ \\
\hline $\begin{array}{l}31 \\
32 \\
33 \\
34 \\
35 \\
36 \\
37 \\
38 \\
39 \\
40\end{array}$ & $\begin{array}{r}9.784612 \\
.784776 \\
.784941 \\
.785105 \\
.785269 \\
.785433 \\
.785597 \\
.785761 \\
.785925 \\
.786089\end{array}$ & $\begin{array}{l}2.73 \\
2.75 \\
2.73 \\
2.73 \\
2.73 \\
2.73 \\
2.73 \\
2.73 \\
2.73 \\
2.72\end{array}$ & $\begin{array}{r}9.899370 \\
.899273 \\
.899176 \\
.899078 \\
.898981 \\
.898884 \\
.898787 \\
.898689 \\
.898592 \\
.898494\end{array}$ & $\begin{array}{l}1.62 \\
1.62 \\
1.63 \\
1.62 \\
1.62 \\
1.62 \\
1.63 \\
1.62 \\
1.63 \\
1.62\end{array}$ & $\begin{array}{r}9.885242 \\
.885504 \\
.885765 \\
.886026 \\
.886288 \\
.886549 \\
.886811 \\
.887072 \\
.887333 \\
.887594\end{array}$ & $\begin{array}{l}\text { 4. } 37 \\
4.35 \\
4.35 \\
4.37 \\
4.35 \\
4.37 \\
4.35 \\
4.35 \\
4.35 \\
4.35\end{array}$ & $\begin{array}{r}10.114758 \\
.114496 \\
.114235 \\
.113974 \\
.113712 \\
.113451 \\
.113189 \\
.112928 \\
.112667 \\
.112406\end{array}$ & $\begin{array}{l}29 \\
28 \\
27 \\
26 \\
25 \\
24 \\
23 \\
22 \\
21 \\
20\end{array}$ \\
\hline $\begin{array}{l}41 \\
42 \\
43 \\
44 \\
45 \\
46 \\
47 \\
48 \\
49 \\
50\end{array}$ & $\begin{array}{r}9.786252 \\
.786416 \\
.786579 \\
.786742 \\
.781006 \\
.787069 \\
.787232 \\
.787395 \\
.787557 \\
.787720\end{array}$ & $\begin{array}{l}2.73 \\
2.72 \\
2.72 \\
2.73 \\
2.72 \\
2.72 \\
2.72 \\
2.70 \\
2.72\end{array}$ & $\begin{array}{r}9.898397 \\
.898299 \\
.898202 \\
.898104 \\
.898006 \\
.897908 \\
.897810 \\
.897712 \\
.897614 \\
.897516\end{array}$ & $\begin{array}{l}1.63 \\
1.62 \\
1.63 \\
1.63 \\
1.63 \\
1.63 \\
1.63 \\
1.63\end{array}$ & $\begin{array}{r}9.887855 \\
.885116 \\
.885378 \\
.885639 \\
.888900 \\
.889161 \\
.889421 \\
.889682 \\
.889943 \\
.890204\end{array}$ & $\begin{array}{l}4.35 \\
4.37 \\
4.35 \\
4.35 \\
4.35 \\
4.33 \\
4.35 \\
4.35 \\
4.35 \\
4.35\end{array}$ & $\begin{array}{r}10.112145 \\
.111884 \\
.111622 \\
.111361 \\
.111100 \\
.110839 \\
.110579 \\
.110318 \\
.110057 \\
.109796\end{array}$ & $\begin{array}{l}19 \\
18 \\
17 \\
16 \\
15 \\
14 \\
13 \\
12 \\
11 \\
10\end{array}$ \\
\hline $\begin{array}{l}51 \\
52 \\
53 \\
54 \\
55 \\
56 \\
57 \\
58 \\
59 \\
60\end{array}$ & $\begin{array}{r}9.787883 \\
.785045 \\
.78 \times 208 \\
.785370 \\
.788532 \\
.785694 \\
.756856 \\
.753018 \\
.789180 \\
9.789342\end{array}$ & $\begin{array}{l}2.70 \\
2.72 \\
2.70 \\
2.70 \\
2.70 \\
2.70 \\
2.70 \\
2.70 \\
2.70\end{array}$ & $\begin{array}{r}9.897418 \\
.897320 \\
.897222 \\
.897123 \\
.897025 \\
.896926 \\
.896828 \\
.896729 \\
.896631 \\
0.896532\end{array}$ & $\begin{array}{l}1.63 \\
1.63 \\
1.65 \\
1.63 \\
1.65 \\
1.63 \\
1.65 \\
1.63 \\
1.65\end{array}$ & $\begin{array}{r}9.890465 \\
.890725 \\
.890056 \\
.891247 \\
.891507 \\
.891768 \\
.892028 \\
.892259 \\
.892549 \\
9.892810\end{array}$ & $\begin{array}{l}4.33 \\
4.35 \\
4.35 \\
4.33 \\
4.35 \\
4.33 \\
4.35 \\
4.33 \\
4.35\end{array}$ & $\begin{array}{r}10.109535 \\
.109275 \\
.109014 \\
.108753 \\
.108493 \\
.108232 \\
.107972 \\
.107711 \\
.107451 \\
10.107190\end{array}$ & $\begin{array}{l}1 \\
0\end{array}$ \\
\hline ' & Cosine. & D. $1 "$. & Sine. & D. $1^{\prime \prime}$. & Cotang. & D. 1". & Tang. & ' \\
\hline
\end{tabular}




\begin{tabular}{|c|c|c|c|c|c|c|c|c|}
\hline , & Sine. & D. $1^{\prime \prime}$. & Cosine. & D. $1^{\prime \prime}$ & Tang. & D. $1^{\prime \prime}$ 。 & Cotang. & , \\
\hline $\begin{array}{r}0 \\
1 \\
2 \\
3 \\
4 \\
5 \\
6 \\
7 \\
8 \\
9 \\
10\end{array}$ & $\begin{array}{r}9.789342 \\
.789504 \\
.789665 \\
.789827 \\
.789988 \\
.790149 \\
.790310 \\
.790471 \\
.790632 \\
.790793 \\
.790954\end{array}$ & $\begin{array}{l}2.70 \\
2.68 \\
2.70 \\
2.68 \\
2.68 \\
2.68 \\
2.68 \\
2.68 \\
2.68 \\
2.68 \\
2.68\end{array}$ & $\begin{array}{r}9.896532 \\
.896433 \\
.836335 \\
.896236 \\
.896137 \\
.896038 \\
.895939 \\
.895840 \\
.895741 \\
.895641 \\
.895542\end{array}$ & $\begin{array}{l}1.65 \\
1.63 \\
1.65 \\
1.65 \\
1.65 \\
1.65 \\
1.65 \\
1.65 \\
1.67 \\
1.65 \\
1.65\end{array}$ & $\begin{array}{r}9.892810 \\
.893070 \\
.893331 \\
.893591 \\
.893851 \\
.894111 \\
.894372 \\
.894632 \\
.894892 \\
.895152 \\
.895412\end{array}$ & $\begin{array}{l}4.33 \\
4.35 \\
4.33 \\
4.33 \\
4.33 \\
4.35 \\
4.33 \\
4.33 \\
4.33 \\
4.33 \\
4.33\end{array}$ & $\begin{array}{r}10.107190 \\
.106930 \\
.106669 \\
.106409 \\
.106149 \\
.105589 \\
.105628 \\
.105368 \\
.105108 \\
.104848 \\
.104588\end{array}$ & \\
\hline $\begin{array}{l}11 \\
12 \\
13 \\
14 \\
15 \\
16 \\
17 \\
18 \\
19 \\
20\end{array}$ & $\begin{array}{r}9.791115 \\
.791275 \\
.791436 \\
.791596 \\
.791757 \\
.791917 \\
.792077 \\
.792237 \\
.792397 \\
.792557\end{array}$ & $\begin{array}{l}2.67 \\
2.68 \\
2.67 \\
2.68 \\
2.67 \\
2.67 \\
2.67 \\
2.67 \\
2.67 \\
2.65\end{array}$ & $\begin{array}{r}9.895443 \\
.895343 \\
.895244 \\
.895145 \\
.895045 \\
.894945 \\
.894846 \\
.894746 \\
.894646 \\
.894546\end{array}$ & $\begin{array}{l}1.67 \\
1.65 \\
1.65 \\
1.67 \\
1.67 \\
1.65 \\
1.67 \\
1.67 \\
1.67 \\
1.67\end{array}$ & $\begin{array}{r}9.895672 \\
.895932 \\
.896192 \\
.896452 \\
.896712 \\
.896971 \\
.897231 \\
.897491 \\
.897751 \\
.898010\end{array}$ & $\begin{array}{l}4.33 \\
4.33 \\
4.33 \\
4.33 \\
4.32 \\
4.33 \\
4.33 \\
4.33 \\
4.32 \\
4.33\end{array}$ & $\begin{array}{r}10.104328 \\
.104068 \\
.103898 \\
.103548 \\
.103288 \\
.103029 \\
.102769 \\
.102509 \\
.102249 \\
.101990\end{array}$ & $\begin{array}{l}9 \\
8 \\
7 \\
6 \\
5 \\
4 \\
3 \\
2\end{array}$ \\
\hline $\begin{array}{l}21 \\
22 \\
23 \\
24 \\
25 \\
26 \\
27 \\
29 \\
29 \\
30\end{array}$ & $\begin{array}{r}9.792716 \\
.792876 \\
.793035 \\
.793195 \\
.793354 \\
.793514 \\
.793673 \\
.793832 \\
.793991 \\
.794150\end{array}$ & $\begin{array}{l}2.67 \\
2.65 \\
2.67 \\
2.65 \\
2.67 \\
2.65 \\
2.65 \\
2.65 \\
2.65 \\
2.63\end{array}$ & $\begin{array}{r}9.894446 \\
.894346 \\
.894246 \\
.89 .1146 \\
.894046 \\
.893946 \\
.893846 \\
.893745 \\
.893645 \\
.893544\end{array}$ & $\begin{array}{l}1.67 \\
1.67 \\
1.67 \\
1.67 \\
1.67 \\
1.67 \\
1.68 \\
1.67 \\
1.68 \\
1.67\end{array}$ & $\begin{array}{r}9.898270 \\
.895530 \\
.898759 \\
.899049 \\
.899308 \\
.8995668 \\
.899827 \\
.900087 \\
.900346 \\
.900605\end{array}$ & $\begin{array}{l}4.33 \\
4.32 \\
4.33 \\
4.32 \\
4.33 \\
4.32 \\
4.33 \\
4.32 \\
4.32 \\
4.32\end{array}$ & $\begin{array}{r}10.101730 \\
.101470 \\
.101211 \\
.100951 \\
.100692 \\
.100432 \\
.100173 \\
.099913 \\
.099654 \\
.099395\end{array}$ & $\begin{array}{l}39 \\
38 \\
37 \\
36 \\
35 \\
34 \\
33 \\
32 \\
31 \\
30\end{array}$ \\
\hline $\begin{array}{l}31 \\
32 \\
33 \\
34 \\
35 \\
36 \\
37 \\
38 \\
39 \\
40\end{array}$ & $\begin{array}{r}9.794308 \\
.794467 \\
.794626 \\
.794784 \\
.794942 \\
.795101 \\
.795255 \\
.795417 \\
.795575 \\
.795733\end{array}$ & $\begin{array}{l}2.65 \\
2.65 \\
2.63 \\
2.63 \\
2.65 \\
2.63 \\
2.63 \\
2.63 \\
2.63 \\
2.63\end{array}$ & $\begin{array}{l}9.893444 \\
.893343 \\
.893243 \\
.893142 \\
.893041 \\
.892940 \\
.892839 \\
.892739 \\
.892638 \\
.892536\end{array}$ & $\begin{array}{l}1.68 \\
1.67 \\
1.68 \\
1.68 \\
1.68 \\
1.68 \\
1.67 \\
1.68 \\
1.70 \\
1.68\end{array}$ & $\begin{array}{r}9.900864 \\
.901124 \\
.901383 \\
.901642 \\
.901901 \\
.902160 \\
.902420 \\
.902679 \\
.902938 \\
.903197\end{array}$ & $\begin{array}{l}4.33 \\
4.32 \\
4.32 \\
4.32 \\
4.32 \\
4.33 \\
4.32 \\
4.32 \\
4.32 \\
4.32\end{array}$ & $\begin{array}{r}10.099136 \\
.098876 \\
.098617 \\
.098358 \\
.098099 \\
.097840 \\
.097580 \\
.097321 \\
.097062 \\
.096803\end{array}$ & $\begin{array}{l}9 \\
8 \\
7 \\
6 \\
6 \\
5 \\
4 \\
3 \\
2 \\
2 \\
1 \\
0\end{array}$ \\
\hline $\begin{array}{l}41 \\
42 \\
43 \\
44 \\
45 \\
46 \\
47 \\
43 \\
49 \\
50\end{array}$ & $\begin{array}{r}9.795891 \\
.796049 \\
.796206 \\
.796364 \\
.796521 \\
.796679 \\
.796836 \\
.796993 \\
.797150 \\
.797307\end{array}$ & $\begin{array}{l}2.63 \\
2.62 \\
2.63 \\
2.62 \\
2.63 \\
2.62 \\
2.62 \\
2.62 \\
2.62 \\
2.62\end{array}$ & $\begin{array}{r}9.802435 \\
.892334 \\
.892233 \\
.892132 \\
.892030 \\
.891929 \\
.891827 \\
.891726 \\
.891624 \\
.891523\end{array}$ & $\begin{array}{l}1.68 \\
1.68 \\
1.68 \\
1.70 \\
1.68 \\
1.70 \\
1.68 \\
1.70 \\
1.68 \\
1.70\end{array}$ & $\begin{array}{r}9.903456 \\
.903714 \\
.903973 \\
.904232 \\
.904491 \\
.904750 \\
.905008 \\
.905267 \\
.905526 \\
.905785\end{array}$ & $\begin{array}{l}4.30 \\
4.32 \\
4.32 \\
4.32 \\
4.32 \\
4.30 \\
4.32 \\
4.32 \\
4.32 \\
4.30\end{array}$ & $\begin{array}{r}10.096544 \\
.096286 \\
.096027 \\
.095768 \\
.095509 \\
.095250 \\
.094992 \\
.094733 \\
.094474 \\
.094215\end{array}$ & $\begin{array}{l}9 \\
8 \\
7 \\
6 \\
5 \\
4 \\
3 \\
2 \\
1 \\
0\end{array}$ \\
\hline $\begin{array}{l}51 \\
52 \\
53 \\
54 \\
55 \\
56 \\
57 \\
58 \\
59 \\
60\end{array}$ & $\begin{array}{r}9.797464 \\
.797621 \\
.797777 \\
.797934 \\
.795091 \\
.795247 \\
.795403 \\
.795560 \\
.798716 \\
9.798872\end{array}$ & $\begin{array}{l}2.62 \\
2.60 \\
2.62 \\
2.62 \\
2.60 \\
2.60 \\
2.62 \\
2.60 \\
2.60\end{array}$ & $\begin{array}{r}9.891421 \\
.891319 \\
.891217 \\
.891115 \\
.891013 \\
.890911 \\
.890809 \\
.890707 \\
.890605 \\
9.890503\end{array}$ & $\begin{array}{l}1.70 \\
1.70 \\
1.70 \\
1.70 \\
1.70 \\
1.70 \\
1.70 \\
1.70 \\
1.70\end{array}$ & $\begin{array}{r}9.906043 \\
.906 i 302 \\
.906560 \\
.906819 \\
.907077 \\
.907336 \\
.907594 \\
.907853 \\
.908111 \\
9.900369\end{array}$ & $\begin{array}{l}4.32 \\
4.30 \\
4.32 \\
4.30 \\
4.32 \\
4.30 \\
4.32 \\
4.30 \\
4.30\end{array}$ & $\begin{array}{r}\text { 10. } 093957 \\
.093698 \\
.093440 \\
.093181 \\
.092923 \\
.092664 \\
.092406 \\
.092147 \\
.091599 \\
10,091631\end{array}$ & $\begin{array}{l}9 \\
8 \\
7 \\
6 \\
5 \\
4 \\
3 \\
2 \\
1 \\
0\end{array}$ \\
\hline ' & Cosine. & $1^{\prime \prime}$. & e. & D. $1^{\prime \prime}$. & Cotang. & D. $1^{\prime \prime}$. & Tang. & \\
\hline
\end{tabular}




\begin{tabular}{|c|c|c|c|c|c|c|c|c|}
\hline ' & Sine. & D. $1^{\prime \prime}$. & Cosine. & D. $1^{\prime \prime}$. & Tang. & D. $1^{\prime \prime}$. & Cotang. & ' \\
\hline $\begin{array}{r}0 \\
1 \\
2 \\
3 \\
4 \\
5 \\
6 \\
7 \\
8 \\
9 \\
10\end{array}$ & $\begin{array}{r}9.798872 \\
.799028 \\
.799184 \\
.799339 \\
.799495 \\
.799651 \\
.799806 \\
.799962 \\
.800117 \\
.800272 \\
.800427\end{array}$ & $\begin{array}{l}2.60 \\
2.60 \\
2.58 \\
2.60 \\
2.60 \\
2.58 \\
2.60 \\
2.58 \\
2.58 \\
2.58 \\
2.58\end{array}$ & $\begin{array}{r}9.890503 \\
.890400 \\
.890298 \\
.890195 \\
.890093 \\
.889990 \\
.889888 \\
.889785 \\
.889682 \\
.889579 \\
.889477\end{array}$ & $\begin{array}{l}1.72 \\
1.70 \\
1.72 \\
1.70 \\
1.72 \\
1.70 \\
1.72 \\
1.72 \\
1.72 \\
1.70 \\
1.72\end{array}$ & $\begin{array}{r}9.908369 \\
.905628 \\
.908856 \\
.909144 \\
.909402 \\
.909660 \\
.909918 \\
.910177 \\
.910435 \\
.910693 \\
.910951\end{array}$ & $\begin{array}{l}4.32 \\
4.30 \\
4.30 \\
4.30 \\
4.30 \\
4.30 \\
4.32 \\
4.30 \\
4.30 \\
4.30 \\
4.30\end{array}$ & $\begin{array}{r}10.091631 \\
.091372 \\
.091114 \\
.090856 \\
.090598 \\
.090340 \\
.090082 \\
.089823 \\
.089565 \\
.089307 \\
.089049\end{array}$ & $\begin{array}{l}60 \\
59 \\
58 \\
57 \\
56 \\
55 \\
54 \\
53 \\
52 \\
51 \\
50\end{array}$ \\
\hline $\begin{array}{l}11 \\
12 \\
13 \\
14 \\
15 \\
16 \\
17 \\
18 \\
19 \\
20\end{array}$ & $\begin{array}{r}9.800582 \\
.800737 \\
.800592 \\
.801047 \\
.801201 \\
.801356 \\
.801511 \\
.801665 \\
.801819 \\
.801973\end{array}$ & $\begin{array}{l}2.58 \\
2.58 \\
2.58 \\
2.57 \\
2.58 \\
2.58 \\
2.57 \\
2.57 \\
2.57 \\
2.58\end{array}$ & $\begin{array}{r}9.889374 \\
.889271 \\
.889168 \\
.889064 \\
.888961 \\
.888858 \\
.888755 \\
.888651 \\
.888548 \\
.888444\end{array}$ & $\begin{array}{l}1.72 \\
1.72 \\
1.73 \\
1.72 \\
1.72 \\
1.72 \\
1.73 \\
1.72 \\
1.73 \\
1.72\end{array}$ & $\begin{array}{r}9.911209 \\
.911467 \\
.911725 \\
.911982 \\
.912240 \\
.912498 \\
.912756 \\
.913014 \\
.913271 \\
.913529\end{array}$ & $\begin{array}{l}4.30 \\
4.30 \\
4.28 \\
4.30 \\
4.30 \\
4.30 \\
4.30 \\
4.28 \\
4.30 \\
4.30\end{array}$ & $\begin{array}{r}10.088791 \\
.088533 \\
.088275 \\
.088018 \\
.087760 \\
.087502 \\
.087244 \\
.086986 \\
.086729 \\
.086471\end{array}$ & $\begin{array}{l}49 \\
48 \\
47 \\
46 \\
45 \\
44 \\
43 \\
42 \\
41 \\
40\end{array}$ \\
\hline $\begin{array}{l}21 \\
22 \\
23 \\
24 \\
25 \\
26 \\
27 \\
28 \\
29 \\
30\end{array}$ & $\begin{array}{r}9.802128 \\
.802282 \\
.802436 \\
.802589 \\
.802743 \\
.802897 \\
.803050 \\
.803204 \\
.803357 \\
.803511\end{array}$ & $\begin{array}{l}2.57 \\
2.57 \\
2.55 \\
2.57 \\
2.57 \\
2.55 \\
2.57 \\
2.55 \\
2.57 \\
2.55\end{array}$ & $\begin{array}{r}9.888341 \\
.888237 \\
.888134 \\
.888030 \\
.887926 \\
.887822 \\
.887718 \\
.887614 \\
.887510 \\
.887406\end{array}$ & $\begin{array}{l}1.73 \\
1.72 \\
1.73 \\
1.73 \\
1.73 \\
1.73 \\
1.73 \\
1.73 \\
1.73 \\
1.73\end{array}$ & $\begin{array}{r}9.913787 \\
.914044 \\
.914302 \\
.914560 \\
.914817 \\
.915075 \\
.915332 \\
.915590 \\
.915847 \\
.916104\end{array}$ & $\begin{array}{l}4.28 \\
4.30 \\
4.30 \\
4.28 \\
4.30 \\
4.28 \\
4.30 \\
4.28 \\
4.28 \\
4.30\end{array}$ & $\begin{array}{r}10.086213 \\
.085956 \\
.085698 \\
.085440 \\
.085183 \\
.084925 \\
.084668 \\
.084410 \\
.084153 \\
.083896\end{array}$ & $\begin{array}{l}39 \\
38 \\
37 \\
36 \\
35 \\
34 \\
33 \\
32 \\
31 \\
30\end{array}$ \\
\hline $\begin{array}{l}31 \\
32 \\
33 \\
34 \\
35 \\
36 \\
37 \\
38 \\
39 \\
40\end{array}$ & $\begin{array}{r}9.803664 \\
.803817 \\
.803970 \\
.804123 \\
.804276 \\
.804428 \\
.804581 \\
.804734 \\
.804886 \\
.805039\end{array}$ & $\begin{array}{l}2.55 \\
2.55 \\
2.55 \\
2.55 \\
2.55 \\
2.55 \\
2.55 \\
2.53 \\
2.55 \\
2.53\end{array}$ & $\begin{array}{r}9.887302 \\
.857198 \\
.887093 \\
.886989 \\
.886885 \\
.886780 \\
.886676 \\
.886571 \\
.886466 \\
.886362\end{array}$ & $\begin{array}{l}1.73 \\
1.75 \\
1.73 \\
1.73 \\
1.75 \\
1.73 \\
1.75 \\
1.75 \\
1.73 \\
1.75\end{array}$ & $\begin{array}{r}9.916362 \\
.916619 \\
.916877 \\
.917134 \\
.917391 \\
.917648 \\
.917906 \\
.918163 \\
.918420 \\
.918677\end{array}$ & $\begin{array}{l}4.28 \\
4.30 \\
4.28 \\
4.28 \\
4.28 \\
4.30 \\
4.28 \\
4.28 \\
4.28 \\
4.28\end{array}$ & $\begin{array}{r}10.083638 \\
.083381 \\
.083123 \\
.082866 \\
.082609 \\
.082352 \\
.082094 \\
.081837 \\
.081580 \\
.081323\end{array}$ & $\begin{array}{l}29 \\
28 \\
27 \\
26 \\
25 \\
24 \\
23 \\
22 \\
21 \\
20\end{array}$ \\
\hline $\begin{array}{l}41 \\
42 \\
43 \\
44 \\
45 \\
46 \\
47 \\
48 \\
49 \\
50\end{array}$ & $\begin{array}{r}9.805191 \\
.805343 \\
.805495 \\
.805647 \\
.805799 \\
.805951 \\
.806103 \\
.806254 \\
.806406 \\
.806557\end{array}$ & $\begin{array}{l}2.53 \\
2.53 \\
2.53 \\
2.53 \\
2.53 \\
2.53 \\
2.52 \\
2.53 \\
2.52 \\
2.53\end{array}$ & $\begin{array}{r}9.886257 \\
.856152 \\
.886047 \\
.885942 \\
.885837 \\
.885732 \\
.885627 \\
.885522 \\
.885416 \\
.885311\end{array}$ & $\begin{array}{l}1.75 \\
1.75 \\
1.75 \\
1.75 \\
1.75 \\
1.75 \\
1.75 \\
1.77 \\
1.75 \\
1.77\end{array}$ & $\begin{array}{r}9.918934 \\
.919191 \\
.919448 \\
.919705 \\
.919962 \\
.920219 \\
.920476 \\
.920733 \\
.920990 \\
.921247\end{array}$ & $\begin{array}{l}4.28 \\
4.28 \\
4.28 \\
4.28 \\
4.28 \\
4.28 \\
4.28 \\
4.28 \\
4.28 \\
4.27\end{array}$ & $\begin{array}{r}10.081066 \\
.080809 \\
.080552 \\
.080295 \\
.080038 \\
.079781 \\
.079524 \\
.079267 \\
.079010 \\
.078753\end{array}$ & $\begin{array}{l}19 \\
18 \\
17 \\
16 \\
15 \\
14 \\
13 \\
12 \\
11 \\
10\end{array}$ \\
\hline $\begin{array}{l}51 \\
52 \\
53 \\
54 \\
55 \\
56 \\
57 \\
58 \\
59 \\
60\end{array}$ & $\begin{array}{r}9.806709 \\
.806860 \\
.807011 \\
.807163 \\
.807314 \\
.807465 \\
.807615 \\
.807766 \\
.807917 \\
9.805067\end{array}$ & \begin{tabular}{l|}
2.52 \\
2.52 \\
2.53 \\
2.52 \\
2.52 \\
2.50 \\
2.52 \\
2.52 \\
2.50
\end{tabular} & $\begin{array}{r}9.885205 \\
.885100 \\
.884994 \\
.884889 \\
.884783 \\
.884677 \\
.884572 \\
.884466 \\
.884360 \\
9.884254\end{array}$ & $\begin{array}{l}1.75 \\
1.77 \\
1.75 \\
1.77 \\
1.77 \\
1.75 \\
1.77 \\
1.77 \\
1.77\end{array}$ & $\begin{array}{r}9.921503 \\
.921760 \\
.922017 \\
.922274 \\
.922530 \\
.922787 \\
.923044 \\
.923300 \\
.023557 \\
0.923814\end{array}$ & $\begin{array}{l}4.28 \\
4.28 \\
4.28 \\
4.27 \\
4.28 \\
4.28 \\
4.27 \\
4.28 \\
4.28\end{array}$ & $\begin{array}{r}10.078497 \\
.078240 \\
.077983 \\
.077726 \\
.077470 \\
.077213 \\
.076956 \\
.076700 \\
.076443 \\
10.076186\end{array}$ & $\begin{array}{l}9 \\
8 \\
7 \\
6 \\
5 \\
4 \\
3 \\
2 \\
1 \\
0\end{array}$ \\
\hline , & Cosine. & D. 1". & Sine. & D. $1^{\prime \prime}$. & Cotang. & D. $1^{\prime \prime}$. & Tang. & ' \\
\hline
\end{tabular}


TANGEXTS, AND COTANGEXTS.

\begin{tabular}{|c|c|c|c|c|c|c|c|c|}
\hline ' & Sine. & D. $1^{\prime \prime}$. & Casine. & D. $1 "$. & Tang. & D. $1^{\prime \prime}$. & Cotang. & , \\
\hline $\begin{array}{r}0 \\
1 \\
2 \\
3 \\
4 \\
5 \\
6 \\
7 \\
8 \\
9 \\
10\end{array}$ & $\begin{array}{r}9.808067 \\
.808218 \\
.808368 \\
.808519 \\
.808669 \\
.806819 \\
.808969 \\
.809119 \\
.809269 \\
.809419 \\
.809569\end{array}$ & $\begin{array}{l}2.52 \\
2.50 \\
2.52 \\
2.50 \\
2.50 \\
2.50 \\
2.50 \\
2.50 \\
2.50 \\
2.50 \\
2.48\end{array}$ & $\begin{array}{r}9.884254 \\
\$ 84149 \\
.884042 \\
.883936 \\
.883829 \\
.883723 \\
.883617 \\
.853510 \\
.883404 \\
.883297 \\
.883191\end{array}$ & $\begin{array}{l}1.77 \\
1.77 \\
1.77 \\
1.78 \\
1.77 \\
1.77 \\
1.78 \\
1.77 \\
1.78 \\
1.77 \\
1.78\end{array}$ & $\begin{array}{r}9.923814 \\
.924070 \\
.924327 \\
.924583 \\
.924840 \\
.925096 \\
.925352 \\
.925609 \\
.925865 \\
.926122 \\
.926378\end{array}$ & $\begin{array}{l}4.27 \\
4.28 \\
4.27 \\
4.28 \\
4.27 \\
4.27 \\
4.28 \\
4.27 \\
4.28 \\
4.27 \\
4.27\end{array}$ & $\begin{array}{r}10.076186 \\
.075930 \\
.075673 \\
.075417 \\
.075160 \\
.074904 \\
.074648 \\
.074391 \\
.074135 \\
.073878 \\
.073622\end{array}$ & $\begin{array}{l}60 \\
59 \\
58 \\
57 \\
56 \\
55 \\
54 \\
53 \\
52 \\
51 \\
50\end{array}$ \\
\hline $\begin{array}{l}11 \\
12 \\
13 \\
14 \\
15 \\
16 \\
17 \\
18 \\
19 \\
20\end{array}$ & $\begin{array}{r}9.809718 \\
.809868 \\
.810017 \\
.810167 \\
.810316 \\
.810465 \\
.810614 \\
.810763 \\
.810912 \\
.811061\end{array}$ & $\begin{array}{l}2.50 \\
2.48 \\
2.50 \\
2.48 \\
2.48 \\
2.49 \\
2.49 \\
2.49 \\
2.48 \\
2.48\end{array}$ & $\begin{array}{r}9.883084 \\
.882977 \\
.882871 \\
.882764 \\
.882657 \\
.882550 \\
.882443 \\
.882336 \\
.852229 \\
.852121\end{array}$ & $\begin{array}{l}1.78 \\
1.77 \\
1.78 \\
1.78 \\
1.78 \\
1.78 \\
1.78 \\
1.78 \\
1.80 \\
1.78\end{array}$ & $\begin{array}{r}9.926634 \\
.92 t 890 \\
.927147 \\
.927403 \\
.927659 \\
.927915 \\
.928171 \\
.928427 \\
.928684 \\
.928940\end{array}$ & $\begin{array}{l}4.27 \\
4.28 \\
4.27 \\
4.27 \\
4.27 \\
4.27 \\
4.27 \\
4.28 \\
4.27 \\
4.27\end{array}$ & $\begin{array}{r}10.073366 \\
.073110 \\
.072853 \\
.072597 \\
.072341 \\
.072085 \\
.071829 \\
.071573 \\
.071316 \\
.071060\end{array}$ & $\begin{array}{l}49 \\
48 \\
47 \\
46 \\
45 \\
44 \\
43 \\
42 \\
41 \\
40\end{array}$ \\
\hline $\begin{array}{l}21 \\
22 \\
23 \\
24 \\
25 \\
26 \\
27 \\
23 \\
29 \\
30\end{array}$ & $\begin{array}{r}9.811210 \\
.811358 \\
.811507 \\
.811655 \\
.811804 \\
.811952 \\
.812100 \\
.812248 \\
.812396 \\
.812544\end{array}$ & $\begin{array}{l}2.47 \\
2.48 \\
2.47 \\
2.48 \\
2.47 \\
2.47 \\
2.47 \\
2.47 \\
2.47 \\
2.47\end{array}$ & $\begin{array}{r}9.852014 \\
.851907 \\
.881799 \\
.881692 \\
.881584 \\
.851477 \\
.851369 \\
.881261 \\
.881153 \\
.851046\end{array}$ & $\begin{array}{l}1.78 \\
1.80 \\
1.78 \\
1.80 \\
1.79 \\
1.80 \\
1.80 \\
1.80 \\
1.79 \\
1.80\end{array}$ & $\begin{array}{r}9.929196 \\
.929452 \\
.929708 \\
.929964 \\
.930220 \\
.930475 \\
.930731 \\
.930987 \\
.931243 \\
.931499\end{array}$ & $\begin{array}{l}4.27 \\
4.27 \\
4.27 \\
4.27 \\
4.25 \\
4.27 \\
4.27 \\
4.27 \\
4.27 \\
4.27\end{array}$ & $\begin{array}{r}10.070804 \\
.070548 \\
.070292 \\
.070036 \\
.069780 \\
.069525 \\
.069269 \\
.069013 \\
.068757 \\
.068501\end{array}$ & $\begin{array}{l}39 \\
38 \\
37 \\
36 \\
35 \\
34 \\
33 \\
32 \\
31 \\
30\end{array}$ \\
\hline $\begin{array}{l}31 \\
32 \\
33 \\
34 \\
35 \\
36 \\
37 \\
38 \\
39 \\
40\end{array}$ & $\begin{array}{r}9.812692 \\
.812810 \\
.812988 \\
.813135 \\
.813283 \\
.813430 \\
.813578 \\
.813725 \\
.813872 \\
.814019\end{array}$ & $\begin{array}{l}2.47 \\
2.47 \\
2.45 \\
2.47 \\
2.45 \\
2.47 \\
2.45 \\
2.45 \\
2.45 \\
2.45\end{array}$ & $\begin{array}{r}9.880938 \\
.850830 \\
.850722 \\
.880613 \\
.880505 \\
.850397 \\
.850259 \\
.850180 \\
.850072 \\
.879063\end{array}$ & $\begin{array}{l}1.80 \\
1.80 \\
1.82 \\
1.80 \\
1.80 \\
1.80 \\
1.82 \\
1.80 \\
1.82 \\
1.80\end{array}$ & $\begin{array}{r}9.931755 \\
.932010 \\
.932266 \\
.932522 \\
.932778 \\
.933033 \\
.933289 \\
.933545 \\
.933500 \\
.934056\end{array}$ & $\begin{array}{l}4.25 \\
4.27 \\
4.27 \\
4.27 \\
4.25 \\
4.27 \\
4.27 \\
4.25 \\
4.27 \\
4.25\end{array}$ & $\begin{array}{r}10.068245 \\
.067990 \\
.067734 \\
.067478 \\
.067222 \\
.066967 \\
.066711 \\
.066455 \\
.066200 \\
.065944\end{array}$ & $\begin{array}{l}29 \\
23 \\
27 \\
26 \\
25 \\
24 \\
23 \\
22 \\
21 \\
20\end{array}$ \\
\hline $\begin{array}{l}41 \\
42 \\
43 \\
44 \\
45 \\
46 \\
47 \\
48 \\
49 \\
50\end{array}$ & $\begin{array}{r}9.814166 \\
.814313 \\
.814460 \\
.814607 \\
.814753 \\
.814900 \\
.815046 \\
.815193 \\
.815339 \\
.815485\end{array}$ & $\begin{array}{l}2.45 \\
2.45 \\
2.45 \\
2.43 \\
2.45 \\
2.43 \\
2.45 \\
2.43 \\
2.43 \\
2.45\end{array}$ & $\begin{array}{r}9.879555 \\
.879746 \\
.879637 \\
.879529 \\
.879420 \\
.879311 \\
.879202 \\
.879093 \\
.878984 \\
.878975\end{array}$ & $\begin{array}{l}1.82 \\
1.82 \\
1.80 \\
1.82 \\
1.82 \\
1.82 \\
1.82 \\
1.82 \\
1.82 \\
1.82\end{array}$ & $\begin{array}{r}9.934311 \\
.934567 \\
.934822 \\
.935078 \\
.9353333 \\
.935559 \\
.935844 \\
.936100 \\
.936355 \\
.936611\end{array}$ & $\begin{array}{l}4.27 \\
4.27 \\
4.25 \\
4.27 \\
4.25 \\
4.27 \\
4.25 \\
4.27 \\
4.25 \\
4.27 \\
4.25\end{array}$ & $\begin{array}{r}10.065689 \\
.065433 \\
.065178 \\
.064922 \\
.0646667 \\
.064411 \\
.064156 \\
.063900 \\
.0633645 \\
.063359\end{array}$ & $\begin{array}{l}19 \\
18 \\
17 \\
16 \\
15 \\
14 \\
13 \\
12 \\
11 \\
10\end{array}$ \\
\hline $\begin{array}{l}51 \\
52 \\
53 \\
54 \\
55 \\
56 \\
57 \\
58 \\
59 \\
60\end{array}$ & $\begin{array}{r}9.815632 \\
.815778 \\
.815924 \\
.816069 \\
.816215 \\
.816361 \\
.816507 \\
.816652 \\
.816793 \\
9.816943\end{array}$ & $\begin{array}{l}2.43 \\
2.43 \\
2.42 \\
2.43 \\
2.43 \\
2.43 \\
2.42 \\
2.43 \\
2.42\end{array}$ & $\begin{array}{r}9.878766 \\
.878656 \\
.878547 \\
.878439 \\
.878328 \\
.878219 \\
.878109 \\
.877999 \\
.877800 \\
9.877780\end{array}$ & $\begin{array}{l}1.83 \\
1.82 \\
1.82 \\
1.83 \\
1.82 \\
1.83 \\
1.83 \\
1.82 \\
1.83\end{array}$ & $\begin{array}{r}9.9368666 \\
.937121 \\
.937377 \\
.937632 \\
.937857 \\
.938142 \\
.938398 \\
.938655 \\
.938908 \\
9.939163\end{array}$ & $\begin{array}{l}4.25 \\
4.27 \\
4.25 \\
4.25 \\
4.25 \\
4.27 \\
4.25 \\
4.25 \\
4.25\end{array}$ & $\begin{array}{r}10.063134 \\
.062579 \\
.062623 \\
.062368 \\
.062113 \\
.061858 \\
.061602 \\
.061347 \\
.061092 \\
10.060837\end{array}$ & $\begin{array}{l}9 \\
8 \\
7 \\
6 \\
5 \\
4 \\
3 \\
2 \\
1 \\
0\end{array}$ \\
\hline , & Cosine. & D. $1^{\prime \prime}$. & Sine. & D. $1^{\prime \prime}$. & Co & D. $1^{\prime \prime}$. & Tang. & \\
\hline
\end{tabular}


TABLE 9.-LOGARITHMIC SINES, COSINES,

\begin{tabular}{|c|c|c|c|c|c|c|c|c|}
\hline , & Sine. & D. $1^{\prime \prime}$. & Cosine. & D. $1^{\prime \prime}$. & Tang. & D. 1". & Cotang. & , \\
\hline $\begin{array}{r}0 \\
1 \\
2 \\
3 \\
4 \\
5 \\
6 \\
7 \\
8 \\
9 \\
10\end{array}$ & $\begin{array}{r}9.816943 \\
.817085 \\
.817233 \\
.817379 \\
.817524 \\
.817668 \\
.817813 \\
.817958 \\
.818103 \\
.818247 \\
.818392\end{array}$ & $\begin{array}{l}2.42 \\
2.42 \\
2.43 \\
2.42 \\
2.40 \\
2.42 \\
2.42 \\
2.42 \\
2.40 \\
2.42 \\
2.40\end{array}$ & $\begin{array}{r}9.877780 \\
.877670 \\
.877560 \\
.877450 \\
.877310 \\
.877230 \\
.877120 \\
.877010 \\
.876899 \\
.876789 \\
.876678\end{array}$ & $\begin{array}{l}1.83 \\
1.83 \\
1.83 \\
1.83 \\
1.83 \\
1.83 \\
1.83 \\
1.85 \\
1.83 \\
1.85 \\
1.83\end{array}$ & $\begin{array}{r}9.939163 \\
.939418 \\
.939673 \\
.939928 \\
.940183 \\
.940439 \\
.940694 \\
.940949 \\
.941204 \\
.941459 \\
.941713\end{array}$ & $\begin{array}{l}4.25 \\
4.25 \\
4.25 \\
4.25 \\
4.27 \\
4.25 \\
4.25 \\
4.25 \\
4.25 \\
4.23 \\
4.25\end{array}$ & $\begin{array}{r}10.060837 \\
.060582 \\
.060327 \\
.060072 \\
.059817 \\
.059561 \\
.059306 \\
.059051 \\
.058796 \\
.058541 \\
.058287\end{array}$ & $\begin{array}{l}56 \\
55 \\
54 \\
53\end{array}$ \\
\hline $\begin{array}{l}11 \\
12 \\
13 \\
14 \\
15 \\
16 \\
17 \\
18 \\
19 \\
20\end{array}$ & $\begin{array}{r}9.818536 \\
.818681 \\
.818825 \\
.818969 \\
.819113 \\
.819257 \\
.819401 \\
.819545 \\
.819689 \\
.819832\end{array}$ & $\begin{array}{l}2.42 \\
2.40 \\
2.40 \\
2.40 \\
2.40 \\
2.40 \\
2.40 \\
2.40 \\
2.38 \\
2.40\end{array}$ & $\begin{array}{r}9.876568 \\
.876457 \\
.876347 \\
.876236 \\
.876125 \\
.876014 \\
.875904 \\
.875793 \\
.875682 \\
.875571\end{array}$ & $\begin{array}{l}1.83 \\
1.85 \\
1.83 \\
1.85 \\
1.85 \\
1.85 \\
1.83 \\
1.85 \\
1.85 \\
1.85 \\
1.87\end{array}$ & $\begin{array}{r}9.941968 \\
.942223 \\
.942478 \\
.942733 \\
.942988 \\
.943243 \\
.943498 \\
.943752 \\
.944007 \\
.944262\end{array}$ & $\begin{array}{l}4.20 \\
4.25 \\
4.25 \\
4.25 \\
4.25 \\
4.25 \\
4.25 \\
4.23 \\
4.25 \\
4.25 \\
4.25\end{array}$ & $\begin{array}{r}10.058032 \\
.057777 \\
.057522 \\
.057267 \\
.057012 \\
.056757 \\
.056502 \\
.056248 \\
.055993 \\
.055738\end{array}$ & 49 \\
\hline $\begin{array}{l}21 \\
22 \\
23 \\
24 \\
25 \\
26 \\
27 \\
28 \\
29 \\
30\end{array}$ & $\begin{array}{r}9.819976 \\
.820120 \\
.820263 \\
.820406 \\
.820550 \\
.820693 \\
.820836 \\
.820979 \\
.821122 \\
.821265\end{array}$ & $\begin{array}{l}2.40 \\
2.38 \\
2.38 \\
2.40 \\
2.38 \\
2.38 \\
2.38 \\
2.38 \\
2.38 \\
2.37\end{array}$ & $\begin{array}{r}9.875459 \\
.875348 \\
.875237 \\
.875126 \\
.875014 \\
.874903 \\
.874791 \\
.874680 \\
.874568 \\
.874456\end{array}$ & $\begin{array}{l}1.85 \\
1.85 \\
1.85 \\
1.87 \\
1.85 \\
1.87 \\
1.85 \\
1.87 \\
1.87 \\
1.87\end{array}$ & $\begin{array}{r}9.944517 \\
.944771 \\
.945026 \\
.945281 \\
.945535 \\
.945790 \\
.946045 \\
.946299 \\
.946554 \\
.946808\end{array}$ & $\begin{array}{l}4.23 \\
4.25 \\
4.25 \\
4.23 \\
4.25 \\
4.25 \\
4.23 \\
4.25 \\
4.23 \\
4.25\end{array}$ & $\begin{array}{r}10.055483 \\
.055229 \\
.054974 \\
.054719 \\
.054465 \\
.054210 \\
.053955 \\
.053701 \\
.053446 \\
.053192\end{array}$ & $\begin{array}{l}39 \\
38 \\
37 \\
36 \\
35 \\
34 \\
33 \\
32 \\
31 \\
30\end{array}$ \\
\hline $\begin{array}{l}31 \\
32 \\
33 \\
34 \\
35 \\
36 \\
37 \\
38 \\
39 \\
40\end{array}$ & $\begin{array}{r}9.821407 \\
.821550 \\
.821693 \\
.821835 \\
.821977 \\
.822120 \\
.822262 \\
.822404 \\
.822546 \\
.822688\end{array}$ & $\begin{array}{l}2.38 \\
2.38 \\
2.37 \\
2.37 \\
2.38 \\
2.37 \\
2.37 \\
2.37 \\
2.37 \\
2.37\end{array}$ & $\begin{array}{r}9.874344 \\
.874232 \\
.874121 \\
.874009 \\
.873896 \\
.873784 \\
.873672 \\
.873560 \\
.873448 \\
.873335\end{array}$ & $\begin{array}{l}1.87 \\
1.85 \\
1.87 \\
1.88 \\
1.87 \\
1.87 \\
1.87 \\
1.87 \\
1.88 \\
1.87\end{array}$ & $\begin{array}{r}9.947063 \\
.947318 \\
.947572 \\
.947827 \\
.948081 \\
.948335 \\
.948590 \\
.948844 \\
.949099 \\
.949353\end{array}$ & $\begin{array}{l}4.25 \\
4.23 \\
4.25 \\
4.23 \\
4.23 \\
4.25 \\
4.23 \\
4.25 \\
4.23 \\
4.25\end{array}$ & $\begin{array}{r}10.052937 \\
.052682 \\
.052428 \\
.052173 \\
.051919 \\
.051665 \\
.051410 \\
.051156 \\
.050901 \\
.050647\end{array}$ & 。 \\
\hline $\begin{array}{l}41 \\
42 \\
43 \\
44 \\
45 \\
46 \\
47 \\
49 \\
49 \\
50\end{array}$ & $\begin{array}{r}9.822830 \\
.822972 \\
.823114 \\
.823255 \\
.823397 \\
.823539 \\
.823680 \\
.823821 \\
.823963 \\
.824104\end{array}$ & $\begin{array}{l}2.37 \\
2.37 \\
2.35 \\
2.37 \\
2.37 \\
2.35 \\
2.3 .5 \\
2.37 \\
2.35 \\
2.35\end{array}$ & $\begin{array}{r}9.873223 \\
.873110 \\
.872998 \\
.872855 \\
.872772 \\
.872659 \\
.872547 \\
.872434 \\
.872321 \\
.872208\end{array}$ & $\begin{array}{l}1.88 \\
1.87 \\
1.89 \\
1.88 \\
1.88 \\
1.87 \\
1.88 \\
1.88 \\
1.88 \\
1.88\end{array}$ & $\begin{array}{r}9.949608 \\
.949862 \\
.950116 \\
.950371 \\
.950625 \\
.950879 \\
.951133 \\
.951388 \\
.951642 \\
.951896\end{array}$ & $\begin{array}{l}4.23 \\
4.23 \\
4.25 \\
4.23 \\
4.23 \\
4.23 \\
4.25 \\
4.23 \\
4.23 \\
4.23\end{array}$ & $\begin{array}{r}10.050392 \\
.050138 \\
.049884 \\
.049629 \\
.049375 \\
.049121 \\
.048567 \\
.048612 \\
.048358 \\
.048104\end{array}$ & 19 \\
\hline $\begin{array}{l}51 \\
52 \\
53 \\
54 \\
55 \\
56 \\
57 \\
58 \\
59 \\
60\end{array}$ & $\begin{array}{r}9.824245 \\
.824386 \\
.824527 \\
.824668 \\
.824808 \\
.824949 \\
.825090 \\
.825230 \\
.825371 \\
9.825511\end{array}$ & $\begin{array}{l}2.35 \\
2.35 \\
2.35 \\
2.33 \\
2.35 \\
2.35 \\
2.33 \\
2.35 \\
2.33\end{array}$ & $\begin{array}{r}9.872095 \\
.871981 \\
.871868 \\
.871755 \\
.871641 \\
.871528 \\
.871414 \\
.871301 \\
.871187 \\
9.871073\end{array}$ & $\begin{array}{l}1.90 \\
1.88 \\
1.88 \\
1.90 \\
1.88 \\
1.90 \\
1.88 \\
1.90 \\
1.90\end{array}$ & $\begin{array}{r}9.952150 \\
.952405 \\
.952653 \\
.952913 \\
.953167 \\
.053421 \\
.953675 \\
.953929 \\
.954183 \\
9.954437\end{array}$ & $\begin{array}{l}4.25 \\
4.23 \\
4.23 \\
4.23 \\
4.23 \\
4.23 \\
4.23 \\
4.23 \\
4.23\end{array}$ & $\begin{array}{r}10.047850 \\
.047595 \\
.047341 \\
.047087 \\
.046833 \\
.046579 \\
.046325 \\
.046071 \\
.045817 \\
10.015563\end{array}$ & $\begin{array}{l}9 \\
8 \\
7 \\
6 \\
5 \\
4 \\
4 \\
3\end{array}$ \\
\hline ' & Cosine. & D. $1^{\prime \prime}$. & Sine. & D. $1^{\prime \prime}$. & Cotang. & D. $1^{\prime \prime}$. & Tang. & ' \\
\hline
\end{tabular}




\begin{tabular}{|c|c|c|c|c|c|c|c|c|}
\hline , & Sine. & D. $1^{\prime \prime}$. & Cosine. & D. $1^{\prime \prime}$. & Tang. & D. $1^{\prime \prime}$. & Cotang. & ' \\
\hline $\begin{array}{r}0 \\
1 \\
2 \\
3 \\
4 \\
5 \\
6 \\
7 \\
8 \\
9 \\
10\end{array}$ & $\begin{array}{l}9.825511 \\
.825651 \\
.825791 \\
.825931 \\
.826071 \\
.826211 \\
.826351 \\
.826491 \\
.826631 \\
.826770 \\
.826910\end{array}$ & $\begin{array}{l}2.33 \\
2.33 \\
2.33 \\
2.33 \\
2.33 \\
2.33 \\
2.33 \\
2.33 \\
2.32 \\
2.33 \\
2.32\end{array}$ & $\begin{array}{r}9.871073 \\
.870960 \\
.870946 \\
.870732 \\
.870618 \\
.870504 \\
.870390 \\
.870276 \\
.870161 \\
.870047 \\
.869933\end{array}$ & $\begin{array}{l}1.88 \\
1.90 \\
1.90 \\
1.90 \\
1.90 \\
1.90 \\
1.90 \\
1.92 \\
1.90 \\
1.90 \\
1.92\end{array}$ & $\begin{array}{r}9.954437 \\
.954691 \\
.954946 \\
.955200 \\
.055454 \\
.955708 \\
.955961 \\
.956215 \\
.956469 \\
.956723 \\
.956977\end{array}$ & $\begin{array}{l}4.23 \\
4.25 \\
4.23 \\
4.23 \\
4.23 \\
4.22 \\
4.23 \\
4.23 \\
4.23 \\
4.23 \\
4.23\end{array}$ & $\begin{array}{r}\text { 10. } 045563 \\
.045309 \\
.045054 \\
.044500 \\
.044546 \\
.044292 \\
.0440339 \\
.043785 \\
.043531 \\
.043277 \\
.043023\end{array}$ & $\begin{array}{l}60 \\
59 \\
58 \\
57 \\
56 \\
55 \\
54 \\
53 \\
52 \\
51 \\
50\end{array}$ \\
\hline $\begin{array}{l}11 \\
12 \\
13 \\
14 \\
15 \\
16 \\
17 \\
18 \\
19 \\
20\end{array}$ & $\begin{array}{r}9.827049 \\
.827189 \\
.827328 \\
.827467 \\
.827606 \\
.827745 \\
.827884 \\
.825023 \\
.828162 \\
.828301\end{array}$ & $\begin{array}{l}2.33 \\
2.32 \\
2.32 \\
2.32 \\
2.32 \\
2.32 \\
2.32 \\
2.32 \\
2.32 \\
2.30\end{array}$ & $\begin{array}{l}9.869518 \\
.869704 \\
.860559 \\
.869474 \\
.869360 \\
.869245 \\
.869130 \\
.869015 \\
.868900 \\
.868785\end{array}$ & $\begin{array}{l}1.90 \\
1.92 \\
1.92 \\
1.90 \\
1.92 \\
1.92 \\
1.92 \\
1.92 \\
1.92 \\
1.92\end{array}$ & $\begin{array}{r}9.957231 \\
.957485 \\
.957739 \\
.057993 \\
.058247 \\
.958500 \\
.958754 \\
.959008 \\
.959262 \\
.959516\end{array}$ & $\begin{array}{l}4.23 \\
4.23 \\
4.23 \\
4.23 \\
4.23 \\
4.22 \\
4.23 \\
4.23 \\
4.23 \\
4.23 \\
4.22\end{array}$ & $\begin{array}{r}10.042769 \\
.042515 \\
.042261 \\
.042007 \\
.041753 \\
.041500 \\
.041246 \\
.040992 \\
.040738 \\
.040484\end{array}$ & $\begin{array}{l}49 \\
48 \\
47 \\
46 \\
45 \\
44 \\
43 \\
42 \\
41 \\
40\end{array}$ \\
\hline $\begin{array}{l}21 \\
22 \\
23 \\
24 \\
25 \\
26 \\
27 \\
28 \\
29 \\
30\end{array}$ & $\begin{array}{r}9.828439 \\
.828578 \\
.828716 \\
.828555 \\
.828993 \\
.829131 \\
.829269 \\
.829407 \\
.822545 \\
.829683\end{array}$ & $\begin{array}{l}2.32 \\
2.30 \\
2.32 \\
2.30 \\
2.30 \\
2.30 \\
2.30 \\
2.30 \\
2.30 \\
2.30\end{array}$ & $\begin{array}{r}9.868670 \\
.868555 \\
.868440 \\
.868324 \\
.868209 \\
.865093 \\
.867978 \\
.867862 \\
.867747 \\
.867631\end{array}$ & $\begin{array}{l}1.92 \\
1.92 \\
1.93 \\
1.92 \\
1.93 \\
1.92 \\
1.93 \\
1.92 \\
1.93 \\
1.93\end{array}$ & $\begin{array}{r}9.959769 \\
.960023 \\
.960277 \\
.960530 \\
.960784 \\
.961038 \\
.961292 \\
.961545 \\
.961799 \\
.962052\end{array}$ & $\begin{array}{l}4.23 \\
4.23 \\
4.22 \\
4.23 \\
4.23 \\
4.23 \\
4.22 \\
4.23 \\
4.22 \\
4.23\end{array}$ & $\begin{array}{r}10.040231 \\
.039977 \\
.039723 \\
.039470 \\
.039216 \\
.038962 \\
.038708 \\
.035455 \\
.035201 \\
.037948\end{array}$ & $\begin{array}{l}39 \\
38 \\
37 \\
36 \\
35 \\
34 \\
33 \\
32 \\
31 \\
30\end{array}$ \\
\hline $\begin{array}{l}31 \\
32 \\
33 \\
34 \\
35 \\
36 \\
37 \\
38 \\
39 \\
40\end{array}$ & $\begin{array}{r}9.829521 \\
.829059 \\
.830097 \\
.830234 \\
.830372 \\
.830509 \\
.830646 \\
.830784 \\
.830921 \\
.831058\end{array}$ & $\begin{array}{l}2.30 \\
2.30 \\
2.28 \\
2.30 \\
2.28 \\
2.28 \\
2.30 \\
2.28 \\
2.28 \\
2.28\end{array}$ & $\begin{array}{l}9.867515 \\
.867399 \\
.867283 \\
.867167 \\
.867051 \\
.866935 \\
.866819 \\
.866703 \\
.866586 \\
.866470\end{array}$ & $\begin{array}{l}1.93 \\
1.93 \\
1.93 \\
1.93 \\
1.93 \\
1.93 \\
1.93 \\
1.95 \\
1.93 \\
1.95\end{array}$ & $\begin{array}{r}9.962306 \\
.9625 f 0 \\
.962813 \\
.963067 \\
.9133320 \\
.963574 \\
.963828 \\
.964081 \\
.964335 \\
.964558\end{array}$ & $\begin{array}{l}4.23 \\
4.22 \\
4.23 \\
4.22 \\
4.23 \\
4.23 \\
4.22 \\
4.23 \\
4.22 \\
4.23\end{array}$ & $\begin{array}{r}10.037694 \\
.037440 \\
.037157 \\
.036933 \\
.036650 \\
.036426 \\
.036172 \\
.035919 \\
.035665 \\
.035412\end{array}$ & $\begin{array}{l}29 \\
23 \\
27 \\
26 \\
25 \\
24 \\
23 \\
22 \\
21 \\
20\end{array}$ \\
\hline $\begin{array}{l}41 \\
42 \\
43 \\
44 \\
45 \\
46 \\
47 \\
48 \\
49 \\
50\end{array}$ & $\begin{array}{r}9.831195 \\
.831332 \\
.831469 \\
.831606 \\
.831742 \\
.831879 \\
.832015 \\
.832152 \\
.832289 \\
.832125\end{array}$ & $\begin{array}{l}2.28 \\
2.28 \\
2.28 \\
2.27 \\
2.28 \\
2.27 \\
2.28 \\
2.27 \\
2.28 \\
2.27\end{array}$ & $\begin{array}{l}9.866353 \\
.8666237 \\
.866120 \\
.866004 \\
.865587 \\
.865770 \\
.865653 \\
.865536 \\
.8655419 \\
.865302\end{array}$ & $\begin{array}{l}1.93 \\
1.95 \\
1.93 \\
1.95 \\
1.95 \\
1.95 \\
1.95 \\
1.95 \\
1.95 \\
1.95\end{array}$ & $\begin{array}{r}9.964842 \\
.965095 \\
.965349 \\
.9655602 \\
.065855 \\
.966109 \\
.966362 \\
.960616 \\
.9665669 \\
.967123\end{array}$ & $\begin{array}{l}4.22 \\
4.23 \\
4.22 \\
4.22 \\
4.23 \\
4.22 \\
4.23 \\
4.22 \\
4.23 \\
4.22\end{array}$ & $\begin{array}{r}10.035159 \\
.034905 \\
.034651 \\
.034398 \\
.034145 \\
.033591 \\
.033635 \\
.033384 \\
.033131 \\
.032877\end{array}$ & $\begin{array}{l}19 \\
18 \\
17 \\
16 \\
15 \\
14 \\
13 \\
12 \\
11 \\
10\end{array}$ \\
\hline $\begin{array}{l}51 \\
52 \\
53 \\
54 \\
55 \\
56 \\
57 \\
58 \\
59 \\
60\end{array}$ & $\begin{array}{r}9.832561 \\
.832697 \\
.832833 \\
.832969 \\
.833105 \\
.833241 \\
.833377 \\
.833512 \\
.833648 \\
9.833783\end{array}$ & \begin{tabular}{l|}
2.27 \\
2.27 \\
2.27 \\
2.27 \\
2.27 \\
2.27 \\
2.25 \\
2.27 \\
2.25
\end{tabular} & $\begin{array}{r}\text { 9. } 865185 \\
.865068 \\
.864050 \\
.864833 \\
.864716 \\
.864598 \\
.864451 \\
.864363 \\
.864245 \\
9.864127\end{array}$ & $\begin{array}{l}1.95 \\
1.97 \\
1.95 \\
1.95 \\
1.97 \\
1.95 \\
1.97 \\
1.97 \\
1.97\end{array}$ & $\begin{array}{r}9.967376 \\
.9676729 \\
.967853 \\
.968136 \\
.968389 \\
.968643 \\
.968596 \\
.969149 \\
.969403 \\
9.969656\end{array}$ & $\begin{array}{l}4.22 \\
4.23 \\
4.22 \\
4.22 \\
4.23 \\
4.22 \\
4.22 \\
4.23 \\
4.22\end{array}$ & $\begin{array}{r}10.032624 \\
.032371 \\
.032117 \\
.031564 \\
.031611 \\
.031357 \\
.031104 \\
.030851 \\
.030597 \\
10.030344\end{array}$ & $\begin{array}{l}9 \\
8 \\
7 \\
6 \\
5 \\
4 \\
3 \\
2 \\
1 \\
0\end{array}$ \\
\hline ' & Cosine. & D. $1^{\prime \prime}$. & Sine. & D. $1^{\prime \prime}$. & Cotang. & D. $1^{\prime \prime}$. & Tang. & ' \\
\hline
\end{tabular}


TABLE 9.-LOGARITHMIC SINES, COSINES,

\begin{tabular}{|c|c|c|c|c|c|c|c|c|}
\hline , & Sine. & D. $1^{\prime \prime}$. & Cosine. & D. $1^{\prime \prime}$. & Tang. & D. $1^{\prime \prime}$. & Cotang. & , \\
\hline $\begin{array}{r}0 \\
1 \\
2 \\
3 \\
4 \\
4 \\
5 \\
6 \\
7 \\
8 \\
9 \\
10\end{array}$ & $\begin{array}{r}9.833783 \\
.833919 \\
.834054 \\
.834189 \\
.834325 \\
.834460 \\
.834595 \\
.834730 \\
.834865 \\
.834999 \\
.835134\end{array}$ & $\begin{array}{l}2.27 \\
2.25 \\
2.25 \\
2.27 \\
2.25 \\
2.25 \\
2.25 \\
2.25 \\
2.23 \\
2.25 \\
2.25\end{array}$ & $\begin{array}{r}9.864127 \\
.864010 \\
.863892 \\
.863774 \\
.863656 \\
.863538 \\
.863419 \\
.863301 \\
.863183 \\
.863064 \\
.862946\end{array}$ & $\begin{array}{l}1.95 \\
1.97 \\
1.97 \\
1.97 \\
1.97 \\
1.98 \\
1.97 \\
1.97 \\
1.98 \\
1.97 \\
1.98\end{array}$ & $\begin{array}{r}9.969656 \\
.969909 \\
.970162 \\
.970416 \\
.970669 \\
.970922 \\
.971175 \\
.971429 \\
.971682 \\
.971935 \\
.972188\end{array}$ & $\begin{array}{l}4.22 \\
4.22 \\
4.23 \\
4.22 \\
4.22 \\
4.22 \\
4.23 \\
4.22 \\
4.22 \\
4.22 \\
4.22\end{array}$ & $\begin{array}{r}10.030344 \\
.030091 \\
.029838 \\
.029584 \\
.029331 \\
.02907 \mathrm{~S} \\
.028825 \\
.028571 \\
.028318 \\
.028065 \\
.027812\end{array}$ & $\begin{array}{l}60 \\
59 \\
58 \\
57 \\
56 \\
55 \\
54 \\
53 \\
52 \\
51 \\
50\end{array}$ \\
\hline $\begin{array}{l}11 \\
12 \\
13 \\
14 \\
15 \\
16 \\
17 \\
18 \\
19 \\
30\end{array}$ & $\begin{array}{r}9.835269 \\
.835403 \\
.835538 \\
.835672 \\
.835807 \\
.835941 \\
.836075 \\
.836209 \\
.836343 \\
.836477\end{array}$ & $\begin{array}{l}2.23 \\
2.25 \\
2.23 \\
2.25 \\
2.23 \\
2.23 \\
2.23 \\
2.23 \\
2.23 \\
2.23\end{array}$ & $\begin{array}{r}9.862827 \\
.862709 \\
.862590 \\
.862471 \\
.862353 \\
.862234 \\
.862115 \\
.861996 \\
.861877 \\
.861758\end{array}$ & $\begin{array}{l}1.97 \\
1.98 \\
1.98 \\
1.97 \\
1.98 \\
1.98 \\
1.98 \\
1.98 \\
1.98 \\
2.00\end{array}$ & $\begin{array}{r}9.972441 \\
.972695 \\
.972948 \\
.973201 \\
.973454 \\
.973707 \\
.973960 \\
.974213 \\
.974466 \\
.974720\end{array}$ & $\begin{array}{l}4.23 \\
4.22 \\
4.22 \\
4.22 \\
4.22 \\
4.22 \\
4.22 \\
4.22 \\
4.23 \\
4.22\end{array}$ & $\begin{array}{r}10.027559 \\
.027305 \\
.027052 \\
.026799 \\
.026546 \\
.026293 \\
.026040 \\
.025787 \\
.025534 \\
.025280\end{array}$ & $\begin{array}{l}49 \\
48 \\
47 \\
46 \\
45 \\
44 \\
43 \\
42 \\
41 \\
40\end{array}$ \\
\hline $\begin{array}{l}21 \\
22 \\
23 \\
24 \\
25 \\
26 \\
27 \\
28 \\
29 \\
30\end{array}$ & $\begin{array}{r}9.836611 \\
.836745 \\
.836878 \\
.837012 \\
.837146 \\
.837279 \\
.837412 \\
.837546 \\
.837679 \\
.837812\end{array}$ & $\begin{array}{l}2.23 \\
2.22 \\
2.23 \\
2.23 \\
2.22 \\
2.22 \\
2.23 \\
2.22 \\
2.22 \\
2.22\end{array}$ & $\begin{array}{r}9.861638 \\
.861519 \\
.861400 \\
.861280 \\
.861161 \\
.861041 \\
.860922 \\
.860802 \\
.860682 \\
.860562\end{array}$ & $\begin{array}{l}1.98 \\
1.98 \\
2.00 \\
1.98 \\
2.00 \\
1.98 \\
2.00 \\
2.00 \\
2.00 \\
2.00\end{array}$ & $\begin{array}{r}9.974973 \\
.975226 \\
.975479 \\
.975732 \\
.975985 \\
.976238 \\
.976491 \\
.976744 \\
.976997 \\
.977250\end{array}$ & $\begin{array}{l}4.22 \\
4.22 \\
4.22 \\
4.22 \\
4.22 \\
4.22 \\
4.22 \\
4.22 \\
4.22 \\
4.22\end{array}$ & $\begin{array}{r}10.025027 \\
.024774 \\
.024521 \\
.024268 \\
.024015 \\
.023762 \\
.023509 \\
.023256 \\
.023003 \\
.022750\end{array}$ & $\begin{array}{l}39 \\
38 \\
37 \\
36 \\
35 \\
34 \\
33 \\
32 \\
31 \\
30\end{array}$ \\
\hline $\begin{array}{l}31 \\
32 \\
33 \\
34 \\
35 \\
36 \\
37 \\
38 \\
39 \\
40\end{array}$ & $\begin{array}{r}9.837945 \\
.838078 \\
.838211 \\
.838344 \\
.838477 \\
.838610 \\
.838742 \\
.838875 \\
.839007 \\
.839140\end{array}$ & $\begin{array}{l}2.22 \\
2.22 \\
2.22 \\
2.22 \\
2.22 \\
2.20 \\
2.22 \\
2.20 \\
2.22 \\
2.20\end{array}$ & $\begin{array}{r}9.860442 \\
.860322 \\
.860202 \\
.860052 \\
.859962 \\
.859842 \\
.859721 \\
.859601 \\
.859480 \\
.859360\end{array}$ & $\begin{array}{l}2.00 \\
2.00 \\
2.00 \\
2.00 \\
2.00 \\
2.02 \\
2.00 \\
2.02 \\
2.00 \\
2.02\end{array}$ & $\begin{array}{r}9.977503 \\
.977756 \\
.978009 \\
.978262 \\
.978515 \\
.978768 \\
.979021 \\
.979274 \\
.979527 \\
.979780\end{array}$ & $\begin{array}{l}4.22 \\
4.22 \\
4.22 \\
4.22 \\
4.22 \\
4.22 \\
4.22 \\
4.22 \\
4.22 \\
4.22\end{array}$ & $\begin{array}{r}10.029497 \\
.022244 \\
.021991 \\
.021738 \\
.021485 \\
.021232 \\
.020979 \\
.020726 \\
.020473 \\
.020220\end{array}$ & $\begin{array}{l}29 \\
28 \\
27 \\
26 \\
25 \\
24 \\
23 \\
22 \\
21 \\
20\end{array}$ \\
\hline $\begin{array}{l}41 \\
42 \\
43 \\
44 \\
45 \\
46 \\
47 \\
48 \\
49 \\
50\end{array}$ & $\begin{array}{r}9.839272 \\
.839404 \\
.839536 \\
.839668 \\
.839800 \\
.839932 \\
.810064 \\
.810196 \\
.840328 \\
.849459\end{array}$ & $\begin{array}{l}2.20 \\
2.20 \\
2.20 \\
2.20 \\
2.20 \\
2.20 \\
2.20 \\
2.20 \\
2.18 \\
2.20\end{array}$ & $\begin{array}{r}9.859239 \\
.859119 \\
.858998 \\
.858877 \\
.858756 \\
.858635 \\
.858514 \\
.858393 \\
.858272 \\
.858151\end{array}$ & $\begin{array}{l}2.00 \\
2.02 \\
2.02 \\
2.02 \\
2.02 \\
2.02 \\
2.02 \\
2.02 \\
2.02 \\
2.03\end{array}$ & $\begin{array}{r}9.980033 \\
.980286 \\
.980538 \\
.980791 \\
.981044 \\
.981297 \\
.981550 \\
.981803 \\
.982056 \\
.982309\end{array}$ & $\begin{array}{l}4.22 \\
4.20 \\
4.22 \\
4.22 \\
4.22 \\
4.22 \\
4.22 \\
4.22 \\
4.22 \\
4.22\end{array}$ & $\begin{array}{r}10.019967 \\
.019714 \\
.019462 \\
.019209 \\
.018956 \\
.018703 \\
.018450 \\
.018197 \\
.017944 \\
.017691\end{array}$ & $\begin{array}{l}19 \\
15 \\
17 \\
16 \\
15 \\
14 \\
13 \\
12 \\
11 \\
10\end{array}$ \\
\hline $\begin{array}{l}51 \\
52 \\
53 \\
54 \\
55 \\
56 \\
57 \\
58 \\
59 \\
60\end{array}$ & $\begin{array}{r}9.840591 \\
.840722 \\
.840554 \\
.840985 \\
.841116 \\
.841247 \\
.841378 \\
.841509 \\
.841640 \\
9.841771\end{array}$ & $\begin{array}{l}2.18 \\
2.20 \\
2.18 \\
2.18 \\
2.18 \\
2.18 \\
2.18 \\
2.18 \\
2.18\end{array}$ & $\begin{array}{r}9.858029 \\
.857903 \\
.857786 \\
.857665 \\
.857543 \\
.857422 \\
.857300 \\
.857178 \\
.857056 \\
9.856934\end{array}$ & $\begin{array}{l}2.02 \\
2.03 \\
2.02 \\
2.03 \\
2.02 \\
2.03 \\
2.03 \\
2.03 \\
2.03\end{array}$ & $\begin{array}{r}9.982502 \\
.982814 \\
.983067 \\
.983320 \\
.983573 \\
.983826 \\
.984079 \\
.984332 \\
.984584 \\
9.984 S 37\end{array}$ & $\begin{array}{l}4.20 \\
4.22 \\
4.22 \\
4.22 \\
4.22 \\
4.22 \\
4.22 \\
4.20 \\
4.22\end{array}$ & $\begin{array}{r}10.017438 \\
.017186 \\
.016933 \\
.016680 \\
.016427 \\
.016174 \\
.015921 \\
.015668 \\
.015416 \\
10.015163\end{array}$ & $\begin{array}{l}9 \\
8 \\
7 \\
6 \\
5 \\
4 \\
3 \\
2 \\
1 \\
0\end{array}$ \\
\hline , & Cosine. & D. $1^{\prime \prime}$ & Sine. & D. $1^{\prime \prime}$. & Cotang. & D. $1^{\prime \prime}$. & Tang. & \\
\hline
\end{tabular}




\begin{tabular}{|c|c|c|c|c|c|c|c|c|}
\hline ' & Sine. & D. $1^{\prime \prime}$. & Cosine. & D. $1^{\prime \prime}$. & Tang. & D. $1^{\prime \prime}$. & Cotang. & , \\
\hline $\begin{array}{r}0 \\
1 \\
2 \\
3 \\
4 \\
5 \\
6 \\
7 \\
8 \\
9 \\
10\end{array}$ & $\begin{array}{r}9.841771 \\
.841902 \\
.842033 \\
.842163 \\
.842294 \\
.842424 \\
.842555 \\
.842655 \\
.842815 \\
.842946 \\
.843076\end{array}$ & $\begin{array}{l}2.18 \\
2.18 \\
2.17 \\
2.18 \\
2.17 \\
2.18 \\
2.17 \\
2.17 \\
2.18 \\
2.17 \\
2.17\end{array}$ & $\begin{array}{r}9.856934 \\
.856812 \\
.856690 \\
.856568 \\
.856446 \\
.856323 \\
.856201 \\
.856078 \\
.855956 \\
.855833 \\
.855711\end{array}$ & $\begin{array}{l}2.03 \\
2.03 \\
2.03 \\
2.03 \\
2.05 \\
2.03 \\
2.05 \\
2.03 \\
2.05 \\
2.03 \\
2.05\end{array}$ & $\begin{array}{r}9.984837 \\
.985090 \\
.985343 \\
.985596 \\
.955848 \\
.986101 \\
.986354 \\
.986607 \\
.986860 \\
.987112 \\
.987365\end{array}$ & $\begin{array}{l}4.22 \\
4.22 \\
4.22 \\
4.20 \\
4.22 \\
4.22 \\
4.22 \\
4.22 \\
4.20 \\
4.22 \\
4.22\end{array}$ & $\begin{array}{r}10.015163 \\
.014910 \\
.014657 \\
.014404 \\
.014152 \\
.013899 \\
.013646 \\
.013393 \\
.013140 \\
.012858 \\
.012635\end{array}$ & $\begin{array}{l}60 \\
59 \\
58 \\
57 \\
56 \\
55 \\
54 \\
53 \\
52 \\
51 \\
50\end{array}$ \\
\hline $\begin{array}{l}11 \\
12 \\
13 \\
14 \\
15 \\
16 \\
17 \\
18 \\
19 \\
20\end{array}$ & $\begin{array}{r}9.843206 \\
.843336 \\
.843466 \\
.843595 \\
.843725 \\
.843855 \\
.843984 \\
.844114 \\
.844243 \\
.844372\end{array}$ & $\begin{array}{l}2.17 \\
2.17 \\
2.15 \\
2.17 \\
2.17 \\
2.15 \\
2.17 \\
2.15 \\
2.15 \\
2.17\end{array}$ & $\begin{array}{r}9.855588 \\
.855465 \\
.855342 \\
.855219 \\
.855096 \\
.854973 \\
.854550 \\
.854727 \\
.854603 \\
.854480\end{array}$ & $\begin{array}{l}2.05 \\
2.05 \\
2.05 \\
2.05 \\
2.05 \\
2.05 \\
2.05 \\
2.07 \\
2.05 \\
2.07\end{array}$ & $\begin{array}{r}9.987618 \\
.987871 \\
.988123 \\
.988376 \\
.988629 \\
.958882 \\
.989134 \\
.989387 \\
.989640 \\
.989893\end{array}$ & $\begin{array}{l}4.22 \\
4.20 \\
4.22 \\
4.22 \\
4.22 \\
4.20 \\
4.22 \\
4.22 \\
4.22 \\
4.20\end{array}$ & $\begin{array}{r}10.012382 \\
.012129 \\
.011877 \\
.011624 \\
.011371 \\
.011118 \\
.010866 \\
.010613 \\
.010360 \\
.010107\end{array}$ & $\begin{array}{l}49 \\
48 \\
47 \\
46 \\
45 \\
44 \\
43 \\
42 \\
41 \\
40\end{array}$ \\
\hline $\begin{array}{l}21 \\
22 \\
23 \\
24 \\
25 \\
26 \\
27 \\
28 \\
29 \\
30\end{array}$ & $\begin{array}{r}9.844502 \\
.844031 \\
.844760 \\
.844889 \\
.845018 \\
.845127 \\
.845276 \\
.845405 \\
.845533 \\
.845662\end{array}$ & $\begin{array}{l}2.15 \\
2.15 \\
2.15 \\
2.15 \\
2.15 \\
2.15 \\
2.15 \\
2.13 \\
2.15 \\
2.13\end{array}$ & $\begin{array}{r}9.854356 \\
.854233 \\
.854109 \\
.853986 \\
.853862 \\
.853738 \\
.853614 \\
.853490 \\
.853366 \\
.853242\end{array}$ & $\begin{array}{l}2.05 \\
2.07 \\
2.05 \\
2.07 \\
2.07 \\
2.07 \\
2.07 \\
2.07 \\
2.07 \\
2.07\end{array}$ & $\begin{array}{r}9.990145 \\
.990398 \\
.990651 \\
.990903 \\
.991156 \\
.991409 \\
.991662 \\
.991914 \\
.992167 \\
.992420\end{array}$ & $\begin{array}{l}4.22 \\
4.22 \\
4.20 \\
4.22 \\
4.22 \\
4.22 \\
4.20 \\
4.22 \\
4.22 \\
4.20\end{array}$ & $\begin{array}{r}10.009855 \\
.009602 \\
.009349 \\
.009097 \\
.008944 \\
.008591 \\
.008338 \\
.008086 \\
.007833 \\
.007580\end{array}$ & $\begin{array}{l}39 \\
38 \\
37 \\
36 \\
35 \\
34 \\
33 \\
32 \\
31 \\
30\end{array}$ \\
\hline $\begin{array}{l}31 \\
32 \\
33 \\
34 \\
35 \\
36 \\
37 \\
38 \\
39 \\
40\end{array}$ & $\begin{array}{r}9.845790 \\
.845919 \\
.846047 \\
.846175 \\
.846304 \\
.846432 \\
.846560 \\
.846688 \\
.846816 \\
.846944\end{array}$ & $\begin{array}{l}2.15 \\
2.13 \\
2.13 \\
2.15 \\
2.13 \\
2.13 \\
2.13 \\
2.13 \\
2.13 \\
2.12\end{array}$ & $\begin{array}{r}9.853118 \\
.852994 \\
.852869 \\
.852745 \\
.852620 \\
.852496 \\
.852371 \\
.852247 \\
.852122 \\
.851997\end{array}$ & $\begin{array}{l}2.07 \\
2.08 \\
2.07 \\
2.08 \\
2.07 \\
2.08 \\
2.07 \\
2.08 \\
2.08 \\
2.08\end{array}$ & $\begin{array}{r}9.992672 \\
.992925 \\
.993178 \\
.993431 \\
.993683 \\
.993936 \\
.994189 \\
.994441 \\
.994694 \\
.994947\end{array}$ & $\begin{array}{l}4.22 \\
4.22 \\
4.22 \\
4.20 \\
4.22 \\
4.22 \\
4.20 \\
4.22 \\
4.22 \\
4.20\end{array}$ & $\begin{array}{r}10.007328 \\
.007075 \\
.006522 \\
.006569 \\
.006317 \\
.006064 \\
.005811 \\
.005559 \\
.005306 \\
.005053\end{array}$ & $\begin{array}{l}29 \\
23 \\
27 \\
26 \\
25 \\
24 \\
23 \\
22 \\
21 \\
20\end{array}$ \\
\hline $\begin{array}{l}41 \\
42 \\
43 \\
44 \\
45 \\
46 \\
47 \\
48 \\
49 \\
50\end{array}$ & $\begin{array}{r}9.847071 \\
.847199 \\
.847327 \\
.847454 \\
.8475 \$ 2 \\
.847709 \\
.847 \times 36 \\
.847964 \\
.848091 \\
.848218\end{array}$ & $\begin{array}{l}2.13 \\
2.13 \\
2.12 \\
2.13 \\
2.12 \\
2.12 \\
2.13 \\
2.12 \\
2.12 \\
2.12\end{array}$ & $\begin{array}{r}9.851872 \\
.851747 \\
.851622 \\
.851497 \\
.851372 \\
.851246 \\
.851121 \\
.850996 \\
.850870 \\
.850745\end{array}$ & $\begin{array}{l}2.08 \\
2.08 \\
2.08 \\
2.08 \\
2.10 \\
2.08 \\
2.08 \\
2.10 \\
2.08 \\
2.10\end{array}$ & $\begin{array}{r}9.995199 \\
.995452 \\
.995705 \\
.995957 \\
.996210 \\
.996463 \\
.996715 \\
.996968 \\
.997221 \\
.997473\end{array}$ & $\begin{array}{l}4.22 \\
4.22 \\
4.20 \\
4.22 \\
4.22 \\
4.20 \\
4.22 \\
4.22 \\
4.20 \\
4.22\end{array}$ & $\begin{array}{r}10.004801 \\
.004548 \\
.004295 \\
.004043 \\
.003790 \\
.003537 \\
.003285 \\
.003032 \\
.002779 \\
.002527\end{array}$ & $\begin{array}{l}19 \\
18 \\
17 \\
16 \\
15 \\
14 \\
13 \\
12 \\
11 \\
10\end{array}$ \\
\hline $\begin{array}{l}51 \\
52 \\
53 \\
54 \\
55 \\
56 \\
57 \\
58 \\
59 \\
60\end{array}$ & $\begin{array}{r}9.848345 \\
.848472 \\
.84 \$ 599 \\
.848726 \\
.848852 \\
.848979 \\
.849106 \\
.849232 \\
.849359 \\
9.849485\end{array}$ & $\begin{array}{l}2.12 \\
2.12 \\
2.12 \\
2.10 \\
2.12 \\
2.12 \\
2.10 \\
2.12 \\
2.10\end{array}$ & $\begin{array}{r}9.850619 \\
.850493 \\
.850368 \\
.850242 \\
.850116 \\
.849990 \\
.819564 \\
.819738 \\
.849611 \\
9.819485\end{array}$ & $\begin{array}{l}2.10 \\
2.08 \\
2.10 \\
2.10 \\
2.10 \\
2.10 \\
2.10 \\
2.12 \\
2.10\end{array}$ & $\begin{array}{r}9.997726 \\
.997979 \\
.998231 \\
.998484 \\
.998737 \\
.998959 \\
.999242 \\
.990495 \\
.999747 \\
10.000000\end{array}$ & $\begin{array}{l}4.22 \\
4.20 \\
4.22 \\
4.22 \\
4.20 \\
4.22 \\
4.22 \\
4.30 \\
4.22\end{array}$ & $\begin{array}{r}10.002274 \\
.002021 \\
.001769 \\
.001516 \\
.001263 \\
.001011 \\
.000755 \\
.000505 \\
.000253 \\
10.000000\end{array}$ & $\begin{array}{l}9 \\
8 \\
7 \\
6 \\
5 \\
4 \\
3 \\
2 \\
1 \\
0\end{array}$ \\
\hline , & Cosine. & 10.1 & sine. & D. $1^{\prime \prime}$. & Cotang. & D. $1^{\prime \prime}$ & I ang. & , \\
\hline
\end{tabular}


No. 100 L. 000.]

[No. 109 L. 040.

\begin{tabular}{|c|c|c|c|c|c|c|c|c|c|c|c|}
\hline N. & 0 & 1 & 2 & 8 & 4 & 5 & 6 & 7 & 8 & 9 & Diff. \\
\hline \multirow{2}{*}{$\begin{array}{r}100 \\
1 \\
2\end{array}$} & \multirow{2}{*}{$\begin{array}{r}000000 \\
4321 \\
8600\end{array}$} & \multirow{2}{*}{$\begin{array}{l}0434 \\
4751 \\
9026\end{array}$} & \multirow{2}{*}{$\begin{array}{l}0868 \\
5181 \\
9451\end{array}$} & \multirow{2}{*}{$\begin{array}{l}1301 \\
5609 \\
9876\end{array}$} & $\begin{array}{l}1734 \\
6038\end{array}$ & & $\begin{array}{l}2598 \\
6894\end{array}$ & $\begin{array}{l}3029 \\
7321\end{array}$ & $\begin{array}{l}3461 \\
7748\end{array}$ & & \multirow{2}{*}{$\begin{array}{l}432 \\
428\end{array}$} \\
\hline & & & & & \multirow{3}{*}{$\begin{array}{l}0300 \\
4521 \\
8700\end{array}$} & & & & & & \\
\hline 3 & 012837 & & & & & \multirow{2}{*}{$\begin{array}{l}0724 \\
4940 \\
9116\end{array}$} & \multirow{2}{*}{$\begin{array}{l}1147 \\
5360 \\
9532\end{array}$} & \multirow{3}{*}{$\begin{array}{l}1570 \\
5779 \\
9947\end{array}$} & \multirow{2}{*}{$\begin{array}{l}1993 \\
6197\end{array}$} & & \multirow{2}{*}{$\begin{array}{l}424 \\
420\end{array}$} \\
\hline 4 & 7033 & 7451 & 7868 & $\begin{array}{l}4100 \\
8284\end{array}$ & & & & & & & \\
\hline & \multirow{3}{*}{$\begin{array}{r}021189 \\
5306 \\
9384\end{array}$} & \multirow{4}{*}{$\begin{array}{l}1603 \\
5715 \\
9789\end{array}$} & & & & & & & \multirow{3}{*}{$\begin{array}{l}0361 \\
4486 \\
8571\end{array}$} & 0775 & \multirow{3}{*}{$\begin{array}{l}416 \\
412 \\
408\end{array}$} \\
\hline 6 & & & 2010 & 272 & 284 & 32 & 36 & 4075 & & & \\
\hline & & & & & & & & & & & \\
\hline \multirow{3}{*}{$\begin{array}{l}8 \\
9\end{array}$} & \multirow{3}{*}{$\begin{array}{l}033124 \\
7426 \\
04\end{array}$} & & \multirow{3}{*}{$\begin{array}{l}0195 \\
4227 \\
8223\end{array}$} & \multirow{3}{*}{$\begin{array}{l}0600 \\
4628 \\
8620\end{array}$} & \multirow{3}{*}{$\begin{array}{l}1004 \\
5029 \\
9017\end{array}$} & \multirow{3}{*}{$\begin{array}{l}1408 \\
5430 \\
9414\end{array}$} & \multirow{3}{*}{$\begin{array}{l}1812 \\
5830 \\
9811\end{array}$} & 2216 & \multirow{2}{*}{$\begin{array}{l}2619 \\
6629\end{array}$} & 30 & \multirow{2}{*}{$\begin{array}{l}404 \\
400\end{array}$} \\
\hline & & \multirow{2}{*}{$\begin{array}{l}3826 \\
7825\end{array}$} & & & & & & 6230 & & 70 & \\
\hline & & & & & & & & 0207 & 0602 & 0998 & 397 \\
\hline
\end{tabular}

Proportional Parts.

\begin{tabular}{|c|c|c|c|c|c|c|c|c|c|}
\hline Dift. & 1 & 2 & 3 & 4 & 5 & 6 & 7 & 8 & 9 \\
\hline $\begin{array}{l}434 \\
433 \\
432 \\
431 \\
430 \\
429 \\
428 \\
427 \\
426 \\
425\end{array}$ & $\begin{array}{l}43.4 \\
43.3 \\
43.2 \\
43.1 \\
43.0 \\
42.9 \\
42.8 \\
42.7 \\
42.6 \\
42.5\end{array}$ & $\begin{array}{l}86.8 \\
86.6 \\
86.4 \\
86.2 \\
86.0 \\
85.8 \\
85.6 \\
85.4 \\
85.2 \\
85.0\end{array}$ & $\begin{array}{l}130.2 \\
129.9 \\
129.6 \\
129.3 \\
129.0 \\
128.7 \\
128.4 \\
128.1 \\
127.8 \\
127.5\end{array}$ & $\begin{array}{l}173.6 \\
173.2 \\
172.8 \\
172.4 \\
172.0 \\
171.6 \\
171.2 \\
170.8 \\
170.4 \\
170.0\end{array}$ & $\begin{array}{l}217.0 \\
216.5 \\
216.0 \\
215.5 \\
215.0 \\
214.5 \\
214.0 \\
213.5 \\
213.0 \\
212.5\end{array}$ & $\begin{array}{l}260.4 \\
259.8 \\
259.2 \\
258.6 \\
258.0 \\
257.4 \\
256.8 \\
256.2 \\
255.6 \\
255.0\end{array}$ & $\begin{array}{l}303.8 \\
303.1 \\
302.4 \\
301.7 \\
301.0 \\
300.3 \\
299.6 \\
298.9 \\
298.2 \\
297.5\end{array}$ & $\begin{array}{l}347.2 \\
346.4 \\
345.6 \\
344.8 \\
344.0 \\
343.2 \\
342.4 \\
341.6 \\
340.8 \\
340.0\end{array}$ & $\begin{array}{l}390.6 \\
389.7 \\
388.8 \\
387.9 \\
387.0 \\
386.1 \\
385.2 \\
384.3 \\
383.4 \\
382.5\end{array}$ \\
\hline $\begin{array}{l}424 \\
423 \\
422 \\
421 \\
420 \\
419 \\
418 \\
417 \\
416 \\
415\end{array}$ & $\begin{array}{l}42.4 \\
42.3 \\
42.2 \\
42.1 \\
42.0 \\
41.9 \\
41.8 \\
41.7 \\
41.6 \\
41.5\end{array}$ & $\begin{array}{l}84.8 \\
84.6 \\
84.4 \\
84.2 \\
84.0 \\
83.8 \\
83.6 \\
83.4 \\
83.2 \\
83.0\end{array}$ & $\begin{array}{l}127.2 \\
126.9 \\
126.6 \\
126.3 \\
126.0 \\
125.7 \\
125.4 \\
125.1 \\
124.8 \\
124.5\end{array}$ & $\begin{array}{l}169.6 \\
169.2 \\
168.8 \\
168.4 \\
168.0 \\
167.6 \\
167.2 \\
166.8 \\
166.4 \\
166.0\end{array}$ & $\begin{array}{l}212.0 \\
211.5 \\
211.0 \\
210.5 \\
210.0 \\
209.5 \\
209.0 \\
208.5 \\
208.0 \\
207.5\end{array}$ & $\begin{array}{l}254.4 \\
253.8 \\
253.2 \\
252.6 \\
252.0 \\
251.4 \\
250.8 \\
250.2 \\
249.6 \\
249.0\end{array}$ & $\begin{array}{l}296.8 \\
296.1 \\
295.4 \\
294.7 \\
294.0 \\
293.3 \\
292.6 \\
291.9 \\
291.2 \\
290.5\end{array}$ & $\begin{array}{l}339.2 \\
338.4 \\
337.6 \\
336.8 \\
336.0 \\
335.2 \\
334.4 \\
333.6 \\
332.8 \\
332.0\end{array}$ & $\begin{array}{l}381.6 \\
380.7 \\
379.8 \\
378.9 \\
378.0 \\
377.1 \\
376.2 \\
375.3 \\
374.4 \\
373.5\end{array}$ \\
\hline $\begin{array}{l}414 \\
413 \\
412 \\
411 \\
410 \\
409 \\
408 \\
407 \\
406 \\
405\end{array}$ & $\begin{array}{l}41.4 \\
41.3 \\
41.2 \\
41.1 \\
41.0 \\
40.9 \\
40.8 \\
40.7 \\
40.6 \\
40.5\end{array}$ & $\begin{array}{l}82.8 \\
82.6 \\
82.4 \\
82.2 \\
82.0 \\
81.8 \\
81.6 \\
81.4 \\
81.2 \\
81.0\end{array}$ & $\begin{array}{l}124.2 \\
123.9 \\
123.6 \\
123.3 \\
123.0 \\
122.7 \\
122.4 \\
122.1 \\
121.8 \\
121.5\end{array}$ & $\begin{array}{l}165.6 \\
165.2 \\
164.8 \\
164.4 \\
164.0 \\
163.6 \\
163.2 \\
162.8 \\
162.4 \\
162.0\end{array}$ & $\begin{array}{l}207.0 \\
206.5 \\
206.0 \\
205.5 \\
205.0 \\
204.5 \\
204.0 \\
203.5 \\
203.0 \\
202.5\end{array}$ & $\begin{array}{l}248.4 \\
247.8 \\
247.2 \\
246.6 \\
246.0 \\
245.4 \\
244.8 \\
244.2 \\
243.6 \\
243.0\end{array}$ & $\begin{array}{l}289.8 \\
289.1 \\
288.4 \\
287.7 \\
287.0 \\
286.3 \\
285.6 \\
284.9 \\
284.2 \\
283.5\end{array}$ & $\begin{array}{l}331.2 \\
330.4 \\
329.6 \\
328.8 \\
328.0 \\
327.2 \\
326.4 \\
325.6 \\
324.8 \\
324.0\end{array}$ & $\begin{array}{l}372.6 \\
371.7 \\
370.8 \\
369.9 \\
369.0 \\
368.1 \\
367.2 \\
366.3 \\
365.4 \\
364.5\end{array}$ \\
\hline $\begin{array}{l}404 \\
403 \\
402 \\
401 \\
400 \\
399 \\
398 \\
397 \\
396 \\
395\end{array}$ & $\begin{array}{l}40.4 \\
40.3 \\
40.2 \\
40.1 \\
40.0 \\
39.9 \\
39.8 \\
39.7 \\
39.6 \\
39.5\end{array}$ & $\begin{array}{l}80.8 \\
80.6 \\
80.4 \\
80.2 \\
80.0 \\
79.8 \\
79.6 \\
79.4 \\
79.2 \\
79.0\end{array}$ & $\begin{array}{l}121.2 \\
120.9 \\
120.6 \\
120.3 \\
120.0 \\
119.7 \\
119.4 \\
119.1 \\
118.8 \\
118.5\end{array}$ & $\begin{array}{l}161.6 \\
161.2 \\
160.8 \\
160.4 \\
160.0 \\
159.6 \\
159.2 \\
158.8 \\
158.4 \\
158.0\end{array}$ & $\begin{array}{l}202.0 \\
201.5 \\
201.0 \\
200.5 \\
200.0 \\
199.5 \\
199.0 \\
198.5 \\
198.0 \\
197.5\end{array}$ & $\begin{array}{l}242.4 \\
241.8 \\
241.2 \\
240.6 \\
240.0 \\
239.4 \\
238.8 \\
238.2 \\
237.6 \\
237.0\end{array}$ & $\begin{array}{l}282.8 \\
282.1 \\
281.4 \\
280.7 \\
280.0 \\
279.3 \\
278.6 \\
277.9 \\
277.2 \\
276.5\end{array}$ & $\begin{array}{l}323.2 \\
322.4 \\
321.6 \\
320.8 \\
320.0 \\
319.2 \\
318.4 \\
317.6 \\
316.8 \\
316.0\end{array}$ & $\begin{array}{l}363.6 \\
362.7 \\
361.8 \\
360.9 \\
360.0 \\
359.1 \\
358.2 \\
357.3 \\
356.4 \\
355.5\end{array}$ \\
\hline
\end{tabular}


TABLE 10.-LOGARITHMS OF NUMBERS.

No. 110 L. 041.]

[No. 119 L. 078.

\begin{tabular}{|c|c|c|c|c|c|c|c|c|c|c|c|}
\hline N. & 0 & 1 & 2 & 3 & 4 & 5 & 6 & 7 & 8 & 9 & Difl. \\
\hline \multirow{3}{*}{$\begin{array}{r}110 \\
1 \\
2\end{array}$} & \multirow{3}{*}{$\begin{array}{r}041393 \\
5323 \\
9218\end{array}$} & \multirow{3}{*}{$\begin{array}{l}1757 \\
5714 \\
9606\end{array}$} & \multirow{3}{*}{$\begin{array}{l}2182 \\
6105 \\
9993\end{array}$} & \multirow{2}{*}{$\begin{array}{l}2576 \\
6495\end{array}$} & \multirow{2}{*}{$\begin{array}{l}2969 \\
6855\end{array}$} & \multirow{2}{*}{$\begin{array}{l}3362 \\
7275\end{array}$} & \multirow{2}{*}{$\begin{array}{l}3755 \\
7664\end{array}$} & \multirow{2}{*}{$\begin{array}{l}4148 \\
8053\end{array}$} & \multirow{2}{*}{$\begin{array}{l}4540 \\
8442\end{array}$} & \multirow{2}{*}{$\begin{array}{l}4932 \\
8530\end{array}$} & \multirow{2}{*}{$\begin{array}{l}393 \\
390\end{array}$} \\
\hline & & & & & & & & & & & \\
\hline & & & & \multirow{3}{*}{$\begin{array}{l}0350 \\
4230 \\
80+6\end{array}$} & \multirow{3}{*}{$\begin{array}{l}0766 \\
4613 \\
8426\end{array}$} & \multirow{3}{*}{$\begin{array}{l}1153 \\
4996 \\
8505 \\
\end{array}$} & \multirow{3}{*}{$\begin{array}{l}1538 \\
5378 \\
9185\end{array}$} & \multirow{3}{*}{$\begin{array}{l}1924 \\
5760 \\
9563\end{array}$} & \multirow{3}{*}{$\begin{array}{l}2309 \\
6142 \\
9942 \\
\end{array}$} & \multirow{2}{*}{$\begin{array}{l}2694 \\
6524\end{array}$} & \multirow{2}{*}{$\begin{array}{l}386 \\
383\end{array}$} \\
\hline 3 & $\begin{array}{r}053078 \\
6905\end{array}$ & 340 & 3846 & & & & & & & & \\
\hline & & 10 & 00 & & & & & & & \multirow{3}{*}{$\begin{array}{l}0320 \\
4083 \\
7815\end{array}$} & \multirow{3}{*}{$\begin{array}{l}379 \\
376 \\
373\end{array}$} \\
\hline \multirow{3}{*}{$\begin{array}{l}5 \\
6 \\
7\end{array}$} & 060693 & 1075 & 1452 & 1829 & 2206 & \multirow{2}{*}{$\begin{array}{l}2552 \\
6326\end{array}$} & 2958 & 3333 & 3709 & & \\
\hline & 4458 & 4832 & 5206 & 5580 & 595 & & 6699 & 7071 & 7443 & & \\
\hline & & & & & & \multirow{3}{*}{$\begin{array}{l}0038 \\
3718 \\
\end{array}$} & 0407 & 0776 & 1145 & \multirow{2}{*}{$\begin{array}{l}1514 \\
5182\end{array}$} & \multirow{2}{*}{$\begin{array}{l}370 \\
366\end{array}$} \\
\hline 8 & 071882 & 225 & 2617 & 298 & 335 & & 4085 & 44 & & & \\
\hline 9 & 5547 & 5912 & 6276 & 6040 & 7004 & & 7731 & 8094 & 8157 & $8 \$ 19$ & 363 \\
\hline
\end{tabular}

Proportional Parts.

\begin{tabular}{|c|c|c|c|c|c|c|c|c|c|}
\hline Diff. & 1 & 2 & 3 & 4 & 5 & 6 & 7 & 8 & 9 \\
\hline 395 & 39.5 & 79.0 & 118.5 & 158.0 & 197.5 & 237.0 & 276.5 & 316.0 & 355.5 \\
\hline 394 & 39.4 & 78.8 & 118.2 & 157.6 & 197.0 & 236.4 & 275.8 & 315.2 & 354.6 \\
\hline 393 & 39.3 & $7 \varepsilon .6$ & 117.9 & 157.2 & 196.5 & 235.8 & 275.1 & 314.4 & 353.7 \\
\hline 392 & 39.2 & 78.4 & 117.6 & 156.8 & 196.0 & 235.2 & 274.4 & 313.6 & 352.8 \\
\hline 391 & 39.1 & 78.2 & 117.3 & 156.4 & 195.5 & 234.6 & 273.7 & 312.8 & 351.9 \\
\hline 390 & 39.0 & 78.0 & 117.0 & 156.0 & 195.0 & 234.0 & 273.0 & 312.0 & 351.0 \\
\hline 359 & 35.9 & 77.8 & 116.7 & 155.6 & 194.5 & 233.4 & 272.3 & 311.2 & 350.1 \\
\hline 389 & 38.8 & 77.6 & 116.4 & 15.5. 2 & 194.0 & 232.8 & 271.6 & 310.4 & 349.2 \\
\hline 387 & 38.7 & 77.4 & 116.1 & 154.8 & 193.5 & 232.2 & 270.9 & 309.6 & 348.3 \\
\hline 356 & 38.6 & 77.2 & 115.8 & 154.4 & 193.0 & 231.6 & 270.2 & 308.8 & 347.4 \\
\hline 385 & 38.5 & 77.0 & 115.5 & 154.0 & 192.5 & 231.0 & 209.5 & 308.0 & 346.5 \\
\hline 354 & 38.4 & 76.8 & 115.2 & 153.6 & 192.0 & 230.4 & 268.8 & 307.2 & 345.6 \\
\hline 383 & 38.3 & 76.6 & 114.9 & 153.2 & 191.5 & 229.8 & 268.1 & 306.4 & 344.7 \\
\hline 382 & 38.2 & 76.4 & 114.6 & 152.8 & 191.0 & 229.2 & 267.4 & 305.6 & 343.8 \\
\hline 381 & 39.1 & 76.2 & 114.3 & 152.4 & 190.5 & 228.6 & 266.7 & 304.8 & 342.9 \\
\hline 380 & 38.0 & 76.0 & 114.0 & 152.0 & 190.0 & 228.0 & 266.0 & 304.0 & 342.0 \\
\hline 379 & 37.9 & 75.8 & 113.7 & 151.6 & 189.5 & 227.4 & 265.3 & 303.2 & 341.1 \\
\hline 378 & 37.8 & 75.6 & 113.4 & 151.2 & 189.0 & 226.8 & 264.6 & 302.4 & 340.2 \\
\hline 377 & 37.7 & 75.4 & 113.1 & 150.8 & 188.5 & 226.2 & 263.9 & 301.6 & 339.3 \\
\hline 376 & 37.6 & 75.2 & 112.8 & 150.4 & 188.0 & 225.6 & 263.2 & 300.8 & 32.4 \\
\hline 375 & 37.5 & 75.0 & 112.5 & 150.0 & 187.5 & 225.0 & 262.5 & 300.0 & 337.5 \\
\hline 374 & 37.4 & 74.8 & 112.2 & 149.6 & 157.0 & 224.4 & 261.8 & 299.2 & 336.6 \\
\hline 373 & 37.3 & 74.6 & 111.9 & 149.2 & 186.5 & 223.8 & 261.1 & 298.4 & 335.7 \\
\hline 372 & 37.2 & 74.4 & 111.6 & 148.8 & 186.0 & 223.2 & 260.4 & 297.6 & 334.8 \\
\hline 371 & 37.1 & 74.2 & 111.3 & 148.4 & 185.5 & 222.6 & 259.7 & 296.8 & 333.9 \\
\hline 370 & 37.0 & 74.0 & 111.0 & 148.0 & 185.0 & 222.0 & 259.0 & 296.0 & 333.0 \\
\hline 369 & 36.9 & 73.8 & 110.7 & 147.6 & 184.5 & 221.4 & 258.3 & 295.2 & 332.1 \\
\hline $3 \mathrm{cs}$ & 36.8 & 73.6 & 110.4 & 147.2 & 184.0 & 220.8 & 257.6 & 294.4 & 331.2 \\
\hline 367 & 36.7 & 73.4 & 110.1 & 146.8 & 183.5 & 220.2 & 256,9 & 293.6 & 330.3 \\
\hline 366 & 36.6 & 73.2 & 109.8 & 146.4 & 183.0 & 219.6 & 256.2 & 292.8 & 329.4 \\
\hline 365 & 36.5 & 73.0 & 109.5 & 140.0 & 182.5 & 219.0 & 255.7 & 292.0 & 328.5 \\
\hline 364 & 36.4 & 72.8 & 109.2 & 145.6 & 182.0 & 218.4 & 254.8 & 291.2 & 327.6 \\
\hline 363 & 36.3 & 72.6 & 108.9 & 145.2 & 181.5 & 217.8 & 254.1 & 290.4 & 326.7 \\
\hline 362 & 36.2 & 72.4 & 108.6 & 144.8 & 181.0 & 217.2 & 253.4 & 289.6 & 325.8 \\
\hline 361 & 36.1 & 72.2 & 108.3 & 144. 4 & 180.5 & 216.6 & 252.7 & 254.8 & 324.9 \\
\hline 360 & 36.0 & 72.0 & 108.0 & 144.0 & 180.0 & 216.0 & 252.0 & 285.0 & 324.0 \\
\hline 359 & 35.9 & 71.8 & 107.7 & 143.6 & 179.5 & 215.4 & 251.3 & 37.2 & 323.1 \\
\hline 358 & 35.8 & 71.6 & 107.4 & 143.2 & 179.0 & 214.8 & 250.6 & 256.4 & 322.2 \\
\hline 357 & 35.7 & 71.4 & 107.1 & 142.8 & 178.5 & 214.2 & 219.9 & $2 \times 5.6$ & 321.3 \\
\hline 356 & 35.6 & 71.2 & 106.8 & 142.4 & 178.0 & 213.6 & 249.2 & 254.8 & 320.4 \\
\hline
\end{tabular}


TABLE 10.-LOGARITIMS OF NUMBERS.

\begin{tabular}{|c|c|c|c|c|c|c|c|c|c|c|c|}
\hline \multicolumn{9}{|c|}{ No. 120 L. 079.] } & \multicolumn{3}{|c|}{ [No. 134 L. 130.} \\
\hline N. & 0 & 1 & 2 & 3 & 4 & 5 & 6 & 7 & 8 & 9 & Diff. \\
\hline \multirow[t]{2}{*}{120} & \multirow[t]{2}{*}{079181} & \multirow[t]{2}{*}{9543} & \multirow[t]{2}{*}{9904} & & & & & & & & \multirow{4}{*}{$\begin{array}{l}360 \\
357 \\
355\end{array}$} \\
\hline & & & & \multirow{2}{*}{$\begin{array}{l}0266 \\
3861\end{array}$} & \multirow{2}{*}{$\begin{array}{l}0626 \\
4219\end{array}$} & \multirow{2}{*}{$\begin{array}{l}0387 \\
4576 \\
8136\end{array}$} & \multirow{3}{*}{$\begin{array}{l}1347 \\
4934 \\
8490\end{array}$} & \multirow{3}{*}{$\begin{array}{l}1707 \\
5291 \\
8845\end{array}$} & \multirow{3}{*}{$\begin{array}{l}2067 \\
5647 \\
9198 \\
\end{array}$} & \multirow{3}{*}{$\begin{array}{l}2426 \\
6004 \\
9552\end{array}$} & \\
\hline \multirow{2}{*}{$\begin{array}{l}1 \\
2 \\
3\end{array}$} & \multirow{3}{*}{$\begin{array}{r}082785 \\
6360 \\
9905\end{array}$} & $\begin{array}{l}3144 \\
6716\end{array}$ & 3503 & & & & & & & & \\
\hline & & & & & & & & & & & \\
\hline & & 0258 & 0611 & 0963 & 1315 & 1667 & 2018 & 2370 & 2721 & 3071 & 352 \\
\hline 4 & $\begin{array}{r}093422 \\
6910\end{array}$ & $\begin{array}{l}3772 \\
7257\end{array}$ & 4122 & $\mathbf{4 4 7 1}$ & 4820 & 5169 & 5518 & 5866 & 6215 & 6562 & 349 \\
\hline 5 & & & & & & & & & & 0026 & 346 \\
\hline 6 & 100371 & 0715 & 1059 & 1403 & 1747 & 2091 & 2434 & 2777 & 3119 & 3462 & 343 \\
\hline $\begin{array}{l}7 \\
8\end{array}$ & $\begin{array}{l}3804 \\
7210\end{array}$ & $\begin{array}{l}4146 \\
7549\end{array}$ & $\begin{array}{l}4487 \\
7588\end{array}$ & 4828 & 5169 & 5510 & 5851 & 6191 & 6531 & 6871 & 341 \\
\hline 8 & 7210 & 7549 & $7 S 88$ & & & & & & 9916 & 0253 & 338 \\
\hline 9 & 110590 & 0926 & $1263^{\circ}$ & 1599 & 1934 & 2270 & 2605 & 2940 & 3275 & 3609 & 335 \\
\hline 130 & 3943 & 4277 & 4611 & 4944 & 5278 & 5611 & 5943 & 6276 & 6608 & 6940 & 333 \\
\hline 1 & 7271 & 7603 & 7934 & 8265 & 8595 & 8926 & 9256 & 9586 & 9915 & 0245 & 330 \\
\hline 2 & 120574 & 0903 & 1231 & 1560 & 1888 & 2216 & 2544 & 2871 & 3198 & 3525 & 328 \\
\hline 3 & 3852 & 4178 & 4504 & 4830 & 5156 & 5481 & 5806 & 6131 & 6456 & 6781 & 325 \\
\hline 4 & $13^{7105}$ & 7429 & 7753 & 8076 & 8399 & 8722 & 9045 & 9368 & 9690 & 0012 & 323 \\
\hline & & & & $\mathrm{B}$ & 0 & $\mathrm{P}$ & s. & & & & \\
\hline Diff. & 1 & 2 & 3 & 4 & & 5 & 6 & 7 & & 8 & 9 \\
\hline 355 & 35.5 & 71.0 & 106.5 & 142 & & 177.5 & 213.0 & 248.5 & & 84.0 & 319.5 \\
\hline 354 & 35.4 & 70.8 & 106.2 & 141 & 1.6 & 177.0 & 212.4 & 247.8 & & 33.2 & 318.6 \\
\hline 353 & 35.3 & 70.6 & 105.9 & 141 & 1.2 & 176.5 & 211.8 & 247.1 & & 32.4 & 317.7 \\
\hline 352 & 35.2 & 70.4 & 105.6 & 140 & .8 & 176. 0 & 211.2 & 246.4 & & 31.6 & 316.8 \\
\hline 351 & 35.1 & 70.2 & 105.3 & 140 & 0.4 & 175.5 & 210.6 & 245.7 & & 30.8 & 315.9 \\
\hline 350 & 35.0 & 70.0 & 105.0 & 140 & 0.0 & 175.0 & 210.0 & 245.0 & & 30.0 & 315.0 \\
\hline 349 & 34.9 & 69.8 & 104.7 & 139 & 6.6 & 5 & 209.4 & 244.3 & & 2 & 314.1 \\
\hline 348 & 34.8 & 69.6 & 104.4 & 139 & 2 & 17 & 208 & 243.6 & & 78.4 & 313.2 \\
\hline 347 & 34.7 & 69.4 & 104.1 & 138 & 3.8 & 173.5 & 208. 2 & 242.9 & & 6 & 312.3 \\
\hline 346 & 34.6 & 69.2 & 10 & 13 & 8.4 & 0 & 207.6 & 242. 2 & & 76.8 & 311.4 \\
\hline 345 & 34.5 & 69.0 & 103.5 & 138 & 3.0 & 172.5 & 207.0 & 241.5 & & 76.0 & 310.5 \\
\hline 344 & 34.4 & 68.8 & 103.2 & 137 & 7.6 & 172.0 & 206. 4 & 240.8 & & & 309.6 \\
\hline 343 & 34.3 & 68.6 & 102 & 137 & 7.2 & 171.5 & 205.8 & 240.1 & & 74.4 & 308.7 \\
\hline 342 & 34.2 & 68.4 & 102.6 & 130 & 8.8 & 171. 0 & 205.2 & 239.4 & & 6 & 307.8 \\
\hline 341 & 34. 1 & 68.2 & 102.3 & 13 & 6. 4 & 17 & 204.6 & 238.7 & & 72.8 & 306.9 \\
\hline 340 & 34.0 & 68.0 & 102.0 & 136 & 3.0 & 17 & 204.0 & 238.0 & & 0 & 306.0 \\
\hline 339 & 33.9 & 67.8 & 101 & 1 & 6 & 16 & 203 & 237.3 & & 71.2 & 305.1 \\
\hline 338 & 33.8 & 67.6 & 101.4 & 135 & 5.2 & 169.0 & 202.8 & 236.6 & & 70.4 & 304.2 \\
\hline 337 & 33.7 & 67.4 & 101.1 & & 8 & 168.5 & 202.2 & 235.9 & & 69. 6 & 303.3 \\
\hline 336 & 33.6 & 67.2 & 100.8 & 13 & 4.4 & 168.0 & 201.6 & 235.2 & & 38. 8 & 302.4 \\
\hline 335 & 33.5 & 67.0 & 100.5 & 134 & 4.0 & 167.5 & 201.0 & 234.5 & & 8. 0 & 301.5 \\
\hline 334 & 33.4 & 66.8 & 100.2 & 133 & 6 & 167.0 & 200.4 & 233. 8 & & 77. 2 & 300.6 \\
\hline 333 & 33.3 & 66.6 & 99.9 & 133 & 3.2 & 166.5 & 199.8 & 233.1 & & 36.4 & 299.7 \\
\hline 332 & 33.2 & 66.4 & 99.6 & 132 & .8 & 166.0 & 199.2 & 232.4 & & 5.6 & 298.8 \\
\hline 331 & 33.1 & 66.2 & 99.3 & 132 & 2.4 & 165 & 198 & 231.7 & & & 297.9 \\
\hline 330 & 33.0 & 66.0 & 99.0 & 132 & 2.0 & 165.0 & 198.0 & 231.0 & & 4. 0 & 297.0 \\
\hline 329 & 32.9 & 65.8 & 98.7 & 131 & 1.6 & 164 & 197. & 230.3 & & 2 & 296.1 \\
\hline 328 & 32.8 & 65.6 & 98.4 & 131 & 1.2 & 164.0 & 196,8 & 229.6 & & 22.4 & 295.2 \\
\hline 327 & 32.7 & 65.4 & 98.1 & 130 & 8 & 163.5 & 196. 2 & 228.9 & & 1.6 & 294.3 \\
\hline 326 & 32.6 & 65.2 & 97.8 & 13 & .4 & 163.0 & 195.6 & 228.2 & & 0.8 & 293.4 \\
\hline 325 & 32.5 & 65.0 & 97.5 & 130 & .0 & 162.5 & 195.0 & 227.5 & & 0.0 & 292.5 \\
\hline 324 & 32.4 & 64.8 & 97.2 & & & 162.0 & 194.4 & 226.8 & & 8.2 & 291. 6 \\
\hline 323 & 32.3 & 04.6 & 96.9 & 129 & .2 & 161.5 & 193.8 & 226.1 & & 8.4 & 290.7 \\
\hline 322 & 32.2 & 64.4 & 96.6 & 125 & 8.8 & 161.0 & 193. 2 & 225.4 & & 7.6 & 289.8 \\
\hline
\end{tabular}


TABLE 10.-LOGARITIMS OF NUMBERS.

No. 135 L. 130.]

[No. 149 L. 175.

\begin{tabular}{|c|c|c|c|c|c|c|c|c|c|c|c|}
\hline N. & 0 & 1 & 2 & 8 & 4 & 5 & 6 & 7 & 8 & 9 & Diff. \\
\hline \multirow{4}{*}{$\begin{array}{r}135 \\
6 \\
7 \\
8\end{array}$} & \multirow{4}{*}{$\begin{array}{r}130334 \\
3539 \\
6721 \\
9579\end{array}$} & 0655 & 97 & & & 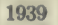 & & o & 0 & 9 & 321 \\
\hline & & & & & & & & & ; & & 315 \\
\hline & & 7037 & & & & & 010 & 934 & 9249 & 9564 & 316 \\
\hline & & \multirow{2}{*}{$\begin{array}{l}0194 \\
3327\end{array}$} & \multirow{2}{*}{$\begin{array}{l}0508 \\
3635\end{array}$} & \multirow{2}{*}{$\begin{array}{l}0822 \\
3951\end{array}$} & \multirow{2}{*}{$\begin{array}{l}1136 \\
4263\end{array}$} & \multirow{2}{*}{$\begin{array}{l}1450 \\
4574\end{array}$} & \multirow{2}{*}{$\begin{array}{l}1763 \\
4855\end{array}$} & \multirow{2}{*}{$\begin{array}{l}2076 \\
5196\end{array}$} & \multirow{2}{*}{$\begin{array}{l}2389 \\
5507\end{array}$} & \multirow{2}{*}{$\begin{array}{l}2702 \\
5818\end{array}$} & \multirow{2}{*}{$\begin{array}{l}314 \\
311\end{array}$} \\
\hline 9 & 143015 & & & & & & & & & & \\
\hline \multirow{2}{*}{$\begin{array}{r}140 \\
1\end{array}$} & \multirow{2}{*}{$\begin{array}{l}6128 \\
9219\end{array}$} & \multirow{2}{*}{$\begin{array}{l}6438 \\
0527 \\
\end{array}$} & \multirow{2}{*}{$\begin{array}{l}6748 \\
9335 \\
\end{array}$} & 7055 & 7367 & 7676 & 7985 & 8294 & 8603 & 8911 & 309 \\
\hline & & & & \multirow{4}{*}{$\begin{array}{l}0142 \\
3205 \\
6246 \\
9266\end{array}$} & \multirow{4}{*}{$\begin{array}{l}0449 \\
3510 \\
6549 \\
9567\end{array}$} & \multirow{4}{*}{$\begin{array}{l}0756 \\
3815 \\
6852 \\
9868\end{array}$} & \multirow{3}{*}{$\begin{array}{l}1063 \\
4120 \\
7154\end{array}$} & \multirow{3}{*}{$\begin{array}{l}1370 \\
4424 \\
7457 \\
\end{array}$} & \multirow{3}{*}{$\begin{array}{l}1676 \\
4728 \\
7759\end{array}$} & \multirow{3}{*}{$\begin{array}{l}1952 \\
5032 \\
8061\end{array}$} & \multirow{3}{*}{$\begin{array}{l}307 \\
305 \\
303\end{array}$} \\
\hline 2 & 152258 & 2594 & 29 & & & & & & & & \\
\hline & 5336 & 5640 & 59 & & & & & & & & \\
\hline & & & & & & & \multirow{4}{*}{$\begin{array}{l}0168 \\
3161 \\
6134 \\
9086\end{array}$} & \multirow{4}{*}{$\begin{array}{l}0469 \\
3460 \\
6430 \\
9380\end{array}$} & \multirow{4}{*}{$\begin{array}{l}0769 \\
3758 \\
6726 \\
9674\end{array}$} & \multirow{4}{*}{$\begin{array}{l}1068 \\
4055 \\
7022 \\
9068\end{array}$} & 301 \\
\hline 5 & 16130 & 16 & & & & & & & & & 0 \\
\hline 6 & 4353 & 465 & 49 & 52 & 55 & & & & & & 297 \\
\hline 7 & 73 & 7613 & 79 & 820 & 849 & 879 & & & & & 295 \\
\hline 8 & 1702 & te. & & & & & & & & & 293 \\
\hline 9 & & & & & & & 4932 & 5222 & 5512 & $5 \mathrm{~S} 02$ & 291 \\
\hline
\end{tabular}

Proportional Parts.

\begin{tabular}{|c|c|c|c|c|c|c|c|c|c|}
\hline Diff. & 1 & 2 & 3 & 4 & 5 & 6 & 7 & 8 & 9 \\
\hline $\begin{array}{l}321 \\
320 \\
319 \\
318 \\
317 \\
316 \\
315 \\
314 \\
313 \\
312\end{array}$ & $\begin{array}{l}32.1 \\
32.0 \\
31.9 \\
31.8 \\
31.7 \\
31.6 \\
31.5 \\
31.4 \\
31.3 \\
31.2\end{array}$ & $\begin{array}{l}64.2 \\
64.0 \\
63.8 \\
63.6 \\
63.4 \\
63.2 \\
63.0 \\
62.8 \\
62.6 \\
62.4\end{array}$ & $\begin{array}{l}96.3 \\
96.0 \\
95.7 \\
95.4 \\
95.1 \\
94.8 \\
94.5 \\
94.2 \\
93.9 \\
93.6\end{array}$ & $\begin{array}{l}128.4 \\
128.0 \\
127.6 \\
127.2 \\
126.8 \\
126.4 \\
126.0 \\
125.6 \\
125.2 \\
124.8\end{array}$ & $\begin{array}{l}160.5 \\
160.0 \\
159.5 \\
159.0 \\
158.5 \\
158.0 \\
157.5 \\
157.0 \\
156.5 \\
156.0\end{array}$ & $\begin{array}{l}192.6 \\
192.0 \\
191.4 \\
190.8 \\
190.2 \\
189.6 \\
189.0 \\
188.4 \\
187.8 \\
187.2\end{array}$ & $\begin{array}{l}224.7 \\
224.0 \\
223.3 \\
222.6 \\
221.9 \\
221.2 \\
220.5 \\
219.8 \\
219.1 \\
218.4\end{array}$ & $\begin{array}{l}256.8 \\
256.0 \\
255.2 \\
254.4 \\
253.6 \\
252.8 \\
252.0 \\
251.2 \\
250.4 \\
249.6\end{array}$ & $\begin{array}{l}258.9 \\
258.0 \\
257.1 \\
256.2 \\
255.3 \\
254.4 \\
253.5 \\
282.6 \\
281.7 \\
250.8\end{array}$ \\
\hline $\begin{array}{l}311 \\
310 \\
309 \\
305 \\
307 \\
306 \\
305 \\
304 \\
303 \\
302\end{array}$ & $\begin{array}{l}31.1 \\
31.0 \\
30.9 \\
30.8 \\
30.7 \\
30.6 \\
30.5 \\
30.4 \\
30.3 \\
30.2\end{array}$ & $\begin{array}{l}62.2 \\
62.0 \\
61.8 \\
61.6 \\
61.4 \\
61.2 \\
61.0 \\
60.8 \\
60.6 \\
60.4\end{array}$ & $\begin{array}{l}93.3 \\
93.0 \\
92.7 \\
92.4 \\
92.1 \\
91.8 \\
91.5 \\
91.2 \\
90.9 \\
90.6\end{array}$ & $\begin{array}{l}124.4 \\
124.0 \\
123.6 \\
123.2 \\
122.8 \\
122.4 \\
122.0 \\
121.6 \\
121.2 \\
120.8\end{array}$ & $\begin{array}{l}155.5 \\
155.0 \\
154.5 \\
154.0 \\
153.5 \\
153.0 \\
152.5 \\
152.0 \\
151.5 \\
151.0\end{array}$ & $\begin{array}{l}186.6 \\
186.0 \\
185.4 \\
184.8 \\
184.2 \\
183.6 \\
183.0 \\
182.4 \\
181.8 \\
181.2\end{array}$ & $\begin{array}{l}217.7 \\
217.0 \\
216.3 \\
215.6 \\
214.9 \\
214.2 \\
213.5 \\
212.8 \\
212.1 \\
211.4\end{array}$ & $\begin{array}{l}248.8 \\
248.0 \\
247.2 \\
246.4 \\
245.6 \\
244.8 \\
244.0 \\
243.2 \\
242.4 \\
241.6\end{array}$ & $\begin{array}{l}279.9 \\
279.0 \\
278.1 \\
277.2 \\
276.3 \\
275.4 \\
274.5 \\
273.6 \\
272.7 \\
271.8\end{array}$ \\
\hline $\begin{array}{l}301 \\
300 \\
299 \\
298 \\
297 \\
296 \\
295 \\
294 \\
293 \\
292\end{array}$ & $\begin{array}{l}30.1 \\
30.0 \\
29.9 \\
29.8 \\
29.7 \\
29.6 \\
29.5 \\
29.4 \\
29.3 \\
29.2\end{array}$ & $\begin{array}{l}60.2 \\
60.0 \\
59.8 \\
59.6 \\
59.4 \\
59.2 \\
59.0 \\
59.8 \\
58.6 \\
55.4\end{array}$ & $\begin{array}{l}90.3 \\
90.0 \\
89.7 \\
89.4 \\
89.1 \\
88.8 \\
85.5 \\
85.2 \\
87.9 \\
87.6\end{array}$ & $\begin{array}{l}120.4 \\
120.0 \\
119.6 \\
119.2 \\
118.8 \\
118.4 \\
118.0 \\
117.6 \\
117.2 \\
116.8\end{array}$ & $\begin{array}{l}150.5 \\
150.0 \\
149.5 \\
149.0 \\
148.5 \\
148.0 \\
147.5 \\
147.0 \\
146.5 \\
146.0\end{array}$ & $\begin{array}{l}180.6 \\
180.0 \\
179.4 \\
178.8 \\
178.2 \\
177.6 \\
177.0 \\
176.4 \\
175.8 \\
175.2\end{array}$ & $\begin{array}{l}210.7 \\
210.0 \\
209.3 \\
208.6 \\
207.9 \\
207.2 \\
206.5 \\
205.8 \\
205.1 \\
204.4\end{array}$ & $\begin{array}{l}240.8 \\
240.0 \\
239.2 \\
238.4 \\
237.6 \\
236.5 \\
236.0 \\
235.2 \\
234.4 \\
233.6\end{array}$ & $\begin{array}{l}270.9 \\
270.0 \\
269.1 \\
265.2 \\
267.3 \\
266.4 \\
265.5 \\
264.6 \\
263.7 \\
262.8\end{array}$ \\
\hline $\begin{array}{l}291 \\
290 \\
259 \\
258 \\
257 \\
256\end{array}$ & $\begin{array}{l}29.1 \\
29.0 \\
28.9 \\
28.8 \\
28.7 \\
28.6\end{array}$ & $\begin{array}{l}58.2 \\
58.0 \\
57.8 \\
57.6 \\
57.4 \\
57.2\end{array}$ & $\begin{array}{l}87.3 \\
87.0 \\
86.7 \\
86.4 \\
86.1 \\
85.8\end{array}$ & $\begin{array}{l}116.4 \\
116.0 \\
115.6 \\
115.2 \\
114.8 \\
114.4\end{array}$ & $\begin{array}{l}145.5 \\
145.0 \\
144.5 \\
144.0 \\
143.5 \\
143.0\end{array}$ & $\begin{array}{l}174.6 \\
174.0 \\
173.4 \\
172.8 \\
172.2 \\
171.6\end{array}$ & $\begin{array}{l}203.7 \\
203.0 \\
202.3 \\
201.8 \\
300.9 \\
200.2\end{array}$ & $\begin{array}{l}232.8 \\
232.0 \\
231.2 \\
230.4 \\
229.6 \\
228.8\end{array}$ & $\begin{array}{l}2031.9 \\
201.0 \\
200.1 \\
259.2 \\
255.3 \\
257.4\end{array}$ \\
\hline
\end{tabular}


TABLE 10.-LOGARITHMS OF NUMBERS.

\begin{tabular}{|c|c|c|c|c|c|c|c|c|c|c|c|}
\hline \multicolumn{9}{|c|}{ No. 150 L. 176.] } & \multicolumn{3}{|c|}{ [No. 169 L. 230.} \\
\hline N. & 0 & 1 & 2 & 3 & 4 & 5 & 6 & 7 & 8 & 9 & Diff. \\
\hline \multirow{2}{*}{$\begin{array}{r}150 \\
1\end{array}$} & \multirow{2}{*}{$\begin{array}{r}176091 \\
8977\end{array}$} & \multirow{2}{*}{$\begin{array}{l}6381 \\
9264\end{array}$} & \multirow{2}{*}{$\begin{array}{l}6670 \\
9552\end{array}$} & \multirow{2}{*}{$\begin{array}{l}6959 \\
9539\end{array}$} & 7248 & 7536 & 7825 & 8113 & 8401 & 8689 & 289 \\
\hline & & & & & \multirow{4}{*}{$\begin{array}{l}0126 \\
2985 \\
5825 \\
8647 \\
\end{array}$} & 0413 & 0699 & 0986 & \multirow{4}{*}{$\begin{array}{l}1272 \\
4123 \\
6956 \\
9771 \\
\end{array}$} & 1558 & 287 \\
\hline 2 & & \multirow{3}{*}{$\begin{array}{l}2129 \\
4975 \\
7803 \\
\end{array}$} & \multirow{3}{*}{$\begin{array}{l}2415 \\
5259 \\
8084 \\
\end{array}$} & \multirow{3}{*}{$\begin{array}{l}2700 \\
5542 \\
8366 \\
\end{array}$} & & & 3555 & 3839 & & 4407 & 285 \\
\hline \multirow{2}{*}{$\begin{array}{l}3 \\
4\end{array}$} & \multirow{2}{*}{$\begin{array}{r}4691 \\
7521 \\
\end{array}$} & & & & & 6108 & 6391 & 6674 & & 7239 & 283 \\
\hline & & & & & & 8928 & 9209 & 9490 & & & \\
\hline 5 & 190332 & 0612 & 0892 & 1171 & 1451 & 1730 & 2010 & 2289 & 2567 & 2846 & 279 \\
\hline 6 & 3125 & 3403 & 3681 & & 4237 & 4514 & 4792 & 5069 & 5346 & 5623 & 278 \\
\hline 7 & 5900 & $\begin{array}{l}6176 \\
8932\end{array}$ & $\begin{array}{l}6453 \\
9206\end{array}$ & $\begin{array}{l}6729 \\
9481\end{array}$ & $\begin{array}{l}7005 \\
9755\end{array}$ & 7281 & 7556 & 7832 & 8107 & 8382 & 276 \\
\hline & & & & & & 0029 & 0303 & 0577 & 0850 & 1124 & 274 \\
\hline 9 & 201397 & 1670 & 1943 & 2216 & 2488 & 2761 & 3033 & 3305 & 3577 & 3848 & 272 \\
\hline 160 & 4120 & 4391 & 4663 & 4934 & 5204 & 5475 & 5746 & 016 & 6286 & 6556 & 271 \\
\hline $\begin{array}{l}1 \\
2\end{array}$ & $\begin{array}{l}6826 \\
9515\end{array}$ & $\begin{array}{l}7096 \\
9783\end{array}$ & 73 & 7634 & 7904 & 8173 & 8441 & 8710 & 8979 & 9247 & 269 \\
\hline 3 & 212188 & 245 & $\begin{array}{l}0051 \\
2720\end{array}$ & $\begin{array}{l}0319 \\
2986\end{array}$ & $\begin{array}{l}0586 \\
3252\end{array}$ & $\begin{array}{l}0853 \\
3518\end{array}$ & $\begin{array}{l}1121 \\
3783\end{array}$ & $\begin{array}{l}1388 \\
4049\end{array}$ & $\begin{array}{l}1654 \\
4314\end{array}$ & $\begin{array}{l}1921 \\
4579\end{array}$ & $\begin{array}{l}267 \\
266\end{array}$ \\
\hline 4 & 4844 & 5109 & 5373 & 5638 & 5902 & 6166 & 6430 . & 6694 & 6957 & 7221 & 264 \\
\hline 5 & 7484 & 7747 & 8 & 8273 & 8536 & $\$ 798$ & $9060^{\circ}$ & 9323 & 9585 & $98 \div 6$ & 262 \\
\hline 6 & 220108 & 0370 & 0631 & 0892 & 1153 & 1414 & 1675 & 936 & 2196 & 2456 & 261 \\
\hline 7 & 2716 & 2976 & ? & & 37 & & 4 & 3 & 4792 & 5051 & 259 \\
\hline $\begin{array}{l}8 \\
9\end{array}$ & $\begin{array}{l}5309 \\
7887\end{array}$ & $\begin{array}{l}5.568 \\
8144\end{array}$ & 6 & & 6342 & & 8 & 5 & $\begin{array}{l}7372 \\
9938\end{array}$ & 7630 & 258 \\
\hline & $23^{100}$ & & +0 & 8000 & 0910 & 9110 & $y \pm 20$ & 9082 & 9338 & 0193 & 256 \\
\hline & & & & & & & & & & & \\
\hline Diff. & 1 & 2 & 3 & 4 & & 5 & 6 & 7 & $\varepsilon$ & 8 & 9 \\
\hline 285 & & 5 & 85 & & & 2.5 & 171.0 & 199.5 & & & 6.5 \\
\hline & & & & & & & & & & & 6 \\
\hline 2 & & 5 & & & & 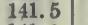 & & & & & 7 \\
\hline & & 5 & & & & & & 19 & & & 8 \\
\hline ? & & & & & & & & & & & 9 \\
\hline & & & & & & & & & & & 22.0 \\
\hline 279 & & & & & & & & & & & 51.1 \\
\hline 2 & & & & & & & & & & & 2 \\
\hline 277 & & & & & & & & & & & 9.3 \\
\hline 276 & & & & & & & & & & & 8.4 \\
\hline 275 & 27.5 & 55.0 & 82.5 & 1 & & 7.5 & 0 & 19 & & & 47.5 \\
\hline & & & & & & & & & & & \\
\hline & & & & & & & & & & & 7 \\
\hline & & & & & & & & & & & 8 \\
\hline & & & & & & & & & & & 9 \\
\hline & & & & & & & & & & & \\
\hline & & & & & & & & & & & 1 \\
\hline & & & & & & & & & & & 15 \\
\hline & & 53 & & & & & & & & & .3 \\
\hline 260 & & & & & & & & & & & 239.4 \\
\hline 205 & 26 & 53. & 78 & 10 & & 2.5 & 0 & 5 & & 0 & 238.5 \\
\hline & & & & & & & & & & & \\
\hline 2 & & & & & & & & & & & 236.7 \\
\hline & & & & & & & & & & & 235.8 \\
\hline & & & & & & & & & & & 234.9 \\
\hline 2 & 26 & & & & & & & & & & 234.0 \\
\hline 2 & 25 & & & & & & & & & & 233.1 \\
\hline 25 & 25. & & & & & & & & & & 232.2 \\
\hline & 25. & & & & & & & & & & 231.3 \\
\hline 2 & 25 & & & & & & & & & & 230.4 \\
\hline 255 & 25.5 & & & & & & 153.0 & & & 4.0 & 229.5 \\
\hline
\end{tabular}


TAIBLE 10,-LOGAIRTHS OF NCMBEIS.

No. 1\%0 L. 230.]

[No. 189 L. 278.

\begin{tabular}{|c|c|c|c|c|c|c|c|c|c|c|c|}
\hline N. & 0 & 1 & 2 & 3 & 4 & 5 & 6 & 7 & 8 & 9 & Diff. \\
\hline \multirow{4}{*}{$\begin{array}{r}170 \\
1 \\
2 \\
3\end{array}$} & \multirow{4}{*}{$\begin{array}{r}230449 \\
2996 \\
5528 \\
8046 \\
\end{array}$} & \multirow{4}{*}{$\begin{array}{l}0704 \\
3250 \\
5781 \\
8297 \\
\end{array}$} & \multirow{4}{*}{$\begin{array}{l}0560 \\
3504 \\
6033 \\
8548 \\
\end{array}$} & \multirow{4}{*}{$\begin{array}{l}1215 \\
3757 \\
6285 \\
8799 \\
\end{array}$} & \multirow{4}{*}{$\begin{array}{l}1470 \\
4011 \\
6537 \\
9049\end{array}$} & \multirow{4}{*}{$\begin{array}{l}1724 \\
4264 \\
6789 \\
9299 \\
\end{array}$} & \multirow{4}{*}{$\begin{array}{l}1979 \\
4517 \\
7041 \\
9550\end{array}$} & \multirow{4}{*}{$\begin{array}{l}2234 \\
4770 \\
7292 \\
9800 \\
\end{array}$} & \multirow{4}{*}{$\begin{array}{l}2488 \\
5023 \\
7544 \\
\end{array}$} & \multirow{3}{*}{$\begin{array}{l}2742 \\
5276 \\
7795 \\
\end{array}$} & \multirow{3}{*}{$\begin{array}{l}255 \\
253 \\
252\end{array}$} \\
\hline & & & & & & & & & & & \\
\hline & & & & & & & & & & & \\
\hline & & & & & & & & & & \multirow{4}{*}{$\begin{array}{l}0300 \\
2790 \\
5266 \\
7728 \\
\end{array}$} & \multirow{2}{*}{$\begin{array}{l}250 \\
249\end{array}$} \\
\hline \multirow{4}{*}{$\begin{array}{l}4 \\
5 \\
6 \\
7\end{array}$} & 240 & & & & & & & & \multirow{4}{*}{$\begin{array}{l}0050 \\
2541 \\
5019 \\
7482 \\
9932\end{array}$} & & \\
\hline & 8 & & 35 & & & & & & & & 248 \\
\hline & 5513 & 57 & 600 & & 6499 & 67 & 6991 & 7237 & & & 246 \\
\hline & 7973 & 82 & 846 & 8709 & 8954 & 91 & 9443 & 968 & & & \\
\hline 8 & 2 & 0 & 0908 & 11 & 1395 & 1638 & 1881 & 2125 & 2368 & & 243 \\
\hline 9 & 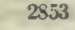 & & 33 & & 3822 & & 4306 & 8 & 4790 & 5031 & 242 \\
\hline 180 & 527 & 55 & 5755 & 59 & 6237 & 6477 & 67 & 69 & 71 & 7439 & 241 \\
\hline & 7679 & & 8158 & 8 & 8637 & 8877 & 9116 & 9355 & 9594 & 9833 & 239 \\
\hline 2 & 260071 & & & & & & & & & & 238 \\
\hline 3 & & & & & & & & & & & 237 \\
\hline 4 & 4818 & 5054 & 5290 & 5525 & 57 & 59 & 62 & & & 6937 & 23 \\
\hline 5 & 7172 & 7406 & 7641 & 7875 & 8110 & 8344 & 8578 & 8812 & 9046 & 9279 & 234 \\
\hline & & & & 021 & 94 & 06 & & & & & 23 \\
\hline & 2718 & & & & & & & & & 3927 & 23 \\
\hline 8 & 4158 & 43 & 46 & 48 & 5081 & 53 & 55 & 5772 & 60 & 6232 & 230 \\
\hline 9 & 6462 & 6692 & 6921 & 7151 & 7350 & 7609 & 7838 & 8067 & 8296 & 8525 & 229 \\
\hline
\end{tabular}

Proportional Parts.

\begin{tabular}{|c|c|c|c|c|c|c|c|c|c|}
\hline Diff. & 1 & 2 & 3 & 4 & 5 & 6 & 7 & 8 & 9 \\
\hline 255 & 25.5 & 51.0 & 76.5 & 102.0 & 127.5 & 153.0 & 178.5 & 204.0 & 229.5 \\
\hline 2 & & 50. & 76.2 & 101.6 & 127.0 & 152.4 & 177.8 & 203.2 & 228.6 \\
\hline 25 & 2 & 50. & 75.9 & 101.2 & 126.5 & 151.8 & 177.1 & 202.4 & 227.7 \\
\hline 2.52 & 25.2 & 50.4 & 75.6 & 100.8 & 126.0 & 151.2 & 176.4 & 201.6 & 226.8 \\
\hline 251 & 25.1 & 50.2 & 75.3 & 100.4 & 125.5 & 150.6 & 175.7 & 200.8 & 225.9 \\
\hline 250 & 25.0 & 50.0 & 75. & 100.0 & 125.0 & 150.0 & 175.0 & 200.0 & 225.0 \\
\hline 249 & 24.9 & 49.8 & 74. & 99.6 & 124.5 & 149.4 & 174.3 & 199.2 & 224.1 \\
\hline 24 & 24.8 & 49.6 & 74. & 99.2 & 124.0 & 148.8 & 173.6 & 198.4 & 223.2 \\
\hline 24 & 24.7 & 49.4 & 74. & 98.8 & 123.5 & 148.2 & 172.9 & 197.6 & 222.3 \\
\hline 24 & 24.6 & 49.2 & 73. & 98,4 & 123.0 & 147.6 & 172.2 & 196.8 & 221.4 \\
\hline 245 & 24. & 49.0 & 7 & 98.0 & 122.5 & 147.0 & 171.5 & 196.0 & 220.5 \\
\hline 244 & 24.4 & 48.8 & 73.2 & 97.6 & 122.0 & 146.4 & 170.8 & 195. 2 & 219.6 \\
\hline 24 & 24. & 48.6 & 72 & 97.2 & 121.5 & 14 & 170.1 & 194.4 & 218.7 \\
\hline 242 & 24. & 48.4 & 72 & 96.8 & 121.0 & 14 & 169.4 & 193.6 & 217.8 \\
\hline 24 & 24. & 48.2 & 72.3 & 96.4 & 120.5 & 144.6 & 168.7 & 192.8 & 216.9 \\
\hline 24 & 24. & 48.0 & 72.0 & 96.0 & 120.0 & 14 & 168.0 & 192.0 & 216.0 \\
\hline 23 & 23.9 & 47.8 & 71.7 & 95.6 & 119.5 & 143.4 & 167.3 & 191.2 & 215.1 \\
\hline 23 & 23.8 & 47.6 & 71.4 & 95.2 & 119.0 & 142.8 & 166.6 & 190.4 & 214.2 \\
\hline 23 & 23.7 & 47. & 71. & 94.8 & 118.5 & 142.2 & 165.9 & 189.6 & 213.3 \\
\hline 236 & 23. 6 & 47.2 & 70.8 & 94.4 & 118.0 & 141.6 & 165.2 & 188.8 & 212.4 \\
\hline 235 & 23.5 & 47.0 & 70.5 & 94.0 & 117.5 & 141.0 & 164.5 & 188.0 & 211.5 \\
\hline 234 & 23.4 & 46. 8 & 70.2 & 93.6 & 117.0 & 140.4 & 163.8 & 187.2 & 210.6 \\
\hline 23 & 23. & 46.6 & 69.9 & 93.2 & 116.5 & 139.8 & 163.1 & & 209.7 \\
\hline 232 & 23.2 & 46.4 & 69.6 & 92.8 & 116.0 & 139. 2 & 162.4 & 185.6 & 208.8 \\
\hline 231 & 23.1 & 46.2 & 69.3 & 92.4 & 115.5 & 135. 6 & 161.7 & 184.8 & 207.9 \\
\hline 230 & 23.0 & 46.0 & 69.0 & 92.0 & 115.0 & 138.0 & 161.0 & 184.0 & 207.0 \\
\hline 229 & 22.9 & 45.8 & 68.7 & 91.6 & 114.5 & 137.4 & 160.3 & 183.2 & 206.1 \\
\hline 229 & 22.8 & 45.6 & 68.4 & 91.2 & 114.0 & 136.8 & 159.6 & 182.4 & 205.2 \\
\hline 227 & 22.7 & 45.4 & 68.1 & 90.8 & 113.5 & 136. 2 & 158.9 & 181.6 & 204.3 \\
\hline 226 & 22.6 & 45.2 & 67.8 & 90.4 & 113.0 & 135.6 & 158.2 & 150.8 & 203.4 \\
\hline
\end{tabular}


TABLE 10.-LOGARITHMS OF NUMBERS.

No. 190 L. 278.]

[No. 214 L. 332.

\begin{tabular}{|c|c|c|c|c|c|c|c|c|c|c|c|}
\hline N. & 0 & 1 & 2 & 3 & 4 & 5 & 6 & 7 & 8 & 9 & Diff. \\
\hline 190 & 278754 & 8982 & 9211 & 9439 & 9667 & 9895 & \multirow{2}{*}{$\begin{array}{l}0123 \\
2396\end{array}$} & \multirow[b]{2}{*}{$\begin{array}{l}0351 \\
2622\end{array}$} & & & \\
\hline 1 & 2810 & 12 & 1458 & 17 & 1942 & 2169 & & & $\begin{array}{l}0578 \\
2849\end{array}$ & $\begin{array}{l}0806 \\
3075\end{array}$ & 228 \\
\hline 2 & & & & & & & 4656 & $\begin{array}{l}2022 \\
4882\end{array}$ & $\begin{array}{l}2849 \\
5107\end{array}$ & $\begin{array}{l}3075 \\
5332\end{array}$ & 226 \\
\hline 3 & 57 & 5782 & 6007 & 232 & 6456 & 6681 & 6905 & 7130 & 7354 & 7578 & 225 \\
\hline 4 & 7802 & 8026 & 8249 & 8473 & 8696 & 8920 & 9143 & 9366 & 9589 & 9812 & 223 \\
\hline 5 & 290035 & 025 & 30 & 2 & 0925 & 147 & 69 & 591 & 13 & 2034 & 222 \\
\hline 6 & 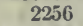 & & & & 31 & & & & & 42 & 221 \\
\hline 7 & 4466 & 46 & 4907 & 5127 & 53 & 5567 & 5787 & 6007 & 6226 & 6446 & 220 \\
\hline 8 & 66 & 68 & 7104 & 7323 & $75+2$ & 7761 & 7979 & 8198 & 8416 & 8635 & 219 \\
\hline 9 & 8853 & 9071 & 9289 & 9507 & 9725 & 9943 & 0161 & 0378 & 0595 & $0 S 13$ & 218 \\
\hline 200 & 301030 & 1247 & 1 & 1681 & 1898 & 2114 & 2331 & 2547 & 2764 & 2980 & 217 \\
\hline 1 & 319 & 34 & & & 40 & 42 & & & & 5136 & 216 \\
\hline 2 & 5351 & 550 & 5781 & 59 & 6211 & 6425 & $6 x$ & 68 & 7 & 7282 & 215 \\
\hline 3 & 7496 & 7710 & 7924 & 8137 & 8351 & $\$ 564$ & 8778 & 8991 & 9204 & 9417 & 213 \\
\hline & & & U & 026 & 04 & 00 & Os & 11 & 13 & 1542 & 212 \\
\hline 5 & 3117 & 19 & 21 & 238 & 26 & 28 & 30 & 32 & 34 & 3656 & 211 \\
\hline . & & 40 & 42 & 44 & 47 & 49 & 51 & 53 & 55 & 5760 & 210 \\
\hline 7 & 597 & 618 & 6390 & 65 & 68 & 7018 & 7227 & 74 & 76 & 7854 & 209 \\
\hline 8 & 8063 & 8272 & 848 & 8689 & 8898 & 9106 & 9314 & 9522 & 9730 & 9938 & 208 \\
\hline 9 & 320146 & 03 & 0 & 9 & 0977 & 4 & 1 & 10 & 5 & 2012 & 207 \\
\hline 210 & 2 & 2 & 268 & 28 & 30 & 32 & 34 & 36 & 38 & 40 & 206 \\
\hline & & & & & 5 & 53 & 55 & 57 & 5 & 6131 & 205 \\
\hline$\pi$ & 6336 & 65 & 6745 & 69 & 71 & 7359 & 7563 & 7767 & 7972 & 8176 & 204 \\
\hline & & & & & & & & & & 1 & 203 \\
\hline 4 & 330414 & 0617 & 0819 & 1022 & 1225 & 1427 & 1630 & 1832 & 2034 & 2236 & 202 \\
\hline
\end{tabular}

Proportional Parts.

\begin{tabular}{|c|c|c|c|c|c|c|c|c|c|}
\hline Diff. & 1 & 2 & 3 & 4 & 5 & 6 & 7 & 8 & 9 \\
\cline { 1 - 8 } 225 & 22.5 & 45.0 & 67.5 & 90.0 & 112.5 & 135.0 & 157.5 & 180.0 & 202.5 \\
224 & 22.4 & 44.8 & 67.2 & 89.6 & 112.0 & 134.4 & 156.8 & 179.2 & 201.6 \\
223 & 22.3 & 44.6 & 66.9 & 89.2 & 111.5 & 133.8 & 156.1 & 178.4 & 200.7 \\
222 & 22.2 & 44.4 & 66.6 & 88.8 & 111.0 & 133.2 & 155.4 & 177.6 & 199.8 \\
221 & 22.1 & 44.2 & 66.3 & 88.4 & 110.5 & 132.6 & 154.7 & 176.8 & 198.9 \\
220 & 22.0 & 44.0 & 66.0 & 88.0 & 110.0 & 132.0 & 154.0 & 176.0 & 198.0 \\
219 & 21.9 & 43.8 & 65.7 & 87.6 & 109.5 & 131.4 & 153.3 & 175.2 & 197.1 \\
218 & 21.8 & 43.6 & 65.4 & 87.2 & 109.0 & 130.8 & 152.6 & 174.4 & 196.2 \\
217 & 21.7 & 43.4 & 65.1 & 86.8 & 108.5 & 130.2 & 151.9 & 173.6 & 195.3 \\
216 & 21.6 & 43.2 & 64.8 & 86.4 & 108.0 & 129.6 & 151.2 & 172.8 & 194.4 \\
215 & 21.5 & 43.0 & 64.5 & 86.0 & 107.5 & 129.0 & 150.5 & 172.0 & 193.5 \\
214 & 21.4 & 42.8 & 64.2 & 85.6 & 107.0 & 123.4 & 149.8 & 171.2 & 192.6 \\
213 & 21.3 & 42.6 & 63.9 & 85.2 & 106.5 & 127.8 & 149.1 & 170.4 & 191.7 \\
212 & 21.2 & 42.4 & 63.6 & 84.8 & 106.0 & 127.2 & 148.4 & 169.6 & 190.8 \\
211 & 21.1 & 42.2 & 63.3 & 84.4 & 105.5 & 126.6 & 147.7 & 168.8 & 189.9 \\
210 & 21.0 & 42.0 & 63.0 & 84.0 & 105.0 & 126.0 & 147.0 & 168.0 & 189.0 \\
209 & 20.9 & 41.8 & 62.7 & 83.6 & 104.5 & 125.4 & 146.3 & 167.2 & 188.1 \\
208 & 20.8 & 41.6 & 62.4 & 83.2 & 104.0 & 124.8 & 145.6 & 166.4 & 187.2 \\
207 & 20.7 & 41.4 & 62.1 & 82.8 & 103.5 & 124.2 & 144.9 & 165.6 & 186.3 \\
206 & 20.6 & 41.2 & 61.8 & 82.4 & 103.0 & 123.6 & 144.2 & 164.8 & 185.4 \\
205 & 20.5 & 41.0 & 61.5 & 82.0 & 102.5 & 123.0 & 143.5 & 164.0 & 184.5 \\
204 & 20.4 & 40.8 & 61.2 & 81.6 & 102.0 & 122.4 & 142.8 & 163.2 & 183.6 \\
203 & 20.3 & 40.6 & 60.9 & 81.2 & 101.5 & 121.8 & 142.1 & 162.4 & 182.7 \\
202 & 20.2 & 40.4 & 60.6 & 80.8 & 101.0 & 121.2 & 141.4 & 161.6 & 181.8 \\
\hline
\end{tabular}


No. 215 L. 332.]

[N้o. 239 L. 380.

\begin{tabular}{|c|c|c|c|c|c|c|c|c|c|c|c|}
\hline N. & 0 & 1 & 2 & 3 & 4 & 5 & 6 & 7 & 8 & 9 & Difr. \\
\hline \multirow{4}{*}{$\begin{array}{r}215 \\
6 \\
7 \\
8\end{array}$} & \multirow{4}{*}{$\begin{array}{r}332438 \\
4454 \\
6460 \\
8456\end{array}$} & \multirow{4}{*}{$\begin{array}{l}2640 \\
4655 \\
6660 \\
8650\end{array}$} & \multirow{4}{*}{$\begin{array}{l}2 S 42 \\
4856 \\
6 S 60 \\
8855\end{array}$} & \multirow{4}{*}{$\begin{array}{l}3044 \\
5057 \\
7060 \\
9054\end{array}$} & \multirow{4}{*}{$\begin{array}{l}3246 \\
5257 \\
7260 \\
9253\end{array}$} & \multirow{4}{*}{$\begin{array}{l}3447 \\
5458 \\
7459 \\
9451\end{array}$} & \multirow{4}{*}{$\begin{array}{l}3649 \\
5658 \\
7659 \\
9650\end{array}$} & \multirow{4}{*}{$\begin{array}{l}3850 \\
5859 \\
7858 \\
9849\end{array}$} & \multirow{4}{*}{$\begin{array}{l}4051 \\
6059 \\
8058\end{array}$} & 4253 & \multirow{4}{*}{$\begin{array}{l}202 \\
201 \\
200\end{array}$} \\
\hline & & & & & & & & & & & \\
\hline & & & & & & & & & & 8257 & \\
\hline & & & & & & & & & & & \\
\hline 9 & 340444 & 0642 & 0841 & 1039 & 1237 & 1435 & 1632 & 1830 & $\begin{array}{l}0 C 47 \\
2028\end{array}$ & 2225 & $\begin{array}{l}199 \\
198\end{array}$ \\
\hline \multirow{4}{*}{$\begin{array}{r}220 \\
1 \\
2 \\
3\end{array}$} & 2423 & \multirow{4}{*}{$\begin{array}{l}2620 \\
4589 \\
6549 \\
8500\end{array}$} & \multirow{4}{*}{$\begin{array}{l}2817 \\
4785 \\
6744 \\
8694\end{array}$} & \multirow{4}{*}{$\begin{array}{l}3014 \\
4981 \\
6939 \\
8859\end{array}$} & \multirow{4}{*}{$\begin{array}{l}3212 \\
5175 \\
7135 \\
9083\end{array}$} & \multirow{4}{*}{$\begin{array}{l}3409 \\
5374 \\
7330 \\
9278\end{array}$} & \multirow{4}{*}{$\begin{array}{l}3606 \\
5570 \\
7525 \\
9472\end{array}$} & \multirow{4}{*}{$\begin{array}{l}3802 \\
5766 \\
7720 \\
8666\end{array}$} & \multirow{4}{*}{$\begin{array}{l}3999 \\
5962 \\
7915 \\
9860\end{array}$} & 4196 & \multirow{3}{*}{$\begin{array}{l}197 \\
196 \\
195\end{array}$} \\
\hline & 4392 & & & & & & & & & 6157 & \\
\hline & 6353 & & & & & & & & & 8110 & \\
\hline & 8305 & & & & & & & & & & \\
\hline \multirow{6}{*}{$\begin{array}{l}4 \\
5 \\
6 \\
7 \\
8 \\
9\end{array}$} & \multirow{6}{*}{$\begin{array}{r}350248 \\
2183 \\
4108 \\
6026 \\
7935 \\
9835\end{array}$} & \multirow{5}{*}{$\begin{array}{l}0442 \\
2375 \\
4301 \\
6217 \\
8125\end{array}$} & \multirow{5}{*}{$\begin{array}{l}0636 \\
2568 \\
4493 \\
6408 \\
8316 \\
\end{array}$} & \multirow{5}{*}{$\begin{array}{l}0829 \\
2761 \\
4685 \\
6599 \\
8506\end{array}$} & \multirow{5}{*}{$\begin{array}{l}1023 \\
2954 \\
4876 \\
6790 \\
8696\end{array}$} & 1216 & 1410 & 1603 & 1796 & & 193 \\
\hline & & & & & & & & 3 & & 39 & 193 \\
\hline & & & & & & 5 & & 5 & 5 & & 192 \\
\hline & & & & & & 69 & 71 & & & $77+4$ & 191 \\
\hline & & & & & & 6 & 90 & 9 & 9456 & 9646 & 190 \\
\hline & & 0025 & 0215 & 0404 & 0593 & 0783 & 0972 & 1161 & 1350 & 1539 & 189 \\
\hline 230 & 361728 & 19 & 2 & 2 & 24 & 1 & 2 & 8 & 6 & 3424 & 188 \\
\hline & 3612 & 380 & 3 & 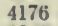 & 43 & & 9 & 6 & 5113 & 5301 & 188 \\
\hline & 5458 & 567 & 5 & & 62 & 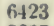 & 65 & 6796 & & 7169 & 187 \\
\hline & 7356 & 7542 & 7729 & 79 & 8101 & 8287 & 8473 & 8659 & 8845 & 9030 & 186 \\
\hline & & & & & & & 03 & 3 & 0698 & 0883 & 18 \\
\hline 5 & 371068 & 125 & 14 & 16 & 18 & & & & & & 18 \\
\hline & 2912 & 3096 & 3280 & 34 & 3647 & 3831 & 4015 & 4198 & 4382 & 4565 & 184 \\
\hline & $47+8$ & 4932 & 51 & 52 & 54 & 56 & 58 & 6029 & 6212 & 6394 & 183 \\
\hline & 6577 & 67 & 69 & 71 & 7306 & 74 & 7670 & 7852 & 8034 & 8216 & 182 \\
\hline & $38^{8398}$ & 8 & 8 & 89 & 9124 & 9 & 9487 & 9 & $98+9$ & 0030 & is \\
\hline & & & & & & & & 47 & & 0.50 & 15 \\
\hline
\end{tabular}

Proportional Parts.

\begin{tabular}{|c|c|c|c|c|c|c|c|c|c|}
\hline Diff. & 1 & 2 & 3 & 4 & 5 & 6 & 7 & 8 & 9 \\
\hline 202 & 20.2 & 40.4 & 60.6 & 80.8 & 101.0 & 121.2 & 141.4 & 161.6 & 181.8 \\
\hline 201 & 20.1 & 40.2 & 60.3 & 80. & 100.5 & 120.6 & 140.7 & 160.8 & 180.9 \\
\hline 200 & 20.0 & 40.0 & 60.0 & 80.0 & 100.0 & 120.0 & 140.0 & 160.0 & 180.0 \\
\hline 199 & 19.9 & 39.8 & 59.7 & 79.6 & 99.5 & 119.4 & 139.3 & 159.2 & 179.1 \\
\hline 198 & 19.8 & 39.6 & 59.4 & 79.2 & 99.0 & 115.8 & 138.6 & 159.4 & 178.2 \\
\hline 197 & 19.7 & 39.4 & 59.1 & 78.8 & 98.5 & 118.2 & 137.9 & 157.6 & 177.3 \\
\hline 196 & 19.6 & 39.2 & 58.8 & 78.4 & 98.0 & 117.6 & 137.2 & 156.8 & 176.4 \\
\hline 195 & 19.5 & 39.0 & 58.5 & 78.0 & 97.5 & 117.0 & 136.5 & 156.0 & 175.5 \\
\hline 194 & 19.4 & 38.8 & 58.2 & 77. & 97.0 & 116.4 & 135.8 & 155.2 & 174.6 \\
\hline 193 & 19.3 & 38.6 & 57.9 & 77. 2 & 96.5 & 115.8 & 135.1 & 154.4 & 173.7 \\
\hline 192 & 19.2 & 38.4 & 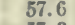 & .8 & 98.0 & 115 & 13 & 53.6 & 172.8 \\
\hline 191 & 19.1 & 38. 2 & 57.3 & 76. & 95.5 & 114.6 & 133.7 & 152.8 & 171.9 \\
\hline 190 & 19.0 & 38.0 & 57.0 & 76.0 & 95.0 & 114.0 & 133. 0 & 152.0 & 171.0 \\
\hline 189 & 18.9 & 37.8 & 56.7 & 75.6 & 94.5 & 113.4 & 132.3 & 151.2 & 170.1 \\
\hline 188 & 18.8 & 37.6 & 56.4 & 75.2 & 94.0 & 112.8 & 131.6 & 150.4 & 169.2 \\
\hline 187 & 18.7 & 37.4 & 56.1 & 74.8 & 93.5 & 112.2 & 130.9 & 149.6 & 168.3 \\
\hline 186 & 18.6 & 37.2 & 55.8 & 74. & 93.0 & 111.6 & 130.2 & 148.8 & 167.4 \\
\hline 185 & 18. 5 & 37.0 & 55.5 & 74. & 92.5 & 111.0 & 129.5 & 148.0 & 166. 5 \\
\hline 194 & 18. 4 & 36.8 & 55. 2 & 73. & 92.0 & 110.4 & 128 & 147.2 & 165. 6 \\
\hline 183 & 18.3 & 36.6 & 54.9 & 73.2 & 91.5 & 109.8 & 128.1 & 146.4 & 164.7 \\
\hline 182 & 18. 2 & 36.4 & 51. 6 & 72.8 & 91.0 & 109.2 & 127.4 & 145.6 & 163.8 \\
\hline 181 & 18.1 & 36. 2 & 54.3 & 72.4 & 90.5 & 108.6 & 126.7 & 144.8 & 162.9 \\
\hline 180 & 18.0 & 35.0 & 54.0 & 72.0 & 90.0 & 108. 0 & 126.0 & 144.0 & 162.0 \\
\hline 179 & 17.9 & 35.8 & 53.7 & 71.6 & 89.5 & 107.4 & 125.3 & 143.2 & 161.1 \\
\hline
\end{tabular}


TABLE 10.-LOGARITHMS OF NUMBERS.

No. 240 L. 380.$]$

[No. 269 L. 431.

\begin{tabular}{|c|c|c|c|c|c|c|c|c|c|c|c|}
\hline N. & 0 & 1 & 2 & 3 & 4 & 5 & 6 & 7 & 8 & 9 & Diff. \\
\hline 240 & 80211 & 0392 & 0573 & 754 & 0934 & 5 & 1296 & 1476 & 1656 & 1837 & 181 \\
\hline 1 & 2017 & & & & 2737 & & 3097 & 3277 & 6 & 36 & 180 \\
\hline 2 & 3815 & 3995 & 74 & 3 & 4533 & 4712 & 4891 & 5070 & 5249 & 5428 & 179 \\
\hline 3 & 5606 & 5785 & 44 & & 6321 & - & 6677 & 6856 & 7034 & 7212 & 178 \\
\hline \multirow{2}{*}{$\begin{array}{l}4 \\
5\end{array}$} & \multirow{2}{*}{9166} & \multirow{2}{*}{9343} & \multirow{2}{*}{$\begin{array}{l}7746 \\
9520\end{array}$} & \multirow{2}{*}{$\begin{array}{l}7924 \\
9698\end{array}$} & \multirow{2}{*}{$\begin{array}{l}8101 \\
9875\end{array}$} & 8279 & 8456 & 8634 & 8811 & 8959 & 178 \\
\hline & & & & & & & 0228 & 04 & 2 & 07.59 & 177 \\
\hline 6 & 390935 & 1112 & 288 & 1464 & 1641 & & 1993 & & & & 176 \\
\hline 7 & 2697 & 2873 & & 3224 & 3400 & 2 & 3751 & 3926 & 41 & 4277 & 176 \\
\hline 8 & 4452 & 4627 & 802 & 4977 & 5152 & 53. & 5501 & 5676 & 58 & 6025 & 175 \\
\hline 9 & 6199 & 6374 & 548 & 6722 & 6896 & 7071 & 7245 & 7419 & 7592 & 7766 & 174 \\
\hline \multirow{2}{*}{$\begin{array}{r}250 \\
1\end{array}$} & \multirow{2}{*}{$\begin{array}{l}7940 \\
9674\end{array}$} & \multirow{2}{*}{$\begin{array}{l}8114 \\
9847\end{array}$} & 8287 & 8461 & 8634 & 8808 & 8981 & 9154 & 9328 & 9501 & 173 \\
\hline & & & 0020 & 0192 & 0365 & 05 & 0711 & 0883 & 1056 & 1228 & 173 \\
\hline \multirow{6}{*}{$\begin{array}{l}2 \\
3 \\
4 \\
5 \\
6 \\
7\end{array}$} & \multirow{6}{*}{$\begin{array}{r}401401 \\
3121 \\
4834 \\
6540 \\
8240 \\
9933\end{array}$} & \multirow{5}{*}{$\begin{array}{l}1573 \\
3292 \\
5005 \\
6710 \\
8410\end{array}$} & 1715 & 1917 & 2089 & & 2433 & 26 & 27 & 2949 & 172 \\
\hline & & & 3464 & 36 & $380^{\circ}$ & 3 & 4149 & 43 & 4492 & 4663 & 171 \\
\hline & & & 6 & 53 & 551 & & 58 & 60 & 61 & 6370 & 171 \\
\hline & & & 6881 & 705 & 7221 & 7 & 7561 & 7731 & 7901 & 8070 & 170 \\
\hline & & & 8579 & 87 & 8918 & 9 & 9257 & 9426 & 9595 & 9764 & 169 \\
\hline & & \multirow{3}{*}{$\begin{array}{l}0102 \\
1788\end{array}$} & 0271 & 07 & 0005 & & U & & 12 & 14 & 1 \\
\hline 8 & 411620 & & 1956 & 212 & 2293 & & 2629 & 27 & 29 & 31 & 168 \\
\hline 9 & 3300 & & 3 & 38 & 3970 & 41 & 4305 & 4472 & 4639 & 4806 & 167 \\
\hline \multirow{4}{*}{$\begin{array}{r}260 \\
1 \\
2 \\
3\end{array}$} & \multirow{4}{*}{$\begin{array}{l}4973 \\
6641 \\
8301 \\
9956\end{array}$} & 514 & 53 & 5474 & 5 & 5308 & 5 & 61 & 63 & 6474 & 167 \\
\hline & & 68 & 69 & & 73 & & 7038 & 78 & 79 & 8135 & 166 \\
\hline & & 8467 & 8633 & 8798 & 8964 & 9129 & 9295 & 9460 & 9625 & 9791 & 165 \\
\hline & & \multirow{7}{*}{$\begin{array}{l}0121 \\
1768 \\
3410 \\
5045 \\
6674 \\
8297 \\
9914\end{array}$} & 0 & 045 & 061 & & 5 & 0 & & 1 & 1 \\
\hline 4 & 421604 & & 1933 & 20 & & & & & & 3082 & 164 \\
\hline & 3246 & & 357 & 37 & 39 & & 42 & & & 4718 & 164 \\
\hline f & 4882 & & 52 & 53 & 55 & 50 & 58 & 60 & 61 & 6349 & 163 \\
\hline 7 & 6511 & & 6 & 69 & 71 & & 74 & 76 & & 7973 & 162 \\
\hline \multirow{2}{*}{$\begin{array}{l}8 \\
9\end{array}$} & \multirow{2}{*}{$\begin{array}{r}8135 \\
9752 \\
43\end{array}$} & & 8459 & 8621 & 8783 & 8944 & 9106 & 9268 & 9429 & 9591 & 162 \\
\hline & & & 0075 & 0236 & 0398 & 0559 & 0720 & 0881 & 1042 & 1203 & 161 \\
\hline
\end{tabular}

Proportional Parts.

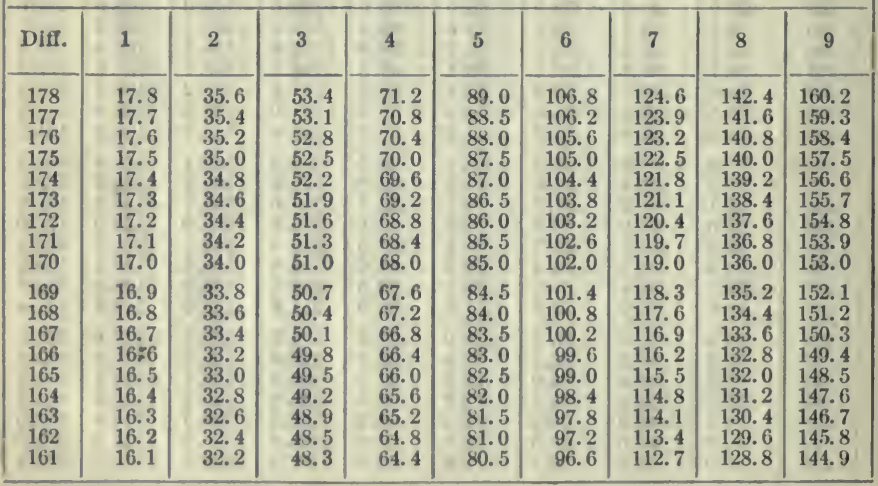


TAB LE 10.-LOGARITIMS OF NUMBERS.

No. 270 L. 431.]

[No. 299 L. 476.

\begin{tabular}{|c|c|c|c|c|c|c|c|c|c|c|c|}
\hline N. & 0 & 1 & 2 & 3 & 4 & 5 & 6 & 7 & 8 & 9 & Diff. \\
\hline 270 & 431364 & 15 & 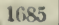 & 6 & 007 & 167 & 223 & 2458 & 2649 & 2809 & 161 \\
\hline 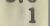 & & & & & & & & 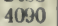 & & & 160 \\
\hline 2 & 9 & & & 5048 & 52 & & 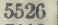 & 56 & 5844 & 6004 & 159 \\
\hline 3 & 61 & & & & 679 & 37 & 110 & 7275 & 7433 & 7592 & 159 \\
\hline \multirow{2}{*}{$\begin{array}{l}4 \\
5\end{array}$} & \multirow{2}{*}{$\begin{array}{l}7751 \\
9333\end{array}$} & 7909 & 8067 & \multirow{2}{*}{$9 S 06$} & \multirow{2}{*}{9964} & 8542 & 8701 & 8859 & 9017 & 9175 & 158 \\
\hline & & 9491 & 9648 & & & 0122 & 0279 & 0437 & 0594 & 0752 & 158 \\
\hline 6 & 440909 & 1066 & 12. & 13 & 153 & & & 20 & & 2323 & 157 \\
\hline 7 & & & & & & 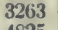 & & 35 & 3732 & 3859 & 157 \\
\hline 8 & 40 & 420 & 4 & 45 & 46 & $\$ 25$ & 49 & 51 & 5293 & 5449 & 156 \\
\hline 9 & 1 & 5 & j’ & 6 & 60 & 6382 & 60 & 66 & 6848 & 7003 & 155 \\
\hline \multirow{2}{*}{$\begin{array}{r}280 \\
1\end{array}$} & \multirow{2}{*}{$\begin{array}{l}7158 \\
8706\end{array}$} & \multirow{2}{*}{$\begin{array}{l}7313 \\
8861\end{array}$} & \multirow{2}{*}{$\begin{array}{l}7468 \\
9015\end{array}$} & \multirow{2}{*}{$\begin{array}{l}7623 \\
9170\end{array}$} & \multirow{2}{*}{$\begin{array}{l}7779 \\
9324 \\
\end{array}$} & \multirow{2}{*}{$\begin{array}{l}7933 \\
9478\end{array}$} & \multirow{2}{*}{$\begin{array}{l}8088 \\
9633 \\
\end{array}$} & \multirow{2}{*}{$\begin{array}{l}8242 \\
9787\end{array}$} & \multirow{2}{*}{$\begin{array}{l}8397 \\
9941\end{array}$} & 8552 & \multirow[t]{2}{*}{155} \\
\hline & & & & & & & & & & & \\
\hline 2 & 450249 & 403 & & & $86^{5}$ & 8 & 172 & 13 & 1479 & & 154 \\
\hline 3 & & 1 & & 7 & $2 \times$ & & & 28 & 3012 & 3165 & 153 \\
\hline 4 & 3 & 3471 & & & & & 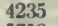 & 43 & 4540 & 4692 & 153 \\
\hline 5 & 45 & $49 ?$ & s & b3 & 545 & 5606 & 57 & 59 & 6062 & 6214 & 152 \\
\hline 6 & 6366 & 651 & 6 & $6 \mathrm{~s}$ & 69 & 7125 & 7276 & 74 & 7579 & 7731 & 152 \\
\hline \multirow{3}{*}{8} & \multirow{2}{*}{9392} & $\begin{array}{l}8033 \\
9543\end{array}$ & $\begin{array}{l}8184 \\
9694\end{array}$ & \multirow{2}{*}{9845} & \multirow{2}{*}{9995} & 8638 & 8789 & 89 & 9091 & 9242 & 151 \\
\hline & & 9543 & 9694 & & & \multirow{2}{*}{$\begin{array}{l}0146 \\
1649\end{array}$} & \multirow{2}{*}{$\begin{array}{l}0296 \\
1799\end{array}$} & \multirow{2}{*}{$\begin{array}{l}0447 \\
1948\end{array}$} & \multirow{2}{*}{$\begin{array}{l}0597 \\
2098\end{array}$} & 0748 & \multirow{2}{*}{$\begin{array}{l}151 \\
150\end{array}$} \\
\hline & 460898 & 1048 & & 18 & & & & & & 2248 & \\
\hline & 2398 & 254 & 2 & 28 & 2997 & 3146 & 3296 & 34 & 3594 & 3744 & 150 \\
\hline & 3 & 40 & A & 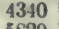 & 44 & & 4 & 49 & 5085 & 5234 & 149 \\
\hline & 58 & 553 & 56 & $5 S$ & $5 ?$ & 61 & 62 & 64 & 6571 & 6719 & 149 \\
\hline & 83 & $\begin{array}{l}701 \\
849\end{array}$ & $\begin{array}{l}7164 \\
8643\end{array}$ & $\begin{array}{l}7312 \\
8790\end{array}$ & $\begin{array}{l}7460 \\
8938\end{array}$ & $\begin{array}{l}7608 \\
9085\end{array}$ & $\begin{array}{l}7756 \\
9233\end{array}$ & $\begin{array}{l}7904 \\
9380\end{array}$ & $\begin{array}{l}8052 \\
9527\end{array}$ & $\begin{array}{l}8200 \\
9675\end{array}$ & 148 \\
\hline & & & & & & & & & & & \\
\hline 6 & 4712 & & & & & & & & $24 t$ & & 146 \\
\hline 7 & 2756 & 29 & 30 & 31 & 33 & 3487 & & 37 & 3925 & 4071 & 146 \\
\hline 8 & 42 & 43 & 45 & 46 & 47 & 49 & 50 & 52 & 5381 & 5526 & 146 \\
\hline 9 & 5671 & 5816 & 5962 & 6107 & 6252 & 6397 & 6542 & 6687 & 6832 & 6976 & 145 \\
\hline
\end{tabular}

Proportional Parts.

\begin{tabular}{|c|c|c|c|c|c|c|c|c|c|}
\hline Diff. & 1 & 2 & 3 & 4 & 5 & 6 & 7 & 8 & 9 \\
\hline 161 & 16.1 & 32.2 & 48.3 & 64.4 & 80.5 & 96.6 & 112.7 & 128.8 & 144.9 \\
\hline 160 & 16.0 & 32.0 & 48.0 & 64.0 & 80.0 & 96.0 & 112.0 & 128.0 & 144.0 \\
\hline 159 & 15.9 & 31.8 & 47.7 & 63.6 & 79.5 & 05.4 & 111.3 & 127.2 & 143.1 \\
\hline 158 & 15. 8 & 31.6 & 47.4 & 63.2 & 79.0 & 94.8 & 110.6 & 126.4 & 142.2 \\
\hline 157 & 15.7 & 31.4 & 47.1 & 62.8 & 78.5 & 94.2 & 109.9 & 125.6 & 141.3 \\
\hline 156 & 15.6 & 31.2 & 46.8 & 62.4 & 78.0 & 93.6 & 109.2 & 124.8 & 140.4 \\
\hline 155 & 15.5 & 31.0 & 46.5 & 62.0 & 77.5 & 93.0 & 108.5 & 124.0 & 139.5 \\
\hline 154 & 15.4 & 30.8 & 46.2 & 61.6 & 77. & 92.4 & 107.8 & 123. 2 & 138.6 \\
\hline 153 & 15.3 & 30.6 & 45.9 & 61.2 & 76.5 & 91.8 & 107.1 & 122.4 & 137.7 \\
\hline 152 & 15.2 & 30.4 & 45.6 & 60.8 & 76.0 & 91.2 & 108.4 & 121.6 & 136.8 \\
\hline 151 & 15.1 & 30.2 & 45.3 & 60.4 & 75.5 & 90.6 & 105.7 & 120.8 & 135.9 \\
\hline 150 & 15.0 & 30.0 & 45.0 & 60.0 & 75.0 & 90.0 & 105.0 & 120.0 & 135.0 \\
\hline 149 & 14. & 29.8 & 44. & 59.6 & 74.5 & 89.4 & 104.3 & 119.2 & 134.1 \\
\hline 148 & 14. & 29.6 & 44. & 59.2 & 74. & 88. & 10.3 .6 & 118.4 & 133.2 \\
\hline 147 & 14.7 & 29.4 & 44. & 58.8 & 73.5 & 88.2 & 102.9 & 117.6 & 132.3 \\
\hline 146 & 14.6 & 29.2 & 43. & 5.8. 4 & 73.0 & 87.6 & 102.2 & 116.8 & 131.4 \\
\hline 145 & 14.5 & 29.0 & 43. & 58.0 & 72.5 & 87.0 & 101.5 & 116.0 & 130.5 \\
\hline 144 & 14.4 & 28.8 & 43.2 & 57.6 & 72.0 & 86.4 & 100.8 & 115.2 & 129.6 \\
\hline 143 & 143 & 28.6 & 42. & 57.2 & 71.5 & 85.8 & 100.1 & 114.4 & 128.7 \\
\hline 142 & 14.2 & 28.4 & 42.6 & 56.8 & 71.0 & 85.2 & 99.4 & 113.6 & 127.8 \\
\hline 141 & 14. 1 & 28.2 & 42.3 & 56.4 & 70.5 & 84.6 & 99.7 & 112.8 & 128.9 \\
\hline 140 & 14.0 & 28.0 & 42.0 & 56.0 & 70.0 & 84.0 & 98.0 & 112.0 & 126.0 \\
\hline
\end{tabular}


TABLE 10.-LOGARITHMS OF NUMBERS.

\begin{tabular}{|c|c|c|c|c|c|c|c|c|c|c|c|}
\hline \multicolumn{9}{|c|}{ No. 3CO L. 477.] } & \multicolumn{3}{|c|}{ [No. 339 L. 531.} \\
\hline N. & 0 & 1 & 2 & 3 & 4 & 5 & 6 & 7 & 8 & 9 & Diff. \\
\hline \multirow{2}{*}{$\begin{array}{r}300 \\
1\end{array}$} & 77121 & 7206 & 411 & 7555 & 7700 & 7844 & 7989 & 8133 & 8278 & 8422 & 145 \\
\hline & 85 & & & & & & & & 9719 & 9863 & 144 \\
\hline \multirow{8}{*}{8} & $480<07$ & 0151 & 0294 & 0438 & SS2 & $0 \pi 25$ & 0869 & 1012 & 1156 & 299 & 144 \\
\hline & 143 & & & & & & & & & & 143 \\
\hline & 2874 & 6 & & & & & 37 & & 4 & & 143 \\
\hline & 4300 & & & & & & & & & & 142 \\
\hline & 5721 & 5863 & & & & 0 & & 67 & 68 & & 142 \\
\hline & 7128 & 7280 & 742 & & & & 19 & & 02 & 0 & 141 \\
\hline & 8551 & 8692 & 883 & 8974 & 9114 & 55 & 9396 & 9537 & 9677 & 9818 & 141 \\
\hline & & 0099 & 0239 & 0380 & 0520 & 0661 & 0801 & 0941 & 1081 & 1222 & 140 \\
\hline \multirow{7}{*}{$\begin{array}{r}310 \\
1 \\
2 \\
3 \\
4 \\
5 \\
6\end{array}$} & 491362 & 1502 & 16 & 82 & 22 & 62 & 2201 & 1 & 2481 & 621 & 140 \\
\hline & 2760 & & & & & & & & $3 S$ & & 139 \\
\hline & 4155 & 4294 & & & & & & 5 & 5267 & & 139 \\
\hline & 5514 & 56 & & & 60 & & & & 60 & & 139 \\
\hline & 6930 & 7068 & 72 & & 74 & 1 & 77 & 78 & 8035 & 73 & 138 \\
\hline & 8311 & 8448 & 85 & 8724 & 8862 & 8999 & 9137 & 9275 & 9412 & 9550 & 138 \\
\hline & & & & \multirow{4}{*}{$\begin{array}{l}0099 \\
1470 \\
2837 \\
4199\end{array}$} & \multirow{4}{*}{$\begin{array}{l}0236 \\
1607 \\
2973 \\
4335 \\
\end{array}$} & \multirow{4}{*}{$\begin{array}{l}0374 \\
1744 \\
31 C 9 \\
4471\end{array}$} & \multirow{4}{*}{$\begin{array}{l}0511 \\
1880 \\
3246 \\
4607\end{array}$} & \multirow{4}{*}{$\begin{array}{l}0648 \\
2017 \\
3332 \\
4743\end{array}$} & \multirow{4}{*}{$\begin{array}{l}0785 \\
2154 \\
3518 \\
4878\end{array}$} & \multirow{4}{*}{$\begin{array}{l}0922 \\
2291 \\
3655 \\
5014\end{array}$} & \\
\hline 7 & 501059 & 1. & 13 & & & & & & & & 137 \\
\hline 8 & & & & & & & & & & & 136 \\
\hline 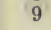 & & 39 & & & & & & & & & 136 \\
\hline \multirow{4}{*}{$\begin{array}{r}320 \\
1 \\
2 \\
3\end{array}$} & \multirow{4}{*}{$\begin{array}{r}5150 \\
6505 \\
7856 \\
9203 \\
\end{array}$} & \multirow{4}{*}{$\begin{array}{l}5286 \\
6640 \\
7991 \\
9337 \\
\end{array}$} & \multirow{4}{*}{$\begin{array}{l}5421 \\
6776 \\
8126 \\
9471 \\
\end{array}$} & \multirow{4}{*}{$\begin{array}{l}5557 \\
6911 \\
8260 \\
9606 \\
\end{array}$} & \multirow{4}{*}{$\begin{array}{l}5693 \\
7046 \\
8395 \\
9740 \\
\end{array}$} & \multirow{4}{*}{$\begin{array}{l}5828 \\
7181 \\
8530 \\
9874 \\
\end{array}$} & \multirow{3}{*}{$\begin{array}{l}5964 \\
7316 \\
8664\end{array}$} & 6099 & \multirow{3}{*}{$\begin{array}{l}6234 \\
7586 \\
8934 \\
\end{array}$} & \multirow{3}{*}{$\begin{array}{l}6370 \\
7721 \\
9068 \\
\end{array}$} & \\
\hline & & & & & & & & & & & 135 \\
\hline & & & & & & & & 8799 & & & 135 \\
\hline & & & & & & & & & 02 & & 134 \\
\hline 4 & 510545 & 0 & 081 & 947 & 1 & 12 & & & & & 134 \\
\hline 5 & & & & 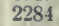 & & & & & & & 133 \\
\hline 6 & 321 & 3 & & & & & & & 42 & & 133 \\
\hline 7 & - & 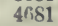 & & 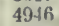 & sin & 52 & 53 & 5 & 54 & & 133 \\
\hline 8 & & f & & & 64 & & 60 & & 69 & & 132 \\
\hline 9 & & & & & 7724 & 5.5 & 7987 & 8119 & 8 & 82 & 132 \\
\hline 330 & 8514 & 8646 & 8777 & 8909 & 9040 & 9171 & 9303 & 9434 & 9566 & 9697 & 131 \\
\hline & & & 00 & 0 & & & & & & & \\
\hline 2 & 5211 & 1 & & & & & & & & & 131 \\
\hline 3 & 24 & & & & & & & & & & \\
\hline 4 & $37: 16$ & 38 & & 41 & 42 & & 45 & 46 & 47 & & 130 \\
\hline & $=0$ & 51 & 53 & 51 & 55 & & 58 & & 60 & 6210 & 129 \\
\hline 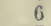 & 63 & 64 & & 67 & & 69 & 71 & & 73 & 7501 & 129 \\
\hline 7 & 76 & & & & & & 84 & & 86 & 8788 & 129 \\
\hline & & & & 93 & 943 & & & 9 & & & \\
\hline 9 & 530200 & 0328 & 0456 & 0584 & 0712 & 0810 & C368 & 1096 & 1223 & 1351 & 128 \\
\hline
\end{tabular}

Proportional Parts.

\begin{tabular}{|c|c|c|c|c|c|c|c|c|c|}
\hline Diff. & 1 & 2 & 3 & 4 & 5 & 6 & 7 & 8 & 9 \\
\hline 139 & 13.9 & 27.8 & 41.7 & 55.6 & 69.5 & 83.4 & 97.3 & 111.2 & 125.1 \\
138 & 13.8 & 27.6 & 41.4 & 55.2 & 69.0 & 82.8 & 96.6 & 110.4 & 124.2 \\
137 & 13.7 & 27.4 & 41.1 & 51.8 & 68.5 & 82.2 & 95.9 & 103.6 & 123.3 \\
136 & 13.6 & 27.2 & 40.8 & 51.4 & 68.0 & 81.6 & 95.2 & 108.8 & 122.4 \\
135 & 13.5 & 27.0 & 40.5 & 54.0 & 67.5 & 81.0 & 94.5 & 108.0 & 121.5 \\
134 & 13.4 & 26.8 & 40.2 & 53.6 & 67.0 & 80.4 & 93.8 & 107.2 & 12.6 \\
133 & 13.3 & 26.6 & 39.9 & 53.2 & 66.5 & 79.8 & 93.1 & 106.4 & 119.7 \\
132 & 13.2 & 26.4 & 39.6 & 52.8 & 66.0 & 79.2 & 92.4 & 105.6 & 118.8 \\
131 & 13.1 & 26.2 & 39.3 & 52.4 & 65.5 & 79.6 & 91.7 & 104.8 & 117.9 \\
130 & 13.0 & 26.0 & 39.0 & 52.0 & 65.0 & 78.0 & 91.0 & 164.0 & 117.0 \\
123 & 12.9 & 25.8 & 38.7 & 51.6 & 64.5 & 77.4 & 90.3 & 103.2 & 116.1 \\
128 & 12.8 & 25.6 & 38.4 & 51.2 & 64.0 & 76.8 & 89.6 & 102.4 & 115.2 \\
127 & 12.7 & 25.4 & 38.1 & 50.8 & 63.5 & 76.2 & 89.9 & 101.6 & 114.3 \\
\hline 127
\end{tabular}


TABLE 10.-LOGAIRTIMS OF NUMBEIR.

No. 340 L. 531.$]$

[No. 379 L. 579.

\begin{tabular}{|c|c|c|c|c|c|c|c|c|c|c|c|}
\hline N. & 0 & 1 & 2 & 3 & 4 & 5 & 6 & 7 & 8 & 9 & Diff. \\
\hline $\begin{array}{r}340 \\
1 \\
2 \\
3 \\
4 \\
5 \\
5 \\
6\end{array}$ & $\begin{array}{r}531479 \\
2754 \\
4026 \\
5294 \\
6558 \\
7819 \\
9076\end{array}$ & $\begin{array}{l}1607 \\
2882 \\
4153 \\
5421 \\
6685 \\
7945 \\
9202\end{array}$ & $\begin{array}{l}1734 \\
3009 \\
4280 \\
5547 \\
6811 \\
8071 \\
9327\end{array}$ & $\begin{array}{l}1862 \\
3136 \\
4407 \\
5074 \\
6937 \\
8197 \\
9452\end{array}$ & $\begin{array}{l}1950 \\
3264 \\
4534 \\
5800 \\
7063 \\
8322 \\
9578\end{array}$ & $\begin{array}{l}2117 \\
3391 \\
4661 \\
5927 \\
7189 \\
8448 \\
9703\end{array}$ & $\begin{array}{l}2245 \\
3518 \\
4787 \\
6053 \\
7315 \\
8574 \\
9829\end{array}$ & $\begin{array}{l}2372 \\
3645 \\
4914 \\
6180 \\
7441 \\
8699 \\
9954\end{array}$ & $\begin{array}{l}2500 \\
3772 \\
5041 \\
6306 \\
7567 \\
8825\end{array}$ & $\begin{array}{l}2627 \\
3899 \\
5167 \\
6432 \\
7693 \\
8951\end{array}$ & $\begin{array}{l}128 \\
127 \\
127 \\
126 \\
126 \\
126\end{array}$ \\
\hline $\begin{array}{l}7 \\
8 \\
9\end{array}$ & $\begin{array}{r}540329 \\
1579 \\
2 S 25\end{array}$ & $\begin{array}{l}0455 \\
1704 \\
2950\end{array}$ & $\begin{array}{l}0580 \\
1829 \\
3074\end{array}$ & $\begin{array}{l}0705 \\
1953 \\
3199\end{array}$ & $\begin{array}{l}0830 \\
2078 \\
3323\end{array}$ & $\begin{array}{l}0955 \\
2203 \\
3447\end{array}$ & $\begin{array}{l}1080 \\
2327 \\
3571\end{array}$ & $\begin{array}{l}1205 \\
2452 \\
369 j\end{array}$ & $\begin{array}{l}0079 \\
1330 \\
2576 \\
3820\end{array}$ & $\begin{array}{l}0204 \\
1454 \\
2701 \\
3944\end{array}$ & $\begin{array}{l}125 \\
125 \\
125 \\
124\end{array}$ \\
\hline $\begin{array}{r}350 \\
1 \\
2 \\
3 \\
4\end{array}$ & $\begin{array}{l}4068 \\
5307 \\
6543 \\
7775 \\
9003\end{array}$ & $\begin{array}{l}4192 \\
5431 \\
6666 \\
7898 \\
9126\end{array}$ & $\begin{array}{l}4316 \\
5555 \\
6789 \\
8021 \\
9249\end{array}$ & $\begin{array}{l}4440 \\
5678 \\
6913 \\
8144 \\
9371\end{array}$ & $\begin{array}{l}4564 \\
5802 \\
7036 \\
8267 \\
9494\end{array}$ & $\begin{array}{l}4689 \\
5925 \\
7159 \\
8389 \\
9616\end{array}$ & $\begin{array}{l}4812 \\
6049 \\
7252 \\
8512 \\
9739\end{array}$ & $\begin{array}{l}4936 \\
6172 \\
7405 \\
8635 \\
9861\end{array}$ & $\begin{array}{l}5060 \\
6296 \\
7529 \\
8758 \\
9984\end{array}$ & $\begin{array}{l}5183 \\
6419 \\
7652 \\
8581\end{array}$ & $\begin{array}{l}124 \\
124 \\
123 \\
123\end{array}$ \\
\hline $\begin{array}{l}5 \\
6 \\
7 \\
8 \\
9\end{array}$ & $\begin{array}{r}550228 \\
1450 \\
2668 \\
3553 \\
5094\end{array}$ & $\begin{array}{l}0351 \\
1572 \\
2790 \\
4004 \\
5215\end{array}$ & $\begin{array}{l}0473 \\
1694 \\
2911 \\
4126 \\
5336\end{array}$ & $\begin{array}{l}0595 \\
1816 \\
3033 \\
4247 \\
5457\end{array}$ & $\begin{array}{l}0717 \\
1938 \\
3155 \\
4368 \\
5578\end{array}$ & $\begin{array}{l}0540 \\
2060 \\
3276 \\
4489 \\
5699\end{array}$ & $\begin{array}{l}0962 \\
2181 \\
3398 \\
4610 \\
5820\end{array}$ & $\begin{array}{l}1084 \\
2303 \\
3519 \\
4731 \\
5940\end{array}$ & $\begin{array}{l}1206 \\
2425 \\
3640 \\
4 S 52 \\
6061\end{array}$ & $\begin{array}{l}1358 \\
2547 \\
3762 \\
4973 \\
6182\end{array}$ & $\begin{array}{l}122 \\
122 \\
121 \\
121 \\
121\end{array}$ \\
\hline $\begin{array}{r}360 \\
1 \\
2\end{array}$ & $\begin{array}{l}6303 \\
7507 \\
8709\end{array}$ & $\begin{array}{l}6423 \\
7627 \\
8829\end{array}$ & $\begin{array}{l}6544 \\
7748 \\
8948\end{array}$ & $\begin{array}{l}6664 \\
7868 \\
9068\end{array}$ & $\begin{array}{l}6785 \\
7988 \\
9188\end{array}$ & $\begin{array}{l}6905 \\
8108 \\
9308\end{array}$ & $\begin{array}{l}7026 \\
8228 \\
9428\end{array}$ & $\begin{array}{l}7146 \\
8349 \\
9548\end{array}$ & $\begin{array}{l}7267 \\
8469 \\
9667\end{array}$ & $\begin{array}{l}7387 \\
8559 \\
9787\end{array}$ & $\begin{array}{l}120 \\
120 \\
120\end{array}$ \\
\hline $\begin{array}{l}4 \\
5 \\
6 \\
7 \\
8 \\
9\end{array}$ & $\begin{array}{r}561101 \\
2293 \\
34 S 1 \\
4666 \\
5848 \\
7026\end{array}$ & $\begin{array}{l}0026 \\
1221 \\
2412 \\
3600 \\
4784 \\
5966 \\
7144\end{array}$ & $\begin{array}{l}0146 \\
1340 \\
2531 \\
3718 \\
4903 \\
6084 \\
7262\end{array}$ & $\begin{array}{l}0265 \\
1459 \\
2650 \\
3837 \\
5021 \\
6202 \\
7379\end{array}$ & \begin{tabular}{l|l|}
0385 \\
1578 \\
2769 \\
3955 \\
5139 \\
6320 \\
7497
\end{tabular} & $\begin{array}{l}0504 \\
1698 \\
2887 \\
4074 \\
5257 \\
6137 \\
7614\end{array}$ & $\begin{array}{l}0624 \\
1817 \\
3006 \\
4192 \\
53376 \\
6555 \\
7732 \\
\end{array}$ & $\begin{array}{l}0743 \\
1936 \\
3125 \\
4311 \\
5494 \\
6973 \\
7849\end{array}$ & $\begin{array}{l}0863 \\
2055 \\
3244 \\
4429 \\
5612 \\
6791 \\
7967\end{array}$ & $\begin{array}{l}0982 \\
2174 \\
3362 \\
4548 \\
5730 \\
6909 \\
8084\end{array}$ & $\begin{array}{l}119 \\
119 \\
119 \\
119 \\
118 \\
118 \\
118\end{array}$ \\
\hline $\begin{array}{r}370 \\
1\end{array}$ & $\begin{array}{l}8202 \\
9374\end{array}$ & $\begin{array}{l}8319 \\
9491\end{array}$ & $\begin{array}{l}8436 \\
9608\end{array}$ & $\begin{array}{l}8554 \\
9725\end{array}$ & $\begin{array}{l}8671 \\
9842\end{array}$ & $\begin{array}{l}8783 \\
9959\end{array}$ & 8905 & 9023 & 9140 & 9257 & 117 \\
\hline $\begin{array}{l}2 \\
3 \\
4 \\
5 \\
6 \\
6 \\
7 \\
8 \\
9\end{array}$ & $\begin{array}{r}570543 \\
1709 \\
2872 \\
4031 \\
5188 \\
6341 \\
7492 \\
8639\end{array}$ & $\begin{array}{l}0660 \\
1825 \\
2958 \\
4147 \\
5303 \\
6457 \\
7607 \\
8754\end{array}$ & $\begin{array}{l}0776 \\
1942 \\
3104 \\
4263 \\
5419 \\
6572 \\
7722 \\
8868\end{array}$ & $\begin{array}{l}0593 \\
2059 \\
3220 \\
4379 \\
5534 \\
66987 \\
7836 \\
8983\end{array}$ & $\begin{array}{l}1010 \\
2174 \\
3336 \\
4494 \\
5650 \\
6 S 02 \\
7951 \\
9097\end{array}$ & $\begin{array}{l}1126 \\
2291 \\
3452 \\
4610 \\
5765 \\
6917 \\
8066 \\
9212\end{array}$ & $\begin{array}{l}1243 \\
2407 \\
3568 \\
4726 \\
5530 \\
7032 \\
8181 \\
9326\end{array}$ & $\begin{array}{l}1359 \\
2523 \\
3654 \\
4841 \\
5996 \\
7147 \\
8295 \\
9441\end{array}$ & $\begin{array}{l}1476 \\
2639 \\
3500 \\
4957 \\
6111 \\
7262 \\
8410 \\
9555\end{array}$ & $\begin{array}{l}0420 \\
1592 \\
2755 \\
3915 \\
5072 \\
6226 \\
7377 \\
8525 \\
9669\end{array}$ & $\begin{array}{l}117 \\
116 \\
116 \\
116 \\
115 \\
115 \\
115 \\
114 .\end{array}$ \\
\hline
\end{tabular}

Proportional Parts.

\begin{tabular}{|c|c|c|c|c|c|c|c|c|c|}
\hline Diff. & 1 & 2 & 3 & 4 & 5 & 6 & 7 & 8 & 9 \\
\hline 128 & 12.8 & 25.6 & 38.4 & 51.2 & 64.0 & 76.8 & 89.6 & 102.4 & 115.2 \\
127 & 12.7 & 25.4 & 35.1 & 50.8 & 63.5 & 76.2 & 89.9 & 101.6 & 114.3 \\
126 & 12.6 & 25.2 & 37.8 & 50.4 & 63.0 & 75.6 & 89.2 & 100.8 & 113.4 \\
125 & 12.5 & 25.0 & 37.5 & 50.0 & 62.5 & 75.0 & 87.5 & 100.0 & 112.5 \\
124 & 12.4 & 24.8 & 37.2 & 49.6 & 62.0 & 74.4 & 86.8 & 99.2 & 111.6 \\
123 & 12.3 & 24.6 & 36.9 & 49.2 & 61.5 & 73.8 & 86.1 & 98.4 & 110.7 \\
122 & 12.2 & 24.4 & 36.6 & 49.8 & 61.0 & 73.2 & 85.4 & 97.6 & 109.3 \\
121 & 12.1 & 24.2 & 36.3 & 49.4 & 60.5 & 72.6 & 84.7 & 96.8 & 108.9 \\
120 & 12.0 & 24.0 & 36.0 & 45.0 & 60.0 & 72.0 & 84.0 & 96.0 & 108.0 \\
119 & 11.9 & 23.8 & 35.7 & 47.6 & 59.5 & 71.4 & 83.3 & 95.2 & 107.1 \\
\hline
\end{tabular}


TABLE 10.-LOGARITHMS OF NUMBERS.

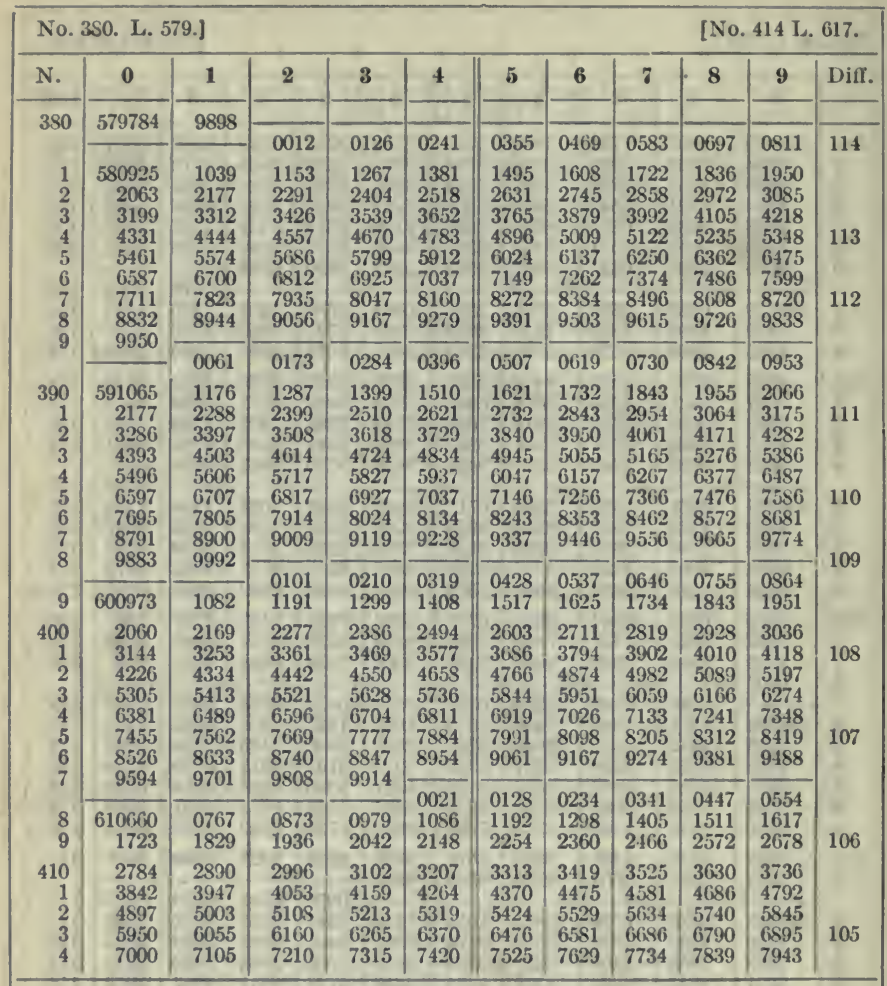

Proportional Parts.

\begin{tabular}{|c|c|c|c|c|c|c|c|c|c|}
\hline Diff. & 1 & 2 & 3 & 4 & 5 & 6 & 7 & 8 & 9 \\
\hline 118 & 11.8 & 23.6 & 35.4 & 47.2 & 59.0 & 70.8 & 82.6 & 94.4 & 106. 2 \\
\hline 117 & 11.7 & 23.4 & 35. 1 & 46.8 & 58. & 70.2 & 81.9 & 93.6 & 105.3 \\
\hline 116 & 11.6 & 23.2 & 34.8 & 46.4 & 58.0 & 69.6 & 81.2 & 92.8 & 104.4 \\
\hline 115 & 11.5 & 23.0 & 34.5 & 46.0 & 57.5 & 69.0 & 80.5 & 92.0 & 103.5 \\
\hline 114 & 11.4 & 22.8 & 34.2 & 45.6 & 57.0 & 68.4 & 79.8 & 91.2 & 102.6 \\
\hline 113 & 11.3 & 22.6 & 33.9 & 45.2 & 56.5 & 67.8 & 79.1 & 90.4 & 101.7 \\
\hline 112 & 11.2 & 22.4 & 33.6 & 44.8 & 56.0 & 67.2 & 78.4 & 89.6 & 100.8 \\
\hline 111 & 11.1 & 22.2 & 33.3 & 44.4 & 55.5 & 66.6 & 77.7 & 88.8 & 99.9 \\
\hline 110 & 11.0 & 22.0 & 33.0 & 44.0 & & 66. & 77.0 & 88.0 & 99.0 \\
\hline 109 & 10.9 & 21.8 & 32.7 & 43.6 & 54. & 65.4 & 76.3 & 87.2 & 98.1 \\
\hline 108 & 10.8 & 21.6 & 32.4 & 43.2 & 54.0 & 64.8 & 75.6 & 86.4 & 97.2 \\
\hline 107 & 10.7 & 21.4 & 32.1 & 42.8 & 53.5 & 64.2 & 74.9 & 85. 6 & 96.3 \\
\hline 106 & 10.6 & 21.2 & 31.8 & 42.4 & 53.0 & 6.3 .6 & 74.2 & 84.8 & 95.4 \\
\hline 105 & 10.5 & 21. & 31.5 & 42.0 & & 63.0 & 73.5 & 84.0 & 94.5 \\
\hline 105 & 10.5 & 21.0 & 31.5 & 42.0 & 52.5 & 63.0 & 73.5 & 84.0 & 94.5 \\
\hline 104 & 10.4 & 20.8 & 31.2 & 41.6 & 52.0 & 62.4 & 72.8 & 83.2 & 93.6 \\
\hline
\end{tabular}


TABLE 10.-LOGARITIMS OF NUMBEIS.

\begin{tabular}{|c|c|c|c|c|c|c|c|c|c|c|c|}
\hline \multicolumn{9}{|c|}{ No. 415 L. 618.] } & \multicolumn{3}{|c|}{ [No. 459 L. 662.} \\
\hline N. & $\mathbf{0}$ & 1 & 2 & 3 & 4 & 5 & 6 & 7 & 8 & 9 & Diff. \\
\hline \multirow{2}{*}{$\begin{array}{r}415 \\
6\end{array}$} & \multirow{2}{*}{$\begin{array}{r}618048 \\
9093\end{array}$} & \multirow{2}{*}{$\begin{array}{l}8153 \\
9198\end{array}$} & \multirow{2}{*}{$\begin{array}{l}8257 \\
9302\end{array}$} & \multirow{2}{*}{$\begin{array}{l}8362 \\
9406\end{array}$} & \multirow{2}{*}{$\begin{array}{l}81666 \\
9511\end{array}$} & \multirow{2}{*}{$\begin{array}{l}8571 \\
9615\end{array}$} & \multirow{2}{*}{$\begin{array}{l}8676 \\
9719\end{array}$} & \multirow{2}{*}{$\begin{array}{l}8780 \\
9524\end{array}$} & \multirow{2}{*}{$\begin{array}{l}8884 \\
9928\end{array}$} & 8989 & \multirow[t]{2}{*}{105} \\
\hline & & & & & & & & & & \multirow{4}{*}{$\begin{array}{l}0032 \\
1072 \\
2110 \\
3146\end{array}$} & \\
\hline 7 & 620136 & 0240 & 0344 & 0443 & 0552 & 0656 & 0760 & 0864 & 0968 & & \multirow[t]{3}{*}{104} \\
\hline 8 & 1176 & 1280 & 1354 & 148.8 & 1592 & 1695 & 1799 & 1903 & 2007 & & \\
\hline 9 & 2214 & 2318 & 2421 & 2525 & 2628 & 2732 & 2835 & 2939 & 3042 & & \\
\hline \multirow{6}{*}{$\begin{array}{r}420 \\
1 \\
2 \\
3 \\
4 \\
5 \\
6\end{array}$} & $\begin{array}{l}3249 \\
4282\end{array}$ & $\begin{array}{l}3353 \\
4385\end{array}$ & $\begin{array}{l}3456 \\
4458\end{array}$ & $\begin{array}{l}3559 \\
4591\end{array}$ & $\begin{array}{l}3663 \\
46,95\end{array}$ & $\begin{array}{l}3766 \\
4799\end{array}$ & $\begin{array}{l}3869 \\
4901\end{array}$ & $\begin{array}{l}3973 \\
5004\end{array}$ & $\begin{array}{l}4076 \\
5107\end{array}$ & $\begin{array}{l}4179 \\
5210\end{array}$ & \\
\hline & 5312 & 5415 & 5518 & 5621 & 5724 & 5827 & $\begin{array}{l}4901 \\
5929\end{array}$ & $\begin{array}{l}5004 \\
6032\end{array}$ & 6135 & 6238 & 103 \\
\hline & 6340 & 6443 & 6546 & 6648 & 6751 & 6853 & 6956 & 7058 & 7161 & 7263 & \\
\hline & 7366 & 7468 & 7571 & 7673 & 7775 & 7878 & 7980 & 8082 & 8185 & 8287 & \\
\hline & 8389 & 8191 & 8593 & 8695 & 8797 & 8900 & 9002 & 9104 & 9206 & 9308 & 102 \\
\hline & 9410 & 9512 & 9613 & 9715 & 9817 & 9919 & 0021 & 0123 & 0224 & 0326 & \\
\hline 7 & 630428 & 0530 & 0631 & 0733 & 0835 & 0936 & 10.38 & 1139 & 1241 & 1342 & \\
\hline 8 & 1444 & 1545 & 1647 & $174 \mathrm{~S}$ & 1849 & 1951 & 2052 & 2153 & 2255 & 2356 & t \\
\hline 9 & 2457 & 2559 & 2660 & 2761 & 2862 & 2963 & 3064 & 3165 & 3266 & 3367 & \\
\hline 430 & 3468 & 3569 & 3670 & 3771 & 3572 & 3973 & 4074 & 4175 & 4276 & 4376 & 101 \\
\hline 1 & 4477 & 4578 & 4679 & 4779 & $45 s 0$ & 4981 & 5081 & 5182 & 5283 & 5383 & \\
\hline 2 & 5481 & 5584 & 5685 & 5785 & $5 S 56$ & 5986 & 6057 & 6187 & 6287 & 6388 & \\
\hline 3 & 6488 & 6588 & Gons & 6789 & 6859 & 6989 & 7059 & 7189 & 7290 & 7390 & \\
\hline 4 & 7490 & 7590 & 7690 & 7790 & 7890 & 7990 & 8090 & 8190 & 8290 & 8389 & 100 \\
\hline 5 & 8159 & 8589 & 8689 & 8789 & 8SS9 & 8988 & 9088 & 9188 & 9287 & 9387 & \\
\hline 6 & 9486 & 9586 & 9656 & 9780 & 9505 & 9981 & 0084 & 0183 & 0283 & 0382 & \\
\hline 7 & 640481 & 0581 & 0680 & 0779 & $0 \times 79$ & 0978 & 1077 & 1177 & 1276 & 1375 & \\
\hline 8 & 1474 & 1573 & 1672 & 1771 & 1871 & 1970 & 2069 & 2168 & 2267 & 2366 & \\
\hline 9 & 2465 & 2563 & 2662 & 2761 & 2860 & 2959 & 3058 & 3156 & 3255 & 3354 & 99 \\
\hline 440 & $\begin{array}{l}3453 \\
4439\end{array}$ & 3551 & 3650 & 3749 & 3547 & $3946-$ & 4044 & 4143 & $\begin{array}{l}4242 \\
5 ? 26\end{array}$ & 4340 & \\
\hline 2 & $\begin{array}{l}4439 \\
5422\end{array}$ & $\begin{array}{l}4537 \\
5521\end{array}$ & $\begin{array}{l}4636 \\
5619\end{array}$ & $\begin{array}{l}473-1 \\
5717\end{array}$ & $\begin{array}{l}4832 \\
5 \times 15\end{array}$ & $\begin{array}{l}4931 \\
5913\end{array}$ & $\begin{array}{l}5029 \\
6011\end{array}$ & $\begin{array}{l}5127 \\
6110\end{array}$ & $\begin{array}{l}5226 \\
6208\end{array}$ & $\begin{array}{l}0324 \\
6306\end{array}$ & \\
\hline 3 & 6104 & $650^{2}$ & 6000 & 6698 & 6796 & 6S94 & 6992 & 7089 & 7187 & 7255 & 98 \\
\hline 4 & 7383 & 7481 & 7579 & 7676 & 7774 & 7872 & 7969 & 8067 & 8165 & 8262 & \\
\hline 5 & 8360 & 8158 & 8555 & 8653 & 8750 & $8 \$ 48$ & 8945 & 9043 & 9140 & 9237 & 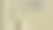 \\
\hline 6 & 9335 & 9432 & .2530 & 9627 & 9721 & $9 \$ 21$ & 9919 & 0016 & 0113 & 0210 & \\
\hline 7 & 650308 & 0405 & 0502 & 0599 & 0696 & 0793 & 0890 & 0 & 10 & 1181 & \\
\hline 8 & 1278 & 1375 & 1472 & 1569 & 1666 & 1762 & 1859 & 1956 & 2053 & 2150 & 97 \\
\hline 9 & 2246 & 2343 & 2440 & 2536 & 2633 & 2730 & 2826 & 2923 & 3019 & 3116 & \\
\hline 450 & 3213 & 3309 & 3405 & 3502 & 3598 & 3625 & 3791 & $3 s s 8$ & 3984 & 4080 & \\
\hline 1 & 4177 & 4273 & 4369 & 4465 & 4562 & 4658 & 4754 & 4850 & 4946 & 5042 & \\
\hline 2 & 5138 & 5235 & 5331 & 5427 & 5523 & 5619 & 5715 & 5810 & 5906 & 6002 & 96 \\
\hline 3 & 6098 & 6194 & 6290 & 6386 & 6452 & 6577 & 6673 & 6769 & 6864 & 6960 & \\
\hline 4 & 7056 & 7152 & 7247 & 7343 & 7438 & 7534 & 7629 & 7725 & 7820 & 7916 & \\
\hline 5 & 8011 & 8107 & 8202 & 8298 & 8393 & 8188 & 8584 & 8679 & 8774 & 8870 & \\
\hline 6 & 8955 & 9060 & 9155 & 9250 & 9346 & 9441 & 9536 & 9631 & 9726 & 9821 & \\
\hline & & 0011 & 0106 & 0201 & 0296 & 0391 & 0486 & 0581 & 0676 & $0 \pi 1$ & 95 \\
\hline 8 & 660565 & 0960 & 1055 & 1150 & 1245 & 1339 & 1434 & 1529 & 162 & 1718 & \\
\hline 9 & 1813 & 1907 & 2002 & 2006 & 2191 & 2286 & 2330 & 2475 & 2569 & 2663 & $=$ \\
\hline & & & & ROP & TIONA & $P$ & & & & & \\
\hline Diff. & 1 & 2 & 3 & 3 & & 5 & 6 & 7 & & 8 & 9 \\
\hline 105 & 10.5 & 21.0 & 31.5 & & & 52. & 63. & & & 81.0 & 94.5 \\
\hline 104 & 10. & 20. & 31 & & & & 62. & & & 2 & 93.6 \\
\hline 103 & 10. & 20. & 39.9 & & & & & & & & 92.7 \\
\hline 102 & 10.2 & 20. & 30. & & & 5 & 61. & & & 81.6 & 91.8 \\
\hline 101 & 10. & 20. & & & & & 60.6 & & & & 90.9 \\
\hline 100 & 10.0 & 20. & 30. & & & 5 & 60.0 & & & 80.0 & 90.0 \\
\hline 99 & 9.9 & 19.8 & 39.7 & & & 49.5 & 59.4 & 69. & & 79.2 & 89.1 \\
\hline
\end{tabular}


TABLE 10.-LOGARITHMS OF NUMBERS.

No. 460 L. 662 .]

[No. 499 L. 698.

\begin{tabular}{|c|c|c|c|c|c|c|c|c|c|c|c|}
\hline N. & 0 & 1 & 2 & 3 & 4 & 5 & 6 & 7 & 8 & 9 & Diff. \\
\hline 460 & 662758 & 2852 & 2947 & 3041 & 3135 & 3230 & 3324 & 3418 & 512 & 3607 & \\
\hline 1 & 3701 & 3795 & 3889 & & & 2 & 4266 & & 54 & 4548 & \\
\hline 2 & 4642 & 4736 & 4830 & 4924 & 5018 & 5112 & 5206 & 5299 & 5393 & 5487 & 94 \\
\hline 3 & 5581 & 5675 & 5769 & 5862 & 5956 & 6050 & 6143 & 6237 & 6331 & 6424 & \\
\hline 4 & 6518 & 6612 & 6705 & 6799 & 6892 & 6986 & 7079 & 7173 & 7266 & 7360 & \\
\hline 5 & 7453 & 7546 & 7640 & 7733 & 7826 & 7920 & 8013 & 8106 & 8199 & 8293 & $\mathrm{~T}$ \\
\hline 6 & 8336 & 8479 & 8572 & 8665 & 8759 & 8552 & \multirow[b]{2}{*}{9875} & 9038 & 9131 & 9224 & \\
\hline 7 & 9317 & 9410 & 9503 & 9596 & 9689 & 9782 & & 9967 & & 0153 & \multirow{3}{*}{93} \\
\hline 8 & 670246 & 0339 & 0431 & 0524 & 0617 & 0710 & 0802 & 0895 & & 1080 & \\
\hline 9 & 1173 & 126 & 1358 & 1451 & 15 & 1 & 8 & 1821 & 1913 & 2005 & \\
\hline 470 & 2098 & 2190 & 2283 & 2375 & 2467 & 2560 & 2652 & 2744 & 2836 & 2929 & \\
\hline 1 & 3021 & 3113 & 3205 & 3297 & 3390 & & 3574 & 3666 & 3758 & 3850 & \\
\hline 2 & 3942 & 4034 & 4126 & 4218 & 4310 & 4402 & 4494 & 4586 & 4677 & 4769 & 92 \\
\hline 3 & 4861 & 4953 & 5045 & 5137 & 5228 & 5320 & 5412 & 5503 & 5595 & 5687 & \\
\hline 4 & 5778 & 5870 & 5962 & 6053 & 6145 & 6236 & 6328 & 6419 & 6511 & 6602 & \\
\hline 5 & 6694 & 6785 & 6876 & 6968 & 7059 & 7151 & 7242 & 7333 & 7424 & 7516 & \\
\hline 6 & 7607 & 7698 & 7789 & 7851 & 7972 & 8063 & 8154 & 8245 & 8336 & 8427 & \\
\hline 7 & 8518 & 8609 & 8700 & 8791 & 8582 & 8973 & 9064 & 9155 & 9246 & 9337 & \multirow[t]{3}{*}{91} \\
\hline 8 & 9428 & 9519 & 9610 & 9700 & 9791 & 9882 & 9973 & \multirow{2}{*}{$\begin{array}{l}0063 \\
0970\end{array}$} & 0154 & 0245 & \\
\hline 9 & 680336 & 0426 & 0517 & 0607 & 0698 & 0789 & 0879 & & 1060 & 1151 & \\
\hline 480 & 1241 & 1332 & 1422 & 1513 & 1603 & 1693 & 34 & 1874 & 1964 & 2055 & 1 \\
\hline 1 & 2145 & 2 & 6 & 6 & 2 & 6 & 6 & 2777 & 2 & 2957 & \\
\hline 2 & 3047 & 3137 & 3227 & 3317 & 3407 & 3. & 3587 & 3677 & 3767 & 3857 & 90 \\
\hline 3 & 3947 & 4 & 4127 & 4217 & 4307 & & 4486 & 4576 & 4666 & 4756 & \\
\hline 4 & 4845 & 4935 & 5025 & 5114 & 5204 & 5294 & 5383 & 5473 & 5063 & 5652 & \\
\hline 5 & 5742 & 5831 & 5921 & 6010 & 6100 & & 6279 & 6368 & 6458 & 6547 & \\
\hline 6 & 6636 & 6726 & 6815 & 6904 & 6994 & 7083 & 7172 & 7261 & 7351 & 7440 & \\
\hline 7 & 7529 & 7618 & 7707 & 7796 & 7886 & 7975 & 8064 & 8153 & 8242 & 8331 & 89 \\
\hline 8 & 8420 & 8509 & 8598 & 8687 & 8776 & 8865 & 8953 & 9042 & 9131 & 9220 & \\
\hline \multirow[t]{2}{*}{9} & 9309 & 9398 & 9486 & 9575 & 9664 & 9753 & 9841 & 9930 & 0019 & 0107 & \\
\hline & 690196 & 0285 & 0 & $0+62$ & 0 & 9 & 0728 & 0816 & 0905 & 0993 & \\
\hline & 1081 & 1170 & 12 & 1347 & & & 1612 & 1700 & 1789 & 1877 & \\
\hline 2 & 1965 & 2053 & 2142 & 2230 & 2318 & 2406 & 2494 & 2583 & 2671 & 2759 & \\
\hline 3 & 2847 & 2935 & 3023 & 3111 & 3199 & 32 & 3375 & 3463 & 3551 & 3639 & 88 \\
\hline 4 & 3727 & 3815 & 3903 & 3991 & 40 & 41 & 4254 & 4342 & 4430 & 4517 & \\
\hline 5 & 4605 & 4693 & 4781 & 4868 & 4956 & 5044 & 5131 & 5219 & 5307 & 5394 & \\
\hline 6 & 54 & 5569 & 5657 & 5744 & 5832 & & 6007 & 6094 & 6182 & 6269 & \\
\hline 7 & 6356 & 6444 & 6531 & 6618 & 6706 & 6793 & 6880 & 6968 & 7055 & 7142 & \\
\hline 0 & 7229 & 7317 & 7404 & 7491 & 7578 & 7665 & 7752 & 7839 & 7926 & 8014 & \\
\hline 9 & 8100 & 8188 & 8275 & 8362 & 8449 & 8535 & 8622 & 8709 & 8796 & 8883 & 87 \\
\hline
\end{tabular}

Proportional Parts.

\begin{tabular}{|r|c|c|c|c|c|c|c|c|c|}
\hline Diff. & 1 & 2 & 3 & 4 & 5 & 6 & 7 & 8 & 9 \\
\hline 98 & 9.8 & 19.6 & 29.4 & 39.2 & 49.0 & 58.8 & 68.6 & 78.4 & 88.2 \\
97 & 9.7 & 19.4 & 29.1 & 33.8 & 48.5 & 58.2 & 67.9 & 77.6 & 87.3 \\
96 & 9.6 & 19.2 & 28.8 & 38.4 & 48.0 & 57.6 & 67.2 & 76.8 & 86.4 \\
95 & 9.5 & 19.0 & 28.5 & 38.0 & 47.5 & 57.0 & 66.5 & 76.0 & 85.5 \\
94 & 9.4 & 18.8 & 28.2 & 37.6 & 47.0 & 56.4 & 65.8 & 75.2 & 84.6 \\
93 & 9.3 & 18.6 & 27.9 & 37.2 & 46.5 & 55.8 & 65.1 & 74.4 & 83.7 \\
92 & 9.2 & 18.4 & 27.6 & 36.8 & 46.0 & 55.2 & 64.4 & 73.6 & 82.8 \\
91 & 9.1 & 18.2 & 27.3 & 36.4 & 45.5 & 54.6 & 63.7 & 72.8 & 81.9 \\
90 & 9.0 & 18.0 & 27.0 & 36.0 & 45.0 & 54.0 & 63.0 & 72.0 & 81.0 \\
89 & 8.9 & 17.8 & 26.7 & 35.6 & 44.5 & 53.4 & 62.3 & 71.2 & 80.1 \\
88 & 8.8 & 17.6 & 26.4 & 35.2 & 44.0 & 52.8 & 61.6 & 70.4 & 79.2 \\
87 & 8.7 & 17.4 & 26.1 & 34.8 & 43.5 & 52.2 & 60.9 & 69.6 & 78.3 \\
86 & 8.6 & 17.2 & 25.8 & 34.4 & 43.0 & 51.6 & 60.2 & 68.8 & 77.4 \\
\hline
\end{tabular}


TABLE 10.-LOGARITHMS OF NUMBERS.

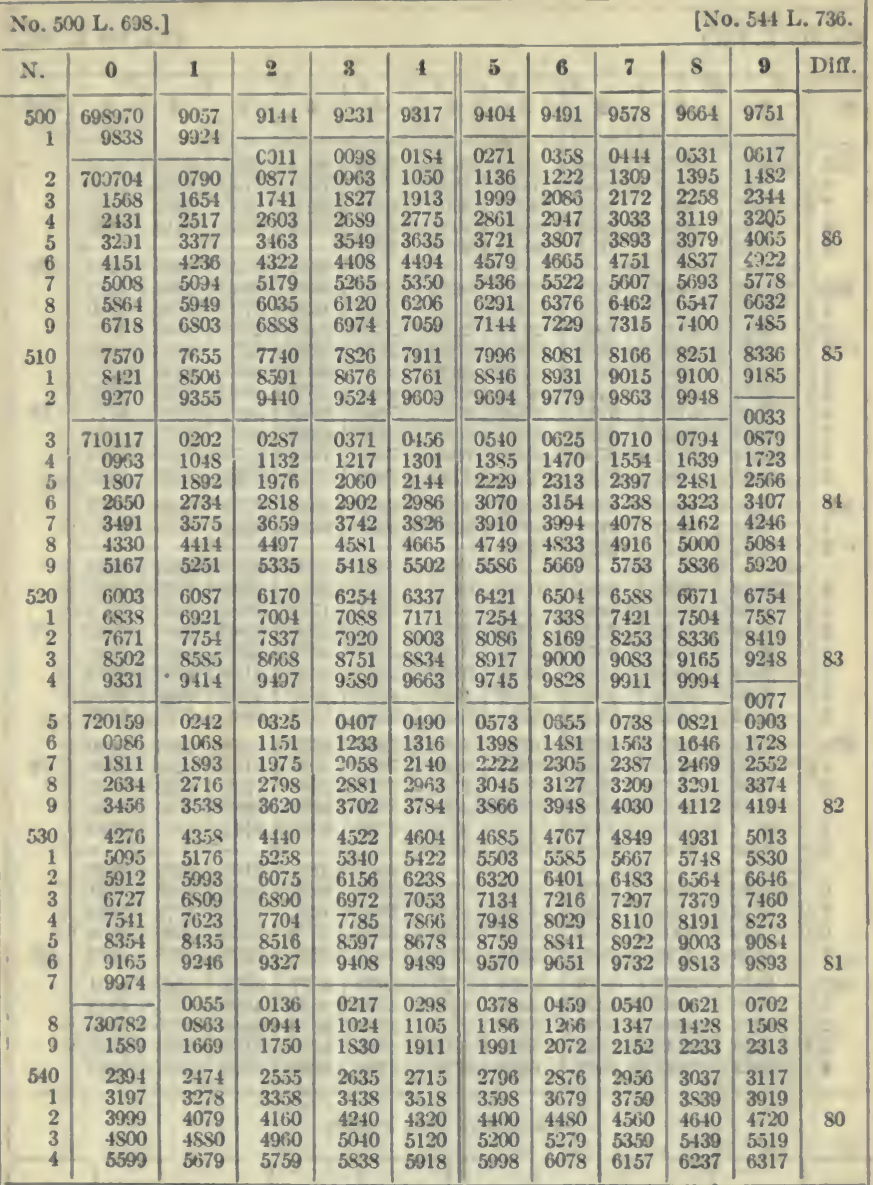

Proportional Parts.

\begin{tabular}{|c|c|c|c|c|c|c|c|c|c|c|}
\hline Diff. & 1 & 2 & 3 & 4 & 5 & 6 & 7 & 8 & 9 \\
\cline { 1 - 9 } 87 & 8.7 & 17.4 & 26.1 & 34.8 & 43.5 & 52.2 & 60.9 & 69.6 & 78.3 \\
86 & 8.6 & 17.2 & 25.8 & 34.4 & 43.0 & 51.6 & 60.2 & 68.8 & 77.4 \\
85 & 8.5 & 17.0 & 25.5 & 34.0 & 42.5 & 51.0 & 59.5 & 68.0 & 76.5 \\
84 & 8.4 & 16.8 & 25.2 & 33.6 & 42.0 & 50.4 & 58.8 & 67.2 & 75.6 \\
\hline
\end{tabular}




\begin{tabular}{|c|c|c|c|c|c|c|c|c|c|c|c|}
\hline \multicolumn{9}{|c|}{ No. 545 L. 736.$]$} & \multicolumn{3}{|c|}{ [No. 581 L. 767.} \\
\hline N. & 0 & 1 & 2 & 3 & 4 & 5 & 6 & 7 & 8 & 9 & Diff. \\
\hline \multirow{5}{*}{$\begin{array}{r}545 \\
6 \\
7 \\
8 \\
9\end{array}$} & \multirow{5}{*}{$\begin{array}{r}736397 \\
7193 \\
7987 \\
8781 \\
9572\end{array}$} & \multirow{5}{*}{$\begin{array}{l}6476 \\
7272 \\
8067 \\
8860 \\
9651\end{array}$} & \multirow{5}{*}{$\begin{array}{l}6556 \\
7352 \\
8146 \\
8939 \\
9731 \\
\end{array}$} & 66335 & \multirow{5}{*}{$\begin{array}{l}6715 \\
7511 \\
8305 \\
9097 \\
9889 \\
\end{array}$} & \multirow{5}{*}{$\begin{array}{l}6795 \\
7590 \\
8384 \\
9177 \\
9968\end{array}$} & 6874 & 6954 & \multirow{4}{*}{$\begin{array}{l}7034 \\
7829 \\
8622 \\
9414\end{array}$} & \multirow{4}{*}{$\begin{array}{l}7113 \\
7908 \\
8701 \\
9493 \\
\end{array}$} & \multirow{5}{*}{79} \\
\hline & & & & 7431 & & & 7670 & 7749 & & & \\
\hline & & & & 8225 & & & 8463 & 8543 & & & \\
\hline & & & & 9018 & & & 9256 & 9335 & & & \\
\hline & & & & 9810 & & & \multirow{2}{*}{$\begin{array}{l}0047 \\
0836\end{array}$} & 0126 & \multirow{2}{*}{0205} & \multirow{2}{*}{0284} & \\
\hline 550 & 740363 & 0442 & 0521 & 0600 & 0678 & 0757 & & 0915 & & & 19 \\
\hline 1 & 1152 & 1230 & 1309 & 1388 & 1467 & 1546 & 1624 & 1703 & 1782 & 1860 & \\
\hline 2 & 1939 & 2018 & 2096 & 2175 & 2254 & 2332 & 2411 & 2489 & 2568 & 2647 & \\
\hline 3 & 2725 & 2804 & 2882 & 2961 & 3039 & 3118 & 3196 & 3275 & 3353 & 3431 & \\
\hline 4 & 3510 & 3588 & 3667 & 3745 & 3823 & 3902 & 3980 & 4058 & 4136 & 4215 & \\
\hline 5 & 4293 & 4371 & 4449 & 4528 & 4606 & 4684 & 4762 & 4840 & 4919 & 4997 & \\
\hline 6 & 5075 & 5153 & 5231 & 5309 & 5387 & 5465 & 5543 & 5621 & 5699 & 5777 & 78 \\
\hline 7 & 5855 & 5933 & 6011 & 6089 & 6167 & 6245 & 6323 & 6401 & 6479 & 6556 & \\
\hline 8 & 6634 & 6712 & 6790 & 6868 & 6945 & 7023 & 7101 & 7179 & 7256 & 7334 & \\
\hline 9 & 7412 & 7489 & 7567 & 7645 & 7722 & 7800 & 7878 & 7955 & 8033 & 8110 & \\
\hline \multirow{3}{*}{$\begin{array}{r}560 \\
1 \\
2\end{array}$} & \multirow{3}{*}{$\begin{array}{l}8188 \\
8963 \\
9736\end{array}$} & & 8343 & 8421 & 8498 & 8576 & 8653 & 8731 & 8808 & 8885 & \\
\hline & & 9040 & 9118 & 9195 & 9272 & 9350 & 9427 & 9504 & 9582 & 9659 & \\
\hline & & & 9891 & 9968 & 0045 & 0123 & 0200 & 0277 & 0354 & 0431 & ter \\
\hline 3 & 750508 & 0586 & 0663 & 0740 & 0817 & 0894 & 0971 & 1048 & 1125 & 1202 & \\
\hline 4 & 1279 & 1356 & 1433 & 1510 & 1587 & 1664 & 1741 & 1818 & 1895 & 1972 & \\
\hline 5 & 2048 & 2125 & 2202 & 2279 & 2356 & 2433 & 2509 & $2586^{\circ}$ & 2663 & 2740 & 77 \\
\hline 6 & 2816 & 2893 & 2970 & 3047 & 3123 & 3200 & 3277 & 3353 & 3430 & 3506 & \\
\hline 7 & 3583 & 3660 & 3736 & 3813 & 3889 & 3966 & 4042 & 4119 & 4195 & 4272 & \\
\hline 8 & 4348 & 4425 & 4501 & 4578 & 4654 & 4730 & 4807 & 4883 & 4960 & 5036 & \\
\hline 9 & 5112 & 5189 & 5265 & 5341 & 5417 & 5494 & 5570 & 5646 & 5722 & 5799 & $t$ \\
\hline 570 & 5875 & 5951 & 6027 & 6103 & 6180 & 6256 & 6332 & 6408 & 6484 & 6560 & \\
\hline & 6636 & 6712 & 6788 & 6864 & 6940 & 7016 & 7092 & 7168 & 7244 & 7320 & 76 \\
\hline 2 & 7396 & 7472 & 7548 & 7624 & 7700 & 7775 & 7851 & 7927 & 8003 & 8079 & \\
\hline 3 & 8155 & 8230 & 8306 & 8382 & 8458 & 8533 & 8609 & 8685 & 8761 & 8836 & \\
\hline 4 & 8912 & 8988 & 9063 & 9139 & 9214 & 9290 & 9366 & 9441 & 9517 & 9592 & \\
\hline & 9668 & 9743 & 9819 & 9894 & 9970 & 0045 & 0121 & 196 & 0272 & 0347 & \\
\hline 6 & 760422 & 0498 & 0573 & 0649 & 0724 & 0799 & 0875 & 0950 & 1025 & 1101 & 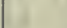 \\
\hline 7 & 1176 & 1251 & 1326 & 1402 & 1477 & 1552 & 1627 & 1702 & 1778 & 1853 & \\
\hline 8 & 1928 & 2003 & 2078 & 2153 & 2228 & 2303 & 2378 & 2453 & 2529 & 2604 & \\
\hline 9 & 2679 & 2754 & 2829 & 2904 & 2978 & 3053 & 3128 & 3203 & 3278 & 3353 & 75 \\
\hline 580 & 3428 & 3503 & 3578 & 3653 & 3727 & 3802 & 3877 & 3952 & 4027 & 4101 & $t$ \\
\hline & 4176 & 4251 & 4326 & 4400 & 4475 & 4550 & 4624 & 4699 & 4774 & 4848 & \\
\hline 2 & 4923 & 4998 & 5072 & 5147 & 5221 & 5296 & 5370 & 5445 & 5520 & 5594 & \\
\hline 3 & 5669 & 5743 & 5818 & 5892 & 5966 & 6041 & 6115 & 6190 & 6264 & 6338 & 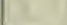 \\
\hline 4 & 6413 & 6487 & 6562 & 6636 & 6710 & 6785 & 6859 & 6933 & 7007 & 7082 & ב \\
\hline & & & & & & PAR & & & & & \\
\hline Diff. & 1 & 2 & 3 & 4 & & 5 & 6 & 7 & & 8 & 9 \\
\hline 83 & 8.3 & 16.6 & 24. & & 2 & 41.5 & 49.8 & 58 & & 66.4 & 74.7 \\
\hline 82 & 8. 2 & 16.4 & 24. & & & 41.0 & 49.2 & & & 65.6 & 73.8 \\
\hline 81 & 8.1 & 16.2 & 24 . & & & 40.5 & 48.6 & & & 64.8 & 72.9 \\
\hline 80 & 8.0 & 16.0 & 24 . & & 0 & 40.0 & 48.0 & & & 64.0 & 72.0 \\
\hline 79 & 7.9 & 15.8 & 23. & & & 39.5 & 47.4 & 55. & & 63.2 & 71.1 \\
\hline 78 & 7.8 & 15.6 & 23. & & .2 & 39.0 & 46.8 & & & 62.4 & 70.2 \\
\hline 77 & 7.7 & 15. & 23. & & 8 & 38.5 & 46.2 & 53. & & 61.6 & 69.3 \\
\hline 76 & & 15. 2 & 22. & & .4 & 38.0 & 45.6 & 53. & & 60.8 & 68.4 \\
\hline 75 & 7.5 & 15.0 & 22. & & 0 & 37.5 & 45.0 & 52. & & 60.0 & 67.5 \\
\hline 74 & 7.4 & 14.8 & 22. & & .6 & 37.0 & 44.4 & 51. & & 59.2 & 66.6 \\
\hline
\end{tabular}


TABLE 10.-LOGARITHMS OF NUMBERS.

No. 585 L. 767.]

[No. 639 L. 799.

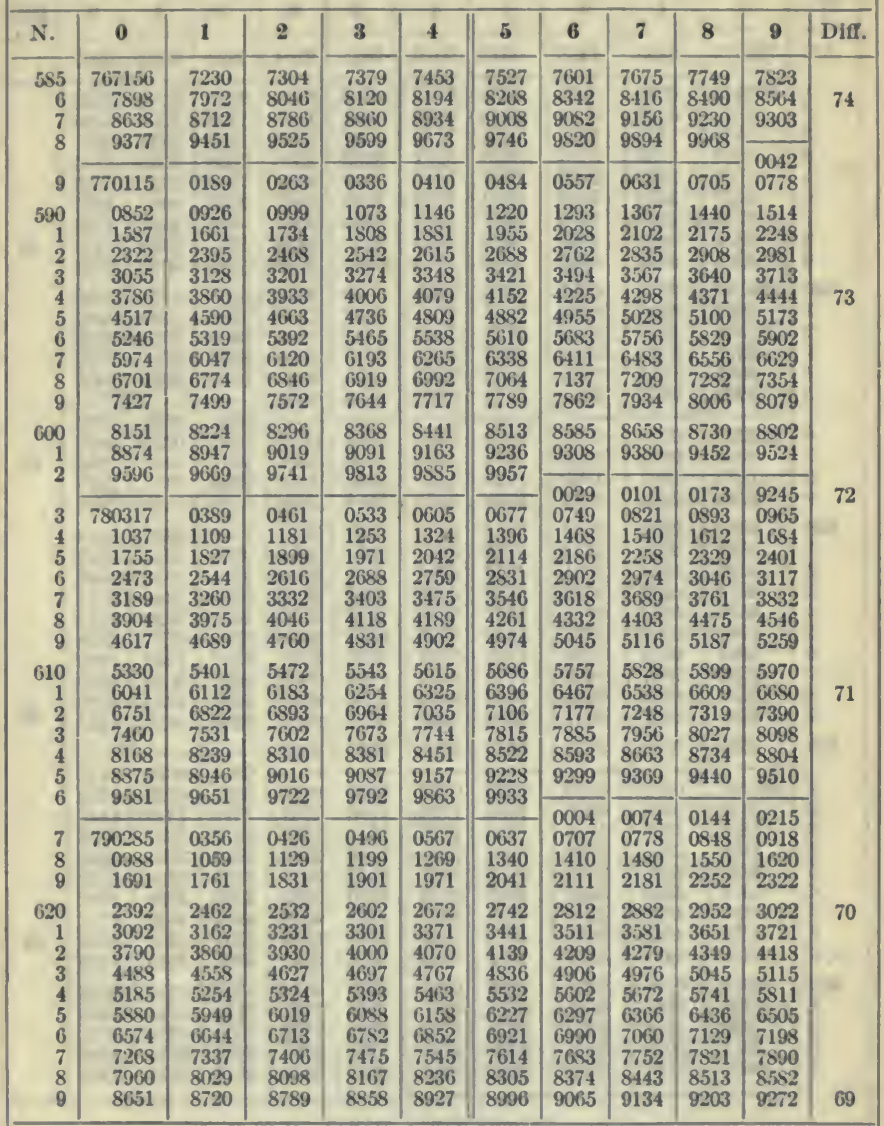

Proportional Parts.

\begin{tabular}{|c|c|c|c|c|c|c|c|c|c|}
\hline Diff. & 1 & 2 & 3 & 4 & 5 & 6 & 7 & 8 & 9 \\
\cline { 1 - 7 } 75 & 7.5 & 15.0 & 22.5 & 30.0 & 37.5 & 45.0 & 52.5 & 60.0 & 67.5 \\
74 & 7.4 & 14.8 & 22.2 & 29.6 & 37.0 & 44.4 & 51.8 & 59.2 & 66.6 \\
73 & 7.3 & 14.6 & 21.9 & 29.2 & 36.5 & 43.8 & 51.1 & 58.4 & 65.7 \\
72 & 7.2 & 14.4 & 21.6 & 28.8 & 36.0 & 43.2 & 50.4 & 57.6 & 64.8 \\
71 & 7.1 & 14.2 & 21.3 & 28.4 & 35.5 & 42.6 & 49.7 & 56.8 & 63.9 \\
70 & 7.0 & 14.0 & 21.0 & 28.0 & 35.0 & 42.0 & 49.0 & 56.0 & 63.0 \\
69 & 6.9 & 13.8 & 20.7 & 27.6 & 34.5 & 41.4 & 48.3 & 55.2 & 62.1 \\
\hline
\end{tabular}


TABLE 10.-LOGARITHMS OF NUMBERS.

\begin{tabular}{|c|c|c|c|c|c|c|c|c|c|c|c|}
\hline \multicolumn{9}{|c|}{ No. 630 L. 799.] } & \multicolumn{3}{|c|}{ [No. 674 L. 829.} \\
\hline N. & $\mathbf{0}$ & 1 & $\mathbf{2}$ & 3 & 4 & 5 & 6 & 7 & 8 & 9 & Diff. \\
\hline 630 & 799341 & 9403 & 9478 & 9547 & 9616 & 9685 & 9754 & 9823 & 9892 & 9961 & \\
\hline $\begin{array}{r}1 \\
2 \\
3 \\
4 \\
5 \\
6 \\
7 \\
8 \\
9\end{array}$ & $\begin{array}{r}800029 \\
0717 \\
1404 \\
2089 \\
2774 \\
3457 \\
4139 \\
4821 \\
5501\end{array}$ & $\begin{array}{l}0098 \\
0786 \\
1472 \\
2158 \\
2842 \\
3525 \\
4208 \\
4889 \\
5569\end{array}$ & $\begin{array}{l}0167 \\
0854 \\
1541 \\
2226 \\
2910 \\
3594 \\
4276 \\
4957 \\
5637\end{array}$ & $\begin{array}{l}0236 \\
0923 \\
1609 \\
2295 \\
2979 \\
3662 \\
4344 \\
5025 \\
5705\end{array}$ & $\begin{array}{l}0305 \\
0992 \\
1678 \\
2363 \\
5047 \\
3730 \\
4412 \\
5093 \\
5773\end{array}$ & $\begin{array}{l}0373 \\
1061 \\
1747 \\
2432 \\
3116 \\
3798 \\
4480 \\
5161 \\
5841\end{array}$ & $\begin{array}{l}0442 \\
1129 \\
1815 \\
2500 \\
3184 \\
3867 \\
4548 \\
5229 \\
5908\end{array}$ & $\begin{array}{l}0511 \\
1198 \\
1884 \\
2568 \\
3252 \\
3935 \\
4616 \\
5297 \\
5976\end{array}$ & $\begin{array}{l}0580 \\
1266 \\
1952 \\
2637 \\
3321 \\
4003 \\
4685 \\
5365 \\
6044\end{array}$ & $\begin{array}{l}0648 \\
1335 \\
2021 \\
2705 \\
3389 \\
4071 \\
4753 \\
5433 \\
6112\end{array}$ & 68 \\
\hline $\begin{array}{r}640 \\
1 \\
2 \\
3 \\
4 \\
5\end{array}$ & $\begin{array}{r}806180 \\
6858 \\
7535 \\
8211 \\
8886 \\
9560\end{array}$ & $\begin{array}{l}6248 \\
6926 \\
7603 \\
8279 \\
8953 \\
9627\end{array}$ & $\begin{array}{l}6316 \\
6994 \\
7670 \\
8346 \\
9021 \\
9694\end{array}$ & $\begin{array}{l}6384 \\
7061 \\
7738 \\
8414 \\
9088 \\
9762\end{array}$ & $\begin{array}{l}6451 \\
7129 \\
7806 \\
8481 \\
9156 \\
9829\end{array}$ & $\begin{array}{l}6519 \\
7197 \\
7873 \\
8549 \\
9223 \\
9896\end{array}$ & $\begin{array}{l}6587 \\
7264 \\
7941 \\
8616 \\
9290 \\
9964\end{array}$ & $\begin{array}{l}6655 \\
7332 \\
800 \mathrm{~S} \\
8684 \\
9358\end{array}$ & $\begin{array}{l}6723 \\
7400 \\
8076 \\
8751 \\
9425\end{array}$ & $\begin{array}{l}6790 \\
7467 \\
8143 \\
8818 \\
9492\end{array}$ & \\
\hline $\begin{array}{l}6 \\
7 \\
8 \\
9\end{array}$ & $\begin{array}{r}810233 \\
0904 \\
1575 \\
2245\end{array}$ & $\begin{array}{l}0300 \\
0971 \\
1642 \\
2312\end{array}$ & $\begin{array}{l}0367 \\
1039 \\
1709 \\
2379\end{array}$ & $\begin{array}{l}0434 \\
1106 \\
1776 \\
2445\end{array}$ & $\begin{array}{l}0501 \\
1173 \\
1843 \\
2512\end{array}$ & $\begin{array}{l}0569 \\
1240 \\
1910 \\
2579\end{array}$ & $\begin{array}{l}0636 \\
1307 \\
1977 \\
2646\end{array}$ & $\begin{array}{l}0031 \\
0703 \\
1374 \\
2044 \\
2713\end{array}$ & $\begin{array}{l}0098 \\
0770 \\
144 \\
2111 \\
2780\end{array}$ & $\begin{array}{l}0165 \\
0837 \\
1508 \\
2178 \\
2847\end{array}$ & 67 \\
\hline $\begin{array}{r}650 \\
1 \\
2 \\
3 \\
4 \\
5 \\
6 \\
7 \\
8 \\
9\end{array}$ & $\begin{array}{l}2913 \\
3581 \\
4248 \\
4913 \\
5578 \\
6241 \\
6904 \\
7565 \\
8226 \\
8585\end{array}$ & $\begin{array}{l}2980 \\
3648 \\
4314 \\
4980 \\
5644 \\
6308 \\
6970 \\
7631 \\
8292 \\
8951\end{array}$ & $\begin{array}{l}3047 \\
3714 \\
4381 \\
5046 \\
5711 \\
6374 \\
7036 \\
7698 \\
8358 \\
9017\end{array}$ & $\begin{array}{l}3114 \\
3781 \\
4447 \\
5113 \\
5777 \\
6440 \\
7102 \\
7764 \\
8424 \\
9083\end{array}$ & $\begin{array}{l}3181 \\
3848 \\
4514 \\
5179 \\
5843 \\
6506 \\
7169 \\
7830 \\
8490 \\
9149\end{array}$ & $\begin{array}{l}3247 \\
3914 \\
4581 \\
5246 \\
5910 \\
6573 \\
7235 \\
7896 \\
8556 \\
9215\end{array}$ & $\begin{array}{l}3314 \\
3981 \\
4647 \\
5312 \\
5976 \\
6639 \\
7301 \\
7962 \\
8622 \\
9281\end{array}$ & $\begin{array}{l}3381 \\
4048 \\
4714 \\
5378 \\
6042 \\
6705 \\
7367 \\
8028 \\
8658 \\
9346\end{array}$ & $\begin{array}{l}3448 \\
4114 \\
4780 \\
5445 \\
6109 \\
6771 \\
7433 \\
8094 \\
8754 \\
9412\end{array}$ & $\begin{array}{l}3514 \\
4181 \\
4847 \\
5511 \\
6175 \\
6838 \\
7499 \\
8160 \\
8820 \\
9478\end{array}$ & 66 \\
\hline 660 & 9544 & 9610 & 9676 & 9741 & 9807 & 9873 & 9939 & & & 66 & \\
\hline $\begin{array}{l}1 \\
2 \\
3 \\
4 \\
5 \\
6 \\
7 \\
8 \\
9\end{array}$ & $\begin{array}{r}820201 \\
0858 \\
1514 \\
2168 \\
2822 \\
3474 \\
4126 \\
4776 \\
5426\end{array}$ & $\begin{array}{l}0267 \\
0924 \\
1579 \\
2233 \\
2887 \\
3539 \\
4191 \\
4841 \\
5491\end{array}$ & $\begin{array}{l}0333 \\
0989 \\
1645 \\
2299 \\
2952 \\
3605 \\
4256 \\
4906 \\
5556\end{array}$ & $\begin{array}{l}0399 \\
1055 \\
1710 \\
2364 \\
3018 \\
3670 \\
4321 \\
4971 \\
5621\end{array}$ & $\begin{array}{l}0464 \\
1120 \\
1775 \\
2430 \\
3083 \\
3735 \\
4386 \\
5036 \\
5686\end{array}$ & $\begin{array}{l}0530 \\
1186 \\
1841 \\
2495 \\
3148 \\
3800 \\
4451 \\
5101 \\
5751\end{array}$ & $\begin{array}{l}0595 \\
1251 \\
1906 \\
2560 \\
3213 \\
3565 \\
4516 \\
5166 \\
5815\end{array}$ & $\begin{array}{l}0661 \\
1317 \\
1972 \\
2626 \\
3279 \\
3930 \\
4581 \\
5231 \\
5850\end{array}$ & $\begin{array}{l}0727 \\
13 \times 2 \\
2037 \\
2691 \\
3344 \\
3996 \\
4646 \\
5296 \\
5945\end{array}$ & $\begin{array}{l}0792 \\
1448 \\
2103 \\
2756 \\
3409 \\
4061 \\
4711 \\
5361 \\
6010\end{array}$ & 65 \\
\hline $\begin{array}{r}670 \\
1 \\
2 \\
3 \\
4\end{array}$ & $\begin{array}{l}6075 \\
6723 \\
7369 \\
8015 \\
8660\end{array}$ & $\begin{array}{l}6140 \\
6787 \\
7434 \\
8080 \\
8724\end{array}$ & $\begin{array}{l}6204 \\
6852 \\
7499 \\
8144 \\
8789\end{array}$ & $\begin{array}{l}6269 \\
6917 \\
7563 \\
8209 \\
8853\end{array}$ & $\begin{array}{l}6334 \\
6981 \\
7628 \\
8273 \\
8918\end{array}$ & \begin{tabular}{l|}
6399 \\
7046 \\
7692 \\
8338 \\
8982
\end{tabular} & $\begin{array}{l}6464 \\
7111 \\
7757 \\
8402 \\
9046\end{array}$ & $\begin{array}{l}6528 \\
7175 \\
7821 \\
8467 \\
9111\end{array}$ & $\begin{array}{l}6593 \\
7240 \\
7886 \\
8531 \\
9175\end{array}$ & $\begin{array}{l}6658 \\
7305 \\
7951 \\
8595 \\
9239\end{array}$ & 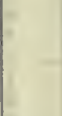 \\
\hline & & & & 然 & 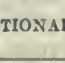 & 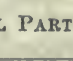 & & & & & \\
\hline Diff. & 1 & 2 & 3 & 4 & & 5 & 6 & 7 & & 8 & 9 \\
\hline $\begin{array}{l}68 \\
67 \\
66 \\
65 \\
64\end{array}$ & $\begin{array}{l}6.8 \\
6.7 \\
6.6 \\
6.5 \\
6.4\end{array}$ & $\begin{array}{l}13.6 \\
13.4 \\
13.2 \\
13.0 \\
12.8\end{array}$ & $\begin{array}{l}20.4 \\
20.1 \\
19.8 \\
19.5 \\
19.2\end{array}$ & 26. & 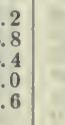 & $\begin{array}{l}34.0 \\
33.5 \\
33.0 \\
32.5 \\
32.0\end{array}$ & $\begin{array}{l}40.8 \\
40.2 \\
39.6 \\
39.0 \\
38.4\end{array}$ & $\begin{array}{l}47.6 \\
46.9 \\
46.2 \\
45.5 \\
44.8\end{array}$ & & $\begin{array}{l}54.4 \\
53.6 \\
52.8 \\
52.0 \\
51.2\end{array}$ & $\begin{array}{l}61.2 \\
60.3 \\
59.4 \\
58.5 \\
57.6\end{array}$ \\
\hline
\end{tabular}


TABLE 10.-LOGARITIMS OF NUMBERS.

No. 675 L. 829.$]$

[No. 719 L. 857.

\begin{tabular}{|c|c|c|c|c|c|c|c|c|c|c|c|}
\hline $\mathbf{N}$ & 0 & 1 & 2 & 3 & 4 & 5 & 6 & 7 & 8 & 9 & Diff. \\
\hline \multirow{2}{*}{$\begin{array}{r}675 \\
6\end{array}$} & \multirow{2}{*}{$\begin{array}{r}829304 \\
9947\end{array}$} & 9368 & 9432 & 9407 & 9561 & 9625 & 9690 & 9754 & 9818 & $98 s 2$ & \multirow{15}{*}{64} \\
\hline & & \multirow{4}{*}{$\begin{array}{l}0011 \\
0653 \\
1294 \\
1934\end{array}$} & \multirow{4}{*}{$\begin{array}{l}0075 \\
0717 \\
1358 \\
1998\end{array}$} & \multirow{4}{*}{$\begin{array}{l}0139 \\
07 \mathrm{~S} 1 \\
1422 \\
2062\end{array}$} & \multirow{4}{*}{$\begin{array}{l}0204 \\
0345 \\
14.66 \\
2126\end{array}$} & \multirow{4}{*}{$\begin{array}{l}0268 \\
0909 \\
1550 \\
2189\end{array}$} & \multirow{4}{*}{$\begin{array}{l}0332 \\
0973 \\
1614 \\
2253\end{array}$} & \multirow{4}{*}{$\begin{array}{l}0396 \\
1037 \\
1678 \\
2317\end{array}$} & \multirow{4}{*}{$\begin{array}{l}0460 \\
1102 \\
1742 \\
2381\end{array}$} & \multirow{4}{*}{$\begin{array}{l}0525 \\
1166 \\
1506 \\
2445\end{array}$} & \\
\hline 7 & 830589 & & & & & & & & & & \\
\hline 8 & $i=0$ & & & & & & & & & & \\
\hline 9 & 1870 & & & & & & & & & & \\
\hline 650 & 2509 & 2573 & 2637 & 2700 & 2764 & 2529 & 2592 & 2956 & 3020 & $30 \mathrm{~S} 3$ & \\
\hline 1 & 3147 & 3211 & 3275 & 3338 & 3402 & 3466 & 3530 & 3593 & 3657 & 3721 & \\
\hline 2 & 3784 & 3548 & 3912 & 3975 & 4039 & 4103 & 4166 & 4230 & 4294 & 4357 & \\
\hline 3 & 4421 & 4454 & 4545 & 4611 & 4675 & 4739 & 4802 & 4566 & 4929 & 4993 & \\
\hline 4 & 5056 & 5120 & $51 \$ 3$ & 5247 & 5310 & 5373 & 5437 & 5500 & 5564 & 5627 & \\
\hline 5 & 5691 & 5754 & 5817 & 5881 & 5944 & 6007 & 6071 & 6134 & 6197 & 6261 & \\
\hline 6 & 6324 & 6357 & 6451 & 6514 & 6577 & 6641 & 6704 & 6767 & 6530 & 6894 & \\
\hline 7 & 6857 & 7020 & 7083 & 7146 & 7210 & 7273 & 7336 & 7399 & 7462 & 7525 & \\
\hline 8 & $75 S 8$ & 7652 & 7715 & 7778 & 7841 & 7904 & 7967 & 8030 & 8093 & 8156 & \\
\hline 9 & 8219 & 8252 & 8345 & 8408 & 8471 & 8534 & 8597 & 8660 & 8723 & 8786 & \\
\hline 690 & 8849 & 8912 & 8975 & 9038 & 9101 & 9164 & 9227 & 9259 & 9352 & 9415 & \\
\hline 1 & 9478 & $95+1$ & 9004 & 9667 & 9729 & 9792 & 9855 & 9918 & 9981 & 004 & \\
\hline 2 & 840106 & 0169 & 0232 & 0294 & 0357 & 0420 & 0482 & 0545 & 0608 & 0671 & is \\
\hline 3 & 0733 & 0796 & 0859 & 0921 & 0254 & 1046 & 1109 & 1172 & 1234 & 1297 & \\
\hline 4 & 1359 & 1422 & 1485 & 1547 & 1610 & 1672 & 1735 & 1797 & 1860 & 1922 & \\
\hline 5 & 1985 & 2047 & 2110 & 2172 & 2235 & 2297 & 2360 & 2422 & 2454 & 2547 & \\
\hline 6 & 2609 & 2672 & 2734 & 2796 & 2559 & 2921 & 2983 & 3046 & 3108 & 3170 & \\
\hline 7 & 3233 & 3205 & 3357 & 3420 & 3452 & 3544 & 3606 & 3669 & 3731 & 3793 & \\
\hline 8 & 3555 & 3918 & 3950 & 4042 & +104 & 4160 & 4229 & 4291 & 4353 & 4415 & \\
\hline 9 & 4477 & 4539 & 4601 & 4664 & 4726 & 4785 & 4550 & 4912 & 4974 & 5036 & \\
\hline 700 & 5098 & 5160 & 5222 & 5254 & 5346 & 5408 & 5470 & 5532 & 5594 & 5656 & 62 \\
\hline 1 & 5718 & 5780 & 5842 & 5904 & 5966 & 6028 & 6090 & 6151 & 6213 & 6275 & \\
\hline 2 & 6337 & 63 & 6461 & 6523 & 6585 & 6646 & 6708 & 6770 & 6832 & 6894 & \\
\hline 3 & 6955 & 7017 & 7079 & 7141 & 7202 & 7264 & 7326 & 7358 & $7+49$ & 7511 & \\
\hline 4 & 7573 & 7634 & 7696 & 7758 & 7819 & 7881 & 7943 & 8004 & 5066 & 8129 & \\
\hline 5 & 8189 & 8251 & 8312 & 8374 & 8435 & 8497 & 8559 & 8620 & 8682 & 8743 & \\
\hline 6 & $\$ 505$ & 8866 & 8928 & 8959 & 9051 & 9112 & 9174 & 9235 & 9297 & 9358 & \\
\hline 7 & $9+19$ & 94 & 9542 & 9604 & 9665 & 9726 & 9788 & $9 S 49$ & 9911 & 9972 & \\
\hline 8 & 850033 & 00 . & 01 & $0=16$ & 0279 & 0340 & 0401 & 0462 & 0524 & 0585 & \\
\hline 9 & 0646 & 070 & 0769 & 0830 & 0891 & 0052 & 1014 & 1075 & 1136 & 1197 & - \\
\hline 710 & 1258 & 1320 & 1381 & 1442 & 1503 & 1564 & 1625 & 1656 & 1747 & 1509 & \\
\hline & $18 \pi 0$ & 1931 & 1992 & 2053 & 2114 & 2175 & 2236 & 2297 & 2358 & 2419 & \\
\hline 2 & 2450 & 25 & 2602 & 2663 & 2724 & $27 \mathrm{~S} 5$ & 2846 & 2907 & 2968 & 3029 & 61 \\
\hline 3 & 3030 & 315 & 3211 & 3272 & 3333 & 3394 & 3455 & 3516 & 3577 & 3637 & - \\
\hline 4 & 3698 & 375 & 3520 & 3551 & 3941 & 4002 & 4063 & 4124 & 4185 & 4245 & \\
\hline 5 & 4306 & 430 & 4425 & 445 & 4549 & 4610 & 4670 & $\$ 731$ & 4792 & $4 \times 52$ & \\
\hline & 4913 & $497 t$ & 5034 & 5025 & 5156 & 5216 & 5277 & 5337 & 5398 & 5459 & 1 \\
\hline$\therefore$ & 551 & 558 & 5640 & 57 & 5761 & 5822 & $58 \mathrm{~S} 2$ & 5943 & 6003 & 6064 & \\
\hline 8 & 6124 & 61 & 6245 & 6306 & 6366 & 6427 & 6487 & 6548 & 66008 & Gitios & \\
\hline 9 & 6729 & $67 \$ 9$ & 6550 & 6910 & 6970 & 7031 & 7091 & 7152 & 7212 & 7272 & \\
\hline
\end{tabular}

Proportional Parts.

\begin{tabular}{|c|c|c|c|c|c|c|c|c|c|}
\hline DIfR. & 1 & 2 & 3 & 4 & 5 & 6 & 7 & 8 & 9 \\
\hline 65 & 6.5 & 13.0 & 19.5 & 26.0 & 32.5 & 39.0 & 45.5 & 52.0 & 58.5 \\
\hline 64 & 6.4 & 12.8 & 19.2 & 25.6 & 32.0 & 38.4 & 44.8 & 51.2 & 57.6 \\
\hline 63 & 6.3 & 12.6 & 18.9 & 25.2 & 31.5 & 37.8 & 4. 1 & 50.4 & 56.7 \\
\hline 62 & 6.2 & 12.4 & 18. 6 & 24.8 & 31.0 & 37.2 & 43. 4 & 49.6 & 55.8 \\
\hline 61 & 6.1 & 12.2 & 18. 3 & 24.4 & 30.5 & 36.6 & 42.7 & 48.8 & 54.9 \\
\hline 60 & 6.0 & 12.0 & 18.0 & 24.0 & 30.0 & 36.0 & 42.0 & 48.0 & 54.0 \\
\hline
\end{tabular}


TABLE 10.-LOGARITHMS OF NUMBERS.

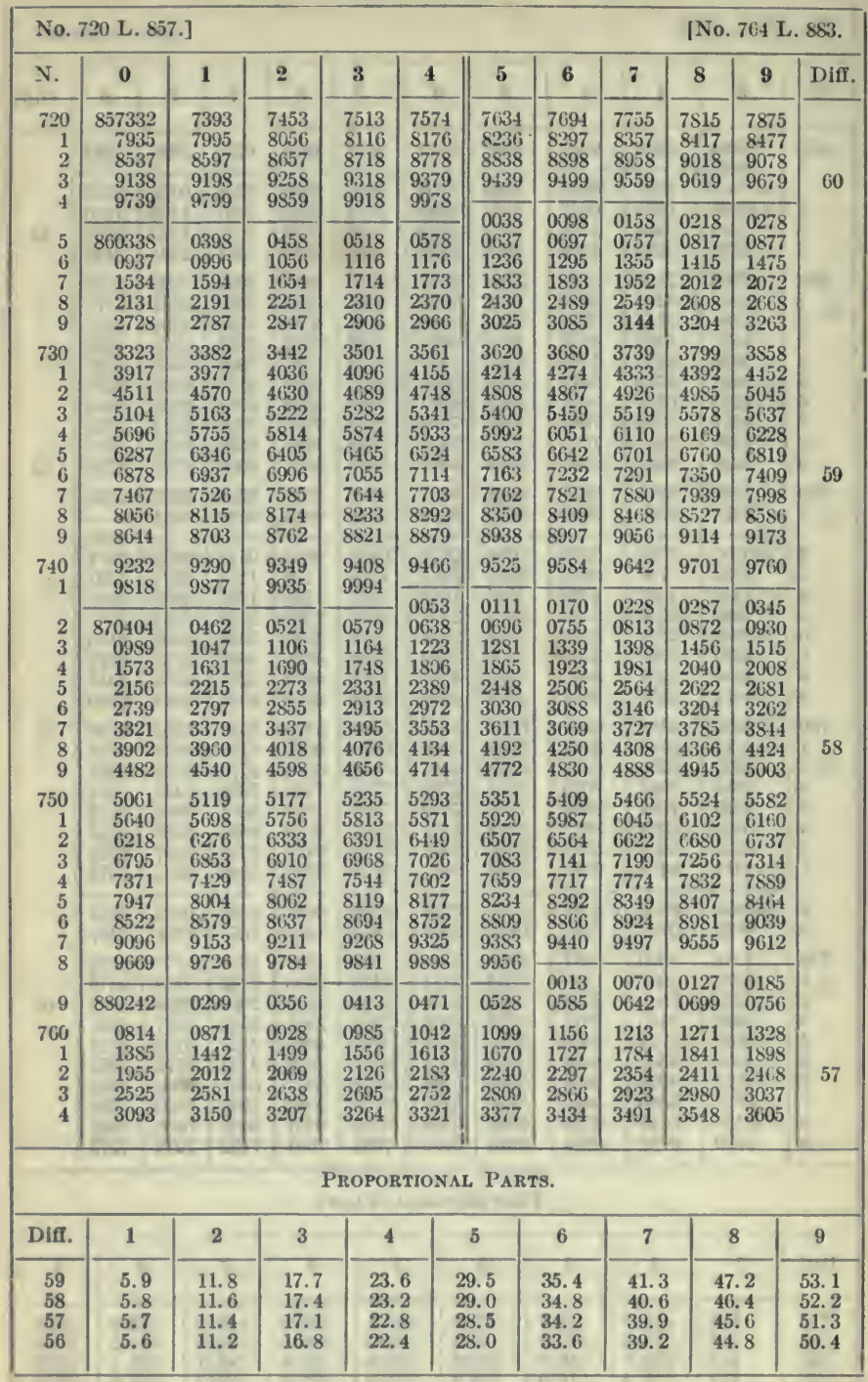


TABLE 10.-LOGARITIMS OF NUMBERS.

No. 765 L. S83.]

INo. 809 L. 908.

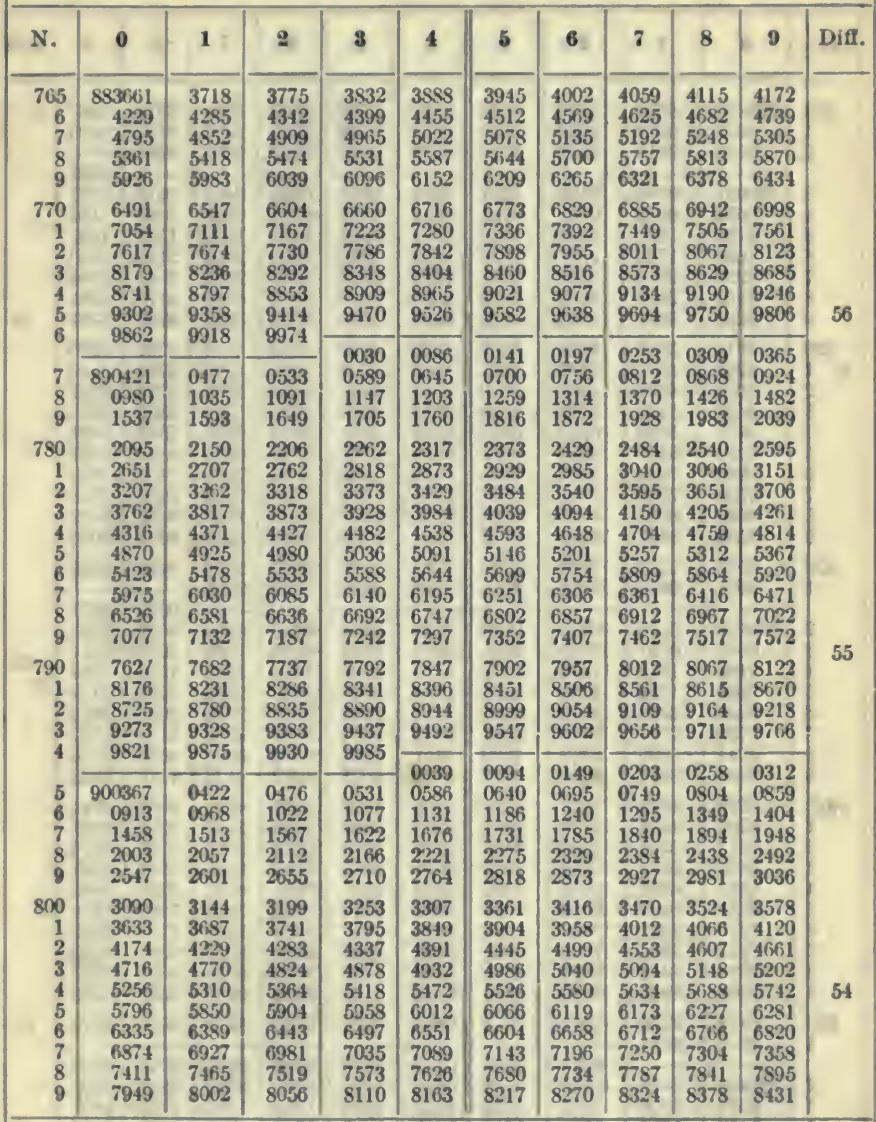

Proportional Parts.

\begin{tabular}{|c|c|c|c|c|c|c|c|c|c|}
\hline Dif. & 1 & 2 & 3 & 4 & 5 & 6 & 7 & 8 & 9 \\
\hline $\begin{array}{l}57 \\
56 \\
55 \\
54\end{array}$ & $\begin{array}{l}5.7 \\
5.6 \\
5.5 \\
5.4\end{array}$ & $\begin{array}{l}11.4 \\
11.2 \\
11.0 \\
10.8\end{array}$ & $\begin{array}{l}17.1 \\
16.8 \\
16.5 \\
16.2\end{array}$ & $\begin{array}{l}22.8 \\
22.4 \\
22.0 \\
21.6\end{array}$ & $\begin{array}{l}28.5 \\
28.0 \\
27.5 \\
27.0\end{array}$ & $\begin{array}{l}34.2 \\
33.6 \\
33.0 \\
32.4\end{array}$ & $\begin{array}{r}39.9 \\
39.2 \\
35.5 \\
37.8\end{array}$ & $\begin{array}{l}45.6 \\
44.8 \\
44.0 \\
43.2\end{array}$ & $\begin{array}{l}51.3 \\
50.4 \\
49.5 \\
48.6\end{array}$ \\
\hline
\end{tabular}


TABLE 10.-LOGARITHMS OF NUMBERS.

No. 810 L. 908.$]$

[No. 854 L. 931.

\begin{tabular}{|c|c|c|c|c|c|c|c|c|c|c|c|}
\hline N. & 0 & 1 & 2 & 3 & 4 & 5 & 6 & 7 & 8 & 9 & Diff. \\
\hline $\begin{array}{r}810 \\
1 \\
2\end{array}$ & $\begin{array}{r}908485 \\
9021 \\
9556\end{array}$ & $\begin{array}{l}8539 \\
9074 \\
9610\end{array}$ & $\begin{array}{l}8592 \\
9128 \\
9663\end{array}$ & $\begin{array}{l}8646 \\
9181 \\
9716\end{array}$ & $\begin{array}{l}8699 \\
9235 \\
9770\end{array}$ & $\begin{array}{l}8753 \\
9289 \\
9823\end{array}$ & $\begin{array}{l}8807 \\
9342 \\
9877\end{array}$ & $\begin{array}{l}8860 \\
9396 \\
9930\end{array}$ & $\begin{array}{l}8914 \\
9449 \\
9984\end{array}$ & $\begin{array}{l}8967 \\
9503\end{array}$ & \\
\hline $\begin{array}{l}3 \\
4 \\
5 \\
6 \\
7 \\
8 \\
9\end{array}$ & $\begin{array}{r}910091 \\
0624 \\
1158 \\
1690 \\
2222 \\
2753 \\
3284\end{array}$ & $\begin{array}{l}0144 \\
0678 \\
1211 \\
1743 \\
2275 \\
2806 \\
3337\end{array}$ & $\begin{array}{l}0197 \\
0731 \\
1264 \\
1797 \\
2328 \\
2859 \\
3390\end{array}$ & $\begin{array}{l}0251 \\
0784 \\
1317 \\
1850 \\
2381 \\
2913 \\
3443\end{array}$ & $\begin{array}{l}0304 \\
0838 \\
1371 \\
1903 \\
2435 \\
2966 \\
3496\end{array}$ & $\begin{array}{l}0358 \\
0891 \\
1424 \\
1956 \\
2488 \\
3019 \\
3549\end{array}$ & $\begin{array}{l}0411 \\
0944 \\
1477 \\
2009 \\
2541 \\
3072 \\
3602\end{array}$ & $\begin{array}{l}0464 \\
0998 \\
1530 \\
2063 \\
2594 \\
3125 \\
3655\end{array}$ & $\begin{array}{l}0518 \\
1051 \\
1584 \\
2116 \\
2647 \\
3178 \\
3708\end{array}$ & $\begin{array}{l}0571 \\
1104 \\
1637 \\
2169 \\
2700 \\
3231 \\
3761\end{array}$ & 53 \\
\hline $\begin{array}{r}820 \\
1 \\
2 \\
3 \\
4 \\
5 \\
6 \\
7 \\
8 \\
9\end{array}$ & $\begin{array}{l}3814 \\
4343 \\
4872 \\
5400 \\
5927 \\
6454 \\
6380 \\
7506 \\
8030 \\
8555\end{array}$ & $\begin{array}{l}3867 \\
4396 \\
4925 \\
5453 \\
5980 \\
6507 \\
7033 \\
7558 \\
8083 \\
8607\end{array}$ & $\begin{array}{l}3920 \\
4449 \\
4977 \\
5505 \\
6033 \\
6559 \\
7085 \\
7611 \\
8135 \\
8659\end{array}$ & $\begin{array}{l}3973 \\
4502 \\
5030 \\
5558 \\
6085 \\
6612 \\
7138 \\
7663 \\
8188 \\
8712\end{array}$ & $\begin{array}{l}4026 \\
4555 \\
5083 \\
5611 \\
6138 \\
6664 \\
7190 \\
7716 \\
8240 \\
8764\end{array}$ & $\begin{array}{l}4079 \\
4608 \\
5136 \\
5664 \\
6191 \\
6717 \\
7243 \\
7768 \\
8293 \\
8816\end{array}$ & $\begin{array}{l}4132 \\
4660 \\
5189 \\
5716 \\
6243 \\
6770 \\
7295 \\
7820 \\
8345 \\
8869\end{array}$ & $\begin{array}{l}4184 \\
4713 \\
5241 \\
5769 \\
6296 \\
6822 \\
7348 \\
7873 \\
8397 \\
8921\end{array}$ & $\begin{array}{l}4237 \\
4766 \\
5294 \\
5822 \\
6349 \\
6875 \\
7400 \\
7925 \\
8450 \\
8973\end{array}$ & $\begin{array}{l}4290 \\
4819 \\
5347 \\
5875 \\
6401 \\
6927 \\
7453 \\
7978 \\
8502 \\
9026\end{array}$ & \\
\hline $\begin{array}{r}830 \\
1\end{array}$ & $\begin{array}{l}9078 \\
9601\end{array}$ & $\begin{array}{l}9130 \\
9653\end{array}$ & $\begin{array}{l}9183 \\
9706\end{array}$ & $\begin{array}{l}9235 \\
9758\end{array}$ & & & $\begin{array}{l}9392 \\
9914\end{array}$ & $\begin{array}{l}9444 \\
9967\end{array}$ & 9496 & 9549 & \\
\hline $\begin{array}{l}2 \\
3 \\
4 \\
5 \\
6 \\
7 \\
8 \\
9\end{array}$ & $\begin{array}{r}920123 \\
0645 \\
1166 \\
1686 \\
2206 \\
2725 \\
3244 \\
3762\end{array}$ & $\begin{array}{l}0176 \\
0697 \\
1218 \\
1738 \\
2258 \\
2777 \\
3296 \\
3814\end{array}$ & $\begin{array}{l}0228 \\
0749 \\
1270 \\
1790 \\
2310 \\
2829 \\
3348 \\
3865\end{array}$ & $\begin{array}{l}0280 \\
0801 \\
1322 \\
1842 \\
2362 \\
2881 \\
3399 \\
3917\end{array}$ & $\begin{array}{l}0332 \\
0853 \\
1374 \\
1894 \\
2414 \\
2933 \\
3451 \\
3969\end{array}$ & $\begin{array}{l}0384 \\
0906 \\
1426 \\
1946 \\
2466 \\
2985 \\
3503 \\
4021\end{array}$ & $\begin{array}{l}0436 \\
0958 \\
1478 \\
1998 \\
2518 \\
3037 \\
3555 \\
4072\end{array}$ & $\begin{array}{l}0489 \\
1010 \\
1530 \\
2050 \\
2570 \\
3089 \\
3607 \\
4124\end{array}$ & $\begin{array}{l}0541 \\
1062 \\
1582 \\
2102 \\
2622 \\
3140 \\
3658 \\
4176\end{array}$ & $\begin{array}{l}0593 \\
1114 \\
1634 \\
2154 \\
2674 \\
3192 \\
3710 \\
4228\end{array}$ & 52 \\
\hline $\begin{array}{r}840 \\
1 \\
2 \\
3 \\
4 \\
5 \\
6 \\
7 \\
8 \\
9\end{array}$ & $\begin{array}{l}4279 \\
4796 \\
5312 \\
5828 \\
6342 \\
6857 \\
7370 \\
7883 \\
8396 \\
8908\end{array}$ & $\begin{array}{l}4331 \\
4848 \\
5364 \\
5879 \\
6394 \\
6908 \\
7422 \\
7935 \\
8447 \\
8959\end{array}$ & $\begin{array}{l}4383 \\
4899 \\
5415 \\
5931 \\
6445 \\
6959 \\
7473 \\
7986 \\
8498 \\
9010\end{array}$ & $\begin{array}{l}4434 \\
4951 \\
5467 \\
5982 \\
6497 \\
7011 \\
7524 \\
8037 \\
8549 \\
9061\end{array}$ & $\begin{array}{l}4486 \\
5003 \\
5518 \\
6034 \\
6548 \\
7062 \\
7576 \\
8088 \\
8601 \\
9112\end{array}$ & $\begin{array}{l}4538 \\
5054 \\
5570 \\
6085 \\
6600 \\
7114 \\
7627 \\
8140 \\
8652 \\
9163\end{array}$ & $\begin{array}{l}4589 \\
5106 \\
5621 \\
6137 \\
6651 \\
7165 \\
7678 \\
8191 \\
8703 \\
9215\end{array}$ & $\begin{array}{l}4641 \\
5157 \\
5673 \\
6188 \\
6702 \\
7216 \\
7730 \\
8242 \\
8754 \\
9266\end{array}$ & $\begin{array}{l}4693 \\
5209 \\
5725 \\
6240 \\
6754 \\
7208 \\
7781 \\
8293 \\
8805 \\
9317\end{array}$ & $\begin{array}{l}4744 \\
5261 \\
5776 \\
6291 \\
6805 \\
7319 \\
7832 \\
8345 \\
8857 \\
9368\end{array}$ & $=$ \\
\hline 850 & 9419 & 9470 & 9521 & 9572 & 9623 & 9674 & 9725 & 9776 & $9 \$ 27$ & 9879 & 51 \\
\hline $\begin{array}{l}2 \\
3 \\
4\end{array}$ & $\begin{array}{r}930440 \\
0949 \\
1458\end{array}$ & $\begin{array}{l}0491 \\
1000 \\
1509\end{array}$ & $\begin{array}{l}0032 \\
0542 \\
1051 \\
1560\end{array}$ & $\begin{array}{l}0083 \\
0592 \\
1102 \\
1610\end{array}$ & $\begin{array}{l}0134 \\
0643 \\
1153 \\
1661\end{array}$ & $\begin{array}{l}0185 \\
0694 \\
1204 \\
1712\end{array}$ & $\begin{array}{l}0236 \\
0745 \\
1254 \\
1763\end{array}$ & $\begin{array}{l}0287 \\
0796 \\
1305 \\
1814\end{array}$ & $\begin{array}{l}0338 \\
0847 \\
1356 \\
1865\end{array}$ & $\begin{array}{l}0389 \\
0898 \\
1407 \\
1915\end{array}$ & \\
\hline
\end{tabular}

Proportional Parts.

\begin{tabular}{|c|c|c|c|c|c|c|c|c|c|}
\hline Diff. & 1 & 2 & 3 & 4 & 5 & 6 & 7 & 8 & 9 \\
\cline { 1 - 5 } 53 & 5.3 & 10.6 & 15.9 & 21.2 & 26.5 & 31.8 & 37.1 & 42.4 & 47.7 \\
52 & 5.2 & 10.4 & 15.6 & 20.8 & 26.0 & 31.2 & 36.4 & 41.6 & 46.8 \\
51 & 5.1 & 10.2 & 15.3 & 20.4 & 25.5 & 30.6 & 35.7 & 40.8 & 45.9 \\
50 & 5.0 & 10.0 & 15.0 & 20.0 & 25.0 & 30.0 & 35.0 & 40.0 & 45.0 \\
\hline
\end{tabular}


No. 855 L. 931.]

[No. 899 L. 954 .

\begin{tabular}{|c|c|c|c|c|c|c|c|c|c|c|c|}
\hline N. & 0 & 1 & 2 & 3 & 4 & 5 & 6 & 7 & 8 & 9 & Diff. \\
\hline $\begin{array}{r}855 \\
6 \\
7 \\
8 \\
9\end{array}$ & $\begin{array}{r}931966 \\
2474 \\
2981 \\
34 S 7 \\
3993\end{array}$ & $\begin{array}{l}2017 \\
2524 \\
3031 \\
3538 \\
4044\end{array}$ & $\begin{array}{l}2068 \\
2575 \\
3052 \\
3589 \\
4094\end{array}$ & $\begin{array}{l}2118 \\
2626 \\
3133 \\
3639 \\
4145\end{array}$ & $\begin{array}{l}2169 \\
2677 \\
3183 \\
3690 \\
4195\end{array}$ & $\begin{array}{l}2220 \\
2727 \\
3234 \\
3740 \\
4246\end{array}$ & $\begin{array}{l}2271 \\
2778 \\
3285 \\
3791 \\
4296\end{array}$ & $\begin{array}{l}2322 \\
2529 \\
3335 \\
3841 \\
4347\end{array}$ & $\begin{array}{l}2372 \\
2879 \\
3386 \\
3892 \\
4397\end{array}$ & $\begin{array}{l}2423 \\
2930 \\
3437 \\
3943 \\
4448\end{array}$ & \\
\hline $\begin{array}{r}860 \\
1 \\
2 \\
3 \\
4 \\
5 \\
5 \\
7 \\
8 \\
9\end{array}$ & $\begin{array}{l}4498 \\
5003 \\
5507 \\
6011 \\
6514 \\
7016 \\
7518 \\
8019 \\
8520 \\
9020\end{array}$ & $\begin{array}{l}4549 \\
5054 \\
5558 \\
6061 \\
6564 \\
7066 \\
7568 \\
8069 \\
8570 \\
9070\end{array}$ & $\begin{array}{l}4599 \\
5104 \\
5608 \\
6111 \\
6614 \\
7116 \\
7618 \\
8119 \\
8620 \\
9120\end{array}$ & $\begin{array}{l}4650 \\
5154 \\
5658 \\
6162 \\
6665 \\
7167 \\
7665 \\
8169 \\
8670 \\
9170\end{array}$ & $\begin{array}{l}4700 \\
5205 \\
5709 \\
6212 \\
6715 \\
7217 \\
7718 \\
8219 \\
8720 \\
9220\end{array}$ & $\begin{array}{l}4751 \\
5255 \\
5759 \\
6262 \\
6765 \\
7267 \\
7769 \\
8269 \\
8770 \\
9270\end{array}$ & $\begin{array}{l}4801 \\
5306 \\
5809 \\
6313 \\
6815 \\
7317 \\
7819 \\
8320 \\
8320 \\
9320\end{array}$ & $\begin{array}{l}4852 \\
5356 \\
5860 \\
6363 \\
6865 \\
7367 \\
7869 \\
8370 \\
8870 \\
9369\end{array}$ & $\begin{array}{l}4902 \\
5406 \\
5910 \\
6413 \\
6916 \\
7418 \\
7919 \\
8420 \\
8920 \\
9419\end{array}$ & $\begin{array}{l}4953 \\
5457 \\
5960 \\
6463 \\
6966 \\
7465 \\
7969 \\
8470 \\
8970 \\
9469\end{array}$ & 50 \\
\hline 870 & 9519 & 9569 & 9619 & 9669 & 9719 & 9769 & 9819 & 9869 & 9918 & 9968 & \\
\hline $\begin{array}{l}1 \\
2 \\
3 \\
4 \\
4 \\
5 \\
6 \\
7 \\
8 \\
9\end{array}$ & $\begin{array}{r}940018 \\
0516 \\
1014 \\
1511 \\
2008 \\
2504 \\
3000 \\
3495 \\
3989\end{array}$ & $\begin{array}{l}0068 \\
0566 \\
1064 \\
1561 \\
2058 \\
2554 \\
3049 \\
3544 \\
4038\end{array}$ & $\begin{array}{l}0118 \\
0616 \\
1114 \\
1611 \\
2107 \\
2603 \\
3099 \\
3593 \\
4088\end{array}$ & $\begin{array}{l}0168 \\
0666 \\
1163 \\
1660 \\
2157 \\
2653 \\
3148 \\
3643 \\
4137\end{array}$ & $\begin{array}{l}0218 \\
0716 \\
1213 \\
1710 \\
2207 \\
2702 \\
3198 \\
3692 \\
4186\end{array}$ & $\begin{array}{l}0267 \\
0765 \\
1263 \\
1760 \\
2256 \\
2752 \\
3247 \\
3742 \\
4236\end{array}$ & $\begin{array}{l}0317 \\
0815 \\
1313 \\
1809 \\
2306 \\
2801 \\
3297 \\
3791 \\
4285\end{array}$ & $\begin{array}{l}0367 \\
0865 \\
1362 \\
1859 \\
2355 \\
2851 \\
3346 \\
3841 \\
4335\end{array}$ & $\begin{array}{l}0417 \\
0915 \\
1412 \\
1909 \\
2405 \\
2901 \\
3396 \\
3890 \\
4384\end{array}$ & $\begin{array}{l}0467 \\
0964 \\
1462 \\
1958 \\
2455 \\
2950 \\
3445 \\
3939 \\
4433\end{array}$ & \\
\hline $\begin{array}{r}880 \\
1 \\
2 \\
3 \\
4 \\
5 \\
6 \\
7 \\
8 \\
9\end{array}$ & $\begin{array}{l}4483 \\
4976 \\
5469 \\
5961 \\
6452 \\
6943 \\
7434 \\
7924 \\
8413 \\
8902\end{array}$ & $\begin{array}{l}4532 \\
5025 \\
5518 \\
6010 \\
6501 \\
6992 \\
7483 \\
7973 \\
8462 \\
8951\end{array}$ & $\begin{array}{l}4581 \\
5074 \\
5567 \\
6059 \\
6551 \\
7041 \\
7532 \\
8022 \\
8511 \\
8999\end{array}$ & $\begin{array}{l}4631 \\
5124 \\
5616 \\
6108 \\
6600 \\
7090 \\
7581 \\
8070 \\
8560 \\
0048\end{array}$ & $\begin{array}{l}4680 \\
5173 \\
5665 \\
6157 \\
6649 \\
7139 \\
7630 \\
8119 \\
8608 \\
9097\end{array}$ & $\begin{array}{l}4729 \\
5222 \\
5715 \\
6207 \\
6698 \\
7189 \\
7679 \\
8168 \\
8657 \\
9146\end{array}$ & $\begin{array}{l}4779 \\
5272 \\
5764 \\
6256 \\
6747 \\
7238 \\
7728 \\
8217 \\
8706 \\
9195\end{array}$ & $\begin{array}{l}4828 \\
5321 \\
5813 \\
6305 \\
6796 \\
7287 \\
7777 \\
8266 \\
8755 \\
9244\end{array}$ & $\begin{array}{l}4877 \\
5370 \\
5862 \\
6354 \\
6845 \\
7336 \\
7826 \\
8315 \\
8804 \\
9292\end{array}$ & $\begin{array}{l}4927 \\
5419 \\
5912 \\
6403 \\
6594 \\
7385 \\
7875 \\
8364 \\
8853 \\
9341\end{array}$ & 49 \\
\hline 890 & 9390 & 9439 & 9488 & 9536 & 9585 & 9634 & 9683 & 9731 & 9780 & 9829 & 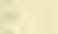 \\
\hline \begin{tabular}{l|l|l}
2 & \\
3 \\
4 \\
5 \\
5 \\
6 \\
7 \\
8 \\
9
\end{tabular} & $\begin{array}{r}950365 \\
0851 \\
1338 \\
1523 \\
2308 \\
2792 \\
3276 \\
3760\end{array}$ & $\begin{array}{l}0414 \\
0900 \\
1336 \\
1872 \\
2356 \\
2841 \\
3325 \\
3808\end{array}$ & $\begin{array}{l}0462 \\
0949 \\
1435 \\
1920 \\
2405 \\
2559 \\
3373 \\
3856\end{array}$ & $\begin{array}{l}0024 \\
0511 \\
0997 \\
1483 \\
1969 \\
2453 \\
2938 \\
3421 \\
3905\end{array}$ & $\begin{array}{l}0073 \\
0500 \\
1046 \\
1532 \\
2017 \\
2502 \\
2983 \\
3470 \\
3953\end{array}$ & $\begin{array}{l}0121 \\
0608 \\
1095 \\
1580 \\
2066 \\
2550 \\
3034 \\
3518 \\
4001\end{array}$ & $\begin{array}{l}0170 \\
0657 \\
1143 \\
1629 \\
2114 \\
2599 \\
3083 \\
3566 \\
4049\end{array}$ & $\begin{array}{l}0219 \\
0706 \\
1192 \\
1677 \\
2163 \\
2647 \\
3131 \\
3615 \\
4098\end{array}$ & $\begin{array}{l}0267 \\
0754 \\
1240 \\
1726 \\
2211 \\
2696 \\
3180 \\
3663 \\
4146\end{array}$ & $\begin{array}{l}0316 \\
0903 \\
1259 \\
1775 \\
2260 \\
2744 \\
3228 \\
3711 \\
4194\end{array}$ & 07 \\
\hline
\end{tabular}

Proportional. Parts.

\begin{tabular}{|c|c|c|c|c|c|c|c|c|c|}
\hline Dif. & 1 & 2 & 3 & 4 & 5 & 6 & 7 & 8 & 9 \\
\hline 51 & 5. 1 & 10.2 & 15.3 & 20.4 & 25.5 & 30.6 & 35. 7 & 40.8 & 45.9 \\
\hline 50 & 5. 0 & 10.0 & 15.0 & 20.0 & 25.0 & 30.0 & 35. 0 & 40.0 & 45. 0 \\
\hline 49 & 4.9 & 9.8 & 14.7 & 19.6 & 24.5 & 29.1 & 34.3 & 39.2 & 44.1 \\
\hline 48 & 4.8 & 9.6 & 14.4 & 19.2 & 24.0 & 28.8 & 33.6 & 38.4 & 43. 2 \\
\hline
\end{tabular}


No. 900 L. 954 .]

[No. 944 L. 975.

\begin{tabular}{|c|c|c|c|c|c|c|c|c|c|c|c|}
\hline N. & 0 & 1 & 2 & 3 & 4 & 5 & 6 & 7 & 8 & D & Diff. \\
\hline $\begin{array}{r}900 \\
1 \\
2 \\
3 \\
4 \\
5 \\
6 \\
7 \\
8 \\
9\end{array}$ & $\begin{array}{r}954213 \\
4725 \\
5207 \\
51388 \\
6168 \\
6649 \\
7128 \\
7607 \\
8086 \\
8564\end{array}$ & $\begin{array}{l}4291 \\
4773 \\
5255 \\
5736 \\
6216 \\
6697 \\
7176 \\
7655 \\
8134 \\
8612\end{array}$ & $\begin{array}{l}4339 \\
4821 \\
5303 \\
5784 \\
6265 \\
6745 \\
7224 \\
7703 \\
8181 \\
8659\end{array}$ & $\begin{array}{l}4387 \\
4 S 69 \\
5351 \\
5832 \\
6313 \\
6793 \\
7272 \\
7751 \\
8229 \\
8707\end{array}$ & $\begin{array}{l}4435 \\
4918 \\
5399 \\
5880 \\
6361 \\
6840 \\
7320 \\
7799 \\
8277 \\
8755\end{array}$ & $\begin{array}{l}4484 \\
4966 \\
5447 \\
5928 \\
6409 \\
6858 \\
7368 \\
7847 \\
8325 \\
8803\end{array}$ & $\begin{array}{l}4532 \\
5014 \\
5495 \\
5976 \\
6457 \\
6936 \\
7416 \\
7894 \\
8373 \\
8850\end{array}$ & $\begin{array}{l}4580 \\
5062 \\
5543 \\
6024 \\
6505 \\
6984 \\
7464 \\
7942 \\
8421 \\
8895\end{array}$ & $\begin{array}{l}4628 \\
5110 \\
5592 \\
6072 \\
6553 \\
7032 \\
7512 \\
7990 \\
8468 \\
8946\end{array}$ & $\begin{array}{l}4677 \\
5158 \\
5640 \\
6120 \\
6601 \\
7080 \\
7559 \\
8038 \\
8516 \\
8994\end{array}$ & 48 \\
\hline $\begin{array}{r}910 \\
1 \\
?\end{array}$ & $\begin{array}{l}9041 \\
9518\end{array}$ & $\begin{array}{l}9089 \\
9566 \\
\end{array}$ & $\begin{array}{l}9137 \\
9614\end{array}$ & $\begin{array}{l}9185 \\
9661 \\
\end{array}$ & $\begin{array}{l}9232 \\
9709 \\
\end{array}$ & $\begin{array}{l}9280 \\
9757\end{array}$ & $\begin{array}{l}9328 \\
9804\end{array}$ & $\begin{array}{l}9375 \\
9852\end{array}$ & $\begin{array}{l}9423 \\
9900\end{array}$ & $\begin{array}{l}9471 \\
9947 \\
\end{array}$ & \\
\hline $\begin{array}{l}3 \\
4 \\
5 \\
6 \\
7 \\
8 \\
9\end{array}$ & $\begin{array}{r}960471 \\
0946 \\
1421 \\
1895 \\
2369 \\
2843 \\
3316\end{array}$ & $\begin{array}{l}0042 \\
0518 \\
0994 \\
1469 \\
1943 \\
2417 \\
2 S 90 \\
3363\end{array}$ & $\begin{array}{l}0090 \\
0566 \\
1041 \\
1516 \\
1990 \\
2464 \\
2937 \\
3410\end{array}$ & $\begin{array}{l}0138 \\
0613 \\
1059 \\
1563 \\
2038 \\
2511 \\
2985 \\
3457\end{array}$ & $\begin{array}{l}0185 \\
0661 \\
1136 \\
1611 \\
2085 \\
2559 \\
3032 \\
3504\end{array}$ & $\begin{array}{l}0233 \\
0709 \\
1184 \\
1658 \\
2132 \\
2606 \\
3079 \\
3552\end{array}$ & $\begin{array}{l}0280 \\
0756 \\
1231 \\
1706 \\
2180 \\
2653 \\
3126 \\
3599\end{array}$ & $\begin{array}{l}0328 \\
0804 \\
1279 \\
1753 \\
2227 \\
2701 \\
3174 \\
3646\end{array}$ & $\begin{array}{l}8376 \\
0851 \\
1326 \\
1801 \\
2275 \\
2748 \\
3221 \\
3693\end{array}$ & $\begin{array}{l}0423 \\
0899 \\
1374 \\
1845 \\
2322 \\
2795 \\
3268 \\
3741\end{array}$ & \\
\hline $\begin{array}{r}920 \\
1 \\
2 \\
3 \\
4 \\
5 \\
6 \\
7 \\
8 \\
9\end{array}$ & $\begin{array}{l}3788 \\
4260 \\
4731 \\
5202 \\
5672 \\
6142 \\
6611 \\
7080 \\
7548 \\
8016\end{array}$ & $\begin{array}{l}3835 \\
4307 \\
4778 \\
5249 \\
5719 \\
6189 \\
6658 \\
7127 \\
7595 \\
8062\end{array}$ & $\begin{array}{l}3882 \\
4354 \\
4825 \\
5296 \\
5766 \\
6236 \\
6705 \\
7173 \\
7642 \\
8109\end{array}$ & $\begin{array}{l}3929 \\
4401 \\
4872 \\
5343 \\
5813 \\
6283 \\
6752 \\
7220 \\
7688 \\
8156\end{array}$ & $\begin{array}{l}5390 \\
5860 \\
6329 \\
6799 \\
7267 \\
7735 \\
8203\end{array}$ & $\begin{array}{l}4024 \\
4495 \\
4966 \\
5437 \\
5907 \\
6376 \\
6845 \\
7314 \\
7782 \\
8249\end{array}$ & $\begin{array}{l}4071 \\
4542 \\
5013 \\
5484 \\
5954 \\
6423 \\
6892 \\
7361 \\
7829 \\
8296\end{array}$ & $\begin{array}{l}4118 \\
4590 \\
5061 \\
5531 \\
6001 \\
6470 \\
6939 \\
7408 \\
7875 \\
8343\end{array}$ & $\begin{array}{l}4165 \\
4637 \\
5108 \\
5578 \\
6048 \\
6517 \\
6986 \\
7454 \\
7922 \\
8390\end{array}$ & $\begin{array}{l}4212 \\
4684 \\
5155 \\
5625 \\
6095 \\
6564 \\
7033 \\
7501 \\
7969 \\
8436\end{array}$ & $16=$ \\
\hline $\begin{array}{r}930 \\
1 \\
2 \\
3\end{array}$ & $\begin{array}{l}8483 \\
8350 \\
9116 \\
989\end{array}$ & $\begin{array}{l}8530 \\
8996 \\
9463 \\
9098\end{array}$ & $\begin{array}{l}8576 \\
9043 \\
9509 \\
9075\end{array}$ & $\begin{array}{l}8623 \\
9090 \\
9556\end{array}$ & $\begin{array}{l}8670 \\
9136 \\
9602\end{array}$ & $\begin{array}{r}8716 \\
9183 \\
9649 \\
\end{array}$ & $\begin{array}{l}8763 \\
9229 \\
9695 \\
\end{array}$ & $\begin{array}{l}8810 \\
9276 \\
9742\end{array}$ & $\begin{array}{l}8856 \\
9323 \\
9789\end{array}$ & $\begin{array}{l}8903 \\
9369 \\
9835\end{array}$ & \\
\hline $\begin{array}{l}4 \\
5 \\
6 \\
7 \\
8 \\
9\end{array}$ & $\begin{array}{r}970347 \\
0 \$ 12 \\
1276 \\
1740 \\
2203 \\
2666\end{array}$ & $\begin{array}{l}0393 \\
0858 \\
1322 \\
1786 \\
2249 \\
2712\end{array}$ & $\begin{array}{l}0440 \\
0904 \\
1369 \\
1832 \\
2295 \\
2758\end{array}$ & $\begin{array}{l}0021 \\
0486 \\
0951 \\
1415 \\
1879 \\
2342 \\
2804\end{array}$ & $\begin{array}{l}0068 \\
0533 \\
0997 \\
1461 \\
1925 \\
2388 \\
2851 \\
\end{array}$ & $\begin{array}{l}0114 \\
0579 \\
1044 \\
1508 \\
1971 \\
2434 \\
2897\end{array}$ & $\begin{array}{l}0161 \\
0626 \\
1090 \\
1554 \\
2018 \\
2481 \\
2943\end{array}$ & $\begin{array}{l}0207 \\
0672 \\
1137 \\
1601 \\
2064 \\
2527 \\
2989\end{array}$ & $\begin{array}{l}0254 \\
0719 \\
1183 \\
1647 \\
2110 \\
2573 \\
3035\end{array}$ & $\begin{array}{l}0300 \\
0765 \\
1229 \\
1693 \\
2157 \\
2619 \\
3082\end{array}$ & \\
\hline $\begin{array}{r}940 \\
1 \\
2 \\
3 \\
4\end{array}$ & $\begin{array}{l}3128 \\
3590 \\
4051 \\
4512 \\
4972\end{array}$ & $\begin{array}{l}3174 \\
3636 \\
4097 \\
4558 \\
5018\end{array}$ & $\begin{array}{l}3220 \\
3682 \\
4143 \\
4604 \\
5064\end{array}$ & $\begin{array}{l}3266 \\
3728 \\
4189 \\
4650 \\
5110\end{array}$ & $\begin{array}{l}3313 \\
3774 \\
4235 \\
4696 \\
5156\end{array}$ & $\begin{array}{l}3359 \\
3820 \\
4281 \\
4742 \\
5: 02\end{array}$ & $\begin{array}{l}3405 \\
3566 \\
4327 \\
4788 \\
5248\end{array}$ & $\begin{array}{l}3451 \\
3913 \\
4374 \\
4834 \\
5294\end{array}$ & $\begin{array}{l}3497 \\
3959 \\
4420 \\
4880 \\
5340\end{array}$ & $\begin{array}{l}3543 \\
4005 \\
4466 \\
4926 \\
5386\end{array}$ & 46 \\
\hline
\end{tabular}

Proportional Parts.

\begin{tabular}{|c|c|c|c|c|c|c|c|c|c|}
\hline Dift. & 1 & 2 & 3 & 4 & 5 & 6 & 7 & 8 & 9 \\
\hline $\begin{array}{l}47 \\
46\end{array}$ & $\begin{array}{l}4.7 \\
\text { 4. } 6\end{array}$ & $\begin{array}{l}9.4 \\
9.2\end{array}$ & $\begin{array}{l}14.1 \\
13.8\end{array}$ & $\begin{array}{l}18.8 \\
18.4\end{array}$ & $\begin{array}{l}23.5 \\
23.0\end{array}$ & $\begin{array}{l}28.2 \\
27.6\end{array}$ & $\begin{array}{l}32.9 \\
32.2\end{array}$ & $\begin{array}{l}37.6 \\
36.8\end{array}$ & $\begin{array}{l}42.3 \\
41.4\end{array}$ \\
\hline
\end{tabular}


TABLE 10.-LOGARITIMS OF NUMBERS.

No. 945 L. 975.]

[No. 989 I.. 995.

\begin{tabular}{|c|c|c|c|c|c|c|c|c|c|c|c|}
\hline N. & 0 & 1 & 2 & 3 & 4 & 5 & 6 & 7 & 8 & 9 & Difl. \\
\hline $\begin{array}{r}945 \\
6 \\
7 \\
8 \\
9\end{array}$ & $\begin{array}{r}975432 \\
5891 \\
6350 \\
6808 \\
7266\end{array}$ & $\begin{array}{l}5478 \\
5937 \\
6396 \\
6854 \\
7312\end{array}$ & $\begin{array}{l}5524 \\
5983 \\
6442 \\
6900 \\
7358\end{array}$ & $\begin{array}{l}5570 \\
6029 \\
6188 \\
6946 \\
7403\end{array}$ & $\begin{array}{l}5616 \\
6075 \\
6533 \\
6992 \\
7449\end{array}$ & $\begin{array}{l}5662 \\
6121 \\
6579 \\
7037 \\
7495\end{array}$ & $\begin{array}{l}5707 \\
6167 \\
6625 \\
7083 \\
7541\end{array}$ & $\begin{array}{l}5753 \\
6212 \\
6671 \\
7129 \\
7586\end{array}$ & $\begin{array}{l}5799 \\
6258 \\
6717 \\
7175 \\
7632\end{array}$ & $\begin{array}{l}5845 \\
6304 \\
6763 \\
7220 \\
7678\end{array}$ & . \\
\hline $\begin{array}{r}950 \\
1 \\
2 \\
3 \\
4\end{array}$ & $\begin{array}{l}7724 \\
8181 \\
8637 \\
9093 \\
9548\end{array}$ & $\begin{array}{l}7769 \\
8226 \\
8683 \\
9138 \\
9594\end{array}$ & $\begin{array}{l}7815 \\
8272 \\
8728 \\
9184 \\
9639\end{array}$ & $\begin{array}{l}7861 \\
8317 \\
8774 \\
9230 \\
9685\end{array}$ & $\begin{array}{l}7906 \\
8363 \\
8819 \\
9275 \\
9730\end{array}$ & $\begin{array}{l}7952 \\
8409 \\
8865 \\
9321 \\
9776\end{array}$ & $\begin{array}{l}7998 \\
8454 \\
8911 \\
9366 \\
9821\end{array}$ & $\begin{array}{l}8043 \\
8500 \\
8956 \\
9412 \\
9867\end{array}$ & $\begin{array}{l}8089 \\
8546 \\
9002 \\
9457 \\
9912\end{array}$ & $\begin{array}{l}8135 \\
8591 \\
9047 \\
9503 \\
9958\end{array}$ & 1 \\
\hline $\begin{array}{l}5 \\
6 \\
7 \\
8 \\
9\end{array}$ & $\begin{array}{r}980003 \\
0458 \\
0912 \\
1366 \\
1819\end{array}$ & $\begin{array}{l}0049 \\
0503 \\
0957 \\
1411 \\
1864\end{array}$ & $\begin{array}{l}0094 \\
0549 \\
1003 \\
1456 \\
1909\end{array}$ & $\begin{array}{l}0140 \\
0594 \\
1048 \\
1501 \\
1954\end{array}$ & $\begin{array}{l}0185 \\
0640 \\
1093 \\
1547 \\
2000\end{array}$ & $\begin{array}{l}0231 \\
0685 \\
1139 \\
1592 \\
2045\end{array}$ & $\begin{array}{l}0276 \\
0730 \\
1184 \\
1637 \\
2090\end{array}$ & $\begin{array}{l}0322 \\
0776 \\
1229 \\
1683 \\
2135\end{array}$ & $\begin{array}{l}0367 \\
0821 \\
1275 \\
1728 \\
2181\end{array}$ & $\begin{array}{l}0412 \\
0867 \\
1320 \\
1773 \\
2226\end{array}$ & \\
\hline $\begin{array}{r}960 \\
1 \\
2 \\
3 \\
4 \\
5 \\
6 \\
7 \\
8 \\
9\end{array}$ & $\begin{array}{l}2271 \\
2723 \\
3175 \\
3626 \\
4077 \\
4527 \\
4977 \\
5426 \\
5875 \\
6324\end{array}$ & $\begin{array}{l}2316 \\
2769 \\
3220 \\
3671 \\
4122 \\
4572 \\
5022 \\
5471 \\
5920 \\
6369\end{array}$ & $\begin{array}{l}2362 \\
2814 \\
3265 \\
3716 \\
4167 \\
4617 \\
5067 \\
5516 \\
5965 \\
6413\end{array}$ & $\begin{array}{l}2407 \\
2559 \\
3310 \\
3762 \\
4212 \\
4662 \\
5112 \\
5561 \\
6010 \\
6458\end{array}$ & $\begin{array}{l}2452 \\
2904 \\
3356 \\
3807 \\
4257 \\
4707 \\
5157 \\
5606 \\
6055 \\
6503\end{array}$ & $\begin{array}{l}2497 \\
2949 \\
3401 \\
3852 \\
4302 \\
4752 \\
5202 \\
5651 \\
6100 \\
6548\end{array}$ & $\begin{array}{l}2543 \\
2994 \\
3446 \\
3897 \\
4347 \\
4797 \\
5247 \\
5696 \\
6144 \\
6593\end{array}$ & $\begin{array}{l}25 \$ 8 \\
3040 \\
3491 \\
3942 \\
4392 \\
4842 \\
5292 \\
5741 \\
6189 \\
6637\end{array}$ & $\begin{array}{l}2633 \\
3885 \\
3536 \\
3987 \\
4437 \\
4857 \\
5337 \\
5786 \\
6234 \\
6682\end{array}$ & $\begin{array}{l}2678 \\
3130 \\
3581 \\
4032 \\
4482 \\
4932 \\
5382 \\
5830 \\
6279 \\
6727\end{array}$ & 45 \\
\hline $\begin{array}{r}970 \\
1 \\
2 \\
3 \\
4 \\
5 \\
6 \\
7\end{array}$ & $\begin{array}{l}6772 \\
7219 \\
7666 \\
8113 \\
8559 \\
9005 \\
9450 \\
9895\end{array}$ & $\begin{array}{l}6817 \\
7264 \\
7711 \\
8157 \\
8604 \\
9049 \\
9494 \\
9939\end{array}$ & $\begin{array}{l}6861 \\
7309 \\
7756 \\
8202 \\
8648 \\
9094 \\
9539 \\
9983\end{array}$ & $\begin{array}{l}6906 \\
7353 \\
7800 \\
8247 \\
8693 \\
9138 \\
9583\end{array}$ & $\begin{array}{l}6951 \\
7398 \\
7845 \\
8291 \\
8737 \\
9183 \\
9628\end{array}$ & $\begin{array}{l}6996 \\
7443 \\
7890 \\
8336 \\
8782 \\
9227 \\
9672\end{array}$ & $\begin{array}{l}7040 \\
7488 \\
7934 \\
8381 \\
8826 \\
9272 \\
9717\end{array}$ & $\begin{array}{l}7085 \\
7532 \\
7979 \\
8425 \\
8871 \\
9316 \\
9761\end{array}$ & $\begin{array}{l}7130 \\
7577 \\
8024 \\
8470 \\
8916 \\
9361 \\
9806 \\
\end{array}$ & $\begin{array}{l}7175 \\
7622 \\
8068 \\
8514 \\
8960 \\
9405 \\
9850\end{array}$ & \\
\hline $\begin{array}{l}8 \\
9\end{array}$ & $\begin{array}{r}990339 \\
0783\end{array}$ & $\begin{array}{l}0383 \\
0827\end{array}$ & $\begin{array}{l}0428 \\
0871\end{array}$ & $\begin{array}{l}0028 \\
0472 \\
0916\end{array}$ & $\begin{array}{l}0072 \\
0516 \\
0960\end{array}$ & $\begin{array}{l}0117 \\
0561 \\
1004\end{array}$ & $\begin{array}{l}0161 \\
0605 \\
1049\end{array}$ & $\begin{array}{l}0206 \\
0650 \\
1033\end{array}$ & $\begin{array}{l}0250 \\
0694 \\
1137\end{array}$ & $\begin{array}{l}0294 \\
0738 \\
1182\end{array}$ & \\
\hline $\begin{array}{r}980 \\
1 \\
2 \\
3 \\
4 \\
5 \\
6 \\
7 \\
8 \\
9\end{array}$ & $\begin{array}{l}1226 \\
1669 \\
2111 \\
2554 \\
2995 \\
3436 \\
3877 \\
4317 \\
4757 \\
5196\end{array}$ & $\begin{array}{l}1270 \\
1713 \\
2156 \\
2598 \\
3039 \\
3480 \\
3921 \\
4361 \\
4801 \\
5240\end{array}$ & $\begin{array}{l}1315 \\
1758 \\
2200 \\
2642 \\
3083 \\
3524 \\
3965 \\
4405 \\
4815 \\
5284\end{array}$ & $\begin{array}{l}1359 \\
1802 \\
2244 \\
2656 \\
3127 \\
3568 \\
4009 \\
4449 \\
4859 \\
5328\end{array}$ & $\begin{array}{l}1403 \\
1546 \\
2298 \\
2730 \\
3172 \\
3613 \\
4053 \\
4493 \\
4933 \\
5372\end{array}$ & $\begin{array}{l}1448 \\
1890 \\
2333 \\
2774 \\
3216 \\
3657 \\
4097 \\
4537 \\
4977 \\
5416\end{array}$ & $\begin{array}{l}1492 \\
1935 \\
2377 \\
2819 \\
3260 \\
3701 \\
4141 \\
4581 \\
5021 \\
5460\end{array}$ & $\begin{array}{l}1536 \\
1979 \\
2421 \\
2863 \\
3304 \\
3745 \\
4185 \\
4625 \\
5065 \\
5504\end{array}$ & $\begin{array}{l}1580 \\
2023 \\
2465 \\
2907 \\
3348 \\
3789 \\
4229 \\
4669 \\
5108 \\
5547\end{array}$ & $\begin{array}{l}1625 \\
2067 \\
2509 \\
2951 \\
3392 \\
3833 \\
4273 \\
4713 \\
5152 \\
5591\end{array}$ & 44 \\
\hline
\end{tabular}

Proportional Parts.

\begin{tabular}{|c|c|c|c|c|c|c|c|c|c|}
\hline Diff. & 1 & 2 & 3 & 4 & 5 & 6 & 7 & 8 & 9 \\
\hline $\begin{array}{l}46 \\
45 \\
44 \\
43\end{array}$ & $\begin{array}{l}4.6 \\
4.5 \\
4.4 \\
4.3\end{array}$ & $\begin{array}{l}9.2 \\
9.0 \\
8.8 \\
8.6\end{array}$ & $\begin{array}{l}13.8 \\
13.5 \\
13.2 \\
12.9\end{array}$ & $\begin{array}{l}18.4 \\
18.0 \\
17.6 \\
17.2\end{array}$ & $\begin{array}{l}23.0 \\
22.5 \\
22.0 \\
21.5\end{array}$ & $\begin{array}{l}27.6 \\
27.0 \\
26.4 \\
25.8\end{array}$ & $\begin{array}{l}32.2 \\
31.5 \\
30.8 \\
30.1\end{array}$ & $\begin{array}{l}36.8 \\
36.0 \\
35.2 \\
34.4\end{array}$ & $\begin{array}{l}41.4 \\
40.5 \\
39.6 \\
38.7\end{array}$ \\
\hline
\end{tabular}


TABLE 10.-LOGARITIMS OF NUMBERS.

\begin{tabular}{|c|c|c|c|c|c|c|c|c|c|c|c|}
\hline \multicolumn{9}{|c|}{ No. 990 L. 995.$]$} & \multicolumn{3}{|c|}{ [No. 999 L. 999.} \\
\hline N. & 0 & 1 & 2 & 3 & 4 & 5 & 6 & 7 & 8 & 9 & Diff. \\
\hline 990 & 995635 & 5679 & 5723 & 5767 & 5811 & 5854 & 5898 & 5942 & 5986 & 6030 & \\
\hline 1 & 6074 & 6117 & 6161 & 6205 & 6249 & 6293 & 6337 & 6380 & 6424 & 6468 & 44 \\
\hline 2 & 6512 & 6555 & 6599 & 6643 & 6687 & 6731 & 6774 & 6818 & 6862 & 6906 & \\
\hline 3 & 6949 & 6993 & 7037 & 7050 & 7124 & 7168 & 7212 & 7255 & 7299 & 7343 & \\
\hline 4 & 7386 & 7430 & 7474 & 7517 & 7561 & 7605 & 7648 & 7692 & 7736 & 7779 & \\
\hline 5 & 7823 & 7867 & 7910 & 7954 & 7998 & 8041 & 8085 & 8129 & 8172 & 8216 & \\
\hline 6 & 8259 & 8303 & 8347 & 8390 & 8434 & 8477 & 8521 & 8564 & 8608 & 8652 & \\
\hline 7 & 8695 & 8739 & 8782 & 8826 & 8869 & 8913 & 8956 & 9000 & 9043 & 9087 & \\
\hline 8 & 9131 & 9174 & 9218 & 9261 & 9305 & 9348 & 9392 & 9435 & 9479 & 9522 & e \\
\hline 9 & 9565 & 9609 & 9652 & 9696 & 9739 & 9783 & 9826 & 9870 & 9913 & 9957 & 43 \\
\hline
\end{tabular}


TABLE 11.-CONVERGENCY OF MERIDIANS, SIX MILES LONG AND SIX MILES APART, AND DIFFERENCES OF LATITUDE AND LONGITUDE.

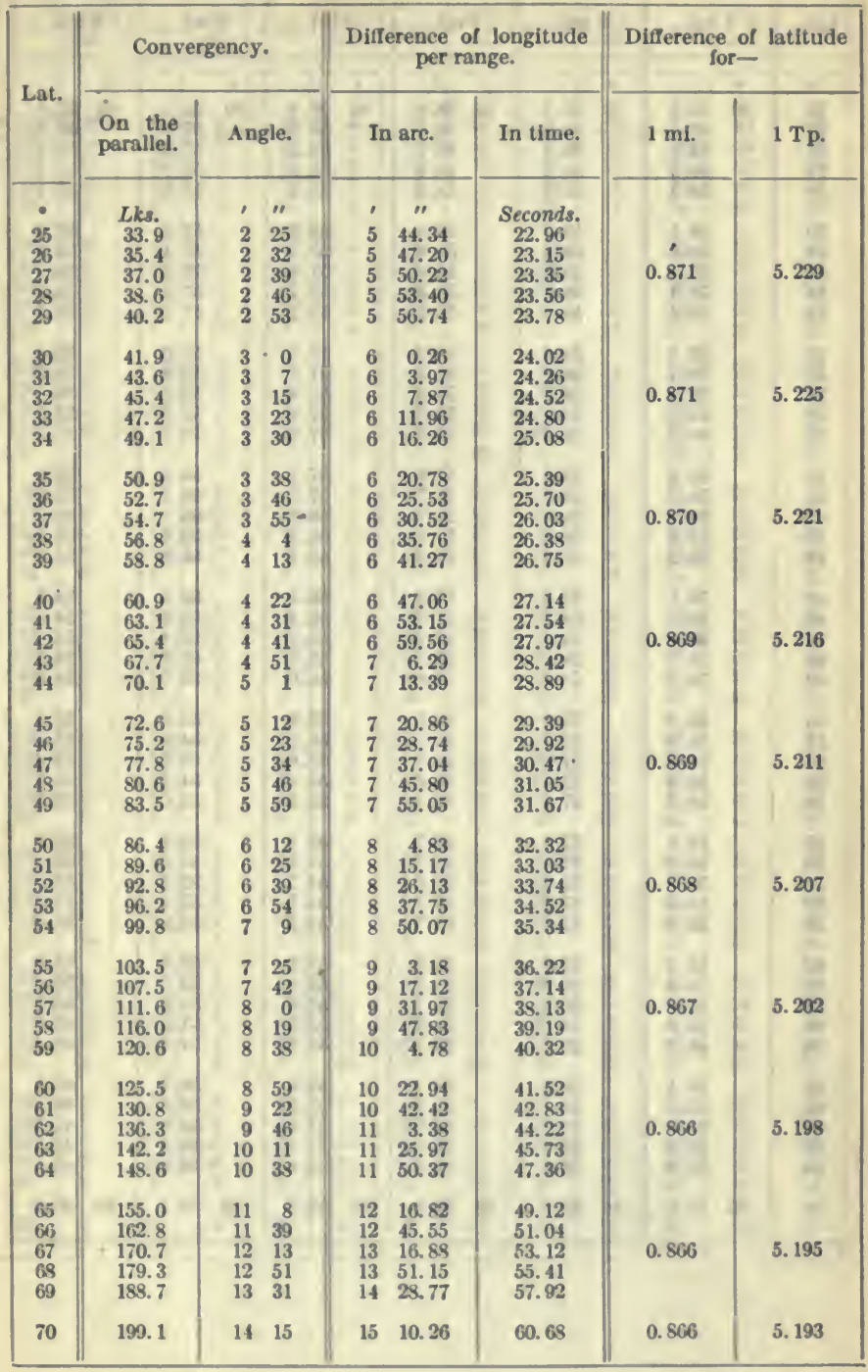


TABLE 12.-AZIMUTHS OF THE TANGENT TO THE PARALLEL.

\begin{tabular}{|c|c|c|c|c|c|c|c|c|c|c|c|c|}
\hline \multirow{2}{*}{ Lat. } & \multicolumn{2}{|c|}{$1 \mathrm{mi}$. } & \multicolumn{2}{|c|}{$2 \mathrm{ml}}$. & \multicolumn{2}{|c|}{$3 \mathrm{mi}}$. & \multicolumn{2}{|c|}{$4 \mathrm{mi}$. } & \multicolumn{2}{|c|}{$5 \mathrm{mi}$} & \multicolumn{2}{|c|}{$6 \mathrm{mi}}$. \\
\hline & - & , & . & , & 。 & , & . & , & . & , & - & , \\
\hline $\begin{array}{l}25 \\
26 \\
27 \\
28 \\
29\end{array}$ & 89 & $\begin{array}{l}59.6 \\
59.6 \\
59.6 \\
59.5 \\
59.5\end{array}$ & 89 & $\begin{array}{l}59.2 \\
59.2 \\
59.1 \\
59.1 \\
59.0\end{array}$ & 89 & $\begin{array}{l}58.8 \\
58.7 \\
58.7 \\
58.6 \\
58.6\end{array}$ & 89 & $\begin{array}{l}58.4 \\
58.3 \\
58.2 \\
58.2 \\
58.1\end{array}$ & 89 & $\begin{array}{l}58.0 \\
57.9 \\
57.8 \\
57.7 \\
57.6\end{array}$ & 89 & $\begin{array}{l}57.6 \\
57.5 \\
57.4 \\
57.2 \\
57.1\end{array}$ \\
\hline $\begin{array}{l}30 \\
31 \\
32 \\
33 \\
34\end{array}$ & & $\begin{array}{l}59.5 \\
59.5 \\
59.5 \\
59.4 \\
59.4\end{array}$ & & $\begin{array}{l}59.0 \\
59.0 \\
58.9 \\
58.9 \\
58.8\end{array}$ & & $\begin{array}{l}58.5 \\
58.4 \\
58.4 \\
58.3 \\
58.2\end{array}$ & & $\begin{array}{l}58.0 \\
57.9 \\
57.8 \\
57.7 \\
57.7\end{array}$ & & $\begin{array}{l}57.5 \\
57.4 \\
57.3 \\
57.2 \\
57.1\end{array}$ & & $\begin{array}{l}57.0 \\
56.9 \\
56.8 \\
56.6 \\
56.5\end{array}$ \\
\hline $\begin{array}{l}35 \\
36 \\
37 \\
38 \\
39\end{array}$ & & $\begin{array}{l}59.4 \\
59.4 \\
59.3 \\
59.3 \\
59.3\end{array}$ & & $\begin{array}{l}58.8 \\
58.7 \\
58.7 \\
58.6 \\
58.6\end{array}$ & & $\begin{array}{l}58.2 \\
58.1 \\
58.0 \\
58.0 \\
57.9\end{array}$ & $\frac{1}{3}$ & $\begin{array}{l}57.6 \\
57.5 \\
57.4 \\
57.3 \\
57.2\end{array}$ & 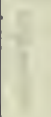 & $\begin{array}{l}57.0 \\
56.9 \\
56.7 \\
56.6 \\
56.5\end{array}$ & & $\begin{array}{l}56.4 \\
56.2 \\
56.1 \\
55.9 \\
55.8\end{array}$ \\
\hline $\begin{array}{l}40 \\
41 \\
42 \\
43 \\
44\end{array}$ & & $\begin{array}{l}59.3 \\
59.2 \\
59.2 \\
59.2 \\
59.2\end{array}$ & & $\begin{array}{l}58.5 \\
58.5 \\
58.4 \\
58.4 \\
58.3\end{array}$ & & $\begin{array}{l}57.8 \\
57.7 \\
57.7 \\
57.6 \\
57.5\end{array}$ & $\frac{7}{8}$ & $\begin{array}{l}57.1 \\
57.0 \\
56.9 \\
56.8 \\
56.7\end{array}$ & 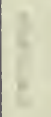 & $\begin{array}{l}56.4 \\
56.2 \\
56.1 \\
56.0 \\
55.8\end{array}$ & & $\begin{array}{l}55.6 \\
55.5 \\
55.3 \\
55.2 \\
55.0\end{array}$ \\
\hline $\begin{array}{l}45 \\
46 \\
47 \\
48 \\
49\end{array}$ & & $\begin{array}{l}59.1 \\
59.1 \\
59.1 \\
59.0 \\
59.0\end{array}$ & & $\begin{array}{l}58.3 \\
58.2 \\
58.1 \\
58.1 \\
58.0\end{array}$ & & $\begin{array}{l}57.4 \\
57.3 \\
57.2 \\
57.1 \\
57.0\end{array}$ & $\frac{1}{2}$ & $\begin{array}{l}56.5 \\
56.4 \\
56.3 \\
56.2 \\
56.0\end{array}$ & f & $\begin{array}{l}55.7 \\
55.5 \\
55.4 \\
55.2 \\
55.0\end{array}$ & & $\begin{array}{l}54.8 \\
54.6 \\
54.4 \\
54.2 \\
54.0\end{array}$ \\
\hline $\begin{array}{l}50 \\
51 \\
52 \\
53 \\
54\end{array}$ & & $\begin{array}{l}59.0 \\
58.9 \\
58.9 \\
58.8 \\
58.8\end{array}$ & & $\begin{array}{l}57.9 \\
57.0 \\
57.8 \\
57.7 \\
57.6\end{array}$ & & $\begin{array}{l}56.9 \\
56.8 \\
56.7 \\
56.6 \\
56.4\end{array}$ & 1 & $\begin{array}{l}55.9 \\
55.7 \\
55.6 \\
55.4 \\
55.2\end{array}$ & 4 & $\begin{array}{l}54.8 \\
54.6 \\
54.5 \\
54.2 \\
54.0\end{array}$ & & $\begin{array}{l}53.8 \\
53.6 \\
53.1 \\
53.1 \\
52.8\end{array}$ \\
\hline $\begin{array}{l}55 \\
56 \\
57 \\
58 \\
59\end{array}$ & & $\begin{array}{l}58.8 \\
58.7 \\
58.7 \\
58.6 \\
58.6\end{array}$ & & $\begin{array}{l}57.5 \\
57.4 \\
57.3 \\
57.2 \\
57.1\end{array}$ & & $\begin{array}{l}56.3 \\
56.2 \\
56.0 \\
55.8 \\
55.7\end{array}$ & 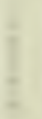 & $\begin{array}{l}55.1 \\
54.9 \\
54.7 \\
54.5 \\
54.2\end{array}$ & $=$ & $\begin{array}{l}53.8 \\
53.6 \\
53.3 \\
53.1 \\
52.8\end{array}$ & & $\begin{array}{l}52.6 \\
52.3 \\
52.0 \\
51.7 \\
51.4\end{array}$ \\
\hline $\begin{array}{l}60 \\
61 \\
62 \\
63 \\
64\end{array}$ & & $\begin{array}{l}58.5 \\
58.4 \\
58.4 \\
58.3 \\
58.2\end{array}$ & & $\begin{array}{l}57.0 \\
56.9 \\
56.7 \\
56.6 \\
56.5\end{array}$ & & $\begin{array}{l}55.5 \\
55.3 \\
55.1 \\
54.9 \\
54.7\end{array}$ & 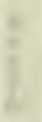 & $\begin{array}{l}54.0 \\
53.8 \\
53.5 \\
53.2 \\
52.9\end{array}$ & r & $\begin{array}{l}52.5 \\
52.2 \\
51.9 \\
51.5 \\
51.1\end{array}$ & & $\begin{array}{l}51.0 \\
50.6 \\
50.2 \\
49.8 \\
49.4\end{array}$ \\
\hline $\begin{array}{l}65 \\
66 \\
67 \\
68 \\
69\end{array}$ & & $\begin{array}{l}58.1 \\
58.1 \\
58.0 \\
57.9 \\
57.8\end{array}$ & & $\begin{array}{l}56.3 \\
56.1 \\
55.9 \\
55.7 \\
55.5\end{array}$ & & $\begin{array}{l}54.4 \\
54.2 \\
53.9 \\
53.6 \\
53.2\end{array}$ & $\begin{array}{l}m \\
y \\
y \\
y\end{array}$ & $\begin{array}{l}52.6 \\
52.2 \\
51.8 \\
51.4 \\
51.0\end{array}$ & 8 & $\begin{array}{l}50.7 \\
50.3 \\
49.8 \\
49.3 \\
48.8\end{array}$ & & $\begin{array}{l}48.9 \\
48.4 \\
47.8 \\
47.2 \\
46.5\end{array}$ \\
\hline 70 & 89 & 57.6 & 89 & 55,3 & 89 & 52.9 & 89 & 50.5 & 89 & 48.1 & 89 & 45.8 \\
\hline
\end{tabular}




\begin{tabular}{|c|c|c|c|c|c|c|c|c|c|c|c|c|}
\hline Lat. & $\begin{array}{l}1 / 9 \\
\text { mi. }\end{array}$ & $1 \mathrm{ml}$. & $\begin{array}{l}11 / 2 \\
\text { mi. }\end{array}$ & $2 \mathrm{mi}$. & $\begin{array}{l}21 / 2 \\
\text { mi. }\end{array}$ & $3 \mathrm{mi}$. & $\begin{array}{l}31 / 2 \\
\mathrm{ml} .\end{array}$ & $4 \mathrm{mi}$. & $\begin{array}{l}41 / 2 \\
\text { mi. }\end{array}$ & $5 \mathrm{mi}$. & $\begin{array}{l}51 / 2 \\
\text { mi. }\end{array}$ & $6 \mathrm{ml}$. \\
\hline • & & & & & & & & & & & & \\
\hline 25 & 0 & 0 & 1 & 2 & 3 & 4 & 6 & 8 & 10 & 12 & 14 & 17 \\
\hline 26 & 0 & 0 & 1 & 2 & 3 & 4 & 6 & 8 & 10 & 12 & 15 & 18 \\
\hline 27 & 0 & 1 & 1 & 2 & 3 & 5 & 6 & 8 & 10 & 13 & 16 & 18 \\
\hline 28 & 0 & 1 & 1 & 2 & 3 & 5 & 7 & 9 & 11 & 13 & 16 & 19 \\
\hline 29 & 0 & 1 & 1 & 2 & 3 & 5 & 7 & 9 & ii & 14 & 17 & 20 \\
\hline 30 & 0 & 1 & 1 & 2 & 4 & 5 & $i$ & 9 & 12 & 15 & 18 & 21 \\
\hline 31 & 0 & 1 & 1 & 2 & 4 & 5 & 7 & 10 & 12 & 15 & 18 & 22 \\
\hline 32 & 0 & 1 & 1 & 3 & 4 & 6 & 8 & 10 & 13 & 16 & 19 & 23 \\
\hline 33 & 0 & 1 & 1 & 3 & 4 & 6 & 8 & 10 & 13 & 16. & 20 & 24 \\
\hline 34 & 0 & 1 & 2 & 3 & 4 & 6 & 8 & 11 & 14 & 17 & 21 & 25 \\
\hline 35 & 0 & 1 & 2 & 3 & 4 & 6 & 8 & 11 & 14 & 18 & 21 & 25 \\
\hline 36 & 0 & 1 & 2 & 3 & 5 & 7 & 9 & 12 & 15 & 18 & 22 & 26 \\
\hline 37 & 0 & 1 & 2 & 3 & 5 & 7 & 9 & 12 & 15 & 19 & 23 & 27 \\
\hline 38 & 0 & 1 & 2 & 3 & 5 & 7 & 10 & 13 & 16 & 20 & 24 & 28 \\
\hline 39 & 0 & 1 & 2 & 3 & 5 & 7 & 10 & 13 & 17 & 20 & 25 & 29 \\
\hline 40 & 0 & 1 & 2 & 3 & 5 & 8 & 10 & 14 & 17 & 21 & 26 & 30 \\
\hline 41 & 0 & 1 & 2 & 4 & 5 & 8 & 11 & 14 & 18 & 22 & 27 & 32 \\
\hline 42 & 0 & 1 & 2 & 4 & 6 & 8 & 11 & 15 & 18 & 23 & 27 & 33 \\
\hline 43 & 0 & 1 & 2 & 4 & 6 & 8 & 11 & 15 & 19 & 24 & 28 & 34 \\
\hline 44 & 0 & 1 & 2 & 4 & 6 & 9 & 12 & 16 & 20 & 24 & 29 & 35 \\
\hline 45 & 0 & 1 & 2 & 4 & 6 & 9 & 12 & 16 & 20 & 25 & 30 & 36 \\
\hline 46 & 0 & 1 & 2 & 4 & 7 & 9 & 13 & 17 & 21 & 26 & 32 & 38 \\
\hline 47 & 0 & 1 & 2 & 4 & 7 & 10 & 13 & 17 & 22 & 27 & 33 & 39 \\
\hline 48 & 0 & 1 & 3 & 4 & 7 & 10 & 14 & 18 & 23 & 28 & 34 & 40 \\
\hline 49 & 0 & 1 & 3 & 5 & 7 & 10 & 14 & 19 & 23 & 29 & 35 & 42 \\
\hline 50 & 0 & 1 & 3 & 5 & 8 & 11 & 15 & 19 & 24 & 30 & 36 & 43 \\
\hline 51 & 0 & 1 & 3 & 5 & 8 & 11 & 15 & 20 & 25 & 31 & 38 & 45 \\
\hline 52 & 0 & 1 & 3 & 5 & 8 & 12 & 16 & 21 & 26 & 32 & 39 & 46 \\
\hline 53 & 0 & 1 & 3 & 5 & 8 & 12 & 16 & 21 & 27 & 33 & 40 & 48 \\
\hline 54 & 0 & 1 & 3 & 6 & 9 & 12 & 17 & 22 & 28 & 35 & 42 & 50 \\
\hline 55 & 0 & 1 & 3 & 6 & 9 & 13 & 18 & 23 & 29 & 36 & 43 & 52 \\
\hline 56 & 0 & 1 & 3 & 6 & 9 & 13 & 18 & 24 & 30 & 37 & 45 & 54 \\
\hline 57 & 0 & 2 & 3 & 6 & 10 & 14 & 19 & 25 & 31 & 39 & 47 & 56 \\
\hline 58 & 0 & 2 & 4 & 6 & 10 & 14 & 20 & 26 & 33 & 40 & 49 & 58 \\
\hline 59 & 0 & 2 & 4 & 7 & 10 & 15 & 20 & 27 & 34 & 42 & 51 & 60 \\
\hline 60 & 0 & 2 & 4 & 7 & 11 & 16 & 21 & 28 & 35 & 44 & 53 & 63 \\
\hline 61 & 0 & 2 & 4 & 7 & 11 & 16 & 22 & 29 & 37 & 45 & 55 & 65 \\
\hline 62 & 0 & 2 & 4 & 8 & 12 & 17 & 23 & 30 & 38 & 47 & 57 & 68 \\
\hline 63 & 0 & 2 & 4 & 8 & 12 & 18 & 24 & 32 & 40 & 49 & 60 & 71 \\
\hline 64 & 1 & 2 & 5 & 8 & 13 & 19 & 25 & 33 & 42 & 52 & 62 & 74 \\
\hline 65 & 1 & 2 & 5 & 9 & 13 & 19 & 26 & 34 & 44 & 54 & 65 & 78 \\
\hline 68 & 1 & 2 & 5 & 9 & 14 & 20 & 28 & 36 & 46 & 57 & 63 & 81 \\
\hline 67 & 1 & 2 & 5 & 9 & 15 & 21 & 29 & 38 & 48 & 59 & 72 & 85 \\
\hline 68 & 1 & 2 & 6 & 10 & 16 & 22 & 30 & 40 & 50 & 62 & 75 & 90 \\
\hline 69 & 1 & 3 & 6 & 10 & 16 & 24 & 32 & 42 & 53 & 66 & 79 & 94 \\
\hline$j 0$ & 1 & 3 & 6 & 11 & 17 & 25 & 34 & 44 & 56 & 69 & 84 & 100 \\
\hline
\end{tabular}


TABLE 14.-AZIMUTIS OF THE SECANT.

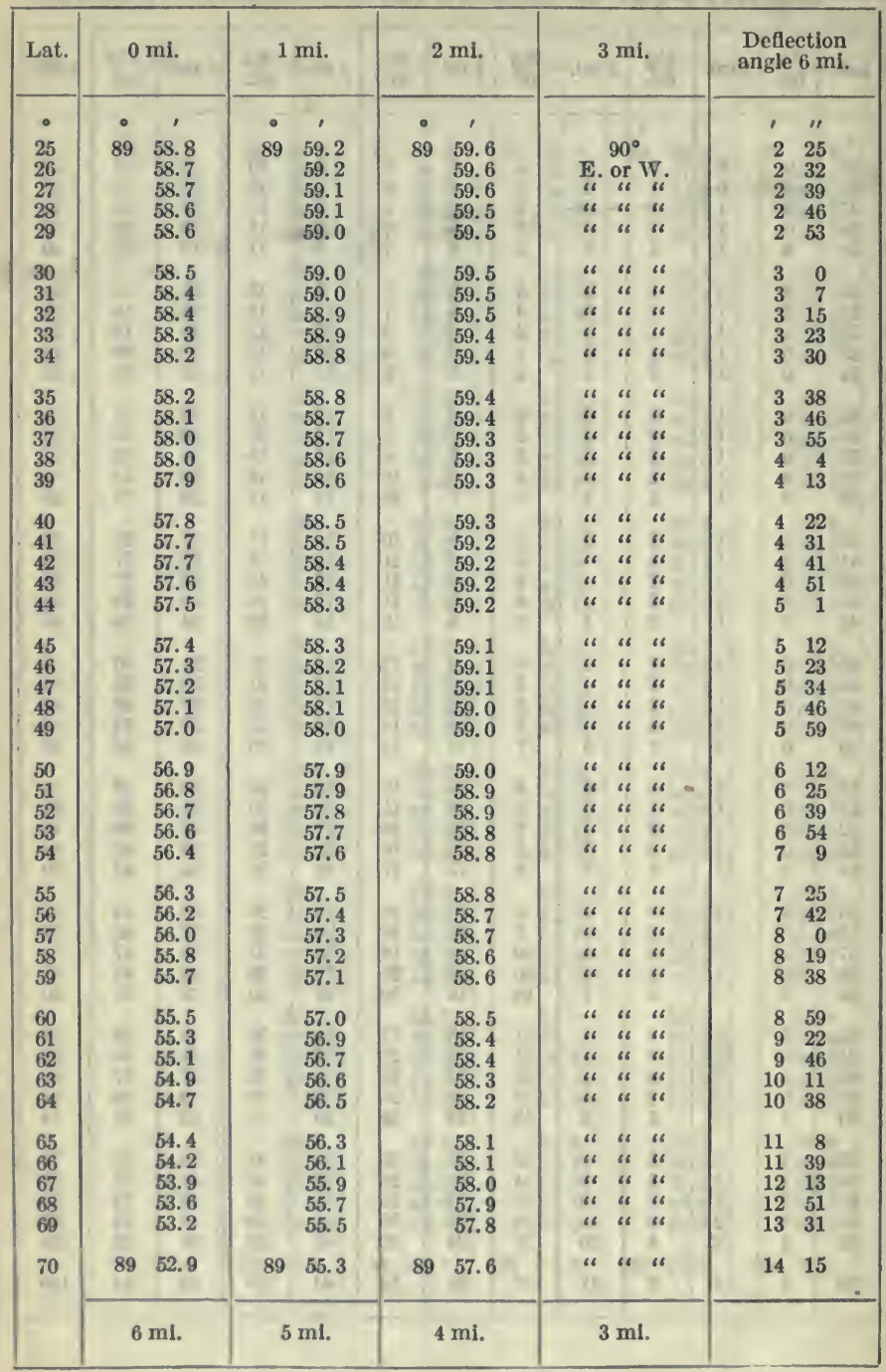


TABLE 15.-OFFSETS, IN LINKS, FIOM TUE SECANT TO THE PARALLEL.

\begin{tabular}{|c|c|c|c|c|c|c|c|}
\hline Lat. & $0 \mathrm{mi}$. & $1 / 2 \mathrm{ml}$. & $1 \mathrm{ml}$. & $11 / 2 \mathrm{ml}$ & $2 \mathrm{ml}$ & $21 / 2 \mathrm{ml}$ & $3 \mathrm{ml}$. \\
\hline - & & & 1 & & & 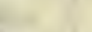 & \\
\hline $\begin{array}{l}25 \\
26 \\
27 \\
29 \\
29\end{array}$ & $\begin{array}{l}2 \mathrm{~N} . \\
2 \\
3 \\
3 \\
3\end{array}$ & $\begin{array}{l}1 \mathrm{~N} . \\
1 \\
1 \\
1 \\
1\end{array}$ & $\begin{array}{l}0 \\
0 \\
0 \\
0 \\
0\end{array}$ & $\begin{array}{l}1 \text { S. } \\
1 \\
1 \\
1 \\
1\end{array}$ & $\begin{array}{l}1 \mathrm{~S} . \\
1 \\
2 \\
2 \\
2\end{array}$ & $\begin{array}{l}2 \mathrm{~S} . \\
2 \\
2 \\
2 \\
2\end{array}$ & $\begin{array}{l}2 \mathrm{~S} . \\
2 \\
2 \\
2 \\
2\end{array}$ \\
\hline 30 & 3 & 1 & 0 & 1 & 2 & 2 & 2 \\
\hline 31 & 3 & $i$ & 0 & 1 & 2 & $\overline{2}$ & 2 \\
\hline 32 & 3 & 1 & 0 & 1 & 2 & 2 & 3 \\
\hline 33 & 3 & 1 & 0 & 1 & 2 & 2 & 3 \\
\hline 34 & 3 & 2 & 0 & 1 & 2 & 3 & 3 \\
\hline 35 & 4 & 2 & 0 & 1 & 2 & 3 & 3 \\
\hline 36 & 4 & 2 & 0 & 1 & 2 & 3 & 3 \\
\hline 37 & 4 & 2 & 0 & 1 & 2 & 3 & 3 \\
\hline 38 & 4 & 2 & 0 & 1 & 2 & 3 & 3 \\
\hline 39 & 4 & 2 & 0 & 1 & 2 & 3 & 3 \\
\hline 40 & 4 & 2 & 0 & 1 & 3 & 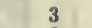 & 3 \\
\hline 41 & 4 & 2 & 0 & 2 & 3 & 3 & 4 \\
\hline 42 & 5 & 2 & 0 & 2 & 3 & 3 & w \\
\hline 43 & 5 & 2 & 0 & 2 & 3 & 4 & 4 \\
\hline 44 & 5 & 2 & 0 & 2 & 3 & 4 & 4 \\
\hline 45 & 5 & 2 & 0 & 2 & 3 & 4 & 4 \\
\hline 46 & 5 & 2 & 0 & 2 & 3 & 4 & 114 \\
\hline 47 & 5 & 2 & 0 & 2 & 3 & 4 & 4 \\
\hline 48 & 6 & 3 & 0 & 2 & 3 & 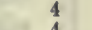 & 4 \\
\hline 49 & 6 & 3 & 0 & 2 & 3 & 4 & 5 \\
\hline 50 & 6 & 3 & 0 & 2 & 4 & 4 & 5 \\
\hline 51 & 6 & 3 & 0 & 2 & 4 & 5 & 5 \\
\hline 52 & 6 & 3 & 0 & 2 & 4 & 5 & 5 \\
\hline 53 & 7 & 3 & 0 & 2 & 4 & $\begin{array}{r}5 \\
5\end{array}$ & $\begin{array}{l}5 \\
6\end{array}$ \\
\hline 54 & 7 & 3 & o & 2 & 4 & 5 & 6 \\
\hline 55 & 7 & 3 & 0 & 3 & 4 & 5 & 6 \\
\hline 56 & 7 & 3 & 0 & 3 & 4 & 6 & 6 \\
\hline 57 & 8 & 3 & 0 & 3 & 5 & 6 & 6 \\
\hline $\begin{array}{l}58 \\
59\end{array}$ & $\begin{array}{l}8 \\
8\end{array}$ & $\begin{array}{l}4 \\
4\end{array}$ & $\begin{array}{l}0 \\
0\end{array}$ & $\begin{array}{l}3 \\
3\end{array}$ & $\begin{array}{l}5 \\
5\end{array}$ & 6 & $\begin{array}{l}6 \\
7\end{array}$ \\
\hline & & & & & & & 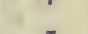 \\
\hline 60 & 9 & 4 & o & 3 & 5 & - & 7 \\
\hline 61 & 9 & 4 & 0 & 3 & 5 & 7 & 7 \\
\hline 62 & 9 & 4 & 0 & $\begin{array}{l}3 \\
3\end{array}$ & 6 & 71. 7 & $\begin{array}{l}8 \\
8\end{array}$ \\
\hline $\begin{array}{l}63 \\
64\end{array}$ & $\begin{array}{l}10 \\
10\end{array}$ & 5 & $\begin{array}{l}0 \\
0\end{array}$ & $\begin{array}{l}3 \\
4\end{array}$ & $\begin{array}{l}6 \\
6\end{array}$ & 8 & 8 \\
\hline 65 & 11 & 5 & 0 & 4 & 6 & 8 & 9 \\
\hline 66 & 11 & 5 & 0 & 4 & 7 & 8 & 9 \\
\hline 67 & 12 & 5 & 0 & 4 & 7 & 9 & 9 \\
\hline 68 & 12 & 6 & 0 & 4 & 7 & 9 & 10 \\
\hline 69 & 13 & 6 & 0 & 5 & 8 & 10 & 10 \\
\hline \multirow[t]{2}{*}{70} & $14 \mathrm{~N}$. & $6 \mathrm{~N}$. & 0 & $5 \mathrm{~S}$. & $8 \mathrm{~S}$ & $10 \mathrm{~s}$ & $11 \mathrm{~s}$ \\
\hline & $6 \mathrm{mi}$. & $51 / 2 \mathrm{ml}$. & $5 \mathrm{mi}$. & $41 / 2 \mathrm{ml}$ & $4 \mathrm{ml}$ & $31 / 2 \mathrm{ml}$. & $3 \mathrm{ml}$. \\
\hline
\end{tabular}


TABLE 16.-LENGTHS OF ARCS OF THE EARTH'S SURFACE.

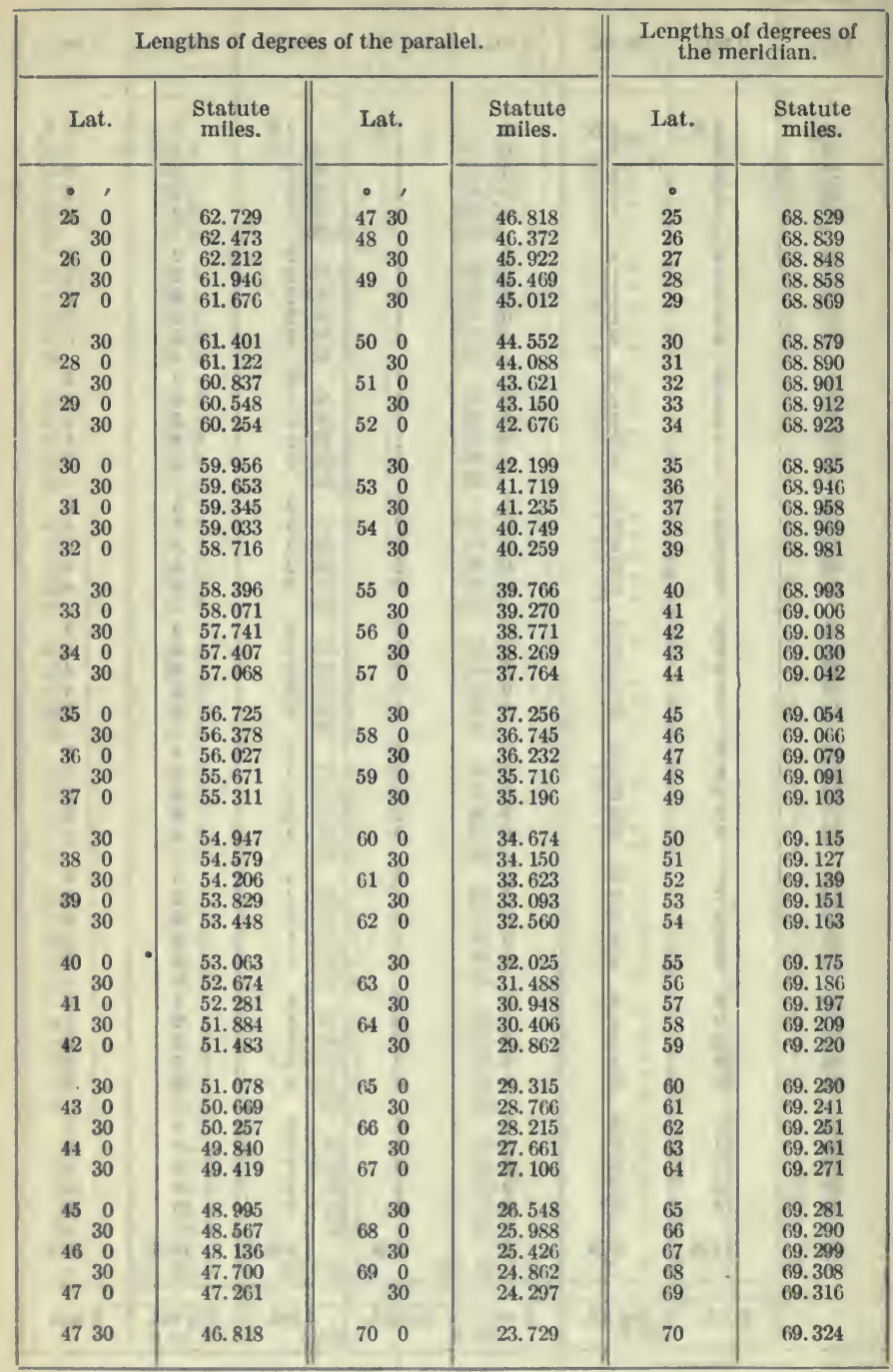

The lengths of degrees of the meridlan are tabulated to correspond to the length of the arc of which the tabulated latltude is the middle, thus the quantlty 68.933 , opposi te latltude $40^{\circ} 0^{\prime}$ is the number of mlles between latitude $39^{\circ} 30^{\prime}$ and $40^{\circ} 30^{\prime}$.

The above table is an abrldgment of a table publlshed by the U. S. Coast and Geodetic Survey based on the values of the Clarke spherold. 
TABLE 17.-APPARENT TIMF OF SUYSET FOR $N$. DECLINATIONS, OR SUNIRISE POIB S. DECLI.NATIONS.

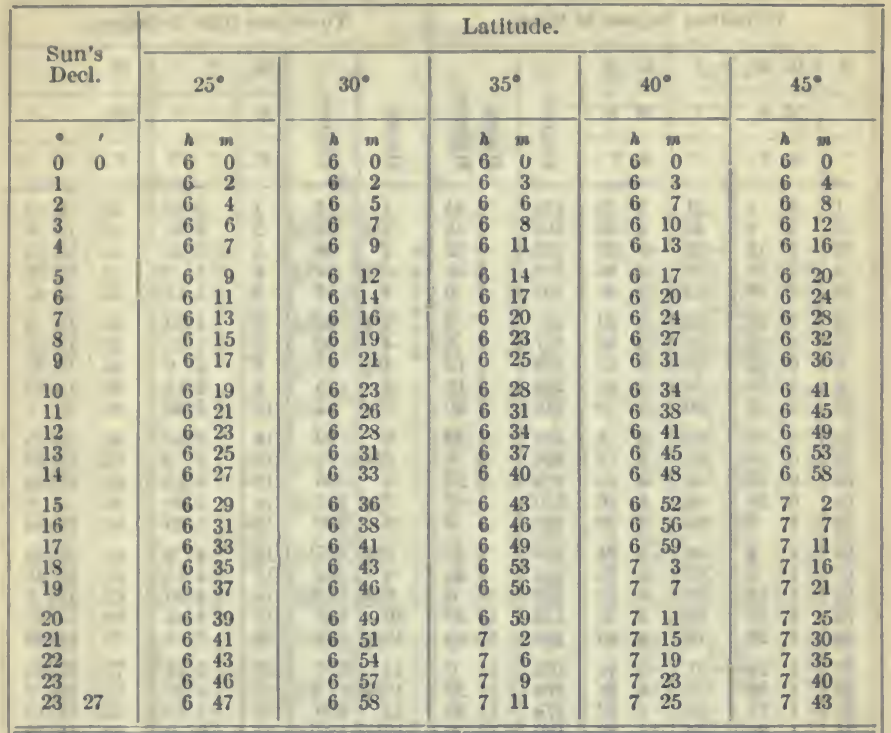

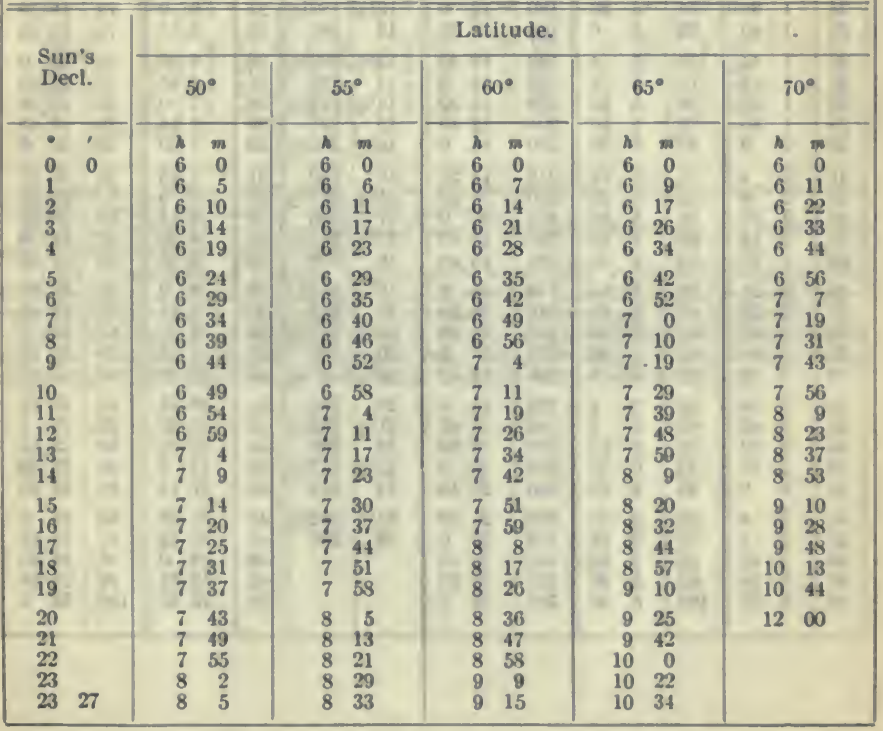




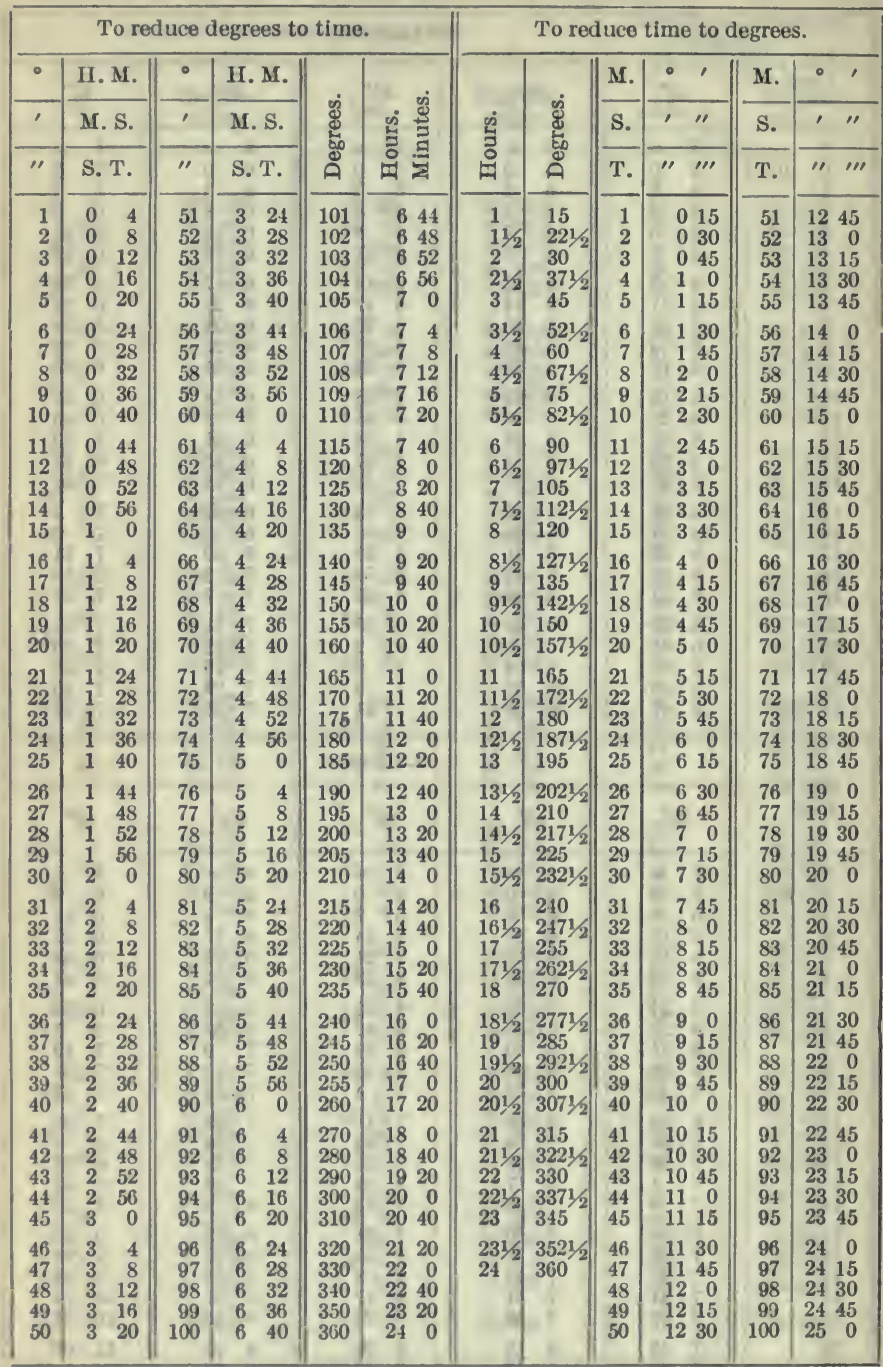


TABLE 19.-SIDEREAL CONVEISIONS.

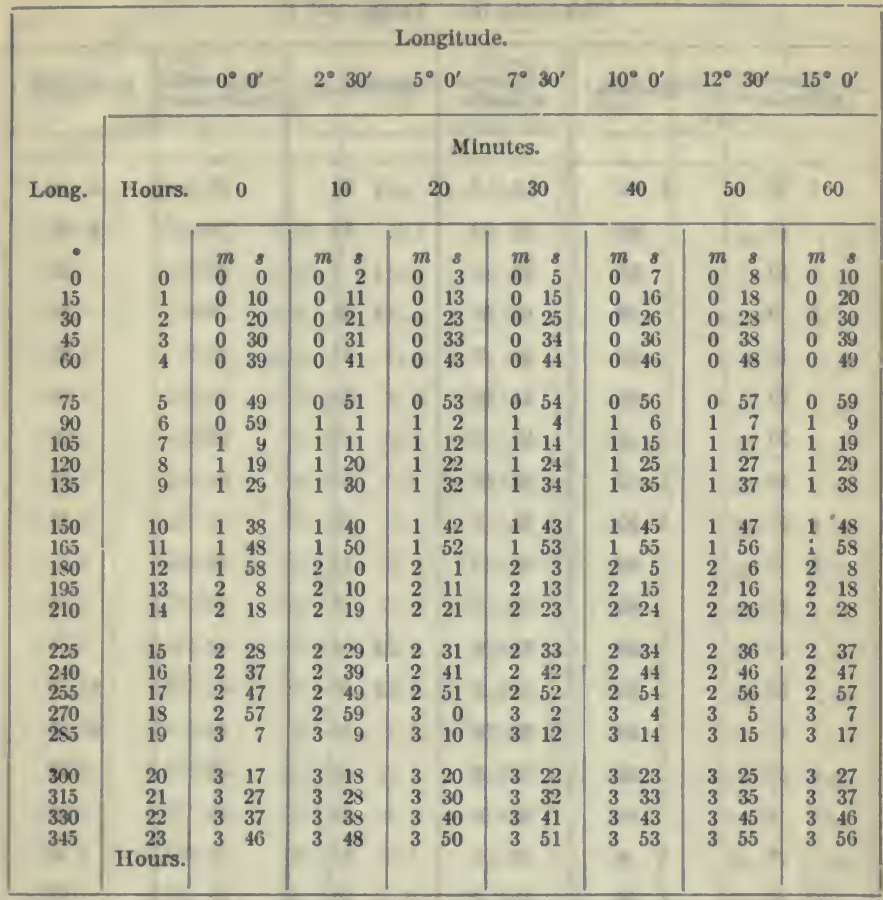

Sidereal into mean soiar time: To be subtracted from a sidereal time interval: A rqument hours and minutes of sidereal interval.

Mean Solar into sidereal time: To be added to a mean time interval: Argument hours and minutes of mean time intervai.

U pper culmination of Polaris: Amount to be subtracted from the Greenwich mean time of upper cuimination of Polaris, or of elongation, to obtain the focal mean time of upper culmination, or of elongation: Argument longitude west from Greenwich.

The above table is an abridged mean of two tables given in the American Ephem. eris and Nautical Almanac for similar conversions; reductions involving a refinement exceeding 0.8 seconds must be made from the more eiaborate tables. 
TABLE 20.-MEAN REFRACTIONS IN ZENITII DISTANCE.

Bar. $=29.6$ Ins. Temp. $=50^{\circ} \mathrm{F}$.

\begin{tabular}{|c|c|c|c|c|c|c|c|c|c|c|}
\hline \multicolumn{2}{|c|}{$\begin{array}{l}\text { Apparent } \\
\text { altitude. }\end{array}$} & \multicolumn{2}{|c|}{ Refraction. } & \multicolumn{2}{|c|}{$\begin{array}{l}\text { A pparent } \\
\text { altitude. }\end{array}$} & \multicolumn{2}{|c|}{ Refraction. } & $\begin{array}{l}\text { Apparent } \\
\text { altitude. }\end{array}$ & \multicolumn{2}{|c|}{ Refraction. } \\
\hline - & , & & & • & , & , & " & • & , & " \\
\hline 7 & 30 & & 53 & 12 & 0 & 4 & 25 & 25 & 2 & 3 \\
\hline & 40 & & 45 & 12 & 30 & & 15 & 26 & 1 & 58 \\
\hline 7 & 50 & $y$ & 37 & 13 & 0 & 4 & 5 & 27 & & 53 \\
\hline 8 & 0 & it & 30 & 13 & 30 & 3 & 56 & 28 & & 48 \\
\hline & 10 & 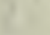 & 22 & 14 & 0 & & 47 & 29 & & 44 \\
\hline & 20 & - & 15 & 14 & 30 & & 39 & 30 & & 40 \\
\hline 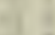 & 30 & 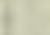 & 8 & 15 & 0 & & 32 & 32 & & 32 \\
\hline 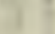 & 40 & 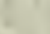 & 2 & 15 & 30 & & 25 & 34 & & 25 \\
\hline$\cdot 8$ & 50 & t & 55 & 16 & 0 & & 19 & 36 & & 19 \\
\hline 9 & 0 & 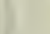 & 49 & 16 & 30 & & 13 & 38 & & 14 \\
\hline & 10 & 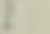 & 43 & 17 & 0 & & 7 & 40 & & 9 \\
\hline 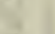 & 20 & 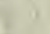 & 38 & 17 & 30 & 3 & 1 & 42 & & 4 \\
\hline & 30 & the & 32 & 18 & 0 & 2 & 56 & 44 & 1 & 0 \\
\hline 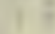 & 40 & 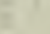 & 26 & 18 & 30 & 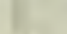 & 51 & 46 & 0 & 56 \\
\hline 9 & 50 & 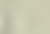 & 21 & 19 & 0 & & 46 & 48 & & 52 \\
\hline 10 & 0 & 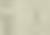 & 16 & 19 & 30 & 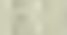 & 42 & 50 & & 48 \\
\hline & 20 & & 6 & 20 & 0 & & 37 & 55 & & 40 \\
\hline 10 & 40 & & 57 & 21 & 0 & . & 29 & 60 & & 33 \\
\hline 11 & 0 & & 48 & 22 & 0 & & 22 & 65 & & 27 \\
\hline & 20 & & 40 & 23 & 0 & & 15 & 70 & A & 21 \\
\hline 11 & 40 & & 32 & 24 & 0 & $r$ & 9 & 80 & & 10 \\
\hline 12 & 0 & in & 25 & 25 & 0 & 2 & 3 & 90 & 0 & 0 \\
\hline
\end{tabular}

SUN'S PARALLAX IN ALTITUDE.

\begin{tabular}{|l|r|r|r|r|r|r|}
\hline Apparent altitude............. & $0^{\circ}$ & $26^{\circ}$ & $48^{\circ}$ & $63^{\circ}$ & $77^{\circ}$ & $90^{\circ}$ \\
Sun's parallax ................ & $8^{\prime \prime} .9$ & $8^{\prime \prime}$ & $6^{\prime \prime}$ & $4^{\prime \prime}$ & $2^{\prime \prime}$ & $0^{\prime \prime}$ \\
\hline
\end{tabular}


TABLE 21.-COEFFICIENTS TO APPLY TO MEAN REFRACTIONS FOI VARIATIONS IX BAROMETER AND TEMPEIRATURE.

\begin{tabular}{|c|c|c|c|c|c|c|c|}
\hline $\begin{array}{c}\text { Barome- } \\
\text { ter. }\end{array}$ & $\begin{array}{l}\text { Eleva- } \\
\text { tion } \\
\text { alove } \\
\text { sea level. }\end{array}$ & $\begin{array}{l}\text { Coeffi- } \\
\text { clent. }\end{array}$ & $\begin{array}{c}\text { Barome- } \\
\text { ter. }\end{array}$ & $\begin{array}{c}\text { Eleva- } \\
\text { tion } \\
\text { above } \\
\text { sea level. }\end{array}$ & $\begin{array}{l}\text { Coefi- } \\
\text { cient. }\end{array}$ & $\begin{array}{l}\text { Temper- } \\
\text { ature } \\
\text { (Fahr.). }\end{array}$ & $\begin{array}{l}\text { Coeffi- } \\
\text { clent. }\end{array}$ \\
\hline Ins. & Feet. & & Ins. & Feet. & $x=$ & - & \\
\hline 30.5 & -451 & 1.03 & 25.4 & 4,535 & 0.86 & -24 & 1.17 \\
\hline 30. 2 & -181 & 1.02 & 25.1 & 4,859 & .85 & 17 & 1.15 \\
\hline 30.0 & 00 & 1.01 & 24.8 & 5,186 & .84 & -9 & 1.13 \\
\hline 29.9 & +91 & 1.01 & 24.5 & 5,518 & .83 & 0 & 1.11 \\
\hline 29.6 & 366 & 1.00 & 24.2 & 5,854 & .82 & +8 & 1.09 \\
\hline 29.3 & 643 & .99 & 23.9 & 6,194 & .81 & 16 & 1.07 \\
\hline 29.0 & 924 & .98 & 23.6 & 6,538 & .80 & 25 & 1.05 \\
\hline 28.7 & 1,207 & .97 & 23.3 & $6,88 \vec{\imath}$ & .79 & 35 & 1.03 \\
\hline 28.4 & 1,493 & .96 & 23.0 & 7,239 & .78 & 40 & 1.02 \\
\hline 28.1 & 1,783 & .95 & 22.7 & 7,597 & .77 & 45 & 1.01 \\
\hline 27.8 & 2,075 & .94 & 22.4 & 7,960 & .76 & 50 & 1.00 \\
\hline 27.5 & 2,371 & .93 & 22.1 & 8,327 & .75 & 55 & .99 \\
\hline 27.2 & 2,670 & .92 & 21.8 & 8,700 & .74 & 60 & .98 \\
\hline 26.9 & 2,972 & .91 & 21.5 & 9,077 & .73 & 66 & .97 \\
\hline 26.6 & 3,277 & .90 & 21.2 & 9,460 & .72 & 77 & .95 \\
\hline 26.3 & 3,556 & .89 & 20.9 & 9,848 & .71 & 88 & .93 \\
\hline 26.0 & 3,899 & .88 & 20.6 & 10,242 & .70 & 100 & .91 \\
\hline 25. 7 & 4,215 & .87 & 20.3 & 10,642 & .69 & 114 & .89 \\
\hline 25.4 & 4,535 & .86 & 20.0 & 11,047 & .68 & 128 & .87 \\
\hline
\end{tabular}

Any true refraction elther in zenith or polar distance-tabulated refraction $X$ coelficient for barometric pressure $X$ coefficient for temperature.

The differenees between the true and the tabulated refractions are generally small and generally negligible excepting for the combined effect of low apparent altitude of observation with high elevation above sea level or extremes of temperature. 

1' ERroRs.

\begin{tabular}{|c|c|c|c|c|c|c|c|}
\hline \multirow{2}{*}{ Lat. } & \multirow{2}{*}{$\begin{array}{l}7+1 \\
x+1\end{array}$} & \multicolumn{4}{|c|}{ Hours from noon. } & tran & \multirow[b]{2}{*}{6} \\
\hline & & 2 & 3 & & 4 & 5 & \\
\hline \multirow{2}{*}{25} & fDeel. & 2.21 & 1.56 & $-2+$ & 1.27 & 1.14 & 1.10 \\
\hline & Lat. & 1.91 & 1.10 & th & 0.64 & 0.30 & 0.00 \\
\hline \multirow{2}{*}{30} & Decl. & 2.31 & 1.68 & $1=$ & 1.33 & 1.20 & 1.15 \\
\hline & Lat. & 2.00 & 1.15 & $1 / 2$ & 0.67 & 0.31 & 0.00 \\
\hline \multirow{2}{*}{35} & Deel. & 2.44 & 1.73 & 6 & 1.41 & 1.26 & 1.22 \\
\hline & \{Lat. & 2.11 & 1.22 & 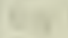 & 0.70 & 0.33 & 0.00 \\
\hline \multirow{2}{*}{40} & fDecl. & 2.61 & 1.85 & +1 & 1.51 & 1.35 & 1.31 \\
\hline & lLat. & 2.26 & 1.31 & H & 0.75 & 0.35 & 0.00 \\
\hline \multirow{2}{*}{45} & Decl. & 2.83 & 2.00 & 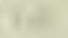 & 1.63 & 1.46 & 1.41 \\
\hline & Lat. & 2.45 & -1.41 & 11 & 0.82 & 0.38 & 0.00 \\
\hline \multirow{2}{*}{50} & fDecl. & 3.11 & 2.20 & 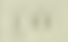 & 1.80 & 1.61 & 1.56 \\
\hline & \{Lat. & 2.69 & 1.56 & -5 & 0.90 & 0.42 & 0.00 \\
\hline \multirow{2}{*}{55} & $\int$ Decl. & 3.49 & 2.47 & 47 & 2.01 & 1.80 & 1.74 \\
\hline & I Lat. & 3.02 & 1.74 & t & 1.01 & 0.47 & 0.00 \\
\hline \multirow{2}{*}{60} & $\int$ Decl. & 4.00 & 2.83 & y & 2.31 & 2.07 & 2.00 \\
\hline & \{Lat. & 3.46 & 2.00 & $+1=$ & 1.15 & 0.54 & 0.00 \\
\hline \multirow{2}{*}{65} & $\int$ Decl. & 4.73 & 3.35 & 1 & 2.73 & 2.45 & 2. 37 \\
\hline & lLat. & 4.10 & 2.37 & in & 1.37 & 0.63 & 0.00 \\
\hline \multirow{2}{*}{70} & $\int$ Deel. & 5.85 & 4.13 & 7 & 3.38 & 3.03 & 2.92 \\
\hline & liat. & 5.06 & 2.92 & - & 1.69 & 0.78 & 0.00 \\
\hline
\end{tabular}

The eoeffieients for noon beeome infinitely large.

Direction of errors in azimuth from true south:

To the east in the a. m., to the west in the p. m.-

Latitude set or assumed to the N. of the true latitude, noon to six hours.

Latitude set or assumed to the $\mathrm{S}$. of the true latitude, beyond six hours.

Declination set or assumed to the $\mathrm{S}$. of the true deelination, all hours.

To the west in the a. m., to the east in the p. m. -

Latitude set or assumed to the $\mathbf{S}$. of the true latitude, noon to six hours.

Latitude set or assumed to the $\mathrm{N}$. of the true latitude, beyond six hours.

Declination set or assumed to the $\mathrm{N}$. of the true declination, all hours.

The error is considered as the erroneous fixation of the south point. 
TABLE 23.-MEAN REFRACTIONS IN POLAR DISTANCE.

Bar. $=29.6$ ins. Temp. $=50^{\circ} \mathrm{F}$.

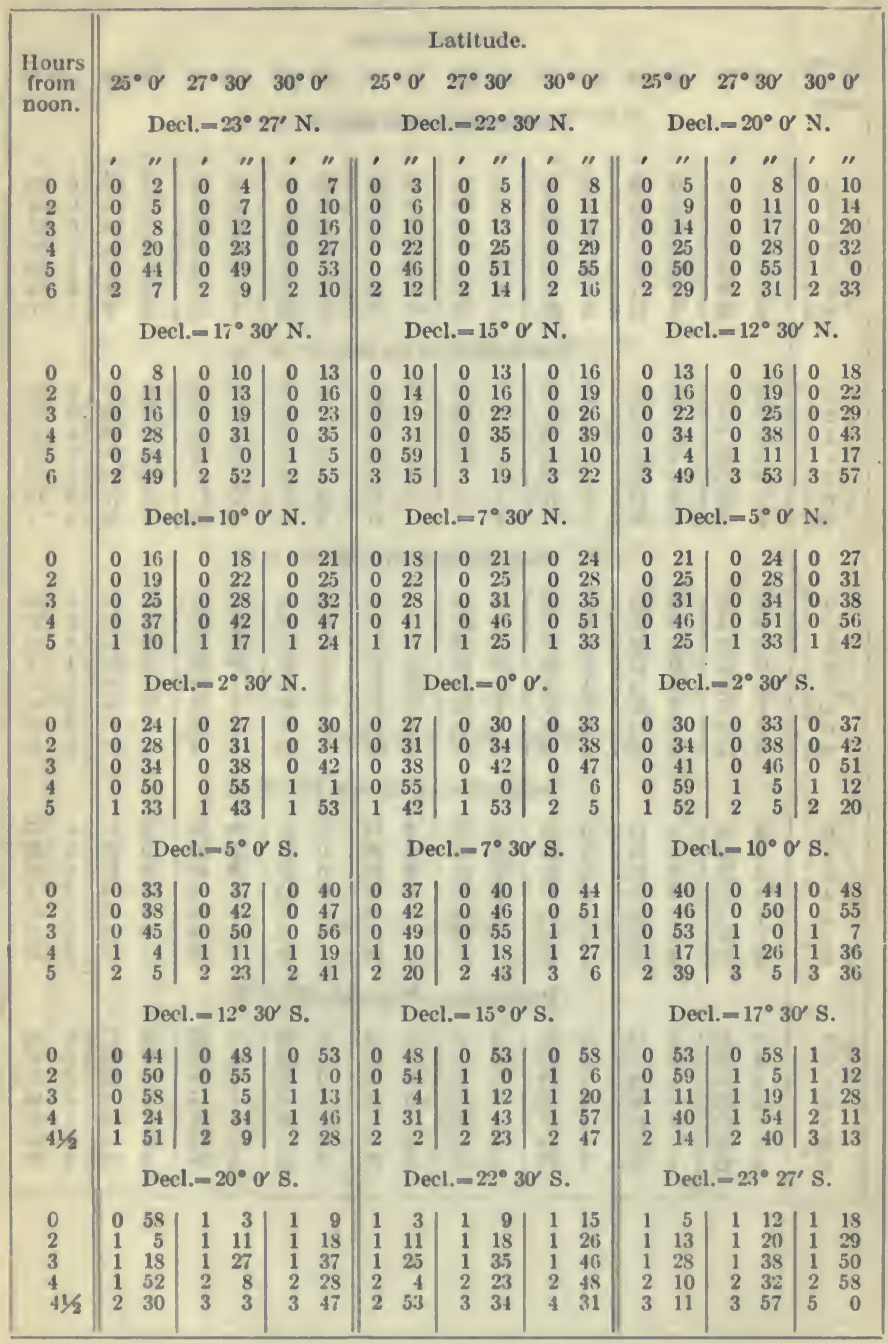


Bar. $=29.6$ ins. Temp. $=50^{\circ} \mathrm{F}$.

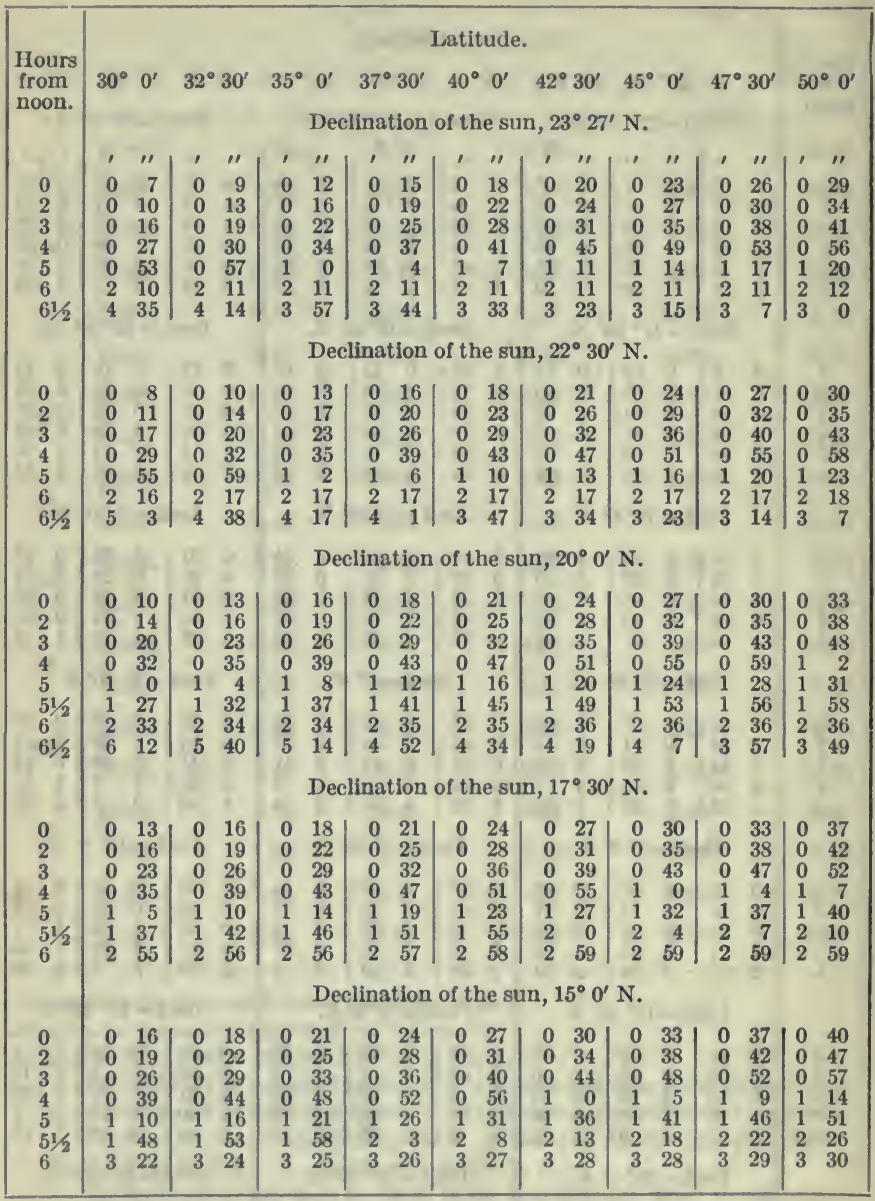


Bar. $=29.6$ ins Temp. $=50^{\circ} \mathrm{F}$.

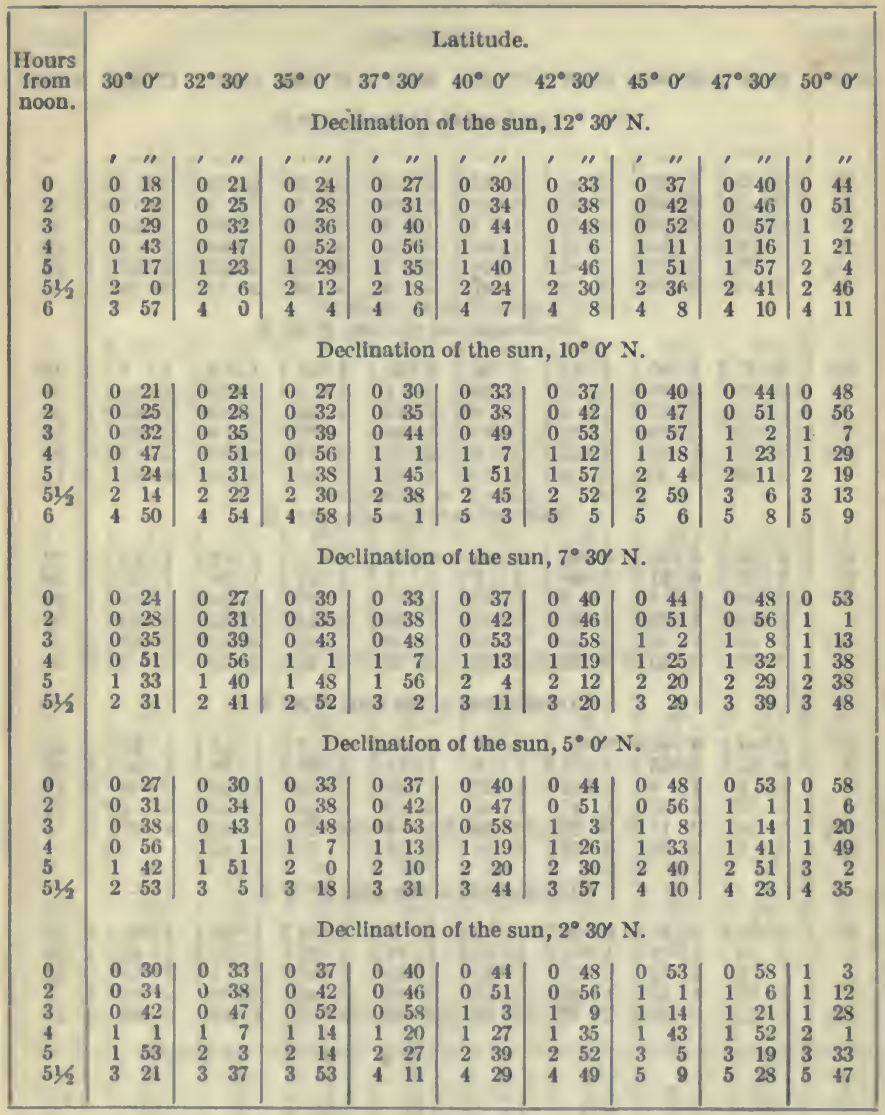


TABLE 23. MEAN REFRICTIONS IN POLAR DISTANCE.

Bar. $=29.6$ ins. Temp. $=50^{\circ} \mathrm{F}$.

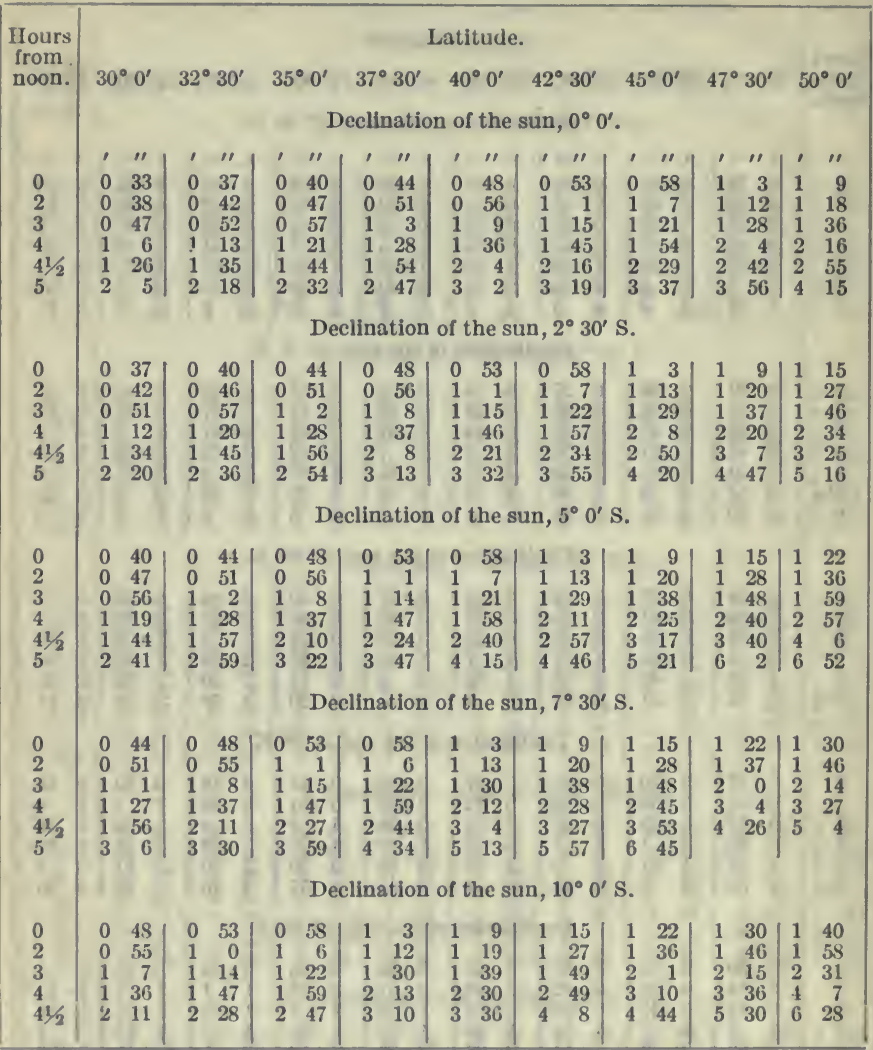


TABLE 23.-MEAN REFRACTIONS IN POIAR DISTANCE.

Bar. -29.6 ins. Temp. $-50^{\circ} \mathrm{F}$.

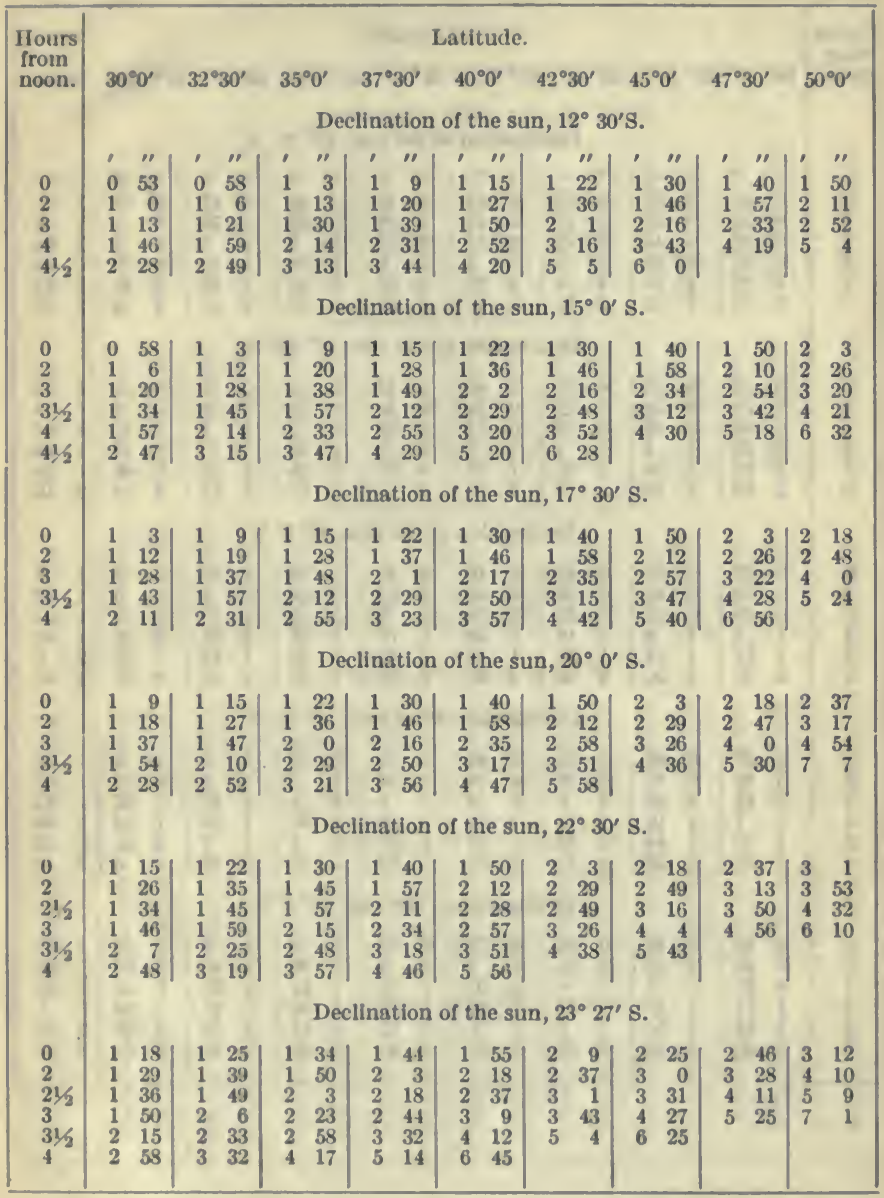


TABLE 23.-MEAN REFRACTIONS IN POLAR DISTANCE.

Bar. $=29.6$ ins. Temp. $=50^{\circ} \mathrm{F}$.

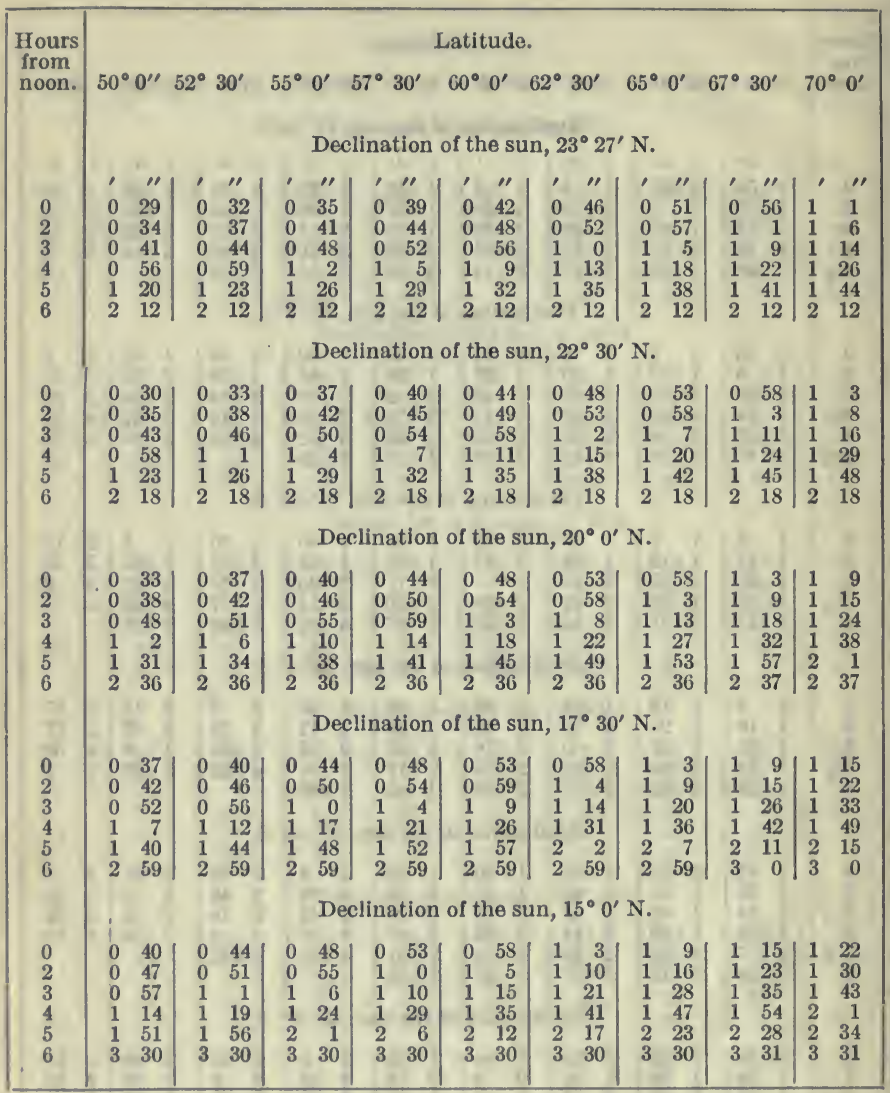


TABLE 23.-MEAN REFRICTIONS IN POLAR DISTANCE.

Bar. $=29.6$ ins. Temp. $=50^{\circ} \mathrm{F}$.

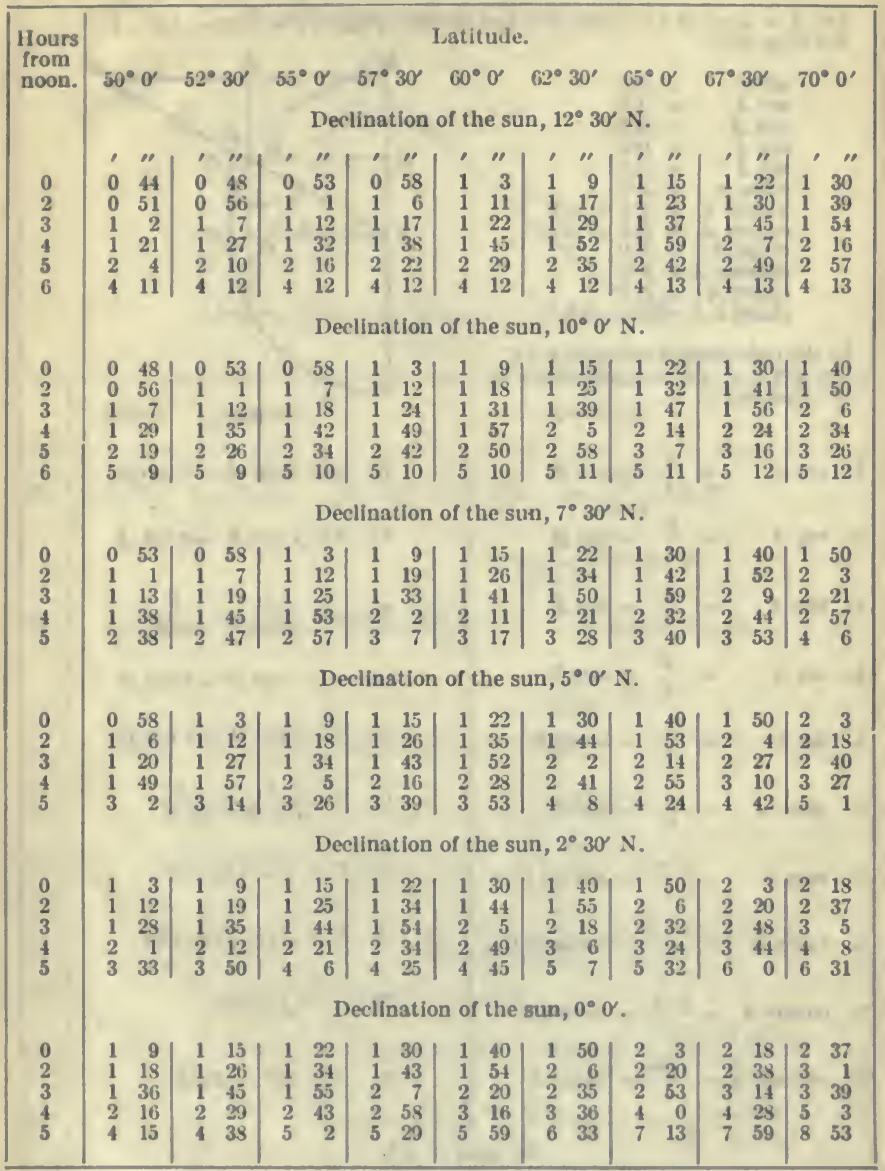


Let $A=$ angle $B A C=\operatorname{arc} B F$, and let the radius $A F=A B=\Lambda H=1$. We then have

$\begin{array}{ll}\sin A & =B C \\ \cos A & =A C \\ \tan A & =D F \\ \cot A & =H G \\ \sec A & =A D \\ \operatorname{cosec} A & =A G \\ \text { versin } A & =C F=B E \\ \operatorname{covers} A & =B K=H L \\ \text { exsec } A & =B D \\ \operatorname{coexsec} A & =B G \\ \operatorname{chord} A & =B F \\ \text { chord } 2 A & =B I=2 B C\end{array}$

In the right-angled triangle $-A B C$ Let $A B=c, A C=b$, and $B C=a$. We then have:

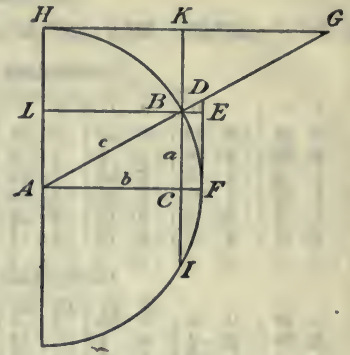

FIG 1.
1. $\sin A=\frac{a}{c}=\cos B$

2. $\cos A=\frac{b}{c}=\sin B$

3. $\tan A=\frac{a}{b}=\cot B$

4. $\cot A=\frac{b}{a}=\tan B$

5. $\sec A=\frac{c}{b}-\operatorname{cosec} B$

6. $\operatorname{cosec} A=\frac{c}{a}=\sec B$.

7. $\operatorname{vers} A=\frac{c-b}{c}=\operatorname{covers} B$

8. $\operatorname{exsec} A=\frac{c-b}{b}=\operatorname{cosexsec} B$

9. $\operatorname{covers} A=\frac{c-a}{c}=\operatorname{versin} B$

10. $\operatorname{coexsec} A=\frac{c-a}{a}=\operatorname{exsec} B$
11. $a=c \sin A=b \tan A$

12. $b=c \cos A=a \cot A$

13. $c=\frac{a}{\sin A}=\frac{b}{\cos A}$.

14. $a=c \cos B=b \cot B$

15. $b=c \sin B=a \tan B$

16. $c=\frac{a}{\cos B}=\frac{b}{\sin B}$

17. $a=\sqrt{(c+b)(c-b)}$

18. $b=\sqrt{(c+a)(c-a)}$

19. $c=\sqrt{a^{2}+b^{2}}$

20. $C=90^{\circ}-A+B$

$$
\text { 21. } \operatorname{area}=\frac{a b}{2}
$$




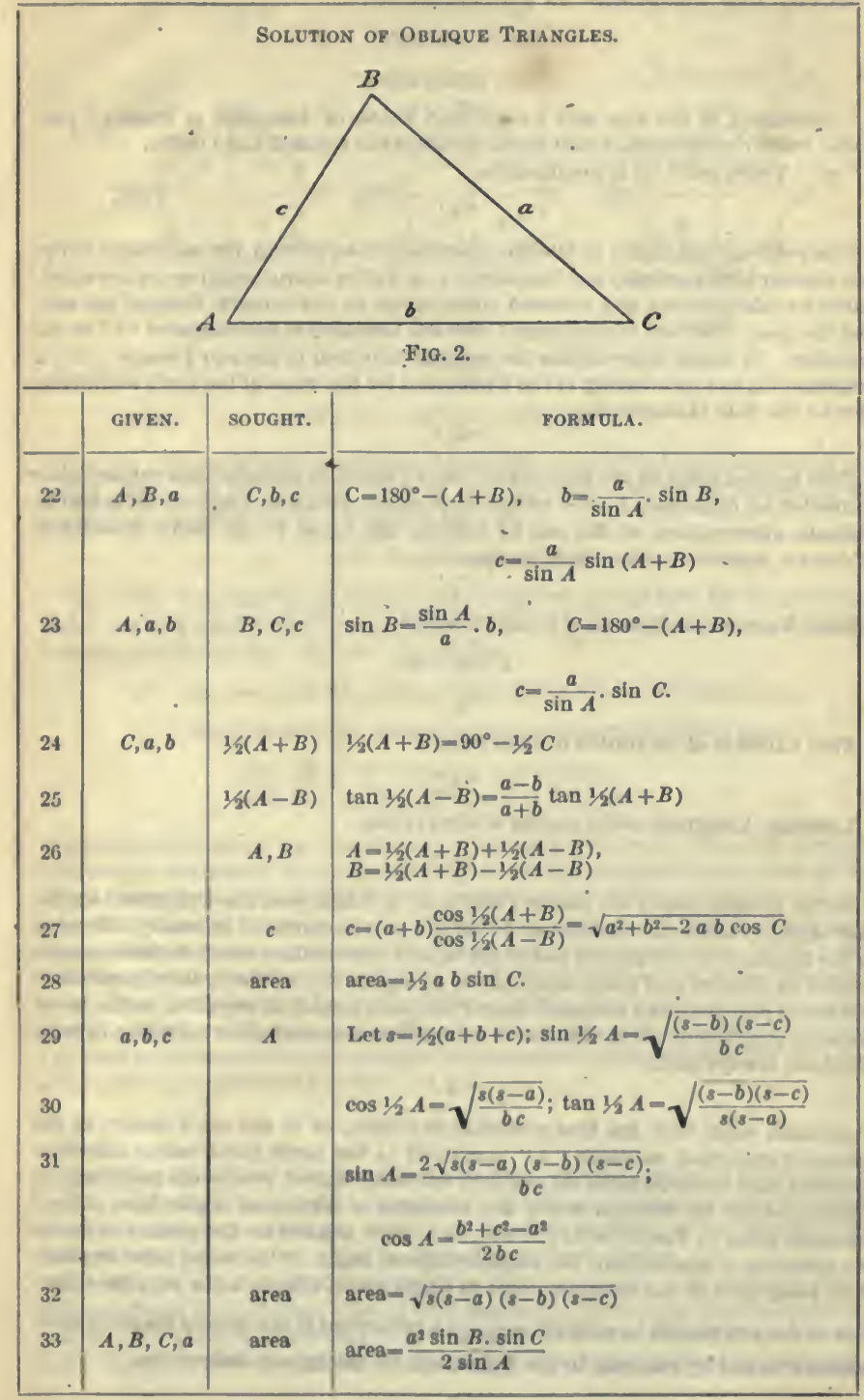



MUTH, AND PROBLEMS IN CONVERGENCY.

\title{
NOTATION.
}

\author{
"THE EPHEMERIS."
}

"Ephemeris of the Sun and Polaris, and Tables of Azimuths of Polaris," pre. pared under the direction of the Commissioner of the General Land Office.

"fl": The symbol for approximation.

$$
\text { " } v . "
$$

Observed vertical angle; in altitude observations on the sun, the reductions to the sun's center both vertically and horizontally, as well as instrumental errors, are eliminated by taking direct and reversed observations on the opposite limbs of the sun, and the mean observed vertical angle to the sun's center is to be considered " $v$ " in the notation. In single observations the vertical reduction to the sun's center $=16^{\prime}$; a refinement is had by referring to the Ephemeris for the value of the sun's semidiameter for the date of observation.

$$
\text { " } h . "
$$

True vertical angle to the sun's center, or to Polaris, in altitude observations, after correction for refraction: $h=v$ - refraction in zenith distance; a refinement is had in altitude observations on the sun by adding the value of the sun's parallax = $8 " .9 \cos \mathrm{v}$, opposite in effect to refraction.

$$
\text { "६." }
$$

Zeta: True zenith distance of the sun's center.

$$
\begin{gathered}
\zeta=90^{\circ}-h . \\
\text { " } \phi . "
\end{gathered}
$$

Phi: Latitude of the station of observation.

$$
\text { " } \lambda . "
$$

Lambda: Longitude of the station of observation.

$$
\text { "g." }
$$

Delta: Declination of the sun or Polaris; to be taken from the Ephemeris for the date of observation; the declination of the sun is to be corrected in hourly difference to the longitude of the station and to the time of observation: north declinations are treated as positive and south declinations as negative; a northerly hourly motion is treated as positive and a southerly hourly motion is treated as negative; in the use of the solar attach ment the declination of the sun is to becorrected for refraction in polar distance, always north.

$$
\text { "A." }
$$

Azimuth angle from the true meridian to Polaris, or to the sun's center; in the following analytical solutions " $\Lambda$ " is referred to the north point unless otherwise modifed, and the reductions are symmetrical either east or west of the meridian; all determinations for azimuth imply the recording of horizontal angles from a fixed reference point to Polaris or to the sun, or a point marked on the ground to define the direction of observation, the mean horizontal angle, or the mean point in direction, being used in the determination; in single observations on the sun, the reduction to the sun's center in azimuth $=\frac{16^{\prime}}{\cos \nabla^{2}}$; a refinement in the value of the sun's semidiameter is had by referring to the Ephemeris for the date of observation. 


\section{Sines}

Cosines

Tangents
and

Cotangents
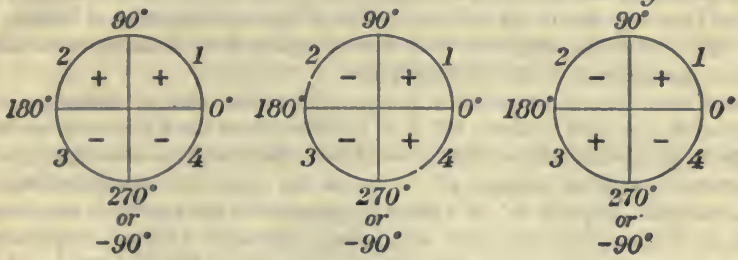

Fig. 3.

STADIA MEASUREMENTS.

Hor. dis.: The true horizontal distance from the center of the instrument to the rod.

Difr. elev.: The true vertical distance from the height of the instrument to the center point between the two targets of the rod.

" $r$ ": Vertical rod reading.

" $K$ ": The wire interval or ratio, to be determined in the field by frequent tests under working conditions in comparison with steel tape measurement, solving the formula given beiow for " $\mathrm{K}$," hor. dis. known.

" $c$ ": Distance from the center of the instrument to the object glass.

"f": Distance from the plane of the cross-wires to the object glass.

Hor. dis. $=\mathrm{K} r \cos ^{2} v+(c+f) \cos v$.

Diff. elev. $=\mathrm{K} r \frac{1}{2} \sin 2 v+(c+f) \sin v$.

In public land surveying it is convenient to have fixed stadia wires with a ratio of 1:132 so that the sum of two rod readings in feet will be equivaient to a ratio of 1:66, or a reduced distance in chains; it is also convenient to reduce the error in the wire Interval to the error in 10 chs., and to eliminate the error by appiying to the reduced distance the proper amount taken from the table of proportional parts. With a ratio of 1:100, using a rod graduated to links, the elimination of the error in the wire inter$\mathrm{val}$ is conveniently made in the same manner. With a ratio of $1: 100$, using a rod graduated to feet, the reduction is simplified by determining the logarithm of the true " $\mathrm{K}$," rod in feet and horizontal distance in chains units, accomplishing the reduction of $\mathrm{K} r \cos v$ by logarithmic functions.

TIME.

Concersion of atandard time into local mean time. - Watch reading \pm watch error in standard time by comparison \pm correction for iongitude; the correction for longitude is additive east and subtractive west of the standard meridian of the time belt; the conversion tabie "degrees to time" is convenient in this reduction.

Conecrsion of apparent lime inlo local mean time. $-\mathbf{A}$ pparent time of observation \pm the equation of time; the equation of time is to be taken from the Ephemeris for the date of observation and corrected for the longitude and time of observation, conveniently interpolated as the interval from Greenwich noon to the time of observation; 
the watch error in local mean time is then found by taking the difference between the watch reading at the epoch of the observation and the reduced local mean time of observation.

Local mean time of upper culmination of Polaris.-The Greenwich mean time of upper culmination of Polaris is to be taken from the Ephemeris for the date of observation; the amount to be subtracted from the Greenwich mean time of upper culmination of Polaris to obtain the local mean time of upper culmination of Polaris, in which the argument is the longitude west from Greenwich, is obtained from the table of sidereal conversions without computation.

Local mcan time of elongation of Polaris.-The mean time of elongation of Polaris, Greenwich meridian, latitude $40^{\circ}$, is to be taken from the Ephemeris for the date of observation; the amount to be subtracted from the mean time of elongation of Polaris, Greenwich meridian, latitude $40^{\circ}$, to obtain the mean time of elongation of Polaris, local meridian, latitude $40^{\circ}$, in which the argument is the longitude west from Greenwich, is obtained from the table of sidereal conversious without computation. The amount to apply to the local mean time of elongation of Polaris latitude $40^{\circ}$ to obtain the local mean time of elongation of Polaris latitude of observation is tabulated in the Ephemeris in connection with the table of azimuths of Polaris at elongation.

Conversion of a mean time interval into a sidereal time interval, or vice versa.-The amount to apply to one time interval to obtain the other time interval is obtained from the table of sidereal conversions without computation.

Hour angles of Polaris. $-\Lambda$ mean time hour angle of Polaris west of the meridian is the mean time interval from the local mean time of upperculmination of Polaris to the local mean time of observation of Polaris; a mean time hour angle of Polaris east of the meridian is the mean time interval from the local mean time of observation of Polaris to the local mean time of upper culmination of Polaris.

Mean time hour angle of Polaris at elongation.- " $t$ " $=$ the sidereal hour angle in angular measure, this converted into time measure, and this in turn converted from a sidereal time interval into a mean time interval gives the mean time hour angle of Polaris at elongation:

$\operatorname{Cos} t=\operatorname{cotan} \delta \tan \phi$

Altitude observation of the sun for apparent time.- " $t$ "=hour angle from apparent noon in angular measure; reverse the signs of " $\delta$ " for south declinations:

$$
\operatorname{Tan} \frac{1}{2} t=\sqrt{\frac{\sin \frac{1}{2}(\zeta+\phi-\delta) \sin \frac{1}{2}(\zeta-\phi+\delta)}{\cos \frac{1}{2}(\zeta+\phi+\delta) \cos \frac{1}{2}(\zeta-\phi-\delta)}}
$$

Mcridian observation of the sun for apparent noon.-With the telescope in the meridian elevated to the sun's altitude, the watch times of transit of the sun's west and east limbs are noted, the mean of which is the watch time of apparent noon; if the observation fails for either limb the reduction to the sun's center is accomplished by adding or subtracting 68 seconds; a refinement in the amount of this time is had by referring to the Ephemeris for the time of the sun's semidiameter passing the meridian for the date of observation; the setting for the approximate altitude of the sun's center is:

$$
\mathrm{V} \neq 90^{\circ}-\phi \pm \delta
$$

LATITUDE.

Meridian altitude observation of the sun for latitude.-Reverse the sign of "g" for south declinations:

$$
\phi=90+8-h
$$

This observation is conveniently combined with the meridian observation of the sun for time, observing simultaneously the sun's lower and west limbs, recording the watch time and the vertical angle and reversing in the interval of about 2$\}$ min- 
utes, and observing simultaneously the sun's upper and east limbs; the sottings for the approximate aititudes of the sun's lower and upper limbs, respectively, are:

$$
\mathrm{v} \neq 180^{\circ}-\phi \pm \delta \mp 16^{\circ}
$$

Altitude observation of Polaris at upper culmination for latitude.-

$$
\phi=h+\delta-90^{\circ}
$$

Altitude observation of Polaris at lovcr culmination for latitude. - The mean time hour angle of Polaris at lower cuimination is 11 hours 58 minutes 2 seconds:

$$
\phi=h+90^{\circ}-\partial
$$

The settings for the approximate altitude of Poiaris at upper and lower culminations, respectively, are:

$$
v \neq \phi \pm\left(90^{\circ}-8\right)
$$

\section{AZIMUTI.}

Altitude observation of the sun for azimuth.-Reverse the signs of "g" for south deciinations: .

$$
\operatorname{Tan} 1 / 2 A=\sqrt{\frac{\cos 1 / 2(\zeta+\phi+\delta) \sin 1 / 2(\zeta+\phi-\delta)}{\cos 1 / 2(\zeta-\phi-\delta) \sin 1 / 2(\zeta-\phi+\delta)}}
$$

The spherical angles " $\zeta$," " $\phi$," and " $g$ " appear in this equation combined as in the formula for the reduction of an altitude observation of the sun for apparent time, and when it is desired to reduce for both time and azimuth, the above equation for azimuth is to be preferred to any that foliow.

Altitude observation of the sun for azimuth.-For south declinations the function "sin 8 " becomes negative by virtue of the sine of a negative angle being treated as negative in analytical reductions: If the algebraic sign of the resuit is positive the azimuth " $\mathrm{A}$ " is referred to the north point, but if negative, the azimuth " $\mathrm{A}$ " is referred to the south point:

$$
\cos A=\frac{\sin \partial}{\cos \phi \cos h}-\tan \phi \tan h
$$

The above equat'on is very con renient in reducing for azimuth oniye

Altitude obscrvation of the sun for azimith.-To many surveyors the following equation is familiariy expressed directly in terms of the sphericai triangle "pole-zenithsun": reverse the sign of "g" for south deciinations:

$$
\begin{aligned}
& \text { Poie to zenith }=90^{\circ}-\phi-\text {-olat.; } \\
& \text { Poie to sun }=90^{\circ}-\delta \text {-codecl.; } \\
& \text { Zenith to sun }=90^{\circ}-h=\text { coalt.; } \\
& \mathrm{S}=1 / 2 \text { sum of the three sides: } \\
& \text { Cos } 1 / 2 \Lambda=\sqrt{\frac{\sin S \sin (S-\text { codeci.) }}{\sin \text { colat. sin coalt. }}}
\end{aligned}
$$

Equal altitude observations of the sun for meridian.-The sun's center at equal alttudes occupies symmetrical positions in azimuth east and west of the mcridian in the morning and in the afternoon except for the correction necessary to be applied due to the change in the sun's declination in the interval between the a. m. and p. m. observations:

" $d \mathrm{~A}_{\jmath}$ ": Correction in azimuth in minutes of angular measure to be applied to the mean position in aximuth to obtain the true south point; the correction is to be appilied to the east with a northeriy hourly change in declination, or to the west with a southerly hourly change.

" $d \delta "$ : Change in declination of the sun from the a. $m$. to the p. m. observation, expressed In minutes of angular measure. 
" $\left(t_{1}+t_{2}\right)$ ": The sum of the hour angles from apparent noon, or the total watch time from the a. m. to the p. m. observation, expressed in angular measure.

$$
d \mathrm{~A}_{\delta}=\frac{1 / 2 d \delta}{\cos \phi \sin 1 / 2\left(t_{1}+t_{2}\right)}
$$

The symmetry of the equal altitude observation is prescrved by observing opposite limbs in azlmuth in the a. $\mathrm{m}$. and $\mathrm{p} . \mathrm{m}$. observations, in connection with the same limb in vertical angle in both observations.

With " $1 / 2 d \delta$ " and " $1 / 2\left(t_{1}+t_{2}\right)$ " calculated, the computation can be concluded by applying to " $1 / 2 d \delta$ " the declination coeflicient obtained by entering the table of coeflicients for computing errors in azimuth due to small errors in declination, arguments: " $\phi$ " and " $1 / 2\left(t_{1}+t_{2}\right)$."

Azimuth of Polaris at elongation.-

$$
\operatorname{Sin} A=\frac{\cos \delta}{\cos \phi}
$$

A table of azimuths of Polaris at elongation, for latitudes from $25^{\circ}$ to $70^{\circ} \mathrm{N}$., appears in the Ephemeris, arguments: " $\gamma$ " and " $\phi . "$

A zimuth of Polaris at any hour angle.- " $t$ " = sidereal hour angle in angular measure; in hour angles exceeding $90^{\circ}$ the function " $-\sin \phi \cos t$ " becomes positive by virtue of the cosine of an angle between $90^{\circ}$ and $270^{\circ}$ being treated as negatlve in analytical reductions:

$$
\operatorname{Tan} A=\frac{\sin t}{\cos \phi \tan \delta-\sin \phi \cos t}
$$

A table of azimuths of Polaris at all hour angles, for latitudes from $30^{\circ}$ to $50^{\circ} \mathrm{N}$., appears in the Ephcmeris, arguments: " $\delta$," mean time hour angle, and " $\phi . "$

For other than the above latitudes the surveyor will be required to perform the above analytical solution, accomplished by the following process: convert the usual mean time hour angle into sidereal hour angle, and convert the sidereal hour angle into angular measure to obtain " $t$ " for the above equation.

Polaris at sunset or sunrise.-Polaris is conveniently observed for azimuth by the hour angle method at sunset or sunrise without artificial illumination; the preparation for the observation consists in computing in advance the approximate settings in azlmuth and altitude in order to find Polaris, and the plan contemplates an approxlmate reference meridian: with the time of sunset or sunrise assumed as the time of observation, the hour angle " $t$ " and azimuth " $A$ " are determined in order to find the position of Polaris in azimuth; the position in altitude is found by the following approximation, the positive sign being used for hour angles less than 6 hours, and the negatlve sign for hour angles exceeding 6 hours:

$$
v \neq \phi \pm 70^{\prime} \cos t
$$

\section{CONVERGENCY OF MERIDIANS.}

" $m_{\lambda}$ ": Measurement along the parallel.

" $m_{\phi}$ ": Measurement along the meridian.

" $a$ ": Equatorial radlus of the earth $=3963.3$ miles $=80 \times 3963.3$ chains.

" $e$ ": Factor of eccentrlcity, $\log e=8.9152515$.

" $d m_{\lambda}$ ": Linear amount of the convergency on the parallel, of two meridians dlstance apart " $m_{\lambda}$," and distance " $m_{\phi}$ " along the meridian: " $d m_{\lambda}$,". " $m_{\lambda}$," " $m_{\phi}$ " and " $a$ " to be expressed in the same unit:

$$
d m_{\lambda}=\frac{m_{\lambda} m_{\phi}}{a} \tan \phi \sqrt{1-e^{2} \sin ^{2} \phi}
$$





$$
\begin{aligned}
& \sim
\end{aligned}
$$

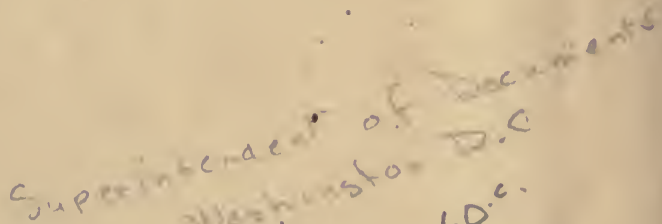

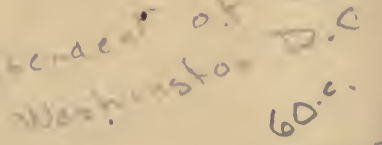

$$
\begin{aligned}
& \begin{array}{l}
60^{\circ} \\
\text { Price }
\end{array}
\end{aligned}
$$




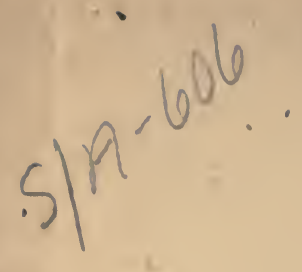

\section{PLEASE DO NOT REMOVE}

CARDS OR SLIPS FROM THIS POCKET

\section{UNIVERSITY OF TORONTO LIBRARY}

S\&M

A

606 
h 